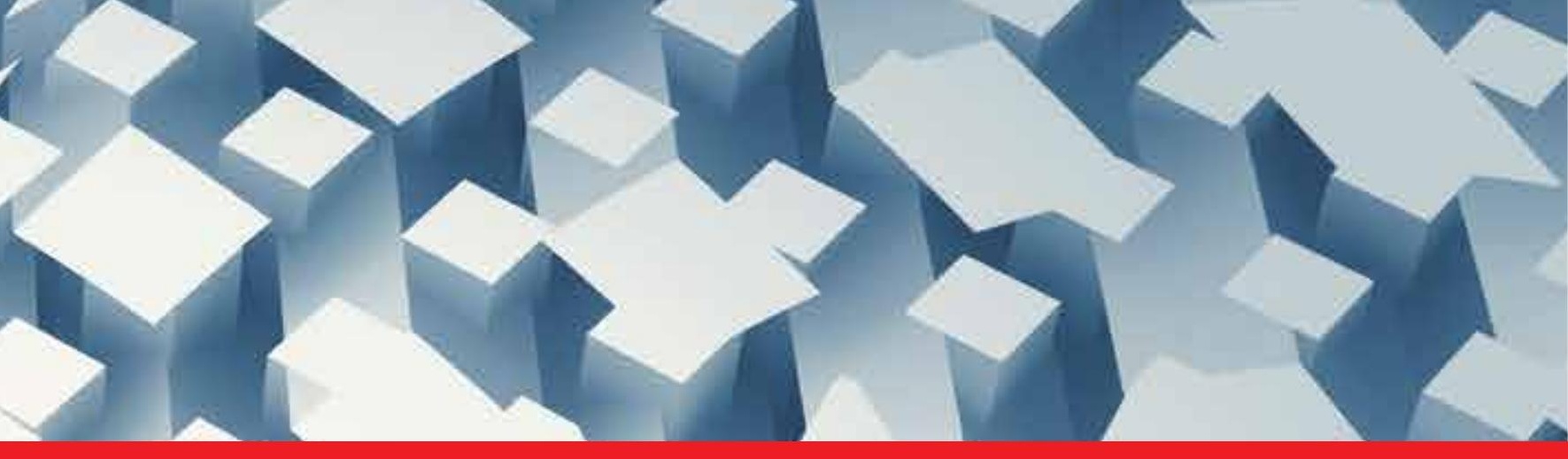

IntechOpen

\title{
Smart Nanoparticles Technology
}

Edited by Abbass Hashim

P

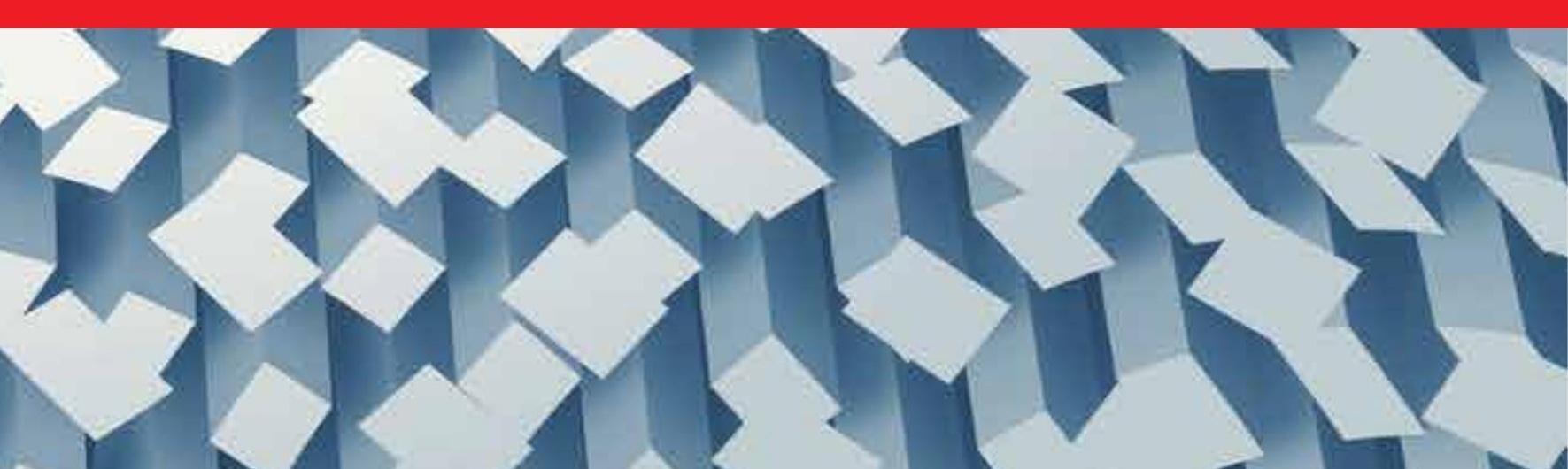





\section{SMART NANOPARTICLES TECHNOLOGY}

Edited by Abbass A. Hashim 


\section{Contributors}

Wei Yu, Udit Surya Mohanty, Kwang Lung Lin, S.Y. Chen, Margarita Sanchez-Dominguez, Carolina Aubery, Conxita Solans, Jelena Tamuliene, Rimas Vaisnoras, Mindaugas Leonas Balevicius, Gonçal Badenes, Miroslaw Dudek, Xuchuan Jiang, Sebastian Mackowski, Akihiko Matsuyama, Venkatathri Narayanan, Domenico Acierno, Giovanni Filippone, Shawn Y. Wu, Sergey Filippov, Jiri Panek, Petr Stepanek, Fei Duan, Hassan Ebadi-Dehaghani, Mehra (Monireh) Mehra Nazempour, Kazutaka Hirakawa, Xinjun Xu, Hon-Man Liu, Jong-Kai Hsiao, Nickolay Kononov, Sergey Dorofeev, Dr. Orhan Yalçın, Mitsunori Yada, Yuko Inoue, Motonari Adachi, Keizo Nakagawa, Yusuke Murata, Masahiro Kishida, Masahiko Hiro, Kenzo Suswa, Fumio Uchida, Jun Adachi, Peter Siffalovic, Eva Majkova, Matej Jergel, Stefan Luby, Martin Weis, Karol Vegso, Hidemi Shigekawa, Shoji Yoshida, Osamu Takeuchi, Gennady Nikolaev, Fernando Moreno, Braulio García-Cámara, Raquel Gómez-Medina, Manuel Nieto-Vesperinas, Juan Jose Saenz, Francisco Gonzalez

\section{(c) The Editor(s) and the Author(s) 2012}

The moral rights of the and the author(s) have been asserted.

All rights to the book as a whole are reserved by INTECH. The book as a whole (compilation) cannot be reproduced, distributed or used for commercial or non-commercial purposes without INTECH's written permission.

Enquiries concerning the use of the book should be directed to INTECH rights and permissions department (permissions@intechopen.com).

Violations are liable to prosecution under the governing Copyright Law.

\section{(c)) BY}

Individual chapters of this publication are distributed under the terms of the Creative Commons Attribution 3.0 Unported License which permits commercial use, distribution and reproduction of the individual chapters, provided the original author(s) and source publication are appropriately acknowledged. If so indicated, certain images may not be included under the Creative Commons license. In such cases users will need to obtain permission from the license holder to reproduce the material. More details and guidelines concerning content reuse and adaptation can be foundat http://www.intechopen.com/copyright-policy.html.

\section{Notice}

Statements and opinions expressed in the chapters are these of the individual contributors and not necessarily those of the editors or publisher. No responsibility is accepted for the accuracy of information contained in the published chapters. The publisher assumes no responsibility for any damage or injury to persons or property arising out of the use of any materials, instructions, methods or ideas contained in the book.

First published in Croatia, 2012 by INTECH d.o.o.

eBook (PDF) Published by IN TECH d.o.o.

Place and year of publication of eBook (PDF): Rijeka, 2019.

IntechOpen is the global imprint of IN TECH d.o.o.

Printed in Croatia

Legal deposit, Croatia: National and University Library in Zagreb

Additional hard and PDF copies can be obtained from orders@intechopen.com

Smart Nanoparticles Technology

Edited by Abbass Hashim

p. cm.

ISBN 978-953-51-0500-8

eBook (PDF) ISBN 978-953-51-5606-2 


\section{We are IntechOpen, \\ the world's leading publisher of Open Access books}

Built by scientists, for scientists

\section{$4,100+$}

Open access books available

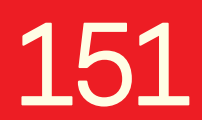

Countries delivered to
$116,000+$

International authors and editors
$120 \mathrm{M}+$

Downloads

Our authors are among the

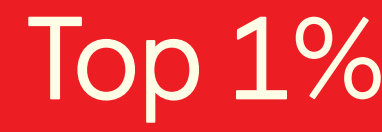

most cited scientists

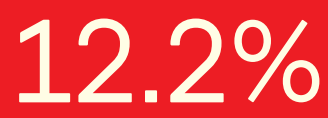

Contributors from top 500 universities

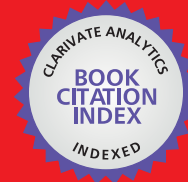

WEB OF SCIENCE ${ }^{\mathrm{TM}}$

Selection of our books indexed in the Book Citation Index in Web of Science ${ }^{\mathrm{TM}}$ Core Collection (BKCI)

Interested in publishing with us?

Contact book.department@intechopen.com

Numbers displayed above are based on latest data collected.

For more information visit www.intechopen.com

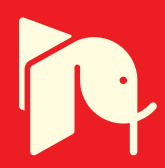





\section{Meet the editor}

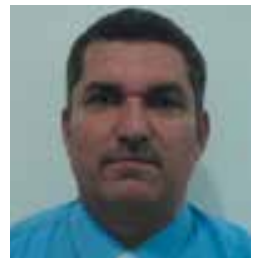

Dr. Abbass A. Hashim is currently working as Head Director of Education Scheme for Training and Developments (ES4TD) British Company. The company is establishing itself in the market of supporting the scientific research and university education programs development. The company has been set up a few years ago. It is well known in the UK and Middle East and has a lot of university and company partners. Within his duties and commitments in this company, Dr. Hashim is in charge of running the research programs of Environmental Pollution Research Group (EPRG), Nanotechnology Research Group (NTRG) and Materials for Engineering Applications Research Group (MEARG). In these fields of research Dr. Hashim published many papers and research reports. He is the editor of more than six books published by InTech Open Access Publisher and a member of many journal editorial boards. 



\section{Contents}

Preface XIII

Part 1 Metallic Nanoparticles 1

Chapter 1 Metallic Nanoparticles Coupled with Photosynthetic Complexes 3

Sebastian Mackowski

Chapter 2 Hollow Nano Silica: Synthesis, Characterization and Applications 29 N. Venkatathri

Chapter 3 Synthesis of Titanate and Titanium Dioxide Nanotube Thin Films and Their Applications to Biomaterials 41 Mitsunori Yada and Yuko Inoue

Chapter 4 Self-Organization of Silver-Core Bimetallic Nanoparticles and Their Application for Catalytic Reaction 67

Kazutaka Hirakawa

Chapter 5 Utilization of Nanoparticles Produced by Aqueous-Solution Methods - Formation of Acid Sites on $\mathrm{CeO}_{2}-\mathrm{TiO}_{2}$ Composite and 1-D $\mathrm{TiO}_{2}$ for Dye-Sensitized Solar Cells 93 Motonari Adachi, Keizo Nakagawa, Yusuke Murata, Masahiro Kishida, Masahiko Hiro, Kenzo Susa, Jun Adachi, Jinting Jiu and Fumio Uchida

Chapter 6 Experimental and Theoretical Study of Low-Dimensional Iron Oxide Nanostructures 119 Jeffrey Yue, Xuchuan Jiang, Yusuf Valentino Kaneti and Aibing Yu

Part 2 Testing Technology 147

Chapter 7 Iron Oxide Nanoparticles Imaging Tracking by MR Advanced Techniques: Dual-Contrast Approaches 149 Shengyong Wu 
Chapter 8 Nanoscale Electrodeposition of Copper on an AFM Tip and Its Morphological Investigations 173 Udit Surya Mohanty, S. Y. Chen and Kwang-Lung Lin

Chapter 9 New Trends on the Synthesis of Inorganic Nanoparticles Using Microemulsions as Confined Reaction Media 195 Margarita Sanchez-Dominguez, Carolina Aubery and Conxita Solans

Chapter 10 Polymeric Nanoparticles Stabilized by Surfactants: Controlled Phase Separation Approach 221

Sergey K. Filippov, Jiri Panek and Petr Stepanek

Chapter 11 Phase Separations in Mixtures of a Nanoparticle and a Liquid Crystal 241

Akihiko Matsuyama

Chapter 12 Laser-Combined STM and Related Techniques for the Analysis of Nanoparticles/Clusters 269

Hidemi Shigekawa, Shoji Yoshida,

Masamichi Yoshimura and Yutaka Mera

Chapter 13 On the Optical Response of Nanoparticles:

Directionality Effects and Optical Forces 287

Braulio García-Cámara, Francisco González, Fernando Moreno, Raquel Gómez-Medina, Juan José Sáenz and Manuel Nieto-Vesperinas

Chapter 14 Deexcitation Dynamics of a Degenerate Two-Level Atom near (Inside) a Body 309 Gennady Nikolaev

Part 3 Properties and Applications 333

Chapter 15 Thermal Property Measurement of $\mathrm{Al}_{2} \mathrm{O}_{3}$-Water Nanofluids 335

Fei Duan

Chapter 16 Magnetic Nanoparticles:

Its Effect on Cellular Behaviour

and Potential Applications $\mathbf{3 5 7}$

Hon-Man Liu and Jong-Kai Hsiao

Chapter 17 Thermal Effects on the Ferromagnetic

Resonance in Polymer Composites with Magnetic Nanoparticles Fillers 373

Mirosław R. Dudek, Nikos Guskos and Marcin Kośmider 
Chapter 18 Nanoparticle Dynamics in Polymer Melts 387

Giovanni Filippone and Domenico Acierno

Chapter 19 Dielectric and Transport Properties

of Thin Films Deposited from Sols

with Silicon Nanoparticles 407

Nickolay N. Kononov and Sergey G. Dorofeev

Chapter 20 Self-Assembly of Nanoparticles at Solid and Liquid Surfaces $\mathbf{4 4 1}$

Peter Siffalovic, Eva Majkova, Matej Jergel, Karol Vegso, Martin Weis and Stefan Luby

Chapter 21 View on the Magnetic Properties of Nanoparticles $\mathrm{Co}_{m}(\mathrm{~m}=6,8,10,12,14)$ and $\mathrm{Co}_{6} \mathrm{O}_{n}(n=1-9) 467$

Jelena Tamulienè, Rimas Vaišnoras,

Goncal Badenes and Mindaugas L. Balevičius

Chapter 22 Nanofluids $\mathbf{4 9 7}$

Wei Yu, Huaqing Xie and Lifei Chen

Chapter 23 Thermal Conductivity of Nanoparticles Filled Polymers

Hassan Ebadi-Dehaghani and Monireh Nazempour

Chapter 24 Magnetic Properties and Size Effects of Spin-1/2 and Spin-1 Models of Core-

Surface Nanoparticles in Different Type Lattices 541

Orhan Yalçın, Rıza Erdem and Zafer Demir

Chapter 25 Organic Semiconductor Nanoparticle Film:

Preparation and Application 561

Xinjun Xu and Lidong Li 



\section{Preface}

In the last few years, Nanoparticles and their applications dramatically diverted science in the direction of brand new philosophy.

The properties of many conventional materials changed when formed from nanoparticles. because nanoparticles have a greater surface area per weight than larger particles which causes them to be more reactive and effective than other molecules.

Currently, Nanoparticle research is a very promising scientific research due to the wide range of potential and promising applications especially in biomedical, optical and electronic fields. Nanoparticles are building the bridge of the scientific knowledge connecting bulk materials to atomic or molecular structures.

This new field of science converts the previous science theories and principles to prove that the properties of materials change as their size approaches the nanoscale and, as a result, the percentage of atoms at the surface of a material becomes significantly smaller. This new concept changes the use of such material in microscale size to be used in absolutely new and advanced applications by using a nanoscale size of the same material.

For instance, the mechanical properties of bulk Copper are in advantage of this material for its use in manufacturing. Copper nanoparticles smaller than $50 \mathrm{~nm}$ show a super hard mechanical property that does not exhibit the same malleability and ductility as bulk copper. On the other hand, Ferroelectric materials which are widely used in memory storage applications smaller than $10 \mathrm{~nm}$ can switch their magnetisation direction using room temperature thermal energy, thus making them useless for memory storage.

In this book, we (InTech publisher, editor and authors) have invested a lot of effort to include 25 most advanced technology chapters. The book is organised into three wellheeled parts. Part one contains 6 chapters discussing the metallic Nanoparticle and reviewing some of the advantages, synthesisation, organization and functioning of a selective metallic Nanoparticle as well as the development of preparation technology. The second part focuses on the Nanoparticle testing technology. Testing techniques are extremely important for the particle within the size of few $\mathrm{nm}$. Experts presented their own experience in this field to be shared, considered and improved by other 
ambitious scientists. Eight chapters describe different techniques with emphasis on the updating knowledge. Preparation and application are also described in part three which contains eleven chapters.

We would like to invite all Nanotechnology scientists to read and share the knowledge and contents of this book.

Dr. Abbass A. Hashim

Head Director of ES4TD

Education Scheme for Training and Developments

UK 


\section{Part 1}

\section{Metallic Nanoparticles}





\title{
Metallic Nanoparticles Coupled with Photosynthetic Complexes
}

\author{
Sebastian Mackowski \\ Optics of Hybrid Nanostructures Group \\ Institute of Physics, Nicolaus Copernicus University, Torun
}

Poland

\section{Introduction}

Plasmon excitations in metallic nanoparticles provide an efficient way to manipulate electromagnetic fields at the nanoscale (Maier, 2004). While the interactions between plasmons and simple nanostructures such as organic dyes or semiconductor nanocrystals is relatively well described and understood, application of metallic nanoparticles to multipigment structures has started just recently (Carmeli, 2010; Govorov, 2008; Kim, 2011; Mackowski, 2008; Nieder, 2010). Light-harvesting complexes, or more generally, photosynthetic complexes, are quite appealing in this regard as they not only provide an interesting biomolecular system for studying plasmon effect on both the optical properties of pigments and the energy transfer between them, but also they could offer attractive potential application route in photovoltaics (Atwater \& Polman, 2010; Mackowski, 2010).

Extending concepts and methods that have been developed for describing the coupling of single organic chromophores with plasmon excitations in metallic nanoparticles (Anger, 2006; Chettiar, 2010; Govorov, 2006) to multi-chromophoric biological systems has not been completely straightforward from both theoretical and experimental points of view. On the one hand, organic molecules or semiconductor quantum dots are much more robust nanostructures than pigment-protein complexes, therefore, the sample preparation in the latter case should be more gentle, so that the protein itself maintains its structure. Preserving protein structure implies that the function of the complex as a whole also remains intact. This assures conservation of the energy transfer pathways between various chromophores comprising the complex as well as identical optical properties, including absorption and fluorescence, to that of the isolated (decoupled from a metallic nanoparticle) biomolecule. On the other hand, from the theory standpoint, biomolecules, and in particular lightharvesting complexes, render themselves a real challenging system to model due to multitude of interactions between chromophores such as chlorophylls and carotenoids (Blankenship 2002), which results in many energy transfer pathways and formation of strongly coupled excitonic systems, as well as conformational changes of the protein itself. Nevertheless, driven by the continuous development of optical spectroscopy and microscopy techniques (Polivka \& Sundstom 2004) as well as more efficient modeling tools, significant progress has been achieved in understanding interactions and functions of lightharvesting complexes. It has also been helped by high-resolution crystal structures of the 
complexes (Hofmann 1996; McDermott, 1995), enabling thus direct association of the pigments as well as their interactions both with themselves and the protein with the actual structure and spatial arrangement of the pigments in these systems.

The purpose of this chapter is to review recent research carried out on hybrid nanostructures composed of metallic nanostructures and light-harvesting complexes. In general, the research is focused on improving the light absorption of the light-harvesting complexes through properly designed plasmonic nanostructures. However, before we start discussing particular hybrid nanostructures fabricated in the context of plasmonically enhanced absorption of light-harvesting complexes, we describe two basic concepts of metal-enhanced fluorescence: distance dependence of the fluorescence intensity and the influence of spectral properties of metallic nanoparticles and placed nearby molecules (Anger, 2006). This brief introductory discussion is essential for understanding the rationale behind designing hybrid nanostructures that involve biological fluorescing complexes.
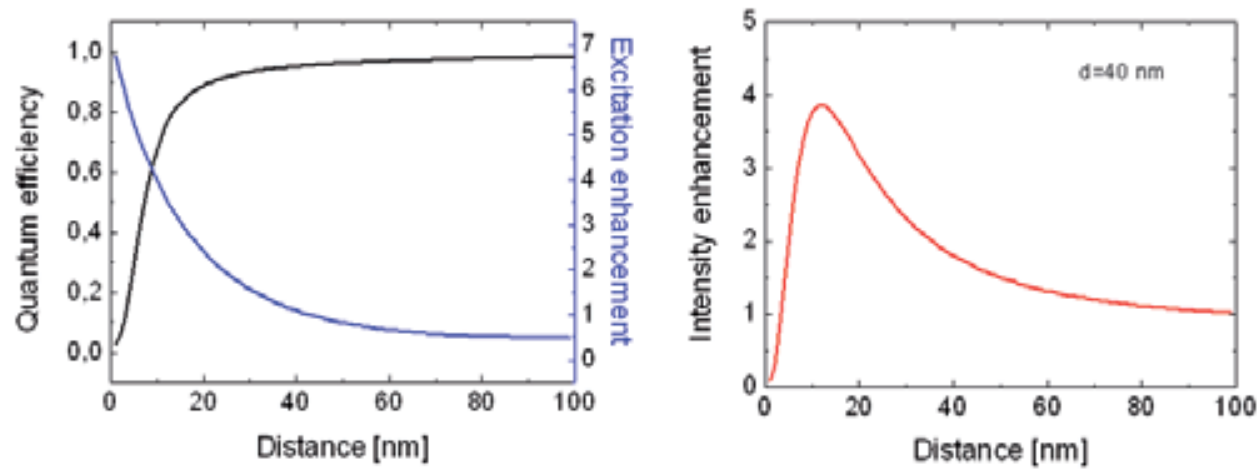

Fig. 1. Dependence of the quantum yield and non-radiative fluorescence quenching upon the distance. Fluorescence intensity of a chromophore displayed as a function of the distance between the chromophore and spherical metallic nanoparticle with $40 \mathrm{~nm}$ diameter.

The optical properties of a fluorophore placed in the vicinity of a metallic nanoparticle are strongly affected by plasmon excitations induced in the latter by a laser light. Without a metallic nanoparticle, a fluorophore is characterized with three rates: absorption rate, radiative rate, and non-radiative rate. Since the oscillation of electrons in the metallic nanoparticle results in creation of local electromagnetic field, in principle all three rates can be changed (Lakowicz, 2006). In addition, another process related to non-radiative energy transfer from the fluorophore to the metallic nanoparticle could also take place in such a hybrid nanostructure. The influence of plasmon excitations upon the quantum yield of a fluorophore and non-radiative energy transfer between the fluorophore and metallic nanoparticle has been recently studied theoretically. In particular, the dependence on the separation distance between the two nanostructures has been analyzed in detail. It turns out that the distance between the fluorophore and metallic nanoparticle is of critical importance in regard to the process that plays dominant role in such a system. In Fig. 1 we show the dependence of the excitation rate and quantum efficiency of a fluorophore upon the distance to the metallic nanoparticle. In this example we consider a metallic nanoparticle with diameter of $40 \mathrm{~nm}$. The excitation efficiency increases exponentially with reducing the distance, which is a clear manifestation of stronger electromagnetic field felt by the 
fluorophore due to plasmon excitation in the metallic nanoparticle. On the other hand, the quantum efficiency, when approaching the range of distances shorter than $20 \mathrm{~nm}$, starts to drop significantly, due to the non-radiative energy transfer from the fluorophore to the nanoparticle. The net result of these two processes in displayed in Fig. 1, where a clear nonmonotonic dependence of the intensity of fluorescence emitted by the fluorophore upon the distance to the metallic nanoparticle can be seen. Importantly, the strongest plasmon induced enhancement of the fluorescence occurs for distances between 10 and $30 \mathrm{~nm}$; for smaller distances non-radiative fluorescence quenching dominates, while for longer distances the fluorophore barely feels the presence of the metallic nanoparticle.
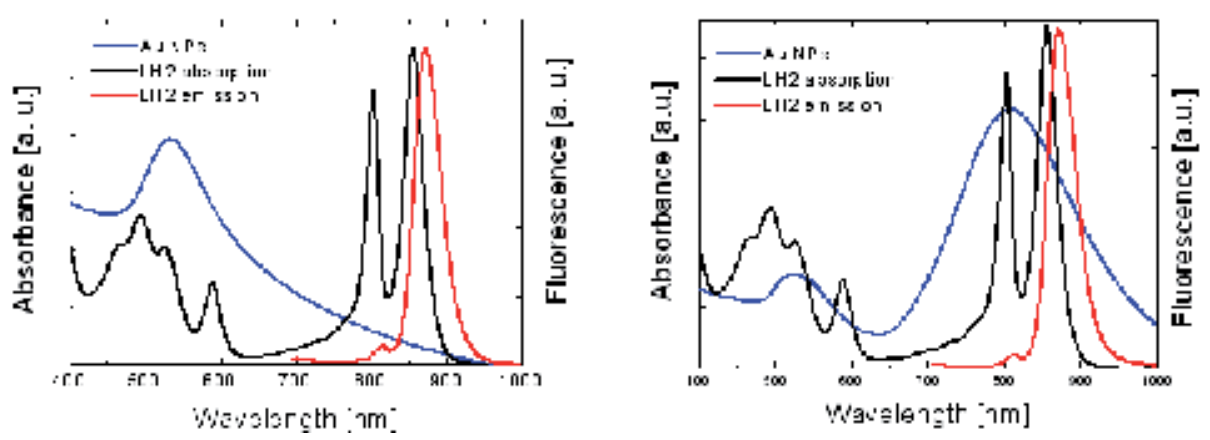

Fig. 2. Comparison between absorption spectra of spherical end elongated gold nanoparticles with the fluorescence and absorption of the LH2 complex. In the first case plasmon excitations should influence mainly the absorption in the visible range, in the second case the effect should be visible for absorption and emission in the infrared.

Another critical parameter that influences the interaction between metallic nanoparticle and fluorophore is the relation of their spectral properties. This is shown schematically in Fig. 2. In the first case scenario the absorption of metallic nanoparticles overlaps significantly with absorption of a biomolecule (in this case this is the LH2 complex from purple bacteria) in the spectral range of about 530-550 $\mathrm{nm}$, while featuring virtually no overlap with the fluorescence. One may expect that for such a combination the major influence of metallic nanoparticles is due to absorption enhancement. In contrast, for a hybrid nanostructures built of components characterized with spectral properties as those displayed in Fig. 2b, there should be absorption enhancement both around $560 \mathrm{~nm}$ as well as in the infrared spectral region, around $800 \mathrm{~nm}$. In addition, since there is a spectral overlap between plasmon band and fluorescence emission, the radiative rate should also increase as a result of plasmon excitation.

Optical spectroscopy provides variety of techniques that allows for distinguishing between various processes that determine the net effect of plasmonic excitations in metallic nanoparticles on a fluorophore. Indeed, in an ideal situation, where only absorption rate is affected by the plasmon excitation, there should be no change in the fluorescence decay time, while an additional band should appear in the fluorescence excitation spectrum. In contrast, when only radiative rate increases as a result of plasmon coupling, the fluorescence excitation spectrum for a hybrid nanostructure should be identical to the reference structure, with much shorter fluorescence decay time. Several experimental configurations exhibiting these various aspects of plasmon coupling with pigment - protein complexes are discussed in this contribution. 


\section{Materials and methods}

In this section we introduce the structure and the optical properties of light-harvesting complexes used in our studies as well as present basic characteristics of metallic nanostructures, including their morphology and plasmon characteristics. Next we present experimental techniques employed for investigating the interactions between plasmon excitations and chromophores embedded in the proteins. These include standard absorption and fluorescence/fluorescence excitation spectroscopy, both in solution and in a layered geometry, as well as confocal fluorescence microscopy coupled with time-resolved capability and spectrally-resolved detection. Combination of all these experimental techniques allows for comprehensive description of plasmon-induced effects on the complex biomolecular systems.

\subsection{Light-harvesting complexes}

Pigment-protein complexes that take part in photosynthesis can be generally divided into two groups: complexes containing reaction centers, which carry out charge separation, and complexes responsible solely for harvesting the sunlight and transferring it to the reaction centers. Large proteins of Photosystem I and Photosystem II fall into the first category, while light harvesting complex 2 (LH2) from purple bacteria and peridinin - chlorophyll - protein (PCP) complex from algae belong to the second group. As the structure and function of all these biomolecules has been described in detail previously, we focus here only on the aspects that are relevant for understanding the influence of plasmon excitations on the optical properties of light-harvesting systems, PCP and LH2.

\subsubsection{Peridinin-chlorophyll-protein}

Light-harvesting complexes were developed in the course of evolution in order to enhance and broaden the absorption of photosystems for the efficient use of sunlight in photosynthesis. Their major function of these pigment-protein complexes is to harvest the sunlight and transfer the energy to the Photosystems. Peridinin-chlorophyll-protein (PCP) found in Dinoflagellates Amphidinium carterae is one of many such complexes. It is a watersoluble protein employed as an antenna external to the membrane. The structure of the PCP complex, shown in Fig. 3, has been determined with $1.3 \AA$ resolution using X-ray crystallography (Hofmann, 1996). The native form of PCP consists of two chlorophyll a (Chl) and eight peridinin (Per) molecules embedded in a protein matrix. All the pigments are arranged in two almost similar clusters and embedded in the hydrophilic protein capsule. The conjugated portion of each Per is close to the chlorophyll tetrapyrrole ring at a van der Waals distance ( 3.3 to $3.8 \AA$ ), the distance between $\mathrm{Mg}$ atoms of the two Chl a in one monomer is $17.4 \AA$ and intercluster edge-to-edge distances between Per are in the range of 4-11 $\AA$. The ratio of Per to Chl a of 4:1 indicates that PCP utilizes the carotenoids as its main light-harvesting pigments. It has been shown that the PCP complex can be reconstituted with other Chl derivatives which exhibit different optical properties (Brotosudarmo, 2006). Importantly, the folding of the protein used in the reconstitution procedure takes place over almost identical pathway as in the native system, which results in very similar structures of the reconstituted systems. Since each of these chlorophyll molecules features specific absorption and emission characteristics, it became possible to construct and study the 
energy transfer dynamics as well as inter-pigment interactions in a well-defined geometry given by the protein (Mackowski, 2007, Polivka, 2005).
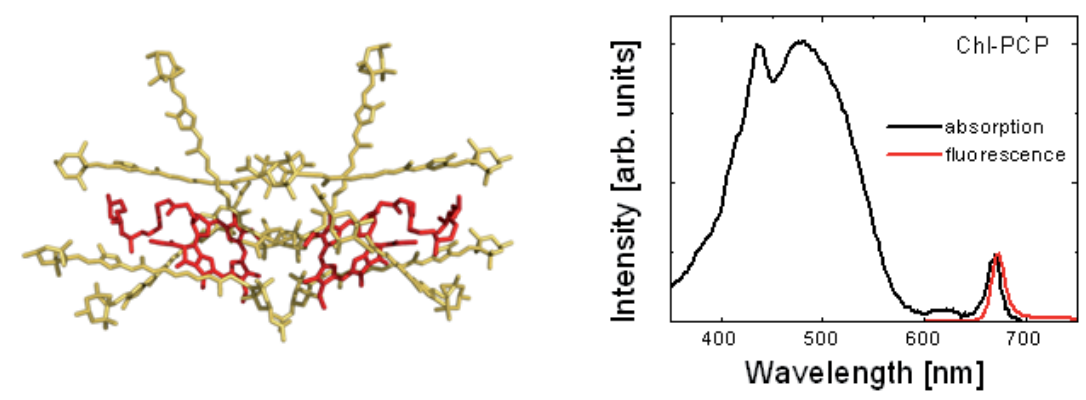

Fig. 3. Pigment structure of the PCP complex reconstituted with Chl $a$ together with absorption (black line) and fluorescence (red line) measured in water solution at room temperature.

The absorption spectrum of the Chl-PCP displayed in Fig. 3 has an intense, broad band between 400 to $550 \mathrm{~nm}$ that is mainly due to Per absorption, and two Chl - related bands at $440 \mathrm{~nm}$ (Soret) and $660 \mathrm{~nm}\left(\mathrm{Q}_{\mathrm{Y}}\right)$. One example of chlorophylls used for reconstituting PCP complexes is [3-acetyl]-chlorophyll a (acChl). Chemically, it differs from $\mathrm{Chl}$ only by the C-3 substituent, but the absorption and fluorescence spectra of acChl-PCP, the PCP complex reconstituted with acChl, are red shifted as compared to the PCP complex containing Chl. At the same time the Per absorption in the blue-green spectral range is affected very slightly. For the PCP complexes reconstituted with acChla the absorption of the Qy band of the chlorophyll molecules is shifted by approximately $20 \mathrm{~nm}$ to the red. The fluorescence emission of the PCP complex originates from weakly coupled $\mathrm{Chl}$ molecules and it appears at $670 \mathrm{~nm}$ for Chl-PCP and $690 \mathrm{~nm}$ for acChl-PCP. Upon absorption of light, peridinins in PCP transfer their electronic excitation to $\mathrm{Chl}$ a. The efficiency of this excitation energy transfer is higher than $90 \%$ [20]. Subsequently, Chl a passes the energy on to membranebound light-harvesting complexes and the Photosystem II. Clearly, the absorption spectrum of PCP enables the photosynthetic apparatus to harness the sunlight not only in the red spectral range, but it extends it into the blue-green spectral region.

Optical spectroscopy studies of both native and reconstituted PCP complexes have been carried out on the ensemble (Akimoto, 1996; Kleima, 2000; Krueger, 2001) and singlemolecule levels (Mackowski, 2007; Wormke, 2007a; Wormke, 2008). Using transient absorption in femtosecond timescale main energy transfer pathways have been described, it has also been demonstrated that the two $\mathrm{Chl}$ a molecules interact relatively weakly with characteristic transfer time between them to be of the order to 12 ps (Kleima, 2000). These observations were also corroborated with fluorescence studies of individual PCP complexes: it has been shown that it is possible to distinguish emission originating from each of the two $\mathrm{Chl}$ a molecules and using the property of sequential photobleaching of the Chl the energy splitting between the two molecules in the monomer were determined (Wormke, 2007a). Recent work on PCP complexes reconstituted with both $\mathrm{Chl}$ a and $\mathrm{Chl} b$ provided coherent description of the energy transfer pathways and dynamics in this unique antenna (Mackowski, 2007). 


\subsubsection{Light-harvesting complex 2}

Another example of a pigment-protein complex employed to harness sunlight energy and transfer it efficiently to reaction centers is a light-harvesting complex 2 (LH2) from purple bacteria Rhodopseudomonas palustris. This protein is placed in the thylakoid membrane, where many of LH2 complexes surround relatively widely spaced LH1 complexes, to which they transfer excitation energy. The BChl a molecules in LH1 have a single strong nearinfrared absorption band of $875 \mathrm{~nm}$, while LH2 has two strong BChl a absorption bands at $800 \mathrm{~nm}$ and $850 \mathrm{~nm}$ (Fig. 2). In this way the energy gradient is formed, which facilitates efficient energy transfer from LH2 to LH1 complex, and then further to the reaction center. Both the structure and the optical properties of the LH2 have been a subject of intense studies in recent years (van Oijen, 1999; Hofmann, 2003; Bopp, 1997). It has been shown using atomic force microscopy technique, that LH2 complexes arrange around a LH1 lightharvesting complex, in the middle of which is a reaction center (Scheuring, 2004). The spatial arrangement of Bacteriochlorophylls (BChls) and carotenoids in this complex is displayed in Fig. 4. The X-ray crystallography studies of the LH2 complex have shown that out of the $27 \mathrm{BChl}$ molecules 18 form a strongly coupled ring with average distances between the molecules less than $1 \mathrm{~nm}$ (McDermott, 1995). This excitonically coupled ring is responsible for the absorption band at $850 \mathrm{~nm}$. The remaining 9 molecules form a ring of weakly coupled BChls as they are spaced by more than $2 \mathrm{~nm}$. All pigments, BChls and carotenoids, are embedded in a hydrophobic protein (not shown). Single molecule investigations (van Oijen, 1999) proved that the B 850 ring are in fact not fully symmetric, and the exciton levels feature significant splitting.
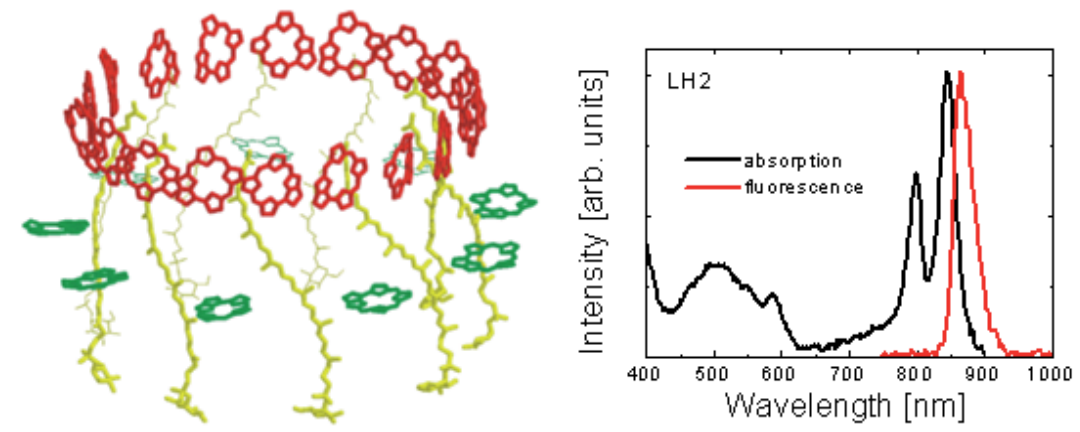

Fig. 4. Pigment structure of the LH2 complex together with absorption (black line) and fluorescence (red line) measured in buffer solution at room temperature.

The absorption spectrum of the LH2 complex is shown in Fig. 4. It consists of two prominent bands at $800 \mathrm{~nm}$ and $850 \mathrm{~nm}$ which correspond to absorption of the two rings of $\mathrm{BChl}$ molecules. The carotenoids are in close contact with both $\mathrm{BChl}$ rings, and are mainly responsible for a broad absorption between $390 \mathrm{~nm}$ and $550 \mathrm{~nm}$. Importantly, the fluorescence of LH2, which originates exclusively from the strongly coupled ring (named B850) has therefore an excitonic character. The presence of strong absorption bands and fluorescence emission in the infrared spectral range requires - in order to influence the optical properties of the LH2 complex - application of metallic nanoparticles that feature plasmon resonances in the near infrared. 


\subsection{Metallic nanostructures}

Metallic structures with nanometric sizes have been the subject of intense research in recent years due to mainly unique optical properties of these systems that can be used for manipulating light at the nanoscale, designing biosensors and artificial chiral nanostructures, enhancing the optical properties of semiconductor nanocrystals and organic fluorescent dyes, enabling detection of emitters characterized with low fluorescence quantum yields, such as carbon nanotubes or DNA. In addition to efforts aimed at exploiting plasmon effect in metallic nanoparticles, significant research have been carried out to achieve almost perfect control of the morphology of metallic nanoparticles, and thus the plasmon properties thereof. Among many techniques of fabrication of metallic nanostructures are evaporation of metallic film on a corrugated substrate (Chettiar, 2010; Mackowski 2008), nanosphere lithography (Hulteen, 1995), electron beam lithography, electrochemical deposition, direct formation of silver island film (Ray, 2006), and chemical synthesis (Link, 1999). Each of these methods requires particular technical capabilities, frequently the experimental setups for nanostructure fabrication is expensive making them hardly accessible. Chemical synthesis of nanoparticles however, is quite simple, at least on the basic level, and, when mastered, provides a way to obtain highly monodisperse nanoparticles with tailored morphology and surface functionalization. This results in welldefined optical properties such as energies of plasmon resonances, and conjugation capabilities to other nanostructures or surfaces. In this contribution we describe the interactions present in hybrid nanostructures composed of light-harvesting complexes PCP and LH2 and metallic nanostructures fabricated using chemical deposition of silver island film on a glass substrate, electron beam assisted deposition of silver island film on a glass substrate, as well as chemically synthesized gold spherical nanoparticles and nanorods. We show that by careful design of a hybrid nanostructure we can control the impact of plasmon excitations in metallic nanoparticles upon the absorption and emission of the chlorophyllcontaining light-harvesting complexes.

\subsubsection{Silver island film}

One of the simplest to fabricate metallic nanostructures is a corrugated metallic film. The method to obtain such a film with islands characterized with sizes of tens of nanometers has been previously applied to study the impact of plasmon interactions upon the fluorescence of various organic dyes, semiconductor quantum dots, and a few proteins, including the green fluorescent protein (Lakowicz, 2006; Ray, 2006). Silver island films used in our experiment were prepared by reducing an aqueous silver nitrate solution. All chemicals were purchased from Sigma-Aldrich and used as received. First, freshly prepared aqueous $\mathrm{NaOH}(1.25 \mathrm{M})$ was added to a silver nitrate solution. The precipitate was re-dissolved by adding $\mathrm{NH}_{4} \mathrm{OH}$, and the solution was cooled to $\sim 5^{\circ} \mathrm{C}$ under stirring. After adding Dglucose, clean microscope cover slips were dipped in the solution, which was then heated up to $30^{\circ} \mathrm{C}$. The resulting Ag-covered glass coverslips were examined using absorption spectroscopy and atomic force microscopy (AFM). In order to change the morphology and thus the properties of the silver island film, we fabricated several samples with varied dipping time in the reaction solution: the coverslips were kept in solution for 1 and 3 minutes. In Fig. 5 we show AFM image of the SIF obtained by dipping the coverslip for 1 minute in the reaction solution. The islands are characterized with average sizes of about 40- 
$50 \mathrm{~nm}$ laterally and the surface density is very high. In addition we also include in Fig. 5 the absorption spectrum measured for the SIF structure which features plasmon resonance with a maximum at $450 \mathrm{~nm}$ and the linewidth of about $150 \mathrm{~nm}$, and thus matches the absorption of Per in the PCP complexes.
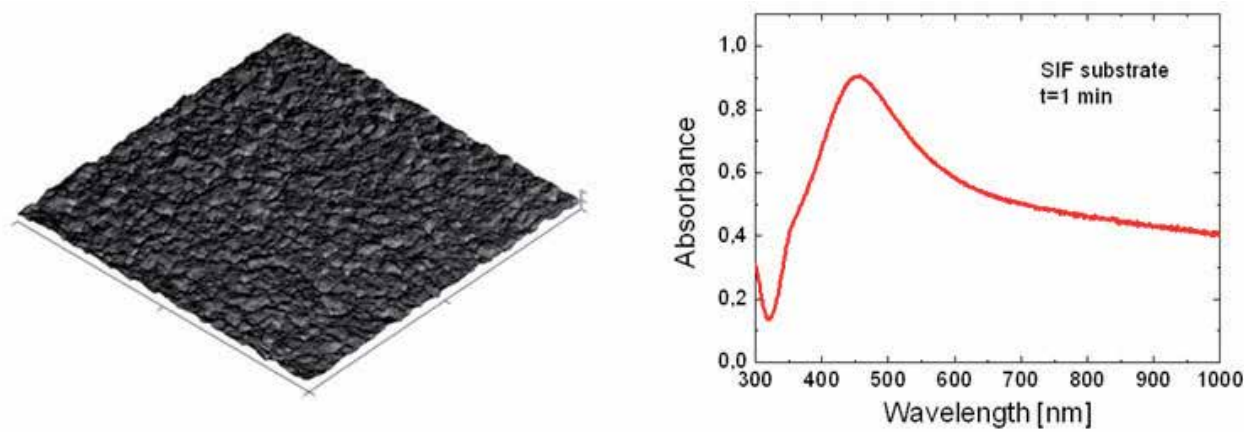

Fig. 5. Atomic force microscopy image of a silver island film fabricated by chemical synthesis together with absorption spectrum of the sample obtained for 1 minute-long dipping time of the glass substrate in the reaction solution. The lateral size of the AFM image is 5 microns.

\subsubsection{Semicontinuous metallic layer}

The semicontinuous silver film has been fabricated using electron beam-assisted evaporation of silver on glass substrate (Chettiar, 2010). While this nanostructure may look similar to the SIF discussed above, the substrates obtained with the e-bam technique are typically more homogeneous, the sizes and shapes of the silver islands is controlled to higher degree. Proper adjustment of the parameters during the evaporation process leads to corrugated metallic films with designed optical properties. For instance, it has been shown that by changing evaporation time it is possible to obtain morphologies ranging from roughly isolated islands to the strongly coalescing ones. Such differences in morphology resulted in strong shift of plasmon energies towards the red and near infrared spectral ranges, opening thus completely new possibilities for applying these structures for controlling the optical properties of infrared - emitting systems. Yet another important advantage of semicontinuous metallic films fabricated using e-beam assisted evaporation is the capability of uniform coating of such films with dielectric layers with thicknesses ranging from a few nanometers up to tens of nanometers. As plasmon induced effects depend crucially upon the separation between metallic nanoparticles and optically active molecules, such structures render themselves a highly suitable system for investigating processes that occur in plasmonic hybrid nanostructures. In particular, the results included in this contribution have been obtained for a semicontinuous silver film covered with a 25nm-thick $\mathrm{SiO}_{2}$ layer evaporated in the same process without exposing the structure to ambient conditions.

\subsubsection{Colloidal metallic nanoparticles}

Among metallic nanostructures, ones of the most studied are colloidal metallic nanoparticles. It is triggered mainly by enormous variety of metallic nanoparticles that can 
be synthesized, even with little resources: sizes of the nanoparticles range from a few to a few hundreds of nanometers. They can also be of essentially any shape: spherical, elongated, triangular, cube or star-like. Furthermore, since the nanoparticles are synthesized in the colloidal form, it is possible to functionalize their surface with functional group suitable for specific attachment to surfaces or conjugation with other nanostructures. Lastly, there have been many examples for self assembly of metallic nanoparticles in complex structures with new properties and functions (Link, 1995).
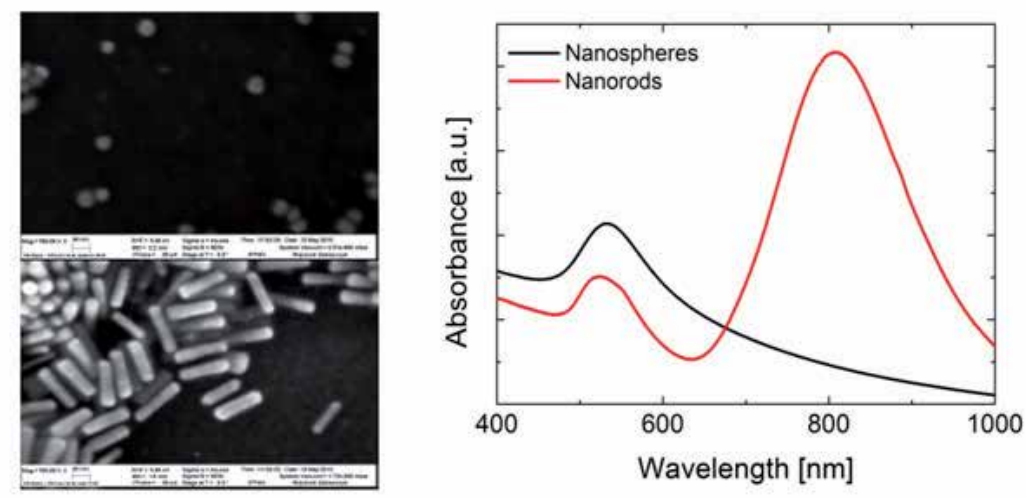

Fig. 6. Scanning electron microscopy images of gold spheres and nanorods. The structural data is accompanied with absorption spectra measured for these two samples at room temperature.

In this contribution we report on synthesis of spherical gold nanoparticles and gold nanorods with the purpose to match absorption bands of light-harvesting complexes. While spherical gold nanoparticles, which feature plasmon resonances around 500-500 nm correspond to carotenoid absorption both in the PCP and LH2 complexes, the nanorods have their resonance also in the infrared, as shown in Fig. 6. By controlling the reaction we tune the energy of the plasmon resonance exactly to $800 \mathrm{~nm}$, thus matching perfectly the B800 absorption of the LH2 complex.

Gold nanoparticles were synthesized using a reduction reaction and dispersed in toluene. The average diameter of the nanocrystals was $5 \mathrm{~nm}$, which results in a plasmon resonance maximum at $530 \mathrm{~nm}$. The synthesis of Au nanorods was based on seed-mediated growth in water solution. All chemicals $\left(\mathrm{HAuCl}_{4} \times 3 \mathrm{H}_{2} \mathrm{O}(99.9 \%), \mathrm{NaBH}_{4}(99 \%), \mathrm{L}\right.$-Ascorbic Acid (99+\%), hexadecyltrimethylammoniumbromide (CTAB) (99\%), and $\mathrm{AgNO}_{3}(99+\%)$ ) were purchased from Aldrich and used without further purification. Deionized water (Fluka) was used in all experiments. In order to prepare Au seeds CTAB solution (4.7 ml, 0.1M) was mixed with $25 \mu \mathrm{l}$ of $0.05 \mathrm{M} \mathrm{HAuCl}_{4}$. To the stirred solution, $0.3 \mathrm{ml}$ of $0.01 \mathrm{M} \mathrm{NaBH}_{4}$ was added, which resulted in the formation of brownish yellow solution. Seeds solution was kept at room temperature until further used. For the synthesis we use Au seeds prepared beforehand. The "seed-mediated" method was developed previously; it is carried out in aqueous solution at atmospheric pressure and near room temperature. Appropriate quantities and molarities of CTAB $(150 \mathrm{ml}, 0.1 \mathrm{M}), \mathrm{HAuCl}_{4}(1.5 \mathrm{ml}, 0.05 \mathrm{M})$, L-Ascorbic acid $(1.2 \mathrm{ml}, 0.1 \mathrm{M}), 0.01 \mathrm{M} \mathrm{AgNO}_{3}(1.6 \mathrm{ml}, 1.8 \mathrm{ml}, 2 \mathrm{ml})$ and seed $(360 \mu \mathrm{l})$ water solutions were added one by one in a flask, followed by a gentle mixing. Addition 
of ascorbic acid, as a mild reduction agent, triggered a mixture color change from dark yellow to colorless. After addition of the seed solution, the mixtures was put into water bath and kept at constant temperature of $28{ }^{\circ} \mathrm{C}$ for 2 hours. Obtained products were separated from unreacted substrate and spherical particles by centrifugation at $9.000 \mathrm{rpm}$ for 60 minutes. The supernatant was removed using a pipette and the precipitate was redissolved in pure water.

\subsection{Sample preparation}

In the research described in this work, we have used several sample architectures, from a very simple ones, where light-harvesting complexes were deposited directly on the surface of the metallic layer being either in the form of silver island film or colloidal gold nanoparticles spin-coated on a glass coverslide, to more advanced, where metallic nanoparticles were separated from the light-harvesting complexes by a thin dielectric layer. For that purpose we used $\mathrm{SiO} 2$ deposited using e-beam assisted evaporation, the thickness of the $\mathrm{SiO} 2$ layer was changed from $5 \mathrm{~nm}$ to $40 \mathrm{~nm}$. The light-harvesting complexes were dispersed in a PVA and then spin-coated on the substrate.

\subsection{Experimental techniques}

The optical properties of hybrid nanostructures comprising light-harvesting complexes and metallic nanostructures have been studied using absorption and fluorescence spectroscopy in the visible and infrared regions. spectral region. Absorption spectra were obtained using a Cary 50 spectrophotometer. Fluorescence and fluorescence excitation spectra of both structures were measured using the FluoroLog 3 spectrofluorimeter equipped with specially designed mount suitable for holding planar samples. A Xenon lamp source with a double grating monochromator was used for excitation and the signal was detected with a thermoelectrically cooled photomultiplier tube characterized by a dark current of less than $100 \mathrm{cps}$.

Fluorescence spectra of samples comprising light-harvesting complexes and $\mathrm{Au}$ nanoparticles were measured in a standard optical setup with a back-scattering geometry. The laser excitation beam ( $\lambda=485 \mathrm{~nm}, 640 \mathrm{~nm}$, or $405 \mathrm{~nm}$ ) was focused, using a lens with a focal length of $30 \mathrm{~mm}$, on the sample surface and the excitation power was controlled using notch filters. Typical excitation powers used were in the range of $200 \mu \mathrm{W}$. The emission was guided through a $150 \mu \mathrm{m}$ pinhole and focused on a slit of a 0.5 monochromator (Shamrock 500, Andor) coupled with a charge coupled device detector (iDus 420BV, Andor). Fluorescence decays were studied using time-correlated single photon counting. For excitation, a diode-pumped solid state laser emitting at $405 \mathrm{~nm}, 640 \mathrm{~nm}$, or $485 \mathrm{~nm}$ and generating 30 ps pulses at $80 \mathrm{MHz}$ repetition rate was used. Detection was carried out with an ultrafast avalanche photodiode detector (idQuantique). The experiment was controlled using a time-correlated single photon counting card (SPC 150 Becker \& Hickl). Emission spectra as well as fluorescence decays were collected for ten different spots across the sample in order to check for the reproducibility and homogeneity of the sample. Fluorescence of light-harvesting complexes was extracted using appropriate long-pass and band-pass optical filters. 


\subsubsection{Fluorescence imaging}

In order to image fluorescence of light-harvesting complexes coupled to metallic nanoparticles we constructed a confocal fluorescence microscope based on Olympus infinity-corrected microscope objective LMPlan 50x, characterized with a numerical aperture of 0.5 and working distance of $6 \mathrm{~mm}$ (Krajnik, 2011). The resulting laser spot size is about 1 $\mu \mathrm{m}$ for the excitation laser of $485 \mathrm{~nm}$. The sample is placed on a XYZ piezoelectric stage (Physik Instrumente) with $1 \mathrm{~nm}$ nominal resolution of a single step, which enables us to raster-scan the sample surface in order to collect fluorescence maps. They are formed by combining fluorescence intensity measurements with the motion of the XY translation stage. For excitation of fluorescence, we use one of four diode-pumped solid-state lasers with wavelengths of 405, 485, 532 and $640 \mathrm{~nm}$. Typical optical power of the laser sources is about $5 \mathrm{~mW}$, but in the case of actual measurements it needs to be strongly reduced in order to prevent photobleaching of the molecules. We used the excitation powers in the range of 0.004 to $0.04 \mathrm{~mW}$. Gaussian beams of the lasers are achieved by using a spatial filter. The fluorescence is detected in a back-scattering geometry and focused on a confocal pinhole $(150 \mu \mathrm{m})$ in order to reduce stray light coming out of the focal plane. The emission of PCP complexes is extracted with HQ 650LP (Chroma) dichroic mirror and HQ 670/10 (Chroma) bandpass filter. In order to extract fluorescence of LH2 complexes we used a longpass filter HQ850LP (Chroma) and a bandpass filter D880/40m (Chroma).

Our experimental configuration, described in detail in (Krajnik, 2011), allows for measuring fluorescence intensity, spectra and lifetimes. The spectrum, dispersed using the Amici prism is measured with a CCD camera (Andor iDus DV 420A-BV). The spectral resolution of the system is about $2 \mathrm{~nm}$. Fluorescence intensity maps are collected with an avalanche photodiode (PerkinElmer SPCM-AQRH-14) with dark count rate of about 80 cps. Fluorescence lifetimes are measured using time-correlated single photon counting module (Becker \& Hickl) equipped with fast avalanche photodiode (idQuantique id100-50) triggered by a laser pulse. Time resolution of the TCSPC setup is about $30 \mathrm{ps}$.

\section{Experimental results}

In this section we describe experimental results obtained for five architectures of hybrid nanostructures comprising metallic nanoparticles and light-harvesting systems. As for metallic nanostructures we used silver island film, semicontinuous silver film, spherical gold nanoparticles and elongated gold nanoparticles (nanorods). We coupled them with chlorophyll and carotenoid molecules embedded in the PCP complex from Amphidinium carterae and in the LH2 complex from Rhodopseudomonas palustris. The results of optical spectroscopy and microscopy show that the optical properties of light-harvesting systems are affected by the plasmon excitations in metallic nanoparticles both in th visible and infrared spectral ranges. Depending on the actual design of a nanostructure, either absorption or fluorescence radiative rate enhancement is obtained.

Generally, the effect of plasmon excitations in metallic nanoparticles on the optical properties of nearby emitters is monitored by measuring the fluorescence intensity. When the geometry of a hybrid nanostructure leads to plasmon-induced enhancement, the fluorescence intensity of such a hybrid structure is increased. When, on the other hand, nonradiative energy transfer from the emitter to the metallic nanoparticles plays the dominant 
role, the emission is efficiently quenched. However, fluorescence spectrum alone gives only limited information about the actual processes responsible for the enhancement of the emission intensity. In order to elucidate the mechanisms in detail, it is important to combine standard fluorescence spectroscopy with fluorescence excitation spectroscopy and timeresolved fluorescence spectroscopy; these two experimental techniques provide a way to separate the plasmon-induced increase of the radiative rate from an induced increase of the absorption.

\subsection{Peridinin-chlorophyll-protein on silver island film}

Initial experiments on hybrid nanostructures composed of light-harvesting complexes and metallic nanoparticles have been carried out on PCP complexes deposited directly onto the silver island film layer (Mackowski, 2008). In order to change the spectral properties of the metallic film, we fabricated SIF substrates with 1 and 3 second long dipping time in the reaction solution. Next, PCP complexes diluted in PVA were spin coated in ensemble concentration on the SIF layer. Since the thickness of the PVA layer is approximately 100 $\mathrm{nm}$, the structure formed in this way spans over all relevant ranges of plasmon-pigment interaction. For PCP complexes located very close to the SIF the non-radiative energy transfer to the metallic nanostructure should play a dominant role and thus fluorescence quenching is expected. In contrast, when the distance between light-harvesting complexes and the SIF is larger than $40-50 \mathrm{~nm}$, there is virtually no interaction between the two components of the hybrid nanostructure. Yet, the optical properties of all in-between molecules should be affected by the plasmon excitations in metallic nanostructure.

In Fig. 7 we display fluorescence images obtained with our confocal fluorescence microscope for the PCP complexes spin-coated onto two SIF substrates characterized with different time of deposition. Bright areas correspond to the higher fluorescence intensity. In the case of the SIF substrate that was kept in the reaction solution for 1 minute only the image is relatively homogeneous, the variation of fluorescence intensity is moderate. On the other hand, for the second structure, which was kept in solution two minutes longer, the areas of high and low fluorescence intensity are clearly separated from each other. We attribute the areas characterized with high fluorescence intensity to regions where the SIF layer was formed during the reaction, while the low fluorescence intensity suggests that the metallic layer detached from the glass substrate during the reaction.

The structure where both SIF and glass surfaces are present at once provide an easy and straightforward means to compare the fluorescence properties of PCP complexes coupled to plasmon excitation to the uncoupled ones. In Fig. 8 we show fluorescence spectra as well as fluorescence decay curves measured with the laser focused on either one of the two areas. As expected, for the PCP complexes placed on the SIF substrate the intensity of the emission is substantially higher that for the reference structure. The enhancement factor estimated from these two spectra is about fourfold. It correspond well to the average enhancement factor obtained for this structure. Importantly, as demonstrated in previous report (Mackowski, 2008), the maximum emission of the PCP complexes as well as the shape of the fluorescence spectrum remain unchanged upon coupling the light-harvesting complexes to the metallic nanoparticles. Also, since we use a $485 \mathrm{~nm}$ laser wavelength for the excitation, the observation of the intact fluorescence emission for both substrates indicates that the efficiency of the energy transfer from carotenoids to Chl molecules is comparable. This 
indicates that the PCP complexes that interact with metallic nanoparticles preserve their overall functionality. We can also see that the change in the fluorescence intensity is accompanied with sharp reduction of the fluorescence lifetime. In fact, the emission of PCP complexes on the glass substrate features a monoexponential decay while upon coupling to the SIF substrate the fluorescence decay curve is more complex. First a rapid decay takes place, which is probably due to efficient quenching of the PCP complexes that are very close to the metallic layer. After a first nanosecond the decay time of fluorescence gets longer, thus becoming similar to the decay observed for the reference structure.
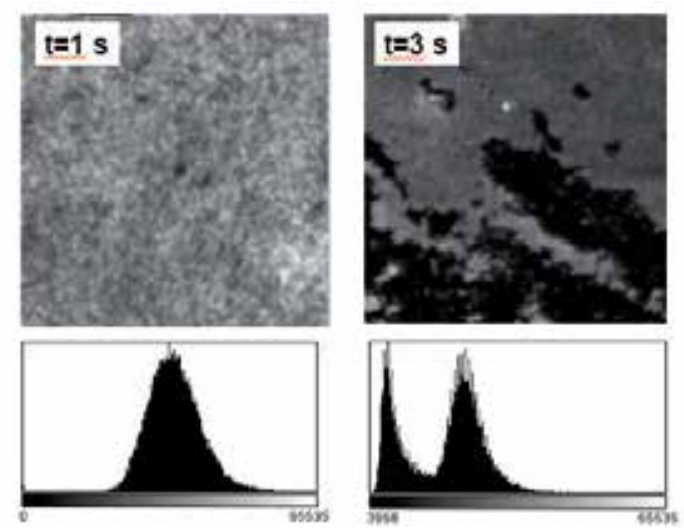

Fig. 7. Images of PCP fluorescence measured for the complexes deposited on the SIF substrate with 1 minute-long dipping time and 3 minute-long dipping time in the reaction solution. The maps were obtained at room temperature for the laser excitation wavelength of $485 \mathrm{~nm}$, the laser power was $40 \mu \mathrm{W}$. The size of the images is 100x100 microns.
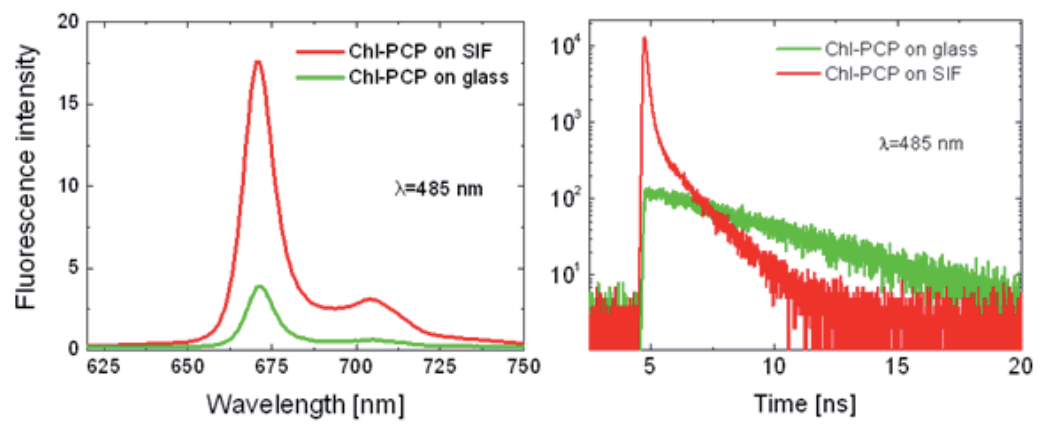

Fig. 8. Comparison between fluorescence spectra and fluorescence decay curves measured for PCP complexes on the glass and SIF substrates. For all measurements the excitation wavelength was $485 \mathrm{~nm}$.

Overall, the results obtained for PCP complexes embedded in PVA matrix on the SIF layer demonstrate that high inhomogeneity of the structure leads to quite complicated behavior. Indeed the enhancement of absorption rate is entangled with enhancement of fluorescence rate, and in addition, signatures of non-radiative energy transfer from the chlorophylls to metallic structure are present. In the case of the hybrid nanostructure studied here, there is no control over the morphology of the SIF itself as well as over the separation between light- 
harvesting complexes and metallic surface. Therefore, other approaches need to be devised, aimed at better control of sizes or shapes of metallic nanoparticles and the distance between the proteins and metallic structures.

\subsection{Peridinin-chlorophyll-protein on semicontinuous silver film}

In previous sections we pointed out the important role of the separation between lightharvesting complexes and the metallic nanoparticles. In the case of sample geometry involving inhomogeneous silver island film and PCP complexes spin-coated directly on top of it in a relatively thick PVA layer, we have observed that the increase of the fluorescence emission is a combined product of absorption and fluorescence rate enhancement. In addition, the signatures of non-radiative energy transfer from the PCP complexes to the SIF layer have been observed in the time-resolved spectra.

In order to minimize the influence of the processes that lead to fluorescence quenching and at the same time to achieve uniform distance from the metallic layer to light-harvesting complexes, we have fabricated a hybrid nanostructure based on semicontinuous silver film (Czechowski, 2011). Such a corrugated metallic surface can be made using e-beam assisted evaporation under high-vacuum conditions. Scanning electron microscopy studies of similarly prepared samples have indicated improved uniformity of the islands, that resulted in narrowing of the plasmon resonance measured in the absorption experiment (Chettiar 2010). Furthermore, on top of the silver film we deposited a $25-\mathrm{nm}$-thick silica layer. The layer serves two purposes: on the one hand it protects the silver surface against oxidation, on the other hand it provides a uniform spacer between metallic nanoparticles and lightharvesting complexes. The final change compared to the structure where PCP complexes were spin-coated in a PVA matrix on top of the SIF, concerned direct deposition of the PCP water solution on the $\mathrm{SiO}_{2}$ surface of the spacer. In this way we can assume that all the complexes are at approximately identical distances from the silver islands. Here we used PCP complexes reconstituted with acChl a as they offer the largest energy separation between their fluorescence and plasmon resonance of silver islands. In addition, the concentration of PCP complexes is much higher than for samples prepared with spincoating, which makes it possible to study the plasmon induced effects using standard fluorescence excitation spectroscopy.

The fluorescence excitation spectrum measured for the detection wavelength of $690 \mathrm{~nm}$ for acChl-PCP on glass substrate is shown in Fig. 9. It is compared with with the result obtained for acChl-PCP complexes placed on the semicontinuous silver film. The excitation spectrum for the reference structure is similar to previously published (Brotosudarmo, 2008) it features strong absorption due to Per in the spectral range from $400 \mathrm{~nm}$ to $550 \mathrm{~nm}$, and corresponds roughly to the absorption spectrum. This suggests that the sample preparation leaves no effect on either the protein or the pigments. In contrast, the maximum of fluorescence excitation spectrum is blue-shifted by $\sim 40 \mathrm{~nm}$ for the PCP complexes deposited on the silver island film and separated from the metallic nanostructures by a 25-nm thick $\mathrm{SiO}_{2}$ layer. The difference between the two cases is seen after subtracting both curves and evaluating the enhancement of the emission. We find that the enhancement curve is a welldefined band with a maximum at $407 \mathrm{~nm}$ and linewidth of about $35 \mathrm{~nm}$. We attribute this enhancement to plasmon excitations in the metallic layer that impact the absorption of the PCP complexes. 

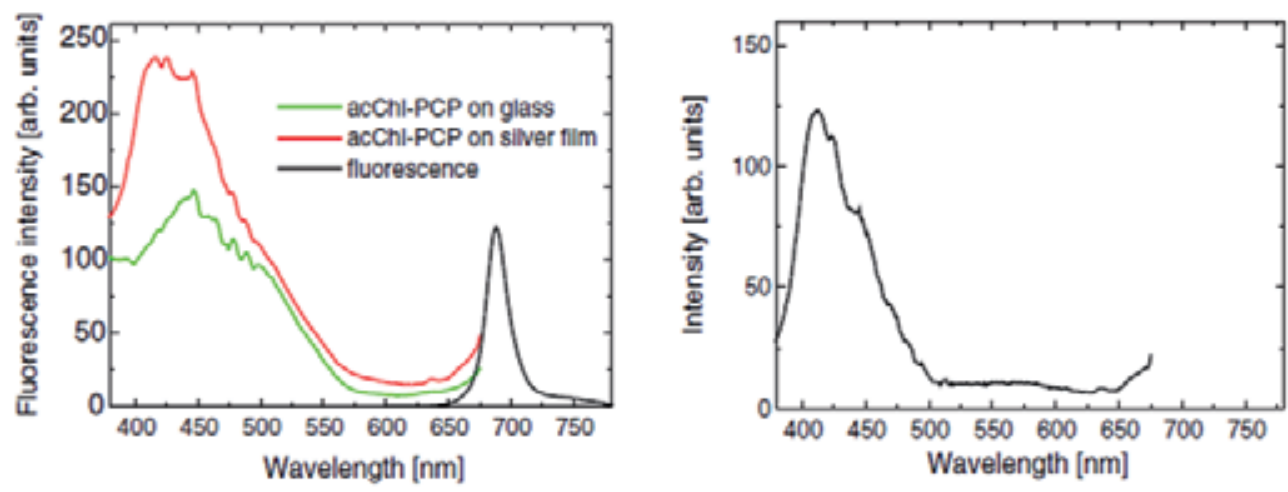

Fig. 9. Comparison between fluorescence excitation spectra measured for acChl-PCP on glass substrate and semicontinuous silver film. The detection energy was $690 \mathrm{~nm}$. An enhancement dependence on the wavelength obtained by subtracting both fluorescence excitation curves is also shown.

It is important to note that the fluorescence excitation spectra measured for the reference structure and for the PCP complexes deposited on the silver film are not in any way adjusted or normalized. Yet, they are very comparable for wavelengths longer than $475 \mathrm{~nm}$, in particular in the absorption range of low energy Per molecules. This suggests that the number of PCP complexes probed in both experiments is almost identical, which makes the estimation of the enhancement factor remarkably straightforward. Also the fluorescence spectrum measured for the hybrid nanostructure is is identical, for all excitation wavelengths, to that of the reference structure, which supports our previous observation that the preparation of the hybrid nanostructure has no measurable effect on the protein or pigment properties.

The fluorescence excitation data point towards increase of the absorption rate of the lightharvesting complex as being the dominant mechanism responsible for the enhancement of the fluorescence intensity. This suggestion is also helped with analysis of the spectral properties of both the PCP complexes and the semicontinuous silver film: they overlap mainly in the blue-green spectral range. In order to verify this we carry out time-resolved fluorescence experiment with the excitation wavelength of $405 \mathrm{~nm}$, which corresponds to the maximum of the enhancement curve displayed in Fig. 9. The result if this experiment in shown in Fig. 10. The decay time of the control sample on glass is equal to 3.2 ns, while for the hybrid nanostructure a shortening of the lifetime to $2.3 \mathrm{~ns}$ when plasmons in the silver island film are excited. This less than 30 percent reduction of the lifetime, while measurable, is relatively small compared to previous results on fluorescent dyes (Dulkeith, 2002) and light-harvesting complexes (Mackowski, 2008), where order-of-magnitude changes have been measured. The small change of the fluorescence lifetimes in the case of the acChl-PCP complexes coupled to the semicontinuous silver film supports our conclusion that the enhancement measured in the fluorescence excitation is predominantly due to the enhancement of the excitation rate in the light-harvesting complexes. We also note that the fluorescence decay curve measured for the hybrid nanostructure features a monoexponential behavior, in contrast to the results obtained for PCP complexes deposited on the SIF. Such a uniform characteristics suggests improved homogeneity of the distance 
between the light-harvesting complexes and the metallic layer, as indeed expected for our preparation procedure. We have also carried out time-resolved experiments with other excitation energies, in particular with $640 \mathrm{~nm}$. This wavelength corresponds to direct excitation of $\mathrm{Chl}$ molecules and excites no plasmons. In this case the fluorescence decay shows no dependence upon either glass or metallic substrate.

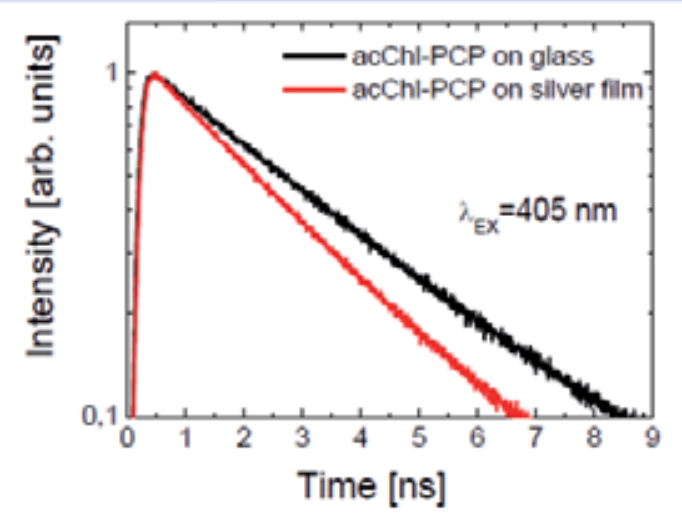

Fig. 10. Comparison of fluorescence decay curves measured for acChl-PCP on glass substrate and semicontinuous silver film. The excitation wavelength was $405 \mathrm{~nm}$.

Finally we comment on another aspect of fluorescence decay time reduction observed for the $405 \mathrm{~nm}$ laser excitation. Since this reduction is attributed to the increase of the radiative rate of emission, it implies that there are plasmons excited in the semicontinuous silver film with wavelengths around $690 \mathrm{~nm}$, where acChl-PCP emits. As $405 \mathrm{~nm}$ laser excites no such plasmons directly, this observation could be indicative of plasmon propagation in terms of energy relaxation. This hypothesis requires further experimental evidence but when proven correct, it could open another pathway in te field of plasmon engineering.

The results of fluorescence spectroscopy on acChl-PCP complexes deposited on semicontinuous silver film spaced by $25 \mathrm{~nm} \mathrm{SiO} 2$ layer confirm that by careful design of plasmonic hybrid nanostructure it is possible to selectively enhance the absorption of the light-harvesting complexes. The next step is to devise and fabricate a hybrid nanostructure, which would allow for systematic studies of plasmon induced effects as a function of the separation layer thickness.

\subsection{Peridinin-chlorophyll-protein on spherical gold nanoparticles}

The influence of the distance upon the interaction between PCP complexes and metallic nanoparticles requires fabrication of structures with precisely controlled thickness of the $\mathrm{SiO}_{2}$ spacer. Such structures were fabricated in an analogous way as described previously, with the thickness of $\mathrm{SiO}_{2}$ layer equal to 4,12 , and $40 \mathrm{~nm}$. In this case however the metallic nanostructure used was a monolayer of uniform gold nanoparticles characterized with plasmon resonance at $530 \mathrm{~nm}$.

While at the distances of $40 \mathrm{~nm}$ the influence of plasmon excitations on the optical properties of light-harvesting complexes is expected to be minimal, for thinner spacers the 
effect should be much stronger. In order to evaluate that we carried out fluorescence imaging experiment on PCP complexes deposited on the three Au nanoparticle samples with varied thickness of the $\mathrm{SiO}_{2}$ spacer. In the first step a fluorescence map was acquired of 100x100 micron sample area. The fluorescence maps were in all cases very uniform, variations of fluorescence intensity were below 15 percent. Next, approximately 50 fluorescence spectra we collected, each off a different spot on the sample surface. Finally, the same procedure was applied for measuring fluorescence decay curves. In this way statistically significant information about fluorescence intensity as well as fluorescence decay time is obtained.
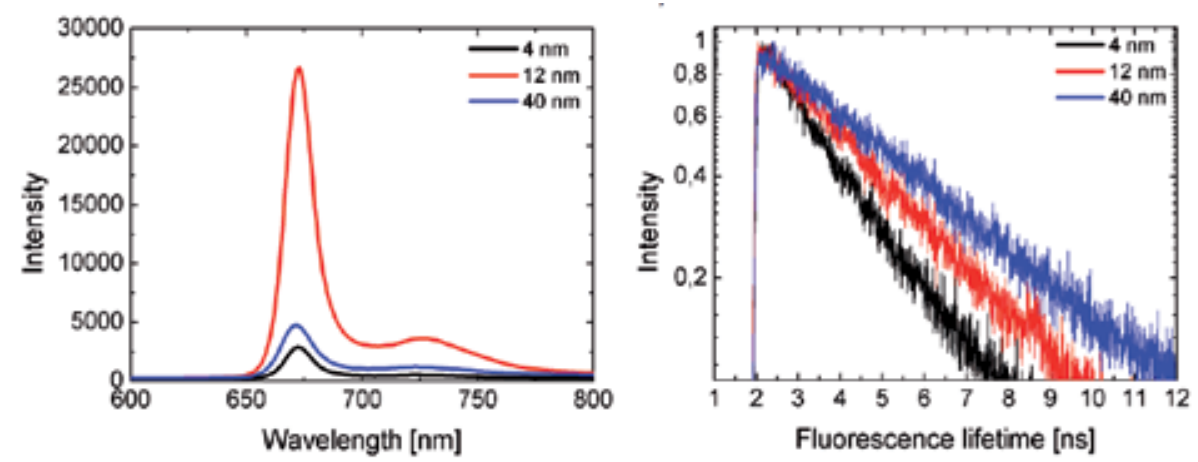

Fig. 11. Typical fluorescence spectra and fluorescence decay curves of Chl-PCP complexes deposited on $\mathrm{Au}$ nanoparticle substrates with different thickness of the $\mathrm{SiO}_{2}$ spacer: $40 \mathrm{~nm}$ (blue), $12 \mathrm{~nm}$ (red), and $4 \mathrm{~nm}$ (black). The laser excitation wavelength was $485 \mathrm{~nm}$.

In Fig. 11 we compare representative fluorescence spectra of Chl-PCP deposited on plasmonic substrates with Au spherical nanoparticles. The continuous-wave results are accompanied with time - resolved data. The intensity of fluorescence emission for $12 \mathrm{~nm}$ spacer is dramatically (fivefold) enhanced as compared to the reference structure with 40 $\mathrm{nm}$ thick $\mathrm{SiO}_{2}$ spacer. For the smallest spacer $(4 \mathrm{~nm})$ the fluorescence decreases rapidly due to non-radiative energy transfer from chlorophylls embedded in the PCP complexes to metallic nanoparticles. Importantly, in analogy to all previously described experiments, the fluorescence spectrum of the light-harvesting complexes remains unchanged, indicating that the biomolecules are intact upon interacting with metallic nanoparticles.

Fluorescence decay curves that accompany the spectra provide means for understanding the mechanism of fluorescence enhancement. The decay time measured for the reference structure $(40 \mathrm{~nm})$ is equal to $3.3 \mathrm{~ns}$, a typical value for PCP complexes reconstituted with Chl $a$ (Mackowski, 2007). As the $\mathrm{SiO}_{2}$ spacer gets thinner, the fluorescence lifetime gets shorter, and for $12 \mathrm{~nm}$ thick spacer is equal to $2.5 \mathrm{~ns}$. Further reduction of the fluorescence lifetime is seen for the thinner, $4 \mathrm{~nm}$, spacer. In this case the decay time is approximately 50 percent of the reference value. However, the mechanism of lifetime reduction is in both cases $(4 \mathrm{~nm}$ and $12 \mathrm{~nm}$ ) completely different. In the first case the shortening of the fluorescence decay time indicates enhancement of radiative rate of PCP complexes. This effects contributes to the observed increase of the emission intensity seen in the fluorescence spectra. On the other hand, for the $4 \mathrm{~nm}$ thick $\mathrm{SiO}_{2}$ spacer, the lifetime reduction is due to excitation quenching. Thee results obtained for PCP complexes coupled to Au nanoparticles demonstrate clear 
dependence of the fluorescence enhancement upon the distance between chlorophyllcontaining proteins and metallic nanoparticles. While most of the effect is due to increase of absorption, there is also significant contribution associated with increase of the radiative rate. This approach can be then used for optimizing the geometry of plasmonic hybrid nanostructure for the most efficient performance.

\subsection{Light-harvesting complex 2 on spherical gold nanoparticles}

Light-harvesting complex LH2 from the purple bacteria is characterized by relatively weak absorption in the visible spectral range with its main absorption bands appearing in the near infrared, at 800 and $850 \mathrm{~nm}$. By coupling LH2 to spherical gold nanoparticles we attempt to enhance the absorption between 400 and $550 \mathrm{~nm}$. The geometry of the hybrid nanostructure was identical to discussed previously: monolayers of $\mathrm{Au}$ nanoparticles were covered with $\mathrm{SiO}_{2}$ dielectric layers with thickness of 4, 12, and $40 \mathrm{~nm}$. During the experiment the fluorescence spectra excited into carotenoid absorption (Wormke, 2007b) were measured at ten different locations across the sample. In this way it was possible to account for any inhomogeneities due to the preparation of the hybrid nanostructures. The fluorescence spectra measured with $\mathrm{SiO}_{2}$ spacers between 4 and $40 \mathrm{~nm}$ and are shown in Fig. 12.

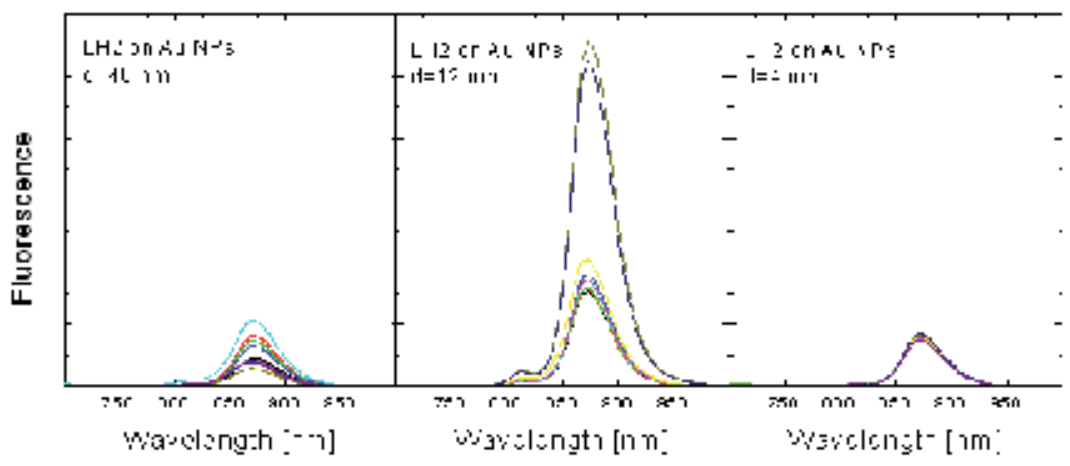

Fig. 12. Fluorescence spectra measured for LH2 complexes deposited on Au spherical nanoparticles on $\mathrm{SiO} 2$ spacers with thicknesses as indicated. The spectra were obtained for ten different locations on each sample.

There are several interesting observations worth pointing out. First of all, for the reference sample with 40 -nm-thick $\mathrm{SiO}_{2}$ spacer the scattering of the measured intensities can be attributed to local fluctuations in the LH2 concentration due to spin-coating approach. In contrast, for the sample with the 12-nm-thick $\mathrm{SiO}_{2}$ spacer the spread of fluorescence intensities is significantly greater and the observed variation cannot be due to fluctuations of the LH2 concentration. Since plasmon interactions are expected to be significant for such a separation between the metallic nanoparticles and light-harvesting complexes, we attribute the distribution of fluorescence intensity to variation in plasmon coupling between the LH2 complexes and Au nanoparticles. Such variations can be caused for instance by interface roughness of the $\mathrm{SiO}_{2}$ layer, even small variations of the spacer thickness would result in measurable changes of the fluorescence intensity. Finally, for the 
thinnest $\mathrm{SiO}_{2}$ layer of $4 \mathrm{~nm}$ the fluorescence intensities are all very similar. In fact, the measured distribution is even less pronounced than in the case of the reference sample. Such behavior may well be due to the dominant role of the fluorescence quenching caused by metallic nanoparticles, which takes over below a certain thickness of the spacer between the metallic nanoparticles and light-harvesting complexes. In such a case any fluctuations of either $\mathrm{LH} 2$ concentration or $\mathrm{SiO}_{2}$ spacer thickness may be of lesser significance.
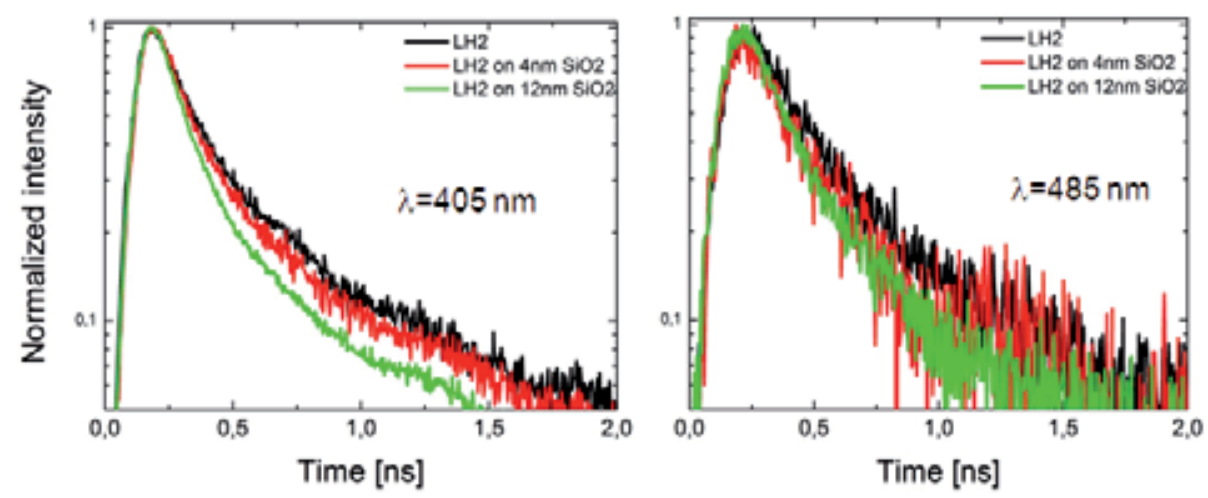

Fig. 13. Fluorescence decay curves measured for LH2 complexes on Au nanoparticles separated by $\mathrm{SiO}_{2}$ spacer. Excitation wavelengths of $405 \mathrm{~nm}$ and $485 \mathrm{~nm}$ were used.

In order to determine the possible origin of the observed fluorescence enhancement, time-resolved fluorescence was measured on identically prepared samples. The fluorescence decay curves obtained for the structure with 4 and $12 \mathrm{~nm}$ thick $\mathrm{SiO}_{2}$ layer is compared in Fig. 13 with the one measured for LH2 complexes deposited directly in glass substrate (Bujak, 2011). Apparently, upon coupling to the plasmons localized in the $\mathrm{Au}$ nanoparticles the fluorescence decays show virtually no change. Therefore, we assume that the fluorescence enhancement is predominantly due to an increase in the absorption in the carotenoid region of the LH2. The observation of exclusive increase of the absorption efficiency in the LH2 complexes coupled to Au nanoparticles was rendered by two factors. On the one hand, the difference in energy between plasmon resonance and the fluorescence emission is almost $400 \mathrm{~nm}$, thus the overlap between low-energy tail of the plasmon resonance with the emission spectrum of the LH2 is minimal. This is much larger energy difference than for PCP complexes deposited on the semicontinuous silver film or Au nanoparticles. On the other hand, spherical gold nanoparticles are very uniform in size. This inhibits any possibility of energy relaxation in plasmonic structure, as it was observed for PCP complexes on the highly inhomogeneous SIF substrate.

The results described so far point clearly towards strong dependence of the plasmon induced effects upon the excitation energy. In most cases achieving strong coupling requires direct excitation of plasmons in metallic nanoparticles. In order to illustrate this, the fluorescence lifetimes were measured for LH2 complexes on Au nanoparticles with the excitation energy of $405 \mathrm{~nm}$. In contrast to the $485 \mathrm{~nm}$ excitation, this energy excites 
plasmons very inefficiently while still populating excited states of carotenoids. The results included in Fig. 13 show that the fluorescence lifetime shows no dependence upon the thickness of the $\mathrm{SiO}_{2}$ spacer. However, the actual enhancement factor measured for $405 \mathrm{~nm}$ laser is substantially reduced compared to $485 \mathrm{~nm}$ laser, which very efficiently excites plasmons in metallic nanoparticles. The comparison is displayed in Fig. 14. For both excitation wavelengths the dependence of the enhancement factor on the distance between light-harvesting complexes and metallic nanoparticles is qualitatively the same. Yet, under the condition of efficient excitation of plasmons the maximum enhancement observed for the spacer with $12 \mathrm{~nm}$ thickness is 2.5 times greater.

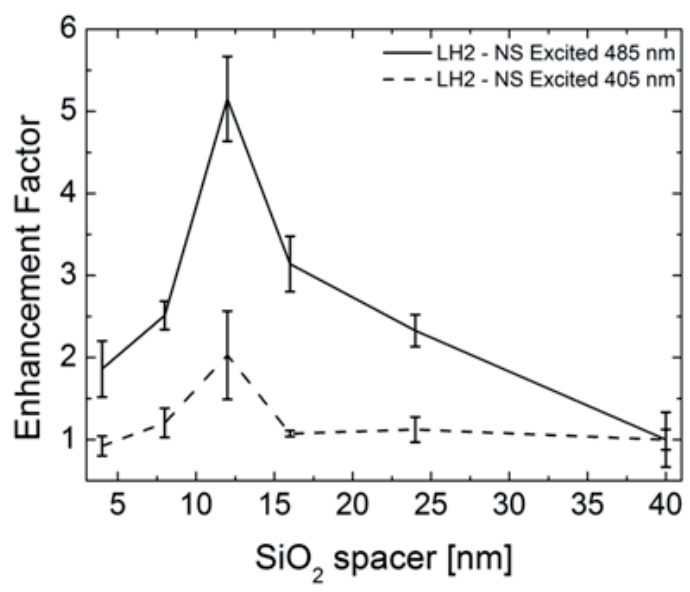

Fig. 14. Comparison of distance dependence of the fluorescence intensity enhancement for PH2 complexes deposited on Au spherical nanoparticles with different spacer thickness. The data was obtained for $485 \mathrm{~nm}$ and $405 \mathrm{~nm}$ laser excitations.

In conclusion, results of fluorescence spectroscopy carried out on hybrid nanostructures composed of light-harvesting complex LH2 and gold nanoparticles demonstrate the strong impact of plasmon excitations upon the optical properties of the biomolecule. For a spacer with a thickness of $12 \mathrm{~nm}$ substantial increase of the fluorescence intensity is observed, which is due to an enhancement of absorption of the carotenoids in this light-harvesting complex. Furthermore, we observe strong dependence of the fluorescence enhancement on the laser wavelength: for efficient excitation of plasmons in metallic nanoparticles $(\lambda=485$ $\mathrm{nm}$ ) the enhancement is approximately 2.5 times stronger than for the out-of-plasmonresonance excitation wavelength $(\lambda=405 \mathrm{~nm})$.

\subsection{Light-harvesting complex 2 on gold nanorods}

The final example of a hybrid nanostructure composed of light-harvesting complexes and metallic nanoparticles is a system where we combine Au nanorods with LH2 complexes from purple bacteria. From the previous discussion we know that by using Au nanorods we gain a tunability of plasmon resonances that reach near infrared spectral region (Bryant, 2008). In this way then we can affect the spectral properties of 800 and B850 absorption bands of the LH2 complex as well as its fluorescence emission. 
In Fig. 15 we show the result of fluorescence imaging experiment carried out on LH2 complexes deposited directly on gold nanorods with plasmon resonances at $550 \mathrm{~nm}$ and 800 $\mathrm{nm}$. The maxima of the resonances match ideally with absorption bands of the LH2 complex, attributed to carotenoids and bacteriochlorophylls, respectively. In the experiment we probe the fluorescence enhancement for these two excitation wavelengths, importantly for these two excitations the same sample area was monitored. It can be seen in particular for the maps shown in the upper row of Fig. 15, areas with low fluorescence intensity are clearly correlated. In the case of LH2 complexes on glass substrate fluorescence maps acquired for both excitation wavelengths are very uniform, as shown below the maps with intensity histograms. In both cases the histograms oare of Gaussian shape with maxima at 7500 and $21000 \mathrm{cps}$ for 556 and $808 \mathrm{~nm}$ excitation, respectively. The picture changes
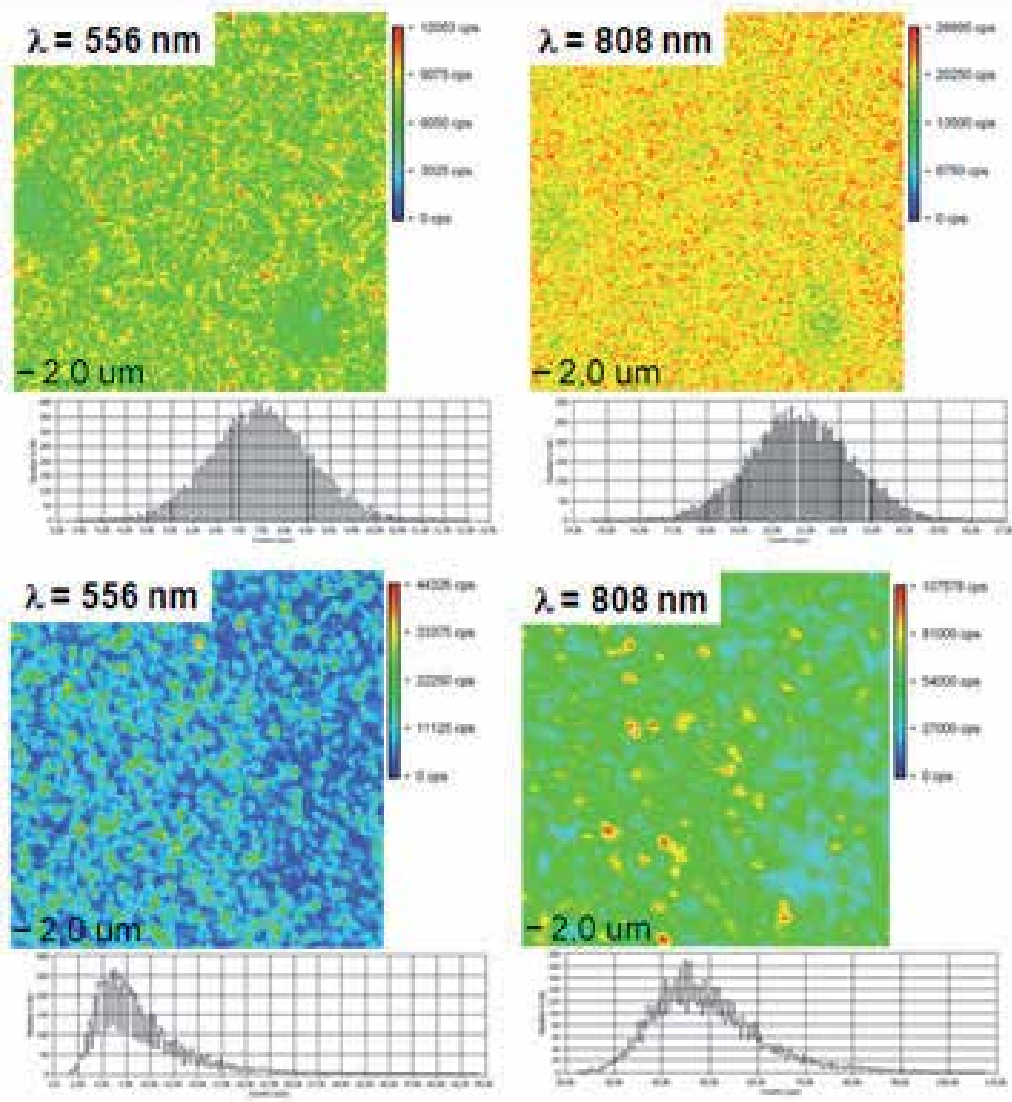

Fig. 15. Fluorescence images of LH2 complexes deposited on glass substrate (upper row) and Au nanorods (lower row). For exciting carotenoid absorption a $556 \mathrm{~nm}$ laser was used, whereas for exciting B800 BChl ring - a $808 \mathrm{~nm}$ laser was used. The maps for a given structure were obtained from the same sample area. The size of the images is $50 \times 50$ microns. 
qualitatively for the LH2 complexes deposited on gold nanorods. The most pronouncing effect is much larger inhomogeneity of the fluorescence maps. There are regions of a few micron size that feature much stronger emission intensity. We can attribute them to either favorable separation between LH2 and Au nanorods or orientation/geometry of gold nanorods that would lead to formation of hot-spots of strongly localized electromagnetic field.

In addition to highly homogeneous fluorescence images, there are also significant differences of the distribution of fluorescence intensity, in spite of using the same excitation powers for a given laser wavelength. Indeed, the maximum of fluorescence intensity measured for $556 \mathrm{~nm}$ appears roughly at the same value as for the reference sample, but the histogram features substantial high-intensity tail of intensities, which is due to plasmoninduced enhancement in the hybrid nanostructure. Conversely, for the excitaiton of $808 \mathrm{~nm}$ the we also observe a broad tail towards higher intensities, but in this case the average intensity is also twice the average intensity measured for the reference sample. These preliminary results demonstrate that by using gold nanorods we are able to modulate the optical properties of multi-chromophoric systems such as light-harvesting complexes, which absorb in the infrared spectral range. Further work is required to coherently describe the complexity of plasmon interactions in this system.

\section{Summary and conclusions}

We have described various geometries of hybrid nanostructures composed of lightharvesting complexes from algae or purple bacteria and metallic nanostructures in the form of silver island films or monolayers of metallic nanoparticles synthesized chemically. The samples were studied with numerous optical spectroscopy and microscopy techniques including fluorescence excitation, time-resolved fluorescence, and fluorescence imaging with high spatial resolution. In all fabricated structures we observe strong effects attributable to plasmon induced effects on the optical properties of the light-harvesting complexes. Depending on the actual geometry we are able to increase fluorescence or absorption rate, in most cases however both effects are entangled. The results demonstrate that plasmon excitations in metallic nanostructures can be efficiently applied for controlling the light-harvesting capability of photosynthetic complexes, possibly paving the road towards novel photovoltaic architectures based - at least in some degree - on natural photosynthesis.

\section{Acknowledgment}

Research in Poland has been supported by the WELCOME project "Hybrid Nanostructures as a Stepping Stone towards Efficient Artificial Photosynthesis" funded by the Foundation for Polish Science and EUROCORES project "BOLDCATS" funded by the European Science Foundation. I am indebted to my friends and colleauges, with whom I have a great pleasure to collaborate on this project: I thank Wolfgang Heiss (Linz University), Eckhard Hofmann (University of Bochum), Richard J. Cogdell (University of Glasgow), Nicholas A. Kotov (University of Michigan), Hugo Scheer (LMU Munich) and the members of their research groups involved in parts of this research. Last but not least, I also acknowledge members of my research group at the Institute of Physics, Nicolaus Copernicus Unviersity in Torun, in 
particular Dr. Dawid Piatkowski, Dr. Radek Litvin, Lukasz Bujak, Nikodem Czechowski, Bartosz Krajnik, Maria Olejnik, Kamil Ciszak, and Mikolaj Schmidt for their excellent work and vital contribution.

\section{References}

Akimoto, S.; Takaichi, S.; Ogata, T.; Nishimura, Y.; Yamazaki, I. \& Mimuro, M. (1996), Excitation energy transfer in carotenoid chlorophyll protein complexes probed by femtosecond fluorescence decays, Chemical Physics Letters, Vol.260, No.1-2, (September 1996), pp.147-152, ISSN 009-2614

Anger, P; Bharadwaj, P. \& Novotny, L. (2006), Enhancement and quenching of singlemolecule fluorescence, Physical Review Letters, Vol.96, No. 11, (March 2006), pp. 113002, ISSN 0556-2813

Atwater, H \& Polman, A.(2010), Plasmonics for improved photovoltaic devices, Nature Materials, Vol.9, (February 2010) pp. 205-213, ISSN 1476-1122

Blankenship, R. (2002), Molecular Mechanisms of Photosynthesis, Wiley-Blackwell, ISBN 9780632-04321-7, Oxford, United Kingdom

Bopp, M.; Jia, Y.; Li, L.; Cogdell, R. \& Hochstrasser R. (1997), Fluorescence and photobleaching dynamics of single light-harvesting complexes, Proceedings of the National Academy of Sciences of the United States of America, Vol.94, No.20, (September 1997), pp.10630-10635, ISSN 0027-8424

Brotosudarmo, T.; Hofmann, E.; Hiller, R.; Wörmke, S.; Mackowski, S.; Zumbusch, A.; Bräuchle, C. \& Scheer, H.(2006), Peridinin-chlorophyll-protein reconstituted with chlorophyll mixtures: preparation, bulk and single molecule spectroscopy, FEBS Letters, Vol.580, No.22, (October 2006), pp.5257-5262, ISSN 0014-5793

Brotosudarmo, T.; Mackowski, S.; Hofmann, E.; Hiller, R.; Bräuchle, C. \& Scheer, H. (2008), Relative Binding Affinities of Chlorophylls in Peridinin - Chlorophyll - Protein Reconstituted with Heterochlorophyllous Mixtures, Photosynthesis Research, Vol.95, No.2-3, (February 2008), pp.247-252, ISSN 0166-8595

Bryant, G.; García de Abajo, F. \& Aizpurua, J. (2008), Mapping the plasmon resonances of metallic nanoantennas, Nano letters, Vol.8, No.2, (January 2008), pp.631-636, ISSN 1530-6984

Bujak, L.; Czechowski, N.; Piatkowski, D.; Litvin, R.; Mackowski, S.; Brotosudarmo, T.; Cogdell, R.; Pichler, S. \& Heiß, W. (2011), Absorption Enhancement of LH2 LightHarvesting Complexes Coupled to Spherical Gold Nanoparticles, Applied Physics Letters, Vol.99, No.17, (October 2011), pp. 173701, ISSN 0003-6951.

Carmeli, I; Liberman, I.; Kraverski, L.; Fan, Z.; Govorov, A.; Markovich, G. \& Richter, S. (2010), Broad band enhancement of light absorption in photosystem I by metal nanoparticle antennas, Nano letters, Vol.10, No.6, (May 2010), pp. 2069-2074, ISSN 1530-6984

Chettiar, U.; Nyga, P.; Thoreson, M.; Kildishev, A.; Drachev, V. \& Shalaev, V. (2010), FDTD modeling of realistic semicontinuous metal films, Applied Physics B: Lasers and Optics, Vol.100, No.1, (March 2010), pp. 159-168, ISSN 1432-0649

Czechowski, N.; Nyga, P.; Schmidt, M.; Brotosudarmo, T.; Scheer, H.; Piatkowski D. \& Mackowski, S. (2011), Absorption Enhancement in Peridinin-Chlorophyll-Protein 
Light-Harvesting Complexes Coupled to Semicontinuous Silver Film, Plasmonics, Vol., (2011), pp.1-7, ISSN 1557-1955

Dulkeith, E.; Morteani, A.; Niedereichholz, T.; Klar, T.; Feldmann, J.; Levi, S.; van Veggel, F.; Reinhoudt, D.; Möller, M. \& Gittins, D. (2002), Fluorescence quenching of dye molecules near gold nanoparticles: radiative and nonradiative effects, Physical Review Letters, Vol.89, No.20, (October 2002), pp.203002, ISSN 0556-2813

Govorov, A.; Bryant, G.; Zhang, W.; Skeini, T.; Lee, J.; Kotov, N.; Slocik, J. \& Naik, R. (2006), Exciton-plasmon interaction and hybrid excitons in semiconductor-metal nanoparticle assemblies, Nano letters, Vol.6, No.5, (April 2006), pp. 984-994, ISSN 1530-6984

Govorov, A. (2008), Enhanced optical properties of a photosynthetic system conjugated with semiconductor nanoparticles: the role of Förster transfer. Advanced Materials, Vol.20, No.22, (April 2008), pp.4330-4335, ISSN 1521-4095

Hofmann, C.; Aartsma, T.; Michel, H. \& Köhler, J. (2003), Direct observation of tiers in the energy landscape of a chromoprotein: a single-molecule study, Proceedings of the National Academy of Sciences of the United States of America, Vol.100, No.26, (December 2003), pp.15534-15538, ISSN 0027-8424

Hofmann, E.; Wrench, P.; Sharples, F.; Hiller, R.; Welte, W. \& Diederichs, K. (1996), Structural basis of light harvesting by carotenoids: peridinin-chlorophyll-protein from Amphidinium carterae, Science, Vol.272, No.5269, (June 1996), pp.1788-1791, ISSN 0036-8075

Hulteen, J. and van Duyne, R. (1995), Nanosphere Lithography: A Materials General Fabrication Process for Periodic Particle Array Surfaces, Journal of Vacuum Science and Technology A, Vol.13, No.3, (1995), pp.1553-1558, ISSN 0022-5355

Kim, I.; Bender, S.; Hranisavljevic, J.; Utschig, L.; Huang, L.; Wiederrecht, G. \& Tiede, D. (2011), Metal nanoparticle plasmon-enhanced light-harvesting in a Photosystem I thin film, Nano letters, Vol.11, No.8, (August 2011), pp.3091-3098, ISSN 15306984

Kleima, F.; Hofmann, E.; Gobets, B.; van Stokkum, I.; van Grondelle, R.; Diederich, K. \& van Amerongen, H. (2000), Förster excitation energy transfer in peridinin-chlorophyll aprotein, Biophysical Journal, Vol.78, No.1, (January 2000), pp.344-353, ISSN 00063495

Krajnik, B.; Schulte, T.; Piatkowski, D.; Czechowski, N.; Hofmann, E. \& Mackowski, S. (2011), SIL-based Confocal Fluorescence Microscope for Investigating Individual Nanostructures, Central European Journal of Physics, Vol.9, No.2, (2011), 293-299, ISSN 1895-1082

Krueger, B.; Lampoura, S.; van Stokkum, I.; Papagiannakis, E.; Salverda, J.; Gradinaru, C.; Rutkauskas, D.; Hiller, R. \& van Grondelle, R. (2001), Energy transfer in the peridinin chlorophyll-a protein of Amphidinium carterae studied by polarized transient absorption and target analysis, Biophysical Journal, Vol.80, No.6, (June 2001), pp.2843-2855, ISSN 0006-3495

Lakowicz, J. (2006), Plasmonics in biology and plasmon-controlled fluorescence, Plasmonics, Vol.1, No1., (2006), pp. 5-33, ISSN 1557-1955 
Link, S. and El-Sayed, M. (1999), Size and Temperature Dependence of the Plasmon Absorption of Colloidal Gold Nanoparticles, Journal of Physical Chemistry B, Vol.103, No.21, (May 1999), pp.4212-4217, ISSN 1520-6106

Mackowski, S.; Wörmke, S.; Brotosudarmo, T.; Jung, C.; Hiller, R.; Scheer, H. \& Bräuchle, C., Biophysical Journal, Vol. 93, No.9, (August 2007), pp. 3249-3258, ISSN 0006-3495

Mackowski, S.; Wörmke, S.; Maier, A.; Brotosudarmo, T.; Harutyunyan, H.; Hartschuh, A.; Govorov, A.; Scheer, H. \& Bräuchle, C. (2008), Metal-enhanced fluorescence of chlorophylls in single light - harvesting complexes, Nano letters, Vol.8, No.2, (February 2008), pp. 558-564, ISSN 1530-6984

Mackowski, S. (2010), Hybrid Nanostructures for Efficient Light Harvesting, Journal of Physics: Condensed Matter, Vol.22, No.19, (April 2010), pp.193102/1-17, ISSN 09538984

Maier, S. (2004). Plasmonics: fundamentals and applications, Springer Science + Business Media LLC, ISBN 978-0387-37825-1, New York, USA

McDermott, G.; Prince, S.; Freer, A.; Hawthornthwaite-Lawless, A.; Papiz, M.; Cogdell, R. \& Isaacs, N. (1995), Crystal structure of an integral membrane light-harvesting complex from photosynthetic bacteria, Nature, Vol.374, (April 1994), pp. 517-521, ISSN 0028-0836

Nieder, J.; Bittl, R. \& Brecht, M. (2010), Fluorescence studies into the effect of plasmonic interactions on protein function, Angewandte Chemie International Edition,Vol.49, No.52, (November 2010), pp. 10217-10220, ISSN 1521-3773

Polivka, T. \& Sundström, V. (2004), Ultrafast dynamics of carotenoid excited states-from solution to natural and artificial system, Chemical Reviews, Vol.104, No.4, (February 2004), pp.2021-2071, ISSN 0009-2665

Polívka, T.; Pascher, T.; Sundström, V. \& Hiller, R. (2005), Tuning energy transfer in the peridinin-chlorophyll complex by reconstitution with different chlorophylls, Photosynthesis Research, Vol.86, No.1-2, (2005), pp.217-227, ISSN 0166-8595

Ray, K.; Badugu, R. \& Lakowicz, J. (2006), Metal-Enhanced Fluorescence from CdTe Nanocrystals: A Single-Molecule Fluorescence Study, Journal of American Chemical Society, Vol.128, No.28, (June 2006), pp.8998-8999, ISSN 0163-3864

Scheuring, S.; Sturgis, J.; Prima, V.; Bernadac, A.; Lévy, D. and Rigaud. J. (2004), Watching the photosynthetic apparatus in native membranes, Proceedings of the National Academy of Sciences of the United States of America, Vol.101, No.31, (August 2004), pp.11293-11297, ISSN 0027-8424

van Oijen, A.; Ketelaars, M.; Köhler, J.; Aartsma T. \& Schmidt, J. (1999), Unraveling the electronic structure of individual photosynthetic pigment-protein complexes, Science, Vol.285, No.5426, (July 1999), pp.400-402, ISSN 0036-8075

Wörmke, S.; Mackowski, S.; Jung, C.; Ehrl, M.; Zumbusch, A.; Brotosudarmo, T.; Scheer, H.; Hofmann, E.; Hiller R. \& Bräuchle, C. (2007a), Monitoring Fluorescence of Individual Chromophores in Peridinin-Chlorophyll-Protein Complex Using Single Molecule Spectroscopy, Biochimica et Biophysica Acta - Bioenergetics, Vol.1767, No.7, (July 2007), pp.956-964, ISSN 0006-3002

Wörmke, S.; Mackowski, S.; Brotosudarmo, T.; Garcia, T.; Braun, P.; Scheer, H.; Hofmann, E. \& Bräuchle, C. (2007b), Detection of single biomolecule fluorescence excited 
through energy transfer: Application to light-harvesting complexes, Applied Physics Letters, Vol.90, No.19, (May 2007), pp.193901, ISSN 0003-6951

Wörmke, S.; Mackowski, S.; Schaller, A.; Brotosudarmo, T.; Johanning, S.; Scheer, H. \& Bräuchle C. (2008), Single Molecule Fluorescence of Native and Refolded PeridininChlorophyll-Protein Complexes, Journal of Fluorescence, Vol.18, No. 3-4, (2008), pp. 611-617, ISSN 1053-0509 


\title{
Hollow Nano Silica: Synthesis, Characterization and Applications
}

\author{
N. Venkatathri \\ Department of Chemistry, National Institute of Technology, Andhra Pradesh, \\ India
}

\section{Introduction}

Since the discovery of mesoporous silica molecular sieves by Beck et al. (Beck et al., 1992; Kresge et al., 1992), mesoporous materials have opened many new possibilities for application in the fields of catalysis (Tanev et al., 1994), separation, and nanoscience (Wu \& Bein, 1994; Agger et al., 1998; Li et al., 2003; Yu et al., 2005]. In recent years, fabrication of silica materials with designed structure (e.g. thin films, monoliths, hexagonal prisms, toroids, discoids, spirals, dodecahedron and hollow sphere shapes) is an important research in modern materials chemistry. Among them the fabrication of monodispersed hollow spheres with control size and shape is fastest developing area (Schacht et al., 1996; Bruinsma et al., 1997; Fowler et al., 2001). It is generally accepted that hollow sphere with mesopores will exhibit more advantages in mass diffusion and transportation as compared with conventional hollow spheres with solid shell. They can serve as a small container for application in catalysis and control release studies (Mathlowitz et al., 1997; Huang \& Remsen, 1999). The methods currently used to fabricate a wide range of stable hollow spheres include nozzle reactor processes, emulsion/phase separation, sol-gel processing, and sacrificial core techniques. The fabrication of hollow spheres has been greatly impacted by the layer-by-layer (LbL) self-assembly technique (Decher, 1997). This method allows the construction of composite multilayer assemblies based on the electrostatic attraction between nanoparticles and oppositely charged polyions. By varying the synthetic methodology and reactants, it is highly probable to achieve the materials with interesting morphology and properties.

The presence of pores of uniform size lined with silanol groups confers these mesoporous materials as a potential candidate for hosting a variety of guest chemical species, such as organic molecules, semiconductor clusters, and polymers (Moller \& Bein, 1998). For example, MCM-41 was reported as a drug delivery system (Vallet-Regi et al., 2001). Ibuprofen has been shown to readily adsorb from an $n$-hexane solution into the porous matrix of MCM-41, and to slowly release into a solution simulating physiological fluid. Furthermore, it has been found that in this host/guest system there is a strong interaction between the silanol groups and the carboxylic acid of the ibuprofen molecule. Having proven the feasibility of this system for drug retention and delivery, further effort should be made in gaining control of the amount of drug delivered, and its release rate. It can be thought that this delivery rate could be modulated by modifying the interaction between the 
confined molecule and the mesoporous matrix with different morphology. Here, one of the advantages of nanocuboids compared to conventional mesoporous materials is reflected in their much higher storage capacity. Ibuprofen with the molecules size of $1.0 \times 0.6 \mathrm{~nm}$ was used to examine the storage capacity.

Nanomaterials are the talk of today's Materials researchers. Mesoporous hollow silica spheres were recently invented. It is important due to the drug storage property. Synthesis of mesoporous silica nano hollow cuboids is the very recent advancement (Venkatathri et al., 2008) in this category. In the present invention, the physicochemical property of mesoporous silica's, Nanocrystalline MCM-41 and Nanohollow cuboids were compared. It is found that Nanohollow cuboids store much more drug molecules say Ibuprofen.

\section{Experimental}

Silica Nanohollow cuboids are synthesized as follows. $3.57 \mathrm{ml}$ of triethanolamine (TEtA, $98 \%$, Aldrich, USA) was added to a solution containing $74 \mathrm{ml}$ of ethanol $(99 \%$, Aldrich, USA) and $10 \mathrm{ml}$ of deionized water. $6 \mathrm{ml}$ of tetraethoxyorthosilicates (TEOS, 98\%, Aldrich, USA) was added to the above prepared mixture at $298 \mathrm{~K}$ with vigorous stirring. The reaction mixture was stirred for another $1 \mathrm{~h}$. A solution containing $5 \mathrm{ml}$ of TEOS and $2 \mathrm{ml}$ of octadecyltrimethoxy silane (C18TMS, $90 \%$, Aldrich, USA) was added to the above solution (11.4 $\mathrm{SiO}_{2}$ : 6 TEtA: 1 C18TMS: $149 \mathrm{H}_{2} \mathrm{O}: 297.5 \mathrm{EtOH}$ ) and further reacted for $24 \mathrm{~h}$. The resulting octadecyl group incorporated silica nanocomposite was retrieved by centrifugation. The sample was washed several times with distilled water, dried and calcined at $823 \mathrm{~K}$ for $8 \mathrm{~h}$ in air to obtain hollow cuboids silica material.

Nanocrystalline Silica MCM-41 is synthesized as follows. Cetyltrimethylammonium bromide was dissolved in $120 \mathrm{~g}$ of deionized water to yield a $0.055 \mathrm{~mol} \mathrm{l}^{-1}$ solution, and $9.5 \mathrm{~g}$ of aqueous ammonia $(25 \mathrm{wt} \%, 0.14 \mathrm{~mol})$ was added to the solution. While stirring, $10 \mathrm{~g}$ of tetraethoxy silane $(0.05 \mathrm{~mol})$ was added slowly to the surfactant solution over a period of 15 min resulting in a gel with the following molar composition: 1 TEOS: 0.152 cetyltrimethylammonium bromide; $2.8 \mathrm{NH}_{3}$ : $141.2 \mathrm{H}_{2} \mathrm{O}$. The mixture was stirred for one hour then the white precipitate was filtered and washed with $100 \mathrm{ml}$ of deionized water. After drying at $363 \mathrm{~K}$ for $12 \mathrm{~h}$, the sample was heated to $823 \mathrm{~K}$ (rate $1 \mathrm{~K} \mathrm{~min}^{-1}$ ) in air and kept at this temperature for $5 \mathrm{~h}$ to remove the template.

X-ray diffractograms (XRD) were recorded on Rigaku Multiplex diffractometer using $\mathrm{Cu}$ $\mathrm{K} \alpha$ radiation and a proportional counter as detector. A divergence slit of $1 / 328$ on the primary optics and an anti-scatter slit of $1 / 168$ on the secondary optics were employed to measure data in the low angle region. The particle size and shape were analyzed by a Scanning electron microscope (SEM), Topcon, SM-300. Transmission electron micrographs (TEM) of the samples were scanned on a on a JEOL JSM-2000 EX electron microscope operated at $200 \mathrm{kV}$. The samples for TEM were dispersed in isopropyl alcohol, deposited on a Cu-grid and dried. Thermogravimetry (TG) analysis of the crystalline phase was performed on an automatic derivatograph (Setaram TG 92). The specific surface area (BET) of the samples was determined using a Micromeritics ASAP 2010 volumetric adsorption analyzer. Before $\mathrm{N}_{2}$ adsorption samples was evacuated in vacuum at $573 \mathrm{~K}$. The data points of $\mathrm{p} / \mathrm{p}_{0}$ in the range of about $0.05-0.3$ were used in the calculations. The 
Fourier transform Infrared (FT-IR) spectra in the framework region were recorded in the diffuse reflectance mode (Nicolet $60 \mathrm{SXB}$ ) using 1:300 ratio of sample with $\mathrm{KBr}$, pellet. Ultraviolet - visible (UV-Vis.) spectroscopic analysis were carried out using Shimadzu, UV-2450 spectrometer.

Ibuprofen (IBU) drug (Ranbaxy Chem. LTD., 99\%) was dissolved in hexane solution at a concentration of $30 \mathrm{mg} / \mathrm{ml} .1 .0 \mathrm{~g}$ nanocuboids or MCM- 41 was added into $50 \mathrm{ml}$ IBU hexane solution at room temperature. Sealing the vials to prevent the evaporation of hexane, then the mixture was stirred for $24 \mathrm{~h}$. The nanocuboids or MCM- 41 adsorbed with IBU was separated from this solution by centrifugation and dried under vacuum at $60{ }^{\circ} \mathrm{C}$. Filtrates $(1.0 \mathrm{ml})$ was extracted from the vial and diluted to $10 \mathrm{ml}$, and then was analyzed by $\mathrm{UV} / \mathrm{vis}$ spectroscopy at a wavelength of $235-320 \mathrm{~nm}$.

\section{Results and discussion}

The X-ray diffraction pattern calcined MCM-41 and hollow cuboids are given in Fig. 1a,b. The pattern shows their identity. The pattern from as-synthesized sample did not change much on calcinations. Both the as-synthesized and calcined patterns of hollow cuboid shows three Bragg diffraction peaks, which can be assigned to the $\left(\begin{array}{lll}1 & 0 & 0\end{array}\right),\left(\begin{array}{lll}1 & 1 & 0\end{array}\right)$ and $\left(\begin{array}{lll}2 & 0 & 0\end{array}\right)$ reflections of a hexagonal symmetry structure $(P 6 \mathrm{~mm})$ typical for MCM-41. $d$ spacing and unit cell parameter $\left(\mathrm{a}_{0}\right)$ calculated from the XRD data are $12.6 \mathrm{~nm}$ and $14.54 \mathrm{~nm}$ respectively (Grun et al., 1999).

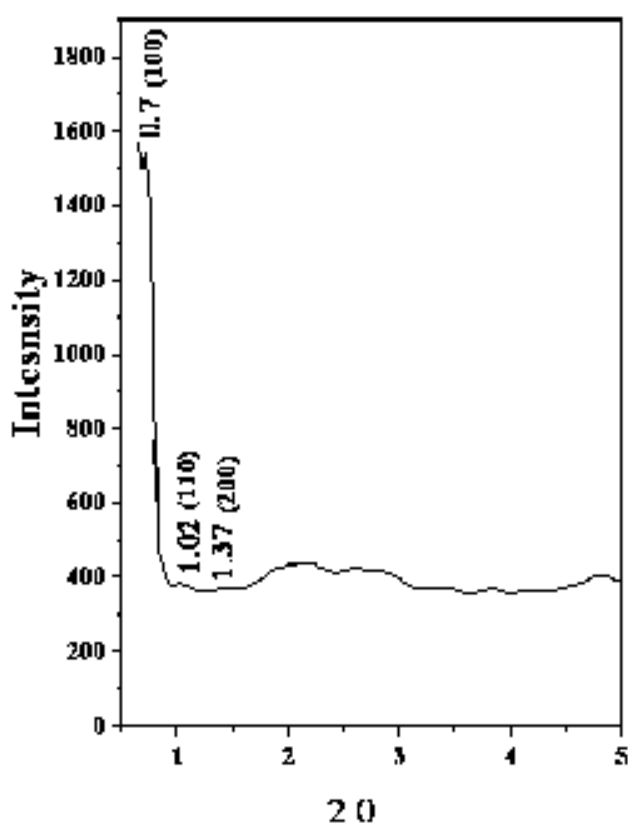

a

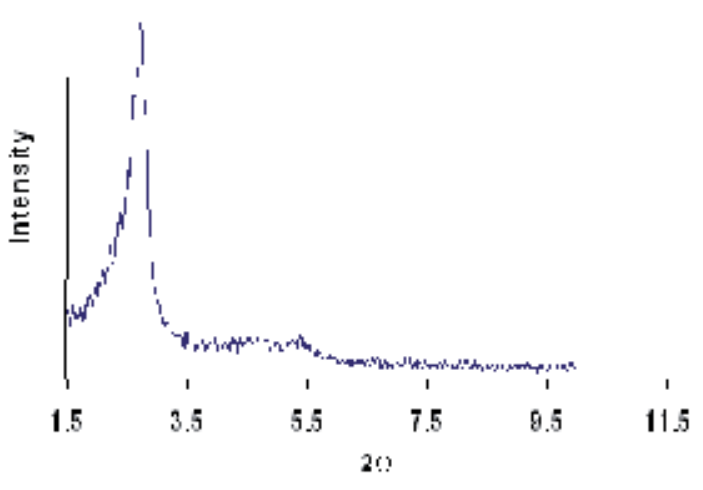

b

Fig. 1. X-ray diffraction pattern of calcined mesoporous silicas a) Nanohollow cuboids and b) Nanocrystalline MCM-41 
Fig.2a,b shows the scanning electron micrograph of MCM-41 and hollow cuboids. MCM-41 particle size is $200-500 \mathrm{~nm}$ with spherical shape. Hollow cuboids are aggregate of cuboids with $500 \mathrm{~nm}$ particle size.

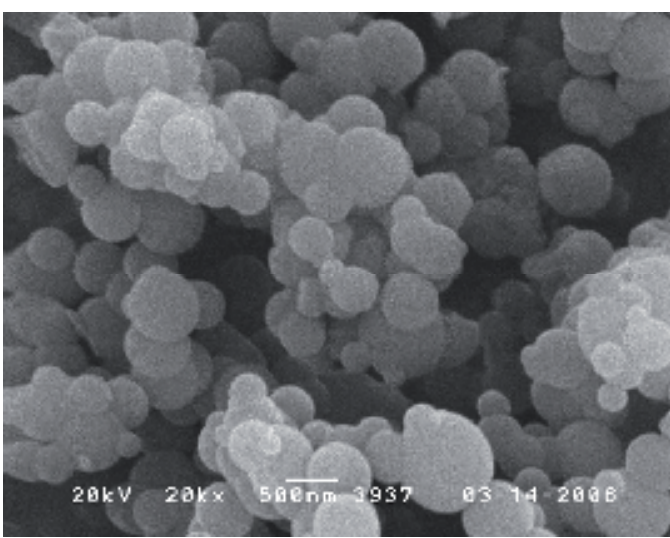

a

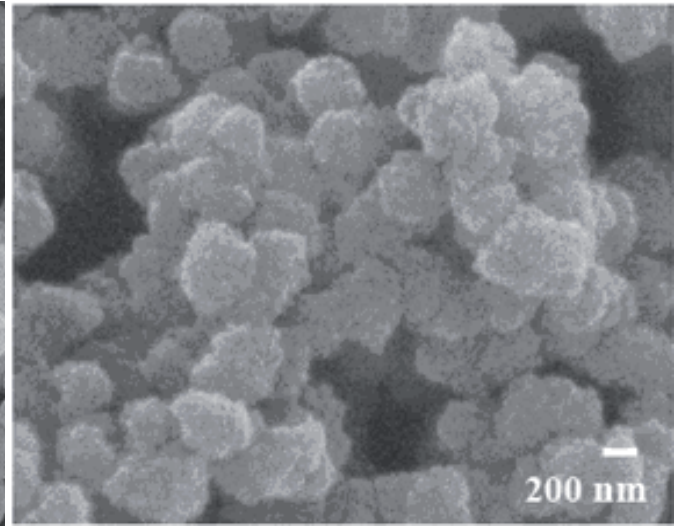

$\mathrm{b}$

Fig. 2. Scanning electron micrograph of calcined mesoporous silicas

a) NanocrystallineMCM-41 and b) Nanohollow cuboids

Transmission electron micrograph of MCM-41 and hollow cuboids are given in Fig. 3a,b. MCM-41 shows hexagonal array of channels characteristic of Mesoporous structure. By Fast Fourier Transform (FFT) of the TEM images, we estimate a unit cell dimension of $3.3 \mathrm{~nm}$. TEM of cuboids shows core and shell structure. It can be seen from the images that the average inner diameter of the cuboids are nearly $100 \mathrm{~nm}$, with outer shell thickness $50 \mathrm{~nm}$. The particle sizes are uniform similar to SEM results. This distinguished pore channel arrangement with most of them running through the shell, are favorable for the access of guest molecules.

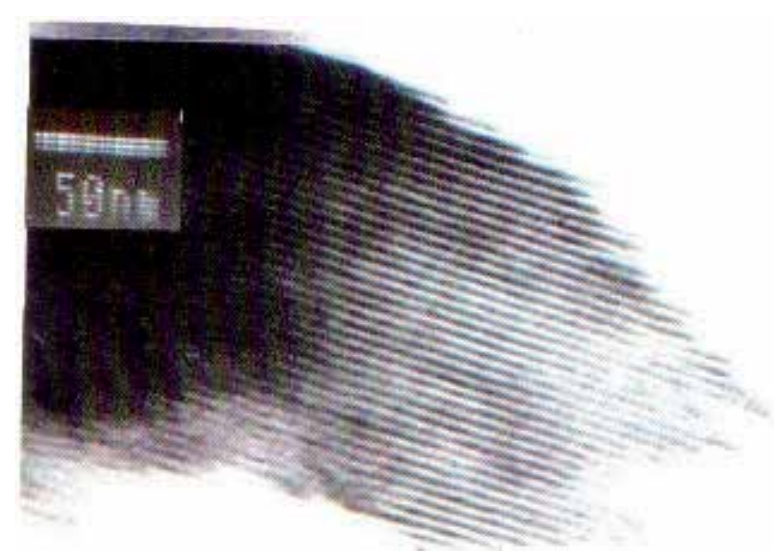

a

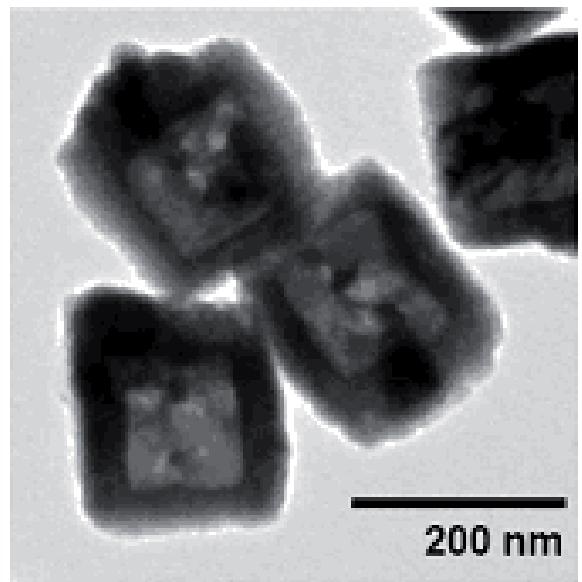

$\mathrm{b}$

Fig. 3. Transmission electron micrograph of calcined mesoporous silicas

a) NanocrystallineMCM-41 and b) Nanohollow cuboids 


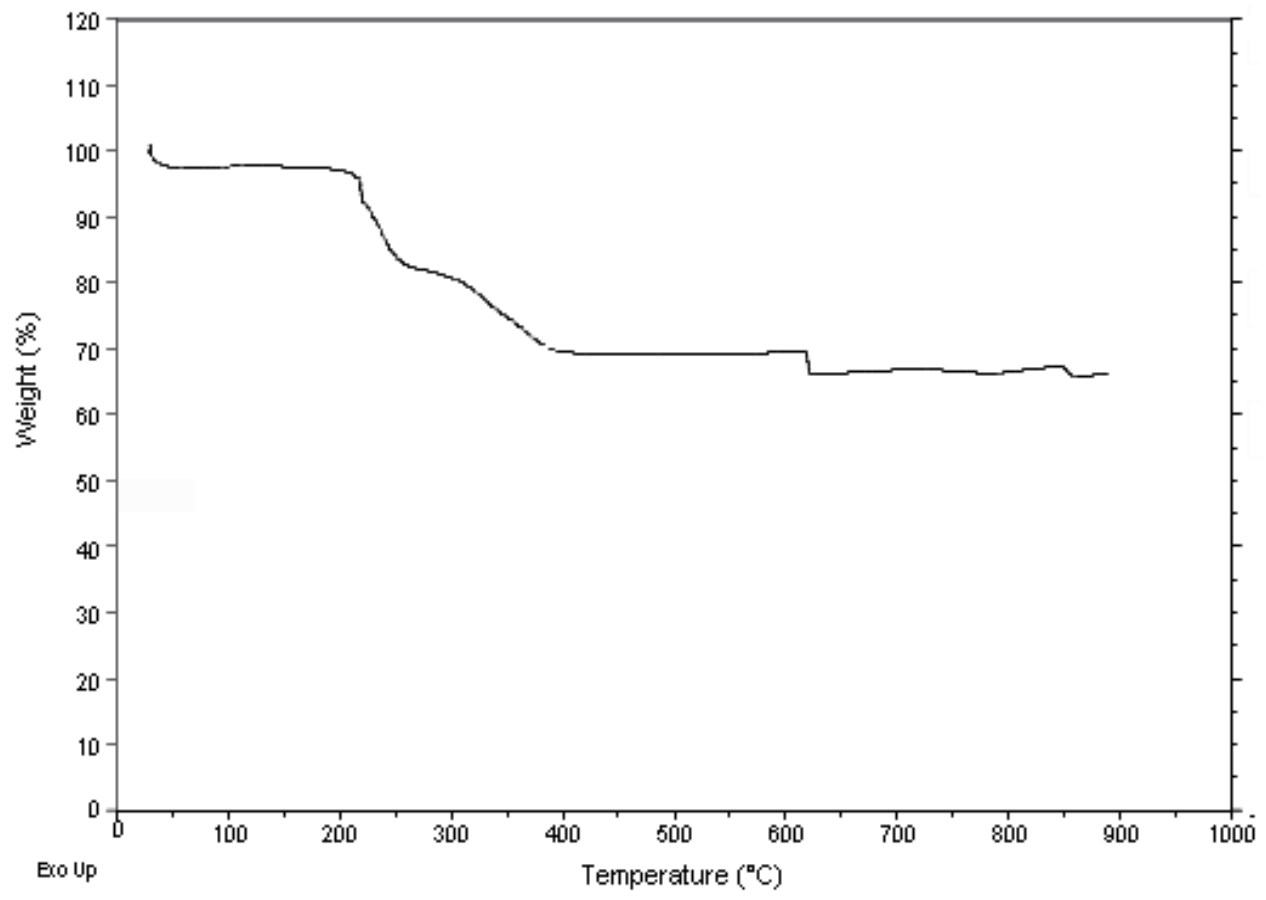

a

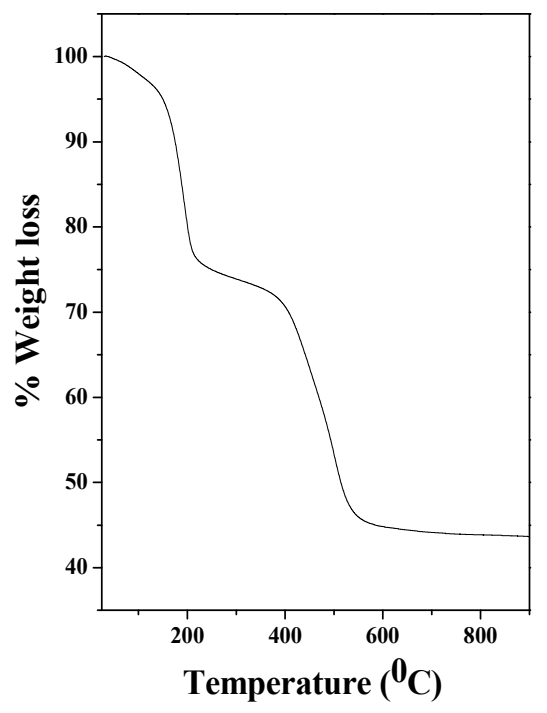

$\mathrm{b}$

Fig. 4. Thermogravimetric profile of as-synthesized mesoporous silicas,

a) Nanocrystalline MCM-41 and b) Nanohollow cuboids. 
The Thermogravimetry of MCM-41 and hollow cuboids were given in Fig. 3a,b. MCM-41 shows the $30 \%$ loss at $25-625{ }^{\circ} \mathrm{C}$ due to the loss of template. The initial endothermic loss is due to loss of physisorbed water. Later the exothermic loss is due to oxidative decomposition of template. According to the curve the cuboids began to lose its weight at the beginning of heating, likely because of desorption of the physisorbed water and ethanol. It eliminates almost $25 \%$ of its weight in the temperature range $25-200^{\circ} \mathrm{C}$ and losses almost $30 \%$ weight in the temperature range of $200-500{ }^{\circ} \mathrm{C}$. The later weight loss is due to the oxidative decomposition of the template.

Typical nitrogen sorption isotherms for MCM-41 and hollow cuboids are shown in Fig. 5a,b. In case of MCM-41, the nitrogen isotherms indicate a linear increase of the amount of adsorbed nitrogen at low pressures $(\mathrm{P} / \mathrm{Po}=0.35)$. The resulting isotherm can be classified as a type IV isotherm with a type $\mathrm{H} 2$ hysteresis, according to the IUPAC nomenclature (Fujiwara et al., 2004; Brunauer et al., 1940; de Boer, 1958; IUPAC, 1957). The steep increase in nitrogen uptake at relative pressures in the range between $\mathrm{P} / \mathrm{Po}=0.40$ and 0.60 is reflected in a narrow pore size distribution. Thus, the variation of the catalyst in the solution during the growth process enables one to adjust and to control pore structural parameters such as the specific surface area $\left(900 \mathrm{~m}^{2} / \mathrm{g}\right)$, the specific pore volume $\left(1.29 \mathrm{~cm}^{3} / \mathrm{g}\right)$, and the average pore diameter $\left(239 \mathrm{~A}^{\circ}\right)$ and medium pore width $\left(302 \mathrm{~A}^{\mathrm{o}}\right)$. The nitrogen adsorption/desorption isotherms of nanocuboid is of type IV nature (Fig. 5b) and exhibited a H1 hysteresis loop, which is typical of mesoporous solids (Wu et al., 2002). Furthermore, the adsorption branch of the isotherm showed a sharp inflection at a relative pressure value of about 0.68 . This is characteristic of capillary condensation within uniform pores. The position of the inflection point indicates mesoporous structure, and the sharpness of these steps indicates the uniformity of the mesoporous size distribution. Correspondingly, the pore size distribution of the calcined sample shows a narrow pore distribution with a mean value of $1.90 \mathrm{~nm}$. The sample with a specific surface area of $792 \mathrm{~m}^{2} / \mathrm{g}$ and pore volume of $0.51 \mathrm{~cm}^{3} / \mathrm{g}$ was obtained using the Brunauer-Emmett-Teller (BET) and Barrett- JoynerHalenda (BJH) methods, respectively.

The Fourier transform Infrared spectra of as-synthesized MCM-41 and hollow cuboids are shown in Fig. 6a,b. Peaks around 1700 and $3430 \mathrm{~cm}^{-1}$ corresponding to the carboxyl and hydroxyl groups ( $\mathrm{Li}$ et al., 2002) respectively. The adsorption peak belonging to the Si-O stretching vibration of Si-OH bond appears at $960 \mathrm{~cm}^{-1}$ (Shan et al., 2004). The weak peaks at 2855 and $2920 \mathrm{~cm}^{-1}$ belong to the stretching vibrations of $\mathrm{C}-\mathrm{H}$ bonds, which show a few organic groups are adsorbed on the spheres. The peaks for carboxyl, hydroxyl and C-H vibrations are weak in MCM-41, shows the lesser organics, resulting of organic template. The strong peaks near 1100, 802 and $467 \mathrm{~cm}^{-1}$ agree to the Si-O-Si bond which implies the condensation of silicon source (Agger et al., 1998).

Fig. 7 shows the UV ray absorbance spectra of $30 \mathrm{mg} / \mathrm{ml}$ ibuprofen hexane solutions (Zhu et al., 2005) before (a) and after (b) the interaction with nanocuboid and (c) MCM-41. The drug put in contact with nanocuboid and MCM-41 does not show any sign of degradation, since the positions of the absorbance maxima remain unchanged after the interaction and no new bands appear. The Ultraviolet ray absorbance intensity of filtrate decreases after Ibuprofen solution interaction with nanocuboids and MCM-41. This shows the remaining Ibuprofen is adsorbed over the molecular sieves. It was calculated that $561.8 \mathrm{mg}$ and $270.5 \mathrm{mg}$ ibuprofen 


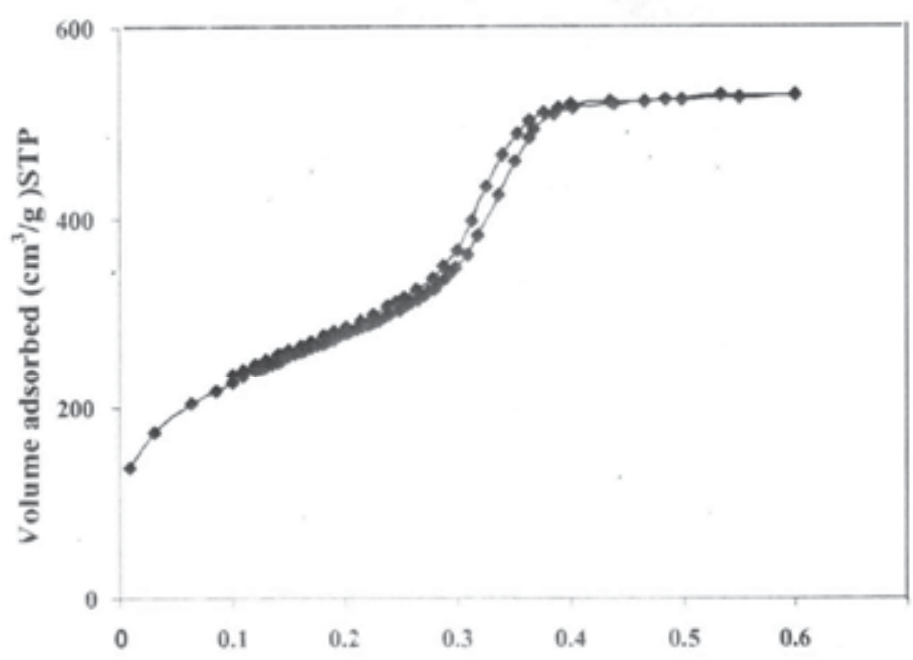

Relative pressure $\left(p / p_{0}\right)$

a

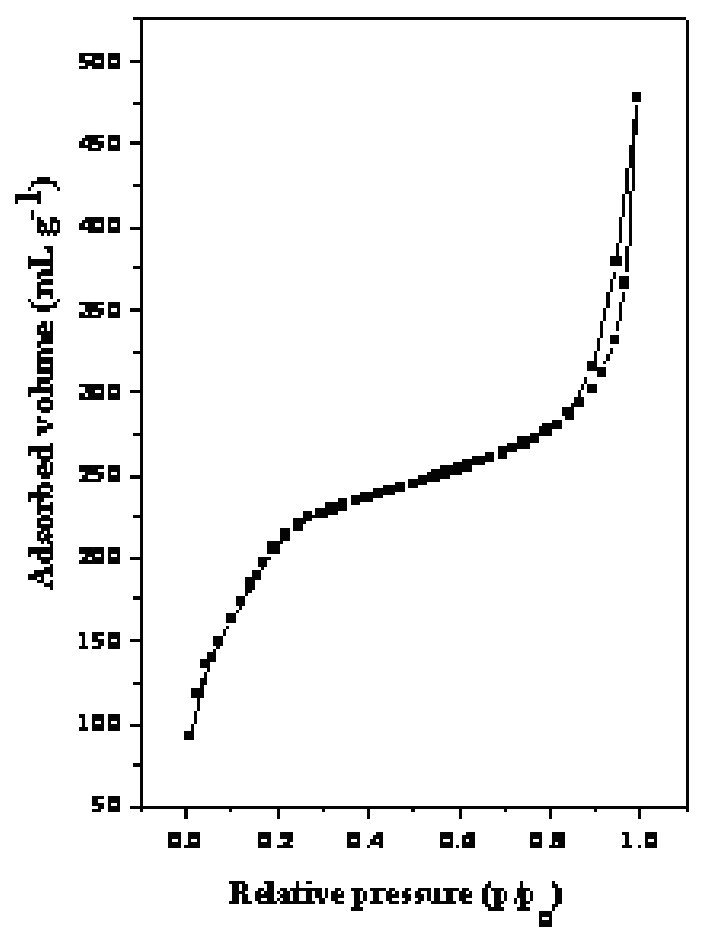

b

Fig. 5. Nitrogen adsorption/desorption isotherms of calcined mesoporous silicas, a) Nanocrystalline MCM-41 and b) Nanohollow cuboids. 

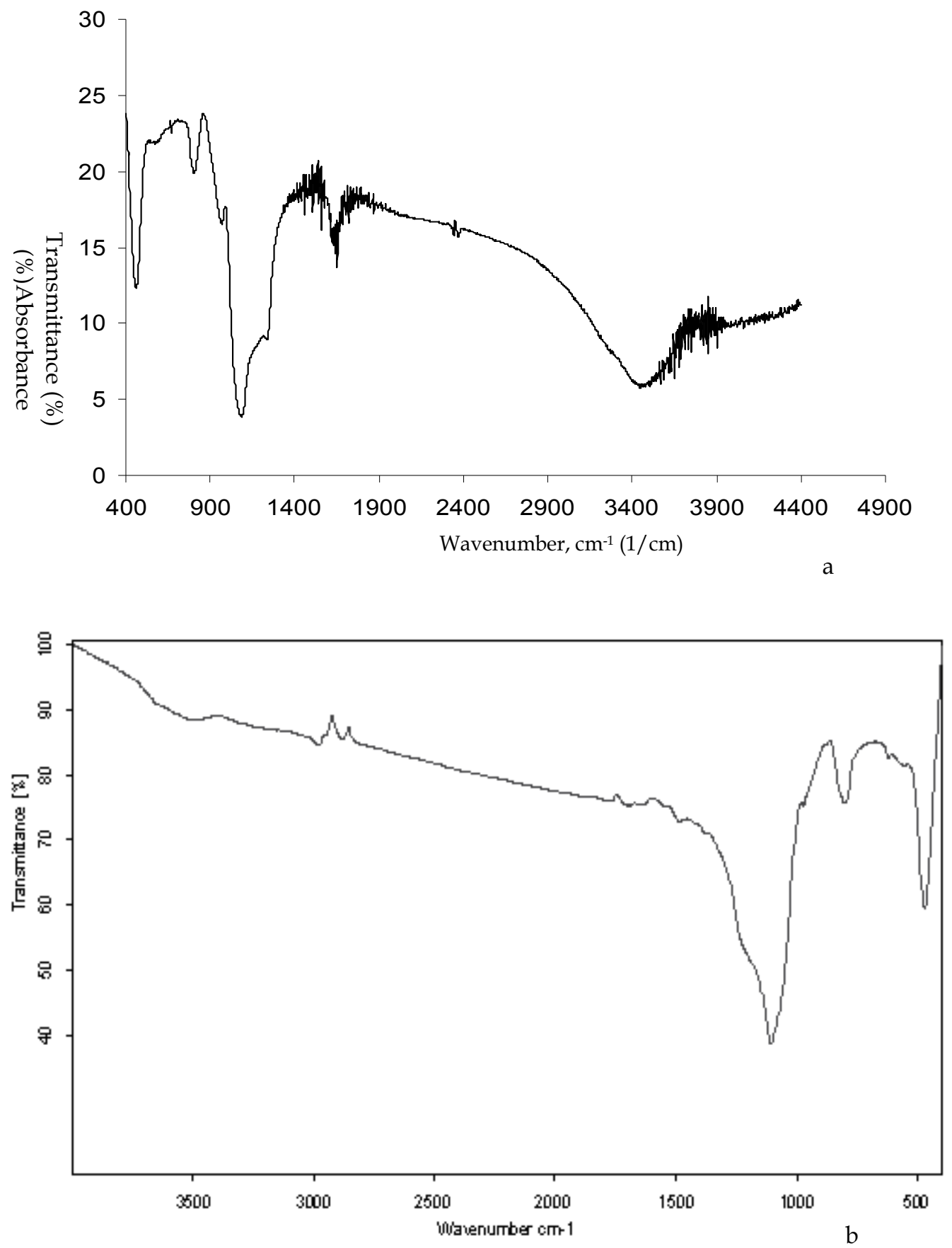

Fig. 6. Fourier transform Infrared spectroscopic analysis of as-synthesized mesoporous silicas, a) Nanohollow cuboids and b) a) Nanocrystalline MCM-41. 


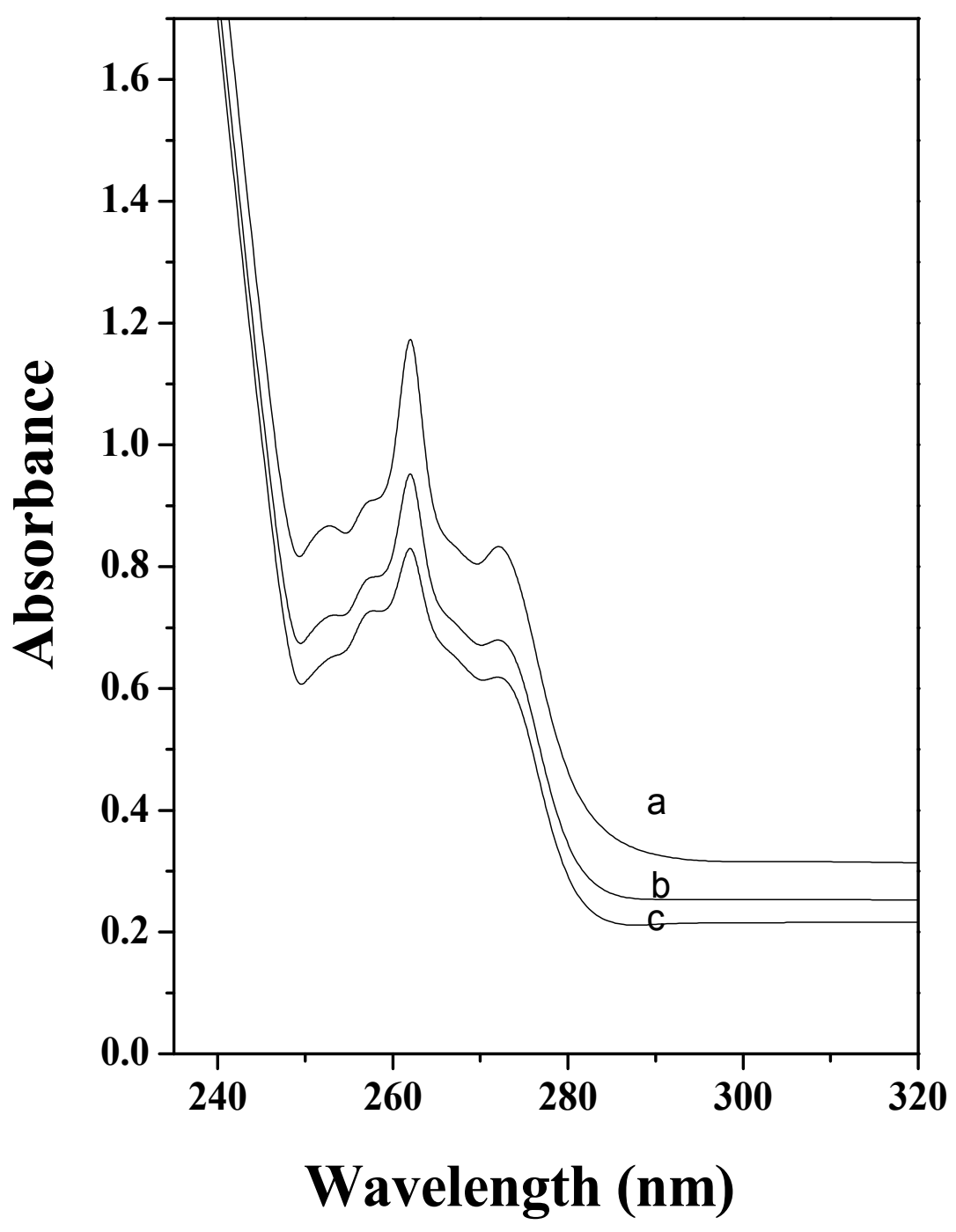

Fig. 7. The Ultraviolet - visible absorbance spectra of $30 \mathrm{mg} / \mathrm{ml}$ ibuprofen hexane solutions before (a) and after (b) the interaction with calcined mesoporous silica Nanohollow cuboids and (c) mesoporous Silica Nanocrystalline MCM-41. 
molecules can be stored in per gram nanocuboid and MCM-41, respectively from Ultraviolet ray absorbance according to Beer-Lambert Law (Jeffery et al., 1997). The surface area and pore volume of MCM-41 and nanocuboid are very close to each other, but much more ibuprofen molecules can be stored into nanocuboid than into MCM-41. This illustrates that the hollow cores could hold more than half drug molecules of total storage amount.

Tetraethylorthosilicate (TEOS) was hydrolyzed in the presence of basic triethanolamine. However the hydrolysis rate of TEOS using triethanolamine is very slow as compared to hydrolysis with $\mathrm{NH}_{3}$. For example, using the molar ratio described above, TEOS can be hydrolyzed in $2 \mathrm{~h}$ using $\mathrm{NH}_{3}$ whereas triethanolamine took $24 \mathrm{~h}$ to hydrolyze the TEOS. In the present synthetic recipe, triethanolamine not only act as a catalyst for the hydrolysis but also it acts as a reactant. The hydrolyzed silica monomers react with triethanolamine to give respective oxide. Such silicate-triethanolamine adduct are held together with hydrogen bonding. The triethanolamine sandwiched silica layer condensed and form nanocuboids. MCM-41 is reported to crystallize by self assembly of surfactant/template (Grun et al., 1999) in similar to nanocuboids.

\section{Conclusion}

A novel procedure was invented to synthesize mesoporous Silica Nano hollow cuboids with uniform size and morphology. It is characterized by various physicochemical techniques. The results are compared with Nanocrystalline silica MCM-41. Transmission electron micrographs shows, $150 \mathrm{~nm}$ hollow diameter and $50 \mathrm{~nm}$ shell thickness in hollow cuboids. Further, the mesoporous silica Nanohollow cuboids were found to store much more guest molecules than conventional mesoporous silica Nanocrystalline MCM-41.

\section{Acknowledgement}

The author thanks Director, National Institute of Technology, Warangal, India for constant encouragement throughout the course of work.

\section{References}

Agger J.R., Anderson M.W., \& Pemble M.E., 1998, Growth of Quantum - confined Indium Phosphide inside MCM-41. J. Phys. Chem. B, vol. 102, 1998, pp. 3345-3353.

Beck J.S., Vartuli J.C., . Roth W.J., Leonawicz M.E., \& Kresge C.T., 1992, A new family of mesoporous molecular sieves prepared with liquid crystal templates. J. Am. Chem. Soc., vol. 114, 1992, pp. 10834-10843.

Bruinsma P.J., Kim A.Y., \& Liu J., 1997, Mesoporous silica synthesized by solvent Evaporation: spun Fibers and spray-Dried Hollow spheres. Chem. Mater., vol. 9, 1997, pp. 2507-2512.

Brunauer S., Deming L.S., Deming W.S., \& Teller E., 1940, On a Theory of the vander waals Adsorption of Gases. J. Am. Chem. Soc., vol. 62, 1940, pp. 1723-1732.

de Boer J.H., 1958, The Structure and Properties of Porous Materials, Butterworths, London, 1958.

Decher G., 1997, Fuzzy Nano assemblies : Toward Layered polymeric Multicomposites. Science, vol. 277, 1997, pp. 1232-1237,. 
Fowler C.E., Khushalani D., \& Mann S., 2001, Interfacial synthesis of hollow microsphere of meso structured silica. Chem. Comm., 2001, pp. 2028-2029.

Fujiwara M., Shiokawa K., Tanaka Y., \& Nakahara Y., 2004, Preparation and formation Mechanism of Silica Microcapsules (Hollow sphere) by water/oil/water Interfacial Reaction. Chem. Mater., vol. 16, 2004, pp. 5420-5426.

Grun M., Unger K.K., Matsumoto A., \& Tsutsumi K., 1999, Novel pathways for the preparation of mesoporous MCM-41 materials : Control of porosity and morphology. Micropor. Mesopor. Mat., vol. 27, 1999, pp. 207-216.

Huang H., \& Remsen E.E., 1999, Nanocages Derived from shell cross-linked Micelle Templates. J. Am. Chem. Soc., vol. 121, 1999, pp. 3805-3806.

IUPAC, Reporting Physisorption Data for Gas/Solid Systems, 1957, Pure Appl. Chem., vol. 87, 1957, pp. 603-608.

Jeffery G.H., Bassett J., Mendham J., \& Denney R.C., 1997, Vogel's Textbook of Quantitative Chemical Analysis, Addition Wesley Longman Limited, Edinburgh Gate, Harlow, Essex CM20 2JE, England, 1997, pp. 649-655.

Kresge C.T., Leonowicz M.E., Roth W.J., Vartuli J.C., \& Beck J.S., 1992, Ordered mesoporous molecular sieves synthesized by a liquid - crystal template mechanism. Nature, vol. 359, 1992, pp. 710-712.

Li Y., Shi J., Hua Z., Chen H., Ruan M., \& Yan D., 2003, Hollow spheres of Mesoporous Aluminosilicate with a three- dimensional pore Network and Extraordinarily High Hydrothermal stability. Nano Lett., vol. 3, 2003, pp. 609-612.

Li Y., Xu C., Wei B., Zhang X., Zheng M., Wu D., \& Ajayan P.M., 2002, Self - organized Ribbons of Aligned carbon Nanotubes. Chem. Mater., vol. 14, 2002, pp. 483-485.

Mathlowitz E., Jacob J.S., Jong Y.S., Carino G.P., Chickering D.E., Chaturvedl P., Santos C.A., Vijayaraghavan K., Montgomery S., Bassett M., \& Morrell C., 1997, Biologically erodable microspheres as potential oral drug delivery systems. Nature, vol. 386, 1997, pp. 410-414.

Moller K., \& Bein T., 1998, Inclusion chemistry in Periodic Mesoporous Hosts, Chem. Mater., vol. 10, 1998, pp. 2950-2963.

Schacht S., Huo Q., Voigt-Martin I.G., Stucky G.D., \& Schuth F., 1996, Oil-Water Interface Templating of Mesoporous Macroscale Structures. Science, vol. 273, 1996, pp. 768771.

Shan Y., Gao L., \& Zheng S., 2004, A facile approach to load CdSe nanocrystallites into mesoporous SBA-15. Mater. Chem. Phys., vol. 88, 2004, pp. 192-196.

Tanev P.T., Chibwe M., \& Pinnavaia P.J., 1994, Titanium - containing mesoporous molecular sieves for catalytic oxidation of aromatic compounds. Nature, vol. 368, 1994, pp. 321-323,

Vallet-Regi M., Ra'mila A., del Real R.P., \& Pe'rez-Pariente J., 2001, A New Property of MCM-41 : Drug Delivery System. Chem. Mater., vol. 13, 2001, pp. 308-311.

Venkatathri N., Srivastava R., Yun D.S., \& Yoo J.W., 2008, Synthesis of a novel class of mesoporous hollow silica from organic templates. Micropor. Mesopor. Mat., vol. 112, 2008, pp. 147-152.

Wu C.G., \& Bein T., 1994, Polyaniline wires in oxidant-containing Mesoporous channel hosts. Chem. Mater., vol. 6, 1994, pp. 1109-1112. 
Wu P., Tatsumi T., Komatsu T., \& Yashima T., 2002, Postsynthesis, Characterization and Catalytic Properties in Alkene Epoxidation of Hydrothermally stable Mesoporous Ti-SBA-15. Chem. Mater., vol. 14, 2002, pp. 1657-1664.

Yu K., Guo Y., Ding X., Zhao J., \& Wang Z., 2005, Synthesis of silica nanocubes by sol-gel method. Mat. Lett., vol. 59, 2005, pp. 4013-4015.

Zhu Y., Shi J., Chen H., Shen W., \& Dong X., 2005, A facile method to synthesize novel hollow mesoporous silica spheres and advanced storage property. Micropor. Mesopor. Mat., vol. 84, 2005, pp. 218-222. 


\title{
Synthesis of Titanate and Titanium Dioxide Nanotube Thin Films and Their Applications to Biomaterials
}

\author{
Mitsunori Yada and Yuko Inoue \\ Saga University \\ Japan
}

\section{Introduction}

Recently, titanium compounds with one-dimensional nanostructures, such as nanotubes and nanofibers, have recently attracted much attention. Among these 1-D compounds, nanotubes composed of titanium dioxide and titanate are now being studied actively. Titanium dioxide nanotubes can be synthesized using porous anodic alumina membranes (Imai et al., 1999; Yamanaka et al., 2004), organic molecules (Jung et al., 2002), or polycarbonate membranes (Shin et al., 2004) as templates, or methods involving anodization of titanium metals (Macak et al., 2005). Since the interesting reports by Kasuga et al. (Kasuga et al., 1998; Kasuga et al., 1999) and Chen et al. (Chen et al., 2002), titanate and titanium dioxide nanotubes synthesized using the hydrothermal method have found a wide range of potential uses in photocatalysis (Tokudome et al., 2004; Jiang et al., 2008), dye sensitizing solar batteries (Uchida et al., 2002), hydrogen storage (Bavykin et al., 2005), electrochromism (Tokudome et al., 2005), bonelike apatite formation (Kubota et al., 2004), proton conductors (Thorne et al., 2005), electron field emission characteristic (Miyauchi et al., 2006), photoinduced hydrophilicity (Tokudome et al., 2004), etc.

In order to maximize the characteristics of the nanotube and to use them efficiently, preventing their excessive aggregation and arrangement at larger than micrometer or centimeter size are considered important. Especially, it is important to fabricate thin films composed of nanotubes. Kasuga et al. (Kasuga et al., 2003) reported the fabrication of titanate nanotube thin films by coating a titanate nanotube dispersion liquid to a substrate, and then calcinating the substrate. Tokudome et al. (Tokudome et al., 2004) and Ma et al. (Ma et al., 2004) also reported the fabrication of titanate nanotube thin films using a layerby-layer method. However, neither study had transformed titanate nanotube thin films into titanium dioxide thin films. Kim et al. (Kim et al., 2007) used electrophoretic deposition (EPD) to fabricate 2 - $\mu \mathrm{m}$-thick titanate nanotube thin films, and they transformed the titanate nanotube thin films into titanium dioxide nanotube thin films by calcination. However, these methods involve complicated processes, including (1) synthesis of nanotubes, (2) preparation of a liquid in which the synthesized nanotubes are dispersed, (3) coating of the nanotubes onto a substrate using the prepared liquid, and (4) fixation of the coated nanotubes onto the substrate surface by calcination. Since it is generally difficult to prepare 
a liquid in which nanotubes are uniformly dispersed and that partial aggregation is inevitable, the homogeneity of thin films thus formed is questionable. Moreover, permanent fixation of the thin films onto the substrates is also doubtful. On the other hand, titanate and titanium dioxide nanotube thin films can also be formed on titanium metal by immersing titanium metal as a raw material into $\mathrm{NaOH}$ aqueous solution and then performing hydrothermal treatment (Miyauchi et al., 2006; Tian et al., 2003; Chi et al., 2007; Yada et al., 2007; Guo et al., 2007). The fabrication of titanate nanotube thin films using titanium metal plates was first reported by Tian et al. (Tian et al., 2003). The thin $(\sim 10 \mu \mathrm{m})$ films were detached from the titanium metal plates by hydrothermal reaction for $20 \mathrm{~h}$. In contrast, thin films obtained by a short $(6 \mathrm{~h})$ hydrothermal reaction strongly adhered to the titanium metal plate. Miyauchi et al. (Miyauchi et al. 2006) obtained a titanium dioxide nanotube thin film by hydrothermal treatment on titanium metal, followed by acid treatment and calcination. Although this thin film was fixed onto the substrate, its thickness was only a few hundred nanometers. Therefore, it is clear that titanate and titanium dioxide nanotube thin films tend to detach from the substrates when they become too thick. Chi et al. also reported the fabrication of a sodium titanate nanotube thin film (Chi et al., 2007). However, the thickness of the film was not mentioned in their report, and the sodium titanate nanotubes were not transformed into titanium dioxide nanotubes.

In this chapter, first, we will report the synthesis and organization of sodium titanate nanotube (hereafter referred to as Na-TNT) of size larger than a micrometer, using various titanium metals with controlled shapes of a micrometer size including plate, wire with a diameter of a micrometer, mesh woven from the titanium wire, microspheres, and microtube (Yada et al., 2007). The titanium metal acts as a template for the organization as well as a titanium source. Therefore, the originality of our study is to use titanium metal as a morphology-directing material. In addition, we will report a novel procedure for fixation of Na-TNT thin film on titanium metal (Yada et al., 2007). As a result, the thickness of the sodium titanate nanotube thin film can be adjusted by changing the duration of the hydrothermal reaction and the obtained films are thicker than those reported in previous studies (Miyauchi et al., 2006; Tian et al., 2003). Furthermore, we will also introduce a novel "hydrothermal transcription method" for forming Na-TNT films on various substrates such as Co-Cr alloy and SUS316L (Yada et al., 2008). Transformation of Na-TNT thin films into thin films consisting of anatase nanotube, anatase nanowires, anatase nanoparticles, and rhomboid-shaped anatase nanoparticles are also introduced (Inoue et al. 2010). To obtain an anatase nanotube thin film, it is necessary to slightly modify previously reported methods for synthesizing titanium dioxide nanotube particles.

Next, in this chapter, obtained titanate and titanium dioxide nanotube thin films will be applied to antibacterial biomaterials (Inoue et al., 2010). The nanotube thin film has several advantages: it can be formed on titanium, titanium alloy, Co-Cr alloy, and SUS316L, which are useful for manufacturing surgical instruments and implants such as artificial joints; its thickness can be controlled up to $20 \mu \mathrm{m}$ or more, in contrast to only $1 \mu \mathrm{m}$ for the thickness of the previously reported sodium titanate thin film with a porous network structure; and medicines can be incorporated into the nanotube. It is well known that bacterial infection may occur during surgery because of several factors. For example, during hip-replacement arthroplasty, bacterial infections occur in $1 \%$ to $2 \%$ of operations and usually cause physical and economic burdens for patients, such as re-implantation. As a conventional method for 
preventing infections, antibiotics are administered even in operation rooms with few pathogens. However, this does not prevent every infection. Therefore, imparting antibacterial properties to implants is currently under investigation. There have been reports of the use of apatite coating containing silver on implants by sputtering (Chen et al., 2006), silver-plated implants (Hardes et al., 2007), and gentamicin-hydroxyapatite coating for cementless joints (Alt et al., 2006), all of which have shown antibacterial properties. However, these methods have drawbacks such as the need for expensive instruments and the use of antibiotics that may cause the emergence of resistant bacteria. Therefore, further research is required. In this study, in order to develop more convenient and inexpensive antibacterial implants, silver ions are studied as an antibacterial component along with titanate nanotube formed on the surface of titanium. Silver is one of the most common antibacterial elements and is considered highly safe with high antibacterial activity. Sodium titanates are composed of a titanate framework with a negative electric charge and $\mathrm{Na}^{+}$ions with a positive electric charge. Since they have a cation exchange property, $\mathrm{Na}^{+}$ions can be exchanged with several cations (Kim et al., 1997; Chen et al., 2002; Sun et al., 2003; Bavykin et al., 20006). Therefore, it is considered that sodium titanate can be transformed into silver titanate by exchange of $\mathrm{Na}^{+}$in sodium titanate with $\mathrm{Ag}^{+}$, and the in vivo elution of silver ions from the titanates would be promising for application to antibacterial implants. In addition, it is suggested that the titanate nanotube thin film would be able to possess a larger amount of silver and allow the amount of silver to be controlled more widely as compared with the titanate thin film previously reported (Kim et al., 1996). In this chapter, we will describe the synthesis and characterization of titanate nanotube thin films with silver and the behavior of silver ion elution of the thin films in vitro. We will also describe the antibacterial properties against methicillin-resistant Staphylococcus aureus (MRSA) with a biofilm-forming gene, which is a major concern in actual infections, to investigate the possibility of using synthesized thin films as antibacterial implants.

Finally, we will describe the apatite-forming abilities of titanium compound nanotube thin films by comparing the apatite deposition behaviors of a sodium titanate nanotube thin film (Yada et al., 2007), a titanium dioxide nanotube thin film (Inoue et al., 2010), and a silver nanoparticle/silver titanate nanotube nanocomposite thin film (Inoue et al., 2010), in simulated body fluid (SBF) (Yada et al., 2010). In evaluating the in vivo apatite-forming ability or the osteoconductive property of a material, researchers commonly perform experiments in SBF (Kokubo et al., 2006)). Kim et al. (Kim et al., 1996) first reported the formation of a sodium titanate thin film with a porous network structure on a titanium metal plate by alkali and heat treatment and demonstrated the osteoconductive property of the obtained sodium titanate thin film. Since then, researchers have actively performed many studies on the applications of sodium titanate thin films in implants (Kokubo et al., 1996; Kim et al., 1997; Kim et al., 1997; Nishiguchi et al., 1999; Jonášová et al., 2003; Kim et al., 2003; Muramatsu et al., 2003; Wang et al., 2007; Wang et al., 2008). Similar studies have also been performed on calcium titanate thin films (Hanawa et al., 1997; Hamada et al., 2002; Nakagawa et al., 2005; Kon et al., 2007; Ohtsu et al., 2008), titanium dioxide thin films (Ohtsuki et al., 1997; Wang et al., 2001; Wang et al., 2003; Byon et al., 2007), and a nanohydroxyapatite thin film (Xiong et al., 2010), and the excellent biocompatibilities of these films have been reported. Therefore, titanium compound thin films show tremendous promise for use as implant materials. 


\section{Synthesis and characterization of titanate and titanium dioxide nanotube thin films}

\subsection{Sodium titanate nanotube thin films formed on various shaped titanium metal templates}

\subsubsection{Sodium titanate nanotube thin film formed a titanium plate}

First, the growth and fixation of Na-TNT on titanium plate were investigated. A titanium plate $(20 \mathrm{~mm} \times 20 \mathrm{~mm} \times 2 \mathrm{~mm}$ ) was immersed in $20 \mathrm{ml}$ of $10 \mathrm{~mol} / \mathrm{l}$ aqueous $\mathrm{NaOH}$ solution in a Teflon container and reactions were carried out at $160^{\circ} \mathrm{C}$. After hydrothermal treatment for $20 \mathrm{~h}$, the surface of the plate changed to pale, indicating the formation of a thin film on the titanium plate. In order to wash out $\mathrm{NaOH}$ and the particles that adhered to the surface of the thin film, the plate was washed with water after the reaction. The thin film immediately exfoliated as shown in Fig. 1a, and then a surface with a metallic luster similar to that of titanium metal appeared on the surface of the plate. The greater part of the film was posited to consist of nanotubes with an outer diameter of approximately $8 \mathrm{~nm}$ (Fig. 1b). Through EDX analysis, the mol fraction of $\mathrm{Na} / \mathrm{Ti} / \mathrm{O}$ for the obtained film was determined to be 1:1.947:4.943. The film was thus assumed to be $\mathrm{Na}_{2} \mathrm{Ti}_{4} \mathrm{O}_{9} \cdot \mathrm{H}_{2} \mathrm{O}$, though some titanate structures, such as $\mathrm{A}_{2} \mathrm{Ti}_{2} \mathrm{O}_{5} \cdot \mathrm{H}_{2} \mathrm{O}$ (Yang et al., 2003), $\mathrm{A}_{2} \mathrm{Ti}_{3} \mathrm{O}_{7}$ (Chen et al., 2002), $\mathrm{H}_{2} \mathrm{Ti}_{4} \mathrm{O}_{9} \cdot \mathrm{H}_{2} \mathrm{O}$ (Nakahira et al., 2004), and lepidocrocite titanates (Ma et al., 2003), have been assigned as nanotube constituents $(\mathrm{A}=\mathrm{Na}$ and /or $\mathrm{H})$ as summarized by Tasi et al (Tasi et al., 2006). Moreover, by detailed SEM observation of the cross-section of this film, the film thickness was determined to be approximately $20.2 \mu \mathrm{m}$, as shown in Fig. 1c. The thickness of the NaTNT phase was determined to be approximately $19.2 \mu \mathrm{m}$, and the thickness of the dense sodium titanate phase without Na-TNT was determined to be approximately $1.0 \mu \mathrm{m}$. Although fibrous morphologies were observed on the surface of the film, the back of the film was flat with no visible fibers. Therefore, the film exfoliation was considered to occur at the interface between the titanium metal phase and the sodium titanate phase without NaTNT. Based on the above results, the formation of the Na-TNT thin film can be explained as follows: (1) titanium dissolves into titanium ions $\left(\mathrm{Ti}^{4+}\right)$ by oxidizers, $\mathrm{H}^{+}$and/or $\mathrm{O}_{2}$; (2) dissolved $\mathrm{Ti}^{4+}$ ions immediately form titanium species (Wu et al., 2006) such as $\mathrm{TiO}_{3}{ }^{2-}$, $\mathrm{TiO}_{2}(\mathrm{OH})_{2}{ }^{2-}$, and $\mathrm{Ti}_{\mathrm{n}} \mathrm{O}_{2 \mathrm{n}+\mathrm{m}^{2 m-}}$ and the concentration of titanium species in the reaction solution increases as the dissolution of titanium is accelerated; (2) titanium species are reprecipitated as sodium titanate with an increase in the concentration of titanium species in the reaction solution; (3) since the concentration of the titanium species in the reaction solution is expected to increase with time, the sodium titanate phase without Na-TNT is formed when the concentration of titanium species is low and the Na-TNT phase is formed after the concentration becomes sufficiently high. The Na-TNT-free sodium titanate phase formed at low concentrations of titanium species may be amorphous sodium titanate. Since the concentration of titanium species in the reaction solution is considered to affect the type of sodium titanate cluster and the formation rate of the sodium titanate phase, the concentration of titanium species, together with temperature and other concentrations, are also considered to be factors in determining the type of phase formed.

Moreover, in order to prevent detachment of the thin Na-TNT film, the as-synthesized plate was slowly dried at room temperature after the hydrothermal treatment without washing it with water. Although $\mathrm{NaOH}$ crystals were observed on the plate, the thin film still adhered 


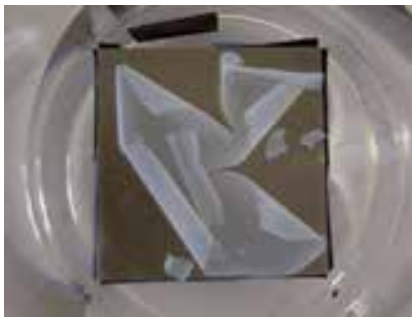

a

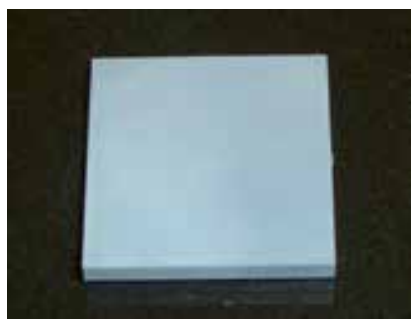

d

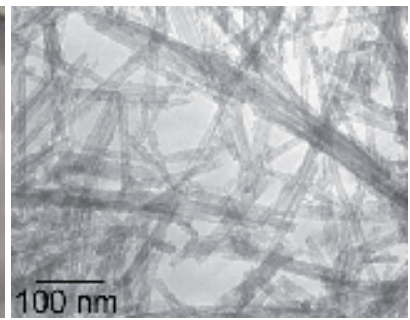

b

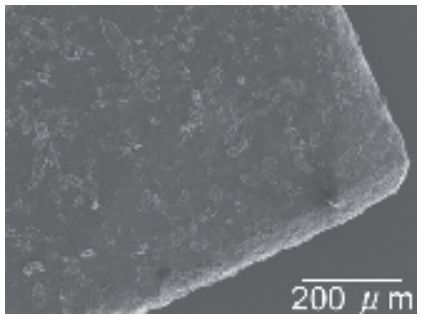

e

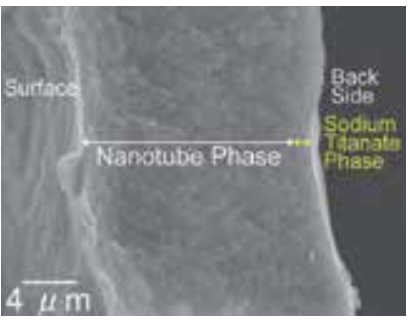

C

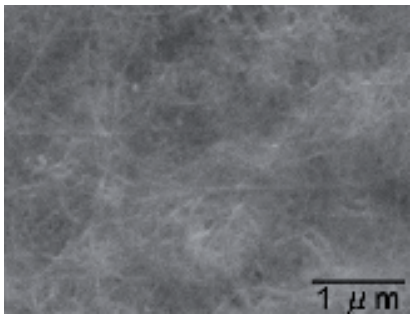

f

Fig. 1. Photograph (a, d), TEM (b), and SEM (c, e, f) images for the as-grown (a-c) and the $300{ }^{\circ} \mathrm{C}$ calcined (d-f) products obtained after the $20 \mathrm{~h}$ reaction.

to the plate. When the plate was washed with water after heat treatment at $300^{\circ} \mathrm{C}$ for $1 \mathrm{~h}$ in air, although the $\mathrm{NaOH}$ crystals dissolved, the thin Na-TNT film still adhered to the plate firmly and no detachment was observed as shown in Fig. 1d. Na-TNT formation was confirmed by the fibrous morphologies observed on the surface of the thin film in an enlarged SEM image (Figs. 1e, f) and nanotubes observed in a TEM image of the thin film. Moreover, in an XRD pattern of the thin film (Fig. 2), only diffraction peaks characteristic of Na-TNT (Chen et al., 2002) were observed along with peaks assigned to titanium metal. The reason for this stable coating is probably because polycondensation of hydroxyl groups in the interface area between the titanium plate and the Na-TNT-free sodium titanate phase occurred by the heat treatment at $300^{\circ} \mathrm{C}$, and Na-TNT being firmly fixed on the plate. The slow drying process is also considered to be important for the fixation of Na-TNT onto the titanium plate, since the thin film detached from the titanium plate by drying at $60^{\circ} \mathrm{C}$. The formation and fixation of the Na-TNT thin film were also observed in the reaction after $3 \mathrm{~h}$. Nanotubular structures similar to those of the $20 \mathrm{~h}$ product were also observed. The thickness of approximately $5 \mu \mathrm{m}$ for the film obtained after the $3 \mathrm{~h}$ reaction was smaller than that of $20.2 \mu \mathrm{m}$ for the film obtained after the $20 \mathrm{~h}$ reaction. The thickness of the film is thus controllable by the reaction time. On the other hand, when an as-synthesized Na-TNT thin film obtained by hydrothermal reaction in $10 \mathrm{~mol} / \mathrm{L}$ $\mathrm{NaOH}$ solution at $160{ }^{\circ} \mathrm{C}$ for $1 \mathrm{~h}$ was washed with large amounts of water, the Na-TNT thin film do not detach from the substrates and remains as thin as approximately $1 \mu \mathrm{m}$. Therefore, it is clear that sodium titanate nanotube thin films tend to detach from the substrates when they become too thick, but remain firmly fixed on substrates when the obtained samples are dried (without washing with water) and subsequently calcined at $300^{\circ} \mathrm{C}$. 


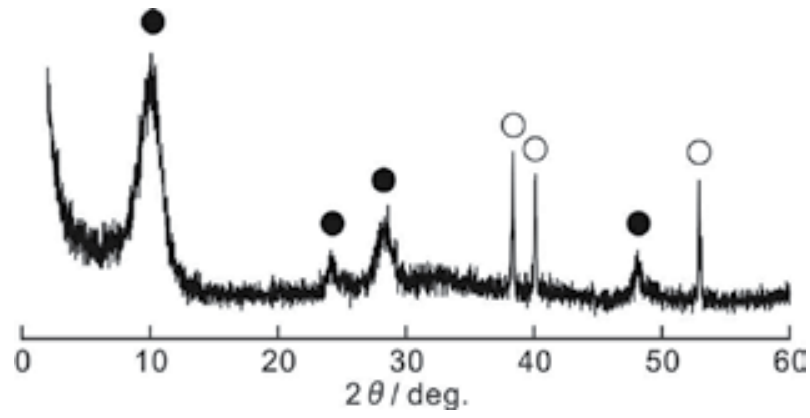

Fig. 2. XRD pattern for the plate obtained after the $20 \mathrm{~h}$ reaction. Peak assignment: $\bigcirc$ titanium metal, sodium titanate nanotube.

\subsubsection{Sodium titanate nanotube thin films formed on titanium wire, titanium mesh, titanium sphere, and titanium microtube}

Titanium wire (lengths: $5 \mathrm{~cm}, 24 \mathrm{~cm}$, and diameters: $53.4 \mu \mathrm{m}, 104.4 \mu \mathrm{m}, 203.7 \mu \mathrm{m}$ ), titanium mesh (woven from the titanium wire with a diameter of $104.4 \mu \mathrm{m}, 20 \mathrm{~mm} \times 20 \mathrm{~mm}$ ), titanium tube (inner diameter: $800 \mu \mathrm{m}$, outer diameter: $1 \mathrm{~mm}$, length: $1 \mathrm{~cm}$ ), and titanium spheres (diameter: $850-1180 \mu \mathrm{m}$, weight: 0.21-0.24 g) were used as metal titanium sources instead of a titanium plate.

After the hydrothermal treatments for $3 \mathrm{~h}$ and $20 \mathrm{~h}$, the surfaces of titanium mesh and titanium sphere were completely covered with Na-TNT thin film. Both outer and inner surfaces of the microtube were also covered with uniform nanotubes with an average diameter of $8 \mathrm{~nm}$. Typical digital camera and SEM images are shown in Figs. 3a-c.

On the other hand, the formation of Na-TNT thin film on a titanium wire requires special procedures which are different from those for plate, mesh, sphere, and microtube. First, the synthesis and fixation of Na-TNT were investigated using titanium metal wires of diameters 53.4 and $104.4 \mu \mathrm{m}$ and length $5 \mathrm{~cm}$ as titanium sources. As a result, after the hydrothermal treatment for $3 \mathrm{~h}$, sodium titanate with an irregular morphology was formed on the surface of the titanium wires, and only small amount of nanotubes was observed in the product synthesized using the titanium wire of diameter $104.4 \mu \mathrm{m}$. The diameters decreased from 53.4 and $104.4 \mu \mathrm{m}$ for the original wires to 36.3 and $93.8 \mu \mathrm{m}$ for the wires after a reaction time of $3 \mathrm{~h}$, respectively. Moreover, after the hydrothermal treatment for $20 \mathrm{~h}$, both wires completely dissolved. In addition, in the experiment using a wire of diameter $53.4 \mu \mathrm{m}$ and length $24 \mathrm{~cm}$, no nanotubes were observed on the surface of the obtained wire at a reaction time of $3 \mathrm{~h}$ and the wire completely dissolved at a reaction time of $20 \mathrm{~h}$. On the other hand, in the experiment using a wire of diameter $104.4 \mu \mathrm{m}$ and length $24 \mathrm{~cm}$, the amount of NaTNT formed increased at $3 \mathrm{~h}$ reaction time, and the surface of the wire was completely covered with Na-TNT thin film at the $20 \mathrm{~h}$ reaction. The reason for the complete dissolution of the original wires is because the dissolution rates of titanium species from the wires were faster than the redeposition rate of sodium titanate on the surface of the wires. On the other hand, the reason for the complete coverage of Na-TNT on the wire without dissolution is that the redeposition rate of sodium titanate nanotubes on the wire's surface became faster than the dissolution rate of titanium species from the wire with an increase in its diameter and length. These differences depending on the diameters of the original wires are 


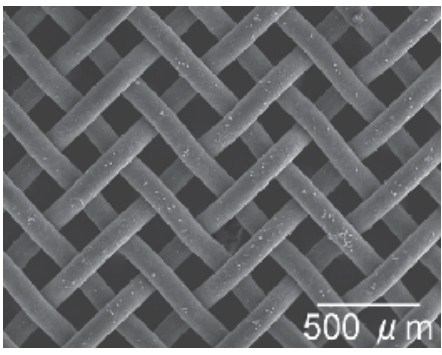

a

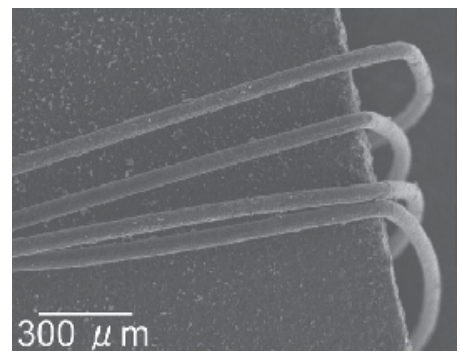

d

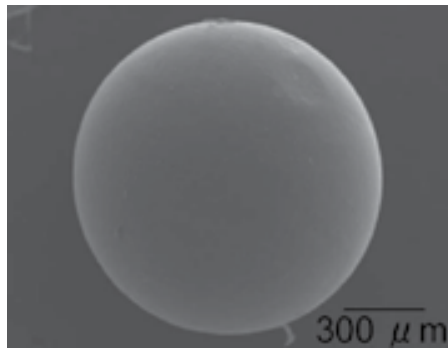

b

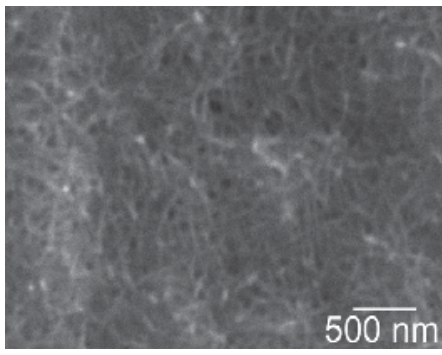

$\mathrm{e}$

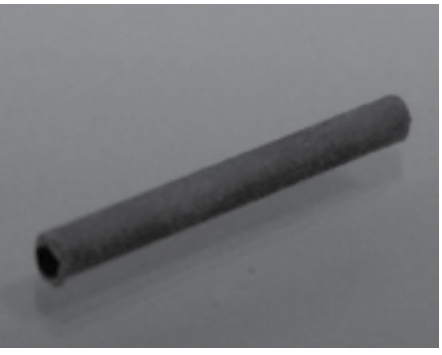

C

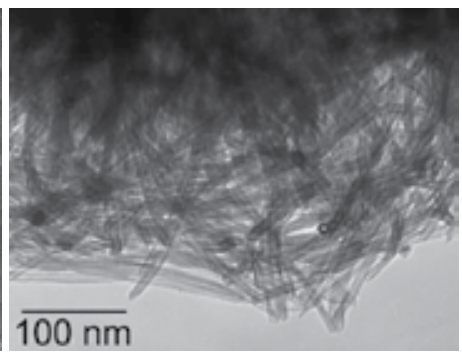

f

Fig. 3. SEM (a, b, d, e), Photograph (c), and TEM (f) images for the mesh (a), micro-sphere (b), microtube (c), and wire (d-f) obtained after the $3 \mathrm{~h}$ reaction.

explained as follows. Surface area and surface texture strongly affect the concentration of titanium species in the reaction solution. The amount of titanium species in the reaction solution increases with an increase in the diameter of the wire, since the surface area of the wire increases with an increase in the diameter. Additionally, the difference in the surface texture of the wires also affects the concentration of titanium species near their surfaces. Detailed SEM observations of the original titanium wires confirmed that the surfaces of the wire of diameter $104.4 \mu \mathrm{m}$ were porous, but the surface of the wire of diameter $53.4 \mu \mathrm{m}$ was relatively smooth. The concentration of titanium species would be higher near the wire and lower as the distance from the wire increases. In particular, the concentration of titanium species near the porous surface would be higher than that near the smooth surface. Therefore, in the experiments using the $104.4 \mu \mathrm{m}$ diameter wire, the amount of titanium species formed per unit of time and the concentration of the titanium species would be large due to their larger diameters and porous surfaces, and consequently the concentration of titanium species would be sufficiently high for the formation of Na-TNT as the dissolution of titanium proceeded. On the other hand, since the surface area of the $53.4 \mu \mathrm{m}$ diameter wire was predicted to be smaller than the $104.4 \mu \mathrm{m}$ diameter wire due to its diameter and smooth surface, the concentration of titanium species formed per unit of time would also be small. Therefore, the concentration of titanium species would be too low for the formation of Na-TNT. Consequently, sodium titanate with irregular morphology was formed without Na-TNT at a reaction time of $3 \mathrm{~h}$ and the original wire completely dissolved at a reaction time of $20 \mathrm{~h}$. Taking into consideration the above discussion, a similar hydrothermal and fixing treatment was performed using a wire of diameter $53.4 \mu \mathrm{m}$ and length $24 \mathrm{~cm}$ wound onto the above mentioned titanium plate, which could act as a source of titanium species. 
Wired morphologies remained for $3 \mathrm{~h}$ (Fig. 3d) and $20 \mathrm{~h}$ reactions, respectively, and the surfaces of both wires were completely covered with uniform Na-TNT thin films (Figs. 3e, f). It is considered that since the amount of titanium species reprecipitated on the wire, supplied by the dissolution from the titanium plate, was larger than the amount of titanium species dissolved from the wire, the surface of the wire was covered with Na-TNT. These results also indicate that dense concentration of titanium species near the titanium surface is required for the formation of Na-TNT on the titanium wire. Based on the above results, NaTNT applications can be largely extended by the hydrothermal treatment of a cloth woven with titanium wires and by weaving a cloth with Na-TNT/Ti wires.

\subsection{Sodium titanate nanotube thin films formed on Co-Cr alloy and SUS316L plates}

We devised a "hydrothermal transcription method" for forming Na-TNT films on various substrates, as shown in Fig. 4. In this method, Na-TNT would be produced by re-depositing or transcribing the titanium species such as $\mathrm{TiO}_{3}{ }^{2-}, \mathrm{TiO}_{2}(\mathrm{OH})_{2^{2-}}$, and $\mathrm{Ti}_{n} \mathrm{O}_{2 n+m^{2 m-}}$ formed near the surface of the titanium plate by hydrothermal treatment in aqueous $\mathrm{NaOH}$ solution on other substrate as Na-TNT, and grown to form dense films on several substrates as well as on the titanium plate. As shown in Fig. 4, under the conditions where a titanium metal plate and a substrate were adjacently placed, the titanium metal plate and substrate were spaced uniformly (about $200 \mu \mathrm{m}$ ) and fixed using titanium wires or SUS316 wires. For the substrate, Co-Cr alloy disk, SUS316L plate, SUS430 plate, tantalum plate, and silicon plate were used. These were immersed in $10 \mathrm{~mol} / 1 \mathrm{NaOH}$ aqueous solution and reacted hydrothermally for $20 \mathrm{~h}$ at $160{ }^{\circ} \mathrm{C}$. After the reaction, the samples were removed from the container and dried. Then, by washing in water following heat treatment at $300{ }^{\circ} \mathrm{C}$, excessive $\mathrm{NaOH}$ adhered on the substrate was removed.

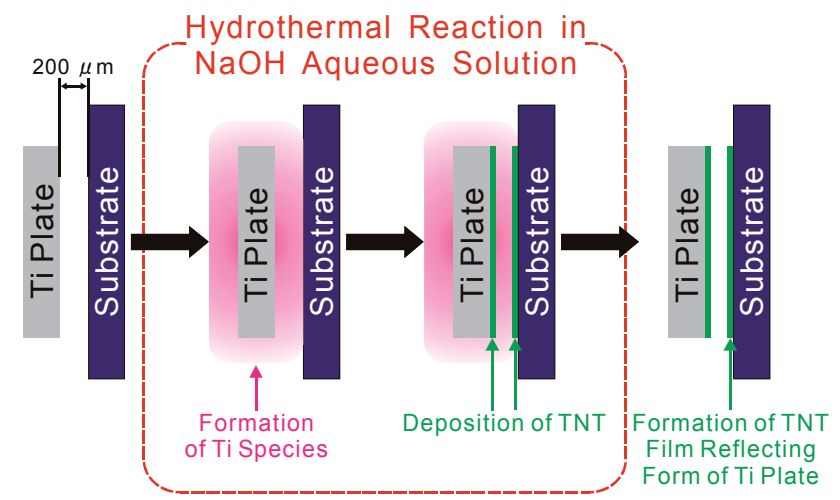

Fig. 4. Schematic representation of a reaction process by the hydrothermal transcription method.

Firstly, the Co-Cr alloy disk was used as a substrate. As shown in Fig. 5a, after the reaction, the formation of a white film whose base is the color of Co-Cr alloy along the square form of counter titanium plate on only the face countered to the titanium plate was observed. This white film strongly adhered to the Co-Cr alloy plate. By SEM images (Figs. 5b, c), the uniform and dense formation of fibrous substances was identified. Also by TEM observation of fibrous substances, nanotubes with an outer diameter of about $8 \mathrm{~mm}$ were identified (Fig. 
5d). The thickness of this film is about $5 \mu \mathrm{m}$. This thickness was less than the $20 \mu \mathrm{m}$ thickness of the Na-TNT film formed on the titanium plate when reacted singly (Yada et al., 2007). As the XRD pattern of the Co-Cr alloy surface countered to the titanium plate, a diffraction peak characteristic to titanate nanotube near $2 \theta=10^{\circ}$ as well as the peaks attributed to the $\mathrm{Co}-\mathrm{Cr}$ alloy of the raw material were observed. From a EDX analysis, it was found that the film contains $\mathrm{Na}, \mathrm{Ti}, \mathrm{O}, \mathrm{Co}, \mathrm{Cr}$, and $\mathrm{Si}$, and the molar ratio for the film was Na:Ti:O:Co:Cr:Si=0.322:1:2.401:0.112:0.052:0.045. Sodium titanate nanotube film is thus thought to be formed on the $\mathrm{Co}-\mathrm{Cr}$ alloy disk. The elements of $\mathrm{Co}, \mathrm{Cr}$, and $\mathrm{Si}$ would dissolve from the Co-Cr alloy disk and would be incorporated into the titanate framework and/or the interlayer spacing of the titanate. Furthermore, as observed above, the white NaTNT film reflecting the square form of the titanium plate was observed on the Co-Cr alloy disk countered to the titanium plate (Fig. 5a). Thus, the titanium species capable of forming $\mathrm{Na}$-TNT were present near the surface of titanium, and it can be considered that Na-TNT patterning reflecting the form of the titanium plate was made on the surface of the $\mathrm{Co}-\mathrm{Cr}$ alloy disk countered to the titanium plate. The above results suggest that by using several forms of the titanium plate as the titanium source, several forms of Na-TNT patterning can be made on heterogeneous substrates.

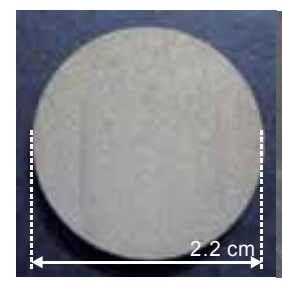

a

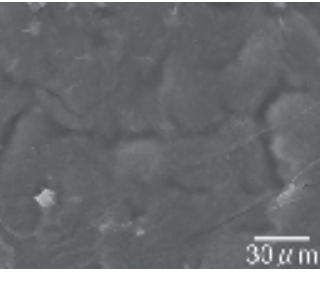

$\mathrm{b}$

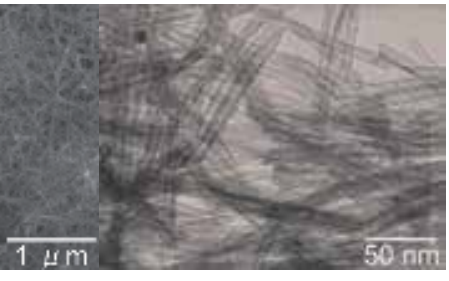

$\mathrm{d}$

Fig. 5. Digital camera (a), SEM (b, c), and TEM (d) images for the obtained thin film formed on the surface of $\mathrm{Co}-\mathrm{Cr}$ alloy countered to titanium metal.

When the same experiment was conducted with SUS316L plate instead of the Co-Cr alloy disk, it was found that as in the case of Co-Cr alloy, diluted white Na-TNT thin film was formed on the surface of SUS316L plate countered to the titanium plate. On the other hand, when the same reaction was performed using the SUS430 plate instead of Co-Cr alloy disk, brown and black iron compounds were formed on the SUS430 plate, although white NaTNT film was formed in part. SUS430 is an industrial grade stainless alloy, whereas SUS316L is a stainless alloy used in implants and has exceptionally high corrosion resistance, and the results reflecting this corrosion resistance were obtained. When the same reaction was performed with the tantalum plate instead of the $\mathrm{Co}-\mathrm{Cr}$ alloy plate, copious amounts of white products were produced on the tantalum plate, and particles other than nanotubes were observed. In addition, when the same experiment was performed using a silicon plate instead of the $\mathrm{Co}-\mathrm{Cr}$ alloy disk, the silicon plate was completely dissolved by the hydrothermal reaction. On considering the differences in the responsiveness of substrates, it is thought that the dissolution rate of the titanium plate and substrates and redeposition rate of chemical species that arose from the dissolved titanium plate and substrates should be considered. Particularly, in this experiment system, it is considered that the dissolution rate of substrates has a large effect on the results of the experiments. The dissolution rate of using metals as substrates, as in this study, can be explained by the 
ionization tendency, i.e., oxidation-reduction potential. It is considered that titanium dissolves into titanium ions $\left(\mathrm{Ti}^{4+}\right)$ by oxidizers, $\mathrm{H}^{+}$and/or $\mathrm{O}_{2}$, and these dissolved ions immediately form chemical species (Wu et al., 2006) such as $\mathrm{TiO}_{3}{ }^{2-}, \mathrm{TiO}_{2}(\mathrm{OH})_{2}{ }^{2-}$, and $\mathrm{Ti}_{n} \mathrm{O}_{2 n+m^{2 m-}}$ which are re-deposited as Na-TNT. When a substrate whose ionization tendency is smaller than titanium, especially materials such as SUS316L and Co-Cr alloy, is hydrothermally reacted with titanium simultaneously, the dissolution rate of titanium is higher than that of the substrate. In this reaction, titanium species are immediately produced following the dissolution of titanium and spread and re-deposited on the substrate as Na-TNT film, which predominates the dissolving reaction of the substrates. As a result, the surface of the substrate is covered by Na-TNT film, and the dissolution of the substrate was further minimally suppressed. On the other hand, it is considered that when the substrates with ionization tendencies larger than titanium, i.e., substrates such as silicon and tantalum, and titanium were hydrothermally reacted at the same time, the dissolution reaction of substrates predominate the dissolution reaction of titanium. Na-TNT film was not thus obtained on the substrates.

\subsection{Hydrogen titanate and anatase-type titanium dioxide nanotube thin films}

$\mathrm{H}^{+}$ion-exchange treatment for the sodium titanate nanotube thin film and the subsequent calcination can produce an anatase-type titanium dioxide nanotube thin film.

\subsection{1 $\mathrm{H}^{+}$ion-exchange treatment for sodium titanate nanotube thin films}

We performed $\mathrm{H}^{+}$ion-exchange treatment for Na-TNT thin film obtained after the $3 \mathrm{~h}$ reaction using $0.01 \mathrm{~mol} / 1$ hydrochloric acid solution at 90 and $140{ }^{\circ} \mathrm{C}$ for $3 \mathrm{~h}$. The thin films resulting from treatment at these two temperatures remain attached over the entire surface of each sample. EDX analysis reveal that because the molar ratio of $\mathrm{Na} / \mathrm{Ti}$ decrease from 0.48 before treatment to 0 after treatment at 90 and $140{ }^{\circ} \mathrm{C}, \mathrm{Na}^{+}$ions between titanate layers are confirmed to be completely exchanged for $\mathrm{H}^{+}$ions. We observe nanotubes with an average outer diameter of $8.3 \mathrm{~nm}$ and inner diameter of $3.3 \mathrm{~nm}$ in the ion-exchange-treated sample at $90{ }^{\circ} \mathrm{C}$ using $0.01 \mathrm{~mol} / 1$ hydrochloric acid solution (Fig. 6a). No change is observed in the porous structure of the thin film before or after treatment. The XRD pattern for the $\mathrm{H}^{+}$ion-exchanged sample at $90^{\circ} \mathrm{C}$ shows four diffraction peaks (near $2 \theta=9,24,29$, and $48^{\circ}$ ) attributed to titanate together with peaks attributed to a-titanium, similar to those for the as-grown sample, as shown in Fig. 7. We therefore believe that the $\mathrm{H}^{+}$ion-exchange treatment at $90{ }^{\circ} \mathrm{C}$ transforms sodium titanate nanotubes into hydrogen titanate nanotubes while maintaining the crystal structure of titanate, nanotubular morphology, and porous thin-film structure. In contrast, the $\mathrm{H}^{+}$ion-exchange treatment at $140{ }^{\circ} \mathrm{C}$ replaces the fibrous morphology with rhomboid-shaped particles (average diameter $21 \mathrm{~nm}$ ) (Fig. 6b) and pores ( $45 \mathrm{~nm}$ diameter) in the interstitial gaps. The XRD pattern of this sample (Fig. 7) shows peaks attributed to anatase. Therefore, a porous thin film consisting of rhomboid-shaped anatase is confirmed to be formed on the titanium metal plate. Change in the crystal structure of sodium titanate nanotubes to anatase nanotubes by acid treatment have been described previously by Tsai et al. (Tsai et al., 2006). They reported that although a nanotube form is maintained by acid treatment at $\mathrm{pH}$ 1.6, only irregular-shaped anatase particles are formed by acid treatment at $\mathrm{pH} 0.38$. Zhu et al. (Zhu et al., 2005) reported that hydrogen titanate nanofiber transforms into anatase nanocrystals in dilute $(0.05 \mathrm{~mol} / \mathrm{L}) \mathrm{HNO}_{3}$ at $80-120^{\circ} \mathrm{C}$. They stated that monodispersed anatase nanocrystals 
are obtained at $80{ }^{\circ} \mathrm{C}$ and aggregates of nanocrystals are obtained at $120^{\circ} \mathrm{C}$. Our results also suggest that the change in the crystal structure change of titanate compounds to anatase is determined not only by $\mathrm{pH}$ but also by the temperature of the ion-exchange treatment. We suggest that the high temperature $\left(140^{\circ} \mathrm{C}\right)$ of the ion-exchange treatment is responsible for the change in the crystal structure of hydrogen titanate to the anatase structure, with a high degree of crystallization, and that this change occurs due to polycondensation and dissolutionredeposition reactions.

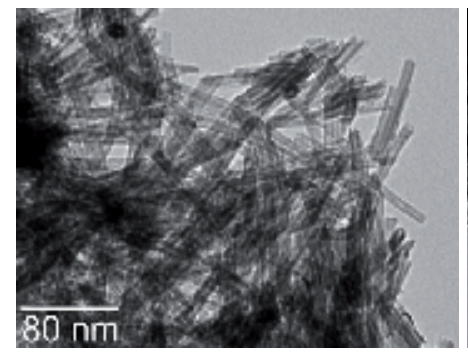

a

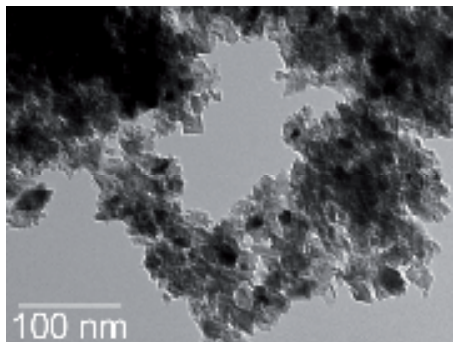

$\mathrm{b}$

Fig. 6. TEM images of the ion-exchange-treated thin films at $90^{\circ} \mathrm{C}(\mathrm{a})$ and $140{ }^{\circ} \mathrm{C}(\mathrm{b})$.

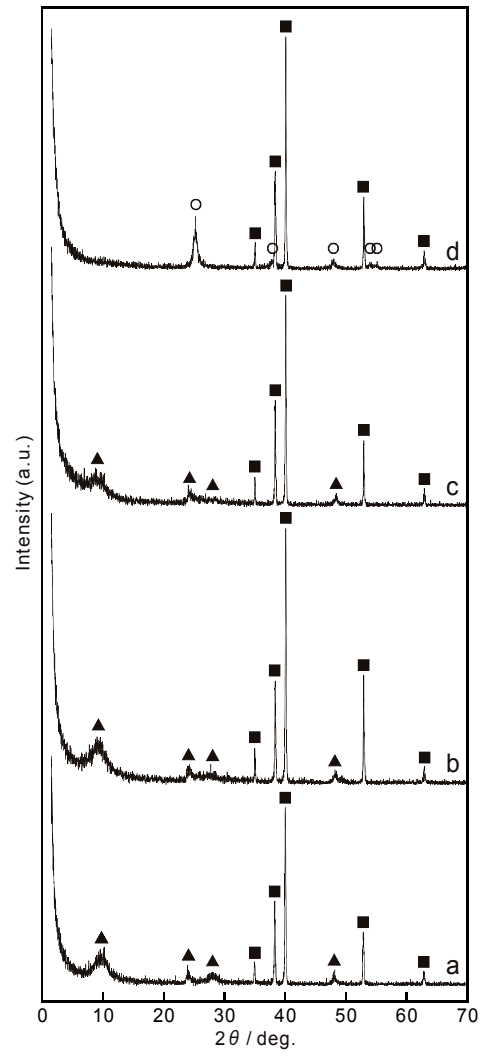

Fig. 7. XRD patterns of the as-grown (a) and the ion-exchange-treated thin films at $40{ }^{\circ} \mathrm{C}(\mathrm{b})$, $90{ }^{\circ} \mathrm{C}(\mathrm{c})$, and $140{ }^{\circ} \mathrm{C}(\mathrm{d})$. Peak assignment: $\boldsymbol{\square}, \alpha$-titanium; $\bigcirc$, anatase; $\boldsymbol{\Delta}$, titanate. 
The temperature required for the complete $\mathrm{H}^{+}$ion-exchange reaction would be higher than that previously reported (Kasuga et al., 1998; Kasuga et al., 1999; Tokudome et al., 2004; Uchida et al., 2002; Tokudome et al., 2005; Thorne et al., 2005; Miyauchi et al., 2006; Tokudome et al., 2004; Kasuga et al., 2003), because the $\mathrm{H}^{+}$ion-exchange treatments at $40{ }^{\circ} \mathrm{C}$ using $0.01,0.1$, and $1 \mathrm{~mol} / 1$ hydrochloric acid solutions were unsuccessful. $\mathrm{H}^{+}$ion-exchange treatment at $40{ }^{\circ} \mathrm{C}$ for $3 \mathrm{~h}$ using 0.01 and $0.1 \mathrm{~mol} / 1$ hydrochloric acid solutions resulted in nanotube films respectively. However, substantial amounts of $\mathrm{Na}^{+}$ions remained in the samples after treatment. It is considered that elevated temperature assists the diffusion of ions, allowing ion-exchange to occur within the deepest regions of the film. After $\mathrm{H}^{+}$ionexchange treatment at $40{ }^{\circ} \mathrm{C}$ using $1.0 \mathrm{~mol} / 1$ hydrochloric acid solution, the nanotube thin films detached from the titanium metal plate.

\subsubsection{Calcination of hydrogen titanate nanotube thin film}

The hydrogen titanate nanotube thin films obtained by $\mathrm{H}^{+}$ion-exchange treatment at $90{ }^{\circ} \mathrm{C}$ using a $0.01 \mathrm{~mol} / \mathrm{L}$ solution of hydrochloric acid were calcined at $300-900{ }^{\circ} \mathrm{C}$ for $3 \mathrm{~h}$ in air to transform them into titanium dioxide nanotube thin films. A uniform thin film formed on each sample surface, similar to the sample before calcination.

TEM images (Fig. 8) and XRD patterns (Fig. 9) show that calcination at 300 and $450{ }^{\circ} \mathrm{C}$ yields anatase nanotubes. Although the average inner diameter of $3.3 \mathrm{~nm}$ for nanotubes synthesized by calcination at $450{ }^{\circ} \mathrm{C}$ is similar to that of the as-grown sodium titanate nanotubes, the average outer diameter of the nanotubes decreased from $8.3 \mathrm{~nm}$ for the asgrown thin film to $8.1 \mathrm{~nm}$ for the anatase nanotubes synthesized by calcination at $450{ }^{\circ} \mathrm{C}$. This slight decrease in the average outer diameter may be due to a phase transition from titanate into anatase. An cross-section image of the thin film calcined at $450{ }^{\circ} \mathrm{C}$ is similar to that of the as-grown sodium titanate nanotube thin film. Although a dense phase is observed at the bottom of the thin film (i.e., at the interface between the nanotube phase and the titanium metal), the porous structure composed of fibrous particles is observed in the film itself. Calcination at $600{ }^{\circ} \mathrm{C}$ yields anatase nanofibers approximately $11 \mathrm{~nm}$ thick, but not nanotubes (Figs. 8c and 9d). The porous structure consisting of fibrous particles are maintained until calcination at $600{ }^{\circ} \mathrm{C}$. Calcination at $750{ }^{\circ} \mathrm{C}$ changes the thin film into a porous thin film consisting of particles (with 50-nm average diameter) and interstitial pores (with 79-nm average size) as shown in Fig. 8d. We attribute these changes in morphology to a progressive sintering reaction caused by the high calcination temperature. Furthermore,

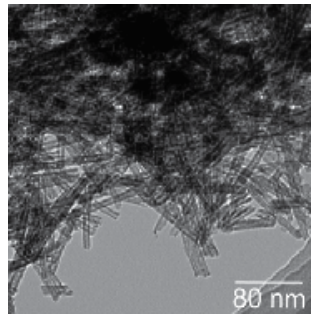

a

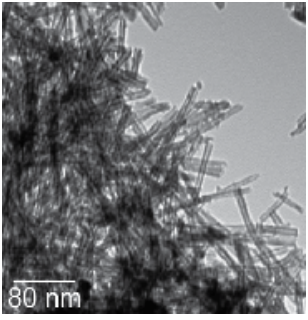

$\mathrm{b}$

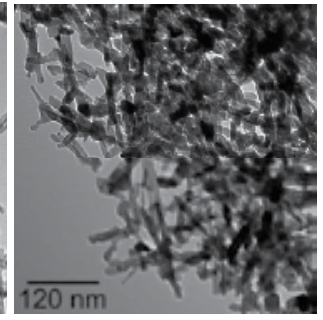

C

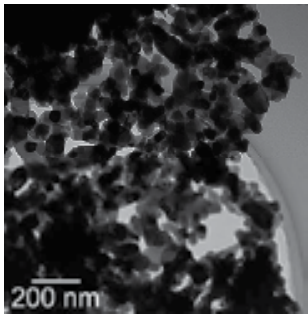

d

Fig. 8. TEM images of the $90{ }^{\circ} \mathrm{C}$ ion-exchange-treated thin films calcined at $300{ }^{\circ} \mathrm{C}(\mathrm{a}), 450{ }^{\circ} \mathrm{C}$ (b), $600{ }^{\circ} \mathrm{C}(\mathrm{c})$, and $750{ }^{\circ} \mathrm{C}(\mathrm{d})$. 
calcination at $900{ }^{\circ} \mathrm{C}$ yields a dense rutile thin film because of the densification and phase transition caused by the sintering of anatase nanoparticles (Fig. 9f).

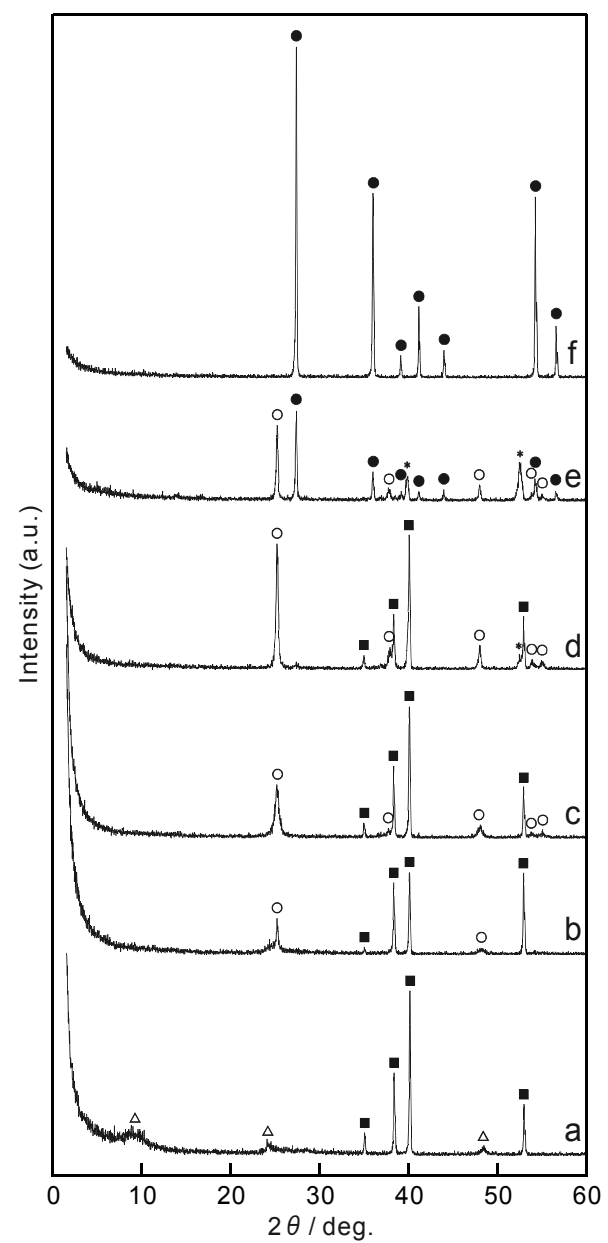

Fig. 9. XRD patterns of the $90{ }^{\circ} \mathrm{C}$ ion-exchange-treated (a) and the $90^{\circ} \mathrm{C}$ ion-exchangetreated thin films calcined at $300^{\circ} \mathrm{C}(\mathrm{b}), 450{ }^{\circ} \mathrm{C}$ (c), $600{ }^{\circ} \mathrm{C}$ (d), $750{ }^{\circ} \mathrm{C}$ (e), and $900{ }^{\circ} \mathrm{C}$ (f). Peak assignment: $\mathbf{\square}, \alpha$-titanium; $\bigcirc$, anatase; $\bigcirc$, rutile; $\triangle$, hydrogen titanate; ${ }^{*}$, distorted titanium.

\subsection{Silver nanoparticle / silver titanate nanotube nanocomposite thin film}

Na-TNT thin film obtained after the $3 \mathrm{~h}$ reaction with dimensions of $20 \mathrm{~mm} \times 20 \mathrm{~mm} \times 2$ $\mathrm{mm}$ (hereafter referred to as Na-TNT-TF) was immersed in $12 \mathrm{~mL}$ of $0.05 \mathrm{M}$ silver acetate solution at $40{ }^{\circ} \mathrm{C}$ for $3 \mathrm{~h}$, then repeatedly washed with distilled water and dried in a cool dark place, to exchange the $\mathrm{Na}^{+}$in the sodium titanate with $\mathrm{Ag}^{+}$. Hereafter, the sample obtained by the silver ion-exchange treatment of Na-TNT-TF was called Ag-TNT-TF.

The EDX spectra of the samples before and after the silver ion-exchange treatment were then compared. Since the peaks attributed to $\mathrm{Na}$, observed in the samples before the silver ion- 
exchange treatment (Na-TNT-TF), disappeared in the samples after the silver ion-exchange treatment (Ag-TNT-TF), and the peaks attributed to Ag appeared after the silver ion-exchange treatment, $\mathrm{Na}^{+}$in the sodium titanate seemed to be exchanged with $\mathrm{Ag}^{+}$during the silver ionexchange treatment. However, for the composition calculated from these spectra, the molar ratio of $\mathrm{Ag} / \mathrm{Ti}$ was 0.67 for $\mathrm{Ag}-\mathrm{TNT}-\mathrm{TF}$, while the molar ratio of $\mathrm{Na} / \mathrm{Ti}$ was 0.50 for Na-TNTTF. This confirmed presence of Ag in an excess compared with the exchangeable cations in the sample. SEM observation at the micrometer scale did not show changes in the morphologies before and after the silver ion-exchange treatment. However, the TEM observations of AgTNT-TF (Fig. 10) show particles with sizes ranging from several nanometers to a few dozen nanometers, which were not observable before the ion-exchange treatment. These are considered to be silver nanoparticles, since the color of Ag-TNT-TF was slightly yellow, indicating the formation of silver nanoparticles. The silver nanoparticles were deposited on titanates by the photoreduction of silver ions that were adsorbed on the titanate surface. The excess silver determined through the exchangeable mass of ions observed via EDX analysis is thus attributed to these silver nanoparticles. In other word, in the silver ion-exchange treatment, $\mathrm{Ag}^{+}$ion was not only incorporated into the titanate by ion exchange with $\mathrm{Na}^{+}$ion, but also deposited on the outer surface of titanate as silver nanoparticles.

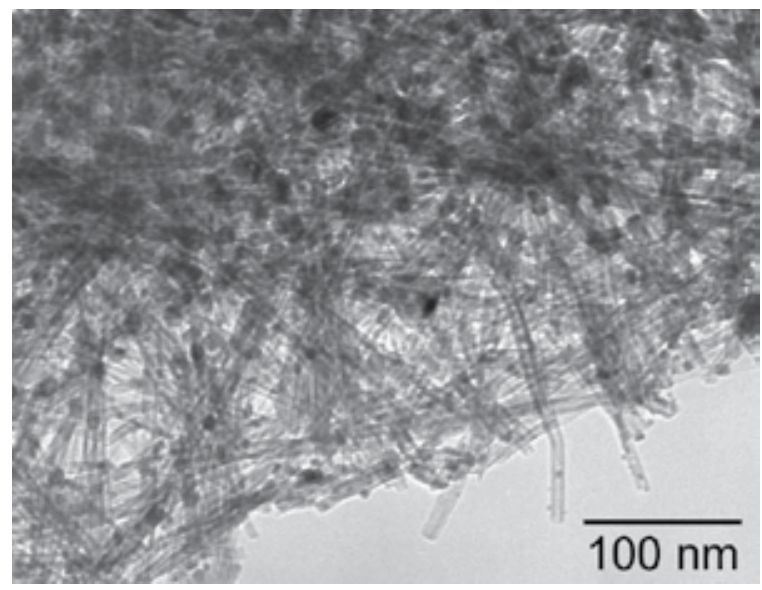

Fig. 10. TEM image of silver nanoparticle/silver titanate nanotube nanocomposite thin film.

Furthermore, TF-XRD patterns of Na-TNT-TF and Ag-TNT-TF shown in Fig. 11 also indicate the transformation of sodium titanate thin film into silver titanate thin film. When silver ionexchange treatment was performed for Na-TNT-TF, a diffraction peak expressing the interlayer distance of $10 \AA$ that was observed in Na-TNT-TF, disappeared in Ag-TNT-TF. The disappearance of the diffraction peak expressing the interlayer distance of $10 \AA$ is considered to be due to the insertion of $\mathrm{Ag}^{+}$ions into an interlayer of titanate and disappearance of the layered structure of titanate. A further reason is a strong and peculiar interaction between the inserted $\mathrm{Ag}^{+}$ions and the titanate layer, which would cause a structural change of the layered structure into a three-dimensional structure. This structural change can also be confirmed, as the diffraction peaks in Na-TNT-TF due to the crystal structure of titanate, observed at $2 \theta=24.2^{\circ}$ and $28.3^{\circ}$, disappeared in Ag-TNT-TF, concomitant with the appearance of a new diffraction peak at $2 \theta=29.3^{\circ}$ in Ag-TNT-TF. These results indicate the formation of silver titanate nanotube. 

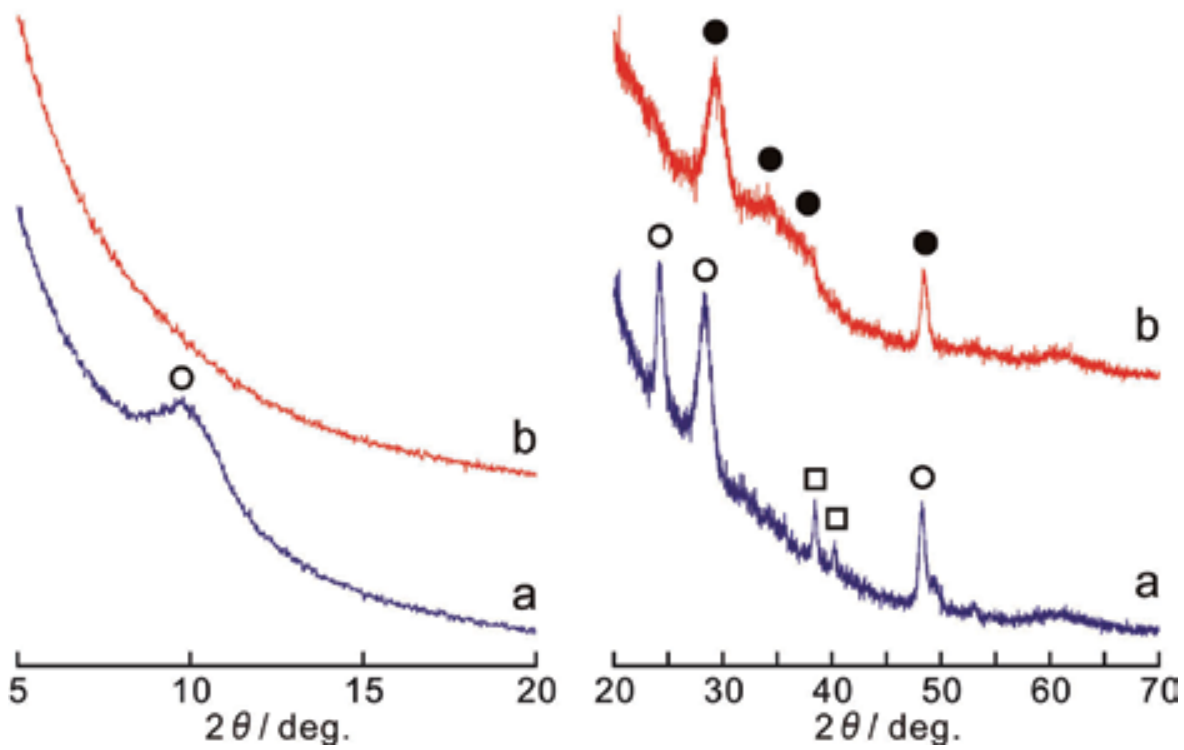

Fig. 11. TF-XRD patterns of sodium titanate nanotube thin film (a) and silver nanoparticle/ silver titanate nanotube nanocomposite thin film (b). Peak assignment: $\square$, titanium; $\bigcirc$, sodium titanate; $\mathbf{O}$, silver titanate.

\section{Antibacterial activities of titanate nanotube thin films}

\subsection{Elution properties of silver ions from the silver nanoparticle / silver titanate nanotube nanocomposite thin film}

The elution properties of silver ions from the samples were examined in various solutions to determine the behavior of silver in MRSA environment or in the body. Ag-TNT-TF with dimensions of $20 \mathrm{~mm} \times 20 \mathrm{~mm} \times 2 \mathrm{~mm}$ was immersed in $15 \mathrm{~mL}$ physiological saline, phosphate buffered saline $\left(^{+}\right)(\operatorname{PBS}(+))$, phosphate buffered saline $(-)(\operatorname{PBS}(-))$, and fetal bovine serum solution, maintained at $37{ }^{\circ} \mathrm{C}$ for $24 \mathrm{~h}$. Then, the eluates were collected, centrifuged, and filtrated through a $0.22-\mu \mathrm{m}$ filter. After filtration, Ag concentration in the eluates was determined by inductively coupled plasma mass spectrometry (ICP-MS). In physiological saline, PBS(+), and PBS(-), almost the same average concentration of eluted silver was measured $-300,320$, and $440 \mathrm{ppb}$, respectively. The eluted silver ions may originate from metallic silver and silver titanate. Since the solubility of metallic silver is known to be very small, the large portion of eluted silver was eluted by exchanging silver ions in titanates with $\mathrm{Na}^{+}, \mathrm{K}^{+}$, and $\mathrm{H}^{+}$in the solutions. On the other hand, in fetal bovine serum, the average eluted silver concentration was measured in large amounts $-82000 \mathrm{ppb}$ for Ag-TNT-TF. This was because a large quantity of a compound composed of silver and a protein was formed together with $\mathrm{AgCl}$, since the protein that exists in fetal bovine serum has very high affinity with $\mathrm{Ag}^{+}$through the $-\mathrm{SH}$ group or $-\mathrm{NH}$ group in the protein, and the amount of exchangeable cations in fetal bovine serum was larger than that in physiological saline and PBS. Moreover, when the silver elution test in fetal bovine serum was performed for a silver metal plate under similar conditions as that for Ag-TNT-TF, silver of $7900 \mathrm{ppb}$ was eluted. This amount was also significantly smaller than that for of Ag-TNT-TF. In the 
silver elution test of Ag-TNT-TF, although silver is eluted from silver nanoparticles deposited on the surface of titanate, its elution amount is thus considered small. Consequently, in the XRD and TF-XRD patterns of the sample obtained by the silver elution test of Ag-TNT-TF, diffraction peaks appeared near $2 \theta=10^{\circ}, 24^{\circ}$ and $28^{\circ}$, which were not observed in Ag-TNT-TF. These diffraction peaks of the sample obtained by the silver elution test of Ag-TNT-TF appeared at the same locations as the diffraction peaks observed in NaTNT-TF. This indicates that the crystal structure of the sample obtained by the silver elution test of Ag-TNT-TF is similar to that of Na-TNT-TF. The likely reasons are as follows: (1) the layered structure of titanate of Na-TNT-TF transformed into a three-dimensional structure because $\mathrm{Ag}^{+}$ions were inserted into the interlayer of titanate by the silver ion-exchange treatment to form silver titanate, (2) $\mathrm{Ag}^{+}$ions were eluted from the silver titanate and $\mathrm{Na}^{+}$ ions were reinserted into the titanate during the silver elution test; and (3) the threedimensional structure of titanate returned to the original condition as in Na-TNT-TF. Thus, it is clearly demonstrated that insertion (intercalation) and elimination (deintercalation) of $\mathrm{Ag}^{+}$ions occurs during the silver ion-exchange treatment and the silver elution test, respectively. Therefore, this experiment indicated that $\mathrm{Ag}^{+}$ions in silver titanate greatly contributed to the elution of $\mathrm{Ag}^{+}$. Diffraction peaks attributable to $\mathrm{AgCl}$ were also observed in the sample obtained by the silver elution test of $\mathrm{Ag}$-TNT-TF, because $\mathrm{AgCl}$ particles were formed by the reaction between eluted $\mathrm{Ag}^{+}$and $\mathrm{Cl}^{-}$in fetal bovine serum. Since fetal bovine serum solution is considered as the system most similar to MRSA environment, silver elution tests in fetal bovine serum solution were repeated (Fig. 12). In Ag-TNT-TF, the elution concentration slowly decreased from $94000 \mathrm{ppb}$ for the first test to $11000 \mathrm{ppb}$ for the tenth, indicating that a large amount of eluted silver was measured in the tenth test for AgTNT-TF. The 2-step elution curve was obtained from Ag-TNT-TF. A rapid elution of a large amount of silver at the initial stage of the repeated elution test (between the first and third time) was considered to be mainly due to $\mathrm{Ag}^{+}$ion elution from the silver titanate based on

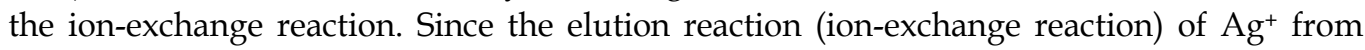
silver titanate is rapid, the elution of $\mathrm{Ag}^{+}$is considered to be almost completed at the initial stage of the repeated elution test. These discussions are also supported by the above described TF-XRD data, indicating that crystal structure of the sample obtained by the silver elution test of Ag-TNT-TF is similar to that of Na-TNT-TF. Therefore, a slow elution of a small amount of silver after the forth repetition of the elution test was considered to be mainly because of silver elution from the silver nanoparticles. This two-step elution curve is difficult to explain if it is considered that only silver nanoparticles are formed in the thin film, but it is reasonably explained if two types of Ag (silver nanoparticles and silver titanate) exhibiting different elution behaviors are present in Ag-TNT-TF. The silver titanates loading silver nanoparticles would be promising as a novel antibacterial material, because they have two silver sources. The silver-ion elution property of silver titanate would be different from that of the silver nanoparticles, i.e., the elution speed of silver ions from silver titanate would be greater than that from silver nanoparticles. Therefore, silver titanate would be effective for short-term bacterial killing, and silver nanoparticles would be effective for long-term antibacterial action. Since we have already found that a thicker (i.e., $20 \mu \mathrm{m}$ thick) titanate nanotube film can be formed after a longer reaction time or $20 \mathrm{~h}$ in $\mathrm{NaOH}$ solution, it would be possible to prolong the elution period of silver ions and to increase the amount of eluted silver ions or the duration. 


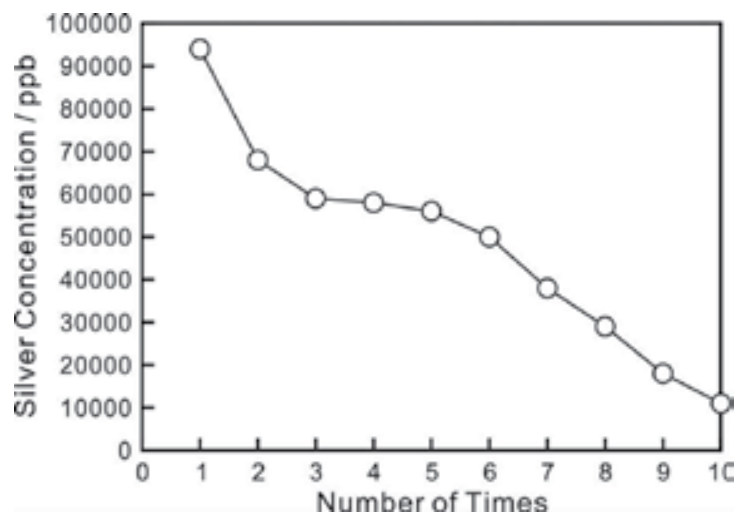

Fig. 12. Repeated silver ion elution test of silver nanoparticle/silver titanate nanotube nanocomposite thin film.

\subsection{Antibacterial property of silver nanoparticle / silver titanate nanotube nanocomposite thin film}

The modified Japanese Industrial Standard test (JIS Z 2801) was performed as an antibacterial test as follows. To approximate an infection environment within an actual organism, an inactivated bovine serum $(0.4 \mathrm{~mL})$ was used as a solvent of bacterial suspension to create a eutrophic condition, and the antibacterial test was conducted using MRSA with a biofilm-forming gene. A bacterial suspension $(0.4 \mathrm{~mL})$ was dropped on a 50 $\mathrm{mm} \times 50 \mathrm{~mm} \times 2 \mathrm{~mm}$ test piece, covered with a $40 \mathrm{~mm} \times 40 \mathrm{~mm}$ polyethylene film (Elmex Corp.), and cultured at $37^{\circ} \mathrm{C}$ for $24 \mathrm{~h}$. The test piece was washed, and the viable MRSA count was determined. The antibacterial test was performed thrice for each of the samples of Na-TNT-TF and Ag-TNT-TF, to obtain averaged values of viable MRSA counts. The antibacterial activity value $(R)$ for the sample was calculated as follows.

$$
R=\{\log (B / A)-\log (C / A)\}=\log (B / C)
$$

Here, $A, B$, and $C$ are the average viable MRSA counts just after inoculation, after $24 \mathrm{~h}$ for a blank and after $24 \mathrm{~h}$ for a sample, respectively. The viable MRSA count just after the inoculation was $2.1 \times 10^{5} \mathrm{CFU} /$ sample, and for a blank, the average viable MRSA count after $24 \mathrm{~h}$ increased to $5.9 \times 10^{8} \mathrm{CFU} /$ sample. In Na-TNT-TF, the average viable MRSA count after $24 \mathrm{~h}$ was slightly less than that of the blank: $1.1 \times 10^{7} \mathrm{CFU} /$ sample. On the other hand, in Ag-TNT-TF, the average viable MRSA count after $24 \mathrm{~h}$ was markedly small: $3.3 \times$ $10^{2} \mathrm{CFU} /$ sample. $R$ increased from 1.7 for Na-TNT-TF to 6.3 for Ag-TNT-TF through the silver ion-exchange treatment. These results indicate that the silver ion-exchanged titanate thin films display high antibacterial activity against MRSA. It was also revealed that although the crystal structure of titanate itself does not have a large antibacterial effect, higher antibacterial activity arises in the silver in the titanate. The conversion of sodium titanates into antibacterial materials through the silver ion-exchange treatment can apply to other nanostructured sodium titanates. For example, by the same silver ion-exchange treatment, porous sodium titanate film calcined at $600{ }^{\circ} \mathrm{C}$ reported by Kim et al. (Kim et al., 1997) can also be converted into silver nanoparticle / silver titanate nanocomposite thin film with high antibacterial activity for MRSA of $R=6.7$. 


\section{Apatite-forming ability of titanate and titanium dioxide nanotube thin films}

The three thin films (Na-TNT-TF, $\mathrm{TiO}_{2}-\mathrm{NT}-\mathrm{FT}$ (anatase type titanium dioxide nanotube thin film formed by the $\mathrm{H}^{+}$ion-exchange treatment and calcination at $450{ }^{\circ} \mathrm{C}$ of Na-TNT-TF), AgTNT-TF) formed on a titanium metal were immersed in simulated body fluid (SBF) and monitored the development of apatite formation on their surfaces. In accordance with ISO 23317, "Implants for surgery-In vitro evaluation for apatite-forming ability of implant materials," we evaluated the apatite-forming ability on the surface of the coating in SBF. A plate was placed in $96.0 \mathrm{~mL} \mathrm{SBF}$ at $36.5^{\circ} \mathrm{C}$. After 2, 4, and 14 days, the plate was removed and gently rinsed with water. The surface of the plate was dried in air.

For Na-TNT-TF, after immersing the film for 4 days, the SEM images showed only nanotubes and no substances with foliaceous morphology peculiar to apatite; the XRD patterns remained unchanged. However, when the SBF immersion was extended to 14 days, the SEM images showed the surface of the film to be completely covered with a domeshaped form consisting of foliaceous particles peculiar to apatite (Figs. 13a, b); the XRD pattern showed diffraction peaks attributable to apatite. Thus, apatite is confirmed to be formed on the sodium titanate thin films. In addition, after immersing the hydrogen titanate nanotube thin film formed by exchanging $\mathrm{Na}^{+}$ions between the layers of the layered sodium titanate for $\mathrm{H}^{+}$ions in SBF for 4 days, no apatite was evident. This lack of apatite indicates that ions $\left(\mathrm{Na}^{+}\right.$and $\left.\mathrm{H}^{+}\right)$between the titanate layers do not particularly contribute to the acceleration of the apatite formation. In contrast, for $\mathrm{TiO}_{2}-\mathrm{NT}-\mathrm{FT}$ formed by the $450^{\circ} \mathrm{C}$ calcination of the hydrogen titanate nanotube thin film and Ag-TNT-TF, after immersing the

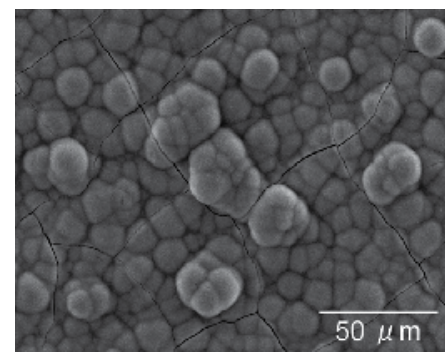

a

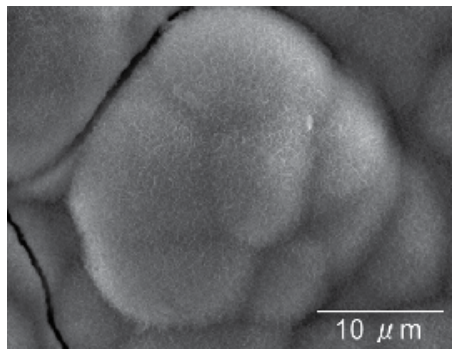

$\mathrm{b}$

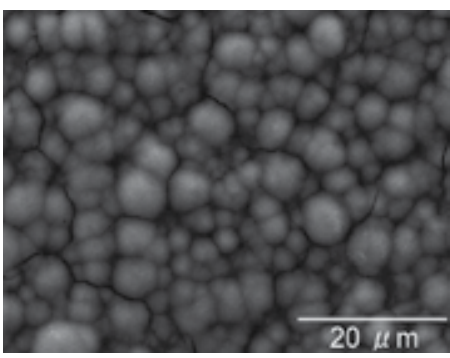

C

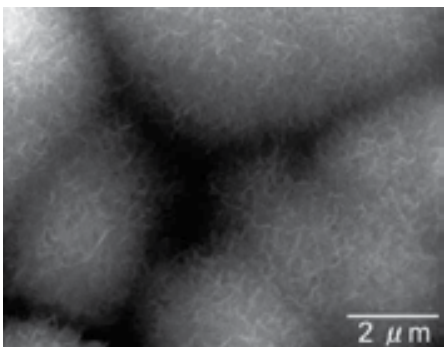

d

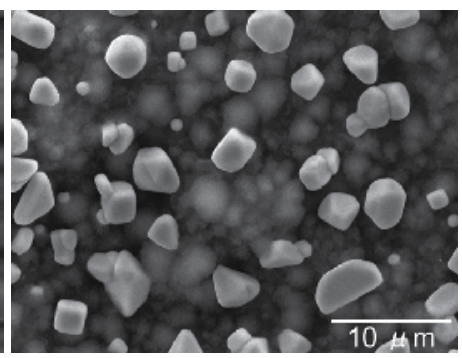

$\mathrm{e}$

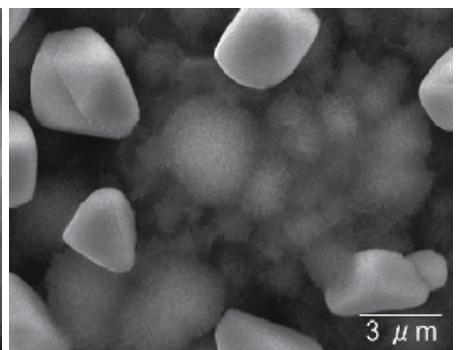

$\mathrm{f}$

Fig. 13. Low magnification (a, c, e) and high magnification (b, d, f) SEM images of Na-TNTTF $(\mathrm{a}, \mathrm{b}), \mathrm{TiO}_{2}-\mathrm{NT}-\mathrm{FT}(\mathrm{c}, \mathrm{d})$, and Ag-TNT-TF (e, f) after immersions in SBF. 
films for 4 days, the SEM images showed a stretch of the dome-shaped form consisting of foliaceous particles peculiar to apatite (Figs. 13c, d). XRD patterns of the two films showed diffraction peaks attributable to apatite, respectively. These results indicate that the surfaces of these two thin films are completely covered with apatite and that the apatite-forming ability of the two films is greater than that of Na-TNT-TF having layered structure. In contrast, for $\mathrm{TiO}_{2}$-NT-FT after immersing the film in SBF for 2 days, the surface is thinly covered with apatite. However immersing Ag-TNT-TF for 2 days, the surface is almost covered with a dome-shaped form consisting of foliaceous particles peculiar to the apatite (Figs. 13e, f). The apatite-forming ability of Ag-TNT-TF is, thus, slightly higher than that of $\mathrm{TiO}_{2}$-NT-FT.

We then investigated the newly observed high apatite-forming ability of silver nanoparticle/silver titanate nanocomposite thin film (Ag-TNT-TF). After immersing the film in SBF for 4 days, we observed bulky particles of a few micrometers in diameter together with apatite. XRD pattern shows diffraction peaks attributable to silver chloride. EDX element mapping analysis shows the bulky particles to be composed of $\mathrm{Ag}$ and $\mathrm{Cl}$ (Fig. 14) and, therefore, to be silver chloride particles. Therefore, $\mathrm{Ag}^{+}$ions are eluted from silver titanate mainly by ion-exchange reaction with cations, resulting in deposition of silver chloride particles. After immersing the film in SBF for 1 day, SEM images reveal only bulky $\mathrm{AgCl}$ particles on the film surface, however immersing the film for 2 days, the surface is almost covered with a dome-shaped form consisting of foliaceous particles peculiar to the apatite (Figs. 13e, f), as mentioned above. We clarified whether silver nanoparticle or silver titanate contributes more to the apatite formation by investigating the apatite-forming ability of a silver metal plate. After immersing the plate in SBF for 4 days, no apatite formation was evident; thus, the silver titanate nanotubes are responsible for the high apatite-forming ability. Researchers have reported that the effects of crystal structure (Uchida et al., 2003) and surface hydroxyl groups such as Ti-OH (Kasuga et al., 2002) influence the apatite formation on the titanium compounds immersed in SBF. Kokubo et al. reported that the apatite-forming ability is improved by the crystal structure transformation of sodium titanate into titanium dioxide (Fujibayashi et al., 2001; Uchida et al., 2002; Takemoto et al., 2006). Although the detailed crystal structure of silver titanate is not yet known, we speculate that the surface atomic arrangement and surface functional groups of silver titanate might be suitable for rapid apatite formation. We further investigated the high apatite forming ability by considering $-\mathrm{OH}$ groups that influence apatite formation using the FT-IR measurements. As shown in Fig. 15, Na-TNT-TF and $\mathrm{TiO}_{2}-\mathrm{NT}-\mathrm{FT}$ exhibited similar absorption spectra in a wide range of $3000-3700 \mathrm{~cm}^{-1}$. These absorption spectra are considered to be mainly due to water molecules adsorbed on the inner and outer surfaces of nanotubes and partially due to $-\mathrm{OH}$ groups on the surface. Unlike Na-TNT-TF and $\mathrm{TiO}_{2}-$ NT-FT, strong absorption was observed at $3000-3400 \mathrm{~cm}^{-1}$ in addition to $3400-3700 \mathrm{~cm}^{-1}$ in Ag-TNT-TF. This absorption at $3000-3400 \mathrm{~cm}^{-1}$ is considered to indicate the existence of surface $-\mathrm{OH}$ groups due to silver titanate. A surface atomic arrangement peculiar to silver titanate would arise and a large number of $-\mathrm{OH}$ groups would be generated on the nanotube surfaces, which would stimulate apatite formation.

Oh et al. (Oh et al., 2005) and Tsuchiya et al. (Tsuchiya et al., 2006) reported anatase-type titanium dioxide nanotube thin films synthesized by anodization and heat treatment of the titanium metal. We compared the apatite-forming ability of these nanotube thin films with 


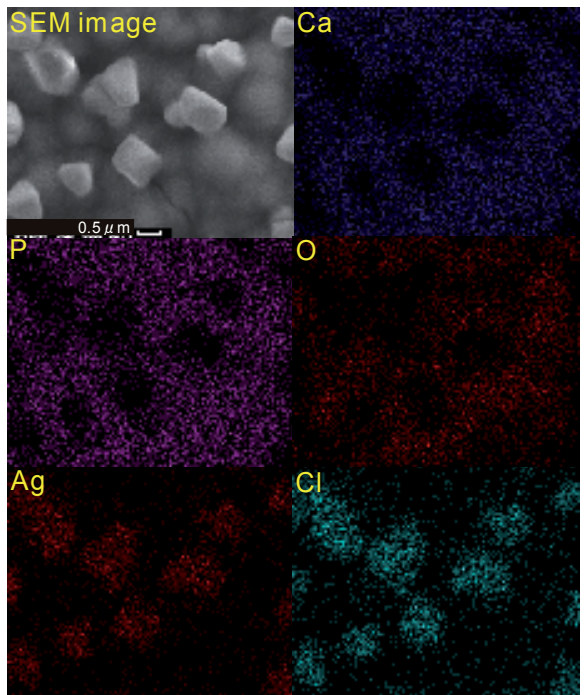

Fig. 14. Elemental mapping performed on Ag-TNT-TF after immersion in SBF for 4 days using EDX analysis.

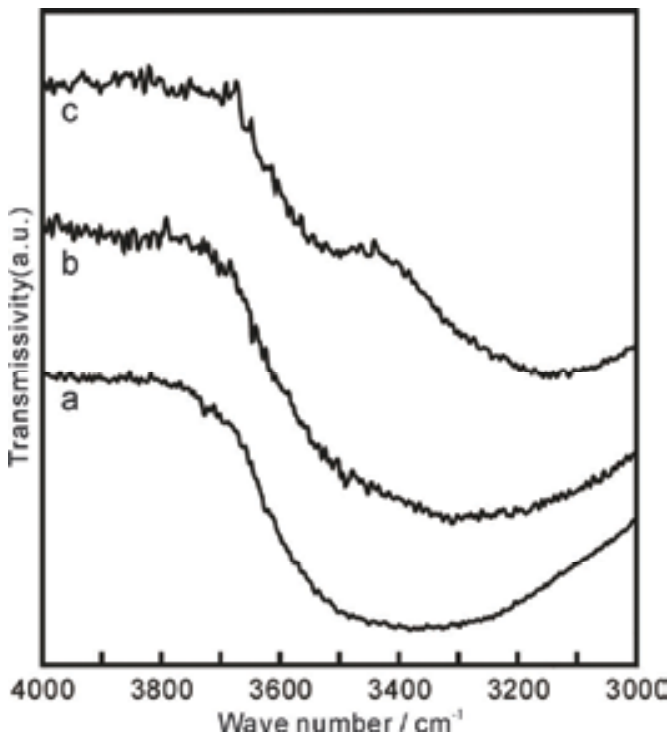

Fig. 15. FT-IR spectra of Na-TNT-TF (a), TiOㄹ-NT-FT (b), and Ag-TNT-TF (c).

that of $\mathrm{TiO}_{2}$-NT-FT obtained in this study by immersing the film in SBF for 2 days. SEM images show not only a small amount of the dome-shaped form consisting of foliaceous particles peculiar to apatite, but also several slightly swelled and whitish areas. EDX element mapping on this thin film revealed that titanium dioxide nanotubes exist in the blackish areas and apatite exists in the whitish areas. At this point, after 2 days of immersion, the apatite phase has grown slightly but not yet achieved its dome-shaped form. Therefore, the apatite-forming ability of $\mathrm{TiO}_{2}-\mathrm{NT}-\mathrm{FT}$ is slightly superior though still similar 
to that of the thin film with 2- $\mu$ m long nanotube synthesized by Tsuchiya et al. (Tsuchiya et al., 2006) and clearly superior to that of the nanotube thin film synthesized by Oh et al. (Oh et al., 2005) and the thin film with 500-nm long nanotube synthesized by Tsuchiya et al. (Tsuchiya et al., 2006). While comparing the ratio of the void parts to the anatase-type titanium dioxide part on the surface using SEM images, we found the proportion consisting anatase-type titanium dioxide to be larger in the surface of our synthesized thin film as compared to that in the surfaces of the thin films reported by Oh et al. and Tsuchiya et al. Hence, the apatite-forming ability of our film is also correspondingly higher.

\section{Conclusion}

In this study, novel procedure of synthesis and fixation of Na-TNT onto titanium metals with various morphologies such as plate, wire, mesh, tube, and sphere was reported. Especially, since the Na-TNT/Ti composite wires have softness and flexibility peculiar to metal titanium because of the existence of titanium metal in its core part, this wire can be fabricated into various shapes of cloth, fiber, etc. with centimeter or meter size by using conventional spinning techniques. The Na-TNT thin films can be transformed into anatasetype titanium dioxide nanotube thin films. Another advantage of the proposed procedure is that the thickness of the thin films produced is greater than that of the thin films reported by other researchers. Therefore, Na-TNT's applications mentioned in the introduction would be remarkably expanded. In addition, the novel growth of the Na-TNT film on substrates such as Co-Cr alloy and SUS316L and simple patterning of the Na-TNT phase by the hydrothermal transcription method were also reported. As these substrates including titanium metal, Co-Cr alloy, and SUS316L have superior mechanical properties and corrosion resistance, they are frequently used as implants such as artificial joints. Generally, the coating of films to implants with complex shapes requires thin films with uniform and controlled thickness, and a high fixing strength to the implants. Because of the direct growth of the nanotubes from the substrate, our proposed method is very simple and the fixing strength to the substrate is expected to be higher. Therefore, the method proposed in the present study has excellent potential for these biomedical applications.

Next, through a silver ion-exchange treatment, $\mathrm{Na}^{+}$ions in sodium titanate nanotube were exchanged with $\mathrm{Ag}^{+}$ions in silver acetate solution, along with the loading of silver nanoparticles on the titanate surfaces, and the layered structure of titanate transformed into a new three-dimensional crystal structure. Results of silver ion elution tests of the obtained thin films in fetal bovine serum solution indicate that the release period and the number of silver ions released from the silver titanate thin films can be controlled. The silver ionexchanged titanate thin films showed high antibacterial activity against MRSA. It was also revealed that although the crystal structure of titanate itself has no large antibacterial effect, higher antibacterial activity mainly arises from the silver ions held in the titanate. The samples coated with apatite containing silver and silver plate have already been reported as possessing antibacterial properties through metallic silver with low solubility. In contrast, in this study, the antibacterial properties were mainly caused by the elution of silver ions from a titanate with an ion-exchange property. Since the thin film obtained by this study has a higher silver-ion elution speed, greater and more rapid antibacterial effects than in metallic silver can be expected. Since we have also revealed that the morphology, thickness, and crystal structure of the titanate phase can be controlled, we think that this can also promise 
control of the antibacterial properties, i.e., the duration and amount of antibacterial activity, for the future. The obtained results should aid the development of more convenient and inexpensive antibacterial implants.

Furthermore, the present study compares the apatite-forming ability of a sodium titanate nanotube thin film, an anatase-type titanium dioxide nanotube thin film, and a silver nanoparticle/silver titanate nanotube nanocomposite thin film. Of these, the apatite-forming ability of the silver titanate nanotube was higher than that of the titanium dioxide nanotubes or the sodium titanate nanotubes, in that order. This superior apatite-forming ability of the silver nanoparticle/silver titanate nanotube nanocomposite thin film is a novel phenomenon and is presumably due to the surface atomic arrangement of silver titanate, the large amount of $\mathrm{Ti}-\mathrm{OH}$ formed on the nanotube surface, or both. In conclusion, the silver nanoparticle/silver titanate nanotube nanocomposite thin film, which have the antibacterial property and the ability to form bone-like apatite, i.e., the osteoconductive property, may have bright prospects for future use in implant materials such as artificial joints.

\section{Acknowledgment}

This research was partially supported by KAKENHI (16685021, 19750172) and Saga University Dean's Grant 2010 For Promising Young Researchers. Figures are reproduced with permission from American Chemical Society, Elsevier, and John Wiley \& Sons.

\section{References}

Alt, V.; Bitschnau, A.; Österling, J.; Sewing, A.; Meyer, C.; Kraus, R.; Meissner, S. A.; Wenisch, S.; Domann, E. \& Schnettler, R. (2006). The effects of combined gentamicin-hydroxyapatite coating for cementless joint prostheses on the reduction of infection rates in a rabbit infection prophylaxis model. Biomaterials, Vol. 27, pp. 4627-4634.

Bavykin, D. V.; Lapkin, A. A.; Plucinski, P. K.; Friedrich, J. M. \& Walsh, F. C. (2005). Reversible storage of molecular hydrogen by sorption into multilayered $\mathrm{TiO}_{2}$ nanotubes. The Journal of Physical Chemistry B, Vol. 109, pp. 19422-19427.

Bavykin, D. V.; Friedrich, J. M. \& Walsh, F. C. (2006). Protonated Titanates and $\mathrm{TiO}_{2}$ Nanostructured Materials: Synthesis, Properties, and Applications. Advanced Materials, Vol. 18, pp. 2807-2824.

Byon, E.; Jeong, Y.; Takeuchi, A.; Kamitakahara, M. \& Ohtsuki, C. (2007). Apatite-forming ability of micro-arc plasma oxidized layer of titanium in simulated body fluids. Surface and Coat Technology, Vol. 201. pp. 5651-5654.

Chen, Q.; Zhou, W.; Du, G. \& Peng, L.-M. (2002). Trititanate nanotubes made via a single alkali treatment. Advanced Materials, Vol. 14, pp. 1208-1211.

Chen, W.; Liu, Y.; Courtney, H. S.; Bettenga, M.; Agrawal, C. M.; Bumgardner, J. D. \& Ong, J. L. (2006). In vitro anti-bacterial and biological properties of magnetron co-sputtered silver-containing hydroxyapatite coating. Biomaterials, Vol. 27, pp. 5512-5517.

Chi, B.; Victorio, E. S. \& Jin, T. (2007). Synthesis of $\mathrm{TiO}_{2}$-Based Nanotube on Ti Substrate by Hydrothermal Treatment. Journal of Nanoscience and Nanotechnology, Vol. 7, pp. 668672. 
Fujibayahsi, S.; Nakamura, T.; Nishiguchi, S.; Tamura, J.; Uchida, M.; Kim, H. M. \& Kokubo, T. (2001). Bioactive titanium : effect of sodium removal on the bone-bonding ability of bioactive titanium prepared by alkali and heat treatment. Journal of Biomedical Materials Research, Vol. 56, pp. 562-570.

Guo, Y.; Lee, N.-H.; Oh, H.-G.; Yoon, C.-R.; Park, K.-S.; Lee, H.-G.; Lee, K.-S. \& Kim, S.-J. (2007). Structure-tunable synthesis of titanate nanotube thin films via a simple hydrothermal process. Nanotechnology, Vol. 18, pp. 295608-295616.

Hanawa, T.; Kon, M.; Ukai, H.; Murakami, K.; Miyamoto, Y. \& Asaoka, K. (1997). Surface Modification of Titanium in Calcium-Ion-Containing Solutions. Journal of Biomedical Materials Research, Vol. 34, pp. 273-278.

Hamada, K.; Kon, M.; Hanawa, T.; Yokoyama, K.; Miyamoto, Y. \& Asaoka, K. (2002). Hydrothermal modification of titanium surface in calcium solutions. Biomaterials, Vol. 23, pp. 2265-2272.

Hardes, J.; Ahrens, H.; Gebert, C.; Streitbuerger, A.; Buerger, H.; Erren, M.; Gunsel, A.; Wedemeyer, C.; Saxler, G \& Winkelmann, W. (2007). Lack of toxicological sideeffects in silver-coated megaprostheses in humans. Biomaterials, Vol. 28, pp. 28692875.

Imai, H.; Takei, Y.; Shimizu, K.; Matsuda, M. \& Hirashima, H. (1999). Direct preparation of anatase $\mathrm{TiO}_{2}$ nanotubes in porous alumina membranes. Journal of Materials Chemistry, Vol. 9, pp. 2971-2972.

Inoue, Y.; Noda, I.; Torikai, T.; Watari, T.; Hotokebuchi, T. \& Yada, M. (2010). $\mathrm{TiO}_{2}$ nanotube, nanowire, and rhomboid-shaped particle thin films fixed on a titanium metal plate. Journal of Solid State Chemistry, Vol. 183, pp. 57-64.

Inoue, Y.; Uota, M.; Torikai, T.; Watari, T.; Noda, I.; Hotokebuchi, T. \& Yada, M. (2010). Antibacterial properties of nanostructured silver titanate thin films formed on a titanium plate, Journal of Biomedical Materials Research Part A, Vol. 92A, pp. 11711180.

Jiang, Z.; Yang, F.; Luo, N.; Chu, B. T. T.; Sun, D.; Shi, H.; Xiao, T. \& Edwards, P. P. (2008). Solvothermal synthesis of $\mathrm{N}$-doped $\mathrm{TiO}_{2}$ nanotubes for visible-light-responsive photocatalysis. Chemical Communication, pp. 6372-6374.

Jung, J. H.; Kobayashi, H.; van Bommel, K. J. C.; Shinkai, S. \& Shimizu, T. (2002). Creation of Novel Helical Ribbon and Double-Layered Nanotube $\mathrm{TiO}_{2}$ Structures Using an Organogel Template. Chemistry of Materials, Vol. 14, pp. 1445-1447.

Jonášová, L.; Müller, F. A.; Helebrant, A.; Strnad, J. \& Greil, P. (2003). Biomimetic apatite formation on chemically treated titanium. Biomaterials, Vol. 25, pp. 1187-1194.

Kasuga, T.; Hiramatsu, M.; Hoson, A.; Sekino, T. \& Niihara, K. (1998). Formation of titanium oxide nanotube. Langmuir, Vol. 14, pp. 3160-3163.

Kasuga, T.; Hiramatsu, M.; Hoson, A.; Sekino, T. \& Niihara, K. (1999). Titania nanotubes prepared by chemical processing. Advanced Materials, Vol. 11, pp. 1307-1311.

Kasuga, T.; Kondo, H. \& Nogami, M. (2002). Apatite formation on $\mathrm{TiO}_{2}$ in simulated body fluid. Journal of Crystal Growth, Vol. 235, pp. 235-240.

Kasuga, T. (2003). Jpn. Kokai Tokkyo Koho, P2003-220127A.

Kim, G.-S.; Ansari, S. G.; Seo, H.-K.; Kim, Y.-S. \& Shin, H.-S. (2007). Effect of annealing temperature on structural and bonded states of titanate nanotube films. Journal of Applied Physics, Vol. 101, pp. 024314-024320. 
Kim, H. M.; Miyaji, F.; Kokubo, T. \& Nakamura, T. (1996). Preparation of bioactive Ti and its alloys via simple chemical surface treatment. Journal of Biomedical Materials Research, Vol. 32, pp. 409-417.

Kim, H. M.; Miyaji, F.; Kokubo, T. \& Nakamura, T. (1997). Effect of heat treatment on apatite-forming ability of Ti metal induced by alkali treatment. Journal of Materials Science: Materials in Medicine, Vol. 8, pp. 341-347.

Kim, H. M.; Miyaji, F.; Kokubo, T.; Nishiguchi, S. \& Nakamura, T. (1997). Graded surface structure of bioactive titanium prepared by chemical treatment. Journal of Biomedical Materials Research, Vol. 45, pp. 100-107.

Kim, H. M.; Himeno, T.; Kawashita, M.; Lee, J. H. \& Kokubo, T. (2003). Surface Potential Change in Bioactive Titanium Metal during the Process of Apatite Formation in Simulated Body Fluid. Journal of Biomedical Materials Research Part A, Vol. 67A, pp. 1305-1309.

Kokubo, T.; Miyaji, F.; Kim, H. M. \& Nakamura, T. (1996). Spontaneous Formation of Bonelike Apatite Layer on Chemically Treated Titanium Metals. Journal of the American Ceramic Society, Vol. 79, pp. 1127-1129.

Kokubo, T. \& Takadama, H. (2006). How useful is SBF inpredicting in vivo bone bioactivity ? Biomaterials, Vol. 27, pp. 2907-2915.

Kon, M.; Sultana, R.; Fujihara, E.; Asaoka, K. \& Ichikawa, T. (2007). Hydroxyapaite Morphology Control by Hydrothermal Treatment. Key Engineering Materials, Vol. 330-332, pp. 737-740.

Kubota, S.; Johkura, K.; Asanuma, K.; Okouchi, Y.; Ogiwara, N.; Sasaki, K. \& Kasuga, T. (2004). Titanium oxide nanotubes for bone regeneration. Journal of Materials Science: Materials in Medicine, Vol. 15, pp. 1031-1035.

Ma, R.; Bando, Y. \& Sasaki, T. (2003). Nanotubes of lepidocrocite titanates. Chemical Physics Letters, Vol. 380, pp.577-582.

Ma, R.; Sasaki, T. \& Bando, Y. (2004). Layer-by-Layer Assembled Multilayer Films of Titanate Nanotubes, Ag- or Au-Loaded Nanotubes, and Nanotubes/Nanosheets with Polycations. Journal of the American Chemical Society, Vol. 126, pp. 10382-10388.

Macak, J. M.; Tsuchiya, H. \& Schmuki, P. (2005). High-Aspect-Ratio $\mathrm{TiO}_{2}$ Nanotubes by Anodization of Titania. Angewandte Chemie International Edition, Vol. 44, pp. 21002102.

Miyauchi, M.; Tokudome, H. Toda, Y.; Kamiya, T. \& Hosono, H. (2006). Electron Field Emission from $\mathrm{TiO}_{2}$ Nanotube Arrays Synthesized by Hydrothermal Reaction. Applied Physics Letters, Vol. 89, pp.043114-043116.

Muramatsu, K.; Uchida, M.; Kim, H. M.; Fujisawa, A. \& Kokubo, T. (2003). Thromboresistance of alkali- and heat-treated titanium metal formed with apatite. Journal of Biomedical Materials Research Part A, Vol. 65A, pp. 409-416.

Nakagawa, M.; Zhang, L.; Udou, K.; Matsuya, S. \& Ishikawa, K. (2005). Effects of hydrothermal treatment with $\mathrm{CaCl}_{2}$ solution on surface property and cell response of titanium implants. Journal of Materials Science: Materials in Medicine, Vol. 16, pp. 985-991.

Nakahira, A.; Kato, W.; Tamai, M.; Isshiki, T.; Nishio, K. \& Aritani, H. (2004). Synthesis of nanotube from a layered $\mathrm{H}_{2} \mathrm{Ti}_{4} \mathrm{O}_{9} \mathrm{H}_{2} \mathrm{O}$ in a hydrothermal treatment using various titania sources. Journal of Materials Science, Vol. 39, pp. 4239-4245. 
Nishiguchi, S.; Nakamura, T.; Kobayashi, M.; Kim, H. M.; Miyaji, F. \& Kokubo, T. (1999). The effect of heat treatment on bone-bonding ability of alkali-treated titanium. Biomaterials, Vol. 20, pp. 491-500.

Oh, S. H.; Finõnes, R. R.; Daraio, C.; Chen, L. H. \& Jin, S. (2005). Growth of nano-scale hydroxyapatite using chemically treated titanium oxide nanotubes. Biomaterials, Vol. 26, pp. 4938-4943.

Ohtsu, N.; Abe, C.; Ashino, T.; Semboshi, S. \& Wagatsuma, K. (2008). Calcium-hydroxide slurry processing for bioactive calcium-titanate coating on titanium. Surface and Coat Technology, Vol. 202, pp. 5110-5115.

Ohtsuki, C.; Iida, H.; Hayakawa, S. \& Osaka, A. (1997). Bioactivity of titanium treated with hydrogen peroxide solutions containing metal chlorides. Journal of Biomedical Materials Research, Vol. 35, pp. 39-47.

Shin, H.; Jeong, D.-K.; Lee, K.; Sung, M. M. \& Kim, J. (2004). Formation of $\mathrm{TiO}_{2}$ and $\mathrm{ZrO}_{2}$ Nanotubes Using Atomic Layer Deposition with Ultra-Precise Wall Thickness Control, Advanced Materials, Vol. 16, pp. 1197-1200.

Sun, X. \& Li, Y. (2003). Synthesis and Characterization of Ion-Exchangeable Titanate Nanotubes. Chemistry - A European Journal, Vol. 9, pp. 2229-2238.

Tsai, C.-C. \& Teng, H. (2006). Structural features of nanotubes synthesized from $\mathrm{NaOH}$ treatment on $\mathrm{TiO}_{2}$ with different post-treatments. Chemistry of Materials, Vol. 18, pp. 367-373.

Takemoto, M.; Fujibayashi, S.; Neo, M.; Suzuki, J.; Matsushita, T.; Kokubo, T. \& Nakamura, T. (2006). Effect of sodium removal by dilute $\mathrm{HCl}$ treatment. Biomaterials, Vol. 27, pp. 2682-2691.

Thorne, A.; Kruth, A.; Tunstall, D.; Irvine, J. T. S. \& Zhou, W. (2005). Formation, Structure, and Stability of Titanate Nanotubes and their Proton Conductivity. The Journal of Physical Chemistry B, Vol. 109, pp. 5439-5444.

Tian, Z. R.; Voigt, J. A.; Liu, J.; Mckenzie, B. \& Xu, H. (2003). Large oriented arrays and continuous films of $\mathrm{TiO}_{2}$-based nanotubes. Journal of the American Chemical Society, Vol. 125, pp. 12384-12385.

Tokudome, H. \& Miyauchi, M. (2004). N-doped $\mathrm{TiO}_{2}$ Nanotube with Visible Light Activity. Chemistry Letter, Vol. 33, pp. 1108-1109.

Tokudome, H. \& Miyauchi, M. (2004). Titanate nanotube thin films via alternate layer deposition. Chemical Communication, pp. 958-959.

Tokudome, H. \& Miyauchi, M. (2005). Electrochromism of titanate-based nanotubes. Angewandte Chemie International Edition, Vol. 44, pp. 1974-1977.

Tsuchiya, H.; Macak, J. M.; Müller, L.; Kunze, J.; Müller, F.; Greil, P.; Virtanen, S. \& Schmuki, P. (2006). Hydroxyapatite growth on anodic $\mathrm{TiO}_{2}$ nanotubes. Journal of Biomedical Materials Research Part A, Vol. 77A, pp. 534-541.

Uchida, S.; Chiba, R.; Tomiha, M.; Masaki, N. \& Shirai, M. (2002). Application of Titania Nanotubes to a Dye-Sensitized Solar Cell. Electrochemistry, Vol. 70, pp. 418-420.

Uchida, M.; Kim, H. M.; Kokubo, T.; Fujibayashi, S. \& Nakamura, T. (2002). Effect of water treatment on the apatite-forming ability of $\mathrm{NaOH}$-treated titanium metal. Journal of Biomedical Materials Research, Vol. 63, pp. 522-530.

Uchida, M.; Kim, H. M.; Kokubo, T.; Fujibayashi, S. \& Nakamura, T. (2003). Structural dependence of apatite formation on titania gels in a simulated body fluid. Journal of Biomedical Materials Research Part A, Vol. 64A, pp. 164-70. 
Wang, F.; Liao, Y.; Wang, M.; Gong, P.; Li, X.; Tang, H.; Man, Y.; Yuan, Q.; Wei, N.; Tan, Z. \& Ban, Y. (2007). Evaluation of Sodium Titanate Coating on Titanium by Sol-Gel Method In Vitro. Key Engineering Materials, Vol. 330-332, pp. 777-780.

Wang, X. J.; Li, Y. C.; Lin, J. G.; Yamada, Y.; Hodgson, P. D. \& Wen, C. E. (2008). In vitro bioactivity evaluation of titanium and niobium metals with different surface morphologies. Acta Biomaterialia, Vol. 4, pp. 1530-1535.

Wang, X. X.; Hayakawa, S.; Tsuru, K. \& Osaka, A. (2001). A comparative study of in vitro apatite deposition on heat-, $\mathrm{H}_{2} \mathrm{O}_{2-}$, and $\mathrm{NaOH}$ - treated titanium surfaces. Journal of Biomedical Materials Research, Vol. 54, pp. 172-178.

Wang, X. X.; Yan, W.; Hayakawa, S.; Tsuru, K. \& Osaka, A. (2003). Apatite deposition on thermally and anodically oxidized titanium surfaces in a simulated body fluid. Biomaterials, Vol. 24, pp. 4631-4637.

Wu, D.; Liu, J.; Zhao, X.; Li, A.; Chen, Y. \& Ming, N. (2006). Sequence of events for the formation of titanate nanotubes, nanofibers, nanowires, and nanobelts. Chemistry of Materials, Vol. 18, pp. 547-553.

Xiong, J.; Li, Y.; Hodgson, P. D. \& Wen, V. (2010). Nanohydroxyapatite coating on a titanium-niobium alloy by a hydrothermal process. Acta Biomaterialia, Vol. 6, pp. 1584-1590.

Yada, M.; Inoue, Y.; Uota, M.; Torikai, T.; Watari, T.; Noda, I. \& Hotokebuchi, T. (2007). Plate, Wire, Mesh, Microsphere, and Microtube Composed of Sodium Titanate Nanotubes on a Titanium Metal Template. Langmuir, Vol. 23, pp. 2815-2823.

Yada, M.; Inoue, Y.; Uota, M.; Torikai, T.; Watari, T.; Noda, I. \& Hotokebuchi, T. (2008). Formation of Sodium Titanate Nanotube Films by Hydrothermal Transcription. Chemistry of Materials, Vol. 20, pp. 364-366.

Yada, M.; Inoue, Y.; Gyoutoku, A.; Noda, I.; Torikai, T.; Watari, T. \& Hotokebuchi, T. (2010). Apatite-forming ability of titanium compound nanotube thin films formed on a titanium metal plate in a simulated body fluid. Colloids and Surfaces B: Biointerfaces, Vol. 80, pp. 116-124.

Yamanaka, S.; Hamaguchi, T.; Muta, H.; Kurosaki, K. \& Uno, M. (2004). Fabrication of Oxide Nanohole Arrays by a Liquid Phase Deposition Method. Journal of Alloys and Compounds, Vol. 373, pp. 312-315.

Yang, J.; Jin, Z.; Wang, X.; Li, W.; Zhang, S.; Guo, X. \& Zhang, Z. (2003). Study on composition, structure and formation process of nanotube $\mathrm{Na}_{2} \mathrm{Ti}_{2} \mathrm{O}_{4}(\mathrm{OH})_{2}$. Journal of the Chemical Society Dalton Transactions, Vol. 3, pp. 3898-3901.

Zhu, H. Y.; Lan, Y.; Gao, X. P.; Ringer, S., P.; Zheng, Z. F.; Song, D. Y. \& Zhao, J. C. (2005). Phase Transition between Nanostructures of Titanate and Titanium Dioxides via Simple Wet-Chemical Reactions. Journal of the American Ceramic Society, Vol. 127, pp. $6730-6736$. 


\title{
Self-Organization of Silver-Core Bimetallic Nanoparticles and Their Application for Catalytic Reaction
}

\author{
Kazutaka Hirakawa \\ Faculty of Engineering, Shizuoka University \\ Japan
}

\section{Introduction}

Metal nanoparticles have received much attentions as a building block of advanced materials for nanoscience and nanotechnology (Bönnemann \& Richards, 2001). Their optical, (Fukumi et al., 1994; Lu et al., 1999; Link et al., 1999; Shipway et al., 2000), magnetic (Sun et al., 1999; Teranishi \& Miyake, 1999), and catalytic (Kiely et al., 1998; Pileni, 1998; Bradley, 1994; Harriman, 1990; Lee et al., 1995; Toshima et al., 1995; Bonilla et al., 2000; Siepen et al., 2000) properties have been reported with great interests. The character of metal nanoparticle can be altered by the addition of other metals. Bimetallic nanoparticles, composed of two different metallic elements, have been reported to show outstanding characters different from the corresponding monometallic nanoparticles (Harriman, 1990; Yonezawa \& Toshima, 1993; Toshima \& Hirakawa, 1997, 1999; Toshima \& Wang, 1994; Lee et al., 1995). For example, catalytic activities of gold $(\mathrm{Au})$-core structured bimetallic nanoparticles, gold/platinum (Au/Pt) (Harriman, 1990; Yonezawa \& Toshima, 1993; Toshima \& Hirakawa, 1999), gold/palladium (Au/Pd) (Toshima \& Hirakawa, 1999; Lee et al., 1995), and gold/rhodium (Au/Rh) (Toshima \& Hirakawa, 1999), for hydrogenation and/or water reduction are higher than platinum $(\mathrm{Pt})$, palladium $(\mathrm{Pd})$, and rhodium $(\mathrm{Rh})$ monometallic nanoparticles, respectively. Surprisingly, in some cases, a physical mixture of monometallic nanoparticles such as $\mathrm{Pt}$ and ruthenium $(\mathrm{Ru})$ nanoparticles in solution shows higher catalytic activity than the corresponding monometallic nanoparticles under a certain condition (Toshima et al., 1995; Toshima \& Hirakawa, 1997). This suggests that an interaction between two kinds of monometallic nanoparticles can produce novel nanoparticles. Further, it has been reported that physical mixture of silver (Ag) and other metal nanoparticles, such as $\mathrm{Pt}, \mathrm{Rh}$, and $\mathrm{Pd}$, spontaneously forms the bimetallic nanoparticles with Ag-core structure in aqueous solution. This reaction can be used to construct the core-shell structured novel bimetallic nanoparticles. The formed nanoparticles demonstrate superior character for certain catalytic reactions.

In this chapter, the simple method of the preparation of core-shell structured bimetallic nanoparticles by the physical mixing and the application of the formed novel metal nanoparticles for catalytic reaction are described. The topics of the catalytic reaction presented in this chapter are the visible light induced hydrogen generation (Toshima \& 
Hirakawa, 2003), the removal of reactive oxygen species (Hirakawa \& Sano, 2009), and its application to the chemoprevention of ultraviolet induced biomolecules damage (Hirakwa et al., 2008, 2009).

\section{Spontaneous formation of silver-core bimetallic nanoparticles}

Much attention has been paid to bimetallic nanoparticles, especially those having a core/shell structure (Toshima et al., 2007). From the view point of Au catalysts, bimetallic nanoparticles have received much attention recently. On the other hand, a physical mixture of monometallic nanoparticles such as $\mathrm{Pt}$ and $\mathrm{Ru}$ nanoparticles in solution shows higher catalytic activity than the corresponding monometallic nanoparticles under a certain condition (Toshima et al., 1995; Toshima \& Hirakawa, 1997). Further, it has been reported that physical mixture of $\mathrm{Ag}$ and other metal nanoparticles, such as $\mathrm{Pt}, \mathrm{Rh}$, and $\mathrm{Pd}$, spontaneously forms the bimetallic nanoparticles with Ag-core structure in aqueous solution (Figure 1). In this section, the spontaneous formation of the Ag-core bimetallic nanoparticles is reviewed.

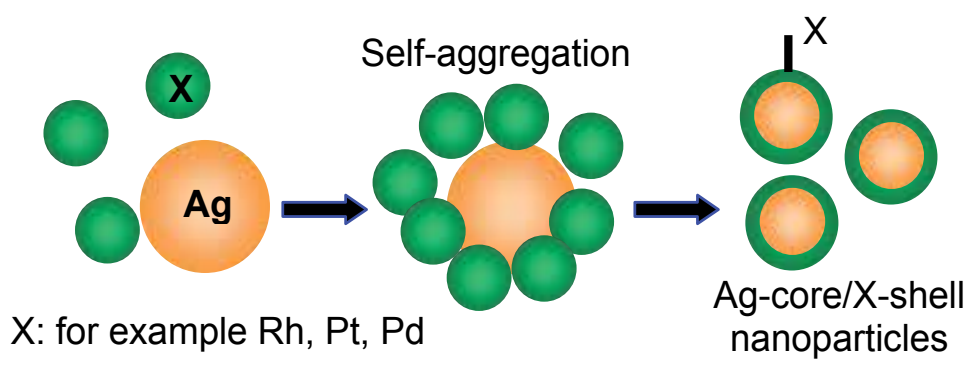

Fig. 1. Schematic diagram of the spontaneous formation of Ag-core bimetallic nanoparticles

\subsection{Siver-core/rhodium-shell bimetallic nanoparticles}

The interaction between $\mathrm{Ag}$ and $\mathrm{Rh}$ monometallic nanoparticles in solution by physical mixing was reported. The main reason for using $\mathrm{Ag}$ and $\mathrm{Rh}$ nanoparticles is the reported prominent characteristics of Rh nanoparticles as a catalyst (Toshima \& Hirakawa, 1999), and the expected electronic effect of Ag similar to Au upon enhancement of the catalytic activity of Rh. Furthermore, Ag is inexpensive metal compared with Au. The colloidal dispersions of $\mathrm{Ag}$ and Rh monometallic nanoparticles protected by poly(N-vinyl-2-pyrrolidone) (PVP), a water soluble polymer, were prepared by an alcohol reduction method (Hirai et al., 1979). Average diameters of $\mathrm{Ag}$ and $\mathrm{Rh}$ monometallic nanoparticles were $7.5 \mathrm{~nm}$ and $2.2 \mathrm{~nm}$, respectively.

\subsubsection{Surface plasmon absorption of siver-core bimetallic nanoparticles}

Colloidal sol of Ag nanoparticles shows characteristic plasmon absorption aeound $400 \mathrm{~nm}$ (Henglein, 1979). The plasmon absorption band of Ag nanoparticles decreased by addition of $\mathrm{Rh}$ nanoparticles and was almost completely extinguished within $30 \mathrm{~min}$ after mixing (Figure 2). The parts of plasmon absorption in larger wavelength region were preferentially 
extinguished within $10 \mathrm{~min}$, suggesting that influences of $\mathrm{Rh}$ nanopartilces on $\mathrm{Ag}$ nanoparticles depend on the size of the Ag nanoparticles. When relatively smaller molar quantity of Rh to Ag was added, the plasmon absorption was not completely extinguished. More than 40 atom-mol\% of $\mathrm{Rh}$ against to $\mathrm{Ag}$ was required to extinguish the plasmon absorption band completely.

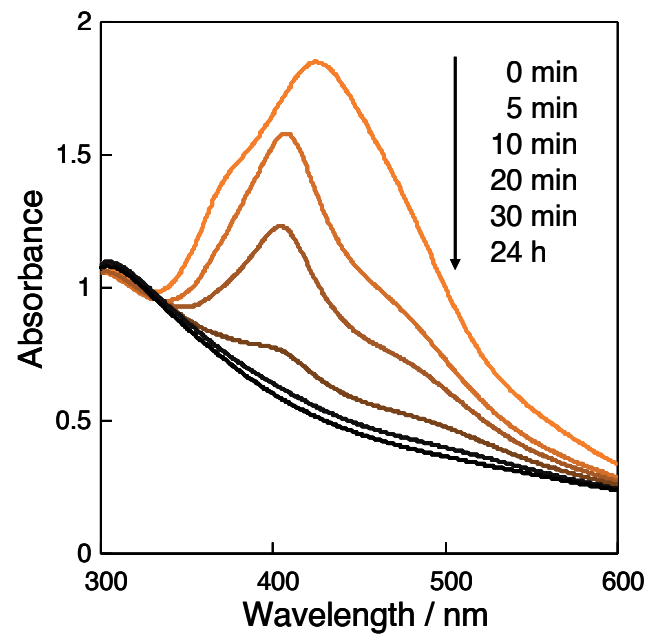

Fig. 2. UV-Vis spectral change of the physical mixtures of dispersions of Ag and Rh nanoparticles. The aqueous solutions of $\mathrm{Ag}(1$ atom-mmol L-1, $50 \mathrm{~mL})$ and $\mathrm{Rh}(1$ atom-mmol $\mathrm{L}^{-1}, 50 \mathrm{~mL}$ ) nanoparticles were mixed.

\subsubsection{Transmission electron microgram of the siver-core bimetallic nanoparticles}

Figure 3 shows transmission electron microscopy (TEM) photographs of the physical mixtures of $\mathrm{Ag}$ and $\mathrm{Rh}$ monometallic nanoparticles. The samples for TEM measurement were prepared by drying the aqueous dispersions of the physical mixtures of $\mathrm{Ag}$ and $\mathrm{Rh}$ nanoparticles under vacuum in 0,10 , and $30 \mathrm{~min}$, and $24 \mathrm{~h}$, respectively, after mixing. Relatively large particles are attributed to Ag nanoparticles, and rather small ones are $\mathrm{Rh}$ nanoparticles. The TEM photographs showed that Rh particles gathered around Ag particle to surround within several minutes, comparable period of the extinction of plasmon absorption. Interestingly, these aggregated particles changed into homogeneous small particles (average diameter $=2.7 \mathrm{~nm}$ ) after $24 \mathrm{~h}$. Preliminary study has shown that the

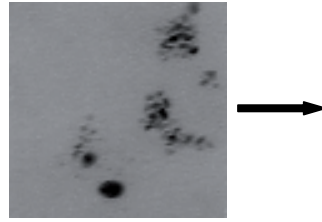

0 min

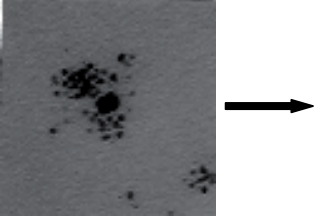

$10 \mathrm{~min}$

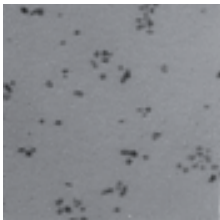

$24 \mathrm{~h}$

Fig. 3. TEM photographs of the physical mixtures of Ag and Rh nanoparticles. The aqueous solutions of Ag and Rh nanoparticles (1/1, atom-mol/atom-mol) were mixed, and dried after indicated periods. 
increase of $\mathrm{Rh} / \mathrm{Ag}$ molar ratio reduces the average diameter and the size distribution of the nanoparticles. The elemental analysis using characteristic X-ray in high-resolution TEM measurement has shown that the particles produced from their physical mixtures in $24 \mathrm{~h}$ are composed of Ag and Rh.

\subsubsection{X-ray diffraction of the of siver-core bimetallic nanoparticles}

Figure 4 shows $\mathrm{X}$-ray diffraction (XRD) patterns of poly( $N$-vinyl-2-pyrrolidone)-protected $\mathrm{Ag}$ and $\mathrm{Rh}$ monometallic nanoparticles, and their physical mixture. The sample of the physical mixture of $\mathrm{Ag}$ and $\mathrm{Rh}$ nanoparticles was prepared by drying the mixtures of their aqueous solutions under vacuum for $24 \mathrm{~h}$ after mixing. The XRD pattern of the mixtures of $\mathrm{Ag}$ and $\mathrm{Rh}$ nanoparticles was similar to that of Rh nanoparticle, suggesting that the surface of the particle produced by mixing $\mathrm{Ag}$ and $\mathrm{Rh}$ nanoparticles is composed of Rh. Similarly, Au-core/Pt-shell and Au-core/Pd-shell structured nanoparticles have shown the XRD pattern quite similar to that of their surface metals (Yonezawa \& Toshima, 1995). These findings suggest that the aggregation of $\mathrm{Rh}$ particles around the Ag particle is involved in the extinction of the plasmon absorption.

\subsubsection{Mechanism of the formation of the siver-core/rhodium shell bimetallic nanoparticles}

Henglein et al. reported that lead $(\mathrm{Pb})$ atoms transfer from $\mathrm{Pb}$ colloidal particle onto the surface of $\mathrm{Ag}$ colloidal particle in physical mixing of $\mathrm{Ag}$ and $\mathrm{Pb}$ colloidal sols (Henglein et al., 1992). If the extinction of the plasmon absorption is due to coating of the surface of $\mathrm{Ag}$ particle by $\mathrm{Rh}$ atoms transferred from $\mathrm{Rh}$ nanoparticle, at least $28 \mathrm{~mol} \%$ of $\mathrm{Rh}$ to $\mathrm{Ag}$ is required assuming that a $\mathrm{Ag}$ particle (average diameter $=7.5 \mathrm{~nm}$ ) is uniformly coated by $\mathrm{Rh}$ atoms in a one-atom layer. In the present experiments about 40 atom-mol\% of Rh to Ag was

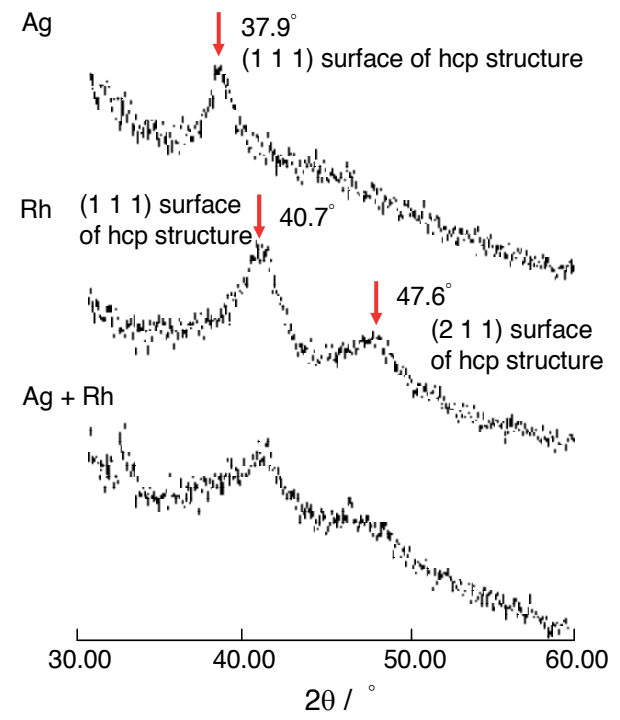

Fig. 4. XRD patterns of Ag and Rh monometallic nanoparticles, and their physical mixture $(1 / 1$, atom-mol/atom-mol) 
required to completely extinguish the plasmon absorption, which is reasonably supporting the above assumption. These observations suggest that the physical mixture of Ag and Rh nanoparticles spontaneously generates $\mathrm{Ag} / \mathrm{Rh}$ bimetallic nanoparticles with an $\mathrm{Ag}$ core/Rh-shell structure. The disappearance of the XRD peak of Ag nanoparticles suggests that the core of this bimetallic nanoparticles is not complete Ag, but possibly has a partial $\mathrm{Ag} / \mathrm{Rh}$ alloy structure. The driving force of the formation of this $\mathrm{Ag} / \mathrm{Rh}$ bimetallic nanoparticles may be due to the larger binding energy between $\mathrm{Ag}$ and $\mathrm{Rh}$ atoms than between Rh atoms (Peiner \& Kopitzki, 1998). Reduction of diameter of the nanoparticle increases not only its surface energy but also number of the binding sites between Ag and $\mathrm{Rh}$ atoms, which stabilizes the total energy. Therefore, the shrinking of $\mathrm{Ag} / \mathrm{Rh}$ bimetallic nanoparticles might be explained by the balance between the binding energy and the surface energy. The size and the rate of formation of the bimetallic nanoparticles can be controlled by the kind and concentration of protective agents. The self-assembling formation of bimetallic nanoparticle using Ag nanoparticle is applicable to construction of novel nanoparticles.

\subsection{Silver-core/noble metal-shell bimatallic nanoparticles}

The above mentioned procedure can be used to prepare the Ag-core/noble metal shell nanoparticles, other than Ag-core/Rh-shell nanoparticles. The physical mixing of Ag and other metal nanoparticles, such as $\mathrm{Au}, \mathrm{Pt}, \mathrm{Rh}$, and $\mathrm{Pd}$ particles, produces $\mathrm{Ag}$-core bimetallic particles. The interaction rate between $\mathrm{Ag}$ and other metal nanoparticles was determined by the extinction of the surface plasmon absorption of Ag nanoparticle. The initial step of this reaction was investigated by isothermal titration calorimetry (Toshima et al., 2005). This study revealed that the strength of the interaction between Ag and other metals increases in the order of $\mathrm{Rh} / \mathrm{Ag}>\mathrm{Pd} / \mathrm{Ag}>\mathrm{Pt} / \mathrm{Ag}$.

The formed Ag-core/Pt-shell nanoparticle catalyzed the decomposition of hydrogen peroxide (described later). On the other hand, $\mathrm{Au}$ and $\mathrm{Au} / \mathrm{Ag}$ nanoparticles showed an activity of photocatalytic decomposition of methylene blue (Hirakawa, 2007), although their activities were significantly smaller than that of well-known titanium dioxide photocatalyst (Fujishima et al., 2000, 2008). The physical mixing method is simple and useful to prepare novel bimetallic nanoparticles. These nanoparticles may be used as catalyst and photocatalyst.

\subsection{Application to the preparation of trimetallic nanoparticles}

This method can be applied to the preparation of trimetallic nanoparticles (Toshima et al., 2007, 2011). It has been reported that the synthesis of trimetallic nanoparticles having a Aucore structure by a combination of the preparation of bimetallic nanoparticles by coreduction with the formation of core/shell-structured bimetallic nanoparticles by selforganization in physical mixture (Figure 5). The formation of trimetallic nanoparticles has been suggested by UV-Vis spectral change, TEM image change, FT-IR spectra of adsorbed carbon monoxide, XPS spectra and calorimetric studies. The catalytic activity of trimetallic nanoparticles in the molar ratio of $\mathrm{Au} / \mathrm{Pd} / \mathrm{Rh}=1 / 4 / 20$ was higher than the corresponding monometallic and bimetallic nanoparticles for hydrogenation of methyl acrylate. This high catalytic activity can be understood by sequential electronic charge transfer from surface Rh atoms to interlayered $\mathrm{Pt}$ atoms and then to core Au atoms (Toshima et al., 2011). 


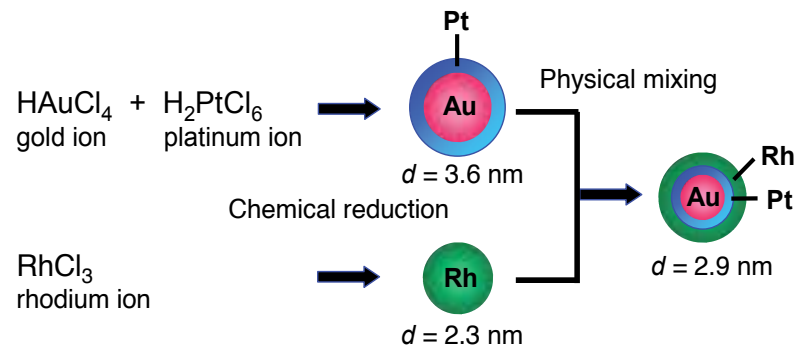

Fig. 5. Schematic diagram of the formation of the trimetallic $\mathrm{Au} / \mathrm{Pt} / \mathrm{Rh}$ nanoparticles

\section{Visible-light-induced hydrogen generation by metal nanoparticle catalytic system}

Metal nanoparticles are very important materials for nanoscience and nanotechnology (Fukumi et al., 1994; Lu et al., 1999; Link et al., 1999; Sun et al., 1999; Teranishi \& Miyake, 1999; Akinaga, 2002). A particularly large number of reports have been published on their applications to catalysts (Kiely et al., 1998; Pileni, 1998; Bradley, 1994; Widegren \& Finke 2003; Willner et al., 1987; Toshima et al., 1995; Yonezawa \& Toshima, 1993). As the catalyst in the homogeneous system, the colloidal dispersions of metal nanoparticles have the advantage that they are soluble or homogeneous in an aqueous solution and transparent to visible light (Kiely et al., 1998; Pileni, 1998; Bradley, 1994; Widegren \& Finke 2003; Willner et al., 1987). Thus, colloidal metal nanoparticles are useful for photocatalytic reaction systems. For example, colloidal metal nanoparticles catalyze the water reduction in the visible-lightinduced electron transfer system composed of ethylenediaminetetraacetic acid disodium salt (EDTA), tris(bipyridine)ruthenium(II) dichloride $\left(\left[\mathrm{Ru}(\mathrm{bpy})_{3}\right]^{2+}\right)$, and 1,1'-dimethyl-4,4'bipyridium dichloride (methyl viologen, $\mathrm{MV}^{2+}$ ) (Yonezawa \& Toshima, 1993) (Figure 6).

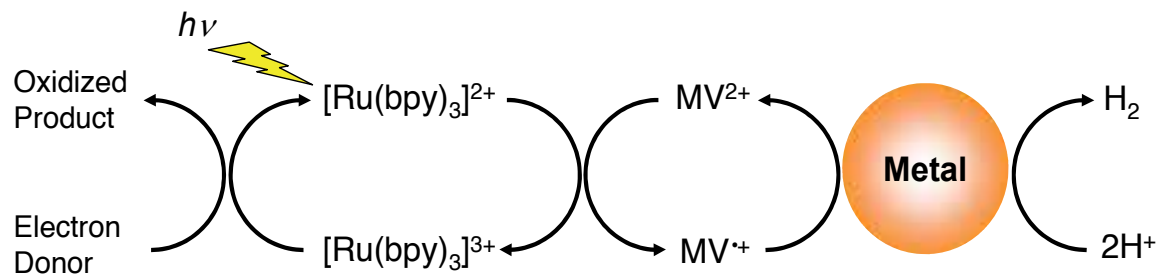

Fig. 6. Schematic diagram of the visible-light induced hydrogen generation using the electron transfer system and metal nanoparticle catalyst

\subsection{Catalytic activity of gold-core/platinum-shell bimetallic nanoparticles}

The bimetallization of metal nanoparticle can improve the catalytic activity of surface metal. Especially, core-shell structured nanoparticles are important. Several study demonstrated the Au-core/Pt shell metal nanoparticles show higher catalytic activity for the visible-lightinduced hydrogen generation than Pt monometallic nanoparticles. The following study is an example of the hydrogen generation using $\mathrm{Au} / \mathrm{Pt}$ nanoparticle catalyst (Yonezawa \& Toshima, 1993). In this study, the Au/Pt bimetallic systems stabilized by polymer and micelle were obtained by alcohol- and photo-reduction of the corresponding metal ions in 
the presence of water-soluble polymers and non-ionic surfactant-micelles, respectively. The $\mathrm{UV}$-Vis spectra and the transmission electron micrographs suggest that the polymerprotected $\mathrm{Au} / \mathrm{Pt}$ bimetallic systems are composed of bimetallic alloy clusters, but the micelle-protected ones are mostly composed of the mixtures of the monometallic $\mathrm{Au}$ and $\mathrm{Pt}$ particles. The in-situ UV-Vis spectra during the reductions can elucidate the formation processes of the bimetallic dispersions which are different from each other depending on the protective reagent. The $\mathrm{Au} / \mathrm{Pt}$ bimetallic systems can be used as the catalyst for visible lightinduced hydrogen generation. The bimetallic system stabilized by the polymer at a molar ratio of $\mathrm{Au} / \mathrm{Pt}=2 / 3$ is the most active catalyst.

\subsection{Application of siver-core/rhodium-shell bimetallic nanoparticles}

It has been reported that the catalytic activity of the $\mathrm{Ag} / \mathrm{Rh}$ bimetallic nanoparticles for visible-light-induced hydrogen generation (Toshima \& Hirakawa, 1999) in an aqueous solution composed of ethylenediaminetetraacetic acid, tris(bipyridine)ruthenium(II), methyl viologen, and metal nanoparticle catalyst. The activity is clearly higher than the corresponding monometallic nanoparticles and alloy-structured $\mathrm{Ag} / \mathrm{Rh}$ nanoparticles, suggesting that the Ag-core shows an electronic effect on the surface Rh as in the case of the Au-core (Yonezawa \& Toshima, 1993) and enhances the catalytic activity of the surface Rh. The highest catalytic activity was observed at 1:9 ratio of $\mathrm{Ag}$ and Rh atoms (Figure 7). Similar results reported on the other catalytic reactions.

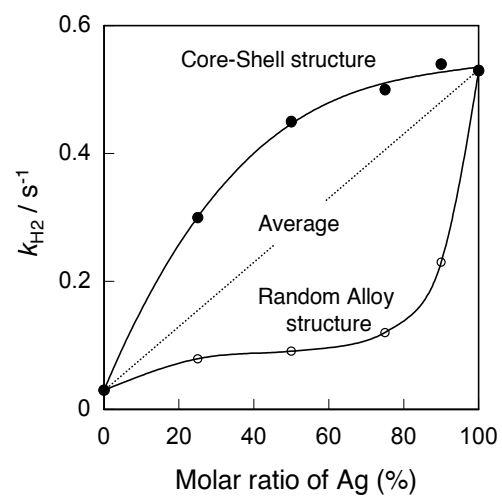

Fig. 7. Hydrogen generation rate coefficient $\left(k_{\mathrm{H} 2}\right)$ depending on the molar ratio of Ag of $\mathrm{Ag} / \mathrm{Rh}$ bimetallic nanoparticles. The $k_{\mathrm{H} 2}$ indicates the number of generated $\mathrm{H}_{2}$ molecules on a surface metal atom per one second. The average is the calculated activity of the simple mixture of Ag and Rh monometallic nanoparticles.

\subsection{Carbon dioxide reduction by visible-light-induced electron transfer system using metal nanoparticle}

A photochemical reduction of $\mathrm{CO}_{2}$ can be applied to a novel energy storage process for the utilization of solar energy in the future. The above mentioned catalytic system can be applied to $\mathrm{CO}_{2}$ reduction. The strategy is the catalytic reduction of $\mathrm{CO}_{2}$ using electrons gathered by an electron transfer system (Willner et al., 1987, Toshima et al., 1995). It has been reported that nanoparticles catalyzes the reduction of $\mathrm{CO}_{2}$ and the generation of methane (Toshima et al., 1995) (Figure 8). 


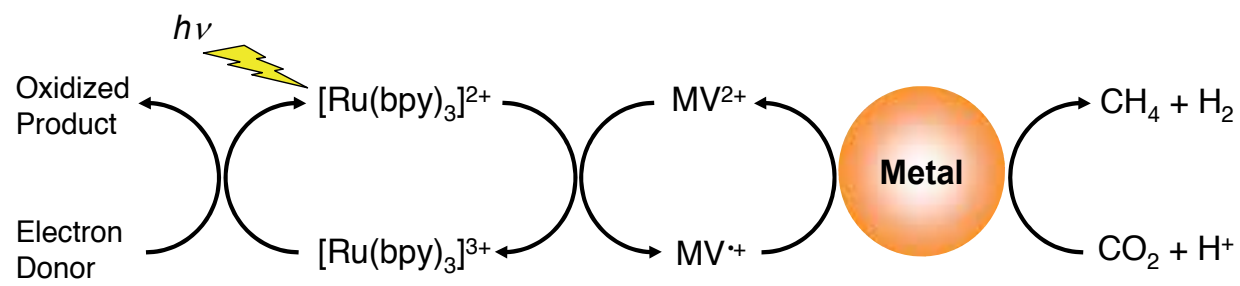

Fig. 8. Schematic diagram of the visible-light induced $\mathrm{CO}_{2}$ reduction using the electron transfer system and metal nanoparticle catalyst

The possible reaction scheme of the $\mathrm{CO}_{2}$ reduction is as follows:

$$
\mathrm{CO}_{2}+8 \mathrm{H}^{+}+8 \mathrm{e}^{-} \rightarrow \mathrm{CH}_{4}+2 \mathrm{H}_{2} \mathrm{O}
$$

This eight-electron reduction of $\mathrm{CO}_{2}$ is advantageous process compared with other possible $\mathrm{CO}_{2}$ reduction process from the thermodynamic point of view. Although it is not a study using the silver-core bimetallic nanoprticles, this topic is closely related to the applications of bimetallic nanoparticles to catalytic reaction. Thus, the topic of the $\mathrm{CO}_{2}$ reduction using metal nanoparticle catalyst is presented here.

Typical reactions were performed by the similar manner to the hydrogen generation. A 20$\mathrm{cm}^{3}$ Pyrex Schlenk tube was charged with a $10 \mathrm{~cm}^{3}$ aqueous solution, containing EDTA (a sacrificial electron donor), $\left[\mathrm{Ru}(\mathrm{bpy})_{3}\right]^{2+}$ (a photosensitizer), $\mathrm{MV}^{2+}$ (an electron mediator), $\mathrm{NaHCO}_{3}$ (a pH adjuster and a $\mathrm{CO}_{2}$ source), and colloidal dispersion of metal nanoparticles. The mixtures were degassed by freeze-thaw cycles and the tubes were then filled with $1 \mathrm{~atm}$ of $\mathrm{CO}_{2}$. The photo-irradiation was carried out for 3 or $4 \mathrm{~h}$ with a $500 \mathrm{~W}$ super-high-pressure mercury lamp through a UV cut filter $(>390 \mathrm{~nm})$ in a water bath maintained at $30^{\circ} \mathrm{C}$. About $100 \mu \mathrm{mol}$ of methane was detected in this system (Toshima et al., 1995). However, it has not been confirmed that methane was actually the reduction product of $\mathrm{CO}_{2}$.

\subsubsection{Strategy for the demonstration of the methane generation from carbon dioxide}

In a heterogeneous system, photoreduction of $\mathrm{CO}_{2}$ was confirmed by experiments using an isotope (Ishitani et al. 1993). To our knowledge, however, the isotopic method has not been applied to the confirmation of the photoreduction of $\mathrm{CO}_{2}$ in a homogeneous system using the colloidal dispersion of metal nanoparticles. To confirm the above mentioned methane generation, the following study was carried out. In this study, photoreduction of $\mathrm{CO}_{2}$ was carried out in a similar system to one reported previously (Toshima et al., 1995), and the generation of methane from $\mathrm{CO}_{2}$ was confirmed by isotopic experiments. As the catalysts, novel metal nanoparticles, i.e., liposome-protected Pt nanoparticles, were prepared and used in the present system. Colloidal dispersions of $\mathrm{Pt}$ and $\mathrm{Ru}$ nanoparticles were prepared by photoreduction without using ethanol (Yamaji et al. 1995). Preparation of nanoparticles without ethanol is required, because the coexisting ethanol is decomposed during the photochemical reaction, leading to the formation methane. This methane formation cannot be distinguished from the actual methane generation from $\mathrm{CO}_{2}$. Protective agents used for the metal nanoparticles were poly( $N$-vinyl-2-pyrrolidone), $\mathrm{C}_{12} \mathrm{EO}$, and liposome. The products in the gas phase were analyzed with a gas chromatograph. The characterization of gaseous products was carried out with a gas chromatograph mass-spectrometer. 


\subsubsection{Methane generation from carbon dioxide reduction}

The formation of methane was then clearly detected by gas chromatography (about 19 nmol in the case of the liposome protected $\mathrm{Pt}$ nanoparticles system). In order to confirm the methane generation from $\mathrm{CO}_{2}$, isotope experiments were carried out using $\mathrm{NaH}^{13} \mathrm{CO}_{3}$ as a $\mathrm{CO}_{2}$ source and analyzed by a gas chromatograph mass-spectrometer. Since $\mathrm{NaHCO}_{3}$ is equilibrated with $\mathrm{CO}_{2}$ in solution and easily treated, it was a good source of $\mathrm{CO}_{2}$ in the present experiments. In this experiment, ${ }^{13} \mathrm{CH}_{4}$ was clearly detected, though the produced methane was not pure ${ }^{13} \mathrm{CH}_{4}$ and it did contain ${ }^{12} \mathrm{CH}_{4}$. In the same experiment, the mole ratio of ${ }^{13} \mathrm{CO}_{2}$ to ${ }^{12} \mathrm{CO}_{2}$ in the gas phase was about $57: 43$, which is nearly the same as the isotopic ratio of the generated methane. EDTA works as an electron donor in the system and is known to decompose into $\mathrm{CO}_{2}$. Therefore, ${ }^{12} \mathrm{CH}_{4}$ generation possibly occurs through the reduction of ${ }^{12} \mathrm{CO}_{2}$ generated from EDTA. The effect of EDTA on methane generation was examined in the Pt-liposome system. Methane was detected on visible-light irradiation of the system involving EDTA without $\mathrm{CO}_{2}$ or $\mathrm{NaHCO}_{3}$ but could not be detected in the absence of EDTA. These results suggest that the detected ${ }^{12} \mathrm{CH}_{4}$ is generated by the reduction of ${ }^{12} \mathrm{CO}_{2}$ originated from EDTA.

\subsubsection{Liposome-protected metal nanoparticle catalyst}

Liposome was better than other protective-colloid of $\mathrm{Pt}$ nanoparticles for methane generation. This is probably explained by assuming that liposome can form a larger and stronger hydrophobic region to concentrate $\mathrm{CO}_{2}$ around a $\mathrm{Pt}$ nanoparticle than $\mathrm{C}_{12} \mathrm{EO}$ micelle and poly( $N$-vinyl-2-pyrrolidone). In addition, $\mathrm{Ru}-\mathrm{C}_{12} \mathrm{EO}$ showed higher catalytic activity than $\mathrm{Pt}-\mathrm{C}_{12} \mathrm{EO}$. Thus, Ru-liposome was considered to be an active catalyst for methane generation in the system tested here. The synthesis of Ru-liposome was tried in a way similar to that of Pt-liposome, but the suspension of the Ru-liposome was not active as a catalyst. The resulting Ru-liposome was not as homogeneous, probably because the Ru ion is not miscible with liposome in water.

\subsubsection{Summary of the carbon dioxide photo-reduction by metal nanoparticle catalyst}

The Pt and Ru nanoparticle catalysts, which were prepared by a photoreduction method of metal salt in water without ethanol, successfully generated methane from $\mathrm{CO}_{2}$. The methane generation suggests that the eight-electron reduction of $\mathrm{CO}_{2}$ easily proceeds on metal nanoparticles possibly due to a thermodynamic advantage. This is different from an electrochemical $\mathrm{CO}_{2}$ reduction using Pt electrodes, on which $\mathrm{CO}_{2}$ is reduced to $\mathrm{CO}$ with adsorbed hydrogen atoms. In the present system using metal nanoparticles, the competition reaction, i.e., the kinetically favorably hydrogen generation, inhibits the methane generation. An increase of $\mathrm{CO}_{2}$ concentration, the electron supply rate, or both may enhance $\mathrm{CO}_{2}$ reduction.

\section{Catalytic decomposition of hydrogen peroxide by metal nanoparticle}

The modification of biomacromolecules upon exposure to reactive oxygen species, including hydrogen peroxide $\left(\mathrm{H}_{2} \mathrm{O}_{2}\right)$, dioxide $(1-)$ (superoxide $\mathrm{O}_{2}{ }^{\bullet}$ ), hydroxyl radical $(\mathrm{HO} \cdot)$, and singlet oxygen $\left({ }^{1} \mathrm{O}_{2}\right)$, is the likely initial event involved in the induction of the mutagenic and lethal effects of various oxidative stress agents (Kawanishi et al. 2001; Cadet et al., 2003; 
Drechsel \& Patel, 2008). Therefore, the activity of reactive oxygen species generation by various chemical compounds is closely related to their toxicity, carcinogenicity, or both. For example, hydroquinone, a metabolite of carcinogenic benzene, causes DNA damage via $\mathrm{H}_{2} \mathrm{O}_{2}$ generation (Hirakawa et al., 2002). Many studies have addressed the role of antioxidants, such as vitamins (Slaga, 1995; Sohmiya et al., 2004) and catechins (Weyant et al., 2001), in protection against cancers and cardiovascular diseases. These antioxidants can scavenge reactive oxygen species and protect against cancer occurrence. On the other hand, every antioxidant is in fact, a redox agent, protecting against reactive oxygen species in some circumstances and promoting free radical or secondary reactive oxygen species generation in others. Indeed, an excess of these antioxidants elevates the incidence of cancer (Nitta et al. 1991; Omenn et al., 1996). Solovieva et al. reported that antioxidants, ascorbic acid (Solovieva et al., 2007) and dithiothreitol (Solovieva et al., 2008), exhibit cytotoxicity via $\mathrm{H}_{2} \mathrm{O}_{2}$ generation. Relevantly, it has been reported that vitamins A (Murata \& Kawanishi, 2000) and E (Yamashita et al., 1998) and catechins (Oikawa et al., 2003) induce DNA oxidation through $\mathrm{H}_{2} \mathrm{O}_{2}$ generation during their oxidation. $\mathrm{H}_{2} \mathrm{O}_{2}$ is a long-lived reactive oxygen species which plays an important role in biomacromolecular damage induced by various chemical compounds (Kawanishi et al., 2001; Hirakawa et al., 2002).

\subsection{Metal catalyzes decomposition of hydrogen peroxide}

Various studies have demonstrated the catalytic decomposition of $\mathrm{H}_{2} \mathrm{O}_{2}$ by noble metals such as Pt (Keating et al., 1965; McKee, 1969; Bianchi et al., 1962), Pd (Keating et al., 1965; McKee, 1969; Bianchi et al., 1962; Eley \& Macmahon, 1972) Ag (Baumgartner et al., 1963; Goszner et al., 1972; Goszner \& Bischof, 1974), and Au (Eley \& Macmahon, 1972; Goszner \& Bischof, 1974). These metals themselves are hardly oxidized by reactive oxygen species, however, it is difficult to use metal powder or foils as anti-oxidative drugs. Recently, Kajita et al. reported that $\mathrm{Pt}$ nanoparticles catalyze the decomposition of reactive oxygen species (Kajita et al., 2007). These nanoparticles can be dispersed in water and used as homogenous solutions. Because this removal mechanism is catalytic decomposition, no oxidized product is formed through this reaction. Platinum metal is used as a food additive and is not considered to be a toxic material. This result led us to the idea that inorganic materials, in particular noble metals, rather than organic antioxidants, can be used as novel chemopreventive agents against reactive oxygen species-mediated biomolecules damage. In this section, the examination of the removal of $\mathrm{H}_{2} \mathrm{O}_{2}$ generated from a chemical compound, hydroquione, using water-soluble polymer-protected $\mathrm{Pt}$ and $\mathrm{Ag} / \mathrm{Pt}$ nanoparticles are reviewed.

\subsection{Catalytic activity of monometallic nanoparticles}

\subsubsection{Preparation of metal nanoparticles for reactive oxygen scavenger}

Colloidal dispersions of poly( $N$-vinyl-2-pyrrolidone)-protected $\mathrm{Pt}, \mathrm{Pd}, \mathrm{Rh}$, and $\mathrm{Au}$ nanoparticles were prepared using an alcohol reduction method (Hirai et al., 1979). $50 \mathrm{~mL}$ of water/ethanol $(1 / 1, \mathrm{v} / \mathrm{v})$ solution containing $1 \mathrm{mM}$ metal salts and $40 \mathrm{mM}$ poly(N-vinyl-2pyrrolidone) (monomer unit) was refluxed for $2 \mathrm{~h}$, resulting in the formation of typical colored sols of metal nanoparticles. The solvent was removed by vacuum evaporation, and the nanoparticles were dispersed into water to prepare $1 \mathrm{mM} /$ atom (atomic concentration) metal colloidal sols. An aqueous solution of poly( $N$-vinyl-2-pyrrolidone)-protected Ag 
nanoparticles (Shiraishi \& Toshima, 1999) was prepared from reduction of $1 \mathrm{mM} \mathrm{AgNO}_{3}$ with $\mathrm{NaBH}_{4}$ in the presence of $40 \mathrm{mM}$ poly( $N$-vinyl-2-pyrrolidone). The obtained $\mathrm{Ag}$ colloidal dispersion was purified with an ultra-filter.

These poly( $N$-vinyl-2-pyrrolidone)-protected metal nanoparticles formed water-soluble sols. The average diameters $(d)$ and standard deviations $(\sigma)$ of monometallic nanoparticles determined by TEM measurement were as follows: $\mathrm{Pt}(d=2.2 \mathrm{~nm}, \sigma=1.0 \mathrm{~nm}), \operatorname{Pd}(d=2.0$ $\mathrm{nm}, \sigma=0.9 \mathrm{~nm}), \operatorname{Rh}(d=2.2 \mathrm{~nm}, \sigma=1.0 \mathrm{~nm}), \operatorname{Ag}(d=10.0 \mathrm{~nm}, \sigma=1.9 \mathrm{~nm})$, and Au $(d=10.2 \mathrm{~nm}$, $\sigma=2.0 \mathrm{~nm})$.

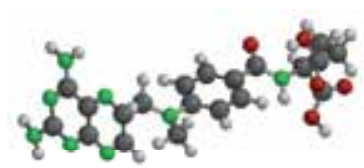

Folic acid
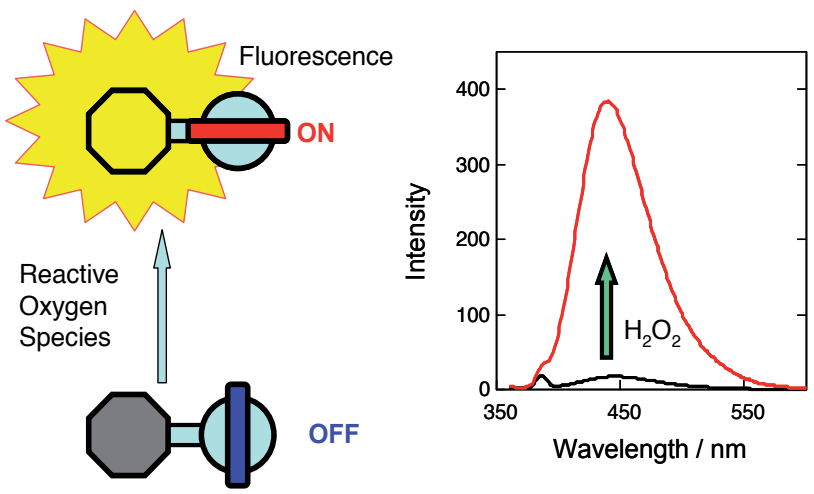

Fig. 9. Fluorometry of reactive oxygen species (hydrogen peroxide) using folic acid

\subsubsection{Method of the detection of hydrogen peroxide}

The generated $\mathrm{H}_{2} \mathrm{O}_{2}$ was measured by a previously reported method using folic acid (Hirakawa, 2006). This assay is based on the fluorescence enhancement of less-fluorescent folic acid via oxidative decomposition by $\mathrm{H}_{2} \mathrm{O}_{2}$ and copper(II) ion into strong-fluorescent 2amino-4-oxo-3H-pterine-6-carboxylic acid (Figure 9). The concentration of $\mathrm{H}_{2} \mathrm{O}_{2}\left(\left[\mathrm{H}_{2} \mathrm{O}_{2}\right]\right)$ can be determined using a calibration curve. A reaction mixture containing folic acid, copper(II) chloride, and the $\mathrm{H}_{2} \mathrm{O}_{2}$ sample (or $\mathrm{H}_{2} \mathrm{O}_{2}$ generator ${ }^{4}$ ) with or without the metal nanoparticle in a sodium phosphate buffer ( $\mathrm{pH} 7.6)$ was incubated in a microtube for $30 \mathrm{~min}$. After incubation at $37{ }^{\circ} \mathrm{C}$, the fluorescence intensity of the reaction mixture at $450 \mathrm{~nm}$ was measured using a fluorescence spectrophotometer with 350-nm excitation.

\subsubsection{Platinum nanoparticles effectively scavenge hydrogen peroxide}

Platinum nanoparticles effectively scavenged $\mathrm{H}_{2} \mathrm{O}_{2}$ in a dose-dependent manner and showed the highest activity among the metal nanoparticles used in this study (Figure 10). A sample solution of $5 \mu \mathrm{M}$ /atom Pt nanoparticles, among which $1 \mu \mathrm{g}$ Pt metal is included, exhibits comparable activity for $\mathrm{H}_{2} \mathrm{O}_{2}$ decomposition to that of 10 units of catalase. One unit of catalase can remove $1.0 \mu \mathrm{mol} \mathrm{H}_{2} \mathrm{O}_{2}$ per min in water $\left(\mathrm{pH} \mathrm{7.0,} 25^{\circ} \mathrm{C}\right)$. Poly $(\mathrm{N}$-vinyl-2pyrrolidone) itself did not scavenge $\mathrm{H}_{2} \mathrm{O}_{2}$. This experiment confirmed that poly( $\mathrm{N}$-vinyl-2pyrrolidone)-protected $\mathrm{Pt}$ nanoparticles can remove $\mathrm{H}_{2} \mathrm{O}_{2}$. The mechanism of $\mathrm{H}_{2} \mathrm{O}_{2}$ removal by $\mathrm{Pt}$ nanoparticles can be explained by catalytic decomposition into water and molecular oxygen as follows: 


$$
\mathrm{H}_{2} \mathrm{O}_{2} \rightarrow \mathrm{H}_{2} \mathrm{O}+1 / 2 \mathrm{O}_{2}
$$

The generation of $\mathrm{O}_{2}$ gas through the $\mathrm{H}_{2} \mathrm{O}_{2}$ decomposition was confirmed with a gas-burette as following procedure. The $10 \mathrm{~mL}$ of aqueous solution containing $0.1 \mathrm{M} \mathrm{H}_{2} \mathrm{O}_{2}$ was treated by $10 \mu \mathrm{g} \mathrm{Pt}$ nanoparticles and generated $\mathrm{O}_{2}$ gas was measured with a gas-burette. The volume of detected gas coincided with that of the theoretically calculated value of $\mathrm{O}_{2}$ generation from the decomposition of $\mathrm{H}_{2} \mathrm{O}_{2}$ in the sample solution.

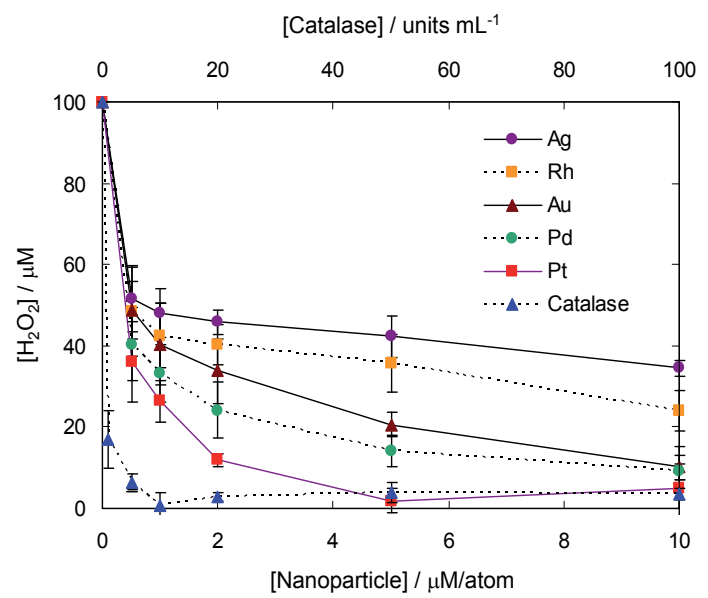

Fig. 10. Removal of $\mathrm{H}_{2} \mathrm{O}_{2}$ by metal nanoparticles and catalase. The $1 \mathrm{~mL}$ of sample solution containing $100 \mu \mathrm{M} \mathrm{H}_{2} \mathrm{O}_{2}, 10 \mu \mathrm{M}$ folic acid, $20 \mu \mathrm{M}$ copper(II) chloride, and indicated concentration of metal nanoparticles or catalase was incubated for $30 \mathrm{~min}$. The concentration of $\mathrm{H}_{2} \mathrm{O}_{2}$ was estimated from the fluorescence measurement.

\subsection{Application of siver-core/platinum-shell bimetallic nanoparticles to catalytic decomposition of hydrogen peroxide generated by chemical compound}

\subsubsection{Preparation of silver-core bimetallic nanoparticles for hydrogen peroxide scavenger}

The catalytic activity of $\mathrm{Pt}$ and its modified particles with $\mathrm{Ag}(\mathrm{Ag} / \mathrm{Pt})$ on the decomposition of $\mathrm{H}_{2} \mathrm{O}_{2}$ generated from chemical compounds was evaluated, since Pt showed the highest activity. The $\mathrm{Ag} / \mathrm{Pt}$ nanoparticles were prepared from the following procedure. The absorption spectrum of the sol of Pt nanoparticles is a flat curve (Figure 11), indicating the formation of homogenous particles. Ag nanoparticles exhibited a typical yellow color due to surface plasmon absorption around $400 \mathrm{~nm}$. It has been reported that a physical mixture of $\mathrm{Ag}$ and Pt nanoparticles spontaneously forms bimetallic nanoparticles, possibly Ag-core/Ptshell structured particles (Toshima et al., 2005). The time-course of the absorption spectra of this physical mixture showed the extinction of Ag surface plasmon absorption, and the absorption was completely extinguished within $24 \mathrm{~h}$ (Figure 11), suggesting that the surface of the formed bimetallic nanoparticles is composed of $\mathrm{Pt}$ atoms. Typical TEM images showed the formation of relatively small particles of $\mathrm{Pt}$ and large particles of Ag (Figure 12). TEM photographs showed that the large Ag particles disappeared through interaction with $\mathrm{Pt}$ particles, resulting in the formation of bimetallic particles smaller than the parent $\mathrm{Ag}$ particles (Figure 12). A similar result has been observed in the case of $\mathrm{Ag} / \mathrm{Rh}$ bimetallic 
nanopaticles (Toshima \& Hirakawa, 2003). These findings suggest the formation of selforganized Ag/Pt bimetallic nanoparticles. These metal nanoparticles are stable in water for several months. The Ag/Pt (Ag-atom/Pt-atom, 1/1) bimetallic nanoparticles were prepared using a self-organization method to mix $\mathrm{Pt}$ and $\mathrm{Ag}$ monometallic nanoparticles according to previous reports (Toshima \& Hirakawa, 2003; Toshima et al., 2002, 2005; Matsushita et al., 2007).

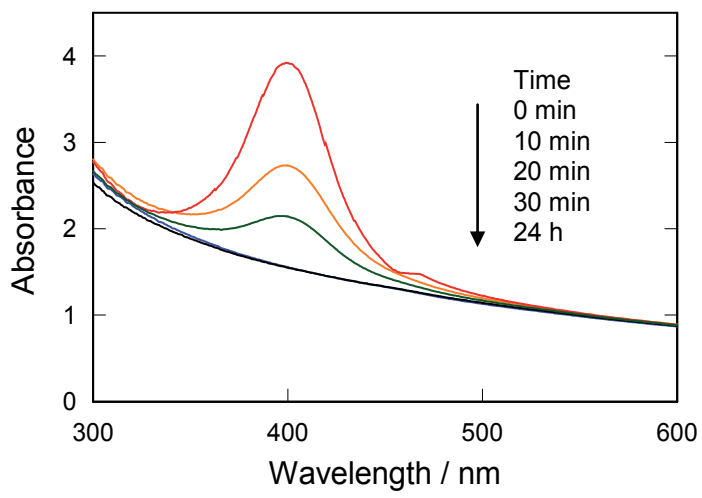

Fig. 11. Absorption spectral change of the physical mixture of dispersions of $\mathrm{Ag}$ and $\mathrm{Pt}$ nanoparticles. The aqueous solutions of $\mathrm{Ag}(1 \mathrm{mM} /$ atom, $10 \mathrm{~mL})$ and $\mathrm{Pt}(1 \mathrm{mM} /$ atom, 10 $\mathrm{mL}$ ) nanoparticles were mixed and measured at $0,10,20$, and $30 \mathrm{~min}$, and $24 \mathrm{~h}$ after mixing.
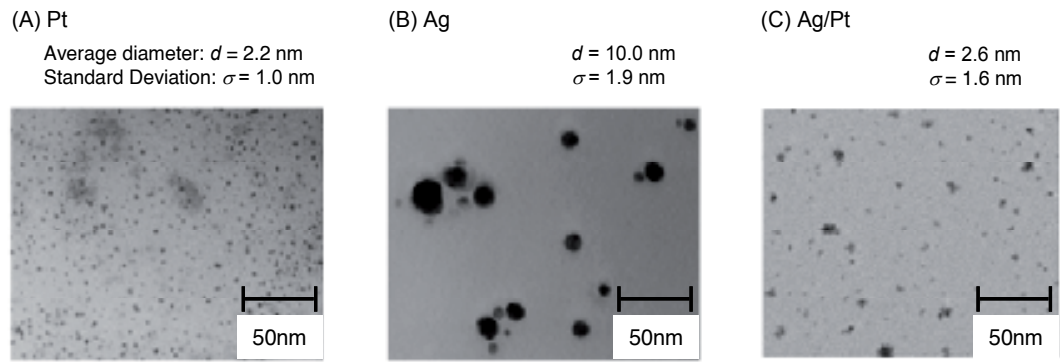

Fig. 12. TEM photographs of metal nanoparticles. The sample of $\mathrm{Ag} / \mathrm{Pt}$ nanoparticles was prepared by drying the mixtures of the aqueous solutions of $\mathrm{Pt}$ and $\mathrm{Ag}$ monometallic nanoparticles 24 hour after mixing.

\subsubsection{Hydrogen peroxide formation from hydroquinone and its removal by metal nanoparticles}

Hydroquinone, which is a metabolite of carcinogenic benzene, was used as $\mathrm{H}_{2} \mathrm{O}_{2}$ source. This compound can generate $\mathrm{H}_{2} \mathrm{O}_{2}$ through autooxidation (Figure 13) (Hirakwa et al., 2002). Under these experimental conditions, hydroquinone generated $\mathrm{H}_{2} \mathrm{O}_{2}$ in a dose-dependent manner (Figure 14). Twenty units/mL catalase effectively removed $\mathrm{H}_{2} \mathrm{O}_{2}$ generated from this system, and $10 \mu \mathrm{M} /$ atom $(2 \mu \mathrm{g} / \mathrm{mL})$ Pt nanoparticles exhibited a comparable activity to that of this catalase. Silver nanoparticles showed apparently weaker activity for $\mathrm{H}_{2} \mathrm{O}_{2}$ removal than $\mathrm{Pt}$ nanoparticles. The bimetallization of $\mathrm{Pt}$ with $\mathrm{Ag}$ apparently suppressed the catalytic activity per unit atom. 


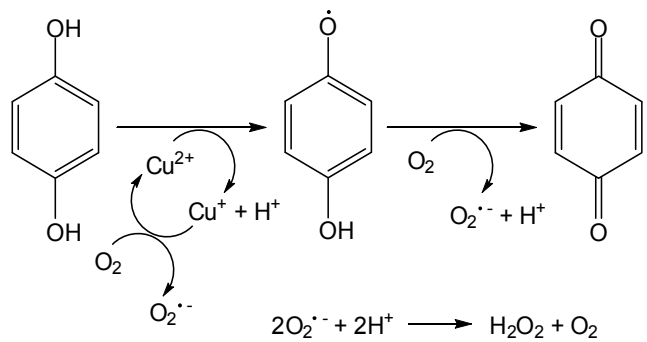

Fig. 13. Schematic diagram of hydrogen peroxide formation by the autooxidation of hydroquinone

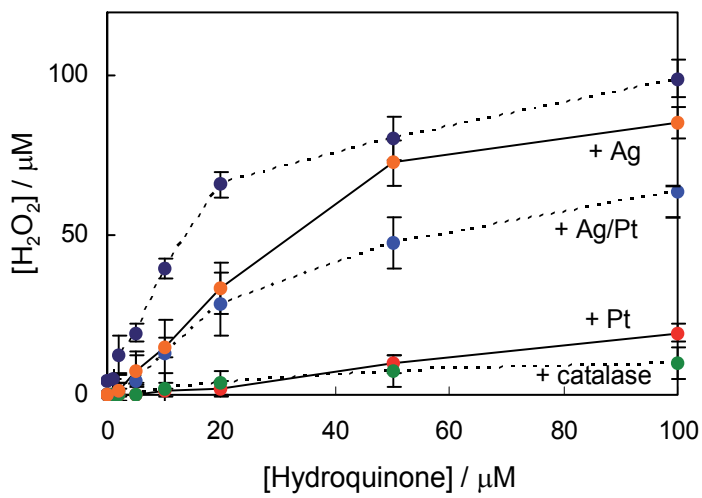

Fig. 14. $\mathrm{H}_{2} \mathrm{O}_{2}$ generation through autooxidation of hydroquinone in the absence or presence of metal nanoparticles and catalase. The $1 \mathrm{~mL}$ of sample solution containing $10 \mu \mathrm{M}$ folic acid, $20 \mu \mathrm{M}$ copper(II) chloride, and indicated concentration of hydroquinone with or without $10 \mu \mathrm{M} /$ atom metal nanoparticles or 20 units/mL catalase was incubated for $30 \mathrm{~min}$. The concentration of generated $\mathrm{H}_{2} \mathrm{O}_{2}$ was estimated from the fluorescence measurement.

\subsubsection{Activity of silver-core/platinum-shell nanoparticles on hydrogen peroxide decomposition}

Figure 15 shows the removal activity of $\mathrm{H}_{2} \mathrm{O}_{2}$ generated from a high concentration of hydroquinone $(50 \mu \mathrm{M})$ by metal nanoparticles. These metal nanoparticles and catalase scavenged $\mathrm{H}_{2} \mathrm{O}_{2}$ in a dose-dependent manner. The activity of the $10 \mu \mathrm{M} /$ atom $(2 \mu \mathrm{g} / \mathrm{mL}) \mathrm{Pt}$ nanoparticles was comparable to that of 20 units/mL catalase, and Pt completely scavenged $\mathrm{H}_{2} \mathrm{O}_{2}$ over $20 \mu \mathrm{M} /$ atom $(4 \mu \mathrm{g} / \mathrm{mL})$. The activity per atom of the $\mathrm{Ag} / \mathrm{Pt}$ bimetallic nanoparticles was almost the same as that of the $\mathrm{Ag}$ monometallic nanoparticles.

To investigate the effect of $\mathrm{Pt}$ nanoparticles on $\mathrm{H}_{2} \mathrm{O}_{2}$ generation through the autooxidation of hydroquinone, NADH consumption during this autooxidation was measured. The consumption of NADH during the autooxidation of hydroquinone was measured by a previously reported method (Oikawa et al., 2003). A sample solution containing $100 \mu \mathrm{M}$ $\mathrm{NADH}, 50 \mu \mathrm{M}$ hydroquinone, and $20 \mu \mathrm{M}$ copper(II) chloride was incubated at $37^{\circ} \mathrm{C}$ in the absence or presence of $20 \mu \mathrm{M}$ /atom Pt nanoparticles. The concentration of NADH was determined by the measurement of absorbance of NADH at $340 \mathrm{~nm}$ using a microplate 
absorbance reader. The oxidized form of hydroquinone can be reduced into the parent hydroquinone by NADH (Hirakwa et al., 2002). The concentration of NADH was gradually decreased through the redox of hydroquinone and Pt nanoparticles hardly inhibited NADH consumption (data not shown). This result indicated that $\mathrm{Pt}$ nanoparticles do not inhibit the $\mathrm{H}_{2} \mathrm{O}_{2}$ generation itself, because $\mathrm{H}_{2} \mathrm{O}_{2}$ is produced through the autooxidation of hydroquinone.

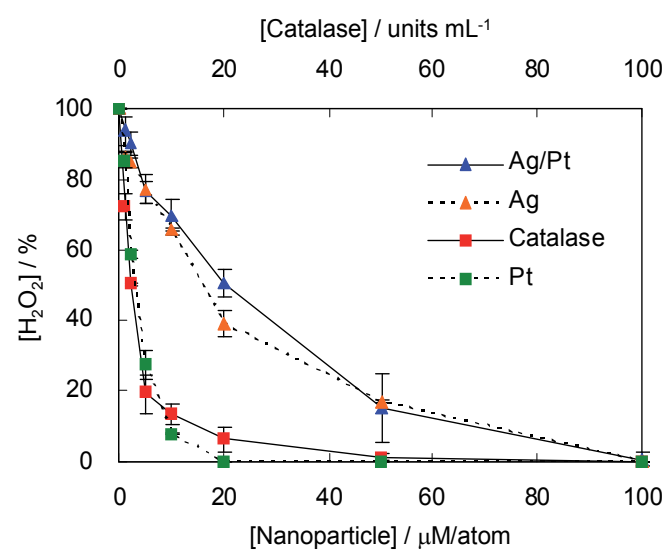

Fig. 15. Removal of $\mathrm{H}_{2} \mathrm{O}_{2}$ generated through the autooxidation of hydroquinone by metal nanoparticles and catalase. The $1 \mathrm{~mL}$ of sample solution containing $10 \mu \mathrm{M}$ folic acid, $20 \mu \mathrm{M}$ copper(II) chloride, $50 \mu \mathrm{M}$ hydroquinone, and indicated concentration of metal nanoparticles or catalase was incubated for $30 \mathrm{~min}$. The concentration of $\mathrm{H}_{2} \mathrm{O}_{2}$ was estimated from the fluorescence measurement.

\subsection{Summary and possible mechanism of hydrogen peroxide decomposition by metal nanoparticles}

Poly(N-vinyl-2-pyrrolidone)-protected metal nanoparticles, in particular Pt nanoparticles, exhibited a removal effect on $\mathrm{H}_{2} \mathrm{O}_{2}$ generated through autooxidation of hydroquinone (Figure 16). The removal of $\mathrm{H}_{2} \mathrm{O}_{2}$ by these metal nanoparticles can be explained by a catalytic reaction similar to that by catalase, which decomposes $\mathrm{H}_{2} \mathrm{O}_{2}$ into $\mathrm{H}_{2} \mathrm{O}$ and $\mathrm{O}_{2}$. The formation of $\mathrm{H}_{2} \mathrm{O}_{2}$ during autooxidation of hydroquinone is through $\mathrm{O}_{2}{ }^{\bullet}$, which is generated from a reduction of $\mathrm{O}_{2}$ by hydroquinone (Hirakawa et al., 2002). Because the lifetime of $\mathrm{O}_{2}{ }^{\bullet}$, which dismutates into $\mathrm{H}_{2} \mathrm{O}_{2}$ through reaction with $\mathrm{H}^{+}$, is short $(\sim 0.1 \mathrm{~ms})$, the scavenging of $\mathrm{O}_{2} \cdot$ - by a metal nanoparticle can be negligible. The $\mathrm{H}_{2} \mathrm{O}_{2}$ removal activity per metal atom of these metal nanoparticles occurred in the following order: $\mathrm{Pt}>$ $\mathrm{Ag} \approx \mathrm{Ag} / \mathrm{Pt}$. The activities of $\mathrm{H}_{2} \mathrm{O}_{2}$ decomposition per metal atom consisting of these metal nanoparticles $\left(\mu \mathrm{M}-\mathrm{H}_{2} \mathrm{O}_{2} / \mu \mathrm{M}\right.$-nanometal $)$ have been estimated, and the resulting values are 4.2, 12.2, and 3.8 for $\mathrm{Ag}, \mathrm{Pt}$, and $\mathrm{Ag} / \mathrm{Pt}$, respectively. Further, the activity on the surface area of the $\mathrm{Ag} / \mathrm{Pt}$ nanoparticles $\left(17 \mu \mathrm{M}-\mathrm{H}_{2} \mathrm{O}_{2} / \mathrm{cm}^{2}\right.$-nanometal) was also smaller than that of $\mathrm{Pt}\left(49 \mu \mathrm{M}-\mathrm{H}_{2} \mathrm{O}_{2} / \mathrm{cm}^{2}\right.$-nanometal). These findings showed that the $\mathrm{Pt}$ nanoparticles have the highest catalytic activity for $\mathrm{H}_{2} \mathrm{O}_{2}$ decomposition in the metal nanoparticles used in this experiment and the activity of Pt nanoparticles is suppressed by modification with Ag. 


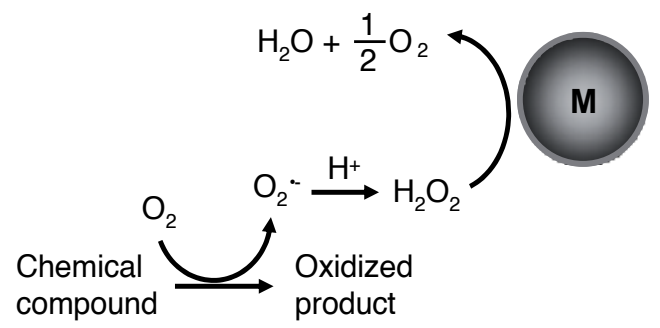

Fig. 16. Hydrogen peroxide generation from an autooxidation of chemical compound and its catalytic decomposition by metal nanoparticle

$\mathrm{H}_{2} \mathrm{O}_{2}$ is a long-lived reactive oxygen species and plays an important role in DNA damage (Kawanishi et al., 2001, Hirakawa et al., 2002). Indeed, various chemical compounds, including carcinogens, generate $\mathrm{H}_{2} \mathrm{O}_{2}$ during redox reaction (Kawanishi et al., 2001, Hirakawa et al., 2002). Molecular oxygen is easily reduced by various compounds, leading to the formation of $\mathrm{O}_{2}{ }^{\bullet}$. Formed $\mathrm{O}_{2}{ }^{-}$- is rapidly dismutated into $\mathrm{H}_{2} \mathrm{O}_{2}$. Although $\mathrm{H}_{2} \mathrm{O}_{2}$ itself is not a strong reactive species, it can generate highly reactive $\mathrm{HO} \cdot$ through a Fenton reaction or a Haber-Weiss reaction. Furthermore, $\mathrm{H}_{2} \mathrm{O}_{2}$ can penetrate a cytoplasm membrane and be incorporated into the cell nucleus. Therefore, $\mathrm{H}_{2} \mathrm{O}_{2}$ is considered to be one of the most important reactive species or a precursor participating in carcinogenesis. The removal of $\mathrm{H}_{2} \mathrm{O}_{2}$ is an effective method for cancer chemoprevention. Furthermore, protective agents against $\mathrm{H}_{2} \mathrm{O}_{2}$ are important to treat acatalasemia, a genetic deficiency of erythrocyte catalase inherited as an autosomal recessive trait. Antioxidants, such as vitamins $\mathrm{A}$ and $\mathrm{E}$, are effective protective agents. However, the oxidized products of antioxidants or these molecules themselves promote the formation of secondary $\mathrm{H}_{2} \mathrm{O}_{2}$ (Yamashita et al., 1998; Murata \& Kawanishi, 2000). Indeed, an excess of these antioxidants elevates the incidence of cancer (Nitta et al., 1991; Omenn et al., 1996). A catalyst consisting of an inorganic stable material is not oxidized and does not generate secondary reactive oxygen species. Water-soluble nanoparticles of noble metal may become novel protective agents against reactive oxygen species.

In summary, $\mathrm{Pt}, \mathrm{Ag}$, and $\mathrm{Ag} / \mathrm{Pt}$ nanoparticles effectively scavenge $\mathrm{H}_{2} \mathrm{O}_{2}$ generated from autooxidation of a highly concentrated hydroquinone. Platinum nanoparticles exhibited the highest catalytic activity among these nanoparticles. Pt is a very stable metal against various chemical compounds and permitted as a food additives. The noble metal nanoparticles may be used as novel chemopreventive agents for cancer or other non-malignant conditions induced by chemical compounds through $\mathrm{H}_{2} \mathrm{O}_{2}$ generation.

\section{Application of metal nanoparticles to prevention of ultraviolet radiation induced biomolecules damage}

Exposure to solar ultraviolet radiation is undoubtedly linked to skin carcinogenesis and phototoxic effect. Photosensitized reaction by ultraviolet radiation, especially ultraviolet-A (UVA) radiation $(320 \sim 400 \mathrm{~nm})$, is considered to cause toxic effect through oxidative biomolecules damage including DNA damage (Hiraku et al., 2007). Photosensitized formation of reactive oxygen species, such as hydrogen peroxide, superoxide, hydroxyl radicals, and singlet oxygen, is involved in UVA-induced biomolecules damage. As 
mentioned above, the application of metal nanoparticles to scavenge reactive oxygen species through catalytic decomposition.

\subsection{Traditional methods of chemoprevention to biomolecules damage by ultraviolet radiation and its problem}

Many studies have addressed the role of antioxidants, such as vitamins and catechins, in protection against cancers and cardiovascular diseases. These antioxidants can scavenge reactive oxygen species and protect against cancer occurrence. On the other hand, every antioxidant is, in fact, a redox agent, protecting against reactive oxygen species in some circumstances and promoting free radical or secondary reactive oxygen species generation in others. Indeed, an excess of these antioxidants elevates the incidence of cancer. It has been reported that antioxidants, ascorbic acid and dithiothreitol, exhibit cytotoxicity via $\mathrm{H}_{2} \mathrm{O}_{2}$ generation, and their toxic effects are significantly enhanced by vitamin $\mathrm{B}_{12} . \mathrm{H}_{2} \mathrm{O}_{2}$ is a longlived reactive oxygen species which plays an important role in biomacromolecules damage induced by various chemical compounds.

\subsection{Preventive action of metal nanoparticles on ultraviolet-sensitized oxidation of molecules}

As mentioned above, metal nanoparticles catalyze the decomposition of reactive oxygen species. Because this removal mechanism is catalytic decomposition, no oxidized product is formed through this reaction. Platinum metal is used as a food additive and is not considered to be a toxic material. This result led us to the idea that inorganic materials, in particular noble metals, rather than organic antioxidants, can be used as novel chemopreventive agents against UVA-induced biomolecules damage.

Recently, it has been reported that the removal of reactive oxygen species generated from a photocatalytic reaction of titanium dioxide $\left(\mathrm{TiO}_{2}\right)$ particles using water-soluble polymerprotected $\mathrm{Pt}, \mathrm{Rh}$, and $\mathrm{Pt} / \mathrm{Ag}$ bimetallic nanoparticles. Silver, a relatively inexpensive noble metal, is also used as a food additive, and bimetallization with Ag may improve the catalytic activity of other metal nanoparticles.

\subsubsection{Preparation of metal nanoparticles for ultraviolet protection}

The colloidal dispersions of poly( $\mathrm{N}$-vinyl-2-pyrrolidone)-protected $\mathrm{Pt}$ and $\mathrm{Rh}$ nanoparticles were prepared from an alcohol reduction. The size (particle diameter) of these nanoparticles is about $2 \mathrm{~nm}$. The aqueous solution of poly( $N$-vinyl-2-pyrrolidone)-protected $\mathrm{Ag}$ nanoparticle was prepared from a reduction of silver nitrate by sodium borohydride in the presence of poly(N-vinyl-2-pyrrolidone). The Ag-core/Pt-shell (Ag-atom/Pt-atom, 1/1) bimetallic nanoparticle was prepared using a physical method to mix $\mathrm{Pt}$ and $\mathrm{Ag}$ monometallic nanoparticles according to the previous reports (Toshima et al., 2005).

\subsubsection{Evaluation model for the biomolecules damage by ultraviolet radiation}

$\mathrm{TiO}_{2}$ (anatase) and methylene blue were used as a model of the UVA-induced reaction. The sample solution containing methylene blue and $\mathrm{TiO}_{2}$ dispersion in sodium phosphate buffer $(\mathrm{pH}$ 7.6) with or without metal nanoparticle was irradiated with a UVA lamp $(365 \mathrm{~nm}, 1$ 
$\mathrm{mW} \mathrm{cm}^{-2}$. The decomposition of methylene blue was evaluated by absorption measurement at $659 \mathrm{~nm} . \mathrm{TiO}_{2}$ is a well-known photocatalyst (Fujishima et al., 2000, 2008). When exposing to UVA light, the reduction-oxidation activity of $\mathrm{TiO}_{2}$ has a significant biological impact, as is exemplified by its bactericidal activity. Photo-irradiated $\mathrm{TiO}_{2}$ effectively decomposed methylene blue (Figure 17). Various reactive oxygen species contribute to the photocatalytic reaction of $\mathrm{TiO}_{2}$. Especially, hydrogen peroxide is long-lived reactive oxygen species and plays an important role in oxidative biomolecules damage. Molecular oxygen is reduced by photoexcited materials, leading to the formation of superoxide. Formed superoxide is rapidly dismutated into hydrogen peroxide. Although hydrogen peroxide itself is not a strong reactive species, it can generate highly reactive hydroxyl radicals through a Fenton reaction or a Haber-Weiss reaction. Furthermore, hydrogen peroxide can penetrate a cytoplasm membrane and be incorporated into the cell nucleous. Therefore, hydrogen peroxide is considered to be one of the most important reactive oxygen species participating in UVA carcinogenesis and phototoxicity. Since other reactive oxygen species, such as directly produced hydroxyl radicals (Hirakawa et al., 2004) and singlet oxygen (Hirakawa \& Hirano, 2006), rapidly quenched in aqueous solution, hydrogen peroxide should be key reactive species in this experiment. The $\mathrm{TiO}_{2}$ and methylene blue could be used as a simple model of UVA-induced oxidation.

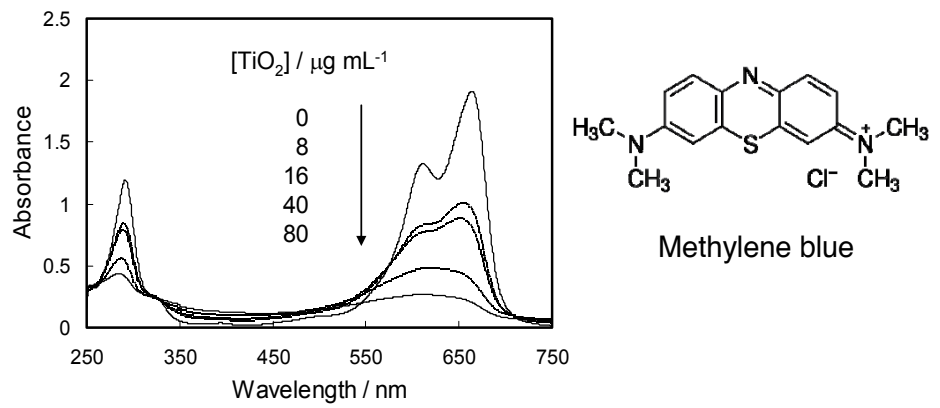

Fig. 17. UV-Vis absorption spectra of methylene blue photocatalyzed by $\mathrm{TiO}_{2}$. The sample solution containing $10 \mu \mathrm{M}$ methylene blue and indicated concentration of $\mathrm{TiO}_{2}$ in $10 \mathrm{mM}$ sodium phosphate buffer ( $\mathrm{pH} 7.6)$ was irradiated $\left(\mathrm{Ex}=365 \mathrm{~nm}, 1 \mathrm{~mW} \mathrm{~cm}^{-2}\right)$ for $30 \mathrm{~min}$.

\subsubsection{Preventive action of metal nanoparticles on ultraviolet radiation induced biomolecules damage}

Poly(N-vinyl-2-pyrrolidone)-protected metal nanoparticles, in particular, the $\mathrm{Pt}$ nanoparticle, inhibited the methylene blue decomposition photocatalyzed by $\mathrm{TiO}_{2}$ (Figure 18). Poly(N-vinyl-2-pyrrolidone) itself did not inhibit the methylene blue decomposition. This inhibitory effect can be explained by the catalytic decomposition of $\mathrm{H}_{2} \mathrm{O}_{2}$ generated through $\mathrm{TiO}_{2}$ photocatalysis. These nanoparticles decomposed $\mathrm{H}_{2} \mathrm{O}_{2}$ into $\mathrm{H}_{2} \mathrm{O}$ and $\mathrm{O}_{2}$ similar to catalase. In the case of $\mathrm{H}_{2} \mathrm{O}_{2}$ decomposition, the $\mathrm{Pt}$ nanoparticle showed the highest catalytic activity per unit atom. The activity of a $1 \mu \mathrm{g}$ Pt nanoparticle was comparable to that of 5 units of catalase. One unit of catalase can remove $1.0 \mu \mathrm{mol} \mathrm{H}_{2} \mathrm{O}_{2}$ per $1 \mathrm{~min}$ in water $\left(\mathrm{pH} 7.0,25^{\circ} \mathrm{C}\right)$. Unexpectedly, the bimetallization with $\mathrm{Ag}$ did not show improvement effect and rather decreased the inhibitory effect of Pt nanoparticle on the decomposition of methylene blue. 
The UV-Vis absorption spectra of these metal nanoparticles were hardly changed by the photocatalytic reaction, suggesting that the noble metal nanoparticles are stable for reactive oxygen species and UVA irradiation. Organic antioxidant undergoes oxidation in the removal process of reactive oxygen species, leading to the formation of various oxidized products and may produce secondary reactive oxygen species. In the case of noble metal catalyst, these effects can be negligible.

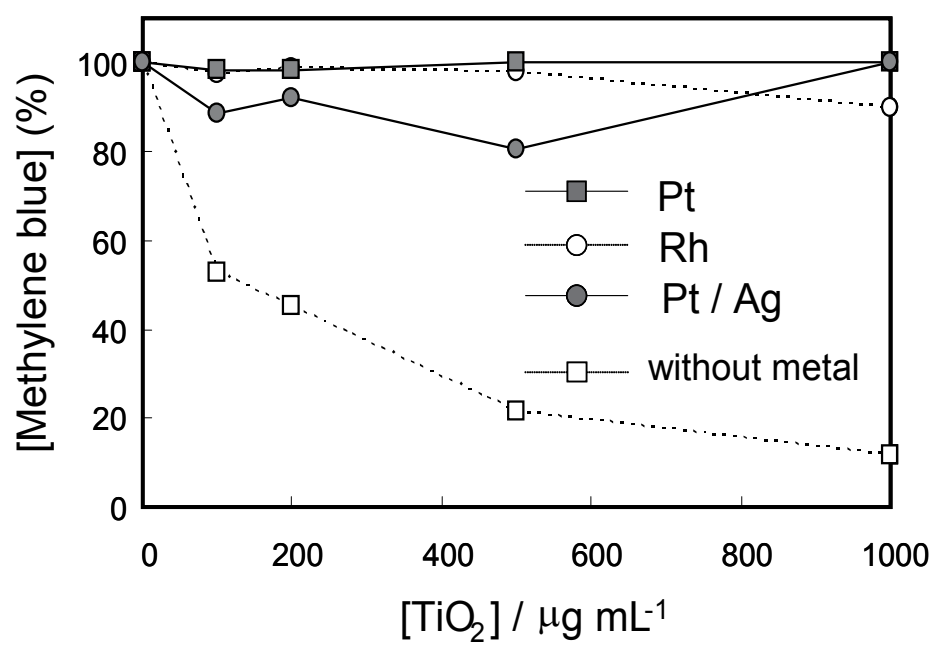

Fig. 18. Inhibitory effect of metal nanoparticles on methylene blue decomposition photocatalyzed by $\mathrm{TiO}_{2}$. The sample solution containing $20 \mu \mathrm{g} \mathrm{mL}^{-1}$ metal nanoparticle, $\mathrm{TiO}_{2}$, and $10 \mu \mathrm{M}$ methylene blue in $10 \mathrm{mM}$ sodium phosphate buffer (pH 7.6) was irradiated

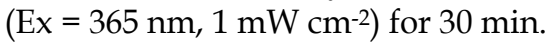

\subsection{Summary of the ultraviolet protection by metal nanoparticles}

$\mathrm{Pt}, \mathrm{Rh}$, and $\mathrm{Pt} / \mathrm{Ag}$ nanoparticles effectively inhibited the methylene blue decomposition photocatalyzed by $\mathrm{TiO}_{2}$. $\mathrm{TiO}_{2}$ photocatalytic system was used as a UVA-induced reactive oxygen species generation. The most important reactive oxygen species in this photocatalytic reaction is $\mathrm{H}_{2} \mathrm{O}_{2}$, because of its long lifetime in aqueous solution. This inhibitory effect of metal nanoparticle can be explained by the removal of $\mathrm{H}_{2} \mathrm{O}_{2}$. Unexpectedly, the activity of $\mathrm{Pt}$ nanoparticle was not improved by the bimetallization with Ag. Platinum is a very stable metal against various chemical compounds and is used as food additive. A poly(N-vinyl-2-pyrrolidone)-protected $\mathrm{Pt}$ nanoparticle may be used as a novel preventive agent for UVA-induced biomolecules damage through reactive oxygen species generation.

\section{Conclusion}

Physical mixture of $\mathrm{Ag}$ and other metal nanoparticles, such as $\mathrm{Pt}, \mathrm{Rh}$, and $\mathrm{Pd}$, spontaneously forms the bimetallic nanoparticles with Ag-core structure in aqueous solution. These monometallic nanoparticles can be easily prepared from an alcohol reduction of the corresponding metal ions in the presence of water-soluble polymer such 
as poly( $N$-vinyl-2-pyrrolidone), a protective colloid. Aqueous sol of Ag nanoparticles exhibits the surface plasmon absorption around $400 \mathrm{~nm}$. The surface plasmon absorption was diminished through interaction with other metal nanoparticle in the physical mixture of these nanoparticles. This phenomenon was explained by that the Ag nanoparticle was coated by other metal. The transmission electron micrograph and X-ray diffraction measurement confirmed the formation of the Ag-core bimetallic nanoparticles. This reaction can be used to construct the core-shell structured novel bimetallic nanoparticles. The formed nanoparticles act superior character for certain catalytic reactions. The catalytic activity of the silver/rhodium bimetallic nanoparticles for visible-light-induced hydrogen generation in an aqueous solution was examined. This system composed of an electron source, a photosensitizer, an electron relay, and metal nanoparticle catalyst. The activity is clearly higher than the corresponding monometallic nanoparticles, suggesting that the silver-core enhances the catalytic activity of the surface rhodium. On the other hand, the catalytic activity of the decomposition of hydrogen peroxide was decreased by this bimetallization. Platinum nanoparticle effectively catalyzes hydrogen peroxide decomposition. The Ag-core/platinum shell bimetallic nanoparticle, which was prepared by the physical mixing of $\mathrm{Ag}$ and $\mathrm{Pt}$ nanoparticles, demonstrated lower activity of the decomposition of hydrogen peroxide than the monometallic Pt nanoparticle. Metal nanoparticles can be applied to various catalytic reactions. The bimetallic and trimetallic nanoparticles demonstrate superior activity in the certain reaction. The self-assembly formation of Ag-cored nanoparticle may be convenient method to prepare novel metal nanoparticle catalyst.

\section{Acknowledgments}

The author wish to thank Professor Naoki Toshima (Tokyo University of Science, Yamaguchi) for his helpful discussion and Professor Kenji Murakami (Research Institute of Electronics, Shizuoka University) for his helpful advice on TEM measurement. These works were supported by a Grant-in-Aid for Scientific Research from the Ministry of Education, Culture, Sports, Science and Technology (MEXT) of the Japanese Government.

\section{References}

Akinaga, H. (2002). Metal-nanocluster Equipped GaAs Surfaces Designed for High-sensitive Magnetic Field Sensors. Surface Science, Vol.514, No.1-3, (August 2002), pp.145-150, ISSN 0039-6028

Baumgartner, H. J.; Hood, G. C.; Monger, J. M.; Roberts, R. M. \& Sanborn, C. E. (1963). Decomposition of Concentrated Hydrogen Peroxide on Silver I. Low Temperature Reaction and Kinetics. Journal of Catalysis, Vol.2, No.5, (October 1963), pp.405-414, ISSN 0021-9517

Bianchi, G.; Mazza, F. \& Mussini, T. (1962). Catalytic Decomposition of Acid Hydrogen Peroxide Solutions on Platinum, Iridium, Palladium and Gold Surfaces. Electrochimica Acta, Vol.7, No.4, (July-August 1962), pp.457-473, ISSN 0013-4686 
Bonilla, R. J.; James, B. R. \& Jessop, P. G. (2000). Colloid-catalyzed Arene Hydrogenation in Aqueous/Supercritical Fluid Biphasic Media. Chemical Communications, No.11, (May 2000), pp.941-942, ISSN 1359-7345

Bönnemann, H. \& Richards, R. M. (2001). Nanoscopic Metal Particles-Synthetic Methods and Potential Applications. Europian Journal of Inorganic Chemistry, Vol.2001, No.10, (October 2001), pp.2455-2480, ISSN 1099-0682

Bradley, J. S. (1994). In: Clusters and Colloids: From Theory and Applications, G. Schmid (Ed), 456-544, VCH, ISBN 3527290435, Weinheim

Cadet, J.; Douki, T.; Gasparutto, D. And Ravanat, J. L. (2003). Oxidative Damage to DNA: Formation, Measurement and Biochemical Features. Mutation Research, Vol.531, No.1-2, (October 2003), pp.5-23, ISSN 0027-5107

Drechsel, D. A. \& Patel, M. (2008). Mechanisms of Environmental Neurotoxicant-induced Oxidative Stress. Free Radical Biology and Medicine, Vol.44, No.11, (June 2008), pp.1873-1886, ISSN 0891-5849

Eley, D. D. \& Macmahon D. M., (1972). The Decomposition of Hydrogen Peroxide Catalyzed by Palladium-gold Alloy Wires. Journal of Colloid and Interface Science, Vol.38, No.2, (February 1972), pp.502-510. ISSN: 0021-9797

Fujishima, A.; Rao, T. N. \& Tryk, D. A. (2000). Titanium Dioxide Photocatalysis. Journal of Photochemistry and Photobiology C: Photochemistry Reviews, Vol.1, No.1, (June 2000), pp.1-21, ISSN 1389-5567

Fujishima, A.; Zhang, X. \& Tryk, D. A. TiO2. (2008). Photocatalysis and Related Surface Phenomena. Surface Science Reports, Vol.63, No.12, (December 2008), pp.515-582, ISSN 0167-5729

Fukumi, K.; Chayahara, A,; Kadono, K.; Sakaguchi, T.; Horino, Y.; Miya, M.; Fujii, K,.; Hayakawa, J. \& Satou, M. (1994). Gold Nanoparticles Ion Implanted in Glass with Enhanced Nonlinear Optical Properties, Journal of Applied Physics; Vol.75, No.6, (March 1994), pp.3075-3080, ISSN 0021-8979

Goszner, K.; Körner, D. \& Hite, R. (1972). On the Catalytic Activity of Silver: I. Activity, Poisoning, and Regeneration during the Decomposition of Hydrogen Peroxide. Journal of Catalysis, Vol.25, No.2, (May 1973), pp.245-253, ISSN 0021-9517

Goszner, K. \& Bischof, H. (1974). The Decomposition of Hydrogen Peroxide on Silver-gold Alloys. Journal of Catalysis, Vol.32, No.2, (February 1974), pp.175-1820, ISSN 00219517

Harriman, A. (1990). Bimetallic Pt-Au Colloids as Catalysts for Photochemical Dehydrogenation. Journal of the Chemical Society, Chemical Communications, No.1, (January 1990), pp.24-26, ISSN 0022-4936

Henglein, A. (1979). Catalysis of the Reduction of Thallium(1+) and of Dichloromethane by Colloidal Silver in Aqueous Solution. The Journal of Physical Chemistry, Vol.83, No.22, (November 1979), pp.2858-2862, ISSN 0022-3654

Henglein, A.; Holzwarth, A. \& Malvaney, P. (1992). Fermi Level Equilibration between Colloidal lead and Silver Particles in Aqueous Solution. The Journal of Physical Chemistry, Vol.96, No.22, (October 1992), pp.8700-8702, ISSN 0022-3654 
Hirai, H.; Nakao, Y. \& Toshima, N. (1979). Preparation of Colloidal Transition Metals in Polymers by Reduction with Alcohols or Ethers. Journal of Macromolecular Science: Part A-Chemistry, Vol.13, No.6, (1979), pp.727-750, ISSN 0022-233X

Yamaji, Y.; Hirakawa, K.; Yonezawa, T. \& Toshima, N. (1995). Visible-light-induced Reduction of Carbon Dioxide Using Platinum Colloidal Catalyst. Proceedings of Annual Meeting on Photochemistry 1995, pp.B1080, Fukuoka, Japan, October 6-9, 1995

Hirakawa, K.; Oikawa, S.; Hiraku, Y.; Hirosawa, I. \& Kawanishi, S. (2002). Catechol and Hydroquinone Have Different Redox Properties Responsible for Their Differential DNA-damaging Ability. Chemical Research in Toxicology, Vol.15, No.1, (January 2002), pp.76-82, ISSN 0893-228X

Hirakawa, K. \& Toshima, N. (2003). Ag/Rh Bimetallic Nanoparticles Formed by Selfassembly from $\mathrm{Ag}$ and $\mathrm{Rh}$ Monometallic Nanoparticles in Solution. Chemistry Letters, Vol.32, No.1, (January 2003), pp.78-79, ISSN 0366-7022

Hirakawa, K.; Mori, M.; Yoshida, M.; Oikawa, S. \& Kawanishi, S. (2004). Photo-irradiated Titanium Dioxide Catalyzes Site Specific DNA Damage via Generation of Hydrogen Peroxide, Free Radical Research, Vol.38, No.5, (May 2004), pp.439-447, ISSN 1071-5762

Hirakawa, K. \& Hirano, T. (2006). Singlet Oxygen Generation Photocatalyzed by $\mathrm{TiO}_{2}$ Particles and Its Contribution to Biomolecule Damage. Chemistry Letters, Vo.35, No.8, (August 2006), pp.832-833, ISSN 0366-7022

Hirakawa, K. (2006). Fluorometry of Hydrogen Peroxide Using Oxidative Decomposition of Folic Acid. Analytical and Bioanalytical Chemistry, Vol.386, No.2, (September 2006), pp.244-248, ISSN 1618-2642

Hirakawa, K. (2007). Preparation of Novel Metal Nanoparticles and Their Catalytic and Photocatalytic Properties. Reports of Researches Assisted by the Asahi Glass Foundation (2007), No.53, (October 2007) pp.1-10, ISSN 0919-9179

Hirakawa, K.; Shiota, K. \& Sano, S. (2008). Preventive Action of Metal Nanoparticles on UVA-sensitized Oxidation through Hydrogen Peroxide Formation. Photomedicine and Photobiology, Vol.30, (July 2008), pp.27-28, ISSN 0912-232X

Hirakawa, K. \& Sano, S. (2009). Platinum Nanoparticle Catalyst Scavenges Hydrogen Peroxide Generated from Hydroquinone. Bulletin of the Chemical Society of Japan, Vol.82, No.10, (October 2009), pp.1299-1303, ISSN 0009-2673

Hirakawa, K. (2009). Evaluation and Chemoprevention of Phototoxic Effect by the Novel Materials. Photomedicine and Photobiology, Vo.31, (July 2009), pp.33-34, ISSN 0912$232 X$

Hiraku, Y.; Ito, K.; Hirakawa, K. \& Kawanishi, S. (2007). Photosensitized DNA Damage and Its Protection via a Novel Mechanism. Photochemistry and Photobiology, Vo.83, No.1, (January 2007), pp.205-212, ISSN 1751-1097

Ishitani, O.; Inoue, C.; Suzuki, Y. \& Ibusuki, T. (1993). Photocatalytic Reduction of Carbon Dioxide to Methane and Acetic Acid by an Aqueous Suspension of MetalDeposited $\mathrm{TiO}_{2}$. Journal of Photochemistry and Photobiology A: Chemistry, Vol.72, No.3, (June 1993), pp.269-271, ISSN 1010-6030 
Kajita, M.; Hikosaka, K.; Iitsuka, M.; Kanayama, A.; Toshima, N. \& Miyamoto, Y. (2007). Platinum Nanoparticle Is a Useful Scavenger of Superoxide Anion and Hydrogen Peroxide. Free Radical Research, Vol.41, No.6, (January 2007), pp.615-626, ISSN 10715762

Kawanishi, S.; Hiraku, Y. \& Oikawa, S. (2001). Mechanism of Guanine-specific DNA Damage by Oxidative Stress and Its Role in Carcinogenesis and Aging. Mutation Research, Vol.488, No.1, (March 2001), pp.65-76, ISSN 0027-5107

Keating, K. B.; Rozner, A. G. \& Youngblood, J. L. (1965). The Effect of Deformation on Catalytic Activity of Platinum in the Decomposition of Hydrogen Peroxide. Journal of Catalysis, Vol.4, No.5, (October 1965), pp.608-619, ISSN 0021-9517

Kiely, C. J.; Fink, J.; Brust, M.; Bethell, D. \& Schiffrin, D. J. (1998). Spontaneous Ordering of Bimodal Ensembles of Nanoscopic Gold Clusters. Nature, Vol.396, pp.444-446, (December 1998), ISSN 0028-0836

Lee, A. F.; Baddeley, C. J.; Hardacre, C.; Ormerod, R. M.; Lambert, R. M.; Schmid, G. \& West, H. (1995). Structural and Catalytic Properties of Novel Au/Pd Bimetallic Colloid Particles: EXAFS, XRD, and Acetylene Coupling. The Journal of Physical Chemistry, Vol.99, No.16, (Aprile 1995), pp.6096-6102, ISSN 0022-3654

Link, S:; Wang, Z. L. \& El-Sayed, M. A. (1999). Alloy Formation of Gold-Silver Nanoparticles and the Dependence of the Plasmon Absorption on Their Composition. The Journal of Physical Chemistry B, Vol.103, No.18, (Aprile 1999), pp.3529-3533, ISSN 1520-6106

Lu, P.; Dong, J. \& Toshima, N. (1999). Surface-Enhanced Raman Scattering of a Cu/Pd Alloy Colloid Protected by Poly( $N$-vinyl-2-pyrrolidone). Langmuir, Vol.15, No.23, (September 1999), pp.7980-7992, ISSN 0743-7463

Matsushita, T.; Shiraishi, Y.; Horiuchi, S. \& Toshima, N. (2007). Synthesis and Catalysis of Polymer-Protected Pd/Ag/Rh Trimetallic Nanoparticles with a Core-Shell Structure. Bulletin of the Chemical Society of Japan, Vol.80, No.6, (June 2007), 12171225, ISSN 0009-2673

McKee, D. W. (1969). Catalytic Decomposition of Hydrogen Peroxide by Metals and Alloys of the Platinum Group. Journal of Catalysis, Vol.14, No.4, (August 1969), pp.355-364, ISSN 0021-9517

Murata, M. \& Kawanishi, S. (2000). Oxidative DNA Damage by Vitamin A and Its Derivative via Superoxide Generation. The Journal of Biological Chemistry, Vol.275, No.3, (January 2000), pp.2003-2008, ISSN 0021-9258

Nitta, Y.; Kamiya, K.; Tanimoto, M.; Sadamoto, S.; Niwa, O. \& Yokoro, K. (1991). Induction of Transplantable Tumors by Repeated Subcutaneous Injections of Natural and Synthetic Vitamin E in Mice and Rats. Japanese Journal of Cancer Research, Vol.82, No.5, (May 1991), pp.511-517, ISSN 0910-5050

Oikawa, S.; Furukawa, A.; Asada, H.; Hirakawa, K. \& Kawanishi, S. (2003). Catechins Induce Oxidative Damage to Cellular and Isolated DNA through the Generation of Reactive Oxygen Species. Free Radical Research, Vol. 37, No.8, pp.881-890, (August 2003), ISSN 1071-5762

Omenn, G. S.; Goodman, G. E.; Thornquist, M. D.; Balmes, J.; Cullen, M. R.; Glass, A.; Keogh, J. P.; Meyskens Jr, F. L.; Valanis, B.; Williams Jr, J. H.; Barnhart, S.; 
Cherniack, M. G.; Brodkin, C. A. \& Hammar, S. (1996). Risk Factors for Lung Cancer and for Intervention Effects in CARET, the Beta-Carotene and Retinol Efficacy Trial. Journal of the National Cancer Institute, Vol.88, No.21, (Novenber 1996), pp.1550-1559, ISSN 0027-8874

Peiner, E. \& Kopitzki, K. (1988). Metastable Phases Formed by Ion Beam Mixing of Binary Metal Systems with Positive Heats of Hormation. Nuclear Instruments and Methods in Physics Research Section B: Beam Interactions with Materials and Atoms, Vol.134, No.2, (August 1988), pp.173-180, ISSN 0168-583X

Pileni, M. P. (1998). Preparation, Characterization and Application, In: Nanoparticles and Nanostructured Films:, J. H. Fendler (Ed.), 71-100, Wiely-VCH, ISBN 3527294430, Weinheim

Shipway, A. N.; Katz, E. \& Willner, I. (2000). Nanoparticle Arrays on Surfaces for Electronic, Optical, and Sensor Applications. ChemPhysChem, Vol.1, No.1, (August 2000), pp.18-52, ISSN 1439-7641

Shiraishi, Y. \& Toshima, N. (1999). Colloidal Silver Catalysts for Oxidation of Ethylene. Journal of Molecular Catalysis A: Chemical, Vol.141, No.1-3, (May 1999), pp.187-192, ISSN 1381-1169

Siepen, K.; Bönnemann, H.; Brijoux, W.; Rothe, J. \& Hormes, J. (2000). EXAFS/XANES, Chemisorption and IR Investigations of Colloidal $\mathrm{Pt} / \mathrm{Rh}$ Bimetallic Catalysts. Applied Organometallic Chemistry, Vol.14, No.10, (October 2000), pp.549-556, ISSN 1099-0739

T. J. Slaga, (1995). Inhibition of Skin Tumor Initiation, Promotion, and Progression by Antioxidants and Related Compounds. Critical Reviews in Food Science and Nutrition, Vol.35, No.1-2, (January 1995), pp.51-57, ISSN 1040-8398

Sohmiya, M.; Tanaka, M.; Okamoto, K.; Fujisawa, A. \& Yamamoto, Y. (2004). Synergistic Inhibition of Lipid Peroxidation by Vitamin E and a Dopamine agonist, Cabergoline. Neurological Research, Vol.26, No.4, (June 2004), pp.418-421, ISSN 17431328

Solovieva, M. E.; Soloviev, V. V. \& Akatov, V. S. (2007). Vitamin B12b Increases the Cytotoxicity of Short-time Exposure to Ascorbic acid, Inducing Oxidative Burst and Iron-Dependent DNA Damage. European Journal of Pharmacology, Vol.566, No.1-3, (July 2007), pp.206-214, ISSN 0014-2999

Solovieva, M. E.; Soloviev, V. V.; Kudryavtsev, A. A.; Trizna, Y. A. \& Akatov, V. S. (2008). Vitamin B12b Enhances the Cytotoxicity of Dithiothreitol. Free Radical Biology and Medicine, Vol.44, No.10, (May 2008), pp.1846-1856, ISSN 0891-5849

Sun, S.; Murray, C. B.; Weller, D.; Folks, L. \& Moser, A. (1999). Monodisperse FePt Nanoparticles and Ferromagnetic FePt Nanocrystal Superlattices. Science, Vol.287, No. 5460, (March 1999), pp.1989-1992, ISSN 0036-8075

Teranishi ,T. \& Miyake, M. (1999). Novel Synthesis of Monodispersed Pd/Ni Nanoparticles. Chemistry of Materials, Vol.11, No.12, (November 1999), pp.3414-3416, ISSN 08974756

Toshima, N. \& Wang, Y. (1994). Polymer-protected Cu/Pd Bimetallic Clusters. Advanced Materials, Vol.6, No.3, (March 1994), pp.245-247, ISSN 1521-4095 
Toshima, N.; Yamaji, Y.; Teranishi, T. \& Yonezawa, T. (1995). Photosensitized Reduction of Carbon Dioxide in Solution Using Noble Metal Clusters for Electron Transfer. Zeitschrift für Naturforschung A, Vol.50a, pp.283-291, ISSN 0932-0784

Toshima, N. \& Hirakawa, K. (1997). Polymer-protected Pt/Ru Bimetallic Cluster Catalysts for Visible-light-induced Hydrogen Generation from Water and Electron Transfer Dynamics. Applied Surface Science, Vol.121/122, (November 1997), pp.534-537, ISSN 0169-4332

Toshima, N. \& Hirakawa, K. (1999). Polymer-Protected Bimetallic Nanocluster Catalysts Having Core/Shell Structure for Accelerated Electron Transfer in Visible-LightInduced Hydrogen Generation. Polymer Journal, Vol.31, No.11, (November 1999), pp.1127-1132, ISSN 0032-3896

Toshima, N.; Shiraishi, Y.; Matsushita, T.; Mukai, H. \& Hirakawa, K. (2002). Selforganization of Metal Nanoparticles and Its Application to Synthesis of $\mathrm{Pd} / \mathrm{Ag} / \mathrm{Rh}$ Trimetallic Nanoparticle Catalysts with Triple Core/Shell Structures. International Journal of Nanoscience, Vol.1, No.5-6, (December 2002), pp.397-401, ISSN 0219-581X

Toshima, N.; Kanemaru, M.; Shiraishi, Y. \& Koga, Y. (2005). Spontaneous Formation of Core/Shell Bimetallic Nanoparticles: A Calorimetric Study. The Journal of Physical Chemistry B, Vol.109, No.24, (August 2005), pp.16326-16331, ISSN 1520-6106

Toshima, N.; Ito, R.; Matsushita, T. \& Shiraishi, Y. (2007), Trimetallic Nanoparticles Having a Au-core Structure. Catalysis Today, Vol.122, No.3-4, (Aprile 2007), pp.239-244, ISSN 0920-5861

Toshima, N. \& Zhang, H. (2011). Preparation of Novel Au/Pt/Ag Trimetallic Nanoparticles and Their High Catalytic Activity for Aerobic Glucose Oxidation. Applied Catalysis A: General, Vol.400, No.1-2, (June 2011), pp.9-13, ISSN 0926-860X

Weyant, M. J.; Carothers, A. M.; Dannenberg, A. J. \& Bertagnolli, M. M. (2001). (+)-Catechin Inhibits Intestinal Tumor Formation and Suppresses Focal Adhesion Kinase Activation in the Min/+ Mouse. Cancer Research, Vol.61, No.1, (January 2001), pp.118-125, ISSN 0008-5472

Widegren, J. A. \& Finke, R. G. (2003). A Review of Soluble Transition-metal Nanoclusters as Arene Hydrogenation Catalysts. Journal of Molecular Catalysis A: Chemical, Vol.191, No.2, (January 2003), pp.187-207, ISSN 1381-1169

Willner, I.; Maiden, R.; Mandler, D.; Dürr, H.; Dörr, G. \& Zengerle, K. (1987). Photosensitized Reduction of Carbon Dioxide to Methane and Hydrogen Evolution in the Presence of Ruthenium and Osmium Colloids: Strategies to Design Selectivity of Products Distribution. Journal of the American Chemical Society, Vol.109, No.20, (September 1987), pp.6080-6086, ISSN 0002-7863

Yamashita, N.; Murata, M.; Inoue, S.; Burkitt, M. J.; Milne, L. \& Kawanishi, S. (1998). Alphatocopherol Induces Oxidative Damage to DNA in the Presence of Copper(II) Ions. Chemical Research in Toxicology, Vol.11, No.8, (August 1998), pp.855-862, ISSN 0893$228 \mathrm{X}$

Yonezawa, T. \& Toshima, N. (1993). Polymer- and Micelle-protected Gold/Platinum Bimetallic Systems. Preparation, Application to Catalysis for Visible-lightinduced Hydrogen Evolution, and Analysis of Formation Process with Optical 
Methods. Journal of Molecular Catalysis, Vol.83, No.1-2, (July 1993), pp.167-181, ISSN 1381-1169

Yonezawa, T. \& Toshima, N. (1995). Mechanistic Consideration of Formation of Polymerprotected Nanoscopic Bimetallic Clusters. Journal of the Chemical Society, Faraday Transactions, Vol.91, No.22, (November 1995), pp.4111-4119, ISSN 0956-5000 


\title{
Utilization of Nanoparticles Produced by Aqueous-Solution Methods - Formation of Acid Sites on $\mathrm{CeO}_{2}-\mathrm{TiO}_{2}$ Composite and 1-D $\mathrm{TiO}_{2}$ for Dye-Sensitized Solar Cells
}

\author{
Motonari Adachi et al. ${ }^{*}$ \\ Fuji Chemical Co., Ltd., 1-35-1 Deyashikinishi-Machi, Hirakata, \\ Japan
}

\section{Introduction}

Nanoparticles with well-defined nanostructures with unique physical properties are assembled into optoelectronic (Colvin et al. 1994), and nano electronic (Fuhrer et al. 2000) devices and other functional materials (Morris et al. 1999). Highly crystallized nanoparticles can be produced by aqueous-solution methods which provide low cost and ease of fabrication.

In this chapter two utilizations of nanoparticles are presented. First one is formation of acid sites on $\mathrm{CeO}_{2}-\mathrm{TiO}_{2}$ composite. Cerium dioxide has an unusual ability to shift easily between the reduced and oxidized states $\left(\mathrm{Ce}^{3+} \rightleftarrows \mathrm{Ce}^{4+}\right)$. This ability coupled with a high oxygen transport capacity gives a unique property of catalysis. Based on the remarkable properties of cerium dioxide, catalytic activity of nanoscale composite of $\mathrm{CeO}_{2}-\mathrm{TiO}_{2}$ was studied with variation in composition and formation temperature, which brought change in the number of Lewis acid site together with morphological changes.

The second one is 1-D $\mathrm{TiO}_{2}$ for dye-sensitized solar cells (DSSCs). We succeeded in the preparation of titania nanorods (Jiu et al. 2006), network structure of titania nanowires (Adachi et al. 2004) and one-dimensional titania nanochains. All cells composed of these highly crystallized 1-dimensional titania nanoscale materials (1DTNM) show high power

\footnotetext{
${ }^{*}$ Keizo Nakagawa², Yusuke Murata ${ }^{3}$, Masahiro Kishida ${ }^{4}$, Masahiko Hiro $^{5}$, Kenzo Susa 6 , Jun Adachi ${ }^{7}$, Jinting Jiu ${ }^{8}$ and Fumio Uchida ${ }^{1}$

${ }^{1}$ Fuji Chemical Co., Ltd., 1-35-1 Deyashikinishi-machi, Hirakata, Japan

2Department of Advanced Materials, Institute of Technology and Science, The University of Tokushima, Minami-josanjima, Tokushima, Japan

${ }^{3}$ Toyo Tanso Co., Ltd., 5-7-12 Takeshima, Nishiyodogawa-ku, Osaka, Japan

${ }^{4}$ Graduate School of Engineering, Kyushu University,744 Motooka, Nishi-ku, Fukuoka, Japan

${ }^{5}$ Hitachi Chemical Co., Ltd., 2-1-1 Nishishinjuku, Shinjuku, Tokyo, Japan

${ }^{6}$ Trial Corporation., 2-195 Asahi, Kitamoto, Japan

${ }^{7}$ National Instituite of Biomedical Innovation, 7-6-8 Asagi Saito, Ibaraki, Japan

${ }^{8}$ The Institute of Scientific and Industrial Research (ISIR), Osaka University, 8-1 Mihogaoka, Ibaraki, Japan
} 
conversion efficiency about $9 \%$. We also present necessity of 1DTNM for attainment high efficiency theoretically based on the consideration of electron transport processes in the titania electrode and then present that it is indispensable to use highly crystallized 1DTNM for attainment of higher efficient DSSCs based on the analysis of experimental results obtained by electrochemical impedance spectroscopy (EIS) and I-V measurements.

\section{2. $\mathrm{CeO}_{2}-\mathrm{TiO}_{2}$ composite as a catalyst}

Ceria-based materials are major compounds of the rare earth family, and these have been extensively studied and found application as ultraviolet absorbers (Masui et al., 1997, 2000), solid electrolytes (Inaba \& Tagawa, 1996), so-called three-way catalysts for automotive exhaust catalysts (Bekyarova et al., 1998), and soot oxidation catalysts (Pisarello et al., 2002; Aneggi et al., 2006). Nanocrystalline ceria materials have received much attention owing to their physical and chemical properties, which are markedly different from those of the bulk materials. Of particularly interest, the electronic conductivity of $\mathrm{CeO}_{2}$ can be enhanced four orders of magnitude when its microstructure is changed from the micro- to nanocrystalline region (Chiang et al., 1996). Various aqueous solution-based methods for synthesizing crystallized $\mathrm{CeO}_{2}$ nanoparticles (Masui et al., 2002a; Hirano et al., 2000; Li et al., 2001; Wu et al., 2002; Zhou et al., 2003; Bumajdad et al., 2004) and 1D, 2D and 3D $\mathrm{CeO}_{2}$ nanostructures with different morphologies (Vantomme et al., 2005, Zhou et al., 2005, Kuiry et al., 2005, Ho et al., 2005; Han et al., 2005; Sun et al., 2006; Zhong et al., 2007) have been investigated. Some of the properties of these materials, such as the dispersibility of the particles (Masui et al., 2002a) and their catalytic properties (Masui et al., 1997; Sun et al., 2006; Zhong et al., 2007) have also been studied.

The features of $\mathrm{CeO}_{2}$ in these applications are mainly due to the unique combination of its elevated oxygen transport capacity, coupled with its ability to shift easily between the reduced and oxidized states $\left(\mathrm{Ce}^{3+} \leftrightarrow \mathrm{Ce}^{4+}\right)$. To increase the temperature stability and ability of ceria to store and release oxygen, other transition and non transition metal ions (such as $\mathrm{Al}^{3+}, \mathrm{Si}^{4+}, \mathrm{Ti}^{4+}$ and $\mathrm{Zr}^{4+}$ ) are normally introduced into the ceria cubic structure (Reddy et al., 2003, 2005; Rynkowski et al., 2000; Masui et al., 2002b). The redox and catalytic properties of $\mathrm{CeO}_{2}$ are strongly influenced when it is combined with other transition metals. In addition, when the particle size is decreased below $100 \mathrm{~nm}$, the materials become nanophasic, where the density of defects increases, such that up to half $(50 \%)$ of the atoms are situated in the cores of the defects, promoting fast catalyst activation and reaction kinetics (Reddy et al., 2005). Thus, a study of the synthesis and reaction characteristics of nano-sized ceria-based mixed oxides is very important for utilizing the oxygen transport capacity and redox properties. One of the main disadvantages of ceria-based nanoparticles prepared in aqueous solution, however, is the resultant hard agglomeration of the fine particles, which has posed a major challenge to the realization of the full potential of nanocrystalline $\mathrm{CeO}_{2}$ powders.

In this section, first we present the preparation of cubic $\mathrm{CeO}_{2}$ nanoparticles using an alkoxide-primary amine surfactant in an aqueous solution and the existence of a clear potential to make $1 \mathrm{D}, 2 \mathrm{D}$ or $3 \mathrm{D} \mathrm{CeO}_{2}$ materials by assembling cubic-shape $\mathrm{CeO}_{2}$ nanoparticle building blocks. Amine surfactant works as a colloidal stabilizer through the adsorption on the $\mathrm{CeO}_{2}$ nanoparticles. Second, the preparation of $\mathrm{CeO}_{2}-\mathrm{TiO}_{2}$ nanocomposite nanostructures is presented. The morphologies and redox reactivities of $\mathrm{CeO}_{2}-\mathrm{TiO}_{2}$ 
composite nanostructures are influenced by changing the mole ratio of cerium/titanium alkoxides and by changing the calcination temperature.

\subsection{Preparation of $\mathrm{CeO}_{2}$ nanoparticles and $\mathrm{CeO}_{2}-\mathrm{TiO}_{2}$ composite nanostructure}

The preparation method of $\mathrm{CeO}_{2}$ nanoparticles and $\mathrm{CeO}_{2}-\mathrm{TiO}_{2}$ composite are based on the aqueous solution system including metal alkoxides and amine surfactant molecules. The experimental procedure has been described in detail in our previous papers (Murata \& Adachi, 2004; Nakagawa et al., 2007). The typical synthesis was as follows: first, laurylamine hydrochloride (LAHC) was dissolved in distilled water. Cerium tri-isopropoxide (CTIP) or cerium $n$-butoxide ( $\mathrm{CeBu}$ ) was used as a cerium source. Tetraisopropyl orthotitanate (TIPT) was used as a titanium source. In the synthesis of $\mathrm{CeO}_{2}$ nanoparticles, $\mathrm{CTIP}$ or $\mathrm{CeBu}$ was mixed with acetylacetone (ACA) in a beaker and immediately added to an aqueous LAHC solution at $\mathrm{pH}$ 4.6. In the case of the synthesis of $\mathrm{CeO}_{2}-\mathrm{TiO}_{2}$ composite nanostructures, the mole ratio of $\mathrm{CeBu}$ to TIPT (CeBu/TIPT) was changed to 100/0, 75/25, 25/75 and 0/100. Each mixed alkoxide solution was mixed with acetylacetone. In all cases, the mole ratio of metal alkoxides to ACA and metal alkoxides to LAHC were 1 and 4 , respectively. After stirring at room temperature for $1 \mathrm{~h}$, the reaction temperature was then changed to $353 \mathrm{~K}$. When the two solutions were mixed, precipitation occurred immediately. After 1 week, the precipitates were separated by centrifugation. After washing with 2-propanol and successive centrifugation, the obtained products were dried through a combination of freeze-drying and vacuum drying, and calcined in air at different temperatures.

\subsection{Cubic $\mathrm{CeO}_{2}$ nanoparticles and their assembled structures}

The formation yield of $\mathrm{CeO}_{2}$ particles for the surfactant assisted-process was $100 \%$ approximately. The structure of $\mathrm{CeO}_{2}$ nanoparticles was studied by TEM image of $\mathrm{CeO}_{2}$ sample in a dried state. During the formation process, we observed systematic changes in color of the precipitated particles. After mixing of the solution of CTIP or CeBu modified with ACA with the aqueous solution of LAHC, the brown transparent original solution immediately became dark brown. The white colloidal suspension was formed after stirring for $1 \mathrm{~h}$ at room temperature. A brown and clear supernatant was formed after the precipitation. Further color change of the precipitate was observed. First, the color of the $\mathrm{CeO}_{2}$ particles changed from white to dark blue in about 1 day at $353 \mathrm{~K}$. Subsequently, the color of the precipitate gradually turned into pale purple for 1 week, but the color change was slower than the first change. Moreover the wet centrifuged precipitate appeared dark blue, and the freeze-dried powders were gray. But final $\mathrm{CeO}_{2}$ particles calcined at $673 \mathrm{~K}$ was light yellow. These changes in color were observed in the cases that the particles were synthesized in LAHC surfactant aqueous solution at $\mathrm{pH}$ 4.2. On the other hand, there was no color change of the precipitate without LAHC surfactant. These color changes are related to the valence state of the $\mathrm{Ce}$; most likely purple corresponds to $\mathrm{Ce}^{3+}$ and yellow corresponds to $\mathrm{Ce}^{4+}$. Therefore, it is clear that the $\mathrm{Ce}^{3+}$ oxide is stabilized by existence of LAHC in aqueous solution.

We succeeded in the preparation of $\mathrm{CeO}_{2}$ nanoparticles with cubic structures and 1D, 2D or $3 \mathrm{D} \mathrm{CeO}_{2}$ nanostructures by assembling the cubic-shape $\mathrm{CeO}_{2}$ nanoparticle building blocks (Murata \& Adachi, 2004, Nakagawa et al., 2007) as shown in Fig. 1, 2 and 3. It is evident 
from this figure that the particle shape was square, and the particle size was calculated to be 2.7-3.8 nm. Furthermore, it seems that the particles were aligned. TEM image of Figure 1a clearly showed the mono-dispersed $\mathrm{CeO}_{2}$ nanoparticles. The inset picture shows the SAED pattern and Debye-Scherrer rings of the nanoparticles, which can be indexed as those of cerium oxide with the cubic fluorite structure. The HRTEM images and FFT pattern as shown in Figure $1 \mathrm{~b}$ show that the $\mathrm{CeO}_{2}$ cubic nanoparticles had a single crystalline structure and high crystallinity; these lattice images were observed for many particles. The main lattice spacing of the crystalline structure was calculated to be $3.11 \AA$ A according to FFT analysis. This lattice spacing corresponds to the (111) planes of $\mathrm{CeO}_{2}$ with a cubic phase, which coincides with the SAED analysis.
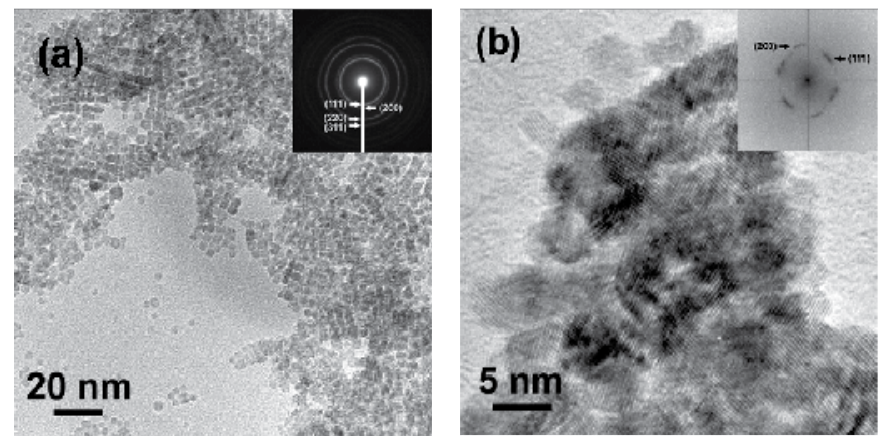

Fig. 1. (a) Low-magnification TEM images of the freeze-dried $\mathrm{CeO}_{2}$ nanoparticles prepared at $353 \mathrm{~K}$ for 1 week. Inset: SAED pattern. (b) High-Resolution TEM images of the aggregated $\mathrm{CeO}_{2}$. The lattice images were observed. Inset: FFT pattern obtained from HRTEM.

1D rod-like $\mathrm{CeO}_{2}$ structures are obtained after calcination at $673 \mathrm{~K}$. Rod-like $\mathrm{CeO}_{2}$ with diameters of $30 \mathrm{~nm}$ and lengths of $180 \mathrm{~nm}$ are observed although the majority of $\mathrm{CeO}_{2}$ samples were assembled into aggregates as shown in Fig.2. The HRTEM image show that the principal axis of the crystal growth of $\mathrm{CeO}_{2}$ was aligned along the rod axis.
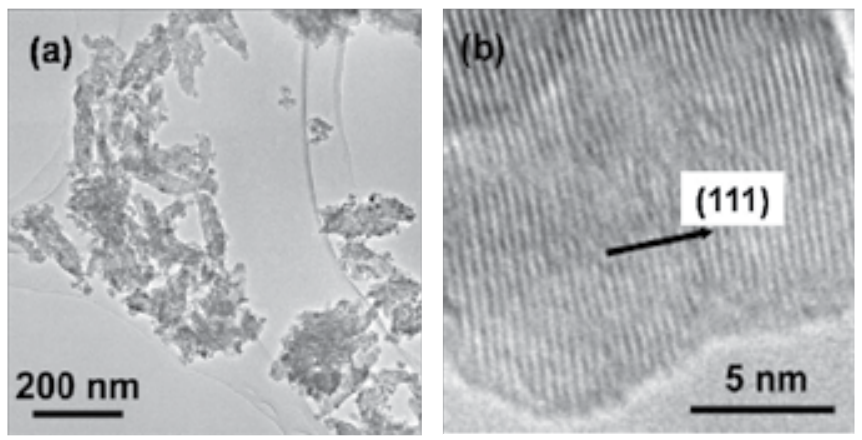

Fig. 2. (a) TEM images of $\mathrm{CeO}_{2}$ calcined at $673 \mathrm{~K}$ for $4 \mathrm{~h}$. (b) HRTEM image of rod-like $\mathrm{CeO}_{2}$ with a clearly lattice image of $(111)$ planes $(\mathrm{d}=3.11 \AA$ ).

An ordered structure (2D or 3D superlattice-like structure) are also obtained from the freezedried $\mathrm{CeO}_{2}$ nanoparticles. Figure 3 shows an array of cubic nanocrystals with a mean interparticle (center-to-center) distance of $2.9 \mathrm{~nm}$, as determined from direct imaging and the FFT pattern. We believe this assembly with an ordered structure is formed to minimize the total 
surface energy, which is attained by the association of the cubic $\mathrm{CeO}_{2}$ with a face-to-face structure.

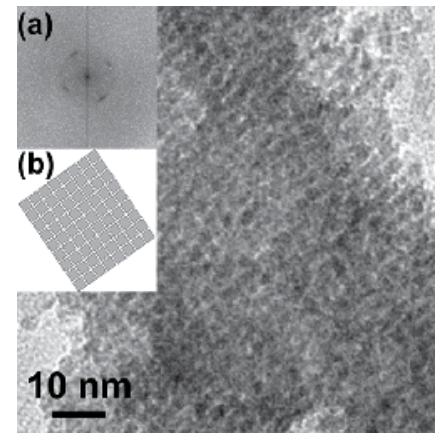

Fig. 3. TEM image of $\mathrm{CeO}_{2}$ nanocrystals self-assembled into a superlattice-like arrangement with dimensions of the order on the nano-scale, Inset a: the FFT pattern confirms the orientational order of the superlattice-like structure, Inset b: the model structure demonstrates the superlattice-like assembled $\mathrm{CeO}_{2}$ nanoparticles.

The thermodynamics of hydrolysis and condensation depend on the strength of the entering nucleophile and electrophilicity of the metal, and on the partial charge. Transition metals are very electropositive, and the hydrolysis and condensation kinetics of the transition metal alkoxides are affected by the positive partial charge $\delta^{+}$(Livage et al., 1988). Positive partial charge $\delta^{+}$for metals in various alkoxides have been reported; for example, cerium alkoxide: 0.75, titanium alkoxides: 0.63 , and silicon alkoxides: 0.32 . Since a large positive partial charge corresponds to a rapid reaction rate, the precursor for the complex formed from cerium alkoxide was not generated gradually, but the nano-sized particles were formed by the rapid hydrolysis and condensation reactions. In our systems using LAHC and CTIP (or $\mathrm{CeBu}$ ) modified with $\mathrm{ACA}$, the resulting suspensions of $\mathrm{CeO}_{2}$ nanoparticles were exceptionally mono-dispersive without aggregation, demonstrating the high power of LAHC as a colloidal stabilizer through the adsorption of LAHC on the surface of the $\mathrm{CeO}_{2}$ nanoparticles, in accordance with the results of Sugimoto et al. who reported the effect of primary amines as shape controllers for the synthesis of $\mathrm{TiO}_{2}$ (Sugimoto et al., 2003). Since the shape of the $\mathrm{CeO}_{2}$ particles is nearly cubic even if the cubic shape has somewhat rounded edges and corners, the LAHC would control the morphology of the $\mathrm{CeO}_{2}$ particles.

For hydrous oxides in aqueous solution systems, the charge-determing ions are $\mathrm{H}^{+}$and $\mathrm{OH}^{-}$, which establish the charge on the particles by protonating or deprotonating the $\mathrm{MOH}$ bonds on the surface of the particles.

$$
\begin{gathered}
\mathrm{M}-\mathrm{OH}+\mathrm{H}^{+} \rightarrow{\mathrm{M}-\mathrm{OH}_{2}{ }^{+}}^{\mathrm{M}-\mathrm{OH}+\mathrm{OH}^{-} \rightarrow \mathrm{M}-\mathrm{O}^{-}+\mathrm{H}_{2} \mathrm{O}}
\end{gathered}
$$

The ease of protonation and deprotonation on the surface of the oxide depends on the metal atom. The $\mathrm{pH}$ at which the particle is neutrally charged is called the point of zero charge (PZC). At $\mathrm{pH}>$ PZC, Eq. 2 predominates, and the particle is negatively charged, whereas at $\mathrm{pH}<\mathrm{PZC}$, Eq.1 makes the particle positive. Value of the PZC for $\mathrm{CeO}_{2}$ particles is 8.1 (De Faria and Trasatti, 1994). The magnitude of the surface potential depends on the departure 
of the $\mathrm{pH}$ from the PZC, and that potential attracts oppositely charged ions that present in the solution. Therefore, at $\mathrm{pH} 4.2$, the hydrolyzed and condensed $\mathrm{CeO}_{2}$ particle is positively charged. LAHC molecules also have a positively charged amine group under acidic condition. Hence, there seems to be no driving force for adsorption by electrostatic attraction. However, chloride ion $\left(\mathrm{Cl}^{-}\right)$mediates the interaction between the laurylamine surfactant and charged $\mathrm{CeO}_{2}$ by weak $\mathrm{H}$-bonding forces, and $\mathrm{CeO}_{2}$ particles are covered by surfactant molecules, resulting in the formation of cube crystals. Since the adsorption of LAHC takes place to a specific crystal face, anisotropic structures such as cubes would be formed.

\subsection{Morphology of $\mathrm{CeO}_{2}-\mathrm{TiO}_{2}$ composite}

A few studies on $\mathrm{CeO}_{2}-\mathrm{TiO}_{2}$ composite nanoparticles (Reddy et al., 2003, 2005; Rynkowski et al., 2000; Masui et al., 2002b) have been reported. Reddy et al. obtained $\mathrm{CeO}_{2}-\mathrm{TiO}_{2}$ composites comprised of relatively larger nanocrystals of $\mathrm{CeO}_{2}$ and $\mathrm{TiO}_{2}$ (anatase), and some overlapped regions (Reddy et al., 2005). Rynkowski et al. studied the redox properties of $\mathrm{CeO}_{2}-\mathrm{TiO}_{2}$ composites (Rynkowski et al., 2000), and stated the existence of the $\mathrm{CeO}_{2}$ $\mathrm{TiO}_{2}$ composite. Masui et al. also synthesized $\mathrm{CeO}_{2}-\mathrm{TiO}_{2}$ composite nanoparticles, and reported the deactivation of the thermal and photocatalytic properties of this species by the formation of the $\mathrm{CeO}_{2}-\mathrm{TiO}_{2}$ composite (Masui et al., 2002b).

We also studied the preparation of $\mathrm{CeO}_{2}-\mathrm{TiO}_{2}$ composite nanostructures by changing the mole ratio of cerium/titanium alkoxides and found the effective redox reactivities of $\mathrm{CeO}_{2}-\mathrm{TiO}_{2}$ composite nanostructures (Nakagawa et al., 2007). During the synthesis of $\mathrm{CeO}_{2}-\mathrm{TiO}_{2}$ composite, the reaction behavior of each solution was observed. When the surfactant solution and metal alkoxide solutions were mixed, precipitation occurred immediately. When the mole ratio of $\mathrm{CeBu} / \mathrm{TIPT}=75 / 25$ and $25 / 75$, dark brown-gels and dark purple-precipitates formed, while purple-precipitates with a transparent liquid layer were observed at the mole ratios of $\mathrm{CeBu} / \mathrm{TIPT}=100 / 0$, that was the same behavior using CTIP. The morphology and crystalline structure of the $\mathrm{CeO}_{2}-\mathrm{TiO}_{2}$ composite nanostructures varied according to the change in the mole ratio of $\mathrm{CeBu}$ to TIPT. When $\mathrm{CeBu} / \mathrm{TIPT}$ was $75 / 25$, the nano-network structure with a diameter of 3-9 $\mathrm{nm}$ was observed and the SAED pattern indicated a cubic fluorite structure (Figure 4). Whereas, when $\mathrm{CeBu} / \mathrm{TIPT}$ was 25/75, aggregate structures of rod-like morphology with an average diameter of $20 \mathrm{~nm}$ and length of $80 \mathrm{~nm}$ were observed, and the SAED pattern showed several spots corresponding to the lattice plane of the anatase phase of $\mathrm{TiO}_{2}$ (Figure 5). In the case of the synthesis with only TIPT, a $\mathrm{TiO}_{2}$ nano-network structure of connecting nanowires with diameter of 5-15 $\mathrm{nm}$ formed by an oriented attachment mechanism (Adachi et al., 2004, Nakagawa et al., 2005).

Figure 6 shows the variation in XRD patterns of the $\mathrm{CeO}_{2}-\mathrm{TiO}_{2}$ composite calcined at $673 \mathrm{~K}$ for $4 \mathrm{~h}$ (Nakagawa et al., 2007). The peaks at $\mathrm{CeBu} / \mathrm{TIPT}=100 / 0$ are sharp and can be indexed to a $\mathrm{CeO}_{2}$ cubic fluorite structure. When $\mathrm{CeBu} / \mathrm{TIPT}$ was $75 / 25$, the XRD peaks were indexed to $\mathrm{CeO}_{2}$ cubic fluorite structure, although the peaks became very broad. The reason for the broad peak is due to the formation of composite materials. In the HRTEM image shown in Figure $4 \mathrm{~b}$, the lattice image of the (111) plane of the cubic fluorite structure could be observed. These observations indicate that the crystalline structure of the nanonetwork at $\mathrm{CeBu} / \mathrm{TIPT}=75 / 25$ consists of a $\mathrm{CeO}_{2}$ cubic fluorite structure, which is different 

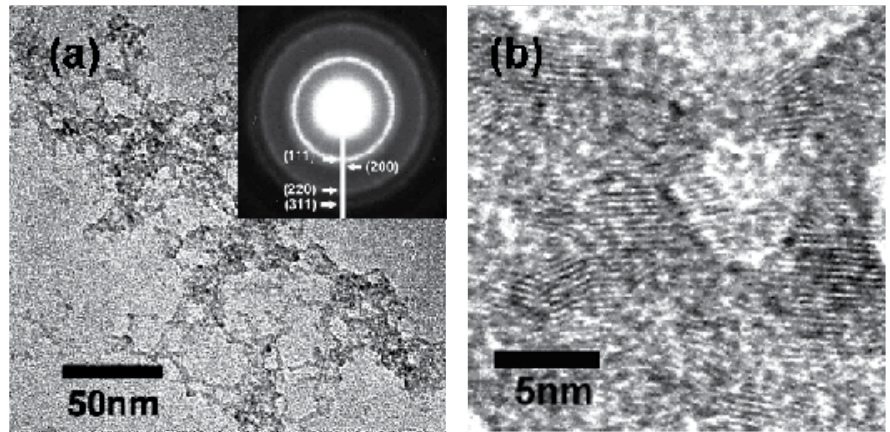

Fig. 4. (a) TEM and (b) HRTEM image of $\mathrm{CeO}_{2}-\mathrm{TiO}_{2}$ composite nanostructures $(\mathrm{CeBu} / \mathrm{TIPT}=75 / 25)$ after reaction at $353 \mathrm{~K}$ for 1 week, inset: SAED patterns.
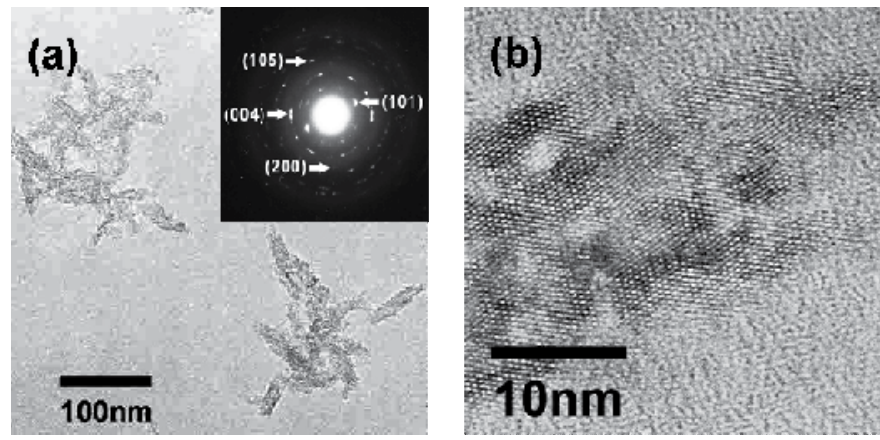

Fig. 5. (a) TEM and (b) HRTEM image of $\mathrm{CeO}_{2}-\mathrm{TiO}_{2}$ composite nanostructures $(\mathrm{CeBu} / \mathrm{TIPT}=25 / 75)$ after reaction at $353 \mathrm{~K}$ for 1 week, inset: SAED patterns.

from that of pure $\mathrm{CeO}_{2}$. No $\mathrm{TiO}_{2}$ anatase peaks were observed. Therefore, the formed materials under $\mathrm{CeBu} / \mathrm{TIPT}=75 / 25$ constitute the composite materials of $\mathrm{CeO}_{2}$ and $\mathrm{TiO}_{2}$, i.e., the formed materials are not a simple mixture of pure $\mathrm{CeO}_{2}$ and $\mathrm{TiO}_{2}$. As a characteristic of our reaction system, the initial solution, including the two metal alkoxides is uniformly well mixed on a molecular scale, easily leading to the formation of composite materials. Since the positive partial charge $\delta^{+}$of cerium alkoxide is larger than that of titanium alkoxide, as mentioned above, it is inferred that the reaction rate of $\mathrm{CeBu}$ is faster than TIPT. Moreover, the content of cerium is much higher than titanium. From these facts, the crystalline structure of the composite materials is inferred as a $\mathrm{CeO}_{2}$ cubic fluorite structure, which is different from that of pure $\mathrm{CeO}_{2}$. The different crystalline structure creates a new morphology, i.e., a nano-network structure, which also leads to the formation of Lewis acid sites, as described later. The XRD patterns at $\mathrm{CeBu} / \mathrm{TIPT}=25 / 75$ show mainly broad peaks of the $\mathrm{TiO}_{2}$ anatase phase and also show a broad peak of $\mathrm{CeO}_{2}$ around $2 \theta=30^{\circ}$. The broad peaks indicate the formation of composite materials, which lead to a nanorod structure. Since the content of titanium is much higher than cerium, the main crystalline structure corresponds to the $\mathrm{TiO}_{2}$ anatase phase, but a small amount of $\mathrm{CeO}_{2}$ crystalline structure is also included, because the reaction rate of $\mathrm{CeBu}$ is faster than TIPT. 


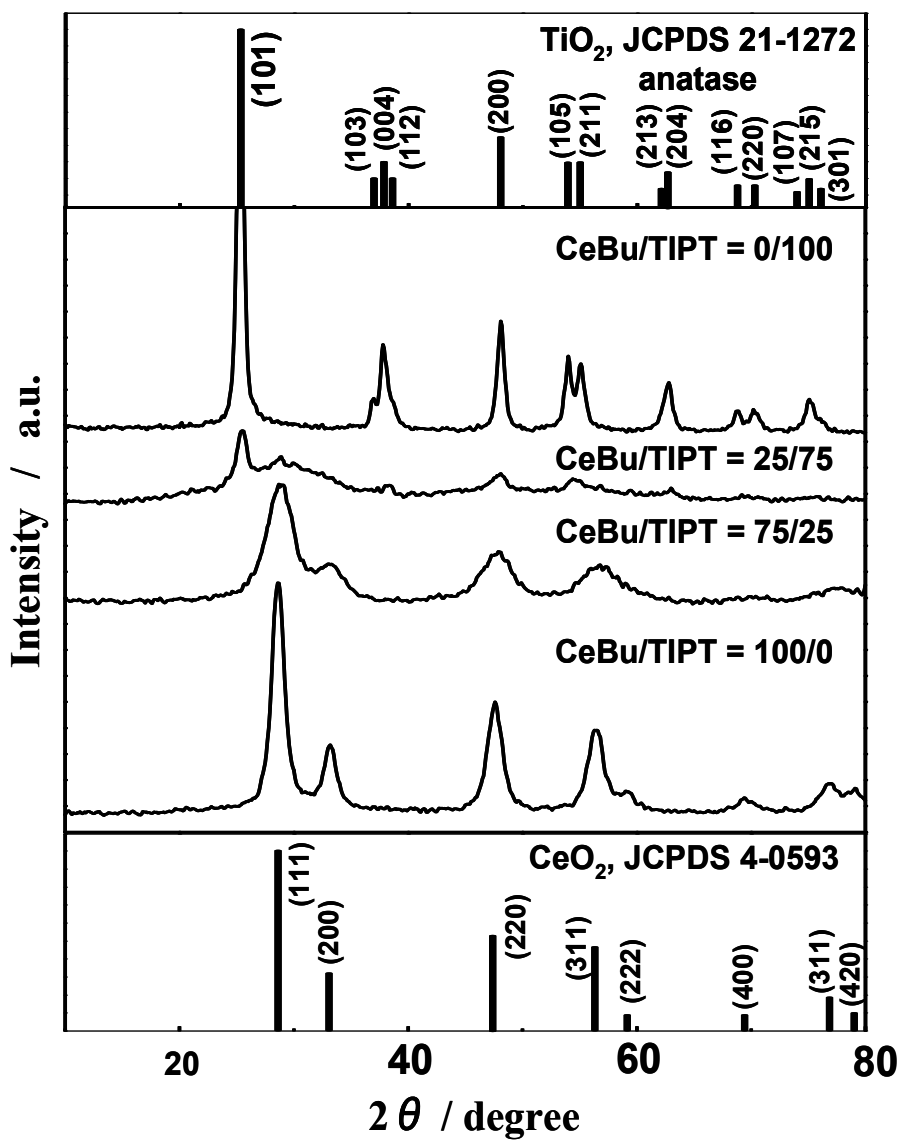

Fig. 6. XRD patterns of the $\mathrm{CeO}_{2}-\mathrm{TiO}_{2}$ composite nanostructures at the mole ratio of $\mathrm{CeBu} / \mathrm{TIPT}=100 / 0,75 / 25,25 / 75$ and $0 / 100$ after calcination at $673 \mathrm{~K}$ for $4 \mathrm{~h}$.

\subsection{Surface properties of $\mathrm{CeO}_{2}-\mathrm{TiO}_{2}$ composites}

The reaction activity of $\mathrm{CeO}_{2}-\mathrm{TiO}_{2}$ composite nanostructures was investigated through the formation rate of $\mathrm{I}_{3}$, formed due to the oxidation of $\mathrm{I}^{-}$to $\mathrm{I}_{2}$ in excess $\mathrm{KI}$ aqueous solution (Nakagawa et al., 2007). Nanostructured $\mathrm{CeO}_{2}-\mathrm{TiO}_{2}(10 \mathrm{mg})$ was suspended by magnetic stirring in $10 \mathrm{ml}$ of $0.2 \mathrm{M} \mathrm{KI}$ aqueous solution without light irradiation. After initiation of the reaction, $0.3 \mathrm{ml}$ of the reaction solution was taken, and the concentration diluted to one tenth. The concentration of $\mathrm{I}_{3}^{-}$was measured using a Shimadzu UV-2450 spectrometer from the absorbance at $288 \mathrm{~nm}$. Figure 7 shows the $\mathrm{I}_{3}$ - formation results of $\mathrm{CeO}_{2}-\mathrm{TiO}_{2}$ composite nanostructures after calcination at $673 \mathrm{~K}$. It was found that $\mathrm{CeO}_{2}$ nanoparticles and $\mathrm{CeO}_{2-}$ $\mathrm{TiO}_{2}$ composite nanostructures have the ability to oxidize $\mathrm{I}^{-}$to $\mathrm{I}_{2}$ although the $\mathrm{TiO}_{2}$ nanostructure shows little activity. The activity of the $\mathrm{CeO}_{2}-\mathrm{TiO}_{2}$ composite nanostructure reaches a maximum at $\mathrm{CeBu} / \mathrm{TIPT}=75 / 25$ at $623 \mathrm{~K}$.

It is known that cerium oxide shows a high oxidation ability and oxygen storage capacity, and the appearance of these functions is attributed to the following two reasons. One is the redox couple $\mathrm{Ce}^{3+} / \mathrm{Ce}^{4+}$, which shows the ability of cerium oxide to shift between $\mathrm{CeO}_{2}$ and 

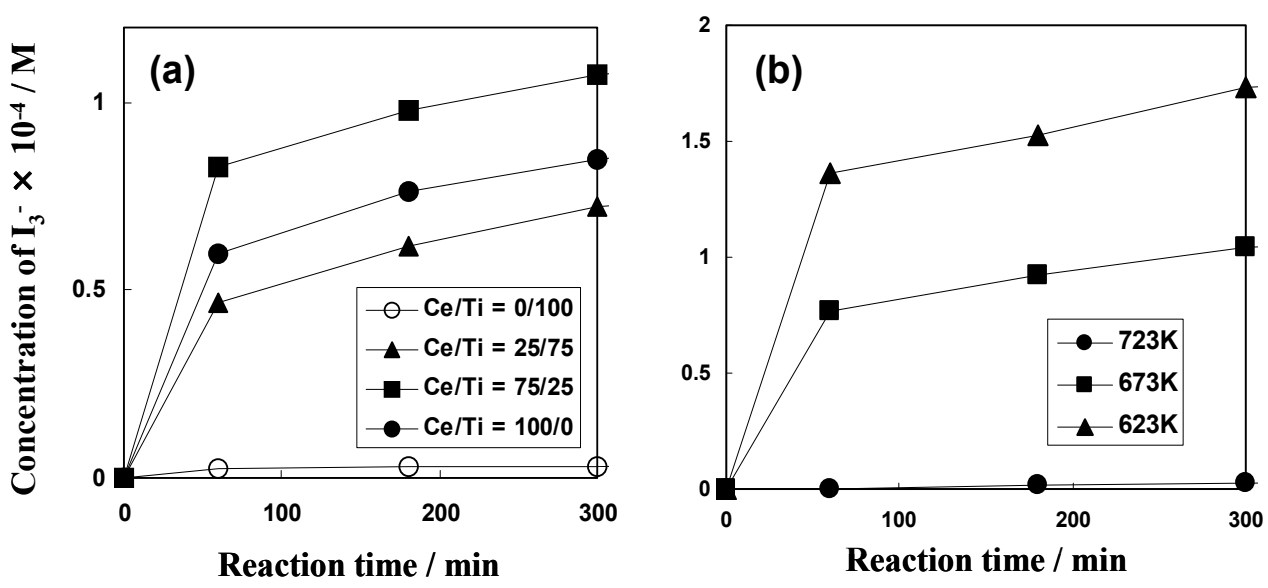

Fig. 7. The variation of concentration of $\mathrm{I}_{3}$ - with reaction time, (a) the effect of the mole ratio of $\mathrm{CeBu} / \mathrm{TIPT}$ calcined at $673 \mathrm{~K},(\mathrm{~b})$ the effect of calcination temperature at the mole ratio of $\mathrm{CeBu} / \mathrm{TIPT}=75 / 25$.

$\mathrm{Ce}_{2} \mathrm{O}_{3}$ under oxidizing and reducing conditions, respectively. Another is its structure: the stable structure of cerium oxide at room temperature under atmospheric pressure is the cubic fluorite structure in which oxygen ions do not have a close-packed structure. Owing to this structure, cerium oxide can easily form many oxygen vacancies while maintaining the basic crystal structure (Reddy et al., 2003). Cerium has a family of related mixed-valency binary oxides, which are anion-deficient and fluorite-related $\mathrm{Ce}_{2} \mathrm{O}_{2 n-2 m}$ between $\mathrm{Ce}_{2} \mathrm{O}_{3}$ and $\mathrm{CeO}_{2}$ at lower temperatures (Kang \& Eyring, 1997). It is considered that many vacant oxygen sites exist in cerium oxide; the cerium cation $\left(\mathrm{Ce}^{\mathrm{n}}\right)$ acts as the Lewis acid site and robs the electron of I-. Additionally, the number of Lewis acid sites could be altered by changing the composition of the $\mathrm{CeO}_{2}-\mathrm{TiO}_{2}$ composite, because mixed oxides, e.g., $\mathrm{SiO}_{2}-\mathrm{TiO}_{2}$ composites, have been frequently reported to exhibit higher catalytic activity than the pure metal oxide (Méndez-Román \& Cardona-Martínez, 1998; Hu et al., 2003). As pointed out above, the uniformly mixed solution of the metal alkoxides led to homogeneously mixed composite oxides on the atomic scale in our preparation method.

We confirmed the formation and number of Lewis acid sites from the pyridine adsorption on the surface of the $\mathrm{CeO}_{2}-\mathrm{TiO}_{2}$ composite nanostructure (Nakagawa et al., 2007) as shown in Figure 8. In the results of IR spectra, two peaks at 1620 and $1350 \mathrm{~cm}^{-1}$ were assigned to the antisymmetric and symmetric stretching vibrations of the carboxyl group, respectively. A peak at $1595 \mathrm{~cm}^{-1}$ and two peaks at 1480 and $1440 \mathrm{~cm}^{-1}$ were observed, and these peaks were assignable to hydrogen-bonded pyridine and pyridine bonded to a Lewis site, respectively (Zaki et al., 1989, 2001). It was found that Lewis acid sites evidently exist in the $\mathrm{CeO}_{2}-\mathrm{TiO}_{2}$ composite nanostructures and these results show a good correlation between the reaction activity (Figure 7a) and the peak area as determined from the Lewis acid sites (Figure 8).

\subsection{Conclusions of $2^{\text {nd }}$ section}

1. The preparation method of cubic $\mathrm{CeO}_{2}$ nanoparticles using an alkoxide-primary amine surfactant in an aqueous solution was presented. In additoion, a clear potential to make 
1D, $2 \mathrm{D}$ or $3 \mathrm{D} \mathrm{CeO}_{2}$ materials by assembling cubic-shape $\mathrm{CeO}_{2}$ nanoparticle building blocks was also revealed.

2. $\mathrm{CeO}_{2}-\mathrm{TiO}_{2}$ composite nanostructures could be prepared by changing the mole ratio of cerium/titanium alkoxides. The morphology and crystalline structure of the $\mathrm{CeO}_{2}-\mathrm{TiO}_{2}$ composite nanostructures were influenced with the mole ratio of the metal alkoxides. These composite nanostructures showed effective reaction activity to oxidize $\mathrm{I}^{-}$to $\mathrm{I}_{2}$ because of the formation of the Lewis acid sites.

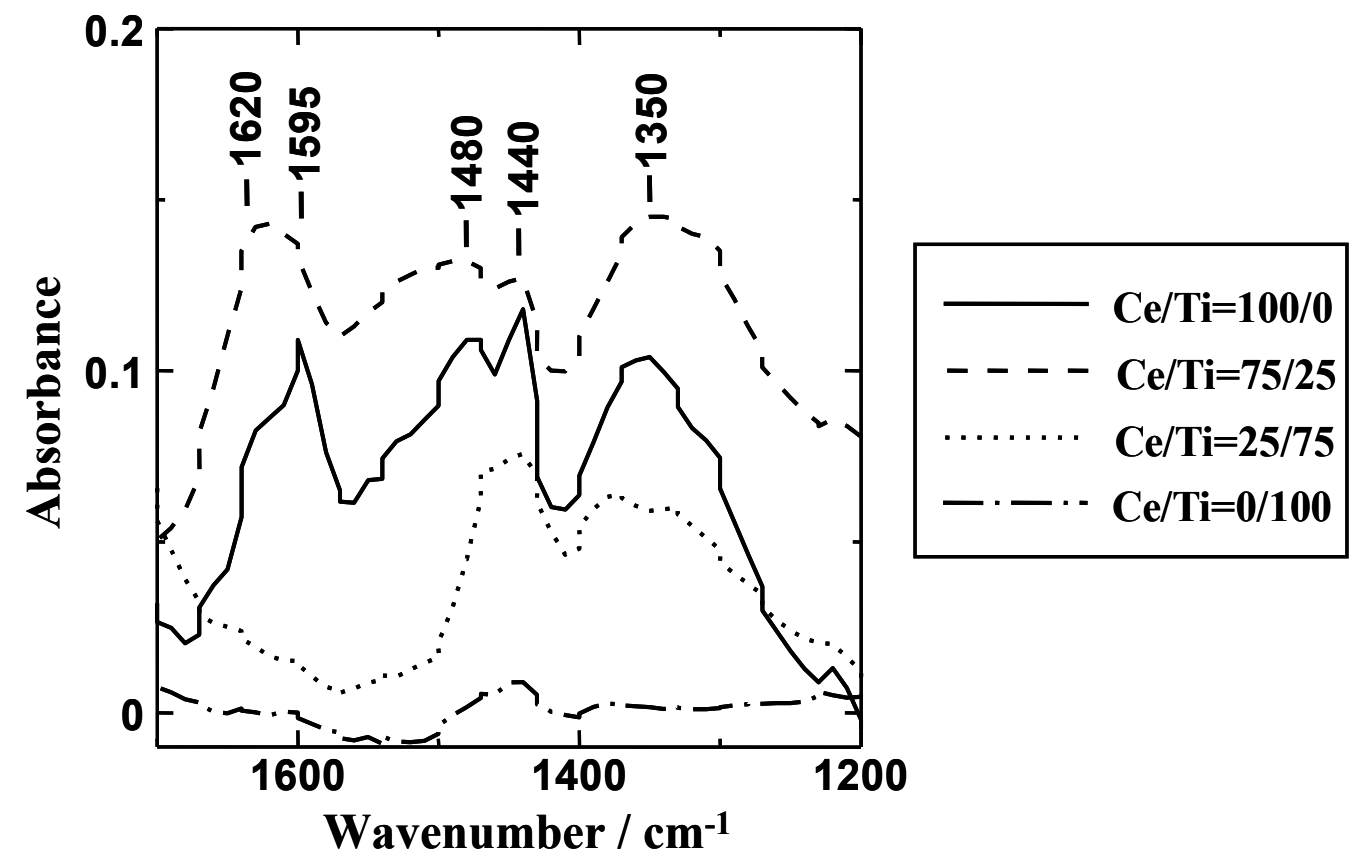

Fig. 8. Pyridine adsorption results (at room temperature) of the $\mathrm{CeO}_{2}-\mathrm{TiO}_{2}$ composite nanostructure at the different mole ratio of $\mathrm{CeBu} / \mathrm{TIPT}$ calcined at $673 \mathrm{~K}$.

\section{1-D $\mathrm{TiO}_{2}$ for dye-sensitized solar cells}

Dye-sensitized solar cells (DSSCs) have attracted much attention as they offer the possibility of extremely inexpensive and efficient solar energy conversion, because light from the sun is the ideal source of energy, and the supply of energy is gigantic, i.e., $3 \times 10^{24} \mathrm{~J} /$ year or about $10^{4}$ times more than what mankind consumes currently. In 1991, O'Regan and Grätzel (O'Regan \& Grätzel 1991) published a remarkable report, and the Grätzel group attained 10 \% efficiency in 1993 (Nazeeruddin et al. 1993). The system already reached conversion efficiency 11.5 \% (Chen, C-Y. et al. 2009), and recently even $12.3 \%$ was reported by Grätzel in Hybrid Organic Photovoltaics Conference in Valencia Spain. These conversion efficiencies exceed the level to supply electricity at the rate of home use, i.e., $10 \%$. Nevertheless, the energy conversion efficiency of the cells for commercial devices has not yet reached the level, which provides lower cost than that of conventional methods of electricity generation using fossil fuel. Therefore, attainment of higher efficient cells is still one of the most important challenges for the dye-sensitized solar cells. 
Titania dioxide is the most promising material for the electrode of DSSCs. Many investigators have improved the anodic electrode over 10 years (Kim et al. 2009, Ito et al. 2008, Grinis et al. 2008, Hamann et al. 2008, Chen, D. et al. 2009, Miyashita et al. 2008, Wang, M. et al. 2009, Youngblood et al. 2009). One-dimensional titania nanoscale materials (1DTNM) have been investigated for attainment of highly efficient solar cells (Colodrero et al. 2009, Kar et al. 2009, Shankar et al. 2009, Wang, D. et al. 2009, Kang, T-S. et al. 2009, Kuang, D. et al. 2008, Shankar et al. 2008, Adachi et al. 2004, Jiu et al. 2006). In this section we present the clear reason for necessity of 1DTNM for attainment of higher efficient dyesensitized solar cells through theoretical consideration and based on the experimental evidences verifying the consideration.

First we present necessity of 1DTNM theoretically based on the consideration of the electron transport processes obtained from electrochemical impedance spectroscopy (EIS), together with $\mathrm{I}-\mathrm{V}$ measurement of the same cell. We present then experimentally that it is indispensable to use highly crystallized 1DTNM for attainment of higher efficient DSSCs based on the analysis of experimental results obtained by EIS and I-V measurements. Also we present that all electrodes composed of our three kinds of 1DTNM showed high light-toelectricity conversion efficiency around $9 \%$.

\subsection{Experimental procedure}

In order to elucidate the relationship between the composition of titania thin film electrode and performance of the electrode, we made three kinds of electrodes, i.e., an electrode made of P-25 only, an electrode made of P-25 with polyethylene glycol (PEG) and an electrode made of network structure of titania nanowires (TNW) mixed with P-25 with PEG (TNW $28 \%$ ) first. Since the electrode containing TNW was the best one, we made electrodes made of various amount of TNW mixed with the mixture of P-25 and PEG. The percentage of TNW to (TNW + P-25) in Ti atom content was varied from $0 \%$ to $100 \%$. Furthermore, we made DSSCs with electrodes composed of all our three kinds of 1DTNM.

\subsubsection{Synthesis of highly crystallized $\mathrm{TiO}_{2}$ nanoscale materials}

The procedure of $\mathrm{TiO}_{2}$ single crystalline nanowires with network structure has been reported in our previous paper (Adachi et al. 2004). The synthesis procedure of highly crystallized titania nanorods has been described in our previous paper (Jiu et al. 2006). The procedure of titania nanochains is almost the same as that of titania nanorods, except usage of $\mathrm{HCl}$ instead of ethylenediamine (EDA) to adjust $\mathrm{pH}$ values to 1.3 to 5 . Titania nanochains can be synthesized using P123 (triblock copolymer of (poly(ethylene oxide)20poly(propylene oxide) 70-poly(ethylene oxide) 20 ) instead of F127 (triblock copolymer of (poly(ethylene oxide) ${ }_{106}$-poly(propylene oxide) 70 -poly(ethylene oxide) $\left.{ }_{106}\right)$ ).

\subsubsection{Preparation of titania electrods and dye-sensitized solar cells}

We synthesized highly crystallized titania nanoparticles (TNP) with diameter of 3-5 nm other than 1DTNM mentioned above (Jiu et al. 2004, Jiu et al. 2007). Titania electrodes with thin film were made by applying titania samples on an electric conducting glass plate. Fluorine doped tin oxide (FTO) was used as an electric conducting oxide. Dilute solution of 
TNP with diameter 3-5 nm was applied on the surface of FTO as a blocking layer. The three kinds of electrodes made of P-25 only, P-25 with PEG and titania nanowire network (TNW) mixed with P-25 with PEG were prepared by coating each gel solution containing these titania materials on the FTO glass by doctor blade method. The gel solution of P-25 only was made by dissolving P-25 powder into water. The aqueous gel solution of P-25 with PEG was made after the procedure reported by Grätzel's group (Nazeeruddin et al. 1993). The gel solution of TNW mixed with P-25 with PEG was made by mixing the gel solution of P-25 with PEG with the reaction products TNW after centrifugation and washing by 2-propanol.

The higher efficient cells constituted with 1DTNM were fabricated as follows. First, the gel solution of TNP with diameter of 3-5 nm was coated three times by doctor blade method on a FTO glass, making 3 layers of TNP. In the case of cells made of TNW, the gel solution of TNW mixed with P-25 with PEG was coated by 8-10 times. The ratio of TNW to P-25 in Ti atom content was around 0.3. In the case of cells made of titania nanorods, the reaction products after centrifugation was mixed with the two gel solutions of P- 25 with PEG and the solution of TNP. The mixed gel solution was coated 7-10 times. In the case of titania nanochains, the procedure was the same as the case of titania nanorods.

After each coating, the sample was calcined at $773 \mathrm{~K}$ for $10 \mathrm{~min}$. The last calcination was made at $773 \mathrm{~K}$ for $30 \mathrm{~min}$. Dye was introduced to the titania thin films by soaking the film $1-3$ days in $3 \times 10^{-4} \mathrm{M}$ solution of ruthenium dye in the mixed solvent of tert-butanol and acetnitryl. Cis-di(thiocyanate) bis(2,2'-bipyridyl-4,4'-di-carboxylate)-ruthenium(II) bis-tetrabutyl-ammonium (N719) (Solaronix SA) produced by Grätzel's group (Nazeeruddin et al. 1993) was used as the dye.

The DSSCs were comprised of a titania thin film electrode on a conducting glass plate, and a platinum electrode made by sputtering on the conducting glass and electrolyte between the titania thin film and the platinum. The composition of the used electrolyte was $0.1 \mathrm{M}$ Guanidium thiocyanate, 0.6 M 1-butyl-3-methylimidazolium iodide, $0.03 \mathrm{M} \mathrm{I}_{2}$, and $0.5 \mathrm{M}$ TBP (4-tert-butyl pyridine) in the mixed solvent of acetonitrile + n-valeronitrile (volume $85: 15$ ).

\subsubsection{Characterization of titania materials and solar cells}

Characterization of the produced materials was made by X-ray diffraction (XRD) (Rigaku Goniometer PMG-A2, CN2155D2), transmission electron microscopy (TEM) (JEOL 200 CX and JEM-2100F), fast Fourier transform (FFT), selected-area electron diffraction (SAED), scanning electron microscopy (SEM) (JEOL JSM 7500FA) and isotherm of nitrogen adsorption (BEL SORP 18 PLUS). The photo-current-voltage characteristics were measured using an AM 1.5 solar simulator (YSS-E40, Yamashita Denso) and in which the light intensity is $100 \mathrm{~mW} / \mathrm{cm}^{2}$ calibrated with a secondary reference solar cell standardized by JET (Japan Electrical Safety \& Environmental Technology Laboratories). Electron transport processes were measured by electrochemical impedance spectroscopy (EIS) (Solartron 1255B). The cell size was $0.25 \mathrm{~cm}^{2}$.

\subsection{Necessity of highly crystallized titania nanoscale materials}

First, let us consider the reason why highly crystallized one-dimensional titania materials are needed. Fig. 9a shows a typical Nyquist plot obtained by EIS. Total direct current (dc) 
resistance is given by the length from 0 to the point at $\omega=0$ on the real axis as shown by Fig. 9a. This fact is confirmed later by reproduction of $\mathrm{I}-\mathrm{V}$ curve using measured total $\mathrm{dc}$ resistances at various bias voltages as shown in Fig. 10. Total dc resistance is also obtained from the slope of the tangent line at the point of Voc. (Fig. 9b) When the total dc resistance becomes small, the slope becomes steep, and the fill factor becomes larger, resulting in a high light-to-electricity conversion efficiency. Thus, the total dc resistance should be small.
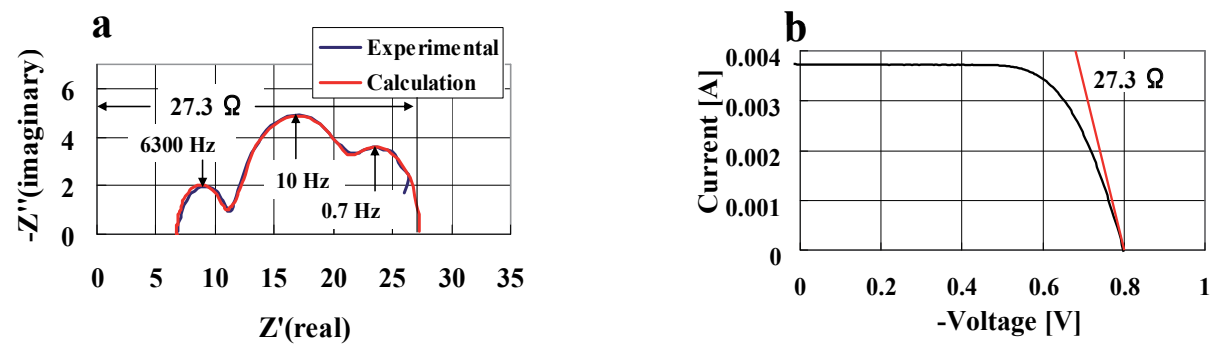

Fig. 9. (a) Typical Nyquist plot obtained by EIS, (b) I-V curve for the same cell

However, the largest arc of around $10 \mathrm{~Hz}$ in Fig. 9a represents the resistance of recombination reactions between electrons in the titania electrode and $\mathrm{I}_{3^{-}}$ions in the electrolyte. Small total dc resistance means small resistance for recombination reactions, indicating rapid reaction rate of recombination. Thus, small total dc resistance seems an obstacle for attainment of highly efficient solar cells. But, whether electrons in the titania electrode are properly collected by the transparent conducting glass electrode or react with $\mathrm{I}_{3}$ - ions in the electrolyte by recombination reactions is determined by the ratio of the resistance for the transport rate to the conducting glass electrode against the resistance for the recombination reactions. When the resistance for the transport rate to the conducting glass electrode is much smaller than that of the recombination reactions, almost all electrons are properly collected by the conducting glass electrode. This means that the transport rate of electrons in the titania electrode should be very rapid, indicating that we need nice titania materials with high electron transport rate, i.e., highly crystallized one-dimensional nanoscale $\mathrm{TiO}_{2}$ materials are needed.

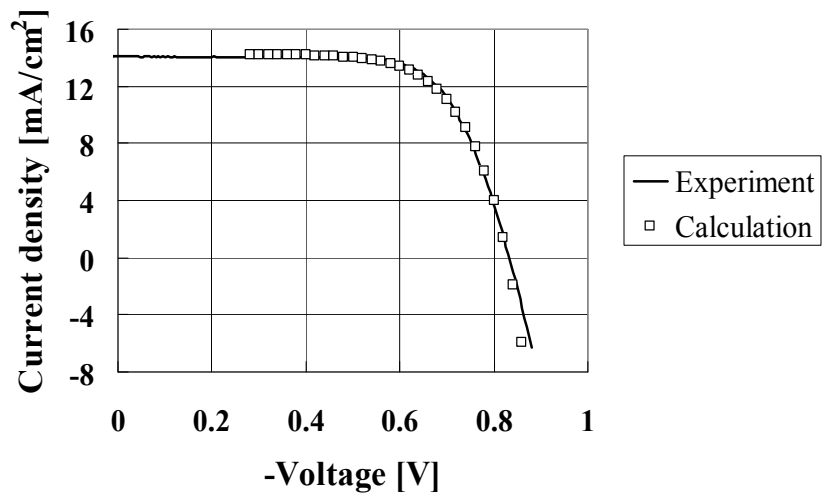

Fig. 10. Reproduction of $\mathrm{I}-\mathrm{V}$ curve by total dc resistances at various bias voltages. 
Solid line in Fig. 10 shows experimentally obtained I-V curve under illumination. The square keys show calculated curve based on the observed total dc resistances at various bias voltages by EIS and the following relationship between current density and voltage,

$$
d i=\frac{d V}{R_{t}}
$$

where $R_{t}$ stands for total dc resistance. The calculated curve reproduces experimentally obtained $\mathrm{I}-\mathrm{V}$ curve very well, confirming that the total $\mathrm{dc}$ resistances can be determined accurately from Nyquist plot of EIS analysis.

\subsection{Comparison of three kinds of electrodes (P-25 only, P-25 with PEG and TNW mixed with mixture of P-25 and PEG)}

Fig. 11 shows $\mathrm{I}-\mathrm{V}$ curves of the cells made of three kinds of electrodes, i.e., (a) P-25 only, (b) P-25 with PEG and (c) titania nanowire network (TNW) mixed with mixture of P-25 and PEG. The cell made of P-25 only showed the lowest power conversion efficiency (PCE) 4.02 \%. PCE of $6.86 \%$ was obtained for the cell made of P-25 with PEG. The highest PCE $8.64 \%$ was obtained for the cell made of TNW mixed with mixture of P-25 and PEG, in which the percentage of titanium atoms of TNW was $28 \%$ for the total titanium atoms, i.e., TNW + P25 . Table 1 shows the current density $J_{\mathrm{sc}}$, open circuit voltage $V_{\mathrm{oc}}$, fill factor $F F$ and power conversion efficiency $\eta$ of the three kinds of cells.

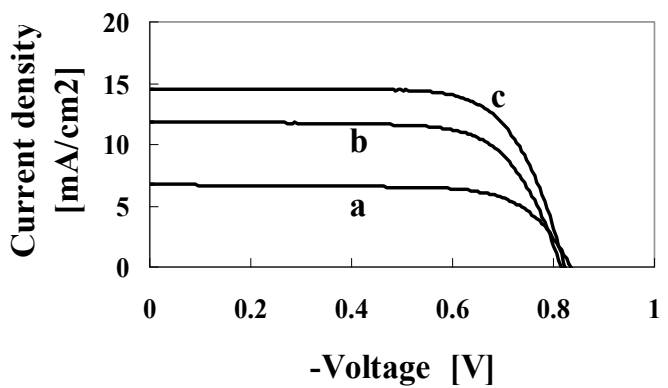

Fig. 11. I-V curves of the cells made of three kinds of electrodes, i.e., (a) P-25 only, (b) P-25 with PEG and (c) titania nanowire network (TNW) mixed with mixture of P-25 and PEG.

\begin{tabular}{ccccc}
\hline & $J_{\text {sc }}[\mathrm{mA} / \mathrm{cm} 2]$ & $V_{\text {oc }}[\mathrm{V}]$ & $F F$ & $\eta[\%]$ \\
\hline P-25 & 6.73 & 0.84 & 0.72 & 4.02 \\
P-25 + PEG & 11.38 & 0.83 & 0.73 & 6.86 \\
$\mathrm{TNW}+(\mathrm{P}-25+\mathrm{PEG})$ & 14.56 & 0.82 & 0.72 & 8.64 \\
\hline
\end{tabular}

Table 1. Current density $J_{\mathrm{sc},}$ open circuit voltage $V_{\mathrm{oc}}$, fill factor $F F$ and power conversion efficiency $\eta$ of the three kinds of cells.

The results of incident photon to current efficiency (IPCE) for the three kinds of cells are shown in Fig. 12. IPCE of the cell made of P-25 only was lowest because of the small amount of dye adsorption. The cell made of TNW with P-25 with PEG showed highest IPCE because 
of the largest amount of dye adsorption. Also IPCE in the range of $600 \mathrm{~nm}$ to $700 \mathrm{~nm}$ shows shoulder like increase because of the strong scattering of TNW.

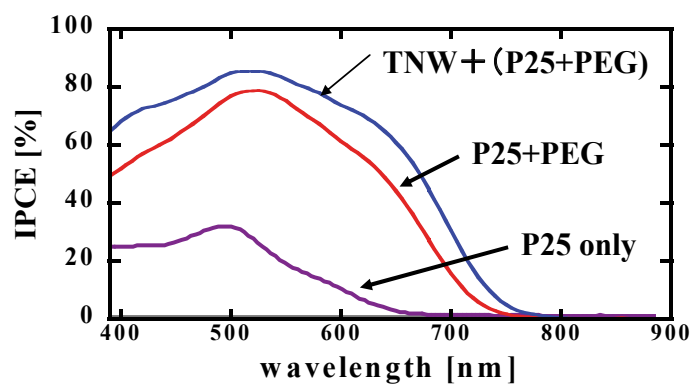

Fig. 12. Results of IPCE for three kinds of cells.

Fig. 13 shows Nyquist plots of the three kinds of cells under open circuit conditions. The total resistance of each cell was obtained as $49 \Omega$ for P-25 only cell, $32 \Omega$ for P-25+PEG cell and $27 \Omega$ for $\mathrm{TNW}+(\mathrm{P}-25+\mathrm{PEG})$ cell, respectively. Since total resistance corresponds to the slope of the tangent line at Voc, the slope of the tangent line in $\mathrm{I}-\mathrm{V}$ curves in Fig. 11 became steeper with decreasing of the total resistance of the cell. The plotted squares in Fig. 13 represent experimental results and the solid curves show the calculated spectra from equations (4) to (7) using parameters shown in Table 2 for each cell (Adachi et al. 2006).
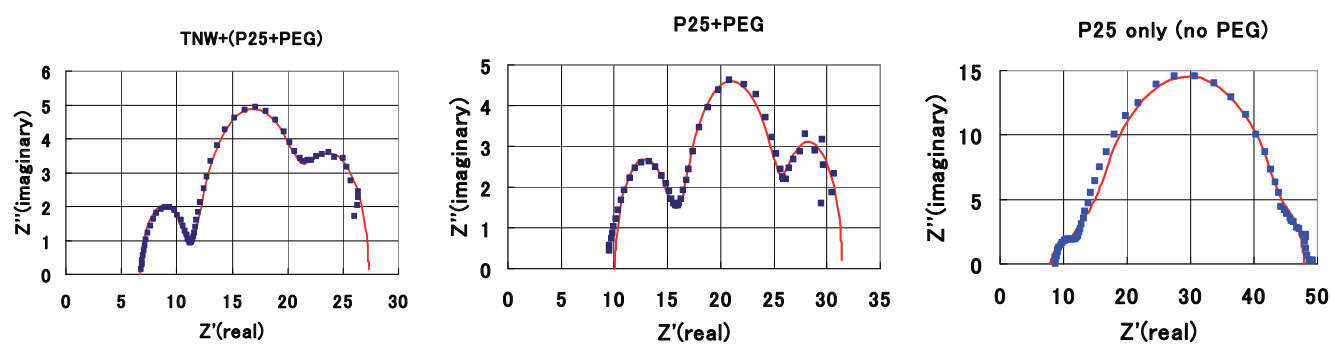

Fig. 13. Nyquist plot of three kinds of cells under open circuit conditions.

Impedance equations for electron transport processes are given as follows (Adachi et al. 2006). For the impedance concerning with titania electrode, equation (4) was derived:

$$
Z=R_{w}\left(\frac{1}{\left(\frac{\omega_{k}}{\omega_{d}}\right)\left(1+\frac{i \omega}{\omega_{k}}\right)}\right)^{1 / 2} \operatorname{coth}\left[\left(\frac{\omega_{k}}{\omega_{d}}\right)\left(1+\frac{i \omega}{\omega_{k}}\right)\right]^{1 / 2}
$$

where,

$$
\omega_{d}=\frac{D_{e f f}}{L^{2}}, \omega_{k}=k_{e f f}, R_{w}=\frac{k_{B} T}{q^{2} A n_{s}} \frac{L}{D_{e f f}}=\operatorname{Con} \frac{L}{D_{e f f}}, R_{k}=\operatorname{Con} \frac{1}{L k_{e f f}}
$$


For the impedance concerning with platinum electrode, equation (6) was assumed:

$$
Z_{P t}=\frac{1}{1+i \omega r_{p t} C_{p t}}
$$

where, $r_{\mathrm{pt}}$ and $C_{\mathrm{pt}}$ represent the resistance at the $\mathrm{Pt}$ surface and the capacitance at the $\mathrm{Pt}$ surface, respectively. For the impedance concerning with tri-iodide diffusion, finite Warburg impedance equation, i.e., equation (7) was assumed:

$$
Z_{N}=R_{I 3-} \frac{1}{\sqrt{\frac{i \omega}{\left(D_{I 3-} / \delta^{2}\right)}}} \tanh \sqrt{\frac{i \omega}{\left(D_{I 3-} / \delta^{2}\right)}}
$$

The calculated solid curves in Fig. 13 agree quite well with the plotted experimental data. The characteristics shown in Table 2 are following three points which show strong tendency for the highly efficient cells. 1) The resistance for the electron transport from the titania electrode to the conducting glass electrode $R \mathrm{w}$ becomes smaller with increasing conversion efficiency. 2) The ratio of the resistance for the recombination reactions against the resistance for the transport rate to the conducting glass electrode $(R \mathrm{k} / R \mathrm{w})$ becomes large, and the rate constant of recombination reactions $k_{\text {eff }}$ becomes smaller with increasing conversion efficiency. 3) The values of Con, which represents constant inversely proportional to the

$\begin{array}{lrrr} & \text { P25 only } & \text { P25+PEG } & \text { TNW+(P25+PEG) } \\ \text { Con }= & 2.65 & 0.28 & 0.163 \\ D_{\text {eff }}= & 0.0006 & 0.00014 & 0.00008 \\ L= & 0.0042 & 0.0025 & 0.002 \\ k_{\text {eff }}= & 23 & 13.8 & 10 \\ R_{\mathrm{l3}-}= & 5.1 & 7 & 7.5 \\ D_{13-}= & 0.000015 & 0.000003 & 0.000005 \\ \delta= & 0.005 & 0.005 & 0.005 \\ R_{\mathrm{Pt}}= & 1.5 & 4.7 & 3.65 \\ C_{\mathrm{Pt}}= & 0.00005 & 0.00007 & 0.00005 \\ R_{\text {sub }}= & 7.9 & 10 & 6.7 \\ R \mathrm{k} / R \mathrm{Ww}= & 1.48 & 1.62 & 2 \\ R \mathrm{~kW}= & 14.8 & 5 & 4.08 \\ R \mathrm{k}= & 27.4 & 8.11 & 8.15 \\ n= & 2.42 \times 10^{17} & 2.29 \times 10^{18} & 3.94 \times 10^{18}\end{array}$

Where, Con $k_{\mathrm{B}} T / q A n\left[\Omega \mathrm{cms}^{-1}\right]$, where $k_{\mathrm{B}}\left[\mathrm{JK}^{-1}\right]$ represents Boltzmann constant, $T[\mathrm{~K}]$ is absolute temperature, $q[\mathrm{C}]$ is elementary charge, $A\left[\mathrm{~cm}^{2}\right]$ is area of the cell and $n\left[\mathrm{~cm}^{-3}\right]$ is electron density. Con: constant inversely proportional to the electron density, $D_{\text {eff }}\left[\mathrm{cm}^{2} \mathrm{~s}^{-1}\right]$ : diffusion coefficient of electron, $L[\mathrm{~cm}]$ : film thickness of $\mathrm{TiO}_{2}$ electrode, $k_{\text {eff }}\left[\mathrm{s}^{-1}\right]$ : reaction rat constant of recombination reactions, $R_{\mathrm{I} 3-}[\Omega]$ : diffusion resistance of $\mathrm{I}_{3}{ }^{-}, D_{\mathrm{I} 3-}\left[\mathrm{cm}^{2} \mathrm{~s}^{-1}\right]$ : diffusion coefficient of $\mathrm{I}_{3}{ }^{-}, \delta[\mathrm{cm}]$ : thickness of the electrolyte phase, $R_{\mathrm{Pt}}[\Omega]$ : resistance of Pt electrode, $C_{\mathrm{Pt}}[\mathrm{F}]$ : capacity of Pt electrode, $R_{\text {sub }}[\Omega]$ : resistance of substrate, $R \mathrm{w}[\Omega]$ : resistance for electron transport in the $\mathrm{TiO}_{2}$ electrode, $R \mathrm{k}[\Omega]$ : resistance for recombination reaction.

Table 2. Determined parameters concerning with electron transport by impedance spectroscopy for three kinds of cells 


\begin{tabular}{cccccc}
\hline & $\begin{array}{c}J \mathrm{sc} \\
{\left[\mathrm{mA} / \mathrm{cm}^{2}\right]}\end{array}$ & $\begin{array}{c}V \text { oc } \\
{[\mathrm{V}]}\end{array}$ & $F F$ & $\begin{array}{c}\eta \\
{[\%]}\end{array}$ & $\begin{array}{c}\text { thickness } \\
{[\mu \mathrm{m}]}\end{array}$ \\
\hline $0 \mathrm{wt} \%$ & 10.93 & 0.85 & 0.74 & 6.85 & 10 \\
$0 \mathrm{wt} \%$ & 11.82 & 0.82 & 0.71 & 6.87 & 26 \\
$0 \mathrm{wt} \%$ & 10.92 & 0.80 & 0.71 & 6.20 & 35 \\
$5 \mathrm{wt} \%$ & 11.98 & 0.85 & 0.73 & 7.48 & 19 \\
$5 \mathrm{wt} \%$ & 10.96 & 0.81 & 0.73 & 6.50 & 32 \\
$10 \mathrm{wt} \%$ & 12.84 & 0.84 & 0.73 & 7.87 & 18 \\
$10 \mathrm{wt} \%$ & 12.68 & 0.84 & 0.72 & 7.64 & 20 \\
$28 \mathrm{wt} \%$ & 14.88 & 0.82 & 0.71 & 8.66 & 24 \\
$28 \mathrm{wt} \%$ & 13.09 & 0.83 & 0.74 & 8.04 & 27 \\
$50 \mathrm{wt} \%$ & 13.39 & 0.85 & 0.71 & 8.02 & 14 \\
$50 \mathrm{wt} \%$ & 15.18 & 0.80 & 0.70 & 8.51 & 22 \\
$100 \mathrm{wt} \%$ & 11.94 & 0.84 & 0.73 & 7.28 & 5 \\
$100 \mathrm{wt} \%$ & 9.93 & 0.82 & 0.71 & 5.75 & 9 \\
$100 \mathrm{wt} \%$ & 10.16 & 0.84 & 0.70 & 5.97 & 11 \\
\hline
\end{tabular}

Table 3. Performance of dye sensitized solar cells with various TNW content.

electron density, becomes smaller with increasing conversion efficiency, i.e., Con value increase in the order of P-25 only $>(\mathrm{P}-25+\mathrm{PEG})>\mathrm{TNW}+(\mathrm{P}-25+\mathrm{PEG})$. Therefore the electron density $n$ increases with increasing conversion efficiency. So, the characteristics of highly efficient cells are high electron density, small resistance for the electron transport to the conducting glass electrode, and large ratio of the resistances $R \mathrm{k} / R \mathrm{w}$ with small rate constant for recombination reactions.

\subsection{Effects of content of TNW on the properties of dye-sensitized solar cells}

Since the cells containing TNW gave high conversion efficiencies, we examined the effects of content of TNW in the electrode composed of TNW and P-25 upon the conversion efficiency with variation in TNW from $0 \%$ to $100 \%$. Content of TNW was defined as percentage of titanium atoms of TNW in the total titanium atoms included in the titania electrode. Table 3 shows performance of DSSCs with various TNW content, i.e., $J_{\mathrm{sc}}, V_{\mathrm{oc}}, F F$ and $\eta$, together with film thickness. Effect of TNW content on PCE is shown in Fig. 14.

PCE of the cells including TNW are higher than those cells without TNW, indicating that TNW is useful to attain high efficiency except $100 \%$ TNW case. When the film thickness of $100 \%$ TNW cells increased larger than $5 \mu \mathrm{m}$, peel off of the films with cracks was observed by SEM images as shown in Fig. 15, resulting that less than 6\% of PCE were observed as shown in Table 3 and Fig. 14. Thus, mixing of TNW with P-25 nanoparticles is important to make robust films.

Since the amount of adsorbed dyes is another important factor to affect PCE, the amounts of adsorbed dyes for the cells with various TNW contents are shown against film thickness in Fig. 16. The amount of adsorbed dye in the cells containing TNW from $0 \%$ to $50 \%$ locates in the same straight line regardless of the difference in TNW contents, except $100 \%$ TNW which shows higher adsorbed amounts. 


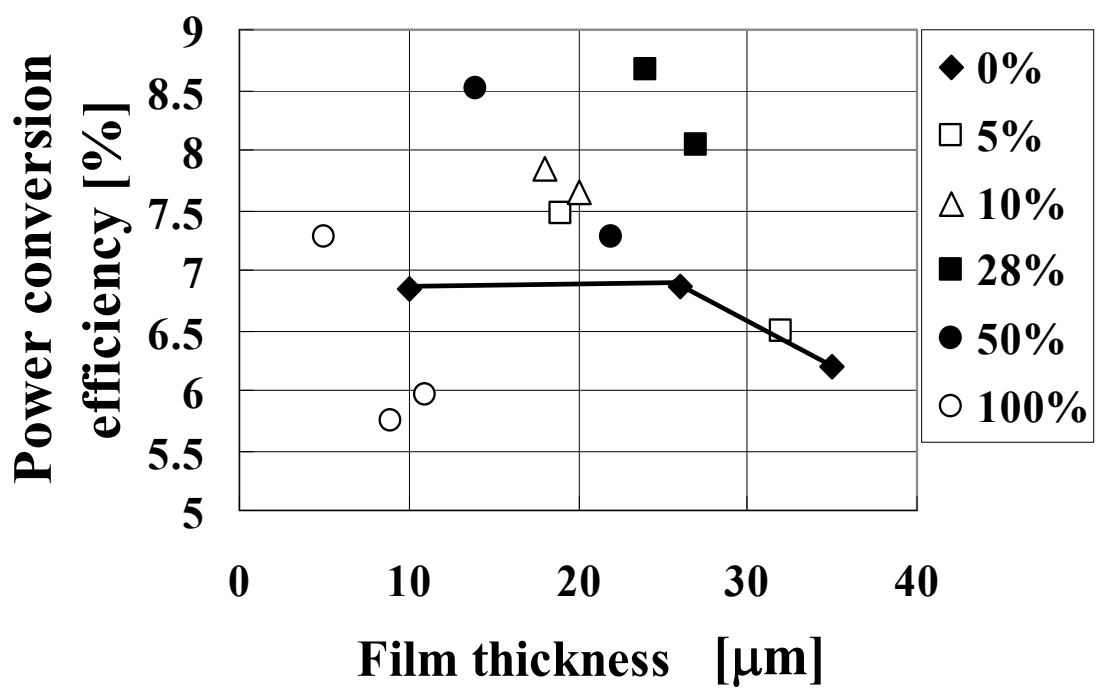

Fig. 14. Effect of content of TNW on power conversion efficiency
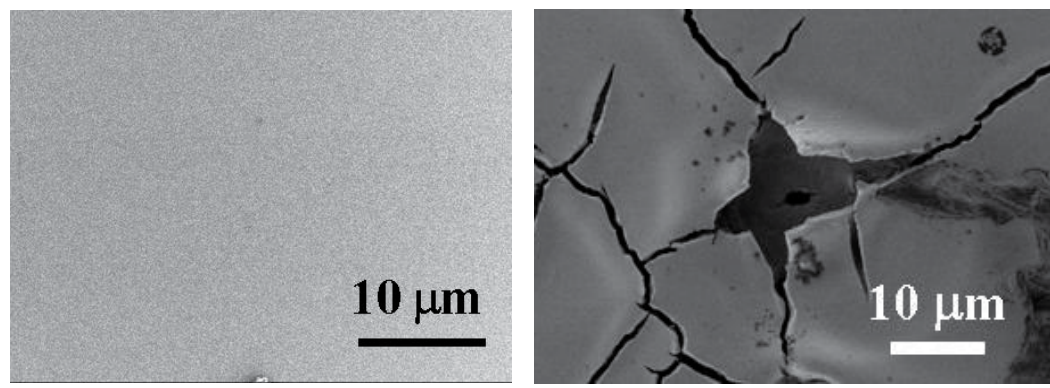

Fig. 15. Top views of $100 \%$ TNW films. Left: $5 \mu \mathrm{m}$, right: $11 \mu \mathrm{m}$ thickness.

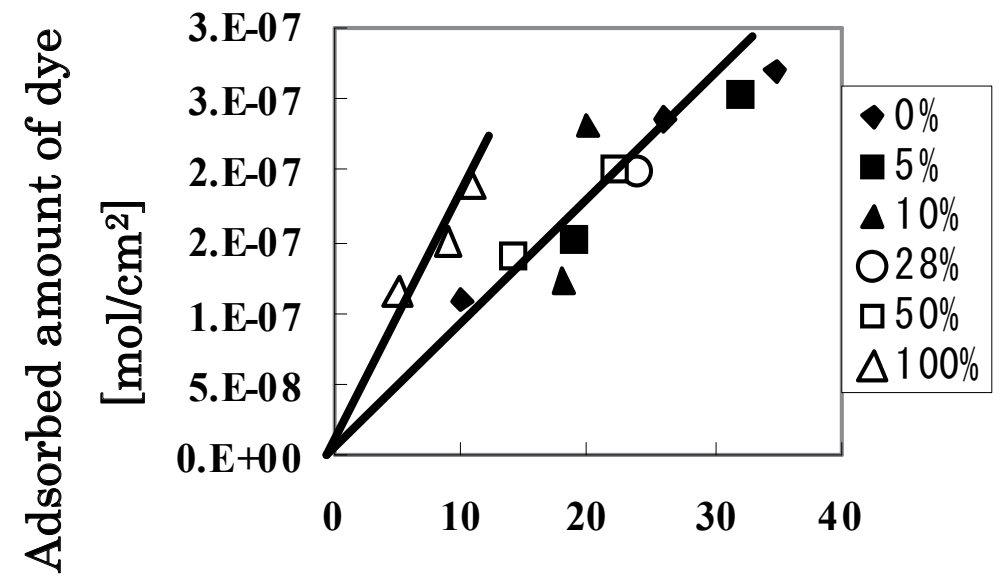

\section{Film thickness $[\mu \mathrm{m}]$}

Fig. 16. Relationship between film thickness and the amount of dye. 


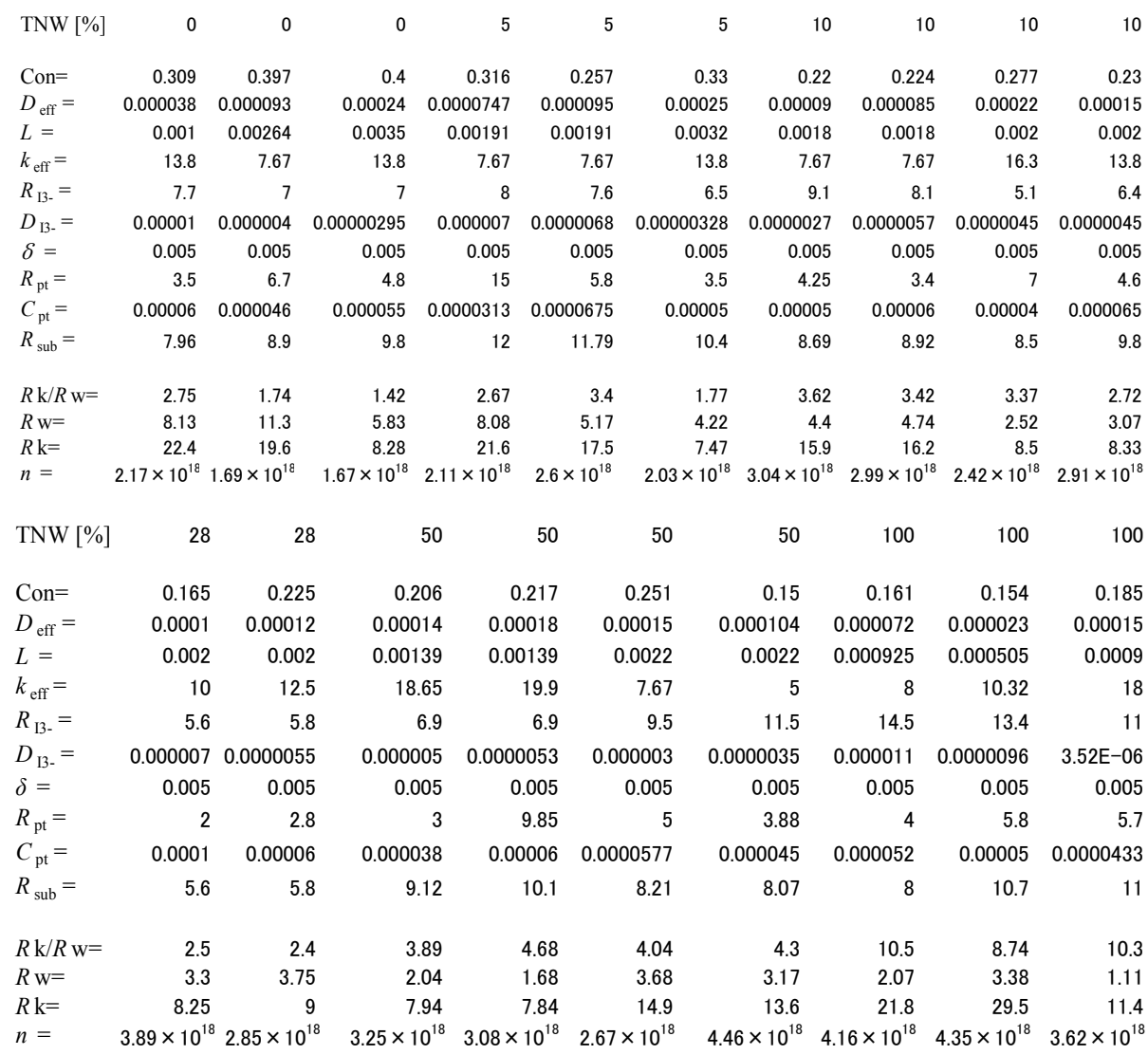

Table 4. Parameters determined by EIS analysis for the cells with various TNW contents.

This higher adsorption of $100 \%$ TNW is attributed to the smaller diameter of TNW of 3-7 $\mathrm{nm}$, which is much smaller than the diameter of P-25 of $23 \mathrm{~nm}$. The specific surface area of P-25 and the mixture of $28 \%$ TNW with P-25 after calcinations at $773 \mathrm{~K}$ for $30 \mathrm{~min}$. were 45 $\mathrm{m}^{2} / \mathrm{g}$ and $48 \mathrm{~m}^{2} / \mathrm{g}$, respectively. These values of specific surface area are much smaller than that of $100 \% \mathrm{TNW}$ which is $78 \mathrm{~m}^{2} / \mathrm{g}$ after calcinations. This difference in specific surface area between $28 \%$ TNW with P-25 and pure $100 \%$ TNW corresponds well to the difference in adsorbed dye amount between from $0 \%$ to $50 \%$ TNW with P-25 and pure $100 \%$ TNW. These findings suggest some interesting structural change in the surface of the mixture of TNW and P-25. However, the reason why the cells containing different TNW content from $0 \%$ to $50 \%$ locates in the same straight line in Fig. 16 is not well understood at present.

Resistance for electron transport from titania electrode to the transparent conducting glass electrode Rw are plotted against TNW content in Fig. 17a. Rw values decrease steeply up to $10 \%$ and become gradual decrease after $20 \%$ of TNW content. This decrease indicates clearly that electron transport in the titania electrode is improved by mixing TNW with P-25 nanoparticles.

The ratios of $R \mathrm{k}$ representing the resistance for the recombination reactions between electrons in the titania electrode and $\mathrm{I}_{3}$ - in the electrolyte to $\mathrm{RW}$ are plotted against TNW 
content in Fig. 17b. The ratio of $R \mathrm{k} / \mathrm{Rw}$ increases with increase in TNW content. This shows that TNW restrains the recombination reactions between electrons and $\mathrm{I}_{3}{ }^{-}$and contributes to collect electrons properly to the transparent conducting glass electrode. The findings shown in Fig. 17 a, b bring the high electron density in the titania electrode as shown in Fig. 17c.
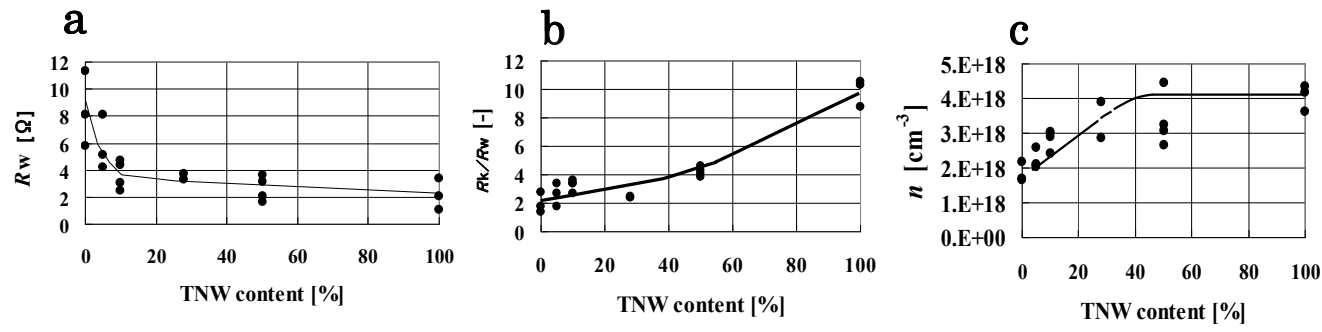

Fig. 17. a) Relationship between $R \mathrm{w}$ and TNW content, $\mathrm{b}$ ) relationship between $R \mathrm{k} / R \mathrm{w}$ and TNW cointent and c) relationship between electron density and TNW content.

Thus, the conclusion deduced from the experiments of three kinds of cells, i.e., small resistance for the electron transport to the conducting glass electrode, large value of resistance ratio $R \mathrm{k} / \mathrm{Rw}$, and high electron density in the titania electrode as the characteristics of highly efficient cells, was confirmed again by the experiments of variation in TNW content.

\subsection{Some examples of our highly crystallized one-dimensional $\mathrm{TiO}_{2}$ nanoscale materials for fabricating highly efficient dye-sensitized solar cells}

We succeeded in the preparation of titania nanorods (TNR) (Jiu et al. 2006), network structure of titania nanowires (Adachi et al. 2004) and one-dimensional titania nanochains (see Fig. 18 ), which have been newly synthesized. We applied these materials for DSSCs.

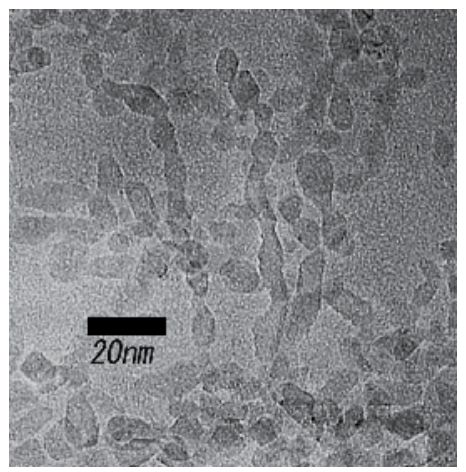

Fig. 18. TEM image of titania nanochains.

We present highly crystallized one-dimensional titania nanoscale materials are effective to attain high light-to-electricity conversion yield. As shown in our previous paper (Adachi et al. 2004), network structure of single crystal-like titania nanowires can be synthesized successfully by the oriented attachment mechanism. We attained $9.33 \%$ conversion 
efficiency with complex titania electrode made of titania nanowires and P-25. Recently, we attained the same conversion efficiency $9.33 \%$ using different electrolyte, i.e., 0.6M 1-butyl 3-methyl imidazolium iodide, $0.1 \mathrm{M}$ guanidium thiocyanate, $0.05 \mathrm{M} \mathrm{I}, 0.5 \mathrm{M}$ tertbutylpyridine in a mixture of acetonitrile and valeronitrile $(85: 15)$ for a complex titania electrode made of titania nanowires, titania nanoparticles (3-5 nm in diameter) and P-25. (Fig. 19)

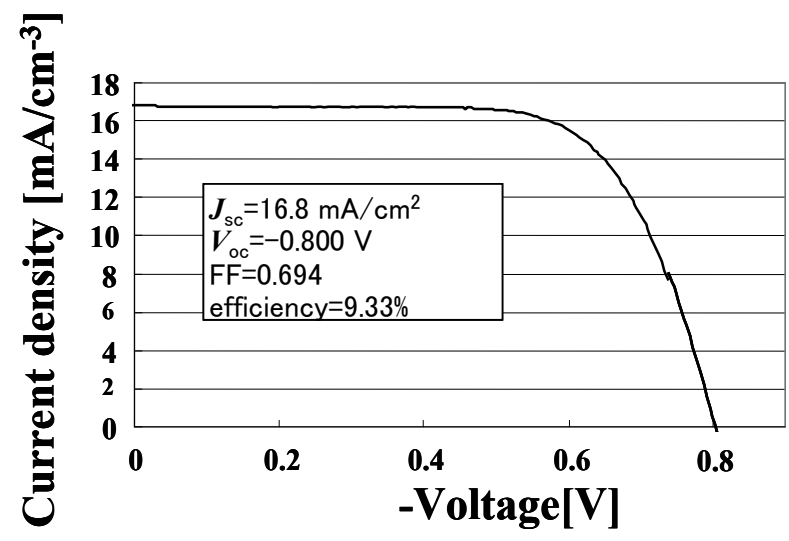

Fig. 19. I-V curve obtained for a cell with a complex electrode composed of network structure of single-crystal-like titania nanowires, titania nanoparticles and P-25

In our previous paper (Adachi et al. 2004), we used an electrolyte composed of $0.1 \mathrm{M}$ of LiI,

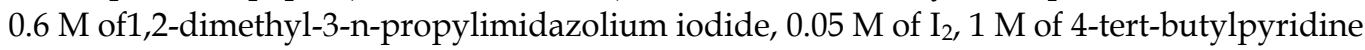
in methoxyacetonitrile and got $9.33 \%$ conversion efficiency with short circuit current density $J \mathrm{sc}=19.2 \mathrm{~mA} / \mathrm{cm}^{2}$, open circuit voltage $V o c=0.72 \mathrm{~V}$ and fill factor 0.675 . In the recent results, $\mathrm{Voc}$ value $0.8 \mathrm{~V}$ is larger than that of previous one $0.72 \mathrm{~V}$, because guanidium thiocyanate decreased redox potential of $\mathrm{I}^{-} / \mathrm{I}_{3}$ - in the electrolyte. Unfortunately, we got lower short circuit current density $J \mathrm{sc}=16.8 \mathrm{~mA} / \mathrm{cm}^{2}$ than that of our previous one, and the same efficiency was obtained.

Highly crystallized titania nanorods (TNR) have been synthesized by hydrothermal process using blockcopolymer (F127) and surfactant cetyltrimethylammonium bromide (CTAB) as a mixed template (Jiu et al. 2006). TNR with 100-300 nm in length and 20-30 $\mathrm{nm}$ in diameter was obtained. A high-resolution TEM (HRTEM) image of single TNR shows that titanium atoms align perfectly in titania anatase crystalline structure with no lattice defect, and the surface of TNR is facetted with the $\mathrm{TiO}_{2}$ anatase $\{101\}$ faces (Yoshida et al. 2008). The fringes are $\{101\}$ planes of anatase $\mathrm{TiO}_{2}$ with a lattice spacing of about $0.351 \mathrm{~nm}$, which agrees with the value recorded in JCPDS card. The highly crystallized titania nanorods prepared successfully were used to fabricate a titania electrode of DSSCs. The complex electrodes were made by the repetitive coating-calcining process: 3 layers of titania nanoparticles (3-5 $\mathrm{nm}$ in diameter) were first coated on FTO conducting glass, followed by 8 layers of mixed gel composed of titania nanorods and titania nanoparticles. A high light-to-electricity conversion efficiency of $8.93 \%$ was achieved (Yoshida et al. 2008). 


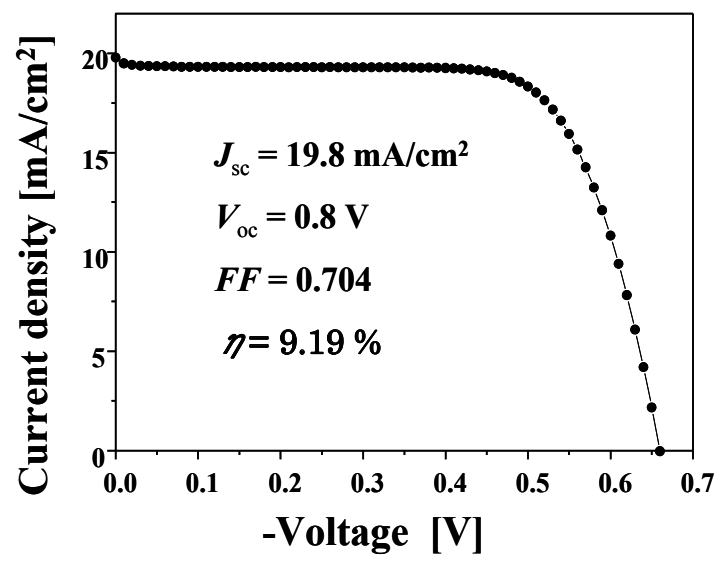

Fig. 20. I-V curve obtained for the cell composed of one-dimensional chains of titania nanoparticles mixed with fine titania nanoparticles (3 - $5 \mathrm{~nm}$ in diameter).

We have newly synthesized titania nanochains as shown in Fig. 18. Highly crystallized titania nanoparticles with diameter of around $10 \mathrm{~nm}$ combine with each other and make chains. The obtained white solid product was mixed with spherical titania nanoparticles (3-5 $\mathrm{nm}$ in diameter) synthesized using F127 reported in our previous paper (Jiu et al. 2004, Jiu et al. 2007) to fabricate titania film electrodes. The I-V curve of the cell is shown in Fig. 20. The obtained light-to-electricity conversion yield of the cell was 9.2\%.

All three kinds of one-dimensional titania nanoscale materials mentioned above show high light-to-electricity conversion yield around 9\%, suggesting strongly that highly crystallized one-dimensional titania materials are essentially important for attainment of high efficient dye-sensitized solar cells.

\subsection{Conclusions of 3rd section}

1. Many researchers familiar with EIS measurement know that highly efficient dyesensitized solar cells show small total resistance of the cell, i.e., small Nyquist spectrum. They also know that largest arc of Nyquist plot represents the resistance for recombination reactions $R \mathrm{k}$. This apparent conflict is solved clearly by theoretical consideration through recognition that the large value of the ratio $R \mathrm{k} / \mathrm{Rw}$ is essentially important for the highly efficient cells, and the absolute value of $R \mathrm{k}$ is not important, i.e., very small $R \mathrm{w}$ is indispensable for the highly efficient cells.

2. The experimental results of $\mathrm{I}-\mathrm{V}$ and EIS measurements of the three kinds of cells made of P-25 only, P-25+PEG, and TNW+P-25+PEG and also cells made of various content of TNW with P-25+PEG clearly showed the following three points as characteristics of highly crystallized 1-dimensional titania nanoscale material TNW. 1) Resistance of electron transport in the titania electrode $R \mathrm{w}$ is small. 2) The ratio of resistance $R \mathrm{k} / R \mathrm{w}$ is large. 3) Electron density $n$ in the titania electrode is high.

3. All cells composed of three kinds of highly crystallized 1-dimensional titania nanoscale materials, i. e., network structure of titania nanowires, titania nanorods, and titania nanochains, show high power conversion efficiency about $9 \%$. 


\section{References}

Adachi, M. Murata, Y. Takao, J. Jiu, J. Sakamoto, M. \& Wang, F. (2004). Highly Efficient DyeSensitized Solar Cells with Titania Thin Film Electrode Composed of Network Structure of Single-Crystal-Like $\mathrm{TiO}_{2}$ Nanowires Made by "Oriented Attachment" Mechanism. J. Am. Chem. Soc., 126, pp. 14943-14949

Adachi, M. Sakamoto, M. Jiu, J. Ogata, Y. Isoda, S. (2006). Determination of Parameters of Electron Transport in Dye-Sensitized Solar Cells Using Electrochemical Impedance Spectroscopy: J. Phys. Chem. B 110, pp. 13872-13880

Aneggi, E.; de Leitenburg, C. Dolcetti, G \& Trovarelli, A. (2006) Promotional effect of rare earths and transition metals in the combustion of diesel soot over $\mathrm{CeO}_{2}$ and $\mathrm{CeO}_{2}-$ $\mathrm{ZrO}_{2}$. Catalysis Today. 114, pp. 40-47.

Bekyarova, E.; Fornasiero, P.; Kaspar, J. \& Graziani, M. (1998) CO oxidation on Pd/ $\mathrm{CeO}_{2-}$ $\mathrm{ZrO}_{2}$ catalysts. Catalysis Today. 45, pp. 179-183.

Bumajdad, A.; Zaki, M. I.; Eastoe, J. \& Pasupulety, L. (2004) Microemulsion-Based Synthesis of $\mathrm{CeO}_{2}$ Powders with High Surface Area and High-Temperature Stabilities. Langmuir. 20, pp. 11223-11233.

Chen, C-Y., Wang, M., Li, J-Y., Pootrakulchote, N., Alibabael, L., Ngoc-le, C-H., Decoppetr, J-D., Tsai, J-H., Grätzel, C., Wu, C-G., Zakeeruddin, M., \& Grätzel, M. (2009) Highly efficient light-harvesting ruthenium sensitizer for thin-film dye-sensitized solar cells. ACS NANO, 3, pp. 3103-3109

Chen, D., Huang, F. Cheng, Y-B. \& Caruso, R. A. (2009) Mesoporous anatase $\mathrm{TiO}_{2}$ beads with high surface areas and controllable pore sizes: A superior candidate for highperformance dye-sensitized solar cells. Adv. Mater., 21, pp. 2206-2210

Chiang, Y. M.; Lavik, E. B., Kosacki, I.; Tuller, H. L. \& Ying, J. Y. (1996) Defect and transport properties of nanocrystalline $\mathrm{CeO}_{2-x}$. Appl. Phys. Lett. 69, pp. 185-187.

Colodrero, S., Mihi, A., Häggman, L. Ocana, M. Boschloo, G. Hagfeldt, A., \& Miguez, H. (2009) Porous one-dimensional photonic crystals improve the power-conversion efficiency of dye-sensitized solar cells. Adv. Mater., 21, pp. 764-770

Colvin, V. L., Schlamp, M. C., \& Alivisatos, A. P. (1994) Light-emitting diodes made from cadmium selenide nanocrystals and a semiconducting polymer Nature, 370, pp. 354-357.

De Faria, L. A. \& Trasatti, S. (1994) The Point of Zero Charge of $\mathrm{CeO}_{2}$. J. Colloid Interface Sci., 167, pp. 352-357.

Fuhrer, M. S., Nygard, J., Shih, L., Forero, M., Yoon, Y. -G., Mazzoni, M. S. C., Choi, H. J., Ihm, J., Louie, S. G., Zettl, A., McEuen, P. L. (2000) Crossed nanotube junctions Science, 288, 494-497.

Grinis, L., Dor, S., Ofir, A., \& Zaban, A. (2008) Electrophoretic deposition and compression of titania nanoparticle films for dye-sensitized solar cells. J. Photochem. Photobio. A: Chemistry, 198, pp. 52-59

Hamann, T. W., Farha, O. K. \& Hupp, J. T. (2008) Atomic layer deposition of $\mathrm{TiO}_{2}$ on aerogel templates: New photoanodes for dye-sensitized solar cells. J. Phys. Chem. C, 112, pp. 19756-19764

Han, W-Q.; Wu, L. \& Zhu, Y. (2005) Formation and Oxidation State of $\mathrm{CeO}_{2-x}$ Nanotubes. J. Am. Chem. Soc. 127, pp. 12814-12815. 
Hirano, M.; Fukuda, Y.; Iwata, H.; Hotta, Y. \& Inagaki, M. (2000) Preparation and Spherical Agglomeration of Crystalline Cerium(IV) Oxide Nanoparticles by Thermal Hydrolysis. J. Am. Ceram. Soc. 83, pp. 1287-1289.

Ho, C.; Yu, J. C.; Kwong, T.; Mak, A. C. \& Lai, S. (2005) Morphology-Controllable Synthesis of Mesoporous CeO2 Nano- and Microstructures. Chem. Mater. 17, pp. 4514-4522.

$\mathrm{Hu}$, S.; Willey, R. J. \& Notari, B. (2003) An investigation on the catalytic properties of titaniasilica materials. J. Catal., 220, pp. 240-248.

Inaba, H. \& Tagawa, H. (1996) Ceria-based solid electrolytes. Solid State Ionics., 83, pp. 1-16.

Ito, S., Murakami, T. N., Comte, P., Liska, P., Grätzel, C., Nazeerudin, M. K. \& Grätzel, M. (2008) Fabrication of thin film dye sensitized solar cells with solar to electric power conversion efficiency over 10\%. Thin Solid Film, 516, pp. 4613-4619

Jiu, J. Isoda, S. Adachi, M. \& Wang, F. (2007). Preparation of TiO2 nanocrystalline with 3-5 $\mathrm{nm}$ and application for dye-sensitized solar cell. J. Photochem. Photobio. A: Cemistry, 189, pp. 314-321

Jiu, J. Isoda, S. Wang, F. \& Adachi, M. (2006). Dye-Sensitized Solar Cells Based on a SingleCrystalline TiO2 Nanorod Film. J. Phys. Chem. B, 110, pp. 2087-2092

Jiu, J. Wang, F., Sakamoto, M., Takao, J. \& Adachi, M. (2004). Preparation of nanocrystal $\mathrm{TiO}_{2}$ with mixed template and application for dye-sensitized solar cell. J. Electrochem. Soc. 151, pp. A1653-A1658

Kang, T-S., Smith, A. P., Taylor, B. E. \& Durstock, M. F. (2009) Fabrication of highly-ordered $\mathrm{TiO}_{2}$ nanotube arrays and their use in dye-sensitized solar cells. Nano Letter, 9, pp. 601-606

Kang, Z. C. \& Eyring, L. (1997) A compositional and structural rationalization of the higher oxides of Ce, Pr, and Tb. J. Alloys and Comp. 249, pp. 206-212.

Kar, A., Smith, Y.R., \& Subramanian, V. (2009) Improved photocatalytic degradation of textile dye using titanium dioxide nanotubes formed over titanium wires. Environmental Sci. and Technol., 43, pp. 3260-3265

Kim, Y. J., Lee, M. H., Kim, H. J., Lim, G., Choi, Y. S., Park, N-G., Kim, K., \& Lee, W. I. (2009) Formation of highly efficient dye-sensitized solar cells by hierarchical pore generation with nanoporous $\mathrm{TiO}_{2}$ spheres. Adv. Mater., 21, pp. 3668-3673

Kuang, D., Brillet, J., Chen, P., Takata, M. Uchida, S. Miura, H., Sumioka, K., Zakeeruddin, S. M. \& Grätzel, M. (2008) Application of highly ordered $\mathrm{TiO}_{2}$ nanotube arrays in flexible dye-sensitized solar cells. ACS Nano, 2, pp. 1113-1116

Kuiry, S.; Patil, S.; Deshpande, S. \& Seal, S. (2005) Spontaneous Self-Assembly of Cerium Oxide Nanoparticles to Nanorods through Supraaggregate Formation. J. Phys. Chem. B, 109, pp. 6936-6939.

Li, J. -G.; Ikegami, T.; Lee, J. -H. \& Mori, T. (2001) Characterization and sintering of nanocrystalline $\mathrm{CeO}_{2}$ powders synthesized by a mimic alkoxide method. Acta. Mater. 49, pp. 419-426.

Livage, J.; Henry, M. \& Sanchez, C. (1988) Sol-gel chemistry of transition metal oxides. Prog. Solid St. Chem., 18, pp. 259-341.

Masui, T.; Fujiwara, K.; Machida, K.; Adachi, G.; Sakata, T. \& Mori, H. (1997) Characterization of Cerium(IV) Oxide Ultrafine Particles Prepared Using Reversed Micelles. Chem. Mater., 9 pp. 2197-2204.

Masui, T.; Yamamoto, M.; Sakata, T.; Mori, H. \& Adachi, G. (2000) Synthesis of BN-coated $\mathrm{CeO}_{2}$ fine powder as a new UV blocking material. J. Mater. Chem., 10, pp. 353-357. 
Masui, T.; Hirai, H.; Imanaka, N. \& Adachi, G. (2002) Synthesis of cerium oxide nanoparticles by hydrothermal crystallization with citric acid. J. Mater. Sci. Lett. 21, pp. 489-491.

Masui, T.; Fukuhara, K.; Imanaka, N.; Sakata, T.; Mori, H. \& Adachi, G. (2002) Effects of Titanium Oxide on the Optical Properties of Cerium Oxide. Chem. Lett. 31, pp. 474745.

Méndez-Román, R. \& Cardona-Martínez, N. (1998) Relationship between the formation of surface species and catalyst deactivation during the gas-phase photocatalytic oxidation of toluene. Catal. Today., 40, pp. 353-365.

Miyashita, M., Sunahara, K., Nishikawa, T. Uemura, Y., Koumura, N., Hara, K., Mori, A., Abe, T. Suzuki, E. \& Mori, S. (2008) Interfacial electron-transfer kinetics in metalfree organic dye-sensitized solar cells: Combined effects of molecular structure of dyes and electrolytes. J. Am. Chem. Soc., 130, pp. 17874-17881

Morris, C. A., Anderson, M. L., Stroud, R. M., Merzbacher, C. I., \& Rolison, D. R., (1999) Silica sol as a nanoglue: Flexible synthesis of composite aerogels Science, 284, 622624.

Murata, Y. \& Adachi, M. (2004) Formation of highly dispersed cerium oxide with cubic structure prepared alkoxide-surfactant system. J. Mater. Sci., 39, 7397-7399.

Nakagawa, K.; Wang, F.; Murata, Y. \& Adachi, M. (2005) Effect of Acetylacetone on Morphology and Crystalline Structure of Nanostructured $\mathrm{TiO}_{2}$ in Titanium Alkoxide Aqueous Solution System. Chem. Lett. 34, pp. 736-737.

Nakagawa, K.; Murata, Y.; Kishida, M.; Adachi, M.; Hiro, M. \& Susa, K. (2007) Formation and reaction activity of $\mathrm{CeO}_{2}$ nanoparticles of cubic structure and various shaped $\mathrm{CeO}_{2}-\mathrm{TiO}_{2}$ composite nanostructures. Mater. Chem. Phys., 104, pp. 30-39.

Nazeeruddin, M. K., Kay, A., Rodicio, I., Humphry, B. R., Mueller, E., Liska, P., Vlachopoulous, N., \& Grätzel, M. (1993) Conversion of light to electricity by cis$\mathrm{X}_{2}$ bis(2,2'-bipyridyl-4,4'-dicarboxylate)ruthenium(II) charge-transfer sensitizers (X $=\mathrm{Cl}^{-}, \mathrm{Br}^{-}, \mathrm{I}-, \mathrm{CN}^{-}$, and $\mathrm{SCN}-$ ) on nanocrystalline $\mathrm{TiO}_{2}$ electrodes. J. Am. Chem. Soc., 115, PP. 6382-6390

O'Regan, B \& Grätzel, M. (1991) A low-cost, high-efficiency solar cell based on dyesensitized colloidal $\mathrm{TiO}_{2}$ films. Nature, 353, pp. 737-740

Pisarello, M. L.; Milt, V.; Peralta, M. A.; Querini, C.A. \& Miró. E. E. (2002) Simultaneous removal of soot and nitrogen oxides from diesel engine exhausts. Catalysis Today. 75, pp. 456-470.

Reddy, B. M.; Khan, A.; Yamada, Y.; Kobayashi, T.; Loridant, S. \& Volta, J-C. (2003) Structural Characterization of $\mathrm{CeO}_{2}-\mathrm{TiO}_{2}$ and $\mathrm{V}_{2} \mathrm{O}_{5} / \mathrm{CeO}_{2}-\mathrm{TiO}_{2}$ Catalysts by Raman and XPS Techniques. J. Phys. Chem. B, 107, pp. 5162-5167.

Reddy, B. M.; Khan, A.; Lakshmanan, P.; Aouine, M.; Loridant, S. \& Volta, J-C. (2005) Structural Characterization of Nanosized $\mathrm{CeO}_{2}-\mathrm{SiO}_{2}, \mathrm{CeO}_{2}-\mathrm{TiO}_{2}$, and $\mathrm{CeO}_{2}-\mathrm{ZrO}_{2}$ Catalysts by XRD, Raman, and HREM Techniques. J. Phys. Chem. B, 109, pp. 33553363.

Rynkowski, J.; Farbotko, J.; Touroude, R. \& Hilaire, L. (2000) Redox behaviour of ceriatitania mixed oxides. Appl. Catal. A, 203, pp.335-348.

Shankar, K., Basham, J. I., Allan, N. K., Varghese, O. K., Mor, G. K., Feng, X., Paulose, M. J., Seabold, A., Choi, K-S., \& Grimes, C. A. (2009) Recent advances In the use of $\mathrm{TiO}_{2}$ 
nanotube and nanowire arrays for oxidative photoelectrochemistry. J. Phys. Chem. C, 113, pp. 6327-6359

Shankar, K., Bandara, J., Paulose, M., Wietasch, H., Varghese, O. K., Mor, G. K., LaTempa, T. J., Thelakkat, M., \& Grimes, C. A. (2008) Vertically aligned single crystal $\mathrm{TiO}^{2}$ nanowire arrays grown directly on transparent conducting oxide coated glass: Synthesis details and applications. Nano Letter, 8, pp. 1654-1659

Sugimoto, T.; Zhou, X. \& Muramatsu, A. (2003) Synthesis of uniform anatase $\mathrm{TiO}_{2}$ nanoparticles by gel-sol method: 4. Shape control. J. Colloid Interface Sci., 259, pp. 53-61.

Sun, C.; Sun, Jie.; Xiao, G.; Zhang, H.; Qiu, X.; Li, H. \& Chen, L. (2006) Mesoscale Organization of Nearly Monodisperse Flowerlike Ceria Microspheres. J. Phys. Chem. B, 110, pp. 13445-13452.

Vantomme, A.; Yuan, Z-Y.; Du, G. \& Su, B-L. (2005) Surfactant-Assisted Large-Scale Preparation of Crystalline $\mathrm{CeO}_{2}$ Nanorods. Langmuir. 21, pp. 1132-1135.

Wang, D., Liu, Y., Yu, B., Zhou, F., \& Liu, W. (2009) $\mathrm{TiO}_{2}$ nanotubes with tunable morphology, diameter, and length: Synthesis and photo-electrical/catalytic performance. Chem. Mater., 21, pp. 1198-1206

Wang, M., Chen, P., Humphry-Baker, R., Zakeeruddin, S. M., \& Grätzel, M. (2009) The influence of charge transport and recombination on the performance of dyesensitized solar cells. ChemPhysChem, 10, pp. 290-299

Wu, N-C.; Shi, E-W.; Zheng, Y-Q. \& Li, W-J. (2002) Effect of pH of Medium on Hydrothermal Synthesis of Nanocrystalline Cerium(IV) Oxide Powders. J. Am. Ceram. Soc. 85, pp. 2462-2468.

Yoshida, K. Jiu, J. Nagamatsu, D. Nemoto, T. Kurata, H. Adachi, M. \& Isoda, S. (2008). Structure of TiO2 Nanorods Formed with Doiuble Surfactants, Molecular Crystals and Liquid Crystals. 491, pp. 14-20

Youngblood, J. W., Lee, S-H. A., Kobayashi, Y., Hernandez-Pagan, E. A., Hoertz, P. G., Moor, T. A. Moor, A. L., Gust, D., \& Mallouk, T. E. (2009) Photoassisted overall water splitting in a visible light-absorbing dye-sensitized photoelectrochemical cell. J. Am. Chem. Soc., 131, pp. 926-927

Zaki, M. I.; M Hussein, G. A.; Mansour, S. A. A. \& El-Ammawy, H. A. (1989) Adsorption and surface reactions of pyridine on pure and doped ceria catalysts as studied by infrared spectroscopy. J. Mol. Catal. 51, pp. 209-220.

Zaki, M. I.; Hasan, M. A. \& Pasupulety, L. (2001) Surface Reactions of Acetone on $\mathrm{Al}_{2} \mathrm{O}_{3}$, $\mathrm{TiO}_{2}, \mathrm{ZrO}_{2}$, and $\mathrm{CeO}_{2}$ : IR Spectroscopic Assessment of Impacts of the Surface Acid-Base Properties. Langmuir., 17, pp. 768-774.

Zhou, X. -D.; Huebner, W. \& Anderson, H. U. (2003) Processing of Nanometer-Scale $\mathrm{CeO}_{2}$ Particles. Chem. Mater. 15, pp. 378-382.

Zhou, K.; Wang, X.; Peng, Q. \& Li, Y. (2005) Enhanced catalytic activity of ceria nanorods from well-defined reactive crystal planes. J. Catal. 229, pp. 206-212.

Zhong, L-S.; Hu, J-S.; Cao, A-M.; Liu, Q.; Song, W-G.; \& Wan, L-J. (2007) 3D Flowerlike Ceria Micro/Nanocomposite Structure and Its Application for Water Treatment and CO Removal. Chem. Mater. 19, pp. 1648-1655. 


\title{
Experimental and Theoretical Study of Low-Dimensional Iron Oxide Nanostructures
}

\author{
Jeffrey Yue, Xuchuan Jiang", Yusuf Valentino Kaneti and Aibing Yu \\ School of Materials Science and Engineering, University of New South Wales, Sydney, \\ Australia
}

\section{Introduction}

Iron oxide has many phases, including 16 pure phases (e.g., $\mathrm{FeO}_{2} \mathrm{Fe}_{3} \mathrm{O}_{4}$ ), 5 polymorphs of $\mathrm{FeOOH}($ e.g., $\alpha-\mathrm{FeOOH}, \beta-\mathrm{FeOOH})$ and 4 kinds of $\mathrm{Fe}_{2} \mathrm{O}_{3}$ (e.g., $\alpha-\mathrm{Fe}_{2} \mathrm{O}_{3}, \gamma-\mathrm{Fe}_{2} \mathrm{O}_{3}$ ). Because of their unique properties (optical, electronic, magnetic), they have found many applications in the areas of catalysts, magnetic recording, sorbents, pigments, flocculants, coatings, gas sensors, lubrications, and biomedical applications (e.g., magnetic resonance imaging, drug delivery and therapy).

Many efforts have been made in the synthesis (co-precipitation, hydrothermal, microemulsion, and sol-gel method), structural characterization, and functional exploration, as well as fundamental understandings of iron oxide nanostructures. Despite some success, several challenges still exist regarding the synthesis, strcture, properties, and fundamental understanding of the iron oxides. the grand challenge is how to efficiently synthesize iron oxides with controlled morphology, size and functionality, and how to fundamentally understand the formation and growth mechanisms, structure, and interaction forces. Therefore, the development of simple but effective experimental and theoretical strategies to overcome the challenges is still imperative.

To fundamentally understand the nanoscale system, theoretical methods should exist. Computational modeling is one of the most important enabling techniques in nanotechnology and material research. It can increase the pace of discovery across the entire scientific scope, and reduce the cost in the development and commercialization of technologies and materials. Various computational approaches have been developed and used to predict the materials properties (e.g., electronic, magnetic, optical) at different length and time scales. For example, at an atomic scale, density functional theory (DFT) is widely used for binding energy calculation, while at a microscopic scale, molecular dynamics (MD) are able to provide insights into atomic/molecular systems.

This Chapter will give a brief overview of the experimental and theoretical methods conducted on iron oxide nanostructures, particularly for low-dimensional iron oxide nanoparticles. This includes: (i) several representative methods for iron oxides nanomaterials in Sections 2 and 3; (ii) surface modified iron oxide nanostructures by

\footnotetext{
${ }^{*}$ Corresponding Author
} 
surfactants, polymers, silica or metals in Section 4; (iii) functional properties of such nanomaterials in gas sensing, catalysis, and biotechnology in Section 5. Moreover, in Section 6, the discussion will be extended to the theoretical modeling and simulation methods that can predict the formation and performance of nanomaterials, such as MD and DFT methods.

\section{Iron oxide materials}

Iron is the fourth most abundant element in the Earth's crust, and iron oxides are commonly found in nature and have become the most plentiful transition metal oxides (Morrissey and Guerinot, 2009; Ilani et al., 1999). The complicated phases and features of iron oxides have been listed in Table 1.

Some crystalline phases of iron oxides are not very stable and can convert into others. Much work has been conducted to convert akaganéite to hematite and/or magnetite phases to pursuit good performance in catalysis and gas sensing applications. Magnetite nanorods can be produced by the conversion of iron oxyhydroxides into a thermally stable structure of hematite by heating above $400{ }^{\circ} \mathrm{C}$ in air, or magnetite in a mixture of $\mathrm{H}_{2}$ and Ar gas (BomatiMiguel et al., 2008). Recently, our group has simplified the phase conversion procedures among the iron oxides. The iron oxyhydroxides can directly convert into magnetite by using hydrazine as a reducing agent, and the morphology was maintained. Using this method, magnetite nanorods could be directly synthesized from akaganéite rather than using hematite as an intermediate (Yue et al., 2010).

By using hydrazine, iron(III) ions can be reduced to iron(II). The change in coordination number to the iron atom will therefore transfer from $\mathrm{Fe}-\mathrm{OH}$ to $\mathrm{Fe}-\mathrm{O}$ following dehydration. The structure change caused by loss of $\mathrm{H}_{2} \mathrm{O}$ will create pores or holes within the nanorod framework. Continuous reaction with hydrazine can form larger defects in the 1-D nanostructure, leading to the collapse of the framework. At the same time, the $\mathrm{FeO}_{6}$ units will reconstruct into other crystals, and the broken fractions could fuse with neighboring particles to form larger ones. However, this does not happen to hematite because of its thermally stable structure under the considered conditions. The nature of such a conversion needs further investigation. Nevertheless, the proposed approach could be used for a controlled conversion of akaganéite to magnetite nanostructures (Fig. 1) without hightemperature treatment. These porous magnetic structures would find more applications in electronic and magnetic areas (Yue et al., 2011).

\section{Synthesis methods}

A variety of methods have been reported to synthesize iron oxide nanoparticles, including solid-state, liquid-phase, and gas-phase syntheses, as listed in Table 2. Among the synthesis approaches, liquid-phase synthesis is the most popular. The iron salts are highly soluble in water and different additives can be used in conjunction to modify the structure of the nanoparticles. Moreover, the liquid-phase synthesis is convenient for understanding ageing, recrystallization, and evolution into other shapes and sizes. It is also available for controlling experimental conditions in liquid (e.g., concentration, salt precursor, $\mathrm{pH}$, temperature, surface modifiers). A few representative synthesis methods are briefly introduced in this Section, such as co-precipitation, hydrothermal and microemulsion. 


\begin{tabular}{|c|c|c|c|}
\hline Iron oxides & $\begin{array}{l}\text { Crystallographic } \\
\text { system }\end{array}$ & Crystallographic structural features & References \\
\hline $\begin{array}{l}\text { Goethite } \\
(\alpha-\mathrm{FeOOH})\end{array}$ & $\begin{array}{l}\text { Orthorhombica = } \\
0.9956 \mathrm{~nm}, \\
\mathrm{~b}=0.30215 \mathrm{~nm} \\
\text { and } \mathrm{c}=0.4608 \mathrm{~nm}\end{array}$ & $\begin{array}{l}\text { 3D-structure built up with } \mathrm{FeO}_{3}(\mathrm{OH}) \\
\text { octahedra spreading along the }(010) \\
\text { direction, with each octahedron linked } \\
\text { to eight neighbouring octahedral by } \\
\text { four edges and three vertices. Oxygen } \\
\text { atoms are in tetrahedral surroundings, } \\
\text { either } \mathrm{OFe}_{3} \mathrm{H} \text { or } \mathrm{OFe}_{3} \mathrm{H} \text { (bond). }\end{array}$ & $\begin{array}{l}\text { Cornell and } \\
\text { Schwertmann, } \\
\text { 1991; } \\
\text { Cudennec and } \\
\text { Lecerf, } 2005\end{array}$ \\
\hline $\begin{array}{l}\text { Akaganéite } \\
(\beta-\mathrm{FeOOH})\end{array}$ & $\begin{array}{l}\text { Monoclinic } \\
\mathrm{a}=1.056 \mathrm{~nm} \\
\mathrm{~b}=0.3031 \mathrm{~nm} \text { and } \\
\mathrm{c}=1.0483 \mathrm{~nm}\end{array}$ & $\begin{array}{l}\text { Double chains of edge linked } \mathrm{Fe}(\mathrm{O}, \\
\mathrm{OH}) \text { octahedral that share corners to } \\
\text { form a framework containing large } \\
\text { tunnels with square cross sections. }\end{array}$ & $\begin{array}{l}\text { Cornell and } \\
\text { Schuwertmann, } \\
\text { 1991; } \\
\text { Garcia et al., } \\
2009\end{array}$ \\
\hline $\begin{array}{l}\text { Lepidocrocite } \\
(\gamma-\mathrm{FeOOH})\end{array}$ & $\begin{array}{l}\text { Orthorhombic } \\
\mathrm{a}=0.3071 \mathrm{~nm}, \\
\mathrm{~b}=1.2520 \mathrm{~nm} \text { and } \\
\mathrm{c}=0.3873 \mathrm{~nm}\end{array}$ & $\begin{array}{l}\text { Arrays of close cubic -packed anions } \\
\left(\mathrm{O}^{2-/} \mathrm{OH}-\right) \text { stacked along the }[150] \\
\text { direction with } \mathrm{Fe}^{3+} \text { ions occupying the } \\
\text { octahedral interstices. }\end{array}$ & $\begin{array}{l}\text { Cornell and } \\
\text { Schuwertmann, } \\
1991\end{array}$ \\
\hline $\begin{array}{l}\text { Feroxyhyte } \\
\left(\delta^{\prime}-\mathrm{FeOOH}\right)\end{array}$ & $\begin{array}{l}\text { Hexagonal } \\
\mathrm{a}=0.293 \mathrm{~nm} \text { and } \\
\mathrm{c}=0.456 \mathrm{~nm}\end{array}$ & $\begin{array}{l}\text { Disordered hexagonally close-packed } \\
\text { array of anions with } \mathrm{Fe}^{3+} \text { ions } \\
\text { distributed over half the octahedral } \\
\text { sites in an orderly manner. }\end{array}$ & $\begin{array}{l}\text { Cornell and } \\
\text { Schuwertmann, } \\
1991\end{array}$ \\
\hline $\begin{array}{l}\text { Hematite } \\
\left(\mathrm{a}-\mathrm{Fe}_{2} \mathrm{O}_{3}\right)\end{array}$ & $\begin{array}{l}\text { Hexagonal } \\
a=0.5035 \mathrm{~nm} \text { and } \\
\mathrm{c}=1.375 \mathrm{~nm}\end{array}$ & $\begin{array}{l}\text { Stacking of sheets of octahedrally (six- } \\
\text { fold) coordinated } \mathrm{Fe}^{3+} \text { ions. Between } \\
\text { two close-packed layers of oxygen } \\
\text { ions. Each oxygen ion is bonded to } \\
\text { only two Fe ions. }\end{array}$ & $\begin{array}{l}\text { Mohapatra and } \\
\text { Anand,2010 }\end{array}$ \\
\hline $\begin{array}{l}\text { Maghemite } \\
\left(\gamma-\mathrm{Fe}_{2} \mathrm{O}_{3}\right)\end{array}$ & $\begin{array}{l}\text { Cubic } \\
a=0.83474 \mathrm{~nm}\end{array}$ & $\begin{array}{l}\text { Each cell of maghemite contains } 32 \mathrm{O}^{2-} \\
\text { ions, } 21(1 / 3) \mathrm{Fe}^{3+} \text { ions and } 2(1 / 3) \mathrm{O} \\
\text { vacancies. Eight cations occupy } \\
\text { tetrahedral sites and the remaining } \\
\text { cations are distributed over the } \\
\text { octahedral sites. The vacancies are } \\
\text { confined to octahedral sites. }\end{array}$ & $\begin{array}{l}\text { Cornell and } \\
\text { Schuwertmann, } \\
\text { 1991; } \\
\text { Weckler and } \\
\text { Lutz, } 1998\end{array}$ \\
\hline $\begin{array}{l}\text { Magnetite } \\
\left(\mathrm{Fe}_{3} \mathrm{O}_{4}\right)\end{array}$ & $\begin{array}{l}\text { Cubic } \\
\mathrm{a}=0.8396 \mathrm{~nm}\end{array}$ & $\begin{array}{l}\text { Inverse spinel structure with a face- } \\
\text { centered cubic cell based on } 32 \mathrm{O}^{2-} \\
\text { ions, regularly close-cubic packed } \\
\text { along }[111] \text {, with } \mathrm{Fe}^{2+} \text { ions and half of } \\
\text { the } \mathrm{Fe}^{3+} \text { occupying the octahedral sites } \\
\text { and the other half of } \mathrm{Fe}^{3+} \text { ions, } \\
\text { occupying the tetrahedral sites. }\end{array}$ & $\begin{array}{l}\text { Cornell and } \\
\text { Schuwertmann, } \\
1991 \\
\text { Mohapatra and } \\
\text { Anand,2010 }\end{array}$ \\
\hline
\end{tabular}

Table 1. Complicated phases and polymorphs of iron oxides in nature 


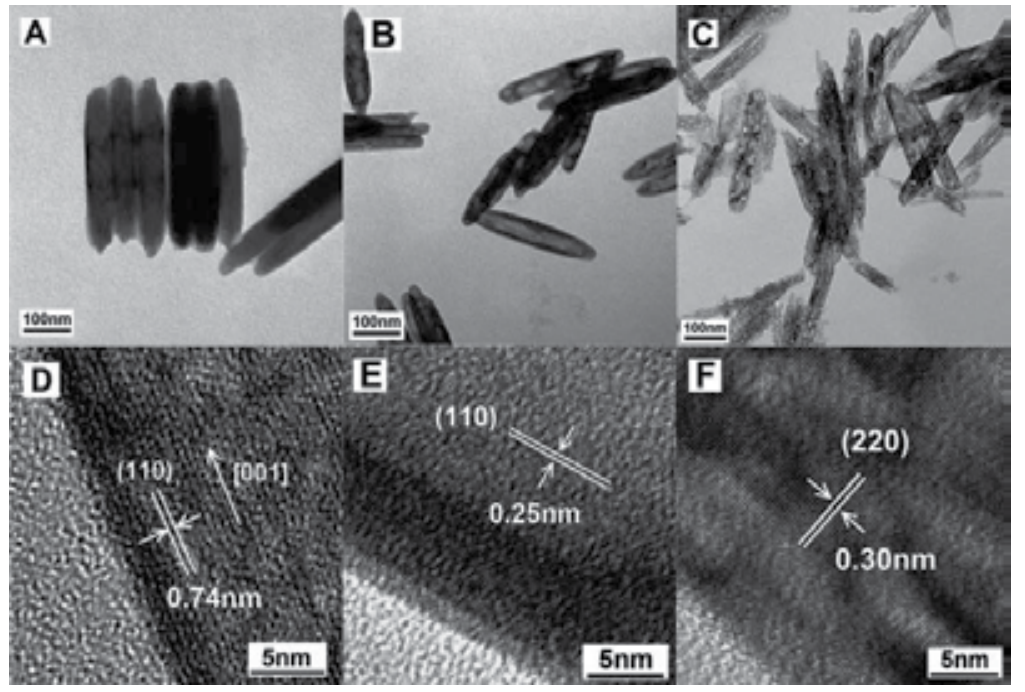

Fig. 1. TEM images showing the conversion of nanorods: (A) $\beta$-FeOOH nanorods, (B) $\beta$-FeOOH nanorods calcined at $300{ }^{\circ} \mathrm{C}$, (C) $\beta$-FeOOH nanorods reduced with $\mathrm{N}_{2} \mathrm{H}_{4}$ at $80^{\circ} \mathrm{C}$; and $(\mathrm{D}-\mathrm{F})$ the corresponding HRTEM images with labeled lattice spacing and crystal planes. Reprinted with permission from (Yue et al., 2011).

\subsection{Co-precipitation}

One simple and efficient way is to use co-precipitation technique in solution. By this approach, iron(II) and/or iron(III) salts are first dissolved in aqueous solution, and then one alkaline media (e.g., $\mathrm{NaOH}, \mathrm{Na}_{2} \mathrm{CO}_{3}$ ) solution is added to form precipitate. The prepared particles can be tuned to be uniform in size, shape as well as pure in its composition. Various crystalline phases of iron oxides can be produced using this method, which is controlled by experimental parameters such as types of iron salts (e.g., chloride, sulphate and nitrate), alkaline media, concentration, temperature, and $\mathrm{pH}$ (Iida et al., 2007).

Moreover, the phase of iron oxide(s) formed through the co-precipitate approach is often reported as goethite or hematite if iron(III) salt is used. However, the initially precipitated material is usually found as ferrihydrite, which is a thermodynamically unstable phase. The precipitate can further convert into other phases (e.g., hematite, magnetite) depending on the $\mathrm{pH}$, ionic medium, and temperature. For example, Varada et al. (2002) prepared monodispersed acicular goethite particles by precipitating $\mathrm{Fe}(\mathrm{III})$ using sodium carbonate. If sodium hydroxide was used, the axial ratio of particles will increase from 60 to $230 \mathrm{~nm}$. It was proposed that different bases have different ability to maintain the solution at a constant $\mathrm{pH}$, where other $\mathrm{pH}$ levels would produce polydispersed and hematite particles. The mechanism of the growth of spherical hematite nanoparticles has been explored by Liu et al.(2007). The variation in the final $\mathrm{pH}$ of the solution plays a key role in the formation of hematite at different sizes. They found that the particles with diameter of $60-80 \mathrm{~nm}$ were obtained at $\mathrm{pH} 7$, while reduced to $30-40 \mathrm{~nm}$ in diameter at $\mathrm{pH} 9$. 


\begin{tabular}{|c|c|c|c|c|c|}
\hline $\begin{array}{l}\text { Synthesis } \\
\text { media }\end{array}$ & $\begin{array}{l}\text { Synthesis } \\
\text { methods }\end{array}$ & $\begin{array}{l}\text { Common } \\
\text { products }\end{array}$ & $\begin{array}{l}\text { Particle } \\
\text { shape/size }\end{array}$ & Features & References \\
\hline Solid state & $\begin{array}{l}\text { Mechanical } \\
\text { milling }\end{array}$ & $\begin{array}{l}\delta-\mathrm{Fe}_{2} \mathrm{O}_{3}, \\
\mathrm{Fe}_{3} \mathrm{O}_{4}\end{array}$ & $\begin{array}{l}\text { Spheres } \\
\mathrm{D}=2.9-3.6 \mathrm{~nm}\end{array}$ & $\begin{array}{l}\text { Mechanical } \\
\text { energy to smash. }\end{array}$ & Lu et al. (2007) \\
\hline \multirow[t]{7}{*}{$\begin{array}{l}\text { Liquid } \\
\text { state }\end{array}$} & Co-precipitation & $\begin{array}{l}\mathrm{a}-\mathrm{Fe}_{2} \mathrm{O}_{3}, \mathrm{\delta}- \\
\mathrm{Fe}_{2} \mathrm{O}_{3} \\
\mathrm{Fe}_{3} \mathrm{O}_{4}\end{array}$ & $\begin{array}{l}\text { Nanospheres } \\
(\mathrm{d}=30-80 \\
\mathrm{nm})\end{array}$ & $\begin{array}{l}\text { Ageing of ferric } \\
\text { and ferrous salts } \\
\text { in a basic } \\
\text { medium. }\end{array}$ & $\begin{array}{l}\text { Liu } \\
\text { et al. (2007) }\end{array}$ \\
\hline & Hydrothermal & $\begin{array}{l}\alpha-\mathrm{Fe}_{2} \mathrm{O}_{3}, \\
\delta-\mathrm{Fe}_{2} \mathrm{O}_{3} \\
\mathrm{Fe}_{3} \mathrm{O}_{4} \\
(\alpha-\mathrm{FeOOH} \\
\beta-\mathrm{FeOOH}\end{array}$ & $\begin{array}{l}\text { Nanorods } \\
(1: 400-600 \mathrm{~nm}, \\
\mathrm{w}=20-30 \mathrm{~nm}) \\
\text { Nanodiscs } \\
(\mathrm{d}=50 \mathrm{~nm}, \\
\text { thickness = } 6.5 \\
\mathrm{~nm})\end{array}$ & $\begin{array}{l}\text { Low } \\
\text { temperature, } \\
\text { reaction, } \\
\text { commonly } \\
\text { conducted in } \\
\text { autoclaves, and } \\
\text { high efficiency. }\end{array}$ & $\begin{array}{l}\text { Li et al. (2006); } \\
\text { Yue et al. } \\
\text { (2010, 2011); } \\
\text { Jiang } \\
\text { et al. (2009) }\end{array}$ \\
\hline & $\begin{array}{l}\text { Thermal } \\
\text { decomposition }\end{array}$ & $\begin{array}{l}\mathrm{a}-\mathrm{Fe}_{2} \mathrm{O}_{3} \\
\delta-\mathrm{Fe}_{2} \mathrm{O}_{3} \\
\mathrm{Fe}_{3} \mathrm{O}_{4}\end{array}$ & $\begin{array}{l}\text { Nanospheres } \\
(\mathrm{d}=16 \mathrm{~nm})\end{array}$ & $\begin{array}{l}\text { High- } \\
\text { temperature } \\
\text { decomposition of } \\
\text { iron organic } \\
\text { precursors. }\end{array}$ & $\begin{array}{l}\text { Sun } \\
\text { et al. (2004) }\end{array}$ \\
\hline & Sol-gel & $\begin{array}{l}\mathrm{a}-\mathrm{Fe}_{2} \mathrm{O}_{3}, \delta- \\
\mathrm{Fe}_{2} \mathrm{O}_{3} \\
\mathrm{Fe}_{3} \mathrm{O}_{4}\end{array}$ & $\begin{array}{l}\text { Nanoparticles } \\
(24-52 \mathrm{~nm})\end{array}$ & $\begin{array}{l}\text { Dissolve, } \\
\text { condensation, } \\
\text { and calcinations } \\
\text { of alkoxides. }\end{array}$ & $\begin{array}{l}\text { Dong and Zhu } \\
\text { (2004) }\end{array}$ \\
\hline & Microemulsion & $\begin{array}{l}\mathrm{a}-\mathrm{Fe}_{2} \mathrm{O}_{3}, \delta- \\
\mathrm{Fe}_{2} \mathrm{O}_{3} \\
\mathrm{Fe}_{3} \mathrm{O}_{4}\end{array}$ & $\begin{array}{l}\text { Nanoparticles } \\
(3-5 \mathrm{~nm})\end{array}$ & $\begin{array}{l}\text { Reaction in two } \\
\text { immiscible } \\
\text { phases (water } \\
\text { and oil). }\end{array}$ & $\begin{array}{l}\text { Vidal-Vidal } \\
\text { et al. (2006) }\end{array}$ \\
\hline & Sonochemical & $\begin{array}{l}\mathrm{Fe}_{2} \mathrm{O}_{3} \\
\mathrm{Fe}_{3} \mathrm{O}_{4}\end{array}$ & $\begin{array}{l}\text { Nanorods } \\
10-80 \mathrm{~nm} \text { in } \\
\text { diameter }\end{array}$ & $\begin{array}{l}\text { Ultrasound to } \\
\text { promote } \\
\text { chemical } \\
\text { reaction. }\end{array}$ & $\begin{array}{l}\text { Vijayakumar } \\
\text { et al. (2000, } \\
\text { 2001) }\end{array}$ \\
\hline & Electrochemical & $\gamma-\mathrm{Fe}_{2} \mathrm{O}_{3}$ & $\begin{array}{l}\text { Nanoparticles } \\
(\mathrm{d}=3-20 \mathrm{~nm})\end{array}$ & $\begin{array}{l}\text { Electrons act as } \\
\text { reactant with no } \\
\text { pollution. }\end{array}$ & $\begin{array}{l}\text { Zhang et al } \\
\text { (2007); Pascal } \\
\text { et al (1999) }\end{array}$ \\
\hline \multirow[t]{2}{*}{ Gas state } & Spray pyrolysis & $\begin{array}{l}\delta-\mathrm{Fe}_{2} \mathrm{O}_{3} \\
\mathrm{Fe}_{3} \mathrm{O}_{4}\end{array}$ & $\begin{array}{l}\text { Hollow } \\
\text { nanospheres, } \\
(\mathrm{d}=300 \mathrm{~nm})\end{array}$ & $\begin{array}{l}\text { Spraying, aerosol } \\
\text { evaporation, } \\
\text { condensation, } \\
\text { drying, and } \\
\text { thermolysis. }\end{array}$ & $\begin{array}{l}\text { González- } \\
\text { Carreño } \\
\text { et al. (1993) }\end{array}$ \\
\hline & $\begin{array}{l}\text { Laser pyrolysis } \\
\text { or deposition }\end{array}$ & $\begin{array}{l}\mathrm{a}-\mathrm{Fe}_{2} \mathrm{O}_{3} \\
\delta-\mathrm{Fe}_{2} \mathrm{O}_{3} \\
\varepsilon-\mathrm{Fe}_{2} \mathrm{O}_{3} \\
\mathrm{Fe}_{3} \mathrm{O}_{4}\end{array}$ & $\begin{array}{l}\text { Nanowires } \\
(30 \mathrm{~nm} \times 1-5 \\
\mu \mathrm{m}) \\
\text { Nanobelts }(100 \\
\mathrm{nm} \times 7 \mu \mathrm{m})\end{array}$ & $\begin{array}{l}\text { Heating of a } \\
\text { gaseous mixture } \\
\text { of iron precursor. }\end{array}$ & $\begin{array}{l}\text { Morber } \\
\text { et al. (2006) }\end{array}$ \\
\hline
\end{tabular}

Table 2. Several typical synthesis methods for iron oxides 


\subsection{Hydrothermal and thermal decomposition methods}

Hydrothermal technique is defined as any heterogeneous reaction in the presence of aqueous solvents or mineralizers under a high pressure and a temperature (6-10 atm, 100$200{ }^{\circ} \mathrm{C}$ ). A hydrothermal reaction requires the iron(III) salt (e.g., iron chloride, nitrate, or sulphate), which can be dissolved in solution followed by reaction with water. This is different from the thermal decomposition reaction that generally takes place for those iron organic precursors $\left(\mathrm{Fe}(\mathrm{CO})_{5}, \mathrm{Fe}(\mathrm{acac})_{3}\right.$, and $\left.\mathrm{Fe}(\mathrm{cup})_{3}\right)$ in an organic solvent at high temperatures (Hyeon et al., 2001; Li et al., 2004; Rockenberger et al., 1999). Both hydrothermal and thermal decomposition methods are commonly used for the synthesis of iron oxide nanoparticles.

The hydrothermal method is often performed in an autoclave, where the reaction system can exceed the boiling point of liquid(s) at normal atmospheric pressure (Jia et al., 2005). The temperature can alter the system in such a way that disrupts the thermodynamics of a material, which is governed by enthalpy $(\Delta \mathrm{H})$ and entropy $(\Delta S)$, and hence Gibbs free energy $(\Delta G)$. The essential role of a fluid under high temperatures is that it changes the vapor pressure of the fluid. This is also beneficial for diverse choices of solvents (polar and non-polar). The morphology and crystalline phase of iron oxides produced through this approach can vary by simply tuning reaction temperature, concentration, and additive(s) (Almeida et al., 2009; Jiang et al., 2010).

The synthesis of iron oxide nanoparticles via a hydrothermal approach can be conducted with or without the use of surfactant(s). Hematite nanoparticles have been prepared by Sahu et al. (1997) under conditions of $\mathrm{pH}(3-10)$ and $180^{\circ} \mathrm{C}$ in autoclaves. In this study, the average particle size of hematite nanoparticles was found to decrease with an increase of $\mathrm{pH}$. In our recent work (Jiang et al. 2010), we reported a facile hydrothermal route for the synthesis of monodispersed hematite nanodiscs with diameters of $\sim 50 \mathrm{~nm}$ and thickness of $\sim 6.5 \mathrm{~nm}$ in the absence of any surfactants in water at around $90{ }^{\circ} \mathrm{C}$ (Fig. 2). The nanodiscs exhibited interesting paramagnetic property at a low temperature $(20 \mathrm{~K})$, but ferromagnetic at room temperature $(\sim 300 \mathrm{~K})$. In addition, the hematite nanodiscs also showed lowtemperature catalytic activity in $\mathrm{CO}$ oxidation to $\mathrm{CO}_{2}$.

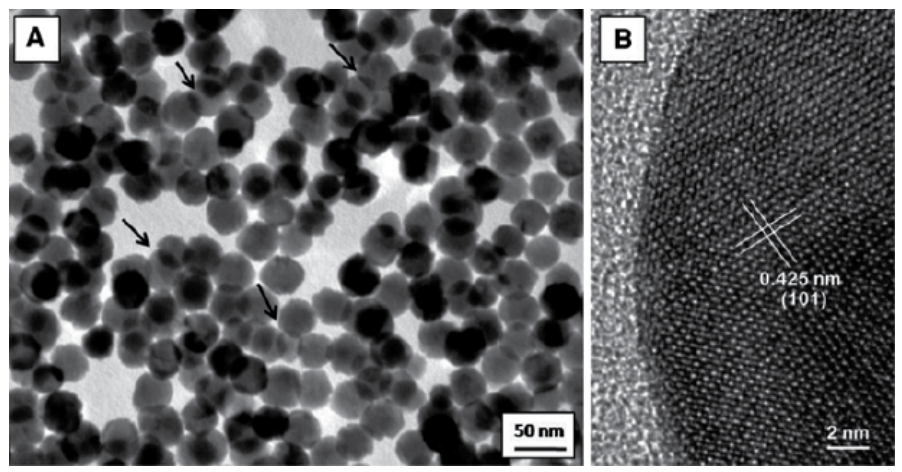

Fig. 2. A) TEM image of $\mathrm{a}-\mathrm{Fe}_{2} \mathrm{O}_{3}$ nanodiscs with overlapping as pointed by arrows; B) HRTEM image showing the lattice fringe of $\{110\}$ plates with spacing between two adjacent planes of $0.411 \mathrm{~nm}$. Reprinted with permission from (Jiang et al. 2010). 


\subsection{Microemulsion}

Microemulsion method (surfactant-stabilized water/oil (W/O) microemulsion) has been widely used to prepare shape- and size-controlled iron oxide nanoparticles. Generally, a microemulsion is transparent, isotropic and thermodynamically stable dispersion of two immiscible phases (e.g., water and oil). When a surfactant is present in W/O system, the surfactant molecules may form a monolayer at the interface of oil and water, with the hydrophobic tails of the surfactant molecules dissolved in the oil phase and the hydrophilic head groups in the aqueous phase ( $\mathrm{Wu}$ et al., 2008). In a binary system such as water/surfactant or oil/surfactant, a variety of self-assembled structures can be formed, ranging from spherical and cylindrical micelles to lamellar phases or bi-continuous microemulsions depending on the molar ratio of water, oil and surfactant(s). This will be useful for the generation of nanoparticles with different shapes and sizes.

For example, magnetite nanoparticles $\sim 4 \mathrm{~nm}$ in diameter have been prepared by the controlled hydrolysis of ammonium hydroxide with $\mathrm{FeCl}_{2}$ and $\mathrm{FeCl}_{3}$ aqueous solution within the reverse micelles nanocavities generated by sodium bis(2-ethylhexyl) sulfosuccinate (AOT) as a surfactant and heptane as a continuous oil phase (López-Quintela and Rivas, 1993). Lee and co-workers (2005) have successfully synthesized uniform and highly crystalline magnetite nanoparticles in microemulsion nanoreactors. The particle size of the prepared magnetite nanoparticles could be adjusted from 2-10 nm by varying the relative concentrations of iron salt, surfactant, and solvent. Li et al (2009) demonstrated the effect of volumetric ratios of aqueous $\mathrm{FeCl}_{3}$ solution to 1,2-propanediamine on the formation of magnetic particles, as shown in Fig. 3. Chin and Yaacob (2007) reported the synthesis of

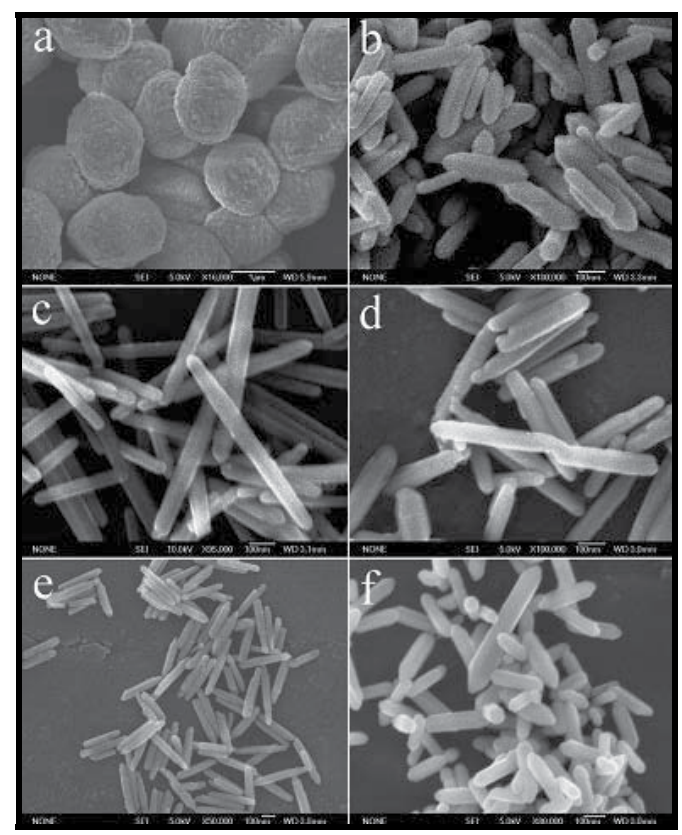

Fig. 3. SEM images of the products obtained at different volume ratios of aqueous $\mathrm{FeCl}_{3}$ solution to 1,2 propanediamine: (a) without 1,2 propanediamine, (b) 3:1, (c) 1:1, (d) 1:2, (e) 1:4, and (f) 1:5. Reprinted with permission from (Li et al., 2009). 
magnetic iron oxide nanoparticles with an average particle size of $<10 \mathrm{~nm}$ by mixing two microemulsion systems, one containing $\mathrm{Fe}^{2+}$ ions and the other containing $\mathrm{OH}^{-}$ions. The study reveals that the nanoparticles prepared by the microemulsion technique were smaller in size and higher in saturation magnetization than those nanoparticles prepared by Massart's procedure (Massart et al., 1981).

Despite some success, this microemulsion approach has some drawbacks, such as the difficulty in scale-up production, the adverse effect of residual surfactants on the properties of the nanoparticles, and the aggregation of the produced nanoparticles. Repeated wash processes and further stabilization treatment are usually required for such a reaction approach (Wu et al., 2008).

\section{Surface modifications}

The surface modifications of nanoparticles have attracted much more attention, which can improve the surface-related properties like hydrophobic or hydrophilic. This can be achieved by using surfactants, polymers, and inorganic materials (silica).

\subsection{Surfactants}

Surface modification with surfactant(s) is widely used for altering surface properties such as hydrophobic or hydrophilic. The use of surfactant molecules, such as oleic acid, oleylamine, or thiols (Wang et al., 2005), can easily functionalize iron oxide nanoparticles to be hydrophobic surfaces. These molecules can covalently bond to the iron atoms or clusters against particle degradation (Soler et al., 2007).

Many researches focus on the synthesis of water-soluble iron oxide nanoparticles with biocompatibility and biodegradability for biological applications. For example, one is to directly introduce the biocompatible organic molecules, e.g., amino acid (Sousa et al., 2001), vitamin (Mornet et al., 2004), and citric acid (Morais et al., 2003). Despite some advantages, the instability of small organic molecules in alkaline or acidic environment may result in agglomeration of the functionalized iron oxide nanoparticles.

Another alternative technique is to transform the oil-soluble type into water-soluble one via a ligand exchange reaction (Chen et al., 2008). The ligand exchange involves the addition of an excess of ligand(s) to nanoparticle suspension, which has stronger interaction with the nanoparticles than the original ones. Sun et al. (2003) converted the synthesized hydrophobic maghemite nanoparticles into hydrophilic ones by mixing with bipolar surfactants such as tetramethylammonium 11-aminoudecanoate. Lattuada and Hatton (2006) reported that the oleic groups initially present on the surface of magnetite nanoparticles were replaced by various capping agents containing reactive hydroxyl moieties. They also tuned the particle size in the range of $6-11 \mathrm{~nm}$ by varying the heating rate.

\subsection{Polymers}

Polymer-functionalized iron oxide nanoparticles have gained much more attention due to the benefits offered by polymeric coating, which may increase repulsive forces to balance the magnetic and van der Waals attractive forces acting on the nanoparticles $(\mathrm{Wu}$ et al. 
2008). It has shown that through careful choice of the passivating and activating polymers and/or reaction conditions, polymer-stabilized iron oxide nanoparticles with tailored and desired properties can be synthesized.

The iron oxide particles by ionic properties can be modified with functional polymer groups with $-\mathrm{COOH},-\mathrm{NH}_{2}$ (Chibowski et al., 2009; Kandori et al., 2005; Li et al., 2004). The polymer coated particles can be synthesized by the ex situ method, i.e. dispersion of the nanoparticles in a polymeric solution, or in situ method, i.e. monomer polymerization in the presence of the synthesized nanoparticles (Mammeri et al., 2005; Guo et al., 2007).

Polymeric coating materials can be classified into two main classes: natural (e.g., dextran, starch, gelatin, chitosan) and synthetic (e.g., polyethylene glycol, PEG; polymethylmethacrylate, PMMA; polyacrylic acid, PAA). However, the saturation magnetization value of iron oxide nanoparticles will decrease after polymer-fictionalization.

Dextran is often utilized as a coating polymer because of its stability and biocompatibility (Laurent et al. 2008). Molday and Mackenzie (Molday and Mackenzie, 1982) have reported the formation of $\mathrm{Fe}_{3} \mathrm{O}_{4}$ in the presence of dextran with molecular weight (MW) of 40,000. In the synthesis of dextran-coated ultra-small superparamagnetic iron oxides (USPIO), the reduction of the terminal glucose of dextran was found to be significant for controlling particle size, stability, and magnetic properties. For low molecular weight dextrans (MW, $<10,000)$, it is difficult to obtain nanoparticles with a small size of $<20 \mathrm{~nm}$.

Polyvinyl alcohol (PVA) is a hydrophilic and biocompatible polymer that can be used for particle surface modification to prevent particle agglomeration (Laurent et al. 2008). Lee et al. (1996) have modified the surface of magnetite nanoparticles with PVA by precipitation of iron salts at a high $\mathrm{pH}(13.8)$ to form stable magnetite colloidal dispersions, and particle size is around $4 \mathrm{~nm}$. The investigators noted that the crystallinity of the magnetite nanoparticles decreased with PVA concentration increasing, although morphology and particle sizes remained. When PVA is introduced, it reacts with the surface through hydrogen bonding between polar functional groups of the polymer and hydroxylated and/or protonated surface of the iron oxide. In addition to the polymer-surface interactions, PVA is known for its hydrogen bonding interaction, resulting in hydrogel structure embedding the nanoparticles. When the PVA concentration is over the critical saturation value, agglomeration may occur for PVA-coated particles via bridging interactions.

\subsection{Polymerized amorphous silica}

Polymerized tetraethoxysilane (TEOS) network is often used as a surface coating material for iron oxide nanoparticles as this coating can prevent aggregation in solution, improve the chemical stability, and provide better protection against toxicity (Laurent et al. 2008). Additionally, polymerized silica-coated iron oxide nanoparticles exhibited good biocompatibility and solubility in water. Silica coating can stabilize the magnetite nanoparticles in two different ways: one is by shielding the magnetic dipole interaction with the silica shell, and another one is by enhancing the coulomb repulsion of the magnetic nanoparticles. Such a silica coating increases the size of the particles and decreases the saturation magnetization value. 
A commonly used method to coat iron oxide nanoparticles with silica is the well-known Stóber method, in which silica is formed in situ via hydrolysis and condensation of a sol-gel precursor such as TEOS. For example, Im et al.(2005) have reported the synthesis of silica colloids loaded with superparamagnetic iron oxide nanoparticles, which revealed that the final size of silica colloids depended upon the concentration of iron oxide nanoparticles because the size of silica was closely related to the number of seeds (emulsion drops). The lower concentration the iron oxide nanoparticles in alcohol, the larger size the obtained colloids.

Another one is aerosol-pyrolysis method, in which silica-coated magnetic nanoparticles were prepared by pyrolysis of a mixed precursor of silicon alkoxides and metal compound in a flame environment (Deng et al. 2005). Tartaj et al.(2001) synthesized silica-coated $\gamma$ $\mathrm{Fe}_{2} \mathrm{O}_{3}$ hollow spheres with size of $150 \pm 100 \mathrm{~nm}$ by aerosol pyrolysis of methanol solution containing iron ammonium citrate and silicon ethoxide.

\subsection{Metals}

Noble metals (e.g., $\mathrm{Au}, \mathrm{Ag}, \mathrm{Pt}$, and $\mathrm{Pd}$ ), possessing unique electronic and catalytic properties, can be utilized to improve the physicochemical properties of magnetic nanoparticles and applications in biomedicine. The coating of iron oxide nanoparticles with noble metals can be helpful to improve stability from aggregation, however, decrease the saturation magnetization value in some cases (Wu et al. 2008).

Several procedures have been employed to synthesize such core-shell nanostructures. For example, Mikhaylova et al. (2004) have prepared gold-coated superparamagnetic iron oxide nanoparticles (SPION) using a reverse micelle method. In their study, the reverse micelles were formed from surfactant, cetyltrimethylammonium bromide (CTAB), octane (the oil phase), butanol (the co-surfactant), and an aqueous mixture of $\mathrm{FeCl}_{3}, \mathrm{FeCl}_{2}$ and $\mathrm{HAuCl}_{4}$ solutions. They found that the Au-coated SPION retained the superparamagnetic properties for a longer period than those of starch-coated and multi-arm polyethylene glycol (MPEG)coated ones. Wang et al.(2005) obtained gold coated iron oxide nanoparticles, in which the pre-synthesized $\mathrm{Fe}_{3} \mathrm{O}_{4}$ nanoparticles were used as seeds during the reduction of gold precursor, $\mathrm{Au}\left(\mathrm{OOCCH}_{3}\right)_{3}$. The average size of $\mathrm{Fe}_{3} \mathrm{O}_{4}$ nanoparticles increases from $5.2 \pm 0.5$ $\mathrm{nm}$ to $6.7 \pm 0.7 \mathrm{~nm}$ after coating with gold (Fig. 4). $\mathrm{Fe}_{3} \mathrm{O}_{4} / \mathrm{Au}$ and $\mathrm{Fe}_{3} \mathrm{O}_{4} / \mathrm{Au} / \mathrm{Ag}$ core/shell nanoparticles with tuneable plasmonic and magnetic properties have been developed by controlling the coating thickness and materials ( $\mathrm{Xu}$ et al. 2007).

A facile and one-pot synthesis approach has been developed by Zhang et al. (Zhang et al., 2010) for generating metal $(\mathrm{Au}, \mathrm{Pt}, \mathrm{Ag}$ and $\mathrm{Au}-\mathrm{Pt}) / \mathrm{Fe}_{2} \mathrm{O}_{3}$ nanocomposites assisted by lysine. Lysine, containing functional groups $-\mathrm{NH}_{2}$ and $-\mathrm{COOH}$, acts as both a linking molecule to the $\mathrm{Fe}_{2} \mathrm{O}_{3}$ matrix and a capping agent to stabilize the noble metal nanoparticles for a good dispersion. Jiang et al. (Jiang and $\mathrm{Yu}, 2009$ ) have demonstrated a facile synthetic method for the preparation of $\mathrm{Pd} / \mathrm{a}-\mathrm{Fe}_{2} \mathrm{O}_{3}$ nanocomposites by adding citric acid into a mixture of iron oxide nanoparticles and palladium precursor, $\left.\mathrm{Pd}\left(\mathrm{CH}_{3} \mathrm{CN}\right)_{2} \mathrm{Cl}_{2}\right)$ under a reflux heating at $90^{\circ} \mathrm{C}$ for 2 hours. The synthesized $\mathrm{Pd} / \mathrm{a}-\mathrm{Fe}_{2} \mathrm{O}_{3}$ nanocomposites inherited the rod-like morphology of the $\mathrm{a}-\mathrm{Fe}_{2} \mathrm{O}_{3}$ nanoparticles and they exhibited superior catalytic activity in $\mathrm{CO}$ oxidation compared with pure $\mathrm{a}-\mathrm{Fe}_{2} \mathrm{O}_{3}$ nanoparticles. UV-vis measurement of the nanocomposites revealed the presence of two plasma bands centered at around 383 and 552 $\mathrm{nm}$, which can be assigned to the synergistic effect of both $\mathrm{Pd}$ and $\mathrm{a}-\mathrm{Fe}_{2} \mathrm{O}_{3}$ nanoparticles. 


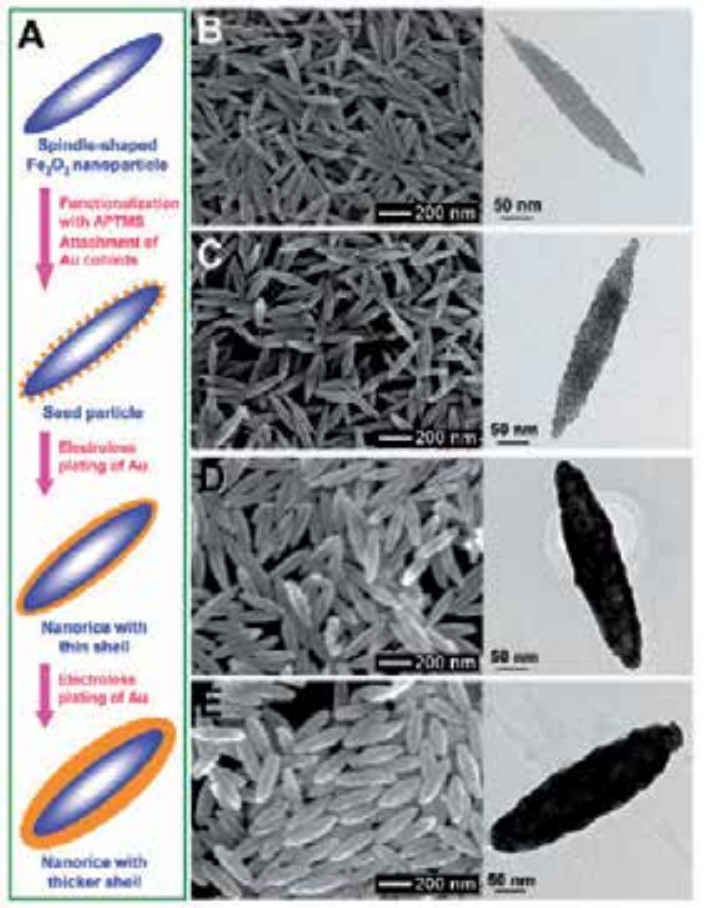

Fig. 4. (A) Schematics of the hematite-gold core-shell nanorice particles. SEM (left) and TEM (right) of (B) hematite core, (C) seed particles, (D) nanorice with thin shells, and (E) nanorice with thick shells. Reprinted with permission from (Wang et al., 2006).

\subsection{Carbon}

Carbon has been widely studied since its poly-morphologies as active carbon, graphite, graphene, carbon nanotubes, and fullerene bucky ball structures. They have exhibited extraordinary tensile strengths and electrical conductivity due to their covalent $s p 2$ hybridized network structure. The combination of semi-conductive iron oxides and carbon may therefore enhance the electrical properties of the nanocomposite material. The method to coat carbon on the surface of iron oxide is often performed by the decomposition of a carbon source (i.e., hydrocarbons, polymer or glucose) at high temperatures under oxygenfree environments (Tristão et al., 2010; Tristão et al., 2009; Zhang et al., 2008, 2010).

Carbon coated iron oxide particles have attracted much more attention. Zhang et al. (2008) demonstrated that carbon coated magnetite nanorods can be synthesized through a series of procedures. In this process, hematite nanorods were firstly synthesized by a hydrothermal method as previously mentioned. Secondly, glucose was coated onto the hematite nanorods by pyrolysis under hydrothermal conditions. Finally, the product was heated at $600{ }^{\circ} \mathrm{C}$ under $\mathrm{N}_{2}$ to carbonize glucose and reduce hematite into magnetite simultaneously. Boguslavsky et al.(2008) reported a similar procedure, in which polydivinylbenzene (PDVB) was used as the carbon source. The PDVB coating was formed by emulsion polymerization of DVB in the presence of $\gamma-\mathrm{Fe}_{2} \mathrm{O}_{3}$, followed by annealing of the powder in a quartz tube at $1050{ }^{\circ} \mathrm{C}$ under flowing Ar gas for 2 hours. The decomposition of the polymer in this case reduced $\gamma-\mathrm{Fe}_{2} \mathrm{O}_{3}$ to metallic $\mathrm{Fe}$, which finally forms carbon coated iron $(\mathrm{Fe} / \mathrm{C})$ nanoparticles. 
In addition, Wang et al. (2006) have reported the synthesis of $\mathrm{Fe}_{3} \mathrm{O}_{4} / \mathrm{C}$ nanocomposites by heating the aqueous solution of glucose and oleic acid-stabilized $\mathrm{Fe}_{3} \mathrm{O}_{4}$ nanoparticles at 170 ${ }^{\circ} \mathrm{C}$ for 3 hours. The results revealed that without prior surface hydrophobic modification, the magnetite nanoparticles could not be encapsulated by the carbon nanospheres, but instead only bare carbon nanospheres with the size of $\sim 200 \mathrm{~nm}$ and $\mathrm{Fe}_{3} \mathrm{O}_{4}$ nanoparticles were obtained. The variation of glucose concentration $(0.3-0.6 \mathrm{M})$ and the reaction temperature $\left(160-180^{\circ} \mathrm{C}\right)$ were found to have no significant effect on the morphology of the product, however, both reaction time and the amount of oleic acid-stabilized $\mathrm{Fe}_{3} \mathrm{O}_{4}$ nanoparticles showed significant effects. The increase in the concentration of oleic-acid stabilized $\mathrm{Fe}_{3} \mathrm{O}_{4}$ nanoparticles from 2.5 to $6 \mathrm{~g} / \mathrm{L}$ was found to generate a product that has more embedded $\mathrm{Fe}_{3} \mathrm{O}_{4}$ nanoparticles increasing from 41 to $63 \%$ ).

Although carbon-coated iron oxide nanoparticles may offer some advantages, such particles are often obtained as agglomerated clusters due to the lack of effective synthetic control, and lack of proper understanding on the formation mechanism. The synthesis of dispersible carbon-coated nanoparticles in isolated forms still remains a challenge in this field.

Moreover, the surface modification of iron oxide allows the attachment of biomolecules such as proteins and drugs (Mohapatra et al. 2007; Sun et al. 2007). The design of the surface modifications may be determined by factors such as ion energy and ion flux of depositing species, interface volume, crystalline size, coating thickness, surface and interfacial energy (Kim et al. 2003; Pinho et al. 2010).

\section{Functionalities of iron oxide nanostructures}

\subsection{Magnetic property}

The magnetic property has been extensively studied since it was discovered and explained through electronic structures of atoms. The magnetic dipole moments generated by the spin and orbital angular momenta of electrons in the Fe atom may vary between each phase of the iron oxide material. In general, magnetic behavior of a material depends on the electron spin vector or the total magnetic dipole moment. One important aspect in iron oxide nanoparticles is the unique form of magnetism called superparamagnetism. At temperature of above the blocking temperature, the magnetization behavior is identical to that of atomic paramagnets. This phenomenon will occur if particles reach below a certain size $(10-20 \mathrm{~nm})$, when the particle consists of a single magnetic domain, even though the material is ferro- or ferri-magnetic in bulk form (Ye et al., 2007), as shown in Fig. 5. Particles with this type of magnetism show high field irreversibility, high saturation field, extra anisotropy contributions, and shifted loops (Pedro et al., 2003).

For noble gold and silver nanoparticles with unique surface plasmon resonance (SPR) properties, they are often used to modify the iron oxide surfaces for generating coupled or multiple functionalities. At the nanoscale, the metallic electron cloud oscillates on the particle surface and absorbs electromagnetic radiation at a particular energy. The surface geometry of the iron oxide particles such as spheres, cubes, triangles, or rods, can therefore influence the absorption of radiation from the ultra-violet up to the near infrared spectrum (350-1200 nm). Other factors that affect the absorption are the solvent and surface functionalization. They are important contributors that can tune the exact frequency and intensity of the plasmon resonance band, which attracts them to the surface enhanced 
resonance spectroscopy (SERS) for sensing devices (Zhai et al., 2009). This effect is also of importance for bimetallic core/shell nanoparticles. As the ratio of gold to iron oxide increases, the gold character increases and the iron oxide becomes buried beneath and suppresses the dielectric effect. The increasing thickness of the shell structure will therefore cause blue-shifting in the surface plasmon resonance (Lyon et al. 2004).

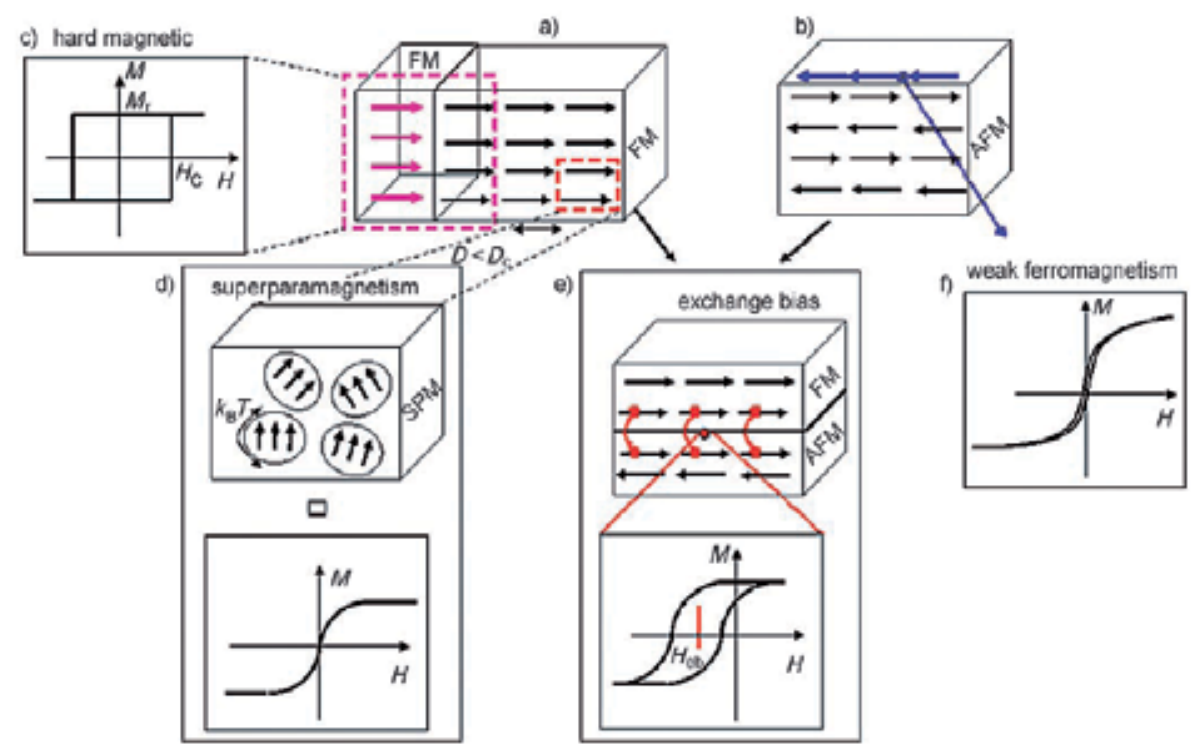

Fig. 5. Diagram of different spin arrangements in magnetic nanoparticles:

a) Ferromagnetism (FM), b) Antiferromagnetism (AFM), D = diameter, $D_{c}=$ critical diameter, c) a combination of two different ferromagnetic phases in permanent magnets, which are materials with high remanence magnetization $\left(\mathrm{M}_{\mathrm{r}}\right)$ and high coercively $\left(\mathrm{H}_{\mathrm{c}}\right)$, d) Superparamagnetism (SPM), e) the interaction at the interface between a ferromagnet and an antiferromagnet producing an exchange bias effect, and f) pure anti-ferromagnetic nanoparticles with superparamagnetic relaxation arising from uncompensated surface spins. Reprinted with permission from (Lu et al., 2007).

\subsection{Biomedical applications}

Many investigations have been reported the application of nanoparticles for biomedicine, such as magnetic nanoparticles for improving the quality of magnetic resonance imaging (MRI), hyperthermic treatment for malignant cells, site-specific drug delivery, cell labeling, and manipulating cell membranes (Babič et al. 2008; Catherine and Adam, 2003). These magnetic particles can also be used for diagnosis, imaging, and drug delivery.

Iron oxide nanocomposites or particle coated with biocompatible polymer(s) have shown some advantages, e.g., reducing aggregation, maintain magnetic stability, slowdown degrading process under physiological conditions, and lower toxicity (Mahmoudi et al. 2009). So far, they have shown promise for monitoring living cells by both MR and fluorescence imaging, as well as for drug delivery (Liong et al., 2008). 
As mentioned previously, iron oxide nanoparticles exhibit paramagnetic or superparamagnetic properties in a limited size range. Particles larger than $50 \mathrm{~nm}$ show superparamagnetic iron oxides (SPIO), whereas particles smaller than $50 \mathrm{~nm}$ show ultrasmall superparamagnetic property (USP). The smaller ones have the ability to enhance signal detection and increase resolution in the MRI (Foy et al., 2010; Tong et al., 2010). Therefore, the SPIO particles can be used for imaging tumors in the liver and spleen, while superparamagnetic particles for contrast agents for lymphography and angiography. However, the superparamagnetic particles do not retain their magnetism when the external magnetic field is removed, while other magnetic materials will become magnetized and aggregate.

In addition, the problem using magnetite or maghemite nanoparticles in clinic is often limited by the biocompatibility and toxicity of these particles (Martin et al., 2008; Pisanic Ii et al., 2007). This happens from the body's defense system, the reticulo-endothelial system (RES), trying to remove these particles from the bloodstream as they pass through the liver, spleen and lymph nodes. The rapid removal of the iron oxide nanoparticles reduces their life-time. This is why it is necessary to produce nanocomposites with special surface modifications. The surface modification of the particles allows the water-insoluble drugs to be loaded and stored for a long time (Liong et al., 2008; Son et al., 2005). Despite some progress, the challenges in using surface modified magnetic iron oxide nanoparticles still exist. More work needs to be performed in the future.

\subsection{Gas sensing}

Gas detection with high sensitivity and selectivity is essential for controlling industrial, waste, and vehicle emissions, household activity and environmental monitoring. In the past decades, many sensor devices have been developed for various gases such as $\mathrm{CO}, \mathrm{CO}_{2}, \mathrm{O}_{2}$, $\mathrm{O}_{3}, \mathrm{H}_{2}, \mathrm{NH}_{3}$ and $\mathrm{SO}_{2}$, as well as various organic vapors e.g., benzene, methanol, ethanol, amines and isopropanol (Jimenez-Cadena et al. 2007). Although semiconducting oxides have been quite useful as gas sensors, the operation at high temperatures often limits their functionality and applications. This has prompted the exploration of new materials that may offer higher sensing and selective capabilities than traditional ones.

Nanostructured metal oxides are one of the most commonly used materials for gas sensing because of the semiconductors make them possible for the electrical conductivity change when the surrounding atmosphere changes. Additionally, nanosized metal oxides exhibit high ratios of surface to volume, which favors the adsorption of gases on the particle surface, and hence increases the sensitivity in detection.

Iron oxide nanoparticles have shown good sensing capabilities toward hydrocarbon gases, CO and alcohols (Jimenez-Cadena et al., 2007; Han et al., 1996, 1999, and 2001). The studies by Zhang et al. (1996) and Tao et al. (1999) showed that $\mathrm{\gamma}-\mathrm{Fe}_{2} \mathrm{O}_{3}$ nanosensors exhibited good sensitivity and selectivity to a range of hydrocarbon gases such as LPG, petrol and $\mathrm{C}_{2} \mathrm{H}_{2}$ at $380{ }^{\circ} \mathrm{C}$, but poor sensitivity to $\mathrm{H}_{2}$ and CO. However, Nakatani and Matsuoka (1983) together with Lee and Choi (1990) reported that the $\mathrm{\gamma}^{-} \mathrm{Fe}_{2} \mathrm{O}_{3}$-based sensors exhibited good sensitivity to $\mathrm{H}_{2}$. This suggests that the gas-sensing characteristics of a nanosensor are related to its preparation process. 
The sensitivity of iron oxide-based nanosensors can be improved by various doping schemes as well as by changing the sensing material structure. For example, the thin film type sensors tend to exhibit higher sensitivity than bulk material sensor(s) (Mohapatra and Anand, 2010). Tao and co-workers (1999) have studied the sensing characteristics of $\mathrm{Y}_{2} \mathrm{O}_{3}$ doped $\gamma-\mathrm{Fe}_{2} \mathrm{O}_{3}$ towards hydrocarbon gases, $\mathrm{H}_{2}$ and $\mathrm{CO}$ and found that the addition of $\mathrm{Y}_{2} \mathrm{O}_{3}$ to $\gamma-\mathrm{Fe}_{2} \mathrm{O}_{3}$ resulted in a little difference in the sensitivity and selectivity compared with those made of pure $\gamma-\mathrm{Fe}_{2} \mathrm{O}_{3}$. Neri et al.(2002) have assessed the gas-sensing properties of $\mathrm{Zn}$ doped $\mathrm{Fe}_{2} \mathrm{O}_{3}$ thin films prepared by liquid phase deposition method. They observed that the addition of metal $\mathrm{Zn}$ can increase the sensitivity of the $\mathrm{Fe}_{2} \mathrm{O}_{3}$ thin film to $\mathrm{NO}_{2}$ below $250{ }^{\circ} \mathrm{C}$.

\subsection{Catalyst}

A catalyst can attract atoms and/or molecules, and then change the surface conductivity and other properties. Different from sensing material, the catalyst often converts itself into a different species through a chemical reaction. The iron oxides (hematite and magnetite) have been applied in industry to produce chemicals with high efficiencies, such as ammonia (Haber process) and hydrocarbons (Fischer-Tropsch process) (Teja and Koh, 2009). It is expected that the nanoparticles with high surface areas can perform much better to enhance the chemical reaction rates than that of bulk states. For hematite, its thermal-dynamically stable structure allows it for high temperature oxidation catalysis (Sivula et al., 2010).

The catalysis effect can also be enhanced by coupling metal nanoparticles on the surface (Jiang and Yu 2009; Zhong et al., 2007). Jiang et al (2009) have reported the synthesis of $\mathrm{Pd} / \mathrm{a}-\mathrm{Fe}_{2} \mathrm{O}_{3}$ nanocomposites at ambient conditions, which displayed superior lowtemperature catalytic activity toward $\mathrm{CO}$ oxidation to the pure $\mathrm{a}-\mathrm{Fe}_{2} \mathrm{O}_{3}$ nanoparticles. It was proposed that the enhanced catalytic activity was due to the reaction between oxygen adsorbed on the reduced sites of the support $\left(\mathrm{Fe}^{2+}\right)$ and $\mathrm{CO}$ adsorbed on $\mathrm{Pd}$ at the metaloxide interface, as shown in Fig. 6.

By using gold deposited iron oxide materials as a catalyst material, the oxidation and hydrogenation reaction of many organic compounds can be performed at much lower temperatures (Kung et al., 2007; Herzing et al., 2008; Lenz et al., 2009; Scirè et al., 2008). For example, Al-Sayari and co-workers (2007) have shown the dependence of the catalytic performance of $\mathrm{Au} / \mathrm{Fe}_{2} \mathrm{O}_{3}$ catalyst that the non-calcined $\mathrm{Au} / \mathrm{Fe}_{2} \mathrm{O}_{3}$ catalyst exhibited a high activity when $\mathrm{pH} \geq 5$, whereas the activity of calcined $\mathrm{Au} / \mathrm{Fe}_{2} \mathrm{O}_{3}$ catalyst was not influenced by the preparation conditions. Furthermore, the authors also noted that the catalytic activity of $\mathrm{Fe}_{2} \mathrm{O}_{3}$ toward $\mathrm{CO}$ oxidation was considerably lower than that of the $\mathrm{Au} / \mathrm{Fe}_{2} \mathrm{O}_{3}$ catalyst.

Maghemite and magnetite/carbon composites have been found to be good catalysts for reducing the concentration of undesirable nitrogen in acrylonitrile-butadiene-styrene (ABS) degradation oil (Brebu et al., 2001), whereas hematite can be used as a photocatalyst for the degradation of chlorophenol and azo dyes (Bandara et al., 2007), as well as a support material for gold in catalysts for the oxidation of carbon monoxide (CO) at low temperatures (Zhong et al., 2007).

The challenge of catalysis research being the reaction mechanism for these systems are still yet to be confirmed or explained, especially for the metal oxide/gold systems (Astruc et al., 2005). The reaction can be compared from titanium oxide/gold. The rutile phase of titania provides a support for gold, in which $\mathrm{CO}$ will convert mostly along the perimeter between the titania and 
gold (Haruta, 2002). In other studies, it was proposed that the nature of the support material has much greater influence on the reactive properties of the deposited nanoparticle, because the active and selective sites are formed by negative gold particles (Milone et al., 2007).
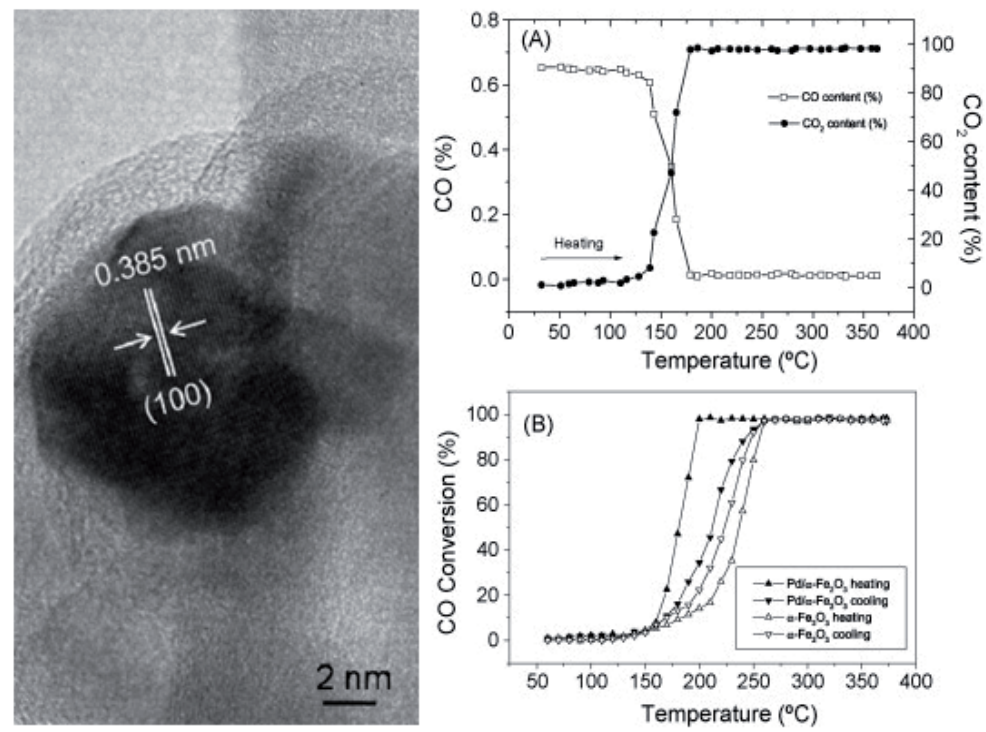

Fig. 6. HRTEM image of Pd particles binding on the surface of iron oxide, in which the

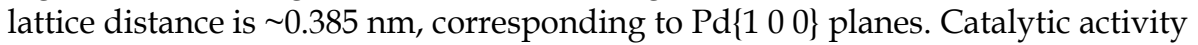
of $\mathrm{Pd} / \mathrm{a}-\mathrm{Fe}_{2} \mathrm{O}_{3}$ nanocomposites showing the dependence of $\mathrm{CO}$ oxidation (A);

(B) comparison of the catalytic activity of nanoparticles with and without doped palladium. Reprinted with permission from (Jiang and Yu, 2009).

\section{Theoretical simulations}

Beyond physical phenomena, theoretical methods have been developed and widely used to understand electronic, structure and forces of nanostructures (Cohen et al., 2008; Freund and Pacchioni, 2008; Hafner et al., 2006; Carter, 2008). Specifically, molecular dynamics (MD) method can be used for calculating interaction energies between surface modifiers and the modified matters, density functional theory (DFT) for binding energies, and Monte Carlo (MC) method for equilibrium properties (e.g., free energy, phase equilibrium) of particles. These methods have allowed researchers to understand and explain the growth mechanisms, structure, and functionalities of nanostructures (Hafner et al., 2006).

\subsection{Molecular dynamics}

MD simulation has been widely used for the study of the molecular behaviours in liquids and solids, examining material properties, and designing new materials, particularly for nanoparticles and nanocomposites. The MD method allows one to predict the time evolution of a system of interacting particles (atoms or molecules) and estimate relevant physicochemical properties. Specifically, it can calculate and simulate the interaction energies among atoms/molecules, which can help understand atomic positions, velocities, 
and forces. Thus, the macroscopic properties (e.g., pressure, energy, heat capacities) can be derived by means of statistical mechanics.

In our recent work, the MD method was used to explain the interactions between various goethite surfaces and surfactants of the nanorods. The simulation results of the side wall $(x y 0)$ surfaces with six different surfactants have been reported (Yue et al. 2010, 2011). The positively charged surfactants, CTAB (Fig. 7) and tetraethylammonium chloride (TEAC), were found to interact greatly with the side wall $(x y 0)$ of the nanorod, while the polymeric polyethylene glycol (PEG) and polyvinylpyrrolidone (PVP) and anionic surfactants (AOT) and Sodium Dodecyl Sulfate (SDS) were not suitable because of the low interaction energies among the surfaces. This is caused by the differences in the active sites on different surfaces (Kim et al. 2007). The ratios of iron and oxygen can vary greatly for different surfaces, in which the packing and exposure of atoms along a particular crystal plane will therefore determine the strength of adsorbed surface molecules. The simulation could provide quantitative information toward the interaction between surfactants and goethite surface(s), and hence understand the particle formation and growth mechanisms.

Through a similar MD simulation, the adsorption of minerals has been explored. Kerisit et al. (2006) simulated the interactions for electrolyte solutions to determine the surface properties of monovalent ions, such as $\mathrm{NaCl}, \mathrm{CsCl}$, and $\mathrm{CsF}$ on the (100) goethite surface. The calculations showed a structured interfacial region is in the first $15 \AA$ on the surface. The structure of the mineral surface will also affect the arrangement and orientation of the water molecules, and hence the diffusive properties and distribution of the ionic species. In comparison, the adsorption of sodium ions is stronger than cesium ions because the former can occupy an interstitial site of mineral(s) due to smaller size.

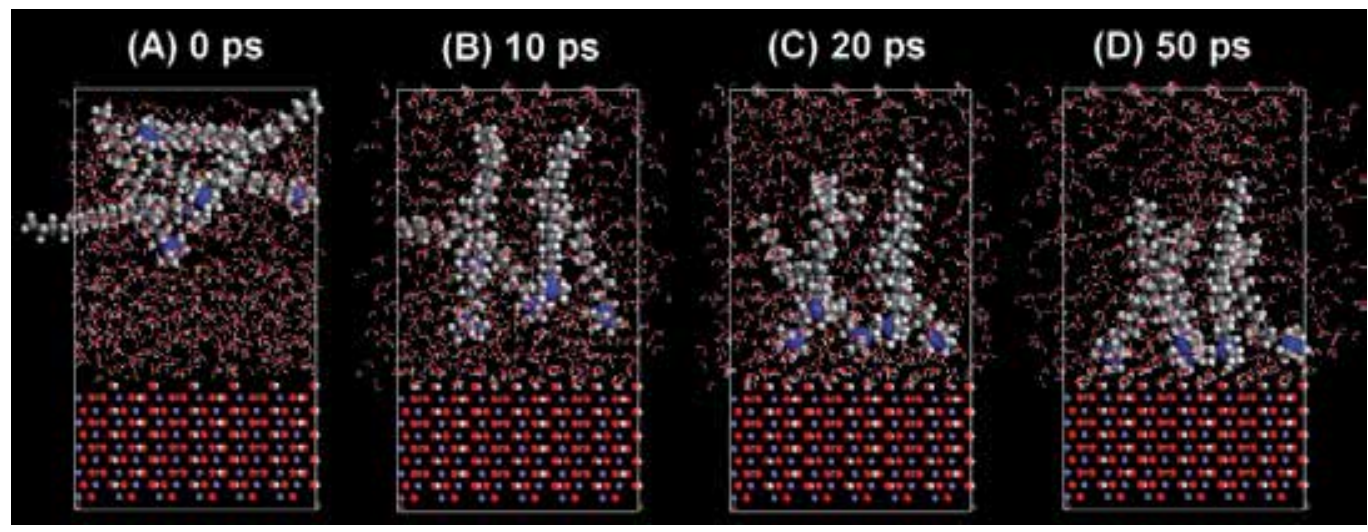

Fig. 7. MD simulation of CTAB molecular adsorption on the goethite crystal (010) surface at different time: (A) 0 ps, (B) 10 ps, (C) 20 ps, and (D) 50 ps. Reprinted with permission from (Yue et al., 2010).

Similarly, MD simulation was also employed to explain the growth mechanisms of akaganéite nanorods (Yue et al., 2011), as shown in Fig. 8, in which the atomic concentration profiles of various anions on different crystalline surfaces were compared. With the assistance of experimental techniques such as transmission electron microscopy (TEM), energy dispersive spectroscopy (EDS), and x-ray diffraction (XRD), the role of chloride ions in the lattice 
structure and forming $\beta$-FeOOH rodlike structure was determined. The analysis showed that the chloride ions were a small size, as well as having an intermediate interaction on the tunnel structure of the (001) surface, while the tight packing of the (100) and (110) surfaces does not allow interaction with any ions. The information was useful for the development of the simulation model, which explained the filling of the tunnel structure along (001) direction.
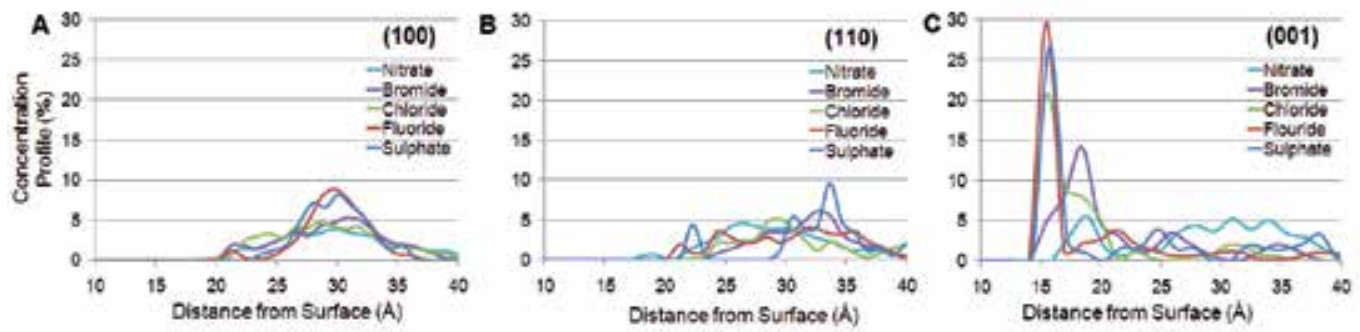

Fig. 8. The concentration profiles of various anions on the crystal surface of akaganéite nanorods: (A) (100); (B) (110); and (C) (001) plane.

Reprinted with permission from (Yue et al., 2011).

This MD method is used not only for small organic molecules but also for metallic nanoclusters. In our recent work (Yue et al., 2011), the $\mathrm{Fe}_{3} \mathrm{O}_{4}(111)$ surface modified with various surfactants, polymers, and silica, followed by the deposition of a Au nanoparticle was simulated by MD method). The results show the dynamic motion of the molecules on the $\mathrm{Fe}_{3} \mathrm{O}_{4}(111)$ surface, followed by the encapsulation of the $\mathrm{Au}$ nanoparticle surface. Through an analysis of the concentration profile, it reveals that $-\mathrm{NH}_{2}$ groups within the molecule(s) are useful for attracting gold atoms, as shown in Fig. 9. Moreover, onedimensional chainlike molecules allow higher flexibility to move toward the Au surface compared with three-dimensional structure (amorphous or polymerized silica)

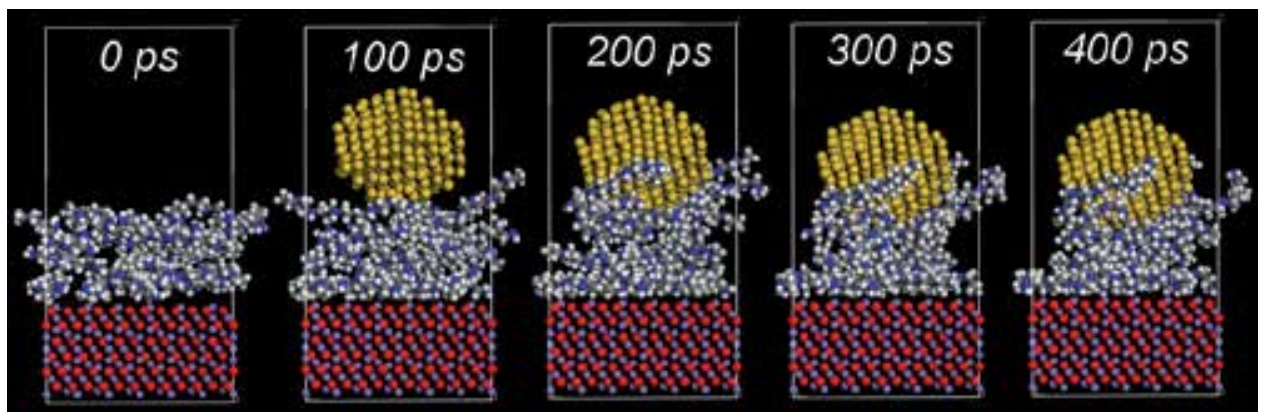

Fig. 9. Snapshots of PEI coating onto the surface of $\mathrm{Fe}_{3} \mathrm{O}_{4}(111)$ and the addition of a AuNP at various times. Reprinted with permission from (Yue et al., 2011).

This theoretical method is available for predicting the interaction energies and adsorption sites of molecules on the iron oxides surfaces. Aquino et al.(2006) simulated various molecules such as water, acetic acid, acetate, 2,4-dichlorophenoxyacetic acid, and benzene on the goethite (110) surface. The results show that two OH types, hydroxo and $\mu$-hydroxo, were able to bend and act as proton acceptors, while the third type, $\mu_{3}$-hydroxo, acts only as proton donor due to its more pronounced rigidity. 
However, MD is a classical simulation method which uses parameterized potentials (or forcefields), which cannot quantify electronic information of nanostructures. This method is limited to its accuracy, although the results can be obtained within a realistic period of time and larger length scales (Rustad et al., 2003; Zeng et al., 2008).

\subsection{Density functional theory}

DFT is another powerful simulation technique for understanding atom/molecular binder energies. The calculation is performed by using approximation method to simplify the Schrödinger's equation (Lado-Touriño and Tsobnang, 2000).

Many DFT studies have emphasized on the structural, electronic, catalytic, and magnetic properties of metal-oxide, such as $\mathrm{Fe}_{2} \mathrm{O}_{3}$, and $\mathrm{Al}_{2} \mathrm{O}_{3}$ (Alvarez-Ramirez et al., 2004; Ma et al., 2006; Mason et al., 2009; Rohrbach et al., 2004; Rollmann et al., 2004; Zhong et al., 2008; Mason et al., 2010). It has been extended into other systems, e.g., carbon nanotubes or graphene ( $\mathrm{Li}$ et al., 2010; Chattaraj et al., 2009), transition metals (Cramer et al., 2009), semiconductors (Jin et al., 2011), and metals (e.g., Pd, Au, Cu) (Yang et al., 2007).

For example, Wong et al.(2011) demonstrated that the electronic and geometric structure of different metald $\left(\mathrm{M}=\mathrm{Au}, \mathrm{Pt}, \mathrm{Pd}\right.$, or $\mathrm{Ru}$ ) bilayers particularly on the $\mathrm{a}-\mathrm{Fe}_{2} \mathrm{O}_{3}(0001)$ support surface (Fig. 10). The analysis shows that the synergistic effect depends on the localized electron gain, electron transfer from Fe atoms to the $\mathrm{dz}^{2}$ orbital of the metal bilayer, and interfacial metallic/ionic bonding. These effects were most pronounced for surfaces modified with $\mathrm{Pt}$ or $\mathrm{Ru}$, while the Au bilayer is the most stable due to its low $\mathrm{a}-\mathrm{Fe}_{2} \mathrm{O}_{3}$ lattice deformation and minimal surface of Fe atom spin quenching. Tuning the Ru bilayer can provide an optimal balance of these factors, and hence enhance the catalytic activity.
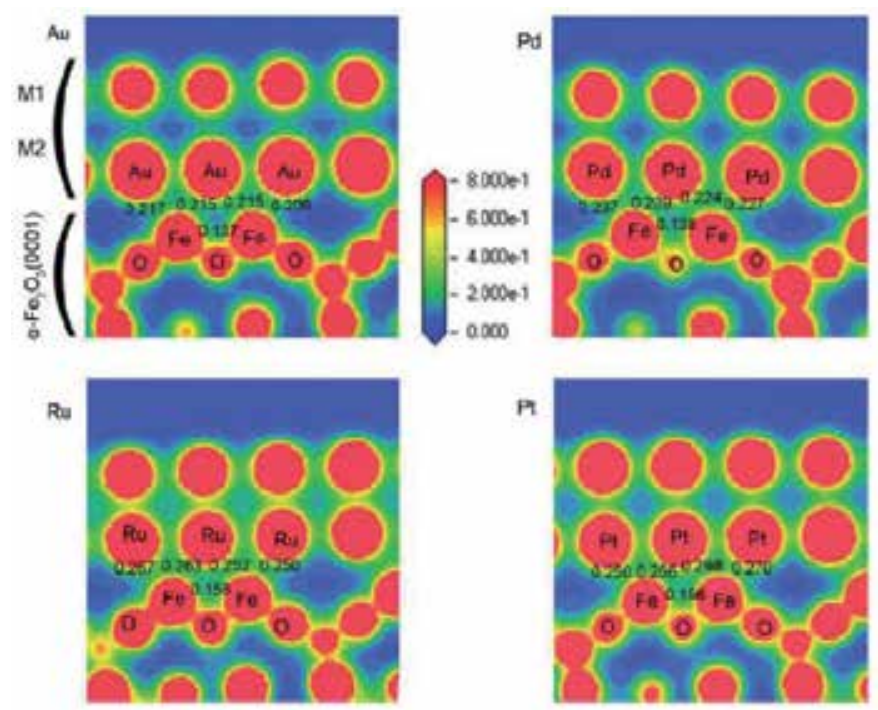

Fig. 10. Electron density contour maps of $\mathrm{M} / \mathrm{a}-\mathrm{Fe}_{2} \mathrm{O}_{3}(0001)$ interfaces, where $\mathrm{M}=\mathrm{Ru}, \mathrm{Pd}$, $\mathrm{Au}$, and $\mathrm{Pt}$, respectively, and the electron density is in the range $0.0-0.8 \mathrm{eV} / \AA^{3}$. Reprinted with permission from (Wong et al., 2011). 
Despite some success, the DFT method still has limitations in accurately describing the van der Waals interactions, phonon dispersion, spin-and space-degenerate states, strongly conjugated $\Pi$ systems, localization and delocalization errors for band gaps. Moreover, the DFT is difficult to solve the problems related to long range interactions and dispersion forces for complex biological systems. So far, the development of DFT technique is still demanded.

Besides DFT and MD simulations, Monte Carlo (MC) method, a stochastic method, has been employed to generate a statistical or probabilistic model for understanding particular systems. The MC method can be used to predict the crystalline structure of $\beta$-FeOOH (Kwon et al. 2006). By combination of quantitative X-ray structural analysis, the MC simulation has been used for characterizing the atomic-scale structure with and without chromium atoms. The results showed that the $\beta$-FeOOH particles containing chromium is distorted, while the particles without chromium is similar to its ideal structure. The combination of the experimental and $\mathrm{MC}$ simulation method can distinguish the differences between $\mathrm{FeO}_{6}$ and $\mathrm{CrO}_{6}$ octahedral units. However, this $\mathrm{MC}$ method can only provide information on equilibrium properties (e.g., free energy, phase equilibrium), but limited to the nonequilibrium systems.

\section{Summary}

This Chapter briefly overviews some experimental methods (hydrothermal, co-precipitate and microemulsion methods) used for the synthesis and surface modifications of lowdimensional iron oxide nanostructures with desirable functional properties (gas sensing, catalytic, magnetic, and biochemical properties), and a few theoretical simulation techniques (MD, DFT, and MC) for fundamental understandings. However, the challenges still exist. Experimentally, one of the big challenges is how to produce iron oxide nanostructures with desired characteristics (shape, size, and surface properties) for target applications. Theoretically, DFT and MD simulations are limited to the large-scale calculations (e.g., mesoscopic structure with size range of $0.1-10 \mu \mathrm{m})$ due to the current restraints in computational capability.

To overcome the limitations, the development of simple, cost-saving, and effective strategies for iron oxide and other nanostructures with desirable functional properties is highly demanded. For the computational modelings and simulation methods, much work needs to be performed in two directions: (i) to develop new and improved simulation techniques for large time and length scales; and (ii) to integrate diverse simulation techniques (DFT, MD, $\mathrm{MC}$ and others) on different levels together to form a powerful tool for exploring the structural, dynamic, and mechanical properties of nanomaterials and nanosystems. This is crucial to predict process-structure-property relationships in material design, optimization, and manufacturing.

\section{Acknowledgement}

We gratefully acknowledge the financial support of the Australia Research Council (ARC) the ARC Centres of Excellence for Functional Nanomaterials and ARC projects. The authors acknowledge access to the UNSW node of the Australian Microscopy \& Microanalysis Research Facility (AMMRF). 


\section{References}

Al-Sayari, S., Carley, A.F., Taylor, S.H. \& Hutchings, G.J. (2007). Au/ $\mathrm{ZnO}$ and $\mathrm{Au} / \mathrm{Fe}_{2} \mathrm{O}_{3}$ catalysts for $\mathrm{CO}$ oxidation at ambient temperature: comments on the effect of synthesis conditions on the preparation of high activity catalysts prepared by coprecipitation. Top. Catal., 44: 123-128.

Almeida, T.P., Fay, M., Zhu, Y. and Brown, P.D. (2009). Process Map for the Hydrothermal Synthesis of $\mathrm{a}-\mathrm{Fe}_{2} \mathrm{O}_{3}$ Nanorods. J. Phys. Chem. C, 113(43): 18689-18698.

Alvarez-Ramirez, F., Martinez-Magadan, J.M., Gomes, J.R.B., \& Illas, F. (2004), On the geometric structure of the (0001) hematite surface, Surface Sci., 558, 4-14.

Aquino, A.J.A., Tunega, D., Haberhauer, G., Gerzabek, M.H. and Lischka, H. (2006). Quantum Chemical Adsorption Studies on the (110) Surface of the Mineral Goethite. J. Phys. Chem. C, 111(2): 877-885.

Astruc, D., Lu, F. and Aranzaes, J.R. (2005). Nanoparticles as Recyclable Catalysts: The Frontier between Homogeneous and Heterogeneous Catalysis. Angew. Chem. Int. Ed., 44(48): 7852-7872.

Babic, M., Horák, D., Trchová, M., Jendelová, P., Glogarová, K.i., Lesný, P., Herynek, V., Hájek, M. and Syková, E. (2008). Poly(l-lysine)-Modified Iron Oxide Nanoparticles for Stem Cell Labeling. Bioconjugate Chem., 19(3): 740-750.

Bandara, J., Klehm, U. and Kiwi, J. (2007). Raschig rings- $\mathrm{Fe}_{2} \mathrm{O}_{3}$ composite photocatalyst activate in the degradation of 4-chlorophenol and Orange II under daylight irradiation. Appl. Catal. B, 76(1-2): 73-81.

Boguslavsky, Y. \& Margel, S. (2008). Synthesis and characterization of poly(divinylbenzene)coated magnetic iron oxide nanoparticles as precursor for the formation of airstable carbon-coated iron crystalline nanoparticles. J. Colloid and Interface Sci., 317(1): 101-114.

Bomati-Miguel, O., Rebolledo, A.F. \& Tartaj, P. (2008). Controlled formation of porous magnetic nanorods via a liquid/liquid solvothermal method. Chem. Commun., (35): 4168-4170.

Brebu, M., Uddin, M.A., Muto, A., Sakata, Y. \& Vasile, C. (2001). Catalytic degradation of acrylonitrile-butadiene-styrene into Fuel Oil 1. The effect of iron oxides on the distribution of nitrogen-containing compounds. Energy \& Fuels, 15(3): 559-564.

Carter, E.A. (2008). Challenges in modeling materials properties without experimental input. Science, 321(5890): 800-803.

Catherine, C.B. \& Adam, S.G.C. (2003). Functionalisation of magnetic nanoparticles for applications in biomedicine. J. Phys. D: Appl. Phys., 36(13): R198.

Chattaraj, P.K. (2009). Chemical reactivity theory: a density functional view, pp. 9781420065435.

Chen, Z.P., Zhang, Y., Zhang, S., Xia, J.G., Liu, J.W., Xu, K. \& Gu, N. (2008). Preparation and characterization of water-soluble monodisperse magnetic iron oxide nanoparticles via surface double-exchange with DMSA. Colloids Surf. A, 316(1-3): 210-216.

Chibowski, S., Patkowski, J. \& Grzadka, E. (2009). Adsorption of polyethyleneimine and polymethacrylic acid onto synthesized hematite. J. Colloid and Interface Sci., 329(1): $1-10$.

Chin, A.B. and Yaacob, I.I. (2007). Synthesis and characterization of magnetic iron oxide nanoparticles via w/o microemulsion and Massart's procedure. J. Mater. Process. Tech., 191(1-3): 235-237. 
Cohen, A.J., Mori-Sánchez, P. \& Yang, W. (2008). Insights into current limitations of density functional theory. Science, 321(5890): 792-794.

Cornell, R.M., Schuwertmann, U. The iron oxides: structure, properties, reactions, occurrences, and uses, Wiley-VCH, Weinheim, 2003.

Cramer, C.J. \& Truhlar, D.G. (2009), Density functional theory for transition metals and transition metal chemistry. Phys. Chem. Chem. Phys. 11: 10757-10816.

Deng, Y.-H., Wang, C.-C., Hu, J.-H., Yang, W.-L. \& Fu, S.-K. (2005). Investigation of formation of silica-coated magnetite nanoparticles via sol-gel approach. Colloids Surf. A, 262(1-3): 87-93.

Di Marco, M., Guilbert, I., Port, M., Robic, C., Couvreur, P. and Dubernet, C. (2007). Colloidal stability of ultrasmall superparamagnetic iron oxide (USPIO) particles with different coatings. Int. J. Pharmaceutics, 331(2): 197-203, 0378-5173.

Dong, W.T., Zhu, C.S. (2002). Use of Ethylene oxide in the sol-gel synthesis of a- $\mathrm{Fe}_{2} \mathrm{O}_{3}$ nanoparticles from Fe (III) Salt. J. Mater. Chem., 12 (6): 1676-1683.

Erogbogbo, F., Yong, K.T., Hu, R., Law, W.C., Ding, H., Chang, C.W., Prasad, P.N. \& Swihart, M.T. (2010). Biocompatible magnetofluorescent probes: luminescent silicon quantum dots coupled with superparamagnetic iron(III) oxide. ACS Nano, 4: 5131-5138.

Foy, S.P., Manthe, R.L., Foy, S.T., Dimitrijevic, S., Krishnamurthy, N. \& Labhasetwar, V. (2010). Optical imaging and magnetic field targeting of magnetic nanoparticles in tumors. ACS Nano, 4(9): 5217-5224.

Freund, H.-J. \& Pacchioni, G. (2008). Oxide ultra-thin films on metals: new materials for the design of supported metal catalysts. Chem. Soc. Rev., 37(10): 2224-2242.

Garcia, K.E., Barrero, C.A, Morales, A.L. \& Greneche, J.M. (2009). Magnetic structure of synthetic akaganeite: A review of Mössbauer data. Rev. Fac. Ing. Univ. Antioquia, 49: 185-191.

González-Carreño, T., Morales M.P., Gracia, M. \& Serna, C.J. (1993). Preparation of uniform amma- $\mathrm{Fe}_{2} \mathrm{O}_{3}$ particles with nanometer size by spray pyrolysis. Mater. Lett., 18: 151155.

Guo, Z., Park, S., Wei, S., Pereira, T., Moldovan, M., BKarki, A., Young, D. P. \& Hahn, H. T. (2007). Flexible high-loading particle-reinforced polyurethane magnetic nanocomposite fabrication through particle-surface-initiated polymerization. Nanotech. 18: 335704.

Hafner, J., Wolverton, C. \& Ceder, G. (2006). Toward computational materials design: the impact of density functional theory on materials research. MRS Bulletin, 31: 659-668

Han, J.S., Yu, A.B., He, F.J. \& Yao, T (1996). A study of the gas sensitivity of alpha- $\mathrm{Fe}_{2} \mathrm{O}_{3}$ sensors to $\mathrm{CO}$ and $\mathrm{CH}_{4}$. J. Mater. Sci. Lett. 15: 434-436.

Han, J.S., Bredow, T., Davey, D.E., Yu, A.B. \& Mulcahy, D.E. (2001). The effect of Al addition on the gas sensing properties of $\mathrm{Fe}_{2} \mathrm{O}_{3}$-based sensors. Sens. and Actuators B 75: 1823.

Han, J.S., Davey, D.E., Mulcahy, D.E. \& Yu, A.B. (1999). An investigation of gas response of alpha- $\mathrm{Fe}_{2} \mathrm{O}_{3}(\mathrm{Sn})$-based gas sensor. Sens. and Actuators B 61: 83-91.

Haruta, M. (2002). Catalysis of gold nanoparticles deposited on metal oxides. CATTECH, 6(3): 102-115. 
Herzing, A.A., Kiely, C.J., Carley, A.F., Landon, P. \& Hutchings, G.J. (2008). Identification of active gold nanoclusters on iron oxide supports for $\mathrm{CO}$ oxidation. Science, 321(5894): 1331-1335.

Hyeon, T., Lee, S.S., Park, J., Chung, Y. \& Na, H.B. (2001). Synthesis of highly crystalline and monodisperse maghemite nanocrystallites without a size-selection Process. J. Am. Chem. Soc., 123(51): 12798-12801.

Iida, H., Takayanagi, K., Nakanishi, T. \& Osaka, T. (2007). Synthesis of $\mathrm{Fe}_{3} \mathrm{O}_{4}$ nanoparticles with various sizes and magnetic properties by controlled hydrolysis. J. Colloid and Interface Sci., 314(1): 274-280.

Ilani, S., Rosenfeld, A. \& Dvorachek, M. (1999). Mineralogy and chemistry of a Roman Remedy from Judea, Israel. J. Archaeological Sci., 26(11): 1323-1326.

Im, S.H., Herricks, T., Lee, Y.T. \& Xia, Y. (2005). Synthesis and characterization of monodisperse silica colloids loaded with superparamagnetic iron oxide nanoparticles. Chem. Phys. Lett., 401(1-3): 19-23.

Ito, A., Shinkai, M., Honda, H. \& Kobayashi, T. (2005). Medical application of functionalized magnetic nanoparticles. J. Biosci. and Bioeng., 100(1): 1-11.

Jia, C.-J., Sun, L.-D., Yan, Z.-G., You, L.-P., Luo, F., Han, X.-D., Pang, Y.-C., Zhang, Z. \& Yan, C.-H. (2005). Single-crystalline iron oxide nanotubes. Angew. Chem. Int. Ed., 44(28): 4328-4333.

Jiang, X.C., Yu, A.B., Yang, W.R., Ding, Y., Xu, C. \& Lam, S. (2010). Synthesis and growth of hematite nanodiscs through a facile hydrothermal approach. J. Nanopart. Res., 12: 877-893.

Jiang, X.C. \&Yu, A.B. (2009). Synthesis of $\mathrm{Pd} /$ alpha- $\mathrm{Fe}_{2} \mathrm{O}_{3}$ nanocomposites for catalytic CO oxidation. J. Mater. Process. Tech., 209(9): 4558-4562.

Jimenez-Cadena, G., Riu, J. \& Rius, F.X. (2007). Gas sensors based on nanostructured materials. Analyst, 132(11): 1083-1099.

Jin, D., Wang, W., Rahman, A., Lizhen, J., Zhang, H., Li, H., He, P., \& Bao, S. (2011), Study on the interface between the organic and inorganic semiconductors, Appl. Surface Sci. 257: 4994-4999.

Kandori, K., Yamoto, Y. \& Ishikawa, T. (2005). Effects of vinyl series polymers on the formation of hematite particles in a forced hydrolysis reaction. J. Colloid and Interface Sci., 283(2): 432-439.

Kerisit, S., Ilton, E.S. \& Parker, S.C. (2006). Molecular Dynamics simulations of electrolyte solutions at the (100) goethite surface. J. Phys. Chem. B, 110(41): 20491-20501.

Kim, D.K., Mikhaylova, M., Zhang, Y. \& Muhammed, M. (2003). Protective coating of superparamagnetic iron oxide nanoparticles. Chem. Mater., 15(8): 1617-1627.

Kim, H.-G., Kim, D.-W., Oh, C., Park, S.-H. \& Oh, S.-G. (2007). Preparation of rod-type ferric oxyhydroxide particles by forced hydrolysis in the presence of a cationic surfactant. J. Ceramic Process Res., 8(3): 172-176.

Kung, M.C., Davis, R.J. \& Kung, H.H. (2007). Understanding Au-Catalyzed low-temperature CO oxidation. J. Phys. Chem. C, 111(32): 11767-11775.

Kwon, S.K., Suzuki, S., Saito, M., Kamimura, T., Miyuki, H. \& Waseda, Y. (2006). Atomicscale structure of beta-FeOOH containing chromium by anomalous $\mathrm{X}$-ray scattering coupled with reverse Monte Carlo simulation. Corrosion Sci., 48(6): 1571-1584.

Lado-Touriño, I. \& Tsobnang, F. (2000). Using computational approaches to model hematite surfaces. Computational Mater. Sci., 17(2-4): 243-248. 
Lattuada, M. \& Hatton, T.A. (2006). Functionalization of monodisperse magnetic nanoparticles. Langmuir, 23(4): 2158-2168.

Laurent, S., Forge, D., Port, M., Roch, A., Robic, C., Eliat, L.V. \& Muller, R.N. (2008). Magnetic iron oxide nanoparticles: Synthesis, stabilization, vectorization physicochemical characterizations and biological applications. Chem. Rev., 108: 2064-2110.

Lee, D.-D. \& Choi, D.-H. (1990). Thick-film hydrocarbon gas sensors. Sens. Actuators B, 1(16): 231-235.

Lee, J., Isobe, T. \& Senna, M. (1996). Preparation of ultrafine $\mathrm{Fe}_{3} \mathrm{O}_{4}$ particles by precipitation in the presence of PVA at high pH. J. Colloid Interface Sci., 177(2): 490-494.

Lee, Y., Lee, J., Bae, C.J., Park, J.G., Noh, H.J., Park, J.H. \& Hyeon, T. (2005). Large-scale synthesis of uniform and crystalline magnetite nanoparticles using reverse micelles as nanoreactors under reflux conditions. Adv. Funct. Mater., 15(3): 503-509.

Lenz, J., Campo, B.C., Alvarez, M. \& Volpe, M.A. (2009). Liquid phase hydrogenation of [alpha],[beta]-unsaturated aldehydes over gold supported on iron oxides. J. Catal., 267(1): 50-56.

Li, Y., Zhou, Z., Yu, G., Chen, W., \& Chen, Z. (2010), CO catalytic oxidation on ironembedded graphene: computational quest for low-cost nanocatalysts, J Phys. Chem. C, 114: 6250-6254.

Li, Z., Chen, H., Bao, H. \& Gao, M. (2004). One-pot reaction to synthesize water-soluble magnetite nanocrystals. Chem. Mater., 16(8): 1391-1393.

Li, Z., Lai, X., Wang, H., Mao, D., Xing, C. \& Wang, D. (2009). Direct hydrothermal synthesis of single-crystalline hematite nanorods assisted by 1,2-propanediamine. Nanotechnology, 20: 1-9.

Liong, M., Lu, J., Kovochich, M., Xia, T., Ruehm, S.G., Nel, A.E., Tamanoi, F. \& Zink, J.I. (2008). Multifunctional inorganic nanoparticles for imaging, targeting, and drug delivery. ACS Nano, 2(5): 889-896.

Liu, H., Wei, Y., Li, P., Zhang, Y. \& Sun, Y. (2007). Catalytic synthesis of nanosized hematite particles in solution. Mater. Chem. Phys., 102(1): 1-6.

López-Quintela, M.A. \& Rivas, J. (1993). Chemical Reactions in Microemulsions: A Powerful Method to Obtain Ultrafine Particles. J. Colloid and Interface. Sci., 158(2): 446-451.

Lu, A.H., Salabas, E. \& Schüth, F. (2007). Magnetic Nanoparticles: Synthesis, Protection, Functionalization, and Application. Angew. Chem. Int. Ed., 46(8): 1222-1244.

Lu. J., Yang. S., Ng, K.M., Su, C.H.,Yeh, C.S., Wu, Y.N. \& Shieh, D.B. (2006). Solid-State synthesis of monocrystalline iron oxide nanoparticle-based ferrofluid suitable for magnetic resonance imaging contrast application. Nanotechnology, 17: 5812-5820.

Lyon, J.L., Fleming, D.A., Stone, M.B., Schiffer, P. \& Williams, M.E. (2004). Synthesis of Fe oxide core/ Au shell nanoparticles by iterative hydroxylamine seeding. Nano Lett., 4(4): 719-723.

Ma, X.Y., Liu, L., Jin, J.J., Stair, P.C., \& Ellis, D.E. (2006), Experimental and theoretical studies of adsorption of $\mathrm{CH}_{3}$ center dot on alpha- $\mathrm{Fe}_{2} \mathrm{O}_{3}(0001)$ surfaces. Surf. Sci., 600: 287485.

Mahmoudi, M., Simchi, A., Milani, A.S. \& Stroeve, P. (2009). Cell toxicity of superparamagnetic iron oxide nanoparticles. J. Colloid and Interface Sci., 336(2): 510518,0021-9797. 
Mammeri, F., Bourhis, E.L., Rozes, L. \& Sanchez, C. (2005). Mechanical properties of hybrid organic-inorganic materials. J. Mater. Chem., 15(35-36): 3787-3811.

Martin, A.L., Bernas, L.M., Rutt, B.K., Foster, P.J. \& Gillies, E.R. (2008). Enhanced cell pptake of superparamagnetic iron oxide nanoparticles functionalized with dendritic guanidines. Bioconjugate Chem., 19(12): 2375-2384.

Mason, S.E., Iceman, C.R., Tanwar, K.S., Trainor, T.P., \& Chaka, A.M. (2009). Pb(II) adsorption on isostructural hydrated alumina and hematite (0001) surfaces: A DFT study, J. Phys. Chem. C, 113: 2159-2170.

Massart, R. (1981). Preparation of aqueous magnetic liquids in alkaline and acidic media. IEEE Trans Magn MAG, I7: 1247-1248.

Milone, C., Crisafulli, C., Ingoglia, R., Schipilliti, L. \& Galvagno, S. (2007). A comparative study on the selective hydrogenation of [alpha],[beta] unsaturated aldehyde and ketone to unsaturated alcohols on Au supported catalysts. Catal. Today, 122: 341351.

Mohapatra, M. \& Anand, S. (2010), Synthesis and applications of nano-structured iron xxides/hydroxides - A Review. Int. J. Eng. Sci. and Tech., 2(8): 127-146.

Mohapatra, S., Pramanik, N., Mukherjee, S., Ghosh, S. \& Pramanik, P. (2007). A simple synthesis of amine-derivatised superparamagnetic iron oxide nanoparticles for bioapplications. J. Mater. Sci., 42(17): 7566-7574.

Molday, R.S. \& Mackenzie, D. (1982). J. Immun. Methods, 52: 353-367.

Morais, P.C., Oliveira, A.C., Tronconi, A.L., Goetze, T. \& Buske, N. (2003). Photoacoustic spectroscopy: a promising technique to investigate magnetic fluids. IEEE Trans. Magn., 39(5): 2654-2656.

Morber, J.R, Ding, Y., Haluska, M. S., Li, Y., Liu, J. P., Wang, Z. L. \& Snyder, R. L. (2006). J. Phys. Chem. B, 110: 21672.

Mornet, S., Vasseur, S., Grasset, F. \& Duguet, E. (2004). Magnetic nanoparticle design for medical diagnosis and therapy. J. Mater. Chem., 14(14): 2161-2175.

Morrissey, J. \& Guerinot, M.L. (2009). Iron uptake and transport in plants: The good, the bad, and the ionome. Chem. Rev., 109(10): 4553-4567.

Nakatani, Y. \& Matsuoka, M. (1983). Some Electrical Properties of $\mathrm{\gamma}^{-} \mathrm{Fe}_{2} \mathrm{O}_{3}$ Ceramics. Jpn. J. Appl. Phys., 22: 232-239.

Neri, G., Bonavita, A., Galvagno, S., Siciliano, P. \& Capone, S. (2002). CO and $\mathrm{NO}_{2}$ sensing properties of doped- $\mathrm{Fe}_{2} \mathrm{O}_{3}$ thin films prepared by LPD. Sens. Actuators B, 82(1): 4047,0925-4005.

Pascal C., Pascal J.L., Favier F., Elidrissi Moubtassim M.L. \& Payen C. (1999). Electrochemical synthesis for the control of $\gamma-\mathrm{Fe}_{2} \mathrm{O}_{3}$ nanoparticle size. Morphology, microstructure, and magnetic behavior. Chem. Mater. 11: 141-147.

Pedro, T. Morales, M. del P., Veintenillas-verdaguer, S., Gonzalez-carreno, T. \& Serma, C.J. (2003). The preparation of magnetic nanoparticles for applications in biomedicine. J. Phys. D: Appl. Phys., 36(13): R182.

Pinho, S.L.C., Pereira, G.A., Voisin, P., Kassem, J., Bouchaud, V.r., Etienne, L., Peters, J.A., Carlos, L., Mornet, S.p., Geraldes, C.F.G.C., Rocha, J.O. \& Delville, M.-H. (2010). Fine tuning of the relaxometry of $\gamma-\mathrm{Fe}_{2} \mathrm{O}_{3} @ \mathrm{SiO}_{2}$ nanoparticles by tweaking the silica coating thickness. ACS Nano, 4(9): 5339-5349. 
Pisanic Ii, T.R., Blackwell, J.D., Shubayev, V.I., Fiñones, R.R. \& Jin, S. (2007). Nanotoxicity of iron oxide nanoparticle internalization in growing neurons. Biomater. 28(16): 25722581.

Rockenberger, J., Scher, E.C. \& Alivisatos, A.P. (1999). A new nonhydrolytic single-precursor approach to surfactant-capped nanocrystals of transition metal oxides. J. Am. Chem. Soc., 121(49): 11595-11596.

Rohrbach, A., Hafner, J., \& Kresse, G. (2004). Ab initio study of the (0001) surfaces of hematite and chromia: Influence of strong electronic correlations. Phys. Rev. B, 70: 125426.

Rollmann, G., Rohrbach, A., Entel, P. \& Hafner, J. (2004), First-principles calculation of the structure and magnetic phases of hematite. Phys. Rev. B, 69: 165107.

Sahu, K.K., Rath, C., Mishra, N.C. \& Anand, S. (1997). Microstructural and magnetic studies on hydrothermally prepared hematite. J. Colloid Interface Sci., 185(2): 402-410.

Scirè, S., Crisafulli, C., Minicò, S., Condorelli, G.G. \& Di Mauro, A. (2008). Selective oxidation of $\mathrm{CO}$ in $\mathrm{H}_{2}$-rich stream over gold/iron oxide: An insight on the effect of catalyst pretreatment. J. Molecular Catal. A: Chem. 284(1-2): 24-32.

Sivula, K., Zboril, R., Le Formal, F., Robert, R., Weidenkaff, A., Tucek, J., Frydrych, J. \& Gratzel, M. (2010). Photoelectrochemical water splitting with mesoporous hematite prepared by a solution-based colloidal approach. J. Am. Chem. Soc., 132(21): 74367444.

Soler, M.A, Alcantara, G.B., Soares, F.Q., Viali, W.R., Sartoratto, P.P., Fernandez, J.R., da Silva, S.W., Garg, V.K., Oliveira, A.C. \& Morais, P.C. (2007). Study of molecular surface coating on the stability of maghemite nanoparticles. Surf. Sci., 601(18): 39213925.

Son, S.J., Reichel, J., He, B., Schuchman, M. \& Lee, S.B. (2005). Magnetic nanotubes for magnetic-field-assisted bioseparation, biointeraction, and drug delivery. J. Am. Chem. Soc., 127(20): 7316-7317.

Sousa, M.H., Rubim, J.C., Sobrinho, P.G. \& Tourinho, F.A. (2001). Biocompatible magnetic fluid precursors based on aspartic and glutamic acid modified maghemite nanostructures. J. Magn. Magn. Mater., 225(1-2): 67-72.

Sun, Q., Reddy, Marquez, M., Jena, P., Gonzalez, C. \& Wang, Q. (2007). Theoretical Study on Gold-Coated Iron Oxide Nanostructure: Magnetism and Bioselectivity for Amino Acids. J. Phys. Chem. C, 111(11): 4159-4163.

Sun, S., Zeng, H., Robinson, D.B., Raoux, S., Rice, P.M., Wang, S.X. \& Li, G. (2003). Monodisperse $\mathrm{MFe}_{2} \mathrm{O}_{4}(\mathrm{M}=\mathrm{Fe}, \mathrm{Co}, \mathrm{Mn})$ nanoparticles. J. Am. Cer. Soc., 126(1): 273279.

Sun, S. \& Zeng, H. (2004). Size-controlled synthesis of magnetite nanoparticles. J. Am. Cer. Soc., 124: 8204-8205.

Tao, S., Liu, X., Chu, X. \& Shen, Y. (1999). Preparation and properties of [gamma- $\mathrm{Fe}_{2} \mathrm{O}_{3}$ and $\mathrm{Y}_{2} \mathrm{O}_{3}$ doped gamma-Fe $\mathrm{O}_{3}$ by a sol-gel process. Sens. Actuators $B, 61(1-3)$ : 33-38.

Tartaj, P., González-Carreño, T. \& Serna, C.J. (2001). Single-step nanoengineering of silica coated maghemite hollow spheres with tunable magnetic properties. Adv. Mater., 13(21): 1620-1624.

Teja, A.S. \& Koh, P.-Y. Synthesis, properties, and applications of magnetic iron oxide nanoparticles. Progress in Crystal Growth and Characterization of Mater., 55(1-2): 22-45. 
Zhang, T., Luo, H, Zeng, H, Zhang, R. \& Shen, Y.(1996). Synthesis and gas-sensing characteristics of high thermostability $\gamma-\mathrm{Fe}_{2} \mathrm{O}_{3}$ powder. Sens. Actuators B, 32: 181184.

Tong, S., Hou, S., Zheng, Z., Zhou, J. \& Bao, G. (2010). Coating optimization of superparamagnetic iron oxide nanoparticles for high T2 relaxivity. Nano Lett., 10: 1530-6984.

Tristão, J., Ardisson, J., Sansiviero, M. \& Lago, R. (2010). Reduction of hematite with ethanol to produce magnetic nanoparticles of $\mathrm{Fe}_{3} \mathrm{O}_{4}$ coated with carbon. Hyperfine Interactions, 195(1): 15-19.

Tristão, J.C., Silva, A.A., Ardisson, J.D. \& Lago, R. (2009). Magnetic nanoparticles based on iron coated carbon produced from the reaction of $\mathrm{Fe}_{2} \mathrm{O}_{3}$ with $\mathrm{CH}_{4}$ : a Mössbauer study. LACAME 2008. J. Desimoni, C. P. Ramos, B. Arcondo, F. D. Saccone and R. C. Mercader, Springer Berlin Heidelberg: 21-25.

Varanda, L.C., Morales, M.P., Jafelicci, J.M. \&Serna, C.J. (2002). Monodispersed spindle-type goethite nanoparticles from FeIII solutions. J. Mater. Chem., 12(12): 3649-3653.

Vidal-Vidal, J., Rivas, J. \& López-Quintela, M.A. (2006). Synthesis of monodispersed maghemite nanoparticles by the microemulsion method. Colloids Surf. A, 288: 44-51.

Vijayakumar, R., Koltypin, Y., Xu, X.N., Yeshurun, Y., Gedanken, A. \& Felner, I. (2001). Fabrication of magnetite nanorods by ultrasonic irradiation. J. Appl. Phys., 89, 632428.

Wang, H., Brandl, D.W., Le, F., Nordlander, P. \& Halas, N.J. (2006). Nanorice: A hybrid plasmonic nanostructure. Nano Lett., 6(4): 827-832.

Wang, L., Luo, J., Maye, M., Fan, Q., Rendeng, Q., Engelhard, M.E., Wang, C., Lin, Y. \& Zhong, C.J. (2005). Iron oxide-gold core-shell nanoparticles and thin film assembly. J. Mater. Chem., 15: 1821-1832

Wang, Z., Guo, H., Yu, Y. \& He, N. (2006). Synthesis and characterization of a novel magnetic carrier with its composition of $\mathrm{Fe}_{3} \mathrm{O}_{4} /$ carbon using hydrothermal reaction. J. Magn. Magn. Mater., 302(2): 397-404.

Weckler, B. \& Lutz, H.D. (1998) Lattice vibration spectra. Part XCV. Infrared spectroscopic studies on the iron oxide hydroxides goethite ([alpha]), akaganéite ([beta]), lepidocrocite ([gamma]), and feroxyhite ([delta]). European J. Solid State and Inorg. Chem., 35(8-9): 531-544.

Wong, K., Zeng, Q.H. \&Yu, A.B. (2011). Electronic structure of metal (M = Au, Pt, Pd, or Ru) bilayer modified a- $\mathrm{Fe}_{2} \mathrm{O}_{3}(0001)$ surfaces. J. Phys. Chem. C, 115(11): 4656-4663.

$\mathrm{Wu}, \mathrm{W} ., \mathrm{He}, \mathrm{Q}$. \& Jiang, C. (2008). Magnetic iron oxide nanoparticles: Synthesis and surface functionalization strategies. Nanoscale Res. Lett., 3: 397-415

$\mathrm{Xu}$, Z., Hou, Y. \& Sun, S. (2007). Magnetic core/shell $\mathrm{Fe}_{3} \mathrm{O}_{4} / \mathrm{Au}$ and $\mathrm{Fe}_{3} \mathrm{O}_{4} / \mathrm{Au} / \mathrm{Ag}$ nanoparticles with tunable plasmonic properties. J. Am. Chem. Soc., 129(28): 86988699.

Yang, Z., Lu, Z., Luo, G. \& Hermansson, K. (2007), Oxygen vacancy formation energy at the $\mathrm{Pd} / \mathrm{CeO}_{2}(111)$ interface. Phys. Lett. A, 369: 132-139.

Ye, Q.-L., Kozuka, Y., Yoshikawa, H., Awaga, K., Bandow, S. \& Iijima, S. (2007). Effects of the unique shape of submicron magnetite hollow spheres on magnetic properties and domain states. Physical Review B, 75(22): 224404

Yue, J., Jiang, X.C. \& Yu, A.B. (2010). Molecular dynamics study on the growth mechanism of goethite ([alpha]-FeOOH) nanorods. Solid State Sciences, 13(1): 263-270. 
Yue, J., Jiang, X.C. \& Yu, A.B. (2011). Experimental and theoretical study on the $\beta$-FeOOH nanorods: growth and conversion. J. Nanoparticle Res., 13(9): 3961-3974.

Yue, J., Jiang, X.C., Zeng, Q. \& Yu, A.B. (2010). Experimental and numerical study of cetyltrimethylammonium bromide (CTAB)-directed synthesis of goethite nanorods. Solid State Sciences, 12(7): 1152-1159.

Yue, J., Jiang, X.C. \& Yu, A.B. (2011). Molecular Dynamics Study on $\mathrm{Au} / \mathrm{Fe}_{3} \mathrm{O}_{4}$ Nanocomposites and Their Surface Function toward Amino Acids. J. Phys. Chem. B., 115:11693-11699.

Zeng, Q.H., Yu, A.B. \& Lu, G.Q. (2008). Multiscale modeling and simulation of polymer nanocomposites. Progress in Polymer Sci., 33(2): 191-269.

Zhai, Y., Zhai, J., Wang, Y., Guo, S., Ren, W. \& Dong, S. (2009). Fabrication of iron oxide core/shell submicrometer spheres with nanoscale surface roughness for efficient surface-enhanced Raman scattering. J. Phys. Chem. C 113(17): 7009-7014.

Zhang, J., Liu, X., Guo, X., Wu, S. \& Wang, S. (2010). A general approach to fabricate diverse noble metal ( $\mathrm{Au}, \mathrm{Pt}, \mathrm{Ag}, \mathrm{Pt} / \mathrm{Au}) / \mathrm{Fe}_{2} \mathrm{O}_{3}$ hybrid nanomaterials. Chem.-Eur. J., 16(27): 8108-8116.

Zhang, S., Niu, H., Hu, Z., Cai, Y. \& Shi, Y. (2010). Preparation of carbon coated $\mathrm{Fe}_{3} \mathrm{O}_{4}$ nanoparticles and their application for solid-phase extraction of polycyclic aromatic hydrocarbons from environmental water samples. J. Chromatography A, 1217(29): 4757-4764.

Zhang, W.M., Wu, X.L., Hu, J.S., Guo, Y.G. \& Wan, L.J. (2008). Carbon coated $\mathrm{Fe}_{3} \mathrm{O}_{4}$ nanospindles as a superior anode material for lithium-ion batteries. Adv. Funct. Mater., 18(24): 3941-3946.

Zhang, Z., Zhang, Q., Xu, L. \& Xia, Y.-B. (2007). Preparation of nanometer $\gamma-\mathrm{Fe}_{2} \mathrm{O}_{3}$ by an electrochemical method in non-aqueous medium and reaction dynamics. Synthesis and Reactivity in Inorg. Metal-Org. and Nano-Metal Chem., 37: 53-56.

Zhong, J. \& Adams, J.B. (2008). Adhesive metal transfer at the $\mathrm{Al}(111) / \mathrm{a}-\mathrm{Fe}_{2} \mathrm{O}_{3}(0001)$ interface: a study with ab initio molecular dynamics, modelling and simulation. Mater. Sci. and Eng., 16: 085001(9 pages).

Zhong, Z., Ho, J., Teo, J., Shen, S. \& Gedanken, A. (2007). Synthesis of porous a- $\mathrm{Fe}_{2} \mathrm{O}_{3}$ nanorods and deposition of very small gold particles in the pores for catalytic oxidation of CO. Chem. Mater., 19(19): 4776-4782.

Zhong, Z., Lin, J., Teh, S.P., Teo, J. \& Dautzenberg, F.M. (2007). A rapid and efficient method to deposit gold particles onto catalyst supports and its application for $\mathrm{CO}$ oxidation at low temperatures. Adv. Funct. Mater., 17(8): 1402-1408. 


\section{Part 2}

Testing Technology 



\title{
Iron Oxide Nanoparticles Imaging Tracking by MR Advanced Techniques: Dual-Contrast Approaches
}

\author{
Shengyong $\mathrm{Wu}$ \\ Medical Imaging Institute of Tianjin
}

China

\section{Introduction}

Recently a number of imaging modalities have been presented for cellular imaging including magnetic resonance imaging (MRI), optical imaging, and positron emission tomography (PET) based on the background of growing demand for molecular imaging to noninvasively and longitudinally visualize cell migration and track transplanted cells in vivo, also to monitor cell biodistribution. Cellular MRI, with its superb ability of resolving soft tissue anatomies in three-dimensions (3D) with high spatial resolution in comparison to other modalities, is particularly important as a noninvasive tool to provide unique information on the dynamics of cell migration in vivo (Modo, 2005; Arbab, 2008a; Zhang, 2008).

In vivo MRI of cells is very useful for studying tumors, inflammation, stem cell therapy, and immune response, etc. Cells labeled with commercially available iron oxide nanoparticles (iron particles) can be imaged for weeks with MRI. The labeling procedure does not exhibit any alteration to cell viability or function (Bulte, 2004; Oude Engberink, 2007). Superparamagnetic iron oxides (SPIO) and ultra-small superparamagnetic iron oxide (USPIO) particles are commercial MR contrast agents for cell labeling due to their biocompatibility and strong effects upon $\mathrm{T}_{2}$ and $\mathrm{T}_{2}{ }^{*}$ relaxation. Several labeling methods have been developed to incorporate sufficient quantities of iron into cells. Cellular MRI has now been widely used for tracking transplanted iron-labeled therapeutic cells in vivo (Bulte, 2004; Oude Engberink, 2007). The technique has recently been introduced into the clinic (de Vries, 2005). The effect from iron particles is seen as hypointensity or negative-contrast on $\mathrm{T}_{2^{-}}$and $\mathrm{T}_{2}{ }^{*}$-weighted images because of the shortening of $\mathrm{T}_{2}$ and $\mathrm{T}_{2}{ }^{*}$ relaxation times. However, concerns have been raised that the negative-contrast could be non-specific and difficult to differentiate from signal hypo-intensities resulting from susceptibility artifacts (i.e. from the presence of air or other field inhomogeneities), flow related signal losses, and calcification. Therefore, several positive-contrast and even dual-contrast imaging techniques have recently been developed for tracking iron-labeled cells. Dual-contrast imaging effectively permits detection of the presence of iron-labeled cells with both negative- and positive-contrast within a single image. This chapter illustrates negative- and positivecontrast MR techniques for tracking iron-labeled cells. Particular attention was paid to 
recently developed positive-contrast cell tracking techniques, the status of dual-contrast approaches of new MRI pulse sequences and image postprocessing techniques and their perspectives. The new advanced technology in imaging contrast of iron oxide NPs on multimodal platform will also be introduced.

\section{Negative-contrast MRI techniques}

Cellular MRI is a newly emerging field of MR research that allows the "non-invasive, quantitative, and repetitive imaging of targeted macromolecules and biological processes in living organisms" (Herschman, 2003). Cellular MRI requires that cells are labeled with MR contrast agent to make them distinct from the surrounding tissues. Iron oxide nanoparticles are regarded as the most extensively applied contrast agent in cell imaging and cell tracking studies based on the fact of their strong negative contrast effect, biocompatibility, variety in core size and coating surface, as well as ease of detection at microscopic level (Muja, 2009). SPIO and USPIO are currently the predominant MRI contrast agents. The description of the physical and chemical properties of SPIO and USPIO can be found in recent reviews (Herschman, 2003; Thorek, 2006; Muja, 2009). The sizes of monocrystalline iron oxide nanoparticles $(\mathrm{MIONs}) \approx 3 \mathrm{~nm}$ in diameter, USPIO particles $\approx 15-30 \mathrm{~nm}$, SPIO particles $\approx 60$ $180 \mathrm{~nm}$ and micron sized iron oxide particles (MPIOs) can be as large as $10 \mu \mathrm{m}$ (Shapiro, 2005). Some of the SPIO and USPIO agents, such as Endorem (SPIO, Guebert), Ferumoxides (SPIO, Berlex) and Resovist (USPIO, Schering), are already approved by the Food and Drug Administration (FDA) and are extensively used for imaging of the liver, central nervous system (CNS) and lymphatic system (Arbab, 2004b; Helmberger, 2005; Manninger, 2005), etc. Cationic transfection agents such as poly-L-lysine or the FDA-approved protamine sulfate are used to increase labeling efficiency in vitro. SPIO particles may decrease $\mathrm{T}_{2}{ }^{*}$ by magnetic susceptibility effect and $\mathrm{T}_{2}$ by dipole-dipole interaction or scalar effect between protons and magnetic centre. A large magnetisation difference occurs as a result of the nonhomogeneous distribution of superparamagnetic particles, which gives rise to local field gradients that accelerate the loss of phase coherence of the spins contributing to the MR signal. Iron-labeled cells cause significant signal dephasing due to the magnetic field inhomogeneity induced in water molecules near the cell such that iron-labeled cells were visualized as signal voids on $\mathrm{T}_{2}$ and $\mathrm{T}_{2}{ }^{*}$ weighted images (negative-contrast $\mathrm{MR}$ imaging). Negative-contrast techniques are the most commonly used approach for the detection of the SPIO-labeled cells.

While cell-based therapies have attracted well attention as novel therapeutics for the treatment of so many kinds of diseases, investigations (Zhang, 2005; Heyn, 2005, 2006) have showed that single, living, highly phagocytic large cells, such as macrophages, or human endothelial cells can be tracked over time in MRI using a 3.0 T even 1.5 T scanner. As an example of stem cell-based studies, investigators (Anderson, 2005) demonstrated that MRI of iron-labeled stem cells was directly identified in neovasculature of a glioma model. The cells were labeled using the ferumoxides/poly-L-lysine complex in vitro and the labeled cells were then injected in the model, and their migration toward and incorporation into the tumor neovasculature was visualized in vivo with negative-contrast MRI. Other studies have shown that ferumoxides-TA labeled human MSCs will home to liver (Arbab, 2004a), tumors (Khakoo, 2006), or heart (Kraitchman, 2005), illustrated at negative-contrast imaging with MR scan and confirmed at histologic evaluation. A group (Zhu, 2006) labeled neural 
stem cells (NSCs) obtained from patients with traumatic brain injury then performed intracerebral injections of either ferumoxide-labeled or unlabeled cells around the injured tissue of them as the first study in the field of noninvasive imaging of stem cell treatment of brain injury, and their serial MRI about 7-10 weeks demonstrated that stem-cell engraftment and migration after implantation can be detected noninvasively with the use of MRI.

Also, in an early study (Kircher, 2003a), a highly derivatized cross-linked iron oxide (CLIO) nanoparticle was used to efficiently label cytotoxic $\mathrm{T}$ lymphocytes (CTLs) for in vivo tracking of the injected cells to melanoma cell line at near single-cell resolution, with MRI and optimized the labeling protocol (three-dimensional nature of the calculated $\mathrm{T}_{2}$ maps), showing no cytotoxic and not influencing cell behavior or effector function. Despite the fact that the high spatial resolution given by MRI provides accurate evaluation of morphology of lymphoid organ, the sensitivity and ability to quantify MR data is still limited when compared with nuclear medicine based techniques. For MR cell tracking to be clinically useful, it should be defined for the detection limits of the MR method which will be utilized. The related clinical studies with 3.0 T scanners suggest that negative-contrast techniques possibly detect 150,000 Feridex labeled cells after directly injected into the lymph nodes of patients (de Vries, 2005). Another recent example of study by Laboratory for Gene Transcript Targeting, Imaging and Repair in Massachusetts General Hospital demonstrated that functionalization allows SPIO nanoparticles to be targeted, and it showed that their phosphorothioate-modified DNA probes linked to SPIO could be used to identify differential gene expression due to amphetamine exposure with high reliability using the calculation of rate of signal reduction $\left(\mathrm{R}_{2}{ }^{*}\right)$ in $\mathrm{T}_{2}{ }^{*}$-weighted MR images (Liu, 2009). There are also extensive published works with detailed descriptions of many aspects of labeled cells for detection with negative-contrast MRI (Ferrucci, 1990; Bulte, 2004b; Hsiao, 2007; Gonzalez-Lare, 2009). Those and many of other preclinical studies have provided evidences for the potential translation of iron oxide NPs labeling and cellular MR imaging to the clinic applications.

An important property of USPIO is its ability to shorten $\mathrm{T}_{1}$ and $\mathrm{T}_{2}$ relaxation times (Small, 1993; $\mathrm{Li}, 2005)$. USPIO-labeled cells can be tracked in $\mathrm{T}_{1}$ and $\mathrm{T}_{2} / \mathrm{T}_{2}{ }^{*}$ weighted images, which should increase the accuracy and the specificity for detection of the labeled cells (Kelloff, 2005), such as in imaging assessment on angiogenesis of tumor (Niu, 2011), atherosclerotic plaques (Metz, 2011), or arthritis (Lefevre, 2011). USPIO nanoparticles recently have shown potential in the imaging of molecular biomarkers, such as integrins that are heterodimeric transmembrane glycoproteins, a family of adhesion molecules playing a major role in angiogenesis and tumor metastasis (Chen, 2009; Tan, 2011).

Much of the progress in detecting individual iron-labeled cells has achieved from improvements in contrast agent design that increases targeting and intracellular uptake properties (Cerdan, 1989; Weissleder, 1990; Bulte, 2001; Zhao, 2002). Improvements in MR hardware and pulse sequence design also have played an important role during recent progress in this area of research. Although negative-contrast MRI has shown promise as a means to visualize labeled cells (Hogemann, 2003; Heyn, 2005), some remaining issues may hamper its wide applications: (1) it is difficult to distinguish the signal voids of labeled cells from those of complex background tissue signals; (2) With the resulting signal void as the means for detection, partial-volume effects are often severe and go far beyond the real cell 
size; (3) it is difficult to discriminate iron-induced susceptibility changes from those caused by other susceptibility artifacts due to i.e. air/tissue interfaces, or peri-vascular effects.

\section{Positive-contrast and dual-contrast MRI techniques}

The "white-marker imaging" positive-contrast mechanism was introduced by Seppenwoolde et al. in 2003 (Seppenwoolde, 2003). Since then, several groups have developed positive-contrast or dual-contrast pulse sequences for tracking iron-labeled cells in vitro and in vivo (Table 1 ).

\subsection{Gradient-dephasing technique: "white-marker" imaging}

"White-marker" imaging was initially presented to create positive-contrast around paramagnetic intravascular device markers used in magnetic-resonance-based interventional procedures (Seppenwoolde, 2003). The gradient-dephasing technique uses a slice gradient to dephase the background water signal followed by an incomplete gradient rephasing pulse which was exploited for the depiction and tracking of paramagnetic susceptibility markers. Local magnetic field inhomogeneities were selectively visualized with positive-contrast, such as those created by iron-labeled cells for "white-marker" imaging. Advanced methods were developed to separate magnetic susceptibility effects from partial volume effects in "white marker" imaging in order to avoid compromising the identification of magnetic structures (Seppenwoolde, 2007). However, this method is only sensitive to macroscopic field inhomogeneities caused by paramagnetic material, to a volume surrounding the paramagnetic material that is free of other field variations (Zurkiya, 2006).

A similar gradient depashing technique termed gradient echo acquisition for superparamagnetic particles (GRASP), by dephasing of the background signal, has been used to detect positive-contrast from superparamagnetic particles based on the phenomena that the z-rephasing gradient is reduced so that dipolar fields generated by the cells are rephrased and positive signal can be observed (Mani, 2006a), also to image ferritin deposition in a rabbit model of carotid injury with relatively low concentrations of iron oxides at $1.5 \mathrm{~T}$ MR scanner (Mani, 2006b). The GRASP technique was used to successfully image low concentrations of ferumoxides $(0.05 \mathrm{mM}$ Fe corresponding to 2.8 $\mu \mathrm{g} \mathrm{Fe} / \mathrm{mL}$ ) and ferritin $(5 \mu \mathrm{g} \mathrm{Fe} / \mathrm{mL})$ in gel phantoms (Mani, 2006). GRASP "whitemarker" imaging has several advantages including ease of implementation, high sensitivity, no influence on positive signal due to both $B_{0}$ and $B_{1}$ field inhomogeneities, and fast acquisition with various TE values. The feasibility of GRASP was tested to aid in dynamically tracking stem cells in a mouse model of myocardial infraction (Mani, 2008). Using $\mathrm{T}_{2}{ }^{*}$-GRE and GRASP techniques at $9.4 \mathrm{~T}$ scanner, iron-labeled embryonic stem cells were visualized in the border zone of infarcted mice at 24 hours, and 1 week following implantation. The positive signal in areas containing iron-loaded stem cells corresponded precisely with the signal loss detected within images produced with conventional GRE sequences. Regions that contained iron-labeled cells were confirmed by histology (Mani, 2008). The presence of the signal loss because of iron-labeled cells would have been difficult to detect on $\mathrm{T}_{2}{ }^{*}$-weighted images without using the positive-contrast sequence. The region of the myocardium containing the iron-labeled cells was clearly visible when both GRASP and $\mathrm{T}_{2}{ }^{*}$-weighted techniques (dual contrast imaging) were applied. Dualcontrast effects act to extend the signal change well beyond the location of the particle or 


\begin{tabular}{|c|c|c|c|c|}
\hline MR sequences & $\begin{array}{l}\text { Contrast } \\
\text { agents }\end{array}$ & $\begin{array}{l}\text { Experimental } \\
\text { conditions }\end{array}$ & Biological target & $\begin{array}{l}\text { Application and } \\
\text { Results }\end{array}$ \\
\hline \multirow{5}{*}{$\begin{array}{l}\text { gradient- } \\
\text { dephasing } \\
\text { technique \& } \\
\text { GRASP }\end{array}$} & Ferritin & $\begin{array}{l}\text { In vitro and } \\
\text { in vivo }\end{array}$ & $\begin{array}{l}\text { Endogeneous } \\
\text { ferritin }\end{array}$ & $\begin{array}{l}\text { Crush injured rabbit } \\
\text { carotid arteries }\end{array}$ \\
\hline & & & & Myocardial infraction \\
\hline & Ferumoxides & $\begin{array}{l}\text { In vitro and } \\
\text { in vivo }\end{array}$ & $\begin{array}{l}\text { Embryonic stem } \\
\text { cell-derived } \\
\text { cardiac precursor }\end{array}$ & \\
\hline & & & cell & $\begin{array}{l}\text { Injected into the hind } \\
\text { limb of mouse }\end{array}$ \\
\hline & Ferumoxides & $\begin{array}{l}\text { In vitro and } \\
\text { in vivo }\end{array}$ & $\begin{array}{l}\text { Embryonic stem } \\
\text { cell line TL-1 }\end{array}$ & \\
\hline $\begin{array}{l}\text { off-resonance } \\
\text { (OR) method }\end{array}$ & Ferumoxides & $\begin{array}{l}\text { In vitro and } \\
\text { in vivo }\end{array}$ & $\begin{array}{l}\text { SPIO-luc-mouse } \\
\text { embryonic stem } \\
\text { cell }\end{array}$ & $\begin{array}{l}\text { Injection into hindlimbs } \\
\text { of mouse }\end{array}$ \\
\hline $\begin{array}{l}\text { Off-resonance } \\
\text { saturation }\end{array}$ & $\begin{array}{l}\text { mMION/ } \\
\text { SPPM }\end{array}$ & $\begin{array}{l}\text { Gel phantom/ } \\
\text { in vivo }\end{array}$ & $\begin{array}{l}\text { the }{ }_{v} \beta_{3} \text {-expressing } \\
\text { microvasculature }\end{array}$ & $\begin{array}{l}\text { molecular imaging of } \\
\text { cancer }\end{array}$ \\
\hline \multirow{3}{*}{$\begin{array}{l}\text { IRON } \\
\text { technique }\end{array}$} & MION-47 & In vivo & Macrophage & Atherosclerotic plaque \\
\hline & & & & MR lymphography \\
\hline & MION-47 & In vivo & Macrophage & \\
\hline $\begin{array}{l}\text { SR-SPSP } \\
\text { sequence }\end{array}$ & Ferumoxides & $\begin{array}{l}\text { In vitro and } \\
\text { in vivo }\end{array}$ & $\begin{array}{l}\text { Human bone } \\
\text { marrow stromal } \\
\text { cells }\end{array}$ & $\begin{array}{l}\text { Injection into the hind } \\
\text { legs of mouse }\end{array}$ \\
\hline $\begin{array}{l}\text { FLAPS } \\
\text { sequence }\end{array}$ & Ferumoxides & $\begin{array}{l}\text { In vitro and } \\
\text { in vivo }\end{array}$ & $\begin{array}{l}\text { GFP-R3230Ac cell } \\
\text { line }\end{array}$ & $\begin{array}{l}\text { Injection into the hind } \\
\text { legs of rat }\end{array}$ \\
\hline UTE imaging & Ferumoxides & $\begin{array}{l}\text { In vitro and } \\
\text { in vivo }\end{array}$ & G6 glioma cells & $\begin{array}{l}\text { Implanted cellular } \\
\text { imaging }\end{array}$ \\
\hline $\begin{array}{l}\text { SWEET } \\
\text { sequence }\end{array}$ & Ferumoxides & in vivo & $\begin{array}{l}\text { Human epidermal } \\
\text { carcinoma cells }\end{array}$ & $\begin{array}{l}\text { Visualization of } \\
\text { magnetically labeled } \\
\text { tumor cells }\end{array}$ \\
\hline
\end{tabular}

Note: GRASP, superparamagnetic particles/susceptibility; IRON, oxide nanoparticles-resonant water suppression; SR-SPSP, self-refocused spatial-spectral; FLAPS, fast low-angle positive contrast steadystate free precession; UTE, ultrashort echo-time

Table 1. Summary of Previously Published Studies of Positive- and Dual-contrast Techniques 
cell itself. This form of signal amplification increases sensitivity in detecting the labeled cells within a complex image background. With the use of signal amplification, potential future applications of (U)SPIO include 'doping' of therapeutic cell preparations with a small fraction of labeled cells, to allow cell tracking without altering the majority of the cells. This would allow for better delineation and identification of labeled cells with both techniques. The challenge for both techniques is the difficulty associated with attempting to quantify the concentration of the labeled cells in vivo because of the susceptibility artifact produced via the iron particles.

Generally, to resolve issues associated with volume averaging and other artifacts that may limit the clinical utility of MRI to detect iron labeled cells (especially in tissues other than the brain), GRASP technique has been developed to differentiate between the signal generated by the cells and signal loss cause by various artifacts (Mani, 2006, 2008), and to specifically avoid the signal loss generated by the iron laden cells to be confused with signal caused by other sources (motion, perivascular effects, coil inhomogeneities, etc.). In the recent study (Briley-Saebo, 2010), the GRASP sequence was also used to both detect and confirm the presence of the Feridex labeled dendritic cells (DCs) in the draining lymph nodes of nude mice $24 \mathrm{~h}$ after footpad injection. The results showed the possibility to detect and longitudinally track ex vivo human DC vaccines in the spleen of mice for up to 2 weeks, with greater lymphoid targeting observed following i.v. injection, relative to subcutaneous foot-pad injection; also showed good correlation between in vivo $\mathrm{R}_{2}{ }^{*}$ values on a 9.47 Tesla dedicated mouse scanner and Feridex concentration, with detection limits of 3.2\% observed for the spleen. But investigators didn't detect the Feridex labeled cells within the liver and spleen using the GRASP sequence while they indicated that, the dipole effects would be limited and signal enhancement would not be observed when the iron particles being homogenously distributed over a large volume (such as the liver or spleen). They further demonstrated the values of nodes the white marker sequence, GRASP, in accurate detection and identification of Feridex labeled DCs in superficial lymph, and indicated that the appropriate utilization of animals models and MR validated imaging strategies might allow for the optimization of human DC vaccine therapies and improved therapeutic success, whereas white marker sequences maybe most effective when the iron laden cells being compartmentalized within a limited volume (such as in lymph nodes, tumors, or myocardium). On the basis of a recent report (Sigovan, 2011) of the feasibility study on a positive contrast technique, GRASP at a relatively high field $4.7 \mathrm{~T}$, for a novel superparamagnetic nanosystem designed for tumor treatment under MRI monitoring, investigators found that the magnetic nanoparticles for drug delivery can be detected using positive contrast, and suggested that the combined negative and susceptibility methods allow good quality images of large magnetic particles and offer their follow-up for theranostic applications.

\subsection{Off-resonance Imaging (ORI)}

Off-resonance MRI approaches have also been developed to produce positive-contrast. With this method, a spectrally selective radio frequency (SSRF) pulse was used to excite only the susceptibility-shifted, or 'off-resonance', water signals (Cunningham, 2005; Foltz, 2006), at the frequency shift induced by the iron particles. Since only the off-resonance signal due to 
iron particles are excited and refocused, the background on-resonance signal is largely eliminated.

Iron-labeled mouse embryonic stem cells were imaged as positive-contrast through suppression of background tissue with these off-resonance methods (Suzuki, 2008). A spinecho sequence was used with million-fold $(120 \mathrm{~dB})$ suppression of on-resonance water by matching the profiles of a $90^{\circ}$ excitation and a $180^{\circ}$ refocusing pulse. The positive-contrast signal from the volume of cells was affected by how well the excitation profile was defined. The method is therefore inherently limited by the complication associated with unwanted magnetization from the regions that suffer from chemical shift or susceptibility-related artifacts (e.g., from fat/lipid present in the region of interest and/or imperfect $\mathrm{B}_{0}$ shimming, due to air/tissue interfaces, etc.) (Farrar, 2008). Although ORI techniques are being increasingly used to image iron oxide imaging agents such as MION, the diagnostic accuracy, linearity, and field dependence of ORI have not been fully characterized. After the sensitivity, specificity, and linearity of ORI were examined as a function of both MION concentration and magnetic field strength (4.7 and $14 \mathrm{~T}$ ), and MION phantoms with and without an air interface as well as MION uptake in a mouse model of healing myocardial infarction were imaged, the linear relationship between MION-induced resonance shifts and with MION concentration were illustrated, whereas $\mathrm{T}_{2}$ showed comparable to the TE and then decreasing after increasing initially with MION concentration and the ORI signal/sensitivity being highly non-linear. Improved specificity of ORI in distinguishing MION-induced resonance shifts and linearity can be expected at lower fields $(4.7 \mathrm{~T}$, onresonance water linewidths $15 \mathrm{~Hz}$ ) with on-resonance water linewidths decreased, airinduced resonance shifts reduced, and longer $\mathrm{T}_{2}$ values observed, thus ORI will be likely optimized at low fields with very short TEs choosing and with moderate MION concentrations. Off-resonance approaches generate positive contrast but have a lower sensitivity than $\mathrm{T}_{2}{ }^{*}$-weighted imaging and are more complex to perform at high field strengths. Superparamagnetic iron-oxide nanoparticles become saturated above 0.5 Tesla and thus have equal sensitivity at clinical field strengths (1.5-3.0 T) and at the higher field strengths often used in preclinical studies (Sosnovik, 2009).

An alternative off-resonance technique termed inversion-recovery with on-resonant water suppression (IRON) sequence was proposed by a serial studies from one lab (Stuber, 2005, 2007). The IRON method used a spectrally-selective saturation pre- pulse to suppress the signal originating from on-resonant protons in the background tissue while preserving the signal from off-resonant spins in proximity to the iron particles. However, since the size of the signal-enhanced region is dependent on the bandwidth of the water suppression pulse, this scheme requires extra steps to adjust the center frequency and bandwidth of the prepulse to locate the exact site proximal to the cells. IRON sequence has been successfully applied for in vivo tracking of iron-loaded stem cells (Stuber, 2007).

The utility of IRON method combined with injection of the long-circulating MION-47 has been recently evaluated by investigators in Johns Hopkins University School of Medicine (Korosoglou, 2008a) for developing a novel contrast-enhanced MR angiography technique. One important aspect of the study was fat suppression for the IRON sequence with an initial radiofrequency pulse offset by $440 \mathrm{~Hz}$ at $3.0 \mathrm{~T}$, and with spin inversion, to cause zero 
longitudinal magnetization of the targeted species for the radiofrequency pulses $\left(105^{\circ}\right.$ for fat, $100^{\circ}$ for water), which obviously shortened the subsequent recovery time. The usage of MION-47 allowed acquisition of multiple image sets over a 1- or 2-day period with high spatial resolution.

IRON techniques with a commercially available MION-47 were recently employed to detect macrophage-rich atherosclerotic plaques in a rabbit model of atherosclerosis (Korosoglou, $2008 \mathrm{~b}$ ), in which pre-contrast imaging was performed in 7 Watanabe rabbits and 4 control New Zealand rabbits, and post-contrast imaging was repeated on days 1 and 3 after intravenous injection of MION-47. A second injection was performed on day 3 after imaging and post-contrast imaging performed again on day 6 . There was a significant increase in signal intensity within aortic atherosclerotic plaques following administration of MION-47 ( $48 \%$ increase on day 3 and $72 \%$ increase on day 6 ) versus hypointensity (negative-contrast) in conventional MR images, but no enhancement was seen in control rabbits that lacked atherosclerosis. The positive-contrast regions corresponded to regions demonstrating deposition of iron particles within macrophage-rich atherosclerotic plaques. These findings not only validated that MION-47 is a successful imaging agent for macrophage-rich atherosclerosis, but also suggested that positive-contrast IRON MRI can be applied to the general class of iron oxide particles. This is significant as USPIO-enhanced MR imaging has been previously studied in human (Trivedi, 2006); enabling IRON MRI sequences to be directly applied to patient care.

Korosoglou et al. also investigated the utility of IRON techniques and MION-47 to create positive-contrast MR-lymphography (Korosoglou, 2008c). After six rabbits received a single bolus injection of $80 \mathrm{mmol} \mathrm{Fe} / \mathrm{kg}$ MION-47, MRI was performed at baseline, 1 day, and 3 days using conventional $\mathrm{T}_{1^{-}}$and $\mathrm{T}_{2}{ }^{*}$-weighted sequences and IRON. On $\mathrm{T}_{2}{ }^{*}$-weighted images, as expected, signal attenuation was observed in areas of para-aortic lymph nodes after MION-47 injection. However, using IRON the para-aortic lymph nodes exhibited very high contrast enhancement, which remained 3 days after injection. IRON in conjunction with iron particles can be therefore used to perform positive-contrast MR-lymphography, particularly 3 days after injection of the contrast agent, when signal is no longer visible within blood vessels. The proposed method may have the potential as an adjunct for nodal staging in cancer screening.

Iron-labeled radioembolization microspheres were visualized for in vivo tracking during trans-catheter delivery to VX2 liver tumors in a rabbit model (Gupta, 2008). The study was performed for real-time observation of microsphere delivery with dual-contrast techniques. The results showed significant changes in post-injection contrast-to-noise ratio (CNR) values from those of pre-injection at positions of microsphere deposition with both negative- and positive-contrast.

The off-resonance MRI method possesses some advantages including no need for dephasing gradients or saturation pulses, high suppression efficiency, and flexible selection of the excited frequency band to encompass spins in the vicinity of the iron particles without fat tissue off-resonance. This technique, however, was not slice-selective such that it can result in interference from insufficiently suppressed background signals or less background signal with regions of greater susceptibility excluded from the selected slice. This technique can also cause less on-resonant signal to be suppressed, has less flexibility in RF pulse design, 
and can lead to less erroneous off-resonant signal detection in a multi-slice manner with individually shimmed slices (Zurkiya, 2006).

The off-resonance saturation method has been developed by Zurkiya and $\mathrm{Hu}$, in which water protons are imaged with and without the presence of an off-resonance saturation pulse (Zurkiya, 2006). This method relies on diffusion-mediated saturation transfer to reduce the on-resonance MRI signal due to the off-resonance saturation (ORS) pulse, similar to chemical exchange saturation transfer techniques (Ward, 2000). This approach has been verified that greatly improved tumor detection accuracy over the conventional $\mathrm{T}_{2}{ }^{*}$-weighted methods because of its ability to turn "ON" the contrast of superparamagnetic polymeric micelles (SPPM) nanoparticles (Khemtong, 2009). SPPM nanoparticles encoded with cyclic (RGDfK) ligand (arginine-glycine-aspartic acid), cRGD, were able to target the $\alpha_{v} \beta_{3}$-expressing microvasculature in A549 non-small cell lung tumor xenografts in mice. The results suggest that the combination of ORS imaging with cancer-targeted SPPM nanoparticles will show promise in detecting biochemical markers at early stages of non-small cell lung tumor development, and could further enhance the sensitivity of contrast and provide new opportunities in imaging biomarkers setting of in vivo tumor target.

The study (Zurkiya, 2008) transfected cells with genes from magnetotactic bacteria (i.e., MagA) under doxycycline-regulated gene expression, resulting in the intracellular production of iron oxide nanoparticles similar to synthetic SPION. MagA-expressing cells could be visualized by MRI after transplantation in the mouse brain after $5 \mathrm{~d}$ of induction with doxycycline. The generalized implementation of these techniques as treatment strategies in stem cell tracking needs to be explored. Investigators have recently inserted magnetic reporter genes into cells. After the expression of iron storage proteins formed stored iron then MRI can be used to detect it. Another transgene reporter, an adenoviral vector carrying a transgene for light- and heavy-chain ferritin protein to transfect cells has been shown that they could be detected by in vivo magnetic resonance imaging (Genove, 2005).

Balchandani et al. recently developed a self-refocused spatial-spectral (SR-SPSP) pulse, which is successful in creating positive-contrast images of SPIO-labeled cells (Balchandani, 2009). This pulse can enable slice-selective, spin-echo imaging of off-resonant spins without an increase in TE, which is essentially a phase-matched $90^{\circ}$ SPSP pulse and a 180 SPSP pulse combined into one pulse. This results in a considerably shorter TE than possible with two separate pulses. The simultaneous spatial and spectral selectivity allows the imaging of offresonant spins while selecting a single slice. The SR-SPSP pulse is also suitable for any application requiring spatial and spectral selectivity, such as tracking metallic devices or replacing standard pulses in MR spectroscopic imaging sequences. More recently a novel combination of off-resonance (ORI) positive-contrast MRI and $\mathrm{T}(2 \rho)$ relaxation in the rotating frame (ORI-T ${ }_{2 \rho}$ method) for positive-contrast MR imaging of USPIO in a mouse model of burn trauma and infection with Pseudomonas aeruginosa (PA), was also reported to have direct implications in the longitudinal noninvasive monitoring of infection, and show promise in testing the new-developed anti-infective compounds (Andronesi, 2010). The same group also reported that ORI- $T_{2 \rho}$ method proved to have slightly higher sensitivity than ORI, and MR imaging clearly showed migration and accumulation of labeled MSCs to the burn area which can be confirmed by histology staining for iron labeled cells (Righi, 2010). 


\subsection{Fast low angle steady-state free precession (FLAPS) sequence}

FLAPS imaging has been proposed for time-efficient acquisition of off-resonance positivecontrast images (Dharmakumar, 2007). The technique takes advantage of the unique spectral response of the steady-state free precession (SSFP) signal to achieve signal enhancement from off-resonant spins while suppressing signal from on-resonant spins at relatively small flip angles (Dharmakumar, 2006). Besides the positive-contrast generated by the weakly offresonant spins, the spins in and around the core of the local magnetic susceptibility (LMS)shifting media (such as labeled cells) experience large deviations from the central frequency leading to intra-voxel dephasing that was observed as negative-contrast in FLAPS images. So this technique has the capability to identify the presence of labeled cells with both negative- and positive-contrast within a single image.

Zhang et al. recently investigated the feasibility of imaging iron-labeled green fluorescent protein (GFP)-expressing cells with the dual-contrast method and compared its measurements with traditional negative-contrast technique (Zhang, 2009). The GFP-cell was incubated for 24 hours using $20 \mathrm{mg} \mathrm{Fe} / \mathrm{mL}$ concentration of SPIO and USPIO nanoparticles. The labeled cells were imaged using the FLAPS technique, and FLAPS images with positive-contrast were compared with negative-contrast $\mathrm{T}_{2}{ }^{*}$-weighted images. The results demonstrated that SPIO and USPIO labeling of GFP cells had no effect on cell function or GFP expression, and the labeled cells were observed as a narrow band of signal enhancement surrounding signal voids in FLAPS images. Positive- and negative-contrast images were both valuable for visualizing labeled GFP-cells. MRI of labeled cells with GFP expression holds great potential for monitoring the temporal and spatial migration of gene markers and cells, and enhances our understanding of cell- and gene-based therapeutic strategies. These findings suggested that the dual-contrast nature of the FLAPS approach offers significant advantages to the field of cellular MRI. A highly sought feature of cellular imaging is the quantification of labeled cells. Past studies have shown that it may be possible to define a relation between number of cells and MR transverse relaxation time constants (apparent $T_{2}$ or $T_{2}{ }^{*}$ ). However, since the specificity of the labeled cells is often compromised in GRE images, it is often difficult to use the time constant thus derived as a reliable metric to quantify the number of cells. These previous FLAPS investigations showed that local contrast was exponentially related to the number of cells. Furthermore, the dual-contrast filter, using an image metric that is analogous to local contrast, can provide additional quantitative information regarding those regions containing the labeled cells. This technique still could be limited by the magnetic perturbations around MNPs. A careful investigation of how the output of dual-contrast image filters can be used to derive quantitative information regarding the concentration of labeled cells from in vivo images has been demonstrated (Dharmakumar, 2009).

\subsection{Ultra short echo time methods}

It has been introduced that ultrashort echo-time (UTE) imaging had capability of imaging materials with extremely short $\mathrm{T}_{2}$ and very fast signal decay (Robson, 2006; Rahmer, 2009), and did as a new and promising approach that allowed the detection of short- $\mathrm{T}_{2}$ signal components, such as tendons, ligaments, menisci, periosteum, and cortical bone before signals within these tissues decay to a level where they were not observable with conventional spin echo pulse sequences. Due to the very short TE (on the order of $1 / 10 \mathrm{~ms}$ ) 
used for UTE imaging, only negligible $\mathrm{T}_{2}$ decay occurs before sampling, and consequently high signal from the short- $\mathrm{T}_{2}$ components can be obtained. Coolen et al. reported that MRI parameters could be optimized for positive-contrast detection of iron-oxide labeled cells using double-echo Ultra-short echo time (d-UTE) sequences (Coolen, 2007). During these studies, there was a linear correlation between signal intensity and concentration USPIO labeled cells. Another group found that the enhancement due to the presence of short $\mathrm{T}_{2}$ USPIO accumulation generally agreed with signal loss within GRE images during ex vivo MR of aorta atherosclerotic rabbit (Crowe, 2005).

Liu et al. recently measured ultrashort $\mathrm{T}_{2}{ }^{*}$ relaxation in tissues containing a focal area of SPIO nanoparticle-labeled cells. MRI experiments in phantoms and rats with iron-labeled tumors demonstrated that these cells can be detected even at ultrashort $\mathrm{T}_{2}{ }^{*}$ down to $1 \mathrm{~ms}$ or less (Liu, 2009). The authors suggested that combining ultrashort $\mathrm{T}_{2}{ }^{*}$ relaxometry with the multiple gradient echo $\mathrm{T}_{2}{ }^{*}$ mapping techniques should improve the ability to measure the relaxation of tissues with high densities of implanted iron- labeled cells. In another investigation, T1-weighted positive contrast enhancement from SPIO particles was achieved from the UTE imaging then this sequence, taking advantage of the unique effect of MNPs on relaxation time domain, was also examined to validate its positive contrast imaging capability of "probe" targeting to U87MG human glioblastoma cells through an SPIO conjugated RDG with high affinity to the cells overexpressing integrin $\alpha_{v} \beta_{3}$ (Zhang, 2011). So the study was regarded as providing a dual contrast imaging method from UTE technique plus $\mathrm{T}_{2}$-weighted TSE images in its application of molecular imaging of glioma with potential quantification of SPIO nanoparticles suggested by previously published report (Liu, 2009).

The more recent study (Girard, 2011) showed that both contrast mechanisms of optimizing $\mathrm{T}_{1}$ contrast from UTE technique with conventional $\mathrm{T}_{2}{ }^{*}$ contrast of SPIO, even an extra subtraction of a later echo signal from the UTE signal, could be powerful both in improving the specificity by providing long $\mathrm{T}_{2}{ }^{*}$ background suppression and increasing detection sensitivity, in molecular imaging application of tumor-targeted IONPs in vivo. A hybrid sequence, PETRA (Pointwise Encoding Time reduction with Radial Acquisition) (Grodzki, 2011), combined the features of single point imaging with radial projection imaging with no need of hardware changes, to show shorter encoding times over the whole k-space and to enable higher resolution for tissue with very short $T_{2}$, compared to the UTE sequence, so that it could avoids problems derived from the UTE but with good image quality and might improve e.g. orthopedic MR imaging as well as MR-PET attenuation correction. A 3D imaging technique (Seevinck, 2011) from the group in University Medical Center Utrecht, The Netherlands, applying center-out RAdial Sampling with Off-Resonance reception (coRASOR) by the using of UTE technique (for the minimization of subvoxel dephasing at locations with high magnetic field gradients in the vicinity of the magnetized objects), and a hard, nonselective RF block pulse and radial sampling of k-space, was also presented to depict and accurately localize small paramagnetic objects with high positive contrast but ideally without background signal.

\subsection{Others new MRI pulse sequences and image postprocessing techniques}

Several other new sequences were reported on positive- and dual-contrast methods of MR cell tracking. Kim et al. recently developed simple means of detecting iron-labeled cells by 
using susceptibility weighted echo-time encoding technique (SWEET) (Kim, 2006). The subtraction of two sets of image volumes acquired at slightly-shifted echo time generates positive-contrast at the cell position. In a more recent study, the SWEET method was employed to selectively enhance the effect of the magnetic susceptibility caused by SPIOlabeled KB cells (KB cell is a cell line derived from a human carcinoma of the nasopharynx, used as an assay for antineoplastic agent). It was also demonstrated that this method could be used to visualize SPIO-labeled KB cells and their tumor formation in mice for at least a 2week period (Kim, 2009).

Dual-contrast images can also be achieved by applying $\mathrm{T}_{2}{ }^{*}$-weighted imaging combined with different post-processing techniques from the magnetic field map (Ward, 2000; Zurkiya, 2006). A susceptibility gradient mapping (SGM) technique has been recently developed, in which a color map of 3D susceptibility-gradient vector for every voxel is generated with calculated echo-shifts, and the map presents a 3D form of a positive-contrast images (Dahnke, 2008; Liu, 2008). Hyperintensities of SGM were seen in areas surrounding the 1×106 ferumoxides/protamine sulfate complex labeled flank C6 glioma cells of experimental rat model. The sensitivity of the method was compared to white-marker and IRON positive-contrast methods for visualizing the proliferation of tumor cells for labeled tumors that were approximately $5 \mathrm{~mm}$ (small), $10 \mathrm{~mm}$ (medium) and $20 \mathrm{~mm}$ (large) in diameter along the largest dimension ( $\mathrm{Liu}, 2008$ ). The number of positive voxels detected around small and medium tumors was significantly greater with the SGM technique than those with the other two techniques, while similar as the "white-marker" technique for large tumors that could not be visualized with the IRON technique. The SGM is a post-processing technique and its positive-contrast images can be derived directly from the $\mathrm{T}_{2}{ }^{*}$-weighted images without requiring dedicated positive-contrast pulse sequences, thereby it can provide the flexibility to display susceptibility gradients or suppress susceptibility artifacts in specific directions; not like the "white marker" or IRON techniques that require specialized pulse sequence designs and extra scans in addition to those obtained for conventional anatomic imaging. With SGM the hyperintense regions on positive-contrast images originating from SPIO labeled cells can be easily differentiated from other signal voids in $\mathrm{T}_{2}$ or $\mathrm{T}_{2}{ }^{*}$-weighted images.

The phase gradient mapping (PGM) techniques have recently developed independently by two groups, one related derived phase gradient maps from standard phase images also including a phase unwrapping procedure to assist the analysis and characterization of object-induced macroscopic phase perturbations (Bakker, 2008); another one utilized fast Fourier transform (FFT) to form phase gradients and develop positive contrast maps by the use of PGM but without need of phase unwrapping, so as to be appropriate technique for the visualization of magnetic nanoparticulate system (Langley, 2011; Zhao, 2011). By the method introduced recently of dual contrast with therapeutic iron nanoparticles at $4.7 \mathrm{~T}$ scanner (Sigovan, 2011), or postprocessing methods, with the measure of the $\mathrm{T}_{2}{ }^{*}$, an efficient estimation of nanoparticle concentration can be made (Langley, 2011). Applications of two kind of approaches, the traditional relaxometry method and model-based method, have demonstrated that, besides the detection of SPIO nanoparticles by positive contrast methods, quantification of the SPIO concentration also play important role in clinical evaluation of results from different treatments with monitoring cellular therapies, and the 
former derives from the signal decay associated with areas containing contrast SPIO particles (Kuhlpeter, 2007; Rad, 2007; Liu, 2009), assuming that the rate varies linearly with contrast agent concentration; the later derives from the formation of magnetic field by SPIOcontaining region (Dixon, 2009).

\section{6 $T_{1} \& T_{2}\left(T_{2}^{*}\right)$ multi-contrast for cell tracking}

As introduced in as earlier as 1990s, it is possible to achieve positive contrast and dual contrast with superparamagnetic particles by employing $\mathrm{T}_{1}$ - and/or $\mathrm{T}_{2}$-weighted sequences (Canet, 1993; Chambon, 1993; Small, 1993). Although most earlier clinical trials with magnetic nanoparticles as contrast agents were evaluated almost exclusively on $T_{2}-\mathrm{W}$ fast spin echo (FSE) and $\mathrm{T}_{2}{ }^{*}-\mathrm{W}$ gradient echo (GRE) sequences, and the strong $\mathrm{T}_{1}$ contrast enhancement effect of magnetic nanoparticles has rarely been used in clinical and molecular imaging (Reimer, 1995; Yamamoto, 1995; Tang, 1999), the effect of SPIO or USPIO on proton relaxation is not confined to $\mathrm{T}_{2}$ and $\mathrm{T}_{2}{ }^{*}$ effect. They should be considered to influence $\mathrm{T}_{1}$ relaxivity with increased $\mathrm{SI}$ on $\mathrm{T}_{1}-\mathrm{W}$ GRE sequences at low concentrations. For in vivo imaging application of MNPs, optimal combination of negative and positive contrast methods is still under evaluation.

Superparamagnetic iron oxide particles (SPIO) were used shortly after gadolinium-chelate magnetic resonance (MR) contrast agent as well known, while USPIO being the strong $\mathrm{T}_{2}$ relaxivity that produces negative contrast also a high $\mathrm{T}_{1}$ relaxivity with an increase in $\mathrm{SI}$ on $\mathrm{T}_{1}$-weighted images (Small, 1993), so that a biphasic imaging sequence protocol (only immediate postadministration and 20-24 hr delayed images) in the in vivo study allowed visualization of the dynamic enhancement patterns of both normal tissue and potentially tumor based on early $\mathrm{T}_{1}$-shortening effects produced by intravascular USPIO particulate agent (BMS 180549, previously AMI-227) and marked $\mathrm{T}_{1}$-shortening produced following agent uptake by liver and spleen, as well as showed markedly less $\mathrm{T}_{2}$-shortening at 20-24 hr within both liver and spleen.

The more recent investigation (Zhang, 2011) demonstrated that an appropriate SPIO core size and concentration range was paid much attention to obtain positive contrast with UTE imaging, and this technique could be used with the receptor targeted SPIO molecular imaging probe so as to provide an opportunity for monitoring cancer cells with overexpression of integrin $\alpha_{v} \beta_{3}$ in addition to negative contrast by the approach of $T_{2}$ relaxometry mapping.

Investigators recently synthesized a biocompatible water-dispersible $\mathrm{Fe}_{3} \mathrm{O}_{4}-\mathrm{SiO}_{2}-\mathrm{Gd}-$ DTPA-RGD nanoparticle with $\mathrm{r}_{1}$ relaxivity of $4.2 \mathrm{~mm}^{-1} \mathrm{~s}^{-1}$ and $\mathrm{r}_{2}$ relaxivity of $17.4 \mathrm{~mm}^{-1} \mathrm{~s}^{-1}$ at the $\mathrm{Gd} / \mathrm{Fe}$ molar ratio of $0.3: 1$, indicating the potential to use this multifunctional agent for dual-contrast MR imaging of tumor cells over-expressing high-affinity $\alpha_{v} \beta_{3}$ integrin in vitro and in vivo (Yang, 2011).

\section{Imaging contrast of IRON-labeled cell on multimodular platform}

MRI can be commonly used to set up a kind of nanomedicine platform for applications of multimodality probe to obtain information about concomitant anatomic, chemical, and physiological features of body. This kind of approach has been found under the 
background that, the nanomedicine platform could capitalize on the availability of specific probes, while achieving an theranostic (integrated diagnostic and therapeutic) design to allow for the visualization of therapeutic efficacy by noninvasive imaging methods such as MRI (Guthi, 2010), for example, in the field of tumor imaging researches, the combination of diagnostic capability with therapeutic intervention is critical to address the challenges of cancer heterogeneity and adaptive resistance, also molecular diagnosis by imaging is important to verify the cancer biomarkers in the tumor tissue and to guide target-specific therapy. It has been thought that ideal multimodality imaging probes enhance capabilities from complementary imaging modalities to enable both noninvasive and invasive molecular imaging (e.g, via probes with MRI and NIR fluorescence reporter capabilities) and to facilitate verification of disease detection and deliver additional evidences for the pathology (eg, probes with reporter capabilities for both positron emission tomography and MRI) (Kircher, 2003b; Lee, 2008). As for the establishment and utilizations of multimodular platform, such as optical and multimodality molecular imaging; multifunctional PET/MRI contrast agent; focused ultrasound/magnetic nanoparticle targeting delivery; design magnetic nanoparticles, etc, some topics are beyond of the scope of this chapter, and some good review papers have already published, so readers are recommended to check them (Jaffer, 2009; Chomoucka, 2010; Liu, 2010; Veiseh, 2010).

Guthi et al. recently introduced a multifunctional methoxy-terminated PEG-b-PDLLA micelle system that was encoded with a lung cancer-targeting peptide (LCP) and loaded with SPIO together with doxorubicin for MR imaging and therapeutic delivery in their in vitro study of a lung cancer (Guthi, 2010), they presented a significantly increased cell targeting, micelle uptake, superb $\mathrm{T}_{2}$ relaxivity for ultrasensitive MR detection and cell cytotoxicity in $\alpha_{v} \beta_{6}$-expressing lung cancer cells, with confocal laser scanning microscopy of Doxo fluorescence also used to study the targeting specificity of LCP-encoded micelles to $\mathrm{a}_{\mathrm{v}} \beta_{6}$-expressing $\mathrm{H} 2009$ over the $\mathrm{a}_{\mathrm{v}} \beta_{6}$-negative $\mathrm{H} 460$ cells. The same micelles were previously conjugated with a cRGD ligand that can target $\alpha_{v} \beta_{3}$ integrins on tumor endothelial (SLK) cells (Nasongkla, 2006), illustrating growth inhibition of tumor SLK cells with ultrasensitive detection by MRI. The same lab in University of Texas Southwestern Medical Center at Dallas has previously demonstrated a multi-functional micelle design that allows for the vascular targeting of tumor endothelial cells, MRI ultrasensitivity, and controlled release of doxorubicin (Doxo) for therapeutic drug delivery (Nasongkla, 2006; Khemtong, 2009). Investigators (Guthi, 2010) found that SPIO-clustered polymeric micelle design has considerably decreased the MR detection limit to subnanomolar concentrations $(<\mathrm{nM})$ of micelles through the increased $\mathrm{T}_{2}$ relaxivity and high loading of SPIO per micelle particle; suggested that, on that multifunctional platform, the application of positive contrast imaging, such as ORS, could further enhance the contrast sensitivity and allow for the in vivo imaging of tumor-specific markers.

The proposed approaches of dual imaging (e.g. with CLIO modified with a NIR fluorophore, therapeutic siRNA sequences, and a cell penetrating peptide for cancer) Medarova, 2007), even multi-modular imaging (e.g. with triple functional iron oxide nanoparticles) (Xie, 2010) demonstrate potential for the creation of targeted multifunctional nanomedicine platforms. 


\section{Perspectives}

There is an increasing interest in using cellular MRI to monitor behavior and physiologic functions of iron-labeled cells in vivo. Iron particles provide good MR probing capabilities and some of these agents are currently available for clinical applications. Based on the fact that iron particles exhibit unique nanoscale properties of super-paramagnetism and have the potential to be utilized as excellent probes for cellular imaging and molecular imaging, several MR techniques have recently been proposed to increase the detection sensitivity for image contrast generated with iron-labeled cells, including negative-, positive- and dualcontrast methods for visualization of iron-labeled cells in vitro and in vivo.

The hyperintense regions on positive-contrast images originating from iron-labeled cells can be easily differentiated from other signal voids on $T_{2}$ or $T_{2}{ }^{*}$-weighted images, therefore providing a greater degree of certainty in the determination of labeled cells. Moreover, the hyperintensities appeared to illustrate a greater sensitivity than the dark spots on regular MR images. Because positive-contrast imaging approaches do not provide sufficient anatomical information, it is necessary to combine positive-contrast techniques with conventional gradient echo or spin echo imaging, to achieve dual-contrast. Also, the combinined gadolinium and SPIO-enhanced imaging in a 'dual contrast' MRI could be the more accurate technique for the detection of rntities, especially of tumors. Additionally, some new applications of agents for MR imaging have been tested so as to obtain dualcontrast agents for noninvasive imaging studies. Dual-contrast MRI techniques for in vivo cell tracking will add to the growing armamentarium for preclinical cellular MR imaging and further demonstrate the value and diagnostic power of molecular MR imaging, and multifunctional iron oxide nanoparticles together with MRI will have unique advantages with diagnostic and therapeutic capabilities. Simutaneously, the "concept" of dual-contrast imaging can be expaned into imaging evaluation on the platform of dual-modality (or even multimodal approach) including the simultaneous MRI-PET of new method for functional and morphological imaging with blooming perspectives for further development.

While much progress has been made to date, many challenges still face cellular MRI approaches aimed at assessing the migration, homing and function of transplanted therapeutic iron-labeled cells in vivo. For cellular MRI techniques to be successful, the combined expertise of basic scientists, clinicians and representatives from industry will undoubtedly be essential.

\section{References}

Anderson, S., Glod, J., Arbab, A., Noel, M., Ashari, P., Fine, H., \& Frank, J. (2005). Noninvasive MR Imaging of Magnetically Labeled Stem Cells to Directly Identify Neovasculature in a Glioma Model. Blood, Vol. 105, No. 1, (August 2004), pp. 420425, ISSN 0006-4971

Andronesi, O., Mintzopoulos, D., Righi, V., Psychogios, N., Kesarwani, M., He, J., Yasuhara, S., Dai, G., Rahme, L., \& Tzika, A. (2010). Combined Off-resonance Imaging and $\mathrm{T}_{2}$ Relaxation in The Rotating Frame for Positive Contrast MR Imaging of Infection in a Murine Burn Model. J Magn Reson Imaging, Vol. 32, No. 5, (November 2010), pp. 1172-1183, ISSN 1053-1807 
Arbab, A., Jordan, E., Wilson, L., Yocum, G., Lewis, B., \& Frank, J. (2004a). In Vivo Trafficking and Targeted Delivery of Magnetically Labeled Stem Cells. Hum Gene Ther, Vol.15, No. 4, (July 2004), pp. 351-360, ISSN 1043-0342

Arbab, A., Yocum, G., Kalish, H., Jordan, E., Anderson, S., Khakoo, A., Read, E., \& Frank, J. (2004b). Efficient Magnetic Cell Labeling with Protamine Sulfate Complexed to Ferumoxides for Cellular MRI. Blood, Vol. 104, No. 4, (April 2004), pp. 1217-1223, ISSN 1053-1807

Arbab, A., \& Frank, J. (2008). Cellular MRI and Its Role in Stem Cell Therapy. Regen Med, Vol. 3, No. 2, (March 2008), pp. 199-215, ISSN 1746-0751

Bakker, C., de Leeuw, H., Vincken, K., Vonken, E., \& Hendrikse, J. (2008). Phase Gradient Mapping as an Aid in The Analysis of Object-induced and System-related Phase Perturbations in MRI. Phys. Med. Biol., Vol. 53, No. 18, (September 2008), pp. N349N358, ISSN 0031-9155

Balchandani, P., Yamada, M., Pauly, J., Yang, P., \& Spielman, D. (2009). Self-refocused Spatial-spectral Pulse for Positive Contrast Imaging of Cells Labeled with SPIO Nanoparticles. Magn Reson Med, Vol. 62, No. 1, (May 2009), pp. 183-192, ISSN 07403194

Briley-Saebo, K., Leboeuf, M., Dickson, S., Mani, V., Fayad, Z., Palucka, A., Banchereau, J., \& Merad, M. (2010). Longitudinal Tracking of Human Dendritic Cells in Murine Models Using Magnetic Resonance Imaging. Magn Reson Med, Vol. 64, No. 5, (October 2010), pp. 1510-1519, ISSN 0740-3194

Bulte, J., Trevor Douglas, T., Witwer, B., Zhang. S, Strable, E., Lewis, B., Zywicke, H., Miller, B., van Gelderen, P., Moskowitz, B., Duncan, I., \& Frank, J. (2001). Magnetodendrimers Allow Endosomal Magnetic Labeling and In Vivo Tracking of Stem Cells. Nature Biotechnology, Vol. 19, No. 12, (December 2001), pp. 1141-1147, ISSN 1087-0156

Bulte, J., \& Kraitchman, D. (2004a). Iron Oxide MR Contrast Agents for Molecular and Cellular Imaging. NMR Biomed, Vol. 17, No. 7, (November 2004), pp. 484-499, ISSN 0952-3480

Bulte, J., \& Kraitchman, D. (2004b). Monitoring Cell Therapy Using Iron Oxide MR Contrast Agent. Curr Pharm Biotechnol, Vol. 5, No. 6, (December 2004) , pp. 567-584, ISSN 1389-2010

Canet, E., Revel, D., Forrat, R., Baldy-Porcher, C., de Lorgeril, M., Sebbag, L., Vallee, J., Didier, D., \& Amiel, M. (1993). Superparamagnetic Iron Oxide Particles and Positive Enhancement for Myocardial Perfusion Studies Assessed by Subsecond T1-weighted MRI. Magn Reson Imaging, Vol. 11, No. 8, (March 2004), pp. 1139-1145, 0730-725X

Cerdan S, Lötscher HR, Künnecke B, Seelig J. (1989). Monoclonal Antibodycoated Magnetite Particles as Contrast Agents in Magnetic Resonance Imaging of Tumors. Magn Reson Med, Vol. 12, No. 2, (November 2005), pp. 151-163, ISSN 0740-3194

Chambon, C., Clement, O., Blanche, R., Schouman-Claeys, E., \& Frija, G. (1993). Superparamagnetic Iron Oxides as Positive MR Contrast Agents: In Vitro and In Vivo Evidence. Magn Reson Imaging, Vol. 11, No. 4, (March 2004), pp. 509-519, ISSN 0730-725X

Chen, K., Xie, J., Xu, H., Behera, D., Michalski, M., Biswal, S., Wang, A., \& Chen, X. (2009). Triblock Copolymer Coated Iron Oxide Nanoparticle Conjugate for Tumor Integrin 
Targeting. Biomaterials, Vol. 30, No. 36, (September 2009), pp. 6912-6919, ISSN 01429612

Chomoucka, J., Drbohlavova, J., Huska, D., Adam, V., Kizek, R., \& Hubalek, J. (2010). Magnetic Nanoparticles and Targeted Drug Delivering. Pharmacol Res, Vol. 62, No. 2, (Febuary 2010), pp. 144-149, ISSN 1043-6618

Coolen, B., Lee, P., Shuter, B., \& Golay, X. (2007). Optimized MRI Parameters for Positive Contrast Detection of Iron-oxide Labeled Cells Using Double-echo Ultra-short Echo Time (d-UTE) Sequences, Proceedings of Intl Soc Magn Reson Med, ISSN 154544362005, Berlin, Germany, May 2007

Crowe, L., Wang, Y., Gatehouse P., Tessier, J., Waterton, J., Robert, P., Bydder, G., \& Firmin, D. (2005). Ex vivo MR Imaging of Atherosclerotic Rabbit Aorta Labelled with USPIO - Enhancement of Iron Loaded Regions in UTE Imaging, Proceedings of Intl Soc Magn Reson Med, ISSN 1545-4436, Miami Beach, Florida, USA, May 2005

Cunningham, C., Arai, T., Yang, P., McConnell, M., Pauly, J., \& Conolly, S. (2005). Positive Contrast Magnetic Resonance Imaging of Cells Labeled with Magnetic Nanoparticles. Magn Reson Med, Vol. 53, No. 5, (April 2005), pp. 999-1005, ISSN 0740-3194

Dahnke, H., Liu, W., Herzka, D., Frank, J., \& Schaeffter, T. (2008). Susceptibility Gradient Mapping (SGM): A New Postprocessing Method for Positive Contrast Generation Applied to Superparamagnetic Iron Oxide Particle (SPIO)-labeled Cells. Magn Reson Med, Vol. 60, No. 3, (August 2008), pp. 595-603, ISSN 0740-3194

de Vries, I., Lesterhuis, W., Barentsz, J., Verdijk, P., van Krieken, J., Boerman, O., Oyen, W., Bonenkamp, J., Boezeman, J., Adema, G., Bulte, J., Scheenen, T., Punt, C., Heerschap, A., \& Figdor, C. (2005). Magnetic Resonance Tracking of Dendritic Cells in Melanoma Patients for Monitoring of Cellular Therapy. Nature Biotechnology, Vol. 23, No. 11, (October 2005), pp. 1407-1413, ISSN 1087-0156

Dharmakumar, R., Koktzoglou, I., \& Li, D. (2006). Generating Positive Contrast from OffResonant Spins with Steady-state Free Precession Magnetic Resonance Imaging: Theory and Proof-of-principle Experiments. Phys. Med. Biol., Vol. 51, No. 17, (August 2006), pp. 4201-4215, ISSN 0031-9155

Dharmakumar, R., Koktzoglou, I., \& Li, D. (2007). Factors Influencing Fast Low Angle Positive Contrast Steady-state Free Precession (FLAPS) Magnetic Resonance Imaging. Phys. Med. Biol., Vol. 52, No. 11, (May 2007), pp. 3261-3273, ISSN 00319155

Dharmakumar, R., Zhang, Z., Koktzoglou, I., Tsaftaris, S., \& Li, D. (2009). Dual-contrast Cellular Magnetic Resonance Imaging. Mol Imaging, Vol. 8, No. 5, (October 2009), pp. 254-263, ISSN 1535-3508

Dixon, W., Blezek, D., Lowery. L, Meyer, D., Kulkarni, A., Bales, B., Petko, D., Foo, T. (2009). Estimating Amounts of Iron Oxide from Gradient Echo Images. Magn Reson Med, Vol. 61, No. 5, (Febuary 2009), pp. 1132-1136, ISSN 0740-3194

Farrar, C., Dai, G., Novikov, M., Rosenzweig, A., Weissleder, R., Rosen, B., Sosnovik, D. (2008). Impact of Field Strength and Iron Oxide Nanoparticle Concentration on The Linearity and Diagnostic Accuracy of Off-resonance Imaging. NMR Biomed, Vol. 21, No. 5, (October 2007), pp. 453-463, ISSN 0952-3480 
Ferrucci, J., \& Stark, D. (1990). Iron Oxide-enhanced MR Imaging of The Liver and Spleen: Review of the First 5 years. AJR Am J Roentgenol, Vol. 155, No. 5, (November 1990), pp. 943-950, ISSN 0361-803X

Foltz, W., Cunningham, C., Mutsaers, A., Conolly, S., Stewart, D., \& Dick, A. (2006). Positive-contrast Imaging in the Rabbit Hind-limb of Transplanted Cells Bearing Endocytosed Superparamagnetic Beads. J Cardiovasc Magn Reson, Vol. 8, No. 6, (November 2006), pp. 817-823, ISSN 1097-6647

Frank, J., Anderson, S., Kalsih, H., Jordan, E., Lewis, B., Yocum, G., \& Arbab, A. (2004). Methods For Magnetically Labeling Stem and Other Cells for Detection by In Vivo Magnetic Resonance Imaging. Cytotherapy, Vol. 6, No. 6, (November 2004), pp. 621625, ISSN 1465-3249

Genove, G., DeMarco, U., Xu, H., Goins, W., \& Ahrens, E. (2005). A New Transgene Reporter for In Vivo Magnetic Resonance Imaging. Nat Med, Vol. 11, No. 4, (April 2005), pp. 450-454, ISSN 1078-8956

Girard, O., Du, J., Agemy, L., Sugahara, K., Kotamraju, V., Ruoslahti, E., Bydder, G., \& Mattrey, R. (2011). Optimization of Iron Oxide Nanoparticle Detection Using Ultrashort Echo Time Pulse Sequences: Comparison of T1, T2*, and Synergistic T1 T2* Contrast Mechanisms. Magn Reson Med, Vol. 65, No. 6, (Febuary 2011), pp. 1649-1660, ISSN 0740-3194

Gonzalez-Lara, L., Xu, X., Hofstetrova, K., Pniak, A., Brown, A., \& Foster, P. (2009). In Vivo Magnetic Resonance Imaging of Spinal Cord Injury in The Mouse. J Neurotrauma, Vol. 26, No. 5, (May 2009), pp. 753-762, ISSN 0897-7151

Grodzki, D., Jakob, P., \& Heismann, B. (2011). Ultrashort Echo Time Imaging Using Pointwise Encoding Time Reduction with Radial Acquisition (PETRA). Magn Reson Med, Article first published online : 30 JUN 2011, ISSN 0740-3194

Gupta, T., Virmani, S., Neidt, T., Szolc-Kowalska, B., Sato, K., Ryu, R., Lewandowski, R., Gates, V., Woloschak, G., Salem, R., Omary, R., \& Larson, A. (2008). MR Tracking of Iron-labeled Glass Radioembolization Microspheres during Transcatheter Delivery to Rabbit VX2 Liver Tumors: Feasibility Study. Radiology, Vol. 249, No. 3, (October 2008), pp. 845-854, ISSN 0033-8419

Guthi, J., Yang, S., Huang, G., Li, S., Khemtong, C., Kessinger, C., Peyton, M., Minna, J., Brown, K., \& Gao, J. (2010). MRI-visible Micellar Nanomedicine for Targeted Drug Delivery to lung cancer cells. Mol Pharm, Vol. 7, No. 1, (Febrary 2010), pp. 32-40, ISSN 1543-8384

Helmberger, T., \& Semelka, R. (2001). New Contrast Agents for Imaging The Liver. Magn Reson Imaging Clin N Am, Vol. 9, No. 4, (November 2001), pp. 745-766, ISSN 10649689

Herschman, H. (2003). Molecular Imaging: Looking At Problems, Seeing Solutions. Science, Vol. 302, No. 5645, (October 2003), pp. 605-608, ISSN 0036-8075

Heyn, C., Bowen, C., Rutt, B., \& Foster, P. (2005). Detection Threshold of Single SPIO-labeled Cells with FIESTA. Magn Reson Med, Vol. 53, No. 2, (Febuary 2005), pp. 312-320, ISSN 0740-3194

Heyn, C., Ronald, J., Mackenzie, L., MacDonald, I., Chambers, A., Rutt, B., Foster, P. (2006). In Vivo Magnetic Resonance Imaging of Single Cells in Mouse Brain with Optical Validation. Magn Reson Med, Vol. 55, No. 1, (January 2006), pp. 23-29, ISSN 07403194 
Högemann-Savellano, D., Bos, E., Blondet, C., Sato, F., Abe, T., Josephson, L., Weissleder, R., Gaudet, J., Sgroi, D., Peters, P., \& Basilion, J. (2003). The Transferrin Receptor: a Potential Molceular Imaging Marker for Human Cancer. Neoplasia, Vol. 5, No. 6, (November 2003), pp. 495- 506, ISSN 1522-8002

Hsiao, J., Tai, M., Chu, H., Chen, S., Li, H., Lai, D., Hsieh, S., Wang, J., \& Liu, H. (2007). Magnetic Nanoparticle Labeling of Mesenchymal Stem Cells Without Transfection Agent: Cellular Behavior and Capability of Detection with Clinical 1.5T Magnetic Resonance at The Single Cell Level. Magn Reson Med, Vol. 58, No. 4, (October 2006), pp. 717-724, ISSN 0740-3194

Jaffer, F., Libby, P., \& Weissleder, R. (2009). Optical and Multimodality Molecular Imaging: Insights Into Atherosclerosis. Arterioscler Thromb Vasc Biol., Vol. 29, No. 7, (July 2009), pp. 1017-1024. ISSN 1079-5642

Kelloff, G., Krohn, K., Larson, S., Weissleder, R., Mankoff, D., Hoffman, J., Link, J., Guyton, K., Eckelman, W., Scher, H., O'Shaughnessy, J., Cheson, B., Sigman, C., Tatum, J., Mills, G., Sullivan, D., \& Woodcock, J. (2005). The Progress and Promise of Molecular Imaging Probes in Oncologic Drug Develpoment. Clin Cancer Research, Vol. 11, No. 22, (November 2005), pp. 7967-7985, ISSN 1078-0432

Khakoo, A., Pati, S., Anderson, S., Reid, W., Elshal, M., Rovira, I., Nguyen, A., Malide, D., Combs, C., Hall, G., Zhang, J., Raffeld, M., Rogers, T., Stetler-Stevenson, W., Frank, J., Reitz, M., \& Finkel, T. (2006). Human Mesenchymal Stem Cells Exert Potent Antitumorigenic Effects in A Model of Kaposi's Sarcoma. J Exp Med, Vol. 203, No. 5, pp. 1235-1247, ISSN 0022-1007

Khemtong, C., Kessinger, C., Ren, J., Bey, E., Yang, S., Guthi, J., Boothman, D., Sherry, A., \& Gao, J. (2009). In Vivo Off-resonance Saturation Magnetic Resonance Imaging of Alphavbeta3-targeted Superparamagnetic Nanoparticles. Cancer Res, Vol. 69, No. 4, (Febrary 2009), pp. 1651-1658, ISSN 0008-5472

Kim, Y., Bae, K., Lee, Y., Park, T., Yoo, S., \& Park, H. (2006). Positive-Contrast Cellular MR Imaging Using Susceptibility Weighted Echo-time Encoding Technique (SWEET), World Congress on Medical Physics and Biomedical Engineering, ISBN 9783540368397, Coex Seoul, Korea, August 2006

Kim, Y., Bae, K., Yoo, S., Park, T., \& Park, H. (2009). Positive Contrast Visualization for Cellular Magnetic Resonance Imaging Using Susceptibility-weighted Echo-time Encoding. Magn Reson Imaging, Vol. 27, No. 5, (December 2008), pp. 601-610, 0730$725 \mathrm{X}$

Kircher, M., Allport, J., Graves, E., Love, V., Josephson, L., Lichtman, A., \& Weissleder, R. (2003a). In Vivo High Resolution Three-dimensional Imaging of Antigen-specific Cytotoxic T-lymphocyte Trafficking to Tumors. Cancer Res, Vol. 63, No. 20, (October 2003), pp. 6838-6846, ISSN 0008-5472

Kircher, M., Mahmood, U., King, R., Weissleder, R., \& Josephson, L. (2003b). A Multimodal Nanoparticle for Preoperative Magnetic Resonance Imaging and Intraoperative Optical Brain Tumor Delineation. Cancer Res, Vol. 63, No. 23, (Deceber 2003), pp. 8122-8125, ISSN 0008-5472

Korosoglou, G., Shah, S., Vonken, E., Gilson, W., Schär, M., Tang, L., Kraitchman, D., Boston, R., Sosnovik, D., Weiss, R., Weissleder, R., \& Stuber, M. (2008a). Off Resonance Angiography: A New Method to Depict Vessels-phantom and Rabbit Studies. Radiology, Vol. 249, No. 2, (September 2008), pp. 501-509, ISSN 0033-8419 
Korosoglou, G., Weiss, R., Kedziorek, D., Walczak, P., Gilson, W., Schär, M., Tang, L., Kraitchman, D., Boston, R., Sosnovik, D., Weiss, R., Bulte, J., Weissleder, R., \& Stuber, M. (2008b). Noninvasive Detection of Macrophage-rich Atherosclerotic Plaque in Hyperlipidemic Rabbits Using "Positive contrast" Magnetic Resonance Imaging. J Am Coll Cardiol, Vol. 52, No. 6, (August 2008), pp. 483-491, ISSN 07351097

Korosoglou, G., Tang, L., Kedziorek, D., Cosby, K., Gilson, W., Vonken, E., Schär, M., Sosnovik, D., Kraitchman, D., Weiss, R., Weissleder, R., \& Stuber, M. (2008c). Positive Contrast MR-lymphography Using Inversion Recovery with ON-resonant Water Suppression (IRON). J Magn Reson Imaging, Vol. 27, No. 5, (May 2008), pp. 1175-1180, ISSN 1053-1807

Kraitchman, D., Tatsumi, M., Gilson, W., Ishimori, T., Kedziorek, D., Walczak, P., Segars, W., Chen, H., Fritzges, D., Izbudak, I., Young, R., Marcelino, M., Pittenger, M., Solaiyappan, M., Boston, R., Tsui, B., Wahl, R., \& Bulte, J. (2005). Dynamic Imaging of Allogeneic Mesenchymal Stem Cells Trafficking to Myocardial Infarction. Circulation, Vol. 112, No. 10, (August 2005), pp. 1451-1461. ISSN 0009-7322

Kuhlpeter, R., Dahnke, H., Matuszewski, L., Persigehl, T., von Wallbrunn, A., Allkemper, T., Heindel, W., Schaeffter, T., \& Bremer, C. (2007). R2 and R2 Mapping for Sensing Cell-Bound Superparamagnetic Nanoparticles. Radiology, Vol. 245, No. 2, (September 2007), pp. 449-457, ISSN 0033-8419

Langley, J., Liu, W., Jordan, E., Frank, J., \& Zhao, Q. (2011). Quantification of SPIO Nanoparticles In Vivo Using the Finite Perturber Method. Magn Reson Med, Vol. 65, No. 5, (November 2010), pp. 1461-1469, ISSN 0740-3194

Lee, H., Li, Z., Chen, K., Hsu, A., Xu, C., Xie, J., Sun, S., \& Chen, X. (2008). PET/MRI DualModality Tumor Imaging Using Arginine-glycine-aspartic (RGD)-conjugated Radiolabeled Iron Oxide Nanoparticles. J Nucl Med, Vol. 49, No. 8, (July 2008), 1371-1379. ISSN 0161-5505

Lefevre S, Ruimy D, Jehl F, Neuville A, Robert P, Sordet C, Ehlinger M, Dietemann JL, Bierry G. (2011). Septic Arthritis: Monitoring with USPIO-enhanced Macrophage MR Imaging. Radiology, Vol. 258, No. 3, (March 2011), pp. 722-728, ISSN 0033-8419

Li, W,, Tutton, S, Vu, A., Pierchala, L., Li, B., Lewis, J., Prasad, P., \& Edelman, R. (2005) First-pass Contrast-enhanced Magnetic Resonance Angiography in Humans Using Ferumoxytol, A Novel Ultrasmall Superparamagnetic Iron Oxide (USPIO)-based Blood Pool Agent. J Magn Reson Imaging, Vol. 1, No. 5, (January 2005), pp. 46-52, ISSN 1053-1807

Liu, C., Ren, J., Yang, J., Liu, C., Mandeville, J., Rosen, B., Bhide, P., Yanagawa, Y., \& Liu, P. (2009). Dna-based Mri Probes for Specic Detection of Chronic Exposure to Amphetamine in Living Brains. The Journal of Neuroscience, Vol. 29, No. 34, (August 2009), pp. 10663-10670, ISSN 0270-6474

Liu, H., Hua, M., Yang, H., Huang, C., Chu, P., Wu, J., Tseng, I., Wang, J., Yen, T., Chen, P., \& Wei, K. (2010). Magnetic Resonance Monitoring of Focused Ultrasound/Magnetic Nanoparticle Targeting Delivery of Therapeutic Agents to the Brain. Proc Natl Acad Sci U S A., Vol. 107, No. 34, (August 2010), pp. 1520515210, ISSN 0027-8424

Liu, W., Dahnke, H., Jordan, E., Schaeffter, T., \& Frank, J. (2008). In Vivo MRI Using Positive-contrast Techniques in Detection of Cells Labeled with Superparamagnetic 
Iron Oxide Nanoparticles. NMR Biomed, Vol. 21, No. 3, (March 2008), pp. 242-250, ISSN 0952-3480

Liu, W., Dahnke, H., Rahmer, J., Jordan, E., \& Frank, J. (2009). Ultrashort T2* Relaxometry for Quantitation of Highly Concentrated Superparamagnetic Iron Oxide (SPIO) Nanoparticle Labeled Cells. Magn Reson Med, Vol. 61, No. 4, (April 2009), pp. 761766, ISSN 0740-3194

Mani, V., Briley-Saebo, K., Hyafil, F., \& Fayad, Z. (2006a). Feasibility of In Vivo Identification of Edogenous Ferritin with Positive Contrast MRI in Rabbit Carotid Crush Injury Using GRASP. Magn Reson Med, Vol. 56, No. 5, (November 2006), pp. 1096-1106, ISSN 0740-3194

Mani, V., Briley-Saebo, K., Itskovich, V., Samber, D., \& Fayad, Z. (2006b). Gradient Echo Acquisition for Superparamagnetic Particles with Positive Contrast (GRASP): Sequence Characterization in Membrane and Glass Superparamagnetic Iron Oxide Phantoms at 1.5 T and 3 T. Magn Reson Med, Vol. 55, No. 1, (January 2006), pp. $126-$ 135, ISSN 0740-3194

Mani, V., Adler, E., Briley-Saebo, K., Bystrup, A., Fuster, V., Keller, G., \& Fayad, Z. (2008). Serial In Vivo Positive Contrast MRI of Iron Oxide-labeled Embryonic Stem Cell Derived Cardiac Precursor Cells in A Mouse Model of Myocardial Infarction. Magn Reson Med, Vol. 60, No. 1, (July 2008), pp. 73-81, ISSN 0740-3194

Manninger, S., Muldoon, L., Nesbit, G., Murillo, T., Jacobs, P., \& Neuwelt, E. (2005). An Exploratory Study of Ferumoxtran-10 Nanoparticles as a Blood-brain barrier Imaging Agent Targeting Phagocytic Cells in CNS Inflammatory Lesions. AJNR Am J Neuroradiol., Vol. 26, No. 9, (October 2005), pp. 2290-2300.

Medarova, Z., Pham, W., Farrar, C., Petkova, V., \& Moore, A. (2007). In Vivo Imaging of siRNA Delivery and Silencing in Tumors. Nat Med, Vol. 13, No. 3, (Febrary2007), pp. 372-377, ISSN 1078-8956

Metz, S., Beer, A., Settles, M., Pelisek, J., Botnar, R., Rummeny, E., \& Heider, P. (2011). Characterization of Carotid Artery Plaques with USPIO-enhanced MRI: Assessment of Inflammation and Vascularity as In Vivo Imaging Biomarkers for Plaque Vulnerability. Int J Cardiovasc Imaging, Vol. 27, No. 6, (October 2010), pp. 901-912, ISSN 1569-5794

Modo, M., Hoehn, M., \& Bulte, J. (2005). Cellular MR imaging. Mol Imaging., Vol. 4, No. 3, (July 2005), pp. 143-164, ISSN 1535-3508

Muja, N., \& Bulte, J. (2009). Magnetic Resonance Imaging of Cells in Experimental Disease Models. Prog Nucl Magn Reson Spectrosc., Vol. 55, No. 1, (July 2009), pp. 61-77, ISSN 0079-6565

Nasongkla, N., Bey, E., Ren, J., Ai, H., Khemtong, C., Guthi, J., Chin, S., Sherry, A., Boothman, D., \& Gao, J. (2006). Multifunctional Polymeric Micelles as Cancertargeted, MRI-ultrasensitive Drug Delivery Systems. Nano Lett., Vol. 6, No. 11, (November 2006), pp. 2427-2430, ISSN 1530-6984

Niu, G., \& Chen, X. (2011). Why Integrin as A Primary Target for Imaging and Therapy. Theranostics, Vol. 1, No. 1, (January 2011), pp. 30-47, ISSN 1838-7640

Oude Engberink, R., van der Pol, S., Döpp, E., de Vries, H., \& Blezer, E. (2007). Comparison of SPIO and USPIO for In Vitro Labeling of Human Monocytes: MR Detection and Cell Function. Radiology, Vol. 243, No. 2, (May 2007), pp. 467-474, ISSN 0033-8419 
Politi, L., Bacigaluppi, M., Brambilla, E., Cadioli, M., Falini, A., Comi, G., Scotti, G., Martino, G., \& Pluchino, S. (2007). Magnetic-resonancebased Tracking and Quantification of Intravenously Injected Neural Stem Cell Accumulation in The Brains of Mice with Experimental Multiple Sclerosis. Stem Cells, Vol. 25, No. 10, (Jun 2007), pp. 25832592, ISSN 0250-6793

Rad, A., Arbab, A., Iskander, A., Jiang, Q., \& Soltanian-Zadeh, H. (2007). Quantification of Superparamagnetic Iron Oxide (SPIO)-labeled Cells Using MRI. J Magn Reson Imaging, Vol. 26, No. 2, (August 2007), pp 366-374, ISSN 1053-1807

Rahmer, J., Bornert, P., \& Dries, S. Assessment of Anterior Cruciate Ligament Reconstruction Using 3D Ultrashort Echo-time MR Imaging. (2009). J Magn Reson Imaging, Vol. 29, No. 2, (Febrary 2009), pp 443-448, ISSN 1053-1807

Reimer, P., Rummeny, E., Daldrup, H., Balzer, T., Tombach, B., Berns, T., \& Peters, P. (1995). Clinical Results with Resovist: A Phase 2 Clinical Trial. Radiology, Vol. 195, No. 2, (May 1995), pp. 489-496, ISSN 0033-8419

Righi, V., Andronesi, O., Mintzopoulos, D., Fichman, A., \& Tzika, A. (2010). Molecular MR Imaging of Labeled Stem Cells in A Mouse Burn Model In Vivo, Proceedings of Intl Soc Magn Reson Med, ISSN 1545-4436, Stockholm, Sweden, May 2010

Robson, M., \& Bydder, G. (2006). Clinical Ultrashort Echo Time Imaging of Bone and Other Connective Tissues. NMR Biomed, Vol. 19, No. 7, (November 2006), pp. 765-780, ISSN 0952-3480

Seevinck, P., de Leeuw, H., Bos, C., \& Bakker, C. (2011). Highly Localized Positive Contrast of Small Paramagnetic Objects Using 3D Center-out Radial Sampling with Offresonance Reception. Magn Reson Med, Vol. 65, No. 1, (January 2008), pp. 146-156, ISSN 0740-3194

Seppenwoolde, J., Viergever, M., \& Bakker, C. (2003). Passive Tracking Exploiting Local Signal Conservation: The White Marker Phenomenon. Magn Reson Med, Vol. 50, No. 4, (October 2003), pp. 784-790, ISSN 0740-3194

Seppenwoolde, J., Vincken, K., \& Bakker, C. (2007). White-marker Imaging-Separating Magnetic Susceptibility Effects from Partial Volume Effects. Magn Reson Med, Vol. 58, No. 3, (September 2007), pp. 605-609, ISSN 0740-3194

Shapiro, E., Skrtic, S., \& Koretsky, A. (2005). Sizing It Up: Cellular Mri Using Micron-sized Iron Oxide Particles. Magn Reson Med, Vol. 53, No. 2, (Febuary 2005), pp. 329-338, ISSN 0740-3194

Sigovan, M., Hamoudeh, M., Al Faraj, A., Charpigny, D., Fessi, H., \& Canet-Soulas, E. (2011). Positive Contrast with Therapeutic Iron Nanoparticles at 4.7 T. MAGMA, Vol. 24, No. 5, (May 2011), pp. 259-265, ISSN 0968-5243

Small, W., Nelson, R., \& Bernardino, M. (1993). Dual-contrast Enhancement of Both T1- and T2-weighted Sequences Using Ultra Small Superparamagnetic Iron Oxide. Magn Reson Med, Vol. 11, No. 5, (May 1993), pp. 645-654, ISSN 0740-3194

Sosnovik, D. (2009). Molecular Imaging of Myocardial Injury: A Magnetofluorescent Approach. Curr Cardiovasc Imaging Rep., Vol. 2, No. 1, (Febuary 2009), pp. 33-39, ISSN 1941-9066

Stuber, M., Gilson, W., Schaer, M., Bulte, J., \& Kraitchman, D. (2005). Shedding Light on the Dark Spot with IRON - A Method That Generates Positive Contrast in The Presence of Superparamagnetic Nanoparticles, Proceedings of Intl Soc Magn Reson Med, ISSN 1545-4436, Miami Beach, Florida, USA, May 2005 
Stuber, M., Gilson, W., Schär, M., Kedziorek, D., Hofmann, L., Shah, S., Vonken, E., Bulte, J., \& Kraitchman, D. (2007). Positive Contrast Visualization of Iron Oxide-labeled Stem Cells Using Inversion-recovery with ON-resonant Water Suppression (IRON). Magn Reson Med, Vol. 58, No. 5, (November 2007), pp. 1072-1077, ISSN 0740-3194

Suzuki, Y., Cunningham, C., Noguchi, K., Chen, I., Weissman, I., Yeung, A., Robbins, R., \& Yang, P. (2008). In Vivo Serial Evaluation of Superparamagnetic Iron-oxide Labeled Stem Cells by Off-resonance Positive Contrast. Magn Reson Med, Vol. 60, No. 6, (December 2008), pp. 1269-1275, ISSN 0740-3194

Tan, M., \& Lu, Z. (2011). Integrin Targeted MR Imaging. Theranostics, Vol. 1, No. 1, (January 2011), pp. 30-47, ISSN 1838-7640.

Tang, Y., Yamashita, Y., Arakawa, A., Namimoto, T., Mitsuzaki, K., Abe, Y., Katahira, K., \& Takahashi, M. (1999). Detection of Hepatocellular Carcinoma Arising in Cirrhotic Livers: Comparison of Gadolinium- and Ferumoxides Enhanced MR Imaging. AJR Am J Roentgenol, Vol. 172, No. 6, pp. 1547-1554, ISSN 0361-803X

Thorek, D., Chen, A., Czupryna, J., \& Tsourkas, A. (2006). Superparamagnetic Iron Oxide Nanoparticle Probes for Molecular Imaging. Ann Biomed Eng, Vol. 34, No. 1, (January 2006), pp. 23-38, ISSN 0090-6964

Trivedi, R., Mallawarachi, C., U-King-Im, J., Graves, M., Horsley, J., Goddard, M., Brown, A., Wang, L., Kirkpatrick, P., Brown, J., \& Gillard, J. (2006). Identifying Inflamed Carotid Plaques Using In Vivo USPIO-enhanced MR Imaging to Label Plaque Macrophages. Arterioscler Thromb Vasc Biol, Vol. 26, No. 7, (July 2006), pp. 16011606, ISSN 1079-5642

Veiseh, O., Gunn, J., \& Zhang, M. (2010). Design and Fabrication of Magnetic Nanoparticles for Targeted Drug Delivery and Imaging. Adv Drug Deliv Rev, Vol. 62, No. 3, (November 2009), pp. 284-304, ISSN 0169-409X

Ward, K., Aletras, A., \& Balaban, R. (2000). A New Class of Contrast Agents for MRI Based on Proton Chemical Exchange Dependent Saturation Transfer (CEST). J Magn Reson., Vol. 143, No. 1, (March 2000), pp. 79-87, ISSN 1090-7807

Weissleder, R., Elizondo, G., Wittenberg, J., Rabito, C., Bengele, H., \& Josephson, L. (1990). Ultrasmall Superparamagnetic Iron Oxide: Characterization of a New Class of Contrast Agents for MR Imaging. Radiology, Vol. 175, No. 2, (May 1990), pp. 489493, ISSN 0033-8419

Xie, J., Chen, K., Huang, J., Lee, S., Wang, J., Gao, J., Li, X., \& Chen, X. (2010). PET/NIRF/MRI Triple Functional Iron Oxide Nanoparticles. Biomaterials, Vol. 31, No. 11, (April 2010), pp. 3016-3022, ISSN 0142-9612

Yamamoto, H., Yamashita, Y. Yoshimatsu, S., Baba, Y., Hatanaka, Y., Murakami, R., Nishiharu, T., Takahashi, M., Higashida, Y., \& Moribe, N. (1995). Hepatocellular Carcinoma in Cirrhotic Livers: Detection with Unenhanced and Iron Oxideenhanced MR Imaging. Radiology, Vol. 195, No. 1, (April 1995), pp. 106-112, ISSN 0033-8419

Yang, H., Zhuang, Y., Sun, Y., Dai, A., Shi, X., Wu, D., Li, F., Hu, H., \& Yang, S. Targeted Dual-ontrast $\mathrm{T}(1)$ - and $\mathrm{T}(2)$-weighted Magnetic Resonance Imaging of Tumors Using Multifunctional Gadolinium-labeled Superparamagnetic Iron Oxide Nanoparticles. Biomaterials, Vol. 32, No. 20, (March 2011), pp. 4584-4593, ISSN 01429612 
Zhang, L., Zhong, X., Wang, L., Chen, H., Wang, Y., Yeh, J., Yang, L., \& Mao, H. T1weighted Ultrashort Echo Time Method for Positive Contrast Imaging of Magnetic Nanoparticles and Cancer Cells bound with the Targeted Nanoparticles. J Magn Reson Imaging, Vol. 33, No. 1, (January 2011), pp. 194-202, ISSN 1053-1807

Zhang, Z., Dharmakumar, R., Mascheri, N., Fan, Z., Wu, S., \& Li, D. (2009). Comparison of SPIO and USPIO Cell Labeling for Tracking GFP Gene Marker with Negative and Positive Contrast MRI. Mol Imaging, Vol. 8, No. 3, (October 2009), pp. 148-155, ISSN 1535-3508

Zhang, Z., Mascheri, N., Dharmakumar, R., \& Li, D. (2008). Cellular Magnetic Resonance Imaging: Potential for Use in Assessing Aspects of Cardiovascular Disease. Cytotherapy, Vol. 10, No. 6, (March 2008), pp. 575-586, ISSN 1465-3249

Zhang, Z., van den Bos, E., Wielopolski, P., de Jong-Popijus, M., Bernsen, M., Duncker, D., \& Krestin, G. (2005). In Vitro Imaging of Single Living Human Umbilical Vein Endothelial Cells with a Clinical 3.0-T MRI Scanner. MAGMA, Vol. 18, No. 4, (August 2005), pp. 175-185, ISSN 0968-5243

Zhao, M., Kircher, M., Josephson, L., \& Weissleder, R. (2002). Differential Conjugation of Tat Peptide to Superparamagnetic Nanoparticles and Its Effect on Cellular Uptake. Bioconjug Chem, Vol. 13, No. 4, (July 2002), pp. 840-844.

Zhao, Q., Langley, J., Lee, S., \& Liu, W. (2011). Positive Contrast Technique for the Detection and Quantification of Superparamagnetic Iron Oxide Nanoparticles in MRI. NMR Biomed, Vol. 24, No. 5, (October 2010), pp. 464-472, ISSN 0952-3480

Zhu, J., Zhou, L., \& XingWu, F. (2006). Tracking Neural Stem Cells in Patients with Brain Trauma. N Engl J Med., Vol. 30, No. 355, (November 2006), pp. 2376-2378, ISSN 0028-4793

Zurkiya, O., Chan, A., \& Hu, X. (2008). MagA is Sufficient for Producing Magnetic Nanoparticles in Mammalian Cells, Making It an MRI Reporter. Magn Reson Med, Vol. 59, No. 6, (Jun 2008), pp. 1225-1231, ISSN 0740-3194

Zurkiya, O., \& Hu, X. (2006). Off-resonance Saturation as a Means of Generating Contrast with Superparamagnetic Nanoparticles. Magn Reson Med, Vol. 56, No. 4, (October 2006), pp. 726-732, ISSN 0740-3194 


\title{
Nanoscale Electrodeposition of Copper on an AFM Tip and Its Morphological Investigations
}

\author{
Udit Surya Mohanty, S. Y. Chen and Kwang-Lung Lin \\ Department of Materials Science and Engineering, \\ National Cheng Kung University \\ Tainan, \\ R.O.C, Taiwan
}

\section{Introduction}

Electrocrystallisation processes occurring at electrochemical solid/liquid interfaces have attracted the interest of many researchers from both fundamental and applied viewpoints. After the pioneering works of Max Volmer at the beginning of the last century (Volmer, $1934 a, 1939 b)$, the processes of electrocrystallisation have been the subject of numerous intensive studies, the results of which have been documented in several books (Bockris \& Razumney 1967; Budevski, et al., 1996; Fischer,1954). The electrochemical method offers several advantages over vapour deposition techniques such as molecular beam epitaxy for depositing nanoscale superlattices. Additional technological advantages over the vapour deposition techniques consist in the relatively low processing temperature and the high selectivity. The low processing temperatures minimizes interdiffusion whereas the high selectivity of electrocrystallisation process allows uniform modification of surfaces and structures with complicated profiles. Phase formation and crystal growth phenomena are the most common morphological parameters observed in many technological important cathodic and anodic electrochemical reactions. One of the most frequently studied electrocrystallisation process is the cathodic metal deposition on foreign and native substrates from electrolytes containing complex metal ions (Fleischmann \& Thirsk, 1963; Milchev, 2002; Paunovic \& Schlesinger, 2006). Some of the typical cited examples are electrocrystallisation of $\mathrm{Ag}$ from $\mathrm{Ag}^{+}$containing electrolytes (Budevski et al., 1980; Fischer, 1969) and the electrodeposition of Cu (Budevski, 1983; Danilov et al., 1994; Hozzle et al., 1995; Michhailova et al. , 1993) which has recently become technologically important for the fabrication of $\mathrm{Cu}$ interconnects on integrated circuit chips (Andricacos et al., 1998; Oskam et al. 1998). Since the electrodeposition of metals is a process of great technological importance, a large number of studies have been carried out to understand the mechanism of electrodeposition of metals on conducting surfaces by employing a variety of electrochemical and spectroscopic techniques (Andricacos, 1999; Markovic \& Ross, 1993). The conventional electrochemical methods such as cyclic voltammetry, impedance spectroscopy have been used to assess the mechanism and kinetics of metal electrocrystallisation. These techniques however provide information on the whole surface. 
To fully understand the process, it is essential to obtain structural information on the substrate and the deposit in pm to the atomic level. Although techniques such as electron microscopy and optical microscopy have been employed to examine the morphology of the substrate and the metal deposit, they can be used only for the ex situ examination. The discovery of scanning tunnelling microscopy and atomic force microscopy (STM and AFM) offered new exciting possibilties for in situ studies of the electrocrystallisation phenomenon down to an atomic level (Binning \& Rohrer, 1982; Lustenberger et al., 1988, Sonnenfeld \& Hannsma, 1986). The application of these techniques in the last two decades has revolutionized the experimental work in this field and led to significant progress in the understanding of the atomistic aspects of the electrocrystallisation process (Gewirth \& Siegenthaler, 1995; Staikov et al., 1994; Stegenthaler, 1992). These processes range from measuring the lateral force using a cantilever tip, measuring magnetic force, electrostatic force, Kelvin potential to the determination of surface conductivity. The invention of scanning probe microscopy (SPM) also provoked a rapid development of the modern nanoscience and nanotechnology dealing with nanoscale structures and objects including single atoms and molecules. Over, the years, many other types of scanning probe microscopic techniques have evolved from the base concept of AFM. Electrochemical fabrication of metal nanostructures has been reported using SPM-based lithography, typically by tip induced electrochemical deposition of metal ions transferred by the STM or AFM tip to the surface. (Allongne, 1995; Benenz et al., 2002). Many studies have been directed towards in situ STM and AFM imaging of metal underdeposition (Hachiya et al.1991; Li et al., 2001) and bulk deposition (Nichols et al., 1992; Yau et al. 1991). Since STM can only be applied to observe conductive surfaces, the existence of anodic oxide films as well as the space charge layer in the depletion condition makes the STM measurements of semiconductor electrodes relatively difficult (Batina \& Nichols, 1992). On the other hand, AFM can image even non-conducting surfaces and electrochemical processes on the tip, which causes serious problems in the STM measurements in electrolyte solutions. AFM is also found to be more useful than STM in studying the electrode surface in situ. AFM works the same way as STM and can transfer materials from tip to substrate at a biased voltage. As AFM tips are normally made of silicon or silicon nitide, metallic materials have to be coated onto the AFM tip in order to make the deposition happen. Once it is coated with metals, it becomes no different from a STM tip, and deposition takes place under high electric field. AS AFM can work in liquid solution, it is possible to initiate electrochemical deposition using an AFM tip. Also the electrochemical reaction rate at the interface can be controlled by application of an external potential to the substrate. In particular, the amount of deposit and the kinetics of the metal deposition onto the surface can be controlled. Its because the electrochemical process is sensitive to the surface properties, in situ local deposition of metal can be made selective by tuning the surface characteristics. Copper has been electrochemically deposited onto GaAs surface by immersing the AFM tip into a mixture of $\mathrm{CuSO}_{4}+\mathrm{H}_{2} \mathrm{SO}_{4}$ solution (Carlsson et al., 1990).

The electrodeposition of $\mathrm{Cu}$ is strongly dependent on the structure of the substrate, applied potential and concentration of $\mathrm{Cu}^{2+}$ ions in the precursor solution. The nanoscale electrodeposition of metal in nanopatterned alkanethiol-modified Au (III) has been reported (Gewirth \& Sigenthaler, 1995). Although the interaction between the tip and the sample enhanced $\mathrm{Cu}$ deposition on the surface, $\mathrm{Cu}$ did not deposit uniformly in the area scanned, but only deposited at the edges of the scanned area as well as in defects in the alkanethiol 
(self assembled monolayer) (SAM). The authors suggested the physical and electrostatic inhibition by the tip, or the diffusion of $\mathrm{Cu}$ ions to the area under the tip, even on a bare $\mathrm{Au}$ (III) surface. In one of the studies (Koinuma \& Uosaki, 1994), AFM and scaling analysis have been employed to investigate the effect of current density, temperature and levelling agent on the morphology of electrolytically produced copper. However, very little information is available on the morphology of the nanostructures formed by the electrodeposition of copper on the AFM tip. In the present chapter an effort has been made to examine various modes of nucleation and growth of copper deposits on an AFM tip of $80 \mathrm{~nm}$. Electron beam lithography techniques have been employed to facilitate selective electrodeposition of copper on the nanosize AFM tip in the presence of photoresist named poly (methyl methacyrlate).

\section{Experimental method}

In the present study an AFM probe NSC/18 / Ti-Pt was used which consisted of a Si base coated by films of Ti (1st layer, $15 \mathrm{~nm}$ ) and Pt (2ndlayer) $10 \mathrm{~nm}$. The Cantilevers employed were $230 \mu \mathrm{m}$ in length and $40 \mu \mathrm{m}$ in diameter with pyramidal tips of diameter $80 \mathrm{~nm}$. The tip height ranged from 20 to $25 \mu \mathrm{m}$. The schematic diagram of the uncoated AFM probe containing the tip is shown in Fig. 1a. The magnified image is demonstrated in Fig. 1b. One of the AFM probes acted as a anode and the other as a cathode. Two connectors were placed on the AFM probes to facilitate the current flow between the power supplier and the probes, as illustrated in Fig. 2. For the preparation of a connector, a $\mathrm{Cu}$ plate of diameter $3.4 \mathrm{~mm}$ and length $12 \mathrm{~mm}$ was first plated with electroless $\mathrm{Au}$ (Fig. 3). The following electrolytic composition was used in the study: $2 \mathrm{~g} / \mathrm{L} \mathrm{KAu}(\mathrm{CN})_{2} ; 75 \mathrm{~g} / \mathrm{L} \mathrm{NH} 4 \mathrm{Cl} ; 50 \mathrm{~g} / \mathrm{L}$ $6 \mathrm{H}_{5} \mathrm{Na}_{3} \mathrm{O}_{7} \cdot 2 \mathrm{H} 2 \mathrm{O}$; and $10 \mathrm{~g} / \mathrm{L} \mathrm{NaH_{2 }} \mathrm{PO}_{2} \cdot \mathrm{H} 2 \mathrm{O}$. The $\mathrm{pH}$ of the electrolytic solution was maintained at 7 and the solution temperature was kept at $92^{\circ} \mathrm{C}$.

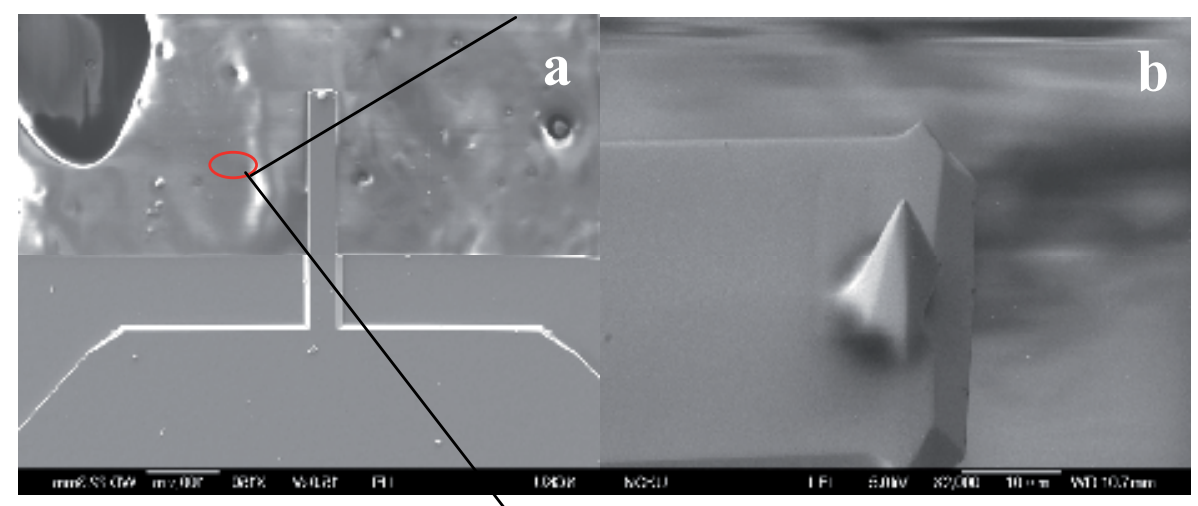

Fig. 1. SEM micrograph illustrating the schematic representation of an uncoated AFM probe

The layer of electroless gold on the copper plate provided good adhesion. The $\mathrm{Cu}$ plate coated with electroless $\mathrm{Au}$ was connected to a $\mathrm{Cu}$ wire as shown in Fig 3 by means of mechanical force. Finally, the connector was electroplated with a hard layer of Au to provide good abrasion ability and a thicker Au layer. The Si holders and the connectors were fixed together and were placed in a rectangular cell containing the electrolyte of composition $80 \mathrm{~g} / \mathrm{L} \mathrm{CuSO}_{4}$ and $200 \mathrm{~mL} / \mathrm{L} \mathrm{H}_{2} \mathrm{SO}_{4}$. The power supply was fixed in the range 
of $10 \mu \mathrm{A}$ to $100 \mathrm{~A}$. Because the AFM probes cannot bear large amounts of current, a large electrode system consisting of Pt anode and Cu cathode was used as shown in Fig. 4.

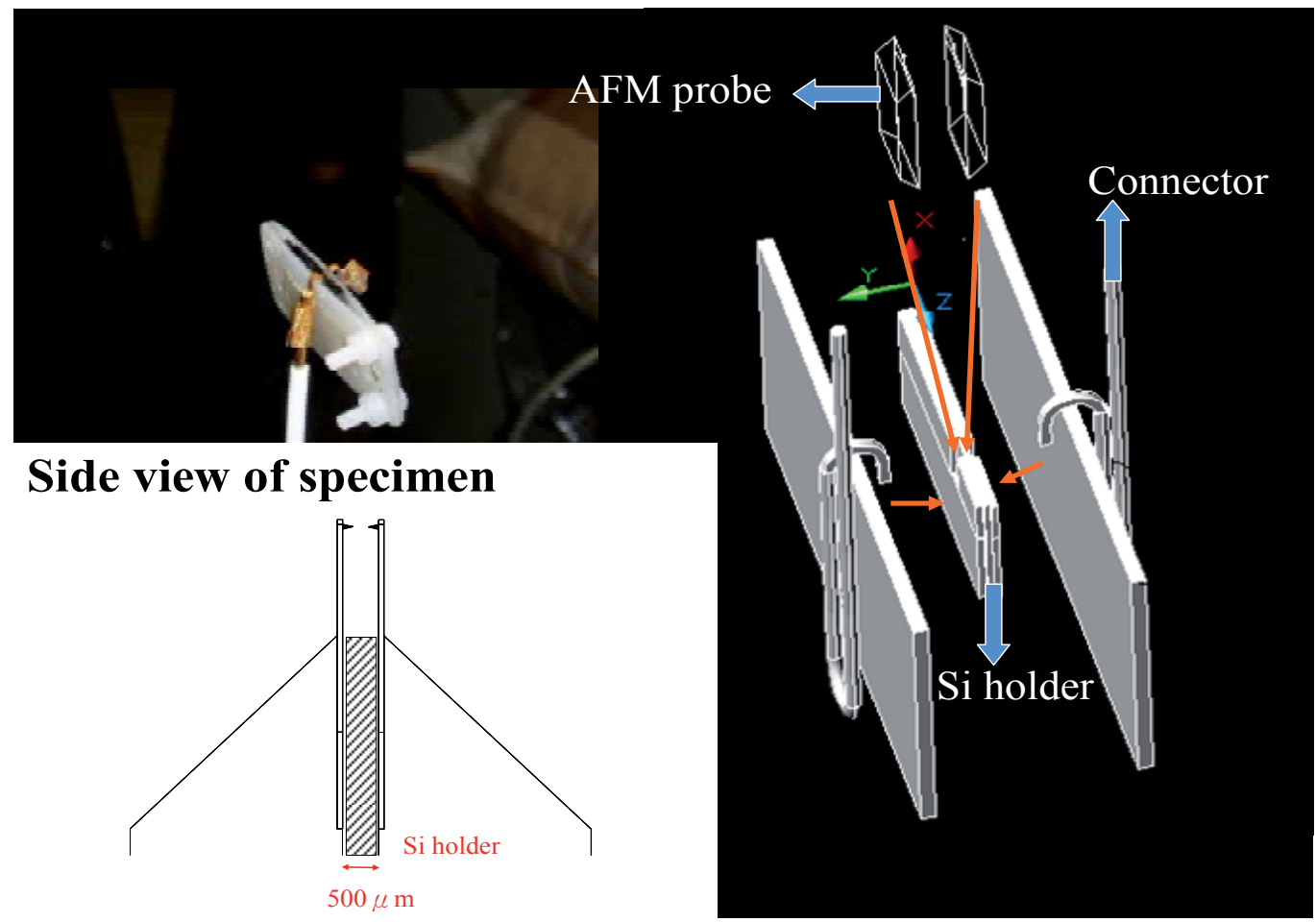

Fig. 2. Schematic diagram of the AFM probe placed on the Si holder

The cathode and anode were placed in parallel connection with the AFM probes. The current from the power supply was controlled between 10 and $0.1 \mathrm{~A}$ to provide a stable current between the AFM probes. Electron-beam lithography (EBL) techniques were used in our present work.

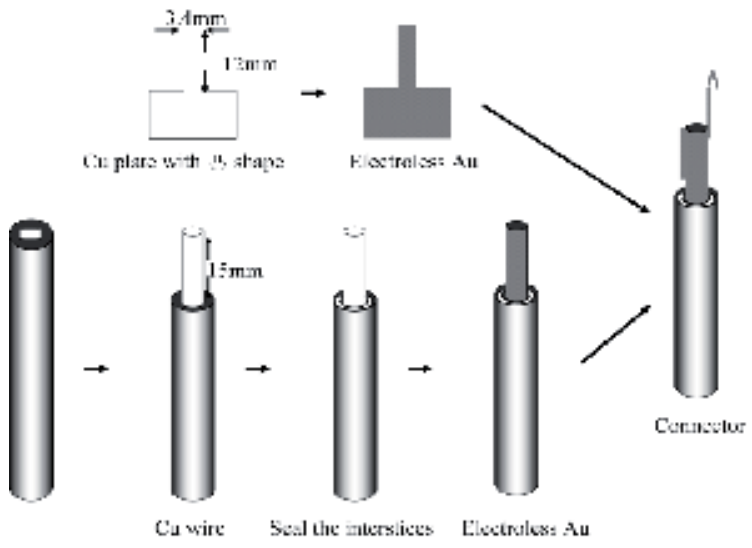

Fig. 3. Schematic diagram of the connector used in the electrodeposition process 


\section{Tank allocation}

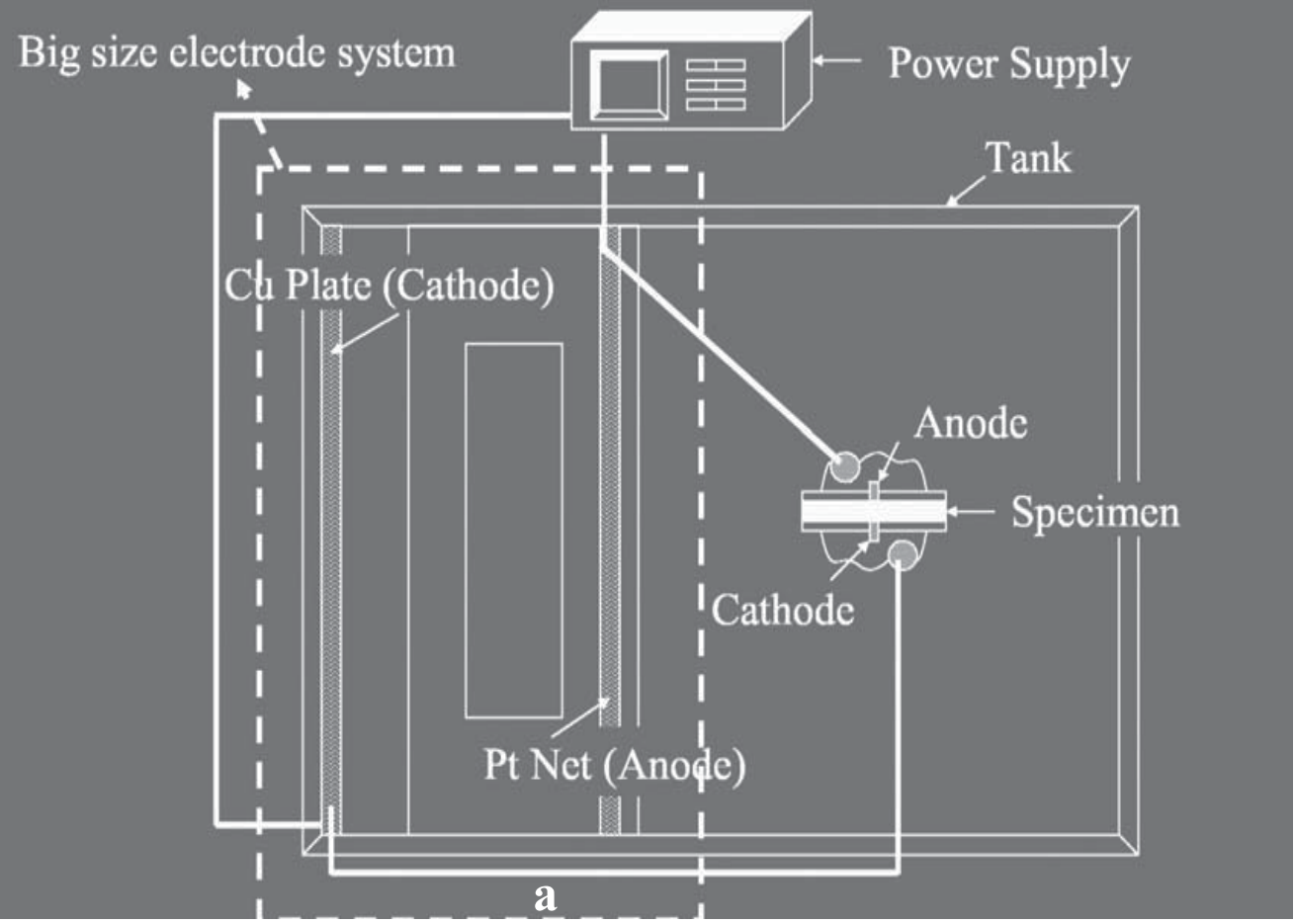

Fig. 4. Schematic diagram demonstrating the electrodeposition of copper on the AFM probe.

The samples were patterned using a JEOL 6400 thermionic emission scanning electron microscope equipped with the lithography software Elphy Quantum. The polymer used for EBL studies was poly_methyl methacrylate (PMMA). PMMA was the standard positive ebeam resist dissolved in a casting solvent anisole. The PMMA solution was spin-coated onto the AFM probe at a rotating speed of $1000 \mathrm{rpm}$ for $60 \mathrm{~s}$. Then baking was performed at $220^{\circ} \mathrm{C}$ to harden the film and to remove the remaining solvent. The EBL system employed a focused electron beam which moved across the sample to selectively expose a pattern in the resist previously designed with the system's built-in computer-assisted design tools.The open area of the AFM tip was selectively exposed to the high energy- beam electrons. The sample was then immersed in the developer solution (3:1 methyl isobutyl ketone: isopropyl alcohol developer) for $30 \mathrm{sec}$ to selectively remove the resist from the exposed areas, whereas the unexposed resist remained insoluble in the developer. The process thus left a patterned resist mask on the sample that could be used for further processing. Finally, $\mathrm{Cu}$ was electrochemically deposited on the AFM tip.

\subsection{Effect of various electrodeposition parameters}

\subsubsection{Effect of current density on copper electrodeposition}

Scanning Electron Microscopy (SEM) was used to investigate the morphologies of the copper deposits nucleated on the AFM probe. SEM micrograph for $\mathrm{Cu}$ deposition on the 
investigated AFM probe for a plating time of $300 \mathrm{~s}$ and a current density of $0.03 \mathrm{~A} / \mathrm{dm}^{2}$ is shown in Fig. 5a . The secondary electron image (SEI) and back scattered electron image (BEI) are displayed in Figs. 5a and 5b. The figure reveals that only a slight amount of copper is electrodeposited on the AFM probe. Further increase in current density to $0.3 \mathrm{~A} / \mathrm{dm}^{2}$ enhanced the copper deposition on the AFM probe however the deposits observed are nonuniform and discontinuous (Fig.6a). The magnified image is seen in Fig. 6b. Furthermore, a gradual increase in the current density to $0.6 \mathrm{~A} / \mathrm{dm}^{2}$ results in uniform deposition of copper on the probe (Fig.7), nevertheless, no copper deposition is noticed on the AFM tip. Similar observations have been reported (Seah et al., 1998). They visualised this morphology on the basis of the fact that formation of more nucleation sites promoted uniform grain growth. In the present study, the formation of uniform copper deposits on the AFM probe could be attributed to the enhanced mass transfer of copper ions with the increase in current density. Litearture reports (Chang, 2001) describe that increase in plating current density increased the surface roughness and reduced the grain size of copper films due to an increase of plating overpotential. Several other researchers have demonstrated that the polarization overpotential increased with increasing the plating current density leading to high copper nucleation rate (Takahashi \& Gross, 1999a, 2000 b; Tean et al., 2003; Teh et al., 2001).

The difficulty in depositing $\mathrm{Cu}$ ions on the AFM tip arises due to the local increase of the ion concentration in the electrolyte around the tip, which makes the effective local Nernst potential for deposition at the surface underneath the AFM tip more positive.

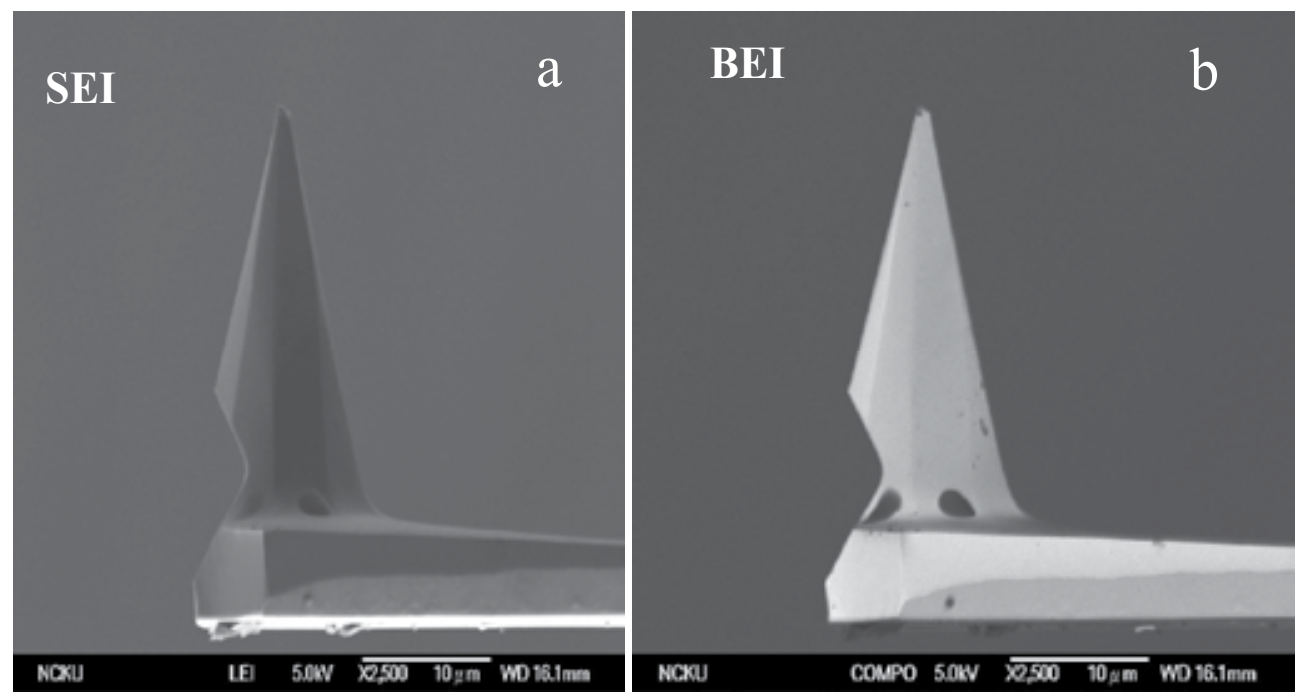

Fig. 5. SEM image obtained after copper deposition on the AFM tip for plating time of $300 \mathrm{~s}$ and current density of $0.03 \mathrm{~A} / \mathrm{dm}^{2}$ (a) SEI image (b) BEI image

Since, the standard electrode potential $\left(\Psi^{\mathrm{e}}\right)$ of $\mathrm{Cu}^{2+}\left[\Psi^{\mathrm{e}}\left(\mathrm{Cu}^{2+}+/ \mathrm{Cu}=+0.337 \mathrm{~V}\right)\right.$ is larger than zero (Fu et al., 1990), from the theoretical point of view, the more positive the $\Psi^{\mathrm{e}}$ value, the more easier it is for the reduction of metal ions, and the more negative the $\Psi^{\mathrm{e}}$ value, the more difficult it is to reduce the metal ions. Our results suggest that the copper ions can be reduced to copper atoms more easily on the surface underneath the AFM tip. It might be possible that the effective Nernst potential which is required to initiate nucleation on the 
AFM tip might be larger due to the deposition overvoltages. Also, the diameter of the AFM tip, which is around $80 \mathrm{~nm}$, might induce high overpotential for deposition of copper on the tip.
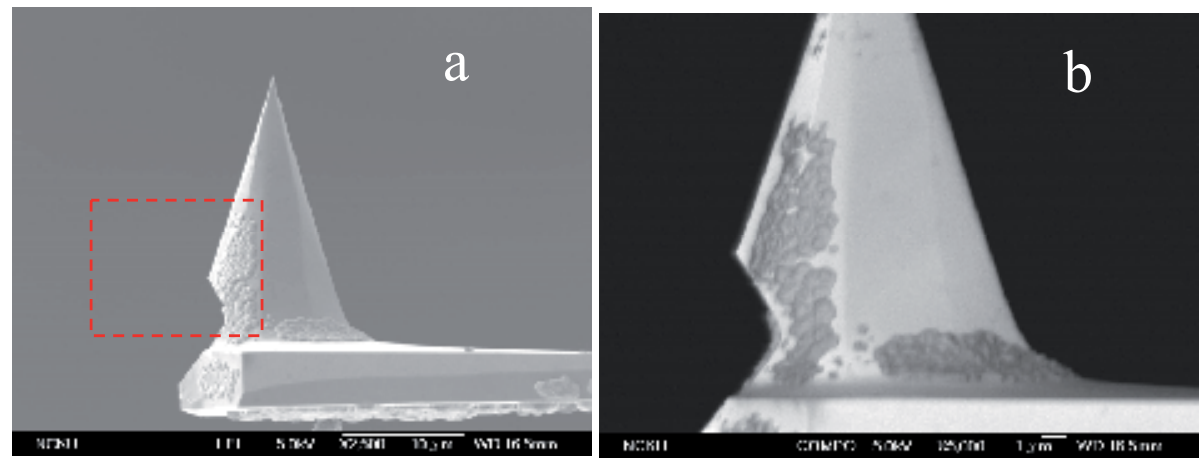

Fig. 6. SEM image obtained after copper deposition on the AFM tip for plating time of $300 \mathrm{~s}$ and current density of $0.3 \mathrm{~A} / \mathrm{dm}^{2}$ (a) SEI image (b) Magnified image (Lin, 2008)

The above results can also be explained on the basis of two reaction schemes which govern the $\mathrm{Cu}$ electrodeposition process on the AFM probe: one is the electrode surface reaction and the other one is the $\mathrm{Cu}^{2+}$ diffusion from the electrolyte solution to the electrode surface.

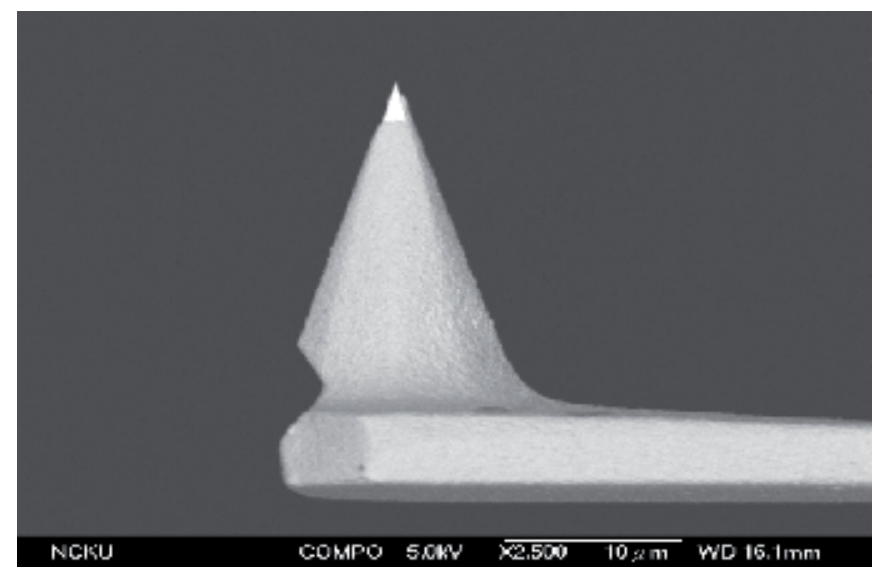

Fig. 7. SEM image obtained after copper deposition on the AFM tip for plating time of $300 \mathrm{~s}$ and current density of $0.6 \mathrm{~A} / \mathrm{dm}^{2}$ (Lin, 2008)

Polarization occurs when the rate of $\mathrm{Cu}^{2+}$ supply from the electrolyte solution is not faster than the rate of reaction at the electrode surface. The film morphology is primarily dependent on the degree of polarization (Seah et al., 1999).Thus higher polarization would make electrodeposition slower resulting in a smoother film. Since the effect of increasing current density is to increase the electrode surface reaction, a faster surface reaction makes $\mathrm{Cu}^{2+}$ undersupplied from the electrolyte solution. Hence, the polarization is higher and smoother film morphology is observed. Nevertheless, when the applied current density is greater than the limiting current density, it is impossible for the electrode to gain any $\mathrm{Cu}$ ions from the electrolyte solution; thereby leading to an increase in the $\mathrm{Cu}$ film surface roughness. 


\subsubsection{Effect of plating time on copper electrodeposition}

The effect of different plating times during copper electrodeposition on the AFM probe is investigated. The plating time was varied from 5 to $900 \mathrm{~s}$ for different current densities. The SEM micrograph in Figs. 8-11 illustrates the morphology of copper deposit formed under current density of $0.3 \mathrm{~A} / \mathrm{dm}^{2}$ and various plating times namely 5, 60, 300, 540 s respectively. The results reveal a random distribution of copper crystals on the cantilever with no trace of copper deposits on the AFM tip. This morphology clearly suggests the case of instantaneous nucleation.

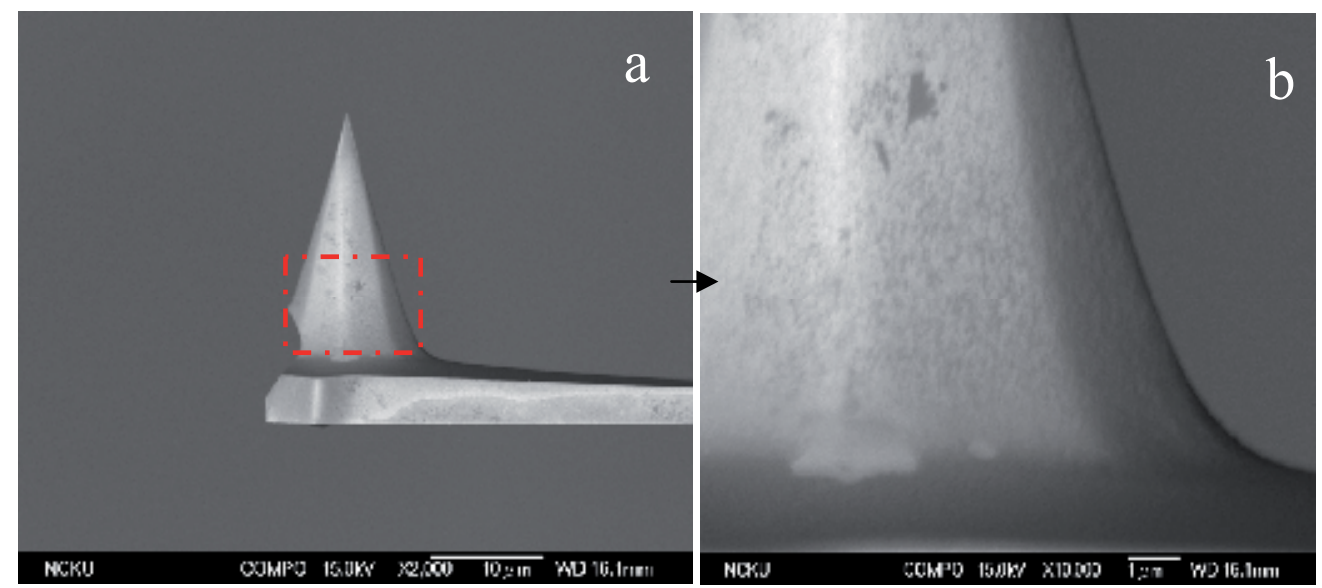

Fig. 8. SEM micrographs illustrating Cu deposition on the AFM tip at a current density of 0.3 $\mathrm{A} / \mathrm{dm}^{2}$ and plating time of $5 \mathrm{~s}$ (a) $2000 \mathrm{~T}$ (b) $10000 \mathrm{~T}$ magnification of the marked area in red

As instantaneous nucleation corresponds to a slow growth of nuclei on a small number of active sites, all activated at the same time. It can be noted from the SEM images displayed in Fig. 8-11, that in most of the samples the nuclei may be nucleated almost simultaneously, as confirmed by their similar size. In other words i.e at high nucleation rates (instantaneous nucleation), all nuclei are formed immediately after imposition of the potential and grow at the same rate. As a result, they are all of the same age and their number remains constant.

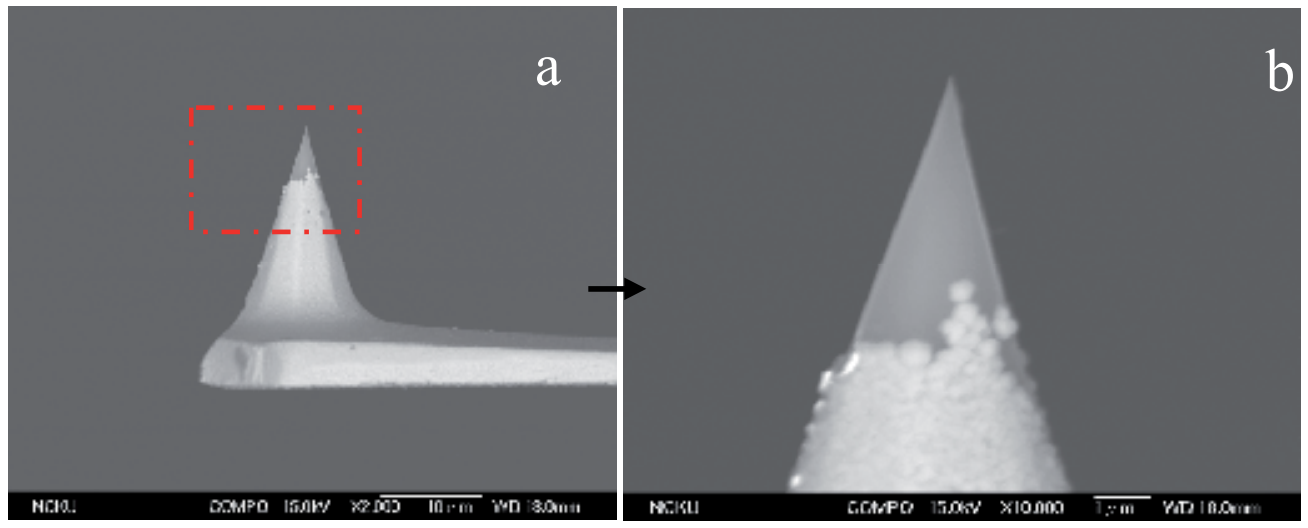

Fig. 9. SEM micrographs illustrating $\mathrm{Cu}$ deposition on the AFM tip at a current density of 0.3 $\mathrm{A} / \mathrm{dm}^{2}$ and plating time of $60 \mathrm{~s}$ (a) $2000 \mathrm{~T}$ (b) $10000 \mathrm{~T}$ magnification of the marked area in red 
The mode of instantaneous nucleation is described by the following equation involving the first-order kinetics law (Budevski et al., 1996; Milchev, 1997)

$$
\mathrm{N}=\mathrm{N}_{0}[1-\exp (-\mathrm{At})]
$$

where $\mathrm{N}$ is the number of sites converted into nuclei at time $\mathrm{t}$ and $\mathrm{A}$ is the nucleation rate constant, $\mathrm{N}_{0}$ is the respective saturation value. Nucleation does not occur simultaneously over the entire cathode surface and a diameter distribution for the crystallites ensues. When A is very high, $\mathrm{N} \equiv \mathrm{N}_{0}$, all surface sites are converted immediately into nuclei and the nucleation is said to be instantaneous. The nonhomogeneity and overgrowth of the $\mathrm{Cu}$ deposits may be due to the existence of low nucleation overpotential in the area beneath the tip. At low overpotentials, the nucleation is described well by the model of instantaneous nucleation for reasonably long time scales (Kelber et al., 2006)

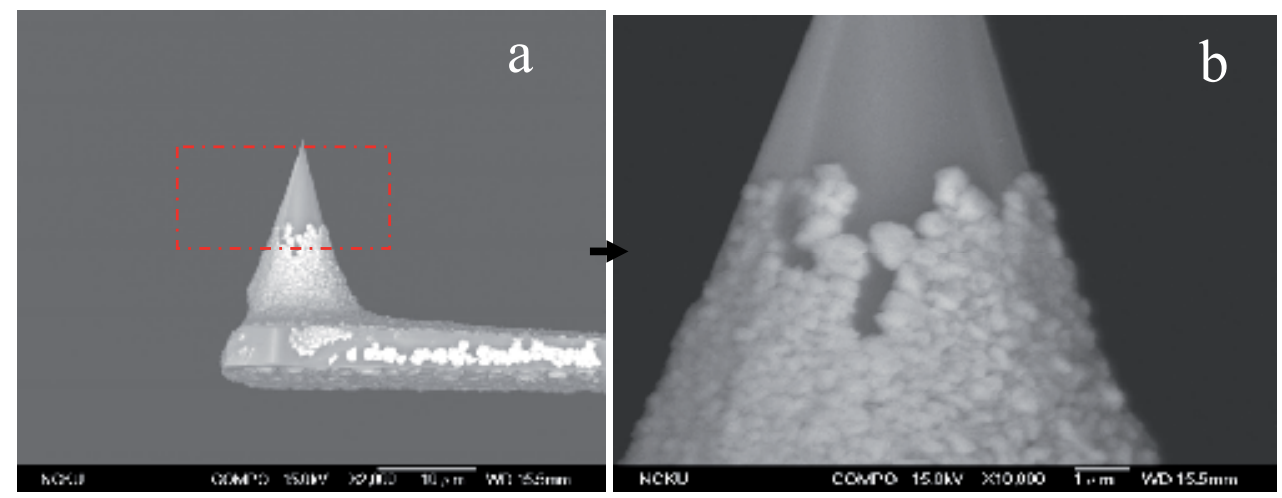

Fig. 10. SEM micrographs illustrating $\mathrm{Cu}$ deposition on the AFM tip at a current density of $0.3 \mathrm{~A} / \mathrm{dm}^{2}$ and plating time of $300 \mathrm{~s} \mathrm{(a)} 2000 \mathrm{~T}$ (b) $10000 \mathrm{~T}$ magnification of the marked area in red

However, the morphology of copper deposits formed under current density of $0.6 \mathrm{~A} / \mathrm{dm}^{2}$ and plating time of $900 \mathrm{~s}$ were found to be totally different. The copper layer on the AFM probe also shows resemblance to a candle base (Fig. 12), and a thicker layer of copper deposits are grown on the whole of the AFM probe containing the tip. Also, on the basis of instantaneous nucleation model, It has been reported (Thirsk \& Harrison, 1972) that under the diffusion controlled three-dimensional growth, the cathodic current density is proportional to $\mathrm{t}^{1 / 2}$.

The growth of copper layer also takes place slowly and farther away from the tip. Also it can be noticed that the growth rate on the side of tip is faster than on the tip (Fig. 12). From the results it could be established that higher current density and higher plating time increases the mass transfer of $\mathrm{Cu}^{2+}$ ions in the open area beneath the tip, thereby enhancing the rate of $\mathrm{Cu}$ deposition between the open area and the tip. The variance of the thickness of copper deposits on the tip and its surrounding area might be attributed to the nanoscale dimension of the AFM tip as compared to the whole of the AFM probe. Literature reports reveal (Seah et al., 1999) that in case of nanocrystalline electrodeposited $\mathrm{Cu}$ the pinhole number-density necessary for full coverage on the substrate can be reduced by increasing the current density. However, abnormal crystallite growth-leading to the formation of bimodal grain 


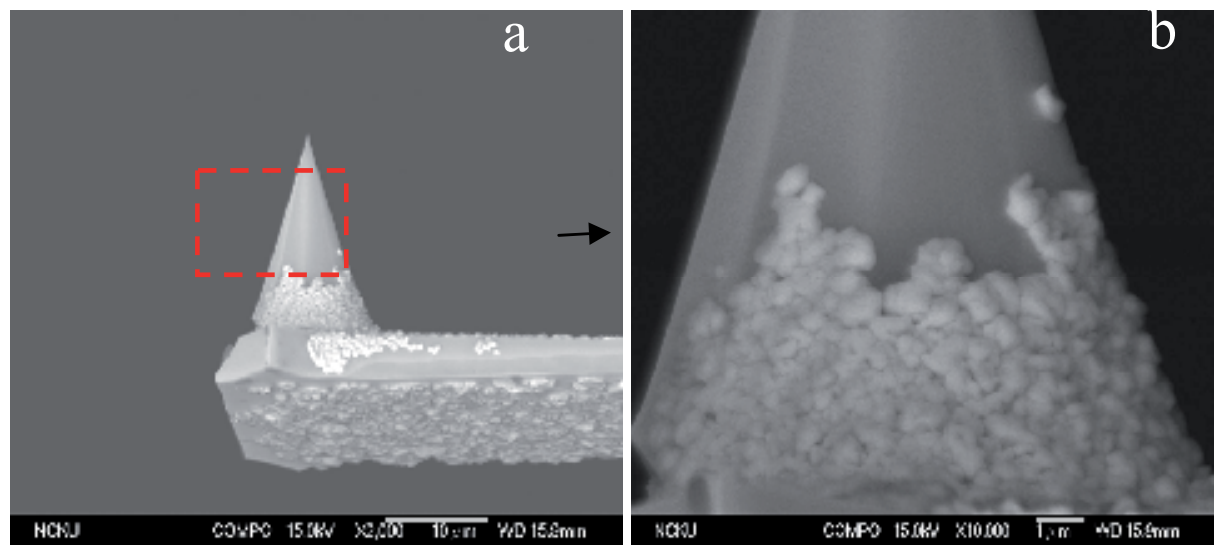

Fig. 11. SEM micrographs illustrating $\mathrm{Cu}$ deposition on the AFM tip at a current density of $0.3 \mathrm{~A} / \mathrm{dm}^{2}$ and plating time of $540 \mathrm{~s}$ (a) $2000 \mathrm{~T}$ (b) $10000 \mathrm{~T}$ magnification of the marked area in red

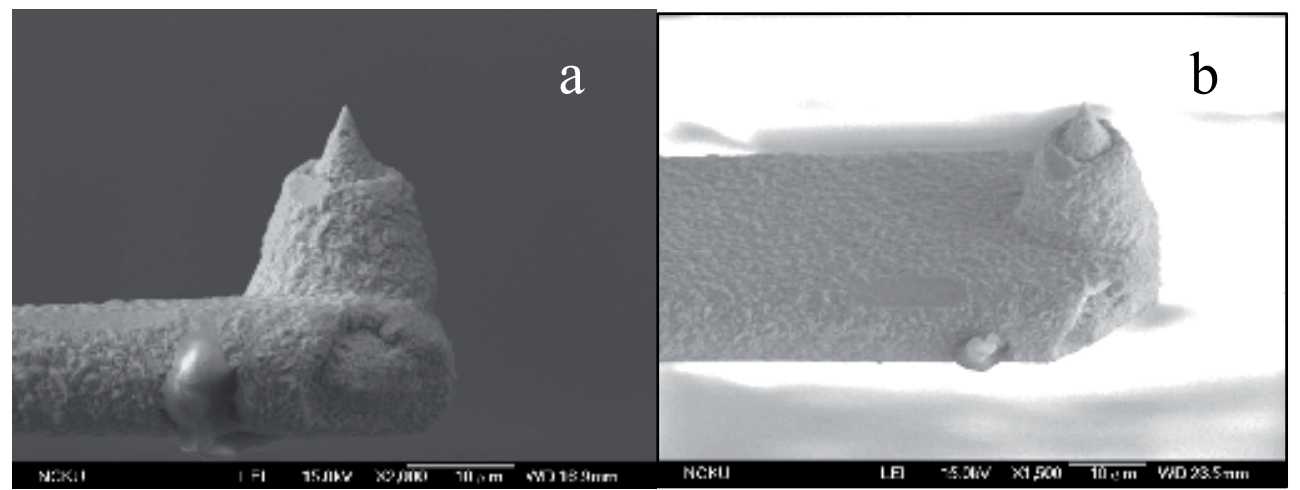

Fig. 12. SEM micrographs illustrating Cu deposition on the AFM tip at a current density of $0.6 \mathrm{~A} / \mathrm{dm}^{2}$ and plating time of $900 \mathrm{~s}$ (a) SEI image (b) image taken at $35^{\circ}$ tilt (Lin, 2008)
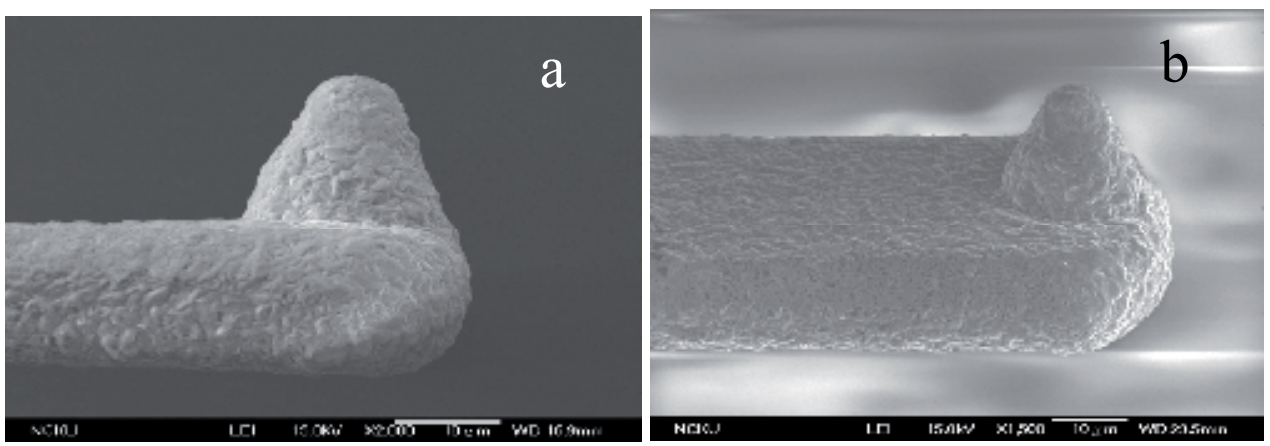

Fig. 13. SEM micrographs illustrating $\mathrm{Cu}$ deposition on the AFM tip at a current density of $0.6 \mathrm{~A} / \mathrm{dm}^{2}$ and plating time of $1200 \mathrm{~s}$ (a) SEI image (b) image taken at $35^{0}$ tilt (Lin, 2008) 
structures- can be suppressed by increasing the electrodeposition current density. In our present case, the crystal growth variation is seen on the open area below the tip and the tip itself. The morphology observed in Fig. 12 is a case of progressive nucleation followed by growth.

As nucleation progresses, the nuclei begins to overlap. Each nucleus is defined by its own diffusion zone through which copper diffuses, thus representing the mass-supply mechanism for continuation of growth. Progressive nucleation corresponds to fast growth of nuclei on many active sites all activated during the course of electroreduction (Pardave et al., 2000). Fig.12b. shows the SEM micrograph for copper deposition at $0.6 \mathrm{~A} / \mathrm{dm}^{2}$ and $900 \mathrm{~s}$ taken at a tilted angle of $35^{\circ}$. Further increase in the plating time to $1200 \mathrm{~s}$ for similar current density resulted in an entirely different morphology from the micrograph shown in Fig. 12. The AFM probe containing the nanoscale AFM tip seems to be entirely covered with copper deposits and also a significant increase in growth and thickness of the deposits are observed in Figs. 13a. Fig. 13b represents the SEM image tilted at an angle of $45^{\circ}$ for clear depiction of the copper deposition on the AFM probe. The copper deposition process on the AFM probe proceeds through instantaneous and progressive nucleation modes for different values of current density. The mechanisms for instantaneous and progressive nucleation modes are described below.

Once nucleation begins, crystals growth may be determined by the rate of charge- transfer or diffusion process. Simple equations have been described (Harrison \& Thirsk, 1971) for two- or three dimensional nucleation and crystal growth processes occurring on a foreign substrate for charge transfer control reactions.

For two-dimensional (2D) instantaneous nucleation and cylindrical growth, current is described by

$$
\mathrm{i}=2 \mathrm{zF} \text { лh } \mathrm{N}_{0} \mathrm{k}^{2}{ }_{2 \mathrm{D}}^{\mathrm{t}} / \rho \exp \left(-л \mathrm{M}^{2} \mathrm{~N}_{0} \mathrm{k}_{2}{ }^{2} \mathrm{D} \mathrm{t}^{2}\right) / \rho^{2}
$$

And for 2D progressive nucleation

$$
\mathrm{i}=\mathrm{z} \text { FлhMK }{ }_{2}{ }^{2} \mathrm{DA}_{2} \mathrm{Dt}^{2} / \rho \exp \left(\text {-л } \mathrm{M}^{2} \mathrm{k}_{2}{ }^{2} \mathrm{D} \mathrm{A}_{2 \mathrm{D} \mathrm{t}}{ }^{3} / 3 \rho^{2}\right)
$$

where $\mathrm{k}_{2 \mathrm{D}}$ represents the lateral growth rate constants $\left(\mathrm{mol} \mathrm{cm}^{-2} \mathrm{~s}^{-1}\right), \mathrm{h}$ is the layer height in $\mathrm{cm}, \mathrm{N}_{0}$ represents the total number of active centers $\left(\mathrm{cm}^{-2}\right), A_{2 D}$ the nucleation rate (nuclei $\left.\mathrm{cm}^{-2} \mathrm{~s}^{-1}\right), \mathrm{M}$ is the atomic weight $\left(\mathrm{g} \mathrm{mol}^{-1}\right)$ and $\rho$ the density $\left(\mathrm{g} \mathrm{cm}^{-3}\right)$ of the deposit. For these type of mechanisms the current usually increases and then decreases to zero when the surface gets completely covered by two dimensional crystals However, for three dimensional (3D) instantaneous nucleation and growth, the current is depicted by the following equations below.

$$
\mathrm{i}=\mathrm{zF} \mathrm{K}^{\prime}\left[1-\exp \left(-л \mathrm{M}^{2} \mathrm{k}^{2} \mathrm{~N}_{0} \mathrm{t}^{2} / \rho^{2}\right)\right]
$$

and for 3D progressive nucleation:

$$
\mathrm{i}=\mathrm{zFK}^{\prime}\left[1-\exp \left(-\pi \mathrm{M}^{2} \mathrm{k}^{2} \mathrm{~A}_{3 \mathrm{D}} \mathrm{t}^{3} / 3 \rho^{2}\right)\right]
$$

Where $\mathrm{k}$ and $\mathrm{k}^{\prime}$ signify the lateral and vertical growth rate constants $\left(\mathrm{mol} \mathrm{cm}^{2} \mathrm{~s}^{-1}\right)$ and $\mathrm{A}_{3 \mathrm{D}}$ the nucleation rate (nuclei $\mathrm{cm}^{2} \mathrm{~s}^{-1}$ ). Hence nucleation and growth phenomena are affected by 
many factors i.e a combination of 2D and 3D growth (Abyaneh \& Fleischmann, 1981; Creus et al., 1992), the death and rebirth of nuclei (Abyaneh \& Fleischmann, 1981) and the secondary three dimensional (3D) growth on top of the first growth layers (Abyaneh et al., 1982).

\subsection{Electron Beam lithography studies}

EBL (Electron-Beam lithography) technique followed soon after the development of the scanning electron microscope (SEM) in 1955 (Smith, 1955) and is one of the earliest processes used for IC fabrication (Buck, 1957). To date, EBL is widely exploited to produce structures in the sub-100 nm range (Allee et al., 1991; Matsui et al., 1989; Sun et al., 2005). Also, as compared with photolithography, the lateral resolution achieved by EBL is higher because the beam of electrons can be focused to produce probe size as small as $1 \mathrm{~nm}$. More over, electrons do not suffer from optical thin-film interference. For ICs, where at present low beam energy and thick conventional resists are employed; electron scattering is the most important factor whereas for nanolithography, which utilizes high beam energy and thin resists, secondary electron emission is the most dominant factor. The resolution of EBL is also dependent on the chemical nature of the resist. Recently, new class of resists such as organic self-assembled mono layers (SAMs) has been developed to fabricate structures below $10 \mathrm{~nm}$ (Golzhauser et al., 2000; Lercel, 1996) Currently, electron beam lithography is used principally in support of the integrated circuit industry, where it has three niche markets. The first is in maskmaking, typically the chrome-on glass masks used by optical lithography tools. It is the preferred technique for masks because of its flexibility in providing rapid turnaround of a finished part described only by a computer. The ability to meet stringent line width control and pattern placement specifications, on the order of 50 $\mathrm{nm}$ each, is a remarkable achievement.

\subsubsection{Principle of EBL}

The principle of pattern transfer based on EBL consists of several process steps. The process steps are essentially the same as those used for photolithography, except that the pattern on the resist is formed by scanning directly the focused particle beam across the surface. The lithographic sequence usually begins with coating of substrates with a positive or negative resist. Positive resists such as poly (methyl-methacrylate) (PMMA) used in the present chapter become more soluble in a developing solvent after exposure because the radiation causes local bond breakages and thus chain scission. This causes the exposed regions containing material of lower mean molecular weight to dissolve after the development. Nevertheless, negative resists become less soluble in solvent after exposure because crosslinking of polymer chains occurs. If in case, a region of a negative resist-covered film is exposed, only the exposed region will be covered by the resist after development. Subsequently, the resist-free parts of the substrate can be selectively coated with metal or etched before removal of the unexposed resist thus leaving the desired patterns at the surface. Fabrication of metallic nanostructures has been widely explored using conventional EBL and lift off techniques. However, this top-down approach cannot be employed for the fabrication of high aspect ratio vertical structures since gradual accumulation of materials at the top of the resist blocks and closes the opening of the structures during the evaporation of metal. Electrodeposition of metals into the holes formed in presence of PMMA resist is a 
convenient alternative to solve this problem (Simon et al., 1997). The fabrication of dense ultra-small magnetic arrays by filling nanoholes with electrodeposited $\mathrm{Ni}$ has been demonstrated (Xu et al., 1995).

\subsubsection{EBL induced Copper deposition}

Electron beam lithography technique is used in the present study to enable selective electrodeposition of $\mathrm{Cu}$ on the AFM tip and the open area beneath it. The selective electrodeposition of $\mathrm{Cu}$ on n-type Si (111) surfaces covered with organic monolayers by using e-beam lithographic techniques has been reported (Balaur et al., 2004). Selective copper deposition on e-beam patterned alkane and biphenylthiols has been reported (Kalten Poth et al., 2002) at suitable deposition potentials. 1-octadecanethiol (ODT) was used as a "positive template" leading to copper deposition only on the irradiated parts, 1,1'-biphenyl4-thiol ( BPT) on the other hand acted as a "negative template," where the irradiated and cross-linked biphenyl layer exhibited a blocking behavior, allowing copper deposition on the non-irradiated parts. In the present study, the open area of the nanosize AFM tip was selectively exposed to the e-beam. It is noticed that copper electrodeposition occurs on the exposed area of the AFM tip. For the copper electrodeposition process, the current density applied was $0.6 \mathrm{~A} / \mathrm{dm}^{2}$, and the electrodeposition time was varied from 300 to $2400 \mathrm{~s}$.
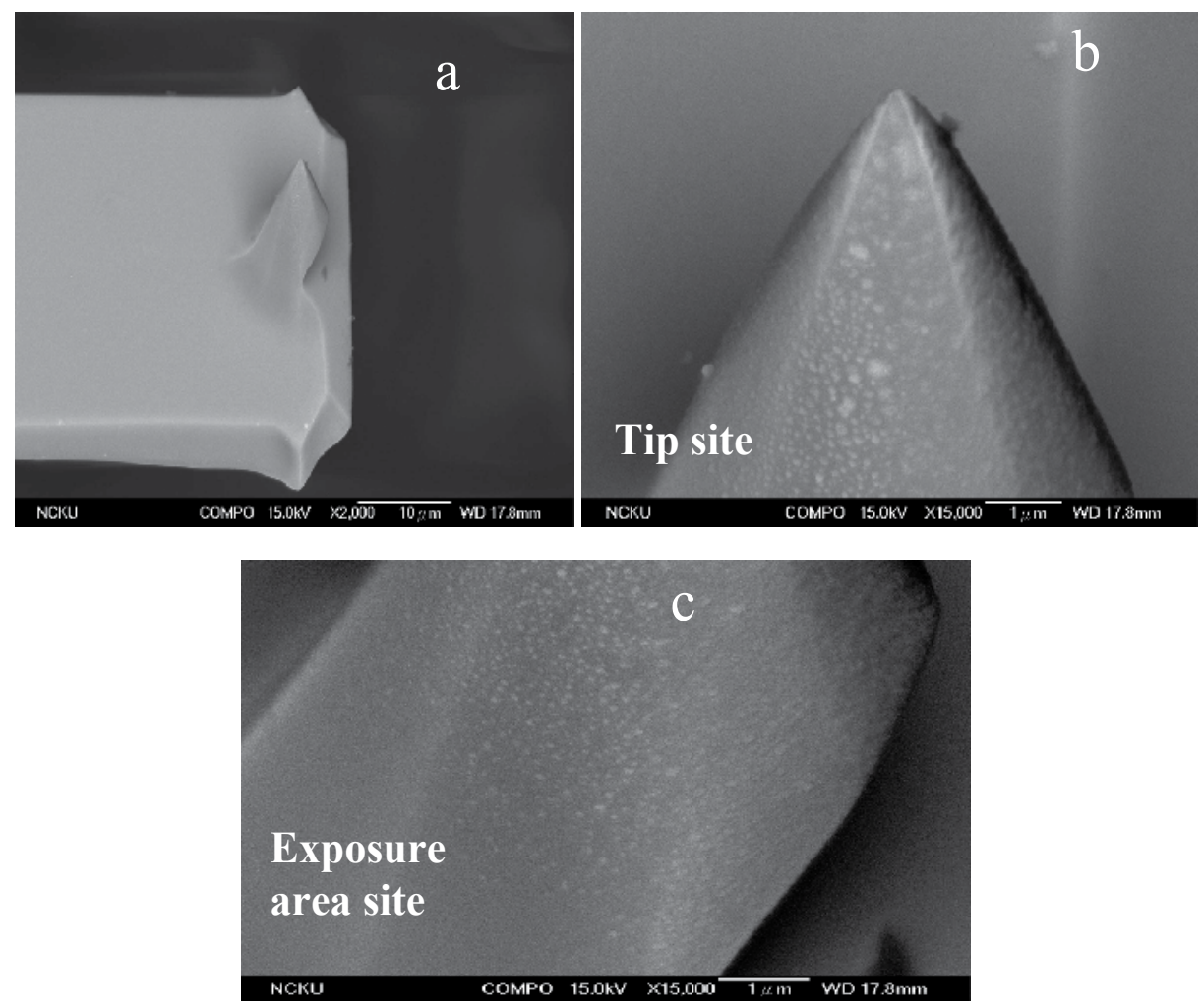

Fig. 14. SEM micrograph demonstrating $\mathrm{Cu}$ deposition on the AFM tip after EBL treatment under current density of $0.6 \mathrm{~A} / \mathrm{dm}^{2}$ and electrodeposition time of $300 \mathrm{~s}$ (a) BEI image

(b) Image taken at the tip site (c) Exposure area site 
SEM micrographs for copper electrodeposition on the AFM tip and the open area beneath it for various deposition times (i.e 300, 600, 1200, $2400 \mathrm{~s}$ ) and current density of $0.6 \mathrm{~A} / \mathrm{dm}^{2}$ are presented in Figs. 14-17.These SEM micrographs were taken after exposure to the electron beam. In Figs. 14 (a) - (c) the micrographs for copper deposition on the AFM tip under current density of $0.6 \mathrm{~A} \mathrm{dm}^{-2}$ and electrodeposition time of $300 \mathrm{~s}$ are clearly depicted. Copper deposition is found to be minimum and non-uniform in these images. Further increase in the electrodeposition time to $600 \mathrm{~s}$ for the similar current density and exposure to the e-beam increases the amount of copper deposits on the nanosize AFM tip and the open area beneath it (Fig. 15 a). SEM micrographs in Fig. 15b and $15 \mathrm{c}$ refers to the magnified images of the AFM tip and the exposed site.
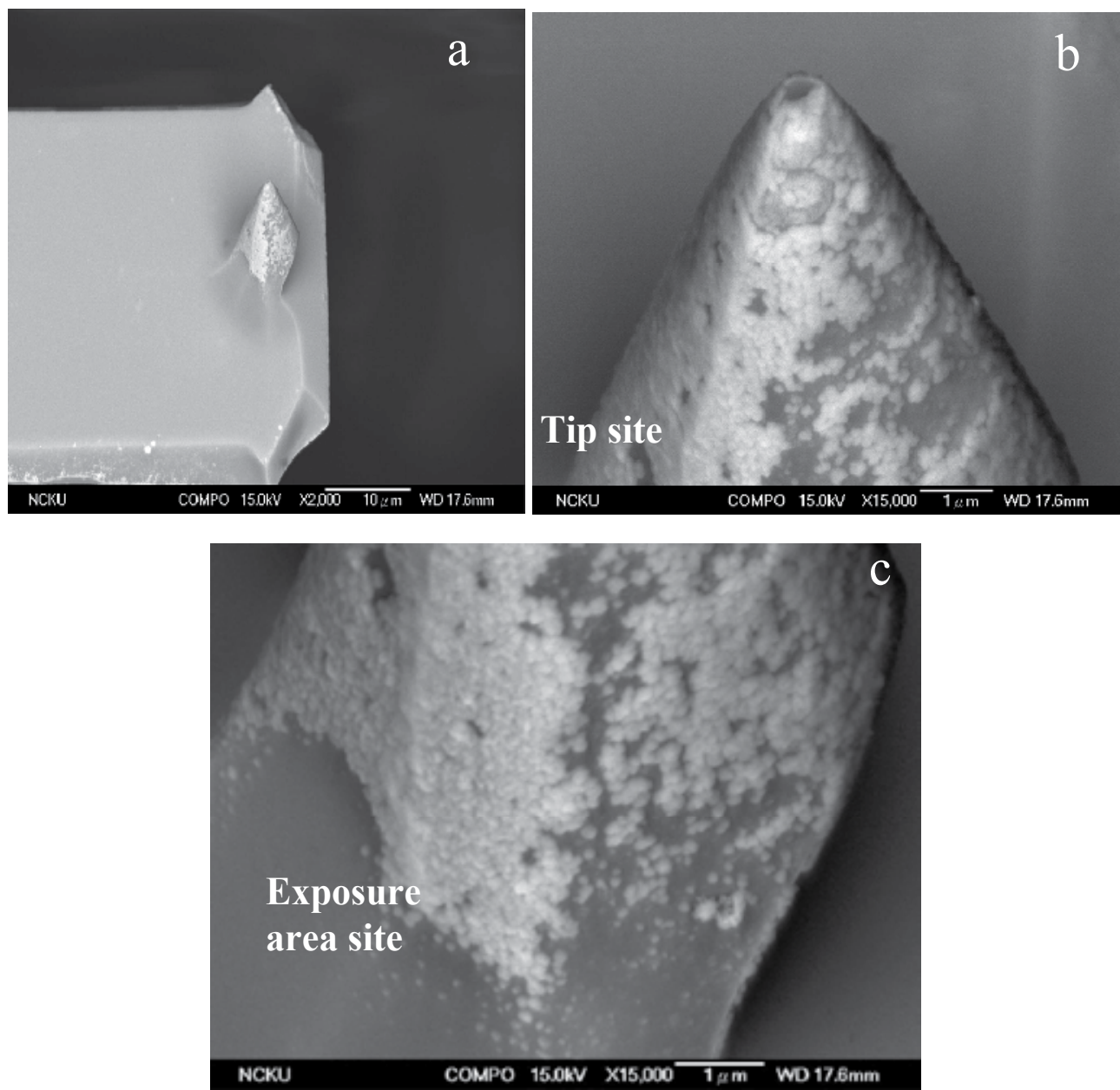

Fig. 15. SEM micrograph demonstrating Cu deposition on the AFM tip after EBL treatmentunder current density of $0.6 \mathrm{~A} / \mathrm{dm}^{2}$ and electrodeposition time of $600 \mathrm{~s}$ (a) BEI image (b) Image taken at the tip site (c) Exposure area site

The micrographs reveal that copper deposition is not uniform in the open area beneath the AFM tip. However a significant change in the morphology of copper deposits is 
observed when the electrodeposition time was increased to $1200 \mathrm{~s}$. SEM micrograph in Fig. 16a shows that an uniform layer of copper is deposited on the AFM tip and the open area beneath the tip. These results indicate that the exposure of the tip to the high energy electron beam might have facilitated the electrodeposition of copper on the tip. The micrographs in Fig. $16 \mathrm{~b}$ reveal that some copper is being deposited on the edges of the cantilever. This is because the PMMA layers on the edges are found to be thinner than on the platform. Those places are not exposed to the e-beam; therefore the developer could dissolve the PMMA layer on the edges and hence copper deposition took place on the edges. The overpotential required to deposit copper on the edges is lower than on the AFM tip.
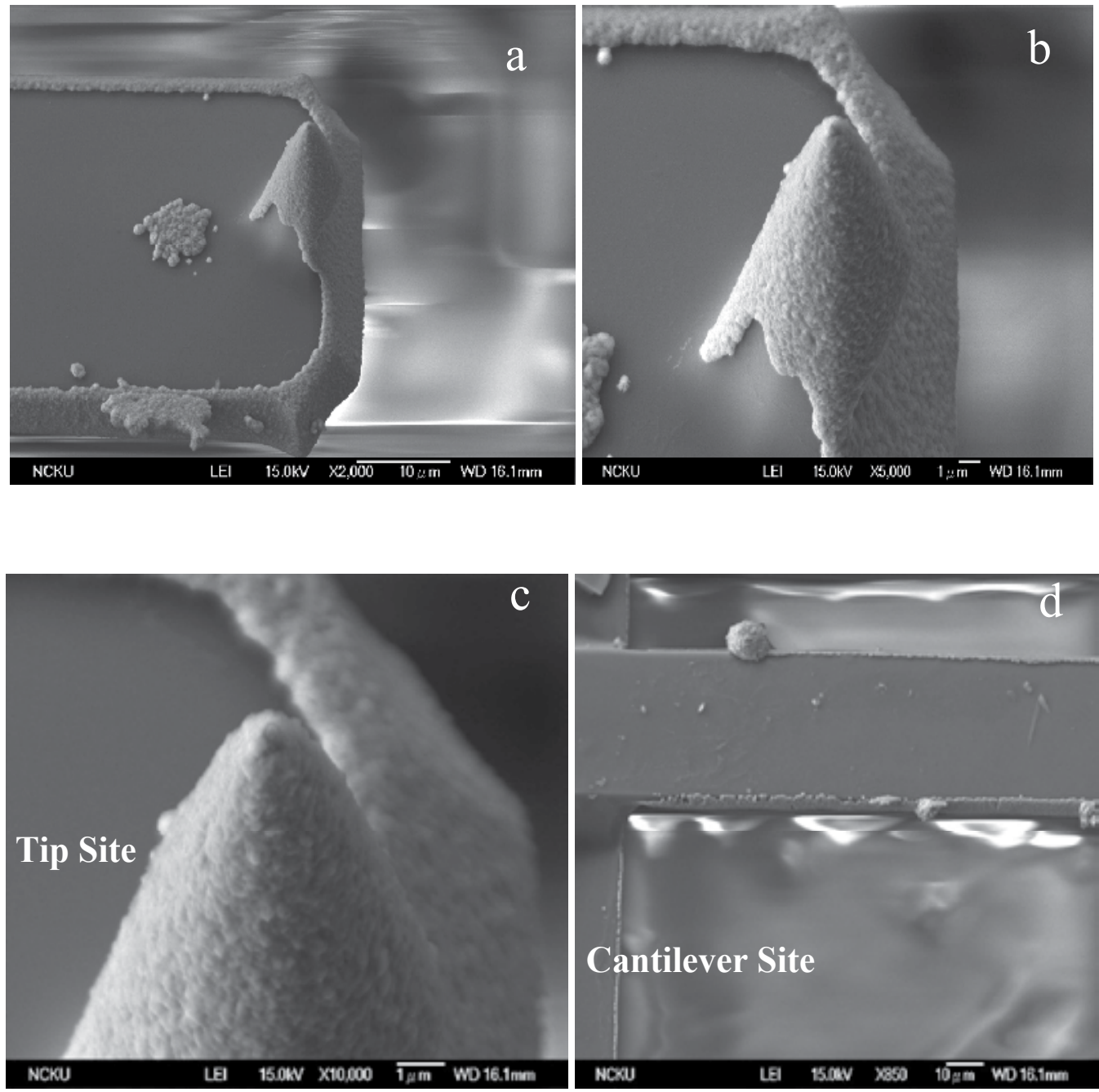

Fig. 16. SEM micrograph demonstrating $\mathrm{Cu}$ deposition on the AFM tip after EBL treatment under current density of $0.6 \mathrm{~A} / \mathrm{dm}^{2}$ and electrodeposition time of $1200 \mathrm{~s}$ (a) SEI image (b) Magnified to $5000 \mathrm{~T}$ (c) Tip site (d) Cantilever site 
Theoretically it has been established (West, 1971) that deposition at low overpotentials is dominated by surface diffusion; hence nucleation and growth occur primarily at step edges and dislocations (Winand, 1975). Fig. 17 illustrates the morphology of copper deposition on the nanosize AFM tip obtained under current density of $0.6 \mathrm{~A} / \mathrm{dm}^{2}$ and electrodeposition time of $2400 \mathrm{~s}$ and after exposure to the e-beam. The micrograph in Fig.17a distinctly shows that copper is deposited on the AFM tip and a very thick growth of copper deposits is seen on the open area beneath the tip. From the series of micrographs obtained at different electrodeposition times and current density of $0.6 \mathrm{~A} / \mathrm{dm}^{2}$, it is noticed that copper gets deposited both on the AFM tip and the open area beneath it, the most uniform deposition seen at $2400 \mathrm{~s}$ of electrodeposition time.
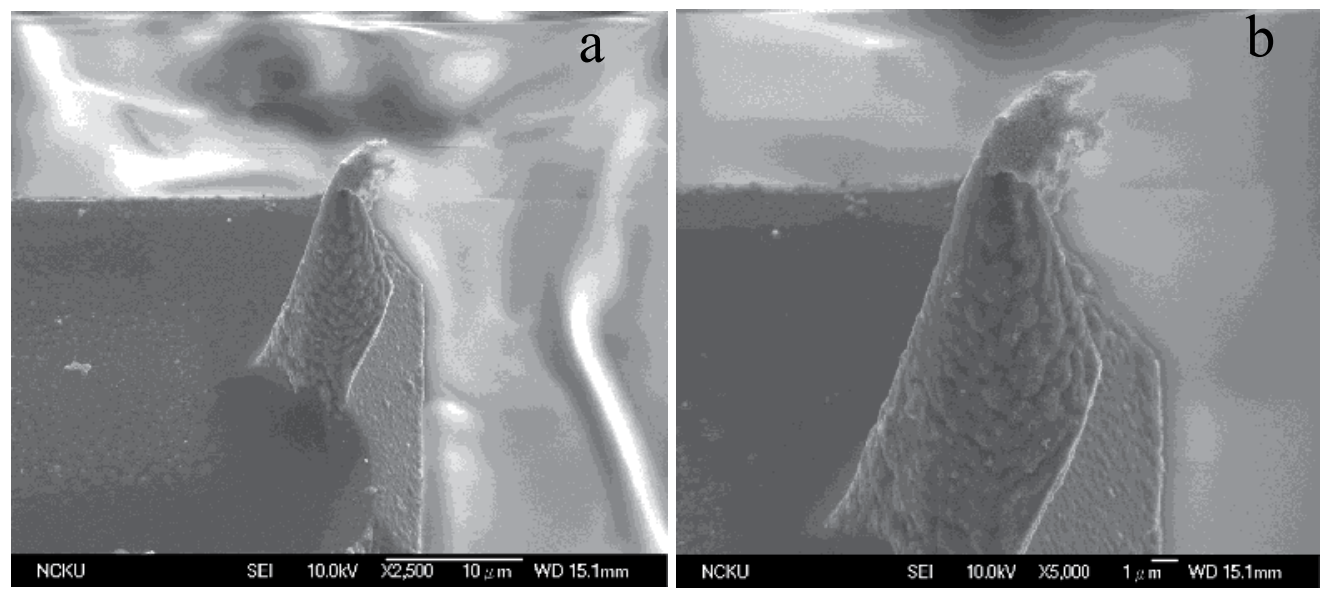

Fig. 17. SEM micrograph demonstrating $\mathrm{Cu}$ deposition on the AFM tip after EBL treatment under current density of $0.6 \mathrm{~A} / \mathrm{dm}^{2}$ and electrodeposition time of $2400 \mathrm{~s}$ (a) SEI image (b) Magnified to $5000 \mathrm{~T}$

The PMMA coated on the AFM tip becomes more soluble in a developing solvent after exposure to the e-beam because the radiation causes local bond breakages and thus chain scission (Djenizian et al., 2006) as mentioned above. It could be clearly seen from Fig. 17b 
that the unexposed areas below the AFM tip remain covered with PMMA. However, the resist free parts of the AFM tip are selectively coated with copper. Reports on the selective electrodeposition of $\mathrm{Cu}$ (Balaur et al., 2004) on n-type Si (llll) surfaces covered with organic monolayers and e-beam modified using e-beam lithographic techniques have also been established. Copper was electrochemically deposited in the e-beam modified regions and the selectivity of the deposition of copper in these regions was strongly dependent on the applied e-beam dose. The selective deposition of copper on the nanosize AFM tip can be described on the basis of Volmer-Weber approach which states that higher numbers of activation sites are triggered with a higher overvoltage. In the Volmer-Weber model, nucleation and growth are strongly potential dependent. At low cathodic potentials, only a few sites are involved because the energy level is not sufficient whereas at high cathodic voltages more initiation sites contribute to the nucleation process. It implies that at low overpotentials the crystallites have to grow extremely large to reach coalescence and form a homogenous deposit. In the present study, higher overpotential existing on the AFM tip might have increased the number of activation sites, leading to the preferential deposition of copper on exposure to the e-beam.

\section{Conclusion}

The investigations made in this chapter have highlighted electrodeposition as an attractive approach for the preparation of nanostructured materials. Copper electrodeposition on a nanosize AFM tip of diameter $80 \mathrm{~nm}$ was established by varying the magnitude of current densities with electrodeposition time and vice versa. Significant changes in the morphology of copper deposits were observed with changes in the above parameters. Morphological investigations by SEM revealed that a nonuniform layer of copper was formed on the open areas surrounding the tip and the AFM probe; however, deposition of copper on the AFM tip could not be achieved in the absence of photoresist. Electron beam lithography technique facilitated the formation of copper deposits on the nanosize AFM tip of diameter $80 \mathrm{~nm}$ in the presence of PMMA. Copper was electrochemically deposited on the e-beam modified regions of the AFM probe at a current density of $0.6 \mathrm{~A} / \mathrm{dm}^{2}$ with electrodeposition times ranging from 300 to $2400 \mathrm{~s}$. The most uniform deposition on the AFM tip was noticed after EBL treatment under current density of $0.6 \mathrm{Adm}^{-2}$ and electrodeposition time of $2400 \mathrm{~s}$.

\section{Acknowledgment}

The authors acknowledge financial support of this study from the National Science Council of China under NSC 94-2811-E-006-021. The Department of Materials Science and Engineering, National Cheng Kung University assisted in meeting the publication costs of this article.

\section{References}

Abyaneh, M.Y.; Hendrikx. J.; Visscher, W. \& Barendrecht. E (1982). Studies of Electroplating using an EQCM. I. Copper and Silver on Gold. Journal of Electrochemical Society, Vol. 129, No.12 (December 1982) pp.2654-2659., ISSN 0013-4651. 
Abyaneh, M.Y.\& Fleischmann, M. (1981), The Electrocrystallisation of Nickel. Journal of Electroanalytical Chemistry, Vol. 119, No. 187, pp. 197, ISSN 1572-6657.

Andricacos, P. C. ; Uzoh, C.; Dukovic, J. O.; Horkans, J. ; Deligianni, H (1998) Damascene copper electroplating for chip interconnections IBM J. Res. Develop Vol 42 (April 2010) No.5, 567-574, ISSN:0018-846.

Andricacos, P. C (1999) Copper on Chip Interconnections -A Breakthrough in Electrodeposition to make better chips, Interface 1999, 8,32.

Allee, D. R.; Umbach, C.P. \& Broers, A.N. (1991). Direct nanometer scale patterning of $\mathrm{SiO}_{2}$ with electron- beam irradiation. Journal of Vaccuum Science and Technology B, Vol. 9, No.16 (November 1991)pp.2838-2841, ISSN 1071-1023.

Allongne, P.A. (1995) in Advances in Electrochemical Science and Engineering, (eds : Gerischer, H. \& Tobias, C. W), ISBN 3-527-28273-4,Weinheim, Germany.

Balaur, E. ; Djenizian, T.; Boukherroub, R.; Chazalviel, J. N. ; Ozanam, F. \& Schmuki, P. Electroplating: an alternative transfer technology in the $20 \mathrm{~nm}$ range. Electrochemistry Communications. Vol.6, No.2, (February 2004), pp. 153-157, ISSN 1366-2481.

Batina, K. \& Nichols, D.M. (1992). An in situ scanning tunneling microscopy study of the initial stages of bulk copper deposition on gold(100): the rim effect. Langmuir, Vol.8, No.10 (Oct 1992) pp.2572-2576, ISSN 0743-7463.

Benenz, P.; Xiao, X. Y.\& Baltruschat, H. (2002). Tip-Induced Nanostructuring of a Clean and Ethene -modified Pt (111) Electrode with Cu. Journal of Physical Chemistry B, Vol. 106, No. 14, ( March 2002) pp 3673-3680, ISSN 1089-5647.

Binning, G. \& Rohrer, H. (1982). Scanning Tunneling Microscopy. Helvetica Physica Acta, Vol. 55, pp.726-735, ISSN 0036-8075.

Bockris J. O.M. \& Razumney, G. A. (1967) Fundamental Aspects of Electrocrystallization, Plenum Press, New York.

Buck, D.A. \& Shoulders. K (1957), In Proceedings Eastern Joint Computer Conference (ATTE, New Yoork), p.55.

Budevski, E. ; Staikov, G. \& Lorenz, W. J. (1996) Electrochemical Phase Formation and Growth An Introduction to the Initial Stages of MetalDeposition, ISBN 3527294228978352729 4220, VCH, Weinheim.

Budevski, E.; Bostanov, V. \& Staikov, G. (1980) Annu. Rev. Mater. Sci. 10, 85.

Budevski E. (1983) Comprehensive Treatise of Electrochemistry, Vol. 7 (Eds: B. E.Conway, J. O’M. Bockris, E. Yeager, S. U. M. Kahn, R. E. White, Plenum Press, New York, p.399.

Budevski, E. ; Staikov, G. \& Lorentz, W. (1996). An Introduction to Initial Stages of MetalDeposition, p. 4, Wiley VCH, ISBN 3527294228, Weinheim, Germany.

Carlsson, P.; Holmstriom, B. ; Kita H. \& Uosaki, V. (1990). Novel application of scanning tunneling microscopy - tip current voltammetry of n-GaAs and $\mathrm{p}-\mathrm{GaP}$ in electrolyte solution.Surface Science, Vol. 237, No.1-3 (November 1990) pp. 280 -290, ISSN 0039-6028.

Chang, S.C. ; Shieh, J.M. ; Lin, K.C.; Dai, B.T. ; Wang, T.C.; Chen, C.F. ; Feng, M.S. ; Li, Y.H. $\& \mathrm{Lu}$, C.P. (2001). Investigations of effects of bias polarization and chemical parameters on morphology and filling capability of $130 \mathrm{~nm}$ damascene 
electroplated copper. Journal of Vacuum Science and Technology B. Vol.19 , No.3 (May 2001) pp. 767-774, ISSN 1071-1023.

Creus ,H. A.; Carro, P.; Gonzalez, S.; Salvarezza, R.C \& Arvia, A.J. (1992). Electrochemical kinetics and growth modes of silver deposits on polyfaceted platinum spherical electrodes Electrochmica Acta, Vol. 37, No. 12, (September 1992), pp. 2215-2227, ISSN 0013-4686.

Danilov, A. I., Molodkina, E. B \& Polukarov Yu. M. (1994) Russ. J.Electrochem. 1994, 30, 674.

Djenizian, T. \& Schmuki, P. (2006) Electron beam lithographic techniques and electrochemical reactions for the micro- and nanostructuring of surfaces under extreme conditions. Journal of Electroceramics, Vol. 16, No.1, (Feb 2006), pp.9-14, ISSN 1385-3449.

Fischer, H. (1954) Elektrolytische Abscheidungund Elektrokristallisation von Metallen,Springer, Berlin

Fischer, H. (1969) Electrocrystallisation of Metals under ideal and real conditions. Angew. Chem. Int. Ed.Engl. , 8, 108-119, ISSN 1521-3773.

Fleischmann, M. \& Thirsk, H. R. (1963). Advances in Electrochemistry and Electrochemical Engineering, Vol. 3, P. Delahay (Ed.), Wiley, New York.

Fu, C. X.; Shen, W. X. \& Yao T. T. (1990) Physical Chemistry, 4th ed., p. 603, Higher Education Press, Beijing.

Gewirth, A.A. \& Siegenthaler (1995), Nanoscale Probes of the Solid/ Liquid Interface, NATO ASI series E: Applied sciences vol. 288 , 334 pp, ISBN 0-7923-3454-X, Kluwer Academic Publishers, Dordrecht/ Boston/ London.

Gileadi, E. \& Tsionsky, V. Studies of Electroplating Using an EQCM. I. Copper and Silver on Gold. Journal of Electrochemical Soc.iety. vol. 147, No.2 (Feb 2000) pp. 567-574, ISSN 0013-4651.

Golzhauser, A.; Geyer, W.; Stadler, V.; Eck, W.; Grunze, M.; Edinger, K.; Weimann, Th.\& Hinz. P (2000). Nanoscale patterning of self-assembled monolayers with electrons. Journal of Vacuum Science and Technology. B, Vol. 18, No.6 (November 2000) 34143418, ISSN 1071-1023.

Hachiya, T.; Honbo, H. \& Iteya, K (1991). Detaled Underpotential Deposition of Copper on Gold (111) in aqueous-Solutions. Journal of Electroanalytical Chemistry, Vol. 315, pp.275-291, ISSN 1572-6657.

Hozzle, M. H.; Zwing, V \& Kolb D. M (1995). The influence of steps on the deposition of Cu on $\mathrm{Au}$ (III) Electrochimica Acta , Vol 40, No. 10,pp 1237-1247, ISSN 0013-4686 (95) 00055-0.

Harrison, J.A. \& Thirsk, H. R. (1971), A guide to the study of electrode kinetics, In :Electroanalytical Chemistry, A. J. Bard, (Ed.), Vol5, 67, Marcel Dekker, London.

KaltenPoth, G.; Volkel, B.; Nottbohm, C.T.; Golzhauser.A \& Buck, M. (2002). Electrode modification by electron-induced patterning of self-assembled monolayers. Journal of Vacuum Science and Technology B, Vol.20 , No.6 (November 2002), pp 2734-2738, ISSN 1071-1023. 
Kelber, J..; Rudenja, J. \&. Bjelkevig, C. (2006) Electrodeposition of copper on Ru $\left(\begin{array}{llll}0 & 0 & 0 & 1\end{array}\right)$ in sulfuric acid solution: Growth kinetics and nucleation behavior. Electrochimica Acta, Vol.51, No.15, (April 2006) pp.3086-3090, ISSN 0013-4686.

Koinuma, M. \& Uosaki, K (1994) In situ observation of anodic dissolution process of n-GaAs in $\mathrm{HCl}$ solution by electrochemical atomic force microscope. Journal of Vaccum Science and Technology B, Vol.12, pp 1543-1546, ISSN 1071-1023.

Lercel, M. J.; Craighead, H. G.; Parikh, A.N.; Seshadri, K. \& Allana, D. L. (1996), Sub-10 nm lithography with self-assembled monolayers. Applied Physics Letters, Vol. 68, No.11, pp.1504-1506, ISSN 0003-6951.

Lin, K.L.; Chen, S.Y. \& Mohanty, U.S (2008) Effect of Current density and Plating Time on the Morphology of Copper Deposits on an AFM tip. Journal of Electrochemical Society. Vol. 155, No. 4, pp. D251-D255, ISSN 0013-4651.

Li, Y.; Maynor, B.W. \& Lu. J (2001). Electrochemical AFM “Dip-Pen” Nanolithography. .Journal of American Chemical Society, Vol. 123, No.9 (March 201) 2105-2106, ISSN 0002-7863.

Lustenberger, P.; Rohrer, H; Christoph, R \& Stegenhaler, H. (1988) Journal of Electroanalytical Chemistry, Vol. 243, pp.225.

Matsui, S.; Ichihashi, T .\& Mito, M. (1989). Electron beam induced selective etching and deposition technology. Journal of Vacuum Science and Technology B, Vol. 7, No.15 September 1989) pp.1182-1190, ISSN 1071-1023 .

Markovic, N.M.; \& Ross, P.N, Jr (1993). Electrodeposition of copper on Pt (111) and Pt (100) single crystal surfaces, Journal of Vacuum Science and Technology A, Vol. 11, No.4, pp. 2225-2232, ISSN 0734-2101.

Michailova, E. ; Vitanova, I.; Stoychev, D. \& Milchev, A. (1993). Initial stages of Copper Electrodeposition in presence of organic additives. Electrochimica Acta 1993, 38, 2455-2458, ISSN 0013-4686 (93) 85116-G.

Milchev, A.. (1997). Electrochemical alloy formation-theory of progressive and instantaneous nucleation without overlap. Electrochimica Acta. Vol. 42, No.10, pp. 1533-1536, ISSN 0013-4686.

Milchev, A (2002) Electrocrystallization:Fundamentals of Nucleation and Growth, ISBN 1-40207090-X, Kluwer Academic Publishers,Boston/Dordrecht/London.

Nichols, R. J.; Beckman.; Mayer, H.; Batina, N \& Kolb, D.M (1992). An in situ scanning tunnelling microscopy study of bulk copper deposition and the influence of an organic additive. Journal of Electroanalytical Chemistry, Vol. 330, No.1-2 (July 1992) pp. 381-394, ISSN 1572-6657.

Oskam, G.; Long, J. G.; Natarajan, A. ; Searson, P. C (1998) Electrochemical deposition of metals onto silicon. J. Phys. D: Appl. Phys. Vol. 31, No.16 (August 1998), pp.19271949, ISSN 0022-3727.

Pardave, M. P.; Ramirez, M. T. ; Gonzalez, I.; \& Scharfiker, B. R. (2000). J. Electrochem. Soc., $147,567(200)$

Paunovic, M. \& Schlesinger, M. (2006) Fundamentals of Electrochemical Deposition, ISBN 978-0471-71221-3, Wiley-Interscience, NewYork.

Seah, C. H.; Mridha, S; Chan, L.H (1998), Growth morphology of electroplated copper: effect of seed material and current density, IITC, 98-158, IEEE, ISSN 0-7803-4285-2/98. 
Seah, C.H.; Mridha, S \& Chan, L.H (1999), Fabrication of D.C.-plated nanocrystalline copper electrodeposits. Journal of Materials Processing Technology, Vol. 89-90 , (May 1999), pp.432-436, ISSN 0924-0136.

Simon, G.; HaghiriGosnet, A.M.; Carcenac, F. \& Lannois, H (1997), Electroplating: An alternative transfer technology in the 20nm range. Microelectronics Engineering, Vol. 35, No. 1-4 (February 1997), pp.51-54, ISSN 0167-9317.

Smith, K.C. A \& Oatley, C.W (1955). Brazilian Journal of Applied Physics, Vol. 6, pp.391.

Sonnenfeld, R. \& Hannsma, P.K (1986) Atomic Resolution Microscopy in Water, Science, Vol. 232, No.4747, pp.211-213,, ISSN 0036-8075.

Staikov, G.; Juttner, K.; Lorenz, W. J \& Budevski, E (1994). Metal depsoition in the nanometer range.,Electrochimica Acta, Vol. 39, No.8-9, (June 1994), pp. 1019-1029, ISSN 0013-4686.

Stegenthaler, H (1992). in Scanning Tunneling Microscopy II, Springer Ser. Sur. Sci, Vol. 28(eds: Ewisendanger, R.; Guntherodt, H. J, Springer, Berlin.

Sun, S.; Chong, K. S. L and Leggett, G.J (2005). Photopatterning of self-assembled monolayers at $244 \mathrm{~nm}$ and applications to the fabrication of functional microstructures and nanostructures, Nanotechnology, 16, pp.1798-1808, ISSN 09574484.

Takahashi, K.M. \& Gross, M.E. (1999). Transport Phenomena That Control Electroplated Copper Filling of Submicron Vias and Trenches. Journal of Electrochemical Society. Vol. 146, No.12 ( December 1999) pp. 4499-4503,.

Takahashi, K.M. (2000). Electroplating Copper onto Resistive Barrier Films. Journal of Electrochemical Society. Vol. 147, No.4 (April 2000) pp 1414- 1417, ISSN 00134651.

Tan, M. \& Harb, J.N. (2003) Additive Behavior during Copper Electrodeposition in Solution Containing Cl-, PEG, and SPS Journal of Electrochemical Soc.iety. Vol.150, pp C420C425, ISSN 0013-4651.

Teh, W.H.; Koh, L.T.; Chen, S.M.; Xie, J.; Li, C.Y. \& Foo, P.D. (2001) Study of microstructure and resistivity evolution for electroplated copper films at near-room temperature. Microelectronics Journal, Vol 32, No.7 (July 2001), pp. 579-585, ISSN 0026-2692.

Thirsk, H. R. \& Harrison, J. A. (1972), A guide to the study of electrode kinetics, ISBN 0126877505, London, New York, Academic Press.

Volmer, M (1934) Das Elektrolytische Kristallwachstum, Hermannet Cie, Paris.

Volmer, M (1939) Kinetik der Phasenbildung, Steinkopf, Dresden.

West, J. M. (1971). Electrodeposition and Corrosion Process, pp. 204, 2nd Edition, ISBN 97804422093525, Van Nostrand Reinhold, New York.

Winand, R (1975). Electrocrystallisation of Copper. Transactions of the Institution of Mining and Metallurgy. Section C, Mineral processing and extractive metallurgy, Vol.84,pp.6775, ISSN 0371-8553.

Xu, W.; Wong, J.; Cheng, C.C.; Johnson, R. \& Scherer.A. (1995), Fabrication of ultrasmall magnets by electroplating . Journal of Vacuum Science and Technology, B. Vol. 13, No.6 (November 1995) pp.2372-2375, ISSN 1071-1023. 
Yau, S.L.; Gao, X; Chang, S.C; Schardt, B.C \& Weaver , M. J.(1991) Atomic-resolution scanning tunneling microscopy and infrared spectroscopy as combined in situ probes of electrochemical adlayer structure: carbon monoxide on rhodium (111). Journal of American Chemical Society, Vol. 113, No.16, pp. 6049-6056, ISSN 00027863. 


\title{
New Trends on the Synthesis of Inorganic Nanoparticles Using Microemulsions as Confined Reaction Media
}

\author{
Margarita Sanchez-Dominguez ${ }^{1}$, Carolina Aubery ${ }^{2}$ and Conxita Solans ${ }^{2}$ \\ ${ }^{1}$ Centro de Investigación en Materiales Avanzados, S. C. (CIMAV), Unidad Monterrey; \\ GENES-Group of Embedded Nanomaterials for Energy Scavenging \\ 2Instituto de Química Avanzada de Cataluña, Consejo Superior de Investigaciones \\ Científicas (IQAC-CSIC); CIBER en Biotecnología, \\ Biomateriales y Nanomedicina (CIBER-BBN) \\ ${ }^{1}$ Mexico \\ ${ }^{2}$ Spain
}

\section{Introduction}

The development of nanotechnology depends strongly on the advances in nanoparticle preparation. Nowadays, there are a number of technologies available for nanoparticle synthesis, from the gas phase techniques such as laser evaporation (Gaertner \& Lydtin, 1994), sputtering, laser pyrolisis, flame atomization and flame spray pyrolisis (Kruis et al. 1998), etc, to the liquid phase techniques such as coprecipitation from homogeneous solutions and sol-gel reactions (Qiao et al. 2011), solvothermal processes (Gautam et al. 2002), sonochemical and cavitation processing (Suslick et al. 1996), and surfactant and polymer-templated synthesis (Holmberg, 2004). Amongst the surfactant-based approaches, the microemulsion reaction method is one of the most used techniques for the preparation of very small and nearly monodispersed nanoparticles. This method offers a series of advantages with respect to other methods, namely, the use of simple equipment, the possibility to prepare a great variety of materials with a high degree of particle size and composition control, the formation of nanoparticles with often crystalline structure and high specific surface area, and the use of soft conditions of synthesis, near ambient temperature and pressure. The traditional method is based on water-in-oil microemulsions $(\mathrm{W} / \mathrm{O})$, and it has been used for the preparation of metallic and other inorganic nanoparticles since the beginning of the 1980's (Boutonnet et al., 1982). The droplets of W/O microemulsions are conceived as tiny compartments or "nanoreactors". The main strategy for the synthesis of nanoparticles in $\mathrm{W} / \mathrm{O}$ microemulsions consists in mixing two microemulsions, one containing the metallic precursor and another one the precipitating agent. Upon mixing, both reactants will contact each other due to droplets collisions and coalescence, and they will react to form precipitates of nanometric size (Figure 1). This precipitate will be confined to the interior of microemulsion droplets. Numerous investigations have been published about the use of $\mathrm{W} / \mathrm{O}$ microemulsions for the preparation of a variety of nanomaterials, 
such as metallic and bimetallic nanoparticles, single metal oxide as well as mixed oxides, quantum dots, and even complex ceramic materials (Boutonnet et al., 1982; Destrée \& Nagy, 2006; Eastoe et al. 2006; Holmberg, 2004; López-Quintela et al. 2004; Pileni 1997 and 2003). Materials synthesized in w/o microemulsions exhibit unique surface properties; for example, nano-catalysts prepared by this method show better performance (activity, selectivity) than those prepared by other methods (Boutonnet et al. 2008).

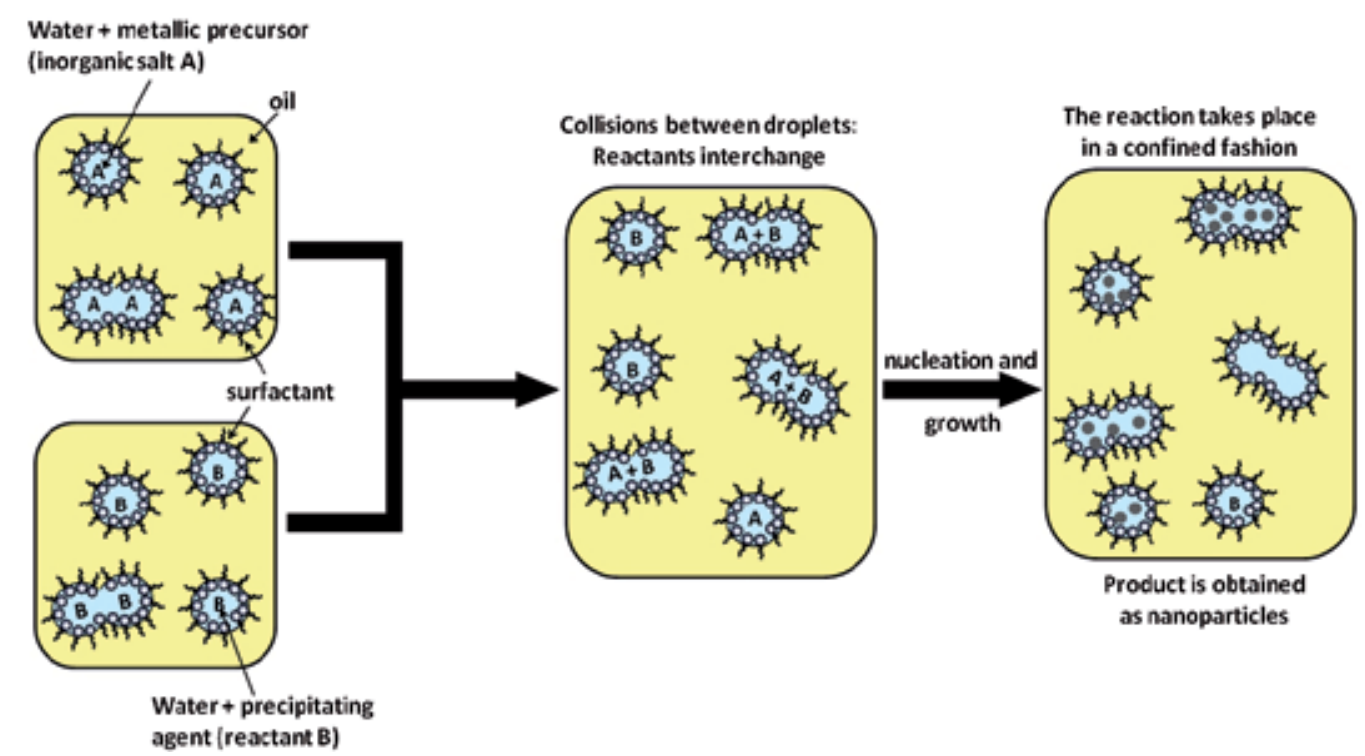

Fig. 1. Scheme of the w/o microemulsion reaction method for the synthesis of inorganic nanoparticles.

In spite of the superior properties and performance of nanoparticles obtained in w/o microemulsions, this method has not found good acceptance at the industrial level, mainly due to the employment of large amounts of oils (solvents) which represent the continuous and hence main component of these systems. In addition, most studies employ relatively low concentration of the metal precursors, leading to small yields of nanoparticles per microemulsion volume. These drawbacks affect negatively from the economic and ecologic point of view. It is the aim of this chapter to review the newest trends in the synthesis of inorganic nanoparticles using microemulsions as confined reaction media, with the objective to identify those alternatives or approaches that make this type of colloidal media more attractive for nanoparticle synthesis from the environmental , economic, technological, and scientific point of view. Some of those approaches are: the synthesis of advanced materials, such as mixed oxides and complex ceramics with nanocrystalline structure, core-shell particles, mixed materials with key nano-heterojunctions, etc, which may be difficult to obtain by other methods; optimization of microemulsion compositions, by making use of advanced phase behaviour knowledge; use of bicontinuous microemulsions in semi-continuous batches, and last but not least, a novel approach based on the use of oil-in-water microemulsions instead of w/o microemulsions as confined reaction media. An introductory section about the generalities and properties of microemulsion systems as well as on the use of microemulsions 
as reaction media for nanoparticle synthesis is first included. In addition, other aspects of nanoparticle synthesis are reviewed, such as study of reaction kinetics; influence of microemulsion dynamics on the characteristics of the obtained materials, as well as phasetransfer and isolation of nanoparticles from the microemulsion reaction media.

\section{General properties and formation of microemulsions}

\subsection{Microemulsions: Definition and basic properties}

Microemulsions are transparent and thermodynamically stable colloidal dispersions in which two liquids initially immiscible (typically water and oil) coexist in one phase due to the presence of a monolayer of surfactant molecules with balanced hydrophilic-lipophilic properties (Danielsson \& Lindman, 1981). They are optically isotropic and transparent. In contrast to emulsions, for which formation requires a considerable energy input, microemulsions form spontaneously upon gentle components mixing, once composition and temperature conditions are right. Depending on the ratio of oil and water and on the hydrophilic-lipophilic balance (HLB) of the surfactant, microemulsions can exist as oilswollen micelles dispersed in water (oil-in-water microemulsions, $\mathrm{O} / \mathrm{W}$ ), or water-swollen inverse micelles dispersed in oil (water-in-oil microemulsions, W/O); at intermediate compositions and HLBs, bicontinuous structures can exist. When a dispersed phase is present, it consists of droplets with a narrow size distribution in the order of 2-50 nm.

The formation of microemulsion depends on the surfactant type and structure, e.g. single hydrocarbon chain ionic surfactants require the incorporation of cosurfactant or electrolytes for microemulsion formation due to their high hydrophilic character; in contrast, double chain ionic surfactants and ethoxylated non-ionic surfactants may form microemulsions without cosurfactant. Lowering the interfacial tension between the oily and aqueous phase $\left(\gamma_{\mathrm{o} / \mathrm{w}}\right)$ is the main role of the surfactant (or surfactant/cosurfactant mixture). The extremely low $\gamma_{\mathrm{o} / \mathrm{w}}$ (in the order of $10^{-2}-10^{-3} \mathrm{mN} \mathrm{m}^{-1}$ ) achieved is one of the main microemulsion characteristics: the decrease on $\gamma_{\mathrm{o} / \mathrm{w}}$ is caused by the surfactant, overcoming the surface energy term caused by the huge increase in interfacial area. In addition, the spontaneous dispersion of numerous water or oil droplets causes an entropy increase, yielding a thermodynamically stable system. The extremely low interfacial tension is decisive for microemulsion formation, and depends on the composition of the system (Kunieda \& Friberg, 1981; Cross, 1987).

Microemulsions are dynamic systems, and it has been shown that droplet content exchange processes can occur in the order of millisecond time scales (Fletcher et al., 1987; Clarke et al., 1990). Collisions are produced due to constant Brownian motion of the droplets. When these collisions are sufficiently violent, the surfactant layer breaks up and the micellar exchange can be produced. It is thought that the micellar exchange process is characterized by an activation energy ( $E_{a}$ or energy barrier), which is affected by the flexibility or rigidity of the surfactant layer (Fletcher \& Horsup, 1992; Lindman \& Friberg, 1999\}, in addition to diffusion processes (Fletcher et al., 1987).

\subsection{Microemulsions and phase equilibria}

Phase behavior studies by means of equilibrium phase diagrams of polar solvent/amphiphile/nonpolar solvent systems provide essential information on 
microemulsion formation and structure. In 1954, Winsor predicted four types of equilibria which was latter experimentally evidenced: i) Winsor I: oil-in-water (o/w) microemulsions are formed, and the surfactant-rich water phase coexists with the oil phase where surfactant is only present as monomers; ii) Winsor II: water-in-oil (w/o) microemulsions are formed and the surfactant-rich oil phase coexists with the surfactant-poor aqueous phase; iii) Winsor III (middle phase): a three-phase system where a bicontinuous middle-phase microemulsion (rich in surfactant) coexists with both excess water and oil phases; and iv) Winsor IV: a single-phase (isotropic) micellar solution (microemulsion), that forms upon addition of a sufficient quantity of amphiphile. Figure 2 shows how this equilibria can be affected by salinity (for ionic surfactants) or temperature (for non-ionic surfactants), and also illustrates the structural variability of microemulsions $(\mathrm{O} / \mathrm{W}, \mathrm{W} / \mathrm{O}$ and bicontinuous $(\mathrm{BC}))$.

Some typical equilibrium phase diagrams are shown in Figure 3 (Destrée \& Nagy, 2006). In each of these diagrams $\mathrm{L}_{2}$ denotes a region where one phase $\mathrm{W} / \mathrm{O}$ microemulsions are formed. AOT (Sodium 2-ethylhexylsulfosuccinate) based systems are amongst the best characterized systems, and it has been found that the size of the inverse microemulsion droplets formed by this type of systems increases linearly with the amount of water added to the system (Pileni, 1998) and can increase from $4 \mathrm{~nm}$ to $18 \mathrm{~nm}$ with $0.1 \mathrm{M}$ sodium AOT surfactant (water/AOT/isooctane). AOT based systems are probably the most used for the synthesis of inorganic nanoparticles in w/o microemulsions, for two reasons: good control of droplet size as explained above and the large microemulsion regions found in water/AOT/alkane systems, which give rise to a great deal of compositions available for nanoparticle synthesis. Systems based on cetyltrimethylammonium bromide (CTAB), usually combine this surfactant with alcohols such as hexanol as the oil phase. This alcohol can act as co-surfactant, adsorbing at the oil/water interface along with the surfactant. As shown in Figure 3 the microemulsion region of water/CTAB/hexanol system is relatively narrow, however, when shorter alcohols such as butanol are added as cosurfactant, the microemulsion regions are considerably enlarged (Košak et al., 2004).

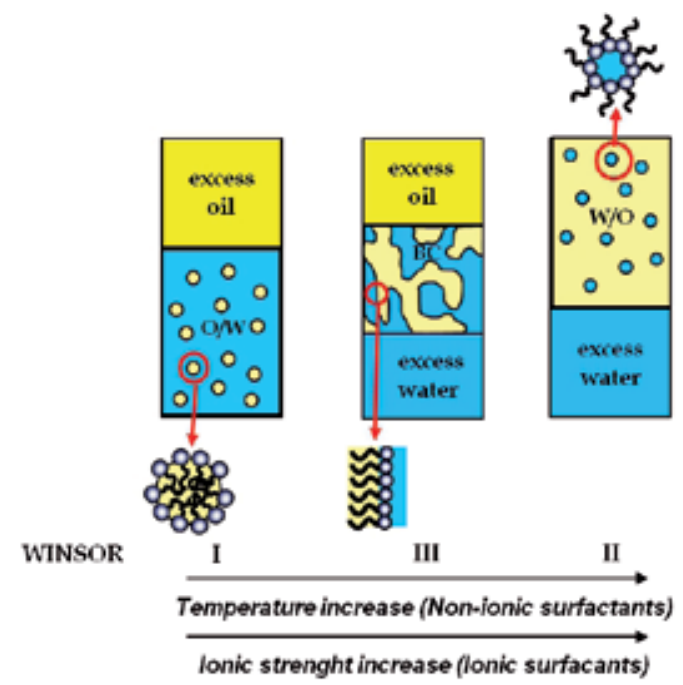

Fig. 2. Winsor classification of microemulsion equilibria. Microemulsion phase sequence as a function of temperature and salinity for non-ionic and ionic surfactants, respectively. 

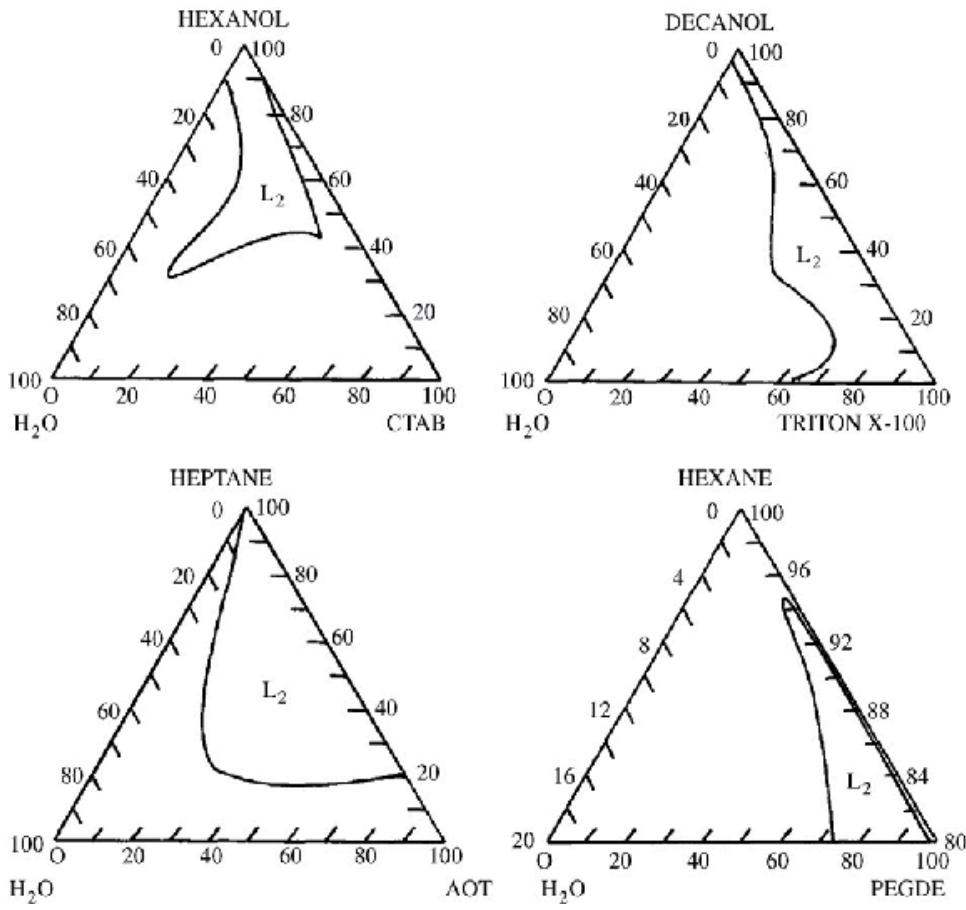

Fig. 3. Water / Nonionic surfactant / Oil pseudo bynary phase diagram, as a function of temperature. Reproduced with permission (Destrée \& Nagy, 2006).

As for non-ionic surfactant - based systems, Triton ${ }^{\circledR}$ X-100 (octyl-phenol ethoxylate) is one of the most used, however, alkyl-phenol ethoxylate surfactants such as this one have a limited biodegradability. Their metabolites of degradation have low solubility and are toxic, for example, nonylphenol has been proven to be an endocrine disrupter (Jobling \& Sumpter, 1993). On the other hand, aliphatic fatty alcohol ethoxylates such as PEGDE (penta(ethylene glycol) dodecyl ether) are more environmentally friendly; for nanoparticle synthesis, the technical-grade options are usually chosen due to their lower cost. A special feature of nonionic surfactant systems is the sensitivity of their hydrophilic-lipophilic properties to temperature, and although sometimes this characteristic is seen as a drawback, the possibility for phase-behavior tuning can be used as an advantage for the formulation of non-ionic microemulsions. In addition, nonionic surfactants have a great capacity of hydration by their ethoxylated (EO) units; hence, an appropriate selection of surfactant, oil and precursor salts/precipitating agent concentration, in combination with the rich structural behavior that such a system may display as a function of temperature, can lead to highly optimized formulations in terms of aqueous phase uptake and hence reactants loading. A good premise to this behavior is the enormous efficiency boost in the formation of middle phase microemulsions by the use of block copolymer surfactants reported by Strey et al. (Jakobs et al., 1999).

\subsection{Effect of precursor salts and additives on the phase behavior}

Although nonionic microemulsion systems are mainly affected by temperature changes, the addition of electrolytes and cosurfactant can also produce shifts in the solubilization and 
$\mathrm{T}_{\mathrm{HLB}}$ (hydrophilic-lipophilic balanced temperature or phase inversion temperature) of the systems (Aramaki et al., 2001; Kunieda et al., 1995; Shinoda, 1968). The use of ionic surfactants may have some drawbacks, as usually the aqueous phase uptake of ionic microemulsions is reduced in the presence of precursor salts due to screening effects, and hence microemulsion regions become smaller (Liu et al., 2000; Gianakas et al., 2006). Additionally, complex species could interfere with particle growth by adsorption to their surface, and contaminations of ceramic nanoparticles with the surfactant counterions aer possible. Often, the effect of addition of precursor salts or precipitating agent on the phase behavior and structure of microemulsion systems is underlooked. Generally two microemulsions with a fixed water/surfactant ratio are prepared without taking into account the influence the added salt has on the size and the structure of the water droplets.

Recently, Stubenrauch et al (Magno et al., 2009) and Sanchez-Dominguez et al. (Aubery et al., 2011) have reported systematic studies on the effects of addition of reactants to nonionic microemulsion systems. It was shown (Magno et al., 2009) that, depending on the aqueous nature of nonionic microemulsion systems, and the salting-in or salting-out effect of the additives, both increase or decrease on the water solubilization could be obtained. The same group studied the effects of different salts on the water solubilization of ionic microemulsions of the system aqueous phase / AOT/butanol / decane (Stubenrauch et al., 2008). They found that depending on the type of precursor (salts of $\mathrm{Pt}, \mathrm{Bi}, \mathrm{or} \mathrm{Pb}$ ) or the reducing agent $\left(\mathrm{NaBH}_{4}\right)$, different behaviors can be obtained, and it was necessary to add different amounts of SDS and 1-butanol in order to keep both the w/o nature of the microemulsion droplets as well as their size (which was only assessed theoretically based on microemulsion composition).

In the studies by our group on the effects of addition of precursor salts and precipitating agent to the non-ionic microemulsion system aqueous solution / Synperonic ${ }^{\circledR}$ 13/6.5 / isooctane (Aubery et al., 2011), several factors were taken into account: phase behavior (pseudoternary phase diagrams at constant temperature), dynamicity (presence or absence of percolation in $\mathrm{W} / \mathrm{O}$ structures, or formation of bicontinuous microemulsions), and droplet size (DLS). It was possible to obtain w/o microemulsions at a wide range of overlapping compositions for both precursor salts and precipitating agent. In fact, the microemulsion regions were considerably enlarged upon addition of precursors and precipitating agent; this behavior is contrary to what is typically obtained with ionic systems which have their microemulsion region reduced with addition of salts. It was difficult to obtain both type of microemulsions in either a non-percolated or percolated state; this was characterized extensively by conductivity, FT PGSE NMR and hydrophilic dye diffusion studies. When pseudobinary phase diagrams as a function of temperature were carried out, there were some compositions and temperatures at which both precursor salts and precipitating agent microemulsions were either percolated, not percolated or bicontinuous.

\subsection{Dynamic processes}

Among the dynamic processes in microemulsions, interactions of droplets components and droplet- droplet interactions must be taken into account (Fletcher et al, 1987; Fletcher \& Horsup, 1992; Moulik \& Paul, 1998). Concerning interactions of droplets components in a $\mathrm{W} / \mathrm{O}$ nonionic microemulsion, a schematic representation is depicted in Figure 4. The example concerns an aqueous droplet stabilized by a mixture of surfactant and cosurfactant 
molecules. The aqueous domain is composed by bounded water (hydrating the surfactant/cosurfactant hydrophilic domains) and free water (forming the droplet core). The exchange process to reach microemulsion equilibria comprises: 1) exchange of water between the bounded and free state; 2 ) exchange of cosurfactants among the interfacial film, the continuous phase and the dispersed phase (depending on its solubility). If ionic species are solubilized in the aqueous solution, they exchange ions between the bounded and free water. The composition of the aqueous droplets, their concentration and the temperature are mayor factors defining further interactions between them.

Droplet-droplet interactions depend strongly on droplet concentration, solvent viscosity, temperature, rigidity or flexibility of interfacial layer, and interactions between surfactant tails (Capek, 2004; Lopez-Quintela, 2003). When water or oil droplet dispersions are present, the droplets continuously collide, break apart, aggregate and break apart giving rise to dynamic processes in microemulsions. These dynamic processes allows microemulsion droplets to continuously exchange their content in microsecond scales. The composition of the aqueous droplets, as explained above, has a great influence on droplet interactions. As an example, the interfacial layer plays an important role on the formation, stability and discrete nature of microemulsion droplets. The film rigidity has been observed to increase with the surfactant hydrocarbon chain, whereas it substantially decreases with cosurfactant addition. The surfactant packing capacity can be also affected by the ionic strength of the droplets (Aramaki et al., 2001; Kunieda et al., 1995). The increase of surfactant molecules in the layer is proportional to the rigidity of the micelles.

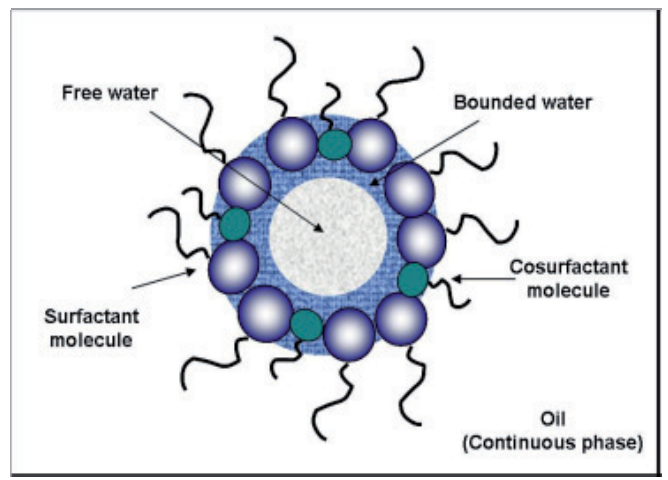

Fig. 4. Schematic representation of W/O microemulsion droplet. (Adapted with permission from Moulik, 1998).

Changes on microemulsion dynamics giving rise to structural transitions can be explained in terms of percolation. Figure 5 refers to a percolation process taking place in $\mathrm{W} / \mathrm{O}$ microemulsions (Borkovec et al., 1988), as the oil to water ratio $\phi_{o}$ is varied. As observed in Figure 5, at high oil concentration, the fraction of water in discrete droplets increases with water composition (decreasing oil concentration) up to a concentration, where it drastically decreases. This concentration is called Percolation Concentration $C_{P}$. Although clusterization occurs below $C_{P}$ (low water concentrations), these clusters remain finite in size respect to the bulk solution. $C_{P}$ represents the concentration at which the first infinite cluster appears. Further increase on water concentration would lead to the disappearance of discrete water droplets to give rise to an increase of infinite water and oily domains, which 
are characteristic of bicontinuous microemulsions. Water percolation can also be induced by temperature, and is defined as the percolation temperature $T_{P}$.

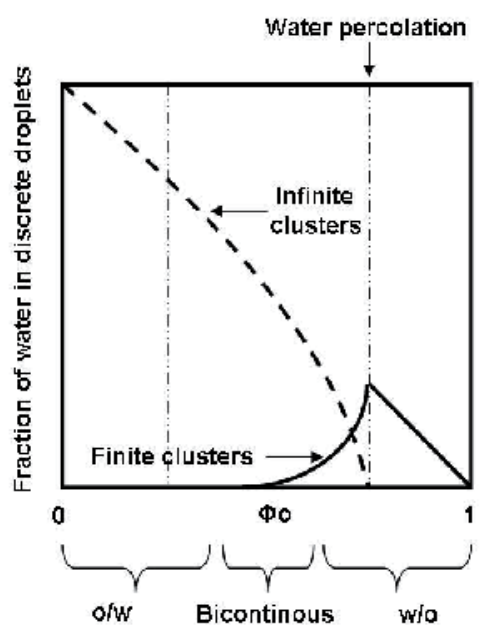

Fig. 5. Schematic representation of structural regimes of microemulsions caused by water percolation as a function of the relative amount of oil. Reprinted (adapted) with permission from Borkovec M., Eicke H.-F., Hammerich H., \& Das Gupta, B. (1988) J. Phys. Chem., 92, 1, pp. (206-211). Copyrigh (1988) American Chemical Society

\section{The microemulsion reaction method: introduction and generalities}

A brief description of the synthesis of nanoparticles in $\mathrm{W} / \mathrm{O}$ microemulsions has already been given in Section 1, along with an explanatory figure (Figure 1). Colloidal nanoparticle formation is a complex process, which includes nucleation and growth steps -giving rise to nanoparticle formation- as well as eventual coagulation and flocculation.

\subsection{Mechanism of nanoparticle formation}

A model of particle precipitation in a homogeneous aqueous medium has been proposed by La Mer (La Mer \& Dinegar, 1950). The model involves particle nucleation at short times. As soon as monomer formation takes place due to chemical reaction, its concentration increases up to the point of spontaneous nucleation, which occurs over a critical supersaturation concentration $[C]_{C}$. Afterwards, growth takes place (Figure 6). The growing step is mainly controlled by the diffusion of monomers in solution (C) onto the particles surface. Thus, $\mathrm{C}$ reaches a maximum and afterwards it begins to decrease. This decrease in monomer concentration is due to the growth of the particles by diffusion. In microemulsions, the number of nucleated sites is expected to be higher, comparing to homogeneous reactions, as illustrated in Figure 6. On the other hand, the diffusion controlled particle growth should occur at lower rate. Another model is based on the thermodynamic stabilization of the particles. In this model the particles are thermodynamically stabilized by the surfactant. The size of the particles remains constant when the precursor concentration and the size of the aqueous droplets vary. Nucleation occurs continuously during the nanoparticle formation. 


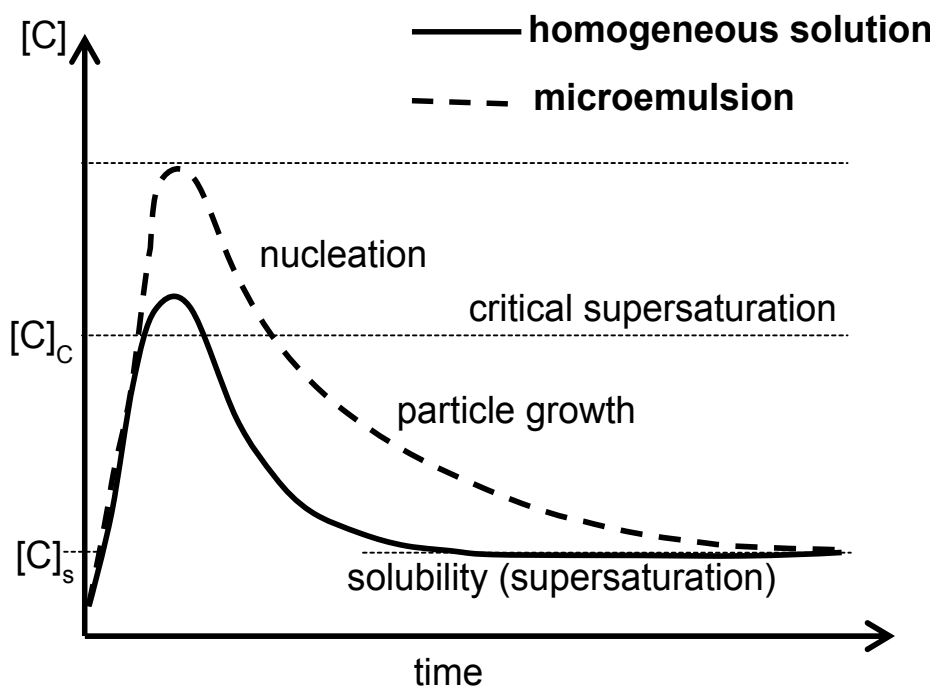

Fig. 6. Monomer concentration [C] as a function of time in microemulsions, compared to a homogeneous system. (Adapted from La Mer \& Dinegar, 1950 and Schmidt, 1999).

\subsection{Reaction kinetics}

Although microemulsions as reaction media for the synthesis of inorganic nanoparticles have been extensively studied, the kinetics of these reactions is still not completely understood. As mentioned above, several types of nanoparticles have been synthesized using a variety of surfactant systems, and relationships between the nanoparticles characteristics and the microemulsion media are not straightforward due to the diversity of variables which can have an influence, and this may be closely related with complex kinetics. An effort to relate the surfactant media with the reaction kinetics was reviewed by Lopez-Quintela et al. (Lopez-Quintela et al., 2004), concerning both inorganic and organic syntheses in microemulsions. Few studies can be cited concerning the follow-up of reactions with time, due to the fast rate of microemulsion reactions. Some of these works are pointed out below:

1. Bandyopadhyaya et al. (Bandyopadhyaya et al., 1997) have modelized $\mathrm{CaCO}_{3}$ formation in microemulsions by carbonation. A time-scale analysis was developed, resulting in a model of reaction kinetic that closely corresponded to results obtained experimentally.

2. Chew et al. (Chew et al., 1990) have studied the effect of alkanes in the formation of $\mathrm{AgBr}$ particles in ionic $\mathrm{W} / \mathrm{O}$ microemulsions (using AOT as surfactant), where the transmittance of the reactions were followed with time with UV-Vis and Stopped-Flow Spectrophotometry. They have found an increase on reaction rate with the chain lenght of the alkane.

3. Curri et al. (Curri et al., 2000) studied the role of cosurfactant on the synthesis of CdS nanoclusters, using CTAB as surfactant. Stopped-Flow Spectrophotometry was used in order to compare a reaction using $\mathrm{CTAB}$ plus cosurfactant and other carried out using AOT. They have summarized two different cosurfactant effects: the influence of the 
surfactant film flexibility on particle growth and the particles stabilization in solution, determined by the adsorption of cosurfactant onto the particle surface.

4. Lopez-Quintela et al. (De Dios et al., 2009) simulated the kinetics of nanoparticles formation in microemulsions. Simulations were carried out by comparing $\mathrm{Ag}, \mathrm{Ag}-\mathrm{Au}$ and $\mathrm{Au}$ formation with experimental data reported by Destrée and Nagy. (Destrée \& Nagy, 2006). The detailed comprehension of the kinetics taking place in microemulsion reactions is limited by the experimental data in this direction. Hence, systematic studies focused on reaction rates are greatly encouraged in order to advance in this field.

\subsection{Parameters influencing on nanoparticle synthesis}

Although complete control of particle characteristics is still far from clear and direct, some results on this field can be pointed out as shown below.

Aqueous solution concentration. It have been described in several publications the particle size dependency with water:surfactant molar ratio $\left(\mathrm{w}_{0}\right)$. In general, it has been observed that, as increasing $\mathrm{w}_{0}$, an increase on particle size is observed (Pileni, 1997; Lopez-Quintela, 2003). However, Cason et al. (Cason et al., 2001) have found that, with different $\mathrm{w}_{0}$, it was possible to obtain constant particle size if the reaction time increases for the synthesis to get completed. They proposed that the growth of the particles is affected by $w_{0}$. It was considered that for low $\mathrm{w}_{0}$ values, the aqueous solution is not enough to completely hydrate the polar groups of the surfactant and the counterion. As a consequence, the film rigidity is higher compared to higher $\mathrm{w}_{0}$ values. This influences on the micellar exchange and, as a consequence, the growth rate decreases. Increasing $\mathrm{w}_{0}$, the micelle rigidity decreases generating an increase in the growth rate up to a certain concentration, where further increase in $\mathrm{w}_{0}$ simply causes reagent dilution, which causes a decrease in the growth rate. Some studies have indicated a decrease on particle size with wo (Bagwe \& Khilar, 1997).

Reagent concentration: Particle size have been determined to be directly dependent on reagent concentration (Lopez-Quintela, 2003). An example is the work carried out by Destrée \& Nagy (Destrée \& Nagy, 2006). They have synthesized Pt nanoparticles, using different concentrations of $\mathrm{K}_{2} \mathrm{PtCl}_{4}$. An increase on particle diameter from 2 to $12 \mathrm{~nm}$ was obtained, by increasing the concentration of the precursor. On the other hand, an increase on the precipitating/precursor ratio generally causes a decrease on particle size (Lisiecki \& Pileni, 2003). It is thought that increasing precipitating agent concentration, particle nucleation can be favored in a higher extent, which further grow simultaneously, resulting in particles with lower size and polydispersity.

Surfactant and cosurfactant: Studies in order to determine the effect of nonionic hydrophilic and lypophilic surfactant groups have been developed. As the lypophilic chain of the surfactant is longer, smaller particles are obtained due to the increased micellar rigidity Generally, the addition of cosurfactant causes an increase micellar exchange, due to the decrease in the interfacial film rigidity. It is thought that the increase in microemulsion droplet size is counteracted with the increase on surfactant film curvature, generating smaller particles than without cosurfactant (Lopez-Quintela et al., 2004).

Solvent: Some studies have shown that low weight oil molecules, with low molecular volumes, can penetrate in the sufactant hydrocarbon chains, increasing the film curvature 
and rigidity (Cason et al., 2001). This effect has been observed to produce micellar exchange decrease and, consequently, smaller particles are obtained.

Electrolytes: Some studies reveal the possible dependence of nanoparticle shape with electrolyte addition (Filankembo et al., 2003). Pileni (Pileni, 2003) has postulated that the selective ion or molecule adsorption over nanocrystal layers can affect their growth in certain directions, which could explain the apparent preference on certain particle shape.

Microemulsion structure: Some studies have claimed about the nanoparticle shape partial dependence on microemulsion structure, where the microemulsion media acts as a template. A particular example is the work carried out by Pileni (Pileni, 2001) on the preparation of Copper nanoparticles from microemulsions by varying the internal structure. Spherical water droplets resulted in spherical particles, water cilinders resulted in cylindrical copper nanocrystals (with spherical particles) and a mixture of W/O microemulsion with lamellar phase resulted in a mixture of particle shape such as spheres, cylinders, etc. It was found that the template was not the only parameter which controls the shape of nanocrystals. There are examples of nonexistent correlation between the microemulsion structure and the nanoparticles obtained, which supports the nanoparticle shape dependence on electrolyte adsorption (Chen \& Lin, 2001).

Even though there is a diversity of studies carried out in order to relate nanoparticle characteristics with microemulsion properties, there is a gap in the effects of microemulsion dynamic behavior on nanoparticle characteristics, as systematic studies in this direction are scarce. The transport and micellar dynamics influence to some extent the nanoparticle formation, and it is important to take this into account in order to understand the basics of nanoparticles synthesis by this route. This type of studies may give rise to improvements on controlled nanoparticle characteristics.

\section{Recent advances in the use of microemulsions as confined reaction media for the synthesis of inorganic nanoparticles}

There have been a number of advances in different aspects of the synthesis of nanoparticles in microemulsions over the last four years. The main ones are: the use of other types of microemulsions for synthesis $(\mathrm{O} / \mathrm{W}$ and bicontinuous microemulsions), the preparation of more complex architectures (core/shell and multishell, hybrid nanocrystals), the synthesis of more complex ceramics (spinels, perovskites, etc), modeling of reactions in microemulsions, and novel approaches for the separation of nanoparticles from the reaction mixtures. The most outstanding examples of each of these aspects are given below.

\subsection{The use of other types of microemulsions for inorganic nanoparticle synthesis}

One of the main drawbacks of the technique reviewed so far (synthesis in W/O microemulsions) and the main reason why it has not been generally accepted for production at the industrial scale, is the fact that these microemulsions employ large quantities of organic solvent, as well as its limited production capacity, since this is restricted to the amount of aqueous phase solubilized and the concentration of precursor which often cannot be that high due to interactions with the surfactant, as discussed in Section 2.3. Some research groups have been working in new approaches to overcome these drawbacks. 


\subsubsection{Synthesis in oil-in-water microemulsions}

Our research group in collaboration with the group of Boutonnet, has developed a novel and straightforward approach based on O/W microemulsions (Sanchez-Dominguez et al., 2009). From a practical and environmental point of view, the possibility of preparing inorganic nanoparticles using $\mathrm{O} / \mathrm{W}$ instead of $\mathrm{W} / \mathrm{O}$ microemulsions may be highly advantageous, since the major (continuous) phase is water. The method consists in the use of organometallic precursors, dissolved in nanometer scale oil droplets of $\mathrm{O} / \mathrm{W}$ microemulsions (Figure 7), and stabilized by a monolayer of hydrophilic surfactant. The first work reported as a proof of concept the synthesis of metallic ( $\mathrm{Pt}, \mathrm{Pd}$, and $\mathrm{Rh}$ ) as well as metal oxide $\left(\mathrm{CeO}_{2}\right)$ nanoparticles (Sanchez-Dominguez et al., 2009). Small (around $3 \mathrm{~nm}$ ), nanocrystalline materials with a narrow size distribution were obtained (Figure 8).

It was followed by the synthesis of the following mesoporous nanocrystalline oxides: $\mathrm{CeO}_{2}$, $\mathrm{ZrO}_{2}, \mathrm{Ce}_{0.5} \mathrm{Zr}_{0.5} \mathrm{O}_{2}$, and $\mathrm{TiO}_{2}$ (Sanchez-Dominguez et al., 2010). Small particle size ( $3 \mathrm{~nm}$ ), and high specific surface area (200-380 $\left.\mathrm{m}^{2} \mathrm{~g}^{-1}\right)$ was obtained for all materials. Nanocrystalline cubic $\mathrm{CeO}_{2}$ and $\mathrm{Ce}_{0.5} \mathrm{Zr}_{0.5} \mathrm{O}_{2}$ were obtained under soft conditions $\left(35^{\circ} \mathrm{C}\right)$. The materials were evaluated as catalyst supports in the $\mathrm{CO}$ oxidation reaction by doping them with $\mathrm{Au}(2 \mathrm{wt} \%$, impregnation technique). The resulting catalysts showed a high Au dispersion (HRTEM/EDX). These materials showed a good activity in $\mathrm{CO}$ oxidation at low temperature $\left(\mathrm{T}_{50}\right.$ of $44^{\circ} \mathrm{C}$ for $\left.\mathrm{TiO}_{2}\right)$. This study demonstrates the feasibility of this approach for the preparation of highly active catalysts.

In a more recent study by the same group (Tiseanu et al., 2011), Eu-doped luminescent $\mathrm{CeO}_{2}$ nanocrystals were prepared by the same method. Several characterization techniques ( X-ray diffraction, RAMAN spectroscopy, UV-Vis diffuse-reflectance, FTIR as well as time-resolved photoluminescence spectroscopy) were used to characterize the nanocrystals, and it was shown that there was a surface enrichment of $\mathrm{Eu}^{3+}$, which diffused progressively to the inner Ceria sites upon calcination. Under excitation into the UV and visible spectral range, the calcined europium doped ceria nanocrystals display a variable emission spanning the orange-red wavelengths. A remarkable result was that the surface area of the powders remained as high as $120 \mathrm{~m}^{2} \mathrm{~g}^{-1}$ even after calcination at $1000^{\circ} \mathrm{C}$.

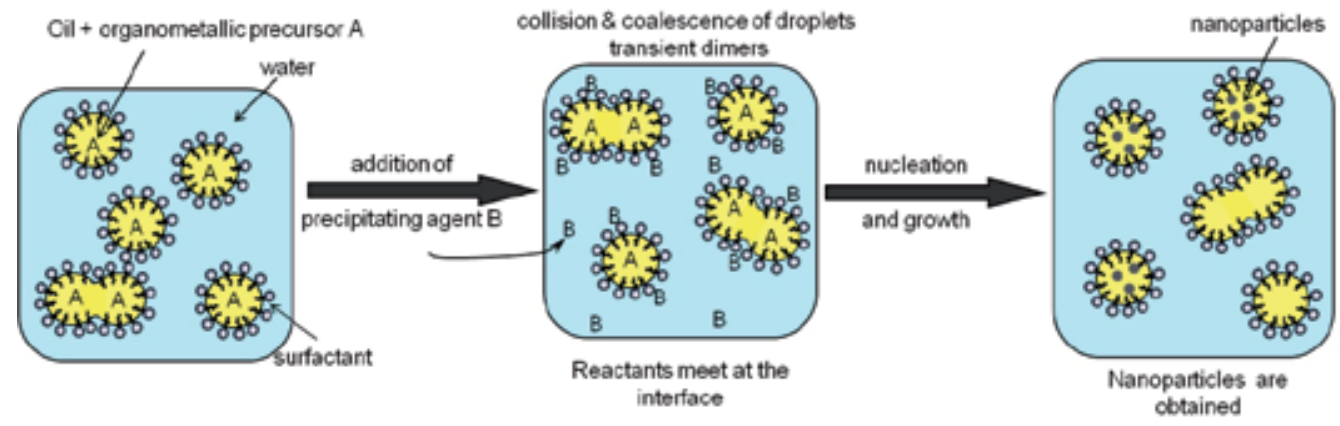

Fig. 7. TEM micrographs and related particle size distribution histograms of nanoparticles prepared in O/W microemulsions: (a) Pt, (b) Pd, (c) $\mathrm{Rh}$ and (d) $\mathrm{CeO}_{2}$. Scale bar: $50 \mathrm{~nm}$, except $\mathrm{d}(10 \mathrm{~nm})$ and inset of $\mathrm{d}(5 \mathrm{~nm})$. Reproduced with permission (Sanchez-Dominguez et al., 2009). 
It should be pointed out that in all of these examples, only one microemulsion is used for synthesis, as opposed to what is typically needed with the W/O method (two microemulsions, one bearing the precursors and another one the precipitating agent in the aqueous phase). Since most precipitating agents are water-soluble, it means that it can be added directly to the microemulsion without affecting its $\mathrm{O} / \mathrm{W}$ structure, and hence only one microemulsion, containing the organometallic precursor is prepared. Hence, the mechanism occurring in this approach is most likely different; possibly, it is an interfacial reaction. Modelization studies in conjunction with kinetic experiments need to be carried out in order to clarify this point. Considering these results, the perspectives of this novel $\mathrm{O} / \mathrm{W}$ microemulsion reaction approach are very positive, and should complement the $\mathrm{W} / \mathrm{O}$ microemulsion method, offering a greener alternative. Finally, it must be highlighted that the typical metal loading in the microemulsions reported, and hence the typical production capacity ranges from 2 to 5 grams of nanoparticles per $\mathrm{kg}$ of microemulsion, which is comparable and in some cases superior to typical metal loadings achieved in $\mathrm{W} / \mathrm{O}$ microemulsions.

\subsubsection{Synthesis in bicontinuous microemulsions}

An interesting approach to boost the metal loading and hence the nanoparticle production capacity of microemulsions is the use of bicontinuous microemulsions. Lopez et al. (Esquivel et al., 2007; Loo et al., 2008) have reported this approach for the synthesis of magnetic nanoparticles. A microemulsion system based on cationic surfactants was used for the synthesis of a mixture of maghemite/magnetite nanoparticles, using bicontinuous microemulsions at $80^{\circ} \mathrm{C}$, with $30-40 \mathrm{wt} \%$ of aqueous phase. They obtained small nanoparticles $(8 \mathrm{~nm})$ with a narrow size distribution, a nanocrystalline structure and superparamagnetic behavior. Furthermore, the yield of the reactions was as high as $1.16 \mathrm{~g}$ of product per 100 grams of microemulsion, which is rather high compared to what can be obtained in most w/o microemulsion systems (0.05 - 0.2 grams per 100 g microemulsion).

(a)

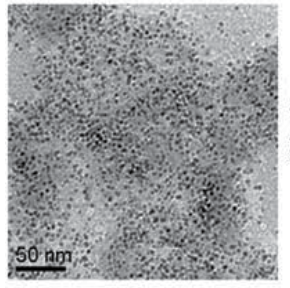

(b)

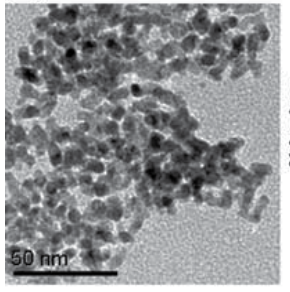

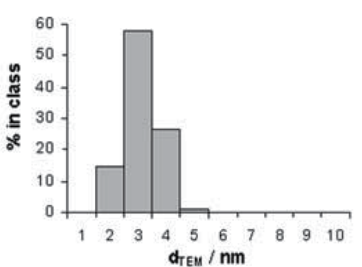

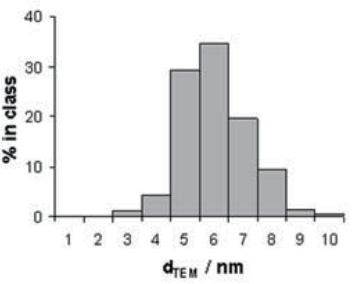

(c)
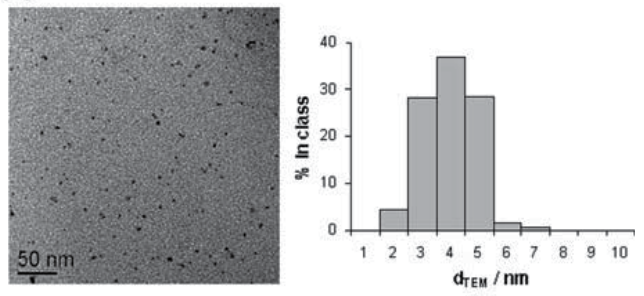

(d)

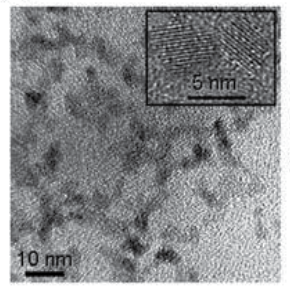

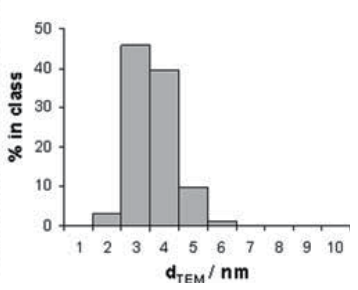

Fig. 8. TEM micrographs and related particle size distribution histograms of nanoparticles prepared in O/W microemulsions: (a) Pt, (b) Pd, (c) Rh and (d) $\mathrm{CeO}_{2}$. Scale bar: 50 nm, except d $(10 \mathrm{~nm})$ and inset of $d(5 \mathrm{~nm})$. Reproduced with permission (Sanchez-Dominguez et al., 2009). 
The same research group reported recently the synthesis of silver nanoparticles by the same approach (Reyes et al., 2010; Sosa et al., 2010), by using a microemulsion system based on AOT/SDS as the surfactant system and toluene as the oil. Depending on the surfactant: oil ratio, the authors found the formation of only globular nanoparticles or a mixture of interconnected, worm-like structures plus globular nanoparticles. The reaction yields for these materials was also remarkably high (up to $1.4 \mathrm{~g}$ of silver nanoparticles per $100 \mathrm{~g}$ of microemulsion). In all of these works, only one microemulsion was necessary for nanoparticle preparation, as the precipitating agent was added directly, as an aqueous solution, to the microemulsion containing the metallic precursors. This aspect also contributes to the greener quality of this approach as compared to the traditional $\mathrm{W} / \mathrm{O}$ microemulsion reaction method.

\subsubsection{Synthesis in microemulsions with an optimized aqueous phase uptake}

In the work carried out by our group concerning a nonionic system (Aubery et al., 2011), large microemulsion regions were obtained when the reactants were incorporated, as mentioned in Section 2.3. Thanks to this high aqueous phase uptake and the overlap of microemulsion regions for both precursor salts and precipitating agent, synthesis of Mn-Zn ferrite nanoparticles could be carried out using a wide range of compositions. Futhermore, different scenarios were available for nanoparticle synthesis: W/O non-percolated, W/O percolated, and bicontinuous microemulsions. Differences were observed in the characteristics of the synthesized nanoparticles depending on the type of microemulsions used, and in all cases spinel nanocrystalline particles with superparamagnetic properties were obtained, directly in the microemulsion, without the need for calcination. The aqueous phase content ranged from $5 \mathrm{wt} \%$ to $50 \mathrm{wt} \%$, which represents a boost in the production capacity. This study should encourage further research into optimized non-ionic microemulsion systems, since although the presence of salts affects their phase behavior, it does so in such a way that aqueous solubilization can be significantly increased at a certain temperature, which can be investigated by phase behavior studies.

\subsection{Preparation of more complex architectures}

In this regard, most of the studies concern core-shell studies, although some other structures include multiple core-shell particles, hollow spheres and nanowires and nanorods.

\subsubsection{Core/shell nanoparticles}

A large majority of the core-shell nanoparticles synthesized in $\mathrm{W} / \mathrm{O}$ microemulsions contain silica, usually as the shell material. In the last few years, the W/O microemulsion approach has been gaining popularity over the well-known adaptation of the Stöber method (Nann \& Mulvaney, 2004), for coating a diversity of nanoparticles with a silica shell. This is because it has been observed that the microemulsion method results in a better shell thickness control (Dong H, 2009), as compared to the adapted Stöber method, which is based on the sol-gel technique. It must be pointed out that in the majority of the studies, the core material was synthesized in a previous step, by a different method, usually hydrothermal or solvothermal techniques (Dong B. et al., 2009; Dong H et al., 2009; Qian et al., 2009; Vogt et al., 2010; Wang J. et al., 2010). Nevertheless, a very interesting point from these investigations is the strategy on 
how this silica shell is deposited onto the core; this sophisticated approach is probably the reason for the high control achieved. The core nanoparticles are usually functionalized for one or two purposes: one is in order to be very well dispersed in one of the microemulsion phases (the oily or the aqueous phase), the other is for very controlled deposition of silica via hydrolytic copolymerization with silanized molecules such as (3-aminopropyl)triethoxysilane (APTES), which were covalently linked to the core particles. By this approach, uniform CdTe@silica nanoparticles with a regular core - shell structure, $48 \pm 3 \mathrm{~nm}$ in diameter were obtained by Dong H. et al. (Dong H. et al., 2009). In their work, the initial core CdTe particles, synthesized by a hydrothermal method, were functionalized with thioglycolic acid, so they could be reacted with APTES and then dispersed in the aqueous phase of the microemulsion. The silica precursor, TEOS, was dissolved in the oily phase of the microemulsion (cyclohexane and octanol), and the silica shell was then formed by addition of ammonia. In the work by Dong B. et al (Dong B. et al., 2009), on the other hand, the core ZnS:Mn particles were functionalized with oleic acid and hence dispersed in the oil phase of the microemulsion, and the silica layer was deposited by reacting TEOS with ammonia in the W/O microemulsion containing the core particles dispersed in the oil. Figure 9 shows TEM results of these coreshell particles.

Fewer examples deal with the formation of core-shell nanoparticles in which both the core and the shell have been synthesized in a W/O microemulsion (Chung et al., 2011; Takenaka et al., 2007). Takenaka et al. prepared Ni nanoparticles in a W/O microemulsion, and afterwards TEOS and ammonia were added in order to form the silica layer. Core-shell nanoparticles with $20-50 \mathrm{~nm}$ diameter and a Ni shell $(5 \mathrm{~nm})$ were formed. For comparison, silica nanoparticles were prepared also in $\mathrm{W} / \mathrm{O}$ microemulsions but the $\mathrm{Ni}$ nanoparticles were prepared by impregnation of these silica nanoparticles. Their catalytic activity in the partial oxidation of methane reaction was evaluated, and the core-shell nanoparticles had a better performance than the impregnated ones (Takenaka et al., 2007). On the other hand, Chung et al. prepared silica nanoparticles coated with a thin layer of $\mathrm{CeO}_{2}$, and the material was also prepared in W/O microemulsions in a two-step procedure (Chung et al., 2011). This reaction turned out to be challenging as the formation of $\mathrm{CeO}_{2}$ shell was competing with bulk precipitation. The problem was overcome by coupling two strategies:

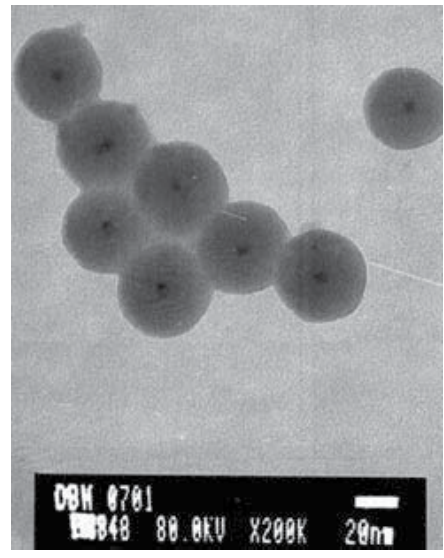

Fig. 9. TEM image of ZnS:Mn@silica nanoparticles with a core - shell structure. (Reproduced with permission, Dong B. et al. 2009). 
functionalization of the surface of the core silica nanoparticles with an organoamine group, and step-wise, semi-batch addition of the second microemulsion containing the Ce precursor. In this way, the silica cores were homogenously coated with a $\mathrm{CeO}_{2}$ shell.

As for core-shell nanoparticles made up of materials different from silica, the synthesis of both the core and the shell is usually carried out in W/O microemulsions, either in a two step process by preparing first the core and the later deposition of the shell (by adding more aqueous phase or more microemulsion comprising the second component), or both precursors are incorporated simultaneously, but the different reaction kinetics for each of the products results in a core-shell structure. The following core-shell nanomaterials can be listed: Pt@ $\mathrm{CeO}_{2}$ (Yeung \& Tsang, 2009 and 2010), Co@Ag (Garcia-Torres et al., 2010), $\mathrm{Fe}_{2} \mathrm{O}_{3} @ \mathrm{Au}$ (Iglesias-Silva et al., 2010), Ni@Au (Chiu et al., 2009), Ag@Polystyrene (Li et al., 2009), and CdS@ $\mathrm{TiO}_{2}$ (Ghows \& Entezari, 2011). So far, the core@shell structures of these materials is not as well defined and controlled as that obtained with core@silica materials.

\subsubsection{Hollow nanospheres}

Jiang et al. have prepared hollow nanospheres of Ni (Jiang et al., 2010) and CuS (Jiang, 2011), by following an approach which resembles that reported by Sanchez-Dominguez et al. (Sanchez-Dominguez et al., 2009). Jiang et al. used an o/w microemulsion in which the precursor (a naphtenate), was dissolved in the oil phase (dimethylbenzene) of a water/SDS/butanol/dimethylbenzene microemulsion. The precipitating agent was added in the water phase. The authors explain that an interfacial reaction occurs, and hollow nanospheres of about 100-200 nm are formed (Figure 10, for Ni hollow spheres). These are made-up of smaller nanoparticles. One difference between Jiang's method and that reported by Sanchez-Dominguez et al is that in the former, the temperatures used for reaction are higher $\left(85^{\circ} \mathrm{C}\right.$ for $\mathrm{Ni}$; post-synthesis hydrothermal treatment for $\left.\mathrm{CuS}\right)$, whereas in the latter the temperatures used are near room temperature $\left(25-35^{\circ} \mathrm{C}\right)$.

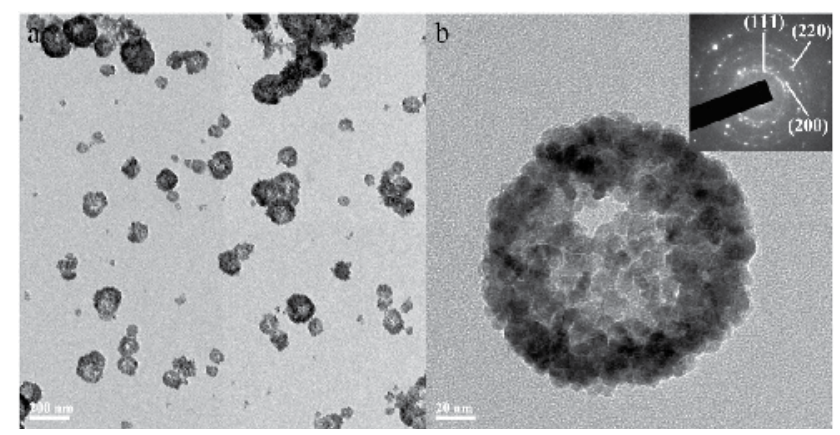

Fig. 10. TEM image of the Ni hollow spheres and (b) a single Ni hollow sphere. Inset: SAED pattern. Reproduced with permission (Jiang et al., 2010).

\subsubsection{Nanowires, nanorods}

Some works describe the formation of nanowire-like or nanorod structures. Usually, in order to obtain such high aspect ratio structures, it is necessary to carry out the synthesis at a relatively high temperature, or include a certain post-synthesis thermal treatment. Wang et 
al. synthesized single-crystalline $\mathrm{ZnO}$ nanowire bundles with a length of about $1 \mu \mathrm{m}$ and a diameter of about 20-30 nm (Wang G. et al., 2010). The approach was by reacting zinc acetate with hydrazine in a w/o microemulsion based on water/dodecylbenzene sulfonic acid sodium salt / xylene. The reaction temperature is not mentioned, however for reflux of xylene is achieved around $140^{\circ} \mathrm{C}$. The relatively high reaction temperature and the heating time is possibly the driving force for the growth of the nanowires, as different structures were obtained at shorter reaction times. Also, it must be pointed out that the precursor used, zinc acetate, is soluble in both water and the oil phase, which is an unusual approach.

$\mathrm{Wu}$ et al. synthesized nanowires of $\mathrm{Zn} / \mathrm{Co} / \mathrm{Fe}$ layered double hydroxides using a w/o microemulsion based on water/CTAB/n-hexanol n-hexane (Wu et al., 2010). In their approach, the sulfate salts were used as precursors, hence these were dissolved in the aqueous phase only, and urea was used as precipitant. The influence of reaction temperature, time, urea concentration and $\mathrm{CTAB}$ to water molar ratio on the structure and

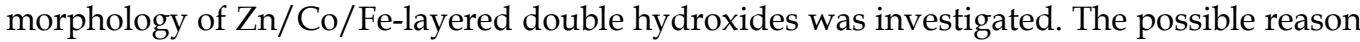
for nanowire growth is the solvothermal treatment of the reaction mixture which was carried out in an autoclave at $80-180^{\circ} \mathrm{C}$ during 6-24 hours. The thermal treatment in the autoclave was a key factor for annealing and therefore obtaining both a crystalline structure and formation of high aspect ratio particles (nanowires).

\subsection{Synthesis of complex ceramics}

The w/o microemulsion reaction method has been used for the synthesis of complex ceramic nanoparticles such as perovskites, spinels, aluminates, and hexaferrites. Often, nanoparticles of precursors such as hydroxides or other amorphous compounds are synthesized in the microemulsions, and these are afterwards calcined at a certain temperature in order to obtain the desired crystalline structure.

He et al. synthesized nanoparticles of perovskite-type oxides $\mathrm{La}_{0.8} \mathrm{Ce}_{0.2} \mathrm{Cu}_{0.4} \mathrm{Mn}_{0.6} \mathrm{O}_{3}$ and $\mathrm{La}_{0.8} \mathrm{Ce}_{0.2} \mathrm{Ag}_{0.4} \mathrm{Mn}_{0.6} \mathrm{O}_{3}$ (He et al., 2007). The microemulsion used was CTAB/butanol / water /heptane, and for comparison purposes, the same materials were synthesized by the sol-gel technique. The precipitation of the precursors was carried out with $\mathrm{NaOH}$ for the microemulsion method, whereas citric acid was used for the sol-gel method. The particle size distribution was smaller and more uniform and the specific surface area was higher for the particles synthesized in microemulsions than those synthesized by sol-gel. Furthermore, the catalytic activity in the NO reduction by $\mathrm{CO}$ was evaluated. Performance of perovskites synthesized in microemulsion was superior than that of materials synthesized by sol-gel.

Gianakas et al. (Gianakas et al., 2007) reported the synthesis of spinel-type metal aluminates $\mathrm{MAl}_{2} \mathrm{O}_{4}$ sutwhere $\mathrm{M}=\mathrm{Mg}, \mathrm{Co}$, o $\mathrm{Zn}$ using $\mathrm{w} / \mathrm{o}$ and bicontinuous microemulsions. They carried out a very complete phase behavior study, which included pseudoternary phase diagrams for each precursor combination as well as the precipitating agent, ammonia. The microemulsion system was: aqueous solution/ CTAB/butanol/ octane. The spinel structure was achieved after calcination at $800^{\circ} \mathrm{C}$. It was found that spinels synthesized in reverse microemulsions showed better surface and textural properties, as well as smaller particle size than spinel synthesized in bicontinuous microemulsions. As for catalytic activity, which was evaluated in the $\mathrm{NO}$ reduction by $\mathrm{CO}$, the spinels synthesized in w/o microemulsions was slightly superior. Similar characteristic size was obtained by Wang et al. (Wang Y. et al., 
2007) for nanoparticles of manganese-doped barium aluminate $\mathrm{BaAl}_{12} \mathrm{O}_{19}: \mathrm{Mn}^{2+}$; calcination at $1300^{\circ} \mathrm{C}$ was carried out in order to obtain the crystalline phase expected. The evaluation of photoluminescent properties of this material showed that this phosphor is a good candidate to replace $\mathrm{Hg}$ lamps.

Other good examples of ceramic materials obtained in w/o microemulsions include: barium hexaferrite $\left(\mathrm{BaFe}_{12} \mathrm{O}_{19}\right)$ nanoparticles $\left(\mathrm{Xu}\right.$ et al., 2007), tungsten oxide $\left(\mathrm{WO}_{3}\right)$ nanoparticles (Asim et al., 2007), and rutile $\mathrm{TiO}_{2}$ nanoparticles (Keswani et al., 2010). In the last example, it is remarkable that the rutile phase was obtained at room temperature, without the need for thermal treatment, hence the size of the rutile nanocrystals remained as small as $4 \mathrm{~nm}$.

\subsection{Modeling of reactions in microemulsions}

There have been a number of studies dealing with the theoretical aspects of nanoparticle formation by the microemulsion reaction method. Most of these studies use the Monte Carlo method. The studies carried out in the last four years are focused on several aspects: kinetics of nanoparticle formation (de Dios et al., 2009), formation of bimetallic nanoparticles (Tojo et al., 2009; Angelescu et al., 2010), droplet exchange (Niemann \& Sundmacher, 2010), cluster coalescence (Kuriyedath et al., 2010), and core-shell nanoparticle formation (Viswanadh et al., 2007).

Kinetics of nanoparticle formation in microemulsion were studied for the $\mathrm{Ag}$ and $\mathrm{Au}$ nanoparticles using Monte Carlo simulations by de Dios et al. (de Dios et al., 2009). It was shown that, although the material interdroplet exchange depends primarily on the flexibility of surfactant film, a slow reaction rate leads to a more effective material interdroplet exchange for a given microemulsion. Two factors contribute to this result. Firstly, a slow reaction implies that autocatalytic growth takes place for a longer period of time, because there are available reactants. If the reaction is faster, the reactants are almost exhausted at early stages of the process. As a consequence, autocatalytic growth is only possible at the beginning. Secondly, a slow reaction rate implies the continuous production of seed nuclei, which can be exchanged between micelles due to their small size, allowing the coagulation of two nanoparticles. This exchange only takes place at early stages of the synthesis. Both factors, autocatalysis and ripening, favor the slow growth of the biggest nanoparticles leading to the production of larger particles when the reaction is slower.

With respect to the formation of bimetallic nanoparticles in microemulsions, the same research group (Tojo et al., 2009), carried out Monte Carlo studies in order to explain the different structures that can be obtained when bimetallic nanoparticles are synthesized in microemulsions. They observed that the difference in reduction rates of both metals is not the only parameter to determine metal segregation; the interdroplet channel size also plays an important role. The reduction rate difference determines nanoparticle structure only in two extreme cases: when both reactions take place at the same rate, a nanoalloy structure is always obtained. In contrast, if both reactions have very different rates, the nanoparticle shows a core-shell structure. However, in the large interval between both extreme cases, the nanoparticle structure is strongly dependent on the intermicellar exchange, which is mainly determined by the flexibility of the surfactant film around the microemulsion droplets. In a related study by Angelescu et al. (Angelescu et al., 2010), it was found that the bimetallic nanoparticle structure is mainly determined by the difference in the reduction rates of the 
two metal ions and the excess of reducing agent. An intermetallic structure is always obtained when both reduction reactions take place at about the same rate. When the metal ions have very different reduction potentials, a core-shell to intermetallic structure transition is found at increasing the excess of the reducing agent. An enhancement of the intermetallic structure at the expense of the core-shell, can be obtained either by decreasing the concentration of both metal salts or by increasing the interdroplet exchange rates. The results obtained by these studies has positive implications in the general formation of bimetallic nanoparticles with a given structure (core-shell or nano-alloy).

\subsection{Novel approaches for the separation of nanoparticles from reaction mixtures}

Often, the nanoparticles formed in a microemulsion are so well dispersed in the reaction media that some solvent has to be added in order to destabilize the microemulsion, which causes desorption of surfactant from the particles, which aggregate and precipitate, making their separation by centrifugation or filtration easier. Sometimes, during this aggressive process the nanoparticles end up so agglomerated that it is difficult to re-disperse them. Some novel and straightforward approaches have been proposed for an improved recovery or phase transfer of nanoparticles from microemulsion media.

Eastoe et al. (Hollamby \& Eastoe, 2009; Myakonkaya et al., 2010, 2011; Nazar et al., 2011; Vesperinas et al., 2007) have proposed three approaches for nanoparticle recovery. One of them is based on the use of a photodestructible surfactant for microemulsion formation, and in the final step, irradiation with UV-light induces microemulsion destabilization and hence separation of Au nanoparticles (Vesperinas et al., 2007). In another approach, excess water is added at the end of the reaction, to the microemulsion containing the nanoparticles, inducing a change in phase behavior and hence microemulsion destabilization, followed by phase separation. Interestingly, by this approach, usually the nanoparticles remain in the oil phase, which can be diluted with organic solvents to form stable nanoparticle dispersions (Nazar et al., 2011). This method shows potential benefits for dispersion, storage, application, and recovery of NPs, with the great advantage that it is not necessary to add organic solvents for nanoparticle separation. In other approach by the same group, nanoparticle separation has been achieved by changing the solvent quality, for example, adding squalene to water/AOT/octane microemulsion containing $\mathrm{Au}$ nanoparticles (Myakonkaya et al., 2010).

Abecassis et al. have proposed nanoparticle separation by thermally inducing the phase separation of the microemulsion media (Abecassis et al., 2009). This was applied to the synthesis of Au NPs, which upon destabilization remained preferentially in the oil phase.

\section{Conclusions and perspectives}

It has been shown that the microemulsion reaction method is a versatile technique, useful for the controlled synthesis of a large variety of nanomaterials, from metals, metal oxides, ceramics, quantum dots, magnetic nanoparticles, etc. The method has now been extended to the synthesis of other types or architectures, such as core-shell, multishell, hollow spheres and nanowires, in addition to the traditional small globular particles. Although for about 25 years only w/o microemulsions were used for the synthesis of inorganic nanoparticles, in the last five years the use of $\mathrm{o} / \mathrm{w}$ and bicontinuous microemulsions has also been 
developed, and their usefulness for the synthesis of a variety of nanomaterials has been demonstrated. These developments are greener than the traditional w/o microemulsion method, so it should contribute to an advance towards the industrial use of microemulsions for nanoparticle synthesis. Furthermore, there have been efforts towards boosting the metal loading in microemulsions, in order to increase their production capacity. The investigations on novel approaches for nanoparticle recovery should also be taken into account by more research groups for the improvement of nanoparticle quality and dispersability in different media. The new developments reviewed here should encourage the preparation of novel materials with different architectures, in order to respond quickly to the demands of Nanotechnology and Materials Science. It is hoped that this chapter is useful to students and researchers who start exploring the microemulsion reaction method for nanoparticle synthesis, as well as for those not new to the field but who are looking for the newest trends in this fascinating technique.

\section{Acknowledgements}

The authors acknowledge financial support by Ministerio Ciencia e Innovación (MICINN Spain, grant number CTQ2008-01979) and Generalitat de Catalunya (Agaur, grant number 2009SGR-961).

\section{References}

Abecassis B., Testard F., \& Zemb T. (2009) Gold nanoparticle synthesis in worm-like catanionic micelles: Microstructure conservation and temperature induced recovery. Soft Matter, 5, 5, (March 2009), pp. (974-978).

Angelescu D. G., Magno L. M., \& Stubenrauch C. (2010) Monte Carlo simulation of the size and composition of bimetallic nanoparticles synthesized in water in oil microemulsions. J. Phys. Chem. C, 114, 50, (Dec. 2010), pp. (22069-22078).

Aramaki K., Hayashi T., Katsuragi T., Ishitobi M., \& Kunieda H. (2001) Effect of Adding an Amphiphilic Solubilization Improver, Sucrose Distearate, on the Solubilization Capacity of Nonionic Microemulsions, J. Colloid Interface Sci., 236, 1, (April 2001), pp. (14-19).

Asim N., Radiman S., \& bin Yarmo M. A. (2007) Synthesis of WO3 in nanoscale with the usage of sucrose ester microemulsion and CTAB micelle solution. Mater. Lett., 61, 13, (May 2007), pp. (2652-2657).

Aubery C., Solans C., \& Sanchez-Dominguez, M. (2011) Tuning high aqueous phase uptake in nonionic w/o microemulsions for the synthesis of Mn-Zn ferrite nanoparticles: phase behavior, characterization and nanoparticle synthesis. Langmuir, 27, 23, (Oct. 2011), pp. (14005-14013).

Bagwe R. P., \& Khilar K. C. (1997) Effects of the Intermicellar Exchange Rate and Cations on the Size of Silver Chloride Nanoparticles Formed in Reverse Micelles of AOT. Langmuir, 13, 24, (Nov. 1997), pp. (6432-6438).

Bandyopadhyaya R., Kumar R., Gandhi K. S., \& Ramkrishna, D. (1997) Modeling of Precipitation in Reverse Micellar Systems, Langmuir, 13, 14, (July 1997), pp. (36103620).

Borkovec M., Eicke H.-F., Hammerich H., \& Das Gupta, B. (1988) Two Percolation Processes in Microemulsions. J. Phys. Chem., 92, 1, (Jan 1988), pp. (206-211). 
Boutonnet M., Kizzling J., \& Stenius P. (1982) The preparation of monodisperse colloidal metal particles from microemulsions. Colloids and Surfaces, 5, 3, (November 1982), pp. (209-225)

Boutonnet M., Lögdberg S., \& Svensson E. E. (2008) Recent developments in the application of nanoparticles prepared from $\mathrm{w} / \mathrm{o}$ microemulsions in heterogeneous catalysis. Curr. Opin. Colloid Interface Sci., 13, 4, (August 2008), pp. (270-286).

Capek, I. (2004) Preparation of metal nanoparticles in water-in-oil (wyo) microemulsions. Adv. Colloid Interface Sci. 2004, 110, 1-2, (June 2004), pp. (49-74)

Cason J. P., Miller M. E., Thompson J. B., \& Roberts C. B. (2001) Solvent Effects on Copper Nanoparticle Growth Behavior in AOT Reverse Micelle Systems. J. Phys. Chem. B., 105, 12, (March 2001), pp. (2297-2302).

Chen C. C., \& Lin J. J. (2001) Controlled Growth of Cubic Cadmium Sulfide Nanoparticles Using Patterned Self-Assembled Monolayers as a Template. Adv. Mater., 13, 2, (Jan, 2001), pp. (136-139).

Chew C. H., Can L. M., \& Shah D. O. (1990) The Effect of Alkanes On The Formation Of Ultrafine Silver Bromide Particles In Ionic W/O Microemulsions. J. Dispersion Sci. Technol., 11, 6, (Dec. 1990), pp. (593-609).

Chiu H. K., Chiang I. C., \& Chen D. H. (2009) Synthesis of NiAu alloy and core-shell nanoparticles in water-in-oil microemulsions. J. Nanopart. Res., 11, 5, (July 2009), pp. (1137-1144).

Chung S. H., Lee D. W., Kim M. S., \& Lee K. Y. (2011) The synthesis of silica and silica-ceria, core-shell nanoparticles in a water-in-oil (W/O) microemulsion composed of heptane and water with the binary surfactants AOT and NP-5. J. Colloid Interface Sci., 355, 1, (March 2011), pp. (70-75).

Clarke S., Fletcher P. D. I., \& Ye X. (1990) Langmuir, 6, 7, (July 1990), pp. (1301-1309).

Cross, J. (1987). Nonionic Surfactants: Chemical Analysis, Surfactant Science Series (1st edition), Marcel Decker Inc., ISBN-10: 0824776267, New York, NY, USA.

Curri M. L., Agostiano A., Manna L., Della Monica M., Catalano, M., Chiavarone L., Spagnolo V., \& Lugara M. (2000) Synthesis and Characterization of CdS Nanoclusters in a Quaternary Microemulsion: the Role of the Cosurfactant J. Phys. Chem. B, 104, 35, (August 2000), pp. (8391-8397).

Cushing B. L., Kolesnichenko V. L., \& O'Connor C. J. (2004) Recent Advances in the LiquidPhase Syntheses of Inorganic Nanoparticles. Chem. Rev., 104, 9, (Nov. 2004), pp. (3893-3946).

Danielsson I., and Lindman B. (1981) The definition of microemulsion. Colloids and Surfaces, 3, 4, (December 1981) pp. (391-392).

De Dios M., Barroso F., Tojo C., \& Lopez-Quintela, M. A. (2009) Simulation of the kinetics of nanoparticle formation in microemulsions. J Colloid Interface Sci., 333, 2, (May 2009), pp. (741-748).

Destrée C., \& Nagy J. B. (2006) Mechanism of formation of inorganic and organic nanoparticles from microemulsions. Adv. Colloid Interface Sci., 123-126, (November 2006), pp. (353-367).

Dong B., Cao L., Su G., Liu W., Qu H., \& Jiang D. (2009) Synthesis and characterization of the water-soluble silica-coated ZnS:Mn nanoparticles as fluorescent sensor for $\mathrm{Cu}^{2+}$ ions. J. Colloid Interface Sci., 339, 1, ( Nov. 2009), pp. (78-82). 
Dong H., Liu Y., Ye Z., Zhang W., Wang G., Liu Z., \& Yuan J. (2009) Luminescent nanoparticles of silica-encapsulated cadmium-tellurium (CdTe) quantum dots with a core-shell structure: Preparation and characterization. Helvetica Chimica Acta, 92, 11, (Nov. 2009), pp.(2249-2256).

Eastoe J., Hollamby M. J., \& Hudson L. (2006) Recent advances in nanoparticle synthesis with reversed micelles, Adv. Colloid Interface Sci., 128-130, (December 2006), pp. (515).

Esquivel J., Facundo I. A., Treviño M. E., \& López R. G. (2007) A novel method to prepare magnetic nanoparticles: precipitation in bicontinuous microemulsions. J. Mater. Sci., 42, 21, (Nov. 2007), pp. (9015-9020)

Filankembo A., Giorgio S., Lisieki I., \& Pileni, M. P. (2003) Is the Anion the Major Parameter in the Shape Control of Nanocrystals? J. Phys. Chem. B, 107, 30, (April 2003), pp. (7492-7500).

Fletcher P. D. I., Howe A. M., \& Robinson B. H. (1987) J. Chem. Soc. Faraday Trans. 1, The kinetics of solubilisate exchange between water droplets of a water-in-oil microemulsion, 83, 4, (April 1987), pp. (985-1006).

Fletcher P. D. I., \& Horsup D. I. (1992) Droplet dynamics in water-in-oil microemulsions and macroemulsions stabilised by non-ionic surfactants. Correlation of measured rates with monolayer bending elasticity. J. Chem. Soc. Faraday Trans. 1, 88, 6, (June 1992), pp. (855-864).

Gaertner, G.F., \& Lydtin H. (1994) Review of ultrafine particle generation by laser ablation from solid targets in gas flows. Nanostructured Mater., 4, 5, (September 1994), pp. (559-568).

Garcia-Torres J., Vallés E., \& Gómez E. (2010) Synthesis and characterization of Co@Ag coreshell nanoparticles. J. Nanopart. Res., 12, 6, (August 2010), pp. (2189-2199).

Gautam U. K., Ghosh M., Rajamathi M., \& Seshadri R. (2002) Solvothermal routes to capped oxide and chalcogenide nanoparticles, Pure Appl. Chem., 74, 9, (September 2002), pp. (1643-1649).

Ghows N., \& Entezari M. H. (2011) Fast and easy synthesis of core-shell nanocrystal $(\mathrm{CdS} / \mathrm{TiO} 2)$ at low temperature by micro-emulsion under ultrasound. Ultrason. Sonochem., 18, 2, (March 2011), pp. (629-634).

Giannakas A. E., Ladavos A. K., Armatas G. S., Petrakis D. E., \& Pomonis P. (2006) Effect of composition on the conductivity of CTAB butanol octane nitrate salts $\left(\mathrm{Al}\left(\mathrm{NO}_{3}\right)_{3}+\right.$ $\left.\mathrm{Zn}\left(\mathrm{NO}_{3}\right)_{2}\right)$ microemulsions and on the surface and textural properties of resulting spinels $\mathrm{ZnAl}_{2} \mathrm{O}_{4}$. J. Appl. Surf. Sci., 252, 6, (Jan. 2006), pp. (2159-2170).

Giannakas A. E., Ladavos A. K., Armatas G. S., \& Pomonis P. J. (2007) Surface properties, textural features and catalytic performance for $\mathrm{NO}+\mathrm{CO}$ abatement of spinels $\mathrm{MAl}_{2} \mathrm{O}_{4} \quad(\mathrm{M}=\mathrm{Mg}$, Co and $\mathrm{Zn})$ developed by reverse and bicontinuous microemulsion method. Appl. Surface Sci., 253, 16, (June 2007), pp. (6969-6979).

He H., Liu M., Dai H., Qiu W., \& Zi X. (2007) An investigation of NO/CO reaction over perovskite-type oxide $\mathrm{La}_{0.8} \mathrm{Ce}_{0.2} \mathrm{~B}_{0.4} \mathrm{Mn}_{0.6} \mathrm{O}_{3}(\mathrm{~B}=\mathrm{Cu}$ or $\mathrm{Ag}$ ) catalysts synthesized by reverse microemulsion. Catal. Today, 126, 3-4, (August 2007), pp. (290-295).

Hollamby M. J., Eastoe J., Chemelli A., Glatter O., Rogers S., Heenan R. K., \& Grillo, I. (2010) Separation and purification of nanoparticles in a single step. Langmuir, 26, 10, (May 2010), pp. (6989-69949. 
Holmberg K. (2004) Surfactant-templated nanomaterials synthesis. J. Colloid Interface Sci., 274, 2, (June 2004), pp. (355-364).

Iglesias-Silva E., Vilas-Vilela J. L., López-Quintela M. A., Rivas J., Rodríguez M., \& León L. M. (2010) Synthesis of gold-coated iron oxide nanoparticles. J. Non-Cryst. Solids, 356, 25-27, (June 2010), pp. (1233-1235).

Jakobs B., Sottman T., Strey R., Allgaier J., Willner L., \& Richter D. (1999) Amphiphilic Block Copolymers as Efficiency Boosters for Microemulsions, Langmuir, 15, 20, (Sep. 1999), pp. (6707-6711).

Jiang D., Deng Y., Wang H., Shen B., Wu Y., Liu L., Zhong C., \& Hu W. (2010) Fabrication of Nickel hollow spheres by microemulsion-template-interface reaction route. Mater. Lett. 64, 6, (March 2010), pp. (746-7489).

Jiang D., Hu W., Wang H., Shen B., \& Deng Y. (2011) Microemulsion template synthesis of copper sulfide hollow spheres at room temperature. Colloids Surface A: Physicochem. Eng. Aspects, 384, 1-3, (July 2011), pp. (228-232).

Jobling S., \& Sumpter J. P. (1993) Detergent components in sewage effluent are weakly oestrogenic to fish: An in vitro study using rainbow trout (Oncorhynchus mykiss) hepatocytes. Aquatic Toxicology. 27, 3-4, (Dec. 1993), pp. (361-372).

Keswani R. K., Ghodke H., Sarkar D., Khilar K. C., \& Srinivasa, R. S. (2010) Room temperature synthesis of titanium dioxide nanoparticles of different phases in water in oil microemulsion. Colloids Surface A: Physicochem. Eng. Aspects, 369, 1-3, (Oct. 2010), pp. (75-81).

Košak A., Makovec D., \& Drofenik M. (2004) The preparation of MnZn-ferrite nanoparticles in a water/CTAB, 1-butanol/1-hexanol reverse microemulsion, Phys. Stat. Sol. C, 1, 12, (Dec. 2004), pp. (3521-3524)

Kruis F. E., Fissan H., \& Peled A. (1998) Synthesis of nanoparticles in the gas phase for electronic, optical and magnetic applications-a review. J. Aerosol Sci. 29, 5-6, (June 1998), pp. (511-535).

Kunieda H., \& Friberg S.E. (1981) Critical Phenomena in a Surfactant/Water/Oil System. Basic Study on the Correlation between Solubilization, Microemulsion, and Ultralow Interfacial Tensions. Bull. Chem. Soc. Jpn, 54, 4, (April, 1981), pp (10101014).

Kunieda, H.; Nakano, A.; Pes, M. A. (1995) Effect of Oil on the Solubilization in Microemulsion Systems Including Nonionic Surfactant Mixtures, Langmuir, 11, 9, (Sep. 1995), pp. (3302-3306).

Kuriyedath S. R., Kostova B., Kevrekidis I. G., \& Mountziaris T. J. (2010) Lattice Monte Carlo simulation of cluster coalescence kinetics with application to template-assisted synthesis of quantum dots. Ind. Eng. Chem. Res. 49, 21, (Nov. 2010), pp. (10442104499.

La Mer V., \& Dinegar R. (1950) Theory, Production and Mechanism of Monodisperse Hydrosols. J. Am.Chem. Soc., 72, 11, (Nov. 1950), pp. (4847-4854).

Li Y. C., Wang C. P., Hu P. F., \& Liu, X. J. (2009) A method for synthesizing the core (Ag)/shell (PSt) composite nanoparticles. Mater. Lett., 63, 20, (August 2009), pp. (1659-1661).

Lindman B., \& Friberg S. E.(1999) Chapter 1: Microemulsions: A Historical Overview, In: Handbook of Microemulsion Science and Technology, Eds. Kumar, P.; Mittal, K.L., pp. (1-12), Marcel Dekker, Inc., ISBN: 0-8247-1979-4, New York, NY, USA. 
Lisiecki I., \& Pileni M. P. (2003) Synthesis of Well-Defined and Low Size Distribution Cobalt Nanocrystals: The Limited Influence of Reverse Micelles, Langmuir, 19, 22, (Sep. 2003), pp. (9486-9489).

Liu C., Rondinone A. J., \& Zhang Z. J. (2000) Synthesis of magnetic spinel ferrite $\mathrm{CoFe}_{2} \mathrm{O}_{4}$ anoparticles from ferric salt and characterization of the size-dependent superparamagnetic properties. Pure Appl. Chem., 72, 1-2, (Jan. 2000), pp. (37-45).

Loo A. L., Pineda M. G., Saade H., Treviño M. E., \& Lopez, R.G. (2008) Synthesis of magnetic nanoparticles in bicontinuous microemulsions. Effect of surfactant concentration. J. Mater. Sci., 43, 10, (May 2008 ), pp. (3649-3654).

López-Quintela M. A. (2003) Synthesis of nanomaterials in microemulsions: formation mechanisms and growth control. Curr. Opin. Colloid Interface Sci., 8, 2 (June 2003), pp. (137-144).

López-Quintela M. A., Tojo C., Blanco M. C., García Rio L., \& Leis J. R. (2004) Microemulsion dynamics and reactions in microemulsions. Curr. Opin. Colloid Interface Sci., 9, 3-4 (November 2004), pp. (264-278).

Magno M., Angelescu D. G, \& Stubenrauch, C. (2009) Phase diagrams of non-ionic microemulsions containing reducing agents and metal salts as bases for the synthesis of bimetallic nanoparticles, Colloids Surfaces A: Physicochem. Eng. Aspects, 348, 1-3, (Sep 2009), pp. (116-123)

Moulik S. P., \& Paul B. K.(1998) Structure, dynamics and transport properties of microemulsions, Adv. Colloid Interface Sci., 78, 2, (Sep. 1998), pp. (99-195).

Myakonkaya O., Deniau B., Eastoe J., Rogers S. E., Ghigo A., Hollamby M., Vesperinas A., Sankar M., Taylor S. H., Bartley J. K., \& Hutchings G. J. (2010) Recycling nanocatalysts by tuning solvent quality. J. Colloid Interface Sci., 350, 2, (Oct. 2010), pp (443-446).

Myakonkaya O., Eastoe J., Mutch K. J., \& Grillo I. (2011) Polymer-induced recovery of nanoparticles from microemulsions. Phys. Chem. Chem. Phys., 13, 8, (Sep. 2010), pp. (3059-3063).

Nann T., \& Mulvaney P. (2004) Single Quantum Dots in Spherical Silica Particles. Angew. Chem. Int. Ed., 43, 40, (Oct. 2004), pp. (5393-5396).

Nazar M. F., Myakonkaya O., Shah, S. S., \& Eastoe J. (2011) Separating nanoparticles from microemulsions. J. Colloid Interface Sci., 354, 2, (Feb. 2011), pp. (624-629).

Niemann B., \& Sundmacher K. (2010) Nanoparticle precipitation in microemulsions: Population balance model and identification of bivariate droplet exchange kernel. $J$. Colloid Interface Sci., 342, 2, (Feb. 2010), pp. (361-371).

Pileni M. P., Zemb T., \& Petit C. (1985) Solubilization by reverse micelles: Solute localization and structure perturbation. Chem. Phys. Lett. 118, 4, (August 1985), pp(414-420).

Pileni M. P. (1997) Nanosized Particles Made in Colloidal Assemblies. Langmuir, 13, 13, (June 1997), pp. (3266-3276).

Pileni M.P. (1998) Fabrication and Properties of Nanosized Material Made by Using Colloidal Assemblies as Templates, Cryst. Res. Technol., 33, 7-8 (Dec. 1998), pp. (1155-1186)

Pileni M. P. (2001) Mesostructured Fluids in Oil-Rich Regions: Structural and Templating Approaches, Langmuir, 17, 24, (Nov. 2001), pp. (7476-7486).

Pileni M. P. (2003) The role of soft colloidal templates in controlling the size and shape of inorganic nanocrystals. Nat. Mater., 2, 3 (March 2003), pp. (145-150). 
Qian L. P., Yuan D., Yi G. S., \& Chow G. M. (2009) Critical shell thickness and emission enhancement of $\mathrm{NaYF}_{4}: \mathrm{Yb}, \mathrm{Er} / \mathrm{NaYF}_{4} /$ silica core/shell/shell nanoparticles. J. Mater. Res., 24, 12, (Jan 2009), pp. (3559-3568).

Qiao, S. Z., Liu J., \& Lu G. Q. M. (2011) Chapter 21: Synthetic Chemistry of Nanomaterials, In: Modern Inorganic Synthetic Chemistry, Eds. Xu, R.; Pang, W.; Huo, Q., pp. (479506) Elsevier, ISBN 13: 978-0-444-53599-3, Amsterdam, The Netherlands.

Reyes P. Y., Espinoza J. A., Treviño M. E., Saade H., \& López R. G. (2010) Synthesis of Silver Nanoparticles by Precipitation in Bicontinuous Microemulsions. J. Nanomaterials, Volume 2010, Article ID 948941, 7 pages. doi:10.1155/2010/948941

Sanchez-Dominguez M., Boutonnet M., \& Solans C. (2009) A novel approach to metal and metal oxide nanoparticle synthesis: the oil-in-water microemulsion reaction method. J. Nanopart. Res., 11, 7, (Oct. 2009), pp. 1823-1829

Sanchez-Dominguez M., Liotta L. F., Di Carlo G., Pantaleo G., Venezia A. M., Solans C., \& Boutonnet M. (2010) Synthesis of $\mathrm{CeO}_{2}, \mathrm{ZrO}_{2}, \mathrm{Ce}_{0.5} \mathrm{Zr}_{0.5} \mathrm{O}_{2}$, and $\mathrm{TiO}_{2}$ nanoparticles by a novel oil-in-water microemulsion reaction method and their use as catalyst support for CO oxidation. Catal. Today, 158, 1-2, (Dec. 2010), pp. (35-43)

Schmidt J., Guesdon C., \& Schomäcker R. (1999). Engineering aspects of preparation of nanocrystalline particles in microemulsions. J. Nanopart.Res., 1, 2, (June 1999), pp.(267-276).

Shinoda K. (1968) Proceedings of 5th Int. Congr. Detergency, Barcelona, Spain, September 1968, p. 275.

Sosa Y. D., Rabelero M., Treviño M. E., Saade H., \& López R. G. (2010) High-Yield Synthesis of Silver Nanoparticles by Precipitation in a High-Aqueous Phase Content Reverse Microemulsion, J. Nanomaterials, Volume 2010, Article ID 392572, 6 pages doi:10.1155/2010/392572

Stubenrauch C., Wielpütz T., Sottmann T., Roychowdhury C., \& Di Salvo F. J. (2008) Microemulsions as templates for the synthesis of metallic nanoparticles. Colloids Surfaces A: Physicochem. Eng. Aspects, 317, 1-3, (March 2008), pp. (328-338)

Suslick K. S., Hyeon T., \& Fang M. (1996) Nanostructured Materials Generated by HighIntensity Ultrasound: Sonochemical Synthesis and Catalytic Studies. Chem. Mater. 8, 8, (August 1996), pp. (2172-2179).

Takenaka S., Umebayashi H., Tanabe E., Matsune H., \& Kishida, M. (2007) Specific performance of silica-coated $\mathrm{Ni}$ catalysts for the partial oxidation of methane to synthesis gas. J. Catal. 245, 2, (Jan. 2007), pp. (392-400).

Tiseanu C., Parvulescu V. I., Boutonnet M., Cojocaru B., Primus P. A., Teodorescu C. M., Solans C., \& Sanchez-Dominguez M. (2011) Surface versus volume effects in luminescent ceria nanocrystals synthesized by an oil-in-water microemulsion method. Phys. Chem. Chem. Phys., 13, 38, (August 2011), pp. (17135-17145)

Tojo C., De Dios M., \& López-Quintela M. A. (2009) On the structure of bimetallic nanoparticles synthesized in microemulsions. J. Phys. Chem. C, 113, 44, (Nov. 2009), pp. (19145-19154).

Vesperinas A., Eastoe J., Jackson S., \& Wyatt P. (2007) Light-induced flocculation of gold nanoparticles. Chem. Commun., 43, 38, (August 2007), pp. (3912-3914).

Viswanadh B., Tikku S., \& Khilar K. C. (2007) Modeling core-shell nanoparticle formation using three reactive microemulsions. Colloids Surfaces A: Physicochem. Eng. Aspects, 298, 3, (May 2007), pp. (149-157). 
Vogt C., Toprak M. S., Muhammed M., Laurent S., Bridot J. L., \& Müller R. N. (2010) High quality and tunable silica shell-magnetic core nanoparticles, J. Nanopart. Res., 12, 4, (May 2010), pp. (1137-1147).

Wang G., Deng Y., \& Guo L. (2010) Single-crystalline ZnO nanowire bundles: Synthesis, mechanism and their application in dielectric composites. Chem. Eur. J., 16, 33, (Sep. 2010), pp. (10220-10225).

Wang, J., Tsuzuki T., Sun L., \& Wang X. (2010) Reverse microemulsion-mediated synthesis of $\mathrm{SiO}_{2}$-coated $\mathrm{ZnO}$ composite nanoparticles: Multiple cores with tunable shell thickness. ACS Appl. Mater. Interfaces, 2, 4, (March 2010), pp. (957-960).

Wang Y. H., \& Li F. (2007) Synthesis of $\mathrm{BaAl}_{12} \mathrm{O}_{19}: \mathrm{Mn}^{2+}$ nanophosphors by a reverse microemulsion method and its photoluminescence properties under VUV excitation. J. Lumin., 122-123, (Jan-April 2007), pp. (866-868).

Wu H., Jiao Q., Zhao Y., Huang S., Li X., Liu H., \& Zhou M. (2010) Synthesis of Zn/Co/Felayered double hydroxide nanowires with controllable morphology in a water-inoil microemulsion. Mater. Charact., 61, 2, (Feb. 2010), pp. (227-232).

Yeung C. M. Y., \& Tsang S. C. (2009) Noble metal core-ceria shell catalysts for water-gas shift reaction. J. Phys. Chem. C, 113, 15, (April 2009), pp. (6074-6087).

Yeung C. M. Y., \& Tsang S. C. (2010) Some optimization in preparing core-shell Pt-ceria catalysts for water gas shift reaction. J. Mol. Catal. A: Chem., 322, 1-2, (May 2010), pp. (17-25).

Xu P., Han X., \& Wang, M. (2007) Synthesis and magnetic properties of $\mathrm{BaFe}_{12} \mathrm{O}_{19}$ hexaferrite nanoparticles by a reverse microemulsion technique. J. Phys. Chem. C, 111, 16, (April 2007), pp. (5866-5870). 


\title{
Polymeric Nanoparticles Stabilized by Surfactants: Controlled Phase Separation Approach
}

\author{
Sergey K. Filippov, Jiri Panek and Petr Stepanek \\ Institute of Macromolecular Chemistry, Academy of Sciences of the Czech Republic, \\ Czech Republic
}

\section{Introduction}

It is accepted nowadays that the self-assembly or self-organization occurs in a system when two types of interactions exist simultaneously between various elements of a system - a short-range attraction and a long-range repulsion. If a combination of such interactions is manifested in a system, equilibrium nanostructures/nanoparticles could occur. This general principle applies for many different systems - e.g., liquid crystals, ferrofluids, lyotropic systems, surfactants and polymers. Polymers and copolymers in good solvent are widely used for creation of self-assembled nanoparticles in solution since they offer an extremely wide range of different monomers and compositions, the possibility to vary the polymer chain length and use tailor-made polymers for producing materials with specific properties and functionalities. For such polymers, no additives are required to form equilibrium nanoparticles.

This chapter reviews another technique of creating self-assembled and self-organized polymeric nanoparticles - controlled phase separation approach. Such approach exploits mutual interactions of a polymers and surface active molecules (surfactants or amphiphilic block copolymers) in a common solvent. We shall explore particularly dilute systems where various types of nanoparticles will be investigated. The nanoparticles will be studied keeping in mind their possible applications, especially for biological purposes encapsulation and delivery of active substances in the case of particles and immobilization.

\section{Background}

The common approach applied to all types of physical systems described below is based on controlling the extent of macrophase separation that occurs in a mixture of two compounds (solvent and polymer) that became immiscible or incompatible as a result of a change of an external variable. This parameter can be temperature, $\mathrm{pH}$ or addition of a another solvent, in principle it could also be a change in pressure but the latter is not very practical since usually large pressure changes are needed to achieve relatively small changes in phase diagrams. 
A phase diagram for a polymer A/polymer B or polymer/solvent system is schematically represented in Fig. 1. In the classical case the energy of the system is given by enthalpic and entropic contributions and the interaction parameter $\chi$ is given by the Flory-Huggins relation $\chi=a / T+b$ where $\mathrm{T}$ is absolute temperature and $a$ and $b$ are specific for the polymer/polymer or polymer/solvent pair. For more complex systems, a third term $\chi_{\mathrm{s}}$ has to be included:

$$
\chi=a / T+b+\chi_{s}
$$

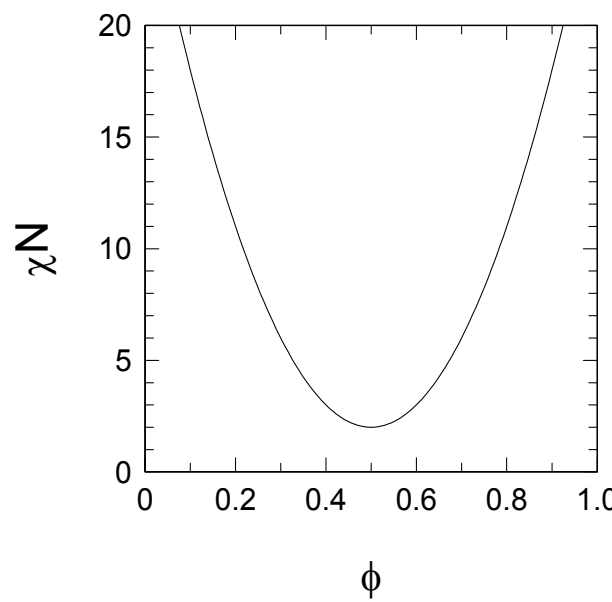

Fig. 1. Schematic phase diagram for a polymer A/polymer B or polymer/solvent. $\phi$ is the volume fraction of the first component of the system, $\mathrm{N}$ the number of monomers in the polymer chain and $\chi$ the interaction parameter describing the strength of interaction between polymer A and B or between the polymer and solvent.

In this simple representation the third term includes all additional interactions in the system, in particular the effect of different temperature expansions of the system components and that of various specific interactions in the system (hydrogen bonds, ionic interactions, ...) that may be dominant compared to the enthalpic/entropic terms $a, b$.

Once a macrophase separation has been initiated, the spatial extent of inhomogeneities produced by nucleation or spinodal decomposition is controlled by addition of amphiphilic molecules chosen in such a way that one part of this amphiphile interacts attractively with the nucleated material while the other part interacts attractively with the surrounding solvent. The surface of the nucleated material then becomes covered with the amphiphilic molecules which effectively terminate the phase separation and stabilizes the system in a dispersed state. The formation of nanoparticles is always driven by thermodynamics (increase of $\chi$-parameter in Eq. (1)) but controlled by specific factors of interaction with the amphiphilic molecules including kinetic and hydrodynamic effects. Assessment of these effects and their importance for nanoparticles preparation is a primary goal in this manuscript. In the following chapter we will describe several systems where this approach can be realized:

- $\quad$ Controlled phase separation induced by a change in temperature. 
- Controlled phase separation induced by a change of solvent

- Controlled phase separation induced by a change in $\mathrm{pH}$

\section{Experimental methods}

To observe and to prove the formation of well defined nanoparticles we have exploited a variety of methods such as static and dynamic light scattering (SLS/DLS), small-angle neutron and X-ray scattering (SANS/SAXS) and Cryo-TEM methods.

Since kinetic factors are involved into formation of nanoparticles created by controlled phase separation method we have examined the nucleation and growth of polymeric nanoparticles using the stopped-flow technique combined with SAXS on the time scale of milliseconds.

\subsection{Small-Angle Neutron Scattering (SANS)}

SANS experiments were performed at CEA-Saclay on the spectrometer PAXY of the Laboratoire Leon-Brillouin. Measurements were run on a $128 \times 128$ multidetector (pixel size $0.5 \times 0.5 \mathrm{~cm}$ ) using a non-polarized, monochromatic (wavelength $\lambda$ set by a velocity selector) incident neutron beam collimated with circular apertures for two sample-to-detector distances, namely, $1 \mathrm{~m}$ (with $\lambda=0.6 \mathrm{~nm}$ ) and $7 \mathrm{~m}$ (with $\lambda=0.8 \mathrm{~nm}$ ). With such a setup, the investigated range of scattering wave vector modulus was $5.9 \times 10^{-2}$ to $4.3 \mathrm{~nm}^{-1}$. In all the cases reported in this paper, the two-dimensional scattering patterns were isotropic so that they were azimuthally averaged to yield the dependence of the scattered intensity $I_{s}(q)$ on the scattering vector $q$. Data were corrected for background scattering and detector efficiency. Intensities of neutron scattering are given in arbitrary units.

\subsection{Dynamic and Static Light Scattering (DLS/SLS)}

Static light scattering measurements were carried out on an ALV-6010 instrument equipped with a $22 \mathrm{~mW}$ He-Ne laser in the angular range $30-150^{\circ}$. Dynamic light scattering measurements were carried out at $90^{\circ}$ angle. The obtained correlation functions were analyzed by REPES (Jakes, 1995) analytical software providing a distribution function, $G\left(R_{\mathrm{h}}\right)$ of hydrodynamic radii $R_{\mathrm{h}}$. To account for the logarithmic scale on the $R_{\mathrm{h}}$ axis, all DLS distribution diagrams are shown in the equal area representation, $R_{\mathrm{h}} G\left(R_{\mathrm{h}}\right)$. The static light scattering data were analyzed by a Zimm plot:

$$
\frac{K c}{R(q)}=\frac{1}{M_{w}}+\frac{R_{G}^{2} q^{2}}{3 M_{w}}
$$

where $R(q)$ is the Rayleigh ratio of the scattering intensity, $q=(4 \pi n / \lambda) \sin \Theta / 2$ is the scattering vector, $\lambda$-wave length in the medium, $\theta$ - scattering angle between the incident and the scattered beam, $K$ is a contrast factor containing the optical parameters, $c$ is a particle concentration, $M_{w}$ is the weight average of the molar mass of the particles, and $R_{G}$ is their radius of gyration. The concentration dependence was neglected which was acceptable because of the low concentrations of the solutions. 


\subsection{Cryo-transmission electron microscopy - Cryo-TEM}

To carry out a Cryo-TEM experiment, a drop of the solution under study was placed on a pretreated copper grid which was coated with a perforated polymer film. Excess solution was removed by blotting with a filter paper. The preparation of the sample film was done under controlled environment conditions, i.e., in a chamber at a constant temperature of $25^{\circ} \mathrm{C}$ and with a relative humidity of $98-99 \%$ to avoid evaporation of the liquid. Rapid vitrification of the thin film was achieved by plunging the grid into liquid ethane held just above its freezing point. The sample was then transferred to the electron microscope, a Zeiss 902A instrument (Carl Zeiss NTS, Oberkochen, Germany), operating at an accelerating voltage of $80 \mathrm{kV}$ and in zero-loss bright-field mode. The temperature was kept below -165 ${ }^{\circ} \mathrm{C}$ and the specimen was protected against atmospheric conditions during the entire procedure to prevent sample perturbation and formation of ice crystals. The resolution in this method was 3-5 nm. Digital images were acquired with a BioVision Pro-SM Slow Scan CCD camera (Proscan electronische systeme, $\mathrm{GmbH}$, Germany). iTEM software (Olympus Soft Imaging Solutions, $\mathrm{GmbH}$, Germany) was used for image processing. The polymer concentration used was $5 \cdot 10^{-3} \mathrm{~g} \mathrm{~mL}^{-1}$.

\subsection{Small angle X-ray scattering - SAXS}

All time-resolved SAXS (TR-SAXS) experiments were performed on the high brilliance beam line ID02 at the ESRF (Grenoble, France). The SAXS setup is based on a pinhole camera with a beam stop placed in front of a two-dimensional detector (X-ray image intensifier coupled to a CCD camera). The X-ray scattering patterns were recorded on the detector that was located $2 \mathrm{~m}$ from the sample, using a monochromatic incident $X$-ray beam $(\lambda=0.1 \mathrm{~nm})$. The available wave vector range was $0.04-2.71 \mathrm{~nm}^{-1}$. Data acquisition and counting of the time $t$ was hardware-triggered within $1 \mathrm{~ms}$ before the final mixing process was initiated. SAXS data were acquired with an exposure time of $50 \mathrm{~ms}$ per frame.

The fast mixing experiments were performed using a stopped-flow device (SFM-3, BioLogic) that has been specifically adapted for SAXS experiments. The device was thermostated at $25.0 \pm 0.5^{\circ} \mathrm{C}$

\section{Controlled phase separation induced by a change in temperature}

To fulfill this task we have exploited the phase separation of thermally sensitive PNIPAM polymer on heating above the lower critical solubility temperature (LCST). PNIPAM is a typical temperature-sensitive polymer that has LCST around of $32^{\circ} \mathrm{C}$ (detailed information on pure PNIPAM, can be found in the review (Aseyev et al., 2010) and references therein). Heating of aqueous solution of PNIPAM above LCST will initialize coil-to-globule transformation with following precipitation of the polymer. Such macrophase separation could be terminated if surface active molecules are presented in solution. Earlier we have demonstrated that well defined nanoparticles of PNIPAM could be prepared in presence of ionic and non-ionic surfactants, (SDS, CTAB, Brij98, Brij97) (Konak et al., 2007). The effect of PNIPAM and surfactant concentration, and molecular weight of PNIPAM on nanoparticle parameters and on the phase transition temperature of PNIPAM solutions was investigated. It was proposed that the structure of particles is supposed to be similar to block copolymer micelles. Hydrophobic PNIPAM molecules form the insoluble core of particles and their 
hydrophilic shell consists of hydrophilic parts of surfactants. An intermediate shell at the core-shell interface contains both the hydrophobic parts of surfactants and PNIPAM chains. The feature of that research is that in contrast to previous studies, where surfactants were used in excess, lower concentrations of surfactants were used.

To validate the proposed model, for PNIPAM and SDS system, a contrast variation study was performed by SANS. It is important to note here that the system studied in our research is different from a so-called mesoglobule state that was also observed (Siu et al., 2003; (Aseyev et al., 2005; Kujawa et al., 2006a; Kujawa et al., 2006b) for PNIPAM. It was established in variety of papers that PNIPAM macromolecules of high molar masses on very diluted solutions might undergo through intermediate mesoglobule state with increasing of temperature above LCST. These mesoglobules are aggregates of PNIPAM molecules that consist of one or more macromolecules. They are metastable particles that are stabilized either by electrostatic or steric interactions (Kujawa et al., 2006b). No surfactant is required. Nevertheless when PNIPAM concentration in solution is rather high macroscopic precipitation occurs. In this case, surface active molecules are needed to create stable polymeric nanoparticles.

Three types of nanoparticles were tested: (i) deuterated d7-PNIPAM + protonated SDS in a $72 \% \mathrm{D}_{2} \mathrm{O} / 28 \% \mathrm{H}_{2} \mathrm{O}$ volume mixture where the coherent scattering originates only from the surfactant. (ii) protonated PNIPAM + protonated SDS in pure $\mathrm{D}_{2} \mathrm{O}$ where the scattering comes from the polymer and the surfactant. The whole nanoparticle should be visible. (iii) protonated PNIPAM + deuterated d25-SDS in $\mathrm{D}_{2} \mathrm{O}$. The scattering length density of the deuterated surfactant is almost matched by $\mathrm{D}_{2} \mathrm{O}$. In this case most of the scattering is produced by the polymer. The experiments have been conducted at $\mathrm{T}=42{ }^{\circ} \mathrm{C}$.

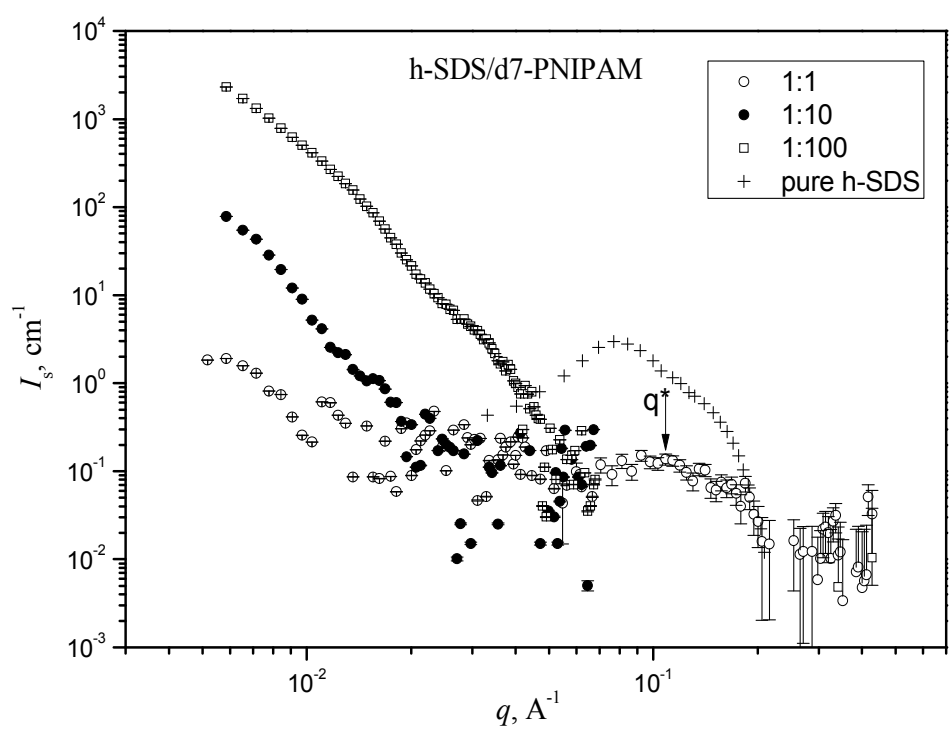

Fig. 2. Scattered intensity $I_{\mathrm{s}}$ as a function of the scattering vector $q$ for systems $\mathrm{d} 7$ -

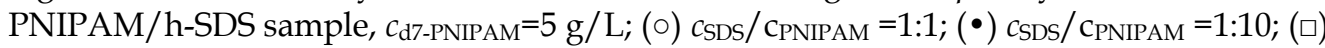
$C_{\text {SDS }} /$ CPNIPAM $=1: 100 ;(+)$ pure SDS. Data are taken from the reference (Lee \& Cabane, 1997). 
Earlier Cabane and Lee in their pioneer work have investigated similar the PNIPAM-SDS system by SANS (Lee \& Cabane, 1997). The polymer molar mass that have been used in their study was $1 \cdot 10^{6} \mathrm{~g} /$ mole and concentration of solution was mainly $30 \mathrm{~g} / \mathrm{L}$. To avoid a mesoglobule state we have selected the h-PNIPAM with $\mathrm{M}_{\mathrm{w}}=1.88 \cdot 10^{5} \mathrm{~g} / \mathrm{mole}$ and $\mathrm{d} 7$ PNIPAM with $\mathrm{M}_{\mathrm{w}}=3.6 \cdot 10^{5} \mathrm{~g} /$ mole that is somewhat smaller than the one used by Cabane et.al. For the same reason, concentration of PNIPAM in all solution was kept of $5 \mathrm{~g} / \mathrm{L}$. Our work is thus a research on a similar system with different conditions.

Fig. 2-4 represents the data for different surfactant-to-polymer ratios. For all systems, the scattered intensity extrapolated to zero $q$ is increasing with decrease of the ratio. In other words, the growth of colloidal nanoparticles is observed with decrease of surfactant-to polymer ratio. One can see continuous evolution of the characteristic features of colloids.

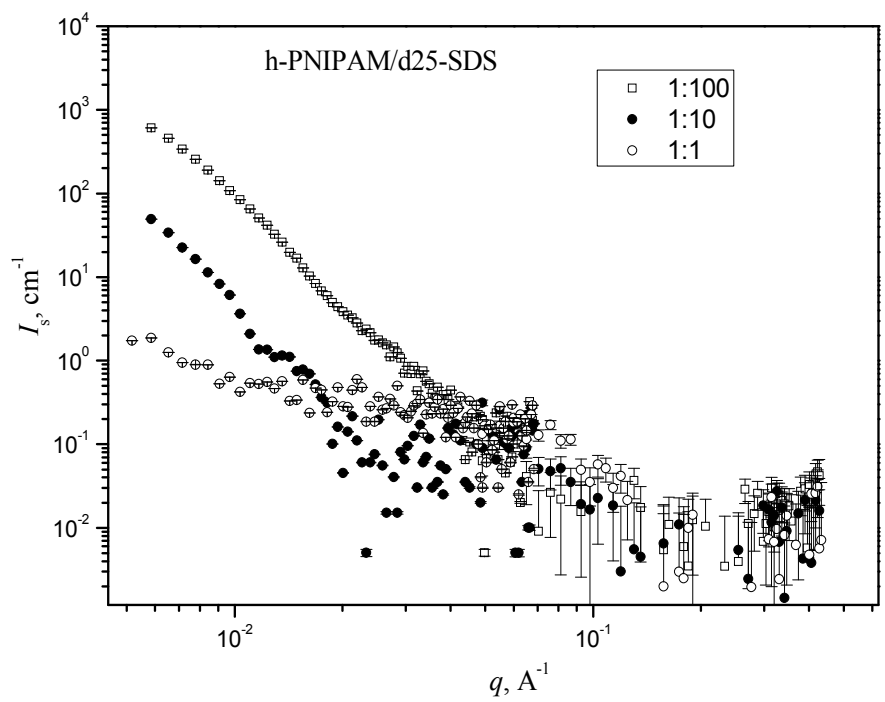

Fig. 3. Scattered intensity $I_{\mathrm{s}}$ as a function of the scattering vector $q$ for systems h-

PNIPAM/d25-SDS sample, $c_{\text {-PNIPAM }}=5 \mathrm{~g} / \mathrm{L} ;(\circ) c_{\text {SDS }} / c_{\text {PNIPAM }}=1: 1 ;(\bullet) c_{\text {SDS }} / C_{\text {PNIPAM }}=1: 10 ;(\square)$ $c_{\text {SDS }} / C_{\text {PNIPAM }}=1: 100$

\section{1 h-SDS/d7-PNIPAM}

When a surfactant is protonated, coherent scattering comes only from the surfactant in a $72 \% \mathrm{D}_{2} \mathrm{O} / 28 \% \mathrm{H}_{2} \mathrm{O}$ volume mixture. At low $q$, a $q^{-4}$ decay is visible at $c_{\text {SDS }} / c_{\text {PNIPAM }}=1: 100$ (Fig. 2). At high $q$ a signal is too low. For comparison reason, the scattering of pure SDS micelles is presented on Fig. 2. No peaks that correspond to the distance between consecutive SDS micelles at high $q$ range observed by Cabanne and Lee[8] (located at $q$ of about $0.1 \AA^{-1}$ ) appear on the graph. We conclude that all surfactant molecules are uniformly incorporated inside of a colloidal particle or on its surface. It is worth to note that such strong q dependence indicates that the surfactant forms big structures. At $c_{S D S} / C_{P N I P A M}=1: 1$, one can see that the scattering at low $q$ is week and simultaneously a peak at high q range appears $\left(q^{*}=0.11 \AA^{-1}\right)$. Obviously, colloidal particles are completely dissolved now; a pearl-necklace complex exists with SDS micelles bound to a polymer chain with the distance of $58 \AA ⿱ ㇒ ⿻ 二 乚\left(d=2 \Pi / q^{*}\right)$. That finding is in good agreement with results of Cabanne where such distance was about $63 \AA$. 


\section{2 h-PNIPAM/d25-SDS}

Similar features are observed for the system where the PNIPAM is only visible. Again, the formation of nanoparticles could be monitored by the growth of the scattering intensity with decrease of the composition ratio. No peaks at high $q$ range observed are visible in this case. We conclude that PNIPAM is also uniformly distributed inside a nanoparticle.

\section{3 h-PNIPAM/h-SDS}

In this case both the polymer and the surfactant are visible in SANS.

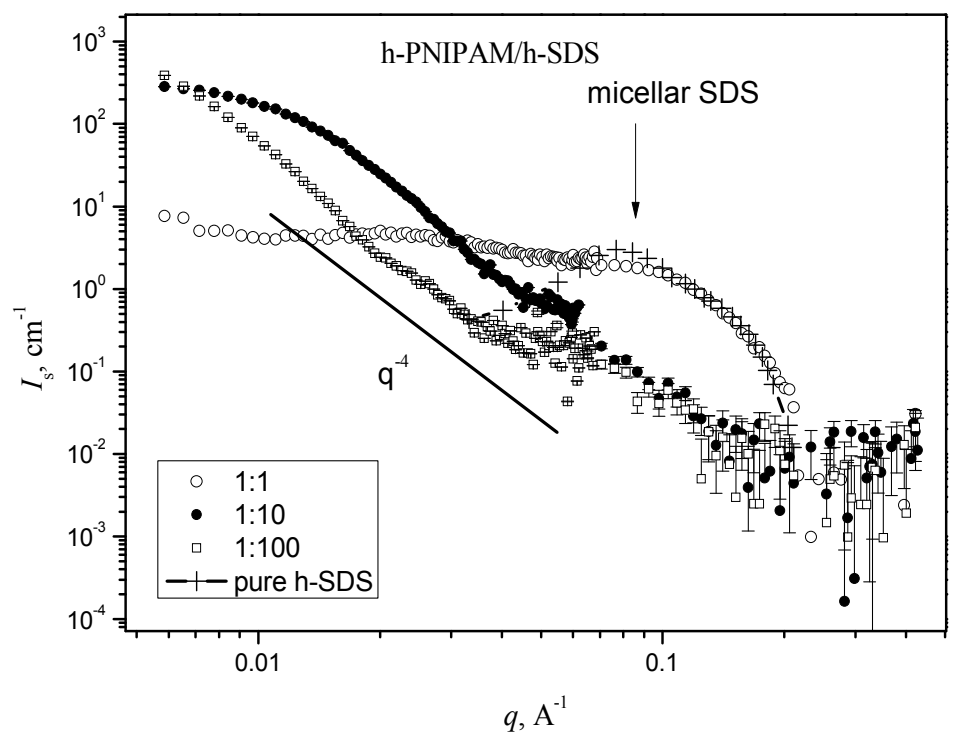

Fig. 4. Scattered intensity $I_{\mathrm{s}}$ as a function of the scattering vector $q$ for systems hPNIPAM/h-SDS sample, $c_{\text {-PNIPAM }}=5 \mathrm{~g} / \mathrm{L} ;(\circ) c_{\text {SDS }} /$ CPNIPAM $=1: 1 ;(\bullet) c_{\text {SDS }} / C_{\text {PNIPAM }}=1: 10 ;(\square)$ $c_{\text {SDS }} / C_{\text {PNIPAM }}=1: 100 ;(+)$ pure SDS. Data are taken from reference (Lee \& Cabane, 1997).

The scattering curve at $c_{\text {SDS }} / C_{\text {PNIPAM }}=1: 100$ begins at low $q$ at high intensity; then it curves downward and continuous with $q^{-4}$ decay (Fig. 4). This part of the scattering curve corresponds the scattering from colloidal particles. Fitting the scattering curves by formfactor of a hard sphere with Schultz-Zimm distribution provides $R_{\mathrm{g}}$ values of nanoparticles. Obtained values nanoparticles are 216, and $96 \AA$ for ratios 1:100 and 1:10, respectively, giving corresponding outer radii 279 , and 124 Á. Polydispersity value obtained from the fitting routine was 0.37 and 0.44 , respectively. Cabanne et. al. reported the similar value of about 0.5 . Such high numbers imply strong polydispersity in size for nanoparticles in solution.

At $c_{\text {SDS }} /$ CPNIPAM $_{\text {PNA }}=1: 1$ the scattering is flat at low $q$ (Fig. $4,5 \mathrm{a}, \mathrm{b}$ ) as it could be visible from a comparison with the spectra of samples made at lower ratios (1:10 and 1:100). At high $q$, one can see a plateau and, beyond $q=0.1 \AA^{-1}$, a steeper decay. This spectrum is identical to the scattering from a micellar solution of SDS at the same concentration in the absence of polymer (Fig. 4, 5a). In particular, the peak position matches the average intermicellar distance in pure SDS solutions. 

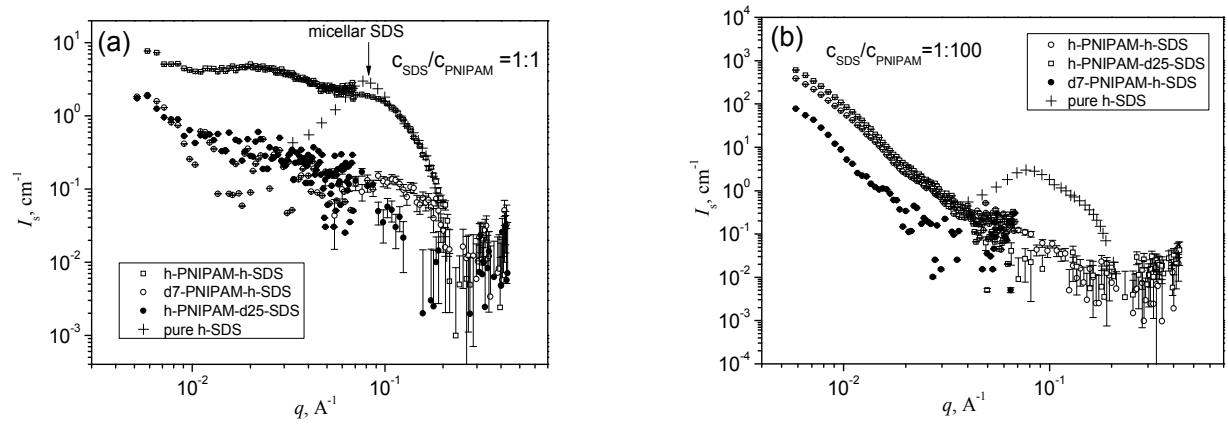

Fig. 5a. Scattered intensity $I_{\mathrm{S}}$ as a function of the scattering vector $q$ for systems

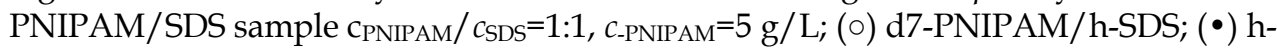
PNIPAM/d25-SDS; ( $\square$ ) h-PNIPAM/h-SDS; (+) pure SDS. Data are taken from reference (Lee \& Cabane, 1997).

Fig. 5b. Scattered intensity $I_{\mathrm{s}}$ as a function of the scattering vector $q$ for systems PNIPAM/SDS sample CPNIPAM $_{\text {CSDS }}=1: 00, c_{\text {-PNIPAM }}=5 \mathrm{~g} / \mathrm{L} ;(\circ) \mathrm{h}$-PNIPAM/h-SDS; $(\bullet) \mathrm{d} 7-$ PNIPAM/h-SDS; ( $\square)$ h-PNIPAM/d25-SDS; (+) pure SDS. Data are taken from reference (Lee \& Cabane, 1997).

In order to determine the shape and geometric size of the particles we performed Cryo-TEM measurements for the samples at composition 1:100. The Cryo-TEM images are shown in Fig. 6.

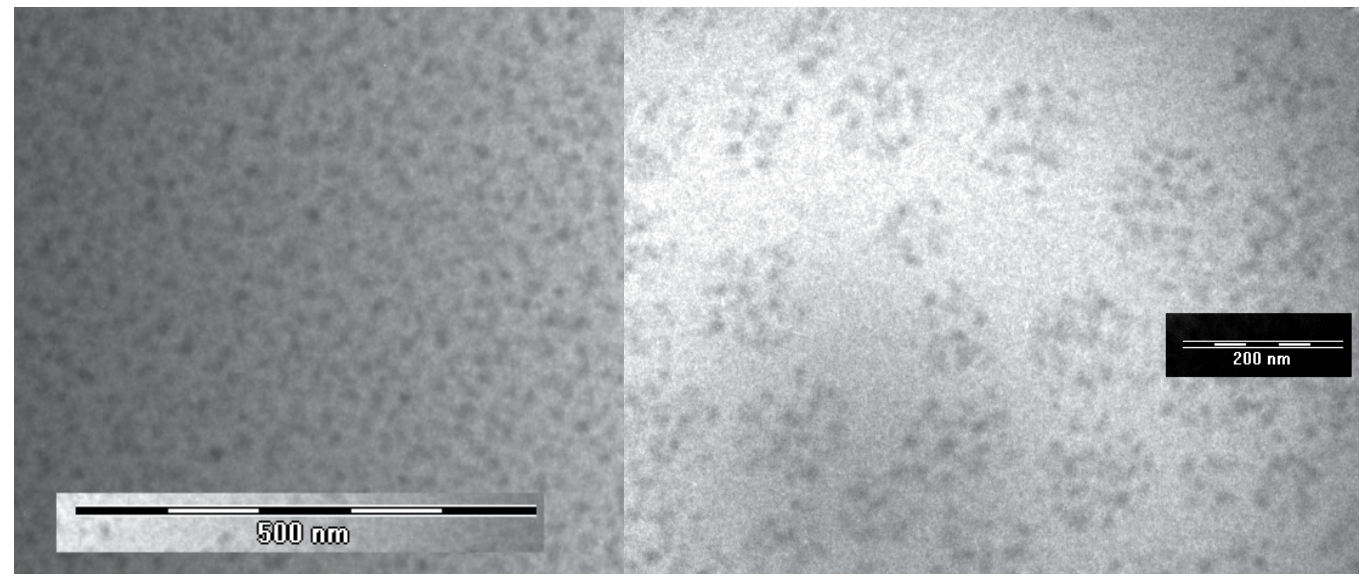

Fig. 6. Cryo-TEM micrographs. h-PNIPAM/h-SDS sample $c_{\text {PNIPAM }}=5 \cdot 10^{-3} \mathrm{~g} \mathrm{~mL}^{-1} ; c_{\mathrm{SDS}}=5 \cdot 10^{-5} \mathrm{~g}$ $\mathrm{mL}^{-1} \cdot \mathrm{c}_{\mathrm{SDS}} / \mathrm{C}_{\mathrm{PNIPAM}}=1: 100$

Fig. 6 shows objects thate are rather polydisperse in size. The average size of moieties is in agreement with SANS data, giving value roughly $20-30 \mathrm{~nm}$ in radius. The TEM images are 2D projection of the particles, observed under different angles. Therefore, we believe that the structures we see in Fig. 6 are of more or less spherical shape. Individual micelles seen in SANS experiments were not imaged in these samples, because of their small size. 


\section{Controlled phase separation induced by a change of solvent}

\subsection{Density}

For a specific polymer/solvent system, phase separation could be induced by a change of the solvent. This particular case of spontaneous macrophase separation leading to formation of nano-sized droplets is frequently referred to as Ouzo effect (Ganachaud \& Katz , 2005), although it has a variety of names. Some authors call this process solvent shifting (Brick et al., 2003; Van Keuren, 2004), solvent displacement(Potineni et al., 2003; Trimaille et al., 2003; Lince et al., 2008; Chu et al., 2008; Vega et al., 2008; Nguyen et al., 2008; Beck-Broichsitter et al., 2009) spontaneous emulsification (Gallardo et al., 1993; QuintanarGuerrero et al., 1997; Baimark et al., 2007; Tan et al., 2008; Katas et al., 2009) or micro/nano precipitation (Leroueil-Le Verger et al., 1998; Peracchia et al., 1999; Bilati et al., 2005; Leo et al., 2006; Legrand et al., 2006). In our previous papers (Panek et al., 2011a; Panek et al., 2011b) we have successfully tested this procedure: polymeric nanoparticles were prepared by mixing a polymer - poly(methyl methacrylate) or polystyrene - dispersed in an organic solvent with an aqueous solution of a surfactant (SDS). Since water is a bad solvent for either of the polymers they start to precipitate but the presence of a surfactant terminates the phase separation and nearly monodisperse nanoparticles appear with a typical size in the range of 50 to $300 \mathrm{~nm}$. Finally the organic solvent is evaporated. We demonstrated that a variety of parameters such as polymer molar mass, surfactant hydrophobicity, solution temperature and composition influence the physico-chemical properties of nanoparticles formed in solution (Panek et al., 2011a; Panek et al., 2011b). Nevertheless, detailed information on nanoparticles structure is still missing.

Here we describe new experiments with static and dynamic light scattering that were conducted to get further insight on the internal structure of the nanoparticles. Using a combination of both methods, for the first time, we calculated such parameters as a structure factor $R_{\mathrm{g}} / R_{\mathrm{h}}$ and density of nanoparticles $(\rho)$. $R_{\mathrm{g}}$ values were measured by static light scattering. In contrast with $R_{\mathrm{h}}$ (Panek et al., 2011a) there is no detectable difference in the factor $\rho=R_{\mathrm{g}} / R_{\mathrm{h}}$ of nanoparticles made from ionic and non-ionic surfactants (Fig. 7).

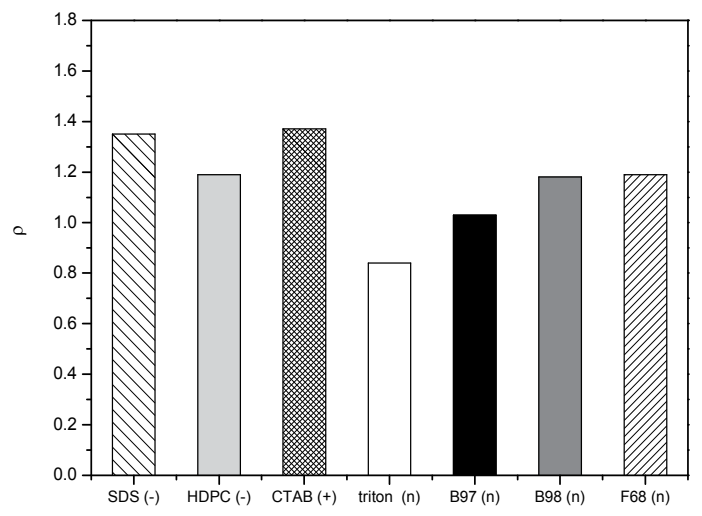

Fig. 7. Histogram of structure factor $\rho=R_{\mathrm{g}} / R_{\mathrm{h}}$ obtained from SLS/DLS data for PMMA(1) $c_{\mathrm{P}}=2 \cdot 10^{-4} \mathrm{~g} \mathrm{~mL}^{-1}$ for various surfactants. 
According to Burchard (Burchard, 1999) this generalized ratio $\rho$ is of special interest for establishing the particle architecture. It is varying in the range 0.8-1.4. The lowest value is for a Triton surfactant, where the particle behaves as a hard sphere ( $\rho$ is close to 0.778 ). The value of $\rho$ for CTAB, SDS, Brij 97 and 98 is about 1.2-1.4. This is characteristic for several models, in particular for branched polymers, soft spheres and dendrimers.

In contrast, the density of nanoparticles is very sensitive to the nature of a surfactant (Fig. 8). The density of nanoparticles was calculated by dividing their mass obtained from static light scattering by their volume based on the $R_{\mathrm{g}}$. Nanoparticles composed of low molecular ionic surfactants have almost two-fold higher density then the ones with polymeric non-ionic surfactants. Since all polymeric surfactants are diblock copolymers, we can assume that polymers can't adopt maximum packing structure due to steric factors.

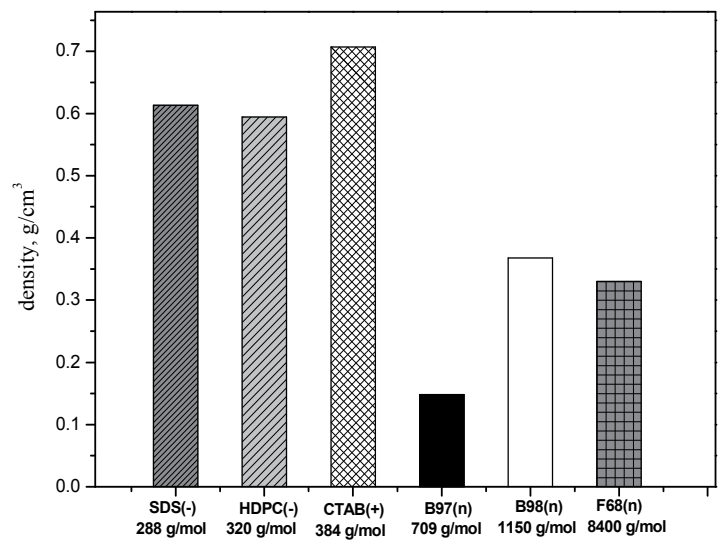

Fig. 8. Histogram of density obtained from SLS/DLS data for PMMA(1) $c_{\mathrm{PMMA}}=2 \cdot 10^{-4} \mathrm{~g}$ $\mathrm{mL}^{-1}$ for various surfactants.

Obviously some voids should be inside. Such conclusion is in agreement with previous SANS and Cryo-TEM (Panek et al., 2011a). Analysis of the SANS curves supports neither a core-shell structure model of the nanoparticles nor a polymeric sphere with surfactant inclusions. Nevertheless a closer inspection of some micrographs reveals the presence of thin white hallo around a nanoparticle. Possible distribution of surfactant inside of a nanoparticle is presented on Fig. 9. The permanent entrapment of a surfactant inside nanoparticle may occur because the polymer (PMMA or PS) is in the glassy state. Plausibility of such scenario has been proven by J. Kriz et al. (Kriz et al., 1996) who demonstrated that the mobility of PS moieties in the core of polystyrene-blockpoly(methacrylic acid) (PS-PMAc) micelles is significantly decreased, which indicates that the polymer including the surfactant inside a micelle is vitrified.

\subsection{Influence of mixing rate}

The effect of mixing rate (i.e. the rate at which the water solution of a surfactant is delivered into the organic solution of a polymer) on the self-assembled nanoparticles formed in the PS/SDS mixed solutions was also investigated (Fig. 10). The molecular weight of PS was varied in the range $0.9-30 \cdot 10^{6} \mathrm{~g} \mathrm{~mol}^{-1}$. At low molecular weights of PS, the mixing rate has 


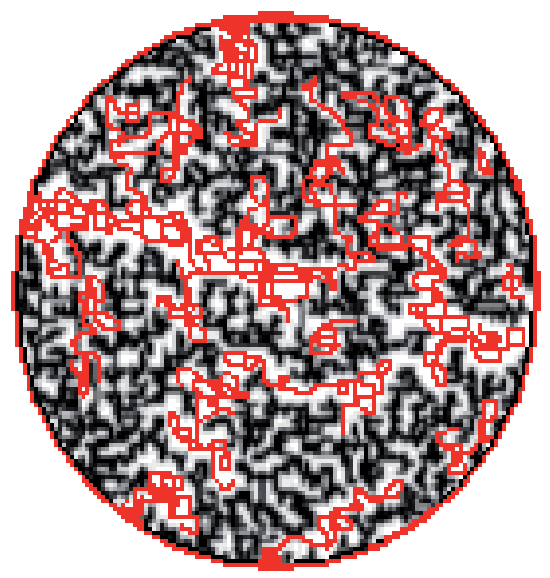

Fig. 9. Possible distribution of a surfactant (red color) and polymer (black color) inside of a nanoparticle by SANS data.

an important influence on the size of nanoparticles. Changing the mixing rate from 0.5 $\mathrm{mL} / \mathrm{min}$ up to $2 \mathrm{~mL} / \mathrm{min}$ makes two times smaller particles. At higher polymer molecular weights the influence of mixing rate is smaller. We conclude that lower mixing rate reduces the number of surfactant molecules in the neighborhood of polymeric nuclei formed after solvent shifting. Smaller number of surfactant molecules slow down stabilization of polymeric nuclei thus leading to forming bigger nanoparticles.

The difference in composition ratio is responsible for molecular weight dependence of nanoparticle dimensions at constant mixing rate and polymer weight concentration (Fig. 10). The bigger molecular weight of a polymer the smaller its molar concentration in mixed solution that leads to a decrease in composition ratio which governs the nanoparticle dimensions. At higher molecular weights of the polymer, the tendency is reverse, showing the growth of sizes (Fig. 10). One of the possible explanations is that macromolecules with

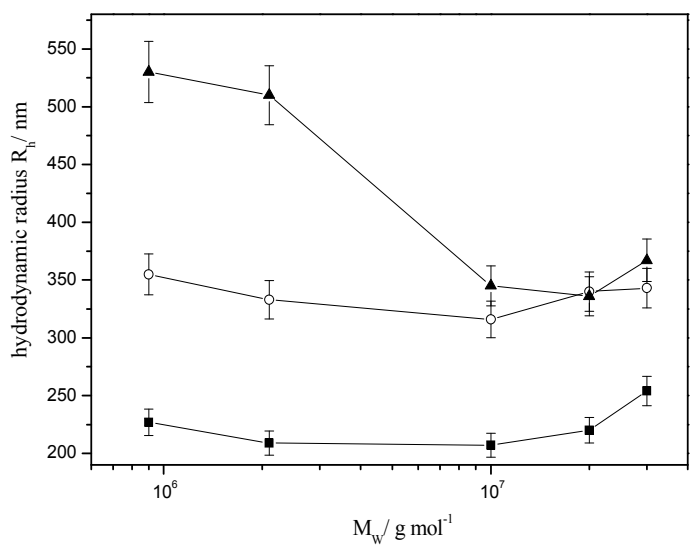

Fig. 10. Dependence of hydrodynamic radius of nanoparticles on molecular weight of polymer for PS $c_{\mathrm{PS}}=2 \cdot 10^{-4} \mathrm{~g} \mathrm{~mL}^{-1}$ and SDS $c_{\mathrm{SDS}}=5 \cdot 10^{-3} \mathrm{~g} \mathrm{~mL}^{-1}$ system at different mixing

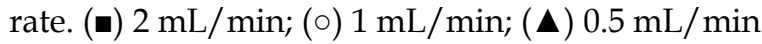


extra large molecular weight have very low diffusion which limits the probability of surfactant molecules to find enough polymer molecules in surroundings. Fast diffusion of water molecules into polymer interface during mixing forms a surfactant-abandoned layer. In these conditions, it's energetically more favorable for a polymer chain to merge with other polymer molecules and form bigger nanoparticles in comparison with solutions of the same surfactant concentration and mixing rate.

The results presented so far show that the self-assembly in the mixed polymer/surfactant systems is rather complex. Mixing of the surfactant solutions with the polymer solutions in organic solvent results in the formation of nanoparticles, whose size can be tuned by changing the relative amounts of surfactant and polymer, as well as mixing rate.

\section{Controlled phase separation induced by a change in $\mathrm{pH}$}

Changing of $\mathrm{pH}$ for a $\mathrm{pH}$ sensitive polymer is another way to construct polymeric nanoparticles. The $\mathrm{pH}$ of the solution is gradually changed so that the polymer which is in the beginning in an environment where it is molecularly soluble (i.e. in the mostly ionized form), starts to precipitate. Adding a surfactant terminates the phase separation in a controlled way leading to formation of well-defined nanoparticles with low polydispersity. We have demonstrated this procedure using a $\mathrm{pH}$-sensitive hydrophobic polymer - i.e. poly(N-methacryloyl-L-valine) (pNMV), the extent of macrophase separation was controlled by the amphiphilic molecule Brij98. We have shown previously (Filippov et al., 2008; Filippov et al., 2010) that in a certain range of concentration and composition of the polymer/amphiphile system very monodisperse particles with size ca. $50 \mathrm{~nm}$ could be reproducibly prepared after a change of $\mathrm{pH}$ from 7 to 3.5. This change is reversible and the nanoparticles can be repeatedly created and dissolved by variation in $\mathrm{pH}$. This type of particles can be very useful, since they may be able to solubilize hydrophobic drugs in large amounts and release them after a change of $\mathrm{pH}$. For example the $\mathrm{pH}$ of stomach is 1 to 3 (nanoparticles associated), while the $\mathrm{pH}$ of duodenum is 7 to 8 (particles dissolved, drug released).

The nucleation of these nanoparticles has not yet been investigated. The early stages of nucleation in such systems determine the nanoscopic structure of the particles that is so far unknown, but important for their envisaged applications. Recently, new technical possibilities to study the kinetics of self-assembly were developed. Primary, it concerns socalled stopped-flow experiments combined with small-angle scattering equipments. (Narayanan et al., 2001; Grillo et al., 2003; Panine et al., 2006). A variety of nanostructures were tested by time-resolved light scattering, SAXS, and SANS. The kinetics of micelles-tovesicles (Schmolzer et al., 2002; Weiss et al., 2005; Weiss et al., 2008; Shen et al., 1989) and lamellar-to-microemulsion (Deen et al., 2009; Tabor et al., 2009) phase transition was studied in details. Another challenging areas for time-resolved experiments are the life time of micelles (Lund et al., 2009) monomers-micelles exchange rate (Eastoe et al., 1998; Tucker et al., 2009), and nucleation of gold (Abecassis et al., 2007; Abecassis et al., 2008) and mineral nanoparticles. (Pontoni et al., 2002; Ne et al., 2003; Bolze et al., 2004). For further details on this topic, the reader is referred to reviews on the application of stopped-flow technique in SANS and SAXS. (Grillo et al., 2009; Gradzielski et al., 2003; Gradzielski et al., 2004) 
We have exploited stopped-flow technique combined with SAXS to monitor early stages of nucleation on the time scale of seconds (Fig. 11). The main difference from solvent-shifting experiments described above is absence of macroscopic fluxes and solution inhomogenuities caused by mixing. In stopped-flow experiments very fast mixing setup provides solution with uniform density where nanoparticles are growing in time.

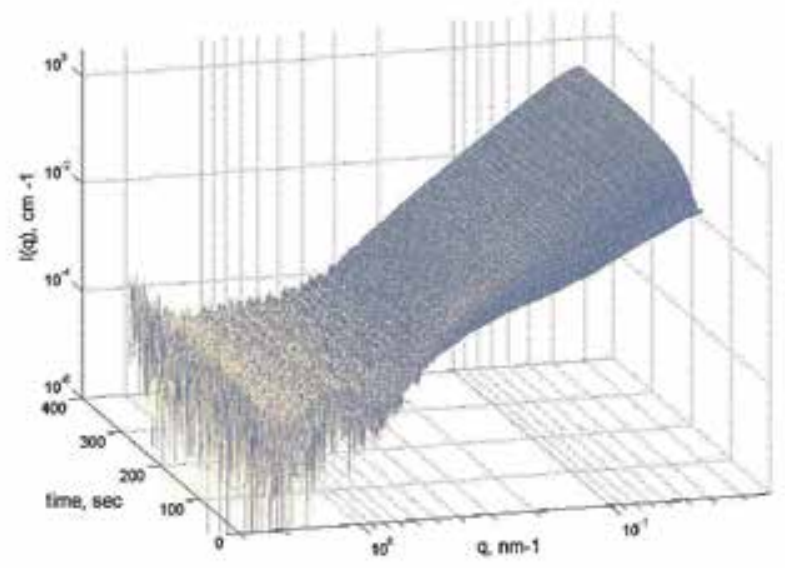

Fig. 11. The temporal evolution of the SAXS intensity for pNMGL $\left(c_{\mathrm{p}} / c_{\text {surf }}=2.0\right)$ system.

The aim of the experiment was to measure the kinetics of self-assembly of $\mathrm{pH}$-sensitive polymeric nanoparticles stabilized by surfactants. The four types of $\mathrm{pH}$-sensitive hydrophobic polymers that have been used in our research for the growth of nanoparticles were: (a) poly(N-methacryloyl-L-valine) (pNMV); (b) poly(N-methacryloyl glycyl-Lphenylalanyl-L-leucinyl-glycine) (pNMGPLG), and (c) poly(N-methacryloyl glycyl-Lleucine) (pNMGL). The extent of macrophase separation was controlled by the surfactants Brij 97, and Brij 98. The surfactants were different in the length of hydrophilic PEO chain.

\subsection{The self-assembly of nanoparticles}

Fig. 11 displays the intensity of scattered X-rays from the mixture of aqueous solution of Brij 98 surfactant and pNMGL $\left(c_{\mathrm{p}} / c_{\text {surf }}=2.0\right)$ solution as a function of time. The SAXS technique is commonly used to extract information on molecular architecture and size of nano-objects in solution that can be performed by the analysis of Kratky or Guinier plots.(Glatter \& Kratky , 1982) The scattering from nanostructures reveals three regions in the dependence of scattering intensity on scattering vector, $I \sim q^{\alpha}$ with different behaviors characteristic for the various length scales. At low $q$-range, the "Guinier" regime $\left(q R_{\mathrm{g}}<1\right)$ is usually attained. Middle $q$-range is usually sensitive to the shape of the scattering object; $a=-4$ stands for hard spheres, -2 stands for planar objects, and $a=-1$ stands for rod-like structures. It was proved experimentally that in some cases, the a value in middle $q$-range is not integral but rather fractional. This situation corresponds to the so-called fractal structure. In the high $q$ range, local stiffness of macromolecules (due to shorter length scales probed) can be revealed with $I(q) \sim q^{-1}$. Nanoparticles with sharp interface and smooth surface obey a $q^{-4}$ law that is usually referred to as "Porod" behavior. 
Several things should be noted. $I(q)$ value at the lowest experimental $q$ grows with time, which is clearly an indication of particle growth (Fig. 11). For the highest composition ratio $\mathrm{C}_{\mathrm{Brij}} 8 / \mathrm{C}_{\mathrm{pNMGL}}=2.0$, in the middle $q$-range, the exponent value $\mathrm{a}$ is growing from -2.2 at the beginning up to -3.4 for the longer time (Fig. 12). The $q^{-2.2}$ dependence of $I(q)$ observed on early stages of self-assembly is attributed to the scattering from a loose, fractal structure. In contrast, a value of -3.4 suggests large compact objects. Thus using TRSAXS we can monitor the self-assembly of nanoparticles when particles transform through fractal structure with loose surface into hard spheres with sharp interface.

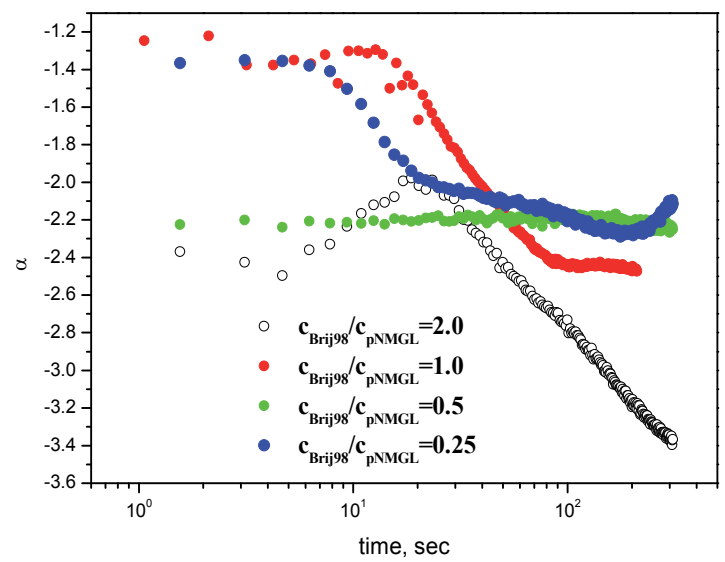

Fig. 12. The temporal evolution of the exponent value $a$ for a pNMGL-Brij 98 system at different composition ratio $\mathrm{c}_{\mathrm{Brij}} \mathrm{g} / \mathrm{C}_{\mathrm{pNMGL}}$.

For the lowest composition ratio $c_{B r i j 98} / c_{p N M G L}=0.25$, the behavior changes greatly. $I(q)$ value at the lowest $q$ as well as the a exponent do not evolve with time (Fig. 12). Obviously, nanoparticles have been already formed prior to the first measurement. Those nanoparticles do not have sharp boundaries and have fractal structure that is a characteristic for loose entities. Surfactant molecules are not enough to cover the whole nanoparticles.

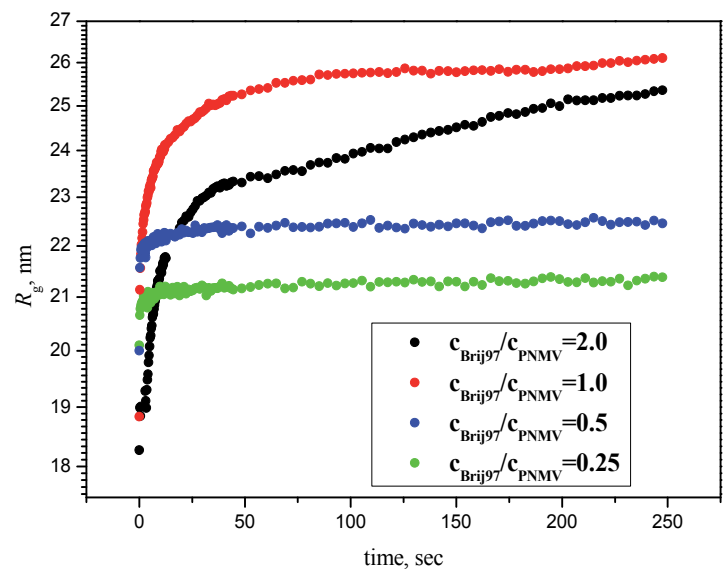

Fig. 13. The temporal evolution of the exponent value a for a pNMV -Brij 97 system at different composition ratio $\mathrm{c}_{\mathrm{Brij}} \mathrm{g} / \mathrm{c}_{\mathrm{pNMV}}$. 
To extract further information on the kinetics of nanoparticle formation, the radius of gyration was calculated and compared for different polymers and surfactants. The results are shown in Fig. 13-15. We observe that the radius strongly depends on the composition ratio. Moreover, two distinct regimes separated in time can be observed. During the first seconds, there is a rapid increase in the $R_{\mathrm{g}}$ value. This behavior could be explained as a nucleation regime when preliminary nuclei are formed. After a short period of time that depends also on composition ratio, the $R_{\mathrm{g}}$ value of nanoparticles increases by consumption of the remaining surfactant molecules in solution, thus defining the growth regime. The higher the composition ratio, the growth regime is more expressive (Fig. 13, 14). Nevertheless, sometimes a decrease in $R_{\mathrm{g}}$ is observed at the first seconds. We can assume that such scenario could be realized when several aggregates of pearl-necklace micelles disassembling prior to formation of original nuclei.

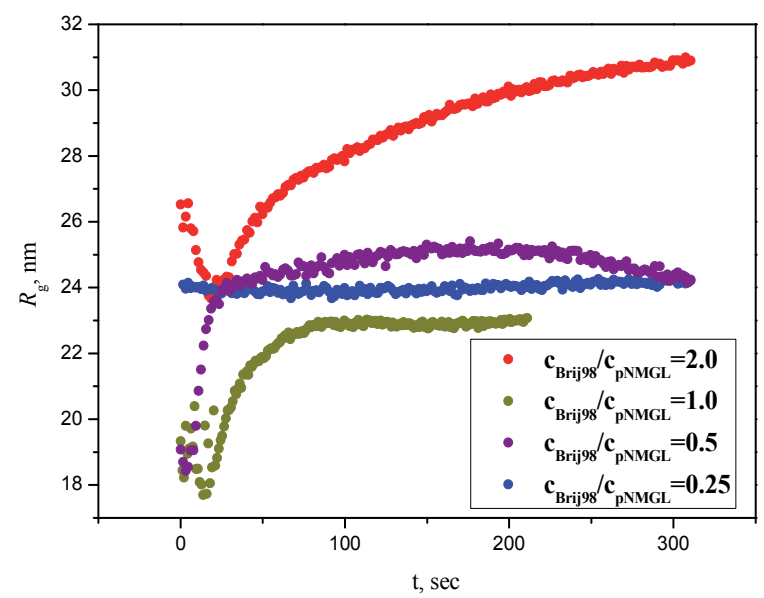

Fig. 14. The temporal evolution of the exponent value a for a pNMV-Brij 98 system at different composition ratio $\mathrm{C}_{\mathrm{Brij}} \mathrm{g} / \mathrm{C}_{\mathrm{pNMGL}}$.

The conclusion that the growth regime is governed by a surfactant only is further supported from a comparison of kinetic curves of different polymers but the same composition ratio (Fig. 15). The polymers of different nature but the same surfactant (Brij 98) and composition ratio (1.0) could be arranged into a master-curve (inset of Fig. 15). 
In contrast, the system with Brij 97 (red circles) is undoubtedly couldn't be superimposed into the master curve. We conclude that the number and hydrophobicity of monomeric units of a polymer determine the final size of a nanoparticle whereas the growth nucleation rates are controlled by the nature and amount of a surfactant.

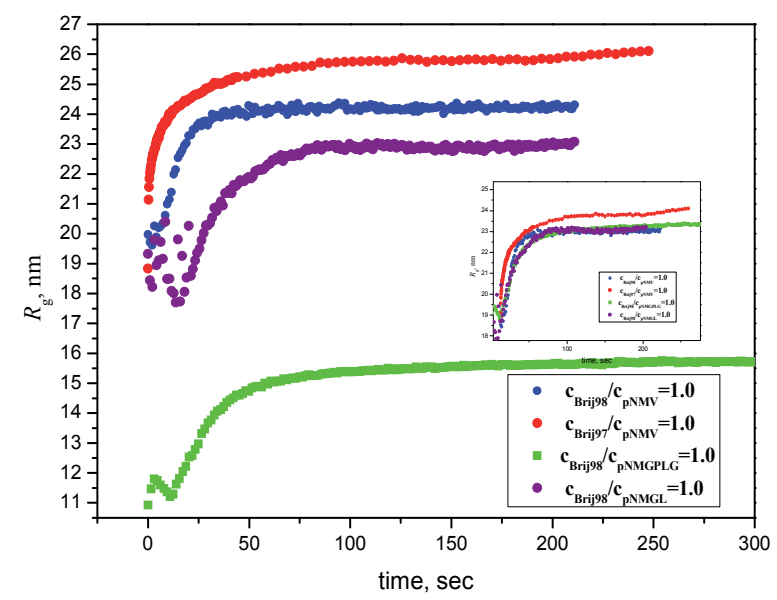

Fig. 15. The temporal evolution of the exponent value a for a pNMV, pNMGL, and pNMGPLG +Brij 98, 97 system at composition ratio 1.0. Inset: master curve.

\section{General conclusions}

We have systematically investigated nanoparticles prepared by controlled phase separation approach. On the basis of our research we have established that the most important parameter for steady-state nanoparticles dimensions is the composition ratio c(surfactant)/c(polymer). Our study demonstrates that full grown nanoparticles have a spherical shape. For the first time we have investigated the architecture of nanoparticles prepared by the solvent shifting method. The density, and factor $\rho=R_{\mathrm{g}} / R_{\mathrm{h}}$ measurement together with SANS experiment shows that nanoparticles are entities with uniform density and without internal structure. Polymeric and surfactant molecules are evenly distributed within a nanoparticle.

When macroscopic non-equilibrium hydrodynamic forces are involved into nanoparticle formation, the nature of the surfactant, its hydrophobicity and charge, insignificantly influences the nanoparticles sizes. A mixing rate is of primary importance for that case.

When hydrodynamic fluxes are eliminated by fast mixing again, the surfactant/polymer composition ratio is of primary importance in nanoparticle formation, thus confirming previous results. Excess of a surfactant results in much faster kinetics in comparison with the solution where a polymer is in excess. Our results suggest that the formation of the 
nanoparticles is a two stage process. In the beginning a nucleation stage occurs followed by a growth regime. The hydrophilicity/hydrophobicity of surfactants plays an important role in the formation of nanoparticles.

\section{Acknowledgements}

We gratefully acknowledge the European Synchrotron Radiation Facility (Grenoble, France) for the provision of synchrotron beam time (SC2883 and SC3113). This work was supported by the Grant Agency of the Czech Republic (202/09/2078) and also by Grant No. IAA400500805 of the Grant Agency of the Academy of Sciences of the Czech Republic. Also, we would like to thank Prof. Katarina Edwards and Dr. Goran Karlsson, Uppsala University, Department of Physical and Analytical Chemistry for help with Cryo-TEM experiments.

\section{References}

Abecassis, B., Testard, F., Spalla, O. \& Barboux, P. (2007). Probing in situ the nucleation and growth of gold nanoparticles by small-angle x-ray scattering. Nano Letters Vol. 7, pp. 1723-1727.

Abecassis, B., Testard, F., \& Spalla, O. (2008). Gold nanoparticle superlattice crystallization probed in situ. Physical Review Letters, Vol. 100, pp. 115504.

Aseyev, V., Hietala, S., Laukkanen, A., Nuopponen, M., Confortini, O., Du Prez, F.E. \& Tenhu, H. (2005). Polymer, Vol. 46, pp. 7118-7131.

Aseyev, V., Tenhu, H. \& Winnik, F. (2010). Non-ionic Thermoresponsive Polymers in Water, Self Organized Nanostrcutures of Amphiphilic Block Copolymers. Springer Berlin Heidelberg, pp. 1-61. (Advances in Polymer Science).

Baimark, Y., Srisaard, M., Threeprom J. \& Narkkong, N. A. (2007). Colloid and Polymer Science, Vol. 285, pp. 1521-1525.

Beck-Broichsitter, M., Gauss, J., Packhaeuser, C.B. , Lahnstein, K. , Schmehl, T. , W. Seeger, T. Kissel \& T. Gessler, (2009). International Journal of Pharmaceutics, Vol. 367, pp. 169178.

Bilati, U., Allemann, E. \& Doelker, E. (2005). European Journal of Pharmaceutical Sciences, Vol. 24, pp. 67-75.

Bolze, J., Pontoni, D., Ballauff, M., Narayanan, T., \& Colfen, H. (2004). Time-resolved SAXS study of the effect of a double hydrophilic block-copolymer on the formation of $\mathrm{CaCO}_{3}$ from a supersaturated salt solution. Journal of Colloid and Interface Science, Vol. 277, pp. 84-94.

Brick, M. C., Palmer, H. J. \& Whitesides T. H. ( 2003). Langmuir, Vol. 19, pp. 6367-6380.

Burchard, W. (1999) Adv Polym Sci, Vol. 143, pp. 113-194.

Chu, B. S. , Ichikawa, S. , Kanafusa S. \& Nakajima, M. (2008). Journal of the Science of Food and Agriculture, Vol. 88, pp. 1764-1769.

Deen, G.R., Oliveira, C.L.P. \& Pedersen, J.S. (2009). Phase Behavior and Kinetics of Phase Separation of a Nonionic Microemulsion of C12E5/Water/1-Chlorotetradecane upon a Temperature Quench. Journal of Physical Chemistry B, Vol. 113, pp. 71387146. 
Eastoe, J., Dalton, J.S., Downer, A., Jones, G. \& Clarke, D. (1998). Breakdown kinetics of fluorocarbon micelles studied by stopped-flow small-angle $\mathrm{X}$-ray scattering. Langmuir, Vol. 14, pp. 1937-1939.

Filippov, S., Hruby, M., Konak, C., Mackova, H., Spirkova, M. Stepanek, P. (2008). Langmuir, Vol. 24, pp. 9295- 9301.

Filippov, S., Starovoytova, L., Koňák, C., Hrubý, M., Macková, H., Karlsson, G. \& Štěpánek, P. (2010). Langmuir, vol. 26, pp. 14450-14457

Gallardo, M. , Couarraze, G. , Denizot, B., Treupel, L. , Couvreur P., and Puisieux, F. (1993). International Journal of Pharmaceutics, Vol. 100, pp. 55-64.

Ganachaud, F. \& Katz, J.L. (2005). Chemphyschem, Vol. 6, pp. 209-216.

Glatter, O. \& Kratky, O. (1982) Small-Angle X-ray Scattering. London: Academic Press

Gradzielski, M. (2003). Kinetics of morphological changes in surfactant systems. Current Opinion in Colloid \& Interface Science, Vol. 8:, pp. 337-345.

Gradzielski, M. (2004). Investigations of the dynamics of morphological transitions in amphiphilic systems. Current Opinion in Colloid \& Interface Science, Vol. 9, pp. 256263.

Grillo, I., Kats, E.I. \& Muratov, A.R. (2003). Formation and growth of anionic vesicles followed by small-angle neutron scattering. Langmuir, Vol.19, pp. 4573-4581.

Grillo, I. (2009). Applications of stopped-flow in SAXS and SANS. Current Opinion in Colloid $\mathcal{E}$ Interface Science, Vol 14, pp. 402-408.

Jakeš, J. (1995). Collect. Czech. Chem. C, Vol. 60, pp. 1781-1797.

Katas, H. , Cevher E. \& Alpara H. O. (2009). International Journal of Pharmaceutics, Vol. 369, pp. 144-154

Koňák, C., Pánek, J. \& Hrubý, M. (2007). Colloid Polym Sci. Vol. 285, pp. 1433-1439.

Kriz, J., Masar, B., Pospisil, H., Plestil, J., Tuzar Z. \& Kiselev, M.A. (1996). Macromolecules, Vol. 29, pp. 7853-7858

Kujawa, P., Tanaka, F. \& Winnik, FM. (2006). Macromolecules, Vol. 39, pp. 3048-3055

Kujawa, P., Aseyev, V., Tenhu, H. \& Winnik, FM. (2006). Macromolecules , Vol. 39, pp. 76867693

Lee, L.T. \& Cabane, B. (1997). Macromolecules, Vol. 30, pp. 6559-6566

Lince, F., Marchisio D. L. \& Barresi A. A. (2008). Journal of Colloid and Interface Science, Vol. 322, pp. 505-515.

Lund, R., Willner, L., Monkenbusch, M., Panine, P., Narayanan, T., Colmenero, J. \& Richter, D. (2009). Structural Observation and Kinetic Pathway in the Formation of Polymeric Micelles. Physical Review Letters, Vol. 102, pp.188301

Leroueil-Le Verger, M., Fluckiger, L., Kim, Y. I. , Hoffman M. \& Maincent, P. (1998). European Journal of Pharmaceutics and Biopharmaceutics, Vol. 46, pp. 137-143.

Leo E., Scatturin, A., Vighi E., \& Dalpia A. (2006). Journal of Nanoscience and Nanotechnology, Vol. 6, pp. 3070-3079.

Legrand, P., Lesieur, S., Bochot, A., Gref, R., Raatjes, W., Barratt G. \& Vauthier C. (2006). Conference on New Trends in Drug Delivery Systems, Paris, FRANCE.

Narayanan, T., Diat, O. \& Bosecke, P. (2001). SAXS and USAXS on the high brilliance beamline at the ESRF. Nuclear Instruments $\mathcal{E}$ Methods in Physics Research Section a- 
Accelerators Spectrometers Detectors and Associated Equipment, Vol. 467, pp. 10051009

Ne, F., Testard, F., Zemb T, \& Grillo, I. (2003). How does ZrO2/surfactant mesophase nucleate? Formation mechanism. Langmuir, Vol.19, pp. 8503-8510.

Nguyen, J. , Steele, T. W. J., Merkel, O., Reul R. \& Kissel, T. (2008). Journal of Controlled Release, Vol. 132, pp. 243-251.

Pánek, J., Filippov, S.K. , Koňák, Č.,Nallet, F., Noirez, L., Karlsson, G. \& Štěpánek, P. (2011). Journal of Dispersion Science and Technology, Vol. 32, N. 6, pp. 888-897

Pánek, J., Filippov, S.K., Koňák, C., Steinhart, M. \& Štěpánek, P. (2011). Journal of Dispersion Science and Technology, Vol. 32, N. 8, pp. 1105-1110

Panine, P., Finet, S., Weiss, T.M. \& Narayanan, T. (2006). Probing fast kinetics in complex fluids by combined rapid mixing and small-angle X-ray scattering. Advances in Colloid and Interface Science, Vol. 127, pp. 9-18.

Peracchia, M. T., Fattal, E., Desmaele, D. , Besnard, M., Noel, J. P. , Gomis, J. M. , Appel, M. ,d'Angelo J. \& Couvreur P. (1999). Journal of Controlled Release, Vol. 60, pp. 121-128.

Potineni, A., Lynn, D. M., Langer R. \& Amiji, M. M. (2003). Journal of Controlled Release, Vol. 86, pp. 223-234.

Pontoni, D., Narayanan, T., \& Rennie AR (2002). Time-resolved SAXS study of nucleation and growth of silica colloids. Langmuir, Vol. 18, pp. 56-59.

QuintanarGuerrero D., Allemann, E., Doelker E. \& Fessi, H. (1997). Colloid and Polymer Science, Vol. 275, pp. 640-647.

Schmolzer, S., Grabner, D., Gradzielski, M. \& Narayanan T (2002). Millisecond-range timeresolved small-angle $x$-ray scattering studies of micellar transformations. Physical Review Letters, Vol. 88, pp. 258301

Shen, L., Du, J.Z., Armes, S.P. \& Liu, S.Y. (1989). Kinetics of pH-induced formation and dissociation of polymeric vesicles assembled from a water-soluble zwitterionic diblock copolymer. Langmuir, Vol. 24, pp.10019-10025.

Siu, M.H., He, C. \&Wu C. (2003). Macromolecules, Vol. 36, pp. 6588-6592

Tabor, R.F., Eastoe, J. \& Grillo, I. (2009) Time-resolved small-angle neutron scattering as a lamellar phase evolves into a microemulsion. Soft Matter, Vol. 5, pp. 21252129.

Trimaille, T. , Chaix, C., Pichot C. \& Delair, T. (2003). Journal of Colloid and Interface Science, Vol. 258, pp. 135-145.

Tucker, I., Penfold, J., Thomas, R.K. \& Grillo, L. (2009). Monomer-Aggregate Exchange Rates in Dialkyl Chain Cationic-Nonionic Surfactant Mixtures. Langmuir, Vol. 25, pp. 2661-2666.

Van Keuren, E. R. (2004). Journal of Dispersion Science and Technology, Vol. 25, pp. 547-553.

Vega, E. , Gamisans, F., Garcia, M. L, Chauvet, A., Lacoulonche F. \& Egea, M. A. (2008). Journal of Pharmaceutical Sciences, Vol. 97, pp. 5306-5317

Weiss, T.M., Narayanan, T., Wolf, C., Gradzielski, M., Panine, P., Finet, S. \& Helsby, W.I. (2005). Dynamics of the self-assembly of unilamellar vesicles. Physical Review Letters, Vol. 94, pp. 038303. 
Weiss, T.M., Narayanan, T. \& Gradzielski, M. (2008). Dynamics of spontaneous vesicle formation in fluorocarbon and hydrocarbon surfactant mixtures. Langmuir, Vol. 24, pp. 3759-3766.

Yan, C. H. , Yuan, X. B., Kang, C. S., Zhao, Y. H. , Liu, J., Guo, Y. S. , Lu, J. , Pu P. Y. \& Sheng J. (2008). Journal of Applied Polymer Science, Vol. 110, pp. 2446-2452. 


\title{
Phase Separations in Mixtures of a Nanoparticle and a Liquid Crystal
}

\author{
Akihiko Matsuyama \\ Department of Bioscience and Bioinformatics, Kyusyu Institute of Technology \\ Japan
}

\section{Introduction}

Liquid crystal suspensions including various micro- and nano-colloidal particles have recently been received great attention for many practical applications such as nanosensors and devices, etc. When large colloidal particles of micronscale are dispersed in a uniform nematic liquid crystal phase, the colloidal particles disturb a long-range orientational order of the nematic phase. For a strong anchoring between the colloidal surface and a liquid crystal, different defect structures such as hedgehogs or Saturn rings can appear around a single colloidal particle, due to strong director deformations.(Fukuda, 2009; Skarabot et.al., 2008; Stark, 2001) Experiments have also shown two-dimensional crystalline structures of colloidal particles.(Loudet et. al., 2004; Musevic et. al., 2006; Nazarenko et. al., 2001; Pouling et.al., 1997; Yada et. al., 2004; Zapotocky et.al., 1999) On the other hand, under a weak surface anchoring between the colloidal surface and a liquid crystal, the coupling to the orientational elasticity of the liquid crystals tends to expel the colloidal particles and the suspension shows a phase separation into an almost pure nematic phase coexisting with a colloidal rich phase.(Anderson et.al., 2001; Pouling et. al., 1994) Such phase separations induced by a nematic ordering have also been discussed in flexible polymers dispersed in a nematic liquid crystal.(Chiu \& Kyu, 1999; Das \& Ray, 2005; Dubaut et.al., 1980; Matsuyama \& Kato, 1996; Shen \& Kyu, 1995)

If the colloidal particles are $\sim 1-10 \mathrm{~nm}$ in diameter, these "nanoparticles" are too small to distort the nematic director and defects do not form. In this case, the system can show a homogeneous single phase or phase separations,(Anderson et.al., 2001; Anderson \& Terentjev, 2001; Caggioni et. al., 2005; Meeker et. al., 2000; Yamamoto \& Tanaka, 2001) depending on the interaction between a colloidal particle and a liquid crystal. Although the theoretical progress on the description of a director around colloidal particles with strong anchoring conditions has been noticeable,(Araki \& Tanaka, 2004; Fukuda \& Yokoyama, 2005; Kuksenok et.al., 1996; Lubensky et. al., 1998; Yamamoto, 2001) little theoretical work exists in phase separations.(Popa-Nita et. al., 2006; Pouling et. al., 1994)

In this chapter, we focus on nanoparticles dispersed in liquid crystals and discuss phase separations and phase behaviors in mixtures of a nanoparticle and a liquid crystal. It is mainly based on authors' original theoretical works obtained within recent years. The nanoparticles have a variety in the shape such as spherical and rodlike. In this chapter, we focus on (1) mixtures of a liquid crystal and a spherical nanoparticle and (2) mixtures of a liquid crystal and a rodlike nanoparticle, such as carbon nanotube. The topics are currently interested in the advanced fields of nanoparticles and fundamental sciences. 
When the nanoparticles are dispersed in isotropic solvents, the system may show phase separations, or sodification, between a liquid and a crystalline phase, depending on temperature and concentration, etc. These phase separations are induced by a balance between steric repulsions and attractive dispersion forces. However, the nature of phase separations of nanoparticles dispersed in liquid crystalline solvents is quite difference. The key point is ordering of nanoparticles induced by liquid crystalline ordering. Depending on the interaction between nanoparticles and liquid crystals, we have a variety of phase separations.

The aim of this chapter is to introduce such a new kind of phase separations. We review recent mean field theories to describe phase separations (or phase diagrams) in mixtures of a nanoparticle and a liquid crystal and summarize the variety of phase separations in such nanoparticle dispersions, where liquid crystalline ordering (nematic and smectic A phases) and nanoparticle ordering compete. In Section 2, We discuss spherical nanoparticles dispersed in liquid crystals. Nanotubes dispersed in liquid crystals are discussed in Section 3. The effects of external forces, such as magnetic and electric fields, on the phase behaviors are also discussed in Section 3.

\section{Spherical nanoparticles dispersed in liquid crystals}

\subsection{Ferroelectric nanoparticles dispersed in nematic liquid crystals}

Small nanoparticles do not significantly perturb the nematic director. However, it has been recently discovered that ferroelectric nanoparticles can greatly enhance the physical properties of nematic liquid crystals. Recent experimental(Copic et.al., 2007; Li et. al., 2006) and theoretical(Kralj et.al., 2008; Lopatina \& Selinger, 2009) studies have shown that low concentrations of ferroelectric nanoparticles $\left(\mathrm{BaTiO}_{3}\right)$ increase the orientational order of a liquid crystal and increase the nematic-isotropic transition temperature, due to the coupling between the ferroelectric nanoparticle with electric dipole moment and the orientational order of liquid crystals.(Lopatina \& Selinger, 2009)
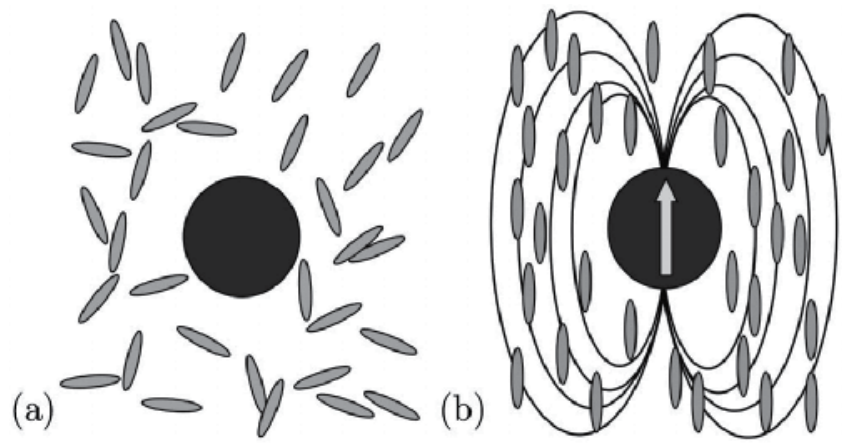

Fig. 1. Nanoparticles dispersed in liquid crystal. (a) Nanoparticle with no electric dipole moment, in an isotropic phase. (b) Ferroelectric particle with dipole moment, which produces an electric field that interacts with orientational order of the nematic phase. Reproduced with permission from (Lopatina \& Selinger, 2009) . Copyright 2009 American Physical Society.

As shown in Fig. 1, the orientational distribution of the nanoparticle dipole moment interacts with the orientational order of liquid crystals and stabilizes the nematic phase. The electric 
field generated by the nanoparticle interacts with the order parameter of the liquid crystal through the free energy

$$
F_{i n t}=-\frac{\Delta \epsilon \rho_{N P} p^{2}}{180 \pi \epsilon_{0} \epsilon^{2} R^{3}} S_{L C} S_{N P}
$$

where $\Delta \epsilon$ is the dielectric anisotropy of the aligned liquid crystal, $\rho_{N P}$ is the number density of nanoparticles, and $p$ is the electric dipole moment, $S_{L C}\left(S_{N P}\right)$ is the scalar orientational order parameters of the liquid crystals (nanoparticles). This free energy can predict the enhancement in the isotropic-nematic transition temperature and in the response to an applied electric field. The attractive interaction between the liquid crystal and the nanoparticle through the order parameters is important to understand the phase behaviors. The next section we consider the free energy to describe the phase separations.

\subsection{Phase ordering in mixtures of a spherical nanoparticle and a liquid crystal}

We consider mixtures of a spherical nanoparticle and a liquid crystal. By taking into account the ordering of liquid crystals and nanoparticles, we can expect six possible phases in this mixture. Figure 2 shows the schematically illustrated six phases. The isotropic (I) phase means both liquid crystals and nanoparticles have no positional and orientational order. In the nematic $(\mathrm{N})$ phase, liquid crystals have an orientational order, while nanoparticles have no positional order. Similarly, in the smectic $A$ (A) phase, liquid crystals have a smectic $A$ order, while nanoparticles have no positional order. When the concentration of nanoparticles is high, we may have a crystalline $(C)$ phase of nanoparticles dispersed in an isotopic matrix of liquid crystals. We can also expect a nematic-crystal (NC) phase and a smectic $A$-crystal (AC) phase, where nanoparticles form a crystalline structure dispersed in a nematic and a smectic $A$ matrix of liquid crystals. To describe these phases, depending on temperature and concentration, we take into account three scalar order parameters: an orientational order parameter for a nematic phase, one-dimensional translational order parameter for a smectic $A$ phase, and a translational order parameter for a crystalline phase of nanoparticles.

\subsection{Free energy of mixtures of a spherical nanoparticle and a liquid crystal}

We consider a binary mixture of $N_{c}$ spherical nano-colloidal particles of the diameter $R_{c}$ and $N_{r}$ low-molecular weight liquid crystal molecules (liquid crystals) of the length $l$ and the diameter $d$. The volume of the liquid crystal and that of the nanoparticle are given by $v_{r}=(\pi / 4) d^{2} l$ and $v_{c}=(\pi / 6) R_{c}^{3}$, respectively. Let $\rho_{r}(\mathbf{u}, \mathbf{r})$ and $\rho_{c}(\mathbf{r})$ be the number density of liquid crystals and colloidal particles with an orientation $\mathbf{u}$ (or its solid angle $\Omega$ ) at a position $\mathbf{r}$, respectively. The free energy $F$ of the dispersion at the level of second virial approximation is given by(Matsuyama \& Hirashima, 2008a; Matsuyama, 2010a)

$$
\begin{aligned}
\beta F / V= & \int \rho_{r}(\mathbf{r}, \mathbf{u})\left[\beta \mu_{r}^{\circ}+\ln \rho_{r}(\mathbf{r}, \mathbf{u})-1\right] d \mathbf{r} d \Omega \\
& +\int \rho_{c}(\mathbf{r})\left[\beta \mu_{c}^{\circ}+\ln \rho_{c}(\mathbf{r})-1\right] d \mathbf{r} \\
& +\frac{1}{2} \iint \rho_{r}(\mathbf{r}, \mathbf{u}) \rho_{r}\left(\mathbf{r}^{\prime}, \mathbf{u}^{\prime}\right) \beta_{r r}\left(\mathbf{r}, \mathbf{u} ; \mathbf{r}^{\prime}, \mathbf{u}^{\prime}\right) d \mathbf{r} d \Omega d \mathbf{r}^{\prime} d \Omega^{\prime}, \\
& +\frac{1}{2} \iint \rho_{c}(\mathbf{r}) \rho_{c}\left(\mathbf{r}^{\prime}\right) \beta_{c c}\left(\mathbf{r} ; \mathbf{r}^{\prime}\right) d \mathbf{r} d \mathbf{r}^{\prime}, \\
& +\iint \rho_{c}(\mathbf{r}) \rho_{r}\left(\mathbf{r}^{\prime}, \mathbf{u}^{\prime}\right) \beta_{c r}\left(\mathbf{r} ; \mathbf{r}^{\prime}, \mathbf{u}^{\prime}\right) d \mathbf{r} d \mathbf{r}^{\prime} d \Omega^{\prime},
\end{aligned}
$$




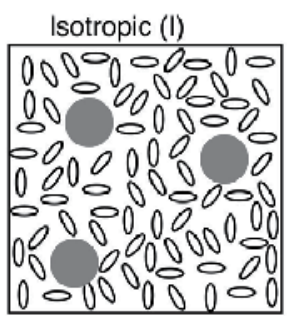

Crystal (C)

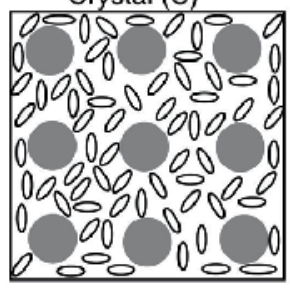

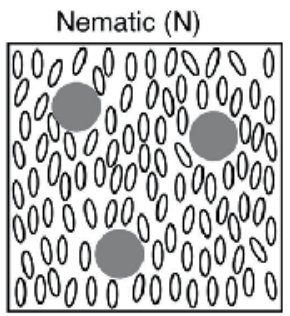

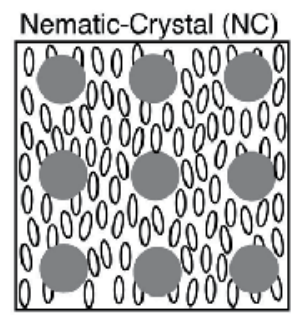

Smectic A (A)

00000000000000 0000000000000 00000000 000000000000000 000000000000000 0000000000000 000000000000000

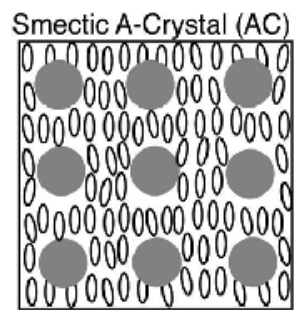

Fig. 2. Ordering of nanoparticles dispersed in liquid crystals. We here take into account three scalar order parameters: an orientational order parameter for a nematic phase, one-dimensional translational order parameter for a smectic $A$ phase, and a translational order parameter for a crystalline phase of nanoparticles.

where $d \Omega$ is the solid angle, $\mu_{i}^{\circ}$ is the standard chemical potential of a particle $i, j(=r, c)$, $\beta \equiv 1 / k_{B} T ; T$ is the absolute temperature, $k_{B}$ is the Boltzmann constant, $\beta_{i j} \equiv 1-\exp \left[-\beta u_{i j}\right]$ is the Mayer-Mayer function, and $u_{i j}$ is the interaction energy between two particles $i$ and $j$.

Let $f_{r}(\mathbf{r}, \mathbf{u})$ be the distribution function of liquid crystals and then the density can be expressed as

$$
\rho_{r}(\mathbf{r}, \mathbf{u})=c_{r} f_{r}(\mathbf{r}, \mathbf{u}),
$$

where $c_{r} \equiv N_{r} / V$ is the average number density of liquid crystals. We here consider a nematic and a smectic $A$ phase of liquid crystals and use the decoupled approximation(Kventsel et.al., 1985) for the distribution function:

$$
f_{r}(\mathbf{r}, \mathbf{u})=f_{r}\left(\tilde{z}_{r}\right) f_{r}(\mathbf{u}),
$$

where $\tilde{z}_{r} \equiv z / l, l$ is the average distance between smectic layers, $f_{r}\left(\tilde{z}_{r}\right)$ is the translational distribution function of liquid crystals for a smectic $A$ phase, and $f_{r}(\mathbf{u})$ is the orientational distribution function of liquid crystals for a nematic phase. Similarly, using the translational distribution function $f_{\mathcal{c}}(\mathbf{r})$ of nanoparticles, the density of nanoparticles can be expressed as

$$
\rho_{\mathcal{c}}(\mathbf{r})=c_{\mathcal{c}} f_{\mathcal{c}}(\mathbf{r}),
$$

where $c_{c} \equiv N_{c} / V$ is the average density of nanoparticles. The total number $N_{r}$ of liquid crystals and $N_{c}$ of nanoparticles must be conserved and then we have the normalization conditions:

$$
\iint \rho_{r}(\mathbf{r}, \mathbf{u}) d \mathbf{r} d \Omega=N_{r} / V
$$

and

$$
\int \rho_{\mathcal{C}}(\mathbf{r}) d \mathbf{r}=N_{c} / V
$$


The orientational order parameter $S$ of a nematic phase is given by(Maier \& Saupe, 1958)

$$
S=\int P_{2}(\cos \theta) f_{r}(\theta) d \Omega,
$$

where $P_{2}(\cos \theta) \equiv 3\left(\cos ^{2} \theta-1 / 3\right) / 2$. The translational order parameter $\sigma_{s}$ of a smectic $\mathrm{A}$ phase is given by(McMillan, 1971)

$$
\sigma_{s}=\int_{0}^{1} \cos \left(2 \pi \tilde{z}_{r}\right) f_{r}\left(\tilde{z}_{r}\right) d \tilde{z}_{r}
$$

In the McMillan's model,(McMillan, 1971) the order parameter for the smectic $A$ phase is given by $\left\langle P_{2}(\cos (\theta)) \cos \left(2 \pi \tilde{z}_{r}\right)\right\rangle$. In Eq. (4), we have used the decoupled approximation: $\left\langle P_{2}(\cos (\theta)) \cos \left(2 \pi \tilde{z}_{r}\right)\right\rangle=S \sigma_{s}$. It has been reported that the decoupled model for the smectic $\mathrm{A}$ phase is in quantitative agreement with the original McMillan's theory.(Kventsel et.al., 1985) In the decoupled model, the smectic $A$ phase is defined by $S \neq 0$ and $\sigma_{S}>0$.

For a crystalline phase, we here consider a face-centered cubic (fcc) structure of nanoparticles for example. The translational order parameter for a fcc crystalline phase can be calculated by(Kirkwood \& Monroe, 1941)

$$
\sigma_{f}=\int_{0}^{1} \int_{0}^{1} \int_{0}^{1} \cos (2 \pi \tilde{x}) \cos (2 \pi \tilde{y}) \cos (2 \pi \tilde{z}) f_{\mathcal{c}}(\tilde{\mathbf{r}}) d \tilde{\mathbf{r}},
$$

where $L$ is the lattice size of a fcc crystal and we define $\tilde{x} \equiv x / L, \tilde{y} \equiv y / L, \tilde{z} \equiv z / L$, and $d \tilde{\mathbf{r}} \equiv d \tilde{x} d \tilde{y} d \tilde{z}$. It is possible to consider the other crystalline structure such as a body-centered cubic and a simple cubic, etc.(Matsuyama, 2006a;b)

When the interaction between liquid crystals is a short-range attractive interaction, the anisotropic part of the interaction can be given by Fourier components of the potential:(McMillan, 1971)

$$
\beta_{r r} \simeq-\left(v_{r} l / d\right) v S\left(1+\gamma \sigma_{s} \cos (2 \pi z / l)\right) P_{2}(\cos \theta)
$$

where we have retained the lowest order of the Fourier components. The $v\left(\equiv U_{a} / k_{B} T\right)$ is the orientational dependent (Maier-Saupe) interaction parameter between liquid crystals(Maier \& Saupe, 1958) and the $\gamma$ shows the dimensionless interaction of a smectic phase(Matsuyama \& Kato, 1998; McMillan, 1971). According to the McMillan theory, the parameter $\gamma$ is given by $\gamma=2 \exp \left[-\left(r_{0} / l\right)^{2}\right]$, which can vary between 0 and 2 , and increases with increasing the chain length of alkyl end-chains of a liquid crystal. The smectic condensation is more favored for larger values of $\gamma$. For the anisotropic interaction between nanoparticles in a fcc crystalline phase, the anisotropic part of the interaction can be given by expanding $\beta_{c c}$ at the lowest order of the Fourier components:(Kirkwood \& Monroe, 1941)

$$
\beta_{c c} \simeq-\left(v_{c} R_{c} / d\right) g \sigma_{f} \cos (2 \pi \tilde{x}) \cos (2 \pi \tilde{y}) \cos (2 \pi \tilde{z}),
$$

where the coefficient $\beta_{c c}$ is proportional to the total surface area $\left(v_{c} R_{c}\right)$ of two particles. The parameter $g\left(\equiv-\beta f_{0}\right)$ is the dimensionless interaction parameter between nanoparticles, where the interaction energy $f_{0}$ consists of an entropic and enthalpic terms. In this paper, we only consider short-range interactions between particles. The long-range interaction, due to 
the presence of surface charges, is not taken into account. Similarly to Eq. (11), the anisotropic interaction between a nanoparticle and a liquid crystal in a nematic and a smectic $A$ phase is given by

$$
\beta_{c r} \simeq \frac{1}{2}\left(v_{c} l / R_{c}\right) \omega S\left(1+\omega_{1} \sigma_{S} \cos (2 \pi z / l)\right) P_{2}(\cos \theta),
$$

where $\beta_{c r}$ is proportional to the surface area $\left(v_{c} / R_{c}\right)$ of a nanoparticle(Stark, 1999). The $\omega \equiv$ $w_{0} / k_{B} T$ shows the dimensionless interaction parameter between a liquid crystal and a particle surface. When $\omega>0$, or repulsive interaction between a liquid crystal and a nanoparticle, doping nanoparticles disturb the orientational ordering of liquid crystals, or the orientational elasticity of the liquid crystals tends to expel the particles to be lower the elastic free energy of a nematic phase(Pouling et. al., 1994). In the mean field level, the elastic distortion cost of a director is taken into account in the order of $\omega S^{2}$. The negative values of $\omega(<0)$ indicate the attractive interactions between a liquid crystal and a nanoparticle and the particles tend to disperse into a liquid crystalline matrix as indicated in Fig. 1. The last term $\omega_{1}(>0)$ is the coupling between a smectic liquid crystal and a colloidal surface.

We here assume the system is incompressible. Let $\phi_{r}=v_{r} N_{r} / V$ and $\phi_{c}=v_{c} N_{c} / V$ be the volume fraction of a liquid crystal and a nano-colloidal particle, respectively. Using the axial ratio $n_{r}(\equiv l / d)$ of a liquid crystal and $n_{c} \equiv R_{c} / d$, the volume of a particle is given by $v_{r}=$ $a^{3} n_{r}$ and $v_{r} \simeq\left(a n_{c}\right)^{3}$, where we define $a^{3} \equiv(\pi / 4) d^{3}$. To describe phase behaviors of the incompressible blends, we calculate the free energy of mixing for the binary mixtures of a liquid crystal and a nanoparticle:

$$
\Delta F=F\left(N_{c}, N_{r}\right)-F\left(N_{c}, 0\right)-F\left(0, N_{r}\right),
$$

where the $F\left(N_{c}, 0\right)$ and $F\left(0, N_{r}\right)$ are the reference free energy of the pure nanoparticles and the pure liquid crystal in an isotropic phase, respectively. Substituting Eqs. (11)-(13) into (14), the mixing free energy is given by

$$
\Delta F=F_{m i x}+F_{c}+F_{n e m}+F_{s m}+F_{a n c}
$$

where the each term is given as following.

The first term in Eq. (15) shows the free energy for mixing of colloids and liquid crystals in the isotropic phase:

$$
a^{3} \beta F_{m i x} / V=\frac{\phi_{r}}{n_{r}} \ln \phi_{p}+\frac{\phi_{c}}{n_{c}^{3}} \ln \phi_{c}+\chi \eta_{c} \phi_{r}
$$

where the first and the second terms in Eq. (16) correspond to the entropy of isotropic mixing for liquid crystals and colloidal particles, respectively. We here have added the third term which shows the isotropic interaction parameter $\chi \equiv U_{0} / k_{B} T$ related to the dispersion force between a colloidal particle and a liquid crystal, where $U_{0}$ is the interaction energy between a colloid and a liquid crystal in an isotropic state. A positive $\chi$ denotes that the colloid-liquid crystal contacts are less favored compared with the colloid-colloid and liquid crystal-liquid crystal contacts. This interaction parameter is well known as the Flory-Huggins parameter in polymer solutions.(Flory, 1953) For a colloidal particle, its surface only can interact with the surrounding solvents and so the probability for the colloid-liquid crystal contact is proportional to $\eta_{c} \phi_{r}$, where the $\eta_{c}$ is the surface fraction of colloidal particles and is 
given by

$$
\eta_{c} \equiv \frac{a^{2} n_{c}^{3} N_{c}}{a^{2}\left(n_{r} N_{r}+n_{c}^{3} N_{c}\right)}=\frac{\phi_{c}}{n_{c}} .
$$

Then the dispersion interaction due to the mixing is given by $\chi \eta_{c} \phi_{r}$. On increasing the diameter $n_{c}$ of a colloid, the interaction term decreases with a fixed $\phi_{c}$. Eq. (16) corresponds to the extended Flory-Huggins free energy for the isotropic mixtures of a liquid crystal, whose the number of segments is $n_{r}$, and a colloidal particle, whose the number of segments is $n_{c}^{3}$. The second term in Eq. (15) shows the free energy for a crystalline ordering of colloidal particles:

$$
\begin{aligned}
a^{3} \beta F_{c} / V= & \frac{\phi_{c}}{n_{c}^{3}} \int_{0}^{1} \int_{0}^{1} \int_{0}^{1} f(\tilde{\mathbf{r}}) \ln f(\tilde{\mathbf{r}}) d \tilde{\mathbf{r}} \\
& -\frac{1}{2} g \eta_{c}^{2} \sigma_{f}^{2},
\end{aligned}
$$

where the first term in Eq. (18) shows the entropy loss due to the crystalline ordering. When the colloidal particles have no positional order, we have the distribution function $f(\tilde{\mathbf{r}})=1$ and the free energy $\left(F_{c}\right)$ becomes zero. The third term in Eq. (15) shows the free energy for nematic ordering of liquid crystals:

$$
\begin{aligned}
a^{3} \beta F_{n e m} / V= & \frac{\phi_{r}}{n_{r}} \int f_{r}(\theta) \ln 4 \pi f_{r}(\theta) d \Omega \\
& -\frac{1}{2} v \phi_{r}^{2} S^{2},
\end{aligned}
$$

where the first term in Eq. (19) shows the entropy change due to the nematic ordering. The forth term in Eq. (15) shows the free energy for smectic A ordering of liquid crystals:

$$
\begin{aligned}
a^{3} \beta F_{s m} / V= & \frac{\phi_{r}}{n_{r}} \int_{0}^{1} f_{r}\left(\tilde{z}_{r}\right) \ln f_{r}\left(\tilde{z}_{r}\right) d \tilde{z}_{r} \\
& -\frac{1}{2} v \gamma \phi_{r}^{2}\left(S \sigma_{s}\right)^{2} .
\end{aligned}
$$

The last term in Eq. (15) shows the anchoring interaction between a colloidal surface and a liquid crystal:

$$
a^{3} \beta F_{a n c} / V=\frac{\omega}{2} \eta_{c} \phi_{r}\left(S^{2}+\omega_{1}\left(S \sigma_{s}\right)^{2}\right)
$$

In a thermal equilibrium state, the distribution functions of nanoparticles and liquid crystals are determined by minimizing the free energy (15) with respect to these functions: $\left(\delta F / \delta f_{c}(\tilde{\mathbf{r}})\right)_{\left\{f_{r}(\theta), f_{r}\left(\tilde{z_{r}}\right)\right\}}=0,\left(\delta F / \delta f_{r}(\theta)\right)_{\left\{f_{c}(\tilde{\mathbf{r}}), f_{r}\left(\tilde{z_{r}}\right)\right\}}=0$, and $\left(\delta F / \delta f_{z}\left(\tilde{z_{r}}\right)\right)_{\left\{f_{c}(\tilde{\mathbf{r}}), f_{r}(\theta)\right\}}=0$. The order parameters $S, \sigma_{s}$, and $\sigma_{f}$ can be determined by Eqs. (8), (9), and (10), respectively. Using these distribution functions and order parameters, we can calculate the free energy of our systems. The chemical potentials of a nanoparticle and a liquid crystal can be obtained from this free energy. 


\subsection{Phase transitions in nanoparticle/liquid crystal mixtures}

In a mixture of a nanoparticle and a liquid crystal, we have some phase transitions, depending on temperature and concentration(Matsuyama, 2009).

One is the nematic-isotropic phase transition of this mixture. The nematic-isotropic transition (NIT) temperature is given by

$$
\tau_{N I}=T / T_{N I}^{\circ}=1-\left(1+\alpha_{a} / n_{c}\right) \phi_{c},
$$

where $T_{N I}^{\circ}$ shows the NIT temperature of a pure liquid crystal. We here define the ratio $\alpha_{a}$ between the anchoring strength $(w)$ and the nematic interaction $(v)$ :

$$
\alpha_{a} \equiv w / \nu .
$$

The value of $\alpha_{a}$ shows the anchoring strength. The negative sign represents attractive interaction between a nanoparticle and a liquid crystal and thus the nanoparticles tend to disperse in a liquid crystalline matrix. On the other hand, the positive sign represents the repulsive interaction and the liquid crystals tend to expel the nanoparticles. The slope of the NIT line on the $T-\phi_{c}$ plane depends on the value of $\alpha_{a} / n_{c}$.

The smectic $\mathrm{A}$ - nematic phase transition (ANT) is given by

$$
\tau_{A N}=T / T_{N I}^{\circ}=2.27 \gamma\left[1-\left(1+\frac{\omega_{1} \alpha_{a}}{n_{c} \gamma}\right) \phi_{c}\right] S^{2} .
$$

Since $\gamma>0$ and $\omega_{1}>0$, the ANT temperature depends on the sign of $\alpha_{a}$. For larger negative values of $\alpha_{a}$, the ANT temperature increases with increasing $\phi_{c}$. It can also be obtained the direct phase transition from an isotropic to a smectic A phase(Matsuyama, 2009).

We also have the isotropic fluid-crystal phase transition (ICT). The ICT temperature is given by

$$
\tau_{I C}=T / T_{N I}^{\circ}=\frac{0.58 \alpha_{c} n_{c} \phi_{c}}{n_{r}\left(1-\phi_{c} / \phi_{c}^{*}\right)}
$$

where

$$
\alpha_{c} \equiv \beta\left|e_{0}\right| / \nu,
$$

shows the strength of the attractive interaction between nanoparticles compared to the nematic interaction parameter $v$. When $\tau<\tau_{I C}$ the crystalline phase is stable. The ICT temperature increases with increasing $\phi_{c}$ and diverges at $\phi_{c}^{*}$. This corresponds to the entropically driven-liquid-solid transition for hard spherical particles due to the excluded volume interactions.(Alder \& Wainwright, 1957; Cates \& Evans, 2000)

Figure 3(a) shows the first-order phase transition lines for NIT (red dotted-line, Eq. (22)), ANT (blue dotted-line, Eq. (24)), and ICT (black dotted-line, Eq. (25)) on the reduced temperature $\left(T / T_{N I}^{\circ}\right)$-concentration $\left(\phi_{c}\right)$ plane. We set $n_{c}=3, n_{r}=2, \alpha_{c}=0.1, \alpha_{a}=-2.5, \alpha_{n}(\equiv v / \chi)=5$, $\gamma=0.87, \omega_{1}=1$ for a typical example. When $\phi_{c}=0$, or pure liquid crystals, the ANT appears at $T / T_{N I}^{\circ} \approx 0.938$, which is consistent with the result of the MacMillan theory. At high temperatures and low concentrations, we have the isotropic (I) liquid phase. On decreasing temperature, the $\mathrm{N}$ phase appears, where nanoparticles are in an isotropic liquid state but liquid crystals are in a nematic state. Further decreasing temperature the smectic $A$ phase 


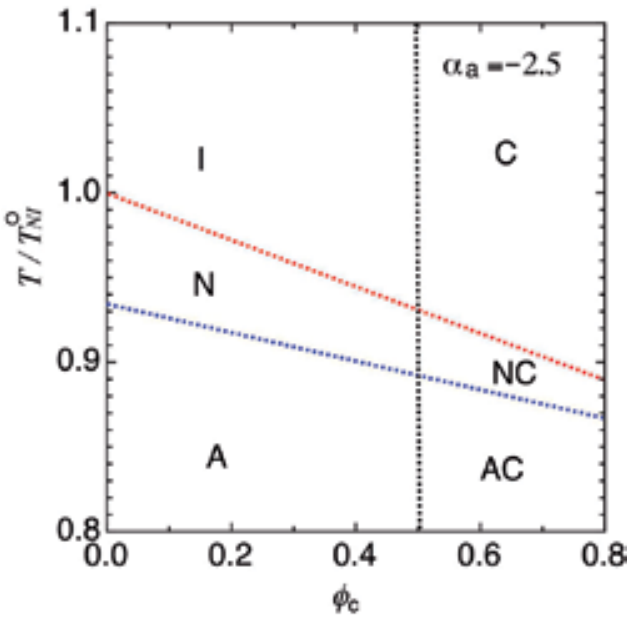

(a)

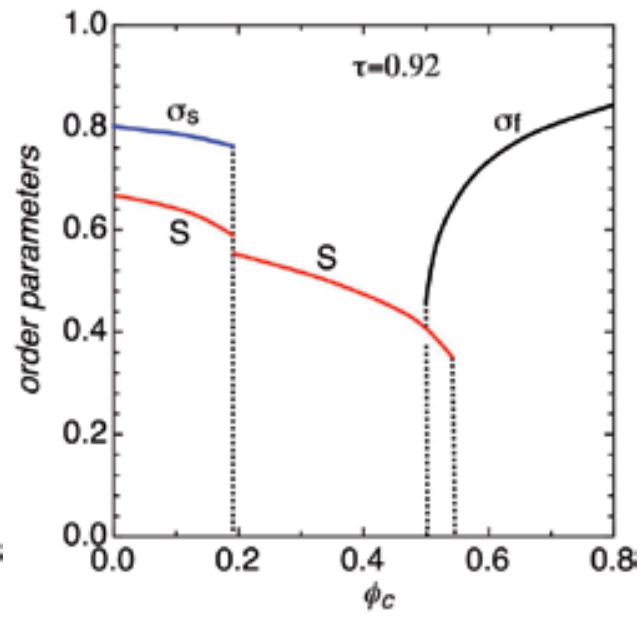

(b)

Fig. 3. (a) The first-order phase transition lines (the red dotted-line for NIT (Eq. (22)), the blue dotted-line for ANT (Eq. (24)), and the black dotted-line for ICT (Eq. (25))) on the reduced temperature $\left(T / T_{N I}^{\circ}\right)$-concentration $\left(\phi_{c}\right)$ plane. (b) Order parameters plotted against the volume fraction of colloidal particles at $T / T_{N I}^{\circ}=0.92$ in Fig. 3(a).

appears, where nanoparticles are in an isotropic liquid state but liquid crystals are in a smectic $A$ phase. At high temperatures and high concentrations, we have the crystalline (C) phase of colloidal particles. On decreasing temperature, the NC phase appears, where colloidal particles are in a crystalline state and liquid crystals are in a nematic state. Further decreasing temperature the AC phase appears, where colloidal particles are in a crystalline state and liquid crystals are in a smectic $A$ phase. The slope of the transition lines depends on the anchoring energy $\left(\alpha_{a}\right)$ as discussed in Eq. (23). For larger negative values of $\alpha_{a}$, the slopes of the NIT and ANT lines become positive on the temperature-concentration plane and the nematic and smectic A phase appear at higher temperatures.

Figure 3(b) shows order parameters plotted against the volume fraction $\phi_{c}$ at $T / T_{N I}^{\circ}=0.92$ in Fig. 3(a). On increasing $\phi_{c}$, we find the first-order phase transition from the smectic $\mathrm{A}$ to nematic $(\mathrm{N})$ phase at $\phi_{c} \simeq 0.2$, where the order parameters $S$ and $\sigma_{s}$ jump. At $\phi_{c} \simeq 0.5$, the first-order phase transition from the $\mathrm{N}$ to $\mathrm{NC}$ phase appears. Further increasing $\phi_{c}$ the first-order phase transition from the NC to $\mathrm{C}$ phase appears at $\phi_{c} \simeq 0.55$.

\subsection{Phase diagrams of nanoparticle/liquid crystal mixtures}

In this subsection we show some phase diagrams calculated from the free energy(15). The coexistence curve (binodal) can be obtained by solving the two-phase coexistence conditions: the chemical potentials of each component are equal in each phase. This binodal curve can also be derived by a double tangent method where the equilibrium volume fractions fall on the same tangent line to the free energy curve.

Figure 4 shows the phase diagrams for $n_{r}=2, n_{c}=3, \omega_{1}=1, \alpha_{c}=0.1, \alpha_{n}=5$, and $\gamma=0.87$ for an example. The value of $\alpha_{a}$ is changed: (a) $\alpha_{a}=1$; (b) $\alpha_{a}=-2$; (c) $\alpha_{a}=-3.5$. 
We here discuss the effects of the anchoring strength $\alpha_{a}$ on the phase behavior. The negative values of $\alpha_{a}$ mean that the nanoparticles prefer to disperse into liquid crystalline phases. The solid curve shows the binodal curve. The red, blue, and black dotted lines show the NIT, ANT, and ICT line, respectively (see Fig. 3(a)). When $\phi_{c}=0$, the smectic $A$ phase appears at $T / T_{N I}^{\circ}=0.94$. When $\alpha_{a}=1$ [Fig. 4(a)], we have the broad nematic-isotropic $(N+I)$ phase separation between $1>T / T_{N I}^{\circ}>0.94$. Below $T / T_{N I}^{\circ}<0.94$, we have the smectic $A$-isotropic $(A+I)$ phase separation. The nematic and smectic $A$ phase at the lower concentrations consist of almost pure liquid crystals. The triple point $(A+I+C)$ appears at $T / T_{N I}^{\circ}=0.89$, where the smectic $A$, isotropic, and crystalline phases can simultaneously coexist. Below the triple point, we have the two-phase coexistence $(A+C)$ between a smectic $A$ and a crystalline phase. Above the triple point, two-phase coexistence $(I+C)$ between an isotropic and a crystalline phase appears.

On decreasing the anchoring parameter $\alpha_{a}$ the phase behavior is drastically changed. When $\alpha_{a}=-2$ [Fig. 4(b)], the NIT (Eq. (22)) and ANT (Eq. (24)) lines shift to higher concentrations and the stable single $\mathrm{N}$ and $\mathrm{A}$ phases appear at low concentrations of nanoparticles. Two tie lines with arrows show the three-phase coexistence: $A+N+I$ and $A+I+C$. Above the triple point $A+N+I$, we have two-phase coexistence $A+N$ and $N+I$. Below the triple point $A+N+I$, we have A+I phase separation. Below the triple point $A+I+C$, we have the broad $\mathrm{A}+\mathrm{C}$ phase separation.

Further decreasing $\alpha_{a}$, Fig. 4(c), the nematic and smectic $A$ ordering are promoted by adding nanoparticles and the NIT and ANT lines shift to higher temperatures. This increase of the NIT and ANT temperature indicates the attractive interactions between a liquid crystal and a colloidal particle. For example, it has been observed that doping low concentrations of ferroelectric $\mathrm{BaTiO}_{3}$ nanoparticles into liquid crystals increases NIT temperature(Li et. al., 2006a). In this case, ferroelectric nanoparticle with electric dipole moment, which produces an electric field, interacts with orientational order of liquid crystals and stabilizes the nematic phase.(Lopatina \& Selinger, 2009) This corresponds to negative anchoring energy in our model. We also have three triple points: $I+C+N C, N+N C+A C$, and $N+A+A C$. Above the $I+C+N C$ triple point, we have the $I+C$ and $C+N C$ phase separations. Below the $I+C+N C$ triple point, the $I+N C$ and $N C+A C$ phase separations appear. Below the triple point $N+N C+A C$, we have the $I+N$ and $N+A C$ phase separations. Below the triple point $N+A+A C$ we have $N+A$ and $A+A C$ phase separations. The anchoring energy between liquid crystals and nanoparticles becomes an important parameter to derive a stable $\mathrm{N}, \mathrm{A}$, $\mathrm{NC}$, and $\mathrm{AC}$ phases in the mixture of nanoparticles and liquid crystals.

Anderson et al. have observed the phase ordering of colloidal (PMMA) particles dispersed in a liquid crystal, 5CB or MBBA.(Anderson et.al., 2001) Particles are covered with chemically grafted short chains, making hairy particles. In a nematic phase, the grafted chains tend to provide a homeotropic (radial) director anchoring. In an isotropic liquid, these particles behave like almost hard spheres and so the $I+C$ phase separation takes place at high concentrations of the colloidal particles. Such $I+C$ phase separation, calculated in Fig. 4, has been observed in colloidal dispersions(Pusey \& van Megen, 1986) and protein solutions(Tanaka et. al, 2020). At dilute concentrations of the colloidal particles, Anderson et al. observed a decrease in the NIT temperature $T_{N I}$ as a function of $\phi_{c}$, which follows a linear law. This is consistent with Eq. (22). The $\mathrm{N}+\mathrm{I}$ and $\mathrm{N}+\mathrm{C}$ phase separations have also been reported in Latex polyballs suspended in an isotropic micellar solution which exhibits 


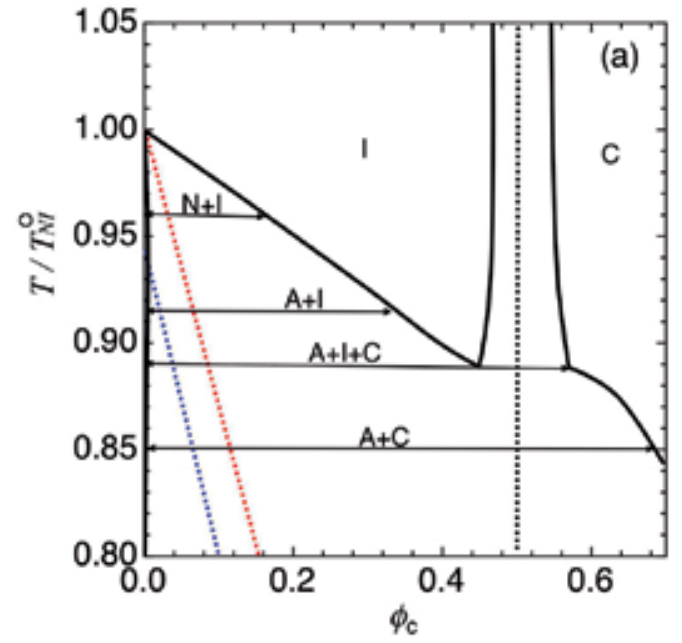

(a)

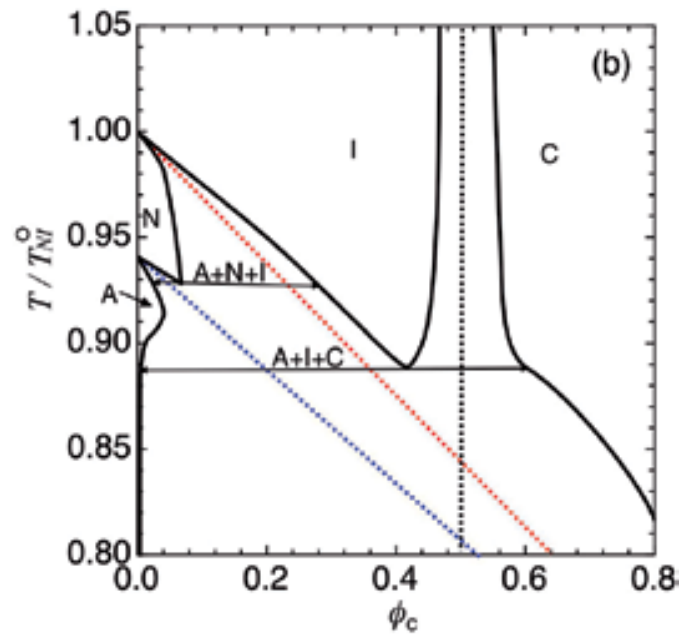

(b)

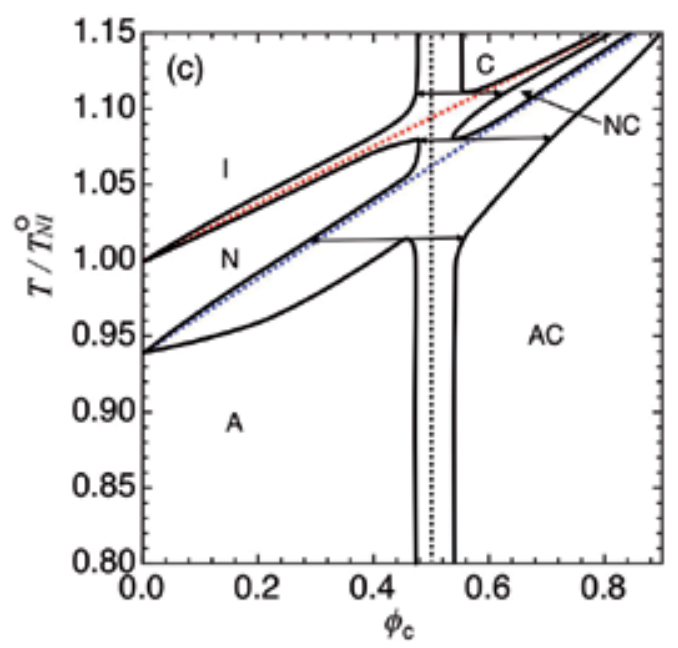

(c)

Fig. 4. Phase diagrams for $\alpha_{c}=0.1, \alpha_{n}=5, \gamma=0.87$. The value of $\alpha_{a}$ is changed: (a) $\alpha_{a}=1$; (b) $\alpha_{a}=-2$; (c) $\alpha_{a}=-3.5$.

a nematic phase at low temperature.(Pouling et. al., 1994) The observed phase diagram are qualitatively consistent with Fig. 4(a).

The binodal lines calculated at high concentrations of nanoparticles may not be experimentally observed because of high viscosity, however, it is important to understand the phase ordering kinetics(Matsuyama et .al., 2000; Matsuyama, 2008b). The cooperative phenomena between liquid crystalline ordering and crystalline ordering induce a variety of phase separations. 


\section{Nanotubes dispersed in liquid crystals}

Since the discovery of carbon nanotubes (CNTs)(Iijima, 1991), extensive studies of physical and chemical properties of CNTs have been received great attention for many practical applications such as nano-sensors and devices. Windle's group first reported the nematic liquid crystalline behavior of an aqueous suspension of CNTs above a certain concentration and the isotropic-nematic phase separations.(Shaffer \& Windle, 1999; Song et. al., 2003). Long nanotubes segregate preferentially to the liquid crystalline phase, whereas shorter nanotubes segregate preferentially to the isotropic phase(Zhang et.al., 2006). Recently such nanotubes as liquid crystalline materials become an important to be used in biological applications such as biosensors, biology imaging, artificial muscles, gene delivery, etc(Woltman et al., 2007).

In order to prepare CNT dispersions, strong van der Waals attractions between nanotubes must be screen out. To do this, the surface of nanotubes can be modified by acid oxidation, acid protonation, polymer or surfactant wrapping, etc.(Zhang \& Kumar, 2008). For example, a water-soluble polymer, biopolymers such as DNA, and surfactant molecules have been used to wrap CNT and to increase the dispersibility in water(Badaire et. al., 2005). The polymer-wrapped nanotubes can be dispersed in a solvent with a considerable concentration. The excluded volume and electro-static repulsion between polymers can overcome the intermolecular van der Waals attractions and therefore the polymer-wrapped CNT can be dispersed in water. Thus it is possible to change the strength of the intermolecular interaction between nanotubes by using polymer-wrapping and negative or positive charging of nanotube surface, etc.

Alignment of such CNTs, or rigid-rodlike polymers (rods), with the aid of low molecular-weight-liquid crystalline molecules is an alternative approach. Indeed thermotropic(Basu \& Iannacchione, 2008; Dierking et.al., 2005; Jayalakshmi \& Prasad, 2009; Lynch \& Patrick, 2002; Russell et.al., 2006) as well as lyotropic nematic liquid crystals(Courty et.al., 2003; Lagerwall et. al., 2007; Schymura et. al., 2009; Weiss et. al., 2006) have been applied as nematic solvents for the alignment of nanotubes. Anisotropic interactions between the nanotube and liquid crystal drastically change the alignments and physical properties of the mixtures. Duran et.al. have observed the nematic-isotopic phase transition temperature $\left(T_{N I}\right)$ is enhanced by the incorporation of a multi-wall CNT within a small composition gap(Duran et.al., 2005).

In this section, we discuss phase separations in binary mixtures of a low molecular-weight-liquid crystal and a nanotube, such as CNT. We discuss uniaxial and biaxial nematic phases.

\subsection{Nematic phases in mixtures of a nanotubes and a liquid crystal}

We here consider the effect of the anisotropic interaction between a nanotube and a liquid crystal and that between rods.(Matsuyama, 2010) Depending on the interaction between a nanotube and a liquid crystal, we can expect various nematic phases. Figure 5 schematically shows the four nematic phases, defined by using the orientational order parameter $\left(S_{1}\right)$ of a liquid crystal and that $\left(S_{2}\right)$ of a nanotube. When the orientational order parameter of one component is positive, determining a nematic director, and the orientational order parameter of the second component is negative, we have planer nematic phase, where the second 


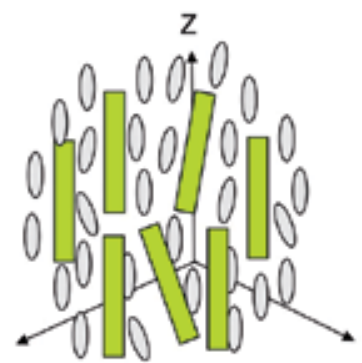

$\mathrm{N}_{0}$

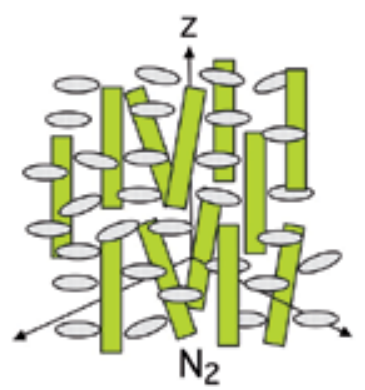

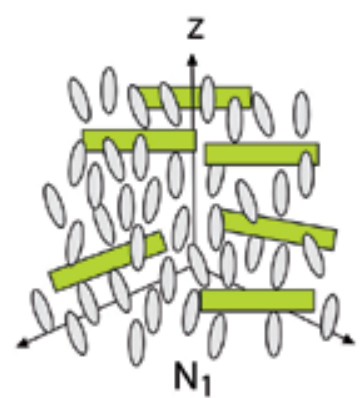

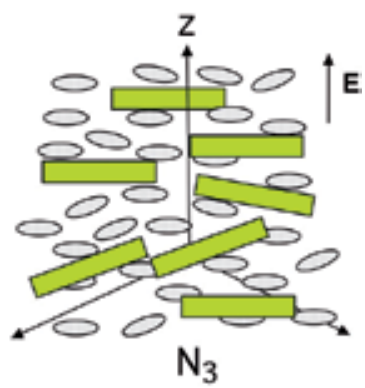

(a)

Fig. 5. Schematically illustrated four possible nematic phases. Four nematic phases are defined using the orientational order parameter $S_{1}$ of the liquid crystal and that $S_{2}$ of the nanotube: the nematic $\mathrm{N}_{0}$ phase with $S_{1}>0$ and $S_{2}>0$, the nematic $\mathrm{N}_{1}$ phase with $S_{1}>0$ and $S_{2}<0$, and the nematic $\mathrm{N}_{2}$ phase with $S_{1}<0$ and $S_{2}>0$. When an external field (E) is applied along to the $z$ axis for the particles of the dielectric anisotropy $\Delta \epsilon_{1}<0$ and $\Delta \epsilon_{2}<0$, the $\mathrm{N}_{3}$ phase with $S_{1}<0$ and $S_{2}<0$ can appear: the nanotubes and liquid crystals are randomly oriented on the plane perpendicular to the direction of the external field.

component is randomly oriented within in the perpendicular plane to the nematic director. The nematic $\mathrm{N}_{0}$ phase shows the nanotube and the liquid crystal are parallel to each other: $S_{1}>0$ and $S_{2}>0$. The nematic $N_{1}$ phase is defined as that the nanotube and the liquid crystal are perpendicular with each other: $S_{1}>0$ and $S_{2}<0$. In this phase, the nematic director $(\mathrm{z}$ axis) can be defined by the orientational direction of the liquid crystals. These perpendicular alignments can be obtained by modifying the surface of a nanotube, or CNT, with polymers or surfactants. The nematic $\mathrm{N}_{2}$ phase is defined as the nanotube and the $\mathrm{LC}$ are perpendicular each other with $S_{1}<0$ and $S_{2}>0$. In this phase, the nematic director ( $\mathrm{z}$ axis) can be defined by the orientational direction of the nanotube. Biaxial nematic phases are discussed in Section 3.3. When an external field (E) is applied along to the $z$ axis for the particles of the dielectric anisotropy $\Delta \epsilon_{1}<0$ and $\Delta \epsilon_{2}<0$, the $\mathrm{N}_{3}$ phase with $S_{1}<0$ and $S_{2}<0$ may appear, where the nanotubes and liquid crystals are randomly oriented on the plane perpendicular to the direction ( $z$ axis) of the external field.

\subsection{Free energy of nanotube/liquid crystal mixtures}

We consider a binary mixture of a liquid crystal of the length $L_{1}$ and the diameter $D_{1}$ and and a nanotube of the length $L_{2}$ and the diameter $D_{2}: L_{1}<L_{2}$. The volume of the liquid crystal and 
that of the nanotube is given by $v_{1}=(\pi / 4) D_{1}^{2} L_{1}$ and $v_{2}=(\pi / 4) D_{2}^{2} L_{2}$, respectively. We here assume $D \equiv\left(D_{1}=D_{2}\right)$. Let $\rho_{1}(\mathbf{r}, \mathbf{u})$ and $\rho_{2}(\mathbf{r}, \mathbf{u})$ be the number density of the liquid crystals and the nanotubes with an orientation $\mathbf{u}$ (or its solid angle $\Omega$ ) at a position $\mathbf{r}$, respectively. The free energy $F$ of the dispersion at the level of second virial approximation is given by Eq. (2). The volume fraction of liquid crystals is given by $\phi_{1}=v_{1} \rho_{1}$ and tat of nanotubes $\phi_{2}=v_{2} \rho_{2}$. As discussed in Eq. (14), we here consider the incompressible fluids: $\phi_{1}+\phi_{2}=1$.

Consider a uniaxial nematic phase, which is spatially uniform but nonuniform for orientation. Let $f_{i}(\mathbf{u})$ be the distribution function of the particle $i(=1,2)$ and then the density can be expressed as

$$
\rho_{i}(\mathbf{r}, \mathbf{u})=c_{i} f_{i}(\mathbf{u}),
$$

where $c_{i} \equiv N_{i} / V$ is the average number density of the particle $i$. The total number $N_{1}$ of the liquid crystals and $N_{2}$ of the nanoparticles must be conserved and then we have the normalization conditions:

$$
\int \rho_{i}(\mathbf{r}, \mathbf{u}) d \mathbf{r} d \Omega=N_{i} / V
$$

where $d \Omega=2 \pi \sin \theta d \theta$ for a uniaxial nematic phase.

For the interaction between liquid crystals in Eq. (2), we take the attractive (Maier-Saupe) interaction:

$$
\beta_{11}=-v_{1} v_{1} P_{2}(\cos \theta) P_{2}\left(\cos \theta^{\prime}\right),
$$

where $v_{1}\left(\equiv U_{1} / k_{B} T>0\right)$ and $U_{0}$ is the anisotropic attractive (Maier-Saupe) interaction between liquid crystals(Brochard et.al., 1984; Maier \& Saupe, 1958). (The subscript symbols $c$ and $r$ in Eq. (2) are changed to 1 and 2, respectively.) For the interaction between nanotubes, we here take into account both the attractive interaction and excluded volume one:(Matsuyama \& Kato, 1996)

$$
\beta_{22}=2 L^{2} D\left|\mathbf{u} \times \mathbf{u}^{\prime}\right|-v_{2} v_{2} P_{2}(\cos \theta) P_{2}\left(\cos \theta^{\prime}\right),
$$

where the first term is the excluded volume interaction between nanotubes, or rods,(Onsager, $1949)$ and the $v_{2}\left(\equiv U_{2} / k_{B} T>0\right)$ is the attractive (Maier-Saupe) interaction between nanotubes. The interaction between a liquid crystal and a nanoparticle is given by

$$
\beta_{12}=-v_{12} v_{12} P_{2}(\cos \theta) P_{2}\left(\cos \theta^{\prime}\right),
$$

where the anisotropic interaction $v_{12}\left(\equiv U_{12} / k_{B} T\right)$ between a liquid crystal and a rod can be positive or negative value. We here assume that the excluded volume interaction of a liquid crystal can be negligible because the length of liquid crystal is short. The volume $v_{12}=(\pi / 4) L_{1} L_{2} D$ is the average excluded volume between a rod and a liquid crystal in an isotropic phase, Using Eqs. (29), (30), and (31), we can obtain the mixing free energy (15) for nanotube/liquid crystal mixtures. We here define an interaction parameter between a nanotube and a liquid crystal:

$$
c_{12}=v_{12} / v_{1},
$$

which becomes an important parameter in the phase behavior. 


\subsection{Phase diagrams of nanotube/liquid crystal mixtures}

In this subsection, we show some phase diagrams calculated from the free energy(Matsuyama, 2010).

3.3.1 Uniaxial nematic $N_{0}$ phase

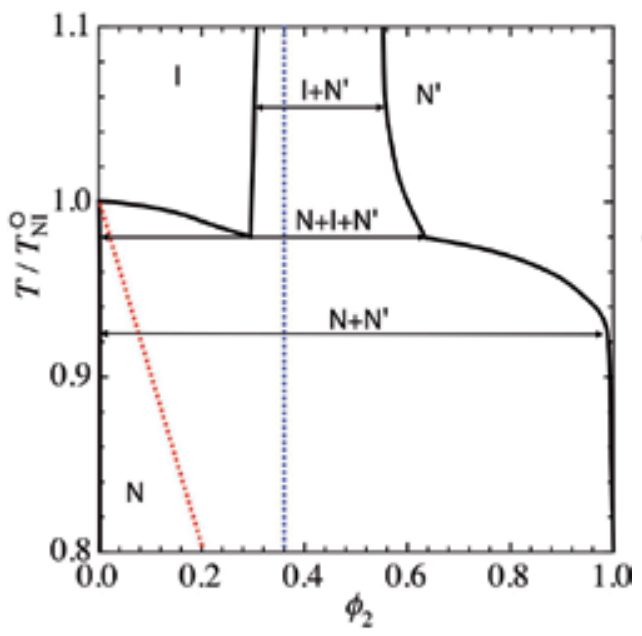

(a)

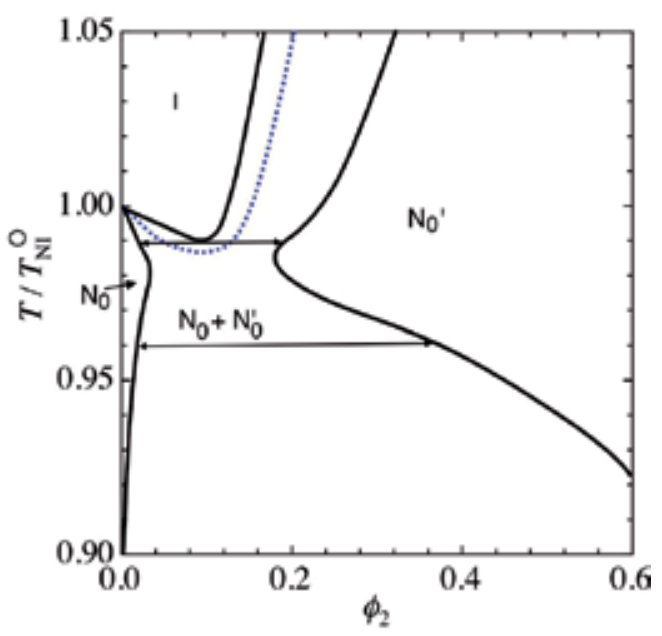

(b)

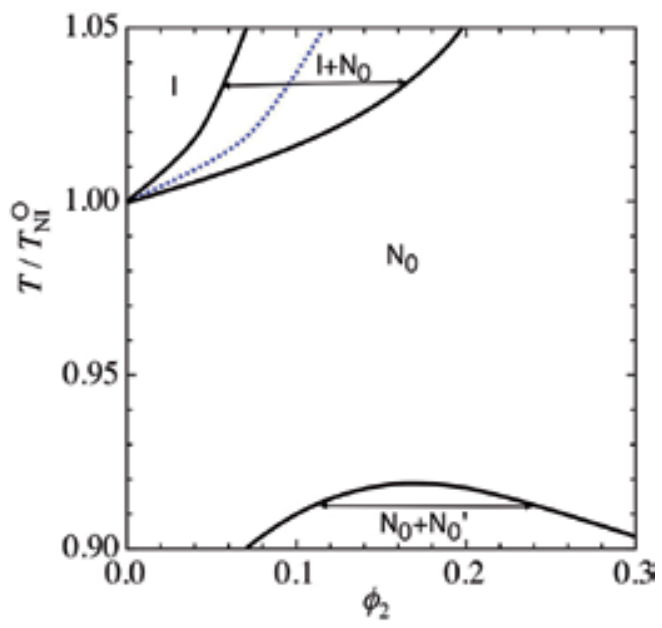

(c)

Fig. 6. Phase diagrams for $c_{12}=0$ (a), $c_{12}=0.3(\mathrm{~b})$, and $c_{12}=0.4$ (c) with $n_{1}=2$ and $n_{2}=10$.

We first show the phase diagram for $c_{12}=0$ (see Fig. 6(a)), where the excluded volume interaction between nanotubes only prevails. The solid curve shows the binodal. The red and blue dotted lines show the first-order NIT line of a liquid crystal and that of a nanotube, respectively. Above $T / T_{N I}^{\circ}=1$, the NIT of nanotubes takes place with increasing the concentration of the nanotube due to the excluded volume interaction between nanotubes, 
and we have the isotropic (I)-nematic $(\mathrm{N})$ phase separation $\left(\mathrm{I}+\mathrm{N}^{\prime}\right)$, which has been obtained by Onsager theory(Onsager, 1949) and Flory's lattice theory(Flory, 1956; 1979). At the low temperatures of the NIT line (red line) of liquid crystals, we have a nematic $(\mathrm{N})$ phase, where liquid crystals are in a nematic state but nanotubes are in an isotropic state. We predict the chimney type phase diagram with a triple point $\left(\mathrm{N}+\mathrm{I}+\mathrm{N}^{\prime}\right)$. Below the triple point, we have the broad nematic-nematic $\left(\mathrm{N}+\mathrm{N}^{\prime}\right)$ phase separation. The nematic $\mathrm{N}$ phase at lower concentrations consists of almost pure liquid crystals and the $\mathrm{N}^{\prime}$ phase are formed by the orientational ordering of rods. Near $T / T_{N I}^{\circ}<1$, we have the $\mathrm{N}+\mathrm{I}$ phase separation.

Figure $6(\mathrm{~b})$ shows the phase diagram for $c_{12}=0.3$. On increasing the coupling constant $c_{12}$, the NIT lines shift to higher temperatures and lower concentrations and two NIT curves appeared in Fig. 6(a) merge. Below the NIT line (blue dotted line), we have a nematic $\mathrm{N}_{0}$ phase, where the rods and the liquid crystals are oriented to be parallel to each other $\left(S_{1}>0\right.$ and $S_{2}>0$ ). The width of the biphasic region $\mathrm{I}+\mathrm{N}_{0}^{\prime}$ decreases with decreasing temperature. We find the triple point $\left(\mathrm{N}_{0}+\mathrm{I}+\mathrm{N}_{0}^{\prime}\right)$, where the nematic $\mathrm{N}_{0}$, isotropic $(\mathrm{I})$, and nematic $\mathrm{N}_{0}^{\prime}$ phases simultaneously coexist. The binodal line of the $\mathrm{N}_{0}$ phase shifts to higher concentrations and that of the $\mathrm{N}_{0}^{\prime}$ phase shifts to lower concentrations with increasing $c_{12}$. Below the triple point we have the phase separation $\mathrm{N}_{0}+\mathrm{N}_{0}^{\prime}$, where the two nematic $\mathrm{N}_{0}$ phases with the different concentrations can coexist.

Figure $6(\mathrm{c})$ shows the phase diagram for $c_{12}=0.4$. The binodal curve sprits into two parts: one is the phase separation $\mathrm{I}+\mathrm{N}_{0}$ with the lower critical solution temperature (LCST) at $T / T_{N I}^{\circ}=1$ and the other is the phase separation $\mathrm{N}_{0}+\mathrm{N}_{0}^{\prime}$ with the upper critical solution temperature (UCST). We have the stable nematic $\mathrm{N}_{0}$ phase between the LCST and UCST. The length of a nanotube is also important to understand the phase diagrams. On increasing the length of the nanotube, the biphasic regions are broadened. Such LCST type phase diagram has been observed in mixtures of a main-chain nematic polyesters (poly[oxy(chloro-1,4-phenylene)oxycarbonyl][(trifluoromethyl)-1,4-phenylene]carbonyl)(PTFC) with a nematic liquid crystal (p-azoxyanisole)(PAAd14)(Ratto et.al., 1991). The theory can qualitatively describe the observed phase diagram.

\subsubsection{Uniaxial nematic $N_{1}$ and $N_{2}$ phases}

When the coupling parameter $c_{12}$ is negative, we can expect that the nanotubes and liquid crystals are oriented to be perpendicular with each other.

Figure 7 shows the phase diagram for $c_{12}=-0.2(a)$ and $c_{12}=-0.5(b)$. The binodal line (solid line) is similar to Fig. 6(a), however, the structure of the nematic phases is different. In Fig. 7(a), the red dotted line at low concentrations shows the first-order nematic $\left(\mathrm{N}_{1}\right)$-isotropic phase transition $\left(1 \mathrm{st}-\mathrm{N}_{1} \mathrm{IT}\right)$ and the red dotted line at high concentrations shows the first-order nematic $\mathrm{N}_{1}-\mathrm{N}_{2}$ phase transition $\left(\mathrm{N}_{1} \mathrm{~N}_{2} \mathrm{~T}\right)$. The blue broken line corresponds to the second-order $\mathrm{N}_{1}$-I phase transition ( $2 \mathrm{nd}-\mathrm{N}_{1} \mathrm{IT}$ ), where the orientational order parameters continuously change. We also find the tricritical point (TCP) at which the 1 st $-\mathrm{N}_{1}$ IT meets the 2nd- $\mathrm{N}_{1} \mathrm{IT}$. The phase diagram shows the three phase coexistence between $\mathrm{N}_{1}$, I and $\mathrm{N}_{2}$ phases at $T / T_{N I}^{\circ} \approx 0.92$. Above the triple point, the $\mathrm{I}+\mathrm{N}_{2}$ and $\mathrm{N}_{1}+\mathrm{I}$ phase separations appear. Below the triple point, we have the $\mathrm{N}_{1}+\mathrm{N}_{2}$ phase separation.

On decreasing $c_{12}(<0)$, the system favors more perpendicular alignment. Figure 7(b) shows the phase diagram for $c_{12}=-0.5$. (Note that the phase diagram is only shown for low 
concentrations.) The two nematic-isotropic phase transitions: 1 st $-\mathrm{N}_{1} \mathrm{IT}$ and 2 nd- $\mathrm{N}_{1} \mathrm{IT}$, shift to higher temperatures and pass the binodal line of the isotropic phase in Fig. 7(a). The 2 nd- $\mathrm{N}_{1}$ IT (blue broken line) appears at lower concentrations than the binodal line and we have the homogeneous $\mathrm{N}_{1}$ phase. Near $T / T_{N I}^{\circ}=1$, the narrow biphasic region $\mathrm{N}_{1}+\mathrm{I}$ appears. Inside the binodal region, we have the $1 \mathrm{st}-\mathrm{N}_{1} \mathrm{IT}$ line (red dotted line). This $\mathrm{N}_{1}+\mathrm{I}$ phase separation disappears at TCP. At higher concentrations, the $\mathrm{N}_{1}+\mathrm{N}_{2}$ phase separation appears. The binodal curve of the coexisting $\mathrm{N}_{2}$ phase exists at $\phi_{2} \approx 0.7$, although it is not depicted in this figure. Further decreasing $c_{12}(<0)$, the 1 st- $\mathrm{N}_{1}$ IT disappears and we have the 2 nd- $\mathrm{N}_{1} \mathrm{IT}$ and the 2 nd $-\mathrm{N}_{1}$ IT temperature increases with increasing $\phi_{2}$.

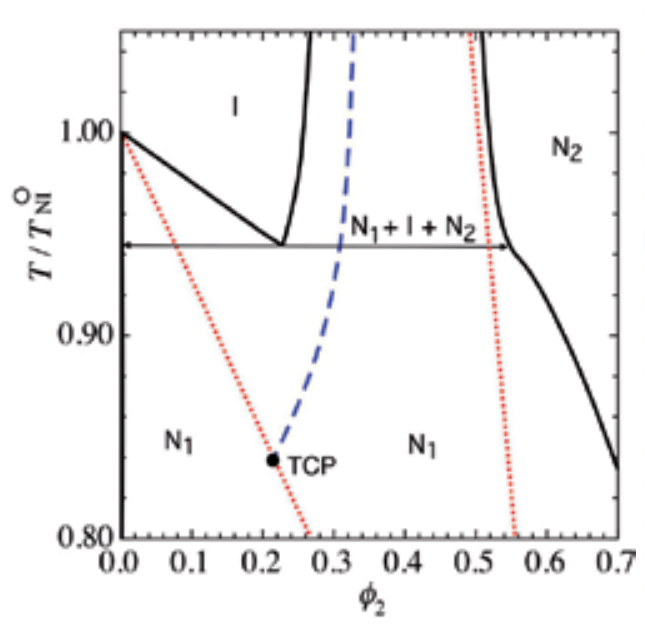

(a)

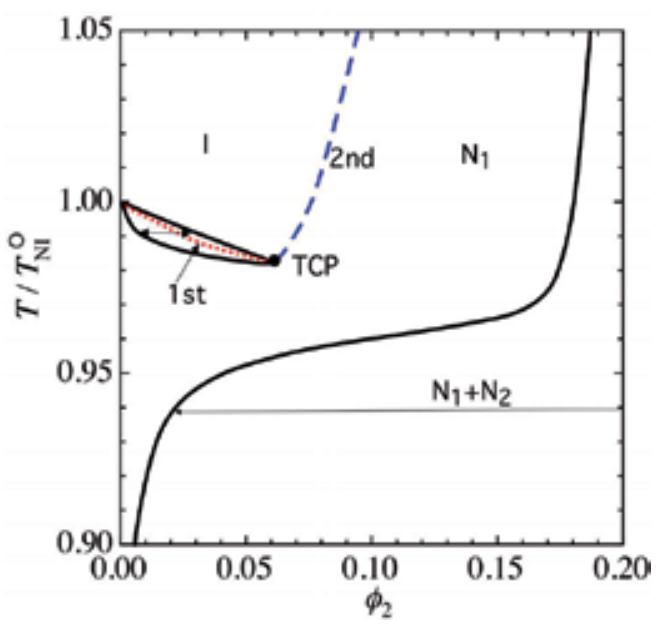

(b)

Fig. 7. Phase diagrams for $c_{12}=-0.2(\mathrm{a})$ and $c_{12}=-0.5(\mathrm{~b})$.

Recent experimental studies of multi-wall carbon nanotube(CNT)/nematic liquid crystal mixtures(Duran et.al., 2005) have observed the NIT temperature of the liquid crystal is enhanced by the incorporation of CNT within a small composition gap and suggested that this enhanced NIT temperature phenomenon is attributed to anisotropic alignment of liquid crystals along the CNT bundles. Our model predicts two kind of phase behavior. When the $\mathrm{CNTs}$ and liquid crystals are parallel, the system shows the first-order isotropic-nematic $\left(\mathrm{N}_{0}\right)$ phase transition. On the other hand, if the CNTs and liquid crystals favor to be perpendicular each other, we have the 1 st- and 2 nd $-\mathrm{N}_{1} \mathrm{IT}$. The appearance of these phase transitions is strongly effected by the orientational order of nanotubes and liquid crystals.

\subsubsection{Effect of external fields}

To form a nematic $N_{3}$ phase, external forces such as electric or magnetic fields will be important. When the external magnetic or electric field $\mathbf{E}$ is applied to the nanotubes and liquid crystals having a dielectric anisotropy $\Delta \epsilon_{i} \equiv \epsilon_{\|, i}-\epsilon_{\perp, i}(i=1,2)$, the free energy changes due to the external field is given by(de Gennes \& Prost, 1993)

$$
a^{3} \beta F_{\text {ext }} / V=-\phi_{1} \beta \Delta \epsilon_{1} \int(\mathbf{n} \cdot \mathbf{E})^{2} f_{1}(\mathbf{u}) d \Omega-\phi_{2} \beta \Delta \epsilon_{2} \int(\mathbf{l} \cdot \mathbf{E})^{2} f_{2}(\mathbf{u}) d \Omega
$$


where $\mathbf{n}$ and $\mathbf{1}$ are the unit orientation vector of the liquid crystal and the nanotube, respectively.

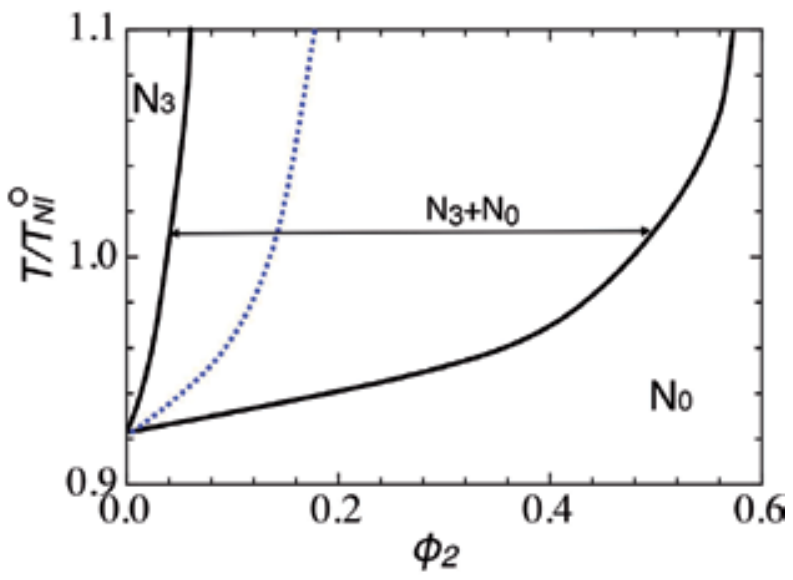

(a)

Fig. 8. Phase diagram under an external field for $\Delta \epsilon_{1}=-1$ and $\Delta \epsilon_{2}=1$, where the liquid crystals tend to align perpendicular to the electric field $\mathbf{E}$, while the nanotubes tend to parallel to $\mathbf{E}$.

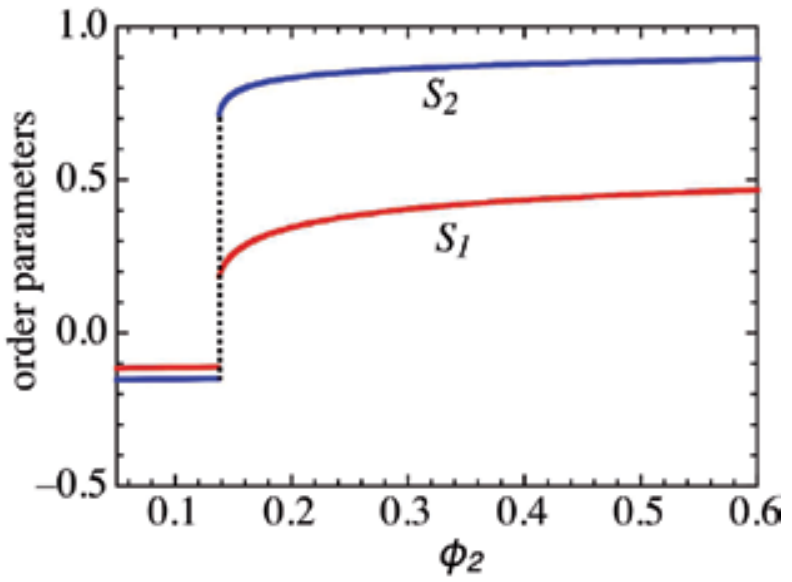

(a)

Fig. 9. Order parameters $S_{1}$ and $S_{2}$ plotted against $\phi_{2}$ at $T / T_{N I}^{\circ}=0.98$ in Fig. 8 . We find the phase transition from the $\mathrm{N}_{3}$ phase with $S_{1}<0$ and $S_{2}<0$ to the $\mathrm{N}_{0}$ phase with $S_{1}>0$ and $S_{2}>0$.

We here consider the case of $\Delta \epsilon_{1}<0$ and $\Delta \epsilon_{2}>0$ : the liquid crystals tend to align perpendicular to the electric field $\mathbf{E}$, while the nanotubes tend to parallel to $\mathbf{E}$. We apply the external field on Fig. 6(c), where the coupling $c_{12}(=0.4)$ between the liquid crystal and nanotube is strong. Figure 8 shows the phase diagram under an external field for $\Delta \epsilon_{1}=-1$ and $\Delta \epsilon_{2}=1$. The binodal line is broadened, compared with Fig. 6(c). We find the $\mathrm{N}_{3}$ phase at low concentrations of nanotubes, where most liquid crystals tend to perpendicular 
to the eternal field and nanotubes favor to be parallel to liquid crystals because of the strong coupling $c_{12}$ even $\Delta \epsilon_{2}=1$. The blue dotted line shows the 1 st-order $\mathrm{N}_{3}-\mathrm{N}_{0}$ phase transition. Figure 9 shows the order parameters plotted against $\phi_{2}$ at $T / T_{N I}^{\circ}=0.98$. We also find the phase separation between $\mathrm{N}_{3}$ and $\mathrm{N}_{0}$ phases. We emphasize that we can control the four nematic phases by applying external fields.

\subsection{Biaxial nematic ordering in nanotube/liquid crystal mixtures}

Biaxial nematic phase has been first theoretically predicted by Freiser(Freiser, 1970). Since then, it has been the subject of much experimental(Galerne \& Marcerou, 1983; Madsen et. al., 2004; Yu \& Saupe, 1980), computational(Biscarini et. al., 1995; Hudson \& Larson, 1993), and theoretical(Alben, 1973; Palffy-Muhoray et. al., 1984; Sharma et. al., 1985; Straley, 1974) work (see a recent review(Tschierske \& Photinos, 2010)). Biaxiality occurs if anisotropic particles orient along a second axis perpendicular to a main director of the particles(Singh, 2000). Recently it has been experimentally observed a biaxial phase in colloidal dispersions of boardlike particles(van den Pol et. al., 2009). Such biaxiality is expected significant advantages in display applications with a fast response.(Luckhurst, 2001)

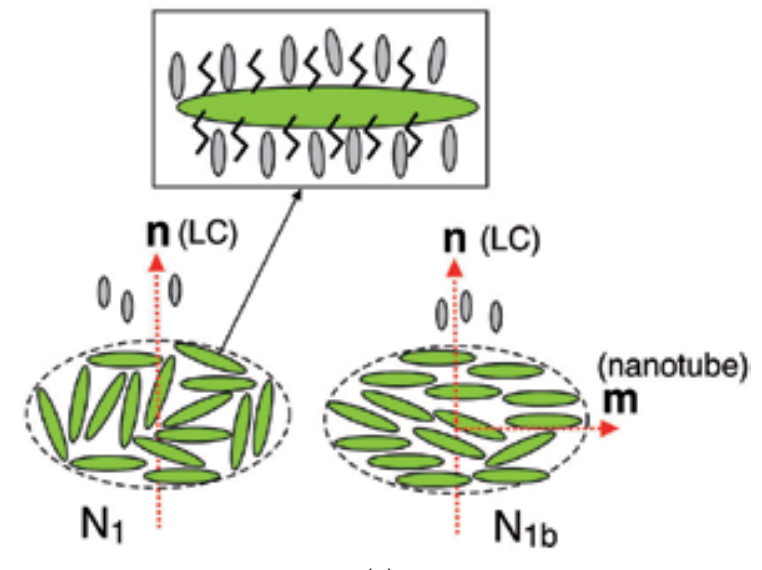

(a)

Fig. 10. Uniaxial planar nematic phase $\left(\mathrm{N}_{1}\right)$ and biaxial nematic phase $\left(\mathrm{N}_{1 b}\right)$ in mixtures of a long nanotube and a short liquid crystal, which favor perpendicular orientations with each other. Nanotubes on an easy plane induce the additional ordering of nanotubes in the direction $\mathbf{m}$ perpendicular to the director $\mathbf{n}$ and yield a biaxial nematic phase $\mathrm{N}_{1 b}$.

As discussed in Section 3.1, when the order parameter of one component is positive, determining the nematic director, and the order parameter of the second component is negative, we have planar nematic phases $\left(\mathrm{N}_{1}\right.$ and $\left.\mathrm{N}_{2}\right)$, where the second component is randomly distributed within the perpendicular plane to the director. In these nematic phases $\left(\mathrm{N}_{1}, \mathrm{~N}_{2}\right)$, we can expect either a uniaxial or a biaxial nematic phase.

Figure 10 schematically shows a novel biaxial nematic in nanotube/liquid crystal mixtures, where the two components favor a mutually perpendicular orientation.(Matsuyama, 2011) The mutually perpendicular alignments of nanotubes and liquid crystals can be achieved by wrapping polymers or surfactants on nanotube's surface(Badaire et. al., 2005; Zhang \& 
Kumar, 2008). To form such mutually perpendicular alignments, the anisotropic interaction (enthalpy) between a nanotube and a liquid crystal is needed. Moreover, in the planar nematic $\mathrm{N}_{1}$ phase, on increasing concentration of nanotubes, we can expect that the excluded volume interaction (entropy) between nanotubes on an easy plane induces the additional ordering of nanotubes in the direction $\mathbf{m}$ (the second "minor" director) perpendicular to the director $\mathbf{n}$ (the first "major" director) of liquid crystals and yields a biaxial nematic phase $\left(\mathrm{N}_{1 b}\right)$. In the $\mathrm{N}_{2}$ phase, we may have a biaxial nematic phase $\left(\mathrm{N}_{2 b}\right)$, where the additional ordering of liquid crystals appears in the direction $\mathbf{m}$ (minor director) perpendicular to the alignment $\mathbf{n}$ (major director) of nanotubes. Such a biaxiality in mixtures of two types of rodlike molecules has been first suggested by Alben(Alben, 1973). In this subsection, we introduce phase diagrams including such biaxial nematic phases. The phase diagrams appeared in Fig. 7 are drastically changed.

Using the distribution function $f_{i}(\theta, \varphi)$ of the component $i(=1,2)$, defined by a polar angle $\theta$ and an azimuthal angle $\varphi$, the biaxial order parameter is given by

$$
\Delta_{i}=\int D(\theta, \varphi) f_{i}(\theta, \varphi) d \Omega,
$$

where $D(\theta, \varphi) \equiv(\sqrt{3} / 2) \sin ^{2} \theta \cos (2 \varphi)$. Using the tensor order parameter

$$
S_{i, \alpha \beta}=(3 / 2) S_{i}\left(n_{\alpha} n_{\beta}-\delta_{\alpha \beta} / 3\right),
$$

$(\alpha, \beta=x, y, z)$, we have $\Delta_{i}=S_{i, y y}-S_{i, x x}$ and $S_{i}=S_{i, z z}\left(\right.$ Singh, 2000). Here $S_{i, z z}$ describes alignment of molecules along the $z$ axis (major director), whereas the nonzero value of $\Delta_{i}$ describes ordering along the $x$ or $y$ axis. Using the order parameters, we can define an isotropic (I) phase with $S_{i}=\Delta_{i}=0$, a uniaxial $\mathrm{N}_{1}$ phase: $S_{1}>0, S_{2}<0, \Delta_{i}=0$, a uniaxial $\mathrm{N}_{2}$ phase: $S_{1}<0, S_{2}>0, \Delta_{i}=0$, a biaxial $\mathrm{N}_{1 b}$ phase: $S_{1}>0, S_{2}<0, \Delta_{i} \neq 0$, and a biaxial $\mathrm{N}_{2 b}$ phase: $S_{1}<0, S_{2}>0, \Delta_{i} \neq 0$. Using the additional theorem of a spherical harmonics in Eqs. (29), (30), and (31), we have $P_{2}(\cos \gamma)=P_{2}(\cos \theta) P_{2}\left(\cos \theta^{\prime}\right)+D(\theta, \varphi) D\left(\theta^{\prime}, \varphi^{\prime}\right)$ and can calculate phase separations(Matsuyama, 2011).

Figure 11 shows the phase diagrams numerically calculated for $c_{12}=-0.5$ (a) and -0.8 (b). Black lines show the binodal line. The red (blue) lines show a first (second)-order phase transition, where the order parameters discontinuously (continuously) change. The biaxial nematic phase $\mathrm{N}_{1 b}$, which includes an unstable biaxial phase, a metastable biaxial, and a stable biaxial phase, is indicated by the yellow area. In Fig. 11(a), at high temperatures, we have the phase separation ( $\left(\mathrm{I}+\mathrm{N}_{2}\right)$ between an isotropic (I) phase at $\phi_{2} \simeq 0.14$ and a uniaxial $\mathrm{N}_{2}$ phase at $\phi_{2} \simeq 0.63$. Such a chimney type's phase diagram with a coexistence between I and $\mathrm{N}$ phases is induced by the excluded volumes between long rods(Flory, 1956; 1979; Matsuyama \& Kato, 1996; Onsager, 1949). Inside the binodal lines, we find the first-order isotropic-biaxial $\mathrm{N}_{1 b}$ phase transition at $\phi_{2} \simeq 0.22$ and the first-order biaxial $\mathrm{N}_{1 b}$-uniaxial $\mathrm{N}_{2}$ phase transition at $\phi_{2} \simeq 0.5$. Above $\phi_{2} \simeq 0.6$, we have a stable uniaxial $N_{2}$ phase. We also find the three phase coexistence, or triple point (TP), between $\mathrm{N}_{1}+\mathrm{I}+\mathrm{N}_{2}$ at $\tau\left(\equiv T / T_{N I}^{\circ}\right) \simeq 0.98$. Below the TP, we have the $\mathrm{N}_{1}+\mathrm{N}_{2}$ phase separation. At low concentrations, the $\mathrm{N}_{1}+\mathrm{I}$ phase separation appears. The biaxial nematic phase is hidden inside the binodal lines.

Further increasing $c_{12}$ (Fig. 11(b)), the coupling between a liquid crystal and a nanotube drastically changes the phase diagram. The biaxial regions shift to lower concentrations and 


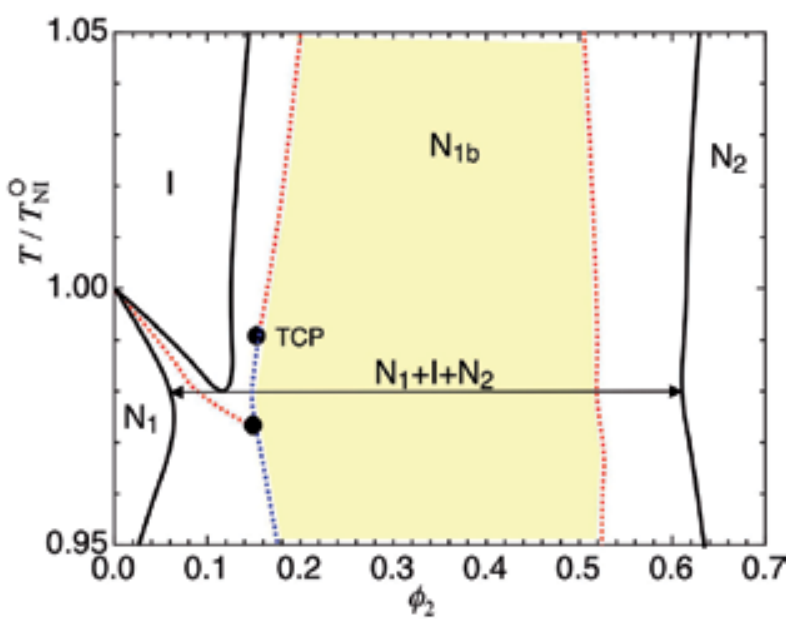

(a)

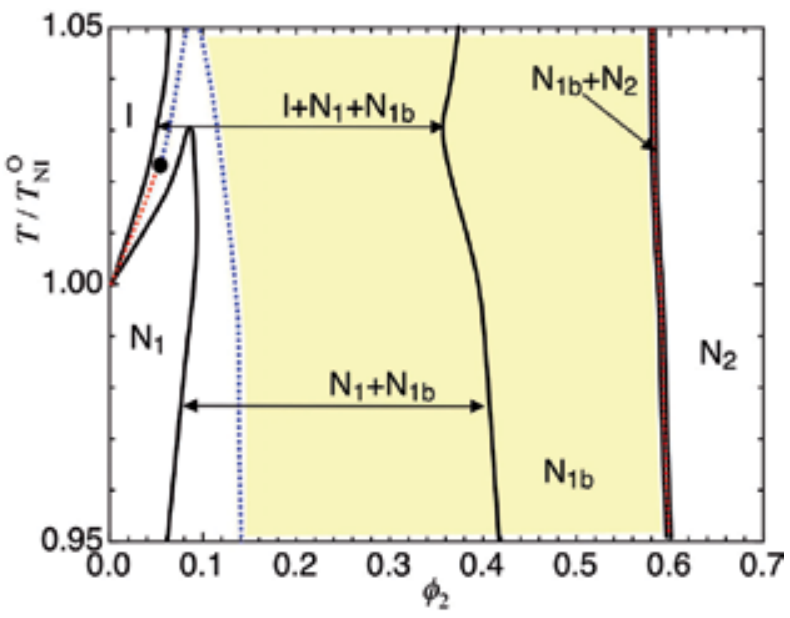

(b)

Fig. 11. Phase diagrams on the temperature $\left(\tau \equiv T / T_{N I}^{\circ}\right)$-volume fraction $\left(\phi_{2}\right)$ plane for $c_{12}=-0.5(\mathrm{a})$ and $-0.8(\mathrm{~b})$. The black lines indicate the binodal. The red (blue) lines show a first (second)-order phase transition, where the order parameters discontinuously (continuously) change. The biaxial nematic phase $\mathrm{N}_{1 b}$, which includes an unstable biaxial phase, a metastable biaxial, and a stable biaxial phase, is indicated by the yellow area. The stable biaxial phase $\mathrm{N}_{1 b}$ appears on (b). 
the thermodynamically stable biaxial $\mathrm{N}_{1 b}$ phase appears between $\phi_{2} \sim 0.4$ and $\phi_{2} \sim 0.6$. We find the phase separations: I+N $\mathrm{N}_{1 b}, \mathrm{~N}_{1}+\mathrm{N}_{1 b}, \mathrm{I}+\mathrm{N}_{1}, \mathrm{~N}_{1 b}+\mathrm{N}_{2}$, and the three phase coexistence $\mathrm{I}+\mathrm{N}_{1}+\mathrm{N}_{1 b}$ at $\tau \simeq 1.03$. Note that the coexistence region $\left(\mathrm{N}_{1 b}+\mathrm{N}_{2}\right)$ at $\phi_{2} \simeq 0.6$ is very narrow. At low concentrations, the I- $\mathrm{N}_{1}$ phase transition temperature increases with increasing $\phi_{2}$ and the TP shifts to higher temperatures. Note that the stable biaxial phase $\mathrm{N}_{1 b}$ appears on (b).

Duran et.al have observed in multiwall CNT/liquid crystal mixtures that the NIT temperature of the liquid crystal is enhanced by the incorporation of CNT(Duran et.al., 2005). Our theory demonstrates that this enhanced NIT temperature phenomena is attributed to anisotropic coupling between CNTs and liquid crystals. A mutually perpendicular orientation between rods and LCs can be achieved by wrapping surfactants on nanotube's surface, like a Langmuir-Blodgett film with liquid crystals(Barbero \& Durand, 1996), where liquid crystals in contact with the surfactants are oriented by steric interaction with the molecules on rods. These modifications can change the strength of the interaction parameter $v_{12}$ in our model and give a possibility of a novel biaxial phase in this mixture. The biaxial $\mathrm{N}_{2 b}$ phase does not appear on the phase diagrams because the length of liquid crystal is too short to form the $\mathrm{N}_{2 b}$ phase.

\section{Summary}

In this chapter we have reviewed the possible phase separations in mixtures of a nanoparticle and a liquid crystal, based on the mean field theory. In Section 2, we have introduced mixtures of a spherical nanoparticle and a liquid crystal. Ferroelectric spherical nanoparticles dispersed in liquid crystal have a possibility of various phase separations, discussed in this chapter. In Section 3, we have introduced phase diagrams in mixtures of a nanotube and a liquid crystal. Novel uniaxial and biaxial nematic phases are theoretically predicted. We also discuss the effect of external fields in nanotube/liquid crystal mixtures. Phase diagrams introduced in this chapter have not been experimentally observed yet, however, it will be a challenging subject from both an experimental and theoretical point of view.

\section{Acknowledgment}

These studies were supported by Grant-in Aid for Scientific Research (C) (Grant No. 23540477) and that on Priority Area "Soft Matter Physics" from the Ministry of Education, Culture, Sports, Science and Technology of Japan (Grant No. 21015025).

\section{References}

Alben, R. (1973).Liquid crystal phase transitions in mixtures of rodlike and platelike molecules,J. Chem. Phys., Vol. 59, No. 3, (Oct. 1973) pp.4299-4304. DOI:10.1063/1.1680625.

Alder, B. J.; Wainwright, T. E. (1957). Phase Transition for a Hard Sphere System,J. Chem. Phys., Vol. 27, No. 5, (Nov. 1957) pp.1208-1209. DOI:10.1063/1.1743957.

Anderson, V. J.; Terentjev, E. M.; Meeker,S.P.; Crain, J.; Poon, W. C. K. (2001). Cellular solid behaviour of liquid crystal colloids 1 . Phase separation and morphology.Eur. Phys. J. E, Vol. 4, No. 1, (Jan. 2001) pp.11-20. 
Anderson, V. J.;Terentjev, E. M. (2001). Cellular solid behaviour of liquid crystal colloids 2. Mechanical properties.Eur. Phys. J. E, Vol. 4, No. 1, (Jan. 2001) pp.21-28.

Araki, T.; Tanaka, H. (2004). Nematohydrodynamic Effects on the Phase Separation of a Symmetric Mixture of an Isotropic Liquid and a Liquid Crystal,Phys. Rev. Lett., Vol. 93, No. 1, (Jun. 2004) pp.015702-1-015702-4. DOI:10.1103/PhysRevLett.93.015702.

Badaire, S.; Zakri, C.; Maugey, M.; Derre, A.; Barisci, J. N.; Wallace, G.; Poulin, P. (2005) Liquid Crystals of DNA-Stabilized Carbon Nanotubes,Adv. Mater., Vol. 17, No. 13, (Jul. 2005) pp.1673-1676. DOI:10.1002/adma.200401741.

Barbero, G. \& Durand,G. (1996). Surface Anchoring of Nematic liquid Crystals, In: Liquid Crystals in Complex Geometries, Crawford, G.P.; Zumer, S., (Ed.), pp.21-52, Taylor \& Francis, ISBN:0-7484-0464-3, London

Basu, R.; Iannacchione, G. S. (2008) Carbon nanotube dispersed liquid crystal: A nano electromechanical system,Appl. Phys. Lett., Vol. 93, No. 18, (Nov. 2008) pp.183105-1-183105-3. DOI: 10.1063/1.3005590.

Biscarini, F.; Chiccoli, C.; Pasini, P.; Semeria, F.; Zannoni, C. (1995) Phase Diagram and Orientational Order in a Biaxial Lattice Model: A Monte Carlo Study,Phys. Rev. Lett., Vol. 75, No. 9, (Aug. 1995) pp.1803-1806. DOI: 10.1103/PhysRevLett.75.1803.

Brochard, F.; Jouffroy, J.; Levinson, P. (1984). Phase diagrams of mesomorphic mixtures, J. de Phys., Vol. 45, No. 7, (Jul. 1984) pp.1125-1136. DOI: 10.1051/jphys:019840045070112500.

Cates, M. E.; Evans, M. R. (2000). Soft and Fragile Matter, Institute of Physics Publishing, ISBN: 0-7503-0724-2, Bristol.

Caggioni, M.; Giacometti, A.; Bellini, T.; Clark, N. A.; Mantegazza, F.; Maritan, A. (2005). Pretransitional behavior of a water in liquid crystal microemulsion close to the demixing transition: Evidence for intermicellar attraction mediated by paranematic fluctuations.J. Chem. Phys., Vol. 122, No. 21, (Jun. 2005) pp.214721-1-214721-19. DOI:10.1063/1.1913444.

Chiu, H. W.; Kyu, T. (1999). Spatio-temporal growth of nematic domains in liquid crystal polymer mixtures.J. Chem. Phys., Vol. 110, No. 12, (Mar. 1999) pp.5998-6006. DOI:10.1063/1.478502.

Copic, M.; Mertelj, A.; Buchev, O.;Reznikov, Y. (2007). Coupled director and polarization fluctuations in suspensions of ferroelectric nanoparticles in nematic liquid crystals, Phys. Rev. E, Vol. 76, No. 1, (Jul. 2007) pp.011702-1-011702-5. DOI:10.1103/PhysRevE.76.011702.

Courty, S.; Mine, J.; Tajbakhsh, A. R.; Terentjev, E. M. (2003). Nematic elastomers with aligned carbon nanotubes: New electromechanical actuators, Europhys. Lett., Vol. 64, No. 5, (Dec. 2003) pp.654-660, DOI:10.1209/epl/i2003-00277-9.

Das, S. K.; Ray, A. D., (2005). Colloidal crystal formation via polymer-liquid-crystal demixing. Europhys. Lett., Vol. 70, No. 5, (May 2005) pp.621-627. DOI: 10.1209/epl/i2005-10034-2.

de Gennes, P. G. \& Prost, J. (1993). The Physics of Liquid Crystals, Oxford University Press, ISBN: 019-851785-8, New York.

Dierking, I.; Scalia, G.; Morales, P., (2005). Liquid crystal?carbon nanotube dispersions, J. Appl. Phys., Vol. 97, No. 4, (Jan. 2005) pp.044309-1-044309-5. DOI: 10.1063/1.1850606. 
Dubaut, A.; Casagrande, C.; Veyssie, M., Deloche, B., (1980). Pseudo Clearing Temperature in Binary Polymer-Nematic Solutions. Phys. Rev. Lett., Vol. 45, No. 20, (Nov. 1980) pp.1645-1648. DOI:10.1103/PhysRevLett.45.1645.

Duran, H.; Gazdecki,; Yamashita, A.; Kyu, T. (2005). Effect of carbon nanotubes on phase transitions of nematic liquid crystals, Liq. Cryst., Vol. 32, No. 7, (Jul. 2005) pp.815-821. DOI:10.1080/02678290500191204.

Flory, P. J. (1953). Principles of Polymer Chemistry, Cornell University, ISBN 0-8014-0134-8, Ithaca, New York.

Flory, P. J. (1956). Statistical Thermodynamics of Semi-Flexible Chain Molecules, Proc. R. Soc. London Ser. A, Vol. 234, No. 1196, (Jan. 1956) pp.60-73. DOI:10.1098/rspa.1956.0015.

Flory, P. J.; Ronca, G. (1979). Theory of Systems of Rodlike Particles: I. Athermal systems, Mol. Cryst. Liq. Cryst., Vol. 54, No. 3, (Mar. 1979) pp.289-309. DOI:10.1080/00268947908084861.

Freiser, M. J. (1970). Ordered States of a Nematic Liquid, Phys. Rev. Lett., Vol. 24, No. 19, (May 1970) pp.1041-1043. DOI:10.1103/PhysRevLett.24.1041.

Fukuda, J.; Yokoyama, H., (2005). Separation-Independent Attractive Force between Like Particles Mediated by Nematic-Liquid-Crystal Distortions, Phys. Rev. Lett., Vol. 94, No. 14, (Apr. 2005) pp.148301-1-148301-4. DOI:10.1103/PhysRevLett.94.148301.

Fukuda, J. (2009). Liquid Crystal Colloids: A Novel Composite Material Based on Liquid Crystals, J. Phys. Soc. Jpn., Vol. 78, No. 4, (Apr. 2009) pp.041003-1-041003-9. DOI: 10.1143/JPSJ.78.041003.

Galerne, Y.; Marcerou, J.P. (1983). Temperature Behavior of the Order-Parameter Invariants in the Uniaxial and Biaxial Nematic Phases of a Lyotropic Liquid Crystal, Phys. Rev. Lett., Vol. 51, No. 23, (Dec. 1983) pp.2109-2112. DOI:10.1103/PhysRevLett.51.2109.

Hudson, S.D.; Larson, R.G. (1993). Monte Carlo simulation of a disclination core in nematic solutions of rodlike molecules, Phys. Rev. Lett., Vol. 70, No. 19, (May 1993) pp.2916-2919. DOI:10.1103/PhysRevLett.70.2916.

Iijima, S. (1991). Helical microtubules of graphitic carbon, Nature, Vol. 354, No. 6348, (Nov. 1991) pp.56-58. DOI:10.1038/354056a0.

Jayalakshmi, V.; Prasad, S. K. (2009). Understanding the observation of large electrical conductivity in liquid crystal-carbon nanotube composites, Appl. Phys. Lett., Vol. 94, No. 20, (May 2009) pp.202106-1-202106-3. DOI: 10.1063/1.3133352.

Kirkwood, G.; Monroe, E. (1941). Statistical Mechanics of Fusion, J. Chem. Phys., Vol. 9, No. 7, (Jul. 1941) pp.514-526. DOI:10.1063/1.1750949.

Kralj, S.; Bradac, Z.; Popa-Nita, V. (2008). The influence of nanoparticles on the phase and structural ordering for nematic liquid crystals, J. Phys.: Condens. Matter, Vol. 20, No. 24, (Jun. 2008) pp.244112. DOI:10.1088/0953-8984/20/24/244112.

Kuksenok, O. V.; Ruhwandl, R. W.; Shiyanovskii, S. V.; Terentjev, E. M., (1996). Director structure around a colloid particle suspended in a nematic liquid crystal, Phys. Rev. E, Vol. 54, No. 5, (Nov. 1996) pp.5198-5203. DOI:10.1103/PhysRevE.54.5198.

Kventsel, G. F.; Luckhurst, G. R.; Zewdie, H. B. (1985). A molecular field theory of smectic A liquid crystals, Molc. Phys., Vol. 56, No. 3, (Mar. 1985) pp.589-610. DOI: 10.1080/00268978500102541.

Lagerwall, J. P. F. ; Scalia, G.; Haluska, M.; Dettlaff-Weglikowska, U.; Roth, S.; Giesselmann, F. (2007). Nanotube Alignment Using Lyotropic Liquid Crystals, Adv. Mater., Vol. 19, No.3, (Feb. 2007) pp.359-364, DOI:10.1002/adma.200600889. 
Li, F.; Buchnev, O.; Cheon, C.; Glushchenko, A.; Reshetnyak, V.;Reznikov, Y.; Sluckin, T. J.; West, J. L. (2006). Orientational Coupling Amplification in Ferroelectric Nematic Colloids, Phys. Rev. Lett., Vol. 97, No.14, (Oct. 2006) pp.147801-1-147801-4, DOI: 10.1103/PhysRevLett.97.147801.

Li, F.; West, J.; Glushchenko, A.; Cheon, C. I.; Reznikov,Y. (2006). Ferroelectric nanoparticle/liquid-crystal colloids for display applications, J. Soc. Info. Disp., Vol. 14, No. 6, (Jun. 2006), pp.523-527, DOI: 10.1889/1.2210802.

Lopatina, L. M.; Selinger, J. V. (2009). Theory of Ferroelectric Nanoparticles in Nematic Liquid Crystals. Phys. Rev. Lett., Vol. 102, No.19, (May 2009) 197802-1-197802-4, DOI: 10.1103/PhysRevLett.102.197802.

Loudet, J. C.; Barois, P.; Auroy, P.; Keller, P.; Richard, H.; Pouling, P. (2004). Colloidal Structures from Bulk Demixing in Liquid Crystals, Langmuir, Vol. 20, No.26, (Sept. 2004) pp.11336-11347, DOI: 10.1021/la048737f.

Lubensky, T. C.; Pettey, D.; Currier, N.;Stark, H. (1998). Topological defects and interactions in nematic emulsions, Phys. Rev. E, Vol. 57, No.1, (Jan. 1998) pp.610-625, DOI: 10.1103/PhysRevE.57.610.

Luckhurst, G. R. (2001). Biaxial nematic liquid crystals: fact or fiction?, Thin Solid Films, Vol. 393, No.1, (Aug. 2001) pp.40-52, DOI: 10.1016/S0040-6090(01)01091-4.

Lynch, M. D.; Patrick, D. L. (2002). Organizing Carbon Nanotubes with Liquid Crystals, Nano Lett., Vol. 2, No.11, (Nov. 2002) pp.1197-625, DOI: 10.1021/nl025694j.

Madsen, L.A.; Dingemans, T.J.; Nakata, M.; Samulski, E.T. (2004). Thermotropic Biaxial Nematic Liquid Crystals, Phys. Rev. Lett., Vol. 92, No.14, (Apr. 2004) pp.145505-1-145505-4, 10.1103/PhysRevLett.92.145505.

Maier, W.; Saupe, A. (1958). A simple molecular theory of the nematic liquid-crystalline state, Z. Naturforsch, Vol. 13a, (Mar. 1958) pp.564-566.

Matsuyama, A. \& Kato, T. (1996). Theory of binary mixtures of a flexible polymer and a liquid crystal. J. Chem. Phys., Vol. 105, No.4, (Jul. 1996) pp. 1654-1660. DOI:10.1063/1.472024

Matsuyama, A.; Kato, T. (1998). Phase diagrams of polymer dispersed liquid crystals, J. Chem. Phys., Vol. 108, No.5, (Feb. 1998), pp. 2067-2072, DOI: 10.1063/1.475585.

Matsuyama, A.; Evans, R. M. L.; Cates, M. E. (2000). Orientational fluctuation-induced spinodal decomposition in polymer-liquid crystal mixtures. Phys. Rev. E, Vol. 61, No.3, (Mar. 2000), pp. 2977-2986, DOI: 10.1103/PhysRevE.61.2977.

Matsuyama, A. (2006a). Mean Field Theory of Crystalline Ordering in Colloidal Solutions, J. Phys. Soc. Jpn., Vol. 75, No.3, (Mar. 2006), pp. 034604-1-034604-9, DOI: 10.1143/JPSJ.75.034604.

Matsuyama, A. (2006b). Spinodal in a Liquid-Face Centered Cubic Phase Separation, J. Phys. Soc. Jpn., Vol. 75, No.8, (Aug. 2006), pp. 084602-1-084602-10, DOI: 10.1143/JPSJ.75.084602.

Matsuyama, A. \& Hirashima, R. (2008a). Phase separations in liquid crystal-colloid mixtures. J. Chem. Phys., Vol. 128, No.4, (Jan. 2008) 044907-1-044907-11, DOI: 10.1063/1.2823737.

Matsuyama, A. (2008b).Morphology of spinodal decompositions in liquid crystal-colloid mixtures. J. Chem. Phys., Vol. 128, No.22, (Jun. 2008) pp. (224907-1)-(224907-8), DOI: 10.1063/1.2936831.

Matsuyama, A. (2009). Phase separations in mixtures of a liquid crystal and a nanocolloidal particle. J. Chem. Phys., Vol. 131, No.20, (Nov. 2009) 204904-1-204904-12, DOI: 10.1063/1.3266509. 
Matsuyama, A. (2010). Theory of binary mixtures of a rodlike polymer and a liquid crystal. J. Chem. Phys., Vol. 132, No.21, (Jun. 2010) 214902-1-214902-10, DOI: 10.1063/1.3447892.

Matsuyama, A. (2010a). Thermodynamics of flexible and rigid rod polymer blends, In: Encyclopedia of polymer blends, Vol. 1: Fundamentals, Isayev, A. I., (Ed.), Chap.2, pp.45-100, WILEY-VCH Verlag GmbH \& Co. KGaA, ISBN: 978-3-527-31929-9, Weinheim.

Matsuyama, A. (2011). Biaxial nematic phases in rod/liquid crystal mixtures. Liq. Cryst., Vol. 38, No.6, (Jun. 2011), pp. 729-736, DOI: 10.1080/02678292.2011.570795.

Meeker,S. P.; Poon, W. C. K.; Crain, J.; Terentjev, E. M. (2000). Colloid?liquid-crystal composites: An unusual soft solid. Phys. Rev. E, Vol. 61, No.6, (Jun. 2000), pp.R6083-R6086, DOI: 10.1103/PhysRevE.61.R6083.

McMillan, W. L. (1971). Simple Molecular Model for the Smectic A Phase of Liquid Crystals, Phys. Rev. A, Vol. 4, No.3, (Sep. 1971), pp.1238-1246, DOI: 10.1103/PhysRevA.4.1238.

Musevic, I.; Skarabot, M.; Tkalec, U.; Ravnik, M.; Zummer, S. (2004). Two-Dimensional Nematic Colloidal Crystals Self-Assembled by Topological Defects. Science, Vol. 313, No.5789, (Aug. 2006), pp.954-958, DOI: 10.1126/science.1129660.

Nazarenko, V. G.; NychA. B.; Lev, B. I. (2001). Crystal Structure in Nematic Emulsion. Phys. Rev. Lett., Vol. 87, No.7, (Jul. 2001), pp.075504-1-075504-4, DOI: 10.1103/PhysRevLett.87.075504.

Onsager, L. (1949). The Effects of Shape on The Interaction of Colloidal Particles, Ann. N. Y. Acad. Sci., Vol. 51, (May 1949), pp.627-659, DOI:10.1111/j.1749-6632.1949.tb27296.x.

Palffy-Muhoray, P. ; Berlinsky, A. J.; De Bruyn, J. R.; Dunmur, D. A. (1984). Coexisting nematic phases in binary mixtures of liquid crystals, Phys. Lett. A, Vol. 104, No.3, (Aug. 1984), pp.159-162, DOI:10.1016/0375-9601(84)90367-0.

Popa-Nita, V.; van-der Schoot,P.; Kralj, S. (2006). Crystal Structure in Nematic Emulsion. Eur. Phys. J. E, Vol. 21, No.3, (Nov. 2006), pp.189-198, DOI:10.1140/epje/i2006-10059-3.

Pouling, P.; Stark, H.; Lubenski, T. C.; Weitz, D. A. (1997). Novel Colloidal Interactions in Anisotropic Fluids, Science, Vol. 275, No. 5307, (Mar. 1997), pp.1770-1773, DOI: 10.1126/science.275.5307.1770.

Pouling, P.; Raghunathan, V. A.; Richetti, P.; Roux, D. J. (1994). On the dispersion of latex particles in a nematic solution. I. Experimental evidence and a simple model, J. Phys. II , Vol. 4, No. 9, (Sept. 1994), pp.1557-1569, DOI: 10.1051/jp2:1994217.

Pusey, P. N.; van Megen, W. (1986). Phase behaviour of concentrated suspensions of nearly hard colloidal spheres, Nature, Vol. 320, No. 6060, (Mar. 1986), pp.340-1569, DOI: 10.1038/320340a0.

Ratto, J. A.; Volino, F.; Blumstein, R. B. (2006). Phase behavior and order in mixtures of main-chain nematic polyesters with small molecules: a combined proton and deuterium NMR study, Macromolecules, Vol. 24, No. 10, (May 1991), pp.2862-2867, DOI: $10.1021 / \mathrm{ma} 00010 \mathrm{a} 035$.

Russell, M.; Oh, S.; Larue, I.; Zhou, O.; Samulski. E. T. (2006). Alignment of nematic liquid crystals using carbon nanotube films, Thin Solid Films, Vol. 509, No. 1-2, (Jun. 2006), pp.53-57, DOI: 10.1016/j.tsf.2005.09.099.

Skarabot, M.; Ravnik, M.; Zumer, S.; Tkalec, U.; Poberaj, I.; Babic, D.; Musevic, I. (2008). Hierarchical self-assembly of nematic colloidal superstructures, Phye. Rev. E, Vol. 77, No. 6, (Jun. 2008), pp.061706-1-061706-4, DOI: 10.1103/PhysRevE.77.061706. 
Shaffer, M. S. P.; Windle, A. H. (1999). Analogies between Polymer Solutions and Carbon Nanotube Dispersions, Macromolecules, Vol. 32, No. 20, (Oct. 1999), pp.6864-6866, DOI:10.1021/ma990095t.

Sharma, S.R.; Palffy-Muhoray, P.; Bergersen, B.; Dunmur, D.A. (1985). Stability of a biaxial phase in a binary mixture of nematic liquid crystals, Phys. Rev. A, Vol. 32, No. 6, (Dec. 1985), pp.3752-3755, DOI:10.1103/PhysRevA.32.3752.

Shen, C.; Kyu, T., (1995). Spinodals in a polymer dispersed liquid crystal, J. Chem. Phys., Vol. 102, No. 1, (Jan. 1995), pp.556-562, DOI:10.1063/1.469435.

Singh, S., (2000). Phase transitions in liquid crystals, Phys. Rep., Vol. 324, No. 2, (Feb. 2000), pp.107-269, DOI:10.1016/S0370-1573(99)00049-6.

Stark, H., (1999). Director field configurations around a spherical particle in a nematic liquid crystal, Eur. Phys. J. B, Vol. 10, No. 2, (Jul. 1999), pp.311-321, DOI:10.1007/s100510050860.

Stark, H., (2001). Physics of colloidal dispersions in nematic liquid crystals, Phys. Rep., Vol. 351, No. 6, (Oct. 2001), pp.387-474, DOI:10.1016/S0370-1573(00)00144-7.

Straley, J.P., (1974). Ordered phases of a liquid of biaxial particles, Phys. Rev. A, Vol. 10, No. 5, (Nov. 1974), pp.1881-1887, DOI:10.1103/PhysRevA.10.1881.

Song, W.; Kinloch, I. A.; Windle, A. H. (2003). Nematic Liquid Crystallinity of Multiwall Carbon Nanotubes, Science, Vol. 302, No. 5649, (Nov. 2003), pp.1363, DOI:10.1126/science.1089764.

Schymura, S.; Enz, E.; Roth, S.; Scalia, G.; Lagerwall, J. P. F. (2009). Macroscopic-scale carbon nanotube alignment via self-assembly in lyotropic liquid crystals, Synth. Mater., Vol. 159, No. 21, (Nov. 2009), pp.2177-2179, DOI:10.1016/j.synthmet.2009.08.021.

Tanaka, S.; Ataka, M.; Ito, K. (2002). Pattern formation and coarsening during metastable phase separation in lysozyme solutions, Phys. Rev. E, Vol. 65, No. 5, (May 2020), pp.051804-1-051804-6, DOI:10.1103/PhysRevE.65.051804.

Tschierske, C.; Photinos, D.J. (2010). Biaxial nematic phases, J. Mater. Chem., Vol. 20, No. 21, (Apr. 2010), pp.4263-4294, DOI:10.1039/B924810B.

van den Pol, E.; Petukhov, A.V.; Thies-Weesie, D.M.E.; Byelov, D.V.; Vroege, G.J. (2009). Experimental Realization of Biaxial Liquid Crystal Phases in Colloidal Dispersions of Boardlike Particles. Phys. Rev. Lett., Vol. 103, No.25, (Dec. 2009), pp.258301-1-258301-4, DOI:10.1103/PhysRevLett.103.258301.

Weiss, V.; Thiruvengadathan, R.; Regev, O. (2006). Preparation and Characterization of a Carbon Nanotubea ¿Lyotropic Liquid Crystal Composite, Langmure, Vol. 22, No. 3, (Jan. 2006), pp.854-856, 10.1021/la052746m.

Woltman, S. J.; Jay, G. D.; Crawdord, G. P. (Eds.) (2007). Liquid Crystals, World Scientific Publishing, ISBN: 10-981-270-545-7, Singapore.

Yada, M.; Yamamoto, J.; Yokoyama, H., (2004). Direct Observation of Anisotropic Interparticle Forces in Nematic Colloids with Optical Tweezers, Phys. Rev. Lett., Vol. 92, No. 18, (May 2004), pp.185501-1-185501-4, DOI:10.1103/PhysRevLett.92.185501.

Yamamoto, J.; Tanaka, H., (2001). Transparent nematic phase in a liquid-crystal-based microemulsion, Nature, Vol. 409, (Jan. 2001), pp.321-325, DOI:10.1038/35053035.

Yamamoto, R. (2001). Simulating Particle Dispersions in Nematic Liquid-Crystal Solvents, Phys. Rev. Lett., Vol. 87, No. 7, (Jul. 2001), pp.075502-1-075502-4, DOI:10.1103/PhysRevLett.87.075502. 
Yu, L. J.; Saupe, A. (1980). Observation of a Biaxial Nematic Phase in Potassium Laurate-1-Decanol-Water Mixtures, Phys. Rev. Lett., Vol. 45, No. 12, (Sept. 1980), pp.1000-1003, DOI:10.1103/PhysRevLett.45.1000.

Zapotocky, M.; Ramos, L.; Pouling, P.; Lubensky, T. C.; Weitz, D. A., (1999). Particle-Stabilized Defect Gel in Cholesteric Liquid Crystals, Science, Vol. 283, No. 5399, (Jan. 1999), pp.209-212, DOI: 10.1126/science.283.5399.209.

Zhang, S.; Kinloch, I. A.; Windle, A. H. (2006). Mesogenicity Drives Fractionation in Lyotropic Aqueous Suspensions of Multiwall Carbon Nanotubes, Nano Lett., Vol. 6, No. 3, (Mar. 2006), pp.568-572, DOI: $10.1021 /$ nl0521322.

Zhang, S.; Kumar, S. (2008). Carbon Nanotubes as Liquid Crystals, Small, Vol. 4, No. 9, (Sep. 2008), pp.1270-1283, 10.1002/smll.200700082. 


\title{
Laser-Combined STM and Related Techniques for the Analysis of Nanoparticles/Clusters
}

\author{
Hidemi Shigekawa ${ }^{1}$, Shoji Yoshida ${ }^{1}$, \\ Masamichi Yoshimura ${ }^{2}$ and Yutaka Mera ${ }^{3}$ \\ ${ }^{1}$ University of Tsukuba \\ ${ }^{2}$ Toyota Technological Institute \\ ${ }^{3}$ University of Tokyo \\ Japan
}

\section{Introduction}

Nanoscale particles and clusters have been attracting considerable attention from researchers and engineers from fundamental and practical viewpoints owing to their high potential for providing an extremely wide range of functional characteristics compared with ordinary solid materials, such as chemical reactivity and electrical, magnetic, optical and mechanical properties [1-5]. In fact, nanoparticles with novel functions have been realized in various fields including catalysis, biology, plasmonics, electronic devices, magnetism and so forth, on the basis of their wide range of properties. The modification of nanoparticle surfaces is producing further advances in the development of functions including those of composite materials.

For the further development of novel functions based on nanoparticles/clusters and to optimize their use, it is essential to understand the physics and chemistry of such materials in relation to their macroscopic functions. However, because nanoparticles/clusters are generally defined as particles with diameters of 1-100 nm (1-10 nm in the field of nanotechnology), conventional analysis techniques are considered to average the information of nanostructures over the ensemble, limiting the understanding of individual characteristics. Furthermore, using conventional methods, analysis of the effect of local structures in each element such as atomic-scale defects, which are considered to determine the overall characteristics of small materials, is difficult. Therefore, the introduction of new methods for the analysis of these highly functional small materials is eagerly awaited.

Scanning tunneling microscopy (STM) is one of the most promising techniques for such purposes. The characteristics of materials can be obtained at the atomic scale not only for their surface but also for their inner structures including the transient dynamics. Furthermore, external perturbation such as by thermal, mechanical or electromagnetic excitation enables advanced measurements. Among the various STM techniques useful for the study of nanoparticles/clusters, STM in combination with optical technologies, which enables probing of the response of local electronic structures to optical treatment, is an interesting approach for considering the future applications of such materials. On the other 
hand, sample preparation is an important factor in the analysis of nanoscale materials. Tunneling current and bias voltage can be used to modify target materials to obtain a deeper understanding of their characteristic properties. In addition, the STM tip plays an essential role in measurement. A Ag tip, for example, is used to enhance the effect of local excitation, and a carbon nanotube (CNT) tip is an excellent probe for observing fine nanoscale structures.

In this chapter, we review and discuss the STM-based techniques developed in combination with optical technologies and their application to the analysis of nanoscale particles and clusters.

\section{Laser-combined STM and related techniques}

\subsection{Probing methods}

In this section, we discuss probing methods of STM combined with optical technologies.

\subsubsection{Photoabsorption spectroscopy}

Photoabsorption spectroscopy is a major branch of optical spectroscopy used to explore the electronic states of materials. Photoabsorption spectroscopy using STM (STM-PAS) provides a high spatial resolution in STM and high spectral accuracy of optical spectroscopy compared with scanning tunneling spectroscopy (STS). The detected signal in STM-PAS is the current flowing through the STM tip in response to the modulation of spectroscopic light. The simplest STM-PAS scheme is based on a lock-in (LI) technique with the intensity modulation of light while the wavelength is swept. Spatially resolved photoabsorption spectroscopy using STM was first demonstrated with a resolution of $\sim 50 \mathrm{~nm}$ [6]. It was then shown that STM-PAS enables the nanometer-scale imaging of isolated subsurface defects in semiconductors through the absorption spectra associated with the defects [7].

Here we introduce two advanced STM-PAS schemes.

\subsubsection{Fourier transform STM-PAS}

The simplest STM-PAS scheme has two inherent technical problems. The first is the spurious spectra that are often generated by temporal instabilities or positional drift of the STM tip, which cause the tunneling current to change with time because acquisition for LI detection requires a long time to sweep the wavelength of spectroscopic light. The second problem is an undesirable excess component in the photomodulated tip current due to the photothermal expansion of the tip material [8].

Fourier transform STM-PAS (STM-PAS-FT) based on the Fourier transformation technique was devised to solve such technical problems [9]. This scheme is essentially the same as that used in Fourier transform near-infrared spectroscopy (FT-NIR). Multiple lights modulated with different frequencies corresponding to their wavelengths generate a tip current with an interferogram caused by the superposition of current components modulated at the different frequencies. The photoabsorption spectrum is computed from the interferogram via Fourier transformation. In this case, the photothermal expansion is suppressed by the simultaneous illumination of multiplexed lights. 
Figure 1(a) shows a schematic diagram of the experimental setup of STM-PAS-FT. Figure 1(b) shows a typical photoabsorption spectrum obtained around the band gap of Si by the STM-PAS-FT scheme (a) and the LI scheme (b). Although it took about $100 \mathrm{~min}$ to acquire the spectrum for a single sweep of photon energy from 0.68 to $1.55 \mathrm{eV}$ in the LI scheme, it took only $16 \mathrm{~min}$ to obtain a. high quality STM-FT-NIR spectrum in the range of $0.25-1.85$ $\mathrm{eV}$ (1s for each scan and 1000 scans totally) [9]. Compared with the long acquisition time for the LI spectrum, that for a single STM-FT-NIR spectrum is much shorter (1 s), which enables us to avoid acquiring spurious spectra.
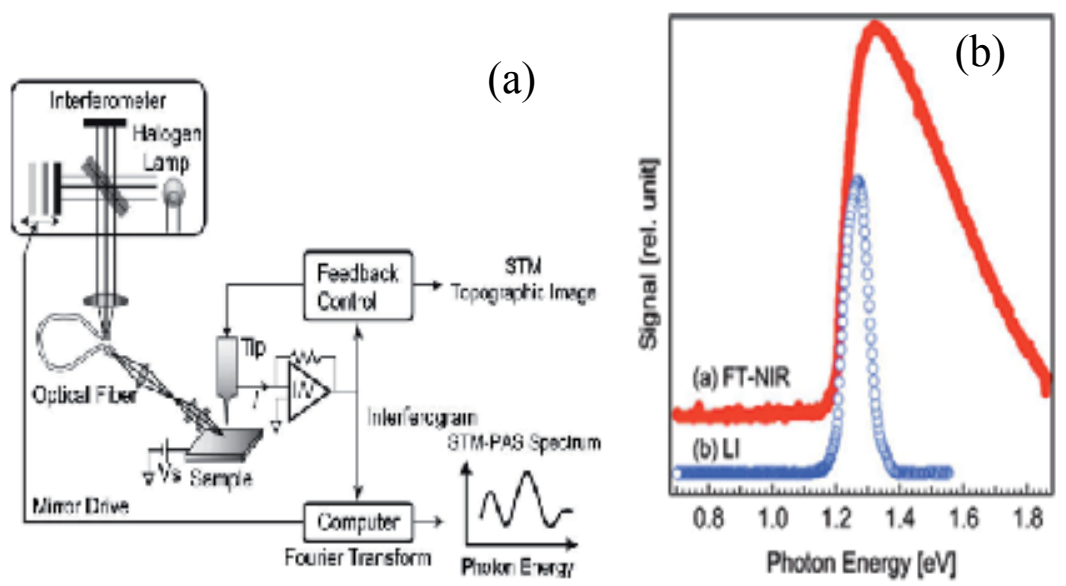

Fig. 1. (a) Experimental setup of Fourier transform near-infrared measurement. (b) STM-FTNIR spectrum and LI spectrum of a Si substrate obtained at $94 \mathrm{~K}$. The acquisition time was $16 \mathrm{~min}$ for FT-NIR measurement (1s for each scan and 1000 scans totally) and $100 \mathrm{~min}$ for LI measurement (1 scan). The decrease in intensity above $1.3 \mathrm{eV}$ in the LI spectrum is due to the cutoff filter used in the grating monochromator. [9]

Figure 2 shows an STM topographic image (a) and a two-dimensional map (b) of Si signals integrated from 1.3 to $1.5 \mathrm{eV}$ in the STM-PAS-FT spectra recorded in the framed area in (a) (8 spectra were measured and averaged at each pixel) [9]. The samples were hemispherical

(a)

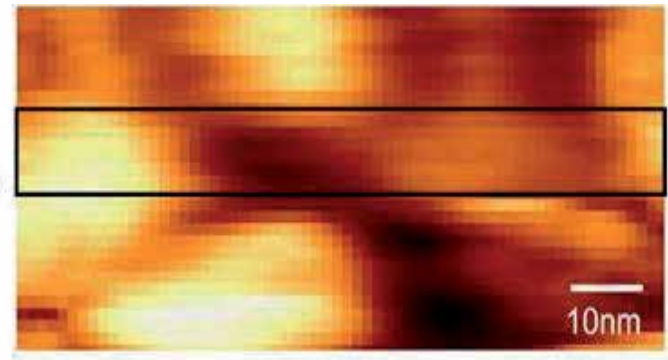

(b)

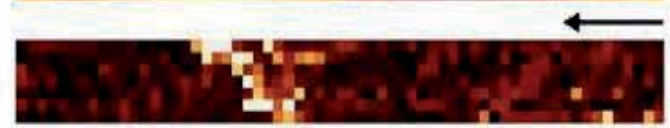

Fig. 2. (a) STM image of GeSn nanodots/Si obtained at 98 K. (b) Map of STM-PAS-FT signal integrated from 1.3 to $1.5 \mathrm{eV}$ for the spectrum obtained from the framed area in (a). [9] 
$\mathrm{Ge}_{1-\mathrm{x}} \mathrm{Sn}_{\mathrm{x}}(\mathrm{x}=0.1)$ nanodots epitaxially grown on $\mathrm{Si}$ substrates with an ultrathin $\mathrm{SiO}_{2}$ film. The deposition of Ge and Sn was controlled to 24 monolayers to grow nanodots with diameters of $\sim 40 \mathrm{~nm}$. After the samples were annealed at $770 \mathrm{~K}$, the surface was terminated with atomic hydrogen to suppress surface states. The region of bright contrast in (b) matches the region without nanodots in (a) reasonably well, i.e., the expected part of the Si substrates. The contrast indicates the spatial resolution of STM-PAS-FT to be $\sim 10 \mathrm{~nm}$.

Figure 3(a) shows a set of photoabsorption spectra obtained on different GeSn nanodots with various lateral diameters [10]. The peak indicated in each spectrum by an arrow is observed at an energy lower than the gap energy of $\mathrm{Si}(\sim 1.2 \mathrm{eV})$ and exhibits a clear blue shift with decreasing dot size, suggesting that the signal is induced by optical transitions between discrete levels in the quantum dots. The photoabsorption energy of a spherical nanodot with radius $R$ is given by

$$
E_{a b s}=E_{b u l k}+\frac{\hbar^{2} \pi^{2}}{2 \mu R^{2}}-1.786 \frac{e^{2}}{\varepsilon R}-0.248 \frac{e^{2}}{8 \pi \varepsilon a_{B}^{*}}
$$

Here, $a_{\mathrm{B}}^{*} \equiv 4 \pi \hbar^{2} \varepsilon / \mu e^{2}$ represents the exciton Bohr radius, $\hbar$ is Planck's constant, $\varepsilon$ is the dielectric constant of the nanodot material, $e$ is the electron charge and $\mu$ is the reduced mass of carriers. The first term $E_{\text {bulk }}$ is the bandgap energy of the bulk crystal [11]. The solid curve in Fig. 3 shows the theoretical curve calculated from Eq. (1). The energy position of the

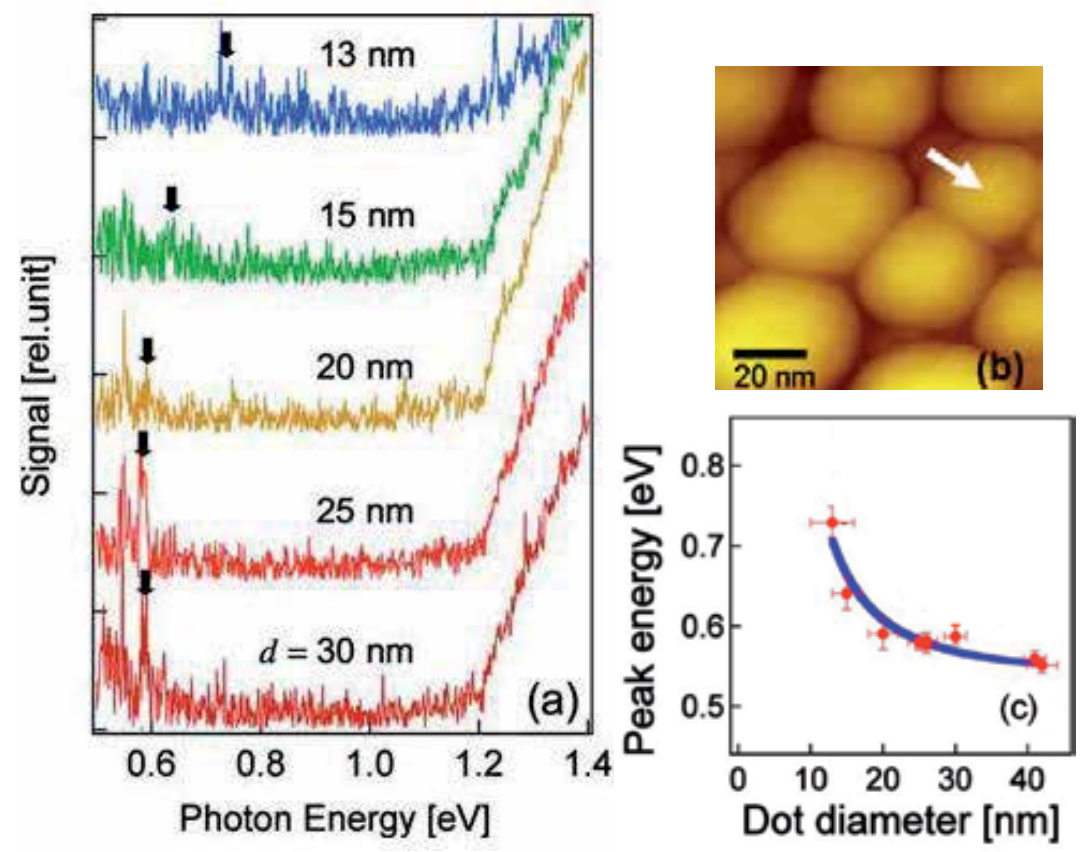

Fig. 3. (a) STM-PAS-FT spectra obtained from individual GeSn nanodots of various sizes. (b) Typical topographic STM image of $\mathrm{Ge}_{1-x} \mathrm{Sn}_{\mathrm{x}}$ nanodots. The white arrow indicates the position where the spectrum for $d=30 \mathrm{~nm}$ was acquired. (c) Peak energy in (a) as a function of the lateral diameter of the nanodots. The solid curve is calculated from Eq. (1). [10] 
peak is in good agreement with the optical transition energy between discrete levels theoretically predicted by the size dependence due to the quantum confinement effect.

\subsubsection{Electric field modulation spectroscopy}

Conventional electric field modulation spectroscopy (EFMS) techniques, such as electroreflectance and photoreflectance, are established tools used for the accurate measurement of interband transition energies in semiconductors [12]. The principle of EFMS is based on the fact that applying an electric field to a semiconducting material causes an oscillatory change in the optical absorption coefficient $\alpha$ depending on the wavelength, i.e., the Franz-Keldysh effect [13]. The spectral line shape of EFMS is closely related to energy derivatives of the unperturbed dielectric function, and represents features corresponding to interband transitions. By combining EMFS with STM (STM-EFMS), we can perform EFMS with nanometer spatial resolution [14].
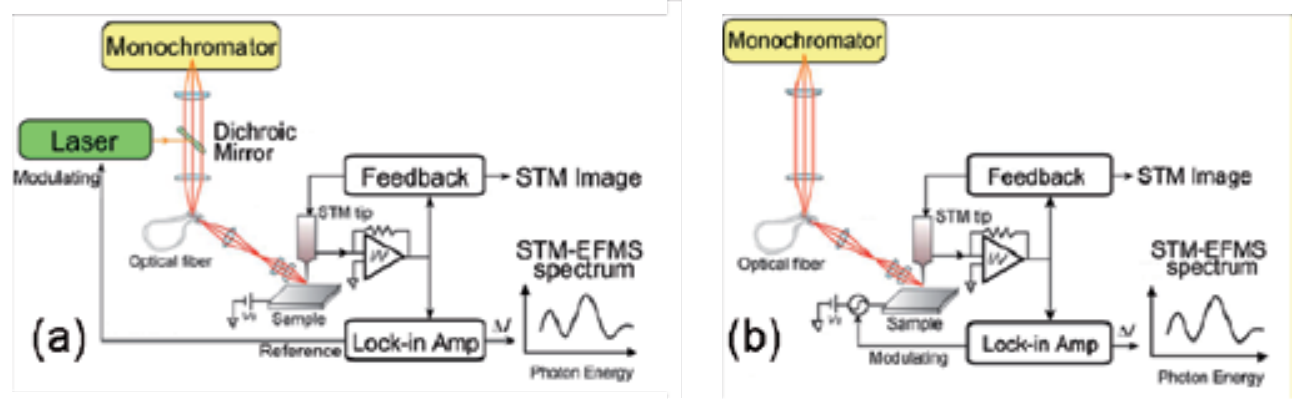

Fig. 4. Schematic illustrations of STM-EFMS measurement: (a) OM scheme, (b) BM scheme.[15]*

Figure 4 illustrates schematics of STM-EFMS measurements using two different schemes for electric field modulation: (a) optical modulation (OM) and (b) bias modulation (BM). In OM, electric field modulation is achieved by an SPV periodically induced by chopped light illumination with energy above the bandgap of the sample from a diode laser. In BM, electric field modulation is achieved by applying a modulated bias voltage to the sample, which directly modulates the tip-induced band bending beneath the tip. In STM-EFMS, the change in $\alpha$ is detected as a change in the STM tip current, which is synchronized with the modulation of the electric field.

Figure 5 shows a typical STM-EFMS spectrum (solid curve) and the spectrum obtained by the conventional EFMS method (dashed curve). The STM-EFMS spectrum reproduces the main features of the band structure. Two distinct structures observed at photon energies approximately $h v=1.41$ and $1.78 \mathrm{eV}$ are ascribed to the interband absorption edge of GaAs and the spin split-off band absorption, respectively. It was demonstrated that the spatial resolution of STM-EFMS measurements was of nanometer scale [15, 16]. A typical STM topographic image of a $\beta-\mathrm{FeSi}_{2}$ nanodot sample is shown in Fig. 6(a). The sample was epitaxially grown on an $\mathrm{n}-\mathrm{Si}(111)$ substrate covered with an ultrathin $\mathrm{SiO}_{2}$ film. After the growth, the surface was terminated with atomic hydrogen. Figure 6(b) shows STM-EFMS spectra at $96 \mathrm{~K}$ obtained by the two schemes in an energy range lower than the absorption edge of $\mathrm{Si}[15]$. 


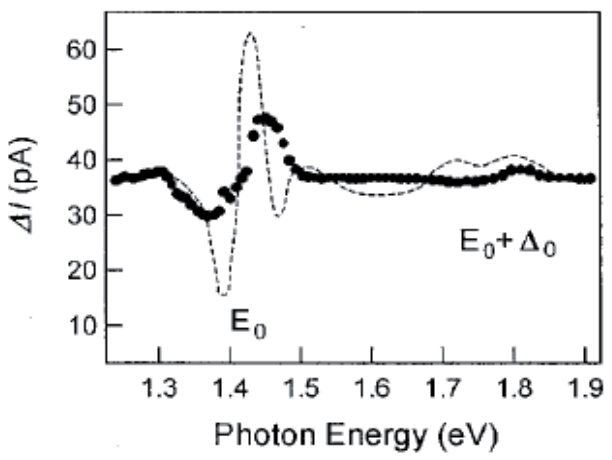

Fig. 5. STM-EFMS spectrum obtained for a perfect (110) cleaved surface of GaAs. The broken curve indicates the EFMS spectrum measured by the conventional electroreflectance method applied to a macroscopic GaAs sample. $\mathrm{E}_{0}$ and $\mathrm{E}_{0}+\Delta_{0}$ denote the interband absorption edge of GaAs and to the spin split-off band absorption, respectively. [14] ${ }^{*}$

For epitaxially grown sufficiently strained $\beta$-FeSi $i_{2}$ nanodots on $\mathrm{Si}$, bandgap crossover, i.e., change from indirect band to direct band, is theoretically predicted [18], which, however, has not been confirmed experimentally despite that the mechanism is of great importance for application. The two spectra exhibit a common feature from 0.72 to $0.76 \mathrm{eV}$. The energy positions of the signals, $0.72-0.76 \mathrm{eV}$, closely match the absorption thresholds detected by macroscopic measurements of the photoabsorption coefficient for a bulk $\beta$-FeSi $i_{2}$ crystal at $100 \mathrm{~K}$ [17] and the energy threshold is attributed to optical transitions across the indirect bandgap. Therefore, these findings strongly indicate that the $\beta-\mathrm{FeSi}_{2}$ nanodot sample examined was an indirect-gap semiconductor, instead of the theoretical prediction.

Using the STM-EFMS scheme, the band structure of individual nanodots can be explored with high accuracy.
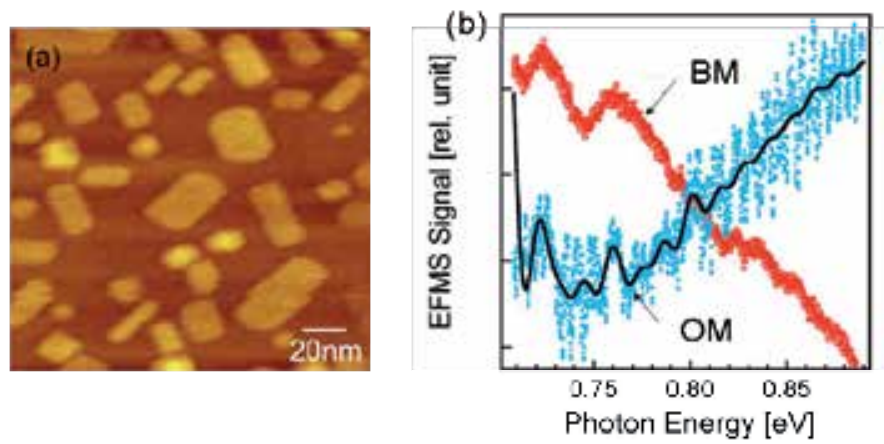

Fig. 6. (a) Typical STM topographic image of $\beta-\mathrm{FeSi}_{2}$ nanodot sample. Bright contrasts with heights of 5-10 nm are H-terminated $\beta-\mathrm{FeSi}_{2}$ nanodots grown on $\mathrm{Si}(111)$. (b) STM-EFMS spectra measured on $\beta-\mathrm{FeSi}_{2}$ nanodots by optical modulation $(\mathrm{OM})$ and bias modulation (BM). The common features near $0.72-0.76 \mathrm{eV}$ agree well with the absorption thresholds detected by macroscopic measurements of the photoabsorption coefficient in a bulk $\beta-\mathrm{FeSi}_{2}$ crystal. [15]* 


\subsubsection{Light-emission spectroscopy}

When carriers are injected from an STM tip to a sample, light emission (LE) is induced in some cases. STM luminescence spectroscopy is a measurement scheme in which the emitted light is collected to explore the local electronic properties of materials (Fig. 7) [19-22].

The mechanism of photon emission depends on the process of measurement such as plasmon polariton (SPP) excitation in conductive samples, carrier recombination in semiconductor samples and the HOMO-LUMO transition in molecular samples. Information on molecular vibrations can be obtained by analyzing the spectrum [22], such as by inelastic tunneling spectroscopy [23], which may be used for the analysis of composite nanoparticles/clusters combined with organic materials.
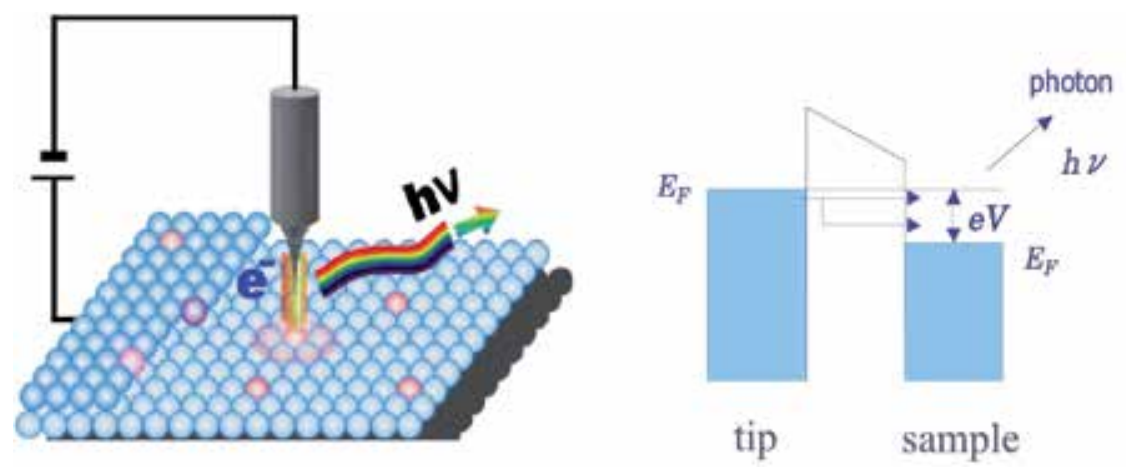

Fig. 7. Schematic illustrations of LE-STM setup and basic mechanism.
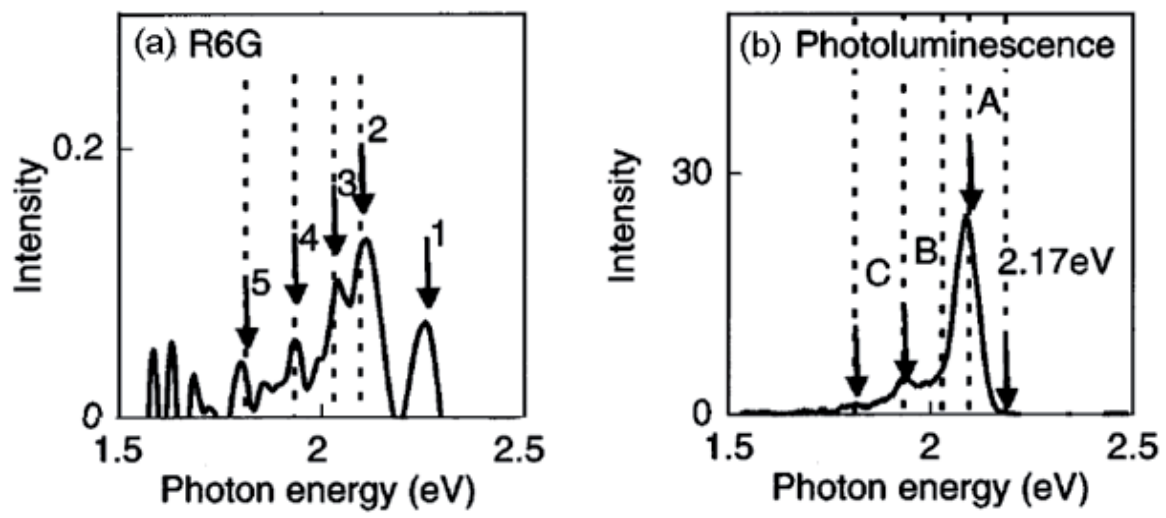

Fig. 8. (a) is the STM light emission spectrum of a single R6G molecule. (b)

Photoluminescence (PL) spectrum of R6G on HOPG. The cutoff of the PL spectrum at 2.17 $\mathrm{eV}$ is due to the short-wavelength-cutoff filter inserted in the collection optics. [24] ${ }^{*}$

From the distribution of emission intensity on a sample surface (photon map), we can investigate the geometry of the electronic structures of the sample. The photon map also enables us to estimate the transport properties of minority carriers by considering the

${ }^{*}$ Reprinted with permission from each reference. Copyright American Institute of Physics. 
diffusion length. When a local spectrum is analyzed as a photon map, more detailed information such as the distribution of elements can be obtained. However, STM luminescence spectra are affected by various factors other than sample properties, such as the tip shape, tip material and the characteristics of the substrate used for the experiment; thus, careful analysis is necessary to determine the physical properties of the target material from the STM luminescence spectra. In the case of organic materials, damages due to carrier injection must be avoided.

Figure 8(a) shows the STM luminescence spectrum obtained from a single rhodamine 6G (R6G) molecule on an HOPG surface. The features of the spectrum are in good agreement with the photoluminescence spectrum of a layer of rhodamine molecules on HOPG (Fig. 8(b)) [24].

\subsubsection{Photoexcitation spectroscopy}

Dynamical processes have often been studied by a laser pump-probe method where a pump pulse excites a sample and a subsequently arriving probe pulse with a delay time of $t_{\mathrm{d}}$ is used to track its temporal evolution $[25,26]$. The temporal resolution attainable in such experiments is limited only by the pulse width, which is generally in the femtosecond range. However, the spatial resolution is determined by the optical diffraction limit, which is large compared with the typical size of materials and devices currently being developed, and therefore, the physical observables are obscured by ensemble averaging. Thus, high spatial resolution in pump-probe experiments would provide new insights into nanoscale structures and materials and unveil a rich variety of dynamical features of light-sensitive phenomena in unexplored regimes such as charge transfer, phase transitions, electronic transitions, carrier or spin transport and quantum coherence.

In contrast, STM easily provides atomic-scale spatial resolution despite its low temporal resolution (typically worse than $100 \mathrm{kHz}$ ) [27-31]. Therefore, if the tunneling process directly produced by optical excitation can be measured, high temporal and spatial sensitivity can be simultaneously achieved with the atomic-scale resolution of STM [32-41]. A promising setup for achieving this is pulse-pair-excited STM (PPX-STM), in which, in analogy with pump-probe experiments, a sequence of paired laser pulses with a certain delay time $t_{\mathrm{d}}$ excites the sample surface beneath the STM tip, and the tunneling current $I$ is measured as a function of $t_{\mathrm{d}}$. To detect a faint time-resolved tunneling current with a high signal-to-noise ratio, the rectangular modulation of delay time with a pulse-picking procedure is used (shaken-pulse-pair excited STM: SPPX-STM), enabling the spatial mapping of time-resolved tunneling current [33].

Figure 9 shows the setup of SPPX-STM. When paired optical pulses arrive at a sample beneath the STM tip, they generate pulses of raw tunneling current $I^{*}$, reflecting the excitation and relaxation of the physical properties of the sample. If these current pulses decay rapidly compared with the time scale of the STM preamplifier bandwidth, they can be temporally averaged in the preamplifier but cannot be detected directly in the signal $I$. Even in this case, the relaxation dynamics can be probed through the $t_{\mathrm{d}}$ dependence of $I$. When $t_{\mathrm{d}}$ is sufficiently long, paired optical pulses with the same intensity independently induce two current pulses with the same $I^{*}$. In contrast, when $t_{\mathrm{d}}$ is short and the second pulse illuminates the sample in the excited state induced by the first pulse, the second current pulse may have a different magnitude, depending on $t_{d}$. A typical process that can be observed using this mechanism is absorption bleaching in semiconductors; when the 
carriers excited by the first optical pulse remain in the excited state, the absorption of the second optical pulse is suppressed. In such a case, the current $I^{*}$ induced by the second current pulse decreases depending on $t_{d}$, reflecting the decay of the excited carriers excitation by the first-pulse. Signal $I$ also depends on $t_{d}$, because the magnitude difference of the second current pulse changes the temporally averaged value of the tunneling current. Accordingly, the relaxation dynamics of the excited carriers of the target material, namely, the decay of carrier density after excitation by the first optical pulse, can be probed by STM at the resolution of the pulse width, that is, in the femtosecond range.

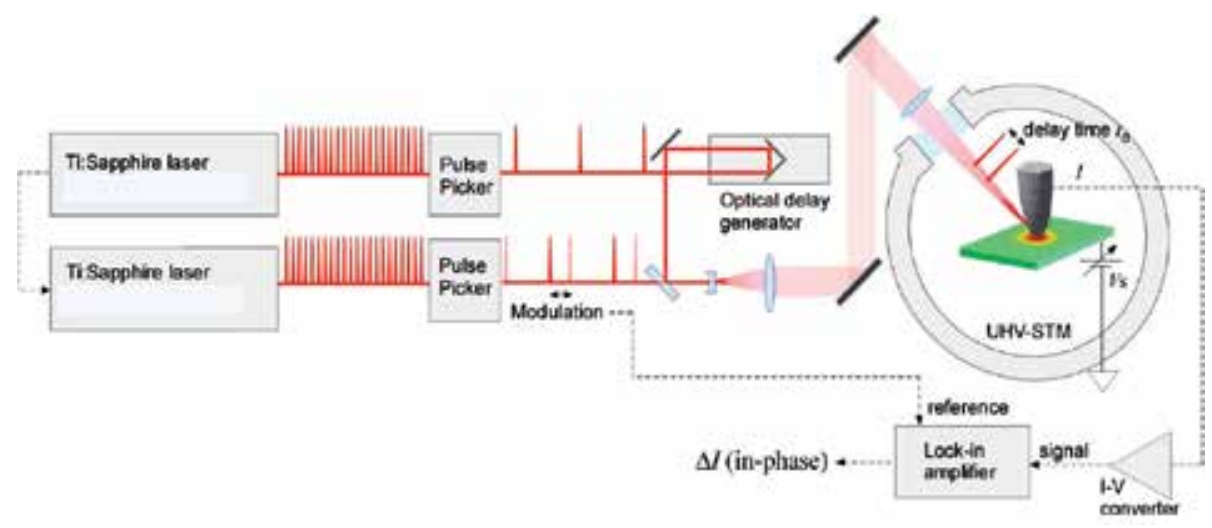

Fig. 9. Schematic illustration of SPPX-STM

In SPPX-STM the sophisticated control of delay-time generation and modulation with a pulse-picking procedure is essential. This enables the probing of nanometer-scale structures with a wide range of relaxation lifetimes. Using the pulse-picking method, a large and discrete modification of $t_{d}$ can be realized by changing the selection of pulses that transmit the pulse pickers, which is suitable for modulating $t_{d}$ in SPPX-STM. In this method, the delay time dependence of the tunneling current, $\Delta I\left(t_{d}\right) \equiv I\left(t_{d}\right)-I(\infty)$, is accurately probed with a high acquisition rate, where $I(\infty)$ is the tunneling current for a delay time sufficiently long for the excited state to be relaxed. Accordingly, SPPX-STM has made it possible to visualize the carrier dynamics in nanometer-scale structures with a wide range of relaxation lifetimes. Figure 10 shows the capability of wide timescale measurement.

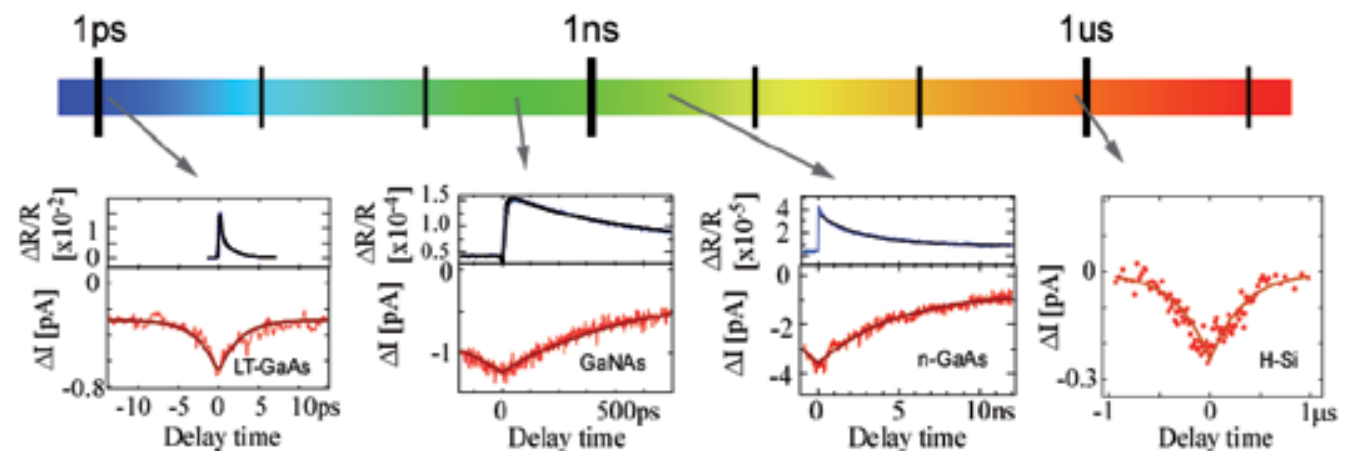

Fig. 10. SPPX-STM signals obtained for various samples. Upper spectra of LT-GaAs, GaNAs and $\mathrm{n}-\mathrm{GaAs}$ were obtained by optical pump-probe method (R: reflectivity of probe pulse). 
As an example, SPPX-STM has been applied to the analysis of carrier dynamics in a Co nanoparticle/GaAs(110) system. When $\mathrm{Co}$ is deposited on a GaAs, nanoparticles are formed (Fig. 11(a)). In this system, photoexcited minority carriers (holes) captured at the surface are recombined with electrons tunneling from the STM tip via the gap states formed by Co as shown in Fig. 11(c). This is considered to be enhanced by the existence of gap states at the Co nanoparticle sites. Understanding such a charge transport mechanism through nanoparticles is of great importance not only for the development of nanoscale electronic devices but also for their application to the finer control of chemical reactions in catalysis.
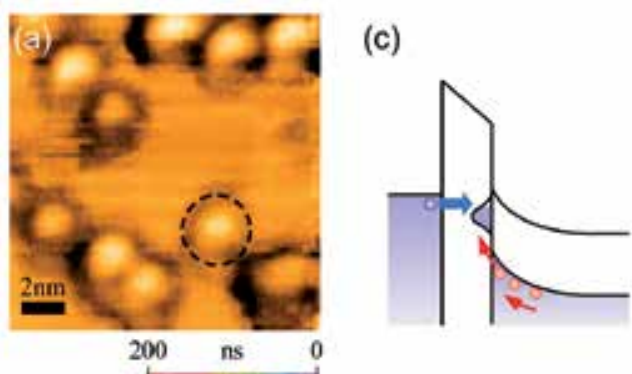

(e)
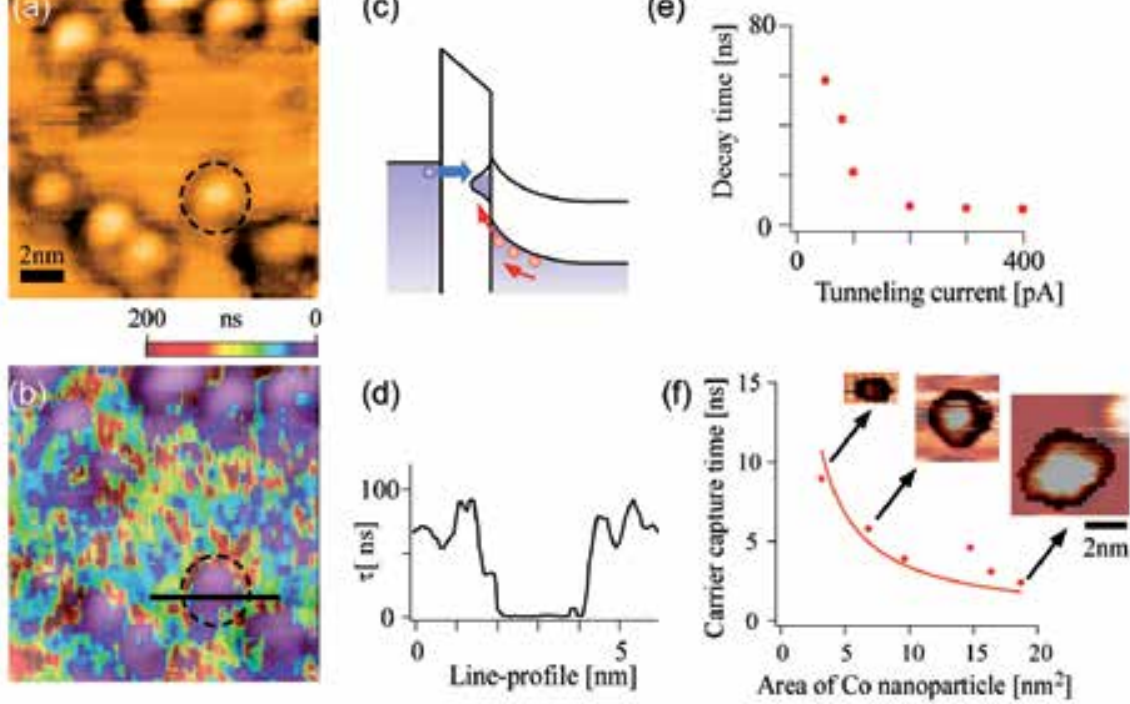

Fig. 11. (a) STM image of Co/GaAs, (b) 2D map of decay constant, (c) schematic model of recombination at gap states, (d) cross section along the line in (b), (e) decay constant as a function of tunneling current, (f) decay constant as a function of Co particle size.

Figure 11(b) shows the overlap of the STM image in Fig. 11(a) with the map of the decay constant obtained over the surface. The two-dimensional (2D) map of the decay constant shown in color scale indicates the decay constant of the photoinduced carrier density at each point. The positional agreement is good. As shown in the cross section in Fig. 11(d) obtained along the line in Fig. 3(b), the decay is rapid in the Co regions. In such regions, photoinduced holes trapped at the surface are recombined with electrons tunneling from the STM tip at the gap states; thus, there are two limitations in this process: the tunneling current and hole-capture rate. When the tunneling current is sufficient, the hole-capture rate becomes the limiting factor of the recombination process. Figure 11(e) shows the relation between the decay constant and tunneling current. As expected, the decay constant decreases with increasing tunneling current and has a saturated value of $6.9 \mathrm{~ns}$, which corresponds to the hole-capture rate of this system. The decay process should depend on the gap-state density. Figure 11(f) shows the decay constant as a function of the Co nanoparticle size. The time constant increases with decreasing nanoparticle size as expected. 
In SPPX-STM, the nonlinear interference between the excitations is essential, which depends on the material we measure. In SPPX-STM applied to a semiconductor, tip-induced band bending and surface photovoltage play important roles in the measurement. However, in general, such as dipole formation, charge transfer, changes in conductance, and vibration that causes the change in the tip-sample distance are possible mechanisms for producing SPPX-STM signals. Therefore, SPPX-STM enables the nanoscale probing of transient dynamics over a wide range of time scales, simultaneously with the observation of local structures by STM.

Another promising technique is STM combined with synchrotron radiation (SR-STM), which probes core-level photoemission, enabling the identification of atomic species of the target materials [42]. The spatial resolution has been improved to $\sim 10 \mathrm{~nm}$, and therefore in the near future, in addition to the analysis of isolated nanostructures, probing of the inner structures of targets may become possible.

\subsection{Manipulation for fine measurement}

The tunneling current and bias voltage in STM, which are the basic parameters of STM measurement, can be used for the modification of target materials. Probing, for example, the effect of atomic-scale defects on local electronic structures enables the clarification of the fundamental mechanism in each element and its relation to macroscopic functions. For nanoparticles/clusters, such effects are essential for determining the characteristic properties of their total systems.
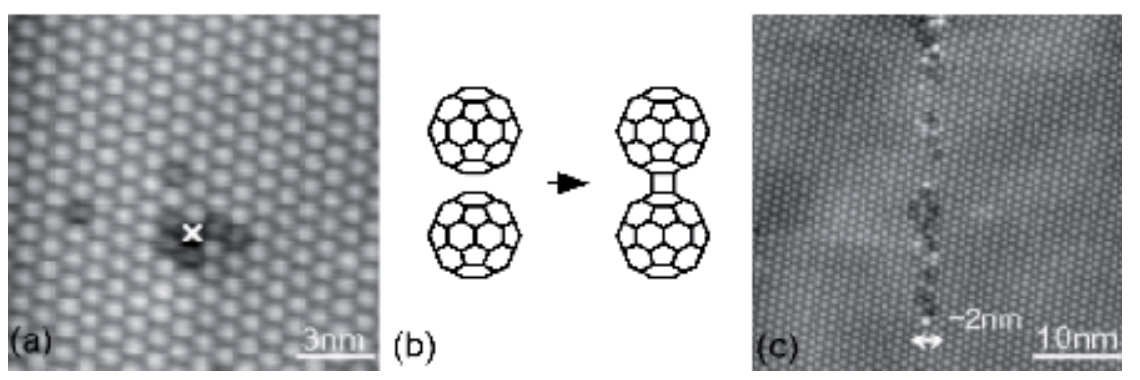

Fig. 12. (a) STM image of current-injection-induced polymerized $\mathrm{C}_{60}$ molecules (dark contrasts), (b) schematic of $\mathrm{C}_{60}$ polymerization, (c) nanoscale patterning of polymerized $\mathrm{C}_{60}$ molecules formed by scanning the tip along the longitudinal direction. [43] ${ }^{\dagger}$

Figure 12(a) shows an STM image of a $\mathrm{C}_{60}$ crystalline film within a thickness of several monolayers grown on an HOPG surfaces in ultrahigh vacuum (UHV). Before acquiring the image, electrons were injected at the point indicated by a cross in Fig. 12(a) at a sample bias voltage of $\mathrm{Vs}=+4.2 \mathrm{~V}$. The dark contrasts around the point represent intracluster structures with a stripe pattern, suggesting the frozen rotation of $\mathrm{C}_{60}$ molecules despite the room temperature. Namely, the dark sites are $\mathrm{C}_{60}$ molecules polymerized with molecules in the underlayer. The polymerization was induced by the injection of low-energy electrons from the STM probe tip. Figure 12(c) shows a line structure consisting of polymerized $\mathrm{C}_{60}$ clusters confined in a width as small as $\sim 2 \mathrm{~nm}$, which is a good example of nanoscale electron-beam

† Copyright The Japan Society of Applied Physics. 
patterning [43]. When a template such as nanoscale cavity is used, individual $\mathrm{C}_{60}$ molecules are stabilized in each cavity even at room temperature. Manipulation of a single $\mathrm{C}_{60}$ molecule using STM tunneling current was successfully carried out (Fig.13) [44.].

(a)

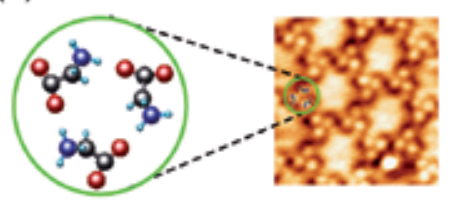

(b)

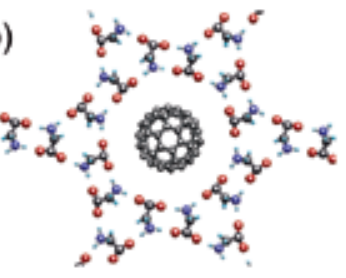

(c)

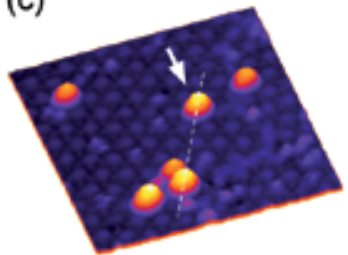

(d)

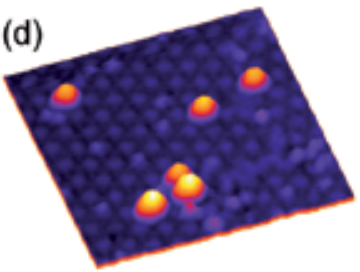

(e)

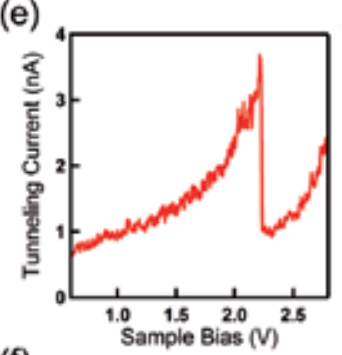

(f)

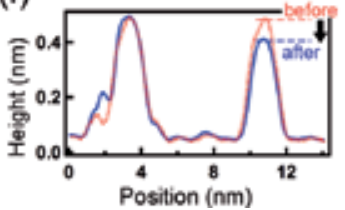

Fig. 13. (a) STM image of glycine-nanocavity array (template). (b) Schematic illustration of $\mathrm{C}_{60}$ molecule stabilized in a nanocavity. STM images of $\mathrm{C}_{60}$ molecules stabilized by a glycine template before (c) and after (d) the injection of tunnel current on the molecule indicated by arrow. (e) Change in tunnel current upon manipulation. (f) Cross sections along the line in (a).

Figures 14(b) and (c) show topographic STM images of a single-walled carbon nanotube (SWNT). After acquiring the image in Fig. 14(b), the STM tip was fixed at the position marked in Fig. 14(b), and a tunneling current of $0.1 \mathrm{nA}$ with $7.0 \mathrm{~V}$ bias voltage was injected. Figure 13(c) shows the defect generated at the probed site. The finite flat LDOS around the Fermi level shown in Fig. 14(c), which was measured before defect generation, indicates that

(a)

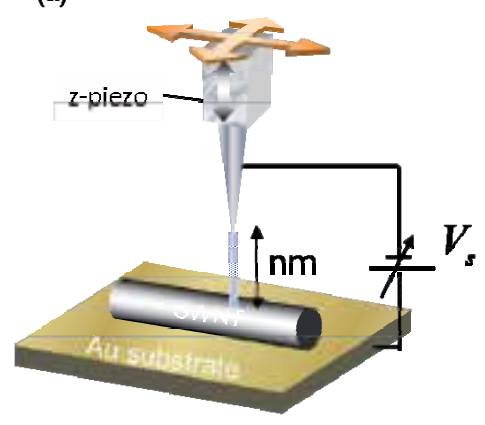

(b)

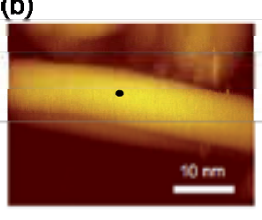

(d)

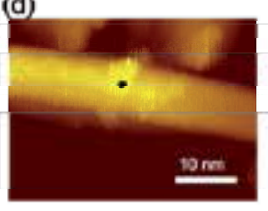

(c)
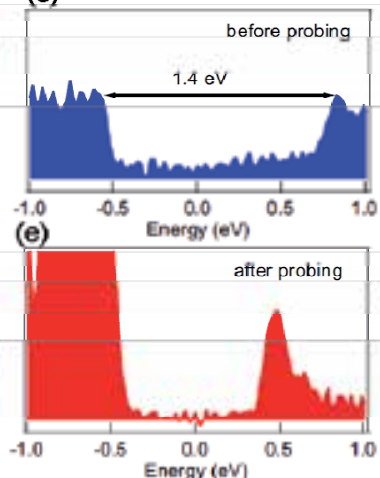

Fig. 14. (a) Schematic illustration of tunnel current injection. (b) STM image of an SWNT acquired with $\mathrm{Vs}=1.0 \mathrm{~V}$ and $\mathrm{It}=0.1 \mathrm{nA}$ at $95 \mathrm{~K}$. (c) dI/dV vs bias-voltage curve, obtained at the position marked in (b), exhibiting the features of a metallic SWNT characterized by a finite flat LDOS in the first van Hove gap. (d) STM image acquired after current injection at the marked position. (e) $\mathrm{dI} / \mathrm{dV}$ vs bias-voltage curve, obtained at the position marked in (d), exhibiting a HOMO-LUMO gap of $\sim 0.7 \mathrm{eV}$. [45] 
the tube was initially metallic. Figure 14(e) shows the LDOS measured at the defect, which is characterized by a HOMO-LUMO gap that opened across the Fermi level. The HOMOLUMO gap was observed to be over $2 \mathrm{~nm}$ along the long axis of the CNT and is considered to act as a barrier to carrier transport along the metallic SWNT. This result indicates that we can modify the local electronic properties of a single cluster in a controlled manner using the STM modification technique $[45,46]$.

Another method of manipulation is mechanical deformation of clusters by an STM tip. For example, the change in the HOMO-LUMO gap of $\mathrm{C}_{60}$ molecule due to deformation was observed thorough the measurement of tunneling current under the compression of the molecule by STM tip [47].

Combination of STM manipulations with optical techniques enables further analysis of nanoscale materials.

\subsection{Probe technology}

For the STM measurement of nanoscale materials, choosing the most suitable STM tip depending on the specific experiment is important. In this section, silver tips for optical measurement, glass-coated tips for photoemission measurements, molecular tip for chemical analysis and CNT probes for high resolution imaging will be described.

\subsubsection{Insulator-coated metal tips for SR-STM}

STM combined with a synchrotron radiation light source (SR-STM) has attracted considerable attention owing to the possibility of elemental analysis at nanometer resolution by detecting the core-level electrons of surface atoms. The fabrication of a tip coated with an insulating thin film is the key to achieving high spatial resolution by reducing the photoinduced current impinging to the side wall of the tip [48, 49]. For example, a W tip was coated with glass except for the region less than $5 \mu \mathrm{m}$ from the tip apex using a focused ion beam (FIB) technique [48]. Using this state-of-the-art STM tip, the photoinduced current was dramatically reduced by a factor of $\sim 40$ compared with that of an untreated tip. Recently, using this tip in combination with the Lock-in (LI) detection method, a spatial resolution of as high as $\sim 10 \mathrm{~nm}$ was demonstrated on checkerboard-patterned $\mathrm{Ni}$ and Fe samples [42].

\subsubsection{Silver tips for TERS and STML}

Tip-enhanced Raman spectroscopy (TERS) [50] is a promising method of chemical analysis at the nanometer level. Under external illumination, a sharp tip is used to create a localized light source and excite a specimen surface. According to classical electromagnetic theory, a sharp metal tip is suitable for enhancing the Raman scattering of nearby molecules. It is known that Ag produces greater enhancement than $\mathrm{Au}$ in the visible range because the imaginary part of its permittivity is much smaller. The silver tip is also used in STMinduced luminescence (STML), where STML intensities are enhanced by about one order of magnitude compared with those obtained using tungsten tips [51]. There have been many reports on the fabrication of Ag tips by electrochemical etching with various electrolytes such as a mixture of perchloric acid and ethanol [52]. Using such a tip, single-molecule tipenhanced Raman spectra from brilliant cresyl blue (BCB) sub-monolayers deposited on a flat 
Au surface were obtained [53]. A highly enhanced electric field was created in the gap of 1 $\mathrm{nm}$ between the tip and sample. For STM imaging, the tip apex should be free of oxidation or contaminants. Atomically resolved STM imaging and STML spectra with a high signal-tonoise ratio are obtained using an electrochemically etched Ag tip followed by tip cleaning by Ar ion sputtering in UHV [54].

\subsubsection{Molecular tips}

Carboxyl-terminated SWNTs from solution phases can be attached onto Au tips through self-assembed monolayers for using in STM [55. 56]. In addition to the high-resolution imaging of molecules (such as diether) on a surface, these CNT tips enable chemically selective observation due to electron tunneling through hydrogen-bond interactions between the atached molecule and carboxyl groups at SWNTs. The differentiation of DNA bases and chiral recognition on a single-molecule basis have also been demonstrated using molecular tips [56]. In a similar way, voltage-induced chemical contrast in an STM image was reported using chemically modified tips with hydrogen-bond donors [57]. Moreover, molecular orbitals of metal phthalocyanines on metal surfaces have been clearly imaged with an $\mathrm{O}_{2}$-functionalized STM tip, where the observations were supported by theoretical calculations [58].

\subsubsection{CNT probes}

CNTs are one of the most intriguing materials in nanotechnology [59]. A CNT has a onedimensional cylindrical structure with sistinct physical characteristics such as a small diameter, high aspect ratio, high stiffness, high conductivity and so forth. In view of the shape and electric conductivity required for a high-resolution STM tip, these properties of CNTs make them ideal as a tip material for probing extremely small objects such as nanoclusters. [60]. Mechanical attachment, direct growth and dielectrophoresis are methods employed to fabricate CNT-STM tips. A single CNT tip can be prepared by mechanical attachment, which enables the high-resolution ghost-free imaging of nanoclusters. However, this method is time-consuming, and the other methods (direct growth and dielectrophoresis) are more suitable for the mass production of CNT tips.

\section{(a) Mechanical attachment method}

Following the first approach to fabricating CNT probes under an optical microscope [61]. a more sophisticated method was proposed, where the attachment of a CNT onto a probe is performed in two independent precise stages under SEM observation, where beam-deposited amorphous carbon is used as a glue [62]. Although the thus mechanically prepared CNT-STM tips exhibited atomic resolution on $\mathrm{Au}(111)$ reconstruction, the cleaning process of CNTs by heating in UHV was necessary for stable observation. [63]. A metal coating method has been proposed for improving the electric conductivity between a CNT and the supporting metal tip [64]. Automotive exhaust catalysts consist of metal nanoclusters supported on metal oxide surfaces. Since catalytic activity can be altered by controlling the size of the nanoclusters because of its strong size dependence, the precise characterization of metal nanoclusters is essential. High-resolution UHV-STM images of size-selected $\mathrm{Pt}(\mathrm{n})(\mathrm{n}=4,7-10,15)$ clusters deposited on $\mathrm{TiO}_{2}(110)-(1 \times 1)$ surfaces were obtained using a CNT tip (Fig. 15) [65]. Clusters of $\mathrm{Pt}$ (7) (Fig.15(b)) and smaller were oriented flat on the surface with a planar structure, and a planar-to-three-dimensional transition was observed at $n=8$ (Fig.15(c)). Color scale shows the 
structures of the top layers. Individual $\mathrm{Pt}$ atoms are clearly identified, especially for (c) $\mathrm{Pt}(8)$ and (d) $\mathrm{Pt}(9)$, indicating that the geometry of the clusters is atomically resolved and the details can be analyzed with a CNT tip.
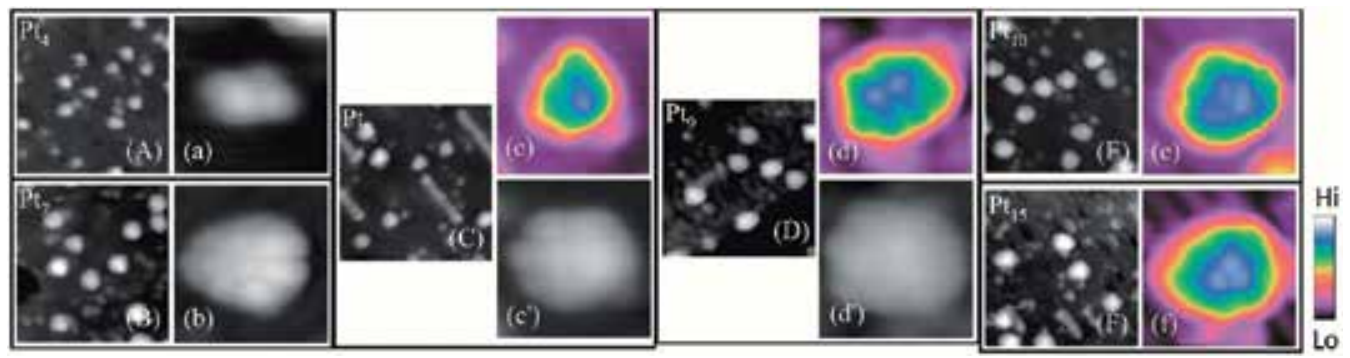

Fig. 15. STM images of $\mathrm{TiO}_{2}(110)$ surface after deposition of size-selected $\mathrm{Pt}_{n}{ }^{+}(\mathrm{n}=4,7-10,15)$ cluster ions. Images with uppercase letters are $20 \times 20 \mathrm{~nm}^{2}$ views and those with lowercase letters are $3.5 \times 3.5 \mathrm{~nm}^{2}$ views of a cluster on the same surface. $\mathrm{TiO}_{2}$ surface after the deposition of [(A), (a)] $\mathrm{Pt}_{4}{ }^{+},[(\mathrm{B})(\mathrm{b})] \mathrm{Pt}_{7}{ }^{+},\left[(\mathrm{C})(\mathrm{c})\left(\mathrm{c}^{\prime}\right)\right] \mathrm{Pt}_{8}{ }^{+},\left[(\mathrm{D})(\mathrm{d})\left(\mathrm{d}^{\prime}\right)\right] \mathrm{Pt}_{9}{ }^{+},[(\mathrm{E})(\mathrm{e})] \mathrm{Pt}_{10}{ }^{+},[(\mathrm{F})(\mathrm{f})]$ $\mathrm{Pt}_{15}+$ [65].

\section{(b) Direct growth method}

This method is suitable for the mass production of CNT probes. Chemical vapor deposition (CVD) is commonly used for the synthesis of CNTs [66]. Tips fabricated by the direct growth method sometimes consist of numerous CNTs, and selective growth at the apex is required for stable operation of the tip. For this purpose, several methods of pinpointing catalysis have been reported $[67,68]$. The growth direction of CNTs is also important in measurements. Plasma CVD is suitable for controlling the alignment of CNTs [69]. By optimizing the reaction at the sharp apex, CNT probes can be directly grown on the apex of a tungsten probe without reducing its sharpness, as shown in Fig.16 [70]. Thin films of Fe or Co $(20-30 \mathrm{~nm})$ are used as a catalyst, and the growth of CNTs with a diameter of $\sim 40 \mathrm{~nm}$ has been observed. Because the magnetic nanoparticles are located at the tip of CNTs, this type of probe can also be utilized to study the magnetic properties of nanoclusters with higher spatial resolution [71]. For spin-polarized STM measurement, a magnetic coating of Fe (1020 monolayers) on a cleaned tungsten tip is conventionally used [72], which may be improved using a CNT tip.
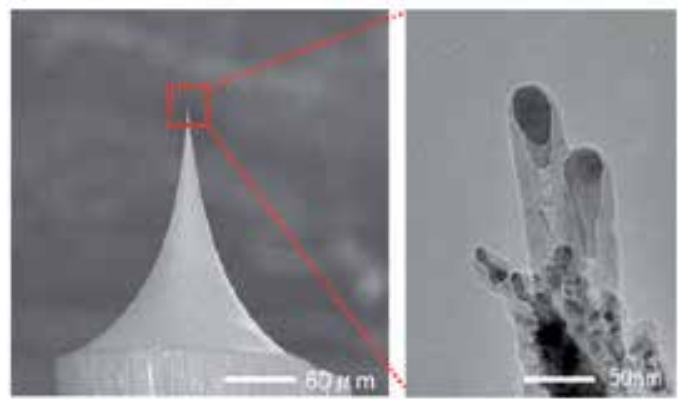

Fig. 16. SEM and TEM images of a grown CNT-STM probe. Two CNTs are grown on the apex of the STM tip. Black contrast corresponds to metal particles used as catalyst. 


\section{(c) Dielectrophoresis}

When an alternating electric field $(\sim \mathrm{MHz})$ is applied between asymmetric electrodes (for example, a metal probe and counter plane electrode) immersed in CNT solution (where solvent is water, alcohol, dichloroethane, etc.), the CNTs are polarized and become attached to the probe (dielectrophoresis). [73]. The high-yield synthesis of conductive CNT tips for the multiprobe microscope [74-76] was reported using the dielectrophoresis method [77]. After Pt-Ir coating, such a tip were successfully applied for electronic transport measurement by multiprobe STM using the four-terminal method.

\section{Summary}

Laser-combined STM and related techniques have been reviewed and discussed focusing on the analysis of nanoscale particles and clusters. The addition of optical technologies to STM provides new approaches to the study of nanoscale-material physics and chemistry. Nearfield optical microscopy (NSOM) and other techniques [78-86], which have not been discussed in this chapter, are expected to play complementary roles in understanding and developing the physics and chemistry of new nanoparticles/clusters for realizing novel functional devices.

\section{References}

[1] M. Haruta, Catal. Today 36, 153 (1997).

[2] P. Jena and A. W. Castleman (Eds), Nanoclusters, A Bridge across Disciplines, Elsevier (2011).

[3] J. A. Alonso, Structure and Properties of Atomic Nanoclusters, Imperial College Press (2006).

[4] J. P. Liu, E. Fullerton, O. Gutfleish and D. J. Sellmyer (Eds), Nanoscale Magnetic Materials and Applications, Springer (2009).

[5] F. J. Owens and C. P. Poole (Eds), The Physics and Chemistry of Nanosolids, Wiley (2008).

[6] J. M. R. Weaver, L. M. Wapita, and H. K. Wickramasinghe, Nature 342 (1989) 783.

[7] A. Hida, Y. Mera, and K. Maeda, Appl. Phys. Lett. 78 (2001) 3190.

[8] S. Grafstrom, P. Schuller, J. Kowalski, and R. Neumann, J. Appl. Phys. 83 (1998) 3453.

[9] N. Naruse, Y. Mera, Y. Fukuzawa, Y. Nakamura, M. Ichikawa, and K. Maeda, J. Appl. Phys., 102 (2007) 114301.

[10] N. Naruse, Y. Mera, Y. Nakamura, M. Ichikawa, and K. Maeda, Appl. Phys. Lett. 94 (2009) 093104.

[11] Y. Kayanuma, Phys. Rev. B 44 (1991) 13085.

[12] D. E. Aspnes, in Handbook on Semiconductors, edited by M. Balkanski (North-Holland, Amsterdam, 1980) Vol.2, p. 109.

[13] D. E. Aspnes, Phys. Rev. 147 (1969) 554.

[14] A. Hida, Y. Mera, and K. Maeda, Appl. Phys. Lett. 78 (2001) 3029.

[15] N. Naruse, Y. Mera, Y. Nakamura, M. Ichikawa, and K. Maeda, J. Appl. Phys. 104 (2008) 074321.

[16] A. Hida, Y. Mera, and K. Maeda, Physica B 308-310 (2001) 1145.

[17] H. Udono et al., Thin Solid Films 461 (2004) 182.

[18] L. Miglio, V. Meregalli, and O. Jepsen, Appl. Phys. Lett. 75 (1999) 385.

[19] C. Thirstrup, M. Sakurai K. Stokbro, and M. Aono, Phys. Rev. Lett. 82 (1999) 1241. 
[20] C. Chen, C. A. Bobisch, and W. Ho, Science 325 (2009) 981.

[21] A. Okada, K. Kanazawa, K. Hayashi, N. Okawa, T. Kurita, O. Takeuchi and H. Shigekawa Appl. Phys. Exp. 3 (2010) 015201.

[22] C. Chen, P. Chu, C. A. Bobisch, D. L. Mills, and W. Ho, Phys. Rev. Lett. 105 (2010) 217402.

[23] Y. Sainoo, Y. Kim, T. Okawa, T. Komeda, H. Shigekawa, and M. Kawai, Phys. Rev. Lett. 95 (2005) 246102.

[24] Y. Uehara and S. Ushioda, Appl. Phys. Lett. 86 (2005) 181905.

[25] A. Othonos, J. Appl. Phys. 83 (1998) 1789 and references therein.

[26] J. Shah, Ultrafast Spectroscopy of Semiconductors and Semiconductor Nanostructures (Berlin: Springer 1999).

[27] H. Mamin, H. Birk, P. Wimmer and D. Rugar, J. Appl. Phys. 75 (1994) 161.

[28] J. Wintterlin, J. Trost, S. Renisch, R. Schuster, T. Zambelli and G. Ertl, Surf. Sci. 394 (1997) 159.

[29] M. Rost et al., Rev. Sci. Instrum. 76 (2005) 053710.

[30] L. Petersen et al., Rev. Sci. Instrum. 72 (2001) 1438.

[31] U. Kemiktarak, T. Ndukum, K. Schwab, K. CandEkinci, Nature 450 (2007) 85.

[32] Y. Terada, S. Yoshida, O. Takeuchi and H. Shigekawa, J. Phys. Condens. Matter 22 (2010) 264008

[33] Y. Terada, S. Yoshida, O. Takeuchi and H. Shigeakawa: Nature photonics, 4 (2010) 869.

[34] H. Shigekawa, S. Yoshida, O. Takeuchi, M. Aoyama, Y. Terada, H. Kondo and H Oigawa, Thin Solid Films, 516 (2008) 2348.

[35] Y. Terada, M. Aoyama, H. Kondo, A. Taninaka, O. Takeuchi and H. Shigekawa, Nanotechnology 18 (2007) 044028.

[36] Y. Terada, S. Yoshida, O. Takeuchi and H. Shigekawa, Advances in Optical Technologies 2011 (2011) 510186.

[37] S. Yoshida, Y. Terada, R. Oshima, O. Takeuchi and H. Shigekawa, Nanoscale 2012 (2012), DOI:10.1039/C2NR11551D.

[38] O. Takeuchi, M. Aoyama, R. Oshima, Y. Okada, H. Oigawa, N. Sano, H. Shigekawa, R. Morita and M. Yamashita, Appl. Phys. Lett. 85 (2004) 3268.

[39] O. Takeuchi, R. Morita, M. Yamashita and H. Shigekawa: Jpn. J. Appl. Phys. 41 (2002) 4994.

[40] H. Shigekawa, O. Takeuchi, Y. Terada and S. Yoshida: Handbook of Nanophysics, Edited by Klaus D. Sattler Taylor \& Francis (2010), vol. 6, Principles and Methods.

[41] M. Yamashita, H. Shigekawa and R. Morita (Eds), Mono-Cycle Photonics and Optical Scanning Tunneling Microscopy-Route to Femtosecond Angstrom Technology(Springer, 2005).

[42] T. Okuda et al., Phys. Rev. Lett. 102 (2009) 105503.

[43] Y. Nakamura, Y. Mera and K. Maeda, Jpn. J. Appl. Phys. 44 (2005) L1373.

[44] K. Kanazawa, A. Taninaka, H. Huang, N Nishimura, S. Yoshida, O. Takeuchi, and H. Shigekawa, Chem. Commun. 47 (2011) 11312.

[45] K. Yamada, H. Sato, T. Komaguchi, Y. Mera, and K. Maeda, Appl. Phys. Lett. 94 (2009) 253103.

[46] M.Berthe, S.Yoshida, Y.Ebine, K.Kanazawa, A.Okada, A.Taninaka, O.Takeuchi, N. Fukui, H. Shinohara, S.Suzuki, K.Sumitomo, Y. Kobayashi, B.Grandidier, D.Stievenard and H.Shigekawa, Nano Lett. 7 (12) (2007) 3623-3627.

[47] C. Joachim, J. K. Gimzewski, R. R. Schlittler, and C. Chavy, Phys. Rev. Lett., 74 (1995) 2102.

[48] K. Akiyama et al., Rev. Sci. Instrum. 76 (2005) 083711. 
[49] A. Saito et al., Surf. Sci. 601 (2007) 5294.

[50] R. M. Stockle, Y. D. Suh, V. Deckert and R. Zenobi, Chem. Phys. Lett. 318 (2000) 131.

[51] R. Berndt, J. K. Gimzewski, and P. Johansson, Phys. Rev. Lett. 71 (1993) 3493.

[52] M. Iwami, Y. Uehara and S. Ushioda, Rev. Sci. Instrum. 69 (1998) 4010.

[53] W. H. Zhang, B. S. Yeo, T. Schmid, R. Zenobi, J. Phys. Chem. C111 (2007) 1733.

[54] C. Zhang et al., Rev. Sci. Instrum. 82 (2011) 083101.

[55] T. Nishino, T. Ito and Y. Umezawa, Anal. Chem. 74 (2002) 4275.

[56] T. Nishino and Y. Umezawa, Anal. Sci. 26 (2010) 1023.

[57] D. Gingery and P. Bühlmann, Surf. Sci. 605 (2011) 1099.

[58] Z. Cheng et al., Nano Res. 4 (2011) 523.

[59] S. Iijima, Nature, 354, 56 (1991).

[60] J. M. Marulanda, 2011, Electronic Properties of Carbon Nanotubes, InTech.

[61] H. Dai, J. H. Hafner, A. G. Rinzler, D. T. Colbert, and R. E. Smalley, Nature 384 (1996) 147.

[62] S. Akita et al., J. Phys D: Appl. Phys. 32 (1999) 1044.

[63] W. Mizutani, N. Choi, T. Uchihashi and H. Tokumoto, Jpn. J. Appl. Phys. 40 (2001) 4328.

[64] T.Ikuno et al., Jpn. J. Appl. Phys. 43 (2004) L644.

[65] N. Isomura, X. Wu, and Y. Watanabe, J. Chem Phys. 131(16) (2009) 164707.

[66] W. Wongwiriyapan et al., Jpn. J. Appl. Phys. 45 (2006) 1880.

[67] C. L. Cheung, J. H. Hafner, and C. M. Lieber, PNAS 97 (2000) 3809.

[68] I. T. Clark, G. Rius, Y. Matsuoka, and M. Yoshimura, J. Vac. Sci. Technol. B28 (2010) 1148.

[69] M. Yoshimura, S. Jo and K. Ueda, Jpn. J. Appl. Phys. 42 (7B) (2003) 4841.

[70] K. Tanaka, M. Yoshimura, and K. Ueda, e-J. Surf. Sci. Nanotech. 4 (2006) 276.

[71] K. Tanaka, M. Yoshimura and K. Ueda, J. Nanomaterials 2009 (2009) 147204.

[72] J. E. Bickel, et al., Phys. Rev. B84 (2011) 054454.

[73] K. Ueda, M. Yoshimura, M. Ishikawa, T. Nagamura, Japan Patent: 3557589

[74] I. Shiraki, F. Tanabe, R. Hobara, T. Nagao, and S. Hasegawa, Surf. Sci. 493 (2001) 633.

[75] J. Onoe, T. Nakayama, M. Aono, and T. Hara, Appl. Phys. Lett. 82 (2003) 595.

[76] M. Ishikawa, M. Yoshimura and K. Ueda, Jpn. J. Appl. Phys. 44 (2005) 1502.

[77] H. Konishi, Y. Murata, W. Wongwiriyapan, M. Kishida, K. Tomita, K. Motoyoshi, S. Honda, M. Katayama, S. Yoshimoto, K. Kubo, R. Hobara, I. Matsuda, S. Hasegawa and M. Yoshimura, Rev. Sci. Instr. 78 (2007) 013703.

[78] Y. Terada, S. Yoshida, A. Okubo, K. Kanazawa, M. Xu, O. Takeuchi and H. Shigekawa, Nano Lett. 8 (11), (2008) 3577-3581.

[79] S. Yoshida, Y. Kanitani, O. Takeuchi and H. Shigekawa, Appl. Phys. Lett. 92 (2008) 102105.

[80] S. Yoshida, Y. Kanitani, R. Oshima, Y. Okada, O. Takeuchi and H. Shigekawa, Phys. Rev. Lett. 98 (2007) 026802.

[81] A. Hagen et al., Phys. Rev. Lett. 95 (2005) 197401.

[82] H. Watanabe, Y. Ishida, N. Hayazawa, Y. Inouye and S. Kawata, Phys. Rev. B 69 (1004) 1.

[83] S. Yasuda, T. Nakamura, M. Matsumoto and H. Shigekawa, J. Am. Chem. Soc. 125 (2003) 16430.

[84] D. Futaba, R. Morita, M. Yamashita, S. Tomiyama and H. Shigekawa, Appl. Phys. Lett. 83 (2003) 2333.

[85] S. Kawata, M. Ohtsu, M. Irie (Eds), Nano-Optics, Springer (2002).

[86] S. Grafstorm (o: umlaut), J. Appl. Phys. 91 (2002) 1717.

[87] S. Loth, M. Etzkorn, C. Lutz, D. Eigler and A. Heinrich, Science 24 (2010) 1628. 


\title{
On the Optical Response of Nanoparticles: Directionality Effects and Optical Forces
}

\author{
Braulio García-Cámara1, Francisco González¹, Fernando Moreno1, \\ Raquel Gómez-Medina², Juan José Sáenz ${ }^{2}$ and Manuel Nieto-Vesperinas ${ }^{3}$ \\ ${ }^{1}$ Grupo de Óptica, Departamento de Física Aplicada, Universidad de Cantabria \\ ${ }^{2}$ Departamento de Física de la Materia Condensada, Universidad Autónoma de Madrid \\ ${ }^{3}$ Instituto de Ciencias de los Materiales de Madrid, CSIC
}

Spain

\section{Introduction}

Nowadays, miniaturization is a general challenge for technology. Researchers in science and technology claim to study ever smaller systems and develop ever smaller devices. The nanometric range is, at present, an important focus of attention of scientists and engineers following the famous prediction by Prof. Feynman: "There's plenty of room at the bottom". Reduction of dimensions, at this level, involves that more specific and more complex tools are needed.

Light has appeared as a convenient solution for these tasks because of its wavelength (hundreds of nanometers) and the large amount of information it contains about systems with which it interacts (Prasad, 2004). The interaction of light with small systems, either particles or structures, gives rise to several scattering phenomena which are strongly dependent on both the characteristics of the incident radiation (frequency, polarization) and those of the object (size, shape, optical properties). These interactions can be used either to obtain information about the interacting object (e.g. particle sizing) (Zhu et al., 2010) or to produce light scattering phenomena "à la carte" by means of suitable nanoobjects.

At the nanoscopic level, the interaction between an incident beam and a metallic system produces an interesting physical phenomenon which is the base of many technological applications in diverse fields like medicine, biology, communications, information storing, energy transformation, photonics, etc (Anker et al., 2008; Maier et al., 2003). This is the excitation of localized surface plasmon resonances (LSPR) (Prasad, 2004). For these, the electromagnetic field experiences a high localization in the scatterer and a strong enhancement out of the scatterer.

These advances have stimulated new research devoted to obtain a greater control over how light is scattered by these systems. Researchers have analyzed emerging structures (nanoholes (Gao et al., 2010), nanocups (Mirin \& Halas, 2009), etc). But, what it is more interesting, new engineered materials, called metamaterials and whose optical properties can be manipulated, have been developed (Boltasseva \& Atwater, 2011). The possibility to obtain structures with 
optical properties "à la carte" allows getting scattering phenomena never observed before in natural media, for instance negative refraction (Shalaev, 2008). The main consequences of negative refraction are the two interesting potential applications: cloacking (Pendry et al., 2006) and perfect lens (Pendry, 2000; Nieto-Vesperinas \& Garcia, 2003).

The control over the values of both the electric permittivity and the magnetic permeability of an object gives us a control over the way it scatters light, and in particular, the angular distribution of the scattered radiation. This control could involve a dramatic evolution on the field of nanodevices. For this reason, the objective of this chapter is to analyze directional effects on both light scattering and optical forces of a nanoparticle with convenient optical constants. The structure of the chapter is as follows: while sections 2, 3 and 4 are devoted to the directional features on light scattering by nanoparticles, section 5 summarizes the main results on optical forces. Finally, the most important conclusions about these results are recapitulated in section 6.

\section{Light scattering by nanoparticles}

\subsection{Mie theory}

The problem of the electromagnetic scattering from an isolated and spherical particle was firstly solved in 1908 by Gustav Mie (Mie, 1908). However, this simple system still involves interesting physical behaviors that are worthy of further study.

Mie theory considers a spherical particle of radius $a$ and optical constants given by an electric permittivity, $\varepsilon_{p}$, and a magnetic permeability, $\mu_{p}$, immersed in a homogeneous and isotropic medium. This is illuminated by a linear polarized plane wave, as in Figure 1. Without loss of generality, we assume that the surrounding medium is vacuum $\left(\varepsilon_{\mathrm{s}}=\mu_{\mathrm{s}}=1\right)$. The scattered electromagnetic field $\left(\mathbf{E}_{s}, \mathbf{H}_{s}\right)$ can be expressed as a multipole expansion of Vector Spherical Harmonics (VSH), called Mie expansion, as follows

$$
\begin{gathered}
\mathbf{E}_{s}=\sum_{n=1}^{\infty} E_{n}\left(i a_{n} \mathbf{N}_{e \ln }^{(3)}-b_{n} \mathbf{M}_{o l n}^{(3)}\right) \\
\mathbf{H}_{s}=\frac{k}{\omega \mu_{p}} \sum_{n=1}^{\infty} E_{n}\left(i b_{n} \mathbf{N}_{o l n}^{(3)}+a_{n} \mathbf{M}_{e l n}^{(3)}\right)
\end{gathered}
$$

where $k=m \omega / c=m 2 \pi / \lambda, \lambda$ being the incident wavelength in vacuum, $m=\sqrt{\varepsilon_{p} \mu_{p}}$ the refractive index of the particle, $c$ the speed of light in vacuum and $\omega$ the angular frequency of the incident wave. $E_{n}$ is defined as $E_{n}=E_{0} i^{n} \frac{2 n+1}{n(n+1)}, E_{0}$ being the amplitude of the incident plane wave. The series are characterized by the $a_{n}$ and $b_{n}$ Mie coefficients which are defined as (Bohren \& Huffman, 1983)

$$
a_{n}=\frac{\mu_{s} m^{2} j_{n}(m x)\left[x j_{n}(x)\right]^{\prime}-\mu_{p} j_{n}(x)\left[m x j_{n}(m x)\right]^{\prime}}{\mu_{s} m^{2} j_{n}(m x)\left[x h_{n}^{(1)}(x)\right]^{\prime}-\mu_{p} h_{n}^{(1)}(x)\left[m x j_{n}(m x)\right]^{\prime}}
$$




$$
b_{n}=\frac{\mu_{p} m^{2} j_{n}(m x)\left[x j_{n}(x)\right]^{\prime}-\mu_{s} j_{n}(x)\left[m x j_{n}(m x)\right]^{\prime}}{\mu_{p} m^{2} j_{n}(m x)\left[x h_{n}^{(1)}(x)\right]^{\prime}-\mu_{s} h_{n}^{(1)}(x)\left[m x j_{n}(m x)\right]^{\prime}}
$$

$x$ being the size parameter, that is defined as

$$
x=k a=\frac{2 \pi a}{\lambda},
$$

In addition, $j_{n}$ are the spherical Bessel functions and $h_{n}(1)$ the spherical Bessel functions of third kind or Hankel functions. As the electric and magnetic dipolar contributions are weighted by coefficients $a_{1}$ and $b_{1}$, respectively, the quadrupolar ones by $a_{2}$ and $b_{2}$ and so on, Mie coefficients $a_{n}$ are associated to the electric part of the scattered electromagnetic radiation, while $b_{n}$ are associated to the magnetic one.

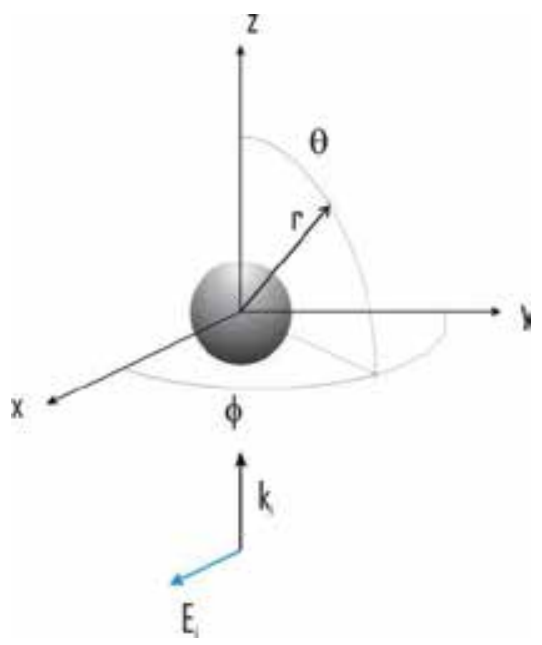

Fig. 1. Scheme of the geometry of the problem.

These coefficients contain the relevant information about essential scattering parameters as the extinction, $C_{\text {ext }}$, and scattering, $C_{s c a}$, cross sections. These can be written as

$$
\begin{gathered}
C_{\text {sca }}=\frac{2 \pi}{k^{2}} \sum_{n=1}^{\infty}(2 n+1)\left(\left|a_{n}\right|^{2}+\left|b_{n}\right|^{2}\right) \\
C_{e x t}=\frac{2 \pi}{k^{2}} \sum_{n=1}^{\infty}(2 n+1) \operatorname{Re}\left(a_{n}+b_{n}\right)
\end{gathered}
$$

\subsection{Details of Mie theory at the nanoscopic level}

If particle size is very small compared with the incident wavelength, that is $a / \lambda<<1$, dipolar contributions ( $n=1$ in Eqs. (1)- (2)) clearly dominate and Mie coefficients of order higher than 1 can be neglected. Thus, the Mie expansion can be simplified and the previous parameters have simple expressions 


$$
\begin{gathered}
C_{\text {sca }}=\frac{2 \pi}{k^{2}}\left[3\left(\left|a_{1}\right|^{2}+\left|b_{1}\right|^{2}\right)\right] \\
C_{\text {ext }}=\frac{2 \pi}{k^{2}}\left[3 \operatorname{Re}\left(a_{1}+b_{1}\right)\right]
\end{gathered}
$$

This is the case of a nanoparticle $(a<50 \mathrm{~nm})$ when it is illuminated by an incident wave in the visible or near infrared (NIR) part of the spectrum $(\lambda>500 \mathrm{~nm})$.

The predominant dipolar conduct, either electric or magnetic, of nanoparticles is usually described by the electric and/or magnetic complex polarizabilities, $\alpha_{e}$ and $\alpha_{m}$, respectively. Both can also be expressed as a function of the two first Mie coefficients

$$
\begin{aligned}
& \alpha_{e}=\frac{\alpha_{e}{ }^{(0)}}{1-i \frac{2}{3} k^{3} \alpha_{e}{ }^{(0)}}=\frac{3 i}{2 k^{3}} a_{1} \\
& \alpha_{m}=\frac{\alpha_{m}{ }^{(0)}}{1-i \frac{2}{3} k^{3} \alpha_{m}{ }^{(0)}}=\frac{3 i}{2 k^{3}} b_{1}
\end{aligned}
$$

where

$$
\begin{gathered}
\alpha_{e}{ }^{(0)}=4 \pi a^{3} \frac{\varepsilon_{p}-1}{\varepsilon_{p}+2} \\
\alpha_{m}{ }^{(0)}=4 \pi a^{3} \frac{\mu_{p}-1}{\mu_{p}+2}
\end{gathered}
$$

are the static polarizabilities, defined in the limit $k a \rightarrow 0$.

\section{Directional effects on light scattering by nanoparticles with arbitrary values of $\varepsilon$ and $\mu$.}

\subsection{Kerker's theory}

In the early eighties, M. Kerker and co-authors (Kerker et al., 1983) presented an interesting study about the scattering properties, in the far field, of a spherical particle much smaller than the incident wavelength, illuminated by a plane wave and without any restriction for the values of its relative optical constants $(\varepsilon$ and $\mu)$. Some interesting electromagnetic scattering effects were described in this work such as the zero-backward and the zeroforward scattering. Although the idea of a magnetic permeability different from 1 in the visible range was hypothetical and the described effects were thought to be impossible to be observed when the work was presented, the engineered metamaterials have currently revitalized these electromagnetic studies (Zhedulev, 2010). 
In this section, the main theoretical aspects described by M. Kerker et al. are briefly reviewed.

\subsubsection{Zero-backward scattering: First Kerker's condition}

When we consider a system, like that of Figure 1, the scattered intensity in the scattering plane can be described by means of two polarized components: $I_{T E}$ and $I_{T M}$. While $I_{T E}$ corresponds to an incident electric field parallel to the scattering plane, $I_{T M}$ corresponds to a perpendicular one. These components can be written as (Bohren \& Huffman, 1983)

$$
\begin{gathered}
I_{T E}=\frac{\lambda^{2}}{4 \pi r^{2}}\left|\sum_{n} \frac{2 n+1}{n(n+1)}\left(a_{n} \pi_{n}+b_{n} \tau_{n}\right)\right|^{2} \\
I_{T M}=\frac{\lambda^{2}}{4 \pi r^{2}}\left|\sum_{n} \frac{2 n+1}{n(n+1)}\left(a_{n} \tau_{n}+b_{n} \pi_{n}\right)\right|^{2}
\end{gathered}
$$

where $r$ is the distance from the particle to the observer $(2 \pi r / \lambda>>1)$ and $\pi_{n}$ and $\tau_{n}$ are angular functions defined in (Bohren \& Huffman, 1983). As we are considering a very small or dipole-like particle $(a \rightarrow 0)$, only the two first Mie coefficients $\left(a_{1}\right.$ and $\left.b_{1}\right)$ are introduced in the expressions. In addition some approximations can be applied to these coefficients in such a way that the scattered intensity components can be approximated by

$$
\begin{aligned}
& I_{T E}=\frac{\lambda^{2} x^{6}}{4 \pi r^{2}}\left|\left(a_{1}+b_{1} \cos \theta\right)\right|^{2}=\frac{\lambda^{2} x^{6}}{4 \pi r^{2}}\left|\left(\left(\frac{\varepsilon^{-1}}{\varepsilon+2}\right)+\left(\frac{\mu^{-1}}{\mu+2}\right) \cos \theta\right)\right|^{2} \\
& I_{T M}=\frac{\lambda^{2} x^{6}}{4 \pi r^{2}}\left|\left(a_{1} \cos \theta+b_{1}\right)\right|^{2}=\frac{\lambda^{2} x^{6}}{4 \pi r^{2}} \mid\left(\left(\frac{\varepsilon^{-1}}{\varepsilon+2}\right) \cos \theta+\left(\frac{\mu^{-1}}{\mu+2}\right)\right)^{2}
\end{aligned}
$$

$\theta$ being the scattering angle, defined as the angle between the incident and the scattered directions (see Figure 1).

For the backward scattering direction $\left(\theta=180^{\circ}\right)$ the previous expressions adopt the following forms

$$
\begin{aligned}
& I_{T E}\left(180^{\circ}\right)=\frac{\lambda^{2}}{4 \pi r^{2}} x^{6}\left|\left(\left(\frac{\varepsilon^{-1}}{\varepsilon+2}\right)-\left(\frac{\mu^{-1}}{\mu+2}\right)\right)\right|^{2} \\
& I_{T M}\left(180^{\circ}\right)=\frac{\lambda^{2}}{4 \pi r^{2}} x^{6} \mid\left(-\left(\frac{\varepsilon^{-1}}{\varepsilon+2}\right)+\left.\left(\frac{\mu-1}{\mu+2}\right)\right|^{2}\right.
\end{aligned}
$$

It easy to observe that when $\varepsilon=\mu$, or equivalently when $\alpha_{e}=\alpha_{m}$, the scattered intensity in the backward direction is zero for both incident polarizations. This is the zero-backward scattering condition and we shall call in the following the first Kerker's condition. In Figure 2 the 
scattering pattern of a dipole-like particle with relative optical properties, $\varepsilon=\mu=3$ is shown. Only a TM polarization is considered because, from Eqs. (18) and (19), the scattered intensity is equal for both polarizations under this condition.

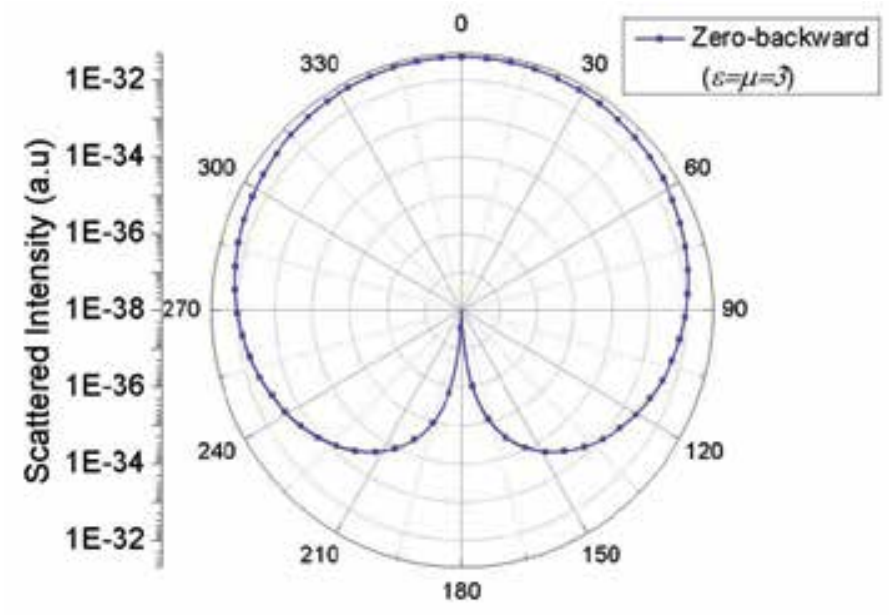

Fig. 2. Scattering diagram of a dipole-like particle $\left(a=10^{-6} \lambda\right)$ with relative optical properties fulfilling the zero-backward condition and for a TM incident polarization

\subsubsection{Zero-forward scattering: Second Kerker's condition}

For $\theta=0^{\circ}$ (forward scattering direction), Eqs. (16)-(17) become

$$
\begin{gathered}
I_{T E}\left(0^{\circ}\right)=\frac{\lambda^{2}}{4 \pi r^{2}} x^{6}\left|\left(\left(\frac{\varepsilon^{-1}}{\varepsilon+2}\right)+\left(\frac{\mu^{-1}}{\mu+2}\right)\right)\right|^{2} \\
I_{T M}\left(0^{\circ}\right)=\frac{\lambda^{2}}{4 \pi r^{2}} x^{6}\left|\left(\left(\frac{\varepsilon^{-1}}{\varepsilon+2}\right)+\left(\frac{\mu^{-1}}{\mu+2}\right)\right)\right|^{2}
\end{gathered}
$$

In this case, the $\varepsilon-\mu$ relation which cancel $I_{T E}\left(0^{\circ}\right)$ and $I_{T M}\left(0^{\circ}\right)$ is not as evident as before. However, Kerker et al. (Kerker et al., 1983) demonstrated that this happens when

$$
\varepsilon=\frac{4^{-} \mu}{2 \mu+1}
$$

which is equivalent to $\operatorname{Re}\left(\alpha_{e}\right)=-\operatorname{Re}\left(\alpha_{e}\right)$ and $\operatorname{Im}\left(\alpha_{e}\right)=\operatorname{Im}\left(\alpha_{e}\right)$. This is the zero-forward scattering condition, that we shall call the second Kerker's condition.

It is interesting to highlight that this condition is symmetric. This means that it remains invariant by interchanging $\varepsilon$ and $\mu$. An example of the angular distribution of the scattered intensity of a very-small particle satisfying this condition is shown in Figure 3 for a TM polarized incident beam (TE polarization produces a similar result). 


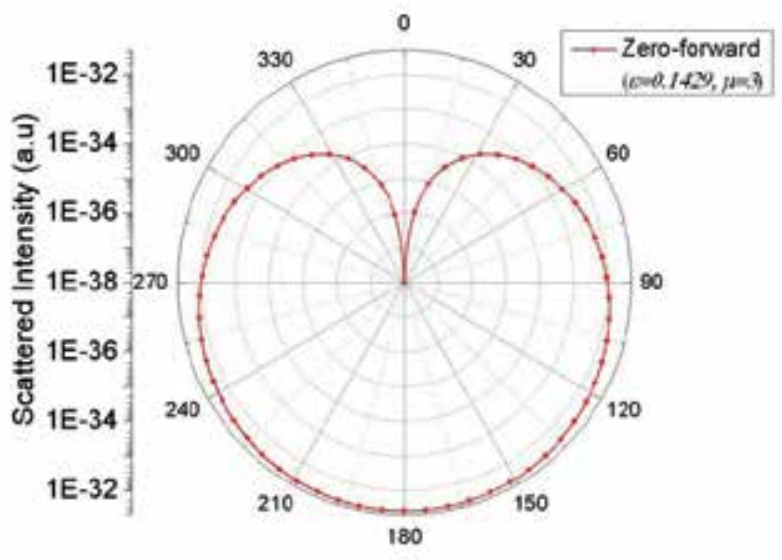

Fig. 3. Scattering diagram of a dipole-like particle $\left(a=10^{-6} \lambda\right)$ with relative optical properties fulfilling the zero-forward condition, $(\varepsilon ; \mu)=(0.1429 ; 3)$, and TM incident polarization.

\subsection{An analysis of Kerker's conditions}

Kerker's theory was developed under the far-field approximation and for the very particular case of dipole-like particles for which only the two first Mie coefficients $\left(a_{1}\right.$ and $\left.b_{1}\right)$ are non negligible. However, as particle size increases or the observer approaches, other multipolar terms become important and the directional features, described previously, can be modified.

\subsubsection{Size effects on the directionality conditions}

One of the responsibles for the appearance of multipolar contributions is the size of the particle, $a$. When this deviates from the condition $a / \lambda<<1$, orders in the Mie expansion greater than 1 start to be non negligible. The purpose of this section is to analyze size effects on the two Kerker's conditions (García-Cámara et al., 2010a).

Zero-backward scattering condition can be extended even for large particle sizes. This is possible because the $\varepsilon-\mu$ symmetry of Mie coefficients (Eqs. (3)-(4)) ensures that all the electric and magnetic multipolar contributions are equal and with opposite sign at backward direction. This produces a destructive interferential effect between both contributions for every multipolar order and for a given particle size, $a$. Figure 4 shows the scattering diagrams for several particles with different size $(a)$ and optical properties satisfying the zero-backward scattering condition $(\varepsilon=\mu)$.

On the contrary, the zero-forward scattering condition is much more sensitive to size effects. In fact, as $a$ increases and multipolar terms, other than the dipolar ones, become important, the electric and magnetic contributions in the forward direction do not interfere destructively anymore, and the zero-forward-scattering tends to disappear. In spite of this, it is possible to find pairs $(\varepsilon ; \mu)$ which minimizes the scattered intensity in the forward direction. In Figure 5, the distribution of the scattered intensity for spherical particles of different sizes is plotted. The values of $\varepsilon$ and $\mu$, which are included in the figure caption, were chosen such that a minimum of the scattered intensity in the forward direction 
appears. For the smallest value of $a$, the scattered intensity in the forward direction is considerably lower compared to other angles. However, as a increases, this minimum becomes less pronounced due to the influence of quadrupolar terms.

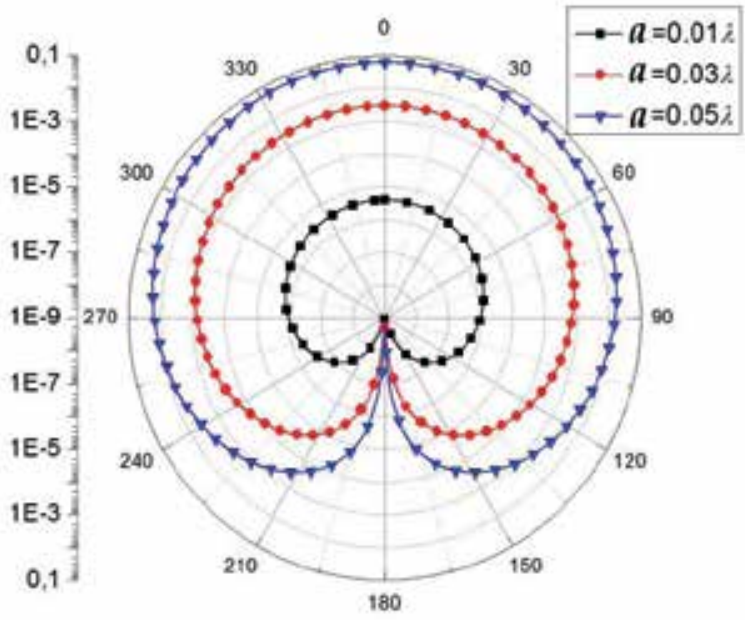

Fig. 4. Scattering diagrams, in logarithmic scale, for a spherical particle with relative optical properties $(\varepsilon ; \mu)=(-3 ;-3)$ and illuminated by a TE-polarized incident light.

Several particlesizes have been considered.

In a recent research (García-Cámara et al., 2010a), it was found that these optical constants which minimize forward scattering don't follow Kerker's conditions but can be fitted to a formally similar expression where fitting coefficients are dependent on particle size.

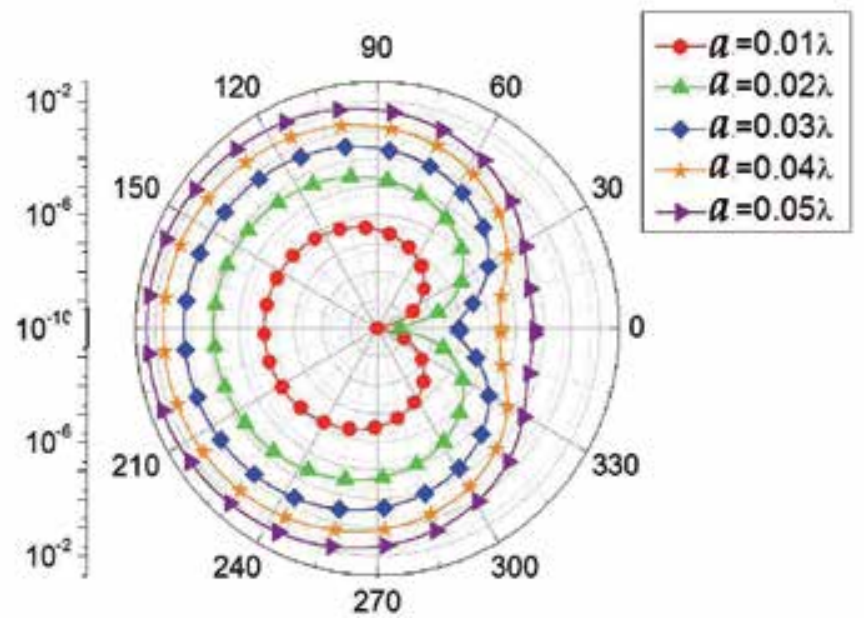

Fig. 5. Scattering diagrams, in logarithmic scale, for a spherical particle illuminated with a TE linearly polarized incident beam. For each particle size, optical properties, in the negative-negative range, are such that the scattered intensity is minimum in the forward direction. In particular, $\mu=-4.55$ for every particle size and $\varepsilon=-1.06(a=0.01 \lambda), \varepsilon=-1.07$ $(a=0.02 \lambda), \varepsilon=-1.09(a=0.03 \lambda), \varepsilon=-1.11(a=0.04 \lambda)$ and $\varepsilon=-1.13(a=0.05 \lambda)$. 


\subsubsection{Distance effects on the directionality conditions: From far to near-field}

Kerker's conditions, as have been remarked above, were deduced under the far-field approximation, that is $(2 \pi r / \lambda>>1)$. If the observer tends to approach $(r / \lambda \leq 1)$, directional effects on light scattering are affected. In a recent work (García-Cámara et al. 2010b), it has been shown that directional effects on light scattering of nanoparticles with optical properties under Kerker's conditions tends to disappear as $r$ decreases. Figure 6 shows the scattered intensity measured on a line crossing a nanoparticle $(a \sim 0.01 \lambda)$ from the backward to the forward direction (Z-axis). Figure 6(a) is devoted to a particle satisfying the first Kerker's condition, while Figure $6(\mathrm{~b})$ shows the same result when its relative optical constants fulfill the second Kerker's condition (eq. (22)). In both cases incident light is P-polarized (an orthogonal polarization produces similar results) and the case of a particle with the same value of $\varepsilon$ and $\mu=1$ (non-directional case) is also plotted, for comparison purposes. For observation distances, $r>0.16 \lambda$, the directionality effects appears through a remarkable drop of the scattered intensity in either the backward (Figure 6a) or the forward direction (Figure 6b). However, as the observer approaches $(r \leq 0.16 \lambda)$, the evolution with the observation distance of the scattered intensity of a nanoparticle with directional features tends to that of a nanoparticle which optical constants do not satisfied any Kerker's condition.

a)

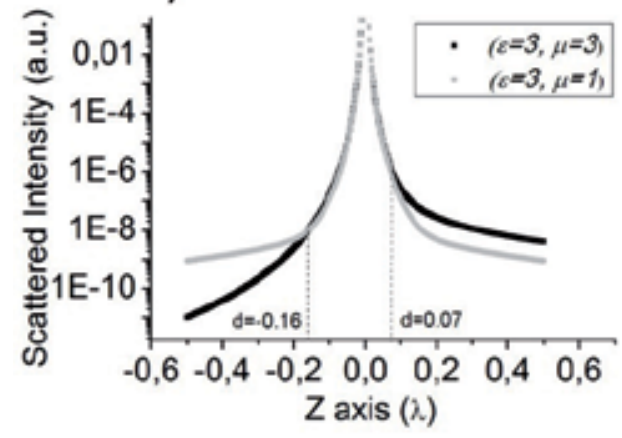

b)

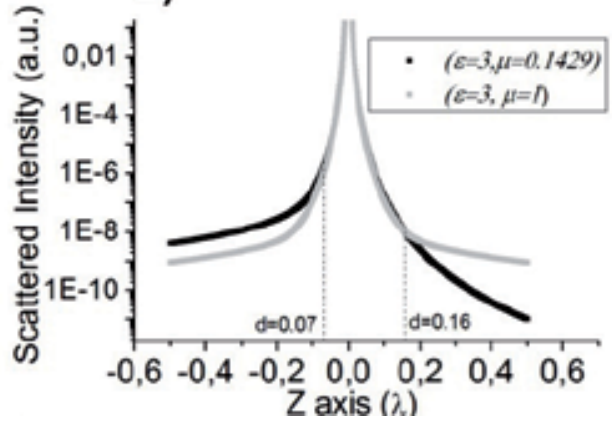

Fig. 6. Scattered intensity by a nanoparticle of radius $a=0.01 \lambda$ and relative optical constants satisfying a) the first Kerker's condition $(\varepsilon=\mu=3)$ or b) the second Kerker's condition $(\varepsilon=3 ; \mu=0.1429)$ as a function of the distance from the particle surface in a direction parallel to the incident direction. For comparison, we have also included the case of a particle with $(\varepsilon=3 ; \mu=1)$. In both cases the incident beam is polarized with the electric field parallel to the scattering plane. 


\subsection{A generalization of the Kerker's conditions}

\subsubsection{The zero-forward scattering condition and the optical theorem}

In a recent research, Alù et al. (Alù \& Engheta, 2011) stated that the zero-forward scattering condition (Eq. 22) is incongruent with the Optical Theorem. This relates the extinction efficiency $\left(Q_{e x t}\right)$ and the scattering amplitude in the forward direction $\left[S\left(0^{\circ}\right)\right]$ as follows (Bohren \& Huffman, 1983)

$$
Q_{e x t}=\frac{4}{x^{2}} \operatorname{Re}\left\{S\left(0^{\circ}\right)\right\}
$$

When the zero-forward scattering condition holds, $S\left(0^{\circ}\right)=0$ and then $Q_{e x t}=0$. This would imply that the particle would not scatter neither absorb electromagnetic radiation. However, in the examples shown in Figures 3 and 5, while the absorption is null because the optical constants are real, light scattering, and then the extinction efficiency, is non-zero at scattering angles other than $\theta=0^{\circ}$.

A first attempt to solve this apparent paradox is found in (Chylek \& Pinnick, 1979) where they conclude that the dipolar approximation used by Kerker and co-workers is a nonunitary approximation because $\operatorname{Re}\left(a_{n}\right) \geq\left|a_{n}\right|^{2}, \operatorname{Re}\left(b_{n}\right) \geq\left|b_{n}\right|^{2}$ are not satisfied, and therefore the Optical Theorem cannot be applied. However, other more specific solutions to this paradox have been proposed recently. Alù et al (Alù \& Engheta, 2011) established that, for a correct estimation of $Q_{e x t}$ it is crucial to include the radiative correction (Draine \& Flatau, 1994) into the two first Mie coefficients $\left(a_{1}\right.$ and $\left.b_{1}\right)$. From these considerations, energy conservation is warranted and, although the forward scattering is not zero, it is minimum with respect to other scattering angles. In addition, if the radiative correction is also included in the deduction of the zero-forward scattering condition (García-Cámara et al., 2011), a new condition can be found where both the Optical Theorem and the zero scattering at $\theta=0^{\circ}$ hold. This condition follows the equation

$$
\varepsilon=\frac{\pi\left(4^{-} \mu\right)^{-} i V k^{3}\left(\mu^{-} 1\right)}{\pi(2 \mu+1)^{-} i V k^{3}\left(\mu^{-1}\right)}
$$

where $V$ is the volume of the particle.

\subsubsection{Directional effects at scattering angles other than forward and backward directions}

Previous analysis on the distribution of the scattered intensity by a nanoparticle at both the forward and the backward direction can also be extended to other scattering angles. In a previous work (García-Cámara, 2010a), it is shown that by choosing a certain scattering angle different from $0^{\circ}$ and $180^{\circ}$, there are pairs $(\varepsilon ; \mu)$, which produce minimum scattered intensity within the scattering plane.

In Figure 7, we plot the scattering diagrams of a nanoparticle $(a=0.01 \lambda)$ illuminated by a TE polarized incident beam. The optical constants are such that the scattered intensity is minimum at representative angles like $30^{\circ}, 60^{\circ}, 120^{\circ}$ and $150^{\circ}$. Each diagram shows a double- 
lobe structure with the position of the minimum depending on the particular values of the relative electric permittivity $(\varepsilon)$ and the relative magnetic permeability $(\mu)$. Therefore, a suitable tuning of the material optical constants serves to control the angular position of the minimum of the scattered intensity.

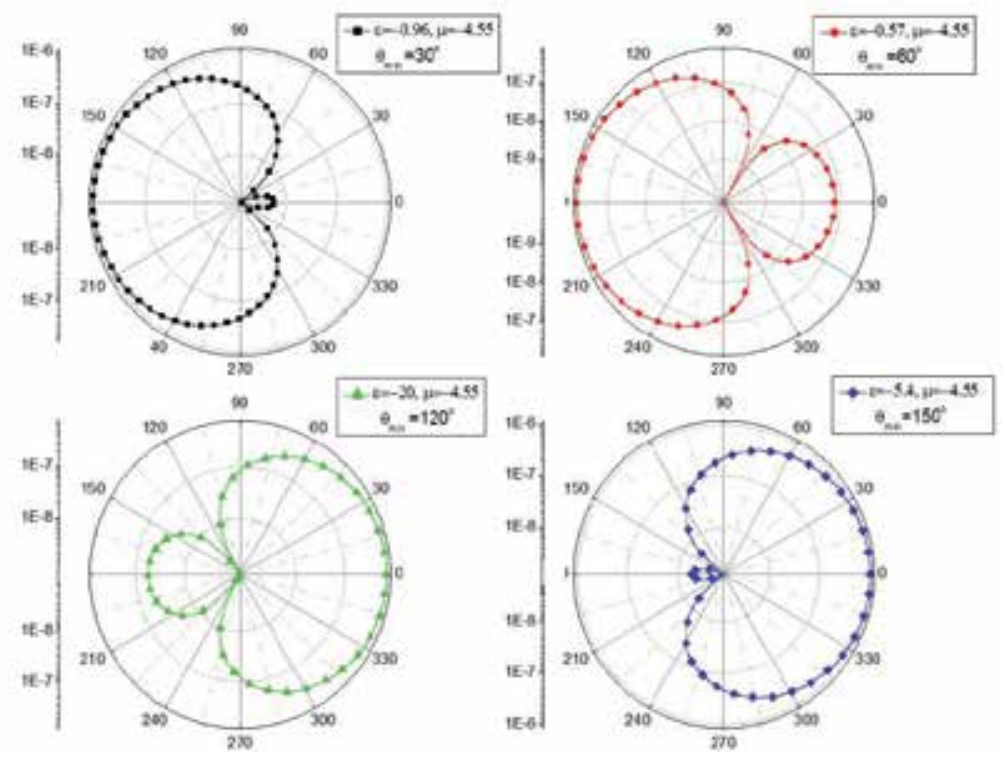

Fig. 7. Scattering diagrams of a spherical particle with $a=0.01 \lambda$ and relative optical constants in the negative-negative range (labeled in the figure) which produce a minimum scattering at certain scattering angles. The particle is illuminated with a linearly polarized incident plane wave with the electric field perpendicular to the scattering plane (TE polarization).

\section{Directional effects on light scattering by dielectric particles}

Previous analysis presented in this chapter about directional effects of light scattering have been done for nanoparticles with arbitrary values of both the relative electric permittivity $(\varepsilon)$ and the relative magnetic permeability $(\mu)$ which do not correspond to any real material. In general, conventional materials do not show any stimulus to the magnetic field of electromagnetic radiation in the visible or the near-infrared region of the electromagnetic spectrum. For this reason, previous analyses have been considered an entelechy, as V. Veselago did in his work to generate and enrich scientific knowledge (Veselago, 1968). Very recently and looking for real situations, it has been shown that submicrometer particles made of Silicon (Evlyukhin et al, 2010 ; García-Extarri et al., 2011) or Germanium (GómezMedina et al, 2011b) present both effective electric and magnetic responses, corresponding to the dipolar contributions characterized by their first-order Mie coefficients, in the nearinfrared range. Either of them can be selected by changing the illumination wavelength.

For this kind of nanoparticles, the spectral proximity of both dipolar electric and magnetic responses allows the appearance of coherent effects between dipolar modes. Consequently, under certain conditions, these scatterers are able to satisfy Kerker's conditions. Following the work made by Gómez-Medina et al. (Gómez-Medina et al, 2011b), in Figure 8, the 
electric $\left(\alpha_{e}\right)$ and the magnetic $\left(\alpha_{m}\right)$ polarizabilities of a Ge nanoparticle of radius $a=240 \mathrm{~nm}$ are plotted as a function of the wavelength $(\lambda)$ of the incident radiation. In the considered spectral range, Germanium has a refractive index which can be well approximated by a real constant $m=4$ (Palik, 1985). Also the spectral evolution of the extinction efficiency $\left(\mathrm{Q}_{\text {ext }}\right)$ has been included in order to show the resonant behaviors that appear in a Ge nanoparticle. A dipolar electric (DE) mode arises at $\lambda=1823 \mathrm{~nm}$, while a dipolar magnetic (DM) resonance is located at $\lambda=2193 \mathrm{~nm}$. The vertical lines point the wavelengths at which either the first $\left(\alpha_{e}=\alpha_{m}\right)$ or the second $\left(\operatorname{Re}\left(\alpha_{e}\right)=-\operatorname{Re}\left(\alpha_{e}\right)\right.$ and $\left.\operatorname{Im}\left(\alpha_{e}\right)=\operatorname{Im}\left(\alpha_{e}\right)\right)$ Kerker's condition are fulfilled.

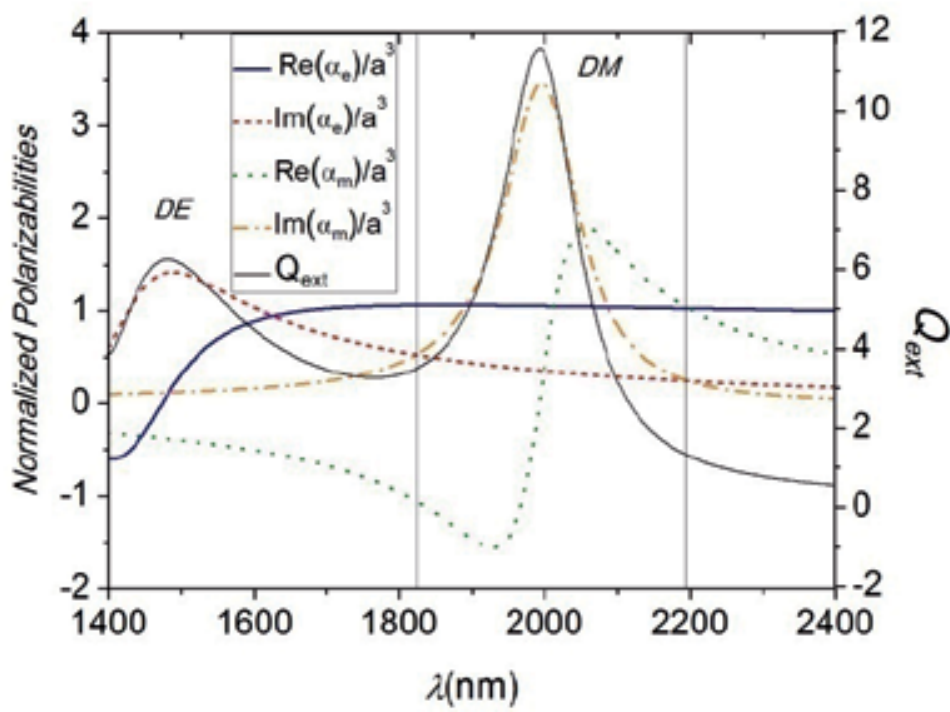

Fig. 8. Real and imaginary parts of the electric $\left(\alpha_{e}\right)$ and the magnetic $\left(\alpha_{m}\right)$ polarizabilities for a Ge nanoparticle $(a=240 \mathrm{~nm})$. The refractive index of Germanium, in the considered range, can be considered as real and constant, $m \simeq 4+0 i$. The wavelengths at which the first and second Kerker's conditions ( $\lambda=2193 \mathrm{~nm}$ and $\lambda=1823 \mathrm{~nm}$, respectively) are satisfied, are identified with vertical lines. Also, for comparison purposes, the extinction efficiency is plotted identifying the dipolar electric (DE) and the dipolar magnetic (DM) resonances.

The fact that a dielectric and non-magnetic particle $(\varepsilon>0$ and $\mu=1)$ presents both dipolar electric and also dipolar magnetic modes is quite interesting and could be useful for potential applications. For instance, this kind of resonances has been currently used for several tasks in a wide range of fields, ranging from the design of nanodevices (Maier et al, 2003; Anker et al., 2008) to biomedical treatments (Zemp, 2009). Unfortunately, they were observed only in metallic materials which present strong absorption losses. One of the advantages of dielectric materials, like Germanium or Silicon, is that they show negligible absorption in the considered range (Palik, 1985) and then losses are almost absent.

The position and shape of the dipolar resonances shown in Fig. 8 for Ge particles (similarly for $\mathrm{Si}$ particles) produces interesting coherent effects between them and consequently a natural way of reproducing Kerker's conditions by means of real materials. In order to verify that these directional features show up, Figure 9 plots the scattering diagrams of a Ge 
nanosphere $(a=240 \mathrm{~nm})$ when the incident wavelengths are those marked by vertical lines in Figure 8 . The zero-backward scattering condition is satisfied for $\lambda=1823 \mathrm{~nm}$, and there is no scattered intensity in this direction (Figure 9a). However, the zero-forward scattering condition is strongly affected by size effects (Figure 9b). As was described in Section 3.2.1, the size of the particle prevents scattered intensity to be completely suppressed in the forward direction. However, its value is very small compared with those at other scattering angles and most part of the scattered intensity is located in the backward hemisphere $(\pi<\theta<2 \pi)$.

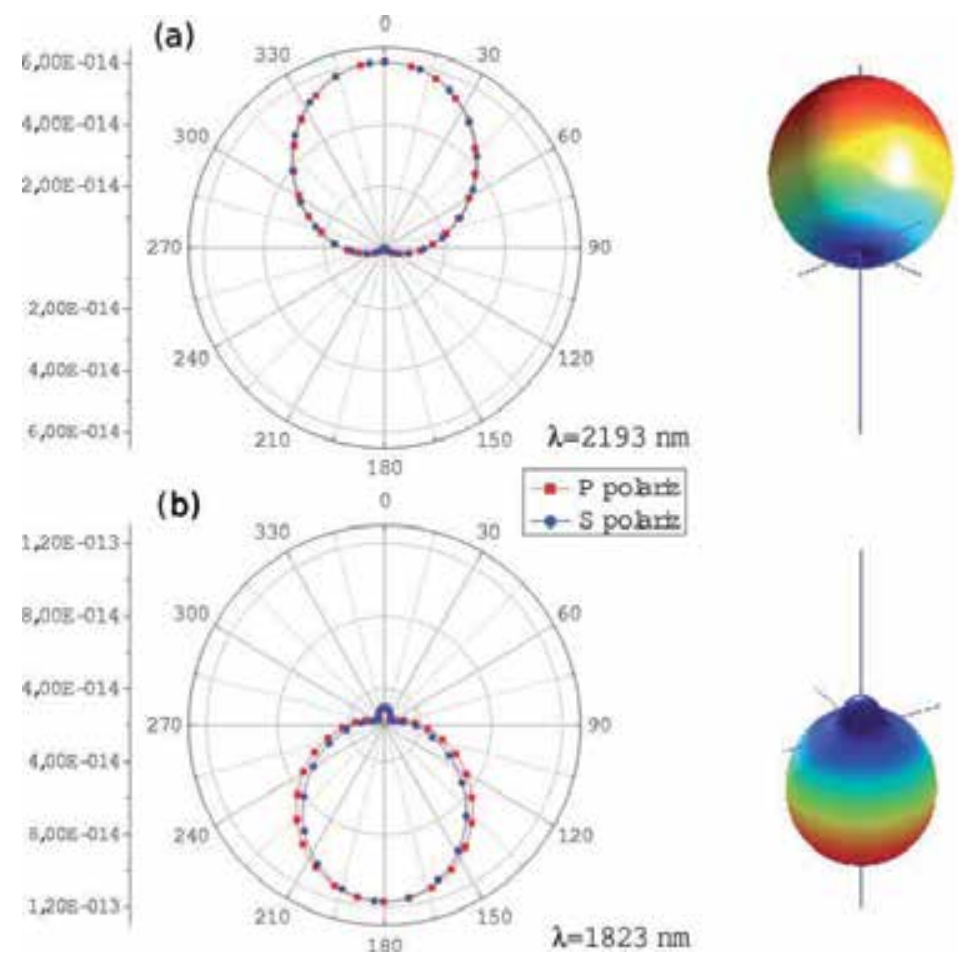

Fig. 9. Scattering diagrams for a Ge nanoparticles $(a=240 \mathrm{~nm})$ illuminated by a linear polarized plane wave. Both polarizations, with the incident electric field parallel (TM or P polarization) or normal (TE or S polarization) to the scattering plane are considered. The incident wavelength is labeled in the figure. From (Gómez-Medina et al., 2011b).

Previous results for Germanium can also be extended to Silicon nanoparticles. These behaviors in Silicon could be even more interesting due to the wide range of applications of this material. Silicon is the base of microelectronics due to its semiconductor character and also to its abundance in Earth. For this reason, the industry of Silicon is very well developed. These new scattering features in the nanometric range could be the base for the development of new silicon applications as, for instance, optical nanocircuits.

\section{Optical forces}

Light carries energy and both linear and angular momenta that can be transferred to atoms, molecules and particles. Demonstration of levitation and trapping of micron-sized particles 
by radiation pressure dates back to 1970 and the experiments reported by Ashkin and coworkers (Ashkin, 1970). Light forces on small particles are usually described as the sum of two terms: the dipole or gradient force and the radiation pressure or scattering force (Askhin et al., 1986; Neuman \& Block, 2004; Novotny \& Hecht, 2006; Chaumet \& NietoVesperinas 2000b; Gómez-Medina et al., 2001; Chaumet \& Nieto-Vesperinas, 2002; NietoVesperinas et al., 2004; Gómez-Medina \& Saénz, 2004). There is an additional nonconservative curl force arising in a light field of non-uniform ellipticity that is proportional to the curl of the spin angular momentum of the light field (Albaladejo et al., 2009a; NietoVesperinas et al., 2010). In analogy with electrostatics, small particles develop an electric (magnetic) dipole moment in response to the light electric (magnetic) field. The induced dipole is then drawn by field intensity gradients which compete with radiation pressure due to momentum transferred from the photons in the beam. By fashioning proper optical field gradients it is possible to trap and manipulate small dielectric particles with optical tweezers (Askhin et al, 1986; Neuman \& Block, 2004) or create atomic arrays in optical lattices (Verkerk et al., 1992; Hemmerich \& $\mathrm{H}^{\prime}$ ansch, 1993). Intense optical fields can also induce significant forces between particles (Burns et al., 1989; Burns et al., 1990; Tartakova et al., 2002; Chaumet \& Nieto-Vesperinas, 2001; Gómez-Medina \& Saénz, 2004). Some previous work focused on optical forces on macroscopic media, either with electric (Mansuripur, 2004) or magnetic response (Kemp et al., 2005; Mansuripur, 2007), or particles with electric response (Kemp et al., 2006a). Radiation pressure forces on dielectric and magnetic particles under plane wave incidence have been computed for both small cylinders (Kemp et al., 2006b) and spheres (Lakhtakia \& Mulholland, 1993; Lakhtakia, 2008). The total force on an electric and magnetic dipolar particle has been shown (Chaumet \& Rahmani, 2009; NietoVesperinas et al., 2010; Nieto-Vesperinas et al., 2011; Gómez-Medina et al., 2011a; GómezMedina et al., 2011b) to have a similarity with that previously obtained for electric dipoles. Moreover, in the presence of both electric and magnetic responses, the force presents an additional term proportional to the cross product of the electric and magnetic dipoles (Chaumet \& Rahmani, 2009; Nieto-Vesperinas et al., 2010; Nieto-Vesperinas et al., 2011; Gómez-Medina et al., 2011a; Gómez-Medina et al., 2011b). The relevance and physical origin of this electric-magnetic dipolar interaction term for a single particle has been recently discussed (Nieto-Vesperinas et al., 2010; Nieto-Vesperinas et al., 2011; Gómez-Medina et al., 2011a; Gómez-Medina et al., 2011b)

\subsection{Force on a small particle with electric and magnetic response to an electromagnetic wave}

We consider a dipolar particle embedded in a non-dissipative medium with relative dielectric permittivity $\varepsilon$ and magnetic permeability $\mu$, subjected to an incident electromagnetic field whose electric and magnetic vectors are $\boldsymbol{E}^{(i)}$ and $\boldsymbol{B}^{(i)}$, respectively. The total time-averaged electromagnetic force acting on the particle is (Chaumet \& NietoVesperinas, 2000; Jackson, 1998; Nieto-Vesperinas et al., 2010):

$$
\langle\mathbf{F}\rangle=\frac{1}{8 \pi} \Re\left\{\int_{S}\left[\varepsilon(\mathbf{E} \cdot \mathbf{s}) \mathbf{E}^{*}+\mu^{-1}(\mathbf{B} \cdot \mathbf{s}) \mathbf{B}^{*}-\frac{1}{2}\left(\varepsilon|\mathbf{E}|^{2}+\mu^{-1}|\mathbf{B}|^{-1}\right) \mathbf{s}\right] d S\right\},
$$

where $\mathfrak{R}$ stands for real part, $d S$ denotes the element of any surface $S$ that encloses the particle. 
The fields in Eq. (25) are total fields, namely the sum of the incident and scattered (reradiated) fields: $\boldsymbol{E}^{(i)}+\boldsymbol{E}^{(r)}, \boldsymbol{B}^{(i)}+\boldsymbol{B}^{(r)}$. s is its local outward unit normal. A time dependence $e^{(-}$ $i w t)$ is assumed throughout. For a small particle, within the range of validity of the dipolar approximation, the scattered field corresponds to that radiated by the induced electric and magnetic dipole moments, $p$ and $m$, respectively. In this case, Eq. (25) leads to the expression

$$
\langle\mathbf{F}\rangle=\frac{1}{2} \mathfrak{R}\left\{\mathbf{p}\left(\nabla \otimes \mathbf{E}^{(i) *}\right)+\mathbf{m}\left(\nabla \otimes \mathbf{B}^{(i) *}\right)-\frac{2 k^{4}}{3} \sqrt{\frac{\mu}{\varepsilon}}\left(\mathbf{p} \times \mathbf{m}^{*}\right)\right\}
$$

Equation (26) represents the generalization of the result of (Chaumet \& Rahmani, 2009) for the time-averaged force on a particle immersed in an arbitrary medium with refractive index: $m=\sqrt{\varepsilon \mu}$. The wavenumber is $k=m \omega / c, \omega$ being the frequency. The symbol $\otimes$ represents the dyadic product so that the matrix operation: $\mathbf{W}(\nabla \otimes \mathbf{V})$ has elements $W_{j} \partial_{j} V_{j}$ for $i, j=1,23$. All variables in Eq. (26) are evaluated at a point $r=r_{0}$ in the particle. The first term of Eq. (26) is the force $\left\langle\mathbf{F}_{e}\right\rangle$ exerted by the incident field on the induced electric dipole, the second and third terms $\left\langle\mathbf{F}_{m}\right\rangle$ and $\left\langle\mathbf{F}_{e m}\right\rangle$ are the force on the induced magnetic dipole and the force due to the interaction between both dipoles (Chaumet \& Rahmani, 2009; Nieto-Vesperinas et al., 2010).

\subsection{Optical theorem and forces on an electric and magnetic dipolar particle}

The question of energy conservation has been recurrently addressed and debated as regards small particles (Chýlek \& Pinnick, 1979; Lock et al., 1995), especially in connection with magnetic particles that produce zero-forward scattering intensity (Alù \& Engheta, 2011; Nieto-Vesperinas et al., 2011; García-Cámara et al., 2011; Gómez-Medina et al., 2011b). It is thus relevant to explore the formal analogy between the force as momentum "absorption" rate and the optical theorem expressing the conservation of electromagnetic energy. From the Poynting's theorem (Bohren \& Huffman, 1983; Jackson, 1998), the rate $-W^{(a)}$ at which energy is being absorbed by the particle is given by

$$
\begin{gathered}
-W^{(a)}=\int_{S}\left\{\langle\mathbf{S}\rangle-\left\langle\mathbf{S}^{(i)}\right\rangle\right\} \mathbf{s} d S \\
=\frac{c}{8 \pi m} \mathfrak{R}\left\{\int_{S} \varepsilon(\mathbf{E} \cdot \mathbf{s}) \mathbf{E}^{*}+\mu^{-1}(\mathbf{B} \cdot \mathbf{s}) \mathbf{B}^{*}-\frac{1}{2}\left(\varepsilon|\mathbf{E}|^{2}+\mu^{-1}|\mathbf{B}|^{-1}\right) d S\right\}
\end{gathered}
$$

By introducing the incident field as a decomposition of plane wave components and taking the sphere $S$ in Eq. (27) so large that $k\left|r-r_{0}\right| \rightarrow \infty$, and using Jones' lemma based on the principle of the stationary phase, (see Appendix XII of Bohren \& Huffman, 1983), and the source-free condition, we get the optical theorem for an arbitrary field (Nieto-Vesperinas et al., 2010):

$$
-W^{(a)}=-\frac{\omega}{2} \mathfrak{J}\left\{\mathbf{p} \cdot \mathbf{E}^{(i)^{*}}\left(r_{0}\right)\right\}-\frac{\omega}{2} \mathfrak{J}\left\{\mathbf{m} \cdot \mathbf{B}^{(i)}\left(r_{0}\right)\right\}+\frac{c}{m} \frac{k^{4}}{3}\left(\varepsilon^{-1}|\mathbf{p}|^{2}+\mu|\mathbf{m}|^{2}\right) .
$$


The first two terms of Eq. (29), coming from the interference between the incident and radiated fields, are the energy analogue of the electric and magnetic dipolar forces given by first two terms in Eq. (26).

The third and fourth terms of Eq. (29) that come from the integral of the third and fourth terms of Eq. (28), now yield the rate $W(s)$ at which the energy is being scattered, which together with the left hand side of this equation contributes to the rate of energy extinction by the particle $W^{(a)+W(s)}$ :

$$
W^{(a)}+W^{(s)}=\frac{\omega}{2} \mathfrak{J}\left\{\mathbf{p} \cdot \mathbf{E}^{(i)^{*}}\left(r_{0}\right)\right\}+\frac{\omega}{2} \mathfrak{J}\left\{\mathbf{m} \cdot \mathbf{B}^{(i)^{*}}\left(r_{0}\right)\right\} .
$$

Analogously as with the rate of scattered energy, the electric-magnetic dipolar interaction term of the force (third term of Eq. (26)) corresponds to the rate at which momentum is being scattered by the particle. We shall explore in some detail this analogy in order to illustrate the physical origin of $\left\langle\mathbf{F}_{e m}\right\rangle$. We notice that the power density of the scattered field can be written as the sum of two terms (Nieto-Vesperinas et al., 2010)

$$
\begin{gathered}
\left\langle\mathbf{S}^{(r)}\right\rangle d S=\frac{c}{8 \pi m} k^{4}\left(\varepsilon^{-1}|\mathbf{p} \times \mathbf{s}|^{2}+\mu|\mathbf{m} \times \mathbf{s}|^{2}\right) \mathbf{s} d \Omega \\
+\frac{c}{4 \pi m} k^{4} \sqrt{\frac{\mu}{\varepsilon}} \Re\left\{(\mathbf{s} \times \mathbf{p}) \cdot \mathbf{m}^{*}\right\} \mathbf{s} d \Omega .
\end{gathered}
$$

where the second term of Eq. (31) corresponds to the interference between the electric and magnetic dipolar fields. After integration over the closed surface $S$, that second term does not contribute to the radiated power, while it is the only contribution to the electricmagnetic dipolar interaction term of the force in Eq. (26). Namely, $<\mathbf{F}_{e m}>$ comes from the interference between the fields radiated by $\mathbf{p}$ and $\mathbf{m}$.

\subsection{Forces on an electric and magnetic dipolar particle for plane wave incidence}

In order to illustrate the relevance of the different terms in the optical forces, we shall next consider the force from a plane wave $E^{(i)}=e^{(i)} e^{i k s_{0} \cdot r}, B^{(i)}=b^{(i)} e^{i k s_{0} \cdot r}$ with $\mathbf{e}^{(i)}=\left\{\mathbf{b}^{(i)} \times \mathbf{s}_{0}\right\} / m$ on a small dielectric and magnetic spherical particle characterized by its electric and magnetic polarizabilities $\alpha_{e}$ and $\alpha_{m}$. When the induced dipole moments are expressed in terms of the incident field, i.e.

$$
\mathbf{p}=\alpha_{e} \mathbf{e}^{(i)} ; \mathbf{m}=\alpha_{m} \mathbf{b}^{(i)}
$$

For plane wave incidence, the total force is given by (Nieto-Vesperinas et al., 2010):

$$
\begin{gathered}
\langle\mathbf{F}\rangle=\left\langle\mathbf{F}_{e}\right\rangle+\left\langle\mathbf{F}_{m}\right\rangle+\left\langle\mathbf{F}_{e m}\right\rangle \\
=\mathrm{s}_{0} \frac{k}{2} \mathfrak{J}\left\{\mathbf{p} \cdot \mathbf{e}^{(i) *}+\mathbf{m} \cdot \mathbf{b}^{(i) *}\right\}-\frac{\mathrm{m}}{\mathrm{c}} \int_{S}\left\langle\mathbf{S}^{(r)}\right\rangle d S
\end{gathered}
$$




$$
=\mathrm{s}_{0} F_{0}\left[\mathfrak{J}\left\{\varepsilon^{-1} \alpha_{e}\right\}+\mathfrak{J}\left\{\mu \alpha_{m}\right\}-\frac{2 k^{3}}{3} \frac{\mu}{\varepsilon} \mathfrak{R}\left\{\alpha_{e} \alpha_{m}^{*}\right\}\right],
$$

where $F_{0}=\varepsilon\left|\mathbf{e}^{(i)}\right|^{2} / 2$. The first two terms, $\left\langle\mathbf{F}_{e}\right\rangle$ and $\left\langle\mathbf{F}_{m}\right\rangle$, correspond to the forces on to the sum of radiation pressures for a pure electric and a pure magnetic dipole, respectively. The third term, $\left\langle\mathbf{F}_{e m}\right\rangle$, is the time-averaged scattered momentum rate, and we shall see below that it also contributes to radiation pressure (Nieto-Vesperinas et al., 2010; GómezMedina et al., 2011a) and it is related to the asymmetry in the scattered intensity distribution (Nieto-Vesperinas et al., 2011; Gómez-Medina et al., 2011b).

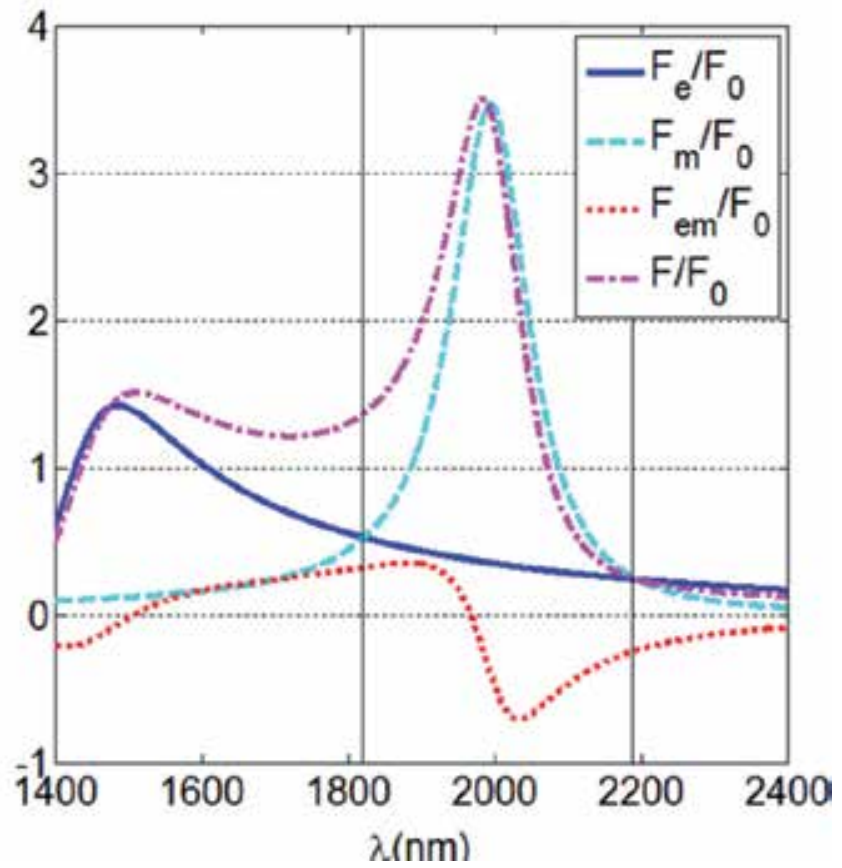

Fig. 10. Different contributions to the total radiation pressure versus the wavelength, for the Ge particle of Figs. 8-9. Normalization is done by $F_{0}=\varepsilon\left|\mathbf{e}^{(i)}\right|^{2} / 2$. The vertical lines mark the first and second generalized Kerker's conditions. Notice that when the first generalized Kerker's condition is fulfilled, i.e., $\mathfrak{R}\left\{\alpha_{e}\right\}=\mathfrak{R}\left\{\alpha_{m}\right\}$ and $\mathfrak{I}\left\{\alpha_{e}\right\}=\mathfrak{I}\left\{\alpha_{m}\right\}$, $\langle\mathbf{F}\rangle=\left\langle\mathbf{F}_{e}\right\rangle=\left\langle\mathbf{F}_{m}\right\rangle=-\left\langle\mathbf{F}_{e m}\right\rangle$. From (Gómez-Medina et al. 2011b).

\subsection{The generalized Kerker's conditions on optical forces}

From Eqs. (6) and (34), one derives for the radiation pressure force (Nieto-Vesperinas et al., 2011):

$$
\langle\mathbf{F}\rangle=\mathrm{s}_{0} F_{0} \frac{1}{6 k}\left[\frac{d C_{s c a}}{d \Omega}\left(0^{\circ}\right)+3 \frac{d C_{s c a}}{d \Omega}\left(180^{\circ}\right)-\frac{3}{2 \pi} C_{a b s}\right] .
$$

Equation (35) emphasizes the dominant role of the backward scattering on radiation pressure forces. 
At the first generalized Kerker's condition, the interference term of Eqs. (34-35) cancels out the magnetic contribution and we obtain $\langle\mathbf{F}\rangle=\left\langle\mathbf{F}_{e}\right\rangle$. At the second generalized Kerker's condition, where the backscattering is enhanced, $\langle\mathbf{F}\rangle=3\left\langle\mathbf{F}_{e}\right\rangle$. Notice that at both generalized Kerker's conditions the scattering cross section is exactly the same; however, the radiation pressures differ by a factor of 3 . These properties are illustrated in Figure 10, where we show the different contributions to the total time averaged force on a submicrometer Ge particle.

The strong peak in the radiation pressure force is mainly dominated by the first "magnetic" Mie resonance. This is striking and in contrast with all previous beliefs about optical forces on dipolar dielectric particles, that assumed that these forces would solely be described by the electric polarizability. It is also common to assume that for dielectric particles the real part of the polarizability is much larger than its imaginary part. As a matter of fact, this is behind the development of optical tweezers, in which gradient forces (that are proportional to $\mathfrak{R}\left(\alpha_{e}\right)$ ), dominate over the radiation pressure or scattering force contribution (which is proportional to $\mathfrak{I}\left(\alpha_{e}\right)$ ) (Volpe et al., 2006). However, as the size of the particle increases, and for any dielectric particle, there is a crossover from electric to magnetic response as we approach the first Mie resonance, the point at which the response is absolutely dominated by the magnetic dipole. Moreover, just at the resonance, and in absence of absorption, $\mathfrak{R}\left(\alpha_{m}\right)=0$ and $\mathfrak{I}\left(\alpha_{m}\right)=3 /\left(\mu 2 k^{3}\right)$. Then, the radiation pressure contribution of the magnetic term dominates the total force $\langle\mathbf{F}\rangle \cong\left\langle\mathbf{F}_{m}\right\rangle \approx \mathbf{S}_{0}\left(\varepsilon\left|\mathbf{e}^{(i)}\right|^{2} / 2\right)\left[3 / 2 k^{3}\right]$. Namely, in resonance the radiation pressure force presents a strong peak, the maximum force being independent of both material parameters and particle radius.

\section{Conclusion}

In this chapter we have analyzed the main aspects of one of the most interesting phenomena of light scattering by nanoparticles: the possibility to control its angular distribution (directionality). As it has been shown, a general magneto-dielectric particle, with suitable values of its relative optical constants $(\varepsilon, \mu)$, could present directional effects resulting from a coherent effect between real and imaginary parts of both electric and magnetic polarizabilities. The control of this effect could improve the characteristics of many current applications which employ nanoparticles. Also, it can be the base of new potential applications related with light guidance in low dimensions, as for instance, intra- or interchip optical communications (García-Cámara; 2011b). In addition, we showed that these scattering effects also affect the radiation pressure on these small particles. Thus, the "nonusual" scattering properties discussed before will strongly affect the dynamics of particle confinement in optical traps and vortex lattices (Albaladejo et al., 2009b; Gómez-Medina et al., 2011a; Albaladejo et al., 2011) governed by both gradient and curl forces.

Finally, we have showed that small dielectric particles made of non magnetic materials present scattering properties similar to those previously reported for hypothetical magnetodielectric particles. In particular, it has been shown that submicrometer Germanium particles present these directional phenomena in light scattering in the near-infrared range. These studies could serve as a stimulus for new experiments which implement these nonconventional phenomena. 


\section{Acknowledgment}

This work has been supported by the EU NMP3-SL-2008-214107-Nanomagma, the Spanish MICINN Consolider NanoLight (CSD2007-00046), FIS2010-21984, FIS2009-13430-C01-C02, and FIS2007-60158, as well as by the Comunidad de Madrid Microseres-CM (S2009/TIC1476). B.G.-C. wants to express his gratitude to the University of Cantabria for his postdoctoral fellowship. Work by R.G.-M. was supported by the MICINN "Juan de la Cierva" Fellowship.

\section{References}

Albaladejo, S.; Marqués, M.I.; Laroche, M. \& Sáenz, J.J. (2009). Scattering forces from the curl of the spin angular momentum of a light field. Physical Review Letters Vol. 102, No. 11 (March 2009), pp. 113602, ISNN 0031-9007.

Albaladejo, S.; Marqués, M.I. \& Sáenz, J.J. (2011). Light control of silver nanoparticle's diffusion. NanoLetters, Vol. 9, No. 10 (October 2009), pp. 3527-3531, ISNN 15306984.

Albaladejo, S.; Marqués, M.I. \& Sáenz, J.J. (2011). Light control of silver nanoparticle's diffusion. Optics Express, Vol. 19, No. 12 (June 2011), pp. 11471-11478, ISNN 10944087.

Alù, A. \& Engheta, N. (2011). How does forward-scattering in magnetodielectric nanoparticles comply with the optical theorem? Journal of Nanophotonics, Vol. 4 (May 2010), pp. 041590, ISSN 1934-2608.

Anker, J.N.; Hall, W.P.; Lyandres, O; Shan, N. C.; Zhao, J. \& Van Duyne, R.P. (2008) Biosensing with plasmonic nanosensors. Nature Materials, Vol. 7, No. 6, pp. 442-453, ISSN 1476-1122.

Ashkin, A. (1970). Acceleration and Trapping of Particles by Radiation Pressure. Physical Review Letters, Vol. 24, No. 4 (January 1970), pp. 156-159, ISNN 1079-7114.

Ashkin, A.; Dziedzic, J. M.; Bjorkholm, J. E. \& Chu, S. (1986). Observation of a single-beam gradient force optical trap for dielectric particles. Optics Letters, Vol. 11, No. 5 (May 1986), pp. 288-290, ISNN 0146-9592.

Bohren, C.F \& Huffman, D.R. (Eds.). (1983). Absorption and Scattering of Light by Small Particles, John Wiley\& Sons, ISBN 0-471-05772-X, New York.

Burns, M.M.; Fournier, J.M. \& Golovchenko, J.A. (1989). Optical Binding. Physical Review Letters, Vol. 63, No. 12 (September 1989), pp. 1233-1236, ISNN 1079-7114.

Burns, M.M.; Fournier, J.M. \& Golovchenko, J.A. (1990). Optical Matter: Crystallization and Binding in Intense Optical Fields. Science, Vol. 249, No. 4970 (August 1990), pp. 749-754, ISNN 0036-8075.

Chaumet, P.C. \& Nieto-Vesperinas, M. (2000). Coupled dipole method determination of the electromagnetic force on a particle over a flat dielectric substrate. Physical Review B, Vol. 61, No. 20 (May 2000), pp. 14119-14127, ISNN 1098-0121.

Chaumet, P.C. \& Nieto-Vesperinas, M. (2000). Electromagnetic force on a metallic particle in the presence of a dielectric surface. Physical Review B, Vol. 62, No. 16 (October 2000), pp. 11185-11191, ISNN 1098-0121.

Chaumet, P.C. \& Nieto-Vesperinas, M. (2001). Optical binding of particles with or without the presence of a flat dielectric surface. Physical Review B, Vol. 64, No. 3 (June 2001), pp. 035422-0354227, ISNN 1098-0121. 
Chaumet, P.C.; Rahmani, A. and \& Nieto-Vesperinas, M. (2002). Optical trapping and manipulation of nano-objects with an apertureless probe. Physical Review Letters, Vol. 88, No. 12 (March 2002), pp. 123601-123604, ISNN 1079-7114.

Chaumet, P.C. \& Rahmani, A. (2009). Electromagnetic force and torque on magnetic and negative-index scatterers. Optics Express, Vol. 17, No. 4 (February 2009), pp. 22242234, ISNN 1094-4087.

Chýlek, P. \& Pinnick, R.G. (1979). Nonunitarity of light scattering approximations. Applied Optics, Vol. 18, No. 8 (April, 1979), pp.1123-1124, ISSN 1559-128X.

Draine, B.T. \& Flatau, P.J. (1994). Discrete-dipole approximation for scattering calculations. Journal of the Optical Society of America A, Vol. 11, No. 4 (April 1994), pp. 1491-1499, ISSN 1084-7529.

Evlyyukhin, A.B.; Reinhardt, C.; Seidel, A.; Luk'yanchuk, B.S. \& Chichkov, B.N. (2010). Optical response features of Si-nanoparticle arrays. Physical Review B, Vol. 82, No. 4 (July 2010), pp. 045404, ISSN 1098-0121.

Gao, H.; Hyun, J.K.; Lee, M.H.; Yang, J.-C.; Lauhon, L.J. \& Odom, T.W. (2010). Broadband plasmonic microlenses based on patches of nanoholes. Nano Letters, Vol 10. No. 10 (September 2010), pp. 4111-4116, ISSN 1530-6984.

García-Cámara, B.; Saiz, J.M.; González, F. \& Moreno, F. (2010). Nanoparticles with unconventional properties: Size effects. Optics Communications, Vol. 283, No. 3 (February, 2010), pp. 490-496, ISNN 0030-4018.

García-Cámara B.; Saiz, J.M.; González, F. \& Moreno, F. (2010). Distance limit of the directionality conditions for the scattering of nanoparticles. Metamaterials, Vol. 4, No. 1 (May 2010), pp. 15-23, ISSN 1873-1988.

García-Cámara, B.; Alcaraz de la Osa, R.; Saiz, J.M.; González, F. \& Moreno, F. (2011). Directionality in scattering by nanoparticles: Kerker's null-scattering conditions revisited. Optics Letters, Vol. 36, No. 5 (February, 2011), pp. 728-730, ISSN 01469592.

García-Cámara, B. (2011), Inta-/Inter-chip optical communications: High speed and low dimensions, In: Communication architecture for systems-on-chip, J.L. Ayala (Ed.), pp. 249-322, CRC Press, ISBN 978-1-4398-4170-9, Florida (USA).

García-Etxarri, A.; Gómez-Medina, R.; Froufe-Pérez, L.S.; López, C.; Chantada, L.; Scheffold F.; Aizpurua, J.; Nieto-Vesperinas, M. \& Sáenz, J.J. (2011). Strong magnetic response of submicrometer silicon particles in the infrared. Optics Express, Vol. 19, No. 6 (February, 2011), pp. 4815-4826, ISSN 1094-4087.

Gómez-Medina, R.; San José, P.; García-Martín, A.; Lester, M.; Nieto-Vesperinas, M. \& Sáenz, J.J. (2001). Resonant radiation pressure on neutral particles in a waveguide Physical Review Letters, Vol. 86, No. 19 (May 2001), pp. 4275-4277, ISNN 1079-7114.

Gómez-Medina, R. \& Saénz J.J. (2004). Unusually Strong Optical Interactions between Particles in Quasi-One-Dimensional Geometries. Physical Review Letters, Vol. 93, No. 24 (December 2004), pp. 243602-243605, ISNN 1079-7114.

Gómez-Medina, R.; Nieto-Vesperinas, M. \& Saénz J.J. (2011). Nonconservative electric and magnetic optical forces on submicrometer dielectric particles. Physical Review A, Vol. 83, No. 3 (March 2011), pp. 033825, ISSN 1050-2947.

Gómez-Medina, R.; García-Cámara, B.; Suárez-Lacalle, I.; González, F.; Moreno, F.; NietoVesperinas, M. \& Saénz J.J. (2011). Electric and Magnetic dipolar response of 
germanium nanospheres: interference effects, scattering anisotropy, and optical forces. Journal of Nanophotonics, Vol. 5 (June, 2011), pp. 053512, ISSN 1934-2608.

Hemmerich A. \& H"ansch, T.W. (1993). Two-dimesional atomic crystal bound by light. Physical Review Letters, Vol. 70, No. 4 (January 1993), pp. 410-413, ISNN 1079-7114.

Jackson, J.D. (1998). Classical Electrodynamics, 3rd edition, John Wiley, New York.

Jessen, P.S.; Gerz, C.; Lett, P.D.; Phillips, W.D.; Rolston, S.L.; Spreeuw, R.J.C. \& Westbrook, C.I. (1992). Observation of quantized motion of $\mathrm{Rb}$ atoms in an optical field. Physical Review Letters, Vol. 69, No. 1 (July 1992), pp. 49-52, ISNN 1079-7114.

Kemp, B.A.; Grzegorczyk, T.M. \& Kong, J.A. (2005). Ab initio study of the radiation pressure on dielectric and magnetic media. Optics Express, Vol. 13, No. 23 (November 2005) pp. 9280-9291, eISNN 1094-4087.

Kemp, B.A.; Grzegorczyk, T.M. \& Kong, J.A. (2006). Optical momentum transfer to absorbing Mie particles. Physical Review Letters, Vol. 97, No. 13 (September 2006), pp. 133902, ISNN 1079-7114.

Kemp, B.A.; Grzegorczyk, T.M. \& Kong, J.A. (2006). Lorentz force on dielectric and magnetic particles. Journal of Electromagnetics Waves and Applications, Vol. 20, No. 6 (June 2006), pp. 827-839, ISNN 0920-5071.

Kerker, M.; Wang, D. \& Giles G. (1983). Electromagnetic scattering by magnetic spheres. Journal of the Optical Society of America, Vol. 73, No. 6 (June, 1983), pp. 756-767, ISSN 0030-3941.

Lakhtakia, A. (2008) Radiation pressure efficiencies of spheres made of isotropic, achiral, passive, homogeneous, negative-phase-velocity materials. Electromagnetics, Vol. 28, No. 5 (June 2008), pp. 346-353, ISNN 0272-6343.

Lakhtakia A. \& Mulholland, G.W. (1993). On two numerical techniques for light scattering by dielectric agglomerated structures. Journal of Research of the National Institute of Standards and Technology, Vol. 98, No. 6 (December 1993), pp. 699-716, ISNN 1044-677X.

Lock, J. A.; Hodges, J.T. \& Gouesbet, G. (1995) Failure of the optical theorem for Gaussianbeam scattering by a spherical particle. Journal of the Optical Society of America A, Vol. 12, No. 12 (December 1995), pp. 2708-2715, ISSN 1084-7529.

Maier, S. A.; Kik, P. G.; Atwater, H. A.; Meltzer, S.; Harel, E.; Koel B. E. \& Requicha, A. G. (2003). Local detection of electromagnetic energy transport below the diffraction limit in metal nanoparticle Plasmon waveguides. Nature Materials, Vol. 2, No. 4 (April 2003), pp. 229-232, ISSN 1476-1122.

Mansuripur, M. (2004). Radiation pressure and the linear momentum of the electromagnetic field. Optics Express, Vol.12, No 22 (November 2004). pp. 5375-5401, eISNN 10944087.

Mansuripur, M. (2007). Radiation pressure and the linear momentum of the electromagnetic field in magnetic media. Optics Express, Vol. 15, No.21 (October 2007), pp. 1350213518, eISNN 1094-4087.

Mirin, N. A. \& Halas, N. J. (2009).Light-bending nanoparticles. Nano Letters, Vol 9, No. 3 (February 2009), pp. 1255-1259, ISSN 1530-6984.

Neuman, K. C. \& Block, S. M. (2004). Optical trapping. Review of Scientific Instruments, Vol. 75, No. 9 (September 2004), pp. 2787-2809, ISSN 0034-6748.

Nieto-Vesperinas, M. \& García, N. Comment on "Negative refraction makes a perfect lens". Physical Review Letters, Vol. 91, No. 9 (August 2003), pp. 099702, ISSN 1079-7114. 
Nieto-Vesperinas, M.; Chaumet, P.C. \& Rahmani, A. (2004). Near field photonic forces. Philosophical Transactions of the Royal Society of London A, Vol. 362, No. 1817 (February 2004), pp. 719-737, ISSN 1471-2962.

Nieto-Vesperinas, M.; Sáenz, J.J.; Gómez-Medina, R.; \& Chantada, L. (2010). Optical forces on small magnetodielectric particles. Optics Express, Vol. 18, No. 11 (May 2010), pp. 11428-11443, eISNN 1094-4087.

Nieto-Vesperinas, M.; Gómez-Medina, R. \& Sáenz, J.J. (2011). Angle-suppressed scattering and optical forces on submicrometer dielectric particles. Journal of the Optical Society of America A, Vol. 28, No. 1 (December 2010), pp. 54-60, ISSN 1084-7529.

Novotny, L. \& Hecht, B. (2006). Principles of Nano-Optics. Cambridge University Press, Cambridge.

Palik, E.D. (Ed.). (1985), Handbook of Optical Constants of Solids, Academis Press, ISBN 0-12544420-6, Orlando, Florida

Pendry, J.B.; Schuring D. \& Smith D.R. (2006). Controlling electromagnetic fields. Science, Vol. 312, N0. 5781 (June 2006), pp. 1780-1782, ISSN 0036-8075.

Pendry, J.B. (2000). Negative refraction makes perfect lens. Physical Review Letters, Vol. 85. No. 18 (October 2000), pp. 3966-3969, ISNN 1079-7114.

Prasad, P. N. (2004). Nanophotonics, John Wiley\& Sons, ISBN 0471649880, New York.

Shalaev, V.M. (2008). Transforming light. Science, Vol. 322, No. 5900 (October 2008), pp. 384386, ISSN 0036-8075.

Tatarkova, S.A.; Carruthers, A.E. \& Dholakia, K. (2002). One-Dimensional Optically Bound Arrays of Microscopic Particles. Physical Review Letters, Vol. 89, No. 28 (December 2002), pp. 283901-283904, ISNN 1079-7114.

Verkerk, P.; Lounis, B.; Salomon, C.; Cohen-Tannoudji, C.; Courtois, J.-Y. \& Grynberg, G. (1992). Dynamics and spatial order of cold cesium atoms in a periodic optical potential. Physical Review Letters, Vol. 68, No. 26 (June 1992), pp. 3861-3864, ISNN 1079-7114.

Veselago, V. (1968). The electrodynamics of substances with simultaneously negative values of $\varepsilon$ and $\mu$. Soviet Physics Uspekhi, Vol. 10, No. 4 (January 1968), pp. 509-514, ISNN 0038-5670.

Volpe, G. ; Quidant, R.; Badenes, G. \& Petrov, D. (2006). Surface plasmon radiation forces. Physical Review Letters, Vol. 96, No. 23 (June 2006), pp. 238101, ISNN 1079-7114.

Zemp, R. J. (2009) Nanomedicine:Detecting rare cancer cells. Nature Nanotechnology, Vol. 4, No. 12 (December 2009), pp. 798-799, ISNN 1748-3387.

Zhedulev, N.I. (2010). The road ahead of metamaterials. Science, Vol. 328, No. 5978 (April, 2010), pp. 582-583, ISNN 0036-8075.

Zhu, J.; Ozdemir, S. K.; Xiao, Y.-F.; Li, L.; He, L.; Chen, D.- R. \& Yang L. (2010). On-chip single nanoparticle detection and sizing by mode splitting in an ultrahigh-Q microresonator. Nature Photonics, Vol. 4, No. 1 (January, 2010), pp. 46-49, ISSN 17494885. 


\title{
Deexcitation Dynamics of a Degenerate Two-Level Atom near (Inside) a Body
}

\author{
Gennady Nikolaev \\ ${ }^{1}$ Institute of Automation and Electrometry, Siberian Branch, \\ Russian Academy of Sciences, Novosibirsk, \\ ${ }^{2}$ Novosibirsk State University, Novosibirsk, \\ Russia
}

\section{Introduction}

It has long been known that atomic radiation processes near a macroscopic body differ from those in free space substantially (Purcell, 1946). In particular, the lifetime of an excited state of an atom or a molecule near surface (Arnoldus \& George, 1988a;b; Barnes, 1998; Chance et al., 1978; Drexhage et al., 1968; Ford et al., 1984; Fort \& Grésillon, 2008; Garrett et al., 2004; Hellen \& Axelrod, 1987; Kreiter et al., 2002; Lukosz \& Kunz, 1977; Macklin et al., 1996; Milonni \& Knight, 1973; Snoeks et al., 1995; Steiner et al., 2005; Yeung \& Gustafson, 1996) or in the vicinity of (or inside) a nanoparticle (Chew, 1987; 1988; Das \& Metiu, 1985; Dung et al., 2000; Gersten \& Nitzan, 1981; Klimov, Ducloy \& Letokhov, 1996; Klimov et al., 2001; Klimov, Ducloy, Letokhov \& Lebedev, 1996; Ruppin, 1982) may be increased or decreased depending on specific conditions. This lifetime change is theoretically calculated in many papers. These calculations made in a variety of ways. Nevertheless all of these papers can be divided into two classes. The first class includes the papers that represent an excited atom as a three-dimensional damped oscillator (Chance et al., 1978; Chew, 1987; 1988; Das \& Metiu, 1985; Hellen \& Axelrod, 1987; Klimov, Ducloy \& Letokhov, 1996; Klimov, Ducloy, Letokhov \& Lebedev, 1996; Ruppin, 1982). The second class includes the papers that consider an excited atom by means of quantum mechanics (Agarwal, 1975a;b; Arnoldus \& George, 1987; 1988a;b; Barnes, 1998; Dung et al., 2000; Wylie \& Sipe, 1984; 1985; Yeung \& Gustafson, 1996).

It is shown in the papers that are in the first class that the atomic oscillator rate of damping take a different value in the case of radial and tangential orientation of the oscillating atomic electric dipole. The magnitude of the rate of damping lies between these values in the case of another atomic dipole orientation. However the atomic or molecule decay rate is measured by the fluorescence detection after light pulse excitation of the atom or molecule. So, fluorescence is two-step process, and hence, orientation of the oscillating atomic dipole in general is not the same as exciting light polarization.

In the second class papers the problem of the atomic dipole orientation is either no discussed explicitly or reduced to partitioning of the dipole matrix element on radial and tangential parts as in the case of the classic atomic oscillator. The ratio between these two parts is either no evaluated or assumed to be in the ratio 1:2 as in the case of free space. This approach 
one cannot consider as correct because of anisotropy of the atomic surroundings. The remark about fluorescence as two-step process mentioned above refers equally to the papers.

To rigorous description of the vector nature of the atomic dipole moment it is necessary to take into account the atomic angular degrees of freedom, that is degeneracy of atomic levels. As far as we know, it was done only in the papers (Arnoldus \& George, 1987; 1988a;b). In the papers the steady-state fluorescence of the atom near an axial symmetrical surface was theoretically investigated and influence of the surface was expressed in terms of electric field correlation function.

The purpose of the chapter is to present the correct description of deexcitation dynamics of a degenerate two-level atom in the vicinity of arbitrary body.

We start with a quantum mechanical expression for the atomic deexcitation probability expressed in terms of the normal correlation function of the atomic dipole moment operator and the antinormal correlation function of the electric field strength operator. Then the antinormal correlation function is expressed in terms of the field susceptibility by use of the fluctuation-dissipation theorem. The atomic dipole moment operator as well as the atomic density matrix operator is expressed in terms of irreducible tensor operators. Finally, it is shown that the atomic deexcitation rate at the instant immediately after pulse excitation is proportional to a linear combination of the products of the so-called atomic polarization moments, population and alignment, and anisotropic relaxation matrix.

To find out deexcitation dynamics, a master equation for atomic density matrix is derived from an evolution equation for the total density matrix describing both atom and field. A consistent system of linear first-order ordinary differential equations for the atomic polarization moments is obtained from the master equation. Components of the anisotropic relaxation matrix describing the consistent system are expressed in terms of the field susceptibility tensor. Symmetries of the anisotropic relaxation matrix are found. It is shown that atomic deexcitation in general is multi-exponential. The simple exponential decay of the excited energy level takes place only if its total angular momentum is less then one. Deexcitation dynamics is considered in more detail for the case when the total angular momenta of the upper and lower levels are equal to 1 and 0 respectively. It is shown that in this case deexitation dynamics also may be exponential at certain polarizations of the exciting light.

In conclusion, an intriguing issue that is why the simple model of classical oscillating dipole for description of fluorescence is in good agreement with observational evidence(Amos \& Barnes, 1997; Chance et al., 1978; Drexhage et al., 1968; Fort \& Grésillon, 2008; Kreiter et al., 2002; Snoeks et al., 1995; Vallée et al., 2001), is clarified.

\section{Atomic transition rate of a degenerate two-level atom in the vicinity of a material body}

To investigate deexcitation of a degenerate two-level atom in the vicinity of a nanoparticle we consider more general problem of deexcitation of the atom in the vicinity of a material body at first.

Our approach to the problem is based on using correlation functions that appear in linear-response theory. It is about the same as used in number of works (Agarwal, 1975a; Wylie \& Sipe, 1984) concerning the quantum electrodynamics and life time of a non-generate atom near an interface. It is most of all close to approach developed in (Klyshko, 2011). 


\subsection{Transition rate in dipole approximation vs atomic and fluctuating electric field correlation functions}

We will assume that both the atom and the electromagnetic field are quantized.

Let the atom and the field be independent at the initial time moment $t_{0}$. Therefore at that instant the quantum state of the system $\left|m k_{i}\right\rangle$ is equal to $|m\rangle\left|k_{i}\right\rangle$, where $|m\rangle,\left|k_{i}\right\rangle$ are the initial states of the atom and field, respectively. In the first order of the perturbation theory, the amplitude $c_{n k}(t)$ of the transition into some state $|n k\rangle$ is proportional to the matrix element of the interaction operator $\hat{V},\left\langle n k|\hat{V}| m k_{i}\right\rangle$, where $|n\rangle,|k\rangle$ are states of the atom and field at the final time moment $t$, respectively. In the dipole approximation, $\hat{V}=-\hat{\vec{d}}(t) \hat{\vec{E}}(t)$, so,

$$
c_{n k}=-\frac{1}{i \hbar} \int_{t_{0}}^{t} d t^{\prime}\left\langle n k\left|\hat{\vec{d}}\left(t^{\prime}\right) \hat{\vec{E}}\left(t^{\prime}\right)\right| m k_{i}\right\rangle,
$$

where the operators of the atomic dipole moment $\hat{\vec{d}}(t)$ and the electric field strength $\hat{\vec{E}}(t)$ are considered in the interaction picture, i.e., without the account for the perturbation.

In the rotating-wave approximation (Allen \& Eberly, 1975), we have

$$
-\hat{V}(t) \approx \hat{\vec{d}}^{(-)}(t) \hat{\vec{E}}^{(+)}(t)+\hat{\vec{d}}^{(+)}(t) \hat{\vec{E}}^{(-)}(t),
$$

where $\hat{\vec{d}}^{(+)}$and $\hat{\vec{E}}^{(+)}$are the positive-frequency parts of the operators, whereas $\hat{\vec{d}}^{(-)}$and $\hat{\vec{E}}^{(-)}$ are negative-frequency ones. At $t-t_{0} \equiv T \gg 1 / \bar{\omega}$, fast oscillating (with approximately twice the mean frequency $\bar{\omega}$ ) products $\hat{\vec{d}}^{(+)} \hat{\vec{E}}^{(+)}$and $\hat{\vec{d}}^{(-)} \hat{\vec{E}}^{(-)}$have no contribution into the integral (1).

The initial atomic state $|m\rangle$ has more high energy than the final atomic state $|n\rangle$ for the deexcitation process under consideration. That is why only the second term in (2) gives a nonzero contribution for this process. Hence, the probability of the atomic deexcitation is given by

$$
\begin{aligned}
P\left(n k \mid m k_{i}\right) & =\frac{1}{\hbar^{2}} \int_{t_{0}}^{t} \int_{t_{0}}^{t} d t^{\prime} d t^{\prime \prime} \sum_{\alpha \beta}\left\langle m\left|\hat{d}_{\alpha}^{(-)}\left(t^{\prime}\right)\right| n\right\rangle\left\langle n\left|\hat{d}_{\beta}^{(+)}\left(t^{\prime \prime}\right)\right| m\right\rangle \\
& \times\left\langle k_{i}\left|\hat{E}_{\alpha}^{(+)}\left(t^{\prime}\right)\right| k\right\rangle\left\langle k\left|\hat{E}_{\beta}^{(-)}\left(t^{\prime \prime}\right)\right| k_{i}\right\rangle,
\end{aligned}
$$

where we have used the equality $\left\langle r\left|\hat{A}^{(+)}\right| s\right\rangle=\left\langle s\left|\hat{A}^{(-)}\right| r\right\rangle^{*}$ for the matrix element of an operator $\hat{A}$ between states $|r\rangle$ and $|s\rangle$. We also have used the Greek letters in subscripts for the notation of the Descartes's components of the vector operators.

One should sum the expression (3) over all possible states $|n k\rangle$ if we are not interested in what specific state the system under consideration has came. These states constitute the complete set and satisfy the completeness condition

$$
\sum_{n k}|n k\rangle\langle n k|=\hat{I}
$$


Thus we can represent the total probability of the atomic deexcitation in the following way

$$
P=\hbar^{-2} \int_{t_{0}}^{t} \int_{t_{0}}^{t} d t^{\prime} d t^{\prime \prime} \sum_{\alpha \beta} f_{\alpha \beta}^{(+)}\left(t^{\prime}, t^{\prime \prime}\right) g_{\alpha \beta}^{(-)}\left(t^{\prime}, t^{\prime \prime}\right),
$$

where

$$
\begin{aligned}
& f_{\alpha \beta}^{(+)}\left(t^{\prime}, t^{\prime \prime}\right) \equiv\left\langle\hat{d}_{\alpha}^{(-)}\left(t^{\prime}\right) \hat{d}_{\beta}^{(+)}\left(t^{\prime \prime}\right)\right\rangle, \\
& g_{\alpha \beta}^{(-)}\left(t^{\prime}, t^{\prime \prime}\right) \equiv\left\langle\hat{E}_{\alpha}^{(+)}\left(t^{\prime}\right) \hat{E}_{\beta}^{(-)}\left(t^{\prime \prime}\right)\right\rangle
\end{aligned}
$$

are normally and anti-normally ordered correlation function (CF) of the atomic dipole moment and the electric field strength in an initial state, respectively. The initial state may be pure as well as mixed, of course.

We suppose that initial unperturbed states of both interacting systems are stationary. In this case correlation functions (6) depend only on the difference of their arguments:

$$
\begin{aligned}
& f_{\alpha \beta}^{( \pm)}(\tau) \equiv f_{\alpha \beta}^{( \pm)}(t, t+\tau)=\left\langle\hat{d}_{\alpha}^{(\mp)}(0) \hat{d}_{\beta}^{( \pm)}(\tau)\right\rangle=\left(f_{\beta \alpha}^{( \pm)}(-\tau)\right)^{*}, \\
& g_{\alpha \beta}^{( \pm)}(\tau) \equiv g_{\alpha \beta}^{( \pm)}(t, t+\tau)=\left\langle\hat{E}_{\alpha}^{(\mp)}(0) \hat{E}_{\beta}^{( \pm)}(\tau)\right\rangle=\left(g_{\beta \alpha}^{( \pm)}(-\tau)\right)^{*} .
\end{aligned}
$$

Hence, the total probability of the atomic deexcitation (5) becomes

$$
P=\hbar^{-2} \int_{0}^{T} d \tau(T-\tau) \sum_{\alpha \beta}\left[f_{\alpha \beta}^{(+)}(\tau) g_{\alpha \beta}^{(-)}(\tau)+(\tau \rightarrow-\tau)\right],
$$

where $T \equiv t-t_{0}$ is observation time. When it is much more then the atomic and field correlation time, the total probability of the atomic deexcitation (9) becomes proportional to $T$. So, atomic transition rate $W \equiv P / T$ independent on time one may introduce

$$
W=\hbar^{-2} \int_{-\infty}^{\infty} d \tau \sum_{\alpha \beta} f_{\alpha \beta}^{(+)}(\tau) g_{\alpha \beta}^{(-)}(\tau),
$$

where limits of integration $\pm T$ are extended to $\pm \infty$. It is convenient rewrite (10) in terms of the Fourier components of the correlation functions in the following way

$$
W=\left(1 / 2 \pi \hbar^{2}\right) \int_{-\infty}^{\infty} d \omega \sum_{\alpha \beta} f_{\alpha \beta}^{(+)}(\omega) g_{\alpha \beta}^{(-)}(-\omega),
$$

where the Fourier transform $A(\omega)$ of a function $A(\tau)$ is defined by

$$
A(\omega)=\int_{-\infty}^{\infty} d \tau e^{i \omega \tau} A(\tau)
$$




\subsection{Transition rate in terms of electric field susceptibility}

It is known that total correlation function is represented as a sum of normally and anti-normally ordered correlation function in the case of stationary process. Indeed, the total correlation function of the electric field strength may be written as

$$
\begin{aligned}
g_{\alpha \beta}(t, t+\tau) & \equiv\left\langle\left[\hat{E}_{\alpha}^{(+)}(t)+\hat{E}_{\alpha}^{(-)}(t)\right]\left[\hat{E}_{\beta}^{(+)}(t+\tau)+\hat{E}_{\beta}^{(-)}(t+\tau)\right]\right\rangle \\
& =\sum_{s^{\prime}, s= \pm 1}\left\langle\hat{E}_{\alpha}^{\left(s^{\prime}\right)}(t) \hat{E}_{\beta}^{(s)}(t+\tau)\right\rangle
\end{aligned}
$$

Expressing $\hat{E}_{\alpha}^{\left(s^{\prime}\right)}(t)$ and $\hat{E}_{\beta}^{(s)}(t+\tau)$ in terms of Fourier transforms, we obtain

$$
g_{\alpha \beta}(t, t+\tau) \equiv(2 \pi)^{-2} \int_{-\infty}^{\infty} \int_{-\infty}^{\infty} d \omega^{\prime} d \omega e^{-i \omega \tau} e^{-i\left(\omega^{\prime}+\omega\right) t} \sum_{s^{\prime}, s= \pm 1}\left\langle\hat{E}_{\alpha}^{\left(s^{\prime}\right)}\left(\omega^{\prime}\right) \hat{E}_{\beta}^{(s)}(\omega)\right\rangle .
$$

Note, that

$$
\hat{E}_{\alpha}^{(s)}(\omega) \equiv \theta(s \omega) \hat{E}_{\alpha}(\omega)
$$

by definition, where $\theta(\omega)$ is step function.

It is clear that (14) is independent on $t$ only when expression in the angle brackets is proportional to Dirac function:

$$
\left\langle\hat{E}_{\alpha}^{\left(s^{\prime}\right)}\left(\omega^{\prime}\right) \hat{E}_{\beta}^{(s)}(\omega)\right\rangle \equiv 2 \pi g_{\alpha \beta}^{(s)}(\omega) \delta\left(\omega^{\prime}+\omega\right),
$$

where spectral density of the normally ordered correlation function $g_{\alpha \beta}^{(+)}(\omega)$ and anti-normally ordered one $g_{\alpha \beta}^{(-)}(\omega)$ are introduced respectively. In turn, (16) and (15) imply $s^{\prime}=-s$. Hence, in (14) only two terms are nonzero, and we have

$$
g_{\alpha \beta}(\tau)=g_{\alpha \beta}^{(+)}(\tau)+g_{\alpha \beta}^{(-)}(\tau) .
$$

Note that from (14), (16), and (17) it is follows that relationship between $g_{\alpha \beta}^{( \pm)}(\omega)$ and $g_{\alpha \beta}^{( \pm)}(\tau)$ is given by the ordinary formula (12). It is clear also that ordered correlation functions $g_{\alpha \beta}^{( \pm)}(\omega)$ are expressed in terms of the ordinary correlation function $g_{\alpha \beta}(\omega)$ similar to relation (15):

$$
g_{\alpha \beta}^{( \pm)}(\omega)=\theta( \pm \omega) g_{\alpha \beta}(\omega)
$$

At thermal equilibrium the correlation function $g_{\alpha \beta}(\tau)$ is simply related with symmetrized correlation function $\{g\}_{\alpha \beta}(\tau)$ defined by

$$
\{g\}_{\alpha \beta}(\tau) \equiv \frac{1}{2}\left\langle\hat{E}_{\alpha}(0) \hat{E}_{\beta}(\tau)+\hat{E}_{\beta}(\tau) \hat{E}_{\alpha}(0)\right\rangle=\frac{1}{2}\left\{g_{\alpha \beta}(\tau)+g_{\beta \alpha}(-\tau)\right\} .
$$

There is a simple Kubo-Martin-Schwinger's boundary condition

$$
g_{\beta \alpha}(-\tau)=g_{\alpha \beta}(\tau+i \hbar \xi),
$$


where $\xi \equiv 1 /(k T), k$ and $T$ are Boltzmann's constant and temperature respectively. It is easily proofed by using the invariance of the trace under a cyclic permutation of the operators:

$$
\begin{aligned}
g_{\beta \alpha}(-\tau) & =\left\langle\hat{E}_{\beta}(\tau) \hat{E}_{\alpha}(0)\right\rangle \equiv \operatorname{tr}\left\{\hat{\rho}_{0} e^{i \hat{H} \tau / \hbar} \hat{E}_{\beta} e^{-i \hat{H} \tau / \hbar} \hat{E}_{\alpha}\right\} \\
& =Z^{-1} \operatorname{tr}\left\{e^{-\xi \hat{H}} e^{i \hat{H} \tau / \hbar} \hat{E}_{\beta} e^{-i \hat{H} \tau / \hbar} \hat{E}_{\alpha}\right\} \\
& =Z^{-1} \operatorname{tr}\left\{\hat{E}_{\alpha} e^{i(i \xi+\tau / \hbar)} \hat{H} \hat{E}_{\beta} e^{-i(i \xi+\tau / \hbar)} \hat{H} e^{-\xi \hat{H}}\right\}=g_{\alpha \beta}(\tau+i \hbar \xi),
\end{aligned}
$$

where $\hat{\rho}_{0}=Z^{-1} e^{-\xi \hat{H}}$ is the thermal equilibrium density operator, $Z=\operatorname{tr}\left\{e^{-\xi \hat{H}}\right\}$, and $\hat{H}$ is unperturbed Hamiltonian of the system.

Using (20), we rewrite relation (19) as follows

$$
\{g\}_{\alpha \beta}(\tau)=\frac{1}{2}\left\{g_{\alpha \beta}(\tau)+g_{\alpha \beta}(\tau+i \hbar \xi)\right\} .
$$

In turn, taking the Fourier transform, we obtain

$$
\{g\}_{\alpha \beta}(\omega)=\frac{1}{2}\left\{1+e^{\hbar \omega \xi}\right\} g_{\alpha \beta}(\omega) .
$$

The Fourier transform of symmetrized correlation function $\{g\}_{\alpha \beta}\left(\vec{r}, \vec{r}^{\prime} ; \omega\right)$ is related with dynamical value $G_{\alpha \beta}\left(\vec{r}, \vec{r}^{\prime} ; \omega\right)$, the Fourier transform of the electric field susceptibility $G_{\alpha \beta}\left(\vec{r}, \vec{r}^{\prime} ; \tau\right)$, by the fluctuation-dissipation theorem as follows (Bernard \& Callen, 1959; Callen et al., 1952; Callen \& Welton, 1951; Landau \& Lifshitz, 1980)

$$
\{g\}_{\alpha \beta}\left(\vec{r}, \vec{r}^{\prime} ; \omega\right)=\frac{1}{2} i \hbar\left[G_{\beta \alpha}^{*}\left(\vec{r}^{\prime}, \vec{r} ; \omega\right)-G_{\alpha \beta}\left(\vec{r}, \vec{r}^{\prime} ; \omega\right)\right] \operatorname{coth}\left(\frac{\hbar \omega \xi}{2}\right),
$$

where tensor $G_{\alpha \beta}\left(\vec{r}, \vec{r}^{\prime} ; \omega\right)$ relates Fourier transforms of the electric dipole $\hat{d}_{\beta}\left(\vec{r}^{\prime} ; \omega\right)$ and induced electric field $\hat{E}_{\alpha}(\vec{r} ; \omega)$ as follows

$$
\hat{E}_{\alpha}(\vec{r} ; \omega)=\sum_{\beta} G_{\alpha \beta}\left(\vec{r}, \vec{r}^{\prime} ; \omega\right) \hat{d}_{\beta}\left(\vec{r}^{\prime} ; \omega\right),
$$

and the electric field susceptibility tensor $G_{\alpha \beta}\left(\vec{r}, \vec{r}^{\prime} ; \tau\right)$ is defined by

$$
G_{\alpha \beta}\left(\vec{r}, \vec{r}^{\prime} ; \tau\right) \equiv \frac{i}{\hbar} \theta(\tau)\left\langle\left[\hat{E}_{\alpha}(\tau), \hat{E}_{\beta}(0)\right]\right\rangle .
$$

Note that the same tensor $G_{\alpha \beta}\left(\vec{r}, \vec{r}^{\prime} ; \omega\right)$ relates classical, not quantum, values $E_{\alpha}(\vec{r} ; \omega)$ and $d_{\beta}\left(\vec{r}^{\prime} ; \omega\right)$ by the same way $(25)$. So it can be found from the solution of the classical electrodynamic problem in the same condition.

Using (18), (23), and (24), we obtain

$$
g_{\alpha \beta}^{(-)}(-\omega)=i \hbar \theta(\omega) \frac{1}{2}\left[1+\operatorname{coth}\left(\frac{\hbar \omega \xi}{2}\right)\right]\left[G_{\beta \alpha}^{*}\left(\vec{r}^{\prime}, \vec{r} ; \omega\right)-G_{\alpha \beta}\left(\vec{r}, \vec{r}^{\prime} ; \omega\right)\right],
$$


When there is no external magnetic field, tensor $G_{\alpha \beta}\left(\vec{r}, \vec{r}^{\prime} ; \omega\right)$ is symmetrical one, and its imaginary part is odd in $\omega$. In this case (27) goes over into (Agarwal, 1975a) ${ }^{1}$

$$
g_{\alpha \beta}^{(-)}(-\omega)=\hbar \theta(\omega)\left[1+\operatorname{coth}\left(\frac{\hbar \omega \xi}{2}\right)\right] \Im\left[G_{\alpha \beta}\left(\vec{r}^{\prime}, \vec{r} ; \omega\right)\right],
$$

We are interesting in only local field response because of point atom approximation used. Substituting (28) in (11) we find

$$
W=(1 / 2 \pi \hbar) \int_{0}^{\infty} d \omega \sum_{\alpha \beta} f_{\alpha \beta}^{(+)}(\omega)\left[1+\operatorname{coth}\left(\frac{\hbar \omega \xi}{2}\right)\right] \Im\left[G_{\alpha \beta}\left(\vec{r}_{0}, \vec{r}_{0} ; \omega\right)\right],
$$

where $\overrightarrow{r_{0}}$ is radius vector of the atom.

\subsection{Transition rate of a degenerate two-level atom}

The explicit form of the atomic CF $f_{\alpha \beta}^{(+)}(\omega)$ depends on the atomic model used. Here we consider a degenerate two-level atom. Its energy levels are degenerate on the total angular momentum projection on any axis. Suppose the excited upper energy level $m$ and lower one $n$ have quantum numbers $J_{m} M_{m}$ and $J_{n} M_{n}$ respectively, where $J_{j}$ and $M_{j}$ label the total angular momentum of the level $j$ and its projection on the $z$-axis , respectively.

It is convenient describe vector or tensor values in terms of the circular components instead of the Descartes's one. The circular components $v_{\sigma}$ of a vector $\vec{v}$, where $\sigma=0, \pm 1$, are related with the Descartes's one $v_{i}$ as follows (Varshalovich et al., 1988):

$$
\begin{aligned}
v_{0} & =v_{z} \\
v_{ \pm 1} & =\mp\left(v_{x} \pm v_{y}\right) / \sqrt{2} .
\end{aligned}
$$

The circular components of the atomic dipole operator can be expressed according to the Wigner-Eckart theorem in terms of the so-called unit irreducible tensor operators $\hat{T}_{Q}^{K}\left(J_{m} J_{n}\right)$ in the following way (Biedenharn \& Louck, 1981; Blum, 1996; Fano \& Racah, 1959; Varshalovich et al., 1988):

$$
\begin{aligned}
& \hat{d}_{\sigma}^{(+)}(t)=\frac{d_{n m}}{\sqrt{3}} \hat{T}_{\sigma}^{1}\left(J_{n} J_{m}\right) \exp \left(-i \omega_{m n} t\right), \\
& \hat{\bar{d}}_{\sigma}^{(-)}(t)=\left\{(-1)^{\sigma} \hat{\bar{d}}_{-\sigma}^{(+)}(t)\right\}^{+},
\end{aligned}
$$

where $d_{m n}$ and $\omega_{m n}$ are reduced matrix element of the atomic dipole moment and resonant frequency of the atomic transition, respectively. The irreducible tensor operator $\hat{T}_{Q}^{K}\left(J_{m} J_{n}\right)$, where $K$ and $Q$ are its rank and component $(-K \leqslant Q \leqslant K)$ correspondingly, is defined as (Biedenharn \& Louck, 1981; Blum, 1996; Fano \& Racah, 1959; Varshalovich et al., 1988)

$$
\hat{T}_{Q}^{K}\left(J_{m} J_{n}\right)=\sum_{M_{m}, M_{n}}(-1)^{J_{n}-M_{n}}\left\langle J_{m} M_{m} J_{n}-M_{n} \mid K Q\right\rangle\left|J_{m} M_{m}\right\rangle\left\langle J_{n} M_{n}\right|
$$

where $\left\langle J_{m} M_{m} J_{n}-M_{n} \mid K Q\right\rangle$ is the vector coupling (Clebsch-Gordan) coefficient. Quantities $J_{m}$, $J_{n}$, and $K$ of the coefficient obey triangle unequality, so $\left|J_{m}-J_{n}\right| \leqslant K \leqslant J_{m}+J_{n}$.

\footnotetext{
${ }^{1}$ Definition of the ordered correlation functions in this paper differs from ours one by sign of the argument $\tau$ and, hence in sign of $\omega$.
} 


\subsubsection{Properties of irreducible tensor operators and density matrix multipole components}

The operators $\hat{T}_{Q}^{K}\left(J J^{\prime}\right)$ are orthonormal in the following sense

$$
\operatorname{tr}\left[\hat{T}_{Q^{\prime}}^{K^{\prime}}\left(J^{\prime} J\right) \hat{T}_{Q}^{K \dagger}\left(J^{\prime} J\right)\right] \equiv \sum_{M^{\prime} M}\left\langle J^{\prime} M^{\prime}\left|\hat{T}_{Q^{\prime}}^{K^{\prime}}\left(J^{\prime} J\right)\right| J M\right\rangle\left\langle J M\left|\hat{T}_{Q}^{K \dagger}\left(J^{\prime} J\right)\right| J^{\prime} M^{\prime}\right\rangle=\delta_{K^{\prime} K} \delta_{Q^{\prime} Q^{\prime}}
$$

where the Hermitian conjugate operator $\hat{T}_{Q}^{K \dagger}\left(J^{\prime} J\right)$ is expressed in terms of $\hat{T}_{Q}^{K}\left(J J^{\prime}\right)$ as follows

$$
\hat{T}_{Q}^{K \dagger}\left(J^{\prime} J\right) \equiv(-1)^{J^{\prime}-J-Q} \hat{T}_{-Q}^{K}\left(J J^{\prime}\right) .
$$

Set of the operators $\hat{T}_{Q}^{K}\left(J^{\prime} J\right)$ is complete. So, density operator can be decomposed into irreducible parts as follows

$$
\hat{\rho}=\sum_{J^{\prime} J K Q} \rho^{K Q}\left(J^{\prime} J\right) \hat{T}_{Q}^{K}\left(J^{\prime} J\right) .
$$

In turn, coefficients $\rho^{K Q}\left(J^{\prime} J\right)$ known as multipole components are expressed in terms of $\hat{T}_{Q}^{K}\left(J^{\prime} J\right)$ and density operator by using (33) and (32) in the following way

$$
\rho^{K Q}\left(J^{\prime} J\right)=\operatorname{tr}\left[\hat{\rho} \hat{T}_{Q}^{K \dagger}\left(J^{\prime} J\right)\right]=\sum_{M^{\prime} M}(-1)^{J-M}\left\langle J^{\prime} M^{\prime} J-M \mid K Q\right\rangle\left\langle J^{\prime} M^{\prime}|\hat{\rho}| J M\right\rangle .
$$

It is seen that multipole components $\rho^{K Q}\left(J^{\prime} J\right)$ satisfy the following relations similar to relations (34):

$$
\left[\rho^{K Q}\left(J^{\prime} J\right)\right]^{*}=(-1)^{J-J^{\prime}-Q} \rho^{K-Q}\left(J J^{\prime}\right),
$$

so multipole components $\rho^{K 0}(J J)$ is real. Note also that $\rho^{K Q}\left(J^{\prime} J\right)$ transform under rotations like $\hat{T}_{Q}^{K+}\left(J^{\prime} J\right)$, and hence, are contravariant to $\hat{T}_{Q}^{K}\left(J^{\prime} J\right)$ because of property (34).

We are interesting only in states of the excited level $m$, so the relevant density operator $\hat{\rho}\left(J_{m}\right)$ is

$$
\hat{\rho}\left(J_{m}\right)=\sum_{K Q} \rho^{K Q}\left(J_{m} J_{m}\right) \hat{T}_{Q}^{K}\left(J_{m} J_{m}\right)
$$

In this decomposition the rank $K$ is in the range $0 \leqslant K \leqslant 2 J_{m}$ as was noted after definition (32). All multipole components $\rho^{K Q}\left(J_{m} J_{m}\right)$ have clear physical sense (see, for example, (Biedenharn \& Louck, 1981; Blum, 1996; Omont, 1977; Varshalovich et al., 1988)). In particular, $\sqrt{2 J_{m}+1} \rho^{00}\left(J_{m} J_{m}\right)$ is equal to the total population of the level $m$, the $\rho^{1 Q}\left(J_{m} J_{m}\right)^{\prime}$ s are the three standard components of what is generally called "orientation" proportional to the mean magnetic dipole of the state, and the $\rho^{2 Q}\left(J_{m} J_{m}\right)^{\prime}$ s are the five standard components of the "alignment" proportional to the mean electric quadrupole moment of the state.

\subsubsection{Transition rate and material body symmetry}

Finally, after some manipulation using the relations (7), (31), and (38), and also properties of irreducible tensor operators, one can represent relation (29) in the form

$$
W=\frac{1}{2}\left[1+\operatorname{coth}\left(\frac{\hbar \omega_{m n} \xi}{2}\right)\right] \sum_{K Q} \gamma_{Q}^{K} \rho^{K Q}\left(J_{m} J_{m}\right),
$$


where

$$
\gamma_{Q}^{K} \equiv 2 \frac{\left|d_{m n}\right|^{2}}{\hbar}(-1)^{J_{m}+J_{n}}\left\{\begin{array}{ccc}
1 & 1 & K \\
J_{m} & J_{m} & J_{n}
\end{array}\right\}\left[\mathbf{G}^{\prime \prime}\left(\vec{r}_{0}, \vec{r}_{0} ; \omega_{m n}\right)\right]_{Q}^{K}
$$

is irreducible relaxation tensor of the multipole $\rho^{K Q}\left(J_{m} J_{m}\right),\left\{\begin{array}{ccc}1 & 1 & K \\ J_{m} & J_{m} & J_{n}\end{array}\right\}$ is $6-j$ coefficient, and $\left[\mathbf{G}^{\prime \prime}\left(\vec{r}_{0}, \vec{r}_{0} ; \omega_{m n}\right)\right]_{Q}^{K}$ is irreducible spherical tensor of the imaginary part of the electric field susceptibility in the $\omega$-representation. Irreducible spherical tensor $G_{Q}^{K}\left(\vec{r}_{0}, \vec{r}_{0} ; \omega_{m n}\right)$ is related with circular components $G_{\sigma \sigma^{\prime}}\left(\vec{r}_{0}, \vec{r}_{0} ; \omega_{m n}\right)$ as follows

$$
G_{Q}^{K}\left(\vec{r}_{0}, \vec{r}_{0} ; \omega_{m n}\right) \equiv \sum_{\sigma \sigma^{\prime}}\left\langle 1 \sigma 1 \sigma^{\prime} \mid K Q\right\rangle G_{\sigma \sigma^{\prime}}\left(\vec{r}_{0}, \vec{r}_{0} ; \omega_{m n}\right)
$$

It is follows from properties of the Clebsch-Gordan coefficient $\left\langle 1 \sigma 1 \sigma^{\prime} \mid K Q\right\rangle$ that $0 \leqslant K \leqslant 2$. Furthermore, symmetry of the tensor $G_{\sigma \sigma^{\prime}}\left(\vec{r}_{0}, \vec{r}_{0} ; \omega_{m n}\right)$ under the interchange $\sigma \leftrightarrows \sigma^{\prime}$ requires that $K$ have to be even, so $K=0,2$. In other words, deexcitation rate depends on the total population of excited level $(K=0)$ and its alignment $(K=2)$. Their relative contribution depends according to (39) and (40) on quantum numbers of combining levels $m$ and $n$, on the excitation type determining the value of $\rho^{K Q}\left(J_{m} J_{m}\right)$, and on the atom surroundings by $G_{Q}^{K}\left(\vec{r}_{0}, \vec{r}_{0} ; \omega_{m n}\right)$. Let us consider these factors in more detail.

As was noted after (38), $K$ is in the range of values defining by $0 \leqslant K \leqslant 2 J_{m}$. Consequently, if the total momentum $J_{m}$ of the the excited level is equal to 0 , or $1 / 2$, there is no alignment of the level. So, deexcitation is governed only by $\gamma_{0}^{0}$ and does not depend on excitation type. In the case of $J_{m}>1 / 2$, the ratio of two deexcitation rates corresponding to some two fixed excitation types, differing in initial values of $\rho^{K Q}\left(J_{m} J_{m}\right)$, is not universal but depends on $J_{m}$, $J_{n}$.

One can diagonalize symmetrical tensor $G_{\alpha \beta}\left(\vec{r}_{0}, \vec{r}_{0} ; \omega_{m n}\right)$. Let us label its principal axes of coordinate by $X, Y, Z$. In this proper basis only the following irreducible components of the tensor $\mathbf{G}$ are not zero:

$$
\begin{aligned}
G_{0}^{0} & =-\frac{1}{\sqrt{3}} \operatorname{tr}(\mathbf{G})=-\frac{1}{\sqrt{3}}\left(G_{X X}+G_{Y Y}+G_{Z Z}\right), \\
G_{0}^{2} & =\sqrt{\frac{2}{3}}\left[G_{Z Z}-\frac{1}{2}\left(G_{X X}+G_{Y Y}\right)\right], \\
G_{ \pm 2}^{2} & =\frac{1}{2}\left(G_{X X}-G_{Y Y}\right) .
\end{aligned}
$$

As is seen from (44), components $G_{ \pm 2}^{2}=0$ if surroundings of the atom is axial symmetric (symmetry axis along $Z$ ). In particular, this case is realized when atom is near a half-space boundary or near a spherical particle.

When surroundings of the atom is isotropic, the only nonzero component of the tensor $\mathrm{G}$ is $G_{0}^{0}$ one. It is just the case of an isotropic infinite medium (in particular, vacuum) or when atom is in the center of spherical particle or cavity. In this case $(-1)^{J_{m}+J_{n}}\left\{\begin{array}{ccc}1 & 1 & 0 \\ J_{m} & J_{m} & J_{n}\end{array}\right\}=$ $-1 / \sqrt{3\left(2 J_{m}+1\right)}$ in (40). So, using relations (42), (40) we obtain from (39)

$$
W_{i s}=\frac{2}{3} \frac{\left|d_{m n}\right|^{2}}{\hbar\left(2 J_{m}+1\right)} \Im\left(\sum_{i=X, Y, Z} G_{i i}\right) \sum_{M_{m}=-J_{m}}^{J_{m}}\left\langle J_{m} M_{m}|\hat{\rho}| J_{m} M_{m}\right\rangle .
$$


Since we are here interested primarily in atomic transition energies on the order of a Rydberg that implies $\frac{\hbar \omega_{m n} \xi}{2} \gg 1$ at room temperature, we have replaced the expression in square brackets in Eq. (39) by 2 . The total population of the upper level

$$
\sum_{M_{m}=-J_{m}}^{J_{m}}\left\langle J_{m} M_{m}|\hat{\rho}| J_{m} M_{m}\right\rangle=1
$$

because we suppose that atom is excited on level $m$ at the initial time. For free space (Barash, 1988; Lifshitz \& Pitaevskii, 1980; Nikolaev, 2006), we have

$$
\Im\left(\sum_{i=X, Y, Z} G_{i i}\right)=2\left(\frac{\omega_{m n}}{c}\right)^{3} .
$$

Substituting these two expressions in Eq. (45) we immediately obtain the well-known expression for the radiative decay rate of the excited state of an isolated atom (see, i.e., (Berestetskii et al., 2008; Sobelman, 1972)) :

$$
W_{0}=\frac{4}{3} \frac{\left|d_{m n}\right|^{2}}{\hbar\left(2 J_{m}+1\right)}\left(\frac{\omega_{m n}}{c}\right)^{3} .
$$

It should be noted that Eq. (39) describes deexcitation rate at the initial time moment just following the excitation. Density matrix multipole components $\rho^{K Q}\left(J_{m} J_{m}\right)$ will be changed with the passage of time. It is reasonable to suggest that the expression opposite in sign to the right-hand side of Eq. (39) describes the decrease of the upper level population per unit of time. To prove the suggestion let us consider more general problem of the dynamics of the density matrix multipole components caused by interaction of the atom with quantized field.

\section{Master equations for the excited density matrix multipole components}

\subsection{Integro-differential equation for total density matrix operator}

Let us consider a large isolated system consisting of an atom, material body and interacting with them quantum electromagnetic field. Atomic surrounding, electromagnetic field and material body that interact among themselves, we will treat as a large subsystem referred to as the thermostat. In the interaction picture representation, the density matrix $\hat{R}$ of the total isolated system obeys the Liouville equation:

$$
i \hbar \frac{\mathrm{d} R \hat{(} t)}{\mathrm{d} t}=[\hat{V}(t), \hat{R}(t)]
$$

where $\hat{V}$ is the atom-field interaction operator that in the rotating-wave approximation is given by Eq. (2). It is known that this equation can be rewritten in the integro-differential form that is suitable for perturbation technique. Indeed, formal integrating this equation in time, we obtain the integral equation:

$$
\hat{R}(t)=\hat{R}(0)-(i / \hbar) \int_{0}^{t} d t^{\prime}\left[\hat{V}\left(t^{\prime}\right), \hat{R}\left(t^{\prime}\right)\right] .
$$


Substituting this expression into Eq. (48), we get the equation for the total density matrix operator in the following form:

$$
\frac{\mathrm{d} \hat{R}(t)}{\mathrm{d} t}=(-i / \hbar)[\hat{V}(t), \hat{R}(0)]+(-i / \hbar)^{2} \int_{0}^{t} d t^{\prime}\left[\hat{V}(t),\left[\hat{V}\left(t^{\prime}\right), \hat{R}\left(t^{\prime}\right)\right]\right] .
$$

In Eqs. (49) and (50) the lower limit we took 0 since it is assumed that the thermostat and the atom did not interact before this time moment because the atom was unexcited. Consequently, until this moment the thermostat and the atom were uncorrelated, so the total density matrix $\hat{R}$ was equal to the direct product of the density matrices of the system:

$$
\hat{R}(0)=\hat{\rho}(0) \hat{\rho}_{t h}(0),
$$

where $\hat{\rho}$ and $\hat{\rho}_{t h}$ are the density matrix operator of the atom and thermostat, respectively.

\subsection{Large thermostat approximation}

Following the paper (Fano \& Racah, 1959) (see also (Blum, 1996)), we will suppose that thermostat is always in the state of the thermal equilibrium because it has a large number of degrees of freedom and, hence, atom almost do not changes its state. The supposition implies that the total density matrix is always equal to the direct product of the density matrices of the system:

$$
R \hat{(t)}=\hat{\rho}(t) \hat{\rho}_{t h}(0)
$$

This relation is referred to as the main condition of the irreversibility.

Substituting (52) in (50) and taking trace over thermostat variables, we get the equation for the reduced atomic density matrix operator, $\hat{\rho}(t) \equiv \operatorname{tr}_{\mathrm{th}} \hat{R}(t)$,

$$
\frac{\mathrm{d} \hat{\rho}(t)}{\mathrm{d} t}=-(i / \hbar) \operatorname{tr}_{\text {th }}\left[\hat{V}(t), \hat{\rho}(0) \hat{\rho}_{t h}(0)\right]-(1 / \hbar)^{2} \int_{0}^{t} d t^{\prime} \operatorname{tr}_{\text {th }}\left[\hat{V}(t),\left[\hat{V}\left(t^{\prime}\right), \hat{\rho}\left(t^{\prime}\right) \hat{\rho}_{t h}(0)\right]\right] .
$$

\subsection{Integro-differential equation for atomic multipole components}

To obtain dynamics equation for atomic multipole components, we make use of relation (36). Precisely, let us multiply both sides of $(53)$ by $\hat{T}_{Q}^{K+}\left(J_{m} J_{m}\right)$ and take trace over atomic variable. So, we get

$$
\begin{aligned}
\frac{\mathrm{d} \rho^{K Q}\left(J_{m} J_{m}\right)(t)}{\mathrm{d} t}= & -(i / \hbar) \operatorname{tr}_{\text {all }}\left\{\hat{T}_{Q}^{K \dagger}\left(J_{m} J_{m}\right)\left[\hat{V}(t), \hat{\rho}(0) \hat{\rho}_{t h}(0)\right]\right\} \\
& -(1 / \hbar)^{2} \int_{0}^{t} d t^{\prime} \operatorname{tr}_{\text {all }}\left\{\hat{T}_{Q}^{K \dagger}\left(J_{m} J_{m}\right)\left[\hat{V}(t),\left[\hat{V}\left(t^{\prime}\right), \hat{\rho}\left(t^{\prime}\right) \hat{\rho}_{t h}(0)\right]\right]\right\},
\end{aligned}
$$

where $\operatorname{tr}_{\text {all }}$ stands for the trace over all isolated system variables including atomic and thermostat one.

We will now transform this equation in such a way that terms include the trace of the product of $\hat{\rho}\left(t^{\prime}\right) \hat{\rho}_{t h}(0)$ by an operator. 
To do this, we make use of the identity (Il'inskii \& Keldysh, 1994)

$$
\operatorname{tr}\left\{\hat{A}\left[\hat{A}_{1},\left[\hat{A}_{2}, \cdots\left[\hat{A}_{k}, \hat{B}\right] \cdots\right]\right]\right\}=\operatorname{tr}\left\{\left[\cdots\left[\left[\hat{A}, \hat{A}_{1}\right], \hat{A}_{2}\right] \cdots \hat{A}_{k}\right] \hat{B}\right\}
$$

which holds for arbitrary operators $\hat{A}, \hat{A}_{1}, \hat{A}_{2}, \cdots, \hat{B}$.

Using identity (55) and the atomic density matrix decomposition (35), we can rewrite (54) as

$$
\begin{aligned}
& \frac{\mathrm{d} \rho^{K Q}\left(J_{m} J_{m} ; t\right)}{\mathrm{d} t}=-\frac{i}{\hbar} \sum_{J^{\prime} J K^{\prime} Q^{\prime}} \rho^{K^{\prime} Q^{\prime}}\left(J^{\prime} J ; 0\right) \operatorname{tr}_{\text {all }}\left\{\left[\hat{T}_{Q}^{K \dagger}\left(J_{m} J_{m}\right), \hat{V}(t)\right] \hat{T}_{Q^{\prime}}^{K^{\prime}}\left(J^{\prime} J\right) \hat{\rho}_{t h}(0)\right\} \\
& -\frac{1}{\hbar^{2}} \sum_{J^{\prime} J K^{\prime} Q^{\prime}} \int_{0}^{t} d t^{\prime} \rho^{K^{\prime} Q^{\prime}}\left(J^{\prime} J ; t^{\prime}\right) \operatorname{tr}_{\text {all }}\left\{\left[\left[\hat{T}_{Q}^{K \dagger}\left(J_{m} J_{m}\right), \hat{V}(t)\right], \hat{V}\left(t^{\prime}\right)\right] \hat{T}_{Q^{\prime}}^{K^{\prime}}\left(J^{\prime} J\right) \hat{\rho}_{t h}(0)\right\} .
\end{aligned}
$$

Substituting in (56) the interaction Hamiltonian (2), using (31), and also taking into account that scalar product $\vec{d} \hat{E}$ in the circular basis (30) has the form $\sum_{\sigma}(-1)^{\sigma} d_{\sigma} E_{-\sigma}$, we obtain

$$
\begin{array}{r}
\frac{\mathrm{d} \rho^{K Q}\left(J_{m} J_{m} ; t\right)}{\mathrm{d} t}=-\frac{d_{m n} d_{n m}}{3 \hbar^{2}} \sum_{J^{\prime} J K^{\prime} Q^{\prime}} \int_{0}^{t} d t^{\prime} \rho^{K^{\prime} Q^{\prime}}\left(J^{\prime} J ; t^{\prime}\right) \sum_{\alpha \beta}\left\{e ^ { i \omega _ { m n } ( t - t ^ { \prime } ) } \left[g_{\alpha \beta}^{(-)}\left(t^{\prime}-t\right) A_{\alpha \beta}\right.\right. \\
\left.\left.-g_{\alpha \beta}^{(+)}\left(t-t^{\prime}\right) B_{\alpha \beta}\right]+e^{-i \omega_{m n}\left(t-t^{\prime}\right)}\left[g_{\alpha \beta}^{(-)}\left(t-t^{\prime}\right) C_{\alpha \beta}-g_{\alpha \beta}^{(+)}\left(t^{\prime}-t\right) B_{\alpha \beta}\right]\right\},
\end{array}
$$

where $g_{\alpha \beta}^{( \pm)}(\tau)$ are the ordered correlation functions of the fluctuating electromagnetic field (8),

$$
\begin{aligned}
& A_{\alpha \beta} \equiv \sum_{\sigma \sigma^{\prime}}(-1)^{\sigma+\sigma^{\prime}}\langle\alpha \mid 1-\sigma\rangle\left\langle\beta \mid 1-\sigma^{\prime}\right\rangle \operatorname{tr}\left\{\hat{T}_{Q}^{K+}\left(J_{m} J_{m}\right) \hat{T}_{\sigma}^{1}\left(J_{m} J_{n}\right) \hat{T}_{\sigma^{\prime}}^{1}\left(J_{n} J_{m}\right) \hat{T}_{Q^{\prime}}^{K^{\prime}}\left(J J^{\prime}\right)\right\}, \\
& B_{\alpha \beta} \equiv \sum_{\sigma \sigma^{\prime}}(-1)^{\sigma+\sigma^{\prime}}\langle\alpha \mid 1-\sigma\rangle\left\langle\beta \mid 1-\sigma^{\prime}\right\rangle \operatorname{tr}\left\{\hat{T}_{\sigma}^{1}\left(J_{n} J_{m}\right) \hat{T}_{Q}^{K \dagger}\left(J_{m} J_{m}\right) \hat{T}_{\sigma^{\prime}}^{1}\left(J_{m} J_{n}\right) \hat{T}_{Q^{\prime}}^{K^{\prime}}\left(J J^{\prime}\right)\right\}, \\
& C_{\alpha \beta} \equiv \sum_{\sigma \sigma^{\prime}}(-1)^{\sigma+\sigma^{\prime}}\langle\alpha \mid 1-\sigma\rangle\left\langle\beta \mid 1-\sigma^{\prime}\right\rangle \operatorname{tr}\left\{\hat{T}_{\sigma}^{1}\left(J_{m} J_{n}\right) \hat{T}_{\sigma^{\prime}}^{1}\left(J_{n} J_{m}\right) \hat{T}_{Q}^{K+}\left(J_{m} J_{m}\right) \hat{T}_{Q^{\prime}}^{K^{\prime}}\left(J J^{\prime}\right)\right\} .
\end{aligned}
$$

In the definitions (58) - (60) symbols $\langle\alpha \mid 1-\sigma\rangle$ and $\left\langle\beta \mid 1-\sigma^{\prime}\right\rangle$ are transformation matrices from the circular components to the Descartes's one, that are inverse of that given by (30), and symbol tr $\{\cdots\}$ from now on stands for trace over atomic variables. Note that the linear on $\hat{V}(t)$ term in (56) vanishes in our case because of the average fluctuated field is zero at the thermal equilibrium: $\operatorname{tr}_{\text {th }}\left\{\hat{E}_{\alpha}\right\} \equiv\left\langle\hat{E}_{\alpha}\right\rangle=0$.

It should be noted that ratio of $\left|g_{\alpha \beta}^{(+)}\left(t-t^{\prime}\right)\right|$ to $\left|g_{\alpha \beta}^{(-)}\left(t-t^{\prime}\right)\right|$ is proportional to the mean number of photons in the thermal equilibrium, $\left\langle n_{p h}\right\rangle \sim k T / \hbar \omega_{m n} \ll 1$. Therefore terms that proportional to $g_{\alpha \beta}^{(+)}\left(t-t^{\prime}\right)$ can be ignored in (57).

\subsection{Master equation for multipole components in Markov-type approximation}

Fluctuating field correlation functions $g_{\alpha \beta}^{( \pm)}\left(t-t^{\prime}\right)$ are nonzero only for the sufficiently small time difference $|\tau| \equiv\left|t-t^{\prime}\right|$ comparable with the typical field correlation time $\tau_{c}$. We will 
assume following (Loisell, 1973) that this correlation time is much less then typical variation times of the atomic multipole components. Thus, in the case of free space the lifetime of the atomic excited state much more than $\tau_{c} \approx 1 / \omega_{m n}$. So, we can replace $\rho^{K^{\prime} Q^{\prime}}\left(J^{\prime} J ; t^{\prime}\right)$ by $\rho^{K^{\prime} Q^{\prime}}\left(J^{\prime} J ; t\right)$ and to take it out of the integral in (57). It is so-called Markov-type approximation.

It is also important to note that $\hat{T}_{Q^{\prime}}^{K^{\prime}}\left(J J^{\prime}\right)$ incoming in (58) and (60) are nonzero only if $J=J^{\prime}=$ $J_{m}$ because of its definition (32) and invariance of the trace under a cyclic permutation of the operators.

Taking into account assumptions mentioned above, property (8), and by making the change of variable $\tau \equiv t-t^{\prime}$ in integration, we can represent (57) as

$$
\frac{\mathrm{d} \rho^{K Q}\left(J_{m} J_{m} ; t\right)}{\mathrm{d} t}=-\frac{d_{m n} d_{n m}}{3 \hbar^{2}} \sum_{K^{\prime} Q^{\prime}} \sum_{\alpha \beta}\left[I_{\beta \alpha}^{*}\left(\omega_{m n}\right) A_{\alpha \beta}+I_{\alpha \beta}\left(\omega_{m n}\right) C_{\alpha \beta}\right] \rho^{K^{\prime} Q^{\prime}}\left(J_{m} J_{m} ; t\right),
$$

where

$$
I_{\alpha \beta}\left(\omega_{m n}\right) \equiv \int_{0}^{\infty} d \tau g_{\alpha \beta}^{(-)}(\tau) e^{-i \omega_{m n} \tau}
$$

In (62) we extended upper limit from $t$ to $\infty$ because of $g_{\alpha \beta}^{( \pm)}(\tau)$ is in fact zero at $\tau \gg \tau_{c}$. The error of this replacement is negligible in Markov-type approximation.

Now we will show that integral (62) is expressed in terms of retarded Green function $G_{\alpha \beta}\left(\vec{r}, \vec{r}^{\prime} ; \omega_{m n}\right)$. To prove that, let as consider Fourier transform $g_{\alpha \beta}^{(-)}\left(-\omega_{m n}\right)$ of the function $g_{\alpha \beta}^{(-)}(\tau)$ defined by (12):

$$
g_{\alpha \beta}^{(-)}\left(-\omega_{m n}\right)=\int_{-\infty}^{\infty} d \tau g_{\alpha \beta}^{(-)}(\tau) e^{-i \omega_{m n} \tau}
$$

Let us split this integral into two parts

$$
g_{\alpha \beta}^{(-)}\left(-\omega_{m n}\right)=\int_{-\infty}^{0} d \tau g_{\alpha \beta}^{(-)}(\tau) e^{-i \omega_{m n} \tau}+\int_{0}^{\infty} d \tau g_{\alpha \beta}^{(-)}(\tau) e^{-i \omega_{m n} \tau} .
$$

Making the change of variable in integration $\tau \rightarrow-\tau$ in the first integral and utilizing relation (8), we can rewrite (64) as

$$
g_{\alpha \beta}^{(-)}\left(-\omega_{m n}\right)=\int_{0}^{\infty} d \tau\left(g_{\beta \alpha}^{(-)}(\tau)\right)^{*} e^{i \omega_{m n} \tau}+\int_{0}^{\infty} d \tau g_{\alpha \beta}^{(-)}(\tau) e^{-i \omega_{m n} \tau}
$$

The second integral in (65) is just equal to $I_{\alpha \beta}\left(\omega_{m n}\right)$, and the first one to its complex conjugation. So, (65) can be rewritten as follows

$$
g_{\alpha \beta}^{(-)}\left(-\omega_{m n}\right)=I_{\alpha \beta}\left(\omega_{m n}\right)+I_{\beta \alpha}^{*}\left(\omega_{m n}\right) .
$$


Now comparing right-hand sides of (66) and (27), we obtain desired relation

$$
I_{\alpha \beta}\left(\omega_{m n}\right)=-i \hbar \frac{1}{2}\left[1+\operatorname{coth}\left(\frac{\hbar \omega_{m n}}{2 k T}\right)\right] G_{\alpha \beta}\left(\vec{r}, \vec{r}^{\prime} ; \omega_{m n}\right)
$$

It is yet mentioned after (37) that multipole components $\rho^{K Q}\left(J^{\prime} J\right)$ transform under rotations contravariant to $\hat{T}_{Q}^{K}\left(J^{\prime} J\right)$. It is convenient to introduce co-variant multipole components $\rho_{Q}^{K}\left(J^{\prime} J\right)$ by convention

$$
\rho_{Q}^{K}\left(J^{\prime} J\right) \equiv(-1)^{J-J^{\prime}-Q} \rho^{K-Q}\left(J J^{\prime}\right)=\left[\rho^{K Q}\left(J^{\prime} J\right)\right]^{*} .
$$

In these notations, making use of (67) and explicitly calculating traces in (58) and (60), one can finally represent (61) as follows ${ }^{2}$

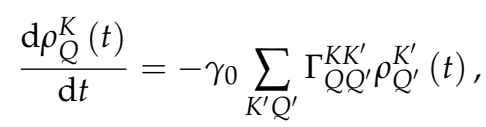

where

$$
\gamma_{0}=W_{0}=\frac{4}{3} \frac{\left|d_{m n}\right|^{2}}{\hbar\left(2 J_{m}+1\right)}\left(\frac{\omega_{m n}}{c}\right)^{3}
$$

is radiation decay rate of the excited degenerate state of the atom in vacuum, dimensionless relaxation tensor $\Gamma_{Q Q^{\prime}}^{K K^{\prime}}$ can be represented as follows:

$$
\Gamma_{Q Q^{\prime}}^{K K^{\prime}}=\gamma_{Q Q^{\prime}}^{K K^{\prime}}+i \Delta_{Q Q^{\prime \prime}}^{K K^{\prime}}
$$

where $\gamma_{Q Q^{\prime}}^{K K^{\prime}}$ and $\Delta_{Q Q^{\prime}}^{K K^{\prime}}$ are in general complex.

Geometrical part of $\gamma_{Q Q^{\prime}}^{K K^{\prime}}$ and $\Delta_{Q Q^{\prime}}^{K K^{\prime}}$ is represented by Clebsch-Gordan coefficient and dynamical one is proportional to retarded Green function:

$$
\begin{aligned}
\gamma_{Q Q^{\prime}}^{K K^{\prime}} & =\sum_{L M}\left\langle K^{\prime} Q^{\prime} L M \mid K Q\right\rangle \bar{G}_{M}^{L}\left(K K^{\prime} L\right) \gamma\left(K K^{\prime} L, J_{m} J_{n}\right), \\
\Delta_{Q Q^{\prime}}^{K K^{\prime}} & =\sum_{L M}\left\langle K^{\prime} Q^{\prime} L M \mid K Q\right\rangle \widetilde{G}_{M}^{L}\left(K K^{\prime} L\right) \gamma\left(K K^{\prime} L, J_{m} J_{n}\right),
\end{aligned}
$$

where scalar coefficient $\gamma\left(K K^{\prime} L, J_{m} J_{n}\right)$ and irreducible tensors $\bar{G}_{M}^{L}\left(K K^{\prime} L\right)$ and $\widetilde{G}_{M}^{L}\left(K K^{\prime} L\right)$ are

$$
\begin{aligned}
\gamma\left(K K^{\prime} L, J_{m} J_{n}\right) & =(-1)^{K+J_{n}-J_{m}} \frac{3}{2}\left(2 J_{m}+1\right) \sqrt{\left(2 K^{\prime}+1\right)(2 L+1)} \\
& \times\left\{\begin{array}{ccc}
K & K^{\prime} & L \\
J_{m} & J_{m} & J_{m}
\end{array}\right\}\left\{\begin{array}{ccc}
1 & 1 & L \\
J_{m} & J_{m} & J_{n}
\end{array}\right\}, \\
\bar{G}_{M}^{L}\left(K K^{\prime} L\right) & =\sum_{\alpha \beta \sigma \sigma^{\prime}}\left\langle 1 \sigma 1 \sigma^{\prime} \mid L M\right\rangle\langle 1 \sigma \mid \alpha\rangle\left\langle 1 \sigma^{\prime} \mid \beta\right\rangle \bar{G}_{\alpha \beta}\left(K K^{\prime} L\right), \\
\widetilde{G}_{M}^{L}\left(K K^{\prime} L\right) & =\sum_{\alpha \beta \sigma \sigma^{\prime}}\left\langle 1 \sigma 1 \sigma^{\prime} \mid L M\right\rangle\langle 1 \sigma \mid \alpha\rangle\left\langle 1 \sigma^{\prime} \mid \beta\right\rangle \widetilde{G}_{\alpha \beta}\left(K K^{\prime} L\right),
\end{aligned}
$$

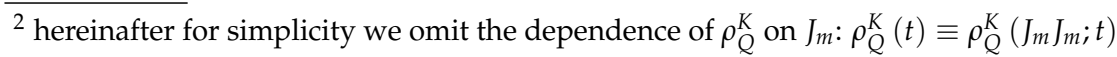


and

$$
\begin{aligned}
& \bar{G}_{\alpha \beta}\left(K K^{\prime} L\right)=\frac{1}{2}\left[G_{\beta \alpha}^{\prime \prime}\left(\omega_{m n}\right)+(-1)^{K+K^{\prime}-L} G_{\alpha \beta}^{\prime \prime}\left(\omega_{m n}\right)\right] /\left(\frac{\omega_{m n}}{c}\right)^{3}, \\
& \widetilde{G}_{\alpha \beta}\left(K K^{\prime} L\right)=\frac{1}{2}\left[G_{\beta \alpha}^{\prime}\left(\omega_{m n}\right)-(-1)^{K+K^{\prime}-L} G_{\alpha \beta}^{\prime}\left(\omega_{m n}\right)\right] /\left(\frac{\omega_{m n}}{c}\right)^{3} .
\end{aligned}
$$

Symbol $G^{\prime}$ and $G^{\prime \prime}$ in (77) and (78) denotes real and imaginary part of $G$, respectively, and symbols $\langle 1 \sigma \mid \alpha\rangle$ and $\left\langle 1 \sigma^{\prime} \mid \beta\right\rangle$ are transformation matrices from the Descartes's components to the circu lar one, that given by (30).

\subsubsection{Relaxation matrix symmetry}

Note that $\bar{G}_{\alpha \beta}\left(K K^{\prime} L\right)$ and $\widetilde{G}_{\alpha \beta}\left(K K^{\prime} L\right)$, and consequently, $\bar{G}_{M}^{L}\left(K K^{\prime} L\right)$ and $\widetilde{G}_{M}^{L}\left(K K^{\prime} L\right)$, are symmetrical with respect to $K$ and $K^{\prime}$. As for the scalar $\gamma\left(K K^{\prime} L, J_{m} J_{n}\right)$, it changes upon permutation of $K$ and $K^{\prime}$ as follows

$$
\gamma\left(K K^{\prime} L, J_{m} J_{n}\right)=(-1)^{K-K^{\prime}} \sqrt{\frac{2 K^{\prime}+1}{2 K+1}} \gamma\left(K^{\prime} K L, J_{m} J_{n}\right)
$$

because of invariance of $6-j$ symbol as regard to permutation of its columns.

Although tensor $G_{\alpha \beta}$ in general has no symmetry with respect to permutation of subscripts, tensors $\bar{G}_{\alpha \beta}\left(K K^{\prime} L\right)$ and $\widetilde{G}_{\alpha \beta}\left(K K^{\prime} L\right)$ have one, as one can see from (77) and (78),

$$
\begin{aligned}
& \bar{G}_{\alpha \beta}\left(K K^{\prime} L\right)=(-1)^{K+K^{\prime}-L} \bar{G}_{\beta \alpha}\left(K K^{\prime} L\right), \\
& \widetilde{G}_{\alpha \beta}\left(K K^{\prime} L\right)=-(-1)^{K+K^{\prime}-L} \widetilde{G}_{\beta \alpha}\left(K K^{\prime} L\right) .
\end{aligned}
$$

Irreducible tensors $\bar{G}_{M}^{L}\left(K K^{\prime} L\right)$ and $\widetilde{G}_{M}^{L}\left(K K^{\prime} L\right)$ in general are complex. Using relation $\langle 1 \sigma \mid \alpha\rangle^{*}=(-1)^{\sigma}\langle 1-\sigma \mid \alpha\rangle$ and Clebsch-Gordan coefficients symmetry, one can show that

$$
\begin{aligned}
& {\left[\bar{G}_{M}^{L}\left(K K^{\prime} L\right)\right]^{*}=(-1)^{L+M} \bar{G}_{-M}^{L}\left(K K^{\prime} L\right),} \\
& {\left[\widetilde{G}_{M}^{L}\left(K K^{\prime} L\right)\right]^{*}=(-1)^{L+M} \widetilde{G}_{-M}^{L}\left(K K^{\prime} L\right) .}
\end{aligned}
$$

This relations allow to find the following symmetry of the relaxation matrix components

$$
\begin{aligned}
& {\left[\gamma_{Q Q^{\prime}}^{K K^{\prime}}\right]^{*}=(-1)^{K^{\prime}-K+Q-Q^{\prime}} \gamma_{-Q-Q^{\prime}}^{K K^{\prime}}} \\
& {\left[\Delta_{Q Q^{\prime}}^{K K^{\prime}}\right]^{*}=(-1)^{K^{\prime}-K+Q-Q^{\prime}} \Delta_{-Q-Q^{\prime}}^{K K^{\prime}}}
\end{aligned}
$$

On the other hand, from hermiticity of density matrix and equation (69) it is easy to obtain

$$
\left[\Gamma_{Q Q^{\prime}}^{K K^{\prime}}\right]^{*}=(-1)^{Q-Q^{\prime}} \Gamma_{-Q-Q^{\prime \prime}}^{K K^{\prime}}
$$

that can be rewrite in terms of $\gamma_{Q Q^{\prime}}^{K K^{\prime}}$ and $\Delta_{Q Q^{\prime}}^{K K^{\prime}}$ as follows

$$
\begin{aligned}
& {\left[\gamma_{Q Q^{\prime}}^{K K^{\prime}}\right]^{*}=(-1)^{Q-Q^{\prime}} \gamma_{-Q-Q^{\prime \prime}}^{K K^{\prime}}} \\
& {\left[\Delta_{Q Q^{\prime}}^{K K^{\prime}}\right]^{*}=-(-1)^{Q-Q^{\prime}} \Delta_{-Q-Q^{\prime}}^{K K^{\prime}}}
\end{aligned}
$$


Comparing (84) and (87) shows that $\gamma_{Q Q^{\prime}}^{K K^{\prime}}$ is different from zero only for even $K+K^{\prime}$. Similarly, comparing (85) and (88) shows that $\Delta_{Q Q^{\prime}}^{K K^{\prime}}$ is different from zero only for odd $K+K^{\prime}$.

These properties can be find more straightforward from symmetries (80) and (81) and definitions (75) and (76) that yield

$$
\begin{aligned}
& \bar{G}_{M}^{L}\left(K K^{\prime} L\right)=(-1)^{K+K^{\prime}} \bar{G}_{M}^{L}\left(K K^{\prime} L\right), \\
& \widetilde{G}_{M}^{L}\left(K K^{\prime} L\right)=(-1)^{K+K^{\prime}+1} \widetilde{G}_{M}^{L}\left(K K^{\prime} L\right) .
\end{aligned}
$$

Taking into account these properties that we can reformulate as $K+K^{\prime}$ is even for $\bar{G}_{\alpha \beta}\left(K K^{\prime} L\right)$ and odd for $\widetilde{G}_{\alpha \beta}\left(K K^{\prime} L\right)$, one can see from (80) and (81) that part of $G_{\alpha \beta}$ which is symmetrical with respect to permutation of subscripts makes a contribution to $\bar{G}_{\alpha \beta}\left(K K^{\prime} L\right)$ and to $\widetilde{G}_{\alpha \beta}\left(K K^{\prime} L\right)$, and hence to $\Gamma_{Q Q^{\prime}}^{K K^{\prime}}$, only when $L$ is even. As for antisymmetrical part of $G_{\alpha \beta}$, it contributes to $\Gamma_{Q Q^{\prime}}^{K K^{\prime}}$ only when $L$ is odd.

When tensor $G_{\alpha \beta}$ is symmetrical (i.e., no external magnetic field), the form of tensor $\bar{G}_{\alpha \beta}\left(K K^{\prime} L\right)$ as well of tensor $\widetilde{G}_{\alpha \beta}\left(K K^{\prime} L\right)$ is simplified

$$
\begin{aligned}
& \bar{G}_{\alpha \beta}\left(K K^{\prime} L\right)=\delta_{L, 2 l} \delta_{K+K^{\prime}, 2 n}\left(\frac{c}{\omega_{m n}}\right)^{3} G_{\alpha \beta^{\prime}}^{\prime \prime} \\
& \widetilde{G}_{\alpha \beta}\left(K K^{\prime} L\right)=\delta_{L, 2 l} \delta_{K+K^{\prime}, 2 n+1}\left(\frac{c}{\omega_{m n}}\right)^{3} G_{\alpha \beta^{\prime}}^{\prime},
\end{aligned}
$$

where $n$ and $l$ are integer. As a consequence, $\bar{G}_{M}^{L}\left(K K^{\prime} L\right)$ and $\widetilde{G}_{M}^{L}\left(K K^{\prime} L\right)$ are also simplified

$$
\begin{aligned}
& \bar{G}_{M}^{L}\left(K K^{\prime} L\right)=\delta_{L, 2 l} \delta_{K+K^{\prime}, 2 n}\left(\frac{c}{\omega_{m n}}\right)^{3}\left[G^{\prime \prime}\right]_{M}^{L}, \\
& \widetilde{G}_{M}^{L}\left(K K^{\prime} L\right)=\delta_{L, 2 l} \delta_{K+K^{\prime}, 2 n+1}\left(\frac{c}{\omega_{m n}}\right)^{3}\left[G^{\prime}\right]_{M}^{L} .
\end{aligned}
$$

As stated above (see Eqs. (42) -(44)), in this case there are only four nonzero components of $G_{M}^{L}$ in the proper coordinate system.

There is additional symmetry of the relaxation tensor $\Gamma_{Q Q^{\prime}}^{K K^{\prime}}$ in the case. Using the fact that $\bar{G}_{M}^{L}\left(K K^{\prime} L\right)$ and $\widetilde{G}_{M}^{L}\left(K K^{\prime} L\right)$ are symmetrical with respect to $K$ and $K^{\prime}$, evenness of $L$, relation (79) and also Clebsch-Gordan coefficient symmetry $\left\langle K^{\prime} Q^{\prime} L M \mid K Q\right\rangle=(-1)^{L+M} \sqrt{\frac{2 K+1}{2 K^{\prime}+1}}\langle K-$ $Q L M\left|K^{\prime}-Q^{\prime}\right\rangle$, one can obtain

$$
\Gamma_{Q Q^{\prime}}^{K K^{\prime}}=(-1)^{K-K^{\prime}+Q-Q^{\prime}} \Gamma_{-Q^{\prime}-Q}^{K^{\prime} K}
$$

that we can rewrite using (86) as follows

$$
\Gamma_{Q Q^{\prime}}^{K K^{\prime}}=(-1)^{K-K^{\prime}}\left[\Gamma_{Q^{\prime} Q}^{K^{\prime} K}\right]^{*} .
$$

This is just the symmetry of $\Gamma_{Q Q^{\prime}}^{K K^{\prime}}$ relative to time reversal (Omont, 1977) that is natural in the absence of magnetic field. 
In case of the atomic surroundings is axial symmetrical in addition, there are only two nonzero components of $G_{M}^{L}$ in the proper coordinate system, $G_{0}^{0}$ and $G_{0}^{2}$. Therefor, only irreducible tensors $\bar{G}_{0}^{L}\left(K K^{\prime} L\right)$ and $\widetilde{G}_{0}^{L}\left(K K^{\prime} L\right)$ are nonzero and real in the system (see relations (82) and (83), and (93) and (94)). Consequently, only $\gamma_{Q Q}^{K K^{\prime}}$ and $\Delta_{Q Q}^{K K^{\prime}}$ are also nonzero and real (see relations (72 and (73)), hence,

$$
\Gamma_{Q Q^{\prime}}^{K K^{\prime}}=\delta_{Q, Q^{\prime}} \Gamma_{Q Q}^{K K^{\prime}}
$$

So, in this case $\Gamma_{Q Q}^{K K^{\prime}}$ is real for even $K+K^{\prime}$, imaginary for odd $K+K^{\prime}$ and

$$
\Gamma_{00}^{K K^{\prime}}=0
$$

for odd $K+K^{\prime}$ because of the following Clebsch-Gordan coefficient symmetry

$$
\left\langle K^{\prime} 0 L 0 \mid K 0\right\rangle=(-1)^{K^{\prime}+L-K}\left\langle K^{\prime} 0 L 0 \mid K 0\right\rangle \text {. }
$$

\section{Deexcitation dynamics}

Deexcitation of upper level is given by (69) with $K=Q=0$

$$
\frac{\mathrm{d} \rho_{0}^{0}(t)}{\mathrm{d} t}=-\gamma_{0} \sum_{K^{\prime} Q^{\prime}} \Gamma_{0 Q^{\prime}}^{0 K^{\prime}} \rho_{Q^{\prime}}^{K^{\prime}}(t) .
$$

Hereinafter we suppose that there is no external magnetic field. In this case $\gamma_{0} \Gamma_{0 Q^{\prime}}^{0 K^{\prime}}=$ $(-1)^{Q^{\prime}} \gamma_{-Q^{\prime}}^{K^{\prime}} / \sqrt{2 J_{m}+1}$, where $\gamma_{-Q^{\prime}}^{K^{\prime}}$ is defined by (40), multiplier $(-1)^{Q^{\prime}}$ transforms covariant component $\rho_{Q^{\prime}}^{K^{\prime}}$ into contravariant one $\rho^{K^{\prime}-Q^{\prime}}$ and denominator $\sqrt{2 J_{m}+1}$ reflect the fact that the right-hand side of $(100)$ is variation in time of $\rho_{0}^{0}$, not of population that is $\sqrt{2 J_{m}+1} \rho_{0}^{0}$ as in (39). To obtain temporal variation of the deexcitation, it is necessary to solve consistent differential equations, involving along with Eq. (100) also differential equations for $\rho_{Q^{\prime}}^{K^{\prime}}(t)$, incoming in its right-hand side.

Let us restrict themselves to the case of axial symmetrical atomic surroundings.

As it mentioned above, this case include half-space boundary and spherical particle. From (97), (98), (100), and also (99), it is follows that consistent differential equations, describing deexcitation dynamics in the proper coordinate system, include only multipole components with even $K$ and $Q=0$. The number of such components is $\left[J_{m}\right]+1$ because of $0 \gtrless K \gtrless 2 J_{m}$ as noted above (symbol $\left[J_{m}\right]$ here and further denotes the integer part of $J_{m}$ ). As the relevant $\Gamma_{00}^{K K^{\prime}}$ are real in our case, from (96) we obtain that they are symmetrical relative to $K$ and $K^{\prime}$

$$
\Gamma_{00}^{K K^{\prime}}=\Gamma_{00}^{K^{\prime} K}
$$

Hence, the number of different relevant components $\Gamma_{00}^{K K^{\prime}}$ is $\left(\left[J_{m}\right]+1\right) \times\left(\left[J_{m}\right]+2\right) / 2$.

As is known, the general solution of $\left[J_{m}\right]+1$ consistent linear homogeneous differential equations is given by a linear combination of $\left[J_{m}\right]+1$ their eigen vectors, each of them varies in time exponentially with its own rate. The rates are eigen values of the consistent equations. The number of the eigen values is also in general equal to $\left[J_{m}\right]+1$. So, the atomic deexcitation is also usually expressed as a linear sum of $\left[J_{m}\right]+1$ exponentials. 
In fact, the eigenvalues are relaxation rates of populations of magnetic sublevels $\left|J_{m} \pm M\right\rangle$ in the case under consideration. Indeed, relevant multipole components $\rho_{0}^{K}$ incoming in the consistent differential equations, describing deexcitation dynamics, are linear combination of the populations of the sublevels $\left|J_{m} M\right\rangle$ (see (36)). In addition, the sublevels $\left|J_{m} M\right\rangle$ and $\left|J_{m}-M\right\rangle$ are transformed one into another (with the sign $(-1)^{P}$, where $P$ is parity of the level $m$ ) under reflection in any plane through the symmetry axis (Landau \& Lifshitz, 1977). Consequently, the relaxation rates of these sublevels are equal. So, the number of different relaxation rates is $\left[J_{m}\right]+1$ as stated above with respect to the eigenvalues.

\subsection{Deexcitation dynamics in the case of $J_{m}=1, J_{n}=0$}

Let us consider in more detail the case when the angular momentums are $J_{m}=1$ and $J_{n}=0$. In the case under study, deexcitation dynamics is described by only two equations

$$
\begin{aligned}
& \frac{\mathrm{d} \rho_{0}^{0}(t)}{\mathrm{d} t}=-\gamma_{0}\left[\Gamma_{00}^{00} \rho_{0}^{0}(t)+\Gamma_{00}^{02} \rho_{0}^{2}(t)\right], \\
& \frac{\mathrm{d} \rho_{0}^{2}(t)}{\mathrm{d} t}=-\gamma_{0}\left[\Gamma_{00}^{02} \rho_{0}^{0}(t)+\Gamma_{00}^{22} \rho_{0}^{2}(t)\right] .
\end{aligned}
$$

The eigen values $\gamma_{ \pm}$of the consistent equations are

$$
\gamma_{ \pm}=\gamma_{0}\left[\Gamma_{+} \pm \Gamma\right]
$$

and fundamental solution matrix are

$$
S(t)=\frac{1}{2}\left(\begin{array}{cc}
\left(1-\frac{\Gamma_{-}}{\Gamma}\right) e^{-\gamma_{-} t}+\left(1+\frac{\Gamma_{-}}{\Gamma}\right) e^{-\gamma_{+} t} & \frac{\Gamma_{00}^{02}}{\Gamma}\left(-e^{-\gamma_{-} t}+e^{-\gamma_{+} t}\right) \\
\frac{\Gamma_{00}^{02}}{\Gamma}\left(-e^{-\gamma_{-} t}+e^{-\gamma_{+} t}\right) & \left(1+\frac{\Gamma_{-}}{\Gamma}\right) e^{-\gamma_{-} t}+\left(1-\frac{\Gamma_{-}}{\Gamma}\right) e^{-\gamma_{+} t}
\end{array}\right),
$$

where dimensionless $\Gamma_{ \pm}$and $\Gamma$ are defined as

$$
\begin{aligned}
\Gamma_{ \pm} & =\frac{1}{2}\left(\Gamma_{00}^{00} \pm \Gamma_{00}^{22}\right), \\
\Gamma & =\sqrt{\left(\Gamma_{-}\right)^{2}+\left(\Gamma_{00}^{02}\right)^{2}} .
\end{aligned}
$$

Specific solution column of the consistent equations (102)-(103), corresponding to initial conditions given by column $\boldsymbol{c}=\operatorname{col}\left(\rho_{0}^{0}(0), \rho_{0}^{2}(0)\right)$, is obtained by multiplication of fundamental solution matrix on the right by column $c$.

It is known that the excited atomic states that are produced by the absorption of anisotropic resonance light are strongly polarized (Alexandrov et al., 1993; Happer, 1972; Omont, 1977). This atomic polarization results from the directionality or polarization of the light beam. So, immediately after excitation there are nonzero both $\rho_{0}^{0}(0)$ and $\rho_{0}^{2}(0)$.

However, let us consider the simplest case of isotropic excitation, when there is only population $\rho_{0}^{0}(0)$ on the upper level at the instant after excitation. So, the solution column 
in the case is given by

$$
\left(\begin{array}{l}
\rho_{0}^{0}(t), \\
\rho_{0}^{2}(t),
\end{array}\right)=\frac{1}{2}\left(\begin{array}{c}
\left(1-\frac{\Gamma_{-}}{\Gamma}\right) e^{-\gamma_{-} t}+\left(1+\frac{\Gamma_{-}}{\Gamma}\right) e^{-\gamma_{+} t} \\
\frac{\Gamma_{00}^{02}}{\Gamma}\left(-e^{-\gamma_{-} t}+e^{-\gamma_{+} t}\right)
\end{array}\right) \rho_{0}^{0}(0) .
$$

In the case under consideration that is $J_{m}=1, J_{n}=0$, dimensionless relaxation matrix elements are following: $\Gamma_{00}^{00}=(1 / 2)\left(c / \omega_{m n}\right)^{3} \operatorname{tr}\left(\mathbf{G}^{\prime \prime}\right), \Gamma_{00}^{02}=-(\sqrt{2} / 2)\left(c / \omega_{m n}\right)^{3}\left(G_{Z Z}^{\prime \prime}-G_{X X}^{\prime \prime}\right)$, $\Gamma_{00}^{22}=\Gamma_{00}^{00}-\Gamma_{00}^{02} / \sqrt{2}$. So, relevant dimensionless $\Gamma_{ \pm}$and $\Gamma$ are

$$
\begin{aligned}
\Gamma_{+} & =\frac{3}{4}\left(\frac{c}{\omega_{m n}}\right)^{3}\left(G_{Z Z}^{\prime \prime}+G_{X X}^{\prime \prime}\right), \\
\Gamma_{-} & =-\frac{1}{4}\left(\frac{c}{\omega_{m n}}\right)^{3}\left(G_{Z Z}^{\prime \prime}-G_{X X}^{\prime \prime}\right), \\
\Gamma & =\frac{3}{4}\left(\frac{c}{\omega_{m n}}\right)^{3}\left(G_{Z Z}^{\prime \prime}-G_{X X}^{\prime \prime}\right) .
\end{aligned}
$$

Substituting (109)-(111) into (105), we obtain

$$
S(t)=\frac{1}{3}\left(\begin{array}{cc}
{\left[2 e^{-\gamma_{-} t}+e^{-\gamma_{+} t}\right]} & \sqrt{2}\left[e^{-\gamma_{-} t}-e^{-\gamma_{+} t}\right] \\
\sqrt{2}\left[e^{-\gamma_{-} t}-e^{-\gamma_{+} t}\right] & {\left[e^{-\gamma_{-} t}+2 e^{-\gamma_{+} t}\right]}
\end{array}\right) .
$$

Eigen values $\gamma_{ \pm}$in the case are

$$
\begin{aligned}
& \gamma_{+}=\frac{3}{2}\left(\frac{c}{\omega_{m n}}\right)^{3} G_{Z Z}^{\prime \prime} \\
& \gamma_{-}=\frac{3}{2}\left(\frac{c}{\omega_{m n}}\right)^{3} G_{X X}^{\prime \prime} .
\end{aligned}
$$

In the case under consideration (i.e., $J_{m}=1, J_{n}=0$ ) it is possible such excitation conditions that upper level deexcitation is pure exponential. Such cases only three.

In the first case the atom is excited by light with linear polarization that is collinear to the symmetry axis. Such light excites only one upper sublevel with angular momentum projection on the symmetry axis $J_{m Z}=0$. In this case the initial conditions column is given by

$$
c_{0} \equiv\left(\begin{array}{c}
\rho_{0}^{0}(0) \\
\rho_{0}^{2}(0)
\end{array}\right)=\frac{1}{\sqrt{3}}\left(\begin{array}{c}
1 \\
-\sqrt{2}
\end{array}\right) \rho_{00}(0),
$$

where $\rho_{00}(0)$ is population of the sublevel mentioned above. If we multiply fundamental solution matrix (112) on the right by column $c_{0}$, we get the variation in the time of the population and alignment of the upper level:

$$
\left(\begin{array}{c}
\rho_{0}^{0}(t) \\
\rho_{0}^{2}(t)
\end{array}\right)=c_{0} e^{-\gamma_{+} t}
$$

In the second case the atom is excited by circular polarized light that propagates along symmetry axis. Now the only upper sublevel with angular momentum projection on the 
symmetry axis $J_{m Z}=+1$ (or $J_{m Z}=-1$ for the opposite circular polarization) is excited. Initial conditions column in the case is given by

$$
\boldsymbol{c}_{\mathbf{1}} \equiv\left(\begin{array}{c}
\rho_{0}^{0}(0) \\
\rho_{0}^{2}(0)
\end{array}\right)=\frac{1}{\sqrt{3}}\left(\begin{array}{c}
1 \\
1 / \sqrt{2}
\end{array}\right) \rho_{11}(0),
$$

where $\rho_{11}(0)$ is population of the exited sublevel. The solution corresponding to this column is

$$
\left(\begin{array}{l}
\rho_{0}^{0}(t) \\
\rho_{0}^{2}(t)
\end{array}\right)=c_{1} e^{-\gamma_{-} t}
$$

Lastly, in the third case the atom is excited by light with linear polarization that is orthogonal to the symmetry axis. It has been known that such polarization can be represented by the sum of the opposite circular polarization with the same amplitude, rotating in the plane that is orthogonal to the symmetry axis. This case is reduced to the previous one because of only two upper sublevels with angular momentum projection on the symmetry axis $J_{m z}= \pm 1$ are excited independently with equal probability, and hence $\rho_{11}(0)=\rho_{-1-1}(0)$. The rates of decay of the both excited sublevels into the only low state are equal due to axial symmetry. Deexcitation dynamics in the case also given by (116).

These three exceptional cases of simple exponetial deexcitation can be physically interpreted as follows. In every case the excited state transforms to the only low state by means of one channel. The decay itself is induced by the optical transition oscillating dipole that arises due to interaction of the excited atom with the electric field quantum oscillations. Both the direction of the dipole oscillation and the direction of the exciting light polarization are the same due to the one and the same channel of excitation and deexcitation (see Fig. 1).

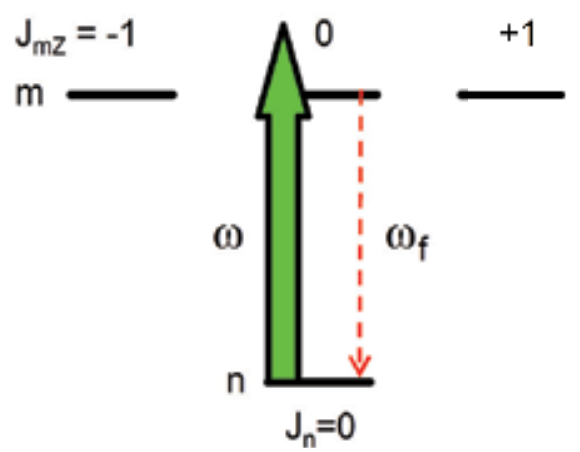

(a) Exciting light is linear polarized along (or transversely to) the symmetry axis passing through the atom and body; $\mathrm{Z}$ - axis is along (or transversely to) this axis

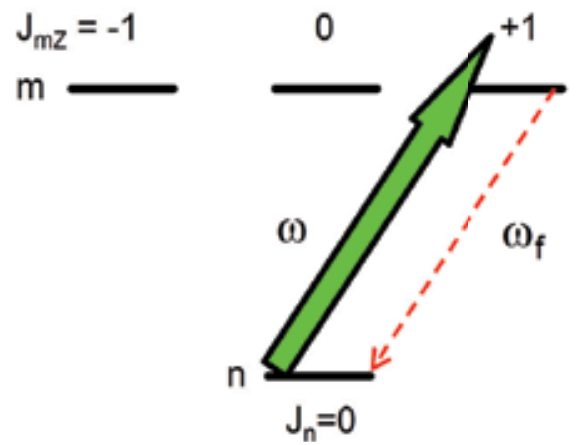

(b) Exciting light is circular polarized and propagates along the symmetry axis that is $\mathrm{Z}$ - axis

Fig. 1. Exceptional polarizations of the exciting light that led to the pure exponential decay of the excited atomic state $\left(\omega\right.$ and $\omega_{f}$ are frequencies of the exciting light and fluorescence respectively)

Precisely owing to this fact, experimental results of the measurement of the decay of the fluorescence signal (Amos \& Barnes, 1997; Chance et al., 1978; Drexhage et al., 1968; Fort \& Grésillon, 2008; Kreiter et al., 2002; Snoeks et al., 1995; Vallée et al., 2001) are in good agreement 
with the simple model of the classic scattering dipole, in spite of the fact that fluorescence is the two-step process, rather than scattering.

It should be noted that consistent equations (102)-(103) describe deexcitation dynamics also in the case $J_{m}=1, J_{n}=1$, or $J_{m}=1, J_{n}=2$, and also in the case $J_{m}=3 / 2, J_{n}=1 / 2$, and either $J_{m}=3 / 2, J_{n}=3 / 2$, or $J_{m}=3 / 2, J_{n}=5 / 2$. Of course, specific values of the dimensionless $\Gamma_{00}^{00}, \Gamma_{00}^{02}$, and $\Gamma_{00}^{22}$ in these cases differ from considered above.

It should be pointed out too that in the case $J_{m}=3 / 2$ and $J_{n}=1 / 2$ there is the only exciting light polarization, namely linear polarization along symmetry axis, that leds to the pure exponential decay of the excited state because of the relaxation rate equality of the excited sublevels $(J m Z= \pm 1 / 2)$ due to the axial symmetry.

\section{Conclusions}

In the chapter we have proposed a general approach to the problem of deexcitation of a degenerate two-level atom near (inside) a body. On the basis of the approach the master equation for density matrix in the polarization moments representation was obtained.

We have shown that relaxation dynamics of a polarization moment is described in general by a consistent linear equations for all $2 J_{m}+1$ polarization moments of the excited level, where $J_{m}$ is the total momentum of the level. We have expressed relaxation matrix elements of the consistent linear equations in terms of the field response tensor that can be found as the electric field of the classic oscillating unit dipole situated near the body.

We have found symmetry of the relaxation matrix.

An additional relaxation matrix symmetry is recognized in the case when there is no external quasistatic magnetic field, and as a result, the field response tensor is symmetrical one. Therefore, the tensor may be diagonalized. We have shown that relaxation matrix depends only on the trace of the field response tensor, on the difference between the most principal value of the diagonal response tensor and the half-sum of two others, and also on the difference between these two.

Axial symmetric atomic surroundings gives rise to one more additional symmetry of the relaxation matrix. In this case it depends only on the trace of the field response tensor and on the difference between its two principal values.

We have shown that deexcitation dynamics of the degenerate two-level atom in the conditions under consideration represents multiexponential decay. In the case of the axial symmetric atomic surroundings, the number of the exponential is equal to $\left[J_{m}\right]+1$, where $\left[J_{m}\right]$ is the integer part of $J_{m}$. So, the simple exponential decay of the atomic excitation is possible only in two cases, namely, when $J_{m}=0$ or $J_{m}=1 / 2$. We have shown that simple exponential decay of the atomic excitation is also possible in the case of $J_{m}=1, J_{n}=0$ and on special polarizations of exciting light, namely on the linear polarization that is collinear or orthogonal to the axial symmetry axis, and on the circular polarizations rotating in the plane that is orthogonal to the symmetry axis. In this exceptional cases both the excitation and decay of the corresponding upper states follow the one and the same respective channel. Simple exponential decay of the atomic excitation is possible too in the case $J_{m}=3 / 2$ and $J_{n}=1 / 2$ when exciting light polarization is linear oriented along symmetry axis.

Our analysis have carried out in the absence of hyperfine structure on the combine energy levels. However, it can be easily expanded straightforward on general case by expanding 
quantum states irreducible basis of the total momentum, including both the total electronic momentum and the nuclear spin, into the direct product of states irreducible bases of the the nuclear spin and the electronic momentum. Just the late basis is involved into the electromagnetic interaction in the course of the allowed optical transition.

We have considered situation when degenerate two-level atom is situated in the vicinity of a body. Nevertheless, it is clear from the consideration that our treatment is more general and results obtained are true for an atom embedded in any anisotropic medium.

\section{References}

Agarwal, G. S. (1975a). Quantum electrodynamics in the presence of dielectrics and conductors. I. electromagnetic-field response functions and black-body fluctuations in finite geometries, Phys. Rev. A 11(1): 230-242.

Agarwal, G. S. (1975b). Quantum electrodynamics in the presence of dielectrics and conductors. IV. general theory for spontaneous emission in finite geometries, Phys. Rev. A 12: 1475-1497. URL: http://link.aps.org/doi/10.1103/PhysRevA.12.1475

Alexandrov, E. B., Chaika, M. P. \& Khvostenko, G. I. (1993). Interference of atomic states, Springer-Verlag, Berlin, New York.

Allen, L. \& Eberly, J. H. (1975). Optical Resonance and Two-Level Atoms, John Wiley and Sons, New York-London-Sydney-Toronto.

Amos, R. M. \& Barnes, W. L. (1997). Modification of the spontaneous emission rate of $\mathrm{Eu}^{3+}$ ions close to a thin metal mirror, Phys. Rev. B 55: 7249-7254.

URL: http://link.aps.org/doi/10.1103/PhysRevB.55.7249

Arnoldus, H. F. \& George, T. F. (1987). Quantum theory of atomic fluorescence near a metal surface, J. Chem. Phys. 87(8): 4263-4272. URL: http://dx.doi.org/10.1063/1.452884

Arnoldus, H. F. \& George, T. F. (1988a). Spontaneous decay and atomic fluorescence near a metal surface or an absorbing dielectric, Phys. Rev. A 37: 761-769.

URL: http://link.aps.org/doi/10.1103/PhysRevA.37.761

Arnoldus, H. F. \& George, T. F. (1988b). Symmetries of spontaneous decay for atoms near any surface, Surface Science 205(3): 617-636.

URL: http://www.sciencedirect.com/science/article/pii/0039602888903056

Barash, S. (1988). Van der Waals Forces, Nauka, Moscow. [in Russian].

Barnes, W. L. (1998). Fluorescence near interfaces: The role of photonic mode density, Journal of Modern Optics 45(4): 661-699.

URL: http://www.tandfonline.com/doi/abs/10.1080/09500349808230614

Berestetskii, V., Lifshitz, E. \& Pitaevskii, L. (2008). Quantum Electrodynamics, Butterworth-Heinemann, Oxford.

Bernard, W. \& Callen, H. B. (1959). Irreversible thermodynamics of nonlinear processes and noise in driven systems, Rev. Mod. Phys. 31(4): 1017-1044.

Biedenharn, L. C. \& Louck, J. D. (1981). Angular Momentum in Quantum Physics. Theory and Application, Addison-Wesley, Massachusetts.

Blum, K. (1996). Density Matrix Theory and Applications, 2nd edn, Plenum Press, New York.

Callen, H. B., Barasch, M. L. \& Jackson, J. L. (1952). Statistical mechanics of irreversibility, Phys. Rev. 88: 1382-1386. URL: http://link.aps.org/doi/10.1103/PhysRev.88.1382

Callen, H. B. \& Welton, T. A. (1951). Irreversibility and generalized noise, Phys. Rev. 83: 34-40. URL: http://link.aps.org/doi/10.1103/PhysRev.83.34 
Chance, R. R., Prock, A. \& Silbey, R. (1978). Molecular fluorescence and energy transfer near interfaces, in I. Prigogine \& S. A. Rice (eds), Advances in Chemical Physics, Vol. 37, Wiley, New York, pp. 1-65.

Chew, H. (1987). Transition rates of atoms near spherical surfaces, The Journal of Chemical Physics 87(2): 1355-1360. URL: http://link.aip.org/link/?JCP/87/1355/1

Chew, H. (1988). Radiation and lifetimes of atoms inside dielectric particles, Phys. Rev. A 8(7): 3410-3416.

Das, P. \& Metiu, H. (1985). Enhancement of molecular fluorescence and photochemistry by small metal particles, J. Phys. Chem. 89(22): 4680-4687.

Drexhage, K., Kuhn, H. \& Schafer, F. (1968). Variation of fluorescence decay time of a molecule in front of a mirror, Berichte Der Bunsen-Gesellschaft Fur Physikalische Chemie 72(2): 329.

Dung, H. T., Knöll, L. \& Welsch, D.-G. (2000). Spontaneous decay in the presence of dispersing and absorbing bodies: general theory and application to a spherical cavity, Phys. Rev. A 62: 053804. URL: http://link.aps.org/doi/10.1103/PhysRevA.62.053804

Fano, U. \& Racah, G. (1959). Irreducible Tensorial Sets, Academic Press, New York.

Ford, G. W., \& Weber, W. H. (1984). Electromagnetic interactions of molecules with metal surfaces, Phys. Rep. 113: 195.

Fort, E. \& Grésillon, S. (2008). Surface enhanced fluorescence, Journal of Physics D: Applied Physics 41(1): 013001.

URL: $h$ ttp://stacks.iop.org/0022-3727/41/i=1/a=013001

Garrett, S. H., Wasey, J. A. E. \& Barnes, W. L. (2004). Determining the orientation of the emissive dipole moment associated with dye molecules in microcavity structures, Journal of Modern Optics 51(15): 2287-2295.

Gersten, J. \& Nitzan, A. (1981). Spectroscopic properties of molecules interacting with small dielectric particles, The Journal of Chemical Physics 75(3): 1139-1152.

URL: http://link.aip.org/link/?JCP/75/1139/1

Happer, W. (1972). Optical pumping, Rev. Mod. Phys. 44: 169-249.

URL: http://link.aps.org/doi/10.1103/RevModPhys.44.169

Hellen, E. H. \& Axelrod, D. (1987). Fluorescence emission at dielectric and metal-film interfaces, J. Opt. Soc. Am. B 4(3): 337-350.

URL: http://josab.osa.org/abstract.cfm? URI=josab-4-3-337

Il'inskii, Y. A. \& Keldysh, L. V. (1994). Electromagnetic Response of Material Media, Plenum Press, New York.

Klimov, V. V., Ducloy, M. \& Letokhov, V. S. (1996). Spontaneous emission rate and level shift of an atom inside a dielectric microsphere, J. Modern Opt. 43(3): 549-563.

Klimov, V. V., Ducloy, M. \& Letokhov, V. S. (2001). Spontaneous emission of an atom in the presence of nanobodies, Quantum Electron. 31(7): 569-586.

Klimov, V. V., Ducloy, M., Letokhov, V. S. \& Lebedev, P. N. (1996). Radiative frequency shift and linewidth of an atom dipole in the vicinity of a dielectric microsphere, J. Modern Opt. 43(11): 2251-2268.

Klyshko, D. (2011). Physical Foundations of Quantum Electronics, World Scientific Publishing Company.

Kreiter, M., Prummer, M., Hecht, B. \& Wild, U. P. (2002). Orientation dependence of fluorescence lifetimes near an interface, J. Chem. Phys 117(20): 9430-9433.

URL: $h t t p: / / d x$.doi.org/10.1063/1.1515732

Landau, L. D. \& Lifshitz, E. M. (1977). Quantum Mechanics: Non-Relativistic Theory, third edn, Pergamon Press, Ltd., New York. 
Landau, L. D. \& Lifshitz, E. M. (1980). Statistical Physics, part 1, third edn, Pergamon Press, Ltd., New York.

Lifshitz, E. M. \& Pitaevskii, L. (1980). Statistical physics : pt. 2: theory of the condensed state, Vol. 9 of Course of Theoretical Physics, third edn, Pergamon Press, Ltd., New York.

Loisell, W. H. (1973). Quantum Statistical Properties of Radiation, Wiley, New York.

Lukosz, W. \& Kunz, R. E. (1977). Light emission by magnetic and electric dipoles close to a plane interface. i. total radiated power, J. Opt. Soc. Am. 67(12): 1607-1615.

URL: http://www.opticsinfobase.org/abstract.cfm? URI=josa-67-12-1607

Macklin, J. J., Trautman, J. K., Harris, T. D. \& Brus, L. E. (1996). Imaging and time-resolved spectroscopy of single molecules at an interface, Science 272(5259): 255-258.

URL: $h$ ttp://www.sciencemag.org/content/272/5259/255.abstract

Milonni, P. \& Knight, P. (1973). Spontaneous emission between mirrors, Optics Communications 9(2): $119-122$.

URL: http://www.sciencedirect.com/science/article/pii/0030401873902393

Nikolaev, G. N. (2006). Effective transfer of light energy to a nanoparticle by means of a resonance atomic lens, JETP 102(3): 394-405.

Omont, A. (1977). Irreducible components of the density matrix: application to optical pumping, 5 in Progress in quantum electronics, Pergamon Press, pp. 69-138.

URL: http://books.google.com/books? id=TmrRQgAACAAJ

Purcell, E. M. (1946). Spontaneous emission probabilities at radio frequencies, Phys. Rev. 69(11-12): 681.

Ruppin, R. (1982). Decay of an excited molecule near a small metal sphere, J. Chem. Phys. 76(4): 1681.

Snoeks, E., Lagendijk, A. \& Polman, A. (1995). Measuring and modifying the spontaneous emission rate of erbium near an interface, Phys. Rev. Lett. 74: 2459-2462.

URL: http://link.aps.org/doi/10.1103/PhysRevLett.74.2459

Sobelman, I. I. (1972). Introduction to the theory of atomic spectra, Vol. 40 of International series of monographs in natural philosophy, Pergamon Press, Oxford, New York.

Steiner, M., Schleifenbaum, F., Stupperich, C., Failla, A. V., Hartschuh, A. \& Meixner, A. J. (2005). Microcavity-controlled single-molecule fluorescence, Chem. Phys. Chem. 6: $2190-2196$.

Vallée, R., Tomczak, N., Gersen, H., van Dijk, E., García-Parajó, M., Vancso, G. \& van Hulst, N. (2001). On the role of electromagnetic boundary conditions in single molecule fluorescence lifetime studies of dyes embedded in thin films, Chemical Physics Letters 348(3-4): 161-167.

URL: http://www.sciencedirect.com/science/article/pii/S0009261401011198

Varshalovich, D. A., Moskalev, A. N. \& Khersonskii, V. K. (1988). Quantum Theory of Angular Momentum, World Scientific Pub Co Inc.

Wylie, J. M. \& Sipe, J. E. (1984). Quantum electrodynamics near an interface, Phys. Rev. A 30(3): 1185-1193.

Wylie, J. M. \& Sipe, J. E. (1985). Quantum electrodynamics near an interface. II, Phys. Rev. A 32(4): 2030-2043.

Yeung, M. S. \& Gustafson, T. K. (1996). Spontaneous emission near an absorbing dielectric surface, Phys. Rev. A 54: 5227-5242.

URL: http://link.aps.org/doi/10.1103/PhysRevA.54.5227 


\section{Part 3}

\section{Properties and Applications}





\title{
Thermal Property Measurement of $\mathrm{Al}_{2} \mathrm{O}_{3}$-Water Nanofluids
}

\author{
Fei Duan \\ School of Mechanical and Aerospace Engineering, Nanyang Technological University \\ Singapore
}

\section{Introduction}

Fluids have been applied in the cooling in the most important industries including microelectronics, manufacturing, metrology, etc. With increasing thermal loads that require advances in cooling the new higher power output devices with faster speeds and smaller feature, the conventional heat transfer fluids, such as water, engine oil, ethylene glycol, etc., demonstrate the relative low heat transfer performance. The use of solid particles as an additive suspended in the base fluid is a potential alternative technique for the heat transfer enhancement, i.e. thermal conductivity of metallic or nonmetallic solids might have two orders of magnitude higher than the conventional fluids. The enhancement of thermal conductivity of conventional fluids with the suspension of solid particles, such as micrometer-sized particles, has been well known for more than 100 years (Choi, 1995). However, the conventional micrometer-sized particle liquid suspensions require high concentrations $(>10 \%)$ of particles to achieve such an enhancement. Because they have the rheological and stability problems such as sedimentation, erosion, fouling, and pressure drop in flow channels, the fluids with the micrometer-sized particle have not been of interest for practical applications. The recent advance in materials technology has made it possible to produce nanometer-sized particles that can overcome these above problems. The innovative fluids suspended with nanometer-sized solid particles can change the transport and thermal properties of the base fluid, and make the fluid stable.

Modern nanotechnology can produce materials with average particle sizes below $50 \mathrm{~nm}$. All solid nanoparticles with high thermal conductivity can be used as additives of nanofluids. These nanoparticles that have been usually used in the nanofluids include: metallic particles $\left(\mathrm{Cu}, \mathrm{Al}, \mathrm{Fe}, \mathrm{Au}, \mathrm{Ag}\right.$, etc.), and nonmetallic particles $\left(\mathrm{Al}_{2} \mathrm{O}_{3}, \mathrm{CuO}, \mathrm{Fe}_{3} \mathrm{O}_{4}, \mathrm{TiO}_{2}, \mathrm{SiC}\right.$, carbon nanotube, etc.). The base media of nanofluids are usually water, oil, acetone, decene, ethylene glycol, etc. ( $\mathrm{Li}$ et al., 2009). A $40 \%$ increase in thermal conductivity was found in the $\mathrm{Cu}$ oil-based nanofluids with $0.3 \%$ volume concentration, while the $\mathrm{Al}_{2} \mathrm{O}_{3}$ water-based nanofluids exhibited a $29 \%$ enhancement of thermal conductivity for the $5 \%$ volume concentration nanofluids (Eastman et al., 1997).

The $\mathrm{Al}_{2} \mathrm{O}_{3}$ nanoparticles were selected to prepare the water-based nanofluids in this study due to their chemical stability. Preparation of nanofluids is the key step in the use of nanoparticles for stable nanofluids. Two kinds of methods have been employed in producing nanofluids: the single-step method and the two-step method. The single-step method is a process combining the preparation of nanoparticles with the synthesis of nanofluids, for which the nanoparticles are directly prepared by the physical vapor deposition technique 
or the liquid chemical method (Choi, 1995; Eastman et al., 1997). The processes of drying, storage, transportation, and dispersion of nanoparticles can be avoided, so the aggregation of nanoparticles is minimized and the stability of fluids is increased. But a disadvantage of the method is that only low vapor pressure fluids are compatible with the process. It limits the applications of the method. The two-step method for preparing nanofluids is a process by dispersing nanoparticles into base liquids. Eastman et al. (1997), Lee et al. (1999), and Wang et al. (1999) used this method to produce the $\mathrm{Al}_{2} \mathrm{O}_{3}$ nanofluids. Nanoparticles used in the method are firstly produced as a dry powder by inert gas condensation, chemical vapor deposition, mechanical alloying, or the other suitable techniques before the nano-sized powder is then dispersed into a fluid in the second processing step. This step-by-step method isolates the preparation of the nanofluids from the preparation of nanoparticles. As a result, aggregation of nanoparticles may take place in both the steps, especially in the process of drying, storage, and transportation of nanoparticles. The aggregation would not only result in the settlement and clogging, but also affect the thermal properties. The techniques such as ultrasonic agitation or the addition of surfactant into the fluids are often used to minimize particle aggregation and improve dispersion behavior. Since nanopowder synthesis techniques have already been commercialized, there are potential economic advantages in using the two-step synthesis method. But an important problem that needs to be solved is the stabilization of the suspension to be prepared.

Nanofluids are a new class of solid-liquid composite materials consisting of solid nanoparticles, with sizes typically in the order of $1-100 \mathrm{~nm}$, suspending in a heat transfer liquid. Nanofluids are expected to have superior properties compared to conventional heat transfer fluids. The much larger relative surface area of nanoparticles should not only significantly improve heat transfer capabilities (Xie et al., 2001), but also increase the stability of the suspensions. In addition, nanofluids can improve abrasion-related properties as compared to the conventional solid/fluid mixtures. Successful applications of nanofluids would support the current trend toward component miniaturization by enabling the design of smaller but higher-power heat exchanger systems (Keblinski et al., 2005). The thermal properties including thermal conductivity, viscosity, and surface tension have been investigated.

\subsection{Thermal conductivity of nanofluids}

Since the model reported by Maxwell (1892), the classical models have been derived by Hamilton \& Crosser (1962), Bruggeman (1935), and Xuan \& Li (2000) for predicting the effective thermal conductivity of a continuum mixture with the assumed well-dispersed solid particles in the base fluid. The Maxwell model was developed to determine the effective thermal conductivity of liquid-solid suspensions for a low volumetric concentration of spherical particles. This model is applicable to statistically homogeneous low volume fraction liquid-solid suspensions with randomly dispersed and uniform spherical particles in size. For non-spherical particles, the thermal conductivity of the nanofluids depends not only on the volume fraction of the particles, but also on the shape of the particles. Hamilton \& Crosser (1962) modified the Maxwell model to determine the effective thermal conductivity of nonspherical particles by applying a shape factor for the effective thermal conductivity of two-component mixtures. The Hamilton-Crosser model considers the nanoparticle aggregation. For spherical particles, the Hamilton-Crosser model reduces to the Maxwell model. In the Bruggeman model, the mean field approach is used to analyze the interactions among the randomly distributed particles (Bruggeman, 1935). The model by Xuan \& Li (2000) is not specified for any particular shape of particles. However, the classical 
models were found to be unable to predict the anomalously high thermal conductivity of nanofluids. This might be because these models do not include the effects of particle size, interfacial layer at the particle/liquid interface, and the Brownian motion of particles (Jang \& Choi, 2004; Keblinski et al., 2002; Wang et al., 1999; Yu \& Choi, 2003). Recently, Yu \& Choi (2003) proposed a modified Maxwell model to account for the effect of the nano-layer by replacing the thermal conductivity of solid particles with the modified thermal conductivity of particles, which is based on the so called effective medium theory (Schwartz et al., 1995). The model can predict the presence of thin nano-layers less than $10 \mathrm{~nm}$ in thickness. Yu \& Choi (2004) proposed a modified Hamilton-Crosser model to include the particle-liquid interfacial layer for nonspherical particles. The model can predict the thermal conductivity of the carbon nanotube-in-oil nanofluids reasonably well. However, it fails to predict the nonlinear behavior of the effective thermal conductivity of general oxide and metal based nanofluids. Xue (2003) presented a model for the effective thermal conductivity of nanofluids considering the effect of the interface between the solid particles and the base fluid based on the Maxwell model and the average polarization theory. Xue (2003) demonstrated that the model predictions were in a good agreement with the experiments of the nanotube oil-based nanofluids at high thermal conductivity and nonlinearity. However, Yu \& Choi (2004) found that the predicted values from the model by Xue are inaccurate by using two incorrect parameters, as same as the finding of Kim et al. (2004). Xue \& Xu (2005) obtained an equation for the effective thermal conductivity based on the Bruggeman model (Bruggeman, 1935). The equation takes account of the effect of interfacial shells by replacing the thermal conductivity of nanoparticles with the assumed value of the "complex nanoparticles", which introduces interfacial shells between the nanoparticles and the base fluids. The model can explain the size dependence of the thermal conductivity of nanofluids (Xuan \& Li, 2000). Xie et al. (2001) considered the interfacial nano-layer with the linear thermal conductivity distribution and proposed an effective thermal conductivity model to account for the effects of nano-layer thickness, nanoparticles size, volume fraction, and thermal conductivities of fluids, and nanoparticles. They claimed that the calculated values could agree well with some available experimental data.

Temperature is one of the important factors influencing the thermal conductivity of nanofluids (Das et al., 2003; Li \& Peterson, 2006; Yang \& Han, 2006). Xuan et al. (2003) considered the Brownian motion of suspended nanoparticles on the basis of the Maxwell model. The prediction from the model is in an agreement with the experiment results, especially when the effect of nanoparticle aggregation is taken into account. But the model may be not accurate for the second term in the equation. Wang et al. (2003) proposed a fractal model for predicting the thermal conductivity of nanofluids based on the effective medium approximation and the fractal theory, developed firstly by Mandelbrot (1982). It can describe the disorder and stochastic process of clustering and polarization of nanoparticles within the mesoscale limit. A comprehensive model considering a large enhancement of thermal conductivity in nanofluids and its strong temperature dependence was deduced from the Stokes-Einstein formula by Kumar et al. (2004). The thermal conductivity enhancement takes into account of the Brownian motion of the particles. However, the validity of the model in the molecular size regime has to be explored and it may not be suitable for a large concentration of the particles where interactions of particles become important. Bhattacharya et al. (2004) developed a technique to compute the effective thermal conductivity of a nanofluid using the Brownian motion simulation. They combined the liquid conductivity and particle conductivity. The model showed a good agreement of the thermal conductivity of nanofluids. Jang \& Choi (2004) combined four modes of energy transport in the nanofluids, collision between base fluid molecules, thermal diffusion of nanoparticles in fluids, collision between nanoparticles 
due to the Brownian motion, and thermal interaction of dynamic nanoparticles with the base fluid molecules in their model, which considered the effects of concentration, temperature, and particle size. The predictions from this model agree with the experimental data of Lee et al. (1999) and Eastman et al. (2001). However, it may not be suitable in the high temperature since the Brownian motion effect was neglected. Prasher et al. (2005) proposed that the convection caused by the Brownian motion of nanoparticles is primarily responsible for the enhancement in the effective thermal conductivity of nanofluids. By introducing a general correlation for the heat transfer coefficient, they modified the Maxwell model by including the convection of the liquid near the particles due to the Brownian motion. The result showed that the model matched well with the experimental data under different fluid temperature in a certain range. A model for nanofluids, which takes account the effects of particle size, particle volume fraction and temperature dependence as well as properties of the base fluid and the particle subject to the Brownian motion, developed by Koo \& Kleinstreuer (2004).

Although many models have been proposed, no theoretical models are available for predicting the thermal conductivity of nanofluids universally up to now. More experimental data are required. Such data should include more studies of the effects of size and shape of the nanoparticles, the interfacial contact resistance between nanoparticles and base fluids, the temperature dependence, the effect of the Brownian motion, or the effect of clustering of particles.

Experimental works have been reported on the thermal conductivity of nanofluids. The main techniques are the transient hot wire (THW) method (Kestin \& Wakeham, 1978), the temperature oscillation technique (Wang et al., 1999), and the steady-state parallel-plate method (Das et al., 2003). Among them, the TWH method has been used most extensively. Since most nanofluids are electrically conductive, a modified hot-wire cell with an electrical system was proposed by Nagasaka \& Nagashima (1981). The advantage of the method is its almost complete elimination of the effect of natural convection. The measuring principle of the THW technique is based on the calculation of the transient temperature field around a thin hot wire as a line source. A constant current is supplied to the wire to raise its temperature. The heat dissipated in the wire increases the temperature of the wire as well as that of the nanofluids. This temperature rise depends on the thermal conductivity of the nanofluids in which the hot wire is at the center. Therefore, the thermal conductivity value of the fluid can be determined. The oscillation method was proposed by Roetzel et al. (1990) and further developed by Czarnetzki \& Roetzel (1995). In principle, the thermal diffusivity of a fluid can be measured very accurately by considering amplitude attenuating of thermal oscillation from the boundary to the center of the fluid. However, for direct measurement of thermal conductivity one has to consider the influence of the reference materials as well. Since the defects of the reference materials might bring out the uncertainty in the thermal conductivity measurement, a direct evaluation of the thermal conductivity of the fluid is less accurate. The apparatus for the steady-state parallel-plate method can be constructed on the basis of the design by Challoner \& Powell (1956). The steady-state parallel-plate method needs to measure the temperature increase accurately in each thermocouple (Das et al., 2003). The difference in temperature readings needs to be minimized when the thermocouples are at the same temperature. In this method, it has to follow the assumption that there is no heat loss from the fluid to the surrounding. As a result, guard heaters would be applied to maintain a constant temperature in the fluid. However, it is challenging to control the conditions in which no heat radiated to the surrounding from the fluid. Thus, the TWH method was selected for this study. 


\subsection{Viscosity of nanofluids}

Viscosity of nanofluids is an important parameter in the fluid transporting. However, the data collected showed that no theoretical models (Batchelor, 1977; Brinkman, 1952; Einstein, 1906; Frankel \& Acrivos, 1967; Graham, 1981; Lundgren, 1967) succeed in predicting the viscosity of nanofluids accurately until now. A few theoretical models were used to estimate particle suspension viscosities. Almost all the formulae were derived from the pioneering work of Einstein (1906), which is based on the assumption of a linearly viscous fluid containing the dilute, suspended, and spherical particles. The Einstein formula is found to be valid for relatively low particle volume fractions less than 0.01 . Beyond this value, it underestimates the effective viscosity of the mixture. Later, many works have been devoted to the "correction" of his formula. Brinkman (1952) has extended the Einstein formula for use with moderate particle concentration. Lundgren (1967) proposed an equation under the form of a Taylor series. Batchelor (1977) considered the effect of the Brownian motion of particles on the bulk stress of an approximately isotropic suspension of rigid and spherical particles. Graham (1981) generalized the work of Frankel \& Acrivos (1967), but the correlation was presented for low concentrations. Almost no model mentioned could predict the viscosity of nanofluids in a wide range of nanoparticle volume fraction so far. According to these correlations the effective viscosity depends only on the viscosity of the base fluid and the concentration of the particles, whereas the experimental studies show that the temperature, the particle diameter, and the kind of nanoparticle can also affect the effective viscosity of a nanofluid. A good understanding of the rheological properties and flow behavior of nanofluids is necessary before nanofluids can be commercialized in the heat transfer applications. These factors influencing the viscosity include concentration, size of nanoparticles, temperature of nanofluids, shear rate, etc. Thus, more thorough investigations should be carried out on the viscosity of nanofluids.

In the measurement, the rotational rheometer, the piston-type rheometer, and the capillary viscometer are the most popular tools used to measure the viscosity of nanofluids. Rotational rheomters use the method that the torque required to turn an object in a fluid is a function of the viscosity (Chandrasekar et al., 2010). The relative rotation determines the shear stress under different rates. The advantage of this type of measurement is it is not affected by the flow rate of the fluids. The operation is simple and high repeatable. The piston type rheometer is based on the Couette flow inside a cylindrical chamber (Nguyen et al., 2007). It composes the magnetic coils installed inside a sensor body. These coils are used to generate a magnetically-induced force on a cylindrical piston that moves back and forth over a very small distance, imposing shear stress on the liquid. By powering the coils with a constant force alternatively, the elapsed time corresponding to a round trip of the piston can then be measured. Since the measurement of the piston motion is in two directions, variations due to gravity or flow forces are minimized. Because of the very small mass of the piston, the induced magnetic force would exceed any disturbances due to vibrations. However, the piston type viscometer is that the duration of the heating phase necessary to raise the fluid sample temperature is relative long, especially under the elevated temperature condition, some base fluids may be evaporated. The capillary viscometer is introduced in U-shaped arms (Li et al., 2007). The capillary viscometer is submerged in a glass water tank. A water tank is maintained at a prescribed constant temperature for the capillary viscometer by the water circulation. The vertical angle of the viscometer is accurately controlled with a special tripod. Li et al. (2007) pointed out that the capillary tube diameter may influence the apparent viscosity and result in inaccurate in the nanofluids at higher nanoparticle mass fractions, especially at a lower temperature. In addition, nanoparticles might stain at the inner wall of 
the bore. Because of the narrow diameter, cleaning is difficult if the nanoparticles are left. In our study, we adopted the rotational rheometer to measure the viscosity of nanofluids because of its simplicity and repeatability.

\subsection{Surface tension of nanofluids}

Interfacial properties such as surface tension play an important role for the fluids having a free surface, however, the studies of the interfacial properties of nanofluids are limited. An understanding of nanofluid properties is essential so that we can optimize the usage of nanofluids and understand their limitations. The temperature dependence of surface tension of the liquid is crucial in the bubble or droplet formation. Wasan \& Nikolov (2003) studied the spreading of nanofluids on solid surfaces and found that the existence of nanoparticles near the liquid/solid contact line can improve its spreading. Vafaei et al. (2009) investigated the effects of size and concentration of nanoparticles on the effective gas-liquid surface tension of the aqueous solutions of the bismuth telluride nanoparticles. Kumar \& Milanova (2009) found that the single-walled carbon nanotube suspensions in a boiling environment can extend the saturated boiling regime and postpone catastrophic failure of the materials even further than that previously reported if the surface tension of the nanofluids is carefully controlled. The surface tension of a liquid strongly depends on the presence of contaminants or dispersion agents such as surfactants.

Pendant droplet analysis is a convenient way to measure surface tension of fluids. It is assumed that the droplet is symmetric and the drop is not in motion. The advantage of the technique is that the calibration is straightforward, only based on the optical magnification. This can lead to a high accuracy. Another advantage is that the cleanliness requirement is not high. Surface tension is determined by fitting the shape of the droplet to the Young-Laplace equation which relates surface tension to droplet shape. Pantzali et al. (2009) used the pendant droplet method to measure the surface tension of the $\mathrm{CuO}$ water-based nanofluids. The other common method to measure surface tension is the capillary method (Golubovic et al., 2009). The main component of the device is a capillary tube in which the liquids would show a significant rise with a meniscus due to the surface tension in order to balance the gravity force. The disadvantage of the capillary method is that cleaning is difficult if the nanoparticles are left in the small diameter capillary. Thus, the pendant drop technique was selected in this study.

In sum, the reported thermal property measurement are scattered, and lack of agreement with the models. It might be due to various factors such as the measuring technique, particle size, base fluid, volume fraction of nanoparticles in fluids, temperature, etc. The lack of reliable experimental data is one of the main reasons for no universal theoretical or empirical models. Therefore, we investigated the thermal properties of the $\mathrm{Al}_{2} \mathrm{O}_{3}$ water-based nanofluids. The thermal conductivity, viscosity, and surface tension were measured. The effects of particle volume fraction, temperature and particle size were discussed at the end of experiments.

\section{Experimental procedure}

\subsection{Preparation of $\mathrm{Al}_{2} \mathrm{O}_{3}$ nanofluids}

As discussed by Kwek et al. (2010), different sizes of the $\mathrm{Al}_{2} \mathrm{O}_{3}$ nanoparticles and the surfactant, Cetyltrimethylammonium Bromide (CTAB), were purchased from Sigma Nanoamor and Aldrich respectively. During the experiments, we dispersed the $\mathrm{Al}_{2} \mathrm{O}_{3}$ nanoparticles with an average diameter of $25 \mathrm{~nm}$ and particle density of $3.7 \mathrm{~g} / \mathrm{cm}^{3}$ into 100 
$\mathrm{ml}$ of the de-ionized water to prepare the different volume concentrations $(1 \%, 2 \%, 3 \%, 4 \%$, and $5 \%$ ). Oxide-particle volume concentrations are normally below $5 \%$ in order to maintain moderate viscosity increases. To investigate the particle size effect on the thermal conductivity and viscosity, additional four sets of nanofluids each with a constant volume concentration of $5 \%$ but with different particle sizes $(10 \mathrm{~nm}, 35 \mathrm{~nm}, 80 \mathrm{~nm}$ and $150 \mathrm{~nm})$ were prepared. Sample preparation is carried out by using a sensitive mass balance with an accuracy of $0.1 \mathrm{mg}$. The volume fraction of the powder is calculated from the weight of dry powder using the density provided by the supplier and the total volume of the suspension.

$$
\operatorname{vol} \%=\frac{m / \rho}{100 m l \text { water }+m / \rho}
$$

where $m$ and $\rho$ are the mass and density of the $\mathrm{Al}_{2} \mathrm{O}_{3}$ nanoparticles respectively.

The surfactant, CTAB with the density is $1.3115 \mathrm{~g} / \mathrm{cm}^{3}$ at volume percentage of around 0.01-0.02 can stabilize the nanofluids (Sakamoto et al., 2002). The amount of $0.01 \mathrm{vol} \%$ CTAB was added into the $\mathrm{Al}_{2} \mathrm{O}_{3}$ water-based nanofluids to keep the nanoparticles well dispersed in the base fluid, water.

The nanofluid was then stirred by a magnetic stirrer for 8 hours before undergoing ultrasonicfication process (Fisher Scientific Model 500) for one and a half hours. This is to ensure uniform dispersion of nanoparticles and also to prevent the nanoparticles from the aggregation in the nanofluids.

\subsection{Thermal conductivity measurement of $\mathrm{Al}_{2} \mathrm{O}_{3}$ nanofluids}

In the study, we adopted the THW technique for measuring thermal conductivity, as shown in Fig. 1. The setup consists of a direct current (DC) power supply, a Wheatstone bridge

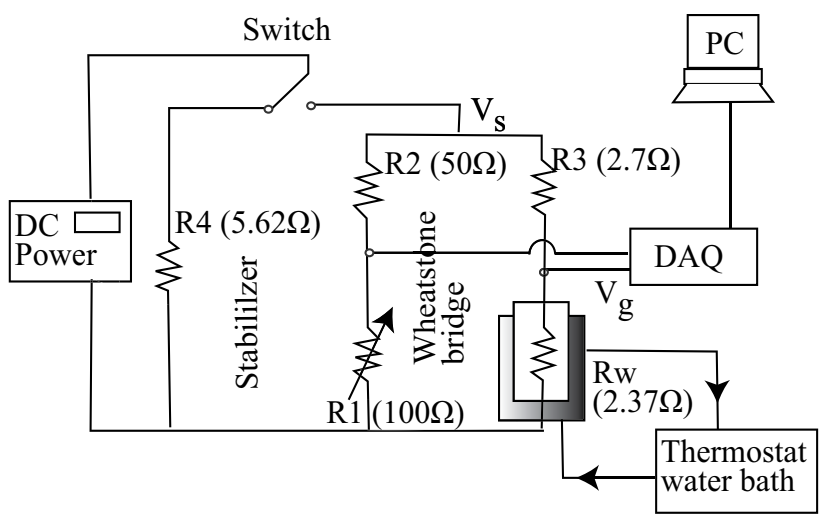

Fig. 1. Schematics of the THW setup (Kwek et al., 2010).

circuit, and a thin platinum wire surrounded by a circular nanofluid container, which is maintained by a thermostat bath. The DC power supply provides a constant voltage source to the Wheatstone bridge circuit at a constant rate to allow a uniform increment of temperature with respect to time. As the resistors used in this experiment have low values of resistance, $\mathrm{V}_{S}$ is adjusted to a value of between 0 to $2.5 \mathrm{~V}$. A data acquisition unit (Yokogawa Electric Corporation, DaqMaster MW100) is applied to capture the readings, recorded in a computer. The voltage supplied by the stabilizer, the voltage supplied in the Wheatstone circuit, the 
voltage for the platinum wire and the voltage across bridge $\left(\mathrm{V}_{g}\right)$ can be monitored during the experiments. The main experimental cell is a part of the Wheatstone bridge circuit since the wire is used as one arm of the bridge circuit. Teflon spray is used for coating a platinum (Pt) wire to act as an electric insulation because the $\mathrm{Al}_{2} \mathrm{O}_{3}$ nanofluids are electrically conductive. The $\mathrm{Pt}$ wire has good resistance as a function of temperature over a wide temperature range. The resistance-temperature coefficient of the $\mathrm{Pt}$ wire is $0.0039092{ }^{\circ} \mathrm{C}$ (Bentley, 1984). The Pt wire of $100 \mu \mathrm{m}$ in diameter and $180 \mathrm{~mm}$ in length was used in the hot-wire cell whose electric resistance was measured. The dimensions of the nanofluid container are chosen to be sufficiently large to be considered as infinite in comparison with the diameter of the Pt wire. The volume and diameter of the nanofluid container are $100 \mathrm{ml}$ and $30 \mathrm{~mm}$ respectively.

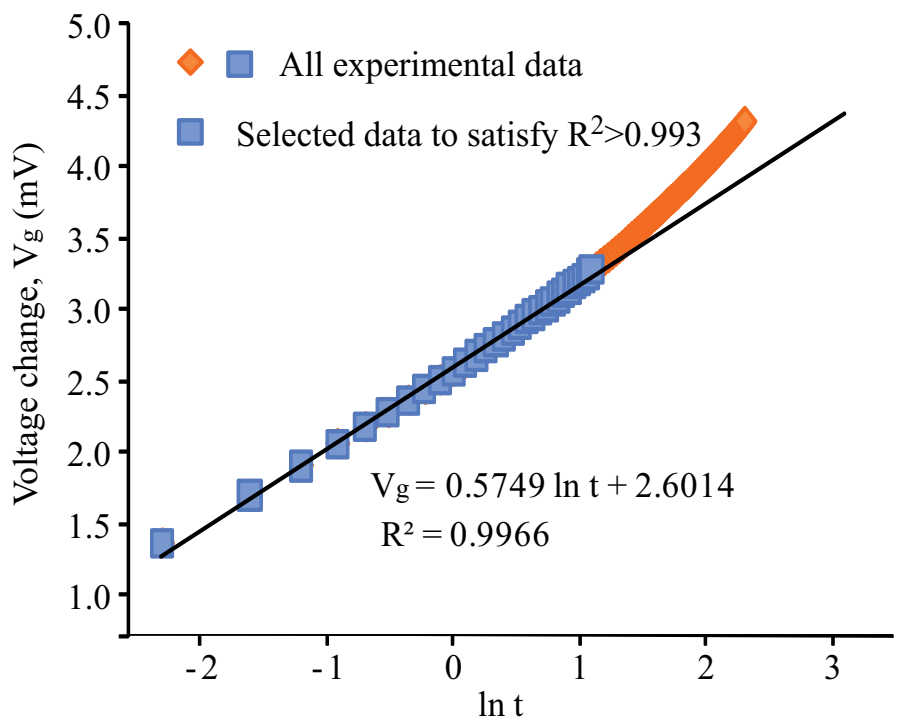

Fig. 2. $V_{g}$ as a function of $(\ln t)$ with the linear fitting curve.

To investigate the effect of temperature from 15 to $55{ }^{\circ} \mathrm{C}$ on the thermal conductivities of the nanofluids, the nanofluid container was enclosed with an acrylic container connected to a thermostat bath. Different temperatures of nanofluids can be reached during the measurement process. The nanofluid temperature was monitored with a thermocouple. In the measurement of the thermal conductivity of the $\mathrm{Al}_{2} \mathrm{O}_{3}$ nanofluids, the cylindrical shaped nanofluid container was filled with $100 \mathrm{ml}$ of the $\mathrm{Al}_{2} \mathrm{O}_{3}$ water-based nanofluid. The required temperature was set at the thermostat to maintain a uniform temperature in the nanofluid. Then the DC power source was switched on with the input voltage $\left(\mathrm{V}_{S}\right)$ being adjusted to 0.5 $\mathrm{V}$ while the switch in the circuit remained on the stabilizer resistor (R4 in Fig. 1) circuit. Thereafter, the switch was turned to the Wheatstone bridge circuit and $V_{g}$ (Fig. 1) was balanced by adjusting manually the variable resistor in circuit. Once there was no voltage change, the circuit was considered as being balanced. Again, it was switched back to the stabilizer resistor circuit and input voltage $\mathrm{V}_{s}$ was then set to the desired value of $2.0 \mathrm{~V}$ before the switch was set back to the Wheatstone bridge circuit. The unbalanced voltage change $\left(\mathrm{V}_{g}\right)$ occurring in the hot wire was recorded for 10 seconds in the computer via a data acquisition unit. The input voltage to the circuit was also recorded for each run. This measured unbalanced voltage over the natural logarithm of time was plotted in Fig. 2 by using Equation (2) (Kwek et al., 2010). The thermal conductivity is then calculated from the 
slope and intersect.

$$
V_{g}=\frac{R_{3}}{\left(R_{3}+R_{w}\right)^{2}}\left(\beta R_{w}\right) \frac{V_{s} q}{4 \pi k}\left(\ln t+\ln \frac{4 \alpha}{a^{2} C}\right)
$$

where $\mathrm{V}_{g}$ can be obtained directly from the Wheatstone bridge circuit, $\mathrm{V}_{s}$ is the voltage supplied, $R_{w}$ is the known resistance of the $\mathrm{Pt}$ wire, $\mathrm{R}_{3}$ is the resistance along same branch of Wheatstone circuit, $q$ is the heat rate per unit length, $\alpha$ is the thermal diffusivity of the surrounding medium, $\beta$ is the resistance-temperature coefficient of the wire, $k$ is the thermal conductivity to be determined, and $C=\exp (0.5772)$.

Figure 2 shows a sample of the unbalanced voltage $\left(\mathrm{V}_{g}\right)$ as a function of the natural logarithm of time. The best fitting with $\mathrm{R}_{2}>0.993$ was applied to determine the thermal conductivity. The average thermal conductivity was then determined.

Before the experiments of nanofluids, the THW setup was calibrated with the de-ionized water, the procedure was as same as the experimental process for measuring the thermal conductivity of nanofluids. The calibration showed that the accuracy of the measured thermal conductivity values is in $\pm 2 \%$ from the documental data.

\subsection{Viscosity measurement of $\mathrm{Al}_{2} \mathrm{O}_{3}$ nanofluids}

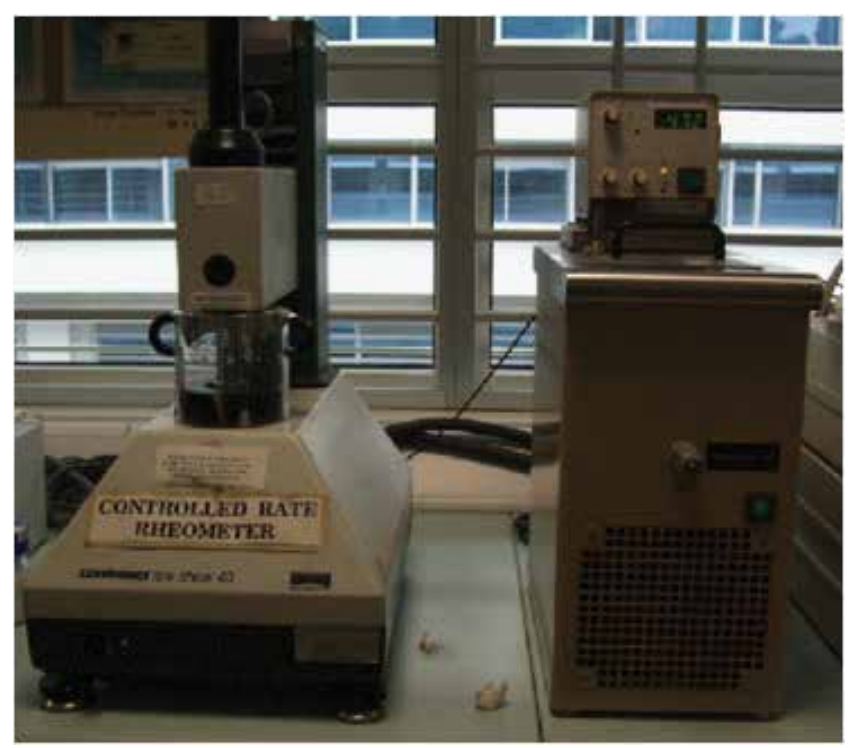

Fig. 3. The image of the controlled shear rate rheometer (Contraves LS 40).

As shown in Fig. 3, the controlled shear rate rheometer (Contraves LS 40) was applied to measure the viscosity of the $\mathrm{Al}_{2} \mathrm{O}_{3}$ nanofluids. The rheometer has a cup and bob geometry. The bob is connected to the spindle drive while the cup is mounted onto the rheometer. As the cup is rotated, the viscous drag of the fluid against the spindle is measured by the deflection of the torsion wire. The cup and bob geometry requires a sample volume of around $5 \mathrm{ml}$, hence, the temperature equilibrium can be achieved quickly within 5 minutes. The spindle type and speed combination would produce satisfactory results when the applied torque is up to $100 \%$ of the maximum permissible torque. In the measurement, the cup was placed onto the rheometer while the bob was inserted into the top shaft. The nanofluids were then transferred to the cup in preventing any bubbles forming. Afterwards, the bob was lowered down until 
it was completely inserted into the cup and immersed in the nanofluids. The lever knob was then adjusted until the bob and cup were concentric. After the measuring settings such as the minimum and maximum shear rates were set, the experiment was run. The viscosity as a function of the shear rate was plotted.

For the temperature effect, the rheological property of the nanofluids was measured by the viscometer with the thermostat, which controls temperature in Fig. 3. The viscosity measurement was started at $15{ }^{\circ} \mathrm{C}$, and temperature was gradually increased to $55^{\circ} \mathrm{C}$ at an interval of $10^{\circ} \mathrm{C}$. The nanofluid temperature was also measured by using a thermocouple. All the viscosity measurements were recorded at steady state conditions.

Before the measurement of nanofluids, the viscometer was calibrated with the de-ionized water, having an error within $\pm 1 \%$.

\subsection{Surface tension measurement of $\mathrm{Al}_{2} \mathrm{O}_{3}$ nanofluids}

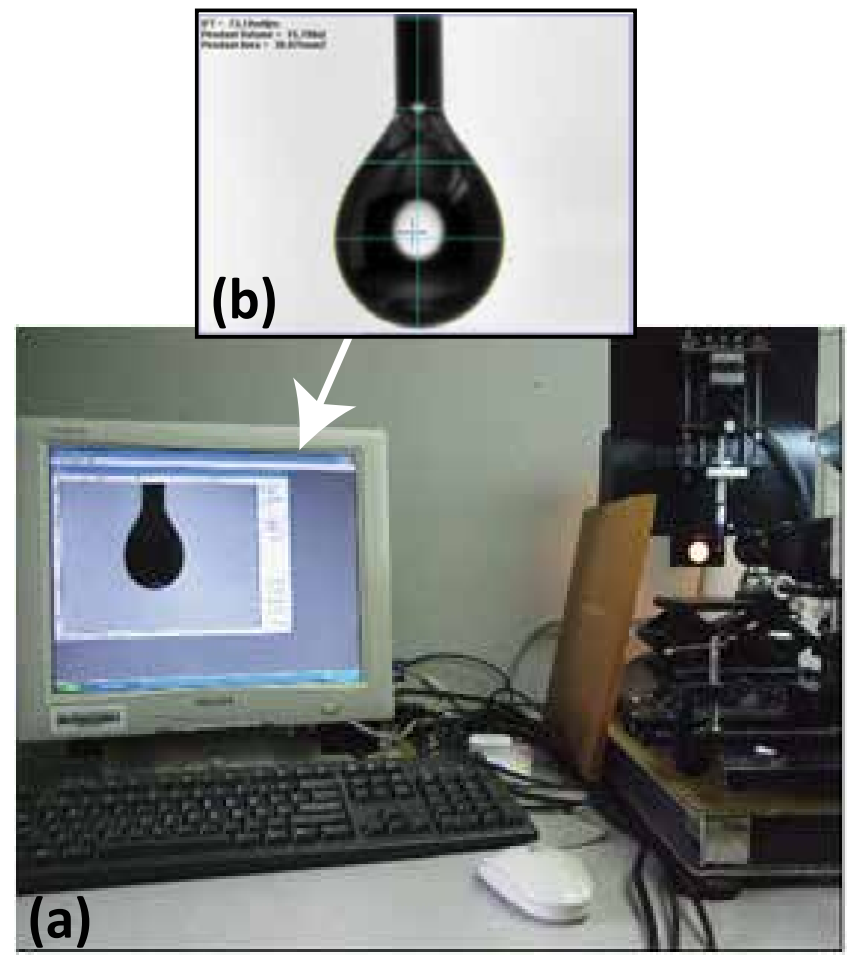

Fig. 4. Surface tension measurement for $\mathrm{Al}_{2} \mathrm{O}_{3}$ water-based nanofluids, (a) FTA 200 system; (b) a pendant droplet of the fluid for measurement.

The surface tension of the $\mathrm{Al}_{2} \mathrm{O}_{3}$ water-based nanofluids under different volume concentrations was measured with First Ten Angstroms (FTA) 200, illustrated in Fig. 4a. The precision syringe pumps (KD Scientific Inc., USA) was used to drive the $\mathrm{Al}_{2} \mathrm{O}_{3}$ water-based nanofluids to form a pendant droplet as shown in Fig. 4b. An epi-fluorescent inverted microscope with a filter set (Nikon B-2A, excitation filter for 450 - $490 \mathrm{~nm}$, dichroic mirror for $505 \mathrm{~nm}$ and emission filter for $520 \mathrm{~nm}$ ) was used to monitor the hanging droplet. A sensitive interline transfer CCD camera (HiSense MKII, Dantec Dynamics, Denmark) was employed for recording the droplet shape. 
In the experiments, the $\mathrm{Al}_{2} \mathrm{O}_{3}$ nanofluids with a certain volume concentration were filled into the syringe, which was held at the loading platform as shown in Fig. 4a. Once a pendant nanofluid droplet was formed, the image of droplet was taken. The surface tension, the droplet volume, and the surface area were then computed.

The calibration was conducted with the de-ionized water before the surface tension of nanofluid was measured. It was found that the surface tension of pure water was $72.93 \pm 1.01$ $\mathrm{mN} / \mathrm{m}$ at room temperature. The value is very close to the standard value at $71.97 \mathrm{mN} / \mathrm{m}$ (Vargaftik et al., 1983).

\section{Results and discussion}

\subsection{Thermal conductivity of $\mathrm{Al}_{2} \mathrm{O}_{3}$ nanofluids}

\subsubsection{Effect of volume concentration on thermal conductivity}

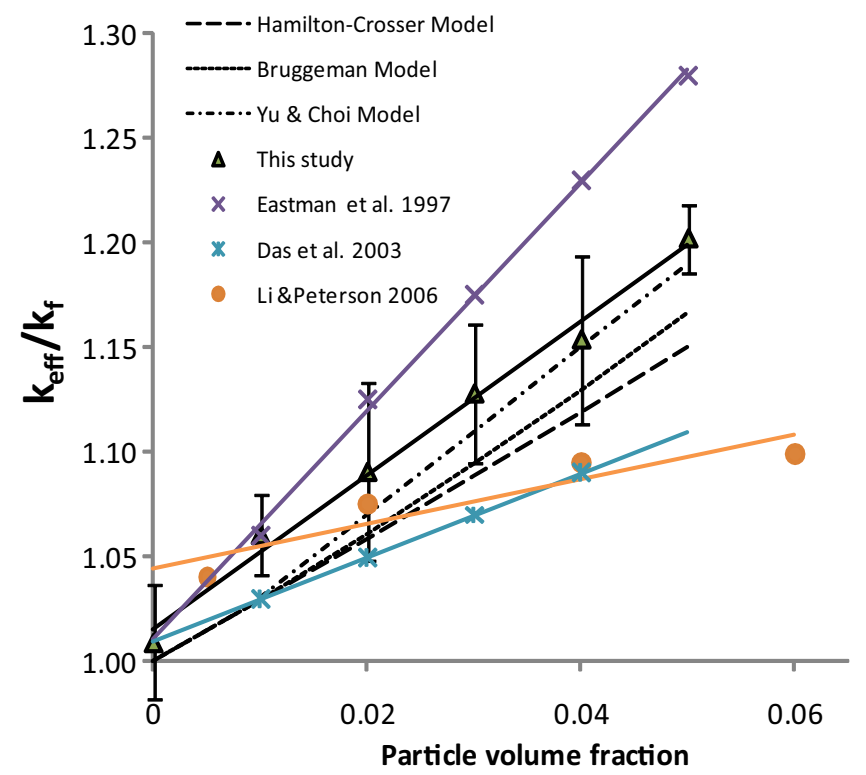

Fig. 5. Thermal conductivity enhancement as a function of volume concentrations of $\mathrm{Al}_{2} \mathrm{O}_{3}$ water-based nanofluids at $25^{\circ} \mathrm{C}$.

Each of the experimental data represents the average of six measurements at a specific concentration under room temperature. As shown in Fig. 5, the effective thermal conductivity ratio $\left(\mathrm{k}_{e f f} / \mathrm{k}_{f}\right)$ of the nanofluids is plotted as a function of nanoparticle volume fraction for a series of the $\mathrm{Al}_{2} \mathrm{O}_{3}$ nanofluids prepared from $25 \mathrm{~nm} \mathrm{Al}_{2} \mathrm{O}_{3}$ powders and measured at $25{ }^{\circ} \mathrm{C}$. $\mathrm{k}_{\text {eff }}$ is the measured thermal conductivity of the nanofluids and $\mathrm{k}_{f}$ is the thermal conductivity of pure water. Figure 5 also illustrates the data reported by Eastman et al. (1997) $(33 \mathrm{~nm})$, Das et al. (2003) $(38.4 \mathrm{~nm}), \mathrm{Li} \&$ Peterson (2006) $(36 \mathrm{~nm})$, and the prediction from the Hamilton-Crosser model (Hamilton \& Crosser, 1962), Bruggeman model (Bruggeman, 1935), and the modified model by Yu \& Choi (2003). Direct quantitative comparisons are not possible in this case as the particle size used by the other researchers differs from this experimental results $(25 \mathrm{~nm})$. It can be noted that the previous experimental results, the predicted thermal conductivity, and the measured values in the study increase with an 
increase of nanoparticle concentration in a distinct linear fashion. However, the slopes are not same. From our experimental results, it is found that a small volume percentage at $1-5 \%$ addition of the $\mathrm{Al}_{2} \mathrm{O}_{3}$ nanoparticles in the water significantly increases the effective thermal conductivity of the $\mathrm{Al}_{2} \mathrm{O}_{3}$ water-based nanofluids by 6 to $20 \%$ respectively. If we disregard the minor differences in the particles size, clear discrepancies were found between the previous experimental data and ours on the amount of enhancement in Fig. 5. This difference may be caused by the various factors such as the different particle preparation, the particle source, or even the measurement technique. Up to now, there are no standard guidelines on the preparation of nanofluids such as the amount and type of surfactant added, the time duration for ultrasonification process, the measurement method and procedures, and the size and shape of nanoparticles in use. All these might add up to account for the difference in the experimental data.

By comparing the percentage difference in the effective thermal conductivity ratio with the measured values, our data are more consistent with the predicted values of the $\mathrm{Yu} \&$ Choi correlation than those of the other correlations, especially at a high volume concentration where the percentage difference at 0.04 and 0.05 volume fraction is around $0.4 \%$ and 1 $\%$ respectively. Thus, the conventional models underestimate the thermal conductivity enhancement when compared against the measured values. The reason may be that the present proposed models did not take into account the additional mechanisms such as the interfacial layer, the Brownian motion, the size and the shape of nanoparticles, and the nanoparticle aggregation. At this stage, most of these aforementioned mechanisms are neither well established nor well understood. Therefore, more experimental works are required before the concrete conclusions can be inferred from the thermal behavior of nanofluids.

\subsubsection{Effect of temperature on thermal conductivity}

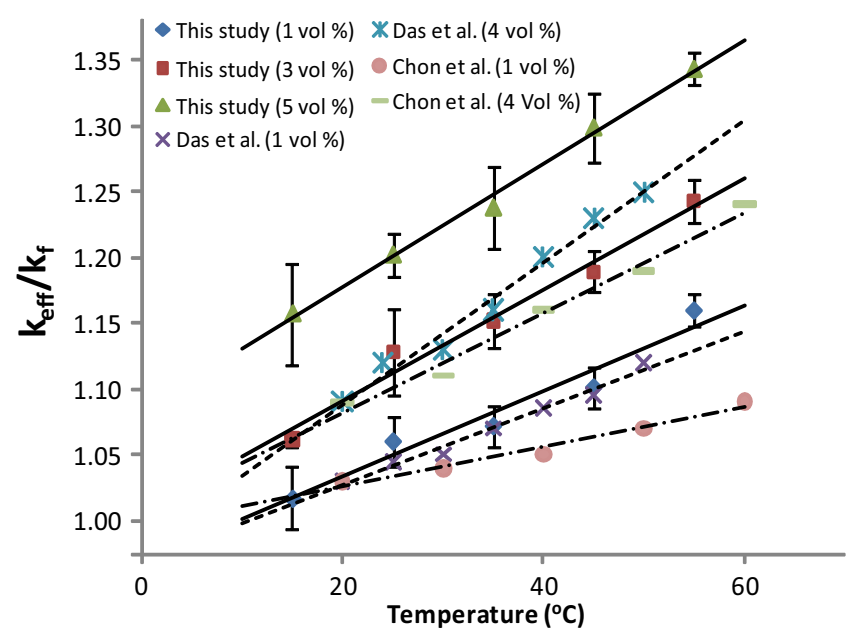

Fig. 6. Temperature dependence of thermal conductivity enhancement for the $\mathrm{Al}_{2} \mathrm{O}_{3}$ water-based nanofluids.

The effective thermal conductivity ratio $\left(\mathrm{k}_{e f f} / \mathrm{k}_{f}\right)$ is expressed with a reference of the measured value of water at the related temperatures. The measurement was made for the $\mathrm{Al}_{2} \mathrm{O}_{3}$ water-based nanofluids with the given particle concentrations at different temperatures. Figure 6 shows the enhancement of thermal conductivity of $\mathrm{Al}_{2} \mathrm{O}_{3}$ nanofluids with temperature. There is a considerable increase in the enhancement from 15 to $55^{\circ} \mathrm{C}$ in 
the nanofluids of $1 \mathrm{vol} \%, 3 \mathrm{vol} \%$, and $5 \mathrm{vol} \%$. With $1 \mathrm{vol} \%$ particles at about $15{ }^{\circ} \mathrm{C}$, the enhancement is only about $1.7 \%$, but about $16 \%$ at $55{ }^{\circ} \mathrm{C}$. The present measurement shows that a higher enhancement can be achieved in the nanofluid having small volume ratio of nanoparticles in the fluids at a higher temperature. The measurement of $3 \mathrm{vol} \%$ and 5 vol \% nanofluids shown in Fig. 6 demonstrates the enhancement goes from $6 \%$ to $24 \%$ and $15 \%$ to $34 \%$ respectively as a function of temperature from 15 to $55{ }^{\circ} \mathrm{C}$. The average rate of enhancement in these cases is higher compared with that of $1 \mathrm{vol} \%$ nanofluids. The increasing slope of the fitted line of the $1 \mathrm{vol} \%, 3 \mathrm{vol} \%$, or $5 \mathrm{vol} \%$ nanofluids has a gradient of $0.003575,0.0045$ or 0.00475 respectively. Thus it can be said that the enhancement of thermal conductivity with increases of temperature depends on the concentration of nanoparticles. The above trends are also explained by the experimental results of Das et al. (2003) (38.4 nm) and Chon et al. (2005) $(47 \mathrm{~nm})$ in Fig. 6. From the data of Das et al., the increasing rates are 0.002 and 0.005 for $1 \mathrm{vol} \%$ and $4 \mathrm{vol} \%$, whereas the results of Chon et al. show the increasing rates of 0.001 and 0.003 for the nanofluids at $1 \mathrm{vol} \%$ and $4 \mathrm{vol} \%$. The increasing trends observed are quite similar.

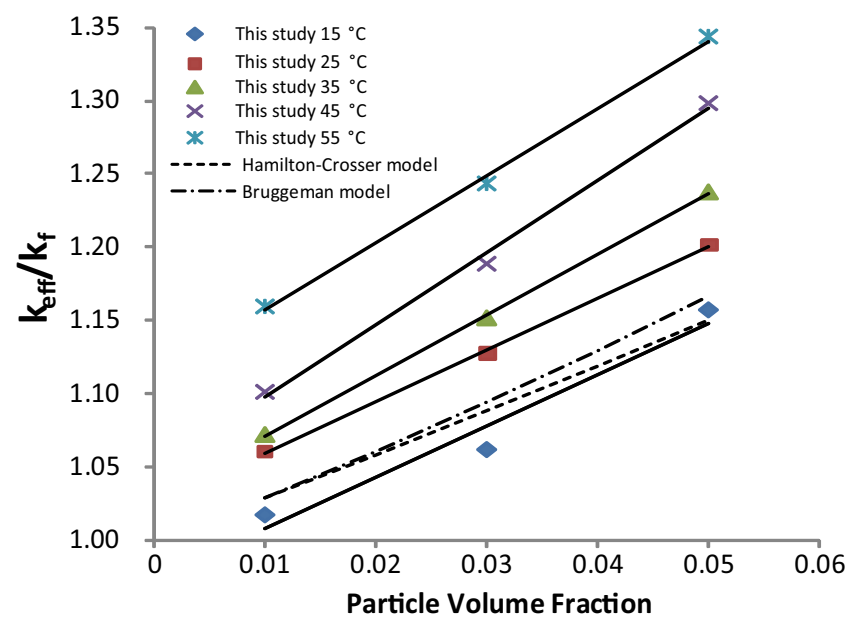

Fig. 7. Enhancement of thermal conductivity of the $\mathrm{Al}_{2} \mathrm{O}_{3}$ water-based nanofluids against particles concentration and comparison with models.

Figure 7 shows that there is a close agreement between the measured thermal conductivity and the Hamilton-Crosser and the Bruggeman models at $15{ }^{\circ} \mathrm{C}$. However, this agreement is only at the low temperature. At higher temperature, the experiments of the $\mathrm{Al}_{2} \mathrm{O}_{3}$ water-based nanofluids disagree with the models. It is suggested that the present models cannot reflect on the effective conductivity with temperature. Das et al. (2003) stated that the main mechanism of the thermal conductivity enhancement in nanofluids can be thought as the stochastic motion of nanoparticles, and that the Brownian motion would depend on the fluid temperature. This enhancement in our experiments can be supported by the results of Das et al. (2003) and Chon et al. (2005). Their data have the maximum enhancements of $25 \%$ and $19 \%$ for $4 \mathrm{vol} \%$ at $55{ }^{\circ} \mathrm{C}$ whereas the Hamilton-Crosser model (Hamilton \& Crosser, 1962) and the Bruggeman model (Bruggeman, 1935) predict only $12 \%$ and $13 \%$, regardless of the temperature effect. At the low temperature, the Brownian motion was less significant. Thus the present results indicate that it is possible to have a threshold temperature at which the effective thermal conductivity of nanofluids starts deviating from that of the usual suspension and the enhancement through the stochastic motion of the particles starts 
dominating. The measurement of the thermal conductivity with the given concentrations at the different temperatures in Fig. 7 indicates the necessity for a better theoretical model for the entire range of temperature.

\subsubsection{Effects of particle size on thermal conductivity}

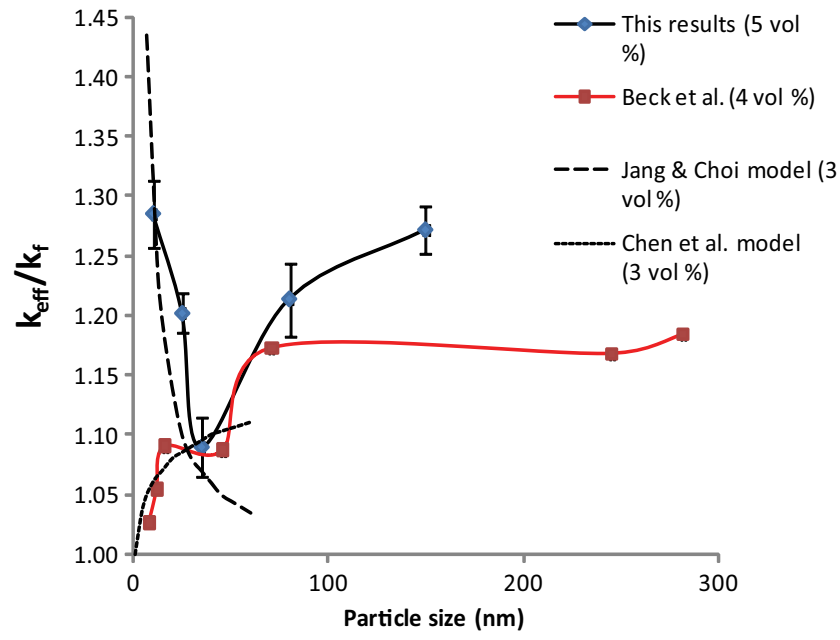

Fig. 8. Effect of diameter of nanoparticle on effective thermal conductivity of the $\mathrm{Al}_{2} \mathrm{O}_{3}$ water-based nanofluids.

As shown in Fig. 8, the experimental data in the study are compared with the predictions from the thermal conductivity model by Jang \& Choi (2004), and a good agreement was found for $10 \mathrm{~nm}, 25 \mathrm{~nm}$ and $35 \mathrm{~nm} \mathrm{Al}{ }_{2} \mathrm{O}_{3}$ water-based nanofluids. Our experimental data indicate that the effective thermal conductivity decreases quickly with the decreasing size of nanoparticles from $10 \mathrm{~nm}$ to $35 \mathrm{~nm}$, however as the nanoparticle size increases, the thermal conductivity deviates from the Jang \& Choi model. As the nanoparticle diameter is reduced, the effective thermal conductivity of nanofluids becomes larger. Jang \& Choi (2004) explained that this phenomenon is based on the Brownian motion, and that the smaller nanoparticles in average might produce a higher velocity of the Brownian motion in the fluid. As a result, the heat transfer by the convection would be enhanced, the effective thermal conductivity of nanofluids increases. However, if the particles approach to the micrometer size, they might not remain well suspended in the base fluid. Thus, large microparticles do not have the Brownian motion any more, and there would be no enhancement of the effective thermal conductivity. Our experimental results for the nanofluids with the $80 \mathrm{~nm}$ and $150 \mathrm{~nm} \mathrm{Al}_{2} \mathrm{O}_{3}$ nanoparticles did not show a similar trend as described in the model of Jang \& Choi (2004). Instead our experimental data shows that the thermal conductivity of the $\mathrm{Al}_{2} \mathrm{O}_{3}$ nanofluids increases as the particle size increases above $35 \mathrm{~nm}$, similar to the data of Beck et al. (2009) above $50 \mathrm{~nm}$. When the particles become larger, it can be better explained by the model of Chen (1996),

$$
k_{p}=k_{b u l k} \frac{0.75 d_{p} / l_{p}}{0.75 d_{p} / l_{p}+1}
$$

where $\mathrm{k}_{p}, \mathrm{~d}_{p}, \mathrm{l}_{p}$ and $\mathrm{k}_{b u l k}$ are the thermal conductivity of nanoparticle, the characteristic length of nanoparticles, the mean free path of nanoparticle, and the thermal conductivity of bulk materials respectively. The correlation of Chen (1996) is built on solving the Boltzmann 
transport equation. The solution approaches the prediction of the Fourier law when the particle radius is much larger than the heat-carrier mean free path of the host medium, which implies that the diffusive heat transport is dominant. The model shows a trend of the thermal conductivity enhancement as the particle size increases. In sum, there may be a threshold in particle size where either the Brownian motion or the diffusive heat transport is more dominant.

\subsection{Viscosity of $\mathrm{Al}_{2} \mathrm{O}_{3}$ nanofluids}

\subsubsection{Effect of volume concentrations on viscosity}

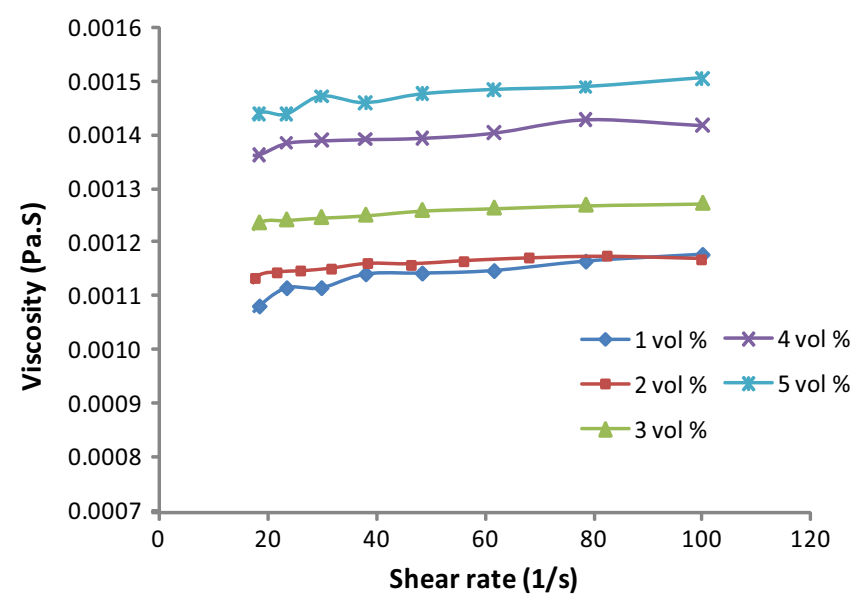

Fig. 9. Viscosity as a function of shear rate for the $\mathrm{Al}_{2} \mathrm{O}_{3}$ nanofluids at different volume concentrations.

The viscosity is illustrated in Fig. 9 as a function of the shear rate. The viscosity of the well-mixed $\mathrm{Al}_{2} \mathrm{O}_{3}$-water nanofluid is independent from the shear rate. The naofluids exhibit a Newtonian behavior. Figure 10 shows that the effective viscosity ratio increases as the volume concentrations increase. The results of Masoumi et al. (2009) (28 nm) and Nguyen et al. (2007)

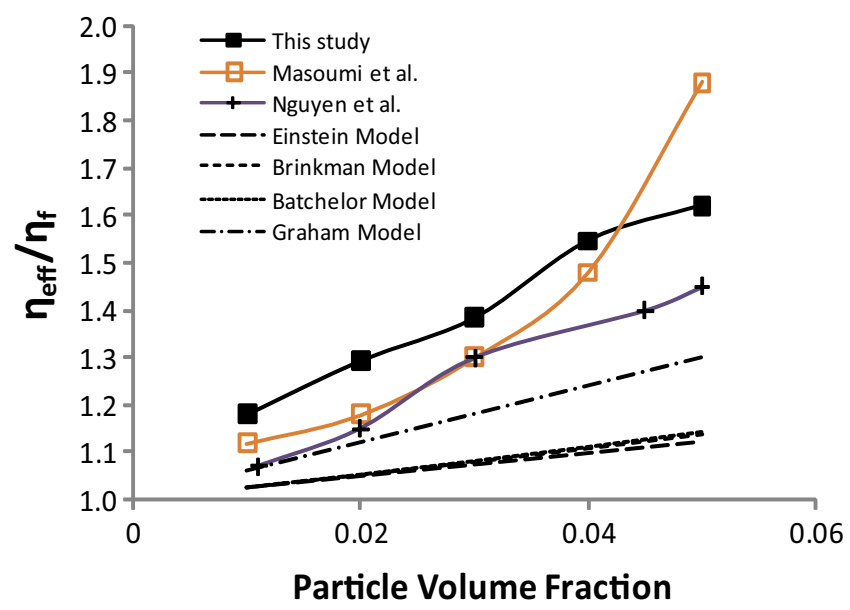

Fig. 10. Relative viscosity of the $\mathrm{Al}_{2} \mathrm{O}_{3}$ nanofluids as a function of volume concentration. 
$(36 \mathrm{~nm})$ show a similar trend. From our experiments, the measured viscosity of the $\mathrm{Al}_{2} \mathrm{O}_{3}$ nanofluids is significantly higher than the base fluid by about $20 \%$ and $61 \%$ at 1 vol $\%$ and 5 vol \% respectively. The results of Masoumi, Nguyen and ours are much higher than those of predicted values using the Einstein, Brinkman, Batchelor and Graham equations, as shown in Fig. 10. It is suggested that these equations have underestimated the nanofluid viscosities. The Einstein formula, and the others originating from it, were obtained based on the theoretical assumption of a linear fluid surrounded by the isolated particles. Such a model may worked under the situation of a liquid that contains a small number of dispersed particles. However, for higher particle concentrations the departure of these formulae from our experimental data is considerable, indicating that the linear fluid theory may be no longer appropriate to represent the nanofluids. Even the Batchelor formula, considering the Brownian effect, also performs poorly. A possible explanation is mentioned by Chandrasekar et al. (2010), the large difference may be a result of the hydrodynamic interactions between particles which become important at higher volume concentrations. Hence the conventional models cannot explain the high viscosity ratio. Noted that there are also discrepancies between our experimental results and the previous studies in Fig. 10. Although the nanofluids prepared have slight differences in the size of particles, it is inappropriate to account for such a large difference in the viscosity ratio. It is difficult to draw any conclusive remarks for such results, unless this intriguing behavior may be attributed to the various factors such as nanofluid preparation methods and how the experiment is conducted.

\subsubsection{Effect of temperature on viscosity}

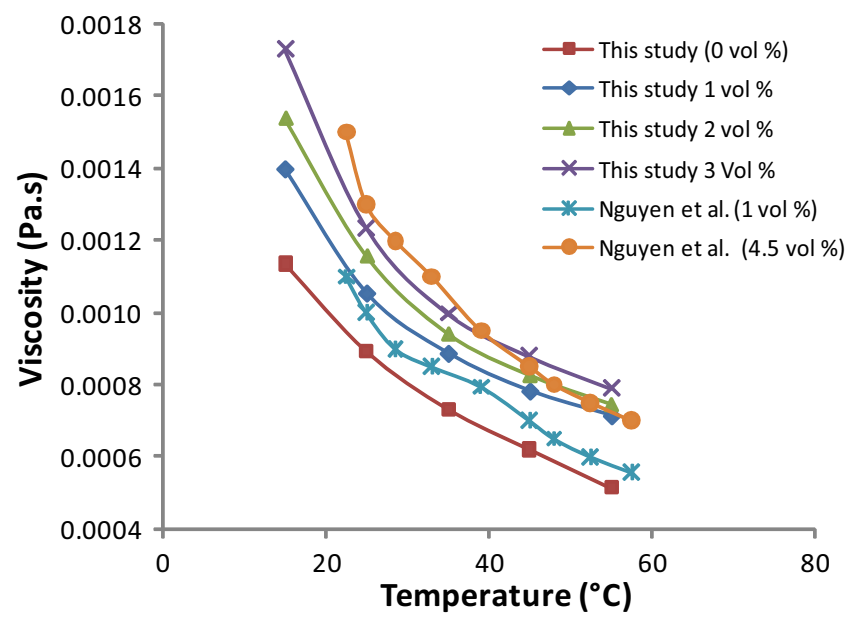

Fig. 11. Viscosity as a function of temperature for $\mathrm{Al}_{2} \mathrm{O}_{3}$ nanofluids.

The viscosities were measured for the nanofluids as a function of temperature. The viscosity under the particle volume fraction ranging at $1 \%, 2 \%$, and $3 \%$ from 15 to $55{ }^{\circ} \mathrm{C}$ is shown in Fig. 11, the nanofluid viscosity decreases with an increase in temperature. The increasing temperature would weaken the inter-particle and inter-molecular adhesion forces. For all the nanofluids measured, the temperature gradient of viscosity is generally steeper at the temperature from 15 to $30^{\circ} \mathrm{C}$. Such the viscosity gradient is particularly more pronounced as the particle volume concentration increases. This observation is supported by Nguyen et al. (2007) results if we compare the gradient from 15 to $30{ }^{\circ} \mathrm{C}$ at $1 \mathrm{vol} \%$ and $4.5 \mathrm{vol} \%$. The results suggest that the temperature effect on the particle suspension properties may be 
different for high particle fractions and for low ones. With an increase of temperature, the measured viscosity data have shown a gentle decrease with an increase of temperature. In our experiments, we have attempted to measure viscosity at the temperature higher than $55{ }^{\circ} \mathrm{C}$, but a critical temperature has been observed, above the temperature, an 'erratic' increase of nanofluid viscosity was observed. The phenomenon may be resulted from the fast evaporation of nanofluids in the related small volume at a relative high temperature. Another possibility is that beyond the critical temperature, the surfactant might be broken down and accordingly the performance was considerably reduced or even destroyed, affecting the suspension capabilities. Thus, the particles have a tendency to form aggregation, resulting in the observed unpredictable increase of the nanofluid viscosity.

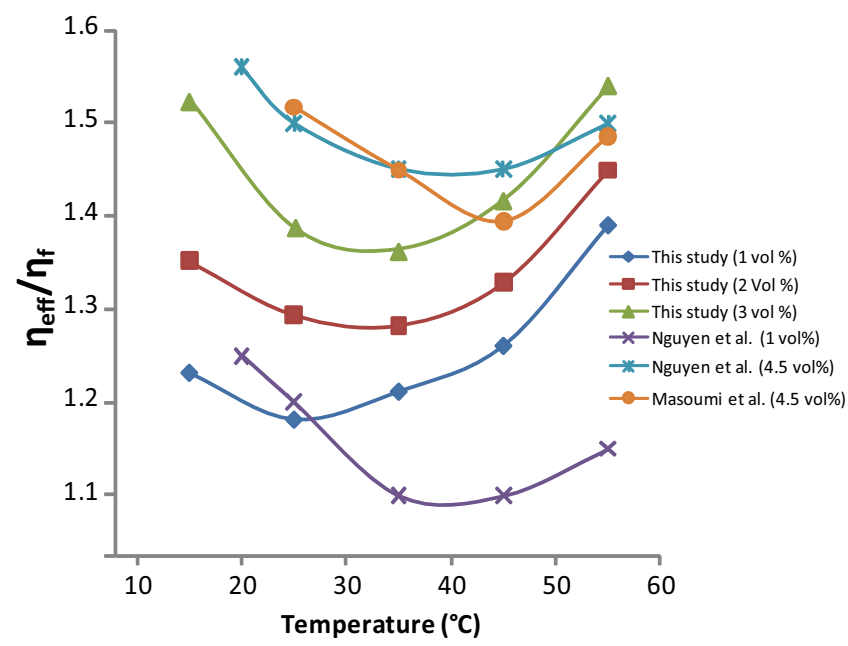

Fig. 12. Relative viscosity as a function of temperature for various concentrations of the $\mathrm{Al}_{2} \mathrm{O}_{3}$-water nanofluids.

As known, the water viscosity decreases with an increase of temperature. The viscosity values of the different concentration nanofluids measured from 15 to $55{ }^{\circ} \mathrm{C}$ are compared with a reference of the viscosity of water at these temperatures. As seen from Fig. 12, the effective viscosity under the different volume concentrations shows similar trends. For a given nanofluid and a particle fraction, the effective viscosity decreases at first and starts to increase at a certain temperature. This value implies that there should be an optimum temperature whereby when temperature increases, the decrease in viscosity is not effective. This observation can be substantiated by Nguyen et al. (2007) and Masoumi et al. (2009).

\subsubsection{Effect of particle size on viscosity}

From Fig. 13, our experimental results show that as the particle sizes increase, the effective viscosity decreases significantly and reaches an almost constant value at the end. This trend is similar to the results of other researchers shown in Fig. 13 except for particle size greater than $100 \mathrm{~nm}$. Timofeeva et al. (2007) suggested the small particle size can form larger aggregates. The Krieger model (Krieger \& Dougherty, 1959) can be used to estimate the relative viscosity between a nanofluid $(n f)$ and its base fluid $(f)$,

$$
\frac{\eta_{n f}}{\eta_{f}}=\left(1-\frac{\phi_{a}}{\phi_{m}}\right)^{-2.5 \phi_{m}}
$$




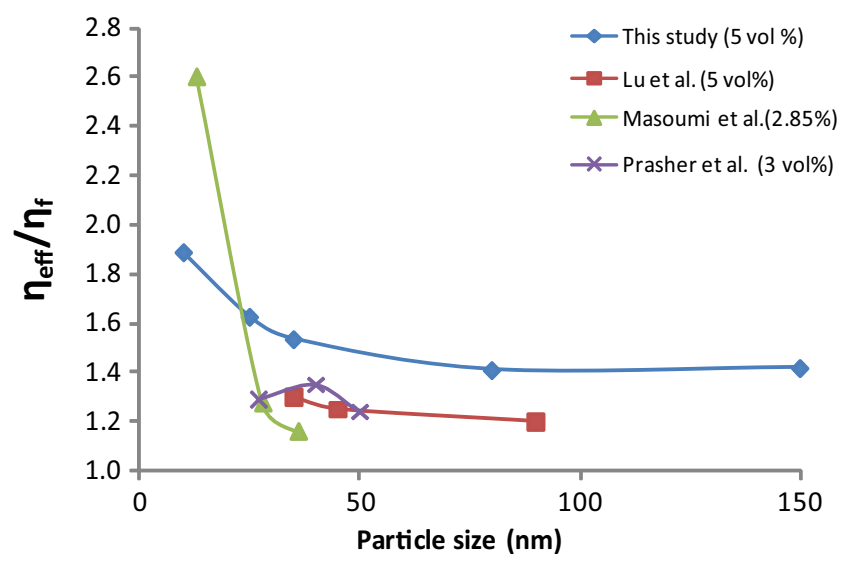

Fig. 13. Relative viscosity as a function of diameter of the $\mathrm{Al}_{2} \mathrm{O}_{3}$ nanoparticles in the base fluids.

where 2.5 is the intrinsic viscosity of spherical particles, $\phi_{a}$ is the volume fraction of aggregates, $\phi_{m}$ is the volume fraction of densely packed spheres and the volume fraction of aggregates is expressed as $\phi_{a}=\phi\left(\frac{d_{a}}{d}\right)^{3-d_{f}}$, in which $d_{a}$ is the diameter of aggregates, $d$ is the nominal diameter of particle, $d_{f}$ is the fractal dimension of the aggregates, $\phi$ is the volume fraction of the well-dispersed individual particles. For well-dispersed individual particles, $\phi_{a}$ is equal to $\phi$, and the Krieger model reduces to the Einstein model. This is a very ideal case where there is zero aggregation. However, none of the researches is able to obey fully the Einstein model until now. The reason may be that it is unlikely to eliminate the aggregation completely (Duan et al., 2011). When nanoparticle size increases, the magnitude of $\frac{d_{a}}{d}$ decreases, thus the volume fraction of the aggregates decreases and relative viscosity ratio decreases. In addition, due to aggregation, the shape of the aggregate is no longer spherical. Theoretically, Einstein obtained the intrinsic viscosity at 2.5 for spherical particles, however the intrinsic viscosity would be greater than 2.5 for the other shapes (Rubio-hernandez et al., 2006) as the aggregate shape becomes disordered. This can also account for the increase of viscosity ratio as the particle diameter decreases.

Slight aggregation is likely to remain in our nanofluids measured just after preparation since the measurements are made for different particle sizes at a constant $5 \%$ volume concentration, which is considered high. Based on Equation (4), the viscosity ratio would be higher after aggregation.

\subsection{Surface tension measurements of nanofluid}

Figure 14 shows the surface tension as a function of the volume concentration. The results demonstrate that the surface tensions of the $\mathrm{Al}_{2} \mathrm{O}_{3}$ water-based nanofluids are significantly lower than those of the base fluid, pure water. At each point, the error bars are too small to be observed. However, as the volume concentration increases, the surface tension remains almost unchanged in the $\mathrm{Al}_{2} \mathrm{O}_{3}$ nanofluids. Hence we can deduce that particle volume concentration does not have a major effect on the surface tension of the nanofluids. The experimental results of Golubovic et al. (2009) and Kim et al. (2007) have shown that the surface tensions of the $\mathrm{Al}_{2} \mathrm{O}_{3}$ nanofluids without surfactant is independent on concentration and has the same values as that of pure water. In our prepared nanofluids, the surfactant, CTAB was added to obtain 
a well-dispersed suspension. The addition of a small amount of surfactant into the liquid reduced the surface tension (Binks, 2002; Bresme \& Faraudo, 2007).

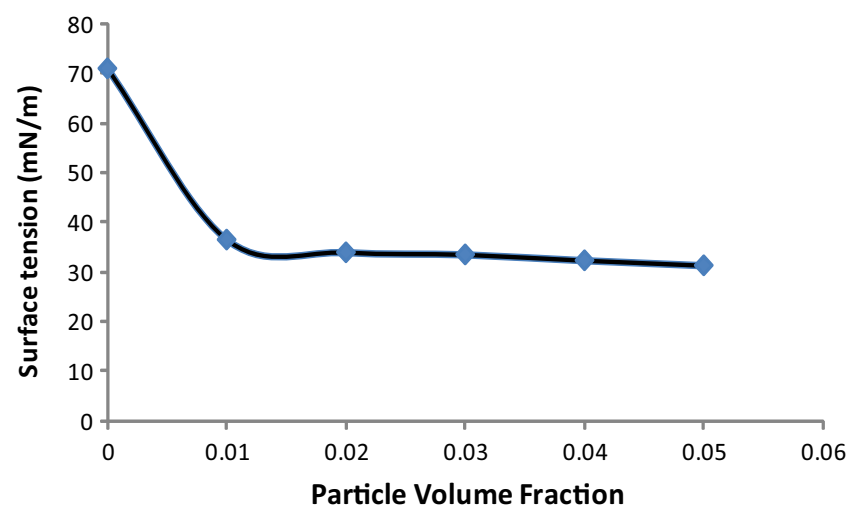

Fig. 14. Surface tension measurement of the $\mathrm{Al}_{2} \mathrm{O}_{3}$ nanofluids as a function of the volume concentration.

\section{Conclusion}

The thermal conductivity, viscosity, and surface tension of the $\mathrm{Al}_{2} \mathrm{O}_{3}$ water-based nanofluids were measured. It is found the thermal conductivity increases significantly with the nanoparticle volume fraction. With an increase of temperature, the thermal conductivity increases for a certain volume concentration of nanofluids, but the viscosity decreases. The size of nanoparticle also influences the thermal conductivity of nanofluids. It is indicated that existing classical models cannot explain the observed enhanced thermal conductivity in the nanofluids. Similarly, the viscosity increases as the concentration increases at room temperature. At the volume concentrations of $5 \%$, the viscosity has an increment of $60 \%$. The effect of particle sizes on the viscosity is limited. The addition of surfactant is believed to be the reason behind the decrease in surface tension in comparison with the base fluid. The significant deviation between the experimental results and the existing theoretical models is still unaccounted for. More comprehensive models therefore need to be developed. Particles sizes, particle dispersions, clustering, and temperature should be taken into account in the model development for nanofluids. Hence, to reach universal models for the thermal properties, more complete experiments involving a wide range of nanoparticle sizes would be conducted in future.

\section{Acknowledgments}

The research mainly depends on the experimental work of Mr. D. Kwok under the support of NTU-SUG and AcRF Tier 1 funding. The author would like to thank Profs. Kai Choong Leong and Charles Yang for their generosity in sharing their HWT and surface tension equipment. The author would also like thank to Dr. Liwen Jin for sharing his knowledge on the thermal conductivity measurement.

\section{References}

Batchelor, G.K. (1977). The effect of Brownian motion on the bulk stress in the suspension of spherical particles. J. Fluid Mech., 83, 97-117. 
Beck, M.P.; Yuan, Y.; Warrier, P. \& Teja, A.S. (2009). The effect of particle size on the thermal conductivity of alumina nanofluids. J. Nanopart. Res., 11, 1129-1136.

Bruggeman, D.A.G. (1935). Calculation of various physics constants in heterogenous substances. I. Dielectricity constants and conductivity of mixed bodies from isotropic substances. Ann. Phys. (Paris), 24, 636-664.

Bentley, J.P. (1984). Temperature sensor characteristics and measurement system design. J. Phys. E, 17, 430-439.

Bhattacharya, P.; Saha, S.K.; Yadav, A.; Phelan, P.E. \& Prasher, R.S. (2004). Brownian dynamics simulation to determine the effective thermal conductivity of nanofluids. J. Appl. Phys., 95, 6492-6494.

Binks, B. (2002). Particles as surfactants - similarities and differences. Curr. Opin. Colloid Interface Sci., 7, 21-41.

Bresme, F. \& Faraudo, J. (2007). Particles as surfactants - similarities and differences. J. Phys.: Condens. Matter, 19, 375110.

Brinkman, H.C. (1952). The viscosity of concentrated suspensions and solution. J. Chem. Phys., $20,571$.

Chandrasekar, M.; Suresh, S. \& Chandra Bose, A. (2010). Experimental investigations and theoretical determination of thermal conductivity and viscosity of Al2O3/water nanofluid. Exp. Therm. Fluid Sci., 34, 210-216.

Challoner, A.R. \& Powell. R.W. (1956). Thermal conductivity of liquids: new determinations for seven liquids and appraisal of existing values. Proc. R. Soc. Lond., 238, 90-106.

Chen, G. (1996). Nonlocal and nonequilibrium heat conduction in the vicinity of nanoparticles. ASME J. Heat Transfer, 118, 539-545.

Choi, S.U.S. (1995). Enhancing thermal conductivity of fluids with nanoparticles, In: Developments and Applications of Non-Newtonian Flows, Sinfiner, D.A. \& Wang, H.P., PP 99-105, ASME, FED-vol.231 MD-vol.66, USA.

Chon, C.H.; Kihm, K.D.; Lee, S.P. \& Choi, S.U.S. (2005). Empirical correlation finding the role of temperature and particle size for nanofluid (Al2O3) thermal conductivity enhancement. Appl. Phys. Lett., 87, 1-3.

Czarnetzki, W. \& Roetzel W. (1995). Temperature oscillation techniques for simultaneous measurement of thermal diffusivity and conductivity. Int. J. Thermophys., 16, 413-422.

Das, S.K.; Putta, N.; Thiesen, P. \& Roetzel W. (2003). Temperature dependence of thermal conductivity enhancement for nanofluids. J. Heat Transfer, 125, 567-574.

Duan, F; Kwek D \& Crivoi A. (2011). Viscosity affected by nanoparticle aggregation in Al2O3-water nanofluids. Nanoscale Res. Lett., 6, 248.

Eastman, J.A.; Choi, S.U.S.; Li, S.; Thompson, L.J. \& Lee, S. (1997). Enhanced thermal conductivity through the development of nanofluids. Mater. Res. Soc. Symp. Proc., 457, 3-11.

Eastman, J.A.; Choi, S.U.S.; Li, S.; Yu, W. \& Thompson, L.J. (2001). Anomalously increased effective thermal conductivities of ethylene glycol-based nanofluids containing copper nanoparticles. Appl. Phys. Lett., 78, 718-720.

Einstein, A. (1906). A new determination of the molecular dimensions. Ann. Phys., 19, 289-306.

Frankel, N.A. \& Acrivos, A. (1967). On the viscosity of a concentrated suspension of solid spheres. Chem. Eng. Sci., 22, 847-853.

Golubovic, M.N.; Madhawa Hettiarachchi, H.D.; Worek, W.M. \& Minkowycz, W.J. (2009). Nanofluids and critical heat flux, experimental and analytical study. Appl. Therm. Eng., 29, 1281-1288.

Graham, A.L. (1981). On the viscosity of suspension of solid spheres. Appl. Sci. Res., 37, 275-286. 
Hamilton, R.L. \& Crosser, O.K. (1962). Thermal conductivity of heterogeneous two component systems. Ind. Eng. Chem. Fundam., 1, 187-191.

Jang, S.P. \& Choi, S.U.S. (2004). Role of Brownian motion in the enhanced thermal conductivity of nanofluids. Appl. Phys. Lett., 84, 4316-4318.

Keblinski, P.; Eastman, J.A. \& Cahill, D.G. (2005). Nanofluids for thermal transport. Mater Today, 8, 36-44.

Keblinski, P.; Phillpot, S.R.; Choi, S.U.S. \& Eastman, J.A. (2002). Mechanisms of heat flow in suspensions of nano-sized particles (nanofluids). Int. J. Heat Mass Transfer, 45, 855-863.

Kestin, J. \& Wakeham W.A. (1978). A contribution to the theory of the transient hot-wire technique for thermal conductivity measurements. Physica A, 92, 102-116.

Kim, J.; Kang, Y.T. \& Choi, C.K. (2004). Analysis of convective instability and heat transfer characteristics of nanofluids. Phys. Fluids, 16, 2395-2401.

Kim, S.J.; Bang, I.C.; Buongiorno, J. \& Hu, L.W. (2007). Surface wettability change during pool boiling of nanofluids and its effect on critical heat flux. Int. J. Heat Mass Transfer, 50 (2007) 4105-4116.

Koo, J. \& Kleinstreuer, C. (2004). A newthermal conductivity model for nanofluids. J. Nanopart. Res., 6, 577-588.

Krieger, I.M. \& Dougherty, T.J. (1959). A mechanism for non-newtonian flow in suspensions of rigid spheres. Trans. Soc. Rheology, 3, 137-152.

Kumar, D.H. Patel, H.E.; Kumar, V.R.R.; Sundararajan, T.; Pradeep, T. \& Das, S.K. (2004). Model for heat conduction in nanofluids. Phys. Rev. Lett., 93, 1-4.

Kumar, R. \& Milanova, D.(2009). Effect of surface tension on nanotube nanofluids. Appl. Phys. Lett., 94, 073107.

Kwek, D.; Crivoi, A. \& Duan, F. (2010). Effects of temperature and particle size on the thermal property measurements of Al2O3-water nanofluids. J. Chem. Eng. Data, 55, 5690-5695.

Lee, S.; Choi, S.U.S.; Li, S. \& Eastman, J.A. (1999). Measuring thermal conductivity of fluids containing oxide nanoparticles. J. Heat Transfer, 121, 280-289.

Li, C.H. \& Peterson G.P.(2006). Experimental investigation of temperature and volume fraction variations on the effective thermal conductivity of nanoparticle suspensions (nanofluids). J. Appl. Phys., 99, 1-8.

Li, X.; Zhu, D. \& Wang X. (2007). Evaluation on dispersion behaviour of the aqueous copper nano-suspensions. J. Colloid Interface Sci., 310, 456-463.

Li, Y.; Zhou J.; Tung, S.; Schneider, E. \& Xi, S. (2009). A review on development of nanofluid preparation and characterization. Powder Technol., 196, 89-101.

Lundgren, T.S. (1972). Slow flow through stationary random beds and suspensions of spheres. J. Fluid Mech., 51, 273-299.

Maxwell, J.C. (1892). A Treatise on Electricity and Magnetism, 3rd ed., Oxford University Press, London.

Mandelbrot; B.B. (1982). The Fractal Geometry of Nature, W.H. Freeman Press, San Francisco.

Masoumi, N.; Sohrabi, N. \& Behzadmehr, A. (2009). A new model for calculating the effective viscosity of nanofluids. J. Phys. D: Appl. Phys., 42, 055501.

Nagasaka, Y. \& Nagashima A. (1981). Absolute measurement of the thermal conductivity of electrically conducting liquids by the transient hot-wire method. J. Phys. E, 14, $1435-1440$.

Nguyen, C.T.; Desgranges, F.; Roy, G.; Galanis, N.; Mare, T.; Boucher, S. \& Angue Mintsa, H. (2007). Temperature and particle-size dependent viscosity data for water-based nanofluids - hysteresis phenomenon. Int. J. Heat Fluid Flow, 28, 1492-1506. 
Pantzali, M.N.; Kanaris, A.G.; Antoniadis, K.D.; Mouza, A.A. \& Paras, S.V. (2009). Effect of nanofluids on the performance of a miniature plate heat exchanger with modulated surface. Int. J. Heat Fluid Flow, 30, 691-699.

Prasher, R.; Bhattacharya, P.; Phelan, P.E. \& Das, S.K. (2005). Thermal conductivity of nanoscale colloidal solutions (nanofluids). Phys. Rev. Lett., 94, 025901.

Roetzel, W.; Prinzen, S. \& Xuan Y. (1990). Measurement of thermal diffusivity using temperature oscillations, In: Measurement of conductivity 21, Cremers C.J. \& Fine, H.A., Ed., 201-207, Plenum Press, New York.

Rubio-hernandez, F.J.; Ayucar-Rubio, M.F.; Velazquez-Navarro J.F. \& Galindo-Rosales, F.J. (2006). Intrinsic viscosity of $\mathrm{SiO} 2, \mathrm{Al} 2 \mathrm{O} 3$ and $\mathrm{TiO} 2$ aqueous suspensions. J. Colloid Interface Sci., 298, 967-972.

Sakamoto, M.; Kanda, Y.; Miyahara, M. \& Higashitani, K. (2002). Origin of long-range attractive force between surfaces hydrophobized by surfactant adsorption. Langmuir, $18,5713-5719$.

Schwartz, L.; Garboczi, E. \& Bentz, D. (1995). Interfacial transport in porous media: application to dc electrical conductivity of mortars. J. Appl. Phys., 78, 5898-5908.

Timofeeva, E.V.; Gavrilov, A.N.; McCloskey, J.M. \& Tolmachev, Y.V. (2007). Thermal conductivity and particle agglomeration in alumina nanofluids: Experiment and theory. Phys. Rev. E, 76, 061203.

Vafaei, S.; Purkayastha, A.; Jain, A.; Ramanath, G. \& Borca-Tasciuc, T. (2009). The effect of nanoparticles on the liquid-gas surface tension of Bi2Te3 nanofluids. Nanotechnology, 20, 185702.

Vargaftik, N.B.; Volkov, B.N. \& Voljak, L.D. (1983). International tables of the surface tension of water. Phys. Chem. Ref. Data, 12, 817.

Wang, B.X. Zhou, L.P. \& Peng, X.F. (2003). A fractal model for predicting the eeffective thermal conductivity of liquid with suspension of nnanoparticles. Int. J. Heat Mass Transfer, 46, 2665-2672.

Wang, X.; Xu, X. \& Choi, S.U.S. (1999). Thermal conductivity of nanoparticle - fluid mixture. J. Thermophys. Heat Tr., 13, 474-480.

Wasan, D.T. \& Nikolov, A.D. (2003). Spreading of nanofluids on solids. Nature, 423, 156-159.

Xie, H.; Fujii, M. \& Zhang, X. (2005). Effect of interfacial nanolayer on the effective thermal conductivity of nanoparticle-fluid mixture. Int. J. Heat Mass Transfer, 48, 2926-2932.

Xuan Y. \& Li, Q. (2000). Heat transfer enhancement of nanofluids. Int. J. Heat Fluid Flow, 21, 58-64.

Xuan, Y.; Li, Q. \& Hu, W. (2003). Aggregation structure and thermal conductivity of nanofluids. AICHE J., 49, 1038-1043.

Xue, Q. (2003). Model for effective thermal conductivity of nanofluids. Phys. Lett. A, 307, 313-317.

Xue, Q. \& Xu, W.-M. (2005). A model of thermal conductivity of nanofluids with interfacial shells. Mater. Chem. Phys., 90, 298-301.

Yang, B. \& Han, Z.H. (2006). Temperature-dependent thermal conductivity of nanorod based nanofluids. Appl. Phys. Lett., 89, 1-3.

$\mathrm{Yu}$, W. \& Choi, S.U.S. (2003). The role of interfacial layers in the enhanced thermal conductivity of nanofluids: a renovated Maxwell model. J. Nanopart. Res., 5, 167-171.

$\mathrm{Yu}, \mathrm{W} . \&$ Choi, S.U.S. (2004). The role of interfacial layers in the enhanced thermal conductivity of nanofluids: a renovated Hamilton-Crosser model. J. Nanopart. Res., 6, 355-361. 


\title{
Magnetic Nanoparticles: Its Effect on Cellular Behaviour and Potential Applications
}

\author{
Hon-Man Liu ${ }^{1}$ and Jong-Kai Hsiao² \\ ${ }^{1}$ National Taiwan University, Department of Radiology \\ ${ }^{2}$ Tzu-Chi University, Department of Radiology
}

Taiwan

\section{Introduction}

With the advancement of nanotechnology and the development of molecular medicine, molecular and imaging becomes one of the most popular researches in the latest medicine. Molecular imaging can be defined as the imaging of targeted molecules non-invasively and repetitively in living organisms and cellular imaging can be defined similarly as the imaging of cells or cellular process non-invasively and repetitively in living organisms. At present, the common imaging tools for clinical study include ultrasound, computed tomography (CT), and magnetic resonance imaging (MRI). However, MRI is superior to CT for its better in soft tissue contrast, more sensitive in pathology detection, and lack of ionization irradiation.

In clinical practice, gadolinium-contained compound is the commonest contrast medium used in MRI study. Molecular imaging differs from traditional imaging in that contrast agents are typically used to help identify particular biomarkers or pathways with high sensitivity and selectivity (Achilefu, 2010). However, gadolinium (Gd) is not proper for the molecular imaging and cellular imaging due to its low relaxivity, that further decrease upon cellular internalization; not biocompatible and potential toxicity following cellular dechelation over time. Iron oxide (IO) nanoparticle contrast medium is another contrast medium used in clinical study. They provide the most significant signal change per unit of metal atom, especially on $\mathrm{T} 2{ }^{*} \mathrm{MR}$ imaging. Iron oxide nanoparticle are made of thousands of iron atoms in $\mathrm{Fe}_{3} \mathrm{O}_{4}$ or $\gamma-\mathrm{Fe}_{2} \mathrm{O}_{3}$ form so that they can increase the contrast-to-noise ratio and make its sensitivity superior to Gd contrast agent on MR examination. Their main component, oxidized iron, can be metabolized in liver and recycled as important component of red blood cells. Iron oxide nanoparticle have a relatively long circulation time and low toxicity (Bradbury and Hricak 2005; Funovics et al., 2004; Harisinghani et al., 2003; Jain et al., 2005; Montet et al., 2006). Their surfaces coating may strategically contain chemical linkage of functional groups and ligands for multimodal imaging purpose (Rogers \& Basu, 2005). They can be easily detected by light and electron microscopy. Iron oxide nanoparticle posses some novel properties not seen with the other macromolecules. They can be manipulated by conjugating both targeting ligands or peptide and therapeutic components such as photosensitizer to help in diagnosis and treatment. Iron oxide nanoparticles can be used to monitor cellular migration, molecular events, and signal pathway associated with different 
pathological status. Owing to its magnetic character, iron oxide nanoparticle can be manipulated magnetically and altered their magnetic character according to size of core and the condition of the coating. In this assay, we are going to review the characteristic and types of magnetic nanoparticles (MNP), especially the IO nanoparticles, the mechanism of internalization of MNP into the cell, the impact to cellular and other behaviour of macrophage and stem cell after labelling with MNP, and the future of application of MNP in nanomedicine.

\section{Magnetic resonance imaging and magnetic nanoparticles}

When we put a body into a strong magnetic field and then apply an external radiofrequency (RF) for a period. The RF may causes disturbance of the thermal equilibrium in the body system. After the RF stopped, MR imaging detects the different signals due to the different proton relaxation times $(\mathrm{T})$ of hydrogen atom of the tissue in different body part. This makes MR offers great contrast between different soft tissues in the body. There are two types of MR imaging mechanisms: T1-weighted and T2-weighted.

$\mathrm{T} 1$, the "longitudinal" (spin-lattice) relaxation time is defined as the time required for a substance to regain the $63 \%$ of its original longitudinal magnetization after an RF pulse. It represents the correlation of frequency between molecular motions and the Larmor frequency. The frequencies of small molecule (e.g. water) and large molecule (e.g. protein) are significantly different from the Larmor frequency and thereby have long T1 and present as low signal intensity (dark) on T1-weighted images. The motion frequency of medium-sized molecule such as cholesterol close to those used for MR imaging, thereby it has a short T1 relaxation time and thus appear high signal intensity (bright) on T1weighted images. $\mathrm{T} 1$ relaxation time can be shortened from the interaction between the unpaired electrons in the paramagnetic iron such as Gd ions in contrast medium and the protons in water. This makes those pathology with pooling of $\mathrm{Gd}$ contrast agent appear bright on T1-weighted images.

T2 is the "transverse" (spin-spin) relaxation time. Following a 90 degree RF pulse, the protons lose their coherence and transverse magnetization. The tissue inhomogeneity causes fluctuations of the magnetic field randomly, leading to variations in the precession frequency of different spins on $x-y$ plane. Consequently, the net $x-y$ magnetization is lost since the initial phase coherence is lost. This results in T2 relaxation. Thus T2 relaxation is a measure of how long the resonating protons of a substance can be changed from coherent to de-coherent and then back to coherent stage following 90 degree RF pulse in $\mathrm{x}-\mathrm{y}$ plane. T2 relaxation time is defined as the time needed for the transverse magnetization decreases to $37 \%$ of its original magnitude after a 90 degree RF pulse. Generally, T2 relaxation is much less dependent on the magnetic field strength than T1 relaxation time. However, the magnetic field is not homogenous, and the process is depending on the exact location of the molecules in the magnet. In such circumstances, a special transverse relaxation time constant is defined as T2*, which is usually much smaller than T2 and highly sensitive to magnetic field strength.

The MR contrast medium can be divided into positive and negative contrast media according to their characteristic appearance on T1- weighted or T2-weighted images. 
Positive contrast media appear brighter on MR images owing to a reduction in $\mathrm{T} 1$ relaxation time. They include those containing Gd, manganese or iron ions. Negative contrast agents appear dark on MR imaging due to shortening T1, T2, and T2* relaxation times. Iron oxide is the most common negative contrast medium used clinically.

As mentioned before, gadolinium agent is not suitable for molecular or cellular imaging. In the last 10 years, most research of molecular imaging using MRI is focused on the application of IO nanoparticle.

Compared to larger particles of the same chemical composition, nanoparticles can pass some biological barriers such as capillaries. Human albumin, a circulatory macromolecule, is similar to nanoparticles with a diameter of 5-10 nm (Wiwanitkit, 2006). Enzymes and receptors are also ranged in the similar size (Rawat, 2006). A nanoparticle of such size can have in excess of 1500 potential sites for chemical modification (Debbage et al., 2008; Harris et al., 2003) without loss of biological functionality. It is 150 times more than antibody has. The high capacity for nanoparticle modification has led to their use as amplifiers for in vivo imaging. Both the surface properties and size of nanoparticles are important for their interaction with biological systems and therefore for their distribution in the circulation.

In considering the use in in vivo imaging, the ideal IO nanoparticles is with small size (5-150 $\mathrm{nm}$ ) (table 1), high mass magnetization value, and great surface functionality. If the diameter of the MNPs is larger than $200 \mathrm{~nm}$, they are usually taken up by the liver, spleen, and reticuloendothelial system and resulting in decreased blood circulation times. If their diameters are less than $5 \mathrm{~nm}$, they are rapidly removed through the kidney (Gupta \& Gupta, 2005). Different sizes of IO nanoparticles including SPIO (superparamagnetic IO, 60-150 $\mathrm{nm}$ ), USPIO (ultrasmall SPIO, 10-50 nm), and MION (monocrystalline IO, 5-10 nm) can lead to different magnetic properties and function differently in various applications (Choi et al., 2006; Corot et al., 2006; de Vries et al., 2005; Thorek et al., 2006; Wang et al. 2001;).

The magnetism of MNP and its effect on MR imaging can depend significantly on their morphology, crystal structure, size and uniformity. The crystal structure of SPIO nanoparticle has the general formula of $\mathrm{Fe}^{3+} \mathrm{O}_{3} \mathrm{M}^{2+} \mathrm{O}$, where $\mathrm{M}^{2+}$ represents a divalent metal ion (i.e., iron, manganese, nickel, cobalt or magnesium) (Kateb et al., 2011). The ferric iron $\left(\mathrm{Fe}^{3+}\right)$ makes the complex magnetic (Daldrup-Link et al., 2003; Wang et al., 2001) and large, unpaired, thermodynamically independent spines (single domain particles) makes the complex superparamagnetic. Single domain particles or magnetic domains have a net magnetic dipole. External magnetic fields can cause the magnetic domain to re-orient. The signal intensity of these MNP is related to the size of the particle, its position, its concentration within a given voxel, data acquisition parameters, the magnetic field, and dosage of the SPIO (Wang et al., 2001). In order to achieve higher relaxivity, types of MNPs have also been designed and included those doped with alternative metals such as $\mathrm{CoFe}_{2} \mathrm{O}_{4}$, $\mathrm{NiFe}_{2} \mathrm{O}_{4}, \mathrm{MnFe}_{2} \mathrm{O}_{4}, \mathrm{Gd}_{2} \mathrm{O}_{3}$ and gold-coated cobalt nanoparticles (Bouchard, et al., 2009; Bridot et al., 2007; Lee et al., 2007). Magnetism in MNPs is highly sensitive to its size because it arises from the collective interaction of atomic magnetic dipoles. At a critical size, MNPs will change from a state that has multiple magnetic domains to only a single domain. Below this critical size, the thermal energy becomes comparable to what is needed for spins to flip, and the magnetic dipoles are in status of rapid randomization. Such MNPs do not have 
permanent magnetic moments in the absence of an external field but can quickly respond to an external magnetic field and are referred to as superparamagnetic.

\begin{tabular}{|l|l|c|c|c|}
\hline Name & Coating & $\begin{array}{c}\text { Size } \\
(\mathrm{nm})\end{array}$ & $\begin{array}{c}\text { Relaxivity } \\
\left(\mathrm{mM}^{-1} \mathrm{sec}^{-1}\right) \mathrm{r}_{1}\end{array}$ & $\begin{array}{c}\text { Relaxivity } \\
\left(\mathrm{mM}^{-1} \mathrm{sec}^{-1}\right) \mathrm{r}_{2}\end{array}$ \\
\hline $\begin{array}{l}\text { Feridex/Endorem, } \\
\text { Ferumoxides AMI-25 }\end{array}$ & Dextran T10 & $120-128$ & 10.1 & 120 \\
\hline $\begin{array}{l}\text { Resovist, Ferucarbotran } \\
\text { SHU-555 A }\end{array}$ & Carboxydextran & 60 & 9.7 & 189 \\
\hline $\begin{array}{l}\text { Combidex/ Sinerem } \\
\text { Ferumoxtran-10 } \\
\text { AMI-227 }\end{array}$ & Dextran T10, T1 & $15-30$ & 9.9 & 65 \\
\hline Supravist SHU- 555 C & Carboxydextran & 21 & 10.7 & 38 \\
\hline $\begin{array}{l}\text { Clariscan, Feruglose } \\
\text { NC-100150 }\end{array}$ & Pegylated starch & 20 & n.a. & n.a. \\
\hline
\end{tabular}

Table 1. Examples of available SPIO and USPIO agents. Modified from Corot et al., 2006.

MION has a magnetically labeled cell probe MR imaging agent with size about 5-10nm. It has monocrystallinity and can be used for receptor-directed MR imaging. Its small size make MION can easily pass through capillary endothelium without changing its supermagnetism. It has been stated that it is possible to be detected by MR imaging at concentration as low as $1 \mathrm{ug} \mathrm{Fe} / \mathrm{g}$ tissue. Though it is still in the experimental state, the preliminary targeted MR imaging with MION prove to be a powerful tool for cellular and molecular MR imaging in the future.

Many different chemical methods can be used for synthesizing magnetic nanoparticles. The most commonly used are precipitation-based approaches, either by co-precipitation or reverse micelle synthesis (Nitin et al., 2004; Shen et al., 1993). MNP without any surface coating are not stable in aqueous media, readily aggregate, and precipitate. For in vivo applications via intravenous route, these particles aggregates in blood frequently and are recognized and phagocytosed by macrophages (Lee et al., 2006). Therefore, the surface of MNP should be coated with a variety of different moieties that can eliminate or minimize their aggregation under physiological conditions. Usually, two main approaches are used for coating MNP, including in situ coatings and post-synthesis coatings (Berry et al., 2004; Horak et al., 2007; Jodin et al., 2006). With in situ coating, the MNP are coated during the synthesis process. This coating approach involves a co-precipitation process in the presence of the polysaccharide dextran and a cross linked chemically to increase its stability. This particular coating approach has been very successful in producing dextran SPIOs which are biocompatible and water - soluble. Other coatings in this class include carboxydextran coating, starch-based coating, and dendrimer-based coatings. The post-synthesis coatings can be used for coating MNP with a variety of materials, including, monolayer ligands, polymers, combinations of polymers and biomolecules such as phospholipids and carbohydrates, and silica.

Multiple MNP can also be encapsulated in liposomes to create magnetoliposomes (De Cuyper \& Joniau, 1988). Polyethylene glycol (PEG)-modified, phospholipid micelles coating is favourable since this can results in satisfactory solubility and stability in aqueous 
solutions, well biocompatibility, and also with prolonged blood circulation time when they are delivered intravenously. The PEG can be modified for bioconjugation of various moieties such as antibody, oligonucleotides, and peptides and may allow for molecular specific intracellular targeting of specific proteins and nucleic acid (Gupta \& Gupta, 2005; Kohler et al., 2004; Kumagai et al., 2007; Lee et al., 2006, 2007a; Mikhaylova et al., 2004; Nitin et al., 2004; Veiseh et al., 2005). PEG-coated MNP has the disadvantage such as limited binding sites available for further ligand binding (Gupta \& Gupta, 2005), and the coating thickness can significantly affect their relaxivity (Laconte et al., 2007). In addition to PEG coating, other materials such as antibiofouling poly(TMSMA-r-PEGMA) (Lee et al., 2006), hyaluronic acid layers (Kumar et al., 2007) and carboxylfunctionalized poly(amidoamine) dendrimers of generation 3 (Shi et al., 2007) have also been used to coat the surface of IO nanoparticles for either increasing circulation time in the blood or delivering peptides at high efficiency.

\section{Impact of magnetic nanoparticles in immunologic cell}

For most of the clinical imaging application on magnetic nanoparticles, the delivery route is intravenous injection. The human immune system, mostly reticuloendothelial system, recognizes these magnetic nanoparticles and ingests them. The size and surface charge of the magnetic nanoparticles determine which kind of cells that interact with magnetic nanoparticles (Moghimi \& Bonnemain 1999) For particles larger than $20 \mathrm{~nm}$, macrophage and Kuppfer cell is the corresponding cells that deal with MNP (Moghimi \& Hunter 2001, 2005). If the MNPs are less than $20 \mathrm{~nm}$, these MNPs have greater opportunity to reside in lymph nodes, after they extravasate into interstitial spaces (Moghimi \& Bonnemain 1999). Currently clinical approved iron oxide nanoparticles for MR images ranged mostly from 50$100 \mathrm{~nm}$, in which macrophages play important roles in ingestion of these MNPs. Macrophages are cells that prevent invading bacteria, viruses by phagocytosis of these microbes. It initiates inflammatory change by secreting cytokines such as tumor necrosis factor-alpha and interleukin 2-beta which recruits more circulating cells for repairing damaged tissue. Recent studies reveal macrophages also play important roles in tumor invasion. Consequently, alteration of macrophage behaviour has potential influence on human immunity, inflammatory process and cancer invasion. Understanding of impacts of macrophages toward ingested magnetic nanoparticles is herein clinically important.

Two different MNPs are now under clinical use. Ferucarbotran is composed of both Fe3O4 (magnetite) and g-Fe2O3 (maghemite) and coated with carboxydextran that is negatively charged. Ferumoxides is also composed of iron oxide that coated with dextran. Protamine sulfate is usually added in cell culture for more efficient ferumoxides labelling (Arbab et al., 2006).

Studies on clinically used MNPs, ferucarbotran, toward murine macrophage cell line revealed MNPs ingestion stimulates TNF-alpha and IL-2 Beta secretion. The migratory ability of MNPs laden macrophage increased but the phagocytotic activity of macrophages decreased (Hsiao et al., 2008) However, these findings are based on $100 \mathrm{ug} \mathrm{Fe} / \mathrm{mL} \mathrm{MNP}$ concentration that is 11 times higher than plasma concentration (Metz et al., 2004). Similar findings could be observed on murine peritoneal macrophage cultured with $100 \mathrm{ug} \mathrm{Fe} / \mathrm{mL}$ MNPs. The secretion of TNF-alpha, IL-2 Beta and Nitric oxide, a bactericidal chemical, are 
all increased in conjunction with the promotion of macrophage migration ability (Yeh et al., 2010).

Long term exposure to MNPs has significant influence on macrophages. Research on human macrophages treated with ferucarbotran show increased apoptosis after 120 hours of incubation even at the concentration of $1 \mathrm{ug} \mathrm{Fe} / \mathrm{mL}$. Human macrophage also shows apoptotic change when facing smaller MNPs, supravist, a smaller particle of $20.8 \mathrm{~nm}$ in diameter, for 120 hours at the concentration of $0.1 \mathrm{ug} \mathrm{Fe} / \mathrm{mL}$ (Lunov et al., 2010a). The apoptotic event is inducted by N-terminal kinase (JNK) pathway that is activated by reactive oxygen species (Lunov et al., 2010a; 2010b). There is evidence that elevated TNF-alpha induce human macrophage apoptosis after these macrophages expose to ferucarbotran for 3- 5 days. However, there is no evidence that support ferucarbotran stimulate TNF-alpha secretion on human macrophage. All of studies performed above are in vitro experiments that intravenous injection of MNPs and collecting of circulating macrophage are still pending. Moreover, under intravenous injection condition, all of clinical MNPs are eliminated by reticuloendothelial system within 30 minutes in which no toxic event are observed.

Human monocyte cell line, THP-1, is a precursor of macrophage and it has been evaluated for its interaction with ferumoxides. The ferumoxides has been mixed with $1 \mathrm{mg} / \mathrm{mL}$ of protamine sulfate for higher labeling efficiency. Under incubation concentration of $4.5 \mathrm{ug}$ $\mathrm{Fe} / \mathrm{mL}$ of ferumoxides-protamine complex for 2 hours, there is no significant TNF-alpha secretion level change upon lipopolysaccharide stimulation (Janic et al., 2008). The CD-54 and CD-83 is not upregulated in response to lipopolysaccharide.

Lymphocytes are important immune cells that regulate both cellular and humoral immunity against invading organism and cancer cells. Although lymphocytes are not easily labeled with MNPs, it is still possible by modifying surface of MNPs with tat peptide, a HIV membrane translocating peptide that is specific to CD4+ lymphocytes (Garden et al., 2006). The synthesized Tat linked MNPs are $31.3 \pm 8.5 \mathrm{~nm}$ which is slightly larger than original MNPs that is $25.7 \pm 6.1 \mathrm{~nm}$. Under TEM, these particles located at both cytoplasm and nucleus, which is different from other MNPs that only located at lysosomes. There is neither proliferation ability nor IL-2 secretion capability change of CD4+ CD25+ lymphocytes after labelling with tat-linked MNPs (Garden et al., 2006). Dendritic cells are antigen presenting cells that express antigens to other immune cells, mostly lymphocytes, to continue immune response. Labelling of dendritic cells allows monitoring migration of these cells in vivo (Tavaré et al., 2011; Noh et al., 2011) The mouse dendritic cells were labelled with endorem, a clinically proved MNPs in Europe with corresponding product named ferumoxide in USA. There is no drastic effect of labelled dendritic cells such as T lymphocyte proliferation, in vivo growth rate of lymph nodes after labelled or unlabeled dendritic cells labelling. Under B16 melanoma lung metastatic model, both labelled and unlabeled dendritic cells show protective effect upon pulmonary metastasis (Tavaré et al., 2011).

In conclusion, the effects of MNPs toward immune cells are diverse, the cell type, particle size, charge and labelling amount all contribute to cell behaviour change. Although some reports show immunological response change after MNPs labelling, most of the MNPs exceeds the daily clinical practice. However, systemically analysis of MNPs and immune cells interaction is important and this study may have potential impact on immune therapy. 


\section{Impact of magnetic nanoparticles in stem cell}

Stem cells play promising roles in tissue regeneration and engineering. They could be used for tissue transplantation and it is now understand that stem cells also interact with cancer cells. Some of the stem cell promotes the growth of cancer cells whereas some animal model shows stem cell suppresses the tumor growth.

There are different types of stem cells. Embryonic stem cells are pluripotent, which means the cells could differentiate into almost all cells. However, the ethics concern and current stem cell technology progress makes it less interesting for cell labelling. Mesenchymal stem cells are multi-potent cells that could differentiate into different kinds of cells of medical interest such as bone tissue and cartilage.

Bone marrow derived mesenchymal stem cells are capable of differentiating into many tissue that is essential for tissue repair. However, when these cells delivered into damaged tissue, it is hard to differentiate where these cells are. Labelling cells with MNPs are then important to monitor the location, migration in vivo. It has been proved that MNPs labelled mesenchymal stem cells can be visualized for implantation into damaged cardiac tissue in porcine model (Kraitchman et al., 2002). In the study, ferumoxides incorporated with polyL-lysine were incubated with swine mesenchymal stem cells and injected into myocardiocytes under X-ray guidance. Post-mortem histology shows injected cells resides in designated myocardial tissue. The labelled mesenchymal stem cells are also applied for monitoring the repair of lipopolysaccarides induced damaged brain tissue in rat model by using MNPs-tat peptide conjugate. The result shows cell migratory behaviour into the damaged brain (Jackson et al., 2010). For understanding of interaction between mesenchymal stem cells and tumor, labelled mesenchymal stem cells is also monitored for its interaction with glioma in mouse model by using ferucarbontran in conjunction with protamine sulfate and proved that mesenchymal stem cells reduce glioma growth and mesenchymal stem cells is capable of migration into glioma tissue (Chien et al., 2010).

The mechanism of MNPs uptake by different kinds of stem cells are not fully investigated but recent study shows endocytosis by clathrin receptor is one of the mechanisms (Huang et al., 2005; Lu et al., 2007). These study shows inhibitor of clathrin receptor, phenylarsine oxide, can block the ingestion of mesoporous iron oxide nanoparticles into human mesenchymal stem cells. Macropinocytosis also play significant role once if protamine sulfate is used. It is also proved that tat peptide linked MNPs enter cell by macropinocytosis (Arbab et al., 2006).

Most of stem cell labelling for MR imaging is based on T2 weighted contrast. However, some efforts aiming on T1 contrast agent such as gadolinium based chelates conjugating into mesoporous silica nanoparticles has been proved for its imaging capability in animals injected with human mesenchymal stem cells. The viability and differentiating capacity of these mesenchymal stem cells are preserved (Hsiao et al., 2008; Tsai et al., 2008). The mesoporous nanoparticles has also been labelled with fluorescent dyes that monitoring the cells with fluorescent imaging modality is also possible.

In addition to cell viability, labelled mesenchymal stem cells has been verified for its mitochondrial potential and reactive oxygen species, both of which represents intracellular stress. Neither mitochondrial potential nor reactive oxygen species change under 
ferucarbotran incubation at the concentration of $100 \mathrm{ug} \mathrm{Fe} / \mathrm{mL}$ for 24 hours (Hsiao et al., 2007). Long term incubation up to 72 hours has also been investigated and shows no adverse effect upon mesenchymal stem cells (Yang et al., 2011). Similar results are found on ferumoxide-polylysine and ferumoxides-protamine sulfate complex toward mesenchymal stem cells (Arbab et al., 2003; Pawelczyk et al., 2006).

Stem cells are valuable for its differentiation capacity. Concerns for preserving its differentiating capacity are essential. For clinical used MNPs such as ferucarbotran for directly labelling, it has been showed that labelled mesenchymal stem cells is still capable of differentiating to adipose tissue, and bone tissue at the labelling period of 24 hours (Hsiao et al., 2007). The long term effect has also been evaluated for its cartilage differentiation capacity (Yang et al., 2011). The activity of chondrogenesis of ferucarbontran labelled mesenchymal stem cells decreased as iron content increases (Hinning et al., 2009). Similar finding upon osteogenesis is also found. Dose dependent osteogenesis inhibition is observed on human mesenchymal stem cells (Chen et al., 2010). The labelling dose is consequently very important.

Labelling of mesenchymal stem cells with ferumoxides in conjunction with transfection agent is also popular. The differentiation capacity has also been studied. The adipogenesis and osteogenesis capacity is preserved but there is debate upon chondrogenesis (Kostura et al., 2004; Arbab et al., 2005). The model of ferumoxides-polylysine shows inhibition of chondrogenesis whereas ferumoxide-protamine sulfate shows no inhibitory effect. Although there is no study comparing these two labelling method, the ferumoxide-protamine sulfate and ferumoxide-polylysine complex, labelling mesenchymal stem cells with ferumoxideprotamine sulfate might be better for further investigation. Besides, labelling dose of MNPs should be suitable for preserving imaging capability and differentiating capacity.

In conclusion, labelling of stem cells for imaging is medically important that could be used for cell trafficking and potentially tumor inhibition. Although imaging capability of these labelled mesenchymal stem cells is concerned, the differentiation capacity of these cells should be preserved. Meanwhile, no satisfactory methods or consensus about labelling stem cell with MNP established though direct labelling using ferucarbotran or labelling ferumoxides with protamine sulfate are popular. Efforts on designing novel MNPs for cell labelling is still demanding.

\section{Bifunctional, multi-functional, and theragnostic magnetic nanoparticles}

Nanoparticles have advantages for their multi-conjugating capability that makes it possible to exhibit imaging and therapeutic character in one particle. The capability of imaging is mostly rely on the core that is magnetic. Either the shell or the core itself exhibit therapeutic effect. The therapeutic effects include gene delivery, hyperthermia, chemotherapy and photodynamic therapy. The benefits of theragnostic design are based on the following advantages. First, the magnetic core plays both imaging and magnetic guidance character. Targeting to specific organ or tissue is theoretically possible once if a guiding magnetic is applied. Secondly, the location where MNPs resides and acts as therapeutic agent can be visualized. Unlike most drugs that are small molecules, MNPs has different, specific organ and cell distribution that makes it possible for different treatment strategy. Lastly, MNPs 
can traverse vasculature barrier and go into intercellular space or even cell surface once if recognizing molecules has been conjugated at the MNPs surface.

Hyperthermia with MNPs is based on the fact that tumor cells are more liable toward temperature change. It have been investigated that temperature between $41^{\circ} \mathrm{C}$ and $42^{\circ} \mathrm{C}$ can induce tumor cell death by destruction of cell membrane (Sellins \& Cohen, 1991). The enzymatic system is also influenced. The hyperthermia is achieved by alternating current magnetic field system around the frequency of $100 \mathrm{KHz}$ at the magnetic field intensity in $30.6 \mathrm{kA} / \mathrm{m}$ (Silva et al., 2011). Limited clinical trial was done and showed controversial effect (Maier-Hauff et al., 2007; 2011). In one study, 66 patients of glioblastoma, a high grade brain tumor, were enrolled and MNPs were injected into tumor of these patient. Hyperthermia associated with radiotherapy was done and there is statistical difference between hyperthermia group and traditional radiotherapy group. The survival after first diagnosis is 8.6 months longer in hyperthermia group compared with conventional treatment group. In addition, the adverse effect of hyperthermia is not significant according to the observation of the study (Maier-Hauff et al., 2011).

Photodynamic therapy is one of the cancer treatment methods that have also been used for theragnostic purpose. The mechanism of photodynamic therapy is based on synthesis of singlet oxygen at the expense of photon activation of photosensitizer. The produced singlet oxygen is capable of destruct adjacent cells by oxidation. Some of the photosensitizers are clinically available. Efforts trying to conjugate MNPs with photosensitizers have potential benefits such as understanding the location of drugs accumulation and MNPs can also be guided by magnetic fields. The model of multi-functional MNPs has been proved possible in vitro. Hela cells can be imaged and killed by iridium complexes conjugated iron oxide nanoparticles (Lai et al., 2008). The iridium complexes have been also conjugated to $\mathrm{MnO}$ based mesoporous silicate nanoparticles that exhibit T1 weighted contrast enhancement. The photodynamic therapy effect is proved efficacious at in vitro HeLa cell model (Peng et al., 2011).

Gene therapy is at the edge of new strategy for cancer therapy. MNPs is capable of serving gene delivery carrier and also used for magnetic guidance. Studies focused on cancer related gene such as E1A has been successfully delivered into HeLa cells after E1A gene incoporated with iron oxide nanoparticles. Intratumoral injection of the plasmid-MNPs complex results in tumor size reduction compared with control group, whereas only radiation therapy was done (Shen et al., 2010).

In conclusion, multifunctional MNPs are at the initial stage of development. The benefits of biodistribution and magnetic character make theragnostic strategy different from other treatment. However, more efforts upon toxicity and therapeutic range should be done before it has been used widely in the clinical medical fields.

\section{Future}

Cellular Imaging can be an application of MNP as cellular marker for imaging of macrophage activity and as cellular marker for imaging of cell migration and cell trafficking. With the advancement of modern molecule design, we can also have the capability of design a MNP with the role of both diagnostic and treatment. 
A major limitation of IO MNP is the loss of signal on T2-weighted MRI and creating 'black holes' on images; that (1) prevent direct anatomical MR evaluation of the tissue in question (requiring comparison of pre- and post-contrast images), and (2) make it difficult to discriminate between targeted cells and image artefacts (i.e. as caused by susceptibility artifacts or imperfect pulse sequences). One such approach could be the use of a white marker' MR T1-weighted sequence, that creates positive MNP contrast. For cellular imaging, as labelling is not permanent and self-replicable like reporter genes, with dilution of label upon cell division, iron oxide detection may rapidly become impossible. Finally, careful iron oxide titration and cellular differentiation studies need to be performed. Short- and longterm toxicity studies are warranted. It needs a comprehensive study on the fate of the particles in vivo following biodegradation; quantify the number of iron oxide labelled molecules or cells per voxel and to increase the specificity of detection of iron oxides.

Perhaps the least studied limitation is the potential acute and chronic systemic toxicity of the particles themselves. Toxicity can result from the MNP themselves or the individual components of the MNP that can be released during degradation in vivo. Nanomaterials may influence a living organism through different biological pathways ( $\mathrm{Nel}$ et al., 2006). From previous limited report IO MNP and gold colloids seem to be less of a concern in terms of toxicity and $\mathrm{IO}$ can be cleared from the body via various routes with minimal toxicity (Briley-Saebo et al., 2004; Corot et al., 2006; Jain et al., 2008). Different types of nanoparticles have been shown to be cytotoxic to human cells (Lewinski et al., 2008), induce oxidative stress (Long et al., 2006), or elicit an immune response (Dobrovolskaia et al., 2007). After administration, nanoparticles must traverse a complex and often hostile environments that have evolved to seek out and exclude foreign material (Minchin et al., 1999). The first few steps of this dangerous journey include the interacting with plasma proteins and accumulating in macrophages or the reticuloendothelial system of the liver, spleen, or lymph nodes. The types of proteins that absorb to the surface are affected by size, shape, and surface characteristics. Importantly, there is now strong evidence that the proteins that surround the nanoparticles play a critical role in determining their fate in vivo (Kreuter et al., 2002; Owens et al.; 2006, Lynch et al., 2006). Dextran is clinically approved for modifying IO MNP but liver accumulation is still evident. Silica nanoparticles have been evaluated for potential hepatoxicity because of their propensity to be taken up by the liver (Nishimori et al., 2009). Whereas large particles (>300nm) showed little adverse effects, particles less than $100 \mathrm{~nm}$ induced acute liver damage and cytokine release.

\section{Conclusion}

The nanotechnology offer great opportunities for molecular imaging and future medicine. However, they are difficulty in designing and administration. The possible acute or chronic toxicity associated with the nanoparticle is still under investigated. The implementation of nanotechnology in medicine will depend on more understand and depth knowledge about them.

\section{References}

Arbab, A.S., Bashaw, L.A., Miller, B.R., Jordan, E.K., Lewis, B.K., Kalish, H., \& Frank, J.A. (2003). Characterization of biophysical and metabolic properties of cells labeled 
with superparamagnetic iron oxide nanoparticles and transfection agent for cellular MR imaging. Radiology, 229(3),838-846.

Arbab, A.S., Yocum, G.T., Rad, A.M., Khakoo, A.Y., Fellowes, V., Read, E.J., \& Frank J.A. (2005). Labeling of cells with ferumoxides-protamine sulfate complexes does not inhibit function or differentiation capacity of hematopoietic or mesenchymal stem cells. NMR in Biomed, 18(8), 553-559.

Arbab, A.S., Liu, W., \& Frank, J.A. (2006).Cellular magnetic resonance imaging: current status and future prospects. Expert Rev Med Devices, 3(4), 427-439.

Achilefu, S. (2010) Introduction to concept and strategies for molecular imaging. Chem. Rev. $110,2575-2578$

Berry, C.C., Wells, S., Charles, S., Aitchison, G., \& Curtis, A.S. (2004). Cell response to dextranderivatised iron oxide nanoparticles post internalisation. Biomaterials, 25, 5405-5413.

Bouchard, L.S., Anwar, M.S., Liu, G.L., Hann, B., Xie, Z.H., Gray, J.W., Wang, X., Pines, A., \& Chen, F.F. (2009). Picomolar sensitivity MRI and photoacoustic imaging of cobalt nanoparticles. Proc. Natl. Acad. Sci. 106, 4085-4089

Bradbury, M. \& Hricak, H. (2005). Molecular MR imaging in oncology. Magn Reson Imaging Clin N Am, 13,225-240.

Bridot, J.L., Faure, A.C., Laurent, S., Rivière, C., Billotey, C., Hiba, B., Janier, M., Josserand, V., Coll, J.L., Elst, L.V., Muller, R., Roux, S., Perriat, P., \& Tillement, O. (2007). Hybrid gadolinium oxide nanoparticles: multimodal contrast agents for in vivo imaging. J. Am. Chem. Soc. 129, 5076-5084

Briley-Saebo, K., Bjørnerud, A., Grant, D., Ahlstrom, H., Berg, T., \& Kindberg, G.M. (2004). Hepatic cellular distribution and degradation of iron oxide nanoparticles following single intravenous injection in rats: implications for magnetic resonance imaging. Cell Tissue Res. 316, 315-323.

Chen, Y.C., Hsiao, J.K., Liu, H.M., Lai, I.Y., Yao, M., Hsu, S.C., Ko, B.S., Chen, Y.C., Yang, C.S., \& Huang, D.M. (2010). The inhibitory effect of superparamagnetic iron oxide nanoparticle (Ferucarbotran) on osteogenic differentiation and its signaling mechanism in human mesenchymal stem cells. Toxicology and Applied Pharmacology, 245(2), 272-279.

Chien, L.Y., Hsiao, J.K., Hsu, S.C., Yao, M., Lu, C.W., Liu, H.M., Chen, Y.C., Yang, C.S., \& Huang, D.M. (2011). In vivo magnetic resonance imaging of cell tropism, trafficking mechanism, and therapeutic impact of human mesenchymal stem cells in a murine glioma model. Biomaterials, 32(12), 3275-3284.

Choi, S.H., Han, M.H., Moon, W.K., Son, K.R., Won, J.K., Kim, J.H., Kwon, B.J., Na, D.G., Weinmann, H.J., \& Chang, K.H. (2006). Cervical lymph node metastases: MR imaging of gadofluorine $\mathrm{M}$ and monocrystalline iron oxide nanoparticle-47 in a rabbit model of head and neck cancer. Radiology, 241 (3), 753-762.

Corot, C. Robert, P., Idée, J.M., \& Port, M. (2006) Recent advances in iron oxide nanocrystal technology for medical imaging. Adv. Drug Deliv. Rev. 58, 1471-1504.

Daldrup-Link, H.E., Rudelius, M., Oostendorp, R.A., Settles, M., Piontek, G., Metz, S., Rosenbrock, H., Keller, U., Heinzmann, U., Rummeny, E.J., Schlegel, J., \& Link, T.M., (2003). Targeting of hematopoietic progenitor cells with MR contrast agents. Radiology, 228, 760-767. 
Debbage, P., \& Jaschke,W. (2008). Molecular imaging with nanoparticles: giant roles for dwarf actors. Histochem Cell Biol, 130,845-875.

De Cuyper, M., \& Joniau, M. (1988). Magnetoliposomes. Formation and structural characterization. Eur Biophys J, 15,311-319.

de Vries, I.J., Lesterhuis, W.J., Barentsz, J.O., Verdijk, P., van Krieken, J.H., Boerman, O.C., Oyen, W.J., Bonenkamp, J.J., Boezeman, J.B., Adema, G.J., Bulte, J.W., Scheenen, T.W., Punt, C.J., Heerschap, A., \& Figdor, C.G., (2005). Magnetic resonance tracking of dendritic cells in melanoma patients for monitoring cellular therapy. Nat. Biotechnol, 23 (11), 1407-1413.

Dobrovolskaia, M.A., \& McNeil, S.E. (2007). Immunological properties of engineered nanomaterials. Nat Nanotechnol, 2,469-478.

Funovics, M.A., Kapeller, B., Hoeller, C., Su, H.S., Kunstfeld, R., Puig, S., \& Macfelda, K. (2004). MR imaging of the her2/neu and 9.2.27 tumor antigens using immunospecific contrast agents. Magn Reson Imaging, 22,843-850.

Garden, O.A., Reynolds, P.R., Yates, J., Larkman, D.J., Marelli-Berg, F.M., Haskard, D.O., Edwards, A.D., \& George, A.J. (2006). A rapid method for labelling CD4+ T cells with ultrasmall paramagnetic iron oxide nanoparticles for magnetic resonance imaging that preserves proliferative, regulatory and migratory behaviour in vitro. Journal of Immunological Methods, 314(1-2), 123-133.

Gupta, A.K., \& Gupta, M. (2005). Synthesis and surface engineering of iron oxide nanoparticles for biomedical applications. Biomaterials, 26, 3995-4021.

Harisinghani, M.G., Barentsz, J., Hahn, P.F., Deserno, W.M., Tabatabaei, S., van de Kaa, C.H., de la Rosette, J., \& Weissleder, R. (2003). Noninvasive detection of clinically occult lymph-node metastases in prostate cancer. N Engl J Med, 348,2491-2499.

Harris, L.A., Goff, J.D., Carmichael, A.Y., Riffle, J.S., Harburn, J.J., St. Pierre, T.G., \& Saunders, M. (2003) Magnetite nanoparticle dispersions stabilized with triblock copolymers. Chem Mater, 15,1367-1377.

Henning, T.D., Sutton, E.J., Kim, A., Golovko, D., Horvai, A., Ackerman, L., Sennino, B., McDonald, D., Lotz, J., \& Daldrup-Link, H.E. (2009). The influence of ferucarbotran on the chondrogenesis of human mesenchymal stem cells. Contrast media $\mathcal{E}$ molecular imaging, 4(4),165-173.

Horak, D., Babic, M., Jendelova, P., Herynek, V., Trchová, M., Pientka, Z., Pollert, E., Hájek, M., \& Syková, E. (2007). D-mannose-modified iron oxide nanoparticles for stem cell labeling. Bioconjug Chem, 18,635-644.

Hsiao, J.K., Tai, M.F., Chu, H.H., Chen, S.T., Li, H., Lai, D.M., Hsieh, S.T., Wang, J.L., \& Liu, H.M. (2007). Magnetic nanoparticle labeling of mesenchymal stem cells without transfection agent: cellular behavior and capability of detection with clinical $1.5 \mathrm{~T}$ magnetic resonance at the single cell level. Magn Reson Med, 58(4),717-724.

Hsiao, J.K., Chu, H.H., Wang, Y.H., Lai, C.W., Chou, P.T., Hsieh, S.T., Wang, J.L., \& Liu, H.M. (2008). Macrophage physiological function after superparamagnetic iron oxide labeling. NMR Biomed, 21(8),820-829.

Hsiao, J.K., Tsai, C.P., Chung, T.H., Hung, Y., Yao, M., Liu, H.M., Mou, C.Y., Yang, C.S., Chen, Y.C., \& Huang, D.M. (2008). Mesoporous silica nanoparticles as a delivery system of gadolinium for effective human stem cell tracking. Small, 4(9),1445-1452.

Huang, D.M., Hung, Y., Ko, B.S., Hsu, S.C., Chen, W.H., Chien, C.L., Tsai, C.P., Kuo, C.T., Kang, J.C., Yang, C.S., Mou, C.Y., \& Chen, Y.C. (2005). Highly efficient cellular 
labeling of mesoporous nanoparticles in human mesenchymal stem cells: implication for stem cell tracking. FASEB J., 19(14),2014-2016.

Jackson, J.S., Golding , J.P., Chapon, C., Jones, W.A., \& Bhakoo, K.K. (2010) Homing of stem cells to sites of inflammatory brain injury after intracerebral and intravenous administration: a longitudinal imaging study. Stem Cell Res Ther. 1(2),17.

Jain, K.K. (2005). Role of nanobiotechnology in developing personalized medicine for cancer. Technol Cancer Res Treat, 4,645-650.

Jain, T.K. Reddy, M.K., Morales, M.A., Leslie-Pelecky, D.L., \& Labhasetwar, V. (2008). Biodistribution, clearance, and biocompatibility of iron oxide magnetic nanoparticles in rats. Mol. Pharm., 5, 316-327.

Janic, B., Iskander,,A.S., Rad, A.M., Soltanian-Zadeh, H., \& Arbab, A.S. (2008). Effects of Ferumoxides - Protamine Sulfate Labeling on Immunomodulatory Characteristics of Macrophage-like THP-1 Cells. PLoS ONE, 3(6), e2499.

Jodin, L., Dupuis, A.C., Rouviere, E., \& Reiss, P. (2006). Infl uence of the catalyst type on the growth of carbon nanotubes via methane chemical vapor deposition. J Phys Chem $B$, 110,7328-7333.

Kateb, B., Chiu, K., Black, K.L., Yamamoto, V., Khalsa, B., Ljubimova, J.Y., Ding, H. , Patil, R., Portilla-Arias, J.A. , Modo, M. , Moore, D.F., Farahani, K., Okun, M.S., Prakash, N., Neman, J., Ahdoot, D., Grundfest, W., Nikzad, S., \& Heiss, J.D. (2011). Nanoplatforms for constructing new approaches to cancer treatment, imaging, and drug delivery: What should be the policy? NeuroImage, 54, S106-S124

Kohler, N., Fryxell, G.E., \& Zhang, M. (2004). A bifunctional poly(ethylene glycol) silane immobilized on metallic oxide-based nanoparticles for conjugation with cell targeting agents. J Am Chem Soc, 126,7206-7211.

Kostura, L., Kraitchman, D.L., Mackay, A.M., Pittenger, M.F., \& Bulte, J.W. (2004). Feridex labeling of mesenchymal stem cells inhibits chondrogenesis but not adipogenesis or osteogenesis. NMR in Biomed, 17(7),513-517.

Kraitchman, D.L., Heldman, A,W, Atalar, E,, Amado, L.C., Martin, B.J., Pittenger, M.F., Hare, J.M.,\& Bulte, J.W. (2003). In Vivo Magnetic Resonance Imaging of Mesenchymal Stem Cells in Myocardial Infarction. Circulation, 107(18), 2290-2293.

Kreuter, J., Shamenkov, D., Petrov, V., Ramge, P., Cychutek, K., Koch-Brandt, C., \& Alyautdin, R. (2002). Apolipoprotein-mediated transport of nanoparticle-bound drugs across the blood-brain barrier. J Drug Target, 10,317-325.

Kumagai, M., Imai, Y., Nakamura, T., Yamasaki, Y., Sekino, M., Ueno, S., Hanaoka, K., Kikuchi, K., Nagano, T., Kaneko, E., Shimokado, K., \& Kataoka, K. (2007). Iron hydroxide nanoparticles coated with poly(ethylene glycol)-poly(aspartic acid) block copolymer as novel magnetic resonance contrast agents for in vivo cancer imaging. Colloids Surf B Biointerfaces, 56,174-81.

Kumar, A., Sahoo, B., Montpetit, A., Behera, S., Lockey, R.F., \& Mohapatra, S.S. (2007). Development of hyaluronic acid-Fe2O3 hybrid magnetic nanoparticles for targeted delivery of peptides. Nanomedicine, 3,132-137.

Laconte, L.E., Nitin, N., Zurkiya, O., Caruntu, D., O'Connor, C.J., Hu, X., \& Bao, G. (2007). Coating thickness of magnetic iron oxide nanoparticles affects $\mathrm{R}(2)$ relaxivity. $J$ Magn Reson Imaging, 26,1634-41.

Lai, C.W., Wang, Y.H., Lai, CH., Yang, M.J., Chen, C.Y., Chou, P.T., Chan, C.S., Chi, Y., Chen, Y.C., \& Hsiao, J.K. (2008). Iridium-complex-functionalized Fe3O4/SiO2 
core/shell nanoparticles: a facile three-in-one system in magnetic resonance imaging, luminescence imaging, and photodynamic therapy. Small, 4(2), 218-224.

Lee, H., Lee, E., Kim, do K., Jang, N.K., Jeong, Y.Y., \& Jon, S. (2006). Antibiofouling polymercoated superparamagnetic iron oxide nanoparticles as potential magnetic resonance contrast agents for in vivo cancer imaging. J Am Chem Soc, 128,73837389.

Lee, J.H., Huh, Y.M., Jun, Y.W., Seo, J.W., Jang, J.T., Song, H.T., Kim, S., Cho, E.J., Yoon, H.G., Suh, J.S., \& Cheon, J. (2007) Artificially engineered magnetic nanoparticles for ultra-sensitive molecular imaging. Nat. Med. 13, 95-99.

Lee, H., Yu, M.K., Park, S., Moon, S., Min, J.J., Jeong, Y.Y., Kang, H.W., \& Jon, S. (2007). Thermally cross-linked superparamagnetic iron oxide nanoparticles: synthesis and application as a dual imaging probe for cancer in vivo. J Am Chem Soc, 129,1273912745.

Lewinski, N., Colvin, V., \& Drezek, R. (2008). Cytotoxicity of nanoparticles. Small, 4,26-49.

Long, T.C., Saleh, N., Tilton, R.D., Lowry, G.V., \& Veronesi, B. (2006). Titanium dioxide (P25) produces reactive oxygen species in immortalized brain microglia (BV2): implications for nanoparticle neurotoxicity. Environ Sci Technol, 40,4346-4352.

Lu, C.W., Hung, Y., Hsiao, J.K., Yao, M., Chung, T.H., Lin, Y.S., Wu, S.H., Hsu, S.C., Liu, H.M., Mou, C.Y., Yang, C.S., Huang, D.M., \& Chen, Y.C. (2007). Bifunctional magnetic silica nanoparticles for highly efficient human stem cell labeling. Nano Lett, 7(1), 149-154.

Lunov, O., Syrovets, T., Büchele, B., Jiang, X., Röcker, C., Tron, K., Nienhaus, G.U., Walther, P., Mailänder, V., Landfester, K., \& Simmet, T. (2010a). The effect of carboxydextran-coated superparamagnetic iron oxide nanoparticles on c-Jun $\mathrm{N}$ terminal kinase-mediated apoptosis in human macrophages. Biomaterials, 31(19),5063-5071.

Lunov, O., Syrovets, T., Röcker, C., Tron, K., Nienhaus, G.U., Rasche, V., Mailänder, V., Landfester, K., \& Simmet, T. (2010b). Lysosomal degradation of the carboxydextran shell of coated superparamagnetic iron oxide nanoparticles and the fate of professional phagocytes. Biomaterials, 31(34), 9015-9022.

Lynch, I., Dawson, K.A., \& Linse, S. (2006). Detecting cryptic epitopes created by nanoparticles. Sci STKE, 2006, pe14

Maier-Hauff, K., Rothe, R., Scholz, R., Gneveckow, U., Wust, P., Thiesen, B., Feussner, A., von Deimling, A., Waldoefner, N., Felix, R., \& Jordan, A. (2007). Intracranial thermotherapy using magnetic nanoparticles combined with external beam radiotherapy: results of a feasibility study on patients with glioblastoma multiforme. Journal of Neuro-Oncology, 81(1), 53-60.

Maier-Hauff, K., Ulrich, F., Nestler, D., Niehoff, H., Wust, P., Thiesen, B., Orawa, H., Budach, V., \& Jordan, A. (2011). Efficacy and safety of intratumoral thermotherapy using magnetic iron-oxide nanoparticles combined with external beam radiotherapy on patients with recurrent glioblastoma multiforme. Journal of NeuroOncology, 103(2), 317-24.

Metz, S., Bonaterra, G., Rudelius, M., Settles, M., Rummeny, E.J., \& Daldrup-Link, H.E. (2004). Capacity of human monocytes to phagocytose approved iron oxide MR contrast agents in vitro. Eur Radiol, 14(10), 1851-1858. 
Mikhaylova, M., Kim, D.K., Bobrysheva, N., Osmolowsky, M., Semenov, V., Tsakalakos, T., \& Muhammed, M. (2004). Superparamagnetism of magnetite nanoparticles: dependence on surface modification. Langmuir, 20,2472-2477.

Minchin, R.F., Orr, R.J., Cronin, A.S., \& Puls, R.L. (1999) The pharmacology of gene therapy. Croat Med J 40,381-391.

Moghimi, S.M. \& Bonnemain, B. (1999). Subcutaneous and intravenous delivery of diagnostic agents to the lymphatic system: applications in lymphoscintigraphy and indirect lymphography. Advanced Drug Delivery Reviews, 37(1-3), 295-312.

Moghimi, S.M. \& Hunter, A.C. (2001). Recognition by Macrophages and Liver Cells of Opsonized Phospholipid Vesicles and Phospholipid Headgroups. Pharmaceutical Research, 18(1), 1-8.

Moghimi, S.M., Hunter, A.C., \& Murray, J.C. (2005). Nanomedicine: current status and future prospects. Faseb J., 19(3),311-330.

Montet, X., Montet-Abou, K., Reynolds, F., Weissleder, R., \& Josephson, L. (2006). Nanoparticle imaging of integrins on tumor cells. Neoplasia, 8,214-222.

Nel, A., Xia, T., Mädler, L., \& Li, N. (2006). Toxic potential of materials at the nanolevel. Science 311, 622-627.

Nishimori, H., Kondoh, M., Isoda, K., Tsunoda, S., Tsutsumi, Y., Yagi, K. (2009) Silica nanoparticles as hepatotoxicants. Eur J Pharmaceut Biopharmaceut, 72,496-501.

Nitin, N., LaConte, L.E., Zurkiya, O., Hu, X., Bao, G. (2004). Functionalization and peptidebased delivery of magnetic nanoparticles as an intracellular MRI contrast agent. J Biol Inorg Chem, 9,706-712.

Noh, Y.-W., Jang, Y.S., Ahn, K.J., Lim, Y.T., \& Chung, B.H. (2011). Simultaneous in vivo tracking of dendritic cells and priming of an antigen-specific immune response. Biomaterials, 32(26), 6254-6263.

Owens, 3rd D.E., \& Peppas, N.A. (2006). Opsonization, biodistribution, and pharmacokinetics of polymeric nanoparticles. Int J Pharmaceut, 307,93-102.

Pawelczyk, E., Arbab, A.S., Pandit, S., Hu, E., \& Frank, J.A. (2006). Expression of transferrin receptor and ferritin following ferumoxides-protamine sulfate labeling of cells: implications for cellular magnetic resonance imaging. NMR in Biomed, 19(5), 581592.

Peng, Y.K., Lai, C.W., Liu, C.L., Chen, H.C., Hsiao, Y.H., Liu, W.L., Tang, K.C., Chi, Y., Hsiao, J.K., Lim, K.E., Liao, H.E., Shyue, J.J., \& Chou, P.T. (2011). A new and facile method to prepare uniform hollow $\mathrm{MnO} /$ functionalized $\mathrm{mSiO}$ core/shell nanocomposites. ACS nano, 5(5), 4177-4187.

Rawat, M., Singh, D., Saraf, S., \& Saraf, S. (2006). Nanocarriers: promising vehicle for bioactive drugs. Biol. Pharm. Bull., 29, 1790-1798.

Rogers, W.J. \& Basu, P. (2005). Factors regulating macrophage endocytosis of nanoparticles: implications for targeted magnetic resonance plaque imaging. Atherosclerosis, 178, 67-73.

Sellins, K.S. \& Cohen, J.J. (1991). Hyperthermia induces apoptosis in thymocytes. Radiation Research, 126(1), 88-95.

Shen, L.-F., Chen, J., Zeng, S., Zhou, R.R., Zhu, H., Zhong, M.Z., Yao, R.J., \& Shen, H. (2010). The Superparamagnetic Nanoparticles Carrying the E1A Gene Enhance the Radiosensitivity of Human Cervical Carcinoma in Nude Mice. Molecular Cancer Therapeutics, 9(7), 2123-2130. 
Shen, T., Weissleder, R., Papisov, M., Bogdanov, A. Jr, \& Brady, T.J. (1993). Monocrystalline iron oxide nanocompounds (MION): physicochemical properties. Magn Reson Med, 29,599-604.

Shi, X., Thomas, T.P., Myc, L.A., Kotlyar, A., \& Baker, J.R. Jr. (2007). Synthesis, characterization, and intracellular uptake of carboxyl-terminated poly(amidoamine) dendrimer-stabilized iron oxide nanoparticles. Phys Chem Chem Phys, 9, 5712-5720.

Silva, A.C., Oliveira, T.R., Mamani, J.B., Malheiros, S.M., Malavolta, L., Pavon, L.F., Sibov, T.T., Amaro, E. Jr, Tannús, A., Vidoto, E.L., Martins, M.J., Santos, R.S., \& Gamarra, L.F. (2011). Application of hyperthermia induced by superparamagnetic iron oxide nanoparticles in glioma treatment. International journal of nanomedicine, 6, 591-603.

Tavaré, R., Sagoo, P., Varama, G., Tanriver, Y., Warely, A., Diebold, S.S., Southworth, R., Schaeffter, T., Lechler, R.I., Razavi, R., Lombardi, G., \& Mullen, G.E. (2011). Monitoring of in vivo function of superparamagnetic iron oxide labelled murine dendritic cells during anti-tumour vaccination. PLoS ONE, 6(5), e19662.

Thorek, D.L., Chen, A.K., Czupryna, J., \& Tsourkas, A. (2006). Superparamagnetic iron oxide nanoparticle probes for molecular imaging. Ann Biomed Eng, 34,23-38.

Tsai, C.P., Hung, Y., Chou, Y.H., Huang, D.M., Hsiao, J.K., Chang, C., Chen, Y.C., \& Mou, C.Y. (2008). High-contrast paramagnetic fluorescent mesoporous silica nanorods as a multifunctional cell-imaging probe. Small, 4(2),186-191.

Veiseh, O., Sun, C., Gunn, J., Kohler, N., Gabikian, P., Lee, D., Bhattarai, N., Ellenbogen, R., Sze, R., Hallahan, A., Olson, J., \& Zhang, M. (2005). Optical and MRI multifunctional nanoprobe for targeting gliomas. Nano Lett, 5,1003-1008.

Wang, Y.X., Hussain, S.M., \& Krestin, G.P. (2001). Superparamagnetic iron oxide contrast agents: physicochemical characteristics and applications in MR imaging. Eur. Radiol., 11, 2319-2331.

Wiwanitkit, V. (2006). Glomerular pore size corresponding to albumin molecular size, an explanation for underlying structural pathology leading to albuminuria at nanolevel. Renal Fail, 28,101.

Yang, C.Y., Hsiao, J.K., Tai, M.F., Chen, S.T., Cheng, H.Y., Wang, J.L., \& Liu, H.M. (2011). Direct labeling of hMSC with SPIO: the long-term influence on toxicity, chondrogenic differentiation capacity, and intracellular distribution. Mol Imaging Biol. 13(3), 443-451.

Yeh, C.-H., Hsiao, J.K., Wang, J.L., \& Fuu Sheu, F. (2010). Immunological impact of magnetic nanoparticles (Ferucarbotran) on murine peritoneal macrophages. Journal of Nanoparticle Research, 12(1), 151-160. 


\title{
Thermal Effects on the Ferromagnetic Resonance in Polymer Composites with Magnetic Nanoparticles Fillers
}

\author{
Mirosław R. Dudek ${ }^{1}$, Nikos Guskos ${ }^{2,3}$ and Marcin Kośmider ${ }^{1}$ \\ ${ }^{1}$ Institute of Physics, University of Zielona Góra, Zielona Góra \\ ${ }^{2}$ Solid State Physics Section, Department of Physics, \\ University of Athens, Panepistimiopolis \\ ${ }^{3}$ Institute of Physics,West Pomeranian University of Technology, Szczecin \\ 1,3 Poland \\ ${ }^{2}$ Greece
}

\section{Introduction}

Magnetic nanopowders placed in the nonmagnetic polymer matrices become a new type of smart materials which combine mechanical properties of temperature responsive polymer matrix and magnetic response of nanoparticles. These properties are used in some biotechnological and medical applications like hyperthermia treatment, nanocolloids, magnetic nanocapsules for drug targeting, magnetic resonance imaging (MRI), intracellular manipulation etc. (e.g. (Gao \& Xu, 2009; Liu et al., 2009)), in the processes of mechanical and electrical micropower generation, in nanoelectromechanical systems as MEMS/NEMS devices (e.g. (Zahn, 2001)), electromagnetic interference suppression (Wilson et al., 2004). Recently, the unusual polymer/magnetic nanoparticles systems with a negative Poisson's ratio (e.g. ferrogels Dudek \& Wojciechowski (2008); Wood \& Camp (2011)) have begun to be studied. They belong to the so-called auxetic materials Evans et al. (1991); Lakes (1987); Smith \& Wojciechowski (2008).

Ferromagnetic resonance experiment (FMR) (Vleck, 1950) is one of the basic tools to study the magnetic properties of magnetic agglomerates in viscoelastic nonmagnetic polymer matrix. As a particular example, we consider the FMR experiment with the $\gamma-\mathrm{Fe}_{2} \mathrm{O}_{3}$ (maghemite) ferrimagnetic nanoparticles embedded in a multiblock poly(ether-ester) copolymer nonmagnetic matrix which has been studied both experimentally (Guskos et al., 2006; 2008) and theoretically (Dudek et al., 2010). However, the obtained results are general and applicable to other nanoparticles and other viscous materials. Note that in medical applications magnetic iron oxides are used due to their low toxicity to human. Their saturation magnetization is practically equal to the bulk value at high temperatures, with negligible coercivity and no exchange bias below the blocking temperature. These properties of the iron oxide magnetic nanoparticles suggest nearly perfect nanocrystals without significant structural disorder (Dutta et al., 2004). 


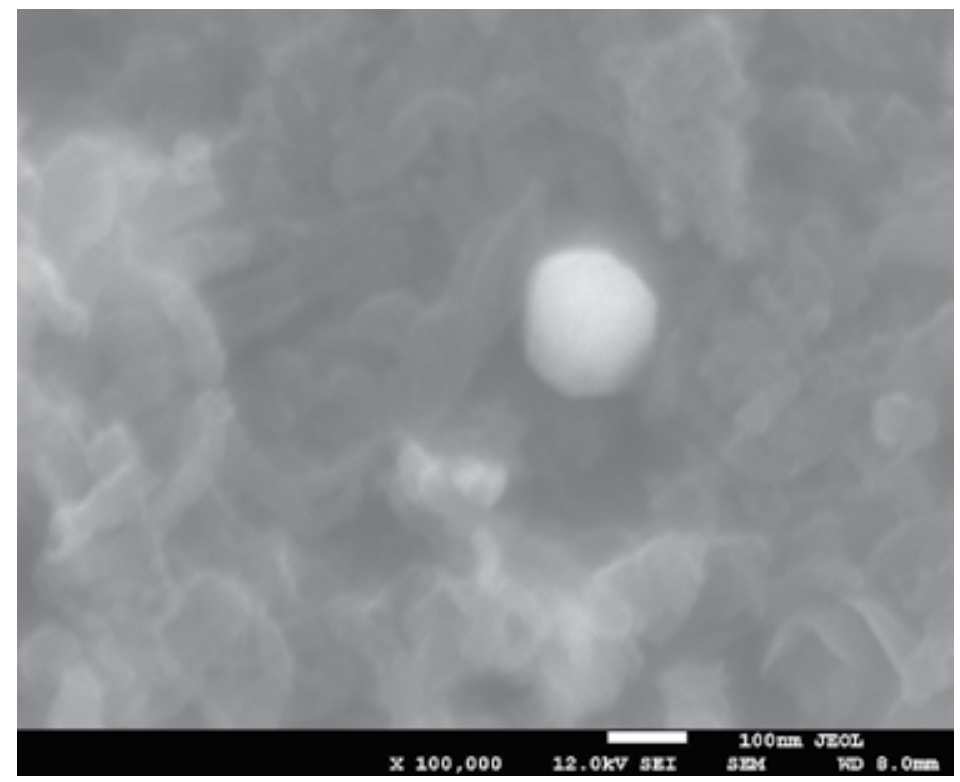

Fig. 1. SEM picture: an example of stucking a single magnetic nanoparticle in a pore - here, a carbon coated nickel nanoparticle in the porous sodium borosilicate glass.

The peculiar feature of the synthesized PEN-block-PTMO copolymer is that the magnetic fillers form agglomerates numbering from several to tens of nanoparticles. In the agglomerates the interparticle dipole - dipole magnetic interaction becomes important as well as the interaction of the magnetic nanoparticles with a non-magnetic matrix. Although the agglomerates are uniformly dispersed in the matrix their FMR spectra show additional peaks in low temperatures which originate from the orientational anisotropy of the frozen polymer blocks. The orientational dependence of the FMR spectra has been found earlier by Owens (Owens, 2003) for a colloidal suspension of $\gamma-\mathrm{Fe}_{2} \mathrm{O}_{3}$ nanoparticles which have been solidified in a static magnetic field (dc magnetic field). Similar observation has been found theoretically in a recent paper by Sukhov et al. (Sukhov et al., 2008). There is very instructive discussion on the shape of the ferromagnetic resonance spectra for the ensemble of the randomly distributed magnetic anisotropy axes as well as the discussion of the dependence of these spectra on temperature in terms of a stochastic model. The model is restricted to the case when the orientation of each anisotropy axis is frozen during computer simulation but it shares many features common with the experimental results, like the broadening of the FMR signal for the randomly distributed magnetic anisotropy axes as compared to the magnetic nanoparticles which all have the same orientation of the magnetic anisotropy. In paper (Dudek et al., 2008) it has been shown directly that blocking the rotational freedom of the magnetic nanoparticles, e.g. when the nanoparticles are stuck in the pores as it is suggested in Fig. 1, can produce additional resonance peaks in the FMR spectrum. In the latter case stochastic equations were used both for the magnetic nanoparticles magnetization and the rotational oscillations of the magnetic nanoparticles as a whole. The influence of the magnetic anisotropy orientation and temperature on the FMR spectra of magnetic agglomerates in polymer matrix was discussed in (Dudek et al., 2010). The most important property of the FMR spectrum depending on temperature will be discussed in the sections below. 


\section{Modeling ferromagnetic resonance experiment}

Theoretical basis of ferromagnetic resonance can be found in the paper by van Vleck (Vleck, 1950) in which a magnetic resonance condition (Kittel's formula) for ferromagnetic materials has been derived with the help of a simple quantum model. It has been noted in the paper the importance of the effect of magnetic anisotropy on the resonance frequency. In our considerations we restrict to the case when an uniaxial anisotropy is the dominating magnetic anisotropy of the magnetic nanoparticles (Shliomis, 1975). Then, the term magnetic anisotropy axis is substituted for the easy axis of magnetization. The magnetization of magnetic nanoparticles changes after an external magnetic field is switched on and there are two mechanisms of this change: the reorientation process of magnetic nanoparticle as a whole (Brownian motion) and the Néel relaxation process of the magnetization itself. A magnetic nanoparticle, and by this the magnetic anisotropy axis, can rotate freely in a liquid carrier, but not in the case when the nanoparticle is part of a large agglomerate or its surrounding is a solid phase. The dominant interparticle interactions in the agglomerate are dipole interactions unless the nanoparticles do not form dense agglomerates where exchange interactions become important. So if we take into account the FMR experiment and we consider the agglomerate consisting of $\mathrm{N}$ single-domain magnetic nanoparticles, each of them experiences an effective magnetic field $\vec{H}_{\text {eff,i }}$ of the form (Füzi, 2006):

$$
\vec{H}_{\mathrm{eff}, \mathrm{i}}=\vec{H}_{\mathrm{dc}}+\vec{H}_{\mathrm{ac}}+\frac{H_{a}}{\left|\vec{M}_{i}\right|}\left(\vec{M}_{i} \cdot \vec{n}_{i}\right) \vec{n}_{i}+\vec{H}_{i, \text { dipole }}
$$

where $\vec{H}_{\mathrm{dc}}$ is the external direct current (dc) magnetic field, $\vec{H}_{\mathrm{ac}}=\vec{H}_{\mathrm{ac}}^{0} \cos (2 \pi f t)$ is the external alternating current (ac) magnetic field of frequency $f, \vec{H}_{i \text {,dipole }}$ represents dipolar magnetic field produced by the magnetic nanoparticles of the agglomerate

$$
\vec{H}_{i, \text { dipole }}=-\frac{1}{4 \pi} \sum_{j=1, j \neq i}^{N}\left(\frac{\vec{M}_{j}}{r_{i j}^{3}}-3 \frac{\left(\vec{M}_{j} \cdot \vec{r}_{j i}\right) \vec{r}_{j i}}{r_{j i}^{5}}\right),
$$

$\vec{M}_{i}$ denotes magnetization of the nanoparticle $i\left(M=M_{S} V\right.$ for the nanoparticle of volume $V$ and saturation magnetization $\left.M_{s}\right), r_{i j}$ is the distance between nanoparticles $i$ and $j, H_{a}$ represents the magnetic anisotropy field which is defined as

$$
H_{a}=\frac{2 K_{a}}{\mu_{0} M_{s}}
$$

where $K_{a}$ is magnetic anisotropy constant, and $\mu_{0}$ is constant of permeability. The symbol $\vec{n}_{i}$ in Eq. (1) is a unit vector along the magnetic anisotropy direction of the nanoparticle $i$ with the components

$$
\begin{aligned}
& n_{x, i}=\sin \left(\varphi_{i}\right) \cos \left(\theta_{i}\right), \\
& n_{y, i}=\sin \left(\varphi_{i}\right) \sin \left(\theta_{i}\right), \\
& n_{z, i}=\cos \left(\varphi_{i}\right) .
\end{aligned}
$$

and $\varphi_{i}$ and $\theta_{i}$ are the angles vector $\vec{n}_{i}$ makes with the $z$-axis and $x$-axis, respectively. 
A rotating external magnetic field $\vec{H}_{\mathrm{ac}}$ is transverse to $\vec{H}_{\mathrm{dc}}$. We assume that the external dc magnetic field is oriented in the $z$-direction and the external ac magnetic field in the $x$-direction (Jung et al., 2002). Then, for the effective magnetic field defined in Eq. (1) the ferromagnetic resonance condition can be expressed as follows:

$$
f=\frac{\gamma}{2 \pi} H_{\mathrm{eff}}
$$

where $\gamma=2.21 \times 10^{5} \mathrm{~s}^{-1}(\mathrm{~A} / \mathrm{m})^{-1}$ denotes the gyromagnetic ratio. In practice, the spectrometers EPR/FMR are built for one value of frequency $f$ and then the dc magnetic field becomes a parameter to be changed to get the resonance condition. In our case the ac magnetic field frequency $f=9.37 \mathrm{GHz}$. Note that even in the case of a single magnetic nanoparticle $(\mathrm{N}=1)$ its resonance frequency strongly depends on the orientation of the magnetic anisotropy axis with respect to the dc magnetic field direction.

It turns out that the magnetic nanoparticle's magnetization dynamics can be modeled with the help of the classical spin model which represents stochastic version of the Landau-Lifshitz equation ((Gilbert, 1955; Landau \& Lifshitz, 1953)) :

$$
\frac{d \vec{M}_{i}}{d t}=-\gamma \vec{M}_{i} \times\left[\vec{H}_{\mathrm{eff}, \mathrm{i}}+\vec{B}_{i}\right]-\alpha \frac{\gamma}{M_{s} V} \vec{M}_{i} \times\left(\vec{M}_{i} \times\left[\vec{H}_{\mathrm{eff}, \mathrm{i}}+\vec{B}_{i}\right]\right),
$$

where $i=1,2, \ldots, N$, and $\alpha$ denotes the damping constant. The symbol $\vec{B}_{i}$ represents the white-noise field fluctuations (e.g. (Jönsson, 2003), (Usadel, 2006)). Then the thermal averages of $\vec{B}_{i}=\left(B_{x, i}, B_{y, i}, B_{z, i}\right)$ fulfill the relations:

$$
\begin{gathered}
\left\langle B_{q, i}(t)\right\rangle=0, \quad q=x, y, z, \\
\left\langle B_{q, i}(t) B_{p, i}\left(t^{\prime}\right)\right\rangle=\frac{2 \alpha k_{B} T}{\gamma M_{s} V} \delta_{q, p} \delta\left(t-t^{\prime}\right), \quad p=x, y, z .
\end{gathered}
$$

The magnetic properties of the magnetic nanoparticles can be described with the help of the solutions $\vec{M}_{i}(t)$ of this set of equations. They strongly depend on the magnetic anisotropy axis orientation. In particular, the shape of the magnetic hysteresis loop can change from the almost square like to the case when it vanishes depending on the orientation of the external dc magnetic field with respect to the orientation of the magnetic anisotropy axis. This can be seen in Fig. 2 and Fig. 3 in which the magnetic hysteresis loops are presented for the $z$-component of the nanoparticle's magnetization in the case when the anisotropy axis orientation oscillations are close to the $z$-direction (parallel to dc magnetic field) and close to the $x$-direction (transverse to dc magnetic field), respectively. The hysteresis loops, which are shown in the figures, result from the computer simulation of a simplified model of a carbon coated magnetic nanoparticle where the coating is represented by $\mathrm{C}_{60}$ molecule. In the model, the magnetic nanoparticle is represented by magnetic anisotropy axis and its magnetization follows the Landau-Lifshitz equation (Eq. (8)). The carbon atoms in $\mathrm{C}_{60}$ molecule vibrate according to the molecular dynamics method. The rotational oscillations of a fullerene (and by this the magnetic anisotropy axis rotations) are harmonically bonded to the $z$-direction and $x$-direction, respectively, with a given spring constant. The latter means that the magnetic nanoparticle cannot rotate freely. The larger temperature is the larger the rotational oscillations are. Besides the spring force the anisotropy axis experiences the 


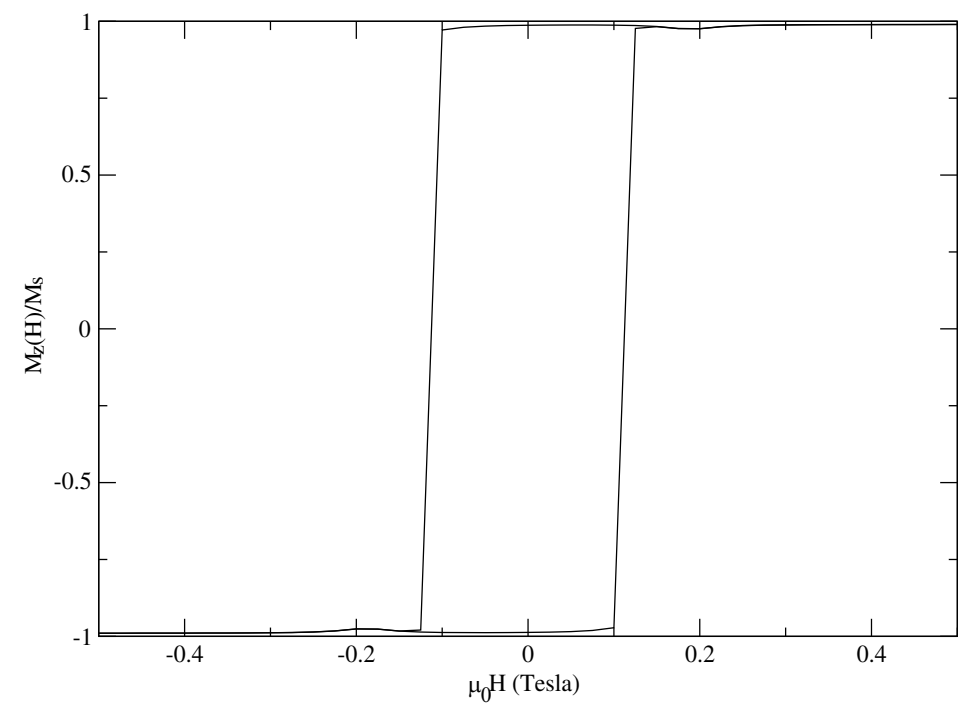

Fig. 2. Magnetic hysteresis loop of the $z$-component of the magnetic nanoparticle's magnetization in the case when the orientation of the magnetic anisotropy axis undergoes the small oscillations around the $z$-direction (parallel to the external dc magnetic field). Some parameters of the computer simulation: demagnetizing factor in shape anisotropy $D=0.15$ $\left(K_{a}=\mu_{0}(1-3 D) M_{s}^{2} / 4\right), \alpha=0.066, R=2 \mathrm{~nm}, M_{s}=450 \mathrm{kA} / \mathrm{m}, \mathrm{T}=10 \mathrm{~K}$.

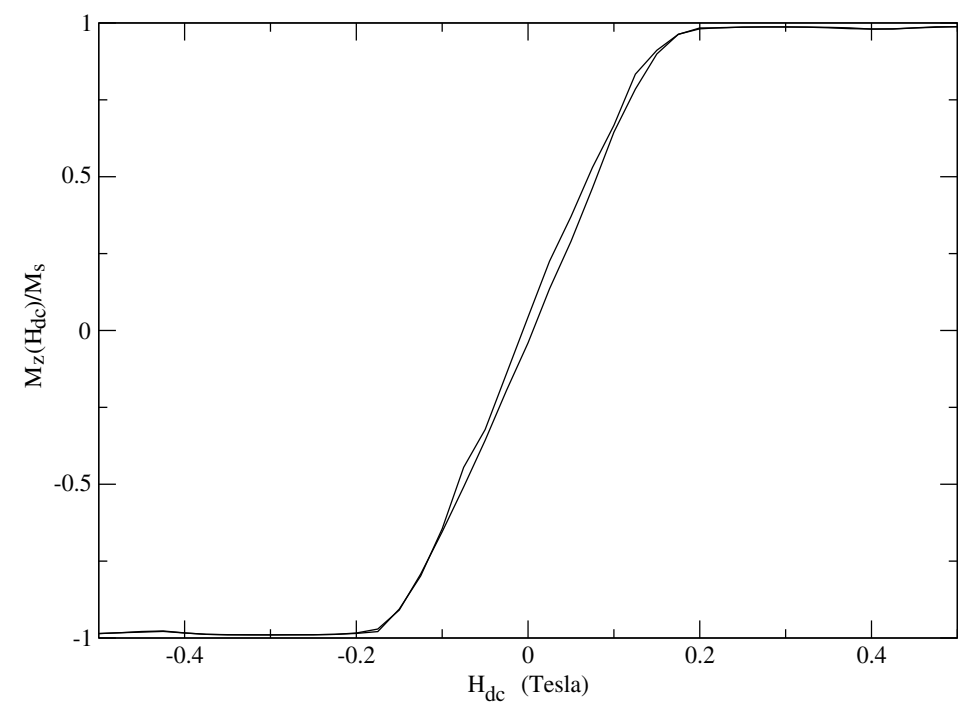

Fig. 3. The same as in Fig. 2 but the magnetic anisotropy axis orientation oscillates around the $x$-direction (transverse to the external dc magnetic field). 
magnetic torque which is represented by two opposite point forces (Dudek et al., 2010) applied to the anisotropy axis with the strength

$$
|\vec{F}|=\frac{1}{R}\left|K_{a} V \sin (2 \Psi)\right|
$$

where $R$ is the fullerene's radius, and the greater the angle $\Psi$ between the easy axis, represented by vector $\vec{n}$, and magnetization $\vec{M}$, the greater the magnetic torque. The model of the magnetic nanoparticles used in the computer simulations has been shown in Fig. 4 in the case when the magnetic anisotropy axis is, respectively, parallel and perpendicular to the external dc magnetic field.
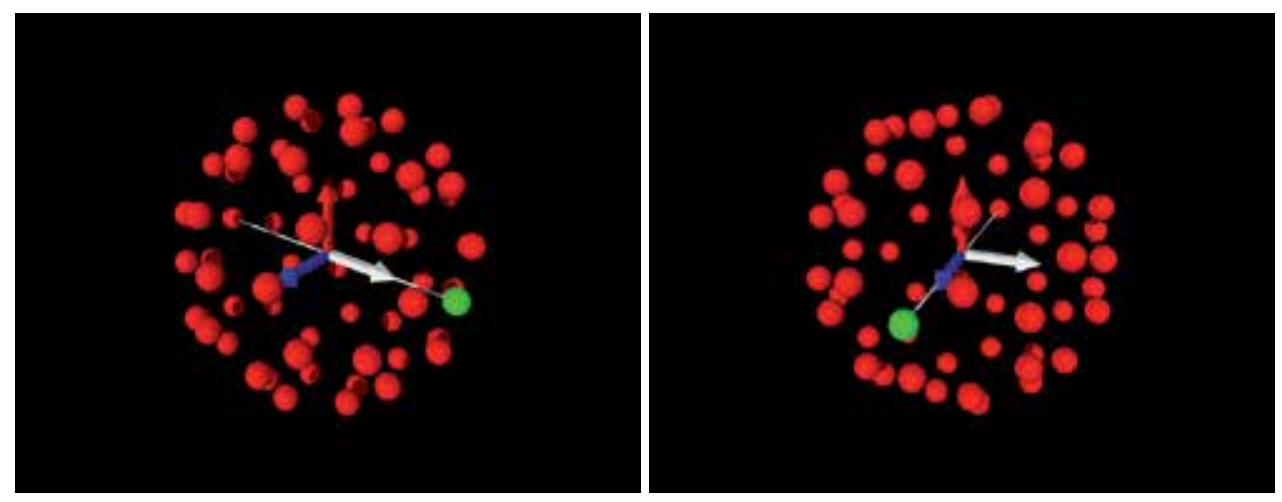

Fig. 4. Model of carbon coated magnetic nanoparticle in the case when the coating is represented by $\mathrm{C}_{60}$ molecule. The magnetic nanoparticle is represented by the anisotropy axis which, in the model, passes through the center of the fulleren and the carbon atom painted green, in the figure. The magnetic nanoparticle was not drawn with clarity reasons. The cartesian coordinate system has been plotted and the blue axis represents the $z$-direction which is the direction of the external dc magnetic field and the white axis represents the $x$-direction.

The same features of the magnetic hysteresis loops as those presented in Fig. 2 and Fig. 3 can be observed in magnetic nanowires, e.g. (Sorop et al., 2004), where the similar relationship between the shape anisotropy of a nanowire and the external dc magnetic is observed. In low temperatures, much below $100 \mathrm{~K}$, the large agglomerates of the $\gamma-\mathrm{Fe}_{2} \mathrm{O}_{3}$ (maghemite) ferrimagnetic nanoparticles embedded in a multiblock poly(ether-ester) copolymer nonmagnetic matrix Guskos et al. $(2006 ; 2008)$ are practically frozen into the matrix with a random orientation of the magnetic anisotropy axes. Then, the observed magnetic hysteresis loop represents the averaged one which is approximately a mixture of the cases discussed in Fig. 2 and Fig. 3. It is worth to add that in high temperatures, where the block copolymer is dissolved, the magnetic properties resemble the properties of ferrofluids and there is no observed magnetic hysteresis loop.

Much more information on the magnetic properties of the polymers filled with nanoparticles can be get from the analyses of the absorption lines in FMR experiment. They can be represented by the imaginary part of the dynamic magnetic susceptibility

$$
\chi=\chi^{\prime}-i \chi^{\prime \prime},
$$


where $\chi^{\prime \prime}$ denotes total hysteresis losses per volume of magnetic nanoparticle through a cycle of the magnetization. For the chosen magnetic fields $H_{z}=H_{d c}$ and $H_{x}=H_{a c}$ the components of the complex ac susceptibility (Eq. (12)) can be calculated by performing the Fourier transform on the time averaged $x$-component of the magnetization, i.e.,

$$
\chi=\frac{1}{\tau H_{\mathrm{ac}}^{0}} \int_{0}^{\tau} d t M_{x}(t) e^{-i 2 \pi f t},
$$

where $\tau=1 / f$. In the case of theoretical modeling, the values $M_{x}(t)$ can be obtained from the Landau-Lifshitz equation (Eq. 8). In real FMR experiments, the absorption lines derivatives, $d \chi^{\prime \prime} / d H_{\mathrm{dc}}$ (the derivative of the out-of-phase susceptibility) are measured instead of direct measuring $\chi^{\prime \prime}$. In Fig. 5 there are presented the absorption lines derivatives obtained for the
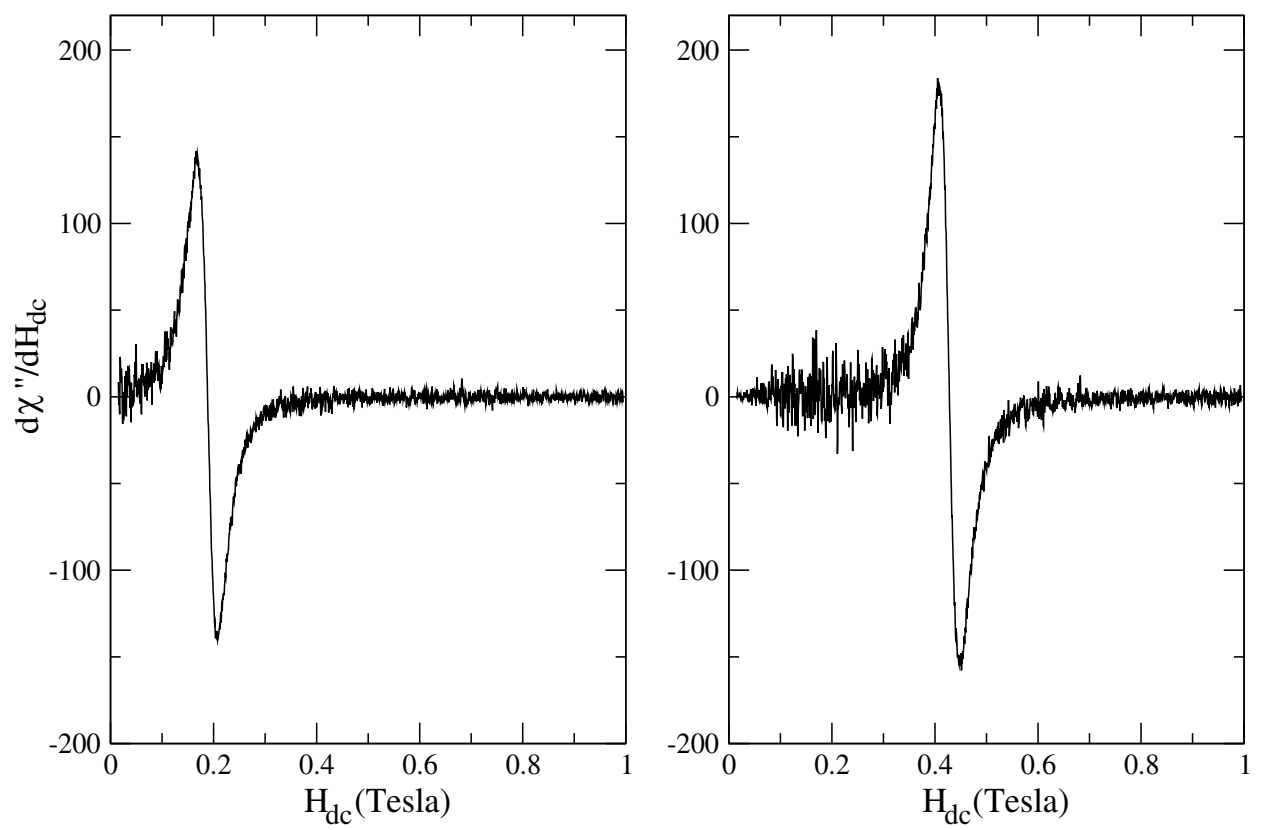

Fig. 5. Absorption lines derivatives, $d \chi^{\prime \prime} / d H_{\mathrm{dc}}$ resulting from the computer simulations in the case when the magnetic anisotropy axis orientation oscillates around the direction parallel and transverse to the external dc magnetic field. The parameters of the computer simulation are the same as in Fig. 2.

model of the carbon coated nanoparticles shown in Fig. 4 in the case when their magnetic anisotropy axis oscillations are controlled by the harmonic forces applied to the ends of the axis and the forces are coupled with the $z$-direcion and $x$-direction, respectively. Note that if the magnetic anisotropy axis is linked to the direction which is tranverse to the direction of $H_{\mathrm{dc}}$ then the corresponding resonance magnetic field $H_{r}$ becomes shifted to higher values of $H_{\mathrm{dc}}$ compared with the case when it is linked to the direction which is parallel. At the value of $H_{\mathrm{dc}}=H_{r}$ the dynamic susceptibility $\chi^{\prime \prime}$ takes its maximum value. It is worth emphasizing that the energy absorbed by the magnetic nanoparticles from the external AC magnetic field 
is proportional to $\chi^{\prime \prime}$. After the energy is converted into heat there is observed an increase $\Delta T$ of temperature which can be estimated at each cycle of the applied AC magnetic field with a given frequency $\omega=2 \pi f$ as follows (Sellmyer \& Skomski, 2006):

$$
\Delta T=\frac{\mu_{0} V H_{0}^{2}}{c m_{\text {ferro }}} f \chi^{\prime \prime}
$$

where $c$ is the average specific heat of carbon and magnetic nanoparticle $c=\left(c_{\text {carbon }}+\right.$ $\left.c_{\text {ferro }}\right) / 2, m_{\text {ferro }}$ represents mass of magnetic nanoparticle. The remote heating of the magnetic nanoparticles can be important in viscous materials in low temperatures for reorientation processes among the magnetic nanoparticles. This particular feature of the magnetic nanoparticles to posses the different values of $H_{r}$ for different orientations of their magnetic anisotropy axis (Fig. 5) becomes another interesting property of materials filled with magnetic nanoparticles where the static magnetic field $H_{\mathrm{dc}}$ can be used as a remote switcher for the local heating different groups of nanoparticles.

\section{Temperature dependence of the spectral lines in viscous magnetic materials}

In the case when the magnetic nanoparticles are placed in a viscous material for which the viscosity varies significantly depending on temperature, magnetic properties of such materials are also beginning to significantly depend on temperature. This special property of viscous magnetic materials has been studied experimentally for maghemite nanoparticles embedded in a multiblock poly(ether-ester) copolymer nonmagnetic matrix Guskos et al. (2006; 2008). The experiments were performed in a wide range of temperatures, 3.5-288 K. In addition to the experimental results were also carried out theoretical studies Dudek et al. (2010). Several examples of spectral lines discussed in Dudek et al. (2010) are presented in Fig. 6 for concetration of $0.1 \%$ of $\gamma-\mathrm{Fe}_{2} \mathrm{O}_{3}$ nanoparticles dispersed in the polymer matrix. The figure shows the completely different FMR spectra in the range of low temperatures and high temperatures. These two ranges of temperatures are also evident in the resonance field $H_{r}$ (Fig. 7) as a function of temperature, where at low temperatures a marked decrement of $H_{r}$ is observed. In the latter case the experimental results for two different concentrations of magnetic nanoparticles are presented and up to $50 \mathrm{~K}$ there is no signicant difference between them. This could mean that the thermal properties of non-magnetic matrix, in this case of a multiblock poly(ether-ester) copolymer, are of decisive importance for the magnetic properties of the magnetic agglomerates and not the reorientation processes between magnetic nanoparticles.

In low temperature region we have solid-like nonmagnetic matrix where magnetic relaxation takes place through the process of magnetization relaxation (Neél relaxation). Once the magnetic anisotropy axes are oriented randomly some additional peaks appear on the spectral lines at the higher values of $H_{\mathrm{dc}}$. This property of spectral lines is shown in the previous section on the example of a single magnetic nanoparticle. The presence of many magnetic agglomerates consisting of different numbers of magnetic nanoparticles is one of the mechanisms for observing the broadening of the spectral lines. At higher temperatures the magnetic relaxation takes place both through the magnetization relaxation and rotation of the whole nanoparticle in a nonmagnetic surrounding and the additional peaks observed on spectral lines at low temepratures do vanish.

A simplified theoretical model was constructed in (Dudek et al., 2010) where a cluster consisting of $N$ magnetic nanograins is placed randomly into a non-magnetic polymer matrix. 


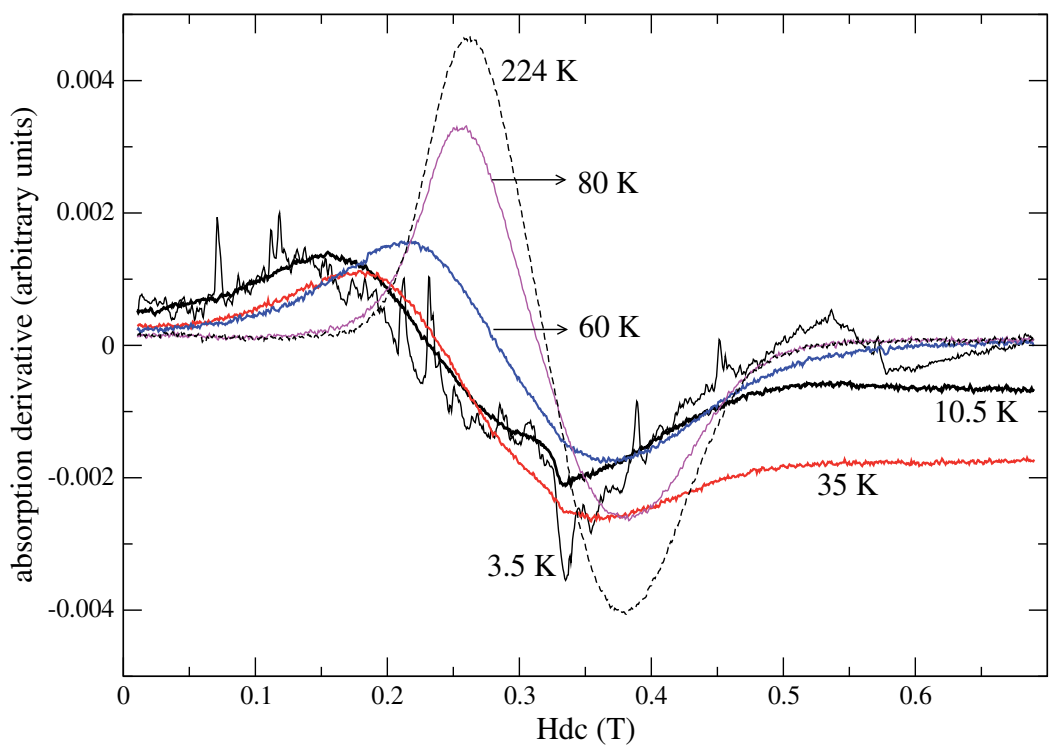

Fig. 6. The temperature dependence of $d \chi^{\prime \prime} / d H_{\mathrm{dc}}$ for $0.1 \% \gamma-\mathrm{Fe}_{2} \mathrm{O}_{3}$ dispersed in PEN-block-PTMO matrix.

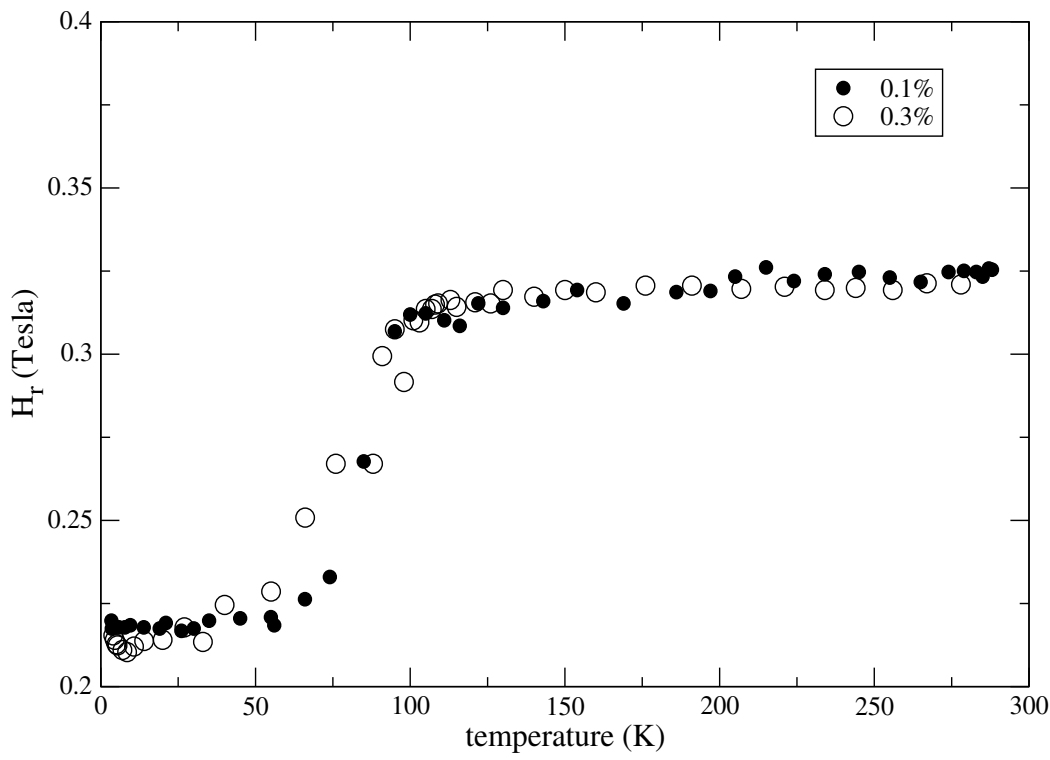

Fig. 7. An example of the dependence of the resonance field $H_{r}$ on temperature for magnetic nanoparticles $\gamma-\mathrm{Fe}_{2} \mathrm{O}_{3}$ in PEN-block-PTMO matrix. The plots correspond to two concentrations of nanoparticles of $0.1 \%$ and $0.3 \%$. 
They occupy a permanent position but may rotate. Each of the magnetic nanograins $i=$ $1,2, \ldots, N$ has magnetization $M_{i}$ which dynamics is described with the help of the stochastic version of the Landau-Lifshitz equation in Eq. (8). The rotational dynamics of magnetic nanoparticles is described with the help of the Langevin equations for the magnetic anisotropy axis orientation. These equations take the following form (Dudek et al., 2010; 2008):

$$
\begin{gathered}
\frac{d \varphi_{i}}{d t}=-\frac{2}{R \xi}\left|K_{a} V \sin \left(2 \psi_{i}\right)\right| \sin \left(\varphi_{i}-\varphi_{i}^{\prime}\right)-\frac{K_{\mathrm{el}}}{\xi} \sin \left(\varphi_{i}-\varphi_{0, i}\right)+\frac{1}{\xi} \lambda_{\varphi_{i},} \\
\frac{d \theta_{i}}{d t}=-\frac{2}{R \xi}\left|K_{a} V \sin \left(2 \psi_{i}\right)\right| \sin \left(\theta_{i}-\theta_{i}^{\prime}\right)-\frac{K_{\mathrm{el}}}{\xi} \sin \left(\theta_{i}-\theta_{0, i}\right)+\frac{1}{\xi} \lambda_{\theta_{i}} .
\end{gathered}
$$

in the diffusion limit, where $\xi$ represents the friction of the $i$-th nanoparticle in the elastic non-magnetic polymer matrix and $\lambda_{\varphi, i}$ and $\lambda_{\theta, i}$ represent the white-noise driving torque (Coffey et al., 1984; Gardiner, 1983) for $i$-th nanoparticle, and $K_{\mathrm{el}}$ represents the spring constant which controls the rotational oscillations of the magnetic anisotropy axis. In the above stochastic equations the thermal rotational fluctuations of the $i$-th magnetic nanoparticle are characterized by temperature $T$ and $\lambda_{\varphi_{i}}$ and $\lambda_{\theta_{i}}$ and they fulfill the relations:

$$
\begin{gathered}
\left\langle\lambda_{q}(t)\right\rangle=0 \\
\left\langle\lambda_{q}(t) \lambda_{q^{\prime}}\left(t^{\prime}\right)\right\rangle=2 k_{B} T \xi \delta\left(t-t^{\prime}\right),
\end{gathered}
$$

where $q=\varphi_{i}, \theta_{i}$. The angles $\varphi^{\prime}$ and $\theta^{\prime}$ represent the angles which the magnetization $\vec{M}$ makes with $z$-axis and $x$-axis, and the angles $\varphi_{0}$ and $\theta_{0}$ are the initial angles of the easy axis after the magnetic nanoparticle has been built into polymer matrix. The numerical scheme applied to the stochastic equations in (Dudek et al., 2010) is the Euler-Maruyama method. The theoretical model introduced in (Dudek et al., 2010) reproduces qualitatively the results of the experiment (Guskos et al., 2006; 2008). In particular, the FMR spectrum and the dependence of $H_{r}$ on temperature have qualitatively the same properties. It is evident from Fig. 8 and Fig. 9 if we compare them with Fig. 6 and Fig. 7.

The results of the theoretical model have been obtained by two assumptions. The first one is assuming an empirical model for the viscosity parameter $v$, the Arrhenius law,

$$
v(T)=v_{0} \mathrm{e}^{\mathrm{E} / \mathrm{k}_{\mathrm{B}} \mathrm{T}}
$$

where $\mathrm{E}$ is the activation energy. In the model, the viscosity parameter $v(T)$ is related to the rotational friction parameter $\xi$ of magnetic nanoparticles in a polymer surounding (used in Eqs. (15) and (16)), as follows:

$$
\xi=8 \pi v(T) r^{3},
$$

where $r=R / 2$ denotes the radius of a sphere representing magnetic nanoparticle and its polymer coating. Hence the simple assumption in Eq. (19) and not the presence of a phase transition is responsible for the qualitatively different behavior of $H_{r}$ in the low and high temperatures in the theoretical model ( Fig. 9).

The second assumption is introducing the Bloch law approximation

$$
M_{S}(T)=M_{0}(0)\left(1-\left(\frac{T}{T_{0}}\right)^{\delta}\right)
$$




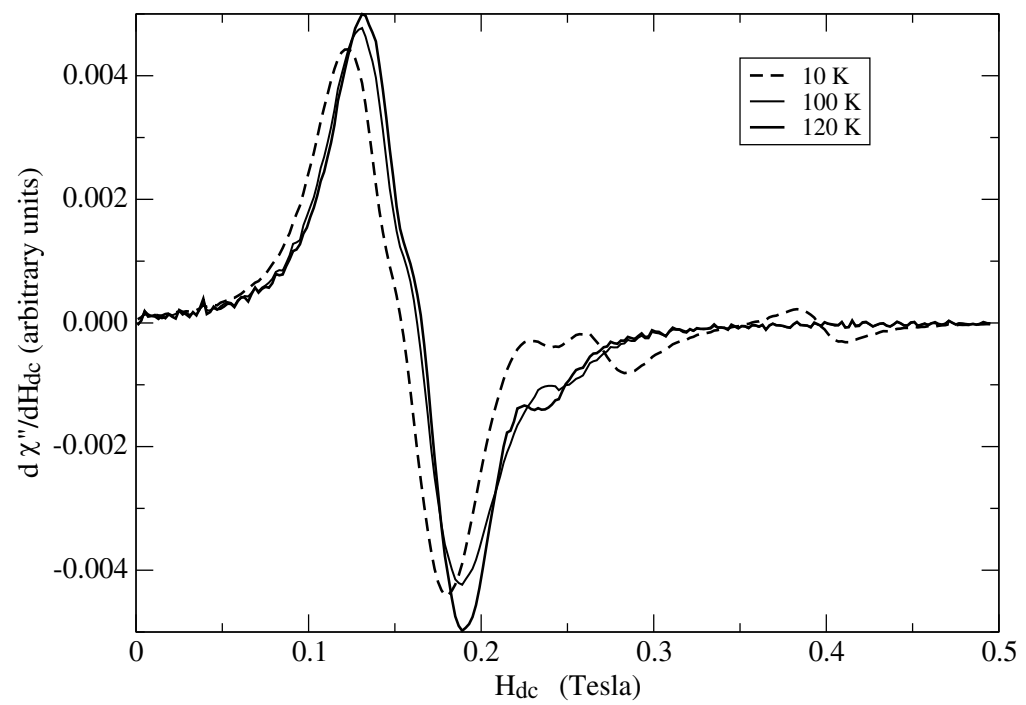

Fig. 8. Computer simulations of the temperature dependence of $d \chi^{\prime \prime} / d H_{\mathrm{dc}}$ calculated for $\mathrm{N}=30$ magnetic nanoparticles in the case when their magnetic anisotropy axes are randomly oriented (Dudek et al., 2010). The parameters of the computer simulation are the same as in Fig. 2.

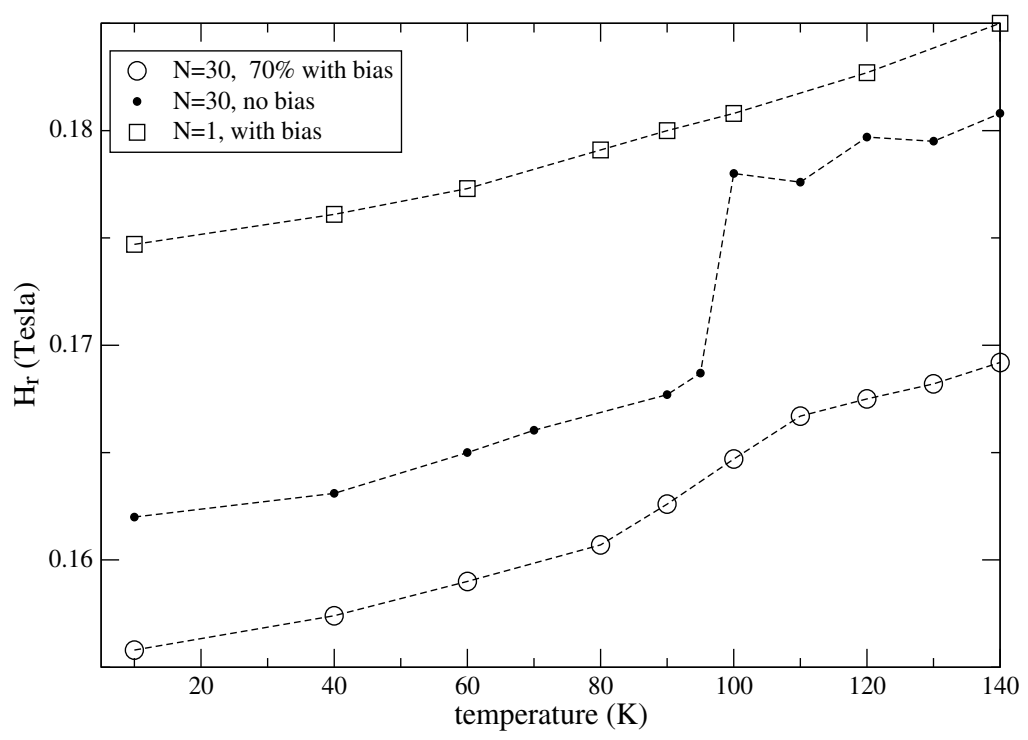

Fig. 9. Computer simulations of the dependence of the resonance field $H_{r}$ on temperature for agglomerates of magnetic nanoparticles (Dudek et al., 2010). The agglomerates consisting of $N=30$ magnetic nanoparticles represent two cases: when all magnetic nanoparticles are randomly oriented and when $70 \%$ of them is aligned with the dc magnetic field. In the case of a single magnetic nanoparticle $(\mathrm{N}=1)$ its magnetic anisotropy axis is aligned with the dc magnetic field. 
for the magnetization of the magnetic nanoparticles where $\mathrm{T}$ is temperature, $\delta=1 / 3$ and $T_{0}$ is some constant. The value of $\alpha$ is a parameter of the model under consideration. Another value of $\alpha$ can be also found in publications on magnetic materials.

The complexity of the FMR spectral lines can be seen in the example in Fig. 10 where the absorption lines derivatives $d \chi^{\prime \prime} / d H_{\mathrm{dc}}$ have been plotted for a single magnetic nanoparticle in the case when its easy magnetic axis oscillates around the direction perpendicular to the external dc magnetic field in a surrounding with temperature-dependent viscosity $v(T)$. In low temperatures the magnetic resonance field $H_{r}$ moves towards the lower values of $H_{\mathrm{dc}}$ with increasing temperature instead to move towards the higher values of $H_{\mathrm{dc}}$ as it is in the case of magnetic nanoparticles oscillating around the direction of the dc magnetic field. Only above a certain temperature there is no qualitative difference in the FMR spectrum for magnetic nanoparticles with magnetic easy axis oriented parallel or perpendicular to the external dc magnetic field.

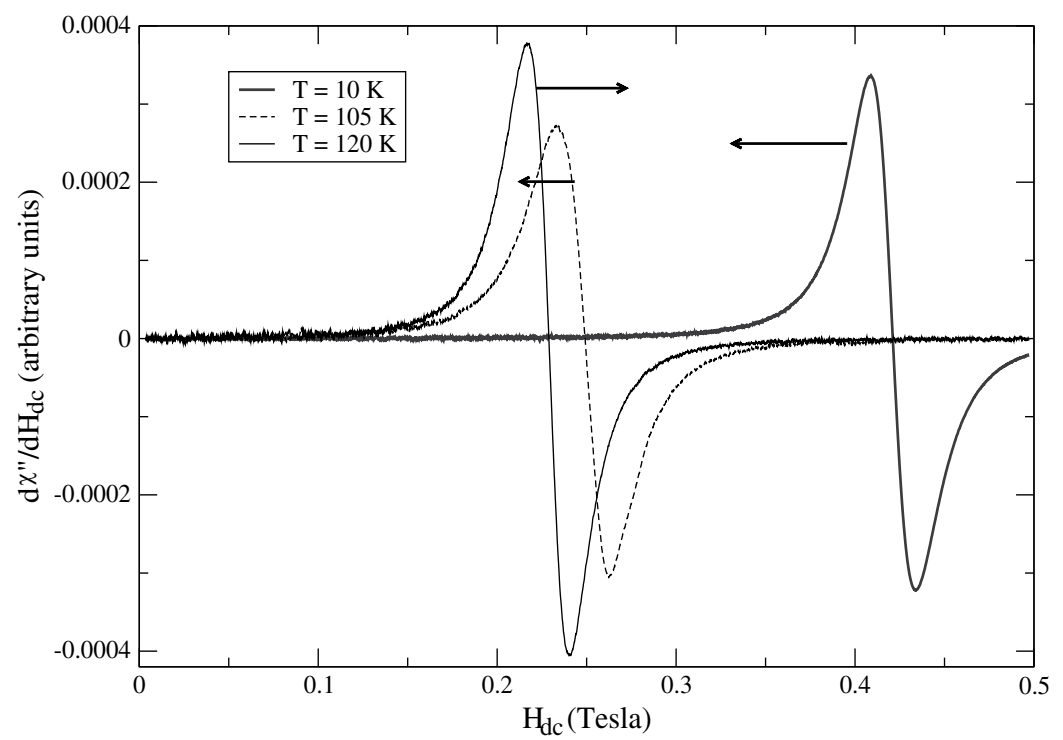

Fig. 10. Absorption lines derivatives, $d \chi^{\prime \prime} / d H_{\mathrm{dc}}$ in theoretical model (Dudek et al., 2010) for a single $(N=1)$ magnetic nanograin in the case when its magnetic anisotropy axis oscillates around the direction transverse to the direction of the external dc magnetic field. The plotted curves correspond to temperatures $T=10,105,120 K$, respectively. In low temperatures the magnetic resonance field $H_{r}$ moves towards the lower values of $H_{\mathrm{dc}}$ with increasing temperature and only above a certain temperature it begins to move toward the higher values of $H_{\mathrm{dc}}$.

In the case of magnetic agglomerates dispersed in a viscous medium and which consist of a large number of magnetic nanoparticles with randomly oriented axes relative to the field $H_{\mathrm{dc}}$ the mechanism shown in Fig. 10 can be important in low temperatures.

\section{Conclusions}

Both the discussion in section 2 and FMR spectrum in Fig. 5 show that the static magnetic field $H_{\mathrm{dc}}$ can be used as a remote switcher for the local heating different groups of nanoparticles 
corresponding to different orientations of their magnetic easy axis. This property of the dependence of a maximum of $\chi^{\prime \prime}$ on the orientation of the magnetic anisotropy axis with respect to the external dc magnetic field may be useful in designing new materials such as multi-functional magnetic nanocapsules. The thermal effects on the FMR spectrum in polymer composites filled with magnetic nanoparticles provide additional information about the magnetic structure of the material.

\section{Acknowledgments}

Some of the computer simulations have been performed in Wroclaw Centre for Networking and Supercomputing, Poland.

\section{References}

Coffey, W., Evans, M. \& Grigolini, P. (1984). Molecular Diffusion and Spectra, Wiley, New York.

Dudek, M., N.Guskos, Senderek, E. \& Rosłaniec, Z. (2010). Temperature dependence of the fmr absorption lines in viscoelastic magnetic materials, J. Alloy Compd. Vol.504: 289-295.

Dudek, M. R., Guskos, N., Grabiec, B. \& Maryniak, M. (2008). Magnetization dynamics in landau-lifshitz-gilbert formulation. fmr experiment modeling, J. Non-Cryst. Solids Vol.354: 4146-4150.

Dudek, M. \& Wojciechowski, K. (2008). Magnetic films of negative poisson's ratio in rotating magnetic fields, J. Non-Cryst. Solids Vol.354: 4304-4308.

Dutta, P., Manivannan, A., Seehra, M. S., Shah, N. \& Huffman, G. P. (2004). Magnetic properties of nearly defect-free maghemite nanocrystals, Phys. Rev. B Vol.70: 174428.

Evans, K., Nikansah, M., Hutchinson, I. \& Rogers, S. (1991). Molecular network design, Nature Vol.353: 124.

Füzi, J. (2006). Magnetic characteristics of dipole clusters, Physica B Vol.372: 239-242.

Gao, J. \& Xu, B. (2009). Applications of nanomaterials inside cells, Nano Today Vol.4: 37-51.

Gardiner, C. (1983). Handbook of Stochastic Methods for Physics, Chemistry and the Natural Sciences, Springer-Verlag.

Gilbert, T. (1955). A lagrangian formulation of the gyromagnetic equation of the magnetization field, Phys. Rev. Vol.100: 1243.

Guskos, N., Glenis, S., Likodimos, V., Typek, J., Maryniak, M., Rosłaniec, Z., Kwiatkowska, M., Baran, M., Szymczak, R. \& Petridis, D. (2006). Matrix effects on the magnetic properties of $\gamma-\mathrm{fe}_{2} \mathrm{O}_{3}$ nanoparticles dispersed in a multiblock copolymer, J. Appl. Phys. Vol.99: 084307.

Guskos, N., Likodimos, V., Glenis, S., Maryniak, M., M.Baran, Szymczak, R., Roslaniec, Z., Kwiatkowska, M. \& Petridis, D. (2008). Magnetic properties of $\gamma$-fe $\mathrm{fe}_{2} \mathrm{O}_{3} /$ poly(esther-ester) copolymer nanocomposites, Nanosci. Nanotech. (No.8): 2127.

Jönsson, P. E. (2003). Superparamagnetism and spin glass dynamics of interacting magnetic nanoparticle systems, arXiv:cond-mat/0310684v2 .

Jung, S., Ketterson, J. \& Chandrasekhar, V. (2002). Micromagnetic calculations of ferromagnetic resonance in submicron ferromagnetic particles, Phys. Rev. B Vol.66: 132405.

Lakes, R. (1987). Foam structures with a negative poisson's ratio, Science Vol.235: 1038-1040.

Landau, L. \& Lifshitz, E. (1953). On the theory of the dispersion of magnetic permeability in ferromagnetic bodies, Phys. Z. Sowjetunion Vol.8: 153. 
Liu, T.-Y., Hu, S.-H., Liu, D.-M., Chen, S.-Y. \& Chen, I.-W. (2009). Biomedical nanoparticle carriers with combined thermal and magnetic responses, Nano Today Vol.4: 52-65.

Owens, F. J. (2003). Ferromagnetic resonance of magnetic field oriented fe3o4 nanoparticles in frozen ferrofluids, J. Phys. Chem. Solids Vol.64: 2289-2292.

Sellmyer, D. \& Skomski, R. (2006). Advanced Magnetic Materials, Springer Science+Business Media, Inc., Place of publication.

Shliomis, M. I. (1975). Magnetic fluids, Sov. Phys. Usp. Vol.17: 153-169.

Smith, C. \& Wojciechowski, K. (2008). Preface: phys. stat. sol. (b), Phys. Status Solidi b Vol.245: 486-488.

Sorop, T., Nielsch, K., Göring, P., Kröll, M., Blau, W., Werspohn, R., Gösele, U. \& de Jongh, L. (2004). Study of the magnetic hysteresis in arrays of ferromagnetic fe nanowires as a function of the template filling fraction, J. Magn. Magn. Mater. Vol.272-276: 1656-1657.

Sukhov, A., Usadel, K. \& Nowak, U. (2008). Ferromagnetic resonance in an ensemble of nanoparticles with randomly distributed anisotropy axes, J. Magn. Magn. Mater. Vol.320: 31-35.

Usadel, K. D. (2006). Temperature-dependent dynamical behavior of nanoparticles as probed by ferromagnetic resonance using landau-lifshitz-gilbert dynamics in a classical spin model, Phys. Rev. B Vol.73: 212405.

Vleck, J. V. (1950). Concerning the theory of ferromagnetic resonance absorption, Phys. Rev. Vol.78: 266-274.

Wilson, J., Poddar, P., Frey, N., Srikanth, H., Mohomed, K., Harmon, J., Kotha, S. \& Wachsmuth, J. (2004). Synthesis and magnetic properties of polymer nanocomposites with embedded iron nanoparticles, J. Appl. Phys. Vol.95: 1439-1443.

Wood, D. \& Camp, P. (2011). Modeling the properties of ferrogels in uniform magnetic fields, Phys. Rev. E Vol.83: 011402.

Zahn, M. (2001). Magnetic fluid and nanoparticle applications to nanotechnology, J. Nanopart. Res. Vol.3: 73-78. 


\title{
Nanoparticle Dynamics in Polymer Melts
}

\author{
Giovanni Filippone and Domenico Acierno \\ Dept. of Materials and Production Engineering, University of Naples Federico II
}

Italy

\section{Introduction}

Adding solid particles to polymeric materials is a common way to reduce the costs and to impart desired mechanical and transport properties. This makes polymers potential substitutes for more expensive non-polymeric materials. The advantages of filled polymers are normally offset by the increased complexity in the rheological behaviour of the resulting composite. Usually, a compromise has to be made between the benefits ensured by the filler, the increased difficulties in melt processing, the problems in achieving a uniform dispersion of the solid particulate, and the economics of the process due to the added step of compounding [Shenoy, 1999]. Filled polymers can be described as a suspension of particles and particle aggregates dispersed in the polymer matrix. Interactions between individual particles or aggregates and the matrix, as well as between particles, hinder the material deformability modifying both the solid- and melt-state behaviour of the host polymer. In polymer-based microcomposites, these effects only become significant at relatively high filler contents, i.e. when the filler particles are sufficiently close to each other to form a network that spans large sections of the polymer matrix. Over the last fifteen years, the same reinforcing and thixotropic effects have been observed with the use of very small amounts of inorganic nanoparticles, which has resulted in extensive research in the field of polymer-based nanocomposites (PNCs) [Usuki et al., 1993; Kojima et al., 1993]. In order to fully understand the exceptional properties of PNCs, the morphological and structural implications stemming from the nanometric sizes of the filler have to be taken into account. With respect to traditional microcomposites, nanocomposites show very high specific interface area, typically of order of $\sim 10^{2} \mathrm{~m}^{2} \mathrm{~g}^{-1}$. The matrix properties are significantly affected in the vicinity of the reinforcement, varying continuously from the interface towards the bulk polymer. As a consequence, the large amount of reinforcement surface area means that a relatively small amount of nanoscale reinforcement can have remarkable effects on the macroscale properties of the composite material.

A noticeable consequence of the nanometric dimensions of the filler is the extremely high numerical density of particles, or alternatively the very small inter-particles distances. If $\mathrm{N}$ monodisperse spherical particles with radius $r$ are randomly distributed in a volume $V$, the distance between the centres of the particles can be approximated to $h=(V / N)^{1 / 3}$. Introducing the particle volume fraction $\Phi=N v / V$, where $v=4 \Pi r^{3} / 3$ is the volume of the single particle, the wall-to-wall distance between contiguous particles is: 


$$
h=\left[\sqrt[3]{\frac{4 \pi}{3 \Phi}}-2\right] r
$$

Once fixed the filler content, $h$ linearly scales with $r$. In addition, we observe that, for diluted systems $(\Phi<0.1)$ such those we are interested in, Eq. 1 gives $h \sim 2 r$. This means that, if the filler particles are well dispersed within the host polymer, nanometric inter-particles distances are expected for nanocomposites. In such systems a large fraction of polymer is in contact with the filler. At the most, if the particle radius is of the same order as the mean radius of gyration of host polymer chains, $R_{g}$, each single chain potentially interacts with more than one nanoparticle, and there is no bulk polymer [Jancar \& Recman, 2010]. Two scenarios are possible when inter-particles distances are so small: if a good affinity exists between the polymer and the filler, then a polymer-mediated transient network between the particles set up [Ozmusul et al., 2005; Saint-Michel et al., 2003; Zhang \& Archer, 2002]; on the other hand, if the polymer-filler interactions are weak, the nanoparticles aggregate forming flocs, which eventually assemble into a space-spanning filler network [Filippone et al. 2009; Inoubli et al., 2006; Ren et al., 2000]. In both cases, the presence of a threedimensional mesostructure has a profound effect on the composite rheology.

The formation of the network, either polymer-mediated or formed by bare nanoparticles, originates from local rearrangements of the filler occurring in the melt both during flow and at rest. Nanoparticles, in fact, are subjected to relevant Brownian motion even in highly viscous mediums such as polymer melts. To get an idea about the relevance of such a phenomenon, we estimate the self-diffusion time of a spherical particle of radius $r, \tau_{s}$, that is the time required for the particle moves of a length equal to its radius [Russel et al., 1989]:

$$
\tau_{s}=\frac{6 \pi \eta_{s} r^{3}}{k_{B} T}
$$

Here $\eta_{s}$ is the viscosity of the suspending medium, $k_{B}$ is the Boltzmann's constant and $T$ is the temperature. For a simple low viscosity $\left(\eta_{s} \sim 10^{-2} \mathrm{~Pa}^{*} \mathrm{~s}\right)$ Newtonian liquid at room temperature, Eq. 2 gives the well-known result that particles of a few microns in size experience appreciable Brownian motions. Setting $T=400^{\circ} \mathrm{K}$ and $\eta_{s} \sim 10^{3} \mathrm{~Pa}^{*} \mathrm{~s}$ as typical values for melted polymers, we obtain the noteworthy result that particles of a few tens of nanometers display Brownian motions on timescales of order of a $101 \div 10^{2}$ seconds. Such durations are typically accessed in many transforming processes of the polymer industry, as well as during rheological analyses. The result is that, unlike polymer microcomposites, PNCs can be depicted as "living systems", in which the particles are free to move and rearrange in the melt, both in flow and even at rest, towards more favourable thermodynamic states. In this sense, PNCs are reminiscent of colloidal suspensions. Therefore, these simpler systems can be considered as the natural starting point to understand the much more complex rheological behaviour of PNCs.

The simplest case of colloidal dispersion is represented by a Newtonian suspension of hard spheres. The inter-particles interaction is zero at all separations and infinitely repulsive at contact. Coupled with thermal fluctuations, this kind of interaction results in a wide variety of possible structures. The suspension may behave like a gas, a liquid, a crystal or a glass depending on the particle volume fraction $\Phi$ [Pusey \& van Megen, 1986]. In the presence of 
interactions, this phase behaviour is modified due to interplay between $\Phi$ and the energy of interaction, $U$. We are mainly interested in weakly attractive colloidal dispersions, which are reminiscent of a large number of PNCs in which Van der Waals forces between nanoparticles and aggregates are of major importance. In such systems, aggregation results in disordered clusters of particles. These mesostructures may or may not span the whole space depending on $\Phi$ and $U$ [Prasad, 2003]. The rheological behaviour of weakly interacting colloidal dispersions can be rationalized with a simple two-phase model that combines the elasticity of the disordered particle network and the Newtonian viscosity of the suspending liquid [Cipelletti et al., 2000; Trappe \& Weitz, 2000; Trappe et al., 2001]. Despite the complexities stemming from the intrinsic non-Newtonian feature of polymer matrices, in this chapter we show that a similar approach can be successfully applied to a series of model PNCs with weak polymer-filler interaction. We emphasize that many PNCs of technological interest fall in this family. The two-phase model is validated through the building of a master curve of the elastic modulus of samples at different composition. A refinement of the model is also presented, which accounts for hydrodynamic effects. The dynamics of de-structuring and reforming of the filler network are studied by analysing the effects of large amplitude deformations. Besides simplifying the viscoelastic analysis of complex systems such as PNCs, the proposed approach can be extended to a wide variety of complex fluids where the elasticity of the components can be superimposed. In particular, the elastic modulus has been recently suggested to follow a universal behaviour with volume fraction also in case of interacting systems in which polymer bridging mechanisms exist [Surve et al., 2006]. This suggests a possible general feature for the proposed approach.

\section{Viscoelasticity and structure of PNCs}

We start our analysis dealing with the implications of Brownian motion in simple model systems constituted by polymer melts filled with small amounts of different kinds of spherical particles. Specifically, we discuss the effect of particle size and matrix viscosity on the ability of the filler to aggregate and eventually assemble in a three-dimensional network. Then, a two-phase model firstly proposed for weakly attractive particles suspended in a Newtonian medium is presented [Trappe \& Weitz, 2000]. The physical picture of an elastic particle network interspersed in a background fluid qualitatively accounts for the viscoelastic behaviour of the suspension. Afterwards, the relations between structure and viscoelasticity of two model PNCs is described in the framework of the two-phase model. A refinement of the model is therefore presented, which accounts for hydrodynamic effects successfully capturing the frequency dependent viscoelastic behaviour of simple PNCs. Finally, the dynamics of de-structuring and reforming of the filler network are studied by analysing the effects of large amplitude deformations.

\subsection{Brownian motion in polymer melts filled with nanoparticles - Gelation and ageing}

\subsubsection{Preliminary considerations}

Untreated inorganic particles are difficult to disperse in polymer matrices due to the typically poor polymer-filler affinity. Such incompatibility clearly emerges in the case of PNCs, where the specific surface of the particles is very high. The hydrodynamic forces 
developed during intense melt mixing processes breakup the large aggregates down to clusters of few tens of particles [Baird \& Collias, 1998]. Above the melting or glass transition temperature of the polymer matrix, however, these aggregates may reassemble into bigger structures because of the inter-particles attraction forces. Since the refractive indexes of polymers and inorganic fillers are typically very different, Van der Waals forces becomes of major importance leading to formation of aggregates and particle gels. The two most simple experimental techniques to follow the rearrangements of the filler in the melt are: (i) direct visualization of the particles through electron microscopy performed on solid samples; (ii) monitoring of rheological parameters sensitive to the material internal structure. Both these techniques have been applied to several model PNC systems constituted by polymer matrices filled with different kinds of inorganic nanoparticles with spherical symmetry. The rational for selecting such model systems originates from the intrinsic high complexity of other technologically relevant PNCs. The properties of such systems, often based on layered or tubular nanoparticles, are too sensitive to the state of dispersion of the filler and the wide variety of the possible nanostructures achievable during processing. The materials, the compounding procedures and the experimental details about the characterization of the composites are described in detail in the following paragraphs. Many of the results have been taken from papers previously published by our group, wherein further experimental details can be found [Acierno et al., 2007a, 2007b; Romeo et al., 2008; Filippone et al., 2010; Romeo et al., 2009].

\subsubsection{Materials and sample preparation}

Nano- and microcomposites were prepared using two different polymeric matrices. The first one is polypropylene (PP Moplen HP563N by Basell; weight average molecular weight $M_{w}=245 \mathrm{KDa}$; zero shear viscosity $\eta_{0}=1.9^{*} 10^{3} \mathrm{~Pa}^{*} \mathrm{~s}$ at $190^{\circ} \mathrm{C}$; terminal relaxation time $\tau_{t} \approx 0.4 \mathrm{~s}$ ) with glass transition temperature $T_{g}=6^{\circ} \mathrm{C}$ and melting temperature $T_{m}=169^{\circ} \mathrm{C}$. The second polymer matrix is atactic polystyrene (PS, kindly supplied by Polimeri Europa). In particular, we used two PS matrices at different molecular weight, coded as PS-low $\left(M_{w}=125\right.$ $\mathrm{KDa} ; \eta_{0}=1.7^{*} 10^{3} \mathrm{~Pa}^{*} \mathrm{~s}$ at $\left.200^{\circ} \mathrm{C} ; \tau_{\tau} \approx 0.1 \mathrm{~s}\right)$ and PS-high $\left(M_{w}=268 \mathrm{KDa} ; \eta_{0}=2.1^{*} 10^{4} \mathrm{~Pa}^{*} \mathrm{~s}\right.$ at $200^{\circ} \mathrm{C}$; $\tau_{t} \approx 100 \mathrm{~s}$ ), both having glass transition temperatures $T_{g}=100^{\circ} \mathrm{C}$.

Three kinds of nanoparticles were used as fillers: titanium dioxide $\left(\mathrm{TiO}_{2}\right.$ by Sigma Aldrich; density $\rho=3.9 \mathrm{~g} / \mathrm{mL}$; surface area $\sim 190 \div 290 \mathrm{~m}^{2} / \mathrm{g}$; average primary particles diameter $d=15$ $\mathrm{nm})$ and alumina nanospheres $\left(\mathrm{Al}_{2} \mathrm{O}_{3}\right.$ by Sigma Aldrich; $\rho=4.00 \mathrm{~g} / \mathrm{mL}$; surface area: $35-43$ $\mathrm{m}^{2} / \mathrm{g}$; $d \approx 40 \mathrm{~nm}$ ) were used to prepare $\mathrm{PP} / \mathrm{TiO}_{2}$ and $\mathrm{PP} / \mathrm{Al}_{2} \mathrm{O}_{3}$ nanocomposites with filler volume fractions up to $\Phi=0.064$; fumed silica $\left(\mathrm{SiO}_{2}\right.$ by Degussa; $\rho=2.2 \mathrm{~g} / \mathrm{mL}$; surface area 135-165 m²/g; $d=14 \mathrm{~nm}$ ) was mixed with the two PS matrices up to $\Phi=0.041 . \mathrm{PP} / \mathrm{TiO}_{2}$ microcomposites were also prepared by using titanium dioxide microparticles $\left(\mathrm{TiO}_{2}\right.$ by Sigma Aldrich; $\rho=3.9 \mathrm{~g} / \mathrm{mL}$; surface area $\sim 0.14-0.04 \mathrm{~m}^{2} / \mathrm{g}$; $d \approx 4 \mu \mathrm{m}$ ).

Nano- and microcomposites were prepared by melt compounding the constituents using a co-rotating extruder (Minilab Microcompounder, ThermoHaake) equipped with a capillary die (diameter $2 \mathrm{~mm}$ ). The extrusions were carried out at $190^{\circ} \mathrm{C}$. The screw speed was set to $\sim 100 \mathrm{rpm}$, corresponding to an average shear rate of order of $50 \mathrm{~s}^{-1}$ inside the extrusion chamber. A feedback chamber allowed an accurate control of the residence time, which was 
set to $250 \mathrm{~s}$ for all the samples. The polymer and the filler were previously dried under vacuum for sixteen hours at $70^{\circ} \mathrm{C}$. The neat polymers used as reference materials were extruded in the same conditions to allow for an accurate comparison with the filled samples.

\subsubsection{Characterization}

The morphology of the composites was examined by transmission electron microscopy (TEM mod. EM 208, Philips). The observations were performed on slices with thickness $\sim 150 \mathrm{~nm}$, which were randomly cut from the extruded pellets using a diamond knife at room temperature.

Rheological tests were carried out by means of either a strain-controlled rotational rheometer (ARES L.S, Rheometric Scientific) or a stress-controlled rotational rheometer (ARG2, TA Instruments). The tests were carried out using parallel plates with diameter of 25 $\mathrm{mm}$ for the nanocomposites, while plates of $50 \mathrm{~mm}$ were used for the neat polymers because of their low viscosity. All measurements were performed in an atmosphere of dry nitrogen. The testing temperature was $\mathrm{T}=190^{\circ} \mathrm{C}$ for the $\mathrm{PP} / \mathrm{TiO}_{2}$ samples and $\mathrm{T}=200^{\circ} \mathrm{C}$ for the $\mathrm{PS} / \mathrm{SiO}_{2}$ samples. The viscoelastic moduli display a range of strain-independence, i.e. a range of linear viscoelasticity, which depends on the filler content. In order to determine the limits of the linear viscoelastic regime, oscillatory strain scans were performed on each sample at a fixed frequency of $0.063 \mathrm{rad} \mathrm{s}^{-1}$. Low-frequency $\left(\omega=0.063 \mathrm{rad} \mathrm{s}^{-1}\right)$ time-sweep experiments were performed to study the evolution of the linear viscoelastic properties during time. The frequency-dependent elastic $\left(G^{\prime}\right)$ and viscous $\left(G^{\prime \prime}\right)$ moduli of the samples were measured by oscillatory shear scans in the linear regime. To account for the marked sensitivity of the rheological response on filler content, we evaluated the effective amount of filler of each sample used for the rheological experiments through thermogravimetrical analyses (TGA). The filler volume fraction $\Phi$ was estimated as:

$$
\Phi=\frac{c \rho_{p}}{\rho_{f}+c\left(\rho_{p}-\rho_{f}\right)}
$$

where $c$ is filler weight fraction as deduced from TGA and $\rho_{p}$ and $\rho_{f}$ are the densities of polymer and filler, respectively.

\subsubsection{Effect of the filler mobility on the linear viscoelasticity}

The internal structure of the as extruded sample $\mathrm{PP} / \mathrm{TiO}_{2}$ at $\Phi=0.038$ is shown in the TEM micrograph of Figure 1.a. Well distributed nanoparticle aggregates of a few hundreds of nanometers are suspended in the polymer matrix. The magnification of one of these aggregates is reported in Figure 1.b. A few hundreds of individual $\mathrm{TiO}_{2}$ nanoparticles are closely packed into dense clusters with irregular shape.

The sample was subjected to a thermal annealing at $190^{\circ} \mathrm{C}$ in quasi-quiescent conditions, i.e. by submitting it to shear oscillations in the rheometer at small strain amplitude $(\gamma=2 \%)$ and frequency $\left(\omega=0.063 \mathrm{rad} \mathrm{s}^{-1}\right)$. This allows to monitor the evolutions during time of slow dynamic populations, relaxing in timescales of order of $\tau=1 / \omega \approx 16 \mathrm{sec}$, without altering the internal structure of the sample. 


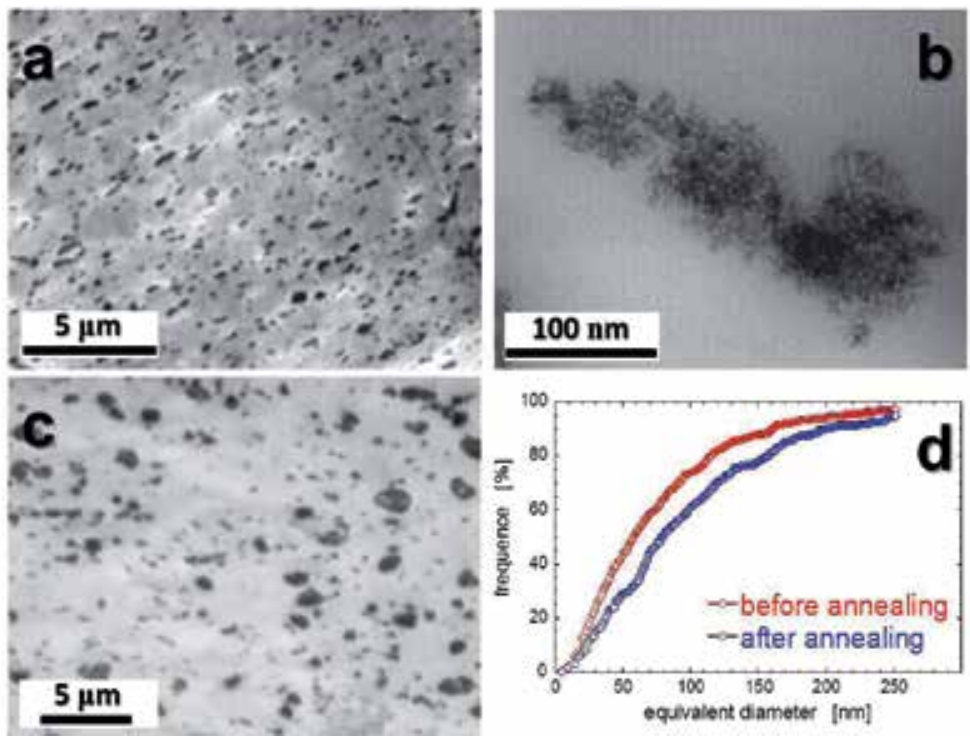

Fig. 1. (a) TEM micrograph of the as extruded sample $\mathrm{PP} / \mathrm{TiO}_{2}$ at $\Phi=0.045$. (b) Magnification of an aggregate of $\mathrm{TiO}_{2}$ nanoparticles. (c) TEM micrograph of the same sample as in (a) after a three-hours thermal annealing at $\mathrm{T}=190^{\circ} \mathrm{C}$. (d) CSD of the samples shown in (a) and (c) (images taken from Acierno et al., 2007a).

The microstructure of the annealed sample is shown in Figure 1.c. A visual comparison with the morphology of the as extruded sample reveals the presence of bigger aggregates and the disappearance of the smaller ones. An analysis of the TEM micrographs was carried out to quantify the effect of the thermal annealing. An equivalent diameter for the aggregates was defined as $D_{i}=\left(\pi A_{i}\right)^{0.5}$, where $A_{i}$ is the measured area of the $i$-th cluster. Once the sizes of the aggregates are available, the cumulative size distribution (CSD) and the number average size of the $\mathrm{TiO}_{2}$ aggregates, $D_{n}=\Sigma n_{i} D_{i} / \Sigma n_{i}\left(n_{i}\right.$ clusters with size $\left.D_{i}\right)$, was determined for each sample. The comparison between the CSDs is shown in Figure 1.d. The lowering of the cumulative CSD curves indicates an increase of the cluster sizes occurred during the thermal conditioning. In particular, the average size of the $\mathrm{TiO}_{2}$ aggregates increases from $D_{n} \approx 125$ $\mathrm{nm}$ to $D_{n} \approx 170 \mathrm{~nm}$.

The coarsening of the microstructure is a consequence of the diffusion of the clusters under the push of Van der Waals attraction. Rheological parameters such as the linear viscoelastic moduli are extremely sensitive to the internal microstructure. Thus, we use them to follow such internal rearrangements. The time evolutions of $G^{\prime}$ and $G^{\prime \prime}$ at $\omega=0.063 \mathrm{rad} \mathrm{s}^{-1}$ are shown in Figure 2.

The elastic modulus, which at the beginning is lower than the viscous one, increases during the first stage and then it reaches a plateau after a certain time; on the other hand, the loss modulus remains essentially constant. Preliminary investigations revealed that the neat matrices display a constant value of the moduli in the analysed time window. Thus, the increase in the sample elasticity is related to the structuring of the inorganic phase. The characteristic timescale for such a phenomenon can be roughly estimated as the Smoluchowski 
time for two clusters of radius $R$ to come in contact, $\tau_{a}$ [Russel et al., 1989]. This characteristic time depends on on the self-diffusion time of each aggregate, given by Equation 2, and on the average inter-aggregates distance, inversely proportional to the filler amount:

$$
\tau_{a}=\frac{\pi \eta_{s} R^{3}}{\bar{\Phi} k_{B} T}
$$

$\bar{\Phi}$ is the actual filler volume fraction, i.e. the volume of the particles in a cluster plus the free volume enclosed between them. These regions are actually inaccessible to the polymer, and depends on how primary particles are assembled together inside the aggregates. As shown in Figure 1.b, the $\mathrm{TiO}_{2}$ clusters appear rather compact. As a consequence, we can reasonably assume that the primary particles are packed at a volume fraction of $\sim 60 \%$, which is close to random close packing. The actual filler volume fraction of the $\mathrm{PP} / \mathrm{TiO}_{2}$ nanocomposites can be consequently estimated as $\bar{\Phi} \approx \Phi / 0.6$. Assuming $R=D_{n} / 2 \approx 65 \mathrm{~nm}$, Equation 4 gives $\tau_{a} \sim 4^{*} 10^{3} \mathrm{~s}$, in good agreement with the data shown in Figure 2. This result suggests that the increasing of the sample elasticity during time is related to cluster-cluster aggregation. In order to support the previous conclusion, we increase $\tau_{a}$ by increasing either the size of primary particles or aggregates or the viscosity of the suspending medium. According to Equation 2, in these conditions we expect that the elasticity of the samples cannot increase significantly because of the reduced particle mobility.

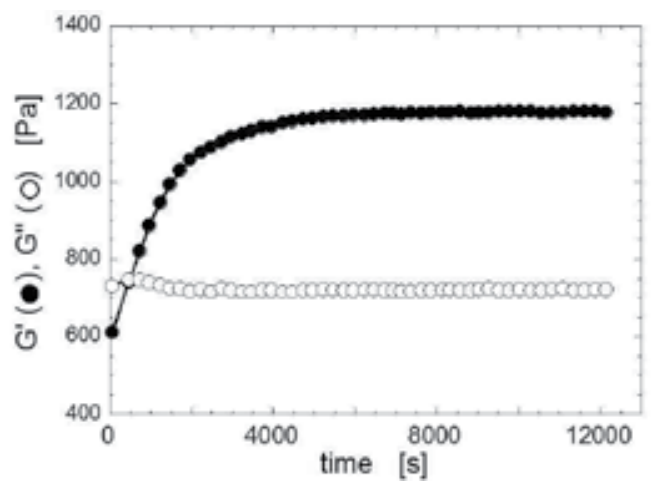

Fig. 2. Time evolution of $G^{\prime}$ (full) and $G^{\prime \prime}$ (empty) at $\omega=0.063 \mathrm{rad} \mathrm{s}^{-1}$ and $T=190^{\circ} \mathrm{C}$ for the nanocomposite $\mathrm{PP} / \mathrm{TiO}_{2}$ at $\Phi=0.038$ (image taken from Romeo et al., 2009).

As first test, we investigate the time evolutions of the linear viscoelastic moduli at $\omega=0.063$ rad $\mathrm{s}^{-1}$ for a $\mathrm{PP} / \mathrm{TiO}_{2}$ microcomposite (particles radius $R \approx 2 \mu \mathrm{m}$ ) at $\Phi \approx 0.035$. Based on Equation 4, we expect that two micron-sized particles should come at contact after timescales of order of $\sim 10^{7} \mathrm{~s}$. As a matter of fact, the results shown in Figure 3.a indicate that both moduli remain stable during the aging test until $\sim 10^{4} \mathrm{~s}$.

As second test, we monitor the moduli of a nanocomposite based on a high viscosity matrix such as PS-high $\left(\eta_{0}=2.1^{*} 10^{4} \mathrm{~Pa}^{*} \mathrm{~s}\right.$ at $\left.200^{\circ} \mathrm{C}\right)$ filled with $\mathrm{SiO}_{2}$ particles at $\Phi \approx 0.035$. Silica aggregates exhibits the typical open and branched structure of fractal objects. In such systems the mass $M$ scales with length $L$ as $M \sim L^{d_{f}}, d_{f}$ being the fractal dimension [Weitz \& Oliveira, 1984]. The actual filler volume fraction thus becomes [Wolthers et al., 1997]: 


$$
\bar{\Phi}=\Phi \times(L / d)^{3-d_{f}}
$$

Setting $L=D_{n} \approx 125 \mathrm{~nm}$ as emerged from the analysis of many TEM micrographs, and taking $d_{f}=2.2$ as a typical fractal dimension of fumed silica aggregates [Kammler et al., 2004], Equation 4 gives $\tau_{a} \sim 10^{5} \mathrm{~s}$. This is in agreement with the results of the time sweep experiment shown in Figure 3.b, which indicate that cluster assembling phenomena, if any, are negligible in the timescale of the test. Obviously, the not structured sample keeps a predominantly viscous connotation, i.e. $G^{\prime \prime}>>G^{\prime}$.
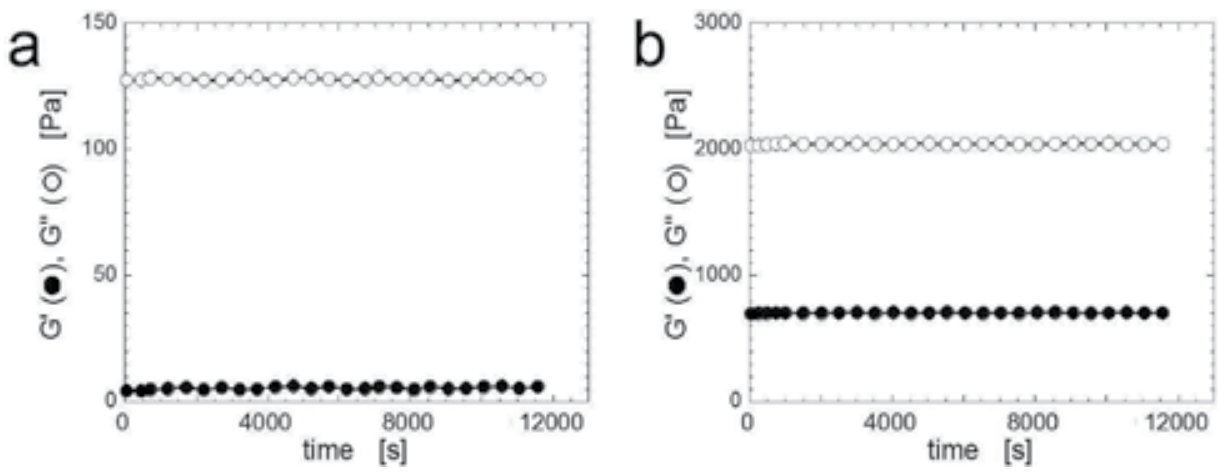

Fig. 3. Time evolution of $G^{\prime}$ (full) and $G^{\prime \prime}$ (empty) at $\omega=0.063 \mathrm{rad} \mathrm{s}^{-1}$ for the microcomposite $\mathrm{PP} / \mathrm{TiO}_{2}$ at $\Phi=0.035$ and $\mathrm{T}=190^{\circ} \mathrm{C}(\mathrm{a})$, and the nanocomposite PS-high $/ \mathrm{SiO}_{2}$ at $\Phi=0.035$ and $\mathrm{T}=200^{\circ} \mathrm{C}(\mathrm{b})$ (images taken from Romeo et al., 2009).

Particle rearrangements eventually give rise to mesoscopic structures, such as branched aggregates or space-spanning filler network, which strongly alter the frequency response of the sample. The $\omega$-dependent $G^{\prime}$ and $G^{\prime \prime}$ of two $\mathrm{PP} / \mathrm{TiO}_{2}$ samples filled with micro- and nanoparticles both at $\Phi \approx 0.035$ are compared in Figure 4.a. In both cases the matrix governs the high-frequency response. This suggests that the relaxation modes of the polymer chains and sub-chains are only slightly affected by the presence of the filler at these high frequencies. The presence of microparticles negligibly affects the whole response of the composite. On the contrary, the nanoparticles significantly alter the low-frequency moduli of the material, and in particular the elastic one.

The flattening of $G^{\prime}$ over long timescales is a general feature characterizing different kinds of PNCs [Krishnamoorti \& Yurekli, 2001; Du et al., 2004]. Such a behaviour, however, is not a direct consequence of the nanometric size of the particles, but rather it originates from particle mobility. To emphasize this point, in Figure 4.b the frequency response of the threehours aged samples PS-low $/ \mathrm{SiO}_{2}$ and $\mathrm{PS}$-high $/ \mathrm{SiO}_{2}$ at $\Phi \approx 0.035$ are compared. The nanocomposite with high viscosity matrix displays a liquid-like behaviour at low frequency reminiscent of that of the neat polymer (not shown). Differently, the PS-low $/ \mathrm{SiO}_{2}$ sample exhibits a predominant elastic connotation, the low-frequency plateau of $G$ ' being indicative of the presence of a space-spanning filler network formed during the ageing process.

To summarize, the viscoelastic response of a filled polymer is greatly affected by particle mobility. When the characteristic diffusion time of the particles and/or aggregates resulting from the extrusion process is too high, either because the matrix is too viscous or the particle 
size is too big, the filler is unable to rearrange and only produce a small perturbation of the composite viscoelastic response. Conversely, when mobility of the inorganic phase is high enough, random motion and attractive Van der Waals forces lead to the structuring of the primary aggregates. This eventually results in the formation of a whole space-spanning filler network. Since this network exhibits the connotation of an elastic solid, a drastic slowing down of relaxation dynamics occurs at low frequencies.
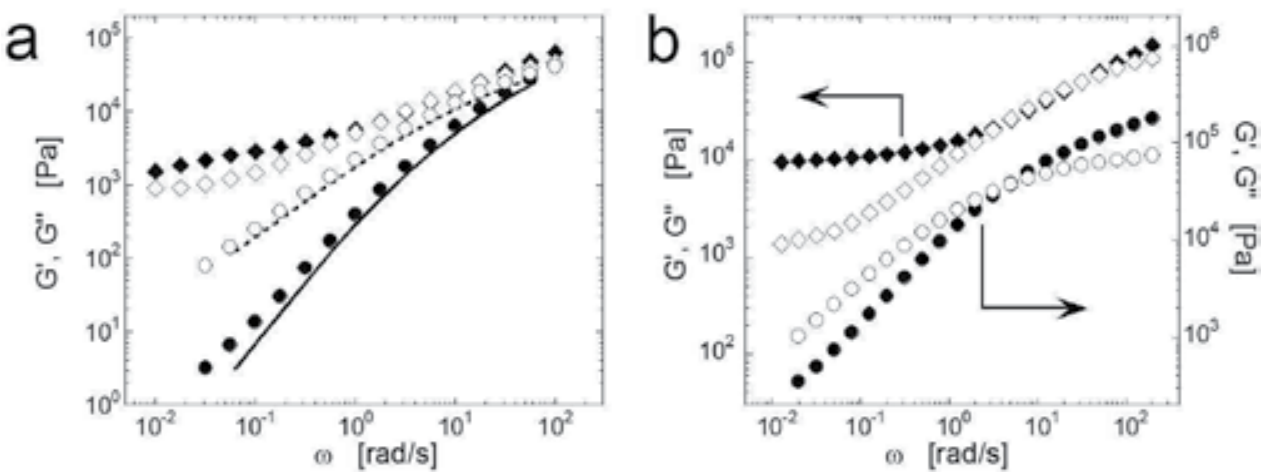

Fig. 4. (a) G' (full) and $G^{\prime \prime}$ (empty) for the samples $\mathrm{PP} / \mathrm{TiO}_{2}$ at $\Phi \approx 0.035$ filled with micrometric (circles) and nanometric (diamonds) particles. Solid and dashed lines represent the elastic and viscous modulus of the neat PP, respectively. (b) G' (full) and G' (empty) for the nanocomposite samples PS-low $/ \mathrm{SiO}_{2}$ (diamonds, left axis) and $\mathrm{PS}-$ high $/ \mathrm{SiO}_{2}$ (circles, right axis) at $\Phi \approx 0.035$ (image taken from Romeo et al., 2009).

\subsection{Linear viscoelasticity of PNCs}

\subsubsection{Weakly attractive particles suspended in Newtonian fluids - A two-phase model}

Colloids are typically nanometer to micron sized particles forming a dispersed phase in a suspending medium. Colloidal dispersions exhibit a wide spectrum of rheological properties, ranging from simply viscous fluids to highly elastic pastes depending on the amount of particles and the sign and magnitude of inter-particles interactions. Here we are interested in weakly attractive systems, where the particles are inclined to assemble together into more or less branched flocs. In these systems, the $\omega$-dependent storage and loss modulus typically exhibit a strong dependence on both $\Phi$ and $U$. Such a high variability makes extremely difficult a general description of the viscoelastic behaviour of colloidal dispersions. A drastic simplification has been introduced by Trappe and Weitz, which showed that modelling the suspension above the particle percolation threshold as an elastic filler network interspersed in a background fluid (two-phase model) qualitatively accounts for the viscoelasticity of their samples [Trappe \& Weitz, 2000]. The authors studied a dispersion of carbon black in base stock oil as a function of particle volume fraction and interaction potential. The $U$ was tuned by adding a dispersant that acts as a surfactant. Without dispersant carbon black particles are rather strongly attractive, and flocs of $\sim 100$ $\mu \mathrm{m}$ in size form even at very low amounts of particles. The linear viscoelastic moduli of samples at different $\Phi$ were measured as a function of frequency with a strain-controlled rheometer with Couette geometry. The authors found that a rheological transition occurs at $\Phi_{c}=0.053$ : at $\Phi>\Phi_{c}$ the suspension is clearly elastic and $G^{\prime}$ is nearly independent on $\omega$; at 
$\Phi<\Phi_{c}$ the viscous feature definitely prevails over the elastic one, and the suspension rheology looks like that of the suspending fluid. Microscopic analyses reveal that the rheological transition reflects the state of dispersion of the filler: isolated carbon black flocs are suspended in the background fluid below $\Phi_{c}$, whereas above this threshold the aggregates assemble in a three-dimensional space spanning network.

Despite their marked differences, the moduli of the samples at $\Phi>\Phi_{c}$ can be scaled onto a single pair of master curves. The authors qualitatively accounted for the observed scaling by assuming that the carbon black forms a solid but tenuous network with a purely elastic, $\omega$ independent modulus. The elasticity of this network, $G_{0}^{\prime}$, increases with $\Phi$ as the network becomes more and more robust. Interspersed throughout this structure is the purely viscous suspending fluid, which $G^{\prime \prime}$ linearly increases with $\omega$ and is substantially independent of $\Phi$. Consequently, the elasticity of the network prevails at low $\omega$, while the viscosity of the fluid dominates at high $\omega$. Within this simplified picture, scaling the elasticity of each sample along the viscosity of the matrix results in the collapse of data of samples at different composition onto a single pair of master curves.

Although the proposed approach can account for the basic scaling behaviour, many issue remain unresolved. For example, the behaviour of the weaker of the two moduli in each regime is not addressed. At low frequencies, $G^{\prime \prime}(\omega)$ must be determined by the loss modulus of the network, which is larger than that of the suspending fluid. Similarly, at the highest frequencies $G^{\prime}(\omega)$ must reflect the storage component of the suspending fluid with the solid network in it. In addition, the model does not take into account hydrodynamic effects. Despite these limitations, the good quality of the scaling supports the reliability of the approach, indicating that there is a strong similarity in the structures of the networks that form at different $\Phi$. This also implies some predictive feature of the model: the tiny elasticity of samples at low $\Phi$ (as long as greater than $\Phi_{c}$ ), which networks are too tenuous to be appreciated through direct dynamic-mechanical analyses, can be predicted with good approximation by simply referring to the master curve of $G^{\prime}$.

\subsubsection{Weakly attractive nanoparticles suspended in non-Newtonian mediums - Recovering the two-phase model}

The relatively high mobility of nanoparticles even in highly viscous fluids such as polymer melts makes PNCs similar to colloidal dispersions. The main difference with these simpler systems is the non-Newtonian feature of the suspending medium. According to Trappe and Weitz, the viscoelasticity of a colloidal suspension above $\Phi_{c}$ originates from the combination of the responses of an elastic particle gel that of the purely viscous background fluid. In the case of a PNC, instead, the suspending medium is viscoelastic by itself, and its response combines with that of the space-spanning network giving rise to a more complex $\omega$ - and $\Phi$ dependent viscoelastic behaviour. It follows that a separation of the effects of the solid and fluid phases is no more possible in the case of PNCs. However, we argue that a recovery of the two-phase model is possible if the elasticity of the polymer is neglected with respect to that of the filler network. Under this assumption, the viscoelasticity of the PNC can be split into the independent responses of an elastic particle network and that of the predominantly viscous polymer. The former contribution depends on the filler content and governs the long timescale response of the composite, whereas the latter is responsible for the high-frequency behaviour (Figure 5). 


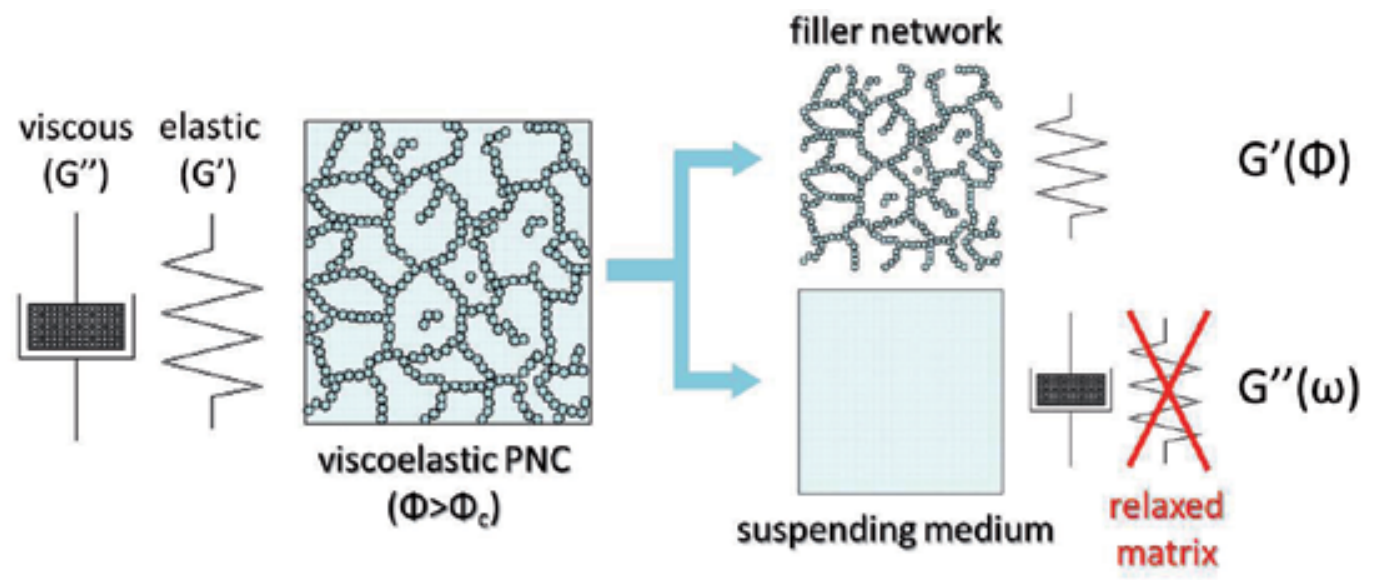

Fig. 5. Scheme of the viscoelasticity of a PNC at $\Phi>\Phi_{c}$. For fully relaxed polymer matrix, the filler network is the only responsible for the elastic connotation of the system.

To test the validity of the previous considerations, we focus on the $\omega$-dependence of the moduli of $\mathrm{PP} / \mathrm{TiO}_{2}$ and PS-low $/ \mathrm{SiO}_{2}$ nanocomposite samples at $\Phi>\Phi_{c}$, i.e. in which the filler rearranges in experimentally accessible timescales forming a space-spanning network. All these samples share a similar pseudo solid-like behaviour at low frequency, with weak $\omega$ dependences of both moduli and $G^{\prime}$ greater than $G^{\prime \prime}$. Since the filler mainly affects the elastic modulus of the samples, $G^{\prime}$ increases with $\Phi$ more rapidly than $G^{\prime \prime}$. As a consequence, a further crossover between $G^{\prime}$ and $G^{\prime \prime}$ occurs at intermediate frequencies in addition to that at high $\omega$ related to the relaxation of the neat polymer. The coordinates of such additional crossing point, $\left(\omega_{c} ; G_{c}\right)$, shift towards higher and higher frequencies and moduli with increasing the filler content. This is shown in Figure 6 for three samples PSlow $/ \mathrm{SiO}_{2}$ at different composition.
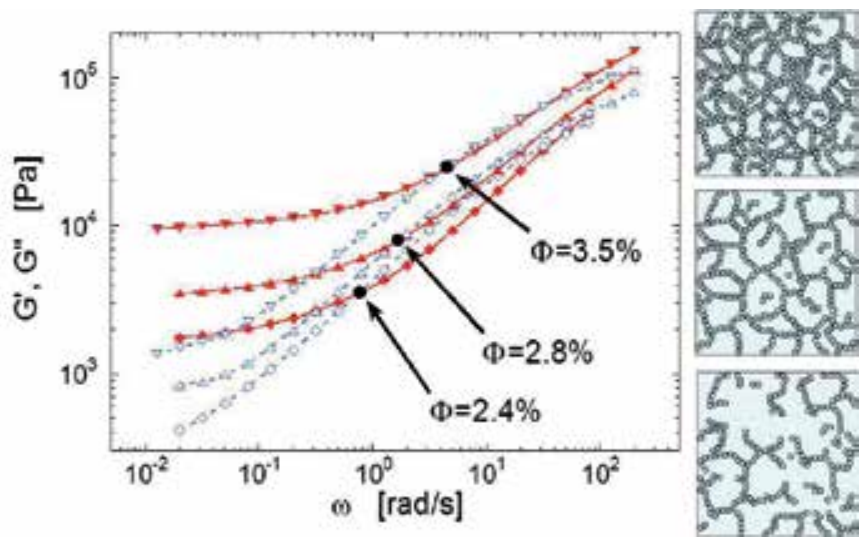

Fig. 6. G' (full, red) and G' (empty, blu) for the nanocomposite samples PS-low $/ \mathrm{SiO}_{2}$ at increasing filler content. The additional crossover is indicated by the arrows.

The additional low-frequency crossover can be interpreted as the point at which the network elasticity equals the viscous contribution of the polymer. As a consequence, 
normalizing the moduli of samples at different $\Phi$ by their elasticity, and doing so along the background fluid viscosity, the curves should collapse onto a single pair of master curves. Accordingly, the scaling has to be done by shifting the curves both horizontally and vertically using as shift factors $a=1 / \omega_{c}$ and $b=1 / G_{c}$, respectively. The resulting master curves are shown in Figure 7 for both the $\mathrm{PP} / \mathrm{TiO}_{2}$ and $\mathrm{PS}$-low $/ \mathrm{SiO}_{2}$ nanocomposites.
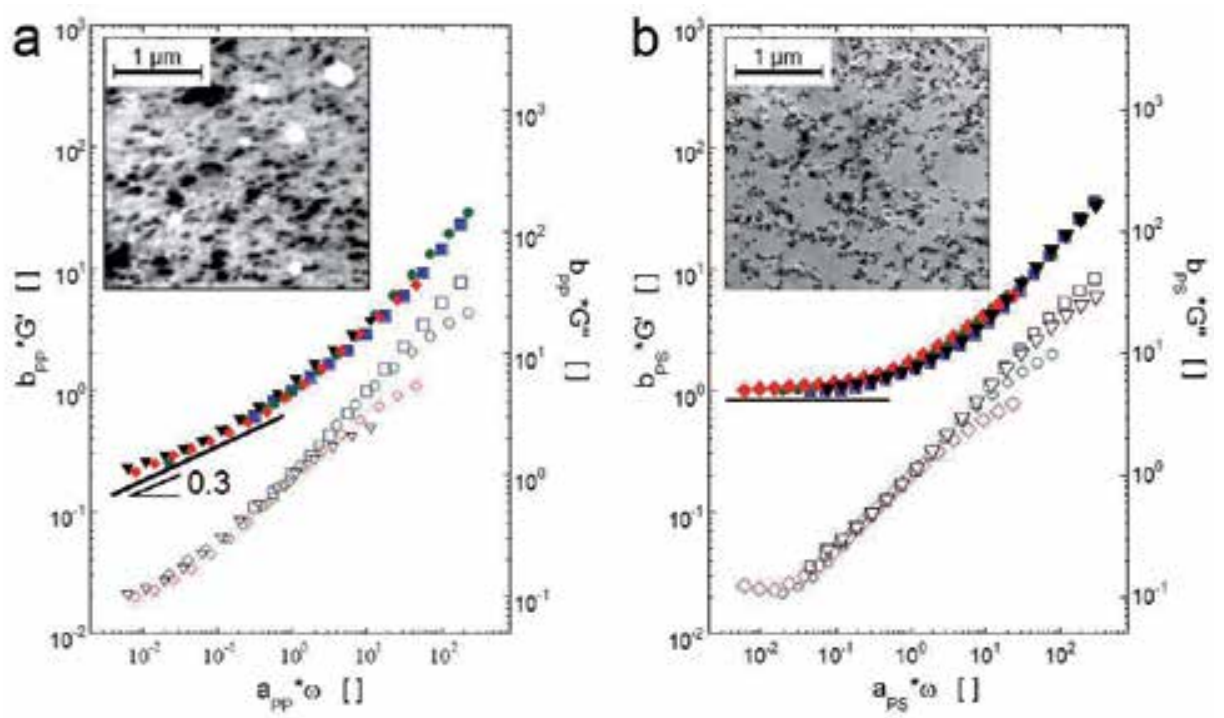

Fig. 7. Master curves of $\mathrm{G}^{\prime}$ (full, left axis) and $\mathrm{G}^{\prime \prime}$ (empty, right axis) for the systems $\mathrm{PP} / \mathrm{TiO}_{2}$ (a) and PS-LOW $/ \mathrm{SiO}_{2}$ (b). Each colour corresponds to a composition. Note that only curves at $\Phi>\Phi_{\mathrm{c}}$ have been used to build the master curves. The TEM micrographs shown in the insets represent the microstructures of samples at $\Phi \approx 0.035$ (image taken from Romeo et al., 2009).

The scaled moduli lie on top of each other in about five decades of frequencies, supporting the validity of the adopted approach. Deviations are observed for the viscous moduli at high scaled frequencies. This is not unexpected, since the relaxation modes of the polymers are independent on the filler content and cannot be scaled using $a$ and $b$ as scaling factors.

Once the master curves are built, the differences in elasticity and dynamic of the particle networks become evident. The $\mathrm{SiO}_{2}$ network is characterized by an $\omega$-independent elastic modulus at low frequency, which emphasizes its truly solid-like feature. Differently, the $\mathrm{TiO}_{2}$ network displays a slow relaxation dynamic with $G^{\prime} \sim \omega^{0.3}$. These differences are related to the differences in network structures formed in the two composites. The TEM images reported in the insets of Figure 7 show that the $\mathrm{SiO}_{2}$ nanoparticles form a tenuous, fractal network of sub-micron sized, branched flocs interspersed within the host PS. Differently, the $\mathrm{TiO}_{2}$ nanoparticles are assembled into dense clusters, which mobility is presumably slowed down by the surrounding aggregates. The transient character of the latter network emerges as a glassy-like decrease of $G^{\prime}$, which reflects the internal rearrangements of the $\mathrm{TiO}_{2}$ clusters. Such slow relaxation dynamics are characteristic of colloidal glasses [Shikata \& Pearson, 1994; Mason \& Weitz, 1995] and has been observed in many other soft materials [Sollich et al., 1997]. 


\subsubsection{Refining the two-phase model - Role of the hydrodynamic effects}

Despite the good quality of the scaling shown in Figure 7, unresolved issues exist regarding the physical meaning of the shift factors. The underlying physics of the model lies on the independent rheological responses of the neat polymer and the particle network. Actually, the coordinates of the crossover point of the moduli of the nanocomposite, identified by Trappe and Weitz as the shift factors for their system, do not rigorously reflect the properties of the two pristine phases of the model. In addition, the presence of the particles implies hydrodynamic effects, which cannot be eluded for a correct scaling of the data. To account for these issues, the procedure to get the shift factors for the building of the master curve has to be revisited. For this aim, hereinafter we only refer to the system PS-low $/ \mathrm{SiO}_{2}$, which particle network exhibits a truly solid-like behaviour at low frequency.

Hydrodynamic effects reflect the perturbation of the flow lines in proximity of the filler. In a liquid filled with a solid particulate, the suspending fluid flows in the narrow gap between contiguous particles or aggregates, locally experiencing a greater flow rate than what externally imposed or measured. Gleissle and Hochstein quantitatively accounted for hydrodynamic effects in oscillatory shear experiments by introducing an empiric amplification factor, representing the ratio between the complex moduli of the filled sample over that of the neat matrix: $B(\Phi)=G^{*}(\Phi) / G_{P S}^{*}$ [Gleissle \& Hochstein, 2003]. In the case of microparticles, $B(\Phi)$ well describe the increase of $G^{*}$ of the suspension in the whole range of accessible frequencies. Differently, non-continuum effects emerge over long timescales in the case of PNCs. Consequently, the hydrodynamic effects only are appreciable at high frequencies, i.e. where the rheological response is governed by the polymer matrix. This is shown in Figure 8, where the complex moduli of various $\mathrm{PS}-\mathrm{low} / \mathrm{SiO}_{2}$ nanocomposites at different composition are reported together with the resulting $B(\Phi)$.
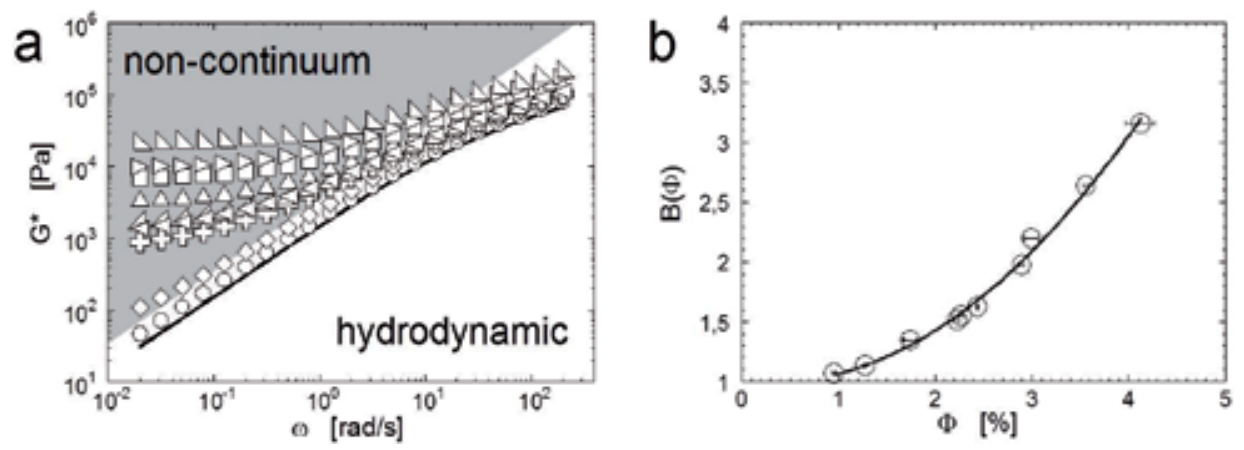

Fig. 8. (a) Complex modulus of $\mathrm{PS}-\mathrm{low} / \mathrm{SiO}_{2}$ nanocomposite at various filler content. The regions in which non-continuum and hydrodynamic effects are dominant are emphasized. (b) Amplification factor for the data shown in (a) (images taken from Filippone et al., 2010).

After the hydrodynamic contribution has been quantified for each sample, then new and more rigorous shift factors can be identified. Specifically, we now refer to the point at which the elasticity of the filler network, given by the plateau modulus of the nanocomposite, $G^{\prime}(\Phi)$, equals the viscous modulus of the neat matrix amplified by $B(\Phi)$ to account for hydrodynamic effects, $B(\Phi) \cdot G^{*}$. 
The comparison between the old $(a ; b)$ and new $\left(a^{\prime} ; b^{\prime}\right)$ shift factors is shown in Figure 9.a for the sample at $\Phi=2.9 \%$; in Figure 9.b the new master curve is reported.
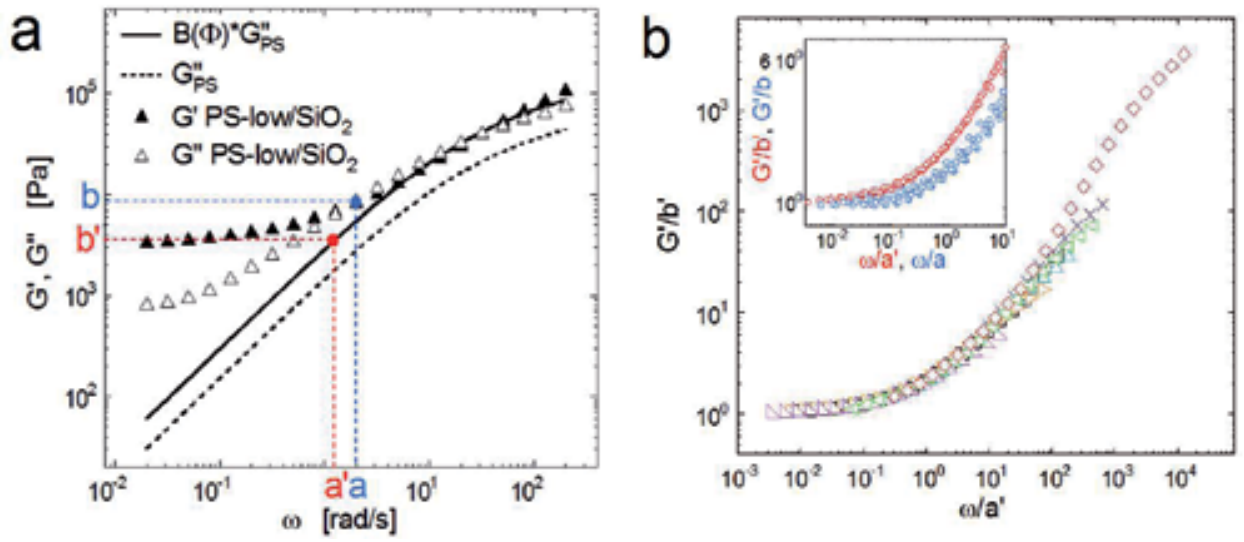

Fig. 9. (a) Comparison of the shift factors for the samples-low $/ \mathrm{SiO}_{2}$ at $\Phi=2.8 \%$. (b) Master curve of $G^{\prime}$ built using $a^{\prime}$ and $b^{\prime}$ as shift factors; the inset shows a magnifications at low scaled frequencies of the master curves obtained using as shift factors ( $\left.a^{\prime} ; b^{\prime}\right)$ (red) and (a; b) (blu) (images taken from Filippone et al., 2010).

The elastic moduli scaled using $a^{\prime}$ and $b^{\prime}$ as shift factors lies on top of each other over about seven decades of scaled frequencies, confirming the validity of the adopted approach. Again, the slight deviations at $\omega / a^{\prime}$ greater than $\sim 10^{1}$ do not invalidate the consistency of the scaling, being a consequence of the intrinsic viscoelastic feature of the suspending fluid.

Besides exactly capturing the underlying physics of the two-phase model, the refined model guarantees a better scaling of the elasticity of samples at different composition. This is shown in the inset of Figure 9.b, where the master curves built using the two pairs of shift factors are compared. The lower scattering of the data scaled using $a^{\prime}$ and $b^{\prime}$ confirms the importance of properly accounting for hydrodynamic contributions when dealing with PNCs.

\subsection{Strength and reversibility of the filler network in $\mathrm{PPIAl}_{2} \mathrm{O}_{3} \mathrm{PNCs}$}

Aim of this paragraph is the study of the relationships between the rheology and structure of $\mathrm{PP} / \mathrm{Al}_{2} \mathrm{O}_{3}$ nanocomposites. The structuring (during a quiescent annealing process) and de-structuring (promoted by large amplitude shear flows) of the filler network are investigated by means of both rheological and TEM analyses. The internal morphology of the sample $\mathrm{PP} / \mathrm{Al}_{2} \mathrm{O}_{3}$ at $\Phi=4.2 \%$ at the end of the extrusion process is shown in Figure 10 .

Although a homogeneous distribution can be observed on microscale, the presence of aggregates of a few hundred nanometers is noticed at higher magnifications. The aggregates appear as open structures formed of tens of nanospheres of different sizes. Such nonequilibrium structures rearrange towards a more favourable thermodynamic state during a subsequent aging above the PP melting temperature. The morphology of a sample at $\Phi=4.2 \%$ after a 3 -hours annealing at $T=190^{\circ} \mathrm{C}$ is shown in Figure 11. 

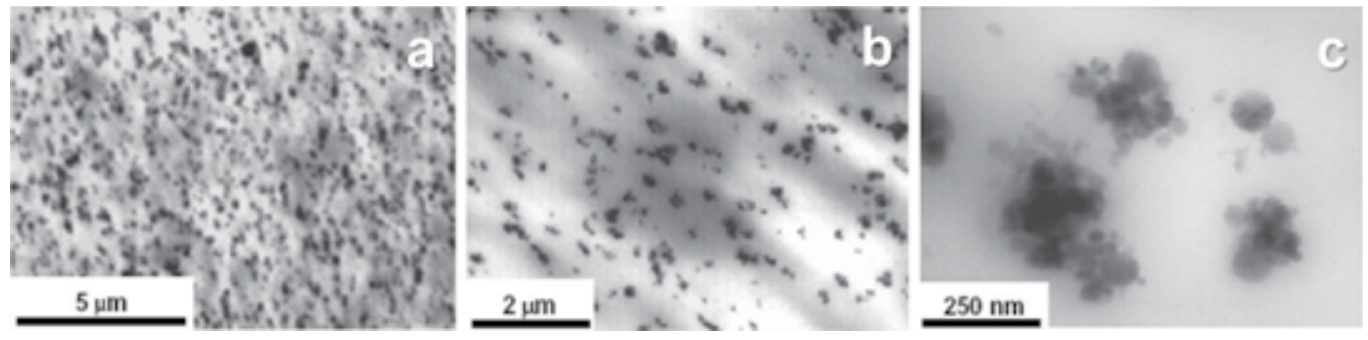

Fig. 10. TEM micrographs of the as extruded $\mathrm{PP} / \mathrm{Al}_{2} \mathrm{O}_{3}$ sample at $\Phi=4.2 \%$ at various magnifications (image taken from Acierno et al., 2007b).
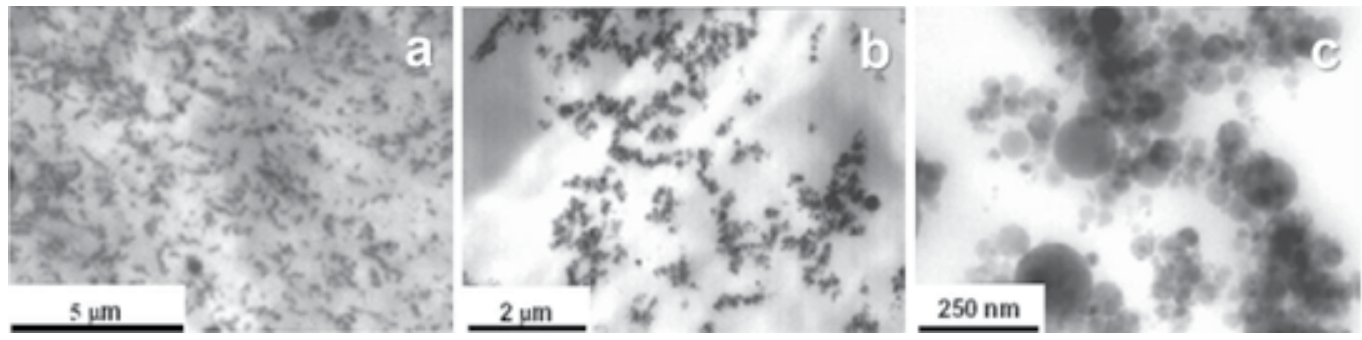

Fig. 11. TEM micrographs of the 3-hours thermal annealed $\mathrm{PP} / \mathrm{Al}_{2} \mathrm{O}_{3}$ sample at $\Phi=4.2 \%$ at various magnifications (image taken from Acierno et al., 2007b).

Pristine individual aggregates are now assembled into a disordered network that spans large sections of the sample. The particles and aggregates are essentially kept together by Van der Waals attractions and/or other kinds of weak bonds between the functional sites located at the particle surfaces. The application of large strains provides an excess energy to overcome such attractive interactions, thus destroying the network. After that, the particles may or may not aggregate again depending on the strength of inter-particle interactions.

The relaxation dynamics of a viscoelastic fluid can be indifferently monitored by frequency scans or stress relaxation tests. In the latter kind of experiment, a constant strain, $r_{0}$, is imposed to the sample in the linear regime, and the transient stress, $\sigma(t)$, is measured as a function of time. The stress relaxation modulus, $G(t)=\sigma(t) / \gamma_{0}$, is shown in Figure 12 after the application of large amplitude oscillatory shear (LAOS) at a constant frequency $\omega=0.0628$ $\mathrm{rad} \mathrm{s}^{-1}$ and different $\gamma_{0}$ on the 3-hours aged sample at $\Phi=4.2 \%$.

Large deformations have a drastic effect on the relaxation spectrum: the bigger the strain amplitude, the faster the relaxation dynamics. Time sweep tests in linear regime were performed after each LAOS to test the viscoelastic behaviour of the sheared sample, and the results are shown in the inset of Figure 12. The elastic feature progressively vanishes with increasing the deformation amplitude. Interestingly, the steadiness of the elastic modulus during time suggests an irreversibility of the network break-up process, at least within the experimental time window. Moreover, a polymer-like behaviour is recovered after the LAOS at the largest amplitude $\left(\gamma_{0}=500 \%\right)$. In such case, the inorganic phase does not affect the rheological response of the nanocomposite at all. 


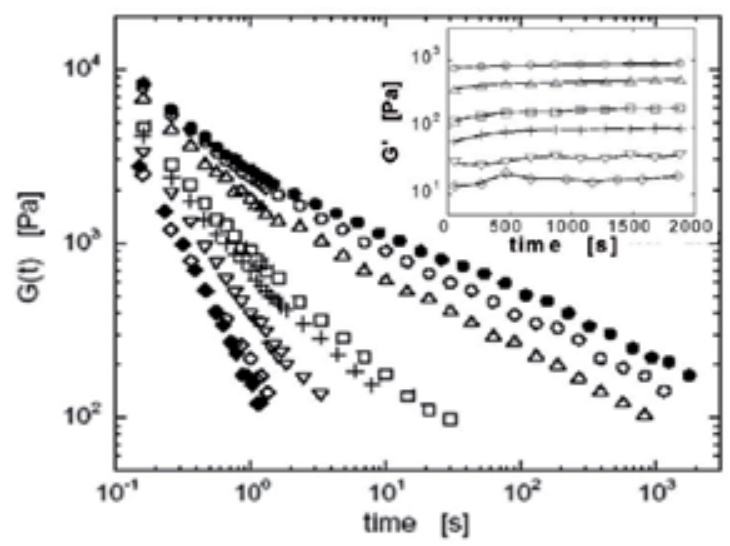

Fig. 12. $G(t)$ of the 3-hours aged $\mathrm{PP} / \mathrm{Al}_{2} \mathrm{O}_{3}$ sample at $\Phi=4,5 \%$ after $\mathrm{LAOS}$ at different strain amplitudes $\gamma_{0}: 0.8 \%$ (solid circles), $10 \%$ (open circles), 25\% (triangles), 50\% (squares), $100 \%$ (crosses), 250\% (reverse triangles), 500\% (diamonds). Solid diamonds represents the $G(t)$ of the neat polymer. The time evolutions of $G^{\prime}$ after each LAOS are shown in the inset.

Symbols are the same of stress relaxation moduli (image taken from Acierno et al., 2007b).

The morphology of the 3-hours aged sample after the LAOS at $\gamma_{0}=500 \%$ is reported in Figure 13. The network formed during aging is no more visible, and the presence of many small clusters characterizes the sheared system. The flocs show a more open structure than that of a not sheared sample, either aged or not, suggesting a weaker tendency to the clustering for the $\mathrm{Al}_{2} \mathrm{O}_{3}$ nanoparticles after the large deformations. The cumulative cluster size distributions were determined through the analysis of TEM micrographs. The results are shown in Figure 14 for the as extruded, 3-hours annealed and sheared after aging samples at $\Phi=4.2 \%$. The number average equivalent diameters of the clusters are reported in the same figure.

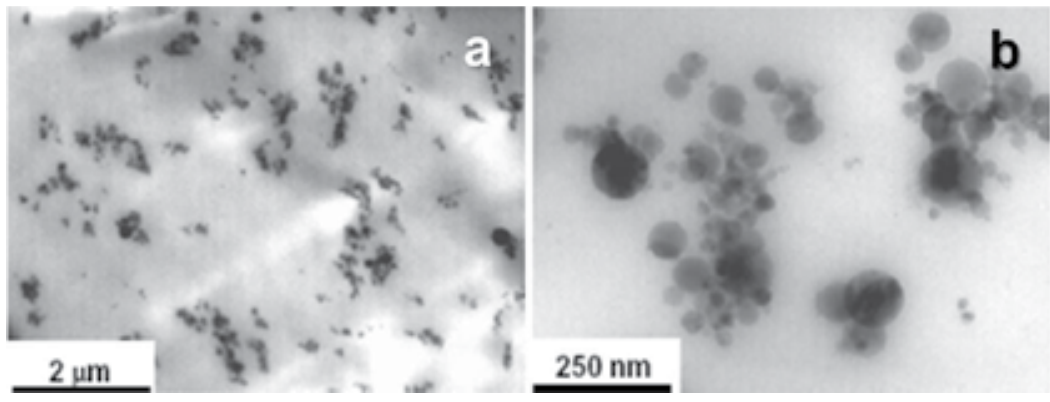

Fig. 13. TEM morphology of the 3-hours aged sample at $\Phi=4.2 \%$ after the LAOS at $\gamma=500 \%$ (image taken from Acierno et al., 2007b).

The CSD of the as extruded sample is rather sharp, indicating a good dispersion efficiency of the extrusion process. The thermal annealing results in a significant widening of the CSD, with the appearance of very large clusters $\left(D_{n}\right.$ greater than $\left.800 \mathrm{~nm}\right)$. This confirms the metastable feature of the samples, which quickly evolve toward states of less free energy 
under the push of the inter-particle attractive interactions. The resulting filler network breaks up when the sample is subjected to LAOS, and a remarkable sharpening of the CSD is observed.

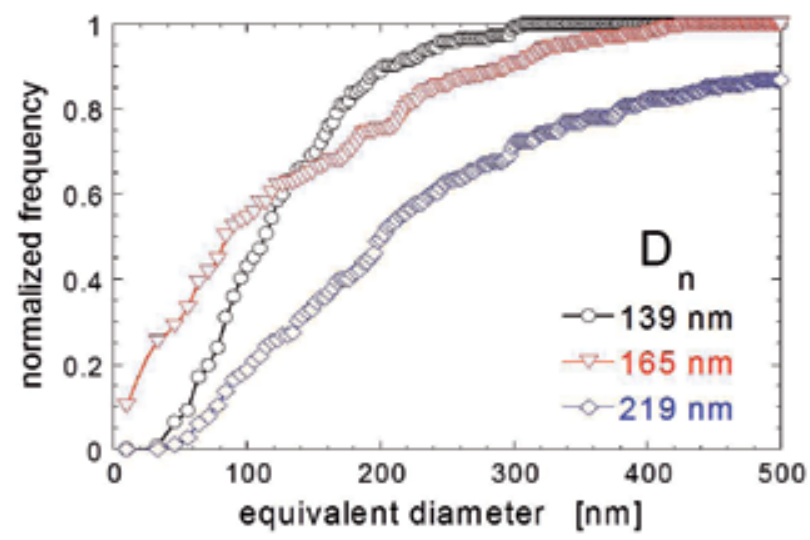

Fig. 14. CSDs for the $\mathrm{PP} / \mathrm{Al}_{2} \mathrm{O}_{3}$ sample at $\Phi=4.2 \%$ as extruded (black circles), 3-hours aged (blue diamonds) and 3-hours aged after LAOS at $\gamma_{0}=500 \%$ (red triangles) (image taken from Acierno et al., 2007b).

Interestingly, the strength of the filler network depends on whether the LAOS is applied before or after the thermal annealing. This is shown in Figure 15, where the loss factor $\tan \delta=G^{\prime \prime} / G^{\prime}(15 . a)$ and the complex moduli (15.b) of the samples at $\Phi=4.2 \%$ submitted to LAOS $\left(\gamma_{0}=500 \%\right)$ are reported before and after the ageing; the curves of the 3-hours aged but not sheared sample are also reported for comparison.

If the LAOS is applied to the sample before the formation of the particle network, the system quickly evolves to a more elastic structure and the $\tan \delta$ asymptotically reaches values close to those of the not sheared sample. However, the comparison between the $G^{*}$ shown in
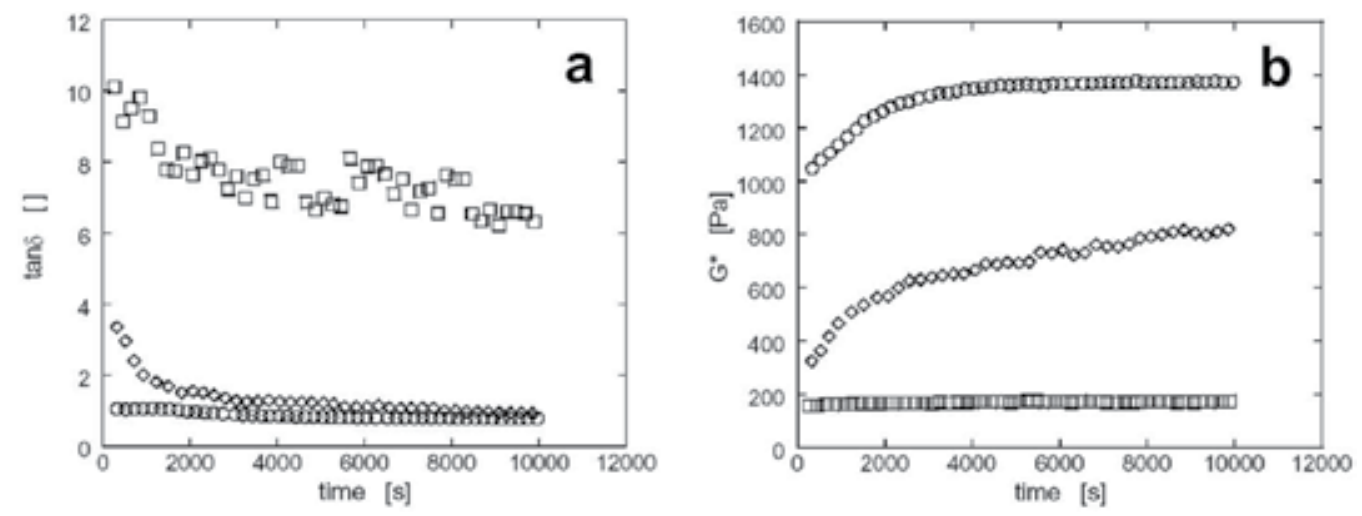

Fig. 15. Loss factor (a) and complex moduli (b) of the samples at $\Phi=4.2 \%$ submitted to LAOS $\left(\gamma_{0}=500 \%\right)$ before (diamonds) and after (squares) the thermal annealing. The curves of the 3-hours aged and not sheared sample (circles) are also reported for comparison (image taken from Acierno et al., 2007b). 
Figure 15.b reveals that the strength of the network formed after the LAOS is much lower than that of the not sheared sample.

Such a result can be explained by assuming some rearrangement of the reactive sites of the particle surfaces after the network break-up, which may weaken the surface activity of the particles. This reduces the intensity of inter-particle interactions and, as a consequence, the strength of the filler network [Bicerano et al., 1999]. On the other hand, if a "strong" network forms and then it is destroyed by LAOS, the restoration of new bonds required for the reformation of the network can results inhibited. This could explain the irreversibility of the structuring process noticed after the LAOS performed on the aged sample.

\section{Conclusions}

The effect of small amounts of nanoparticles on the melt-state linear viscoelastic behaviour has been investigated for different polymer-nanoparticles model systems characterized by poor polymer-particles interactions and low particle contents. The drastic increase of the rheological properties with respect to the matrices has been related to the formation of a filler network above a critical particles volume fraction. This is a consequence of particles and clusters rearrangements taking place during a thermal annealing. The filler mobility depends on both particle size and viscosity of the suspending medium. Once formed, the filler network exhibits an elastic feature that mixes with the intrinsic viscoelastic response of the polymer matrix, resulting in a complex $\Phi$ - and $\omega$-dependent viscoelastic response of the nanocomposite. However, starting from a two-phase model proposed for colloidal suspensions in Newtonian fluids, we have shown that the contributions of filler network and suspending medium can be decoupled due to the weak polymer-particle interactions and the differences in temporal relaxation scales. The adopted approach has been validated through the building of a master curve of the moduli, which reflects the scaling of the elasticity of composites along the viscosity of the suspending medium. The two-phase model well works irrespective of the structure of the filler network, making evident the strict interrelationships between the structure, both on nano- and micro-scale, and the meltstate behaviour of the studied PNCs. The physical meaning of the two-phase model clearly emerges once hydrodynamic effects have been properly taken into account. Besides clarifying the various timescales of PNCs, the proposed model allows for predicting the modulus of particle networks which are too tenuous to be appreciated through simple frequency scans. The application of a large amplitude oscillatory shear flows provides an excess energy for the system to escape from the metastable configuration in which it is trapped. This destroys the network formed during the thermal annealing, leading to a more tenuous structure which is unable to significantly contribute to the system elasticity. After the network has been destroyed the sample cannot recover its previous solid-like feature during a subsequent thermal annealing. This is probably due to some rearrangement of the reactive sites of the particle surfaces occurring after the rupture of the inter-particles bonds formed during annealing.

Besides well describing the behaviour of PNCs in the framework of simpler systems such as Newtonian colloidal suspensions, the analysis proposed in this chapter is expected to be useful to understand a wide variety of complex fluids in which a superposition of the elasticity of the components is possible. The generalization of our approach to such systems 
and to other technologically relevant PNCs, such as nanocomposites based on layered silicates or carbon nanotubes, still remains to be proved.

\section{References}

Acierno, D.; Filippone, G.; Romeo, G.; Russo, P. (2007). Rheological aspects of $\mathrm{PP}^{-\mathrm{TiO}_{2}}$ nanocomposites: a preliminary investigation. Macromol. Symp., Vol. 247, pp. 59-66

Acierno, D.; Filippone, G.; Romeo, G.; Russo, P. (2007). Dynamics of stress bearing particle networks in poly(propilene)/alumina nanohybrids, Macromol. Mater. Eng., Vol. 292, pp. 347-353

Baird, D. G.; Collias D. I. (1995). In: Polymer Processing Principles and Design, John Wiley and Sons, Inc., ISBN: 978-0-471-25453-9, New York (USA)

Bicerano, J.; Douglas, J. F.; Brune, D. A. (1999). Model for the viscosity of particle dispersions. J. Macromol. Sci.: Rev. Macromol. Chem. Phys. Vol. 39, No. 4, pp. 5611-642

Cipelletti, L.; Manley, S.; Ball, R. C.; Weitz, D. A. (2000). Universal aging features in restructuring of fractal colloidal gels. Phys. Rev. Lett., Vol. 84, No. 10, pp. 2275-2278

Du, F.; Scogna, R. C.; Zhou, W.; Brand, S.; Fischer, J. E.; Winey, K. I. (2004). Nanotube networks in polymer nanocomposites: rheology and electrical conductivity, Macromolecules, Vol. 37, pp. 9048-9055

Filippone, G.; Romeo, G.; Acierno, D. (2010). Viscoelasticity and structure of polystyrene/fumed silica nanocomposites: Filler Network and Hydrodynamic Contributions. Langmuir, Vol. 26, No. 4, pp. 2714-2720

Gleissle, W.; Hochstein, B. (2003). Validity of the Cox-Merz rule for concentrated suspensions. J. Rheol., Vol. 47, pp. 897-910

Inoubli, R.; Dagréou, S.; Lapp, A.; Billon, L.; Peyrelasse, J. (2006). Nanostructure and mechanical properties of polybutylacrylate filled with grafted silica particles. Langmuir, Vol. 22, No. 15, pp. 6683-6689

Israelachvili, J. (1991). In: Intermolecular and surface forces, Academic Press, ISBN 0-12-3751810 , London, UK

Jancar, J.; Recman, L. (2010). Particle size dependence of the elastic modulus of particulate filled PMMA near its $\mathrm{T}_{\mathrm{g}}$, Polymer, Vol. 51, No. 17, (August 2010), pp. 3826-3828

Kammler, H. K.; Beucage, G.; Mueller, R.; Pratsinis S. E. (2004). Structure of flame-made silica nanoparticles by ultra-small-angle X-ray scattering. Langmuir, Vol. 20, pp. 1915-1921

Kojima Y, Usuki A, Kawasumi M, Okada A, Fukushima Y, Kurauchi T, Kamigaito O. (1993) Mechanical properties of nylon 6-clay hybrid, J Mat Res Vol. 8, pp. 1185-1189

Krishnamoorti, R.; Yurekli, K. (2001). Rheology of polymer layered silicate nanocomposites, Curr. Opin. Colloid Interface Sci. Vol. 6, No. 5-6, pp. 464-470

Mason, T. G.; Weitz, D. A. (1995) Linear viscoelasticity of colloidal hard sphere suspensions near the glass transition. Phys. Rev. Lett., Vol. 75, pp. 2770-2773

Ozmusul, M. S.; Picu, R. C.; Sternstein, S. S.; Kumar, S. (2005). Lattice Monte Carlo simulations of chain conformations in polymer nanocomposites, Macromolecules, Vol. 38, No. 10, pp. 4495-4500

Prasad, V.; Trappe, V.; Dinsmore, A. D.; Segrè, P. N.; Cipelletti, L.; Weitz, D. A. (2003) Universal features of the fluid to solid transition for attractive colloidal particles. Faraday Discuss., Vol. 123, pp. 1-12 
Pusey, P. N.; van Megen, W. (1986). Phase behaviour of concentrated suspensions of nearly hard colloidal spheres. Nature, Vol. 320, pp. 340-342

Ren, J.; Silva, A. S.; Krishnamoorti, R. (2000). Linear viscoelasticity of disordered polystyrene-polyisoprene block copolymer based layered-silicate nanocomposites. Macromolecules, Vol. 33, No. 10, pp. 3739-3746

Romeo, G.; Filippone, G.; Fernández-Nieves, A.; Russo, P.; Acierno, D. (2008). Elasticiy and dynamics of particle gels in non-Newtonian melts. Rheol. Acta, Vol. 47, pp. 989-997

Romeo, G.; Filippone, G.; Russo, P.; Acierno, D. (2010). Effects of particle dimension and matrix viscosity on the colloidal aggregation in weakly interacting polymernanoparticle composites: a linear viscoelastic analysis. Polym. Bull., Vol. 63, No.6, pp. 883-895

Russel, W. B.; Saville, D. A.; Schowalter, W. R. (1989). In: Colloidal dispersions, Cambridge University Press, ISBN 0-521-34188-4, Cambridge, UK

Saint-Michel, F.; Pignon, F.; Magnin, A. (2003). Fractal behavior and scaling law of hydrophobic silica in polyol. J. Colloid space face Sci., Vol. 267, No. 2, pp. 314-319

Shenoy, A. V. (1999). In: Rheology of filled polymer systems, Kluwer Academic Publishers, ISBN 0-4112-83100-7, Dordrecht, The Netherlands

Shikata, T.; Pearson, D.S. (1994). Viscoelastic behavior of concentrated spherical suspensions. J. Rheol., Vol. 38, pp. 601-613

Sollich, P.; Lequeux, F.; Hébraud, P.; Cates, M. E. (1997). Rheology of soft glassy materials. Phys. Rev. Lett. 78, 2020-2023

Surve, M.; Pryamitsyn, V.; Ganesan, V. (2006). Universality in structure and elasticity of polymer-nanoparticle gels. Phys. Rev. Lett., Vol. 96, No. 17, pp. 1778051-1778054

Trappe, V.; Weitz, D. A. (2000). Scaling of the viscoelasticity of weakly attractive particles. Phys. Rev. Lett., Vol. 85, No. 2, pp. 449-452

Trappe, V.; Prasad, V.; Cipelletti, L.; Segrè, P.N.; Weitz, D. A. (2001). Jamming phase diagram for attractive particles. Nature, Vol. 411, pp. 772-775

Usuki A.; Kojima Y.; Kawasumi M.; Okada A.; Fukushima Y.; Kurauchi T.; Kamigaito O. (1993). Synthesis of nylon 6-clay hybrid. J Mat Res, Vol. 8, pp. 1179-1184

Weitz, D. A.; Oliveira, M. (1984). Fractal structures formed by kinetic aggregation of aqueous gold colloids. Phys. Rev. Lett., Vol. 52, pp. 1433-1436

Wolthers, W.; van den Ende, D.; Bredveld, V.; Duits, M. H. G.; Potanin, A. A.; Wientjens, R. H. W.; Mellema, J. (1997). Linear viscoelastic behavior of aggregated colloidal dispersions. Phys. Rev. E, Vol. 56, pp. 5726-5733

Zhang, Q.; Archer, L. A. (2002). Poly(ethylene oxide)/silica nanocomposites: structure and rheology. Langmuir, Vol. 18, No. 26, pp. 10435-10442 


\title{
Dielectric and Transport Properties of Thin Films Deposited from Sols with Silicon Nanoparticles
}

\author{
Nickolay N. Kononov ${ }^{1}$ and Sergey G. Dorofeev ${ }^{2}$ \\ 1 Prokhorov General Physics Institute, Russian Academy of Sciences, Moscow, \\ ${ }^{2}$ Faculty of Chemistry, Moscow State University, Moscow, \\ Russia
}

\section{Introduction}

Currently, there is steady scientific interest in structures formed by nanocrystalline silicon particles $(n c-\mathrm{Si})$. This interest is to a large extent caused by the fact that efficient methods for fabricating silicon nanoparticles capable of bright and stable photoluminescence in the visible region of the spectrum with high quantum yield were developed over the last decade (Jurbergs at al., 2006). The main carriers of such nanoparticles are colloidal solutions (sols) based on methanol, chloroform, hexane, etc. Such sols are very promising objects for developing technologies for applying highly uniform thin $n c-S i$ films onto various substrates. The use of such films seems very promising for developing light emitting elements based on $n c$-Si electroluminescence (Anopchenko at al., 2009 ). Furthermore, $n c-S i$ films are very promising as elements of solar panels (De la Torre at al., 2006), thin film transistors (Min at al., 2002), and single electronic devices (Tsu, 2000). In the case in which films consist of nanoparticles with a diameter smaller than $10 \mathrm{~nm}$, their total characteristics are controlled not only by their material, but also by properties of atoms on the surface of these particles. In other words, in general, such films should be considered as a multicomponent medium the properties of which are controlled by both crystalline cores of nanoparticles and surface atoms and molecules and air voids being a film component.

In the modern scientific literature, most papers are devoted to the study of properties of amorphous silicon (a-Si) films with introduced silicon nanocrystals (Conte at al., 2006; Wang at al., 2003). Such films can be deposited, e.g., in the high frequency discharge in a mixture of gases SiH4, Ar, or H2 (PECVD method), followed by high temperature annealing (Saadane at al., 2003).

Recently, we showed that homogeneous thin films (with a thickness up to $30 \mathrm{~nm}$ ) can be grown by size selective precipitation sols containing nanocrystalline silicon particles (Dorofeev at al., 2009). Such films (nc-Si) are formed by closely adjacent crystalline Si nanoparticles; therefore, their physical characteristics to a certain extent should be similar to characteristics of films based on porous silicon (por-Si). The optical absorption and photoluminescence ability of por-Si films have been very comprehensively studied to date (see, e.g., Kovalev at al., 1996; Brus at al., 1995); however, the number of studies of transport 
and dielectric properties of such films in an ac electric field is extremely small. Here we indicate papers (Axelrod at al., 2002; Ben-Chorin at al., 1995; Urbach at al., 2007) devoted to such studies of por-Si.

A similar situation exists as applied to nc-Si films; however, we are not aware of results of studies on the conductivity in an ac electric field (ac conductivity and dielectric relaxation in such films).

In this chapter we analyze the dielectric and transport properties of nc-Si films deposited on a glass and quartz substrates from the sol containing nanoparticles of silicon. Silicon nanoparticles were synthesized in the process of laser pyrolysis of silane and placed in ethanol or methanol, repeatedly centrifuged resulting in a colloidal solution (sol) in which the silicon nanoparticles could be a long time (over two years). We analyze three kinds of films. The films deposited on a substrate by centrifugation of sols of nanoparticles in a week after their synthesis. Films deposited on a substrate of sols in which the nanoparticles were 2 years after their synthesis and films deposited from two-year-old sol in which has been added the conductive tetra-aniline. More circumstantial experimental details we will present in the following sections. In the future of the films deposited on a substrate of silicon nanoparticles in a week after their synthesis we call films I, films obtained from similar nanoparticles, but two years after their synthesis (aged nanoparticles) - films II and films deposited of sols with aged nanoparticles and with the tetra aniline addition - films III.

For films I we present measurements of the $n c-S i$ film permittivity in the optical range $\left(5 \times 10^{14} \leq \mathrm{v} \leq 10^{15} \mathrm{~Hz}\right)$ and in the frequency range of $10 \leq \mathrm{v} \leq 10^{6} \mathrm{~Hz}$. In the latter range, the ac conductivity $\left(\sigma_{\mathrm{ac}}\right)$ of $n c$-Si films is also determined.

In the optical region, the real $\varepsilon^{\prime}$ and imaginary $\varepsilon^{\prime \prime}$ components of the complex permittivity were determined from an ellipsometric analysis of light beams incident and reflected from the free boundary of the $n c-S i$ film. In the frequency range of $10 \leq v \leq 10^{6} \mathrm{~Hz}$, the $\varepsilon^{\prime}$ and $\varepsilon^{\prime \prime}$ spectra, were determined from an analysis of the frequency dependence of the nc-Si film impedance.

In an optical spectral region, $\varepsilon^{\prime}$ and $\varepsilon^{\prime \prime}$ varied within 2.1-1.1 and $0.25-0.75$, respectively, as the frequency increased. We attribute such low values of $\varepsilon^{\prime}$ and $\varepsilon^{\prime \prime}$ to the $n c$-Si film structure. The $n c$-Si particles forming such films consist of crystalline cores surrounded by a $\mathrm{SiO}_{\mathrm{x}}$ shell $(0 \leq x \leq 2)$. The $\mathrm{SiO}_{\mathrm{x}}$ shell results from the interaction of the Si nanoparticle surface with ambient air. On the basis of the analysis of the Raman spectra, it is suggested that the amorphous component is involved in the $n c-S i$ powders and films due to oxygen atoms arranged at the nanoparticle surface.

Using the Bruggeman effective medium approximation (EMA) (Bruggeman, 1935), the structural composition of $n c$-Si film was simulated. It was shown that good agreement between the frequency dependences of $\varepsilon^{\prime}$ and $\varepsilon^{\prime \prime}$ obtained from the EMA and the $\varepsilon^{\prime}$ and $\varepsilon^{\prime \prime}$ spectra determined from ellipsometric data is achieved when $n c-S i$ films are considered as a two component medium consisting of $\mathrm{SiO}$ and air voids existing in it. In the frequency range of $10-10^{6} \mathrm{~Hz}$, the $\varepsilon^{\prime}$ and $\varepsilon^{\prime \prime}$ dispersion was determined from an analysis of the frequency dependences of the capacitance of $n c-S i$ films and their impedance spectra. It was found that $\varepsilon^{\prime}$ and $\varepsilon^{\prime \prime}$ vary within 6.2-3.4 and 1.8-0.08, respectively, as the frequency increases. 
It is found that the function $\varepsilon^{\prime}(\omega)$ in this frequency range is well approximated by the semiempirical Cole-Cole dependence (Cole-Cole dielectric relaxation) (Cole, K. S. \& Cole, R. H., 1941). At the same time, the $\varepsilon^{\prime \prime}(\omega)$ spectra of $n c$-Si films are well approximated by the Cole-Cole dependence only at frequencies higher than $2 \times 10^{2} \mathrm{~Hz}$. In the low frequency spectral region, good approximation is achieved by combining the Cole-Cole dependence and the term associated with the presence of free electric charges. From analysis of the approximating dependences, the average room temperature relaxation times of dipole moments in $n c$-Si films were determined as $6 \times 10^{-2} \mathrm{~s}$.

The conductivity $\sigma_{\mathrm{ac}}$ of the studied films $\mathrm{I}$ in an ac electric field depends only on its frequency according to the power law; the exponent is 0.74 in the entire frequency range under study. Such behavior of $\sigma_{\mathrm{ac}}$ suggests that the electrical transport mechanism in films is hopping. Comparison of the measured frequency dependence $\sigma_{\mathrm{ac}}(\mathrm{v})$ with similar dependences following from various models of hopping conductivity shows that the $\sigma_{\mathrm{ac}}(\mathrm{v})$ behavior is most accurately described in the diffusion cluster approximation (DCA) (Dyre \& Schrøder, 2000; Schrøder \& Dyre, 2002; Schrøder \& Dyre, 2008).

Analysis of the dependences of the dark conductivity of films on humidity of ambient air and the temperature dependence of absorption bands caused by associated $\mathrm{Si}-\mathrm{OH}$ groups on the film surface allowed the conclusion to be drawn that conductivity at frequencies lower than $2 \times 10^{2} \mathrm{~Hz}$ is associated with proton transport through the hydrogen bound hydroxyl groups on the silicon nanoparticle surface.

For films II and III we present measurements of the $n c-S i$ film permittivity and ac conductivity $\left(\sigma_{\mathrm{ac}}\right)$ in the frequency range of $1 \leq \mathrm{v} \leq 10^{6} \mathrm{~Hz}$. The dielectric properties of the films II and III were studied by impedance spectroscopy only in the frequency range $1 \leq \mathrm{v} \leq 10^{6} \mathrm{~Hz}$.

We found that in films II and III, a double dielectric relaxation exists and to adequately describe the spectra of $\varepsilon$ 'and $\varepsilon^{\prime \prime}$ of these films should use not only the Cole-Cole relationship, but and the law of Debye's dielectric relaxation.

By a total approximation of the experimental spectra of the films II and III the values of static dielectric constant $\varepsilon_{0}$ have obtained. These values are equal 11.5 and 67 respectively. Value $\varepsilon_{0} \approx 11,5$ characteristic of film II is close to the static permittivity of crystalline silicon, but the magnitude of $\varepsilon_{0} \approx 67$ of films III significantly higher than this value. Next, we analyze this fact.

In contrast to the conductivity of the films I $\sigma_{\mathrm{AS}}$ of films II and III are not subject to a power law over the entire range of measured frequencies. Next, we show that such a deviation from the law $\sigma_{\mathrm{AS}} \sim \omega^{\mathrm{s}}$ associated with the appearance in the spectra $\varepsilon$ " $(\omega)$ of the films II and III Debye's components.

\section{The films from silicon nanoparticles}

\subsection{Films deposited from freshly prepared sols of silicon nanoparticles ( films I)}

\subsubsection{Samples and measurement procedures}

The $n c$-Si films were deposited from silicon nanoparticles produced by $\mathrm{CO}_{2}$ laser pyrolysis of silane. The system for synthesis of the $n c-S i$ powders and conditions of the process are described in detail elsewhere (Kononov at al., 2005; Kuz'min at al., 2000). In what follows, 
we briefly outline the procedure of synthesis of the Si nanoparticles. In a reactor chamber filled with a buffer gas (helium or argon) to the pressure $P=200$ Torr, a fine $\mathrm{SiH}_{4}$ jet is formed and heated by focused $\mathrm{cw} \mathrm{CO}_{2}$ laser radiation beam crossing the jet. During pyrolysis of silane, the $\mathrm{SiH}_{4}$ molecules are decomposed, and free $\mathrm{Si}$ atoms are produced. When colliding with each other and with the atoms of the buffer gas, the $\mathrm{Si}$ atoms form particles, whose average dimensions can be in the range from 10 to $100 \mathrm{~nm}$, depending on the pressure of the buffer gas. The $n c$-Si powders produced in such a manner were dispersed by ultrasonic treatment in ethanol and centrifuged for $30 \mathrm{~min}$ with an acceleration of $2000 \mathrm{~g}$ ( $g$ is the gravitational acceleration). As a result, almost all agglomerates of $n c$-Si particles are precipitated. After preliminary centrifugation, a stable colloidal solution (sol) of nc-Si in ethanol remains. No visible changes in the solution, including precipitations, were observed for two years. For the subsequent deposition of nanoparticles, a water solution of aluminum dihydrophosphate was added to the sol.

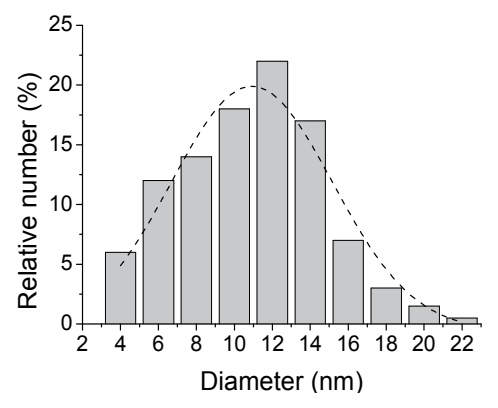

a

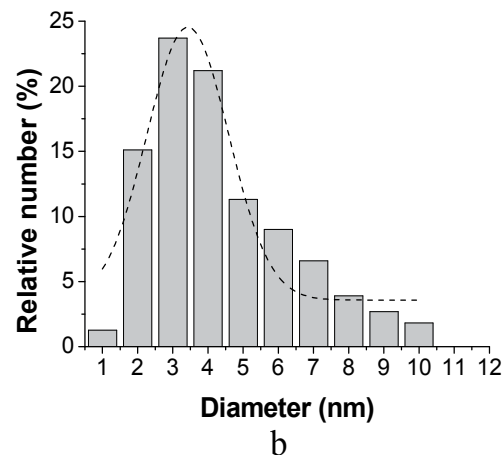

$\mathrm{b}$

Fig. 1. (a) Histogram of the size distribution of particles, as obtained by processing of the TEM images of the $n c$-Si powder (b) Histogram of the size distribution of particles, as obtained by processing of the TEM images of the $n c$-Si powder etched in the $\left(\mathrm{HF}+\mathrm{HNO}_{3}\right)$ acid mixture. The dushed lines represent the normal distribution functions for two different kinds of nc-Si particles

The size distribution of $n c$-Si particles was determined from images obtained using an LEO 912 AV OMEGA transmission electron microscope. The typical spectrum of silicon nanoparticles used for precipitation is shown in the Fig. 1. The nc-Si film thickness was determined using a Taly Step (Taylor-Hobbson) atomic force step profilometer. Ellipsometric spectra were measured using an Ellips 1891 ellipsometer (Institute of Semiconductor Physics, Siberian Branch, Russian Academy of Sciences). The transmission spectra were measured using a Lambda 900 (Perkin-Elmer) spectrophotometer. The Raman spectra of the films were recorded with a microlens equipped T 64000 (Jobin Ivon) Raman triple spectrograph in the backscattering layout of measurements at the power of the excitation argon laser $2 \mathrm{~mW}$.

The impedance spectra were measured using an E7-20 immittance meter (Minsk Research Instrument Making Institute) and a Z-3000X (Elins) impedance meter. Samples for 
measuring impedance spectra were prepared as follows. First, aluminum electrodes separated by a rectilinear gap $1 \mathrm{~mm}$ wide were deposited on a glass substrate. Then $n c-\mathrm{Si}$ particles were precipitated from the sol on the substrate prepared in such a way, which formed a film. The third aluminum electrode was deposited on the obtained $n c$-Si film. As a result, a sandwich like structure similar to that shown in Fig. 2 was obtained. To achieve the ohmic lead contacts, the structure was annealed at a temperature of $400^{\circ} \mathrm{C}$ and a pressure of $10^{-5}$ Torr. Impedance spectra were measured at an amplitude voltage of $100 \mathrm{mV}$; however, the films under study can withstand a voltage to $15 \mathrm{~V}$ without electrical breakdown.

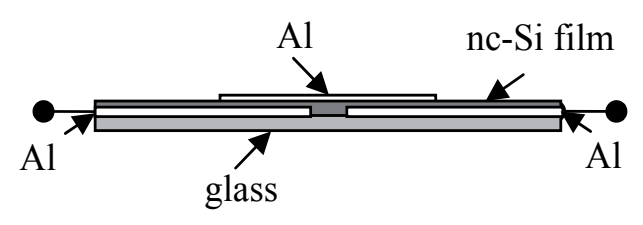

Fig. 2. Diagram of the sandwich like sample structures for measuring impedance spectra.

\subsubsection{Experimental results}

\subsubsection{Raman scattering}

To record the Raman spectra, we first deposited an aluminum film with the thickness $\sim 300$ $\mu \mathrm{m}$ onto the quartz substrate, and then, on top of the film, we deposited the $n c-S i$ film from the sol. We proceeded in such manner in order to avoid the background scattering component produced by the quartz substrate. In this section, we analyze the Raman

spectra recorded for the initial $n c-S i$ powder, for the films deposited at the second stage of centrifuging the sols of the initial $n c-S i$ powder and film deposited from the sol with powder etched in the $\left(5 \mathrm{wt} \% \mathrm{HF}+14 \mathrm{wt} \% \mathrm{HNO}_{3}\right)$ water mixture. The corresponding samples are identified as samples $S_{1}, S_{2}$, and $S_{3}$ respectively.

The typical Raman spectra recorded for these samples are shown in Fig. 3. All of the experimentally recorded spectra are very similar to the Raman spectra obtained for $p$-Si in (Tsang at al., 1992; Tsu at al., 1992) and for the nc-Si clusters in (Ehbrecht at al., 1995).
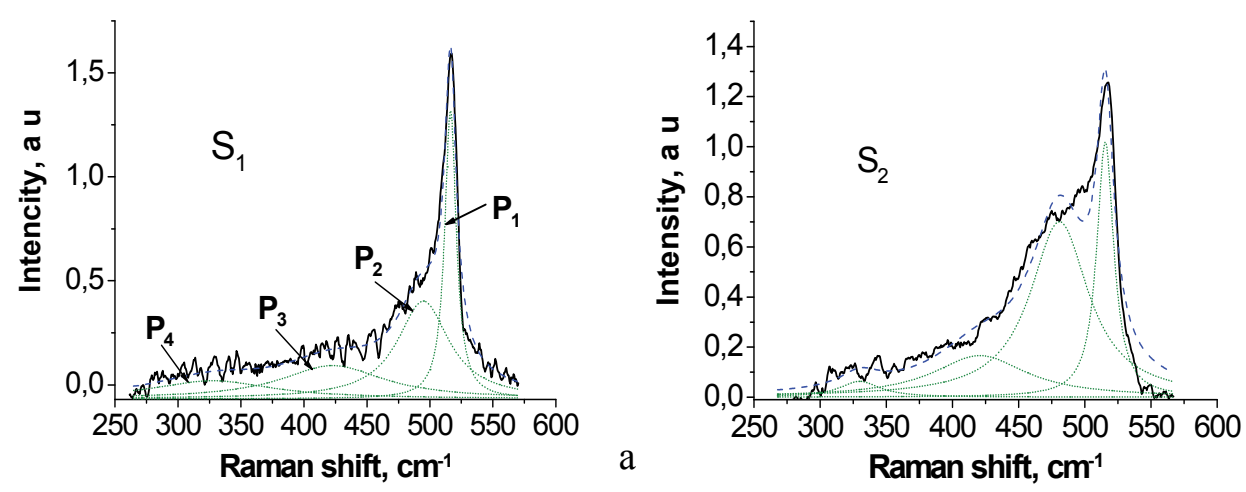


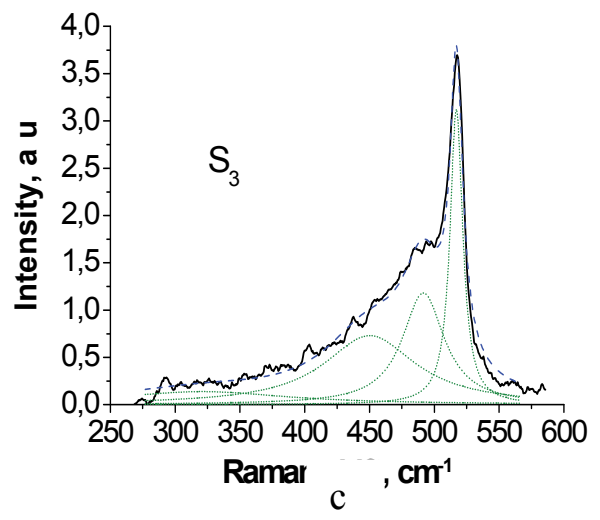

Fig. 3. Raman spectra of: a) nc-Si powder $S_{1}$, b) film deposited from the sol of the initial $n c-S i$ powder- $\left.\mathrm{S}_{2} \mathrm{c}\right)$ film deposited from the sol $n c$-Si powder etched in the $\left(\mathrm{HF}+\mathrm{HNO}_{3}\right)$ acid mixture $-S_{3}$. The dotted line refers to the approximation of the spectrum with Lorentzian contours (the $P 1, P 2, P 3$, and $P 4$ peaks).

The Raman spectra of all of the samples studied here can be fitted with four Lorentzian bands with a rather good accuracy (Fig. 3). In what follows, these bands are referred to as the $P_{1}, P_{2}, P_{3}$, and $P_{4}$ peaks. The Raman shift of the most intense $P_{1}$ peak with respect to the emission frequency of the probing laser is in the range of wave numbers from 515 to $517 \mathrm{~cm}^{-}$ 1 for all of the samples. The Raman shift of the similar peak for $c$-Si corresponds to the wave number $520.5 \mathrm{~cm}^{-1}$. Thus, for all of the films studied here, the $P_{1}$ peak is shifted to smaller wave numbers with respect to the peak for $c$-Si (the red shift). The $P_{1}$ peak in the Raman spectra of the $n c$-Si particles is due to light scattering assisted by longitudinal optical (LO) and transverse optical (TO) phonons at the central point of the Brillouin zone for the $c-S i$ crystal lattice. The red shift of the $P_{1}$ peak and its half width as functions of the nanoparticle dimensions are adequately described in the context of the phonon's confinement model (Campbell \& Faushet 1986; Richter at al., 1981).

The result of application of this model to spherical nanoparticles is shown in Fig. 4. From Fig. 4 , it can be seen that the average dimension of the $n c-S i$ particles in the samples is in the range 4-6 nm, irrespective of whether the particles of the initial $n c$-Si powder were subjected to some treatment or not. For the sols of the $n c-S i$ powders etched in the $\left(\mathrm{HF}+\mathrm{HNO}_{3}\right)$ mixture, the average particle's dimensions determined in the phonon's confinement model are in good agreement with the particle dimensions corresponding to the peak of size distribution obtained for the particles by processing of the TEM images.

However, for the initial $n c$-Si powders, the average particle dimensions determined by the above mentioned two methods differ by a factor of about 2 . There are two possible causes of the difference between the average particle's dimensions determined in the phonon's confinement model and by processing of the TEM images. One of the causes is associated with the fact that, in the phonon's confinement model, the nanoparticles are assumed to be single crystals. Therefore, the magnitude of the phonon wave's vector $q$ in the 
nanoparticle can vary in the range $(0,2 \pi / L)$, where $L$ is the particle diameter. However, if the nanoparticle core is polycrystalline and the average dimension of the elementary crystal lattice in the core is $l$, the confining condition $q \leq 2 \Pi / L$ should be replaced by the condition $q \leq 2 \Pi / l$. Thus, it is possible that the dimensions $l=4-6 \mathrm{~nm}$ calculated in the phonon's confinement model are related to the average dimensions of elementary lattices in the polycrystalline nanoparticle cores rather than to the average nanoparticles' dimensions in the initial $n c-S i$ powder. From this assumption and the fact that, for nanoparticles subjected to etching, the average dimensions determined by the above two methods are the same, it follows that, on such etching of the nanoparticles, the remaining $c$-Si cores are single crystals. The other cause can follow from the well known low contrast of the finest nanoparticles (with the diameter $3 \mathrm{~nm}$ in the case under study) in the TEM images. Because of the low contrast, the processing of the TEM images always reduces the relative portion of the fine grained fraction of nanoparticles in the ensemble of particles under consideration.

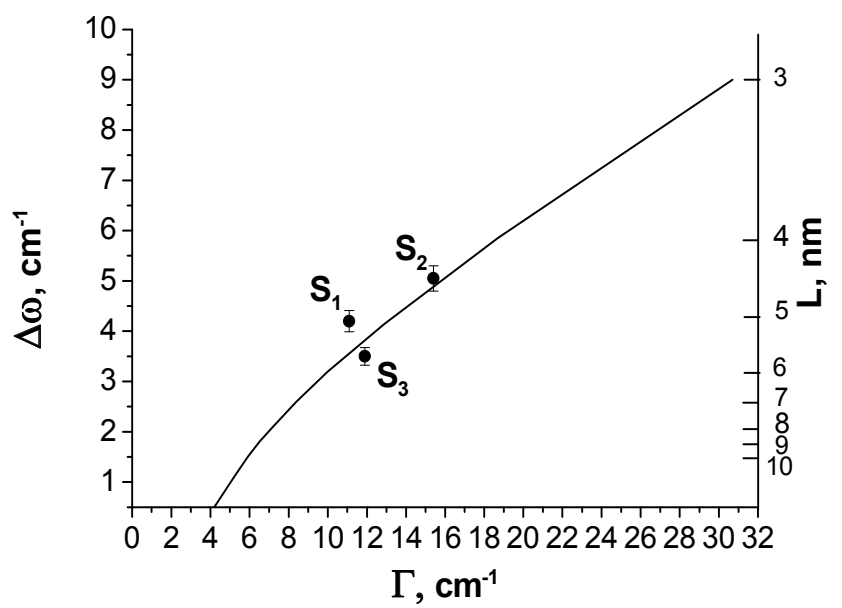

Fig. 4. The half-width and the red shift of the $P_{1}$ Raman peak versus the diameter of the spherical silicon nanoparticles, as obtained (solid line) in the context of the phonon's confinement model $[19,20]$ and (solid circles) from the approximation of the $P_{1}$ peak in samples $S_{1}, S_{2}$ and $S_{3}$, with the Lorentzian contours.

The Raman shift of the $P_{2}$ peak in the samples is in the range from 480 to $495 \mathrm{~cm}^{-1}$. This peak corresponds to the TO-phonon assisted scattering in $a$-Si:H. Similarly to the $P_{2}$ peak, the $P_{3}$ and $P_{4}$ peaks are related to the amorphous component of the structure of the Si particles and result from scattering assisted by LO and longitudinal acoustic (LA) phonons.

From the comparison of the integrated intensities of the $P_{1}$ and $P_{2}$ peaks, $I c$ and $I a$, we can determine the volume fraction of the crystalline phase, Xc, in the Si particles. To do this, we used the expression (Voutsas at al., 1995)

$$
\mathbf{X}_{\mathbf{c}}=\frac{\mathbf{I}_{\mathbf{c}}}{\mathbf{I}_{\mathbf{c}}+\eta \mathbf{I}_{\mathbf{a}}}
$$


where $\eta=\frac{\sigma_{\mathbf{c}}}{\sigma_{\mathbf{a}}}$ is the ratio between the integrated backscattering's cross sections in the crystalline and amorphous fractions (corresponding to the $P_{1}$ and $P_{2}$ peaks). According to (Kakinuma at al., 1991), the quantity $\eta$ for silicon is $\eta=0.8-0.9$. In the calculations, we set $\eta=0.8$. For samples $S_{1}, S_{2}$ and $S_{3}$, the values of the parameter $X c$ are $0.45,0.35$ and 0.50 respectively.

From these values of $X_{c}$, it follows that almost a half of the volume of the particles is characterized by a high degree of disorder of the crystal lattice.

From comparison of the above values, it is evident that, in film $S_{2}$ deposited at the second stage of centrifuging from the sol with the initial $n c$-Si powder, the parameter $X_{c}$ is smaller than $X_{c}$ for the initial powder. The average particle's dimension in film $S_{2}$ is smaller than that in the initial powder. Correspondingly, the surface area to volume ratio for the particles in the $S_{2}$ film is larger than the corresponding ratio in the initial powder. Therefore, the effect of the nanoparticle surface on the general properties of the nanoparticles in film $S_{2}$ is bound to be more pronounced that the corresponding effect in the initial powder. Consequently, the smaller value of $X_{c}$ (the higher degree of amorphization of the particles) in film $S_{2}$ in comparison with $X_{c}$ in sample $S_{1}$ suggests that the disordered regionis at the nanoparticle surface rather than in the nanoparticle core. However, for film $S_{3}$ the value of $X_{c}$ is larger than $X_{c}$ for film $S_{2}$, although the average particle's dimensions in these films are comparable. Such difference suggests that the degree of disorder of particle surfaces in film $S_{3}$ is lower than that in film $S_{2}$.

Since film $S_{3}$ are deposited from the sols of the nc-Si powders subjected to etching, such lower degree of disorder in these films is due to the effect of the $\mathrm{HF}$ and $\mathrm{HNO}_{3}$ acids on the particle surface. Here, it is reasonable to mention the studies ( Luppi \& Ossicini, 2005; Puzder at al., 2002), in which the effect of oxygen atoms on the structure of silicon clusters and on the degree of ordering of the Si crystal lattice in nanoparticles is analyzed, and the studies (Ma at al., 2000 Tsang at al., 1992; ), in which the changes induced in the Raman peak similar to the $P_{2}$ peak (Fig. 3) by the effect of oxygen on the surface of $p$-Si passivated with hydrogen, are reported. The general idea of the above mentioned studies is that the crystal lattice of nanoparticles, whose surface is completely passivated with hydrogen, is practically the same as the lattice of the silicon crystal. However, if oxygen atoms appear at the nanoparticle surface, they can form the $\mathrm{Si}-\mathrm{O}-\mathrm{Si}$ and $(\mathrm{Si}=\mathrm{O})$ bonds and, thus, distort the lattice at the distances up to $0.5 \mathrm{~nm}$. In this space region, the distortions of angles between the $\mathrm{Si}-\mathrm{Si}$ bonds in the crystal lattice can be as large as $10^{\circ}$ (Tsang at al., 1992). Therefore, if the surface of a nanoparticle of a diameter smaller than $3 \mathrm{~nm}$ is coated with the $\mathrm{SiO}_{2}$ oxide, the crystal lattice is distorted within a noticeable volume fraction of such particle. As a consequence, if the $p$-Si surface is etched in the solution of HF, the Raman spectrum involves only one peak similar to the $P_{1}$ peak. If $p$-Si is exposed to oxygen in oxygen containing atmosphere, the Raman spectrum exhibits also the $P_{2}$ peak along with the $P_{1}$ peak. From the above mentioned studies and from the analysis of the Raman spectra discussed here, we can make the statement presented below. At the surface of $n c$-Si nanoparticles in all samples, there is a noticeable number of oxygen atoms, which distort the crystal lattice in these particles and bring about the appearance of the $P_{2}$ peak in the Raman spectra. Since the average nanoparticle's dimensions in film $S_{2}$ are smaller than those in powder $S_{1}$, the effect of these oxygen atoms on the crystal lattice structure in film $S_{2}$ is more pronounced than the 
effect in powder $S_{1}$. As a result, the volume fraction of the crystal phase in film $S_{2}$ is reduced compared to that in $S_{1}$.

Etching of the $n c-\mathrm{Si}$ particles in the solution of the $\left(\mathrm{HF}+\mathrm{HNO}_{3}\right)$ acids results in a decrease in the particle dimensions. However, in this case, the total number of oxygen atoms at the nanoparticle's surface decreases, since a portion of oxygen atoms is replaced with hydrogen atoms. Therefore, in film $S_{3}$ two opposite processes are bound to occur. One process related to the decrease in the nanoparticle's dimensions yields a decrease in $X c$, whereas the other process related to the decrease in the number of oxygen atoms at the nanoparticle surface brings about an increase in $X c$. In film $S_{3}$, we experimentally observe the parameter $X_{c}$ larger than $X_{\mathrm{c}}$ in film $S_{2}$; therefore, we can conclude that, on etching of the $n c$-Si particles, the latter process dominates over the former one.

\subsubsection{Ellipsometric spectra}

In the experiment, the ellipsometric angles $\psi$ and $\Delta$ were measured as functions of the wavelength of a light beam incident at the angle $\Phi_{0}$ on the free flat surface of the $n c$-Si film. The films under study were applied on glass and quartz substrates and on quartz substrates with preliminarily deposited aluminum films. The $n c$-Si film thicknesses $(1-2 \mu \mathrm{m})$ were measured independently. When processing the ellipsometric data, the $n c-S i$ films under study were considered as a 3D medium in air medium. The complex refractive index $N=n-i k$, where $n$ is the film refractive index and $k$ is the extinction coefficient, was determined by the expression (Azzam \& Bashara, 1977)

$$
N=N_{0} \sin \Phi_{0} \sqrt{1+\left(\frac{1-\rho}{1+\rho}\right)^{2} \operatorname{tg}^{2} \Phi_{0}}
$$

Here, $\rho=\mathrm{e}^{\mathrm{i} \Delta .} \operatorname{tg} \Psi$ and $N_{0}$ are the complex refractive index of an ambient medium (air), which was equal to unity in the case at hand. It is known that formula (2) yields accurate values only when light is reflected from a semi-infinite medium with a boundary with an atomically clean surface. If impurities or an oxide filmare on the boundary, they introduce errors to the calculated values. In (Tompkins \& Irene, 2005), the values $n$ and $k$ were compared for crystalline silicon $(c-S i)$ in the absence and presence of the oxide film on its surface. It follows from this comparison that, in the presence of $\mathrm{SiO}_{2}$ film to $2 \mathrm{~nm}$ thick on the silicon surface, the value of $n$ is almost identical to that of $c-S i$ in the incident photon energy range of 1-3.4 eV; in the range of 3.4-5 eV, the refractive index differs from $n$ of $c-S i$ no more than by $20 \%$, as well as $k$. However, in the range of $1-3.4 \mathrm{eV}$, the value of $\mathrm{k}$ in the presence of the $\mathrm{SiO}_{2}$ film almost twice exceeds the $c$-Si extinction.

Since the real $\varepsilon^{\prime}$ and imaginary $\varepsilon^{\prime \prime}$ components of the medium permittivity are related to $n$ and $k$ by the known expressions $\varepsilon^{\prime}=n_{2}-k_{2}$ and $\varepsilon^{\prime \prime}=2 n k$, it can be expected that the values of $\varepsilon^{\prime}$ calculated by Eq. (2) for $n c$-Si films will be slightly systematically underestimated, while the values of $\varepsilon^{\prime \prime}$ will be overestimated.

Nevertheless, representation of the pseudo dielectric functions by relation (2) is very convenient and is quite often used to study the dielectric properties of materials. For example, dielectric parameters of por-Si were studied using this equation in (Pickering, 1984). As applied to the present study, an analysis of the spectra obtained using formula (2) 
was limited by the energy range of incident photons, in which films strongly absorbed incident probe radiation, which could not reach the substrate surface in this case. If probe radiation reached the substrate surface with precipitated film, an interference structure arose in the spectra, which consisted of alternating minima and maxima. Such a structure at energies lower than $2 \mathrm{eV}$ is easily seen in Fig. 5 (curves 3 and 3 ').

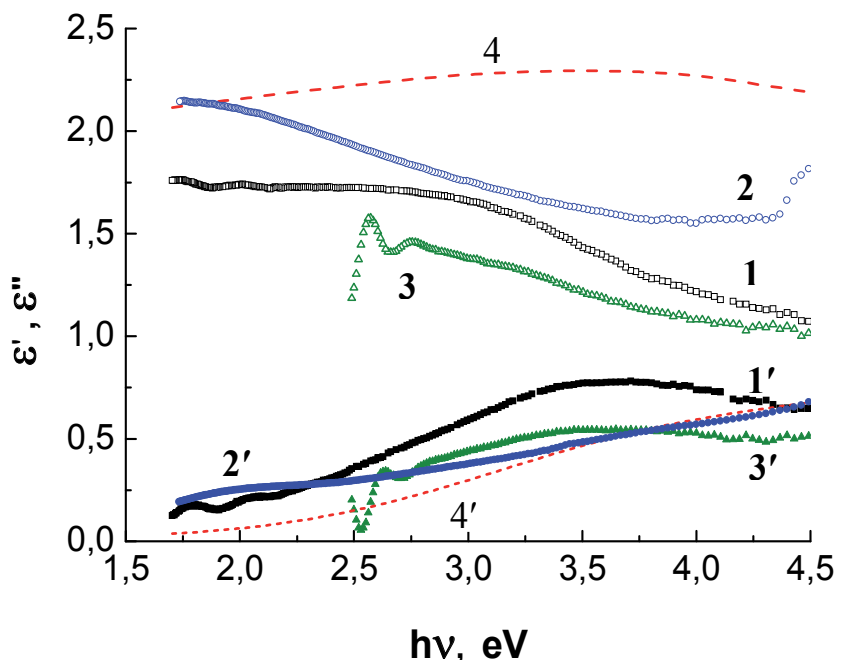

Fig. 5. Spectra of (1-3) real and imaginary $\left(1^{\prime}-3^{\prime}\right)$ permittivity components of $n c-S i$ films precipitated on various substrates: $\left(1,1^{\prime}\right)$ film of initial (unetched) nanoparticles on the glass substrate; $\left(2,2^{\prime}\right)$ film of nanoparticles preliminarily etched in a $\mathrm{HF} / \mathrm{HNO}_{3}$ acid mixture on the quartz substrate; $\left(3,3^{\prime}\right) n c-S i$ film of initial nanoparticles on the glass substrate with a preliminary deposited aluminum film; and $(4,4)$ Bruggeman approximation for $\varepsilon^{\prime}$ and $\varepsilon^{\prime \prime}$, respectively.

We can see the spectra of pseudo dielectric functions $\varepsilon^{\prime}$ and $\varepsilon^{\prime \prime}$ of $n c$-Si films fabricated by precipitating initial silicon nanoparticles on the glass substrate and nanoparticles preliminary etched in a $\mathrm{HF} / \mathrm{HNO}_{3}$ acid mixture in a water for $30 \mathrm{~min}$ on the quartz substrate. Figure 5 also shows the $\varepsilon^{\prime}$ and $\varepsilon^{\prime \prime}$ spectra of $n c$-Si films precipitated on the glass substrate with a preliminarily deposited aluminum film.

It follows from this figure that the obtained values of $\varepsilon^{\prime}$ and $\varepsilon^{\prime \prime}$ are significantly lower than the similar values of $c$-Si.

Figure 6 shows the absorption spectra $a(E)$ of the same films, obtained by the relation:

$$
\alpha(E)=\frac{4 \pi v}{c} k=\frac{4 \pi E}{c h} k
$$

where $E=h \mathrm{v}$ is the energy of the incident photon and $k$ is the experimentally measured extinction coefficient. 


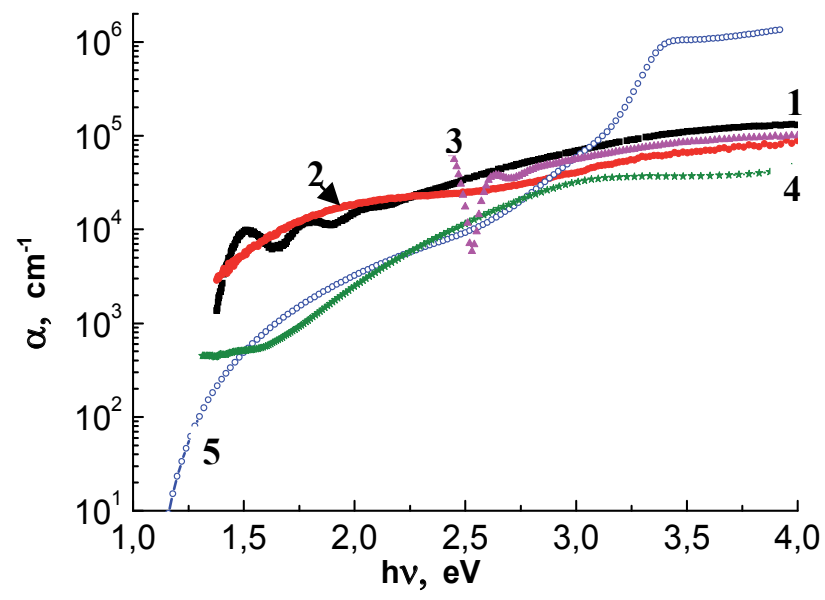

Fig. 6. (1-3) - absorption spectra of $n c$-Si films, obtained from ellipsometric data; (4) - absorption spectrum of film 1, obtained from its transmission spectrum; and (5) absorption spectrum of crystalline silicon.

This figure also shows the absorption spectrum of the nc-Si film formed by unetched nanoparticles, which was calculated from its transmission spectrum. As a reference, the absorption spectrum of crystalline silicon (Aspens \& Studna, 1983) is also shown. The size distribution of unetched and etched $n c$-Si particles used to precipitate films 1 and 3 are shown in Fig. 1.

A comparison of the absorption spectra of the film $n c-S i$ grown from unetched nanoparticles, which were obtained from ellipsometric measurements and by processing the corresponding transmission spectrum, shows that the values of a obtained by ellipsometry are higher than the similar values calculated from transmission spectra, and this difference increases with decreasing the incident photon energies. As noted above, this difference is associated with the error of the extinction coefficient calculation by formula (2). At the same time, both spectra exhibit strong absorption of the nc-Si film in comparison with $c$-Si at energies lower than $1.5 \mathrm{eV}$. Such absorption enhancement in the low energy photon region is also inherent to the film grown by etched nanoparticles. At energies higher than $3 \mathrm{eV}$, all spectra exhibit absorption weaker than that of $c$-Si. In the Fig. 1, we can see that the diameter of an appreciable fraction of particles used to form films is smaller than $10 \mathrm{~nm}$; therefore, the most probable cause of a decrease in the film absorption in the high-energy photon region is widening of the band gap in crystalline cores of silicon nanoparticles due to quantum confinement.

\subsubsection{Dielectric dispersion}

The permittivity spectra of $n c-S i$ films were calculated from the measured frequency dependences of the capacitance of corresponding samples and their impedances, $\mathrm{Z}(\mathrm{v})=\mathrm{Z}^{\prime}-\mathrm{i} \mathrm{Z}^{\prime \prime}, \mathrm{Z}(\mathrm{v})=\mathrm{U}(\mathrm{v}) / \mathrm{I}(\mathrm{v})$, 
where $U(\mathrm{v})$ is the potential difference at sample electrodes and $I(\mathrm{v})$ is the current flowing through the sample.

In what follows, we will analyze the dielectric properties of the $\mathrm{Al}-n c-\mathrm{Si}-\mathrm{Al}$ sandwich system in which the $n$-Si layer was precipitated from the sol with unetched nanoparticles. The thickness of this film was $2 \mu \mathrm{m}$; the geometrical capacitance of this system was $C_{0}=1.15$ $\times 10^{-10} \mathrm{~F}$. The dielectric dispersion of this film is typical of other films obtained in a similar way from similar $n c-S i$ particles.

Figure 7 shows the frequency dependence of the capacitance of this system. The capacitance was measured in parallel connection. The figure also shows the spectrum of the real component $\varepsilon^{\prime}(\mathrm{v})$ of the film permittivity, calculated from the relation $\varepsilon^{\prime}=C(\mathrm{v}) / C_{0}$.

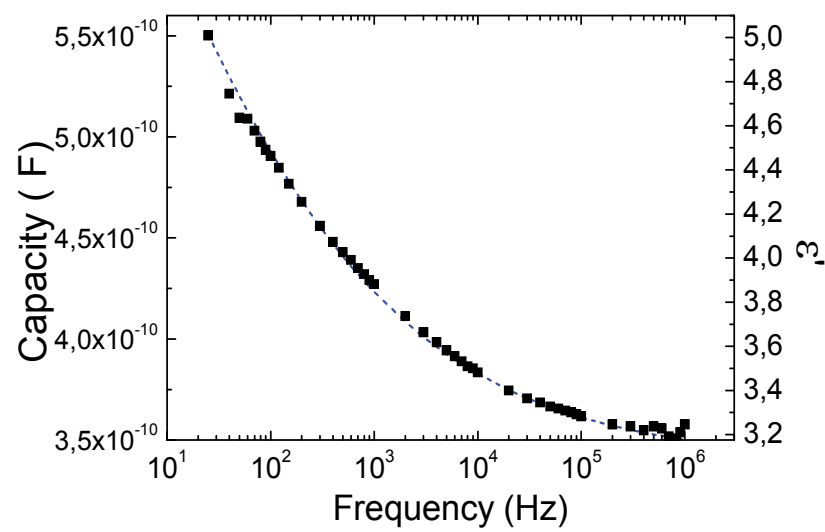

Fig. 7. Frequency dependence of the $n c-S i$ film capacitance. The dashed curve is the approximation by function (3) (see text).

The $\varepsilon^{\prime}(v)$ and $\varepsilon^{\prime \prime}(v)$ spectra of $n c-S i$ films were also determined from the frequency dependence the film impedance by the expression

$$
\varepsilon=\varepsilon^{\prime}-i \varepsilon^{\prime \prime}=\frac{1}{i 2 \pi v C_{0} Z(v)}
$$

Figure 8.a shows the dependences $\varepsilon^{\prime}(\mathrm{v})$ and $\varepsilon^{\prime \prime}(\mathrm{v})$ calculated by the above method for the film under study. A comparison of the values of $\varepsilon^{\prime}(\mathrm{v})$ obtained from $C(\mathrm{v})$ and $Z(\mathrm{v})$ measurements shows good quantitative and qualitative agreement of the values calculated in two different ways; in both cases, in the frequency range of $10 \leq \mathrm{v} \leq 10^{6} \mathrm{~Hz}$, the value of $\varepsilon^{\prime}(\mathrm{v})$ is within 63.4 and decreases with frequency.

\subsubsection{AC conductivity of films I}

The ac conductivity of $n c-S i$ films was determined by the known relation

$$
\sigma_{A C}(v)-\sigma(0)=\varepsilon_{0} \cdot \varepsilon^{\prime \prime}(v) \cdot 2 \Pi v
$$

where $\sigma(0)$ is the dark conductivity of films in a dc electric field and $\varepsilon_{0}=8.85 \times 10^{-12} \mathrm{~F} / \mathrm{m}$ is the permittivity of free space. The value of $\sigma(0)$ of the film under study at room temperature 
$(T=297 \mathrm{~K})$ was $9 \times 10^{-10} \Omega^{-1} \mathrm{~m}^{-1}$ and was used to calculate $\sigma_{\mathrm{AC}}(\mathrm{v})$. The dependence $\sigma_{\mathrm{AC}}(\mathrm{v})$ is shown in Fig. 9 on a log scale.

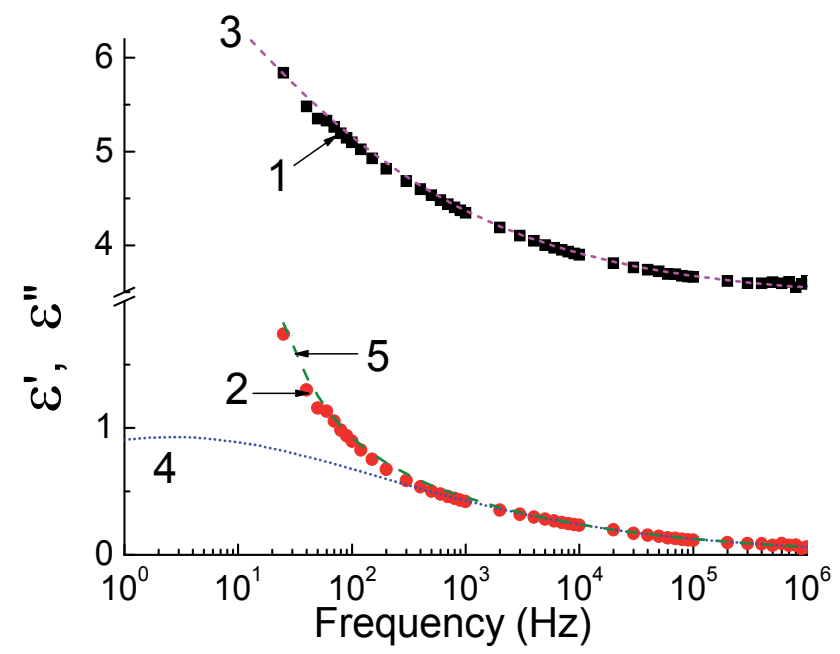

Fig. 8a. $(1,2)$ Frequency dependences of $\varepsilon^{\prime}$ and $\varepsilon^{\prime \prime}$, obtained from impedance spectra. ColeCole approximation of $\varepsilon^{\prime}-(3)$ and $\varepsilon^{\prime \prime}$ spectra (4) without and (5) with consideration of the contribution of free carriers.

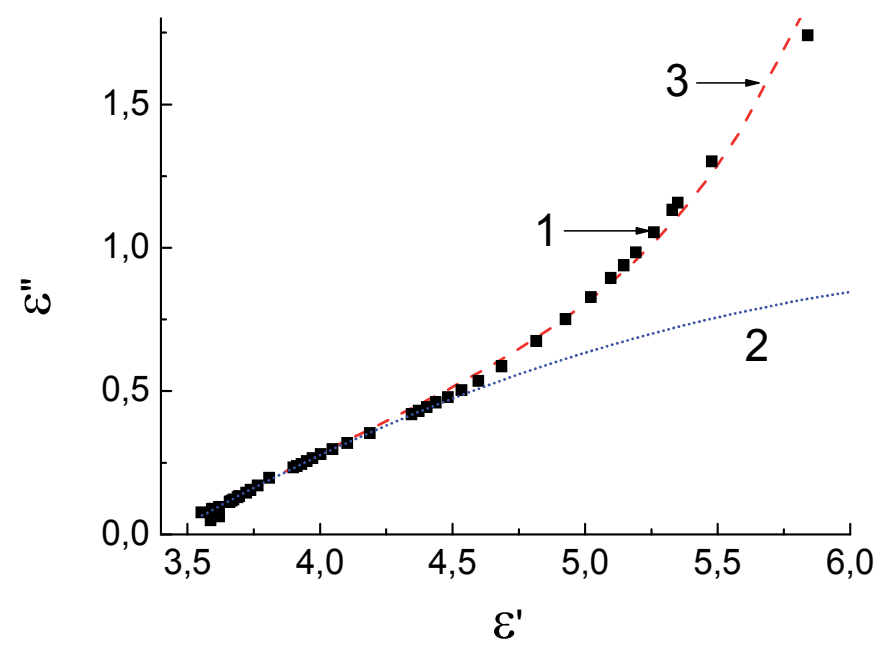

Fig. 8b. (1) Dependence $\varepsilon^{\prime \prime}\left(\varepsilon^{\prime}\right)$ for the $n c$-Si film. Cole-Cole approximation (2) without and (3) with consideration of the contribution of free carriers.

This figure suggests that $\sigma_{\mathrm{AC}}(\mathrm{v})$ can be well approximated by the power law dependence with an exponent of 0.74 . 


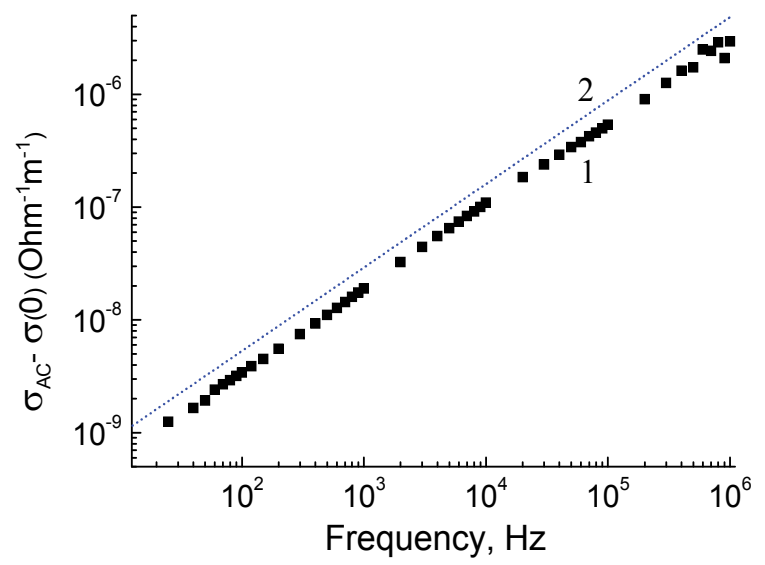

Fig. 9. (1) Frequency dependence of the ac conductivity of the $n c-S i$ film; (2) dependence $\sigma_{\mathrm{ac}}(\mathrm{v})$ defined by the DCA model (see text) using experimentally measured $\varepsilon_{s}, \varepsilon_{\infty}$, and $\sigma(0)$.

\subsection{Films deposited from aged nc-Si sols (Films II) and from aged nc-Si sols with tetraaniline (Films III)}

\subsubsection{Samples}

The previous sections have presented experimental results of a study of thin films obtained from nanoparticles synthesized by one week prior to their deposition on the substrate. As already mentioned silicon nanoparticles could be no precipitation of sols for a long time. By the time of this writing, the silicon nanoparticles used for the deposition of films analyzed in the previous sections, were in sols over two years and in the next section we will report on the results of studies of the properties of the films deposited on substrates of these sols. It should be noted here that the film deposited on a substrate not as a result of centrifugation of sols, and with the spin coating method. Also in the following sections we will analyze the dielectric properties of films deposited from a 2-year nc-Si sols, in which the conductive tetramer - tetraaniline was added (Wang \& MacDiarmid, 2002).

Because pure tetraaniline has low conductivity, for its increase the tetra aniline doped with p-toluensulfonic acid $\left(\mathrm{CH}_{3}\left(\mathrm{C}_{6} \mathrm{H}_{4}\right) \mathrm{SO}_{3} \mathrm{H}\right)$. Briefly the process of doping was as follows. A solution of tetraaniline and dimethyl sulfoxide (DMSO) as a solvent mixed with a DMSO solution of para-toluenesulfonic acid, so that the resulting solution in the molar ratio of tetra aniline and acid was 1.5. At the end of doping the color of resulting solution became green. The conductivity of the film which was deposited on a substrate of resulting tetraaniline solution was at room temperature $10^{-4} \mathrm{Ohm}^{-1} \mathrm{~m}^{-1}$. The resulting solution of tetraaniline in DMSO was added to the sol of silicon nanoparticles in ethanol in a mass ratio 1:10 before deposition of film on substrates.

As we have already reported, the films deposited on a substrate of silicon nanoparticles in a week after their synthesis we call the films I; films, obtained from the same nanoparticles, but two years after their synthesis (aged nanoparticles) - films II and films with aged nanoparticles and addition of tetraaniline - films III. Before the measurement the sandwich 
like structures of the films II and III with $\mathrm{Mg}$ or $\mathrm{Al}$ electrodes were heated to a temperature of $140^{\circ} \mathrm{C}$ and held at that temperature for 30 minutes.

\subsubsection{Experimental results}

\subsubsection{Dielectric dispersion of the films II}

The values of $\varepsilon^{\prime}$ and $\varepsilon^{\prime \prime}$ of films I and II in the frequency range below $10^{4} \mathrm{~Hz}$ are quite close to each other. The main difference between the permittivity's spectra of these films observed in the frequency range higher of $10^{5} \mathrm{~Hz}$

In this frequencies region permittivity spectra of the films II reveal sharp decrease in $\varepsilon^{\prime}$ while the dielectric losses of $\varepsilon^{\prime \prime}$ has a form of enough narrow peak (see Figure 10). Such behavior of the spectrum is typical for the Debye dipole relaxation process, and will be discussed later.

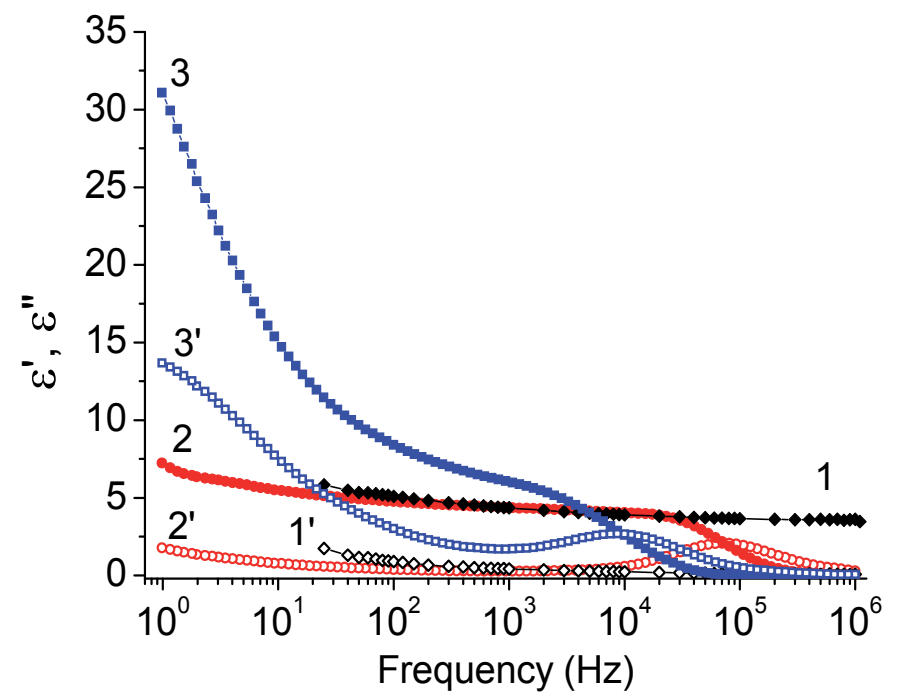

Fig. 10. The frequency dependence of $\varepsilon$ 'and $\varepsilon^{\prime \prime}$ for: Film I: - (1 and 1'), Film II - $\left(2,2^{\prime}\right)$, Film III - $\left(3,3^{\prime}\right)$.

\subsubsection{Dielectric dispersion of the films III}

The $\varepsilon(\omega)$ spectra of the films III, similar to those of the films II, but for the films III decreasing of $\varepsilon$ 'is observed in the frequency of large $10^{4} \mathrm{~Hz}$. The frequency at which a maximum of dielectric loss $\varepsilon^{\prime \prime}$ observed in films III, is $\mathrm{v}_{\max } \approx 9,7 \cdot 10^{3} \mathrm{~Hz}$, while for films II, this frequency is $7,5 \cdot 10^{4} \mathrm{~Hz}$ (see Figure 10). At frequencies $v \leq 10^{4} \mathrm{~Hz}$ the magnitude of $\varepsilon^{\prime}$ of films III reveal a sharp increase with decreasing frequency of the external electric field and greatly exceeds the corresponding values of the films I and II.

\subsubsection{AC conductivity of the films II and III}

In contrast to the film I conductivity of the films II and III may be approximated by a power law $\sigma(\omega) \sim \omega^{\mathrm{s}}$ on the frequency of the alternating electric field only in a very limited range of 
frequencies. So for films II, this area is $1 \leq \mathrm{v} \leq 4 \cdot 10^{2} \mathrm{~Hz}$ in which the exponent is 0.66 , and for films III conductivity satisfactorily approximated by a power law with exponent $s=0,63$ in the frequency range $5 \leq \mathrm{v} \leq 5 \cdot 10^{2} \mathrm{~Hz}$.

For both types of films, a significant increase of the growth rate of the conductivity is observed at frequencies exceeding $2 \cdot 10^{3} \mathrm{~Hz}$, however, the conductivity of the films II and III begins very weakly dependent on frequency of external electric field (see Figure 11) at frequencies larger of $10^{5}$ and $3 \cdot 10^{4} \mathrm{~Hz}$ respectively.

The conductivity of films III containing tetraaniline exceeds the conductivity of the films II in the frequency range $1 \leq \mathrm{v} \leq 3 \cdot 10^{4} \mathrm{~Hz}$, while at higher frequencies observed the opposite picture in which the conductivity of the films II is higher then that of films III (see the same figure).

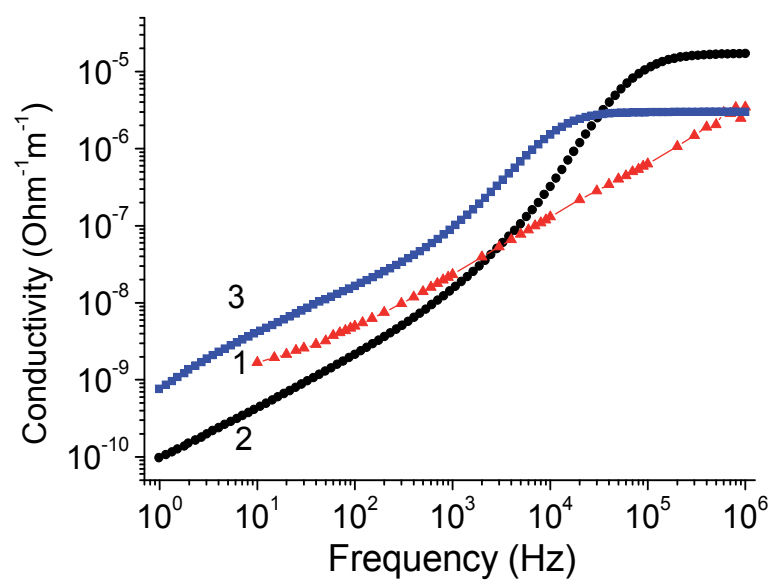

Fig. 11. Frequency dependence of AC conductivity of: films I - (1), film II - (2) and film III - (3)

\subsection{Discussion}

\subsubsection{Ellipsometry of films I}

Analysis of ellipsometric spectra shows that the value of $\varepsilon^{\prime}$ of the $n c$-Si films under study varies in the range of 2.1-1.1 in the energy range of 2-4.4 eV or in the frequency range of $5 \times 10^{14}-1 \times 10^{15} \mathrm{~Hz}$ of the electromagnetic field, respectively, which is significantly below the values typical of $c$-Si in this range. In our opinion, there are two causes resulting in such low $\varepsilon^{\prime}$ and $\varepsilon^{\prime \prime}$. One is that $n c$-Si particles contacted with atmospheric oxygen for some time during film preparation; therefore, their surface was coated with a $\mathrm{SiO}_{x}+\mathrm{SiO}_{2}$ layer $(0 \leq x \leq 2)$. Silicon nanoparticles oxidation was studied by Schuppler at al. (Schuppler at al., 1995), in that study the $\mathrm{SiO}_{x}$ layer thickness on their surface was determined as a function of the nanoparticle diameter. It was shown that the $\mathrm{SiO}_{x}+\mathrm{SiO}_{2}$ layer thickness in the nanoparticle diameter range of $10-3 \mathrm{~nm}$ is $\sim 1 \mathrm{~nm}$. However, this means that the ratio of the volume of the crystalline silicon core to the volume of the $\mathrm{SiO} x$ amorphous shell is from 100 to $40 \%$. In 
other words, the oxidized crystalline silicon nanoparticle with a size smaller than $10 \mathrm{~nm}$ should exhibit amorphous properties to an appreciable extent. We have confirmed this statement previously based on an analysis of Raman spectra of $n c-S i$ thin films (see section 2.A.2.1 and also Dorofeev at al., 2009). The second cause of a decrease in the permittivity is air gaps between nanoparticles, which appear during film formation.

To estimate the relation between crystalline and amorphous film components and their porosity, we use the Bruggeman EMA model. In the EMA approximation, the effective permittivity of the inhomogeneous medium consisting of spherical microobjects with permittivities $\varepsilon_{1}, \varepsilon_{2}, \ldots, \varepsilon_{N-1}$, immersed into a medium with $\varepsilon_{N}\left(\varepsilon_{N} \equiv \varepsilon_{\mathrm{e}}\right)$ is determined from the equation

$$
\sum_{i=1}^{N} f_{i} \frac{\left(\varepsilon_{i}-\varepsilon_{e}\right)}{\left(\varepsilon_{i}+2 \varepsilon_{e}\right)}=0 \sum_{i=1}^{N} f_{i}=1
$$

where: $f_{i}=\frac{V_{i}}{\sum_{i=1}^{N} V_{i}}$

is the degree of medium volume filling with an element with permittivity $\varepsilon_{i}$ and $V_{i}$ is the volume occupied by this element.

Initially, to determine $\varepsilon_{e}$ of the films under study, we assumed that the medium is two-phase and consists of purely crystalline silicon nanoparticles and the air gaps. In this case, Eq. (5) was reduced to a sum of two terms; knowing the dispersion relation of crystalline silicon, it was required to determine $f_{1}$ and $f_{2}$ so that approximating dispersion profiles would be identical to experimental $\varepsilon^{\prime}(v)$ and $\varepsilon^{\prime \prime}(v)$. However, it was impossible to achieve satisfactory approximation at no values of $f_{1}$ and $f_{2}$.

Since the oxidation state of nanoparticles is unknown, we assumed that each particle in the two phase Bruggeman model behaves on average as a $\mathrm{SiO}_{x}$ medium (rather than as crystalline silicon), where $0 \leq x \leq 2$ was a fitting parameter, as well as $f_{1}$ and $f_{2}$. The $\varepsilon^{\prime}(v)$ and $\varepsilon^{\prime \prime}(\mathrm{v})$ spectra for $\mathrm{SiO}_{x}$ in the entire range $0 \leq x \leq 2$ were taken from (Zuter, 1980), in which it was supposed that $\mathrm{SiO}_{x}$ is a mixture of $\mathrm{Si}_{-}-\mathrm{Si}_{y} \mathrm{O}_{4-y}$ tetrahedra; the random parameter takes values from 0 to 4 (random binding model (Hubner, 1980)). Using these spectra, it became possible to achieve good approximation of the experimental dependences $\varepsilon^{\prime}(\mathrm{v})$ and $\varepsilon^{\prime \prime}(\mathrm{v})$ at $x$ $=1$ and $f_{1}=f_{2}=0.5$. The approximating EMA spectra for these parameters are shown in Fig. 5 by dashed curves. Thus, it was shown that the $n c$-Si films under study on average behave as media consisting of $\mathrm{SiO}$ with a porosity of 0.5 . Here we note already mentioned study (Pickering, 1984) in which the $\varepsilon^{\prime}(v)$ and $\varepsilon^{\prime \prime}(v)$ spectra were measured and which are qualitatively and quantitatively rather similar to the spectra analyzed in the present study.

The absorption spectra of $n c$-Si films calculated from ellipsometric data are quite typical. As seen in Fig. 6, the film absorption at incident photon energies below $3 \mathrm{eV}$ is stronger than that of $c-\mathrm{Si}$; at higher energies, it is significantly lower. Such an absorption behavior shows that the $\mathrm{SiO} x$ shell with high density of states of defects near to the phase interface with the crystalline core mainly contributes to absorption for low energy photons; photons with 
energies above $3 \mathrm{eV}$ are mostly absorbed by crystalline cores of nanoparticles with a wider band gap than that of $c$-Si due to quantum confinement.

\subsubsection{Frequency dependence of the capacitance of films I}

There are several models of the interpretation of the results of measurements of the ac conductivity of materials. For semiconductors, the model of (Goswami,A. \& Goswami,A.P. 1973) is a good approximation, according to which a conductive material is a composition of a capacitor with capacitance $C_{1}$ and a resistor with conductance $G_{1}\left(G_{1}=1 / R\right)$ connected in parallel. Furthermore, to take into account the effect of supplying contacts, a resistor with conductance $G_{2}\left(G_{2}=1 / r\right)$ is connected in series with this group. According to this model, $C_{1}$ and $G_{1}$ are independent of the frequency of the applied ac electric field; however, $G_{1}$ depends on the conductive material temperature.

If the sample capacitance is measured in the mode of in parallel connected $C p$, the measured value is related to $C_{1}, G_{1}$, and $G_{2}$ as

$$
C_{p}=\frac{C_{1} G_{2}^{2}}{\left(G_{1}+G_{2}\right)^{2}+\left(2 \pi \nu C_{1}\right)^{2}}
$$

We can see from this equality that the measured nc-Si film capacitance should satisfy the condition $C p \sim \mathrm{v}^{-2}$ while satisfying the conditions of the model of(27Goswami,A. \& Goswami,A.P. 1973). However, it was impossible to approximate the experimental curve for $C_{n c-S i}$ shown in Fig. 7 by such power law dependence. Such a fact suggests that $C_{1}$ and $G_{1}$ should depend on frequency. Indeed, under experimental conditions, $G_{2}>>G_{1}$ and $\mathrm{G}_{2}>>_{\mathrm{v}} C_{1}$, hence, $C p \approx C_{1}$.

Therefore, for approximation, we used the following semi empirical function:

$$
C_{n c-S i}(v)=C_{\infty}+\frac{C}{1+(A v)^{\beta}}
$$

It follows from formula (6) that $\mathrm{C}_{\mathrm{nc}-\mathrm{Si}} \rightarrow \mathrm{C}_{\infty}$, at $\mathrm{v} \rightarrow \infty$ and $\mathrm{C}_{\mathrm{nc}-\mathrm{Si}}=\mathrm{C}_{\infty}+\mathrm{C} \equiv \mathrm{C}(0)$ at $\mathrm{v}=0$.

Thus, the quantity $\mathrm{C}_{\infty}$ entering expression (6) is the film capacitance at an "infinitely high frequency" and $C(0)=C_{\infty}+C$ is the film static capacitance. The dimension of the fitting parameter $A$ in the formula is time; the fitting parameter $\beta$ defines the power law dependence of $C_{n c-S i}$ on the applied ac field frequency. Function (6) appeared to be a very good approximation of the experimental dependence $C_{n c-S i}(v)$ at the following coefficients: $\mathrm{C}_{\infty}=3.9 \times 10^{-10} \mathrm{~F}, \mathrm{C}(0)=11.8 \times 10^{-10} \mathrm{~F}, A=0.5$, and $\beta=0.32$.

The film capacitance is related to the real component of its permittivity by the relation $\mathrm{C}_{\mathrm{nc}-\mathrm{Si}}(\mathrm{v})=\varepsilon^{\prime}{ }_{\mathrm{nc}-\mathrm{Si}}(\mathrm{v}) \cdot \mathrm{C}_{0}$. As noted above, $\mathrm{C}_{0}=1.15 \times 10^{-10} \mathrm{~F}$ for the film under study; the static and optical permittivities $\varepsilon_{s}=\varepsilon(0)=10.3$ and $\varepsilon_{\infty}=3.4$ correspond to the determined capacitances $C(0)$ and $C_{\infty}$.

The static permittivity of the film under study, which is 10.3 , is significantly lower than the permittivity of crystalline silicon, which, as is known, is $\sim 12$. This result will be discussed below. 


\subsubsection{Dielectric relaxation in films I}

The $\varepsilon^{\prime}(\mathrm{v})$ and $\varepsilon^{\prime \prime}(\mathrm{v})$ frequency spectra obtained by measuring the $n c-S i$ film impedance are shown in Fig. 8. The semi empirical Cole-Cole relation ( Cole, K. S. \& Cole, R. H., 1941; Moliton, 2007) appeared to be a good approximation for these spectra,

$$
\varepsilon=\varepsilon_{\infty}+\frac{\varepsilon_{s}-\varepsilon_{\infty}}{1+(i \omega \tau)^{1-h}} 0 \leq \mathrm{h} \leq 1
$$

where $\varepsilon_{\mathrm{s}}$ and $\varepsilon_{\infty}$ are the static and optical permittivities determined above, $\omega=2 \Pi v$ is the cyclic frequency, and $\tau$ is the dipole relaxation time.

As is known, the Cole-Cole relation is valid when a material simultaneously contains several types of dipoles each with a specific relaxation time. Therefore, the quantity $\tau$ entering Eq. (7) is the relaxation time averaged over the ensemble of dipole groups contained by the $n c-S i$ film under study.

The approximating Cole-Cole curves are shown in Fig. 8.a by dashed curves. We can see that $\varepsilon^{\prime}(v)$ is very well approximated in the entire measured frequency range; for $\varepsilon^{\prime \prime}(v)$, the Cole-Cole dependence exhibits good agreement only in the frequency range of $2 \times 10^{2} \leq \mathrm{v} \leq$ $10^{6} \mathrm{~Hz}$. The values $\varepsilon_{s}=10.8, \varepsilon_{\infty}=3.43, \tau=6 \times 10^{-2} \mathrm{~s}$, and $h=0.7$ correspond to the found approximation. It should be noted here that the value of $\varepsilon_{\infty}$ is close to the values of $\varepsilon^{\prime}$ determined in the optical region by the ellipsometry method.

A comparison of the values of $\varepsilon_{s}$ and $\varepsilon_{\infty}$ corresponding to the Cole-Cole approximation with similar values determined from capacitance measurements shows the closeness of their numerical values. The value of $1-h$ is also very close to the exponent $\beta$ in formula (6). Furthermore, if we consider that $A$ in formula (6) is the relaxation time multiplied by $2 \pi$, then $\tau=A / 2 \Pi=6.4 \times 10^{-2} \mathrm{~s}$, which is also close to the average dipole relaxation time corresponding to the Cole-Cole approximation.

The static permittivity $\varepsilon_{s}=10.8$ determined from the Cole-Cole relation is slightly larger than the similar value found from Eq. (6); however, it is also smaller than $\varepsilon_{s}=12$ characteristic of crystalline silicon.

In our opinion, there are two causes resulting in a decrease in $\varepsilon_{s}$ for the $n c-S i$ film in comparison with $\varepsilon_{s}$ of $c$-Si. The first cause is associated with air voids in the film body; the second cause is that the size distribution of nanoparticles composing the film includes a large fraction of particles with sizes smaller than $10 \mathrm{~nm}$ (see the Fig. 1). In (Tsu at al., 1997), the permittivity of silicon nanoparticles was calculated as a function of their size. According to these results, the static permittivity decreases as the particle diameter becomes smaller than $10 \mathrm{~nm}$; for particles $10 \mathrm{~nm}$ in diameter, the permittivity is from 11.2 to 10.1, depending on the used calculation model.

In Fig. 8.a, in the frequency region $\mathrm{v} \leq 2 \times 10^{2} \mathrm{~Hz}$, we can see a notable disagreement between the Cole-Cole approximating function and the experimental dependence $\varepsilon^{\prime \prime}(v)$. This disagreement is caused by the fact that the Cole-Cole relation that describes dipole moment relaxation in dielectrics does not take into account the presence of free electric charges. However, free charges exist in the $n c-S i$ film under study, which is indicated by the nonzero dc conductivity, which, as noted above, is $\sigma(0)=9 \times 10^{-10} \Omega^{-1} \mathrm{~m}^{-1}$ at temperature $T=297 \mathrm{~K}$. 
According to studies by Barton, Nakajima, and Namikawa (Barton 1966; Nakajima, 1972; Namikawa, 1975), the frequency $\mathrm{v}_{m}$ corresponding to the dispersion maximum for $\varepsilon^{\prime \prime}(\mathrm{v})$ is related to $\sigma(0)$ as $\sigma(0)=p\left(\varepsilon_{s}-\varepsilon_{\infty}\right) \cdot \varepsilon_{0} 2 \Pi v_{m}$, where the numerical coefficient $p$ is approximately equal to unity. We can see in Fig. 8.a that the Cole-Cole approximating function reaches a maximum at the frequency $\mathrm{v}_{m}=2.5 \mathrm{~Hz}$, and this value is in good agreement with the experimental value of $\sigma(0)$ when using the Barton-Nakajima-Namikawa formula.

To take into account the conductivity associates with free electric charges, relation (4) should be written as

$$
\varepsilon=\varepsilon_{\infty}+\frac{\varepsilon_{s}-\varepsilon_{\infty}}{1+(i \omega \tau)^{1-h}}+\frac{\sigma(0)}{\varepsilon_{0} \omega}
$$

The approximation of the $\varepsilon^{\prime \prime}(\mathrm{v})$ spectrum of the film under study is shown by the dashed curve in Fig. 8.a (curve 5), from which it is obvious that function (8) is a good approach of the experimental dependence $\varepsilon^{\prime \prime}(v)$.

The effect of free electric charges on dielectric properties of the $n c-S i$ film rather clearly appears in the Nyquist plot in which $\varepsilon^{\prime \prime}$ for each frequency is shown as a function of $\varepsilon^{\prime}$ (see Fig. 8.b).

It follows from the Cole-Cole approximation (see curve 2 in Fig. 8.b) that the $\varepsilon^{\prime \prime}\left(\varepsilon^{\prime}\right)$ should be shaped as a part of a semicircle whose center is below the horizontal axis $\varepsilon^{\prime \prime}$. The intersection of this circle with the $\varepsilon^{\prime}$ axis at $\omega=0$ and $\omega \rightarrow \infty$ yields the values of $\varepsilon_{s}$ and $\varepsilon_{\infty}$.

Figure 8.b shows only the semicircle part corresponding to the measured frequency range; therefore, the value $\varepsilon_{s}=10.8$ is out of sight of the figure; the intersection of the semicircle with the $\varepsilon^{\prime}$ axis at $\omega \rightarrow \infty$ is clearly seen and corresponds to $\varepsilon_{\infty}=3.4$. The same figure shows the approximation corresponding to function (8) (curve 3), similar to the approximation shown in Fig. 8.a.

\subsubsection{AC conductivity of films I}

To determine the nature of electric charge transport in $n c$-Si films, the frequency dependence of the conductivity $\sigma_{A C}(v), \sigma_{A C}(v)-\sigma(0)=\varepsilon_{0} \cdot 2 \Pi v \cdot \varepsilon^{\prime \prime}(v)$, was studied.

The $\sigma_{a c}(v)-\sigma(0)$ plot on a log scale for the film analyzed in this paper is shown in Fig. 10. We can see that $\sigma_{\mathrm{AC}}(\mathrm{v})$ in the entire measured frequency range is well approximated by the power law function: $\sigma_{\mathrm{ac}}(\mathrm{v})=\sigma(0)+A v^{s}$ with $s=0.74$. Such $\sigma_{\mathrm{AC}}(\mathrm{v})$ behavior means that the electric transport in the film has the hopping mechanism, which in turn is a manifestation of the structure disorder in that film region over which charge transport occurs.

Currently, there are several theoretical models describing hopping conductivity in unordered solids. All these models yield the power law dependence of the ac conductivity on the ac electric field frequency: $\sigma(\mathrm{v}) \sim \mathrm{v}^{s}$. However, the numerical values of the exponent $s$ differ. For example, in the models (Austin \& Mott, 1969; Hunt, 2001) according to which the conduction results from electric charge tunneling through energy barriers separating close localized states, the parameter $s$ is given by 


$$
s=1+q \times \ln ^{-1}\left(\frac{v}{v_{p h}}\right)
$$

where $q=4$ or 5 , depending on the theoretical model, and $\mathrm{v}_{\mathrm{ph}} \approx 10^{12} \mathrm{~Hz}$ is the phonon frequency.

It follows from relation (9) that $s$ should decrease with frequency. However, such behavior of $s$ contradicts our experimental data and a large number of other experimental data (Dyre \& Schrøder, 2000).

Currently, it has been sufficiently reliably determined that a large role in conduction processes in unordered solids is played by percolation processes with the result that electric transport occurs along trajectories with the lowest resistance (percolation trajectories) (Hunt, 2001; Isichenko, 1992). Conductive properties of percolation trajectories are controlled by the structure of (percolation) clusters composing the shell of solids.

In highly unordered solids, percolation trajectories at small scales exhibit a fractal structure with the result that their fractal dimension $d_{f}$ appears larger than the topological one $D$ (e.g., the fractal and topological dimensions of the Brownian particle trajectory is $d_{f}=2$ and $D=1$ ) (Isichenko, 1992).

In this regard, we note theoretical studies (Dyre \& Schrøder, 2000; Schrøder \& Dyre, 2002; Schrøder \& Dyre, 2008) in which the diffusion cluster approximation (DCA) model is formulated. As these papers, it is argued that the so-called diffusion clusters with fractal dimensions of 1.1-1.7 make the largest contribution to the ac conductivity in the percolation mode. This statement means that the fractal structure of such clusters is simpler than the structure of multiply connected percolation clusters formed above the percolation threshold in conductive materials (backbone clusters), the fractal dimension of which is 1.7 (Isichenko, 1992). Simultaneously, the structure of diffusion clusters is more branched than the network of singly connected clusters and breaking of each results in disappearance of the current flowing through it (redbonds). The fractal dimension of redbonds clusters is 1.1 (Isichenko, 1992).

In these papers, the universal dependence of the dimensionless complex conductivity

$$
\tilde{\sigma}=\frac{\sigma_{A C}(v)+i \sigma^{\prime \prime}(v)}{\sigma(0)}
$$

on the dimensionless frequency

$$
\tilde{\omega}=\frac{\varepsilon_{0}\left(\varepsilon_{s}-\varepsilon_{\infty}\right)}{\sigma(0)} 2 \pi v
$$

was derived. This dependence is given by

$$
\ln \tilde{\sigma}=\left(\frac{i \tilde{\omega}}{\tilde{\sigma}}\right)^{\frac{d_{f}}{2}}
$$


The fractal dimension $d_{f}$ in formula (10) is a fitting parameter. Processing of a large number of experimental dependences in (Schrøder \& Dyre, 2002; Schrøder \& Dyre, 2008) showed that the best agreement in the frequency region $\mathrm{v}>1 \mathrm{~Hz}$ is achieved at $d_{f}=1.35$.

We compared the experimental dependence $\sigma_{A C}(v)$ obtained in the present study with the values defined by formula (10). Here it should be noted that complex valued equation (10) has no analytical solution and should be solved numerically.

However, in the low frequency region $\omega \rightarrow 0$, Eq. (10) can be written as $\tilde{\sigma}-1=(i \tilde{\omega})^{\frac{d_{f}}{2}}$; accordingly (Kononov at al., 2011):

$$
\sigma_{A C}(v)-\sigma(0)=\sigma(0)^{\left(1-\frac{d_{f}}{2}\right)} \cos \left(\frac{\pi d_{f}}{4}\right)\left(2 \pi \varepsilon_{0} \Delta \varepsilon\right)^{\frac{d_{f}}{2}} v^{\frac{d_{f}}{2}}
$$

where $\Delta \varepsilon=\varepsilon_{s}-\varepsilon_{\infty}$.

Substitution of experimentally determined values of $\varepsilon_{s}, \varepsilon_{\infty}, \sigma(0)$, and $s \equiv d_{f} / 2=0.74$ into formula (11) gives the approximating dependence for $\sigma_{\mathrm{ac}}(\mathrm{v})$ (see Fig. 9) corresponding to the DCA model. We can see that the calculated dependence rather well approximates the experimental curve $\sigma a c(v)$ in the entire measured frequency range. At the same time, the calculated dependence yields values of $\sigma_{\mathrm{AC}}$ larger than the experimental ones by a factor of 1.5. We attribute such disagreement to possible errors when determining the numerical values of $\varepsilon_{s}, \varepsilon_{\infty}$, and $\sigma(0)$.

\subsubsection{Proton conductivity of films I}

One of the possible causes that can result in $\sigma(0)$ measurement errors for nc-Si films is the dependence of $\sigma(0)$ on the ambient air humidity. We qualitatively determines the following systematic feature: the higher the laboratory air humidity, the higher (at a constant temperature) the conductivity $\sigma(0)$ of films similar to the film analyzed in this paper. On the contrary, if the film is preliminarily heated at a temperature of $\sim 200^{\circ} \mathrm{C}$ for a time longer than $15 \mathrm{~min}$ and then it is cooled to its initial temperature, the film conductivity will decrease almost by two orders of magnitude. Thus the presence of water in an atmosphere surrounding the film changes its conductive properties significantly. In (Nogami \& Abe, 1997; Nogami at al., 1998) a similar phenomenon was observed in the study of the ionic conductivity in fused silica glasses. It was shown that, in the presence of $\mathrm{Si}-\mathrm{O}-\mathrm{H}$ bonds on the glass surfaces, $\mathrm{H}_{2} \mathrm{O}$ molecules form complexes with them, confined hydrogen bonds. These complexes can dissociate forming free $\mathrm{H}_{3} \mathrm{O}^{+}$ions and bound $\mathrm{Si}-\mathrm{O}-$ groups according to the scheme:

$$
\mathrm{Si}-\mathrm{O}-\mathrm{H} \cdots \mathrm{OH}_{2} \rightarrow \mathrm{Si}-\mathrm{O}^{-}+\mathrm{H}^{+}: \mathrm{H}_{2} \mathrm{O}
$$

Here, dots denote the hydrogen bond between $\mathrm{H}$ and $\mathrm{O}$ atoms. In this case, the dissociated proton $\mathrm{H}+$ can be trapped by a neighboring $\mathrm{H}_{2} \mathrm{O}$ molecule,

$$
\mathrm{H}_{2} \mathrm{O}: \mathrm{H}_{2} \mathrm{O}_{(1)}: \mathrm{H}^{+}+\mathrm{H}_{2} \mathrm{O}_{(2)} \rightarrow \mathrm{H}_{2} \mathrm{O}_{(1)}+\mathrm{H}^{+}+\mathrm{H}_{2} \mathrm{O}_{(2)} \rightarrow \mathrm{H}_{2} \mathrm{O}_{(1)}+\mathrm{H}^{+}: \mathrm{H}_{2} \mathrm{O}_{(2)} \text { etc. }
$$


Such a scheme allows implementation of proton transport near the glass surface. Returning to nc-Si films, we note that particles used to apply films represent hydrogenized nanocrystalline silicon. However, when exposing these particles to atmospheric air, a $\mathrm{SiO}_{x}$ shell $(0 \leq x \leq 2)$ is formed on their surface. In (Du at al., 2003; Cao at al., 2007), the kinetics of the interaction of $\mathrm{H}_{2} \mathrm{O}$ molecules with $\mathrm{SiO}_{2}$ chain structures was calculated. It was shown that $\mathrm{H}_{2} \mathrm{O}$ molecules very efficiently break $\mathrm{Si}-\mathrm{O}-\mathrm{Si}$ bonds during the interaction with $\mathrm{SiO}_{2}$ surface groups with the formation of $\mathrm{Si}-\mathrm{O}-\mathrm{H}$ groups. The subsequent interaction of $\mathrm{H}_{2} \mathrm{O}$ molecules and $\mathrm{Si}-\mathrm{O}-\mathrm{H}$ groups yields $\mathrm{H}_{3} \mathrm{O}^{+}$ions, which, having high mobility, can appreciably contribute to the proton transport along the $\mathrm{SiO}_{2}$ chain.

In addition to the above process, the collective proton conductivity caused by associated SiO-H groups, i.e., groups linked by hydrogen bond, as shown in Fig. 12.a (Glasser, 1975). Arrows in the diagram indicate the direction of positive charge transport.

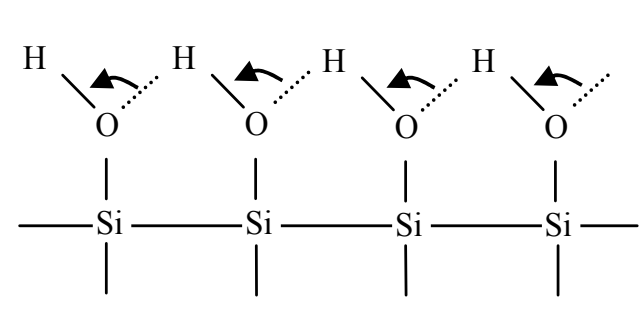

a

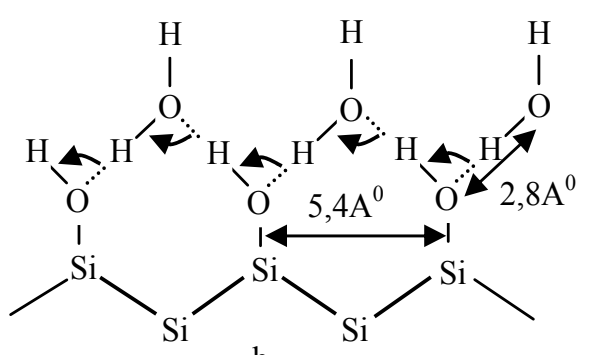

b

Fig. 12. Diagrams illustrating the mechanism of the collective proton conductivity, caused by (a) associated $\mathrm{Si}-\mathrm{O}-\mathrm{H}$ groups and (b) the interaction of water molecules with hydroxyl groups. Arrows indicate the direction of positive charge transport.

The collective proton conductivity is also possible during the interaction of water molecules with hydroxyl groups, which results in the surface structure shown in Fig. 12.b. Since the O...H-O group length is within 2.5-2.9 $\AA$ (Leite at al., 1998) and the angle between $\mathrm{H}-\mathrm{O}-\mathrm{H}$ bonds is $\sim 104^{\circ}$, there is good spatial alignment between the element of this surface structure and the crystalline silicon lattice constant which, as is known, is $5.4 \AA$.

As applied to the nc-Si films analyzed in this paper, there is direct proof of the existence of such structures. Previously, in the investigations of IR transmission spectra of thin wafers (with thickness $\approx 50 \mu \mathrm{m}$ ) made by pressing $\left(\mathrm{P} \sim 10^{9} \mathrm{~Pa}\right.$ ) from nc-Si powders similar to those used in this study, it was shown that the spectra contain a broad intense band with a maximum at $\sim 3420 \mathrm{~cm}^{-1}$ (see Fig. 13 and Kononov at al., 2005). In papers (Wovchko at al., 1995; Stuart, 2004) this band is attributed to $\mathrm{O}-\mathrm{H}$ vibrations in hydrogen bound hydroxyl groups. It was also shown that heating of nc-Si particles to $400^{\circ} \mathrm{C}$ causes an appreciably decrease in the intensity of the band near $3420 \mathrm{~cm}^{-1}$ and an increase in the intensity of the narrow band with a maximum near $3750 \mathrm{~cm}^{-1}$, which is identified with 
$\mathrm{O}-\mathrm{H}$ vibrations in the isolated Si-O-H group ( Kononov at al., 2005). Similar spectra are shown in Figure 13. Such behavior of the intensities of bands at 3420 and $3750 \mathrm{~cm}^{-1}$ means that associated $\mathrm{Si}-\mathrm{O}-\mathrm{H}$ groups become isolated upon heating of nc-Si particles. Accordingly, heating should decrease the proton conductivity associated with these groups.

Thus the dependence of the conductivity $\sigma(0)$ of the $n c-S i$ films under study on the ambient air humidity and the thermal behavior of the absorption bands associated with $\mathrm{Si}-\mathrm{O}-\mathrm{H}$ groups allows the conclusion to be drawn that the proton conductivity makes the main contribution to the dark dc conductivity of $n c-S i$ films.

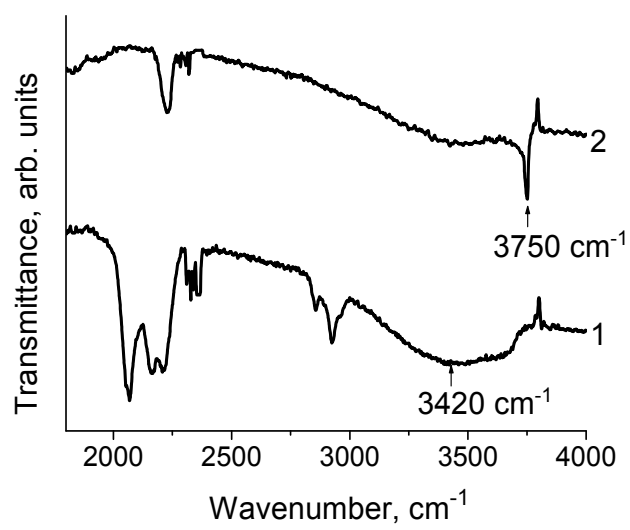

Fig. 13. Infrared transmittance spectra of: (1) - thin wafer from $n c-S i$ particles prepared at a pressure of $5 \times 10^{8} \mathrm{~Pa}$ at $20^{\circ} \mathrm{C}$, (2) - the same $n c-S i$ wafer but annealed at $400^{\circ} \mathrm{C}$ for $30 \mathrm{~min}$.

\subsubsection{Double dielectric relaxation in the films II and III}

Earlier, we noted that the spectra of the $\varepsilon^{\prime}(\mathrm{v})$ and $\varepsilon^{\prime \prime}(\mathrm{v})$ of the films II and III near a frequency $\approx 10^{4} \mathrm{~Hz}$ reveal the structure arising in the Debye dipole relaxation. Following this observation for the numerical approximation of the experimental spectra, we used not only semi-empirical law of Cole-Cole, but the law of Debye dipole relaxation. Thus, all experimental spectra were approximated by the following relation:

$$
\varepsilon=\frac{\varepsilon_{S}-\varepsilon_{\infty}}{1+\left(i \omega \tau_{1}\right)^{1-h}}+\frac{\varepsilon_{\infty}}{1+\left(i \omega \tau_{2}\right)^{2}}+\frac{\sigma(0)}{\varepsilon_{0} \omega}
$$

Here $\tau_{1}$ and $\tau_{2}$ is the relaxation times of dipole moments in the various structural components of the films. With the help of equation (12) was able to accurately approximate the dielectric spectra of the films studied; example of such an approximation for film II is shown in Figure 14.

Furthermore the approximation (12) allowed us to determine the static $\left(\varepsilon_{0}\right)$, high-frequency $\left(\varepsilon_{\infty}\right)$ dielectric constants $\left(\mathrm{v} \sim 10^{5} \mathrm{~Hz}\right)$, the conductivity of the films at constant current $\sigma(0)$, and relaxation times $\tau_{1}$ and $\tau_{2}$. 


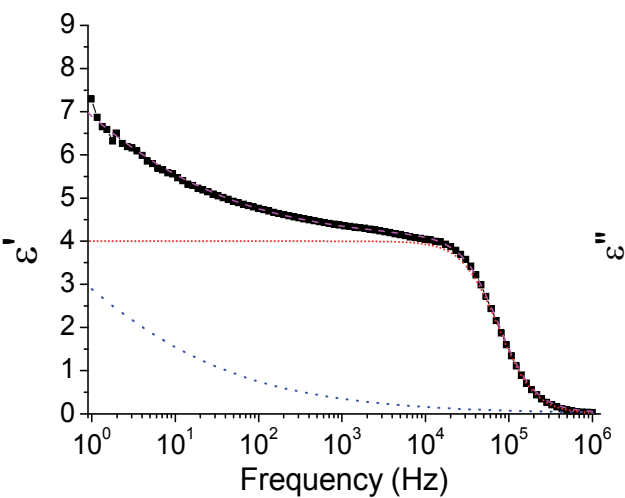

a

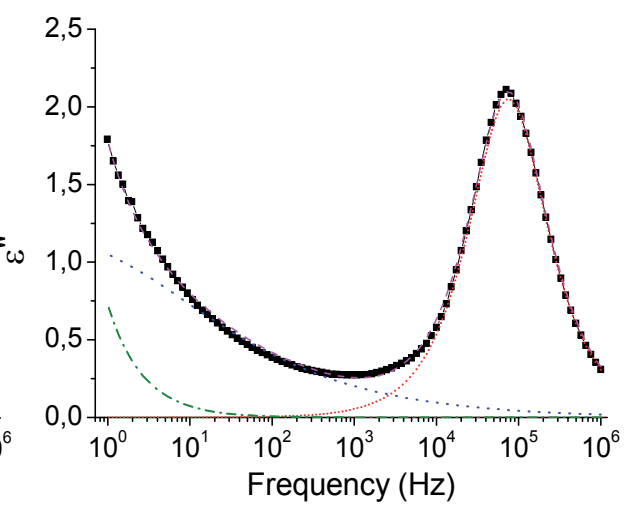

b

Fig. 14. Spectra of the real (a) and imaginary (b) components of permittivity film II. Shortdotted line shows the approximation of the Debye. Dotted line shows the approximation of the Cole-Cole. The dash-dotted line shows the approximation of free charges. The dashed line shows the complete approximation of the spectra.

\begin{tabular}{|l|l|l|l|l|l|l|l|l|}
\hline № & $\varepsilon_{0}$ & $\varepsilon_{\infty}$ & h & $\begin{array}{l}\sigma(0), \\
(\text { Ohm·m) }\end{array}$ & $\begin{array}{l}\sigma_{\mathrm{DC},} \\
(\text { Ohm } \cdot \mathrm{m})^{-1}\end{array}$ & $\begin{array}{l}\sigma_{\mathrm{B}}(0), \\
(\mathrm{Ohm} \cdot \mathrm{m})^{-1}\end{array}$ & $\tau_{1}, \mathrm{~s}$ & $\mathrm{\tau}_{2,} \mathrm{~s}$ \\
\hline I & 10,8 & 3,4 & 0,74 & $1,4 \cdot 10^{-9}$ & $9 \cdot 10^{-10}$ & $10^{-9}$ & 0,06 & - \\
\hline II & 11,5 & 4 & 0,66 & $4 \cdot 10^{-11}$ & $3,5 \cdot 10^{-11}$ & $9 \cdot 10^{-11}$ & 0,72 & $2,12 \cdot 10^{-6}$ \\
\hline III & 66,9 & 4,9 & 0,5 & $9 \cdot 10^{-11}$ & $3,5 \cdot 10^{-9}$ & $2 \cdot 10^{-9}$ & 0,27 & $1,75 \cdot 10^{-5}$ \\
\hline
\end{tabular}

Table 1. Fit parameters for the two dielectric relaxation lows of the films investigated in this study. $\sigma_{\mathrm{DC}}$ and $\sigma_{\mathrm{B}}(0)$ - the conductivites at constant current received from direct measurements of the films resistance and from the Barton-Nakajima-Namikawa formula.

These values for films I, II and III are shown in the table 1 . The table 1 also gives values of $\sigma(0)$ received from direct measurements of the films resistance at constant current at $\mathrm{T}=$ 297K, and those which obtained from the Barton-Nakajima-Namikawa formula. From table 1 it can be seen that the values of the static dielectric constant of films III are about 67 , significantly higher than similar values of the films I and II, which are close to the values characteristic of crystalline silicon. However, the value of $\varepsilon_{0} \approx 67$ is much lower quantities $\varepsilon_{0} \sim 10^{3}$ typical for composites consisting of nanoparticles of tin dioxide and polyaniline which have been reported in (Kousik at al., 2007)

The authors of this work attributed so high $\varepsilon_{0}$ to an anomalously strong polarization of nanoparticle of $\mathrm{SnO}_{2}$ which caused by inhomogeneity of the conductivity of its surface and core. However, the value of $\varepsilon_{0} \approx 67$ which have been measured by us, is quite close to the values of the static dielectric constant of tetraaniline with different degrees of doping it with hydrochloric acid (Bianchi at al., 1999) and which, depending on the degree of doping lies in the range $35-80$.

The presence in equation (12) two different laws of approximation indicates that there are two different dipole relaxation process associated with the various structural components of 
the studied films II and III. Very clear in understanding this phenomenon is a plot of $\varepsilon$ " vs $\varepsilon$ '(Nyquist Plot), shown in Figure 15.

In the inset of Fig. 15 we can see that the dependence of $\varepsilon^{\prime \prime}$ vs of $\varepsilon^{\prime}$ for film III consists of two semicircles, which can be termed as high- and low- frequency components. The film II has a similar structure while the graph $\varepsilon^{\prime \prime}\left(\varepsilon^{\prime}\right)$ of the film I consists of only one semicircle (which is a low-frequency component) and low-frequency tail defined by the presence of free charges. Nanoparticles of silicon used for deposition of films II and III were in ethanol for two years after their synthesis, i.e., they were subjected to natural oxidation significantly longer than the nanoparticles of which consist film I. Therefore we can assume that oxidation of their surface is significantly higher than that of nanoparticles films I.

The previous sections have shown that the optical and electrical properties of films I greatly influenced by the surface of the nanoparticles from which these films are composed. It was found that the average properties of the surface similar to those of $\mathrm{SiO}$ and the component $\varepsilon$ $(\omega)$ is determined by the Cole-Cole law related to the dipole relaxation in $\mathrm{SiO}_{\mathrm{x}}$ shell of silicon nanoparticles.

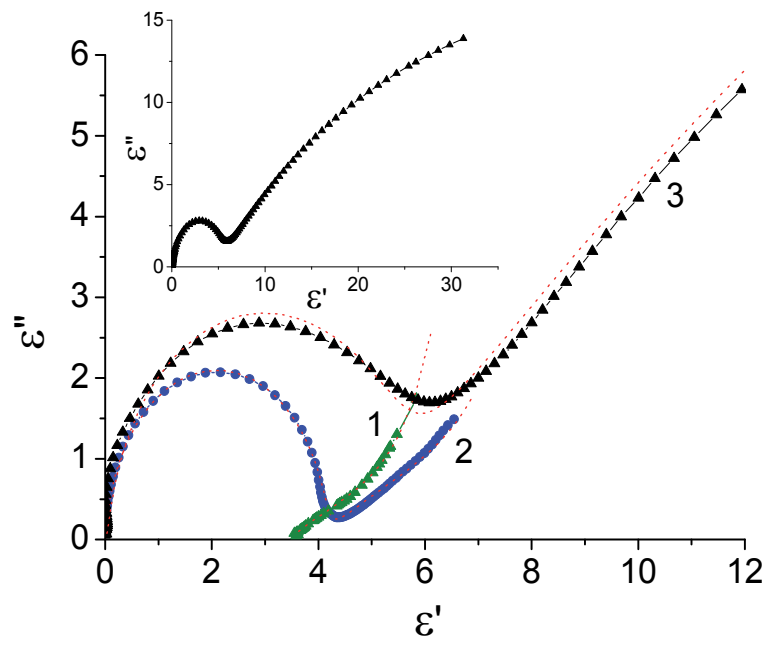

Fig. 15. The graph of dependence $\varepsilon^{\prime \prime}$ vs $\varepsilon^{\prime}$ for: film I - (1), film II - (2) and film III - (3) The inset shows an expanded plot $\varepsilon^{\prime \prime}\left(\varepsilon^{\prime}\right)$ for film III.

Since during the aging process of silicon nanoparticles the $\mathrm{SiO}_{2}$ shell must increase, the appearance of high-frequency components of the Debye spectra $\varepsilon(\omega)$ of the films II and III gives reason to assume that the source of this component is the structure of $\mathrm{SiO}_{2}$ with a narrow distribution of the dipole, which was formed on the surface of nanoparticles in two years of their presence in ethanol.

The fact that the Debye component of the spectrum $\varepsilon(\omega)$ as well as component Cole-Cole connected with the surface of the nanoparticles is confirmed by the fact noted earlier that the maxima of the Debye peak in the spectra of $\varepsilon^{\prime \prime}(\omega)$ of the films II and III correspond to different frequencies $\mathrm{v}_{\mathrm{m}}$. 
The grains of silicon nanoparticles constituting the films II and III are similar to each other, so this difference frequency $\mathrm{v}_{\mathrm{m}}$ can be attributed only to differences in the strength of interaction between the dipoles on the surface of the nanoparticles in these films. In other words, the presence of tetraaniline complexes on the surface of silicon nanoparticles leads to a weakening of the interaction between the dipoles are formed on the surface at the polarization of the particle.

\subsubsection{AC conductivity of the films II and III}

Dependence of the conductivity of the films I, II and III of the frequency of the applied electric field is shown in Figure 11. This figure shows that the conductivity of films I with good accuracy obey the law:

$$
\sigma(\omega)=\sigma(0)+\mathrm{A} \omega^{\mathrm{s}} .
$$

In the entire range of measured frequencies, the exponent $s=0,74$ equal to the value $h$ which obtained from the approximation of the Cole-Cole and given in table 1.

Conductivity of the films II describe such an equation is possible only in very limited region, namely in the frequency range $\mathrm{v} \leq 10^{3} \mathrm{~Hz}$ (let's call it a low-frequency component).

For low-frequency component of the conductivity of the films II as well as for the films I, the value of $s$ coincides with that of $h$, shown in Table 1 . For films of III this statement is incorrect. Indeed, as noted in Section B.2 conductivity of the films III is well approximated by a power law exponent with $\mathrm{s}=0,63$ only in small range of frequencies $5 \leq \mathrm{v} \leq 5 \cdot 10^{2} \mathrm{~Hz}$. As can be seen from Table 1 , this value differs significantly from the values of $h=0,5$ obtained from the approximation of the Cole-Cole.

The coincidence of the values of $\mathrm{s}$ and $\mathrm{h}$ for a film $\mathrm{I}$ is explained as follows circumstance. Spectrum $\varepsilon^{\prime \prime}(\omega)$ of the film over the entire range of measured frequencies is approximated by the Cole-Cole distribution which has the form:

$$
\varepsilon^{\prime \prime}=\frac{A\left(\omega \tau_{1}\right)^{1-h}}{1+B\left(\omega \tau_{1}\right)^{1-h}+\left(\omega \tau_{1}\right)^{2(1-h)}}=\left(\frac{A}{B+\frac{1}{\left(\omega \tau_{1}\right)^{1-h}}+\left(\omega \tau_{1}\right)^{1-h}}\right)
$$

Where $\mathrm{A}$ and $\mathrm{B}$ is constants and $\mathrm{B} \leq 2$

If $\omega \tau_{1}>>1$, this equation takes the form: $\varepsilon^{\prime \prime} \approx \frac{A}{\left(\omega \tau_{1}\right)^{1-h}}$,

and hence the following relation is valid for the conductivity $\sigma(\omega)-\sigma(0)=\varepsilon_{0} \cdot \omega \cdot \varepsilon^{\prime \prime} \sim \omega^{\mathrm{h}}$

As can be seen from Table 1 for the film I $\tau_{1}=0,06 \mathrm{~s}$, hence equation (13) is valid for it, at frequencies $\mathrm{v} \geq 10 \mathrm{~Hz}$. A similar analysis is applicable also to the low frequency component of the film II. For film II $\tau_{1}=0,72 \mathrm{~s}$, therefore, the dependence (13) will be observed if $\mathrm{v} \geq 1$ Hz. This fact is shown in Figure 16, where the conductivity of the films II and III is approximated by the sum of $\sigma(0)$ and two distributions of Cole-Cole and Debye. 
From this figure it is clear that if the $\varepsilon^{\prime \prime}(\omega)$ spectrum of the films II describes only the distribution of the Cole-Cole, they would obey the conductivity relation (13) throughout the frequency range $1 \leq \mathrm{v} \leq 10^{6} \mathrm{~Hz}$ as well as the conductivity of the films I.

For the film III observed more complicated situation, its spectrum is distorted with respect to relation (13), not only at high frequencies $v \geq 10^{3} \mathrm{~Hz}$, but also at frequencies $\mathrm{v} \leq 10 \mathrm{~Hz}$ (see Figure 16, b). According to the vast majority of experimental data, the frequency dependence of the conductivity of disordered media has kind of plateau (low-frequency plateaus) at low frequencies and is a power in excess of a certain critical frequency.

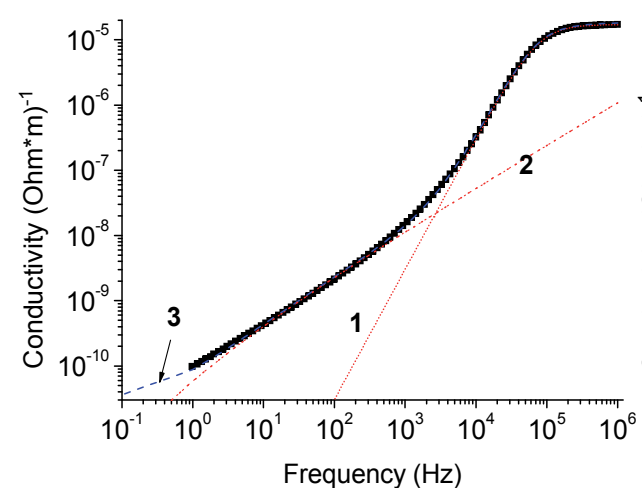

a

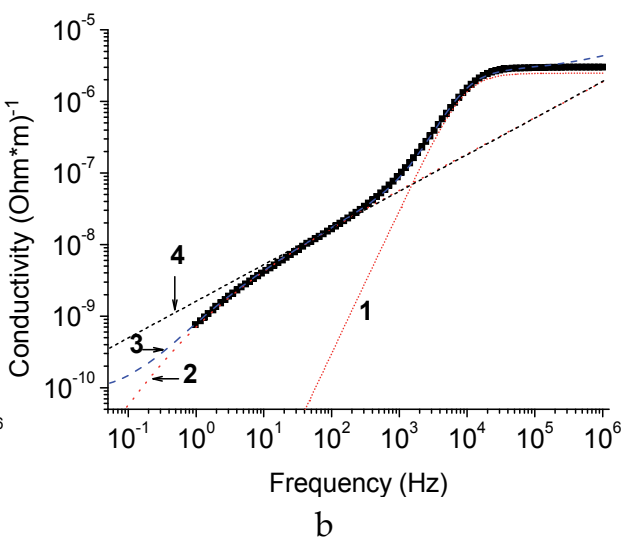

Fig. 16. The frequency dependence of AC conductivity of the films II (a) and III (b), as well as its approximation by: the Debye law - (1), the relation of Cole - Cole - (2) and the total approximation, which takes into account the dc conductivity - (3). (4) - power dependence with an exponent equal to the value of $h$ at the Cole-Cole relation.

For films of III observes the opposite situation, instead, the appearance of a plateau at low frequencies, the conductivity $\sigma(\omega)$ begins to decrease more quickly with decreasing frequency of the external electric field. The reason for the absence of such low frequency plateau may be the existence of significant resistance at the interface of the film-electrode.

Comparison of $\sigma(0), \sigma_{\mathrm{DC}}$ and $\sigma_{\mathrm{B}}(0)$ from Table 1 shows their good agreement for film I. For films II are in good agreement the values $\sigma(0)$ and $\sigma_{D C}$ but somewhat too high the value of $\sigma_{\mathrm{B}}$ $(0)$ with respect to them. For films III good agreement is observed for the values $\sigma_{D C}$ and $\sigma_{B}(0)$ but $\sigma(0)$ is less than these quantities is about 20 times. The fact that $\sigma_{\mathrm{DCI}}$ more than 25 times higher then $\sigma_{\mathrm{DCI}}$ (see Table1) confirms our earlier assumption that the degree of surface oxidation of silicon nanoparticles of films II is significantly higher than that in films I.

At frequencies $\mathrm{v}_{\mathrm{s} 1} \geq 1 \cdot 10^{5} \mathrm{~Hz}$ for films II and $\mathrm{v}_{\mathrm{s} 2} \geq 3 \cdot 10^{4} \mathrm{~Hz}$ for films III conductivity begins to depend very weakly on the frequency of an external electric field. This behavior is usually associated with the manifestation of the nature of hopping conduction (Barsoukov \& Macdonald, 2002), and the frequency $\mathrm{v}_{\mathrm{c}}$ determined by the height of the barriers between potential wells, which are involved in the hopping transport of charge carriers. Because $\mathrm{v}_{\mathrm{s} 1}>$ $\mathrm{V}_{\mathrm{s} 2}$, we can conclude that the presence of tetraaniline on the surface of silicon nanoparticles lowers the barriers separating localized states. 


\section{Conclusion}

Dielectric and transport properties of thin films obtained by deposition of silicon nanoparticles from ethanol sols on a glass, quartz, and aluminum substrates were measured by optical ellipsometry and impedance spectroscopy methods. The real and imaginary permittivities of $n c$-Si films were measured in frequency ranges of $5 \times 10^{14-1015}$ and 10-106 Hz. It was found that the permittivity spectra depend on the time which has elapsed since the synthesis of nanoparticles until their deposition on the substrate.

Only one type of dipole relaxation, which can be described by semi-empirical Cole-Cole equation, exists in films prepared from sols with silicon nanoparticles, synthesized a week before their deposition on a substrate (film I). In films prepared from sols containing aged nanoparticles (film II) there is a double-dipole relaxation, which is revealed in the fact that for the approximation of the experimental spectra of these films not only Cole-Cole relation but the law of Debye dipole relaxation should be used. A similar confirmation is valid also for the films deposited from the sols with aged nanoparticles in which tetra aniline was added (film III).

In the measured frequency ranges, $\varepsilon^{\prime}$ and $\varepsilon^{\prime \prime}$ vary within $2.1-1.1,3.4-6.2$ and $0.25-0.75,0.08-$ 1.8 , respectively. From the EMA analysis of the spectra, it was concluded that the $n c-S i$ film in light reflection processes can on average be considered as a two component medium consisting of $\mathrm{SiO}$ and air gaps with a porosity of $50 \%$.

It was shown that the complex dielectric dispersion of films in the frequency range of 10 $2 \times 10^{6} \mathrm{~Hz}$ is well approximated by the semiempirical Cole-Cole relation, taking into account the effect of free charges controlling the dark dc conductivity of films.

An analysis of the frequency dependences of the ac conductivity of the studied films allowed the conclusion to be drawn that the ac conduction process is well described by the cluster diffusion approximation model.

The dependence of the dark conductivity of films on the ambient air humidity and the temperature dependence of absorption bands related to associated $\mathrm{Si}-\mathrm{O}-\mathrm{H}$ groups allows the conclusion to be drawn that the conductivity at frequencies lower than $2 \times 10^{2} \mathrm{~Hz}$ is controlled by proton transport through hydrogen bound hydroxyl groups on the surface of silicon nanoparticles.

Using Cole-Cole and Debye relations for approximation of experimental spectra $\varepsilon(\omega)$ the values of static permittivity $\varepsilon_{0}$ of films I, II and III have been found. For films I and II quantities $\varepsilon_{0}$ close to the values characteristic of crystalline silicon. For films of III $\varepsilon_{0} \approx 67$, i.e. greatly exceeds $\varepsilon_{0}$ for c-Si. Such a high value $\varepsilon_{0}$ we attribute to increasing polarization of the silicon nanoparticles when the tetraaniline complexes are attached to their surface.

AC conductivity of the films II and III in the whole frequency range of $1-10^{6} \mathrm{~Hz}$ can not be approximated by a power law, which is characteristic of the conductivity of the films I. We show that such deviation from the dependence $\sigma_{\mathrm{AS}} \sim \omega^{\mathrm{s}}$ is associated with a doubledielecrtic relaxation typical for films II and III and with the presence in the spectra $\varepsilon$ " $(\omega)$ of these films Debye components. 


\section{Acknowledgment}

We sincerely thank Dr. Helen Yagudayev, the senior researcher of the Shemyakin Ovchinnicov Institute of Bioorganic Chemistry of RAS, for providing us the conductive tetraaniline solutions.

We also thank our colleagues prof. Plotnichenko V.G., prof. Kuz'min G.P., prof. Ischenko A.A., dr. Koltashev V.V., researcher Tikhonevich O.V. for the fruitful cooperation in investigation of the properties of nano-sized silicon.

\section{References}

[1] Anopchenko,A.; Marconi, A.; Moser, E.; Prezioso, S.; Wang; M., Pavesi; L., Pucker, G. \& Bellutti. P. J. (2009) Low-voltage onset of electroluminescence in nanocrystalline$\mathrm{Si} / \mathrm{SiO}_{2}$ multilayers Journal of Applied Physics, Vol.106, (2009), p.p. 033104, ISSN 0021-8979

[2] Aspens, D. E. \& Studna, A. A. (1983) Dielectric functions and optical parameters of Si, Ge, Gap, GaAs, GaSb, InP, InAs, and InSb from 1.5 to 6.0 eV, Physical Review B, Vol. 27,№2, (January 1983), p.p.985 - 1009

[3] Austin, I. G. \& Mott, N. F. (1069). Polarons in crystalline and non-crystalline materials. Advances in physics, Vol.18, №71, (January 1969), pp. 41-102

[4] Axelrod, E.; Givant, A.; Shappir, J.; Feldman, Y. \& Sa'ar. A. (2002) Dielectric relaxation and transport in porous silicon Physical Review B, Vol. 65, №16, (April 2002) , p.p. 165429(1-7) ,ISSN 0163-1829

[5] Azzam, R.M.A. \& Bashara N.M. (1977) Ellipsometry and polarized light, North-Holland publishing company, ISBN 1704050000 , Amsterdam, New York, Oxford

[6] Barsoukov, E. \& Macdonald, J. R. (2005) Impedance Spectroscopy. Theory, Experiment, and Applications, Second Edition, A John Wiley \& Sons, Inc., Publication, New Jersey, USA and Canada ISBN: 0-471-64749-7

[7] J. L. Barton, (1966). Verres Réfract. Vol. 20, p.p. 328 (1966).

[8] Ben-Chorin, M.; Möller, F.; Koch, F.; Schirmacher, W. \& Eberhard, M. (1995) Hopping transport on a fractal: ac conductivity of porous silicon Physical Review B, Vol. 51, №4, (January 1995) , p.p. 2199(1-15) , ISSN 0163-1829

[9] Bianchi, R.F.; Leal Ferreira, G.F.; Lepienski, C.M. \& Faria, R.M. (1999). Alternating electrical conductivity of polyaniline. Journal of Chemical Physics, Vol.110,№9, (March 1999), p.p. 4602-4607, ISSN 0021-9606

[10] D.A.G. Bruggeman. (1935). Berechung verschiedener physikalisher konstanten von heterogenen substanzen. Annalen der Physik (Leipzig), Vol. 24, (1935), pp. 636-664

[11] Brus, L.E.; Szajowski, P.F.; Wilson, W. L.; Harris, T. D.; Schuppler, S. \& Citrin. P. H. (1995) Electronic Spectroscopy and Photophysics of Si Nanocrystals: Relationship to Bulk c-Si and Porous Si. Journal of American Chemical Society, Vol. 117, (1995) pp. 2915-2922

[12] Campbell, I.H. \& Faushet P.M. (1986) The effect of microcrystalline size and shape on the one phonon Raman spectra of crystalline semiconductors. Solid State Communications. Vol. 58, №10 (February 1986), p.p.739-741, ISSN 0038-1098 
[13] Cao, Chao; He, Yao; Torras, J.; Deumens, E.; Trickey, S. B. \& Cheng, Hai-Ping. (2007) Fracture, water dissociation, and proton conduction in $\mathrm{SiO}_{2}$ nanochains. Journal of Chemical Physics, Vol. 126, №21, (June 2007) p.p. 211101(1-3), ISSN 0021-9606

[14] Cole, K. S. \& Cole, R. H. (1941). Dispersion and Absorption in Dielectrics. Journal of Chemical Physics, Vol. 9, №, (February 1941) p.p. 341-351, ISSN 0021-9606

[15] Conte, G.; Feliciangeli, M. C.; \& Rossi, M. C. (2006) Impedance of nanometer sized silicon structures. Applied Physics Letters, Vol. 89, № 2, (July 2006), p.p.022118(1-3), ISSN0003-6951

[16] Dorofeev, S. G.; Kononov, N. N.; Ishchenko, A. A.; Vasil'ev, R. B.; Goldschtrakh, M. A.; Zaitseva, K. V.; Koltashev, V. V.; Plotnichenko, V. G. and O. V. Tikhonevich. (2009). Optical and Structural Properties of Thin Films Precipitated from the Sol of Silicon Nanoparticles. Rus. Semiconductors, Vol. 43, No. 11, (April 2009), pp. 1420-1427. ISSN 1063-7826.

[17] Du, Mao-Hua; Kolchin, A. \& Chenga, Hai-Ping. (2003). Water-silica surface interactions: A combined quantum-classical molecular dynamic study of energetics and reaction pathways. Journal of Chemical Physics, Vol. 119, №131, p.p.6418 - 6422 (2003) ISSN 0021-9606.

[18] Dyre, J.C. \& Schrøder, T. B. (2000). Universality of ac conduction in disordered solids. Reviews of Modern Physics, Vol.72, №3, (July 2000), p.p. 873 - 892, ISSN 0034-6861.

[19] Ehbrecht, M.; Ferkel, H.; Huisken, F.; Holz, L.; Polivanov, Yu.N.; Smirnov, V.V.; Stelmakh, O.M. \& Schmidt R. (1995) Deposition and analysis of silicon clusters generated by laser-induced gas phase reaction Journal of Applied Physics, Vol.78, № 9, (November 1995), p.p. 5302-5305, ISSN 0021-8979

[20] Glasser, L. (1975) Proton Conduction and Injection in Solids. Chemical Reviews, Vol.75, №1, (January 1974), p.p. 21 - 65.

[21] Goswami, A. \& Goswami, A.P. (1973). Dielectric and optical properties of ZnS films. Thin Solid Films Vol. 16, (January 1973), p.p. 175 - 185

[22] Hubner, K. (1980). Chemical Bond and Related Properties of $\mathrm{SiO}_{2}$. Physica Status Solidi (a) Vol. 61, (May 1980), p.p. 665-673

[23] Hunt, A.G. (2001). Ac hopping conduction: perspective from percolation theory. Philosophical Magazine B Vol. 64, №9, (2001), p.p. 875-913, ISSN 1364-2812 print/ISSN 1463-6417 online

[24] Isichenko, M. B. (1992) Percolation, statistical topography, and transport in random media. Reviews of Modern Physics, Vol. 64, №4, (October 1992), p.p.961 - 1043, ISSN 0034-6861.

[25] Jurbergs, D.; Rogojina, E.; Mongolini, L. \& Kortshagen, U. (2006). Silicon nanocrystalls with ensemble quantum yields exceeding 60\%. Applied Physycs Letters, Vol. 88, №23, (June 2006) 233116(1-3), ISSN 0003-6951.

[26] Kakinuma, H.; Mohri, M.; Sakamoto, M. \& Tsuruoka T. (1991). Sructural properties of polycrystalline silicon films prepared at low temperature by chemical vapor deposition. Journal of Applied Physics, Vol.70, (December 1991), p.p. 7374 - 7381, ISSN 0021-8979

[27] Kononov, N. N.; Kuz'min, G. P.; Orlov, A. N.; Surkov, A. A. and Tikhonevich, O. V. (2005). Optical and Electrical Properties of Thin Wafers Fabricated from Nanocrystalline Silicon Powder. Rus. Semiconductors, Vol. 39, No. 7, (November 2005), pp. 835-839, ISSN 1063-7826 
[28] Kononov, N. N.; Dorofeev, S. G.; Ishenko, A. A.; Mironov, R. A.; Plotnichenko, V. G. \& E. M. Dianov. (2011). Dielectric and Transport Properties of Thin Films Precipitated from Sols with Silicon Nanoparticles Rus. Semiconductors, 2011, Vol. 45, No. 8, (August 2011), pp. 1038-1048. ISSN 1063-7826

[29] Kovalev, D.; Polisski, G.; Ben-Chorin, M.; Diener, J. \& Koch F. (1996) The temperature dependence of the absorption coefficient of porous silicon Journal of Applied Physics, Vol.80, №10, (November 1996) , p.p.5978 -5983, ISSN 0021-8979

[30] Kousik, D. \& De, S.K. (2007). Double dielectric relaxations in $\mathrm{SnO}_{2}$ nanoparticles dispersed in conducting polymer. Journal of Applied Physics, Vol.102,.№ 8, ( October 2007), p.p. $084110-1,084110-7$, ISSN 0021-8979 print | 1089-7550 online

[31] Kuz'min, G.P.; Karasev, M.E.; Khokhlov, E.M. Kononov, N.N.; Korovin, S.B.; Plotnichenko, V.G.; Polyakov, S.N.; Pustovoy, V.I. \& Tikhonevich O.V. (2000). Nanosize Silicon Powders: The Structure and Optical Properties. Rus. Laser Physics Vol.10, №4, (April 2000) p.p. 939-945

[32] Leite, V. B. P.; Cavalli, A. \& Oliveira, O. N. Jr. (1998) Hydrogen-bond control of structure and conductivity of Langmuir films. Physical. Review E, Vol. 57, №6, (June 1998), p.p. 6835 - 6839, ISSN 1063-651X

[33] Luppi, M. \& Ossicini S. (2005). Ab initio study on oxidized silicon clusters and silicon nanocrystals embedded in $\mathrm{SiO}_{2}$ : Beyond the quantum confinement effect. Phys. Rev. B V.71, (January 2005), p.p. 035340(1-15), ISSN 1098-0121

[34] Ma, Zhixun; Liao, Xianbo; Kong, Guanglin \& Chu, Junhao (2000) Raman scattering of nanocrystalline silicon embedded in $\mathrm{SiO}_{2}$. Science in China A, Vol.43, (2000), p.p. 414-420

[35] Min, R .B. \& Wagner, S. (2002), Nanocrystalline silicon thin-film transistors with 50-nmthick deposited channel layer, $10 \mathrm{~cm}^{2} \mathrm{~V}^{-1} \mathrm{~S}^{-1}$ electron mobility and $10^{8}$ on/off current ratio Applied Physics A Materials Science EProcessing, Vol. 74, (August 2001), p.p.541543 (DOI) 10.1007/ s003390100927

[36] Moliton, A. (2007) Applied Electromagnetism and Materials, Ch.1, p.8. Springer Science and Business Media. ISBN-10: 0-387-38062-0

[37] Nakajima, T. (1971) Annual Report, Conference on Electric Insulation and Dielectric Phenomena (Washington, D.C., National Academy of Sciences, 1972), p. 168.

[38] Namikawa. H. (1975).Characterization of the diffusion process in oxide glasses based on the correlation between electric conduction and dielectric relaxation. Journal of Non-Crystalline Solids, Vol.18, №2, (September 1975), p.p.173-195, ISSN 0022-3093.

[39] Nogami, M. \& Abe, Y. (1997). Evidence of water-cooperative proton conduction in silica glasses. Physical Review B, Vol. 55, №18, (May 1997), p.p.12108 - 12112, ISSN 01631829

[40] Nogami, M.; Nagao, R. \& Wong, C. (1998). Proton Conduction in Porous Silica Glasses with High Water Content. Journal of Physical Chemistry B, Vol. 102, (July 1998), p.p. 5772-5775, S1089-5647

[41] Pickering, C.; Beale, M. I. J.; Robbins, D. J.; Pearson, P. J. \& Greef. R. (1984). Optical studies of the structure of porous silicon films formed in p-type degenerate and non- degenerate silicon. Journal Physics C: Solid State Physics, Vol.17, (August 1984) p.p.6535-6552, ISSN 0022-3719 
[42] Puzder, A.; Williamson, A.J.; Grossman, J.C. \& Galli G. (2002). Surface Chemistry of Silicon Nanoclusters. Physical Review Letters, Vol. 88, (2002), p.p.097401(1-4), ISSN 0031-9007

[43] Richter, H.; Wang, Z. \& Ley L. (1981). The one phonon Raman spectrum in microcrystalline silicon. Solid State Communications, Vol.39, №5, (May 1981), p.p.625-629, ISSN 0038-1098

[44] Saadane, O.; Lebib, S.; Kharchenko, A.V.; Longeaud, C. \& R. Roca I Cabarrocas. (2003) Structural, opical and electronic properties of hydrogenated polymorphous silicon films deposited from silane-hydrogen and silane-helium mixtures. Journal of Applied Physics, Vol. 93, №11, (June 2003), p.p.9371 - 9379, ISSN 0021-8979

[45] Schrøder, T. B. \& Dyre. J. C. (2002). Computer simulations of the random barrier model. Physical Chemistry and Chemical Physics, Vol.4, (June 2002), p.p. 3173-3178

[46] Schrøder, T. B. \& Dyre J. C.(2008). AC Hopping Conduction at Extreme Disorder Takes Place on the Percolating Cluster Physical Review Letters, Vol.101, (July 2008) p.p.025901(1-4), ISSN 0031-9007

[47] Schuppler, S.; Friedman, S.L.; Marcus, M.A.; Adler, D.L.; Xie, Y. H.; Ross, F.M.; Chabal, Y.J.; Harris, T.D.; Brus, L.E.; Brown, W.L.; Chaban, E.E.; Szajowski, P.E.; Christman, S.B. \& Citrin, P.H. (1995). Size, shape and composition of luminescent species in oxidized Si nanocrystals and H-passivated porous Si. Physical Review B, Vol.52, p.p. 4910-4925, ISSN 1098-0121

[48] Stuart B. (2004). Infrared Spectroscopy: Fundamentals and Applications, John Wiley \& Sons, Ltd., ISBN 0-470-85427-8

[49] Eds. by Tompkins, G. H. \& Irene, E.A. (2005). Handbook of ellipsometry. p.p. 282-289. William Andrew publishing, Springer-Verlag GmbH \& Co. KG. New York, Heidelberg. ISBN: 0-8155-1499-9 (William Andrew, Inc.), ISBN: 3-540-22293-6 (Springer-Verlag GmbH \& Co. KG)

[50] De la Torre, J.; Bremond, G.; Lemiti, M.; Guillot, G.; Mur, P. \& Buffet. N. (2006) Silicon nanostructured layers for improvement of silicon solar cells efficiency: A promising perspective. Materials Science and Engineering C Vol. 26, (January 2006), p.p. 427430, ISSN 0928-4931

[51] Tsang, J.C.; Tischler, M.A. \& Collins R.T. (1992). Raman scattering from H and O terminated porous Si. Applied Physics Letters, Vol.60, №18, (May 1992) p.p. 22792281, ISSN 0003-6951

[52] Tsu, R.; Shen, H. \& Dutta M. (1992). Correlation of Raman and photoluminescence spectra of porous silicon. Applied Physics Letters, Vol.60, №1, (January1992), p.p. 112-114, ISSN 0003-6951

[53] Tsu, R.; Babić, D. \& Ioriatti, L. Jr. (1997). Simple model for the dielectric constant of nanoscale silicon particle. Journal Applied Physics, Vol. 82, № 3, (August 1997), p.p.1327 - 1329, ISSN 0021-8979

[54] Tsu, R. (2000) Phenomena in silicon nanostructure devices. Applied Physics A Vol.71, (September 2000) p.p.391-402, DOI 10.1007/s003390000552

[55] Urbach, B.; Axelrod, E. \& Sa'ar A. (2007). Correlation between transport, dielectric, and optical properties of oxidized and nonoxidized porous silicon Physical Review B, Vol. 75, №20, (May 2007) , p.p. 205330(11) , ISSN 1098-0121

[56] Voutsas, A.T.; Hatalis, M.K.; Boyce, J. \& Chiang A. (1995). Raman spectroscopy of amorphous and microcrystalline silicon films deposited by low-pressure chemical 
vapor deposition Journal of Applied Physics, Vol.78, №12, (December 1995), p.p. 6999-7005, ISSN 0021-8979

[57] Wang, K.; Chen, H. \& Shen, W.Z. (2003). AC electrical properties of nanocrystalline silicon thin films. Physica B, Vol. 336, (April 2003), p.p. 369-378, ISSN 0921-4526

[58] Wang, W. \& MacDiarmid, A. (2002), New synthesis of phenil/phenil end-capped tetraaniline in the leucoemeraldine oxidation states. Synthetic metals, Vol. (2002) 129, p.p. 199-205, ISSN

[59] Wovchko, E. A.; Camp, J. C.; Glass, J. A. Jr. \& Yates, J. T. Jr. (1995). Active sites on $\mathrm{SiO}_{2}$ : role in $\mathrm{CH}_{3} \mathrm{OH}$ decomposition. Langmuir Vol.11, №7, (1995), p.p.2592-2599, ISSN 0743-7463

[60] G. Zuter.(1980) Dielectric and Optical Properties of $\mathrm{SiO}_{\mathrm{x}}$. Physica Status Solidi (a) Vol. 59, (April 1980), p.p. K109-K113 


\title{
Self-Assembly of Nanoparticles at Solid and Liquid Surfaces
}

\author{
Peter Siffalovic, Eva Majkova, Matej Jergel, \\ Karol Vegso, Martin Weis and Stefan Luby \\ Institute of Physics, Slovak Academy of Sciences \\ Slovakia
}

\section{Introduction}

The research field of nanoparticle synthesis and related nanoparticle applied sciences have been steadily growing in the past two decades. The chemical synthesis of nanoparticles was improved up to the point that the organic and inorganic nanoparticle colloids are produced with a low size dispersion and with a well defined nanoparticle shape in large quantities. A stunning feature of a drying nanoparticle colloidal solution is the ability to create selfassembled arrays of nanoparticles. The self-assembled nanoparticle arrays mimic the natural crystals. The size of perfectly ordered domains is limited by the size dispersion of nanoparticles. Consequently the defects in the self-assembled structure are obvious and unavoidable. Despite these defects, the self-assembled nanoparticle arrays represent a new class of nanostructures built on "bottom-up" technological approach to fabrication. The traditional way of "top-down" fabrication technology primarily based on nano-lithography is complex, including many technological steps, time consuming and expensive. The main advantage is the tight control of all parameters governing the final nanostructures. On the other hand, the emerging fabrication technologies based on the self-assembled nanoparticles are fast, less complex and more price competitive. An extensive research is now focused on a deeper understanding of processes that control the self-assembly. New routes for directed or stimulated self-assembly are studied to achieve a tighter control than readily available in the spontaneous self-assembly. In this chapter we will discuss the spontaneous nanoparticle self-assembly with emphasis on characterization of nanoparticle arrays at various stages of the self-assembly process. The main diagnostic technique used throughout this chapter will be the grazing-incidence small-angle X-ray scattering (GISAXS) that represents a reliable and simple monitor of nanoparticle arrangement. The theoretical background of GISAXS and required instrumentation are described in Section 2. The most flexible surface to study the nanoparticle self-assembly processes is the liquid surface. The Section 3. reviews the latest results of studies combing the GISAXS technique with Langmuir nanoparticle layers on the water subphase. Almost all relevant nanoparticle applications rely on self-assembled arrays on solid surfaces. The Section 4 describes in detail the possibilities of nanoparticle transfer from liquid onto solid surfaces. The post-processing of self-assembled nanoparticle arrays and their applications are reviewed in the last Section 5. 


\section{SAXS/GISAXS techniques and their employment for nanoparticle research}

The transmission (TEM) and scanning (SEM) electron microscopy provide information on the nanoparticle shape, average size and size distribution. However, this information is usually obtained after numerical evaluation of real space micrographs from limited data sets. Alternative approach is based on the angle-resolved analysis of scattered X-rays or neutrons from the nanoparticles and their assemblies. In this chapter we will employ the small-angle X-ray scattering (SAXS) (Guinier and Fournet 1955) for the nanoparticle colloidal solutions. For nanoparticles immobilized at interfaces, a related technique so-called grazing-incidence small-angle X-ray scattering (GISAXS) is used that has been recently reviewed (Renaud, Lazzari et al. 2009). A general scheme of the GISAXS experiment is shown in Fig. 1.

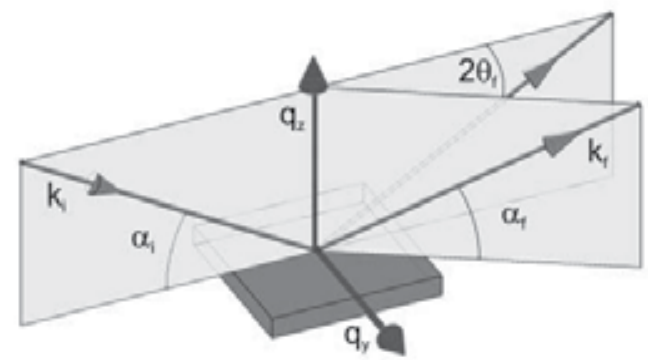

Fig. 1. The GISAXS measurement geometry

The collimated X-ray beam defined by $\vec{k}_{i}$ is incident under a small grazing angle on the sample surface. The scattered radiation is recorded by a two dimensional X-ray detector. Each point at the detector plane receives the scattered radiation given by a set of two angles $\left(2 \theta_{f}, \alpha_{f}\right)$ that corresponds to a unique scattering vector $\vec{q}$ in the reciprocal space. The relationship between the scattering vector in reciprocal space and the scattering angles in the real space is given by the following equations (Müller-Buschbaum 2009)

$$
\begin{aligned}
& q_{x}=\frac{2 \pi}{\lambda}\left[\cos \left(2 \theta_{f}\right) \cos \left(\alpha_{f}\right)-\cos \left(\alpha_{i}\right)\right] \\
& q_{y}=\frac{2 \pi}{\lambda}\left[\sin \left(2 \theta_{f}\right) \cos \left(\alpha_{f}\right)\right] \\
& q_{z}=\frac{2 \pi}{\lambda}\left[\sin \left(\alpha_{f}\right)+\sin \left(\alpha_{i}\right)\right]
\end{aligned}
$$

The SAXS/GISAXS signal is given by constructive interferences of X-ray waves partially scattered on individual nanoparticles. The total scattered intensity (also called the scattering cross-section) at specific $\vec{q}$ vector in the reciprocal space is given as (Feigin, Svergun et al. 1987)

$$
I(\vec{q})=\sum_{i=1}^{N} \sum_{j=1}^{N} F^{i}(\vec{q}) \cdot F^{j, *}(\vec{q}) \cdot \exp \left[i \vec{q} \cdot\left(\vec{r}_{i}-\vec{r}_{j}\right)\right]
$$


where $N$ is the total number of nanoparticles, $F^{i}(\vec{q})$ is the form-factor of the $i^{\text {th }}$ nanoparticle and $\vec{r}_{i}$ defines the position of the $i^{\text {th }}$ nanoparticle. Within the simple Born (kinematic) approximation (BA) the nanoparticle form-factor is simply given by the Fourier transform of the nanoparticle density function $\rho_{i}(\vec{r})$ as follows (Glatter and Kratky 1982)

$$
F^{i}(\vec{q})=\int \rho_{i}(\vec{r}) \cdot \exp (i \vec{q} \cdot \vec{r}) d \vec{r}
$$

For the nanoparticles immobilized at interfaces we have to include the refraction/reflection phenomena at the interfaces and the associated multiple scattering events. This is treated in detail within the framework of the distorted-wave Born approximation (DWBA) which introduces a modified form-factor for each nanoparticle confined near the interface (Holý, Pietsch et al. 1999). A detailed survey of the DWBA theory can be found in the following reference (Renaud, Lazzari et al. 2009). A typical DWBA effect is the presence of the Yoneda enhancement at the critical exit angle in the GISAXS patterns (Yoneda 1963). In many cases we can avoid the DWBA multiple scattering terms by recording the GISAXS pattern at the incident angle several times larger than the critical angle for the total X-ray reflection of the supporting substrate (Daillant and Gibaud 2009). If we assume that the nanoparticles can be described by an average form-factor $\left\langle|F(\vec{q})|^{2}\right\rangle$ than the eq. (2) in BA can be rearranged as follows

$$
I(\vec{q})=N\left\langle|F(\vec{q})|^{2}\right\rangle S(\vec{q})
$$

Here the $S(\vec{q})$ represent the nanoparticle interference function. The nanoparticle interference function is the reciprocal space equivalent of the nanoparticle pair correlation function $P(\vec{r})$ defined in real space (Lazzari 2009). The pair correlation function is proportional to the probability of finding a nanoparticle at the position vector $\vec{r}$ centered at an arbitrarily selected nanoparticle. This function is directly accessible from the TEM/SEM micrographs.

The GISAXS experimental technique was confined for a long time to synchrotron facilities as the scattering cross-section is generally very low. Each synchrotron ring has a dedicated SAXS beamline that can support conventional GISAXS setup. The Fig. 2 shows the typical GISAXS scheme of the BW4 beamline at the DORIS III ring at HASYLAB, Hamburg (Stribeck 2007). The front-end of the experimental setup is a wiggler that generates the X-ray radiation. The crystal monochromator is used to select a single wavelength typically at $0.139 \mathrm{~nm}$. The radiation is further conditioned with slits and two cylindrical mirrors to focus the radiation in both directions at the detector plane. The additional beryllium X-ray lenses can be attached to focus the radiation at the sample position (Roth, Dohrmann et al. 2006).

The distance between the sample and detector can vary between $3 \mathrm{~m}$ and $13 \mathrm{~m}$ that allows flexibility in the accessible range of the reciprocal space. The two-dimensional (2D) X-ray $\mathrm{CCD}$ detector is used to record the $\mathrm{X}$-ray radiation scattered by the sample. The primary and specularly reflected beams are suppressed by the beamstops. 


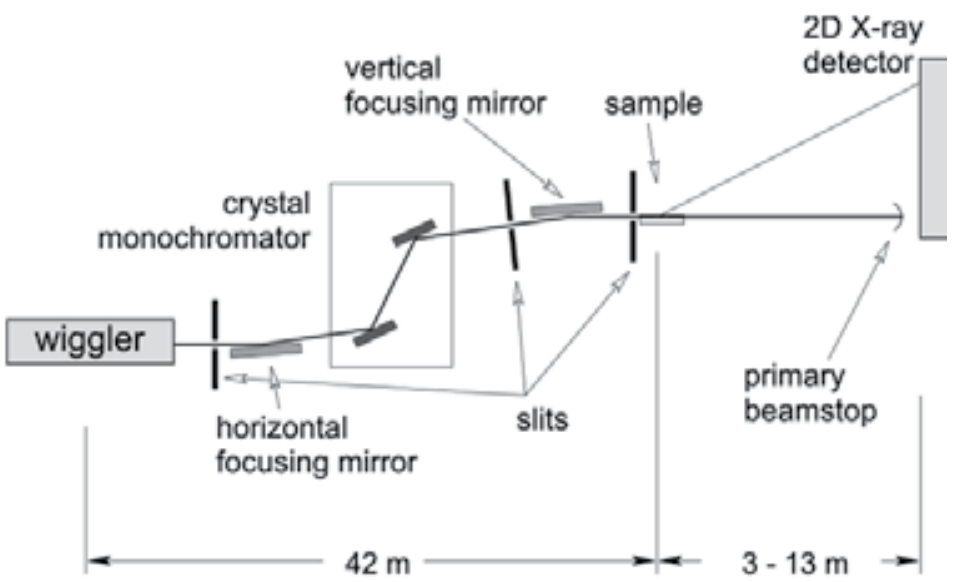

Fig. 2. The sketch of the experimental GISAXS geometry at BW4 beamline, HASYLAB

a)

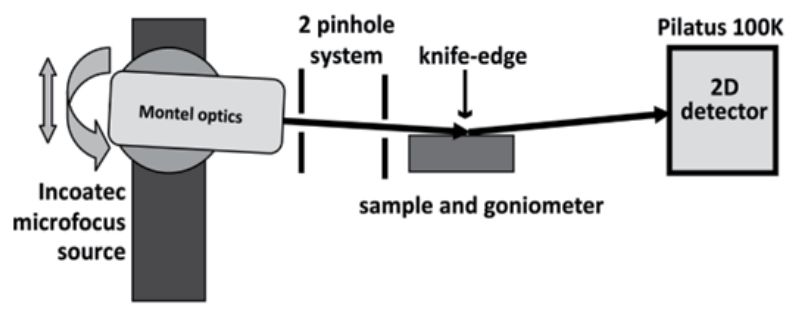

b)

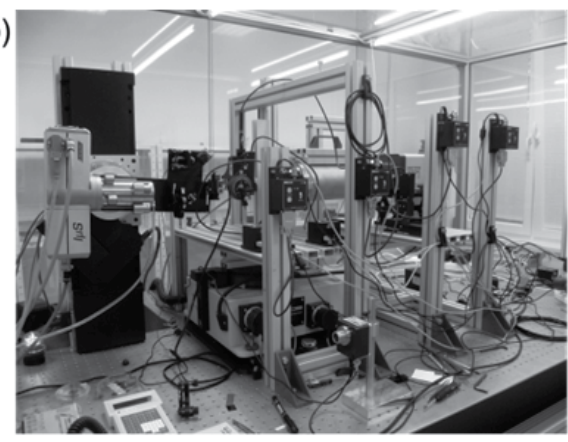

Fig. 3. a) The scheme of the laboratory GISAXS setup and (b) the photograph of its realization at Institute of Physics SAS.

The latest advances in the low-power X-ray generators and the efficient X-ray optics opened a new era of laboratory equipments suitable for GISAXS measurements (Michaelsen, Wiesmann et al. 2002). Nowadays already several companies (Bruker AXS, Anton Paar, Hecus XRS, Rigaku) supply complete X-ray solutions supporting GISAXS measurement modes for solid-state samples. The Fig. 3a and Fig. 3b show the laboratory setup scheme and the photograph of a home-built GISAXS instrumentation developed at the Institute of Physics SAS, respectively (Siffalovic, Vegso et al. 2010). This setup supports GISAXS measurements on solid as well as liquid surfaces. The core of the experimental apparatus is a compact low-power $(30 \mathrm{~W}) \mathrm{X}$-ray source $\left(\mathrm{Cu}-\mathrm{K}_{\alpha}\right)$ equipped with a loosely focusing $\mathrm{X}$-ray Montel optics (Wiesmann, Graf et al. 2009). The source can be freely rotated and translated in the vertical direction. This is important for the precise adjustment of the incident angle in the GISAXS measurements at liquid surfaces. The unwanted scattered radiation is eliminated by laser-beam precisely cut tungsten pinholes. The sample is fixed on a goniometer that allows precise height and tilt adjustments. 
a)

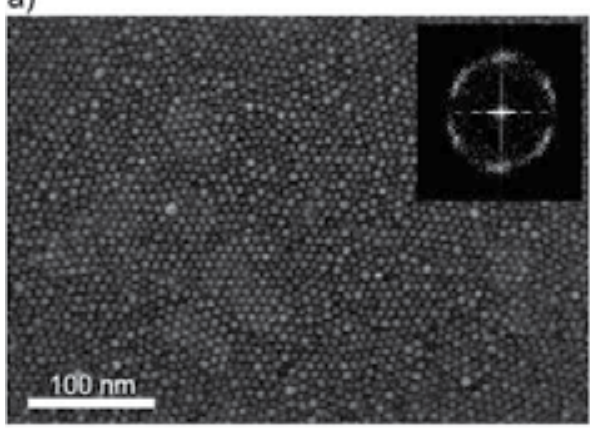

d)

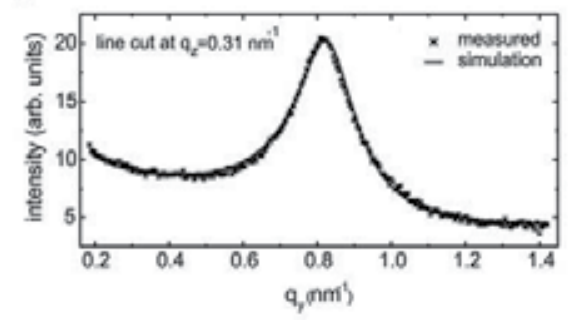

b)
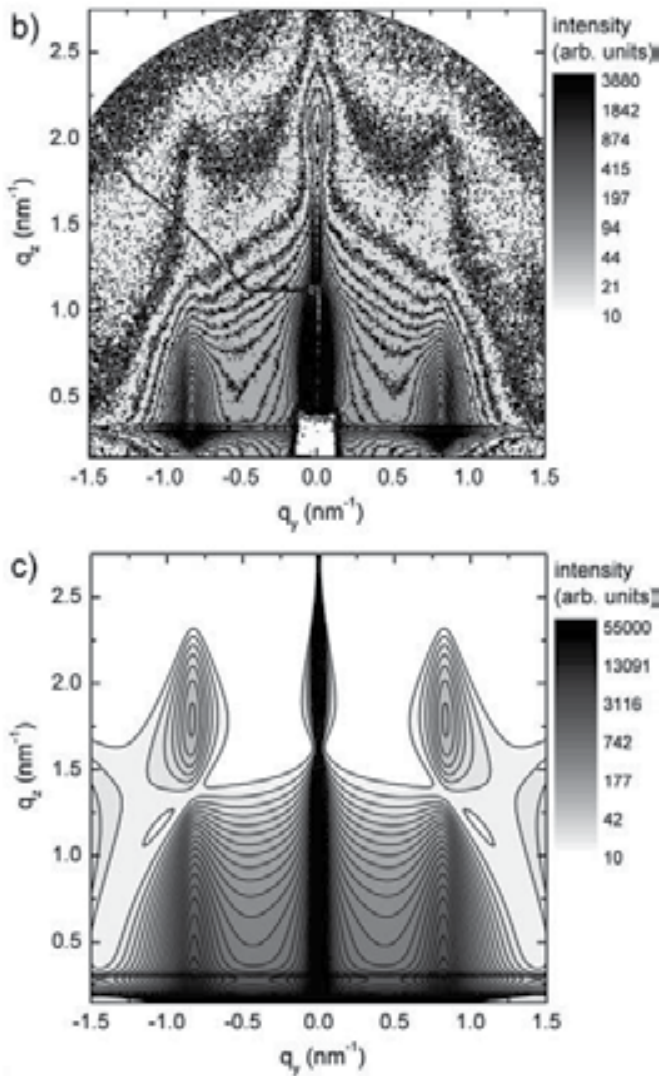

Fig. 4. a) The SEM micrograph of Fe-O self-assembled nanoparticles. Measured (b) and simulated (c) GISAXS pattern of self-assembled nanoparticles. d) The extracted line-cut from the measured GISAXS pattern along with the simulation.

The auxiliary knife-edge blade is used to reduce the parasitic air-scattering. The additional vacuum flight-tube can be inserted between the sample and the X-ray detector to reduce the air scattering and absorption. The detector used is a fast acquisition CMOS based 2D X-ray detector of PILATUS detector family (Kraft, Bergamaschi et al. 2009).

To illustrate the capability of the GISAXS technique to characterize the self-assembled nanoparticle monolayers we use an example of iron oxide nanoparticles (Siffalovic, Majkova et al. 2007). The Fig. 4a shows the SEM image of a self-assembled array of iron oxide nanoparticles. The inset of Fig. 4a shows the Fourier transform of SEM micrograph with partially smeared-out spots corresponding to the hexagonal arrangement. The smearing-out is due to mutually misaligned nanoparticle domains originating from finite nanoparticle size dispersion which is in sharp contrast to natural atomic crystals. The Fig. $4 a$ and $4 \mathrm{~b}$ show the measured and simulated GISAXS pattern, respectively. The characteristic side maxima located at the $q_{y} \approx \pm 0.82 \mathrm{~nm}^{-1}$ are the "finger prints" of the self-assembly in the nanoparticle array. In the first approximation, the mean interparticle separation can be estimated from 
the side maximum position in the reciprocal space as $\Delta \approx 2 \pi / q_{y} \approx 7.7 \mathrm{~nm}$. This simple estimation is valid only for a slowly varying nanoparticle form-factors within the kinematic BA. A precise fitting of the measured GISAXS data using the full DWBA theory can provide further information on the nanoparticle size and size dispersion as well as their correlation length (Lazzari 2002). The Fig. 4d shows a line cut extracted from the measured GISAXS pattern with the corresponding fit. The fitted nanoparticle diameter was $6.1 \pm 0.6 \mathrm{~nm}$ and the lateral correlation length in the nanoparticle array was $87 \mathrm{~nm}$. It has to be noted that colloidal nanoparticles are covered by a surfactant shell to avoid their spontaneous agglomeration in colloidal suspensions. In the case of Fe-O nanoparticles discussed above, oleic acid and oleylamine were used. A GISAXS pattern fitting provides basic information on the metallic-like nanoparticle core size while the organic shell is rather invisible for Xrays. On the other hand, the positions of the side maxima in the GISAXS pattern are always connected with the interparticle distance which is affected by the surfactant shell. This example clearly demonstrates the ability of GISAXS technique to extract main nanoparticle parameters in the self-assembled arrays. The main advantage is that the GISAXS technique does not require any specific sample environment conditions such as vacuum nor special sample preparation. On the other hand it can be applied even in very aggressive environments such as UV/ozone reactor (Siffalovic, Chitu et al. 2010). Moreover, a rapid GISAXS data acquisition in millisecond range can be used for a real-time in-situ probing of nanoparticle reactions and self-assembly processes (Siffalovic, Majkova et al. 2008).

\section{Nanoparticle self-assembly at liquid/air interfaces}

In the last ten years we have seen a tremendous progress in the colloidal nanoparticle chemistry (Feldheim 2002; Nagarajan 2008; Niederberger and Pinna 2009). The refined chemical synthesis routes can produce large quantities of highly monodisperse nanoparticles in colloidal solutions with the size dispersion below $10 \%$ (Park, An et al. 2004). The low nanoparticle dispersion is the "holy grail" of the large-scale nanoparticle selfassembly (Pileni 2005). Being able to prepare nanoparticles with zero size dispersion, we could fabricate genuine artificial nanoparticle crystals competing with natural ones in terms of the structure perfection and long-range order. However the finite nanoparticle size dispersion permits only a limited extent of ordering in nanoparticle self-assembled arrays. A typical model for description of the real nanoparticle assemblies is the paracrystal model (Hosemann and Bagchi 1962; Guinier 1963). Here a paracrystal order parameter summed up with the mean interparticle distance defines degree of the array perfection.

The colloidal nanoparticle solutions can be applied on a solid substrate directly or in two steps, utilizing liquid surface for self-assembly with a subsequent transfer onto a solid substrate. Drop casting followed by solvent evaporation is an example of the former method (Chushkin, Ulmeanu et al. 2003) that proved to be successful e.g. for preparation of largearea self-assembled arrays of noble metal nanoparticles with the diameter of a few tens $\mathrm{nm}$. In addition to the nanoparticle size, surfactant type affects the self-assembly as well. For smaller nanoparticles, such as those presented in this chapter with the diameter below $10 \mathrm{~nm}$, a direct application of the colloidal nanoparticle solutions on solid substrate produces only locally well assembled regions but is not suitable for large-area nanoparticle depositions. Here, the latter above mentioned method is promising as it will be shown later. The GISAXS technique can be employed to track the nanoparticle assemblies in rapidly 
drying colloidal solution at solid surfaces (Siffalovic, Majkova et al. 2007). We used the focused X-ray beam to map the nanoparticle self-assembly at arbitrary selected position within the colloidal drop. The Fig. 5 shows the three typical GISAXS patterns.
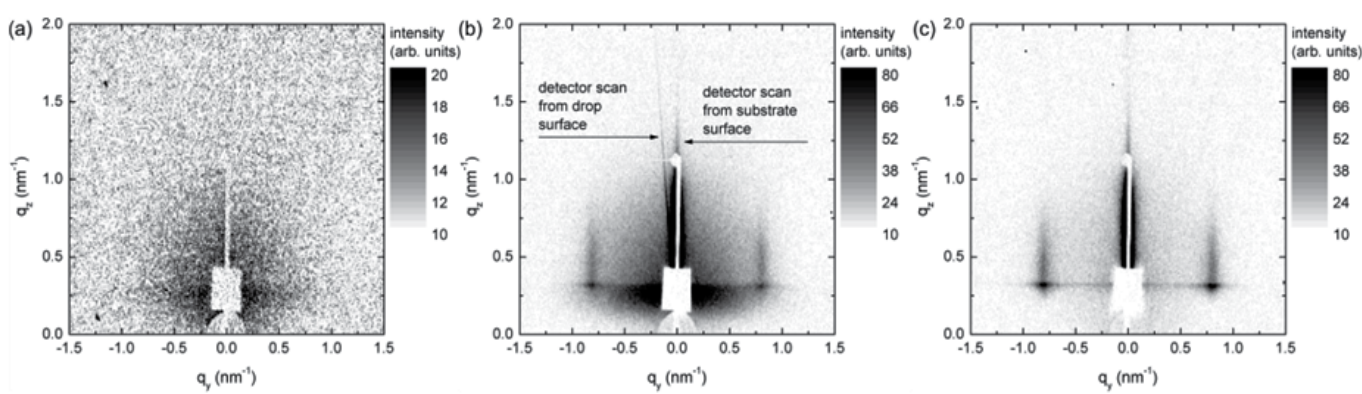

Fig. 5. The GISAXS pattern recorded from a drying colloidal Fe-O nanoparticle drop at three different stages: a) directly after drop casting, b) intermediate phase. c) dried colloidal drop.

The Fig. 5a shows the GISAXS pattern directly after application of a colloidal Fe-O nanoparticle solution onto silicon substrate. The GISAXS pattern does not show any maxima typical for self-assembled nanoparticle layers. The visible scattering in the GISAXS pattern is characteristic for a diluted nanoparticle solution and can be described by the nanoparticle form-factor. The Fig. $5 \mathrm{~b}$ shows the intermediate state when the X-ray beam partially passes through the colloidal drop surface. The scattering streaks originating from interfaces also called "detector scans" are visible. The first one can be attributed to the scattering from the substrate surface and the second one originates from the colloidal drop surface. The angle between the two detector streaks directly maps the angle between the normal of substrate surface and the normal of the probed colloidal drop surface. The side maxima belong to the already dried self-assembled areas. The Fig. $5 c$ shows the final GISAXS pattern after the colloidal solution is completely evaporated. The interparticle distance of final nanoparticle assembly are clearly manifested in the GISAXS pattern by the side maxima.

The spatially static GISAXS technique can track the nanoparticle assembly only in one selected probing volume within the evaporating colloidal drop. In order to monitor various probe volumes inside the colloidal nanoparticle drop during the self-assembly process we introduced a scanning GISAXS technique. The scanning GISAXS method is based on the fast vertical or horizontal scanning of the evaporating colloidal drop by the probing X-ray beam (Siffalovic, Majkova et al. 2008). The sketch of the scanning GISAXS technique is shown in Fig. 6a. The colloidal drop composed of iron oxide nanoparticles dispersed in toluene was applied onto silicon substrate located on a vertically scanning goniometer. As the evaporating drop was gradually scanned across the incoming X-ray beam we continuously recorded X-ray scattering from three different drop zones. In the zone Z0 the X-ray beam passed above the evaporating drop. These data were used for the background correction. In the zone Z1 we recorded exclusively the $\mathrm{X}$-ray scattering originating from the drying drop surface and drop interior. In the zone Z2 we additionally detected the X-ray scattering coming from the substrate surface. The Fig. $6 \mathrm{~b}$ shows the line cuts extracted from the GISAXS frames taken in zone Z1 corresponding to the three different stages of the colloidal drop evaporation process: 1.) directly after drop casting, 2.) intermediate state, and 3.) final state characterized by the complete solvent evaporation. It is important to notice that the 
experimental data for all three evaporation stages can be fitted solely using the nanoparticle form-factor function. According to the eq. (4) the interference function is constant in this case, i.e. $S(\vec{q})=1$.

a)

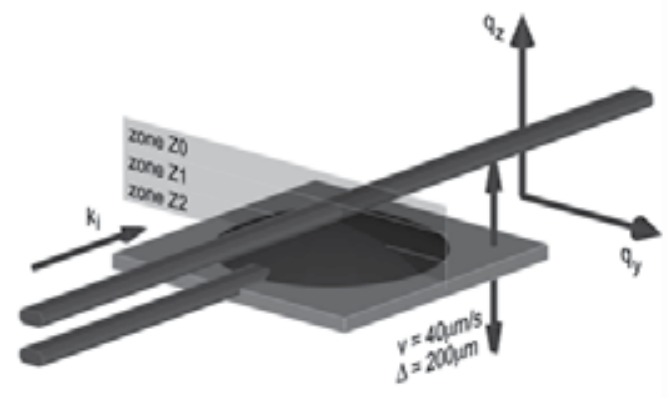

b)

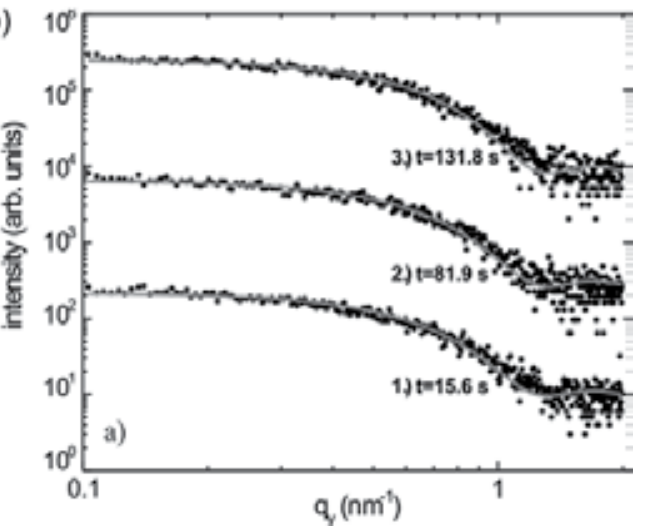

Fig. 6. a) The scheme of the GISAXS scanning technique. b) The GISAXS pattern line cuts at the critical exit angle for the three different stages of the colloidal Fe-O nanoparticle drop evaporation.

This means that the nanoparticles do not create self-assembled domains at the evaporating drop surface or in its volume at any time that suggests the origin of the nanoparticle selfassembly to be located at the three-phase boundary as predicted for a drying drop of dispersed particles (Deegan, Bakajin et al. 1997). The scanning GISAXS technique clearly demonstrates the ability to track the nanoparticle self-assembly process in real-time with millisecond time resolution.

As mentioned above, colloidal nanoparticles are usually terminated by surfactant molecules to avoid spontaneous agglomeration in colloidal suspensions. The nanoparticles with hydrophobic termination allow self-assembly at liquid/air interfaces and formation of Langmuir films in the form of simple 2D systems (Ulman 1991). Controlling the surface pressure by changing the nanoparticle layer area and the temperature of the subphase, we can produce large-area and homogenous self-assembled nanoparticle layers. The electron microscopy techniques including SEM, TEM or scanning probe techniques (AFM, STM) cannot be utilized to monitor the nanoparticle self-assembly at liquid/air interface. The visible/UV optical microscopy and Brewster angle microscopy are limited in resolution due to diffraction limit (Born and Wolf 1999). For a certain kind of metal and metal oxide nanoparticles exhibiting plasmonic properties $(\mathrm{Au}, \mathrm{Ag}, \mathrm{Al}, \mathrm{Cu})$ the interparticle distance can be indirectly monitored by the energy shift in localized surface plasmon resonance due to the dipole-dipole coupling of excited plasmons in the self-assembled nanoparticle arrays (Rycenga, Cobley et al. 2011). On the other hand the GISAXS technique can be employed to directly monitor the interparticle distance in self-assembled arrays directly in the Langmuir trough. The laboratory GISAXS setup shown in Fig. 3 was used to record the GISAXS patterns of Ag nanoparticles $(6.2 \pm 0.7 \mathrm{~nm})$ directly in the Langmuir trough. The GISAXS patterns of self-assembled Ag nanoparticles with oleic acid as surfactant at the surface pressures of $16 \mathrm{mN} / \mathrm{m}$ and $26 \mathrm{mN} / \mathrm{m}$ are shown in Fig. $7 \mathrm{a}$ and Fig. $7 \mathrm{~b}$, respectively. 

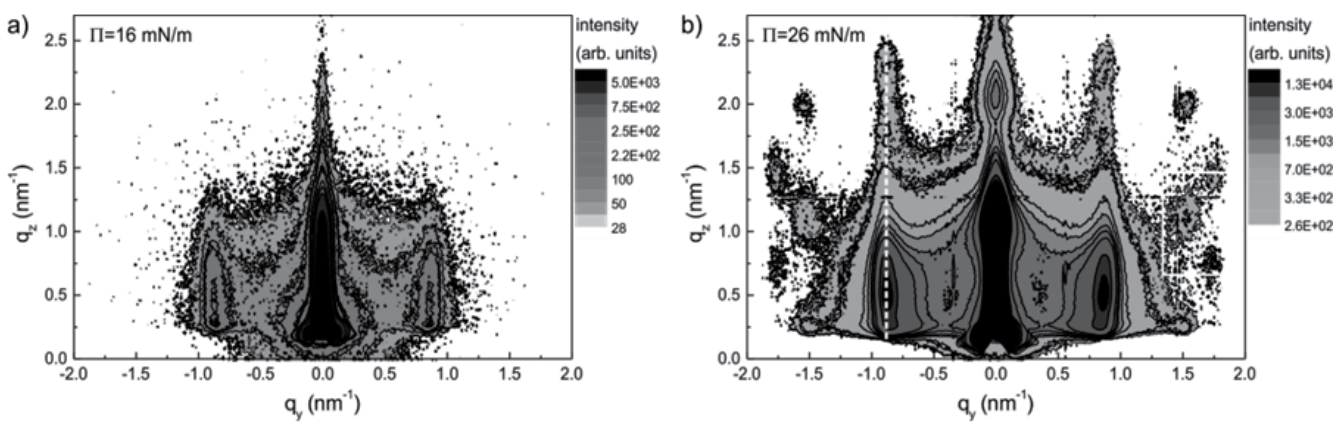

Fig. 7. The GISAXS patterns of self-assembled Ag nanoparticle Lagmuir films at surface pressure a) $16 \mathrm{mN} / \mathrm{m}$ and b) $26 \mathrm{mN} / \mathrm{m}$.

The surface pressure of $16 \mathrm{mN} / \mathrm{m}$ corresponds to a closed nanoparticle monolayer on the water surface. The interference function produces two symmetrical side maxima at $q_{y} \approx \pm 0.87 \mathrm{~nm}^{-1}$ (truncation rods) corresponding to the average interparticle distance of $7.2 \mathrm{~nm}$. The higher order side maxima are absent due to the short exposition time. The twodimensional nanoparticle monolayer has a constant interference function in the $q_{z}$ direction where the modulation visible on the truncation rods is produced solely by the nanoparticle form-factor (Holý, Pietsch et al. 1999). At the surface pressure of $26 \mathrm{mN} / \mathrm{m}$, the second nanoparticle layer forms and changes the observed GISAXS pattern (Vegso, Siffalovic et al. 2011). The newly formed nanoparticle vertical correlation perpendicular to the Langmuir film plane results in the modulation of the observed truncation rod depicted by the dashed white line in Fig. $7 \mathrm{~b}$. It can be shown that the modulation along the truncation rod is associated with the second nanoparticle layer laterally shifted in analogy with the "AB stacking" in solid state crystals (Kittel 2005). The presence of the second layer can be verified also by distinct second order maxima in Fig. $7 \mathrm{~b}$. The presented GISAXS results show the possibility to study not only the lateral but also the vertical nanoparticle correlations in 3D nanoparticle assemblies that is due to the ability of GISAXS to inspect non-destructively buried layers and interfaces. This useful feature of the GISAXS technique to study the buried vertical correlations of interfaces was already applied in studies of multilayered thin films (Salditt, Metzger et al. 1994; Siffalovic, Jergel et al. 2011).

Recently we have performed in-situ real-time studies of compression and decompression of Ag nanoparticle Langmuir films. We were interested in the correlation between the macroscopic elastic properties of nanoparticle layers and microscopic layer parameters like the interparticle distance. As a convenient measure of macroscopic elastic properties we use the surface elastic modulus defined as (Barnes, Gentle et al. 2005)

$$
E=-A\left(\frac{\partial \Pi}{\partial A}\right)_{T}
$$

Here $\Pi$ is the measured surface pressure of the nanoparticle layer with the area $A$ at a constant subphase temperature T. The Fig. 8 shows the evaluated side maximum position along the $q_{y}$ direction in the GISAXS reciprocal space map similar to the one shown in Fig. 7a. 


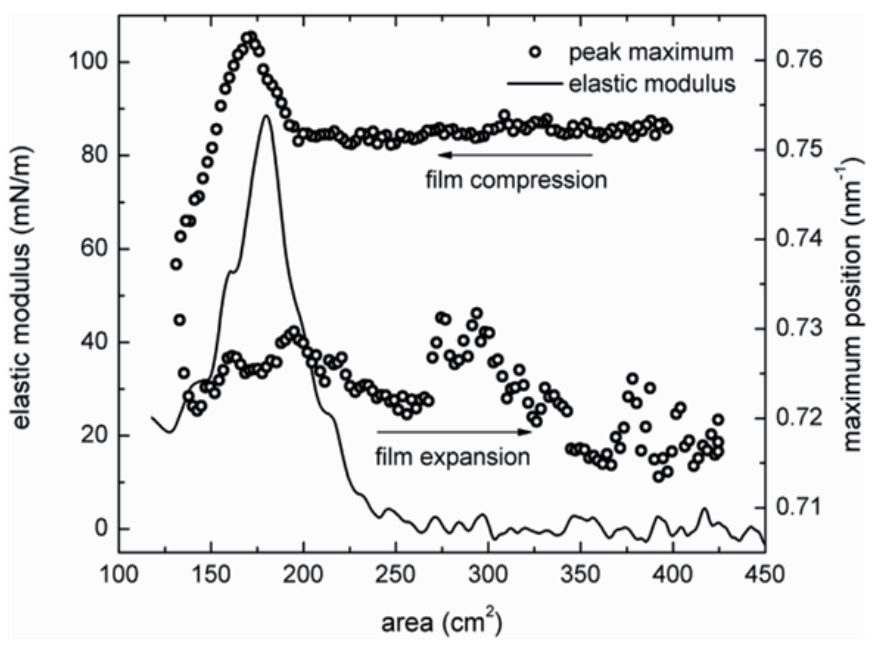

Fig. 8. The evaluated GISAXS peak maximum position and the surface elastic modulus of the Ag nanoparticle layer at water/air interface as a function of the layer area.

After spreading the nanoparticle solution onto the water subphase, the nanoparticles assemble into small clusters with hexagonal ordering that has been identified by independent ex situ experiments (to be published). Increasing the surface pressure by reducing the layer area results in the formation of a continuous monolayer without a change of the interparticle distance. This compression stage is characterized by a constant elastic modulus as the isolated nanoparticle clusters are joining into larger entities. At surface area of approximately $250 \mathrm{~cm}^{2}$ we observe an increase in the elastic modulus peaking at the area of $180 \mathrm{~cm}^{2}$. This stage can be associated with the densification of the nanoparticle layer accompanied by the nanoparticle rearrangements along the individual cluster boundaries and cluster coalescence. At the maximum of surface elastic modulus we observe also a slight compaction of the nanoparticle layer at nanoscale indicated by the change of the interparticle distance. This phase ends up with a compact nanoparticle layer. A further compression of the nanoparticle layer results in the formation of a second nanoparticle layer that induces a sudden drop in the elastic modulus and significant release of the mean interparticle distance. The nanoparticles forming the second layer create vacancies in the first one that is accompanied by deterioration of the order in the first nanoparticle layer. In this case the paracrystal model of the nanoparticle layer predicts a shift of the maximum to lower $q_{y}$ values in the reciprocal space (Lazzari 2009) that was confirmed by this experimental observation. After the decompression the interparticle distance in the nanoparticle layer does not relax to the initial value. It has to be noted that the second layer formation and tendency to form 3D ordered nanoparticle assemblies was demonstrated here for Ag nanoparticles with oleic acid as surfactant, however, other types of metallic nanoparticles with other type of surfactant may behave differently. This example shows the benefit of GISAXS technique to precisely monitor microscopic parameters of the nanoparticle assemblies prior to the deposition onto solid substrates that will be discussed in the following section. 


\section{Transfer of self-assembled layers from liquid onto solid surfaces}

In the previous section we discussed the formation of nanoparticle monolayers at water/air interface. The Langmuir film represented by self-assembled nanoparticle monolayer seems to be the most promising candidate for the homogenous deposition of large-area nanoparticle arrays. The two important questions are remaining. The first one is: "What is the suitable surface pressure for deposition and how to monitor it?" The second one is: "How to transfer the Langmuir film onto solid substrate with a minimum damage of the self-assembled layer?" In this section we try to give answers to them.

The first question was partially addressed in the previous section. We have shown the GISAXS technique gives a precise tool to monitor the monolayer formation at nanoscale. In Fig. 8 we showed the evolution of the interparticle distance with increasing surface pressure and we related formation of the second nanoparticle layer to a sudden drop in the observed surface elastic modulus. Additionally, we can track the evolution of the interference function in the $q_{z}$ direction. We showed that the interference along the $q_{z}$ axis is a constant function for the nanoparticle monolayer. A new vertical correlation between the two layers may appear with the monolayer collapse accompanying the formation of the second nanoparticle layer as discussed in the previous section. This transition is manifested in the modulation of the X-ray scattered intensity along the truncation rod. The Fig. $7 \mathrm{~b}$ shows the GISAXS pattern of the nanoparticle multilayer with a new peak formed along the first truncation rod (marked with dashed white line). For the nanoparticle monolayer, the intensity is at maximum at the critical exit angle, i.e. at the Yoneda peak. The formation of the second layer shifts the maximum intensity upward in the $q_{z}$ direction.

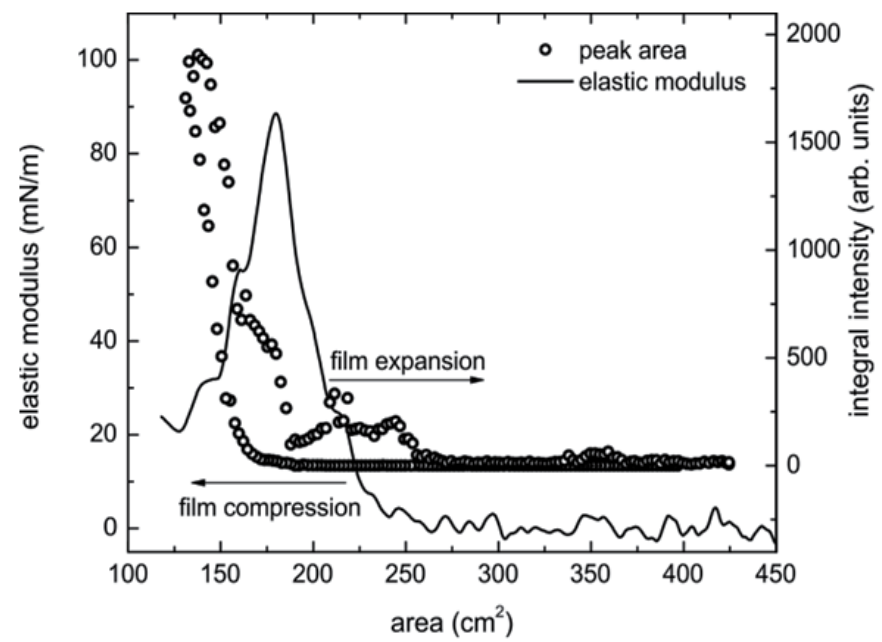

Fig. 9. The integral intensity of the first Bragg peak along the first truncation rod corresponding to the formation of a vertically correlated Ag nanoparticle multilayer as a function of the layer area.

The Fig. 9 shows the integral intensity of the newly formed Bragg peak along the first truncation rod corresponding to the vertically correlated nanoparticles as a function of the surface area. The GISAXS measurement clearly shows that the decrease in the elastic 
modulus is associated with the formation of the second nanoparticle layer. Moreover we observe a hysteretic behavior during the Langmuir film decompression associated with the irreversibility of the expanded nanoparticle layer that is also documented by the interparticle distance behavior shown in Fig. 8. After opening the barriers the nanoparticle layer does not relax into a monolayer but fragments into small islands still exhibiting a certain amount of nanoparticles in the second layer (see also further). The GISAXS measurements confirmed the assumption that the fully closed nanoparticle monolayer forms short before the monolayer collapse evidenced by a maximum in surface elastic modulus.

a)

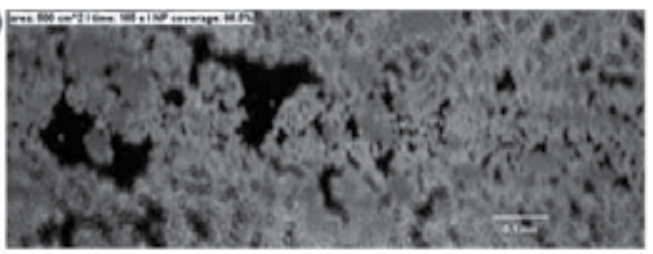

b)

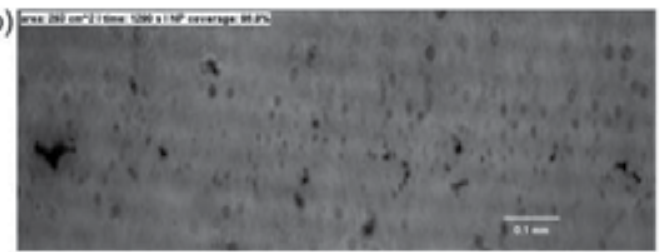

c)

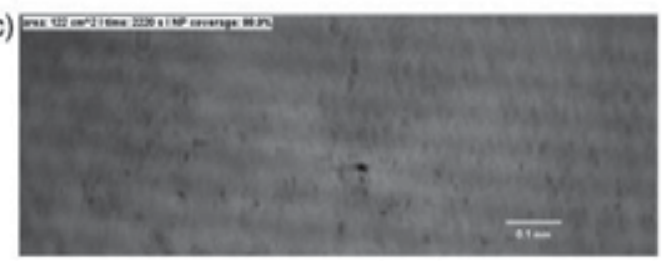

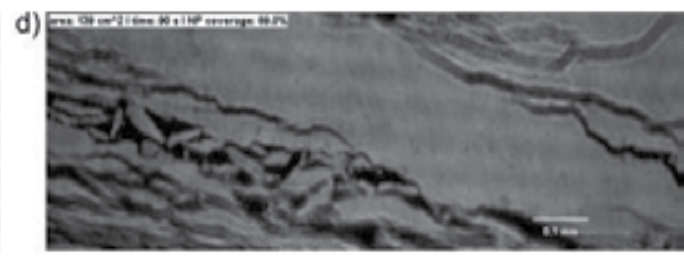
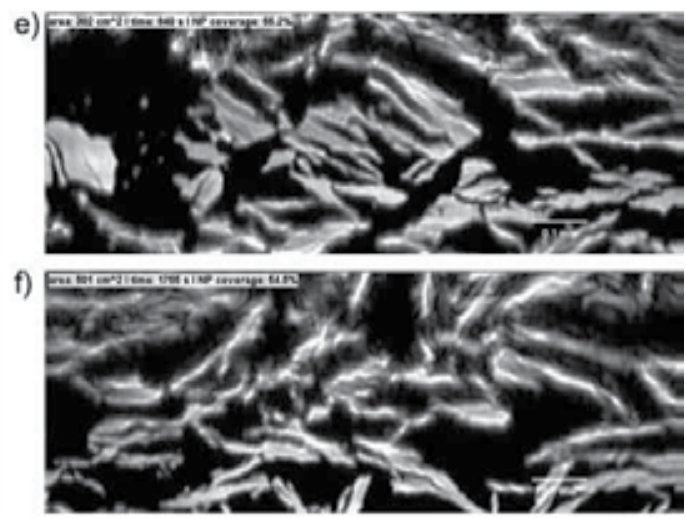

Fig. 10. The BAM images taken at surface areas a) $500 \mathrm{~cm}^{2}$, b) $293 \mathrm{~cm}^{2}$ and c) $122 \mathrm{~cm}^{2}$ taken during the Ag nanoparticle layer compression and BAM images at surface areas d) $139 \mathrm{~cm}^{2}$, e) $302 \mathrm{~cm}^{2}$ and f) $501 \mathrm{~cm}^{2}$ taken during the nanoparticle layer expansion.

The Brewster angle microscopy (BAM) provides further evidence of the nanoparticle monolayer formation at microscale (Henon and Meunier 1991). The laser based BAM provides much better contrast between the nanoparticle monolayer and water subphase than the conventional normal incident microscopy. The Fig. 10a)-10c) show three images taken during the nanoparticle layer compression and Fig. 10d)-10f show three images taken during the nanoparticle layer decompression. The nanoparticle layer was composed of surfactant terminated Ag nanoparticles with a core size of $6.2 \pm 0.7 \mathrm{~nm}$. The nanoparticle surfactant was oleic acid. The nanoparticle layer shows vacant areas in Fig. 10a). Decreasing the film area, we close the vacancies and a compact nanoparticle monolayer forms as shown in Fig. 10c). The subsequent expansion of the nanoparticle layer is accompanied by the generation of millimeters long cracks across the nanoparticle layer as shown in Fig. 10d). A further increase of the area available for the nanoparticle expansion leads to the disruption of nanoparticle layer into micrometer large needle-like clusters as shown in Fig. 10e) and Fig. 10f). The hysteretic behavior of the nanoparticle layer at microscale during the 
compression and decompression cycle is obvious and supports the interpretation of the GISAXS measurements. The Fig. 10 shows selected BAM images during the compression and expansion cycles. However we have recorded a full series of BAM images at 15 second time intervals during the compression cycle. Based on the BAM images we can calculate the average nanoparticle surface coverage based on the ratio between the bright areas that can be attributed to the nanoparticle layer and the black areas corresponding to the water subphase.

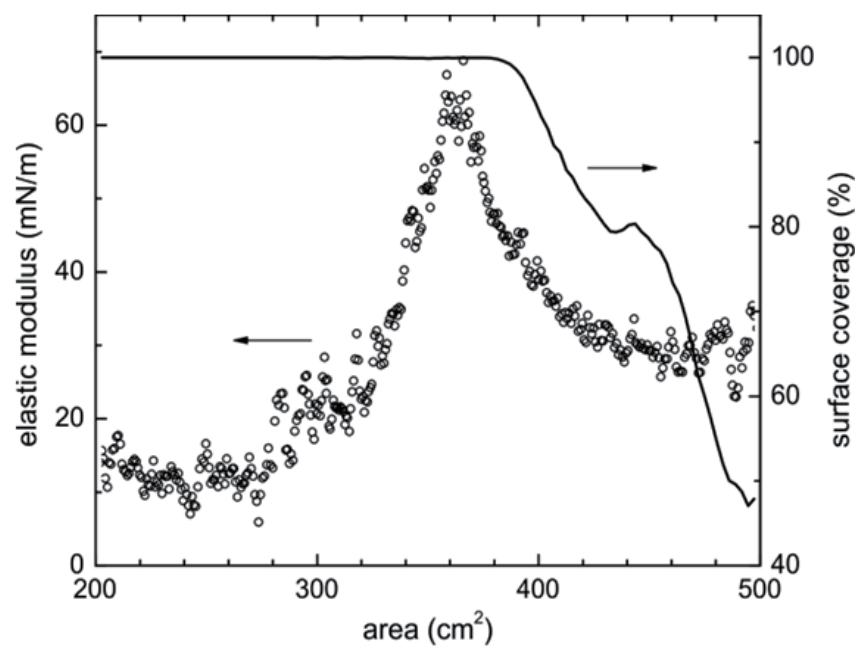

Fig. 11. The nanoparticle surface coverage based on BAM measurement along with the surface elastic modulus as a function of the Ag nanoparticle layer area during compression.

We have to keep in mind that the calculation is correct only at microscale as the nanoscale vacancies are invisible due to the BAM diffraction limit. The Fig. 11 shows the calculated nanoparticle surface coverage as a function of the film area. The graph shows also the calculated elastic modulus based on the measured nanoparticle layer surface pressure. The nanoparticle surface coverage reaches its maximum value of $100 \%$ short before the maximum in the film elastic modulus appears during the compression cycle. This is in a very good correlation with the GISAXS measurement that relates the nanoparticle monolayer collapse to the maximum in elastic modulus. The BAM measurements underestimate the nanometer-sized vacancies in the forming monolayer. This is the reason that the BAM indicate formation of nanoparticle monolayer already before the monolayer collapse. An alternative would be the imaging ellipsometry being able to track the nanoparticle layer formation at microscale more quantitatively than the BAM technique (Roth and et al. 2011).

In order to understand the formation of nanoparticle monolayer at nanoscale we deposited the nanoparticle layers on silicon substrates. The probes were deposited at different surface pressures by simply immersing the substrate into the nanoparticle covered water subphase. The selected areas of nanoparticle layers were studied by the non-contact atomic force microscopy (AFM) rather than the scanning electron microscopy as the latter one cannot provide the information on the layer height. 
a)

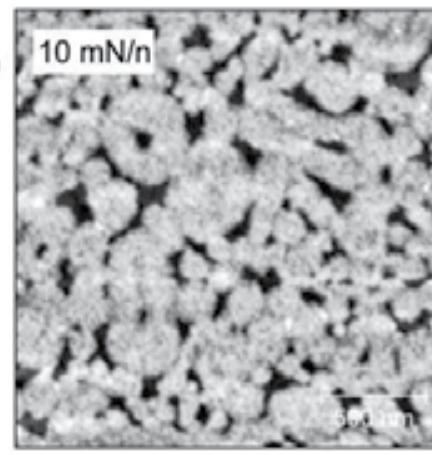

c)

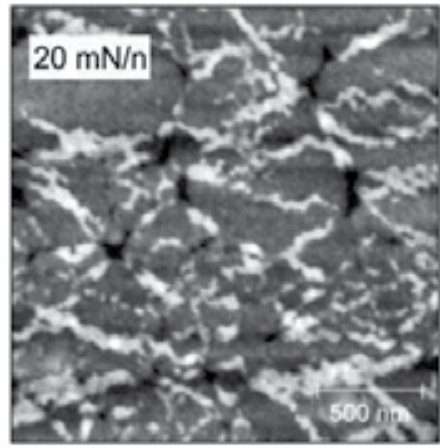

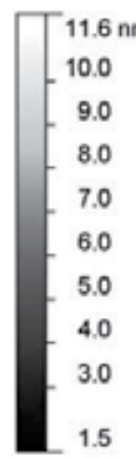
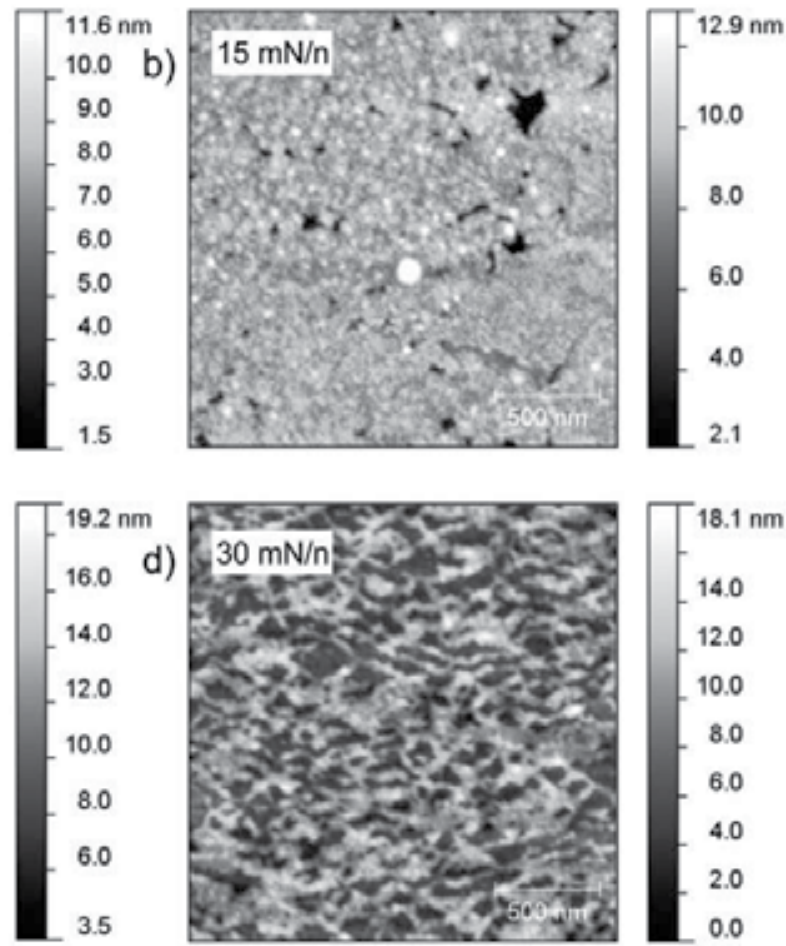

b)

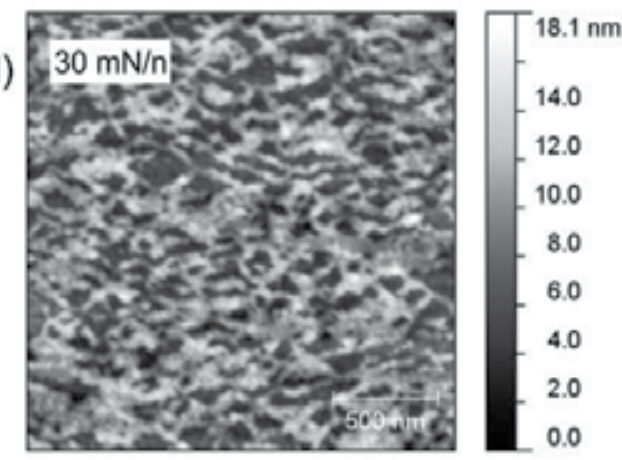

Fig. 12. The AFM images of Ag nanoparticle layers taken at the following surface pressures: a) $10 \mathrm{mN} / \mathrm{m}$, b) $15 \mathrm{mN} / \mathrm{m}, \mathrm{c}) 20 \mathrm{mN} / \mathrm{m}$ and d) $30 \mathrm{mN} / \mathrm{m}$.

The Fig. 12 shows the AFM images of Ag nanoparticle layers deposited at different surface pressures. The nanoparticle monolayer deposited at the $10 \mathrm{mN} / \mathrm{m}$ shown in Fig. 12a displays vacancies in the nanoparticle coverage. At this stage the isolated nanoparticle clusters are coalescing into a single nanoparticle layer. The Fig. $12 \mathrm{~b}$ shows a nanoparticle layer deposited at $15 \mathrm{mN} / \mathrm{m}$. This AFM image shows the nanoparticle clusters forming almost a closed nanoparticle monolayer. The maximum of the surface elastic modulus was reached shortly after $15 \mathrm{mN} / \mathrm{m}$. The AFM image shown in Fig. 12c deposited at the $20 \mathrm{mN} / \mathrm{m}$ clearly demonstrates the formation of the second nanoparticle layer after the monolayer collapse. The preferential sites for the formation of the second layer are located at the boundaries of the nanoparticle clusters. The final AFM image shown in Fig. 12d deposited at the surface pressure of $30 \mathrm{mN} / \mathrm{m}$ exhibits already a significant number of nanoparticles forming the second layer. The Fig. 13 shows calculated AFM height histograms of the nanoparticle layers deposited at different surface pressures. Only a single peak located at $6 \mathrm{~nm}$ corresponding to the height of monolayer is present up to the surface pressure of $15 \mathrm{mN} / \mathrm{m}$. For the sample deposited at $20 \mathrm{mN} / \mathrm{m}$ shown in Fig. 12c, appearance of a shoulder suggests onset of formation of a second nanoparticle layer. For higher surface pressures, the newly formed peak at $12 \mathrm{~nm}$ in the height histogram distribution gives clear evidence of the second nanoparticle layer. 


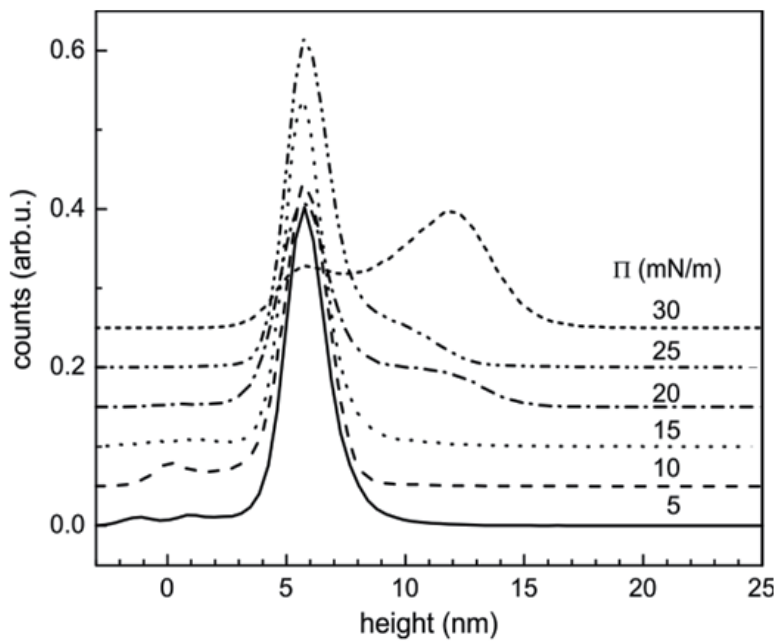

Fig. 13. The height histograms of the Ag nanoparticle layers deposited at different surface pressures obtained by analysis of the AFM images.

The number of nanoparticles occupying the second layer is steadily growing with the increasing surface pressure. At the surface pressure of $30 \mathrm{mN} / \mathrm{m}$ already more than $50 \%$ of the second nanoparticle layer was formed. The ex-situ AFM measurements provide important additional information to the in-situ GISAXS and BAM measurements. However we cannot rule out possible relaxations in the nanoparticle assemblies due to their transfer from the liquid to solid surface.

Based on the previous analyses we can conclude that the optimum deposition conditions for the nanoparticle monolayer deposition occur at the surface pressure slightly below the threshold pressure for the monolayer collapse. To achieve homogenous nanoparticle deposition over large areas of solid substrates, we modified the conventional LangmuirSchaefer deposition (Chitu, Siffalovic et al. 2010). The scheme of the deposition trough is shown in Fig. 14.

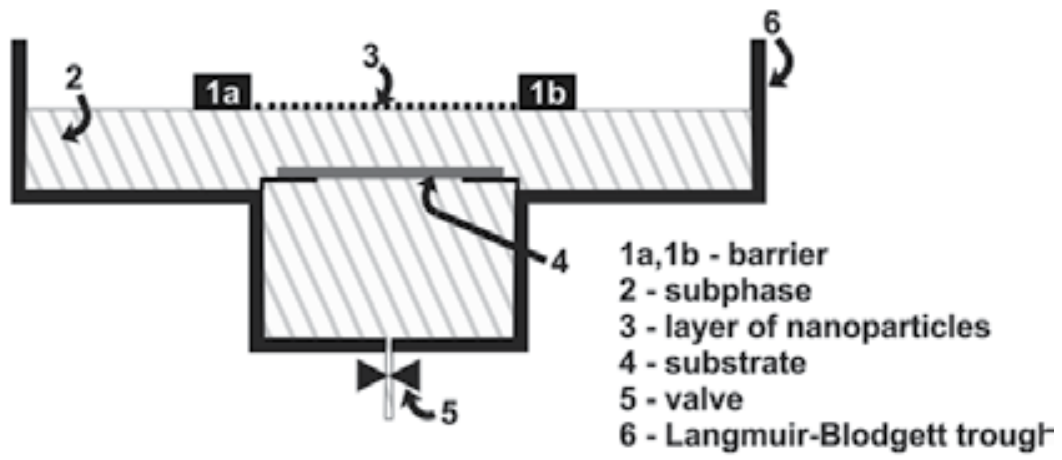

Fig. 14. The scheme of the modified Langmuir-Blodgett trough. 
Differently to the conventional Langmuir-Schaefer deposition, the deposited substrate is immersed into the subphase. After spreading the nanoparticles at the water subphase and adjusting the deposition surface pressure, the water is slowly removed by opening an outlet valve. The moving water/air interface will slowly cross the inclined substrate, depositing the nanoparticle array onto it. This deposition technique produces highly homogenous nanoparticle layers on large substrates. The Fig. 15a shows a silicon wafer with the total area of some $18 \mathrm{~cm}^{2}$ homogenously covered with an iron oxide nanoparticle monolayer $(6.1 \pm 0.6 \mathrm{~nm})$.

a)

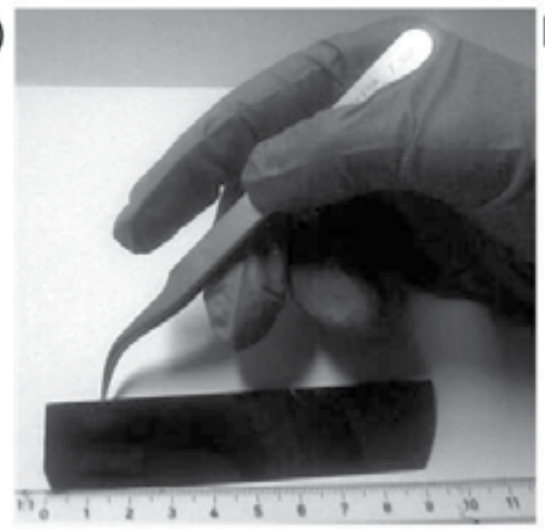

b)
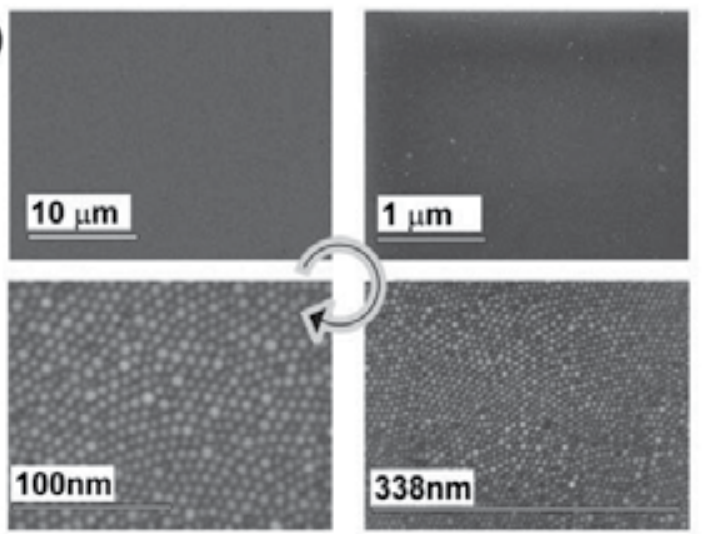

Fig. 15. a) Photograph of the homogenous Fe-O nanoparticle monolayer deposited onto silicon substrate. b) The SEM micrographs of a selected spot at the different magnifications.

To check the monolayer homogeneity we arbitrarily selected one spot at the deposited substrate and analyzed it with the SEM. The Fig. 15b shows four SEM micrographs of the selected spot at different magnification levels.
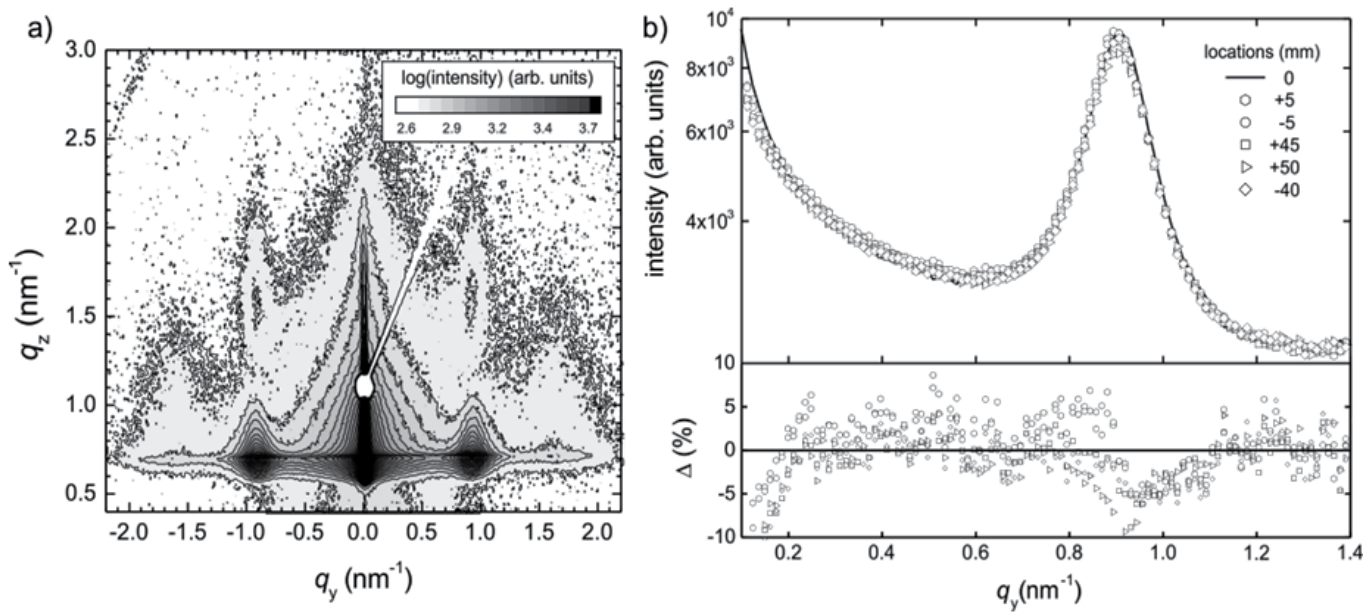

Fig. 16. a) The GISAXS pattern of the Fe-O nanoparticle monolayer. b) The extracted GISAXS line-cuts at the critical exit angle from six different locations at the substrate. 
At the lowest magnification we notice the absence of any cracks in the deposited monolayer. On the contrary the traditional vertical Langmuir-Blodgett deposition is forming a series of long cracks and is not suitable for large-scale deposition. At the highest magnification we can observe a dense hexagonally ordered layer of the iron oxide nanoparticles. The SEM is suitable for detailed analysis of the selected areas of the nanoparticle monolayer but is not convenient for a rapid screening across the large areas. We have already shown that the scanning GISAXS technique provides a fast probe of the nanoparticle order at nanoscale over macroscopic areas. The Fig. 16a shows the GISAXS reciprocal space map of an arbitrarily selected location at the substrate. The integral intensity and the position of the side maxima are the measure of the nanoparticle order in the X-ray probed area. Comparing the GISAXS patterns from the different locations at the substrate we obtain the information on the homogeneity of the deposited nanoparticle monolayer. The Fig. 16b shows six line cuts extracted from the GISAXS patterns measured at different locations. The differences between the measured curves are less than $\pm 5 \%$ that indicates a relatively high homogeneity of the deposited monolayer.

\section{Processing and application of the self-assembled nanoparticle layers}

In this section we focus on the issues connected with applications of deposited selfassembled nanoparticle layers. We discuss possibilities of removing the nanoparticle surfactant to increase the electrical conductivity of the nanoparticle layer as required for many applications. We address deposition of the nanoparticle layers onto thin membranes for sensor applications. We present also embedded self-assembled nanoparticle layers for organic solar cells and spintronic devices.

The surfactant molecules terminating the nanoparticles are inevitable for the synthesis and deposition of nanoparticles. However for many applications the electrical conductivity is required (Schmid 2010) while non-conductive organics is mostly used as surfactant. The surfactant molecules can be eliminated by the vacuum annealing, plasma etching, $\mathrm{UV} /$ ozone cleaning and many other techniques. In this section we analyze the impact of the $\mathrm{UV} /$ ozone cleaning on the Fe-O nanoparticle arrangement in self-assembled arrays. The $\mathrm{UV} /$ ozone cleaning is based on the reaction of UV light $(\lambda=6.7 \mathrm{eV})$ with the oxygen molecules producing the highly reactive ozone. The UV light initiates photo-dissociation of the surfactant molecules that further react with the ozone molecules and are removed from the nanoparticle surface. Also a direct reaction of the surfactant molecules with the ozone molecules also called ozonolysis removes the surfactant molecules from the nanoparticle surface. In our experiment we removed the surfactant molecules from the self-assembled monolayer of iron oxide nanoparticle with the core diameter of $6.1 \pm 0.6 \mathrm{~nm}$. The SEM micrographs along with the calculated nanoparticle pair correlation functions for the asdeposited sample and the sample processed in UV/ozone reactor are shown in Fig. 17a and Fig. 17b, respectively. For the as deposited nanoparticle monolayer the mean interparticle distance is given by the position of the first maximum in the pair correlation function that is $7.4 \mathrm{~nm}$. After removal of the surfactant molecules terminating the nanoparticles the mean interparticle distance decreased to $6.4 \mathrm{~nm}$. Moreover the nanoparticle array re-assembled into a labyrinth-like structure as shown by the SEM micrograph in Fig. 17b. This is very important for the electrical conductivity as the new nanoparticle assembly contains percolated conductive paths across the nanoparticle array. 

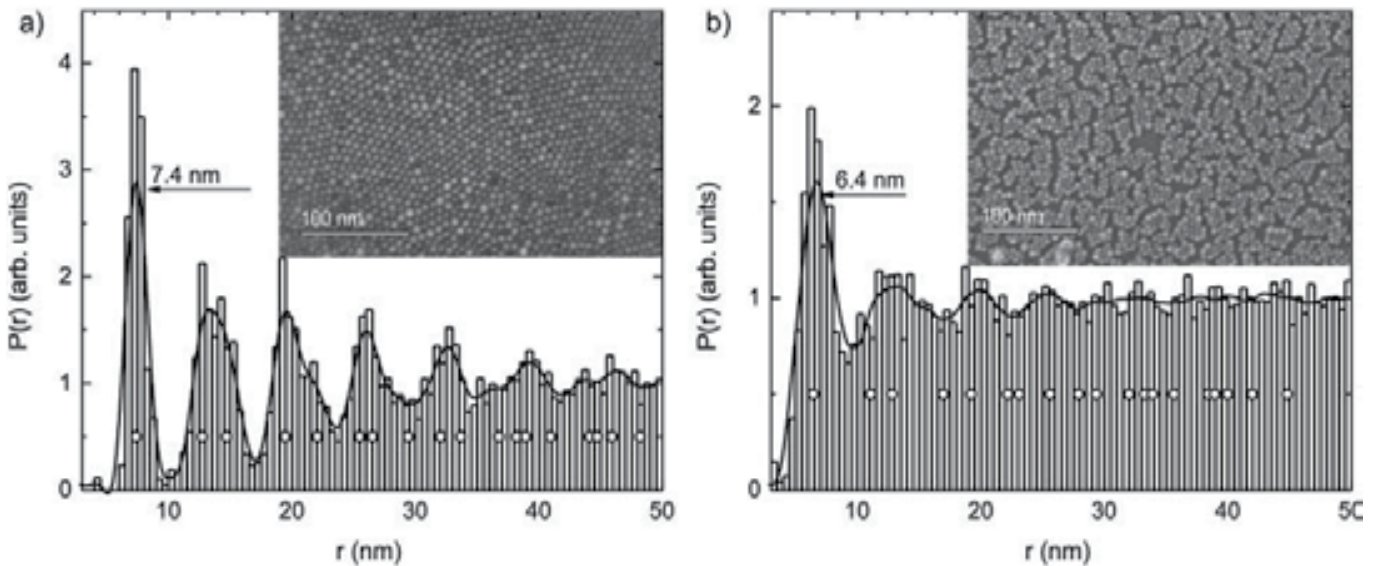

Fig. 17. The SEM micrograph and the corresponding pair correlation function for a) as deposited monolayer and b) monolayer treated in UV/ozone reactor.

We have demonstrated that the GISAXS technique is very suitable as an in-situ probe of the processes at nanoscale. We performed a time-resolved measurement of the nanoparticle reassembly directly in the UV/ozone reactor. The above described changes in the nanoparticle pair correlation function in the direct space are manifested here as changes of the interference function in the reciprocal space. The best way of extracting the shape of the nanoparticle interference function from the GISAXS pattern is its lateral line cut along the $q_{y}$ direction at the critical exit angle. The Fig. 18a shows the temporal evolution of such a line cut constructed from a series of time-resolved GISAXS frames.
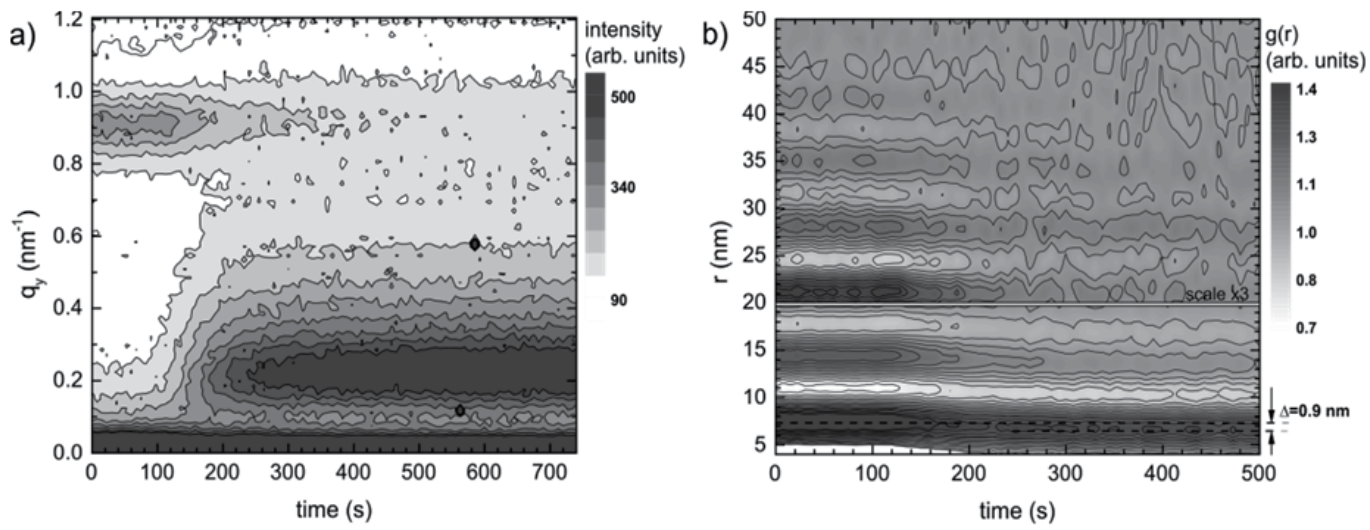

Fig. 18. a) The temporal evolution of the GISXAS line cut along the $q_{y}$ direction at the critical exit angle. b) The corresponding temporal evolution of the nanoparticle pair correlation function.

The initial as-deposited self-assembled state is characterized by a maximum located at $q_{y} \approx 0.9 \mathrm{~nm}^{-1}$. After switching on the UV/ozone reactor the maximum corresponding to the 
initial self-assembled state moves slightly to higher $q_{y}$-values and its integral intensity significantly drops. Simultaneously a new peak located at $q_{y} \approx 0.2 \mathrm{~nm}^{-1}$ develops. The new peak corresponds to the cluster formation that can be seen in the SEM micrograph in Fig. $17 \mathrm{~b}$. The measured GISAXS data can be recalculated into a time-resolved nanoparticle pair correlation function shown in Fig. 18b. This function reflects in detail the nanoparticle reassembly due to the removal of the surfactant molecules. The first maximum of the pair correlation function is shifted by some $0.9 \mathrm{~nm}$ to lower values within the first 200 seconds. This is in full agreement with the change of the interparticle distance calculated from the SEM micrographs in Fig. 17. This example demonstrates the possibilities of GISAXS to track fast temporal changes in the nanoparticle assemblies even in the strongly reducing environments.

Application of the conductive layers composed of metal oxide nanoparticles can be exemplified on the latest generation of the Fe-O nanoparticle-based gas sensors like $\mathrm{SO}_{2}$, $\mathrm{NO}, \mathrm{CO}, \mathrm{O}_{3}$ and $\mathrm{CH}_{4}$. The $\mathrm{NO}_{2}$ sensors are of primary importance for public security as they detect trace amounts of the explosives like EGDN, TNT, PETN, RDX, etc. A large nanoparticle-covered active surface for the gas adsorption is the main advantage when compared to the conventional thin films sensors. The Fig. 19a show a complete sensor based on the metal oxide nanoparticle multilayers (Luby, Chitu et al. 2011).

a)

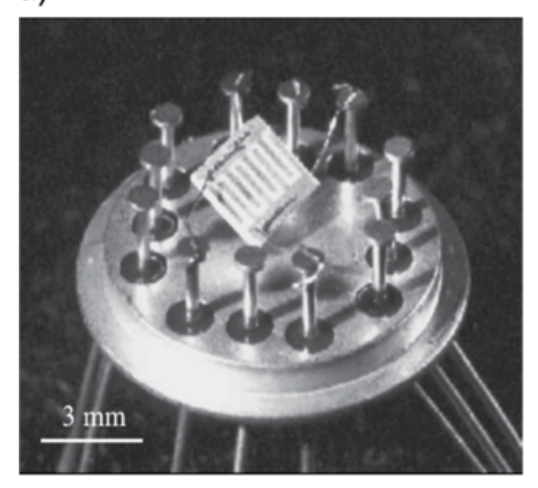

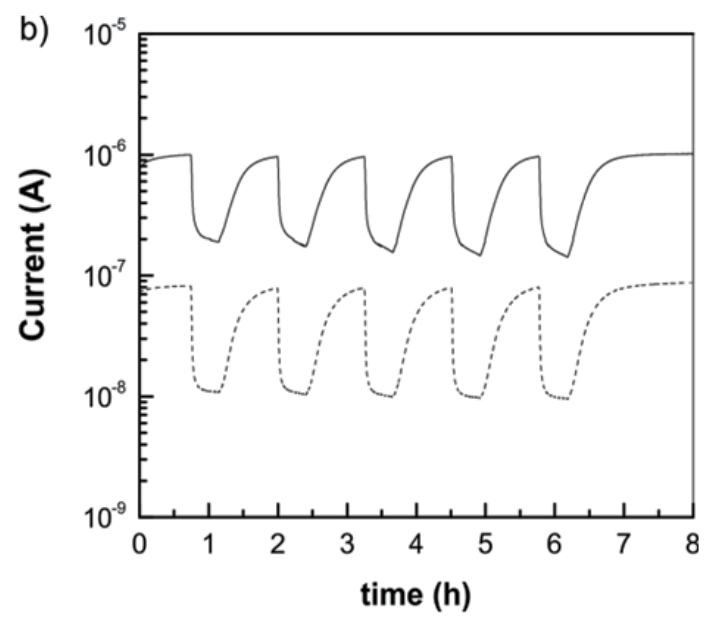

Fig. 19. a) The photograph of a nanoparticle gas sensor. b) The electrical response of the sensors fabricated with iron oxide (full line) or cobalt iron oxide (dashed line) nanoparticles. 
Visible is the heating meander as the sensor working temperature is $350^{\circ} \mathrm{C}$. The active area of the sensor is composed of seven monolayers of $\mathrm{Fe}_{2} \mathrm{O}_{3}$ or $\mathrm{CoFe}_{2} \mathrm{O}_{4}$ nanoparticles. The Fig. 19b shows the dynamic electrical response of the sensors to $5 \mathrm{ppm}$ of $\mathrm{NO}_{2}$ gas.

The nanoparticle layers exhibiting plasmonic properties in the visible and near-infrared parts of the solar spectra are potential candidates for the next generation of plasmonic solar cells (Catchpole and Polman 2008; Atwater and Polman 2010). The enhanced scattering cross-section of the plasmonic nanoparticles can efficiently trap the light into the active layer of the solar cells and to increase their external quantum efficiency.
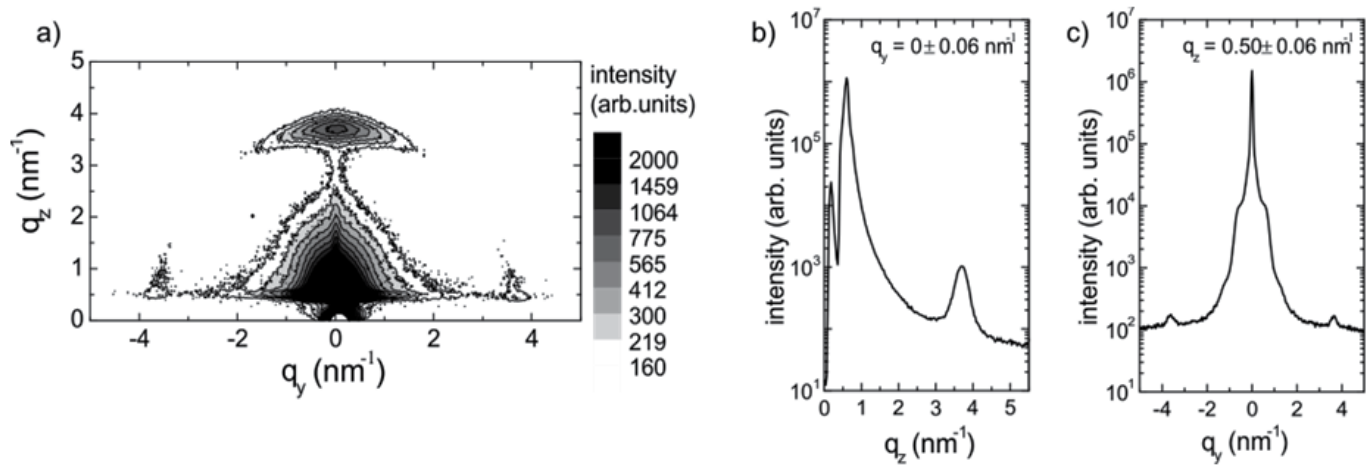

Fig. 20. a) The GISAXS reciprocal space map of the active layer deposited on Ag nanoparticle monolayer. The vertical b) and horizontal c) line-cuts across the GISAXS reciprocal space map.

The Ag nanoparticles fulfill both requirements for application in solar cells. In particular, they exhibit plasmon resonance in visible region and are highly electrically conductive. We deposited a monolayer of Ag nanoparticles $(6.2 \pm 0.7 \mathrm{~nm})$ at the ITO (indium tin oxide) transparent conductive layer supported on a glass substrate. Subsequently an organic active layer composed of polymer blend of P3HT (poly(3-hexylthiophene)) and PCBM (phenylC61-butyric acid methyl ester) of a $100 \mathrm{~nm}$ thickness was spin-coated on the nanoparticle monolayer. The Fig. 20a shows the GISAXS pattern of the final structure.

A prominent Bragg peak at $q_{z}=3.65 \mathrm{~nm}^{-1}$ originates from the molecular P3HT stacking with the inter-molecular distance of $1.7 \mathrm{~nm}$ and is clearly visible also in the vertical line cut in Fig. $20 \mathrm{~b}$ The nanoparticle correlation is visible as a small peak at $q_{y}=0.66 \mathrm{~nm}^{-1}$ in the Fig. 20c that corresponds to the mean interparticle distance of some $9.5 \mathrm{~nm}$. Here the GISAXS method provides the information on the correlations in the nanoparticle monolayer located at the buried interface hardly accessible by other analytical techniques. 
Another example is the embedded nanoparticle monolayer in the hybrid tunnel junction of novel spintronic devices (Siffalovic, Majkova et al. 2009). Here the surfactant shell is inevitable to provide the tunnelling effect. The Fig. 21a shows schematically the multilayer structure containing iron oxide nanoparticle monolayer. The first fabrication step is the vacuum deposition of a metallic layer forming the bottom electrode. The second step is the deposition of the nanoparticle monolayer that is overcoated by another vacuum deposited metallic layer in the final step. The Fig. $21 \mathrm{~b}$ shows the evolution of a line cut in the GISAXS pattern with the growing thickness of the metallic overlayer. The peak at $q_{y}=0.83 \mathrm{~nm}^{-1}$ marked with the dashed line corresponding to the nanoparticle layer can be seen throughout the entire deposition process. These examples demonstrate that the buried nanoparticle monolayer confined to the interface with a thin metallic film can be monitored using the GISAXS technique.

a)

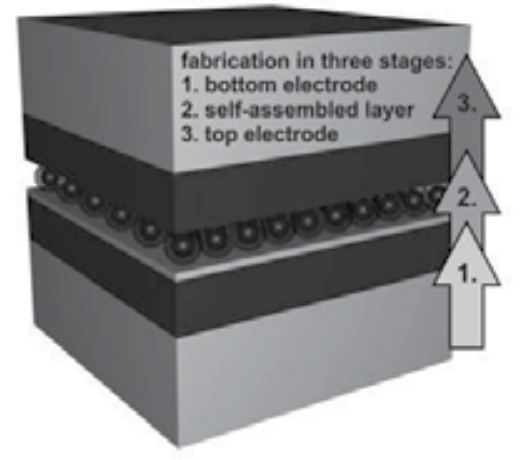

b)

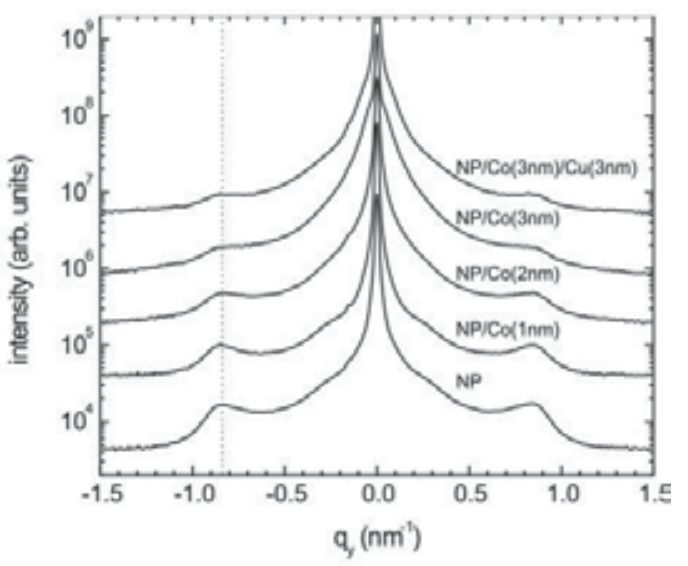

Fig. 21. a) A sketch of the spintronic structure that contains a Fe-O nanoparticle monolayer. b) Extracted line-cuts from the GISAXS reciprocal space maps at the critical exit angle in the different fabrication stages of spintronic structure.

The nanoparticle monolayers and multilayers can be deposited also on flexible membranes to be employed for monitoring mechanical properties like strain (Herrmann, Muiller et al. 2007). The principle of a strain sensor is based on a change of electrical current across the nanoparticle layer as a function of the applied mechanical stress that modifies the interparticle distance in the film and consequently the electrical resistivity. The sensitivity of the nanoparticle-based strain sensors is roughly by two orders of magnitude better than that of the conventional thin metallic film ones. We investigated the nanoscale response of the nanoparticle monolayer to the applied external stress (Siffalovic, Chitu et al. 2010). We deposited a monolayer composed of iron oxide nanoparticles $(6.2 \pm 0.7 \mathrm{~nm})$ onto a mylar foil ( $1 \mu \mathrm{m}$ thickness). The mylar foil was fixed in a stretching device for in-situ SAXS tensile stress measurements as shown in Fig. 22a. 
a)

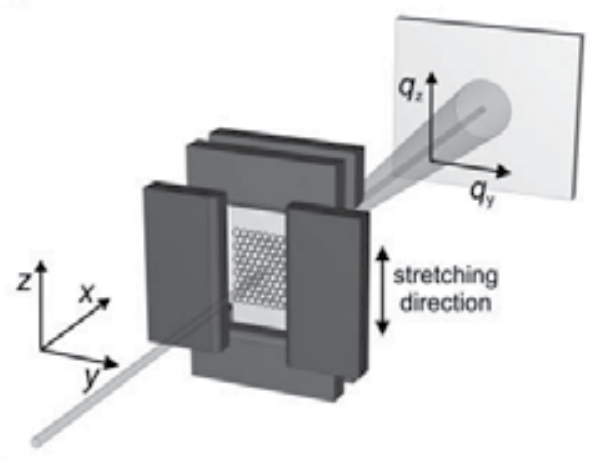

b)

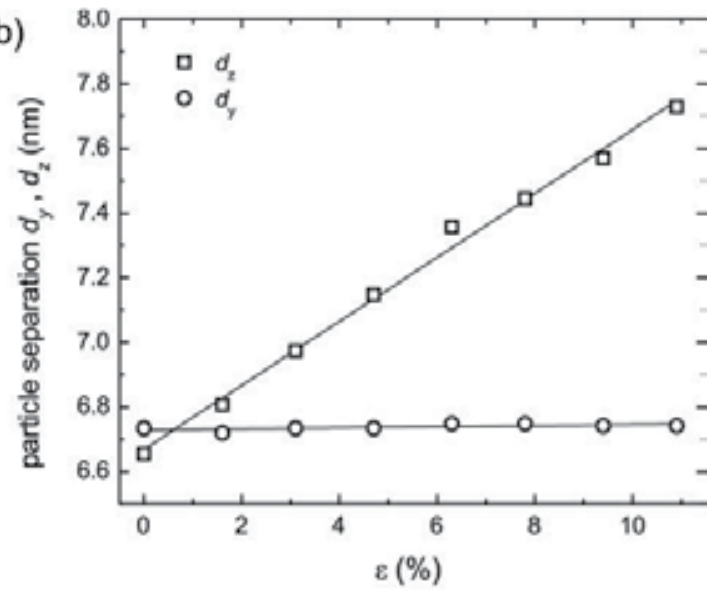

Fig. 22. a) Scheme of the experimental setup with an in-situ SAXS tensile stage. b) The evaluated interparticle separation as a function of the strain in two perpendicular directions.

The mylar foil was strained up to $11 \%$ in the $z$-direction and the SAXS patterns were recorded. Relying on them, the mean interparticle distance was evaluated in the applied stress direction and in the direction perpendicular to it. The results are shown in Fig. 22b. In the direction perpendicular to the applied stress the nanoparticle separation remained constant. However in the direction of the applied stress the interparticle distance followed linearly the measured foil strain. These measurements provide the test basis for the future strain sensors based on the nanoparticle layers.

In this section we included only a few of a large variety of practical applications of the nanoparticle monolayers. The nanoparticle deposition, eventual post-deposition processing of the nanoparticle layer and the test measurements of the macroscopic properties of interest are common for all these applications. The presented SAXS/GISAXS techniques offer an efficient and direct access to the nanoparticle arrangement within the final device.

\section{Conclusion}

The chapter provides an introductory guide to X-ray scattering studies of nanoparticle selfassembly processes at liquid/air and solid/air interfaces. It is primarily intended for graduate and post-graduate students but it is aimed also at other scientific community in the field addressing the issues of general interest. In particular, it shows the latest advances in the rapidly growing field of self-assembled nanoparticle layers. The X-ray scattering diagnostic technique was reviewed that provides an easy access even to buried nanoparticle assemblies. The main advantage of the $\mathrm{X}$-ray scattering analysis is the possibility to track technologically important processes connected with the nanoparticle self-assembly or reassembly in real time. The self-assembly process after colloidal drop casting and 
evaporation was described shortly while a detailed study of the self-assembly process at the liquid/air interface was the core of the chapter. This interface represents an ideal system for the nanoparticle assembling as the nanoparticles are confined to the interface but still keep translational mobility along it. The processes accompanying the formation of a nanoparticle monolayer and its transition to a multilayer were described in detail. Ideal deposition conditions for the nanoparticle monolayer formation were derived relying on the surface pressure and surface elastic modulus measurements. A modified Langmuir-Schaefer technique suitable for large-area deposition of nanoparticle arrays was presented. Selected applications of the deposited self-assembled layers were reviewed.

It has to be stressed that the colloidal nanoparticle self-assembly is a complex process resulting from an interplay between many factors where the nanoparticle type and size as well as the chemical composition of surfactant play a crucial role. Therefore none of the selfassembly techniques described in the chapter is generally applicable to any colloidal nanoparticle solution. It is also the reason why different techniques were presented with different types of nanoparticles.

It has to be also noted that in addition to the spontaneous nanoparticle self-assembly treated in this chapter of limited length, other approaches to assembling based on recent developments are of growing interest in the nanoparticle community. These include e.g. directed self-assembly of nanoparticles on pre-patterned substrates, chemically driven self-assembly, nanoparticle self-assembly stimulated by a magnetic or electro-magnetic field.

\section{Acknowledgment}

This publication is the result of the project implementation Center of Applied Nanoparticle research, ITMS code 26240220011, supported by the Research \& Development Operational Program funded by the ERDF. The support of Grant Agency VEGA Bratislava, project No. $2 / 0041 / 11$, is also acknowledged.

\section{References}

Atwater, H. A. and Polman, A. (2010). Plasmonics for improved photovoltaic devices. Nature Materials 9(3): 205-213.

Barnes, G., Gentle, I., et al. (2005). Interfacial science: an introduction. Oxford [u.a.], Oxford Univ. Press.

Born, M. and Wolf, E. (1999). Principles of optics: electromagnetic theory of propagation, interference and diffraction of light. Cambridge; New York, Cambridge University Press.

Catchpole, K. R. and Polman, A. (2008). Design principles for particle plasmon enhanced solar cells. Applied Physics Letters 93(19): 191113.

Chitu, L., Siffalovic, P., et al. (2010). Modified Langmuir-Blodgett deposition of nanoparticles - measurement of 2D to 3D ordered arrays. Measurement Science Review 10(5): 162-165. 
Chushkin, Y., Ulmeanu, M., et al. (2003). Structural study of self-assembled Co nanoparticles. Journal of Applied Physics 94(12): 7743-7748.

Daillant, J. and Gibaud, A. (2009). X-ray and neutron reflectivity : principles and applications. Berlin Heidelberg, Springer.

Deegan, R. D., Bakajin, O., et al. (1997). Capillary flow as the cause of ring stains from dried liquid drops. Nature 389(6653): 827-829.

Feigin, L. A., Svergun, D. I., et al. (1987). Structure analysis by small-angle X-ray and neutron scattering. New York [etc.], Plenum Press.

Feldheim, D. L. (2002). Metal nanoparticles: synthesis, characterization, and applications. New York [u.a.], Dekker.

Glatter, O. and Kratky, O. (1982). Small angle x-ray scattering. London; New York, Academic Press.

Guinier, A. (1963). X-ray diffraction in crystals, imperfect crystals, and amorphous bodies. San Francisco,, W.H. Freeman.

Guinier, A. and Fournet, G. (1955). Small-angle scattering of X-rays. New York,, Wiley.

Henon, S. and Meunier, J. (1991). Microscope at the Brewster-Angle - Direct Observation of 1st-Order Phase-Transitions in Monolayers. Review of Scientific Instruments 62(4): 936-939.

Herrmann, J., Muiller, K. H., et al. (2007). Nanoparticle films as sensitive strain gauges. Applied Physics Letters 91(18): 183105.

Holý, V., Pietsch, U., et al. (1999). High-resolution X-ray scattering from thin films and multilayers. Berlin; New York, Springer.

Hosemann, R. and Bagchi, S. N. (1962). Direct analysis of diffraction by matter. Amsterdam, North-Holland Publ. Comp.

Kittel, C. (2005). Introduction to solid state physics. Hoboken, NJ, Wiley.

Kraft, P., Bergamaschi, A., et al. (2009). Performance of single-photon-counting PILATUS detector modules. Journal of Synchrotron Radiation 16(3): 368-375.

Lazzari, R. (2002). IsGISAXS: a program for grazing-incidence small-angle X-ray scattering analysis of supported islands. Journal of Applied Crystallography 35: 406-421.

Lazzari, R. (2009). Grazing Incidence Small-Angle X-Ray Scattering from Nanostructures. In: X-ray and Neutron Reflectivity. J. Daillant and A. Gibaud, Springer Berlin / Heidelberg. 770: 283-342.

Luby, S., Chitu, L., et al. (2011). Oxide nanoparticle arrays for sensors of CO and NO2 gases. Vacuum In Press, Corrected Proof.

Michaelsen, C., Wiesmann, J., et al. (2002). Recent developments of multilayer mirror optics for laboratory x-ray instrumentation. X-Ray Mirrors, Crystals, and Multilayers Ii 4782: 143-151.

Müller-Buschbaum, P. (2009). A Basic Introduction to Grazing Incidence Small-Angle X-Ray Scattering. In: Applications of Synchrotron Light to Scattering and Diffraction in Materials and Life Sciences. M. Gomez, A. Nogales, M. C. Garcia-Gutierrez and T. A. Ezquerra, Springer Berlin / Heidelberg. 776: 61-89.

Nagarajan, R. (2008). Nanoparticles: synthesis, stabilization, passivation, and functionalization. Washington, DC, American Chemical Soc. 
Niederberger, M. and Pinna, N. (2009). Metal Oxide Nanoparticles in Organic Solvents Synthesis, Formation, Assembly and Application. Engineering Materials and Processes. London, Springer London.

Park, J., An, K., et al. (2004). Ultra-large-scale syntheses of monodisperse nanocrystals. Nature Materials 3(12): 891-895.

Pileni, M.-P. (2005). Nanocrystals forming mesoscopic structures. Weinheim, Wiley-VCH.

Renaud, G., Lazzari, R., et al. (2009). Probing surface and interface morphology with Grazing Incidence Small Angle X-Ray Scattering. Surface Science Reports 64(8): 255380.

Roth, S. V., Döhrmann, R., et al. (2006). Small-angle options of the upgraded ultrasmallangle x-ray scattering beamline BW4 at HASYLAB. Review of Scientific Instruments 77(8): 085106.

Roth, S. V. and et al. (2011). In situ observation of cluster formation during nanoparticle solution casting on a colloidal film. Journal of Physics: Condensed Matter 23(25): 254208.

Rycenga, M., Cobley, C. M., et al. (2011). Controlling the Synthesis and Assembly of Silver Nanostructures for Plasmonic Applications. Chemical Reviews 111(6): 3669-3712.

Salditt, T., Metzger, T. H., et al. (1994). Kinetic Roughness of Amorphous Multilayers Studied by Diffuse-X-Ray Scattering. Physical Review Letters 73(16): 2228-2231.

Schmid, G. (2010). Nanoparticles from theory to application. Weinheim, Wiley-VCH.

Siffalovic, P., Chitu, L., et al. (2010). Kinetics of Nanoparticle Reassembly Mediated by UVPhotolysis of Surfactant. Langmuir 26(8): 5451-5455.

Siffalovic, P., Chitu, L., et al. (2010). Towards strain gauges based on a self-assembled nanoparticle monolayer-SAXS study. Nanotechnology 21(38).

Siffalovic, P., Jergel, M., et al. (2011). GISAXS - probe of buried interfaces in multilayered thin films. In: X-Ray Scattering. C. M. Bauwens. New York, Nova Science Publishers.

Siffalovic, P., Majkova, E., et al. (2009). Fabrication and Characterization of Hybrid Tunnel Magnetoresistance Structures with Embedded Self-Assembled Nanoparticle Templates. Acta Physica Polonica a 115(1): 332-335.

Siffalovic, P., Majkova, E., et al. (2008). Real-Time Tracking of Superparamagnetic Nanoparticle Self-Assembly. Small 4(12): 2222-2228.

Siffalovic, P., Majkova, E., et al. (2007). Self-assembly of iron oxide nanoparticles studied by time-resolved grazing-incidence small-angle x-ray scattering. Physical Review $B$ 76(19).

Siffalovic, P., Vegso, K., et al. (2010). Measurement of nanopatterned surfaces by real and reciprocal space techniques. Measurement Science Review 10(5): 153-156.

Stribeck, N. (2007). X-ray scattering of soft matter. Berlin, Springer.

Ulman, A. (1991). An introduction to ultrathin organic films : from Langmuir-Blodgett to selfassembly. Boston [u.a.], Acad. Press.

Vegso, K., Siffalovic, P., et al. (2011). In situ GISAXS monitoring of Langmuir nanoparticle multilayer degradation processes induced by UV photolysis. physica status solidi (a): (accepted, in press). 
Wiesmann, J., Graf, J., et al. (2009). X-Ray Diffractometry with Low Power Microfocus Sources - New Possibilities in the Lab. Particle \& Particle Systems Characterization 26(3): 112-116.

Yoneda, Y. (1963). Anomalous Surface Reflection of X Rays. Physical Review 131(5): 2010. 


\title{
View on the Magnetic Properties of Nanoparticles $\mathrm{Co}_{m}(\mathrm{~m}=6,8,10,12,14)$ and $\mathrm{Co}_{6} \mathrm{O}_{\mathrm{n}}(\mathrm{n}=1-9)$
}

\author{
Jelena Tamuliené1, Rimas Vaišnoras², \\ Goncal Badenes ${ }^{3}$ and Mindaugas L. Balevičius ${ }^{4}$ \\ ${ }^{1}$ Vilnius Uinversity, Institute of Theoretical Physics and Astronomy, Vilnius, \\ ${ }^{2}$ Vilnius Pedagogical University, Vilnius, \\ ${ }^{3}$ Institut de Ciences Fotoniques ICFO, Barcelona, \\ ${ }^{4}$ Vilnius University, Vilnius, \\ 1,2,4 Lithuania \\ ${ }^{3}$ Spain
}

\section{Introduction}

Currently there are several potential applications for magnetic nanomaterials in medicine including magnetic resonance imaging contrast agents, magnetic-field-directed drug delivery systems, bio-toxin removal, gene therapy, and magnetic fluid hyperthermia. Cobalt nanoparticles are is one the most promising material for both technological applications and academic studies as model system how effects the nanoparticle size, shape, structure, and surface anisotropy on macroscopic magnetic response. The magnetic behaviour of Co nanoparticles reveals how the magnetic metal nanoparticles can be used to enhance the signal due to their magnetic resonance imaging.

Today it is very well known that in a paramagnetic material there are unpaired electrons, that are free to align their magnetic moment in any direction, while paired electrons by the Pauli Exclusion Principle are to have their intrinsic ('spin') magnetic moments in to opposite directions, causing their magnetic fields to cancel out. It implies, that in many cases, the magnetic properties of the Co nanoparticles are explained by the presence of unpaired electrons because the particles consist of an odd number of cobalt atoms. However, in experimental studies the number of atoms in the particle has never been mentioned only the description of their size and main structure along with their magnetic properties have been provided. It is not a surprise, because a magnetic behaviour of materials depends on their electron configuration that is strongly related with a geometrical structure, and on temperature.

The dependence of magnetic anisotropy energy on crystal symmetry and atomic composition is observed in both ferromagnetic bulk materials and thin films. Even the structural parameters such as the shape of particles or the inter-atomic distances, in some cases, are affected by the above dependence. The importance of the electronic structure of 
particles exhibits the dependence of magnetic anisotropy energy on a single-atom coordination. Current, experiments exhibited that the coercivity of some particles at $10 \mathrm{~K}$ increased from 640 to 1250 Oe while the particle size increased from 1.8 to $4.4 \mathrm{~nm}$. The saturation magnetization increases with decreasing of particle size. Pure $\mathrm{CoO}$ nanoparticles in the 4.5-18 nm exhibit a super-paramagnetic behaviour at room temperature, and a large orbital contribution to the magnetic moment at low temperatures was also observed.

It was mentioned, that an electronic structure of both the materials and particles is strongly related with the geometrical structure. However, there are some difficulties to identify the structure of a cobalt nanoparticle. The crystallinity was evidenced by the transmission electron microscope (TEM) indicating that Co particles sized around $4.7 \mathrm{~nm}$ are a wellcrystallized FCC. While the particles with the average diameter smaller than $4,7 \mathrm{~nm}$ are almost perfectly spherical. The lattice of Co nanoparticles with inter-planar distance of around $0.23 \mathrm{~nm}$ was obtained and explained that such crystalline structure could originate either from BCC cobalt particles observed along the [001] direction or due to Co-FCC particles since the lattice would be formed by two [002] perpendicular planes. Both a highresolution TEM and powder x-ray diffraction profiles reveal the presence of 8-15 nm diameter crystallites that are identified as hcp-Co, FCC-Co nanocrystals. S. Ram reports two crystalline phases of cobalt FCC and BCC structures, while S. P. Gubin and et al. report that hcp and FCC structures or their combination can be realized in Co nanoparticles. C. G. Zimmermann and et al. investigate Co nanoparticles the diameter of which is $13 \mathrm{~nm}$ and the variance of $4 \mathrm{~nm}$; the first four FCC rings were visible in the diffraction pattern. Hence, there is no evidence what a crystalline phase of cobalt is more preferable and it is difficult to define which structure type of $\mathrm{Co}$ is realized in nanoparticles. Theoretical investigations of the Co clusters are not complete. J. Guevara and et al. calculated those Co clusters that are part of FCC or BCC block without distortion of the initial geometry structure. In other works, the structural distortion of the above clusters was performed by moving one or several atoms along the main axis of the clusters, i.e. this operation does not change the symmetry if the configuration of the cluster belongs to a point group with a single main axis. Hence, we begin at the results of the investigation of the structure of the Co nanoparticles aiming to recognize the most important structure features influencing the magnetic properties of the Co nanoparticles.

Other very important results obtained are that the nanoparticle behaviour is influenced by the proximity of neighbouring particles, i.e. dipolar inter-particle interactions lead to the appearance of collective behaviour. Such a collective behaviour due to dipolar interactions has been observed in the low susceptibility measurements corresponding to a highly ordered fine particles system. Puntes and et al. observe that when the density of particles per unit area is higher than a determined threshold, the two-dimensional self-assemblies behave as a continuous ferromagnetic thin film. A weak interaction among the assemblies of the Co nanoparticle is obtained by Park and et al. and this assembly leads to hysteresis disappearance. We shortly explain why the assembly of Co nanoparticles leads to loosing of the particle magnetic properties and make predictions how to avoid the loosing.

One of the reasons of the above assembly of the particles is their stability that is an important factor for the particle application in technology. Small cobalt nanoparticles not only self-assemble, but also easily oxidize in the air and, as a consequence, loose their magnetic properties. Thus, Co nanoparticles need to be coated with organic surfactants 
aiming to prevent them from both irreversible aggregation and loosing of magnetic properties.

For coating of Co nanoparticle different materials such as graphite, nanoroads, nanocapsules and oxygen are used. The core-shell nanoparticles ( $\mathrm{Co}-\mathrm{CoO})$ are examined and, it is established, that the magnetic properties of these particle strongly depend on the plane coverage. The results reported demonstrate the essential role played by shells in stabilizing the magnetism of $\mathrm{Co}-\mathrm{CoO}$ nanoparticles. Few reports on the preparation and properties of pure $\mathrm{CoO}$ in bulk are due to difficulties to obtain the materials in pure form by simple methods. The particles are often contaminated with $\mathrm{Co}_{3} \mathrm{O}_{4}$ or $\mathrm{Co}$ metal. The greater stability of $\mathrm{Co}_{3} \mathrm{O}_{4}$ than $\mathrm{CoO}$ is also established.

Herein, we report on the several very important issues related to magnetic properties of Co nanoparticles such as:

1. What are electronic and geometric structure properties of pure and oxidized Co nanoparticles and how these properties change with the increase of the size of particle ;

2. Could Co nanoparticles consisting of the even number of atoms exhibit magnetic properties because their electronic structure is such that an uncompensated electronmagnetic-moment appears? What are the main reasons of the above appearance?

3. Some Co oxide particles exhibited magnetic properties and have large perspective to be used in electronics.

\section{Description of method}

The structural origin of clusters has been studied by using the generalized gradient approximation for the exchange-correlation potential in the density functional theory (DFT) as it is described by Becke's three-parameter hybrid functional, using the non-local correlation provided by Lee, Yang, and Parr. The DFT method is commonly referred to as B3LYP, - a representative standard DFT method. The 6-31G basis set has been used as well. The basis set was chosen keeping in mind relatively minimum computational costs. The structures of the investigated nanoparticles have been optimized globally without any symmetry constraint and by starting from various initial geometries which have been constructed according to a certain symmetry in order to determine the lowest energy structures of each cluster. The GAMESS and Gaussian program suites were used for all simulations here.

It is necessary to mention that there are different ways to theoretically investigate the magnetic properties of the materials. Aiming to exhibit why closed shell particles could be paramagnetic, we have chosen the most simple method to investigate magnetic properties of the Co nanoparticles. Hence, magnetizability (commonly known as susceptibility) was investigated. The magnetizability is the second-order response to an external magnetic field:

$$
\xi=\left.\frac{-\delta^{2} E(B)}{\delta B^{2}}\right|_{B=0}
$$

Where $\mathrm{E}$ is energy, $\mathrm{B}$ is an external magnetic field. 
When $\xi<0$, the induced magnetic moment is opposite to the applied field, i.e. the investigated materials are diamagnetic; while for paramagnetic materials the magnetizability is larger than zero $(\xi>0)$ in this case the induced magnetic moment enforces the magnetic field. Experimentally, magnetizability is often poorly determined or it is only known in the liquid or solid state, thus it is difficult comparisons between calculated and experimental results, while rotational g-tensors are known as precisely determined. However, a rotational g-tensor behaves in the same manner as magnetizability, with a near cancellation of large nuclear and electronic contributions in a large system.

A calculation of rotational $g$ tensors is closely related to that of magnetizabilities via:

$$
g=-4 \mathrm{~m}_{p}\left(\xi^{L A O}-\xi_{c m}^{d i a}\right) I_{n u c}^{-1}+\frac{1}{2 \mu_{N}} \sum Z_{k}\left(R_{K}^{T} R_{K} I_{3}-R_{K} R_{K}^{T}\right) I_{n u c}^{-1}
$$

where $\mathrm{m}_{\mathrm{p}}$ is the proton mass, $\xi^{L A O}$ is the magnetizability tensor calculated with London orbitals, $\xi_{c m}^{d i a}$ is the diamagnetic contribution to the magnetizability tensor calculated with conventional orbitals and the gauge origin at the centre of mass, and the sum of all nuclei with charges $Z_{K}$ and positions $R_{K}$, while $I_{\text {nuc }}$ is the moment-of-inertia tensor. Although not explored in a large number of studies, obtained theoretical results fit experimental. Hence, the above close relationship allows us to expect that our methods chosen that are well suited to the calculation of rotational $g$ tensors should also be well suited to the calculations of magnetizabilities. Moreover, this simple enough method is suitable to describe general magnetic properties of the investigated particles and to explain the results obtained.

The isotropic magnetizability of the most stable clusters was calculated by adopting quantum mechanical response theory and London atomic orbital to ensure both gaugeorigin independent results and fast basis set convergence by using Dalton program. The approach used allows us to calculate accurate magnetizability even for quite large molecules at a moderate cost of computing time. In this case, the B3LYP method with Ahlrichs-pVDZ basis set was used. These basis sets were obtained by optimizing the exponents and contraction coefficients in the ground state ROHF calculations. There are total 241 contracted functions in the basis mentioned. It is showed, that the isotropic magnezitability and its anisotropy are remarkably constant with respect to the basis set and close to the experiment. So, the performances obtained allow us to foresee how magnetic properties of the particles depend on their structures.

\section{Structure, stability and magnetic properties of $\mathrm{Co}_{m}(m=6,8,10,12,14)$ nanoparticles}

\subsection{Structure and stability}

Let us remember that magnetic properties of the materials are related with an electronic structure. The electronic structure is mostly geometrical-structure-depended. On the other, hand when the geometrical structure of a compound is know, it is possible to predict some properties of the compound. Thus, the first step to understand the nature of the magnetizability of the Co nanoparticles and why this property is shape- and size-depended is to investigate the geometrical structure. 
Let us remember that, there is an infinite number of possible surfaces which can be exposed for every crystal system. In practice, only a limited number of planes are found to exist in any significant amount. Thus, the attention was concentrated on the above surfaces, because it is possible to predict the ideal atomic arrangement for a given surface of a particular metal by considering how the bulk structure is intersected by the surface. It is necessary to remember that investigated nanoparticles consist of a small number of atoms, thus, it is not possible to obtain a very strict crystalline structure; the crystalline structure that is expected to be in the investigated Co nanoparticles was obtained on the basis of the symmetry of a bond and atom location in the planes.

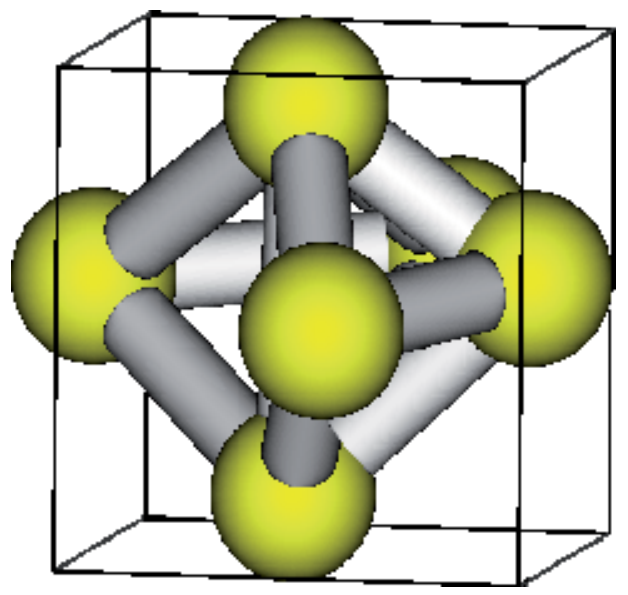

Fig. 1. $\mathrm{Co}_{6}$ nanoparticle located in the cubic cell.

It is necessary to mention that the structure of the $\mathrm{Co}_{4}$ particle was found, too. The results obtained indicate that $\mathrm{Co}_{4}$ is planar and a nice equilateral is formed. In the case of $\mathrm{Co}_{6}$ nanoparticle, we have the three-dimensional structure with a slightly disordered cubic symmetry. The structure was obtained after global optimization of the $\mathrm{D}_{4 \mathrm{~h}}$ isomer of a $\mathrm{Co}_{6}$ particle. It is important that each atom of the $\mathrm{Co}_{6}$ nanoparticle is possible to approximately be located in the centre of the plane of the cubic cell (Fig. 1). The three surfaces are obtained. So, this nanoparticle is the element of a FCC structure.

It is possible to see two planes of the $\mathrm{Co}_{8}$ nanoparticle (Fig. 2). The location of atoms on these planes as well as the symmetry of bonds allows us to predict that the element of FCC structure has been formed, too. This assumption is supported by following: i) each Co atom is four-fold coordinated; ii) the structural element of the $\mathrm{Co}_{6}$ particle is obtained (see the structure that form atoms 5, 6, 7, 8 or 1, 2, 3, 4 in Fig. 2). So, the element of FCC structure has also been obtained in the $\mathrm{Co}_{8}$ nanoparticle. The conformation of $\mathrm{Co}_{8}$ nanoparticle has proved to be the most stable.

One of more interesting situations arises in case of $\mathrm{Co}_{10}$ nanoparticle. In this case, we have the two-dimensional disordered symmetry structure consisting of two planes and two atoms in the middle of each plane (Fig.3). The atoms mentioned join these planes. Roughly speaking, the structure of the $\mathrm{Co}_{10}$ nanoparticle is formed when the planes of the $\mathrm{Co}_{8}$ nanoparticle are rotated in respect of each other when two Co atoms are added and a nice cubic structure is formed. This has also been confirmed by bond order investigations. On the 
other hand, each $\mathrm{Co}$ atom is four-fold-coordinated and a structural element of the $\mathrm{Co}_{6}$ particle could also be foreseen. In case of $\mathrm{Co}_{12}$ and $\mathrm{Co}_{14}$ particles there are three planes where the location of atoms is as in the FCC structure: the atoms lie on the corners of the cube with additional atoms in the center of each of four cube of faces. The structure of $\mathrm{Co}_{6}$ particle is also obtained. The element of BCC structure is also present because some atoms are out of the cube face. The most important for us is that the structure of a $\mathrm{Co}_{6}$ particle was also obtained.
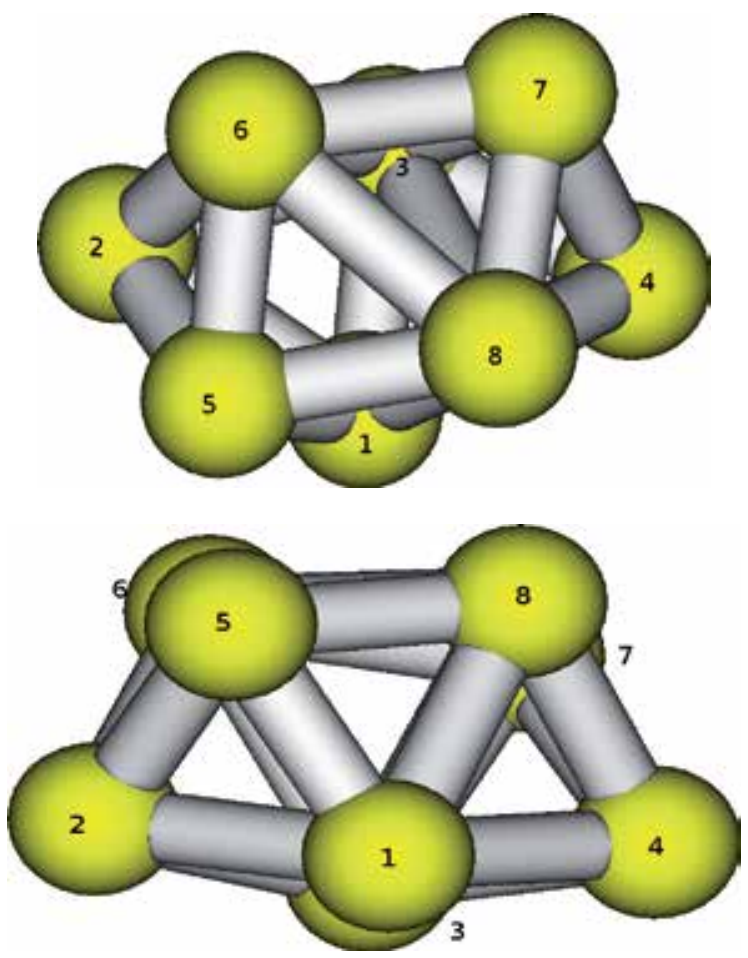

Fig. 2. The view of two planes of $\mathrm{Co}_{8}$ particle from two different sides take places. $\mathrm{T}$

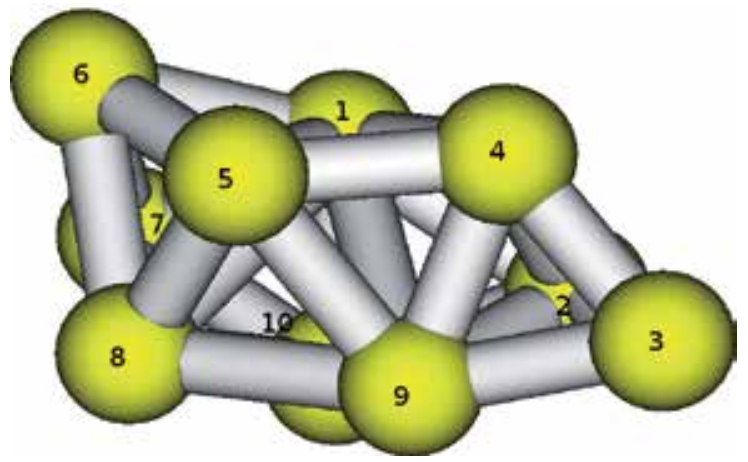

Fig. 3. The views of two formed by atoms 4,5,8,9 and 1,2, 7, 10 planes where atoms 3 and 6 is between the planes in $\mathrm{Co}_{10}$ particle. 
Let us describe the structure of this $\mathrm{Co}_{16}$ particle on the basis of the $\mathrm{Co}_{14}$ particle. Firstly, it is necessary to mention that the additional atoms are joined with three-fold coordinated atoms placed above the centre of the cube face. The joining leads to the deformation of the cube because the above atoms of the $\mathrm{Co}_{14}$ particle are pushed to the centre of the cube face. On the other hand, several structures of the $\mathrm{Co}_{6}$ particle are possible to be seen. Hence, in this case, the deformed FCC structure also takes place, but the BCC structure element tends to disappear in the inner part of the particles although the exterior part the element remains unchanged. So, the tendency to form FCC is possible to predict. It allows us to speculate, that in large particles (particles with the diameter more than $10 \AA$ ) the main structure could be FCC, while in the external part the BCC structure could be present.

When considering the electronic properties of the above $\mathrm{Co}_{n}$ particles, a singlet state is a ground one. The triplet state of these particles lies higher in total energy. These results disagree with the results presented by H.J. Fan and et al. It is necessary to mention that in the paper of H.J. Fan and et al. only high spin multiplicity particles were investigated applying Amsterdam density functional method with STO basis set with no report on how the geometry of the most stable compound was obtained. The calculated binding energies (per atom) of the Co nanoparticles, as a function of the number of these atoms in the particle, indicate that the $\mathrm{Co}_{14}$ particle with the primitive cell of FCC structure is one of the most stable species among those presented in this section (Table 1). We also received, that $\mathrm{Co}_{6}$ and $\mathrm{Co}_{12}$ particles are more stable among the investigated by us particles that consist of less than 12 atoms and this result coincides with that presented by Q. M. Ma and et al. very well.

\begin{tabular}{|c|c|c|}
\hline Number of atom & Binding energy per atom, $\mathbf{e V}$ & HOMO-LUMO gap, $\mathbf{e V}$ \\
\hline 6 & 0.45 & 1.47 \\
\hline 8 & 0.20 & 1.32 \\
\hline 10 & 0.36 & 1.18 \\
\hline 12 & 0.48 & 1.53 \\
\hline 14 & 0.69 & 1.64 \\
\hline 16 & 0.78 & 1.24 \\
\hline
\end{tabular}

Table 1. The dependence of calculated binding energy per atom and HOMO-LUMO gap on the atom number in the particle.

The above results confirm the investigation of HOMO-LUMO gap.

Hence, the main important observations on the geometrical structure of the pure Co nanoparticle are the following:

- The $\mathrm{Co}_{8}, \mathrm{Co}_{10}, \mathrm{Co}_{12}, \mathrm{Co}_{14}, \mathrm{Co}_{16}$ particles consist of $\mathrm{Co}_{6}$, thus this particle can be regarded to as the key element of the large Co nanoparticles.

- $\quad$ The face centered cubic structure which is slightly less close packed occurred in the $\mathrm{Co}_{14}$ nanoparticle, while the other particles described are the elements of the FCC structure. When increasing the number of Co atoms in the particle, the atoms that are above the centre of the cube face are pushed both to the cube face centre and the inner part of the particle. Hence, in the inner part of the particle there is a FCC structure while the BCC structure element is obtained in the exterior part the particle. Thus, the obtained results allow us to speculate that in a large cobalt nanoparticle the FCC structure should be 
clearly seen, while in a smaller one the FCC structure with the element of BCC structure should be obtained.

It is necessary to mention that the bond length and the bond order were also investigated. The obtained results are summarized in Table 2.

\begin{tabular}{|c|c|c|c|}
\hline $\begin{array}{c}\text { Atom number in a } \\
\text { particle }\end{array}$ & $\begin{array}{c}\text { Single bond length, } \\
\AA\end{array}$ & $\begin{array}{c}\text { Double bond length, } \\
\AA\end{array}$ & $\begin{array}{c}\text { Coordinated bond } \\
\text { length }\end{array}$ \\
\hline 6 & 2.2 & 2.0 & 2.3 \\
\hline 8 & $2.1-2.2$ & 2.0 & 2.3 \\
\hline 10 & $2.1-2.2$ & & 2.4 \\
\hline 12 & 2.27 & & 2.3 \\
\hline 14 & $2.15-2.27$ & & 2.3 \\
\hline 16 & $2.15-2.27$ & & 2.3 \\
\hline
\end{tabular}

Table 2. The bond lengths obtained in the investigated particles

It is emphasized, that EXAFS MFT provides a Co-Co inter-atomic distance in the nanoparticle as equal to $2.561 \pm 0.015 \AA$. The comparison of the theoretical investigation of the Co particles with the corresponding experimental data is rather complicated quantitatively. The use of a restricted basis set of Co which can limit the quantitative analysis in the theoretical calculations should be taken into account. Hence, the obtained bond length fits well enough into the above-mentioned results. That obtained structures of the Co nanoparticle are to be mentioned as not fully spherical. The results obtained fit the results of the high magnification TEM image perfectly. In any case it is possible to see that double bonds are ruptured when the number of Co atoms is increased while coordinate bonds remain. The Co-Co bond elongation within the increasing atom number in the particles is not possible to explicitly be exhibited, while the presence of the coordinated bond allow us to foresee that the total electron density in this $\mathrm{Co}-\mathrm{Co}$ bond is smaller than that in the other bonds what leads to non-compensation of the electron spins. On the other hand, the results exhibit that investigated Co nanoparticles are not homogeneous systems, i.e. the systems consist of different-fold-coordinated atoms. The obtained results indicate that a Co atom is three-to-seven-fold coordinated in the most stable nanoparticles. The presence of a coordinated bond, that is a kind of 2-centre, 2-electron covalent bond in which the two electrons derive from the same atom, prove the above results, too. Additionally performed analysis of the molecular orbital nature indicates that in the Co derivatives the number of bonding molecular orbitals, that may be occupied, is insufficient to locate all electrons of the system. It implies that some electrons are displaced on the anti-bonding orbitals, the energy of which is higher than that of the bonding orbitals. This electronic non-uniformity (a different oxidation state of an atom in the particle) of Co atoms, as we will prove below, and the electron displacement on the anti-bonding orbitals are important for the magnetic properties of the Co nanoparticles consisting of the even number of atoms.

\subsubsection{Magnetic properties of the pure Co nanoparticles}

Aiming to explain the results on magnetic properties of the particles investigated as well as particle dependence on the size, we paid our attention to the nature of molecular orbitals and their placements, because the studies on the $\mathrm{Co}_{2} \mathrm{Om}(\mathrm{m}=0-7)$ compound indicate that 
both the increase of the number of oxygen atoms in the compound and the changeability of the oxidation state of the Co atoms led to the increase of the Co-Co bond length and weakening of the Co-Co bonds. The weakening of these bonds is important for the magnetic properties of these compounds. The results obtained indicate that the displacement of the two electrons on $\mathrm{dz}^{2}$ orbitals of Co atoms creates Co-Co bonds. The energy of these orbitals is similar to that of other ones. Thus, the repulsion between the electrons on the $\mathrm{dz}^{2}$ orbitals is larger than in other cases investigated, therefore these electrons tend to be as far as possible from each other and the correlation between them is weakened, resulting in the elongation of $\mathrm{Co}-\mathrm{Co}$ bonds and, as a consequence, presence of an unpaired spin.

Let us remember that in the Co derivatives the number of bonding molecular orbitals that may be occupied is insufficient to locate all electrons of the system. As example, in $\mathrm{Co}_{6}$ compounds all bonding orbitals are occupied and, as it has already been mentioned, some electrons are displaced on the anti-bonding orbitals, the energy of which is higher than that of the bonding orbitals.

It should be mentioned that the increased number of Co atoms in the compound leads to weakening of Co-Co bonds what, as we think, is important for the magnetic properties of these compounds, because magnetic properties depend on the bonds' nature and the number of bonds as well as on the charge distribution. Thus, aiming to explain the magnetic properties of the investigated particles, the attention is paid to the bonds' nature (what orbitals consist of bonds), the dipole moment and its components as well as on the isotropic g-tensor which depends on a spin angular moment.

In Table 3 the data on magnetizability, dipole moment, isotropic $g$ tensor and the number of bonds consisting of anti-bonding orbitals are presented. The analysis of the most important orbitals (HOMO) of the described particles has been performed (Figs. 4-9). Fig.6 represents a full view of the $\mathrm{HOMO}$ of the $\mathrm{Co}_{6}$ particle and the additional schematic presentation of the bond places in the particle is given to better illustrate the results presented.

\begin{tabular}{|c|c|c|c|c|}
\hline Compound & $\begin{array}{c}\text { Magnetizability, } \\
\text { a.u. }\end{array}$ & $\begin{array}{c}\text { Isotropic } \\
\text { g-tensor }\end{array}$ & $\begin{array}{c}\text { Dipole moment, } \\
\text { a.u. }\end{array}$ & $\begin{array}{c}\text { Number of bonds consist of } \\
\text { anti-bonding character }\end{array}$ \\
\hline $\mathrm{Co}_{6}$ & 58.77 & -0.513 & 0.097 & 5 \\
\hline $\mathrm{Co}_{8}$ & 25.79 & -0.289 & 0.468 & 3 \\
\hline $\mathrm{Co}_{10}$ & -26.13 & -0.071 & 0.084 & 20 \\
\hline $\mathrm{Co}_{12}$ & -35.11 & -0.038 & 0.213 & 8 \\
\hline $\mathrm{Co}_{14}$ & -39.27 & -0.046 & 0.157 & 3 \\
\hline $\mathrm{Co}_{16}$ & 69.84 & -0.163 & 0.331 & 5 \\
\hline
\end{tabular}

Table 3. The data on magnetizability, dipole moment, $g$ tensor and the number of bonds consisting of anti-bonding orbitals.

Firstly, it is necessary to mention, that in the g-tensor of a molecule, there is a nuclear contribution and an electronic sum-over-states contribution. The electronic contribution represents an isotropic g-tensor. Here, it should be mentioned, that an isotropic g-tensor along with magnetizability is calculated to recognize what contributions (nuclear or electronic) are more important for the magnetic properties of the particle investigated. 
It is possible to see that only $\mathrm{Co}_{6}, \mathrm{Co}_{8}$ and $\mathrm{Co}_{16}$ particles exhibit paramagnetic properties although the bonds that are of anti-bonding character are present in all the particles investigated. The different number of bonds formed of anti-bonding orbitals is present in the $\mathrm{Co}_{6}, \mathrm{Co}_{8}$ and $\mathrm{Co}_{16}$ particles. The view of the particles and location of the above bonds are presented in Figs. 4-9. The conclusion on the character of bonds was made on the basis of the analysis of the most important atomic orbitals on atoms, bond lengths, bond order and views of the orbitals.
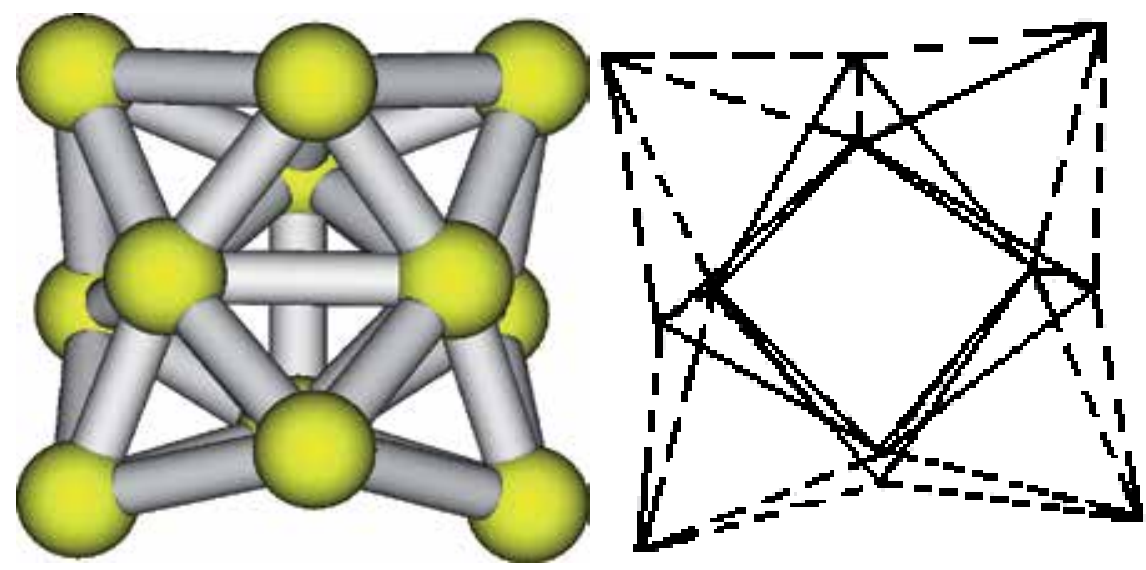

Fig. 4. The views of $\mathrm{Co}_{12}$ particle on the left and the view (on the right) when the bonds form of anti-bonding orbitals are marked by dash lines.

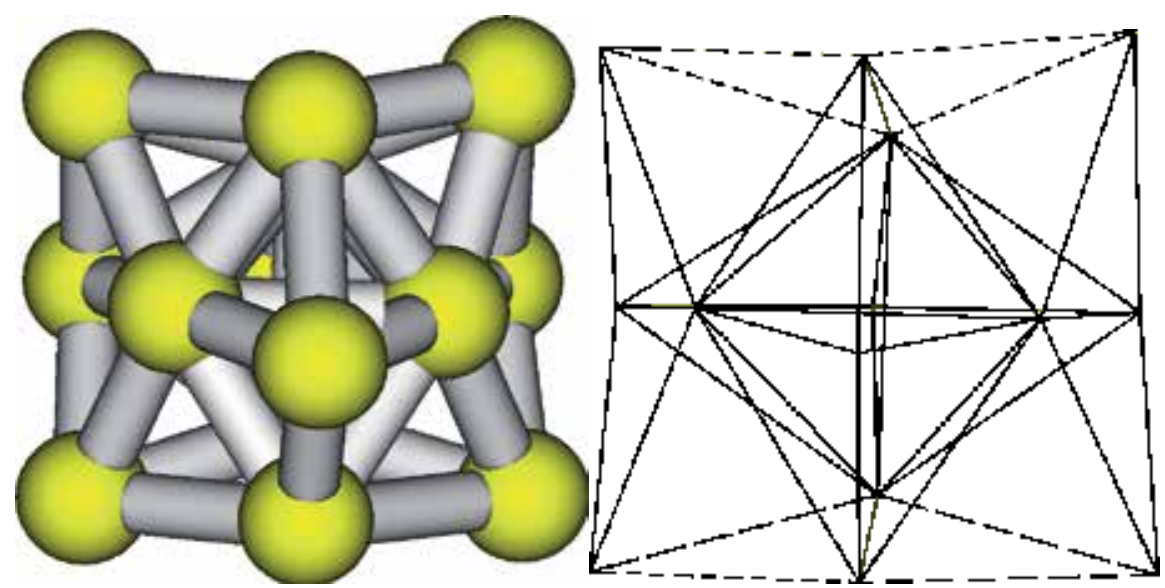

Fig. 5. The view of $\mathrm{Co}_{14}$ particle on the left and the view (on the right) when the bonds form of anti-bonding orbitals are marked by dash lines. 


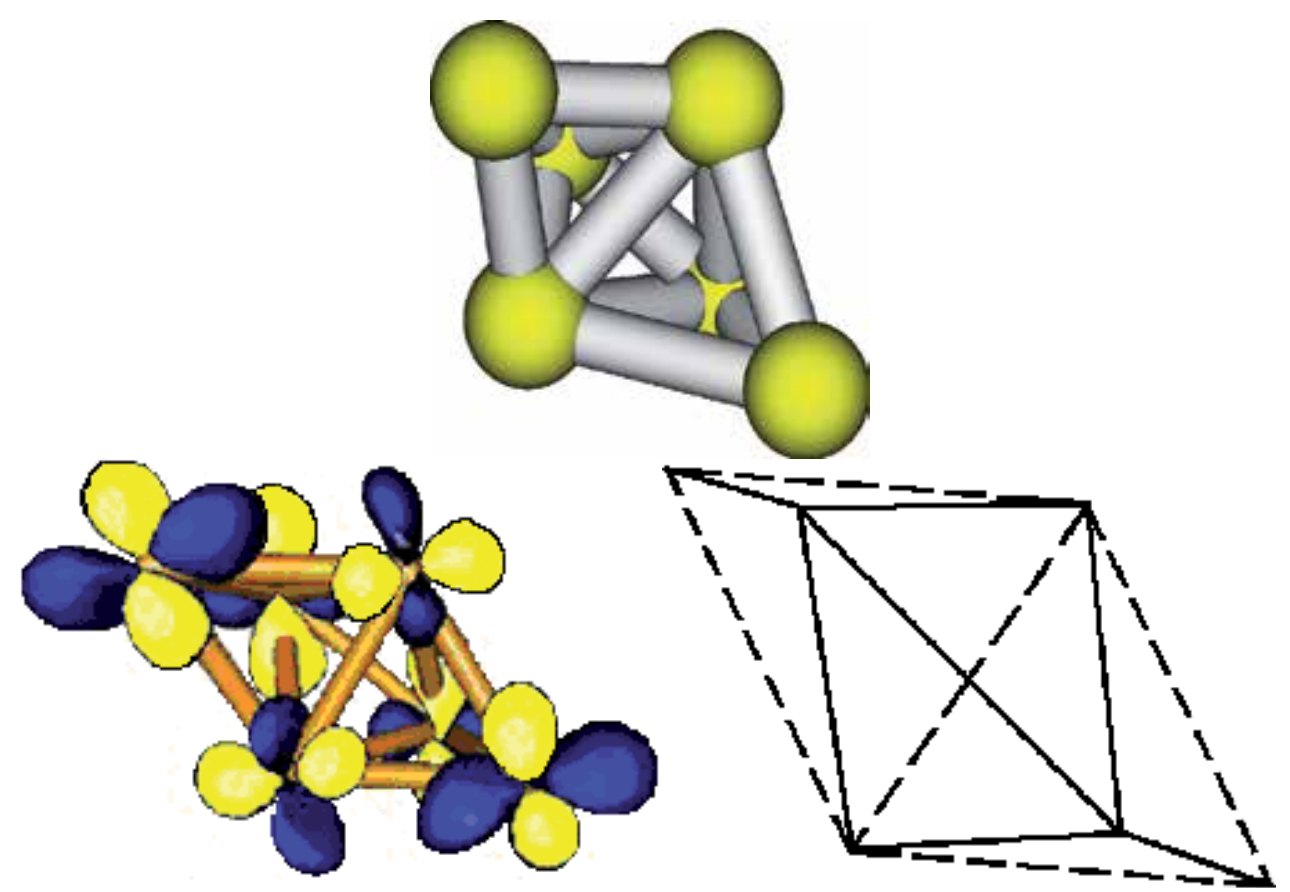

Fig. 6. The views of $\mathrm{Co}_{6}$ particle (on the left) and their most important orbital (HOMO) (in the centre). The same view (on the right) is given when the bonds form of anti-bonding orbitals are marked by dash lines for simple guidance.

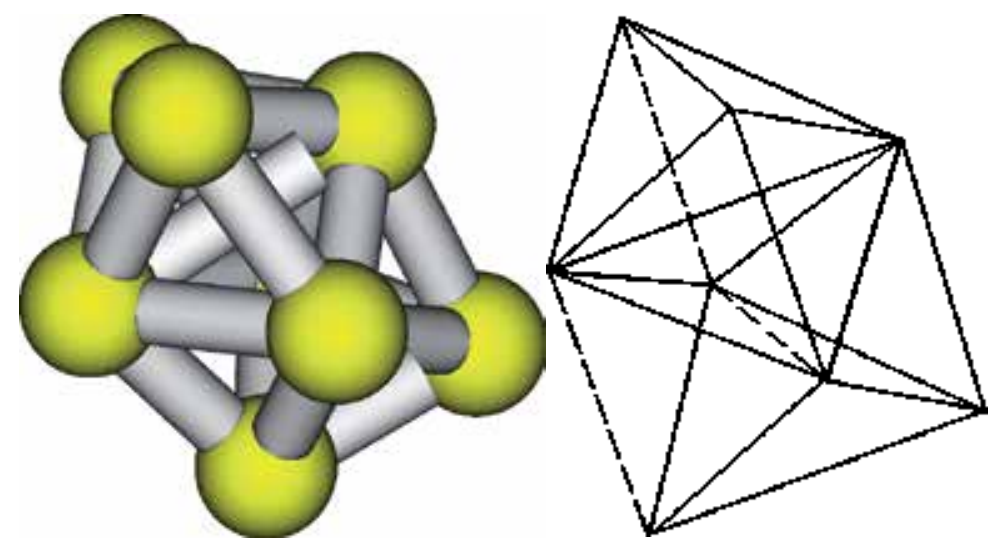

Fig. 7. the views of $\mathrm{Co}_{8}$ particle (on the left) and the same view are given when the bonds consisting of anti-bonding orbitals are marked by dash lines.

Hence, it is possible to see that $\mathrm{CO}_{12}$ and $\mathrm{Co}_{14}$ are diamagnetic because in these particles there are 18 and 4 respectively symmetrically placed bonds with weakly interacting electrons what leads to the disappearance of non-compensate spins. These non-compensated spins quench each other what indicates the isotropic g-tensor value being equal to 0.038 and 0.046 in comparison to the value 2.00 for a free electron and indicates the absence of free electrons or a non-compensate spin. 
In case of $\mathrm{Co}_{12}$, the oxidation state of $\mathrm{Co}$ atoms is even. So, a non-compensate spin can not appear because the atoms of this particle loose the even number of electrons (below, it is exhibited that the oxidation state of atoms is also important to the explanation of Co particle magnetic properties).

The electronic properties of the $\mathrm{CO}_{14}$ particle fit described properties of the bond nature and oxidation state of the atoms very well. In the case the even number of bonds that are of antibonding character is found. Hence, electron spins are compensated and this particle exhibits diamagnetic properties. Additionally, even number (four) of atoms with oxidation state +3 are present

In case of $\mathrm{Co}_{6}, \mathrm{Co}_{8}, \mathrm{Co}_{10}, \mathrm{Co}_{16}$ there are non - symmetrically placed bonds with weakly interacting electrons. Thus, we may suspect that these particles could be paramagnetic.
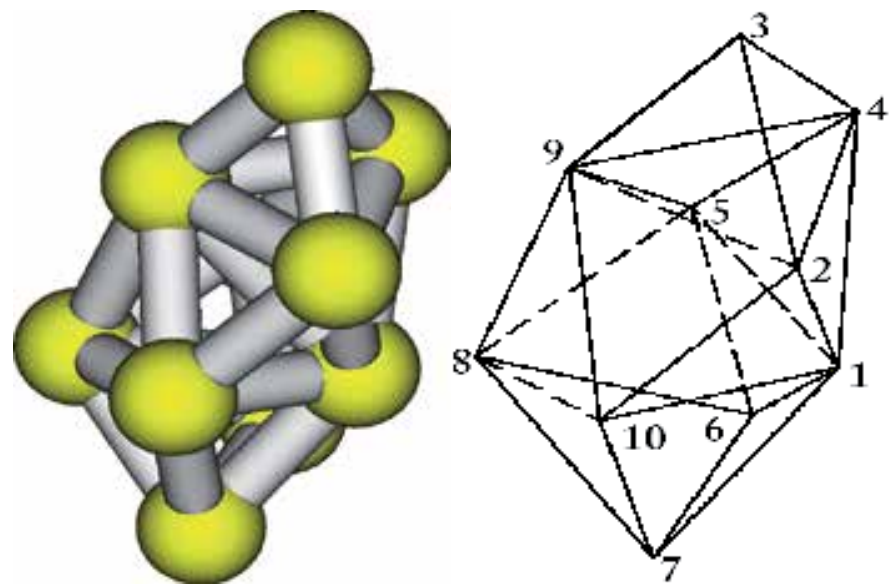

Fig. 8. The views of $\mathrm{Co}_{10}$ particle (on the left) and the view (on the right) when the bonds form of anti-bonding orbitals are marked by dash lines.
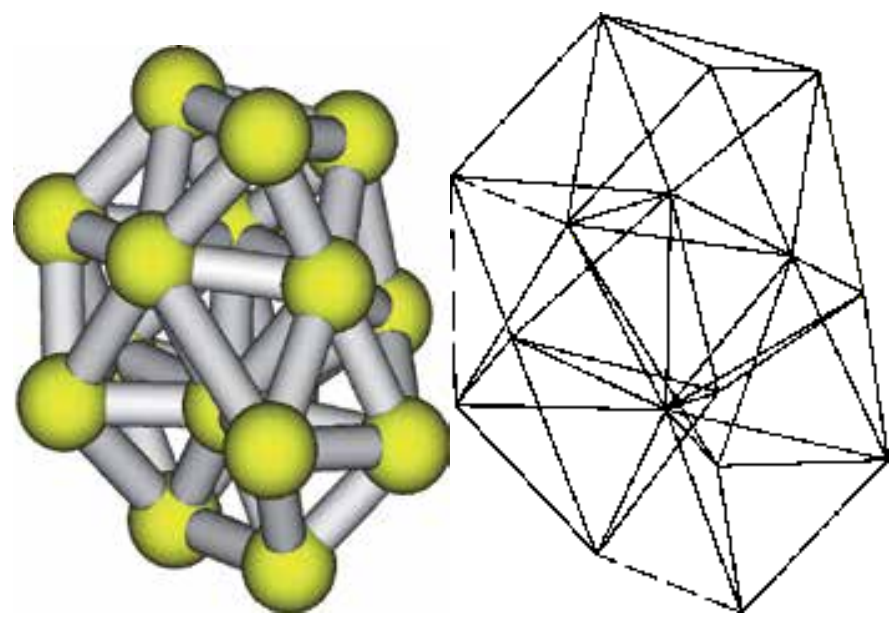

Fig. 9. The views of $\mathrm{Co}_{16}$ particle (on the left) and the view (on the right) when the bonds form of anti-bonding orbitals are marked by dash lines. 
The magnetizability and g-tensor of the $\mathrm{Co}_{6}$ particle are approximately twice larger than those of the $\mathrm{Co}_{8}$ particle. In the $\mathrm{Co}_{6}$ particle the number of bonds of anti-bonding character is five and these bonds are non-parallel. The dipole moment of the particle is approximately zero. It allows us to conclude that this particle is paramagnetic due to the electronic contribution, i.e. the repulsion between the electrons located on the anti-bonding orbital is large, therefore, they tend to be as far as possible from each other and become non-strongly correlated. Thus the spins of the electrons are not compensated, while the unparalleled displacement of the bonds leads to that that spins of all non-strongly-correlated-electrons are not compensated. It implies, that magnetic properties of the $\mathrm{Co}_{6}$ particle are related with an electronic contribution.

A similar situation is obtained in case of $\mathrm{Co}_{8}$ particle. Approximately twice smaller magnetizability of this particle than that of $\mathrm{Co}_{6}$ is present because in the particle the number of non-strongly- correlated electrons is smaller than that in $\mathrm{Co}_{6}$.

The largest magnetizability is the $\mathrm{Co}_{16}$ particle, although, its isotropic g-tensor is approximately twice smaller than that of $\mathrm{Co}_{6}$ particle. To explain the above mentioned contradictions, we investigated a dipole moment of these particles. The dipole moment indicates electron concentration places in a particle. On the other hand, the components of these dipole moments allow us to foresee the distribution of the above places. Both the concentration of electrons and their distribution helps us to find the appearance of the additional spins due to the different oxidation states of the Co atoms, i.e. if the even number of atoms loose the odd number of electrons and the particle possesses a dipole moment, we may suspect the presence of the localization of electrons and non-compensation of their spins. We named the above spin an ion one to simplify the discussion.

The dipole moment components of the particles are presented in Table 4 and indicate the electron charge delocalization in the $\mathrm{Co}_{8}$ and $\mathrm{Co}_{16}$ particles, while in case of the $\mathrm{Co}_{10}$ and $\mathrm{Co}_{12}$ particles, the charge localization occurs (see the component of dipole moment). It is necessary to add, that in $\mathrm{Co}_{8}$ and $\mathrm{Co}_{12}$ cases, the oxidation states of $\mathrm{Co}$ atoms are even. It allows us to predict, that electron spins occurring when the atoms loose an electron are compensated.

In case of $\mathrm{Co}_{16}$ particle, the dipole moment components indicate charge delocalization, while the isotropic $\mathrm{g}$ - tensor value is smaller than that of $\mathrm{Co}_{6}$ and $\mathrm{Co}_{8}$. It allows us to conclude that the magnetic properties of this particle are mostly related with nuclear contribution. However, it is not explicitly possible to recognize the folding of atoms such as 3.49 or 3.51 on the results of these calculations. Thus, it is only speculation based on the comparison of the magnetizability of the investigated results that, in case of $\mathrm{Co}_{16}$ particle, the ion spins and electron spins are not compensated.

The $\mathrm{Co}_{6}, \mathrm{Co}_{8}$ and $\mathrm{Co}_{16}$ particles are paramagnetic, while $\mathrm{Co}_{10}$, that possesses the odd number of anti-bonding character bonds as the particles mentioned, indicates diamagnetic properties. In case of $\mathrm{Co}_{10}$ particle, the oxidation state of the $\mathrm{Co} 1$ atom is +5 (Fig. 8). The four bonds with anti-bonding character are displaced like in case of $\mathrm{Co}_{6}$, however, one bond is in the same direction of the largest component of the dipole moment. Thus, it is possible to suspect, that in this case a weakly interacting electron spin is quenched by the ion spin. It may be concluded that paramagnetic behaviour is dominating when the uncompensated 
spin is present due to the presence of a weakly interacting electron on the anti-bonding orbital and this spin is not quenched by the ion spins.

\begin{tabular}{|c|c|c|c|c|}
\hline Compounds & $\begin{array}{c}\text { Dipole moment, } \\
\text { a.u. }\end{array}$ & \multicolumn{3}{|c|}{$\begin{array}{c}\text { Dipole moment components, } \\
\text { a.u. }\end{array}$} \\
\hline & & $\mathbf{x}$ & $\mathbf{y}$ & $\mathbf{z}$ \\
\hline $\mathrm{Co}_{6}$ & 0.097 & -0.09 & -0.01 & -0.01 \\
\hline $\mathrm{Co}_{8}$ & 0.468 & 0.147 & 0.298 & 0.329 \\
\hline $\mathrm{Co}_{10}$ & 0.084 & 0.076 & 0.031 & 0.021 \\
\hline $\mathrm{Co}_{12}$ & 0.213 & 0.198 & -0.051 & -0.061 \\
\hline $\mathrm{Co}_{14}$ & 0.157 & -0.077 & 0.094 & -0.098 \\
\hline $\mathrm{Co}_{16}$ & 0.331 & -0.107 & 0.167 & -0.264 \\
\hline
\end{tabular}

Table 4. Dipole moments and their components of the investigated particles that are paramagnetic or weakly diamagnetic.

It is possible to see that the investigated systems are very flexible and it is possible to predict that any dipole interaction or Co particle agglomeration could change their magnetic properties. To confirm the above prediction, the magnetic properties of the $\mathrm{Co}_{6}$ and $\mathrm{Co}_{6}$ as well as those of $\mathrm{Co}_{6}$ and $\mathrm{Co}_{12}$ derivatives have also been investigated.

The structure of $\mathrm{Co}_{6}$ and $\mathrm{Co}_{6}$ particles was found after global optimization. The results obtained indicate possible agglomeration of these particles, i.e. the $\mathrm{CO}_{12}$ particle should formed. The magnetizability of this compound is -12.55 a.u., what indicates diamagnetic properties.

In case of the $\mathrm{Co}_{6}$ and $\mathrm{Co}_{12}$ compound, we did not perform any geometry optimization to avoid agglomeration of particles because the changes of geometrical structure lead to dramatical changes of the electronic structure and consequential changes of magnetic properties. The investigated particles were placed randomly. Indeed, a compound consisting of $\mathrm{CO}_{12}$ and $\mathrm{Co}_{6}$ particles is paramagnetic and its magnetizability is equal to 24.65 a.u. The results clearly indicate that dipole interaction and particle agglomeration change magnetic properties of the Co nanoparticle.

\section{Structure, stability and magnetic properties of $\mathrm{Co}_{6} \mathrm{O}_{\mathrm{m}}$ nanoparticles}

\subsection{Structure and stability of $\mathrm{Co}_{6} \mathrm{O}_{\mathrm{m}}$ particles}

As exhibited above, the stability of small Co nanoparticles is not very high. On the basis of our previous investigations it was speculated that those nanoparticles were non-rigid structures. It implies that the geometrical structure of these particles could change very quickly due to the tunnelling effect. Let us remember that $\mathrm{Co}_{6}$ particle is found as the most stable one and it is the key element of other particles investigated. So, $\mathrm{Co}_{6} \mathrm{O}_{n}(n=0-9)$ derivatives were also investigated to establish how the magnetic properties of the Co particles may change due to oxidation. On the other hand, these investigations allow us to foresee the conditions under which the metal Co-Co bond is broken. It is also proved our prediction that the particles consisting of the even number of atoms possess magnetic properties due to the weakly interacting electrons on the anti-bonding orbital and this spin is not quenched by additional spins that occurred because some atoms of the nanoparticle loose the odd number of electrons. 
The most stable structures of the $\mathrm{Co}_{6} \mathrm{O}_{\mathrm{n}}$ derivatives are presented in Fig.10.

Firstly, it is necessary to mention that oxygen stabilizes the Co nanoparticle and the increasing number of oxygen atoms increases the binding energy per atom up to $n=7$ (Table 5). Furthermore, when a certain limit is reached, oxygen atoms do not influence the stability of the $\mathrm{Co}_{6} \mathrm{O}_{\mathrm{n}}$ particles.

The $\mathrm{Co}_{6} \mathrm{O}_{12}$ particle was investigated too. The binding energy per atom of this particle is equal to $3.26 \mathrm{eV}$ what is similar to that of $\mathrm{Co}_{6} \mathrm{O}_{n}(n=7,8,9)$. The difference of the binding energy of the above particles is too small $(0.2 \mathrm{eV}$ or less) to make the conclusion on the most stable particle.

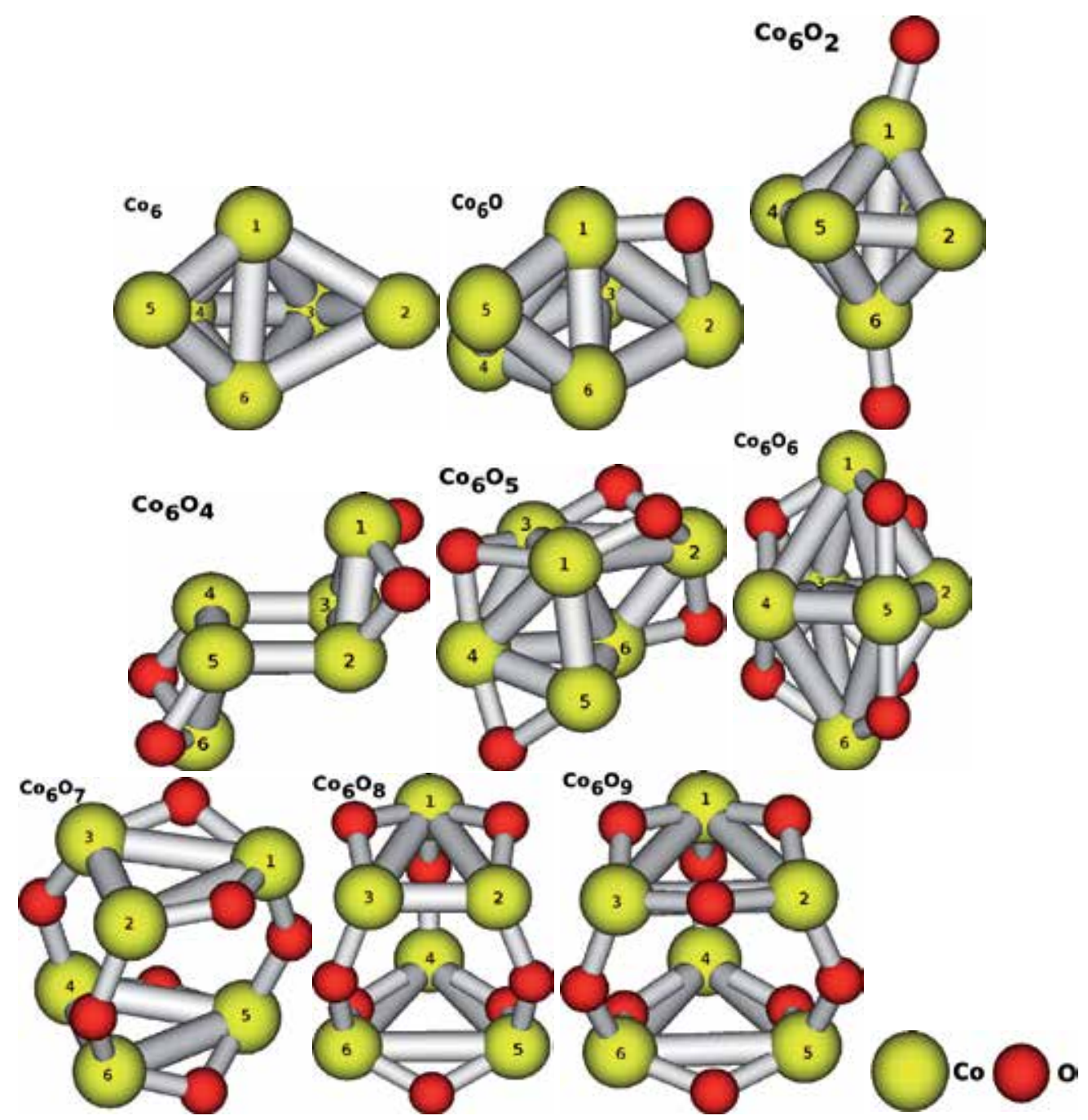

Fig. 10. Views of the particles investigated. Grey lines do not indicate real chemical bonds, but are implemented for the sake simple guidance. 


\begin{tabular}{|c|c|c|c|c|c|c|c|c|c|c|}
\hline Particle & $\mathrm{Co}_{6}$ & $\mathrm{Co}_{6} \mathrm{O}$ & $\mathrm{Co}_{6} \mathrm{O}_{2}$ & $\mathrm{Co}_{6} \mathrm{O}_{3}$ & $\mathrm{Co}_{6} \mathrm{O}_{4}$ & $\mathrm{Co}_{6} \mathrm{O}_{5}$ & $\mathrm{Co}_{6} \mathrm{O}_{6}$ & $\mathrm{Co}_{6} \mathrm{O}_{7}$ & $\mathrm{Co}_{6} \mathrm{O}_{8}$ & $\mathrm{Co}_{6} \mathrm{O}_{9}$ \\
\hline $\begin{array}{c}\text { Binding energy } \\
\text { per atom, eV }\end{array}$ & 0.45 & 0.93 & 1.21 & 2.22 & 2.49 & 2.85 & 3.22 & 3.43 & 3.33 & 3.48 \\
\hline
\end{tabular}

Table 5. Binding energy per atoms for the $\mathrm{Co}_{6} \mathrm{O}_{n}(n=0-9)$ particles

The difference of the binding energy per atom for $\mathrm{Co}_{6}$ and $\mathrm{Co}_{6} \mathrm{O}$ is equal to $0.48 \mathrm{eV}$, while that between $\mathrm{Co}_{6} \mathrm{O}_{6}$ and $\mathrm{Co}_{6} \mathrm{O}_{7}$ is only $0.21 \mathrm{eV}$, i.e. twice less. On the other hand, the changing of the number of oxygen atoms from 2 to 3 leads to the largest increase of the binding energy per atom $(1.01 \mathrm{eV})$, while the binding energy per atom increase only up to $0.27 \mathrm{eV}$ when the oxygen atom number in a particle increases from 3 to 4 . Thus, the results of our investigations allow us to foresee that starting with $n=6$ ( $n$ is the number of oxygen atoms) the further increase of the number of oxygen atoms will not influence the stability of these particles very strongly and the main structure (the key-element) is not considerably changed (Fig. 10). The binding energy per atom of the $\mathrm{Co}_{6} \mathrm{O}_{6}, \mathrm{Co}_{6} \mathrm{O}_{7}, \mathrm{Co}_{6} \mathrm{O}_{8}$ and $\mathrm{Co}_{6} \mathrm{O}_{9}$ is approximately equal and proves these particles to be the most stable. These results coincide with the experimental measurements that indicate the presence of $\mathrm{CoO}$ and $\mathrm{Co}_{3} \mathrm{O}_{4} ; \mathrm{CoO}_{2}$, $\mathrm{Co}_{2} \mathrm{O}_{3}$ and $\mathrm{Co}_{6} \mathrm{O}_{7}$ particles should also be found among them what was proved by the results we obtained.

Such a changeability of the binding energy per atom in some cases could be explained by changes in geometrical structure of Co particle. In case when the additional oxygen atom does not significantly increase the binding energy per atom, the main part of the energy of this atom is used to deform the structure of the key element $\left(\mathrm{Co}_{6}\right)$. Thus, the binding energies per atom of $\mathrm{Co}_{6} \mathrm{O}_{3}$ and $\mathrm{Co}_{6} \mathrm{O}_{4}$ or $\mathrm{Co}_{6} \mathrm{O}_{6}$, and $\mathrm{Co}_{6} \mathrm{O}_{7}$ are approximately equal.

The key element of the $\mathrm{Co}_{6}$ is also present in the $\mathrm{Co}_{6} \mathrm{O}_{n}(\mathrm{n}=0-9)$ derivatives. However, this key element is slightly deformed. The changeability of the initial form is oxygen atom depended. The largest deformation is obtained in $\mathrm{Co}_{6} \mathrm{O}_{7}$, when the distance between the planes (formed of atoms 1, 2, 3 and of 4,5,6) is increased and one plane is rotated in respect of the other one by angle of $\Pi / 4$. Actually, one more structure of the $\mathrm{Co}_{6} \mathrm{O}_{7}$ which looks like $\mathrm{Co}_{6} \mathrm{O}_{6}$ was also obtained, but the energy of this formation of the particle is $1.23 \mathrm{eV}$ higher than that of the particle, the structure of which was described above.

In the $\mathrm{Co}_{6} \mathrm{O}_{4}$ particle the key element $\left(\mathrm{Co}_{6}\right)$ is deformed twice: 1 . firstly, when the distances between the atoms Co2-Co5 decrease; 2. Secondly, when Co1 and Co6 positions in respect of the plane that is formed of atoms 2,3,4,5 is changed. Here, it should be emphasized, that this structure of the particle has been obtained after global geometry optimization starting with several completely different initial geometries. Thus, the geometrical structure of the $\mathrm{Co}_{6} \mathrm{O}_{4}$ particle is confirmed.

Hence, the largest deformations of the $\mathrm{Co}_{6}$ particle are obtained when the number of oxygen atoms is changed from 3 to 4 and from 6 to 7 . In these cases the stabilization energy per atom is smaller than in other cases investigated. Thus, the main part of Oxygen energy is used to deform the key structure of $\mathrm{Co}_{6}$.

It is necessary to mention, when the number of oxygen atom is 2 and 6 , the structure of the $\mathrm{Co}_{6} \mathrm{O}_{\mathrm{m}}$ particle looks like the octahedron, while in case of odd numbers of oxygen the octahedron form is strongly deformed (except the results for $\mathrm{Co}_{6} \mathrm{O}_{4}$ ). It is interesting to note 
that the most stable structure of $\mathrm{Co}_{6} \mathrm{O}_{8}$ (prototype of $\mathrm{Co}_{3} \mathrm{O}_{4}$ ) has a deformed spinel structure. Thus, it is not surprising that a large effective magnetic moment estimated from the inverse susceptibility has not been explained properly.

\begin{tabular}{|c|c|c|c|c|c|c|c|c|c}
\hline Compound & \multicolumn{9}{|c|}{ Co-Co bond length, $\AA$} \\
\hline & $\mathbf{1 - 2}$ & $\mathbf{1 - 3}$ & $\mathbf{1 - 4}$ & $\mathbf{1 - 6}$ & $\mathbf{2 - 3}$ & $\mathbf{2 - 6}$ & $\mathbf{4 - 5}$ & $\mathbf{4 - 6}$ & $\mathbf{3 - 4}$ \\
\hline $\mathrm{Co}_{6}$ & 2.15 & 2.33 & 2.15 & 2.24 & 2.04 & 2.23 & 2.04 & 2.31 & 2.33 \\
\hline $\mathrm{Co}_{6} \mathrm{O}$ & 2.54 & & & & & & & & \\
\hline $\mathrm{Co}_{6} \mathrm{O}_{2}$ & & & & 3.01 & & & & & \\
\hline $\mathrm{Co}_{6} \mathrm{O}_{3}$ & & & & 2.18 & 2.37 & 2.33 & 2.14 & & \\
\hline $\mathrm{Co}_{6} \mathrm{O}_{4}$ & 2.27 & 2.27 & 2.27 & 4.72 & & & & 2.27 & \\
\hline $\mathrm{Co}_{6} \mathrm{O}_{5}$ & 2.25 & & & & 2.61 & 2.25 & & & 2.39 \\
\hline $\mathrm{Co}_{6} \mathrm{O}_{6}$ & 2.83 & 2.67 & 2.89 & & 2.32 & 2.87 & & & 2.44 \\
\hline $\mathrm{Co}_{6} \mathrm{O}_{7}$ & 2.93 & & & 3.11 & 2.93 & & 2.93 & 2.93 & \\
\hline
\end{tabular}

\begin{tabular}{|c|c|c|c|c|c|c|c|c|c|}
\hline & $\mathbf{1 - 2}$ & $\mathbf{1 - 3}$ & $\mathbf{1 - 4}$ & $\mathbf{2 - 3}$ & $\mathbf{2 - 5}$ & $\mathbf{3 . 6}$ & $\mathbf{4 - 5}$ & $\mathbf{4 - 6}$ & $\mathbf{5 - 6}$ \\
\hline $\mathrm{Co}_{6} \mathrm{O}_{7}$ & 2.92 & 2.93 & 3.21 & $2.21^{*}$ & 3.07 & 3.11 & 2.92 & 2.91 & $2.22^{*}$ \\
\hline $\mathrm{Co}_{6} \mathrm{O}_{8}$ & 2.88 & 2.90 & 3.21 & $2.24^{*}$ & 3.18 & 3.16 & 3.03 & 3.03 & 2.93 \\
\hline $\mathrm{Co}_{6} \mathrm{O}_{9}$ & 3.04 & 3.04 & 3.15 & 3.04 & 3.14 & 3.15 & 3.04 & 3.05 & 3.05 \\
\hline
\end{tabular}

* the Co-Co bond is present.

Table 6 . The distance between the $\mathrm{Co}$ atoms which are connected with the $\mathrm{O}$ atom.

According to the results of our investigations, the Co-Co bond length of the single bond is longer $(2.2 \AA)$ than the bond length of a double bond $(2.0 \AA)$ in a $\mathrm{Co}_{6}$ particle. On the other hand, three bonds were obtained where the length is equal to $2.3 \AA$. The bond order of the largest bond is twice smaller than that of a single bond. Here, we the commonly observed that the Co-Co bond lengths are marginally changed only between the atoms that are connected with the oxygen atom (Table 6) and, as a consequence, the bond enlargement leads to $\mathrm{Co}-\mathrm{Co}$ bond dissolving. For example: in the $\mathrm{Co}_{6}$ particle the bond order between Co1-Co5 is equal to 1.018, while that in $\mathrm{Co}_{6} \mathrm{O}_{4}$ is approximately twice smaller and equals to 0.55 . Additionally, the two, one and zero Co-Co bonds are respectively found in the $\mathrm{Co}_{6} \mathrm{O}_{7}$, $\mathrm{Co}_{6} \mathrm{O}_{8}$ and $\mathrm{Co}_{6} \mathrm{O}_{9}$ nanoparticles. To shed some light on the present observation, the analyzes of the most important orbitals of the $\mathrm{Co}_{6}$ particles have been investigated. $\mathrm{HOMO}$ (the highest occupied orbital)- LUMO (the lowest unoccupied orbital) gap dependence on the number of oxygen atoms is represented in Fig. 11. The HOMO-LUMO gap indicates that chemical stability of $\mathrm{Co}_{6}, \mathrm{Co}_{6} \mathrm{O}_{4}$ and $\mathrm{Co}_{6} \mathrm{O}_{6}$ is very low, i.e. they tend to form new chemical bonds. These results coincide well with the results of binding energy per atoms.

However, the electronic structure of the investigated particles is quite different because the oxidation state of Co atoms exchanges when the number of oxygen atoms in the particle is increased. For example, in $\mathrm{Co}_{6} \mathrm{O}$ particle oxidation state of $\mathrm{Co}$ atoms is +3 and +4 ; in the $\mathrm{Co}_{6} \mathrm{O}_{2}$ particle the oxidation state of these atoms is +4 and +5 and in $\mathrm{Co}_{6} \mathrm{O}_{4}$ it is +1 and +3 . We have not observed any relationship between number of oxygen atom in the particle and the oxidation state of Co atoms.

Let us remember that in the Co derivatives the number of bonding molecular orbitals, that may be occupied, is insufficient to locate all the electrons of the system. This leads to the 
presence of electrons on the anti-bonding orbital and, as a consequence to, the dissolution of Co-Co bonds.

On the other hand, the electronic configuration of cobalt for the ground state neutral gaseous atom is $[\mathrm{Ar}] .3 \mathrm{~d}^{7} .4 \mathrm{~s}^{2}$, while that of oxygen is [He].2s $\mathrm{s}^{2} .2 \mathrm{p}^{4}$. The configuration, associated with Cobalt in its compounds, is not necessarily the same, but it could be used to explain formally obtained results.

As it was mentioned above in $\mathrm{Co}_{6}$ compounds some electrons are displaced on the antibonding orbitals, the energy of which is higher than that of the bonding orbitals. Therefore, the stability of the pure cobalt nanoparticle is low. When the $\mathrm{Co}_{6}$ nanoparticle is joined to one or two oxygen atoms, the number of electrons that occupy anti-bonding orbitals, decreases because these electrons occupy the oxygen orbitals (Fig.12)

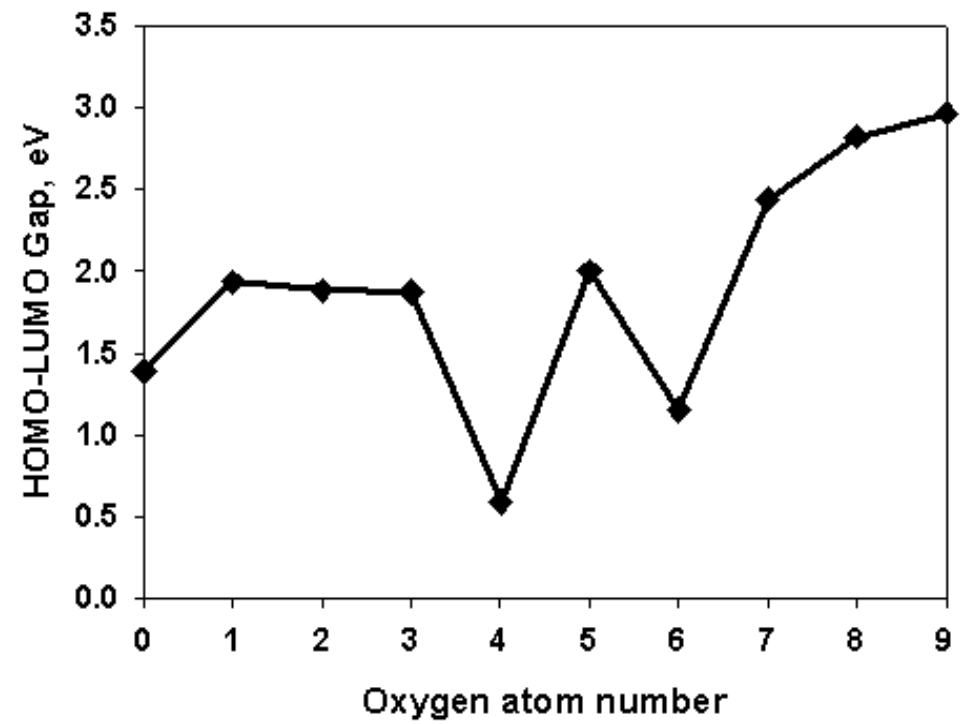

Fig. 11. The HOMO-LUMO gap of the $\mathrm{Co}_{6} \mathrm{O}_{n}(m=1-9)$

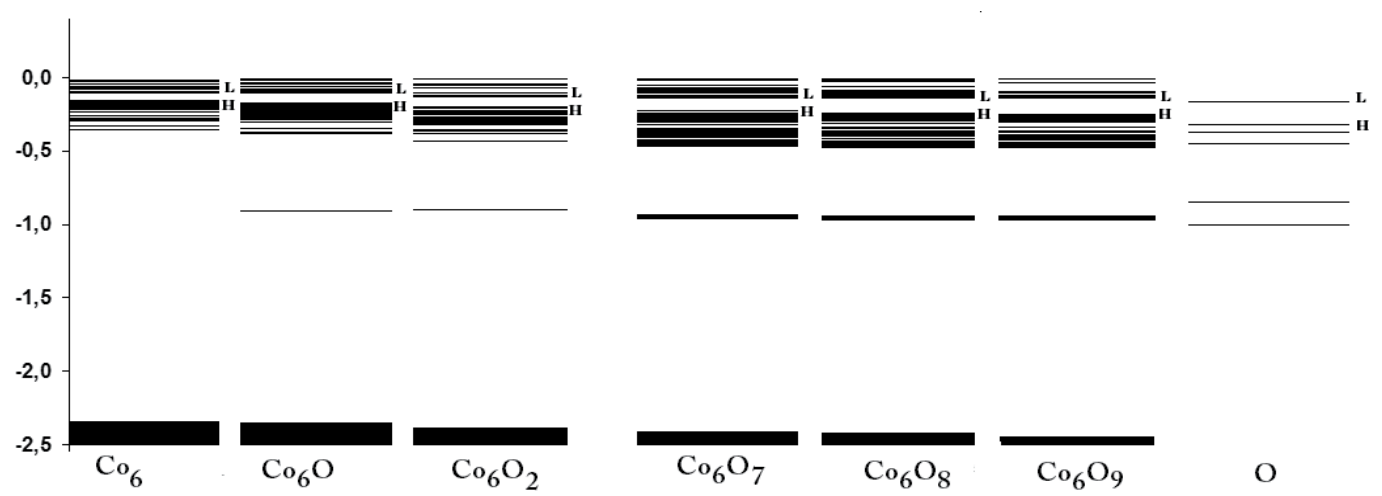

Fig. 12. Displacement of orbitals of several $\mathrm{Co}_{6} \mathrm{O}_{m}(\mathrm{~m}=0,1,2,7,8,9)$ and oxygen atoms in respect of each other. Here, $\mathrm{H}$ and L indicate HOMO and LUMO respectively. Additionally, the ground state (triplet) of oxygen atoms are calculated. It is possible to see that with the 
increasing number of the oxygen atoms in the particle, the number of occupied orbital also increases. i.e. the number of bonds of anti-boding nature decrease.

Oxygen atoms in the $\mathrm{Co}_{6} \mathrm{O}_{4}, \mathrm{Co}_{6} \mathrm{O}_{3}, \mathrm{Co}_{6} \mathrm{O}_{2}$, and $\mathrm{Co}_{6} \mathrm{O}$ particles are joined to atoms between which the anti-bonding orbitals occur. Having in mind that the joining of oxygen atoms leads to the increase of the bond length and dissolution of Co-Co bonds, what confirms the above mentioned prediction. In case of the $\mathrm{Co}_{6} \mathrm{O}_{3}$ particle, one $\mathrm{O}$ atom is joined to $\mathrm{Co} 4-\mathrm{Co} 5$ atoms (Fig.10). The anti-bonding nature of the bonds has not been observed between those atoms. In this case, a steric effect is more preferable because other positions of the oxygen atom should complicate $\mathrm{Co} 1-\mathrm{Co} 2$ and $\mathrm{Co} 2-\mathrm{Co} 6$ elongation or leads to the destruction of this particle. Hence, oxygen atoms stabilize $\mathrm{Co}_{6}$ particles due to dissolving of $\mathrm{Co}-\mathrm{Co}$ bonds that possess anti-bonding character.

It is very well known, that a semiconductor must have at least two characteristics: 1 . the bonding and anti-bonding orbitals must form a delocalized band; 2. the HOMO-LUMO gap in molecular species should be generally of the order of $0.5 \mathrm{eV}$ to $3.5 \mathrm{eV}$. HOMO-LUMO gaps of the investigated derivatives belong to the above range. However, the number of antibonding orbitals decreases with increasing of the number of oxygen atoms. The results allow us to predict that $\mathrm{Co}_{6} \mathrm{O}_{\mathrm{m}}$ are semiconductors but the particles should loose their semiconductor properties if the number of oxygen increases.

\subsubsection{Magnetic properties of $\mathrm{Co}_{6} \mathrm{O}_{\mathrm{m}}$ particles}

In the above chapter we proved that oxygen atoms stabilize cobalt nanoparticles, although, in some cases, the structure of particles changes insufficiently while the electronic structure is dramatically changed because the increasing number of oxygen atoms decreases the difference between the number of electrons and the number of atomic orbitals that they may occupy. Hence, the bonds of anti-bonding nature as well as uncorrelated spins disappear. So, we may suspect, that all Co oxide particles could be diamagnetics. Let us analyze the results presented in Table 7 .

\begin{tabular}{|c|c|c|c|}
\hline Compound & $\begin{array}{c}\text { Magnetizability, } \\
\text { a.u. }\end{array}$ & $\begin{array}{c}\text { Isotropic } \\
\text { g tenzor a.u }\end{array}$ & $\begin{array}{c}\text { Co-Co } \\
\text { bond number }\end{array}$ \\
\hline $\mathrm{Co}_{6}$ & 58,77 & $-0,51$ & 5 \\
$\mathrm{Co}_{6} \mathrm{O}$ & $-11,24$ & $-0,14$ & 6 \\
$\mathrm{Co}_{6} \mathrm{O}_{2}$ & $-15,47$ & $-0,11$ & 6 \\
$\mathrm{Co}_{6} \mathrm{O}_{3}$ & $-3,26$ & $-0,16$ & 5 \\
$\mathrm{Co}_{6} \mathrm{O}_{4}$ & $-9,62$ & $-0,12$ & 4 \\
$\mathrm{Co}_{6} \mathrm{O}_{5}$ & $-15,97$ & $-0,11$ & 2 \\
$\mathrm{Co}_{6} \mathrm{O}_{6}$ & $-2,11$ & $-0,16$ & 1 \\
$\mathrm{Co}_{6} \mathrm{O}_{7}$ & $-25,94$ & $-0,07$ & 2 \\
$\mathrm{Co}_{6} \mathrm{O}_{8}$ & 25,42 & $-0,18$ & 1 \\
$\mathrm{Co}_{6} \mathrm{O}_{9}$ & $-24,28$ & $-0,05$ & 0 \\
$\mathrm{Co}_{6} \mathrm{O}_{12}$ & $-24,25$ & $-0,07$ & 0 \\
\hline
\end{tabular}

Table 7. Data on magnetizability, isotropic $\mathrm{g}$ tensor, and Co-Co bonds number and the number of $\mathrm{Co}-\mathrm{Co}$ bonds that was found based on the electron density investigation results. Only those bonds with unpaired spin electrons are mentioned. 
It is obvious to see, that the particles with odd number of Co-Co bonds are paramagnetic or lightly diamagnetic. On the other hand, the isotropic g-tensor value of the cobalt oxide particles is not large, thus we may suspect that a ion spin in these cases is very important.

It is necessary to mention that based on the results described above, we may divide the described particles into the following groups:

1. The particles that posses shape of $\mathrm{Co}_{6}: \mathrm{Co}_{6}, \mathrm{Co}_{6} \mathrm{O}, \mathrm{Co}_{6} \mathrm{O}_{2}, \mathrm{Co}_{6} \mathrm{O}_{3}, \mathrm{Co}_{6} \mathrm{O}_{5}, \mathrm{Co}_{6} \mathrm{O}_{6}(\mathrm{~A}$ group)

2. The particles $\mathrm{Co}_{6} \mathrm{O}_{7}, \mathrm{C}_{6} \mathrm{O}_{8}, \mathrm{Co}_{6} \mathrm{O}_{9}$ in which the distance between the planes (formed of atoms 1, 2, 3 and of 4,5,6) is increased and one plane is rotated in respect the other one by the $\Pi / 4$ angle (B group).

3. The rest $\left(\mathrm{Co}_{6} \mathrm{O}_{4}\right)$

It has to be pointed out, that a lot of reports concluded that magnetic properties of the nanoparticles depend on their shape. So, we suspected that magnetizability of the particles belonging to one group should be the same. However, the results of our investigations do not prove the above prediction (Table 7).

According to our investigations, the $\mathrm{Co}_{6}$ nanoparticle is a strong paramagnetic, while other particles, belonging to group $\mathrm{A}$, are diamagnetics. The same phenomenon is obtained in case of $\mathrm{B}$ group. In this case, the $\mathrm{Co}_{6} \mathrm{O}_{8}$ particle is paramagnetic, while other particles are diamagnetics. Moreover, the diamagnetic properties of the similarly shaped particles are quite the same only in the following cases: $\mathrm{Co}_{6} \mathrm{O}_{7}, \mathrm{Co}_{6} \mathrm{O}_{12}, \mathrm{Co}_{6} \mathrm{O}_{9} ; \mathrm{Co}_{6} \mathrm{O}_{3}, \mathrm{Co}_{6} \mathrm{O}_{6} ; \mathrm{Co}_{6} \mathrm{O}_{2}$, $\mathrm{C}_{6} \mathrm{O}_{5}$. It implies that the shape of the particle has no influence on the magnetic properties of the nanoparticles. To confirm this conclusion, we have calculated magnetizability of several isomers of $\mathrm{Co}_{6} \mathrm{O}_{8}$ particles (Fig. 13). It is possible to see, that the shapes of isomers II and III are similar, but the shape of isomer I differs. However, the magnetizability of isomers II and I with different shapes is approximately alike, while the magnetizability of isomer III is smaller than that of isomer II with the same shape (Table 8).

\begin{tabular}{|c|c|c|c|}
\hline Isomers & I & II & III \\
\hline Magnetizability, a.u. & 25.42 & 24.76 & 14.24 \\
\hline
\end{tabular}

Table 8. The Magnetizability of different isomers of $\mathrm{Co}_{6} \mathrm{O}_{8}$ particle.

Hence, the magnetic properties of these particles does not depend on their shape.
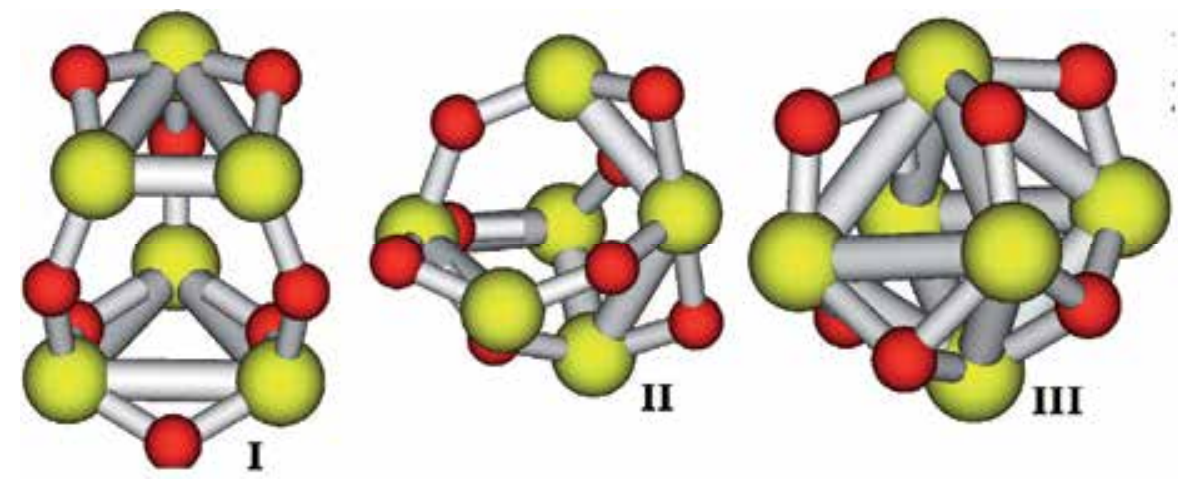

Fig. 13. The view of several isomers of $\mathrm{Co}_{6} \mathrm{O}_{8}$. 
Let us remember, that the nanoparticles could be paramagnetic due to several reasons: 1) the unpaired electron location on the Co-Co bonds; 2) the small total electron charge density between Co atoms which appears due to overlapping of $p$ orbitals of oxygen atoms; 3 ) the significant contribution of atoms that loose odd number of electrons. The second reason mentioned could not be realized in case of the $\mathrm{Co}_{6} \mathrm{O}_{n}$ particles due to their relatively large size and small number of oxygen atoms. The first and third reasons could be realized thus supporting the previously found results. It is necessary to mention, that non-compensation of spin for $\mathrm{CoO} / \mathrm{SiO} 2$ multilayers was also observed.

Now, we shall describe the particles of group B in detail. Firstly, it is necessary to mention, that the particles of this group have the different number of $\mathrm{Co}-\mathrm{Co}$ bonds: $\mathrm{Co}_{6} \mathrm{O}_{7}$, has two, $\mathrm{Co}_{6} \mathrm{O}_{8}$ has one, and $\mathrm{Co}_{6} \mathrm{O}_{9}$ has zero. Only $\mathrm{Co}_{6} \mathrm{O}_{8}$ particle exhibits paramagnetic properties.

Let us remember that in the Co derivatives the number of bonding molecular orbitals, that may be occupied, is insufficient to locate all the electrons of the system. This causes the presence of electrons on the anti-bonding orbital and, as a consequence, a weaker correlation of these electrons. Similar states are obtained in biradicals where the number of atomic orbitals, that may be occupied, is smaller than that of electrons. That leads to the appearance of electrons on the anti-bonding orbitals and serves predicts a large orbital contribution to the magnetic moment of a small $\mathrm{Co}_{\mathrm{m}} \mathrm{O}_{\mathrm{n}}$ particle (Fig.14).

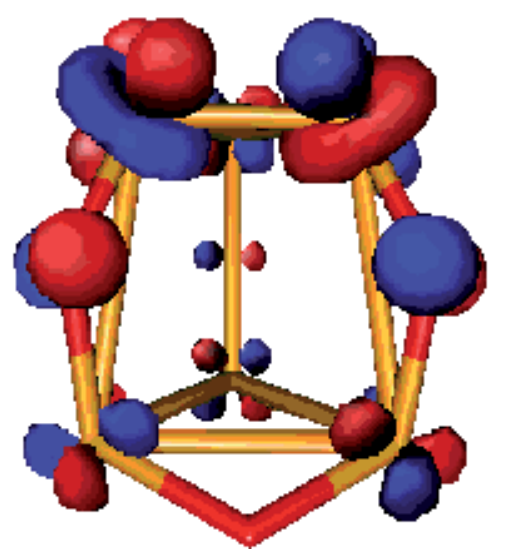

Fig. 14. $\mathrm{HOMO}$ orbital antibonding character of the $\mathrm{Co}_{6} \mathrm{O}_{8}$ particle.

It implies, that a non-compensate electron spin should be obtained. This situation is realized in the $\mathrm{Co}_{6} \mathrm{O}_{7}$ and $\mathrm{Co}_{6} \mathrm{O}_{8}$ particles. However, in the $\mathrm{Co}_{6} \mathrm{O}_{7}$ particle two pairs of weakly correlated electrons are present what leads to the disappearance of non-compensate spins. This is indicated by the isotropic g-tensor value which equals to 0.007 . However, in case of the $\mathrm{Co}_{6} \mathrm{O}_{8}$ particle, only one $\mathrm{Co}-\mathrm{Co}$ bond is present and only one pair of weakly correlated electrons should be found. This weak correlation indicates the nature of HOMO orbital that consists of anti-bonding $\mathrm{dz}^{2}$ type orbitals (Fig.14). Hence, the total spin of electrons is not compensated and, as a consequence, the particle exhibits paramagnetic features. This presumption is also confirmed by the isotropic g-tensor value, that is one of the largest between the particles described (Table 7). The small value of the isotropic g-tensor indicates that the electronic contribution to the magnetic properties of the particle is not very large, but it is essential. 
Additionally, we may supplement the proposition, that $\mathrm{Co}_{6} \mathrm{O}_{n}$ particles should be paramagnetics when the number of $\mathrm{Co}-\mathrm{Co}$ bonds on which the unpaired electrons are located might be odd (Table 7). It should be emphasized, that the number of Co-Co bonds was found on the basis of the electron density investigation results and only the bonds, where unpaired spin electrons could be presented, are mentioned. Indeed, the investigated particles with the odd number of Co-Co bonds exhibit paramagnetic or weak diamagnetic properties. However, it is not clear why the magnetic properties are different, i.e., formally, some, different features should appear.

Aiming to explain the above mentioned discrepancy, we investigated a dipole moment of these particles. The dipole moment indicates electron concentration places in the particle. On the other hand, the components of these dipole moments allow us to foresee the distribution of the above places. Both the concentration of electrons and their distribution helps us find additional spins that appeared due to the different oxidation state of the Co atoms (formally, we call the above spin as an ion one). The exception concerns $\mathrm{Co}_{6}$.

The components of the dipole moment of the particles that are paramagnetics or weak diamagnetics are shown in Table 9.

\begin{tabular}{|c|c|c|c|c|}
\hline Compounds & $\begin{array}{c}\text { Dipole moment, } \\
\text { a.u. }\end{array}$ & \multicolumn{3}{|c|}{ Dipole moment components, a.u. } \\
\hline & & $\mathbf{x}$ & y & z \\
\hline $\mathrm{Co}_{6}$ & 0.096 & -0.09 & -0.01 & -0.01 \\
\hline $\mathrm{Co}_{6} \mathrm{O}_{3}$ & 1.689 & 0.55 & 0.23 & -1.58 \\
\hline $\mathrm{Co}_{6} \mathrm{O}_{6}$ & 1.639 & -1.06 & -1.16 & 0.44 \\
\hline $\mathrm{Co}_{6} \mathrm{O}_{8}(\mathrm{I}$ isomer) & 2.652 & 2.60 & 0.45 & 0.23 \\
\hline $\mathrm{Co}_{6} \mathrm{O}_{8}(\mathrm{II}$ isomer & 2.059 & -1.08 & -0.01 & -1.75 \\
\hline $\begin{array}{c}\mathrm{Co}_{6} \mathrm{O}_{8}(\mathrm{III} \\
\text { isomer) }\end{array}$ & 1.372 & 1.37 & -0.06 & -0.03 \\
\hline
\end{tabular}

Table 9. Dipole moments and their components of the investigated paramagnetic or weak diamagnetical particles.

The $\mathrm{Co}_{6} \mathrm{O}_{8}$ particle is a paramagnetic due to the presence of non-compensate spin what indicates the value of the isotropic g-tensor of 0,51 (a free electron $g$-value is 2.00) because of the appearance of electrons on the anti-bonding orbitals.

So, as it was mentioned, the following different types of magnetic interactions could be obtained in the $\mathrm{Co}_{6} \mathrm{O}_{\mathrm{m}}$ nanoparticles: 1 . an uncompensated spin of weakly interacting electrons on the anti-bonding orbital; 2) the presence of Co ions that looses the odd number of electrons $\left(\mathrm{Co}^{+3}\right.$ and the like) leads to the emergence of the additional non-compensated spin.

The results obtained exhibit that the magnetic properties of nanoparticles could depend on the above interactions. The paramagnetic behaviour dominates when the non-compensated spin is present due to weakly interacting electrons on the anti-bonding orbital and this spin is not quenched by the ion spins. Let us remember, that $\mathrm{Co}_{6} \mathrm{O}_{3}$ and $\mathrm{Co}_{6} \mathrm{O}_{6}$ particles are weak diamagnetics, thought the isotropic g-tensor is not smaller than that of the $\mathrm{Co}_{6} \mathrm{O}_{8}$ particle. In 
these particles the ion spin is also presented what indicates a high dipole moment. The number of the $\mathrm{Co}^{+3}$ ions is 2 and 4 respectively in the $\mathrm{Co}_{6} \mathrm{O}_{3}$ and $\mathrm{Co}_{6} \mathrm{O}_{6}$ particles. However, the components of the dipole moment indicate that the ion spins are delocalized. The interaction between these spins leads to the quench of an electron spin, i.e. both spins (ion and non-compensated spin of electrons located on the anti-bonding orbital of Co-Co bond) are oriented so that the total spin equals to zero.

The opposite situation is realized in the $\mathrm{Co}_{6} \mathrm{O}_{8}$ particle: an ion spin is localized and one $\mathrm{Co}-\mathrm{Co}$ bond is present. In this case, the spins are oriented so that they are relatively parallel to each other. This prediction is supported by additional investigations of the isomers of the $\mathrm{Co}_{6} \mathrm{O}_{8}$ particle. It is necessary to mention, that one Co-Co bond is present in isomer II and a detailed investigation of the dipole moment indicates that it lies approximately in parallel to the Co-Co bonds. Therefore, the unpaired spins of a different nature support each other. Thus, the magnetizability of the I and II isomers of the $\mathrm{Co}_{6} \mathrm{O}_{8}$ particle is the same. In case of isomer III, all Co-Co bonds are dissolved, but an ion non-compensated spin is present. It implies that magnetic properties of the particle are determined by the localized ion spin only. Thus, the magnetizability of isomer III is lower than that of the other isomers investigated.

Hence, the paramagnetic behaviour of the cobalt oxide particle is dominating when the noncompensated spin is present due to weakly interacting electrons on the anti-bonding orbital and this spin is not quenched by the ion spins.

It is necessary to pay attention to other important observations. As it was earlier mentioned, the cobalt oxide particles are semiconductors and $\mathrm{Co}_{6} \mathrm{O}_{8}$ exhibits magnetic properties. It implies that this $\mathrm{Co}_{6} \mathrm{O}_{8}$ particle could be magnetic superconductor and could be implemented in electronic devices to provide a new type of the control of conduction, i.e. of the charge carrier and quantum spin state. Hence, this particle could be used in quantum computing.

\section{Absorption spectra of the $\mathrm{C}_{6} \mathrm{O}_{\mathrm{m}}(\mathrm{m}=1-9)$ nanoparticles}

It is known that the growing metallic particles are stabilized by the absorption of the polymer chains on the surface of the growing metal fragments, lowering their surface energy and creating a barrier to further aggregation. On the other hand, the organic coating of a particle prevents the surface from oxidation, rendering the particle stable over a long period. So, it is necessary to have a tool to investigate and control the process of stabilization of nanoparticles because the stabilization could be related to the oxidation of a metal particle and, as a consequence, it looses its magnetic properties. We believe that the knowledge concerning the shape and the nature of the absorption spectra of the $\mathrm{Co}_{6} \mathrm{O}_{\mathrm{m}}$ particles in the Vis and UV region could be a good tool for the investigation of the oxidation processes of Co nanoparticles. The above assumption is based on the results obtained that indicate the $\mathrm{Co}_{6}$ particle as a key element of larger particles.

As it was to mention in 3.1.1 we may divide the described particles into groups based on the changes of the $\mathrm{Co}_{6}$ structures. The groups are the following:

1. The particles that posses a shape of $\mathrm{Co}_{6}: \mathrm{Co}_{6}, \mathrm{Co}_{6} \mathrm{O}, \mathrm{Co}_{6} \mathrm{O}_{2}, \mathrm{Co}_{6} \mathrm{O}_{3}, \mathrm{Co}_{6} \mathrm{O}_{5}, \mathrm{Co}_{6} \mathrm{O}_{6}$ (group A).

2. The particles in which the distance between the planes (formed by atoms 1, 2, 3 and of 4,5 , and 6) is increased and one plane is rotated in respect of the other one by $\Pi / 4$ angle: $\mathrm{Co}_{6} \mathrm{O}_{7}, \mathrm{C}_{6} \mathrm{O}_{8}, \mathrm{Co}_{6} \mathrm{O}_{9}$ (group B). 
3. The rest $\left(\mathrm{Co}_{6} \mathrm{O}_{4}\right)$.

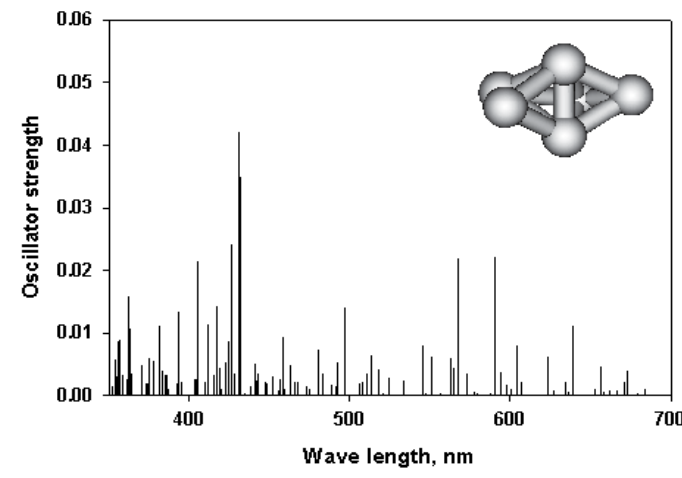

(a) $\mathrm{Co}_{6}$

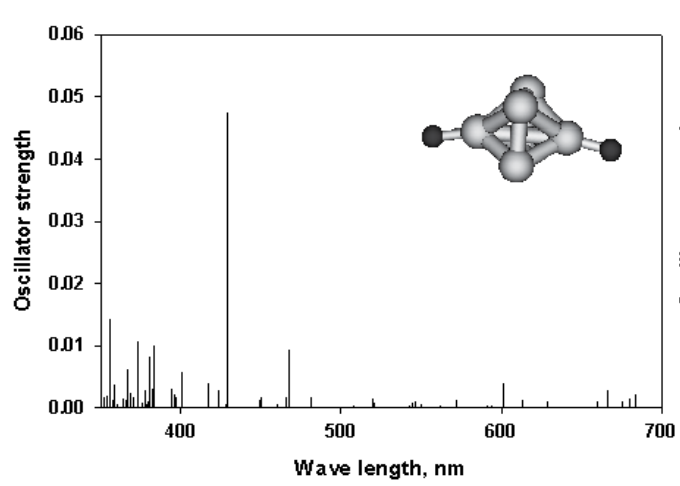

(c) $\mathrm{Co}_{6} \mathrm{O}_{2}$

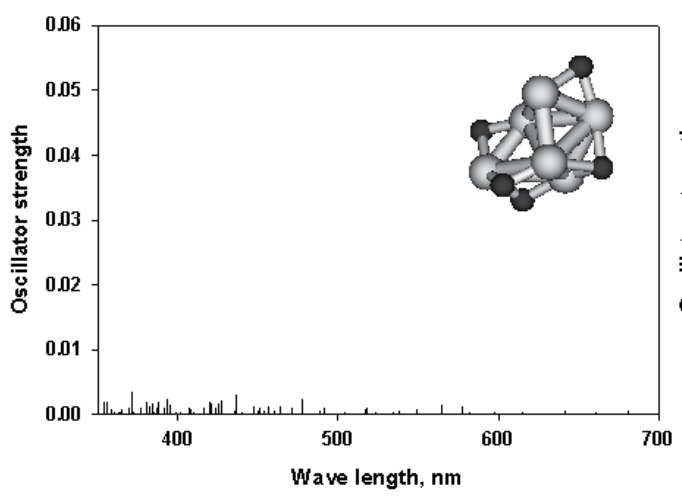

e) $\mathrm{Co}_{6} \mathrm{O}_{5}$

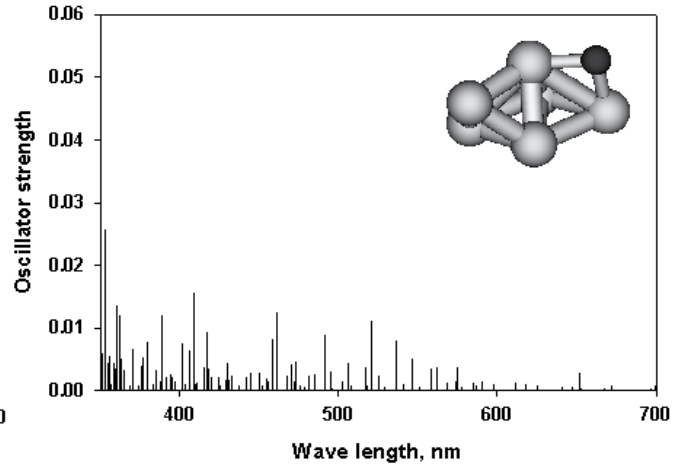

(b) $\mathrm{Co}_{6} \mathrm{O}$

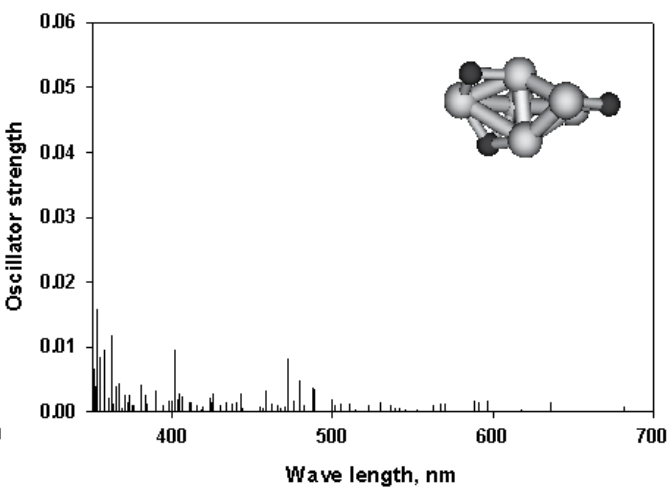

(d) $\mathrm{Co}_{6} \mathrm{O}_{3}$

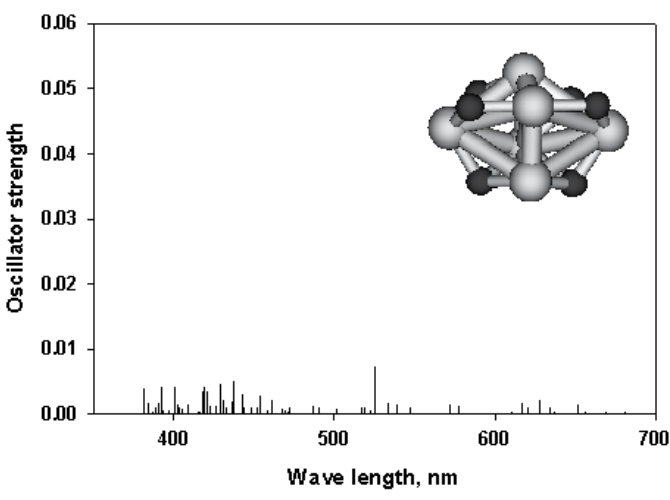

(f) $\mathrm{Co}_{6} \mathrm{O}_{6}$

Fig. 15. Absorption spectra of group A. At (a) there are spectra of a $\mathrm{Co}_{6}$ particle, while (b) - those of $\mathrm{Co}_{6} \mathrm{O}$; (c) in the middle, on the left, there is a spectrum of $\mathrm{Co}_{6} \mathrm{O}_{2}$;

(d) on the right $-\mathrm{Co}_{6} \mathrm{O}_{3}$; (e) at the bottom, on the left, there are spectra of $\mathrm{Co}_{6} \mathrm{O}_{5}$;

(f) on the right - those of $\mathrm{Co}_{6} \mathrm{O}_{6}$. The black circle indicates oxygen atoms,

while the grey one - cobalt atoms. 
The obtained absorption spectra of the particles making up groups A and B and their structures are presented in Fig. 15 and Fig. 16. The case of $\mathrm{Co}_{6} \mathrm{O}_{4}$ particle is different and should be investigated deeper, although the general tendency of absorption spectra changes described below are possible to foresee in the spectra of this particle, too.

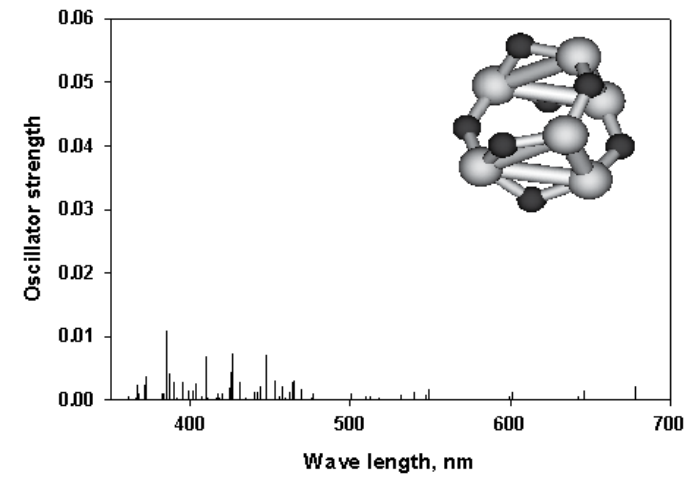

(a) $\mathrm{Co}_{6} \mathrm{O}_{7}$

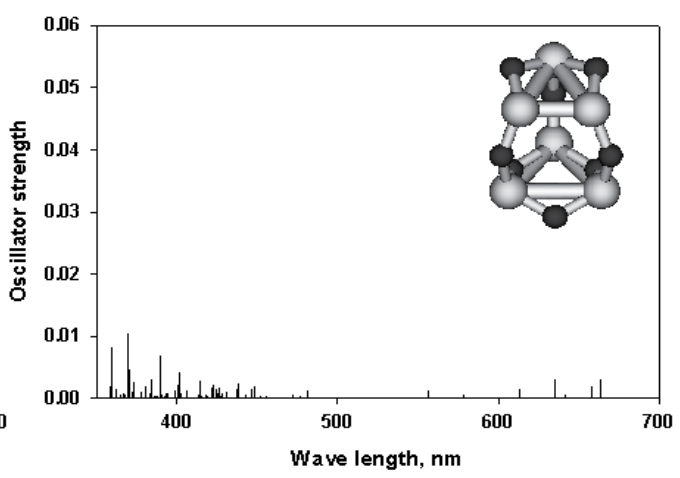

(b) $\mathrm{Co}_{6} \mathrm{O}_{8}$

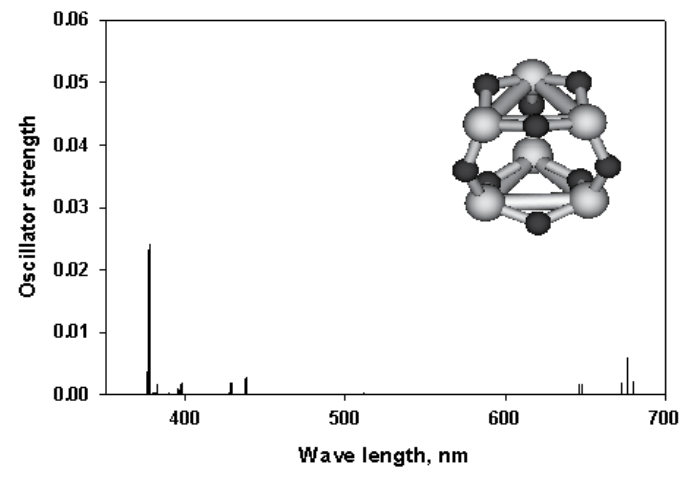

(c) $\mathrm{Co}_{6} \mathrm{O}_{9}$

Fig. 16. Absorption spectra of group $\mathrm{B}$ : (a) at the top on left there is a spectrum of $\mathrm{Co}_{6} \mathrm{O}_{7}$ particle, (b) on the right -those of $\mathrm{Co}_{6} \mathrm{O}_{8} ;$ (c) at the bottom, there are spectra of $\mathrm{Co}_{6} \mathrm{O}_{9}$. The black circle indicates oxygen atoms, while the grey one - those of cobalt.

Let us analyze the spectra of group A. It is obvious, that the intensity of absorption decreases especially in the [500;700] $\mathrm{nm}$ region with increasing of the oxygen number up till 5 , and starts increasing again when the number of oxygen atoms is 6 . The appearance of more intense absorption in the above region of the $\mathrm{Co}_{6} \mathrm{O}_{6}$ is related to the structure of this particle (Fig.15). The structure of the $\mathrm{Co}_{6} \mathrm{O}_{6}$ particle looks like the octahedron, while in the case of other particles investigated, the octahedron form is strongly deformed. The intensity of absorption in the region 300 to $400 \mathrm{~nm}$ increases when the number of oxygen atoms in the particle increases from 7 to 9 (Fig.16).

It is obvious, that with the increasing of the number of the oxygen atoms by one, the number of occupied orbitals in the $[-1 ; 0]$ a.u increase by three (Table 10). Moreover, the gap of the $\mathrm{Co}_{6}$ particle between occupied orbitals in the]-2; -1 [ a.u. region is not filled what is explained 
by the displacement of the orbitals of both $\mathrm{Co}_{6} \mathrm{O}_{\mathrm{m}}(\mathrm{m}=0-9)$ and an oxygen atom in respect of each other (Fig. 12). In case of $\mathrm{Co}_{6}$ particle, only three orbitals (HOMO, HOMO -1 and LUMO) of oxygen interact with the occupied orbitals of the particle, while in case of $\mathrm{Co}_{6} \mathrm{O}_{\mathrm{m}}$ particles, the number of interacting orbitals increases. Starting with $\mathrm{Co}_{6} \mathrm{O}$, the additional occupied level occurred in the gap of the $\mathrm{Co}_{6}$ particle between the occupied orbitals in the region of ]-2; -1[ a.u. However, the HOMO-LUMO gap increases. So, semiconductor properties of the $\mathrm{Co}_{6} \mathrm{O}_{\mathrm{m}}$ particles become stronger.

Naturally, that with the increasing number of oxygen atoms in the $\mathrm{Co}_{6}$ particle, the mixing orbital (the molecular orbital consists of cobalt and oxygen atomic orbitals) increases due to the $\mathrm{Co}$ and $\mathrm{O}$ atomic orbital interaction. The analysis of the contribution of the atomic orbital to the molecular orbitals confirms the predicted interaction. Moreover, due to the above interaction, the orbital splits and several orbitals that are occupied in Co or Co oxide nanoparticles should become virtual and vice versa. Hence, the transitions in the spectra region of [350;700] $\mathrm{nm}$ are of Co3d $\rightarrow$ Co3d type and they are allowed in a pure Co particle or particles with the oxygen number of 1-2 because the above mixing is not very strong.

When the number of oxygen atoms in the Co particle is 3-7, the transitions in the spectra region of $[350 ; 700] \mathrm{nm}$ are of $\mathrm{Co} 3 \mathrm{~d} \rightarrow \mathrm{Co} 3 \mathrm{dO} 2 \mathrm{p}$ orCo3dO2p $\rightarrow \mathrm{Co} 3 \mathrm{~d}$ types. It is emphasized, that starting with the number of six of oxygen atoms, only occupied orbitals of nanoparticles interact with the occupied orbital of oxygen atom, i.e. the above mentioned interaction between LUMO of the oxygen atom and the occupied orbital of $\mathrm{Co}_{6} \mathrm{O}_{\mathrm{m}}$ nanoparticle does not occur. The analysis of the most important orbitals for excitation indicates, that in the spectra of $\mathrm{Co}_{6} \mathrm{O}_{\mathrm{m}}(\mathrm{m}=0-5)$ the most intensive excitations correspond to $\mathrm{Co} 3 \mathrm{~d} \rightarrow \mathrm{Co} 3 \mathrm{~d}$ ones. Other partly allowed excitations correspond to $\mathrm{Co} 3 \mathrm{~d} \rightarrow \mathrm{Co} 3 \mathrm{dO} 2 \mathrm{p}$ ones. So, the number of $\mathrm{Co} 3 \mathrm{~d} \rightarrow \mathrm{Co} 3 \mathrm{dO} 2 \mathrm{p}$ excitations increases with the increased mixture of orbitals. Moreover, when the number of oxygen atoms is up to 7 , the $\mathrm{Co} 3 \mathrm{dO} 2 \mathrm{p} \rightarrow \mathrm{Co} 3 \mathrm{dO} 2 \mathrm{p}$ excitations are more relevant. On the other hand, the symmetry of particles is different what leads to different number of the transitions allowed. It is very well known, that a part of the possible excitations is forbidden when the symmetry group of the particles is high, while all possible excitations are allowed when the symmetry group of the particle is the lowest $\left(C_{1}\right)$.

\begin{tabular}{|c|c|c|c|c|}
\hline Particles & Virtual orbitals & \multicolumn{3}{|c|}{ Occupied orbitals } \\
\hline & {$[-1 ; 0]$ a.u. } & {$[-1 ;-0]$ a.u. } & {$[-2 ;-3]$ a. u. } & {$[-3 ;-4]$ a. u. } \\
\hline $\mathrm{Co}_{6}$ & 11 & 27 & 18 & 6 \\
$\mathrm{Co}_{6} \mathrm{O}$ & 13 & 30 & 18 & 6 \\
$\mathrm{Co}_{6} \mathrm{O}_{2}$ & 13 & 33 & 15 & 5 \\
$\mathrm{Co}_{6} \mathrm{O}_{3}$ & 12 & 36 & 17 & 6 \\
$\mathrm{Co}_{6} \mathrm{O}_{4}$ & 15 & 39 & 14 & 6 \\
$\mathrm{Co}_{6} \mathrm{O}_{5}$ & 13 & 42 & 18 & 4 \\
$\mathrm{Co}_{6} \mathrm{O}_{6}$ & 13 & 45 & 18 & 6 \\
$\mathrm{Co}_{6} \mathrm{O}_{7}$ & 15 & 48 & 18 & 6 \\
$\mathrm{Co}_{6} \mathrm{O}_{8}$ & 15 & 51 & 18 & 6 \\
$\mathrm{Co}_{6} \mathrm{O}_{9}$ & 16 & 54 & 18 & 6 \\
\hline
\end{tabular}

Table 10. The number of states of the $\mathrm{Co}_{6} \mathrm{O}_{m}(m=0-9)$ particles in the regions of different energy. 


\begin{tabular}{|c|ccccccccc|}
\hline Particle & $\mathrm{Co}_{6}$ & $\mathrm{Co}_{6} \mathrm{O}$ & $\mathrm{Co}_{6} \mathrm{O}_{2}$ & $\mathrm{Co}_{6} \mathrm{O}_{3}$ & $\mathrm{Co}_{6} \mathrm{O}_{5}$ & $\mathrm{Co}_{6} \mathrm{O}_{6}$ & $\mathrm{Co}_{6} \mathrm{O}_{7}$ & $\mathrm{Co}_{6} \mathrm{O}_{8}$ & $\mathrm{Co}_{6} \mathrm{O}_{9}$ \\
\hline $\begin{array}{c}\text { Symmetry } \\
\text { group }\end{array}$ & $\mathrm{C}_{2 \mathrm{v}}$ & $\mathrm{C}_{1}$ & $\mathrm{C}_{2 \mathrm{v}}$ & $\mathrm{C}_{1}$ & $\mathrm{C}_{1}$ & $\mathrm{C}_{2 \mathrm{v}}$ & $\mathrm{C}_{2 \mathrm{v}}$ & $\mathrm{C}_{1 \mathrm{~h}}$ & $\mathrm{C}_{3 \mathrm{v}}$ \\
\hline
\end{tabular}

Table 11. Approximate symmetry of the particles investigated.

Hence, the particles with higher symmetry absorb certain wave lengths more intensively, while the absorbance of non-symmetrical particles is not intensive, but a very broad one (Table 11, Figs. 15, 16). It allows us to conclude, that the investigated spectra of the Co nanoparticles in the region of [300; 700] $\mathrm{nm}$ could explain the oxidation of the particles and, as a consequence, their structure changes what lead to changes of magnetic properties.

Basing on the results obtained, we speculate that the dependence on the place of excitation could be related with the particle oxidation when considering the excitation of large particles (approximately of $200 \mathrm{~nm}$ ).

\section{Conclusions}

Herein, we report on the several important results related to magnetic properties of the Co nanoparticle.

The main important observations of the pure Co and oxidized nanoparticle are the following:

- The $\mathrm{Co}_{8}, \mathrm{Co}_{10}, \mathrm{Co}_{12}, \mathrm{Co}_{14}, \mathrm{Co}_{16}$ particles consist of $\mathrm{Co}_{6}$, thus these particles could be regarded to as the key element of the large Co nanoparticles.

- The face centered cubic structure which is slightly less closely packed, occurred in the $\mathrm{Co}_{14}$ and $\mathrm{Co}_{16}$ nanoparticles, while the other particles described are the elements of the FCC structure in the sense of the above conclusions.

- The key element of the $\mathrm{Co}_{6}$ is present in the $\mathrm{Co}_{6} \mathrm{O}_{n}(n=0-9,12)$ particles.

- The present investigations of the magnetic properties of Co and Co oxide particles resulted in the conclusion that a paramagnetic behaviour is dominating when the noncompensated spin is present due to the anti-bonding orbitals and such a spin is not quenched by the ion spins.

- The results of our investigations indicate that both a dipole interaction and particle agglomeration change magnetic properties of the Co nanoparticle.

- The intensity of absorption of $\mathrm{Co}_{6} \mathrm{O}_{m}(\mathrm{~m}=0-9)$ particles should be decreased in the [500;700] nm region with increasing of the number of the oxygen atom up to 5 , and should be increased again when number of oxygen atoms is 6 .

- $\quad$ The spectra of investigated particles become linear when the number of oxygen atoms in the above particle is even, while the absorption lines in spectra should be difficult to observe with odd number of oxygen.

- It is obtained, that in the spectra of $\mathrm{Co}_{6} \mathrm{O}_{\mathrm{m}}(\mathrm{m}=0-3)$ the most intensive excitations correspond to $\mathrm{Co} 3 \mathrm{~d} \rightarrow \mathrm{Co} 3 \mathrm{~d}$ excitations. The $\mathrm{Co} 3 \mathrm{dO} 2 \mathrm{p} \rightarrow \mathrm{Co} 3 \mathrm{dO} 2 \mathrm{p}$ excitations are more relevant in the spectra of the particles where the number of oxygen atoms is up to 7 , while in the rest particles the $\mathrm{Co} 3 \mathrm{~d} \rightarrow \mathrm{Co} 3 \mathrm{dO} 2 \mathrm{p}$ or $\mathrm{Co} 3 \mathrm{dO} 2 \mathrm{p} \rightarrow \mathrm{Co} 3 \mathrm{~d}$ types of excitation are obtained. 


\section{References}

Zalich M. A. and et al. (2006). Structural and Magnetic properties of oxidatively stable cobalt nanoparticles encapsulated in graphite shell. Chemistry of Materials, Vol. 18,p.p. 2648-2655, ISSN 0897-4756

Simeonidis K. and et al. (2008). Shape and composition oriented synthesis of Cobalt nanoparticles, Physics and Advanced materials Winter school, pp.1-8, Thessaloniki, Greece, January 14-18

Neamtu J and et al. (2005). Synthesis and Properties of Magnetic Nanoparticles with Potential Applications in Cancer Diagnostic. Technical Proceedings of the 2005 NSTI Nanotechnology Conference and Trade Show, Vol. 1, p.p. :222 - 224, ISBN:0-9767985-0-6, Anaheim, California, USA,May 8-12, 2005.

Sakurai M.I. and et al. (1998) Magic Numbers in Fe Clusters Produced by Laser Vaporization Source. Journal of the Physical Society of Japan, Vol. 67, No. 8, (August), pp. 2571-2573, ISSN 0031-9015

Gambardella P. and et al. (2003) Giant Magnetic Anisotropy of Single Cobalt Atoms and Nanoparticles. Science, Vol.300, No. 1130, (May), pp. 1130-1133, ISSN 0036-8075

Chen J. P. and et al. (1994) Magnetic properties of nanophase cobalt particles synthesized in inversed micelles. Journal of Applied Physics, Vol. 76, No. 10, (November), pp. 6316 6318 ISSN 021-8979

Ghosh M.; Sampathkumaran E. V.; Rao C.N.R. (2005) Synthesis and Magnetic Properties of CoO Nanoparticles. Chemistry Materials, Vol. 17, No. 9, (March), pp.2348 -2352 ISSN 0897-4756

Zhao Y. W. and et al.(2003) A simple method to prepare Uniform Co nanoparticles. IEEE Transactions on magnetics, Vol.39, No. 5, (September) , pp. 2764-2766, ISSN 0018-9464

Sun X. Ch.; Reyes-Gasga J.; Dong X. L. (2002) Formation and microstructure of Carbo encapsulated superparamagnetic Co nanoparticles. Molecular Physics, Vol. 100, No 19, (March), pp. 314-315, ISSN 0026-8976

Ram S. (2001) Allotropic phase transformations in HCP, FCC and BCC metastable structures in Co- nanoparticles. Material Science and Engineering A, Vol. 304-306, (May), pp. 923-927, ISSN 0921-5093

Gubin S.P. and et al. (2003) Magnetic and structural properties of Co nanoparticles in a polymeric matrix. Journal of Magnetism and Magnetic Materials,Vol. 265, No. 2, (September) pp. 234-242 ISSN 0304-8853

Tsukamoto S.; Koguchi N. (2000) Magic numbers in Ga clusters on GaAs $\left(\begin{array}{lll}0 & 0 & 1\end{array}\right)$ surface. Journal of Crystal Growth, Vol. 209, No.1-2, (February), pp. 258-262, ISSN 0022-0248

Guevara J.and et al. (1997) Electronic properties of transition-metal clusters:Consideration of the spillover in a bulk parametrization. Physical Review B, Vol. 55, No. 19, (May), pp.13283-13287 ISSN 1098-0121

Ma Q. M.and et al. (2006) Structures, stabilities and magnetic properties of small Co clusters. Physics Letter A, Vol. 358, No. 4, (October), pp. 289-296, ISSN 0375-9601

Galvez N. and et al. (2002). Apoferritin-encapsulated Ni and Co superparamagnetic nanoparticles. Erophysics. Letter, Vol. 76, No.5, (December), pp.142-148, ISSN 02955075 
Park I.-W. and et al. (2003). Magnetic properties and microstructure of cobalt nanoparticles in a polymer film. Solid State Communications, Vol. 44, No.7 (May), pp. 385-389 ISSN 0038-1098

Yang H.T.and et al. (2004). Synthesis and magnetic properties of e-Co nanoparticles. Surface and interface analysis, Vol. 36, No. 2, (February), pp.155-160, ISSN 1096-9918

Becke A. D. (1993) Density-functional thermochemistry. iii. the role of exact exchange. Journal of Chemical Physics, Vol. 98, No 7, (April), pp.5648-5652, ISSN 0021-9606

Gordon M. S. and et al. (1982) Self-consistent molecular-orbital methods. 22. Small splitvalence basis sets for second-row elements. Journal of the American Chemical Society, Vol. 104, No.10, (May), pp. 2797-2803, ISSN 0002-7863

Schmidt M.W. and et al. (2004) Gaussian, Inc., Wallingford CT, ISBN 0963676938, Pittsburgh, USA

Lutnæs O. B. and et al. (2005) Benchmarking density-functional-theory calculations of rotational $g$ tensors and magnetizabilities using accurate coupled-cluster calculations . Journal of Chemical Physics, Vol. 131, No.14, (September ), pp.144104144119, ISSN 0021-9606

Schafer, A.; Horn, H.; Ahlrichs, R. (1992) Fully optimized contracted Gaussian basis sets for atom Li to Kr. Journal of Chemical Physics, Vol. 97, No.4, (May), pp. 2571-2577, ISSN 0021-9606

Wu N. and et al. (2004). Interaction of Fatty Acid Monolayers with Cobalt Nanoparticles. Nano Letters, Vol. 4, No. 2, (January), pp. 383-386, ISSN 1530-6984

Fan, H. J.; Liu, Ch. W.; Liao, M. Sh. (1997). Geometry, electronic structure and magnetism of small $\mathrm{Co}_{n}(n=2-8)$ clusters. Chemical Physics Letter, Vol. 273, No. 5-6, (July), pp. 353-359, ISSN 0009-2614.

Meldrum, A.; Boatner, L. A.; Sorge, K. (2003) Nuclear Instruments and Methods in Physics Research Section B: Beam Interactions with Materials and Atoms, Vol. 207,No.1, (May), pp. 36-44, ISSN 0168-583X

Ichiyanaga, Y.; Yamada, S. (2005) The size-depended magnetic properties of $\mathrm{Co}_{3} \mathrm{O}_{4}$ nanoparticles. Polyhedron, Vol.24, No. 16-17, (November), pp. 2813-2816, ISSN 0277-5387,

Graf, Ch. P.; Birringer, R.; Michels, A. (2006) Sythhesis and magnetic properties of cobalt nanocubes. Physical Review B, Vol. 73, No. 21, (April), pp. 212401 - 212404, ISSN 1550-235x

Resnick, D.A and et al. (2006) Magnetic properties of $\mathrm{Co}_{3} \mathrm{O}_{4}$ nanoparticles miniralized in Listeria innoucua Dps. Journal of Applied Physics. Vol. 99 (April), 08Q501-3, ISSN 1089-7550

Salavati-Niasari, M.; Afsaneh Khansari, A.; Davar, F. (2009) Synthesis and characterization of cobalt oxide nanoparticles by thermal treatment process. Inorganica Chimica Acta, Vol. 362, No. 14, (November), p.p. 4937-4942, ISSN: 0020-1693

Papis, E. and et al. (2009) Engineered cobalt oxide nanoparticles readily enter cells, Toxicology letters, Vol. 189 ,(June), p.p.253-259, ISSN: 0378-4274

Sakurai, K. and et al. (1998) Magic numbers in Fe clusters produced by laser vaporization source. Journal of the Physical Society of Japan, Vol. 8 : p.p. 2571-2573.

Gambardella, P. and et al. (2003) Giant magnetic anisotropy of Co atoms and nanoparticles. Science, Vol. 300, No. 5622, (May 16), p.p.1130-1133, ISSN 1095-9203

King, S.; Hyunh, K.; Tannenbaum, R. (2003) Kinetics of Nucleation, Growth, and stabilization of cobalt oxide nanoclusters. Journal of Physical Chemistry B, Vol. 107, No.44, (October 15), p.p. 10297-12104, ISSN 1520-6106 
Nogues, J. and et al. (2006) Shell-driven magnetic stability in core-shell nanoparticles. Physical Review Letter, Vol 97, No. 15, (October 13), p.p.1572031.1572034, ISSN · 0031-9007 


\title{
Nanofluids
}

\author{
Wei Yu, Huaqing Xie and Lifei Chen \\ Shanghai Second Polytechnic University \\ P. R. China
}

\section{Introduction}

Nanofluids are a new class of fluids engineered by dispersing nanometer-sized materials (nanoparticles, nanofibers, nanotubes, nanorods, nanosheet, or droplets) in base fluids. In other words, nanofluids are nanoscale colloidal suspensions containing solid nanomaterials. They are two-phase systems with one phase (solid phase) in another (liquid phase). For a two-phase system, there are some important issues we have to face. One of the most important issues is the stability of nanofluids and it remains a big challenge to achieve desired stability of nanofluids. In this paper we will review the new progress in the methods for preparing stable nanofluids and summarize the stability mechanisms. In recent years, nanofluids have attracted more and more attention. The main driving force for nanofluids research lies in a wide range of applications. Although some review articles involving the progress of nanofluid investigation were published in the past several years [1-6], most of the reviews are concerned on the experimental and theoretical studies of the thermophysical properties or the convective heat transfer of nanofluids. The purpose of this paper will focuses on the new preparation methods and stability mechanisms, especially the new application trends for nanofluids in addition to the heat transfer properties of nanofluids. We will try to find some challenging issues that need to be solved for future research based on the review on these aspects of nanofluids.

\section{Preparation methods for nanofluids}

\subsection{Two-step method}

Two-step method is the most widely used method for preparing nanofluids. Nanoparticles, nanofibers, nanotubes or other nanomaterials used in this method are first produced as dry powders by chemical or physical methods. Then the nanosized powder will be dispersed into a fluid in the second processing step with the help of intensive magnetic force agitation, ultrasonic agitation, high-shear mixing, homogenizing and ball milling. Two-step method is the most economic method to produce nanofluids in large scale, because nanopowder synthesis techniques have already been scaled up to industrial production levels. Due to the high surface area and surface activity, nanoparticles have the tendency to aggregate. The important technique to enhance the stability of nanoparticles in fluids is the use of surfactants. However the functionality of the surfactants under high temperature is also a big concern, especially for high temperature applications. 
Due to the difficulty in preparing stable nanofluids by two-step method, several advanced techniques are developed to produce nanofluids, including one-step method. In the following part, we will introduce one-step method in detail.

\subsection{One-step method}

To reduce the agglomeration of nanoparticles, Choi et al. developed a one-step physical vapor condensation method to prepare $\mathrm{Cu}$ /ethylene glycol nanofluids [7]. The one-step process consists of simultaneously making and dispersing the particles in the fluid. In this method the processes of drying, storage, transportation, and dispersion of nanoparticles are avoided, so the agglomeration of nanoparticles is minimized and the stability of fluids is increased [5]. The one-step processes can prepare uniformly dispersed nanoparticles and the particles can be stably suspended in the base fluid. The vacuum-SANSS (submerged arc nanoparticle synthesis system) is another efficient method to prepare nanofluids using different dielectric liquids $[8,9]$. The different morphologies are mainly influenced and determined by various thermal conductivity properties of the dielectric liquids. The nanoparticles prepared exhibit needle-like, polygonal, square and circular morphological shapes. The method avoids the undesired particle aggregation fair well.

One-step physical method cannot synthesize nanofluids in large scale and the cost is also high, so the one-step chemical method is developing rapidly. Zhu et al. presented a novel one-step chemical method for preparing copper nanofluids by reducing $\mathrm{CuSO}_{4} \cdot 5 \mathrm{H}_{2} \mathrm{O}$ with $\mathrm{NaH}_{2} \mathrm{PO}_{2} \cdot \mathrm{H}_{2} \mathrm{O}$ in ethylene glycol under microwave irradiation [10]. Well-dispersed and stably suspended copper nanofluids were obtained. Mineral oil-based nanofluids containing silver nanoparticles with a narrow size distribution were also prepared by this method [11]. The particles could be stabilized by Korantin, which coordinated to the silver particle surfaces via two oxygen atoms forming a dense layer around the particles. The silver nanoparticle suspensions were stable for about 1 month. Stable ethanol based nanofluids containing silver nanoparticles could be prepared by microwave-assisted one-step method [12]. In the method, polyvinylpyrrolidone (PVP) was employed as the stabilizer of colloidal silver and reducing agent for silver in solution. The cationic surfactant octadecylamine (ODA) is also an efficient phase-transfer agent to synthesize silver colloids [13]. The phase transfer of the silver nanoparticles arises due to coupling of the silver nanoparticles with the ODA molecules present in organic phase via either coordination bond formation or weak covalent interaction.

However there are some disadvantages for one-step method. The most important one is that the residual reactants are left in the nanofluids due to incomplete reaction or stabilization. It is difficult to elucidate the nanoparticle effect without eliminating this impurity effect.

\subsection{Other novel methods}

Wei et al. developed a continuous-flow microfluidic microreactor to synthesize copper nanofluids. By this method, copper nanofluids can be continuously synthesized, and their microstructure and properties can be varied by adjusting parameters such as reactant concentration, flow rate and additive. $\mathrm{CuO}$ nanofluids with high solid volume fraction (up to $10 \mathrm{vol} \%$ ) can be synthesized through a novel precursor transformation method with the 
help of ultrasonic and microwave irradiation [14]. The precursor $\mathrm{Cu}(\mathrm{OH})_{2}$ is completely transformed to $\mathrm{CuO}$ nanoparticle in water under microwave irradiation. The ammonium citrate prevents the growth and aggregation of nanoparticles, resulting in a stable $\mathrm{CuO}$ aqueous nanofluid with higher thermal conductivity than those prepared by other dispersing methods. Phase-transfer method is also a facile way to obtain monodisperse noble metal colloids [15]. In a water-cyclohexane two-phase system, aqueous formaldehyde is transferred to cyclohexane phase via reaction with dodecylamine to form reductive intermediates in cyclohexane. The intermediates are capable of reducing silver or gold ions in aqueous solution to form dodecylamine protected silver and gold nanoparticles in cyclohexane solution at room temperature. Feng et al. used the aqueous-organic phasetransfer method for preparing gold, silver and platinum nanoparticles on the basis of the decrease of the $\mathrm{PVP}^{\prime}$ s solubility in water with the temperature increase [16]. Phase-transfer method is also applied for preparing stable kerosene based $\mathrm{Fe}_{3} \mathrm{O}_{4}$ nanofluids. Oleic acid is successfully grafted onto the surface of $\mathrm{Fe}_{3} \mathrm{O}_{4}$ nanoparticles by chemisorbed mode, which lets $\mathrm{Fe}_{3} \mathrm{O}_{4}$ nanoparticles have good compatibility with kerosene [17]. The $\mathrm{Fe}_{3} \mathrm{O}_{4}$ nanofluids prepared by phase-transfer method do not show the previously reported "time dependence of the thermal conductivity characteristic". The preparation of nanofluids with controllable microstructure is one of the key issues. It is well known that the properties of nanofluids strongly depend on the structure and shape of nanomaterials. The recent research shows that nanofluids synthesized by chemical solution method have both higher conductivity enhancement and better stability than those produced by the other methods [18]. This method is distinguished from the others by its controllability. The nanofluid microstructure can be varied and manipulated by adjusting synthesis parameters such as temperature, acidity, ultrasonic and microwave irradiation, types and concentrations of reactants and additives, and the order in which the additives are added to the solution.

\section{The stability of nanofluids}

The agglomeration of nanoparticles results in not only the settlement and clogging of microchannels but also the decreasing of thermal conductivity of nanofluids. So the investigation on stability is also a key issue that influences the properties of nanofluids for application, and it is necessary to study and analyze influencing factors to the dispersion stability of nanofluids. This section will contain: A) the stability evaluation methods for nanofluids; B) the ways to enhance the stability of nanofluids and C) the stability mechanisms of nanofluids.

\subsection{The stability evaluation methods for nanofluids}

\subsubsection{Sedimentation and centrifugation methods}

Many methods have been developed to evaluate the stability of nanofluids. The simplest method is sedimentation method $[19,20]$. The sediment weight or the sediment volume of nanoparticles in a nanofluid under an external force field is an indication of the stability of the characterized nanofluid. The variation of concentration or particle size of supernatant particle with sediment time can be obtained by special apparatus [5]. The nanofluids are considered to be stable when the concentration or particle size of supernatant particles keeps constant. Sedimentation photograph of nanofluids in test tubes taken by a camera is also a usual method for observing the stability of nanofluids [5]. Zhu et al. used a sedimentation 
balance method to measure the stability of the graphite suspension [21]. The tray of sedimentation balance immerged in the fresh graphite suspension. The weight of sediment nanoparticles during a certain period was measured. The suspension fraction of graphite nanoparticles at a certain time could be calculated. For the sedimentation method, long period for observation is the defect. Therefore centrifugation method is developed to evaluate the stability of nanofluids. Singh et al. applied the centrifugation method to observe the stability of silver nanofluids prepared by the microwave synthesis in ethanol by reduction of $\mathrm{AgNO}_{3}$ with PVP as stabilizing agent [12]. It has been found that the obtained nanofluids are stable for more than 1 month in the stationary state and more than $10 \mathrm{~h}$ under centrifugation at 3,000 rpm without sedimentation. Excellent stability of the obtained nanofluid is due to the protective role of PVP as it retards the growth and agglomeration of nanoparticles by steric effect. $\mathrm{Li}$ et al. prepared the aqueous polyaniline colloids, and used the centrifugation method to evaluate the stability of the colloids [22]. Electrostatic repulsive forces between nanofibers enabled the long-term stability of the colloids.

\subsubsection{Zeta potential analysis}

Zeta potential is electric potential in the interfacial double layer at the location of the slipping plane versus a point in the bulk fluid away from the interface, and it shows the potential difference between the dispersion medium and the stationary layer of fluid attached to the dispersed particle. The significance of zeta potential is that its value can be related to the stability of colloidal dispersions. So, colloids with high zeta potential (negative or positive) are electrically stabilized while colloids with low zeta potentials tend to coagulate or flocculate. In general, a value of $25 \mathrm{mV}$ (positive or negative) can be taken as the arbitrary value that separates low-charged surfaces from highly-charged surfaces. The colloids with zeta potential from 40 to $60 \mathrm{mV}$ are believed to be good stable, and those with more than $60 \mathrm{mV}$ have excellent stability. Kim et al. prepared Au nanofluids with an outstanding stability even after 1 month although no dispersants were observed [23]. The stability is due to a large negative zeta potential of Au nanoparticles in water. The influence of $\mathrm{pH}$ and sodium dodecylbenzene sulfonate (SDBS) on the stability of two water-based nanofluids was studied [24], and zeta potential analysis was an important technique to evaluate the stability. Zhu et al. [25] measured the zeta potential of $\mathrm{Al}_{2} \mathrm{O}_{3}-\mathrm{H}_{2} \mathrm{O}$ nanofluids under different $\mathrm{pH}$ values and different SDBS concentration. The Derjaguin-LaudauVerwey-Overbeek (DLVO) theory was used to calculate attractive and repulsive potentials. Cationic gemini surfactant as stabilizer was used to prepare stable water based nanofluids containing MWNTs [26]. Zeta potential measurements were employed to study the absorption mechanisms of the surfactants on the MWNT surfaces with the help of Fourier transformation infrared spectra.

\subsubsection{Spectral absorbency analysis}

Spectral absorbency analysis is another efficient way to evaluate the stability of nanofluids. In general, there is a linear relationship between the absorbency intensity and the concentration of nanoparticles in fluid. Huang et al. evaluated the dispersion characteristics of alumina and copper suspensions using the conventional sedimentation method with the help of absorbency analysis by using a spectrophotometer after the suspensions deposited for $24 \mathrm{~h}$ [27]. The stability investigation of colloidal FePt nanoparticle systems was done via 
spectrophotometer analysis [28]. The sedimentation kinetics could also be determined by examining the absorbency of particle in solution [25].

If the nanomaterials dispersed in fluids have characteristic absorption bands in the wavelength $190-1100 \mathrm{~nm}$, it is an easy and reliable method to evaluate the stability of nanofluids using UV-vis spectral analysis. The variation of supernatant particle concentration of nanofluids with sediment time can be obtained by the measurement of absorption of nanofluids because there is a linear relation between the supernatant nanoparticle concentration and the absorbance of suspended particles. The outstanding advantage comparing to other methods is that UV-vis spectral analysis can present the quantitative concentration of nanofluids. Hwang et al. [29] studied the stability of nanofluids with the UV-vis spectrophotometer. It was believed that the stability of nanofluids was strongly affected by the characteristics of the suspended particles and the base fluid such as particle morphology. Moreover, addition of a surfactant could improve the stability of the suspensions. The relative stability of MWNT nanofluids [26] could be estimated by measuring the UV-vis absorption of the MWNT nanofluids at different sediment times. From the above relation between MWNT concentration and its UV-vis absorbance value the concentration of the MWNT nanofluids at different sediment times could be obtained. The above three methods can be united to investigate the stability of nanofluids. For example, Li et al. evaluated the dispersion behavior of the aqueous copper nano-suspensions under different $\mathrm{pH}$ values, different dispersant type and concentration by the method of zeta potential, absorbency and sedimentation photographs [20].

\subsection{The ways to enhance the stability of nanofluids}

\subsubsection{Surfactants used in nanofluids}

Surfactants used in nanofluids are also called dispersants. Adding dispersants in the twophase systems is an easy and economic method to enhance the stability of nanofluids. Dispersants can markedly affect the surface characteristics of a system in small quantity. Dispersants consists of a hydrophobic tail portion, usually a long-chain hydrocarbon, and a hydrophilic polar head group. Dispersants are employed to increase the contact of two materials, sometimes known as wettability. In a two-phase system, a dispersant tends to locate at the interface of the two phases, where it introduces a degree of continuity between the nanoparticles and fluids. According to the composition of the head, surfactants are divided into four classes: non-ionic surfactants without charge groups in its head (include polyethylene oxide, alcohols, and other polar groups); anionic surfactants with negatively charged head groups (anionic head groups include long-chain fatty acids, sulfosuccinates, alkyl sulfates, phosphates, and sulfonates); cationic surfactants with positively charged head groups (cationic surfactants may be protonated long-chain amines and long-chain quaternary ammonium compounds); and amphoteric surfactants with zwitterionic head groups (charge depends on $\mathrm{pH}$. The class of amphoteric surfactants is represented by betaines and certain lecithins). How to select suitable dispersants is a key issue. In general, when the base fluid of nanofluids is polar solvent, we should select water soluble surfactants, otherwise we will select oil soluble. For nonionic surfactants, we can evaluate the solubility through the term hydrophilic/lipophilic balance (HLB) value. The lower the HLB number the more oil soluble the surfactants, and in turn the higher the HLB number the more water-soluble the surfactants is. The HLB value can be obtained easily by many handbooks. 


\subsubsection{Surface modification techniques-surfactant free method}

Although surfactant addition is an effective way to enhance the dispersibility of nanoparticles, surfactants might cause several problems [30]. For example, the addition of surfactants may contaminate the heat transfer media. Surfactants may produce foams when heating, while heating and cooling are routinely processes in heat exchange systems. Furthermore surfactant molecules attaching on the surfaces of nanoparticles may enlarge the thermal resistance between the nanoparticles and the base fluid, which may limit the enhancement of the effective thermal conductivity. Use of functionalized nanoparticles is a promising approach to achieve long-term stability of nanofluid. It represents the surfactant free technique. Yang et al. presented a work on the synthesis of functionalized silica $\left(\mathrm{SiO}_{2}\right)$ nanoparticles by grafting silanes directly to the surface of silica nanoparticles in original nanoparticle solutions [31]. One of the unique characteristics of the nanofluids was that no deposition layer formed on the heated surface after a pool boiling process. Chen et al. introduced hydrophilic functional groups on the surface of the nanotubes by mechanochemical reaction [29]. The prepared nanofluids, with no contamination to medium, good fluidity, low viscosity, high stability, and high thermal conductivity, would have potential applications as coolants in advanced thermal systems. A wetmechanochemical reaction was applied to prepare surfactant-free nanofluids containing double- and single-walled CNTs. Results from the infrared spectrum and zeta potential measurements showed that the hydroxyl groups had been introduced onto the treated CNT surfaces [32]. Plasma treatment was used to modify the surface characteristics of diamond nanoparticles [33]. Through plasma treatment using gas mixtures of methane and oxygen, various polar groups were imparted on the surface of the diamond nanoparticles, improving their dispersion property in water. A stable dispersion of titania nanoparticles in an organic solvent of diethylene glycol dimethylether (diglyme) was successfully prepared using a ball milling process [34]. In order to enhance dispersion stability of the solution, surface modification of dispersed titania particles was carried out during the centrifugal bead mill process. Surface modification was utilized with silane coupling agents, (3-acryloxypropyl) trimethoxysilane and trimethoxypropylsilane. Zinc oxide nanoparticles could be modified by polymethacrylic acid (PMAA) in aqueous system [35]. The hydroxyl groups of nano-ZnO particle surface could interact with carboxyl groups of PMAA and form poly (zinc methacrylate) complex on the surface of nano-ZnO. PMAA enhanced the dispersibility of nano- $\mathrm{ZnO}$ particles in water. The modification did not alter the crystalline structure of the $\mathrm{ZnO}$ nanoparticles.

\subsection{Stability mechanisms of nanofluids}

Particles in dispersion may adhere together and form aggregates of increasing size which may settle out due to gravity. Stability means that the particles do not aggregate at a significant rate. The rate of aggregation is in general determined by the frequency of collisions and the probability of cohesion during collision. Derjaguin, Verway, Landau and Overbeek (DVLO) developed a theory which dealt with colloidal stability [36, 37]. DLVO theory suggests that the stability of a particle in solution is determined by the sum of van der Waals attractive and electrical double layer repulsive forces that exist between particles as they approach each other due to the Brownian motion they are undergoing. If the attractive force is larger than the repulsive force, the two particles will collide, and the 
suspension is not stable. If the particles have a sufficient high repulsion, the suspensions will exist in stable state. For stable nanofluids or colloids, the repulsive forces between particles must be dominant. According to the types of repulsion, the fundamental mechanisms that affect colloidal stability are divided into two kinds, one is steric repulsion, and another is electrostatic (charge) repulsion. For steric stabilization, polymers are always involved into the suspension system, and they will adsorb onto the particles surface, producing an additional steric repulsive force. For example, Zinc oxide nanoparticles modified by PMAA have good compatibility with polar solvents [35]. Silver nanofluids are very stable due to the protective role of PVP as it retards the growth and agglomeration of nanoparticles by steric effect. PVP is an efficient agent to improve the stability of graphite suspension [21]. The steric effect of polymer dispersant is determined by the concentration of the dispersant. If the PVP concentration is low, the surface of the graphite particles is gradually coated by PVP molecules with the increase of PVP. Kamiya et al. studied the effect of polymer dispersant structure on electrosteric interaction and dense alumina suspension behavior [38]. An optimum hydrophilic to hydrophobic group ratio was obtained from the maximum repulsive force and minimum viscosity. For electrostatic stabilization, surface charge will be developed through one or more of the following mechanisms: 1) preferential adsorption of ions; 2) dissociation of surface charged species; 3) isomorphic substitution of ions; 4) accumulation or depletion of electrons at the surface and 5) physical adsorption of charged species onto the surface.

\section{Application of nanofluids}

\subsection{Heat transfer Intensification}

Since the origination of the nanofluid concept about a decade ago, the potentials of nanofluids in heat transfer applications have attracted more and more attention. Up to now, there are some review papers, which present overviews of various aspects of nanofluids [1, 3-6, 39-44], including preparation and characterization, techniques for the measurements of thermal conductivity, theory and model, thermophysical properties, convective heat transfer. In this part, we will summarize the applications of nanofluids in heat transfer enhancement.

\subsubsection{Electronic applications}

Due to higher density of chips, design of electronic components with more compact makes heat dissipation more difficult. Advanced electronic devices face thermal management challenges from the high level of heat generation and the reduction of available surface area for heat removal. So, the reliable thermal management system is vital for the smooth operation of the advanced electronic devices. In general, there are two approaches to improve the heat removal for electronic equipment. One is to find an optimum geometry of cooling devices; another is to increase the heat transfer capacity. Recent researches illustrated that nanofluids could increase the heat transfer coefficient by increasing the thermal conductivity of a coolant. Jang et al. designed a new cooler, combined microchannel heat sink with nanofluids [45]. Higher cooling performance was obtained when compared to the device using pure water as working medium. Nanofluids reduced both the thermal resistance and the temperature difference between the heated microchannel wall and the coolant. A combined microchannel heat sink with nanofluids had the potential as the next 
generation cooling devices for removing ultra-high heat flux. Nguyen et al. designed a closed liquid-circuit to investigate the heat transfer enhancement of a liquid cooling system, by replacing the base fluid (distilled water) with a nanofluid composed of distilled water and $\mathrm{Al}_{2} \mathrm{O}_{3}$ nanoparticles at various concentrations [46]. Measured data have clearly shown that the inclusion of nanoparticles within the distilled water has produced a considerable enhancement in convective heat transfer coefficient of the cooling block. With particle loading $4.5 \mathrm{vol} \%$, the enhancement is up to $23 \%$ with respect to that of the base fluid. It has also been observed that an augmentation of particle concentration has produced a clear decrease of the junction temperature between the heated component and the cooling block. Silicon microchannel heat sink performance using nanofluids containing $\mathrm{Cu}$ nanoparticles was analyzed [47]. It was found nanofluids could enhance the performance as compared with that using pure water as the coolant. The enhancement was due to the increase in thermal conductivity of coolant and the nanoparticle thermal dispersion effect. The other advantage was that there was no extra pressure drop since the nanoparticle was small and particle volume fraction was low.

The thermal requirements on the personal computer become much stricter with the increase in thermal dissipation of CPU. One of the solutions is the use of heat pipes. Nanofluids, employed as working medium for conventional heat pipe, have shown higher thermal performances, having the potential as a substitute for conventional water in heat pipe. At a same charge volume, there is a significant reduction in thermal resistance of heat pipe with nanofluid containing gold nanoparticles as compared with water [48]. The measured results also show that the thermal resistance of a vertical meshed heat pipe varies with the size of gold nanoparticles. The suspended nanoparticles tend to bombard the vapor bubble during the bubble formation. Therefore, it is expected that the nucleation size of vapor bubble is much smaller for fluid with suspended nanoparticles than that without them. This may be the major reason for reducing the thermal resistance of heat pipe. Chen et al. studied the effect of a nanofluid on flat heat pipe (FHP) thermal performance [49], using silver nanofluid as the working fluid. The temperature difference and the thermal resistance of the FHP with the silver nanoparticle solution were lower than those with pure water. The plausible reasons for enhancement of the thermal performance of the FHP using the nanofluid can be explained by the critical heat flux enhancement by higher wettability and the reduction of the boiling limit. Nanofluid oscillating heat pipe with ultrahighperformance was developed by Ma et al. [50]. They combined nanofluids with thermally excited oscillating motion in an oscillating heat pipe, and heat transport capability significantly increased. For example, at the input power of $80.0 \mathrm{~W}$, diamond nanofluid could reduce the temperature difference between the evaporator and the condenser from 40.9 to $24.3^{\circ} \mathrm{C}$. This study would accelerate the development of a highly efficient cooling device for ultrahigh-heat-flux electronic systems. The thermal performance investigation of heat pipe indicated that nanofluids containing silver or titanium nanoparticles could be used as an efficient cooling fluid for devices with high energy density. For a silver nanofluid, the temperature difference decreased $0.56-0.65^{\circ} \mathrm{C}$ compared to water at an input power of $30-50$ $\mathrm{W}$ [51]. For the heat pipe with titanium nanoparticles at a volume concentration of $0.10 \%$, the thermal efficiency is $10.60 \%$ higher than that with the based working fluid [52]. These positive results are promoting the continued research and development of nanofluids for such applications. 


\subsubsection{Transportation}

Nanofluids have great potentials to improve automotive and heavy-duty engine cooling rates by increasing the efficiency, lowering the weight and reducing the complexity of thermal management systems. The improved cooling rates for automotive and truck engines can be used to remove more heat from higher horsepower engines with the same size of cooling system. Alternatively, it is beneficial to design more compact cooling system with smaller and lighter radiators. It is in turn benefit the high performance and high fuel economy of car and truck. Ethylene glycol based nanofluids have attracted much attention in the application as engine coolant [53-55], due to the low-pressure operation compared with a 50/50 mixture of ethylene glycol and water, which is the nearly universally used automotive coolant. The nanofluids has a high boiling point, and it can be used to increase the normal coolant operating temperature and then reject more heat through the existing coolant system [56]. Kole et al. prepared car engine coolant $\left(\mathrm{Al}_{2} \mathrm{O}_{3}\right.$ nanofluid) using a standard car engine coolant (HP KOOLGARD) as the base fluid [57], and studied the thermal conductivity and viscosity of the coolant. The prepared nanofluid, containing only $3.5 \%$ volume fraction of $\mathrm{Al}_{2} \mathrm{O}_{3}$ nanoparticles, displayed a fairly higher thermal conductivity than the base fluid, and a maximum enhancement of $10.41 \%$ was observed at room temperature. Tzeng et al. [58] applied nanofluids to the cooling of automatic transmissions. The experimental platform was the transmission of a four-wheel drive vehicle. The used nanofluids were prepared by dispersing $\mathrm{CuO}$ and $\mathrm{Al}_{2} \mathrm{O}_{3}$ nanoparticles into engine transmission oil. The results showed that $\mathrm{CuO}$ nanofluids produced the lower transmission temperatures both at high and low rotating speeds. From the thermal performance viewpoint, the use of nanofluid in the transmission has a clear advantage.

The researchers of Argonne National Laboratory have assessed the applications of nanofluids for transportation [59]. The use of high-thermal conductive nanofluids in radiators can lead to a reduction in the frontal area of the radiator up to $10 \%$. The fuel saving is up to $5 \%$ due to the reduction in aerodynamic drag. It opens the door for new aerodynamic automotive designs that reduce emissions by lowering drag. The application of nanofluids also contributed to a reduction of friction and wear, reducing parasitic losses, operation of components such as pumps and compressors, and subsequently leading to more than $6 \%$ fuel savings. In fact, nanofluids not only enhance the efficiency and economic performance of car engine, but also will greatly influence the structure design of automotives. For example, the engine radiator cooled by a nanofluid will be smaller and lighter. It can be placed elsewhere in the vehicle, allowing for the redesign of a far more aerodynamic chassis. By reducing the size and changing the location of the radiator, a reduction in weight and wind resistance could enable greater fuel efficiency and subsequently lower exhaust emissions. Computer simulations from the US department of energy's office of vehicle technology showed that nanofluid coolants could reduce the size of truck radiators by $5 \%$. This would result in a $2.5 \%$ fuel saving at highway speeds.

The practical applications are on the road. In USA, car manufacturers GM and Ford are running their own research programs on nanofluid applications. A $€ 8.3$ million FP7 project, named NanoHex (Nanofluid Heat Exchange), began to run. It involved 12 organizations from Europe and Israel ranging from Universities to SME's and major companies. NanoHex is overcoming the technological challenges faced in development and application of reliable and safe nanofluids for more sophisticated, energy efficient, and environmentally friendly products and services [60]. 


\subsubsection{Industrial cooling applications}

The application of nanofluids in industrial cooling will result in great energy savings and emissions reductions. For US industry, the replacement of cooling and heating water with nanofluids has the potential to conserve 1 trillion Btu of energy [39, 61]. For the US electric power industry, using nanofluids in closed loop cooling cycles could save about 10-30 trillion Btu per year (equivalent to the annual energy consumption of about 50,000-150,000 households). The associated emissions reductions would be approximately 5.6 million metric tons of carbon dioxide, 8,600 metric tons of nitrogen oxides, and 21,000 metric tons of sulfur dioxide.

Experiments were performed using a flow-loop apparatus to explore the performance of polyalphaolefin nanofluids containing exfoliated graphite nanoparticle fibers in cooling [63]. It was observed that the specific heat of nanofluids was found to be $50 \%$ higher for nanofluids compared with polyalphaolefin and it increased with temperature. The thermal diffusivity was found to be 4 times higher for nanofluids. The convective heat transfer was enhanced by $\sim 10 \%$ using nanofluids compared with using polyalphaolefin. Ma et al. proposed the concept of nano liquid-metal fluid, aiming to establish an engineering route to make the highest conductive coolant with about several dozen times larger thermal conductivity than that of water [64]. The liquid metal with low melting point is expected to be an idealistic base fluid for making super conductive solution which may lead to the ultimate coolant in a wide variety of heat transfer enhancement area. The thermal conductivity of the liquid-metal fluid can be enhanced through the addition of more conductive nanoparticles.

\subsubsection{Heating buildings and reducing pollution}

Nanofluids can be applied in the building heating systems. Kulkarni et al. evaluated how they perform heating buildings in cold regions [65]. In cold regions, it is a common practice to use ethylene or propylene glycol mixed with water in different proportions as a heat transfer fluid. So 60:40 ethylene glcol/water (by weight) was selected as the base fluid. The results showed that using nanofluids in heat exchangers could reduce volumetric and mass flow rates, resulting in an overall pumping power savings. Nanofluids necessitate smaller heating systems, which are capable of delivering the same amount of thermal energy as larger heating systems, but are less expensive. This lowers the initial equipment cost excluding nanofluid cost. This will also reduce environmental pollutants because smaller heating units use less power, and the heat transfer unit has less liquid and material waste to discard at the end of its life cycle.

\subsubsection{Nuclear systems cooling}

The Massachusetts Institute of Technology has established an interdisciplinary center for nanofluid technology for the nuclear energy industry. The researchers are exploring the nuclear applications of nanofluids, specifically the following three [66]: 1) main reactor coolant for pressurized water reactors (PWRs). It could enable significant power uprates in current and future PWRs, thus enhancing their economic performance. Specifically, the use of nanofluids with at least $32 \%$ higher critical heat flux (CHF) could enable a $20 \%$ power density uprate in current plants without changing the fuel assembly design and without 
reducing the margin to $\mathrm{CHF} ; 2$ ) coolant for the emergency core cooling systems (ECCSs) of both PWRs and boiling water reactors. The use of a nanofluid in the ECCS accumulators and safety injection can increase the peak-cladding-temperature margins (in the nominal-power core) or maintain them in uprated cores if the nanofluid has a higher post-CHF heat transfer rate; 3) coolant for in-vessel retention of the molten core during severe accidents in highpower-density light water reactors. It can increase the margin to vessel breach by $40 \%$ during severe accidents in high-power density systems such as Westinghouse APR1000 and the Korean APR1400. While there exist several significant gaps, including the nanofluid thermal-hydraulic performance at prototypical reactor conditions and the compatibility of the nanofluid chemistry with the reactor materials. Much work should be done to overcome these gaps before any applications can be implemented in a nuclear power plant.

\subsubsection{Space and defense}

Due to the restriction of space, energy and weight in space station and aircraft, there is a strong demand for high efficient cooling system with smaller size. You et al. [67] and Vassalo et al. [68] have reported order of magnitude increases in the critical heat flux in pool boiling with nanofluids compared to the base fluid alone. Further research of nanofluids will lead to the development of next generation of cooling devices that incorporate nanofluids for ultrahigh-heat-flux electronic systems, presenting the possibility of raising chip power in electronic components or simplifying cooling requirements for space applications. A number of military devices and systems require high-heat flux cooling to the level of tens of $\mathrm{MW} / \mathrm{m}^{2}$. At this level, the cooling of military devices and system is vital for the reliable operation. Nanofluids with high critical heat fluxes have the potential to provide the required cooling in such applications as well as in other military systems, including military vehicles, submarines, and high-power laser diodes. Therefore, nanofluids have wide application in space and defense fields where power density is very high and the components should be smaller and weight less.

\subsection{Mass transfer enhancement}

Several researches have studied the mass transfer enhancement of nanofluids. Kim et al. initially examined the effect of nanoparticles on the bubble type absorption for $\mathrm{NH}_{3} / \mathrm{H}_{2} \mathrm{O}$ absorption system [69]. The addition of nanoparticles enhances the absorption performance up to 3.21 times. Then they visualized the bubble behavior during the $\mathrm{NH}_{3} / \mathrm{H}_{2} \mathrm{O}$ absorption process and studied the effect of nanoparticles and surfactants on the absorption characteristics [70]. The results show that the addition of surfactants and nanoparticles improved the absorption performance up to 5.32 times. The addition of both surfactants and nanoparticles enhanced significantly the absorption performance during the ammonia bubble absorption process. The theoretical investigations of thermodiffusion and diffusionthermo on convective instabilities in binary nanofluids for absorption application were conducted. Mass diffusion is induced by thermal gradient. Diffusionthermo implies that heat transfer is induced by concentration gradient [71]. Ma et al. studied the mass transfer process of absorption using CNTs-ammonia nanofluids as the working medium [72, 73]. The absorption rates of the CNTs-ammonia binary nanofluids were higher than those of ammonia solution without CNTs. The effective absorption ratio of the CNTs-ammonia binary nanofluids increased with the initial concentration of ammonia and the mass fraction 
of CNTs. Komati et al. studied $\mathrm{CO}_{2}$ absorption into amine solutions, and the addition of ferrofluids increased the mass transfer coefficient in gas/liquid mass transfer [74], and the enhancement extent depended on the amount of ferrofluid added. The enhancement in mass transfer coefficient was $92.8 \%$ for a volume fraction of the fluid of about $50 \%$ (solid magnetite volume fraction of about $0.39 \%$ ). The research about the influence of $\mathrm{Al}_{2} \mathrm{O}_{3}$ nanofluid on the falling film absorption with ammonia-water showed that the sorts of nanoparticles and surfactants in the nanofluid and the concentration of ammonia in the basefluid were the key parameters influencing the absorption effect of ammonia [75].

\subsection{Energy applications}

\subsubsection{Energy storage}

The temporal difference of energy source and energy needs made necessary the development of storage system. The storage of thermal energy in the form of sensible and latent heat has become an important aspect of energy management with the emphasis on efficient use and conservation of the waste heat and solar energy in industry and buildings [76]. Latent heat storage is one of the most efficient ways of storing thermal energy. Wu et al. evaluated the potential of $\mathrm{Al}_{2} \mathrm{O}_{3}-\mathrm{H}_{2} \mathrm{O}$ nanofluids as a new phase change material (PCM) for the thermal energy storage of cooling systems. The thermal response test showed the addition of $\mathrm{Al}_{2} \mathrm{O}_{3}$ nanoparticles remarkably decreased the supercooling degree of water, advanced the beginning freezing time and reduced the total freezing time. Only adding 0.2 $\mathrm{wt} \% \mathrm{Al}_{2} \mathrm{O}_{3}$ nanoparticles, the total freezing time of $\mathrm{Al}_{2} \mathrm{O}_{3}-\mathrm{H}_{2} \mathrm{O}$ nanofluids could be reduced by $20.5 \%$. Liu et al. prepared a new sort of nanofluid phase change materials (PCMs) by suspending small amount of $\mathrm{TiO}_{2}$ nanoparticles in saturated $\mathrm{BaCl}_{2}$ aqueous solution [77]. The nanofluids PCMs possessed remarkably high thermal conductivities compared to the base material. The cool storage/supply rate and the cool storage/supply capacity all increased greatly than those of $\mathrm{BaCl}_{2}$ aqueous solution without added nanoparticles. The higher thermal performances of nanofluids PCMs indicate that they have a potential for substituting conventional PCMs in cool storage applications. Copper nanoparticles are efficient additives to improve the heating and cooling rates of PCMs [78]. For composites with 1 wt \% copper nanoparticle, the heating and cooling times could be reduced by 30.3 and $28.2 \%$, respectively. The latent heats and phase-change temperatures changed very little after 100 thermal cycles.

\subsubsection{Solar absorption}

Solar energy is one of the best sources of renewable energy with minimal environmental impact. The conventional direct absorption solar collector is a well established technology, and it has been proposed for a variety of applications such as water heating; however the efficiency of these collectors is limited by the absorption properties of the working fluid, which is very poor for typical fluids used in solar collectors. Recently this technology has been combined with the emerging technologies of nanofluids and liquid-nanoparticle suspensions to create a new class of nanofluid-based solar collectors. Otanicar et al. reported the experimental results on solar collectors based on nanofluids made from a variety of nanoparticles (CNTs, graphite, and silver) [79]. The efficiency improvement was up to 5\% in solar thermal collectors by utilizing nanofluids as the absorption media. In addition they compared the experimental data with a numerical model of a solar collector with direct 
absorption nanofluids. The experimental and numerical results demonstrated an initial rapid increase in efficiency with volume fraction, followed by a leveling off in efficiency as volume fraction continues to increase. Theoretical investigation on the feasibility of using a nonconcentrating direct absorption solar collector showed that the presence of nanoparticles increased the absorption of incident radiation by more than nine times over that of pure water [80]. Under the similar operating conditions, the efficiency of an absorption solar collector using nanofluid as the working fluid was found to be up to $10 \%$ higher (on an absolute basis) than that of a flat-plate collector. Otanicar et al. evaluated the overall economic and environmental impacts of the technology in contrast with conventional solar collectors using the life cycle assessment methodology [81]. Results showed that for the current cost of nanoparticles the nanofluid based solar collector had a slightly longer payback period but at the end of its useful life has the same economic saving as a conventional solar collector. Sani et al. investigated the optical and thermal properties of nanofluids consisting in aqueous suspensions of single wall carbon nanohorns [82]. The observed nanoparticle-induced differences in optical properties appeared promising, leading to a considerably higher sunlight absorption. Both these effects, together with the possible chemical functionalization of carbon nanohorns, make this new kind of nanofluids very interesting for increasing the overall efficiency of the sunlight exploiting device.

\subsection{Mechanical applications}

\subsubsection{Friction reduction}

Advanced lubricants can improve productivity through energy saving and reliability of engineered systems. Tribological research heavily emphasizes reducing friction and wear. Nanoparticles have attracted much interest in recent years due to their excellent loadcarrying capacity, good extreme pressure and friction reducing properties. Zhou et al. evaluated the tribological behavior of $\mathrm{Cu}$ nanoparticles in oil on a four-ball machine. The results showed that $\mathrm{Cu}$ nanoparticles as an oil additive had better friction-reduction and antiwear properties than zinc dithiophosphate, especially at high applied load. Meanwhile, the nanoparticles could also strikingly improve the load-carrying capacity of the base oil [83]. Dispersion of solid particles was found to play an important role, especially when a slurry layer was formed. Water-based $\mathrm{Al} 2 \mathrm{O} 3$ and diamond nanofluids were applied in the minimum quantity lubrication (MQL) grinding process of cast iron. During the nanofluid MQL grinding, a dense and hard slurry layer was formed on the wheel surface and could benefit the grinding performance. Nanofluids showed the benefits of reducing grinding forces, improving surface roughness, and preventing workpiece burning. Compared to dry grinding, MQL grinding could significantly reduce the grinding temperature [84]. Wear and friction properties of surface modified $\mathrm{Cu}$ nanoparticles as 50CC oil additive were studied. The higher the oil temperature applied, the better the tribological properties of $\mathrm{Cu}$ nanoparticles were. It could be inferred that a thin copper protective film with lower elastic modulus and hardness was formed on the worn surface, which resulted in the good tribological performances of $\mathrm{Cu}$ nanoparticles, especially when the oil temperature was higher [85]. Wang et al. studied the tribological properties of ionic liquid-based nanofluids containing functionlized MWNTs under loads in the range of 200-800 N [86], indicating that the nanofluids exhibited preferable friction-reduction properties under $800 \mathrm{~N}$ and remarkable antiwear properties with use of reasonable concentrations. Magnetic 
nanoparticle $\mathrm{Mn}_{0.78} \mathrm{Zn}_{0.22} \mathrm{Fe}_{2} \mathrm{O}_{4}$ was also an efficient lubricant additive. When used as a lubricant additive in 46\# turbine oil, it could improve the wear resistance, load-carrying capacity, and antifriction ability of base oil, and the decreasing percentage of wear scar diameter was $25.45 \%$ compared to the base oil. This was a typical self-repair phenomenon [87]. Chen et al. reported on dispersion stability enhancement and self-repair principle discussion of ultrafine-tungsten disulfide in green lubricating oil [88]. Ultrafine-tungsten disulfide particulates could fill and level up the furrows on abrasive surfaces, repairing abrasive surface well. What is more, ultrafine-tungsten disulfide particulates could form a WS2 film with low shear stress by adsorbing and depositing in the hollowness of abrasive surface, making the abrasive surface be more smooth, and the FeS film formed in tribology reaction could protect the abrasive surface further, all of which realize the self-repair to abrasive surface. The tribological properties of liquid paraffin with $\mathrm{SiO}_{2}$ nanoparticles additive made by a sol-gel method was investigated by Peng et al. [89]. The optimal concentrations of $\mathrm{SiO}_{2}$ nanoparticles in liquid paraffin was associated with better tribological properties than pure paraffin oil, and an anti-wear ability that depended on the particle size, and oleic acid surface-modified $\mathrm{SiO}_{2}$ nanoparticles with an average diameter of $58 \mathrm{~nm}$ provided better tribological properties in load-carrying capacity, anti-wear and friction-reduction than pure liquid paraffin. Nanoparticles can easily penetrate into the rubbing surfaces because of their nanoscale. During the frictional process, the thin physical tribofilm of the nanoparticles forms between rubbing surfaces, which cannot only bear the load but also separates the rubbing faces. The spherical $\mathrm{SiO}_{2}$ nanoparticles could roll between the rubbing faces in sliding friction, the originally pure sliding friction becomes mixed sliding and rolling friction. Therefore, the friction coefficient declines markedly and then remains constant.

\subsubsection{Magnetic sealing}

Magnetic fluids (Ferromagnetic fluid) are kinds of special nanofluids. They are stable colloidal suspensions of small magnetic particles such as magnetite $\left(\mathrm{Fe}_{3} \mathrm{O}_{4}\right)$. The properties of the magnetic nanoparticles, the magnetic component of magnetic nanofluids, may be tailored by varying their size and adapting their surface coating in order to meet the requirements of colloidal stability of magnetic nanofluids with non-polar and polar carrier liquids [90]. Comparing with the mechanical sealing, magnetic sealing offers a cost-effective solution to environmental and hazardous-gas sealing in a wide variety of industrial rotation equipment with high speed capability, low friction power losses and long life and high reliability [91]. A ring magnet forms part of a magnetic circuit in which an intense magnetic field is established in the gaps between the teeth on a magnetically permeable shaft and the surface of an opposing pole block. Ferrofluid introduced into the gaps forms discrete liquid rings capable of supporting a pressure difference while maintaining zero leakage. The seals operate without wear as the shaft rotates because the mechanical moving parts do not touch. With these unique characteristics, sealing liquids with magnetic fluids can be applied in many application areas. It is reported that an iron particle dispersed magnetic fluids was utilized in the sealing of a high rotation pump. The sealing holds pressure of $618 \mathrm{kPa}$ with a $1800 \mathrm{r} / \mathrm{min}$ [92]. Mitamura et al. studied the application of a magnetic fluid seal to rotary blood pumps. The developed magnetic fluid seal worked for over 286 days in a continuous flow condition, for 24 days (on-going) in a pulsatile flow condition and for $24 \mathrm{~h}$ (electively 
terminated) in blood flow [93]. Ferro-cobalt magnetic fluid was used for oil sealing, and the holding pressure is 25 times as high as that of a conventional magnetite sealing [94].

\subsection{Biomedical application}

\subsubsection{Antibacterial activity}

Organic antibacterial materials are often less stable particularly at high temperatures or pressures. As a consequence, inorganic materials such as metal and metal oxides have attracted lots of attention over the past decade due to their ability to withstand harsh process conditions. The antibacterial behaviour of $\mathrm{ZnO}$ nanofluids shows that the $\mathrm{ZnO}$ nanofluids have bacteriostatic activity against [95]. Electrochemical measurements suggest some direct interaction between $\mathrm{ZnO}$ nanoparticles and the bacteria membrane at high $\mathrm{ZnO}$ concentrations. Jalal et al. prepared $\mathrm{ZnO}$ nanoparticles via a green method. The antibacterial activity of suspensions of $\mathrm{ZnO}$ nanoparticles against Escherichia coli (E. coli) has been evaluated by estimating the reduction ratio of the bacteria treated with $\mathrm{ZnO}$. Survival ratio of bacteria decreases with increasing the concentrations of $\mathrm{ZnO}$ nanofluids and time [96]. Further investigations have clearly demonstrated that $\mathrm{ZnO}$ nanoparticles have a wide range of antibacterial effects on a number of other microorganisms. The antibacterial activity of $\mathrm{ZnO}$ may be dependent on the size and the presence of normal visible light [97]. Recent research showed that $\mathrm{ZnO}$ nanoparticles exhibited impressive antibacterial properties against an important foodborne pathogen, E. coli O157:H7, and the inhibitory effects increased as the concentrations of $\mathrm{ZnO}$ nanoparticles increased. $\mathrm{ZnO}$ nanoparticles changed the cell membrane components including lipids and proteins. $\mathrm{ZnO}$ nanoparticles could distort bacterial cell membrane, leading to loss of intracellular components, and ultimately the death of cells, considered as an effective antibacterial agent for protecting agricultural and food safety [98].

The antibacterial activity research of $\mathrm{CuO}$ nanoparticles showed that they possessed antibacterial activity against four bacterial strains. The size of nanoparticles was less than that of the pore size in the bacteria and thus they had a unique property of crossing the cell membrane without any hindrance. It could be hypothesized that these nanoparticles formed stable complexes with vital enzymes inside cells which hampered cellular functioning resulting in their death [99]. Bulk equivalents of these products showed no inhibitory activity, indicating that particle size was determinant in activity [100]. Lee et al. reported the antibacterial efficacy of nanosized silver colloidal solution on the cellulosic and synthetic fabrics [101]. The antibacterial treatment of the textile fabrics was easily achieved by padding them with nanosized silver colloidal solution. The antibacterial efficacy of the fabrics was maintained after many times laundering. Silver colloid is an efficient antibacterial agent. The silver colloid prepared by a one-step synthesis showed high antimicrobial and bactericidal activity against Gram-positive and Gram-negative bacteria, including highly multiresistant strains such as methicillin-resistant staphylococcus aureus. The antibacterial activity of silver nanoparticles was found to be dependent on the size of silver particles. A very low concentration of silver gave antibacterial performance [102]. The aqueous suspensions of fullerenes and nano- $\mathrm{TiO}_{2}$ can produce reactive oxygen species (ROS). Bacterial (E. coli) toxicity tests suggestted that, unlike nano- $\mathrm{TiO}_{2}$ which was exclusively phototoxic, the antibacterial activity of fullerene suspensions was linked to ROS production. Nano- $\mathrm{TiO}_{2}$ may be more efficient for water treatment involving UV or solar 
energy, to enhance contaminant oxidation and perhaps for disinfection. However, fullerol and $\mathrm{PVP} / \mathrm{C}_{60}$ may be useful as water treatment agents targeting specific pollutants or microorganisms that are more sensitive to either superoxide or singlet oxygen [103]. Lyon et al. proposed that $\mathrm{C}_{60}$ suspensions exerted ROS-independent oxidative stress in bacteria, with evidence of protein oxidation, changes in cell membrane potential, and interruption of cellular respiration. This mechanism requires direct contact between the nanoparticle and the bacterial cell and differs from previously reported nanomaterial antibacterial mechanisms that involve ROS generation (metal oxides) or leaching of toxic elements (nanosilver) [104].

\subsubsection{Nanodrug delivery}

Over the last few decades, colloidal drug delivery systems have been developed in order to improve the efficiency and the specificity of drug action [105]. The small size, customized surface, improved solubility, and multi-functionality of nanoparticles open many doors and create new biomedical applications. The novel properties of nanoparticles offer the ability to interact with complex cellular functions in new ways [106]. Gold nanoparticles provide nontoxic carriers for drug and gene delivery applications. With these systems, the gold core imparts stability to the assembly, while the monolayer allows tuning of surface properties such as charge and hydrophobicity. Another attractive feature of gold nanoparticles is their interaction with thiols, providing an effective and selective means of controlled intracellular release [107]. Nakano et al. proposed the drug delivery system using nano-magnetic fluid [108], which targetted and concentrated drugs using a ferrofluid cluster composed of magnetic nanoparticles. The potential of magnetic nanoparticles stems from the intrinsic properties of their magnetic cores combined with their drug loading capability and the biochemical properties that can be bestowed on them by means of a suitable coating. CNT has emerged as a new alternative and efficient tool for transporting and translocating therapeutic molecules. CNT can be functionalised with bioactive peptides, proteins, nucleic acids and drugs, and used to deliver their cargos to cells and organs. Because functionalised CNT display low toxicity and are not immunogenic, such systems hold great potential in the field of nanobiotechnology and nanomedicine [109, 110]. Pastorin et al. have developed a novel strategy for the functionalisation of CNTs with two different molecules using the 1,3dipolar cycloaddition of azomethine ylides [111]. The attachment of molecules that will target specific receptors on tumour cells will help improve the response to anticancer agents. Liu et al. have found that prefunctionalized CNTs can adsorb widely used aromatic molecules by simple mixing, forming "forest-scrub"-like assemblies on CNTs with PEG extending into water to impart solubility and aromatic molecules densely populating CNT sidewalls. The work establishes a novel, easy-to-make formulation of a SWNT-doxorubicin complex with extremely high drug loading efficiency [112].

In recent years, graphene based drug delivery systems have attracted more and more attention. In 2008, Sun et al. firstly reported the application of nano-graphene oxide (NGO) for cellular imaging and drug delivery [113]. They have developed functionalization chemistry in order to impart solubility and compatibility of $\mathrm{NGO}$ in biological environments. Simple physicosorption via $\Pi$-stacking can be used for loading doxorubicin, a widely used cancer drug onto NGO functionalized with antibody for selective killing of cancer cells in vitro. Functional nanoscale graphene oxide is found to be a novel nanocarrier 
for the loading and targeted delivery of anticancer drugs [114]. Controlled loading of two anticancer drugs onto the folic acid-conjugated NGO via $\Pi-\Pi$ stacking and hydrophobic interactions demonstrated that NGO loaded with the two anticancer drugs showed specific targeting to MCF-7 cells (human breast cancer cells with folic acid receptors), and remarkably high cytotoxicity compared to NGO loaded with either doxorubicin or camptothecin only. The PEGylated (PEG: polyethylene glycol) nanographene oxide could be used for the delivery of water-insoluble cancer drugs [115]. PEGylated NGO readily complexes with a water insoluble aromatic molecule SN38, a camptothecin analogue, via noncovalent van der Waals interaction. The NGO-PEG-SN38 complex exhibits excellent aqueous solubility and retains the high potency of free SN38 dissolved in organic solvents. Yang et al. found $\mathrm{GO}-\mathrm{Fe}_{3} \mathrm{O}_{4}$ hybrid could be loaded with anti-cancer drug doxorubicin hydrochloride with a high loading capacity [116]. This $\mathrm{GO}-\mathrm{Fe}_{3} \mathrm{O}_{4}$ hybrid showed superparamagnetic property and could congregate under acidic conditions and be redispersed reversibly under basic conditions. This $\mathrm{pH}$-triggered controlled magnetic behavior makes this material a promising candidate for controlled targeted drug delivery.

\subsection{Other applications}

\subsubsection{Intensify microreactors}

The discovery of high enhancement of heat transfer in nanofluids can be applicable to the area of process intensification of chemical reactors through integration of the functionalities of reaction and heat transfer in compact multifunctional reactors. Fan et al. studied a nanofluid based on benign $\mathrm{TiO}_{2}$ material dispersed in ethylene glycol in an integrated reactor-heat exchanger [117]. The overall heat transfer coefficient increase was up to $35 \%$ in the steady state continuous experiments. This resulted in a closer temperature control in the reaction of selective reduction of an aromatic aldehyde by molecular hydrogen and very rapid change in the temperature of reaction under dynamic reaction control.

\subsubsection{Nanofluids as vehicular brake fluids}

A vehicle's kinetic energy is dispersed through the heat produced during the process of braking and this is transmitted throughout the brake fluid in the hydraulic braking system [39], and now there is a higher demand for the properties of brake oils. Copper-oxide and aluminum-oxide based brake nanofluids were manufactured using the arc-submerged nanoparticle synthesis system and the plasma charging arc system, respectively [118, 119]. The two kinds of nanofluids both have enhanced properties such as a higher boiling point, higher viscosity and a higher conductivity than that of traditional brake fluid. By yielding a higher boiling point, conductivity and viscosity, the nanofluid brake oil will reduce the occurrence of vapor-lock and offer increased safety while driving.

\subsubsection{Nanofluids based microbial fuel cell}

Microbial fuel cells (MFC) that utilize the energy found in carbohydrates, proteins and other energy rich natural products to generate electrical power have a promising future. The excellent performance of MFC depends on electrodes and electron mediator. Sharma et al. constructed a novel microbial fuel cell (MFC) using novel electron mediators and CNT based electrodes [120]. The novel mediators are nanofluids which were prepared by 
dispersing nanocrystalline platinum anchored CNTs in water. They compared the performance of the new E. coli based MFC to the previously reported E. coli based microbial fuel cells with Neutral Red and Methylene Blue electron mediators. The performance of the MFC using CNT based nanofluids and CNT based electrodes has been compared against plain graphite electrode based MFC. CNT based electrodes showed as high as $\sim 6$ fold increase in the power density compared to graphite electrodes. The work demonstrates the potential of noble metal nanoparticles dispersed on CNT based MFC for the generation of high energies from even simple bacteria like E. coli.

\subsubsection{Nanofluids with unique optical properties}

Optical filters are used to select different wavelengths of light. The ferrofluid based optical filter has tunable properties. The desired central wavelength region can be tuned by an external magnetic field. Philip et al. developed a ferrofluid based emulsion for selecting different bands of wavelengths in the UV, visible and IR regions [121]. The desired range of wavelengths, bandwidth and percentage of reflectivity could be easily controlled by using suitably tailored ferrofluid emulsions. Mishra et al. developed nanofluids with selective visible colors in gold nanoparticles embedded in polymer molecules of polyvinyl pyrrolidone (PVP) in water [122]. They compared the developments in the apparent visible colors in forming the Au-PVP nanofluids of 0.05, 0.10, 0.50, and $1.00 \mathrm{wt} \%$ Au-contents. The surface plasmon bands, which occurs over $480-700 \mathrm{~nm}$, varies sensitively in its position as well as the intensity when varying the Au-content $0-1 \mathrm{wt} \%$.

\section{Conclusions}

Many interesting properties of nanofluids have been reported in the past decades. This paper presents an overview of the recent developments in the study of nanofluids, including the preparation methods, the evaluation methods for their stability, the ways to enhance their stability, the stability mechanisms, and their potential applications in heat transfer intensification, mass transfer enhancement, energy fields, mechanical fields and biomedical fields, etc.

Although nanofluids have displayed enormously exciting potential applications, some vital hinders also exist before commercialization of nanofluids. The following key issues should receive greater attention in the future. Firstly, further experimental and theoretical researches are required to find the major factors influencing the performance of nanofluids. Up to now, there is a lack of agreement between experimental results from different groups, so it is important to systematically identify these factors. The detailed and accurate structure characterizations of the suspensions may be the key to explain the discrepancy in the experimental data. Secondly, increase in viscosity by the use of nanofluids is an important drawback due to the associated increase in pumping power. The applications for nanofluids with low viscosity and high conductivity are promising. Enhancing the compatibility between nanomaterials and the base fluids through modifying the interface properties of two phases may be one of the solution routes. Thirdly, the shape of the additives in nanofluids is very important for the properties, therefore the new nanofluid synthesis approaches with controllable microscope structure will be an interesting research work. Fourthly, Stability of the suspension is a crucial issue for both scientific research and 
practical applications. The stability of nanofluids, especially the long term stability, the stability in the practical conditions and the stability after thousands of thermal cycles should be paid more attention. Fifthly, there is a lack of investigation of the thermal performance of nanofluids at high temperatures, which may widen the possible application areas of nanofluids, like in high temperature solar energy absorption and high temperature energy storage. At the same time, high temperature may accelerate the degradation of the surfactants used as dispersants in nanofluids, and may produce more foams. These factors should be taken into account. Finally, the properties of nanofluids strongly depend on the shape and property of the additive. Therefore nanofluid research can be richened and extended through exploring new nanomaterials. For example, the newly discovered 2-D monatomic sheet graphene is a promising candidate material to enhance the thermal conductivity of the base fluid $[123,124]$. The concept of nanofluids is extended by the use of phase change materials, which goes well beyond simply increasing the thermal conductivity of a fluid [125]. It is found that the indium/polyalphaolefin phase change nanofluid exhibits simultaneously enhanced thermal conductivity and specific heat.

\section{Acknowledgment}

The work was supported by New Century Excellent Talents in University (NECT-10-883), the Program for Professor of Special Appointment (Eastern Scholar) at Shanghai Institutions of Higher Learning, and partly by National Natural Science Foundation of China (51106093).

\section{References}

[1] V. Trisaksri, S. Wongwises, Renew. Sust. Energ. Rev. 11, 512 (2007).

[2] S. Özerinç, S. Kakaç, A.G. Yazıcıoğlu, Microfluid Nanofluid 8, 145 (2009).

[3] X. Wang, A.S. Mujumdar, Int. J. Therm. Sci. 46, 1 (2007).

[4] X. Wang, A.S. Mujumdar, Brazilian J. Chem. Eng. 25, 613 (2008).

[5] Y. Li, J. Zhou, S. Tung, E. Schneider, S. Xi, Powder Technol. 196, 89 (2009).

[6] S. Kakaç, A. Pramuanjaroenkij, Int. J. Heat Mass Transf. 52, 3187 (2009).

[7] J.A. Eastman, S.U. Choi, S. Li, W. Yu, L.J. Thompson, Appl. Phys. Lett. 78, 718 (2001).

[8] C. Lo, T. Tsung, L. Chen, J. Cryst. Growth 277, 636 (2005).

[9] C. Lo, T. Tsung, L. Chen, C. Su, H. Lin, J. Nanopart. Res. 7, 313 (2005).

[10] H. Zhu, Y. Lin, Y. Yin, J. Colloid Interface Sci. 277, 100 (2004).

[11] H. Bönnemann, S.S. Botha, B. Bladergroen, V.M. Linkov, Appl. Organomet. Chem. 19, 768 (2005).

[12] A.K. Singh, V.S. Raykar, Colloid Polym. Sci. 286, 1667 (2008).

[13] A. Kumar, J. Colloid Interface Sci. 264, 396 (2003).

[14] H. Zhu, C. Zhang, Y. Tang, J. Wang, J. Phys. Chem. C 111, 1646 (2007).

[15] Y. Chen, X. Wang, Mater. Lett. 62, 2215 (2008).

[16] X. Feng, H. Ma, S. Huang, W. Pan, X. Zhang, F. Tian, C. Gao, Y. Cheng, J. Luo, J. Phys. Chem. 110, 12311 (2006).

[17] W. Yu, H. Xie, L. Chen, Y. Li, Colloids Surf. A: Physicochemical And Engineering Aspects 355, 109 (2010).

[18] L.Q. Wang, J. Fan, Nanoscale Res. Lett. 5, 1241 (2010).

[19] X. Wei, L. Wang, Particuology 8, 262 (2010).

[20] X. Li, D. Zhu, X. Wang, J. Colloid Interface Sci. 310, 456 (2007).

[21] H. Zhu, C. Zhang, Y. Tang, J. Wang, B. Ren, Y. Yin, Carbon 45, 226 (2007). 
[22] D. Li, R.B. Kaner, Chem. Commun. 14, 3286 (2005).

[23] H.J. Kim, I.C. Bang, J. Onoe, Opt. Laser Eng. 47, 532 (2009).

[24] X. Wang, X. Li, S. Yang, Energy Fuels 23, 2684 (2009).

[25] D. Zhu, X. Li, N. Wang, X. Wang, J. Gao, H. Li, Curr. Appl. Phys. 9, 131 (2009).

[26] L. Chen, H. Xie, Thermochim. Acta 506, 62 (2010).

[27] J. Huang, X. Wang, Q. Long, X. Wen, Y. Zhou, L. Li, Symposium On Photonics And Optoelectronics 1-4 (2009).

[28] M. Farahmandjou, S.A. Sebt, S.S. Parhizgar, P. Aberomand, M. Akhavan, Chin. Phys. Lett. 26, 027501 (2009).

[29] Y. Hwang, J. Lee, C. Lee, Y. Jung, S. Cheong, B. Ku, S. Jang, Thermochim. Acta 455, 70 (2007).

[30] L. Chen, H. Xie, Y. Li, W. Yu, Thermochim. Acta 477, 21 (2008).

[31] X. Yang, Z. Liu, Nanoscale Res. Lett. 5, 1324 (2010).

[32] L. Chen, H. Xie, Thermochim. Acta 497, 67 (2010).

[33] Q. Yu, Y.J. Kim, H. Ma, Appl. Phys. Lett. 92, 103111 (2008).

[34] I.M. Joni, A. Purwanto, F. Iskandar, K. Okuyama, Ind. Eng. Chem. Res. 48, 6916 (2009).

[35] E. Tang, G. Cheng, X. Ma, X. Pang, Q. Zhao, Appl. Surf. Sci. 252, 5227 (2006).

[36] T. Missana, A. Adell, J. Colloid Interface Sci. 230, 150 (2000).

[37] I. Popa, G. Gillies, G. Papastavrou, M. Borkovec, J. Phys. Chem. B 114, 3170 (2010).

[38] H. Kamiya, Y. Fukuda, Y. Suzuki, M. Tsukada, T. Kakui, M. Naito, J. Am. Ceram. Soc. 82,3407 (1999).

[39] K.V. Wong, O. De Leon, Adv. Mech. Eng. 2010, 1 (2010).

[40] G. Donzelli, R. Cerbino, A. Vailati, Phys. Rev. Lett. 102, 1 (2009).

[41] M. Arruebo, R. Fernández-pacheco, M.R. Ibarra, J. Santamaría, Rev. Literature Arts Am. 2, 22 (2007).

[42] W. Yu, D.M. France, D. Singh, E.V. Timofeeva, D.S. Smith, J.L. Routbort, J. Nanosci. Nanotechnol. 10, 4824 (2010).

[43] K. Ma, J. Liu, Phys. Lett. A 361, 252 (2007).

[44] G. Paul, M. Chopkar, I. Manna, P.K. Das, Renew.Sust. Energ Rev. 14, 1913 (2010).

[45] S.P. Jang, S.U.S. Choi, Appl. Therm. Eng. 26, 2457 (2006).

[46] C.T. Nguyen, G. Roy, N. Galanis, S. Suiro, Proceedings of the 4th WSEAS Int. Conf. on heat transfer, thermal engineering and environment, Elounda, Greece, August 2123, 103-108 (2006).

[47] H. Shokouhmand, M. Ghazvini, J. Shabanian, Proceedings of the World Congress on Engineering 2008 Vol III, WCE 2008, July 2 - 4, 2008, London, U.K.

[48] C.Y. Tsaia, H.T. Chiena, P.P. Dingb, B. Chanc, T.Y. Luhd, P.H. Chena, Mater. Lett. 58, 1461 (2004).

[49] Y.T. Chen, W.C. Wei, S.W. Kang, C.S. Yu, 24th IEEE SEMI-THERM Symposium 16 (2008).

[50] H.B. Ma, C. Wilson, B. Borgmeyer, K. Park, Q. Yu, S.U.S. Choi, M. Tirumala, Appl. Phys. Lett. 88, 143116 (2006).

[51] S.W. Kang, W.C. Wei, S.H. Tsai, C.C. Huang, Appl. Therm. Eng. 29, 973 (2009).

[52] P. Naphon, P. Assadamongkol, T. Borirak, Int. Commun. Heat Mass Transf. 35, 1316 (2008).

[53] H.Q. Xie, L.F. Chen, Phys. Lett. A 373, 1861 (2009).

[54] H.Q. Xie, W. Yu, Y. Li, J. Phys. D: Appl. Phys. 42, 095413 (2009).

[55] W. Yu, H.Q. Xie, Y. Li, L.F. Chen, Thermochim. Acta, 491, 92 (2009). 
[56] W. Yu, D.M. France, S.U.S. Choi and J.L. Routbort, "Review and Assessment of Nanofluid Technology for Transportation and Other Applications,"Argonne National Laboratory Technical Report, ANL/ESD/07-9, April 2007, 78 (2007).

[57] M. Kole, T.K. Dey, J. Phys. D: Appl. Phys. 43 315501(2010).

[58] S.Z. Tzeng, C.W. Lin, K.D. Huang, Acta Mechanica, 179, 11 (2005).

[59] D. Singh, J. Toutbort, G. Chen, et al., "Heavy vehicle systems optimization merit review and peer evaluation," Annual Report, Argonne National Laboratory, 2006.

[60] http://www.labnews.co.uk/feature_archive.php/5449/5/keeping-it-cool

[61] J. Routbort, et al., Argonne National Lab, Michellin North America, St. Gobain Corp., 2009, http://www1.eere.energy.gov/industry/nanomanufacturing/pdfs/nanofluids industrial cooling.pdf.

[62] http://96.30.12.13/execsumm/VU0319--Nanofluid\%20for\%20Cooling\%20Enhancement \%20of\%20Electrical\%20Power\%20Equipment.pdf

[63] I.C. Nelson, D. Banerjee, R. Ponnappan, J. Thermophys. Heat Transf. 23, 752 (2009).

[64] K.Q. Ma, J. Liu, Phys. Lett. A 361, 252 (2007).

[65] D.P. Kulkarni, D.K. Das, R.S. Vajjha, Appl. Energy, 86, 2566 (2009).

[66] J. Buongiorno, L.W. Hu, J.K. Sung, R. Hannink, B. Truong, E. Forrest, Nucl. Technol. $162,80(2008)$.

[67] S.M. You, J.H. Kim, and K.H. Kim, Appl. Phys. Lett. 83, 3374 (2003).

[68] P. Vassallo, R. Kumar, S.D. Amico. Int. J. Heat Mass Transf. 47, 407 (2004).

[69] J. Kim, J. Jung, Y. Kang, Int. J. Refrig. 29, 22 (2006).

[70] J. Kim, J. Jung, Y. Kang, Int. J. Refrig. 30, 50 (2007).

[71] J. Kim, Y. Kang, C. Choi, Int. J. Refrig. 30, 323 (2007).

[72] X. Ma, F. Su, J. Chen, Y. Zhang, J. Mech. Sci. Technol. 21, 1813 (2007).

[73] X. Ma, F. Su, J. Chen, T. Bai, Z. Han, Int. Commun. Heat Mass Transf. 36, 657 (2009).

[74] S. Komati, A.K. Suresh, Chem. Technol. 1100, 1094 (2008).

[75] L. Yang, K. Du, B. Cheng, Y. Jiang, 2010 Asia-Pacific Power And Energy Engineering Conference 1-4 (2010).

[76] M.F. Demirbas, Energy Source. Part B: Economics, Planning, and Policy 1, 85 (2006).

[77] S. Wu, D. Zhu, X. Zhang, J. Huang, Energy Fuels 24, 1894 (2010).

[78] Y. Liu, Y. Zhou, M. Tong, X. Zhou, Microfluid. Nanofluid. 7, 579 (2009).

[79] T.P. Otanicar, P.E. Phelan, R.S. Prasher, G. Rosengarten, R.a. Taylor, Renew.Sust. Energ Rev. 2, 033102 (2010).

[80] H. Tyagi, P. Phelan, R. Prasher, J. Solar Energy Eng. 131, 041004 (2009).

[81] T.P. Otanicar, J.S. Golden, Environ. Sci. Technol. 43, 6082 (2009).

[82] E. Sani, S. Barison, C. Pagura, L. Mercatelli, P. Sansoni, D. Fontani, D. Jafrancesco, F. Francini, Opt. Express 18, 4613 (2010).

[83] J. Zhou, Z. Wu, Z. Zhang, W. Liu, Q. Xue, Tribol. Lett. 8, 213 (2000).

[84] B. Shen, A. Shih, S. Tung, Tribology Transactions 51, 730 (2008).

[85] H. Yu, Y. Xu, P. Shi, B. Xu, X. Wang, Q. Liu, T. Nonferr. Metal. Soc. China 18, 636 (2008).

[86] B. Wang, X. Wang, W. Lou, J. Hao, J. Phys. Chem. C 114, 8749 (2010).

[87] W. Li-jun, G. Chu-wen, R. Yamane, J. Trib. 130, 031801 (2008).

[88] S. Chen, D.H. Mao, Adv. Tribol. 995 (2010).

[89] D. Peng, C. Chen, Y. Kang, Y. Chang, S. Chang, Ind. Lubr. Trib. 62, 111 (2010).

[90] L. Vekas, China Particuology 5, 43 (2007).

[91] R.E. Rosensweig, Ann. Rev. Fluid Mech. 19, 437 (1987).

[92] Y.S. Kim, K. Nakatsuka, T. Fujita, T. Atarashi, J. Magn. Magn. Mater. 201, 361 (1999). 
[93] Y. Mitamura, S. Arioka, D. Sakota, K. Sekine, M. Azegami, J Phys.: Condens. Mater. 20, 204145 (2008).

[94] Y. Kim, J. Magn. Magn. Mater. 267, 105 (2003).

[95] L. Zhang, Y. Jiang, Y. Ding, M. Povey, D. York, J. Nanopart. Res. 9, 479 (2006).

[96] R. Jalal, E.K. Goharshadi, M. Abareshi, M. Moosavi, A. Yousefi, P. Nancarrow, Mater. Chem. Phys. 121, 198 (2010).

[97] N. Jones, B. Ray, K.T. Ranjit, A.C. Manna, FEMS Microbiol. Lett. 279, 71 (2008).

[98] Y. Liu, L. He, a. Mustapha, H. Li, Z.Q. Hu, M. Lin, J. Appl. Microbiol. 107, 1193 (2009).

[99] O. Mahapatra, M. Bhagat, C. Gopalakrishnan, K. Arunachalam, J. Exp. Nanosci. 3, 185 (2008).

[100] P. Gajjar, B. Pettee, D.W. Britt, W. Huang, W.P. Johnson, A.J. Anderson, J. Biol. Eng. 3, 9 (2009).

[101] H.J. Lee, S.Y. Yeo, S.H. Jeong, Polym. 8, 2199 (2003).

[102] A. Panacek, L. Kvítek, R. Prucek, M. Kolar, R. Vecerova, N. Pizúrova, V.K. Sharma, T. Nevecna, R. Zboril, J. Phys. Chem. B 110, 16248 (2006).

[103] L. Brunet, D.Y. Lyon, E.M. Hotze, P.J. Alvarez, M.R. Wiesner, Environ. Sci. Technol. 43, 4355 (2009).

[104] D.Y. Lyon, P.J. Alvarez, Environ. Sci. Technol. 42, 8127 (2008).

[105] A. Vonarbourg, C. Passirani, P. Saulnier, J. Benoit, Biomater. 27, 4356 (2006).

[106] R. Singh, J.W. Lillard, Exp. Mol. Pathol. 86, 215 (2009).

[107] P. Ghosh, G. Han, M. De, C.K. Kim, V.M. Rotello, Adv. Drug Deliver. Rev. 171307 (2008).

[108] M. Nakano, H. Matsuura, D. Ju, T. Kumazawa, S. Kimura, Y. Uozumi, N. Tonohata, K. Koide, N. Noda, P. Bian, M. Akutsu, K. Masuyama, K. Makino, Proceedings of the 3rd International Conference on Innovative Computing, Information and Control, ICICIC2008, Dalian, China, June 18-20 (2008).

[109] A. Bianco, K. Kostarelos, M. Prato, Curr. Opin. Chem. Biol. 9, 674 (2005).

[110] C. Tripisciano, K. Kraemer, a. Taylor, E. Borowiak-Palen, Chem. Phys. Lett. 478, 200 (2009).

[111] G. Pastorin, W. Wu, S. Wieckowski, J. Briand, K. Kostarelos, M. Prato, A. Bianco, Chem. Commun. 1, 1182 (2006).

[112] Z. Liu, X. Sun, N. Nakayama-Ratchford, H. Dai, ACS Nano 1, 50 (2007).

[113] X. Sun, Z. Liu, K. Welsher, J.T. Robinson, A. Goodwin, S. Zaric, H. Dai, Nano Res. 1, 203 (2008).

[114] L. Zhang, J. Xia, Q. Zhao, L. Liu, Z. Zhang, Small 6, 537 (2010).

[115] Z. Liu, J.T. Robinson, X. Sun, H. Dai, J. Am. Chem. Soc. 130, 10876 (2008).

[116] X. Yang, X. Zhang, Y. Ma, Y. Huang, Y. Wang, Y. Chen, J. Mater. Chem. 19, 2710 (2009).

[117] X. Fan, H. Chen, Y. Ding, P.K. Plucinski, A.a. Lapkin, Green Chem. 10, 670 (2008).

[118] M.J. Kao, C.H. Lo, T.T. Tsung, Y.Y. Wu, C.S. Jwo, H. M. Lin, J. Alloys Compd. 434, 672 (2007).

[119] M. J. Kao, H. Chang, Y.Y. Wu, T.T. Tsung, H. M. Lin, J. Chin. Soc. Mech. Eng. 28, 123 (2007).

[120] T. Sharma, A.L.M. Reddy, T.S. Chandra, S. Ramaprabhu, Int. J. Hydrogen Energy, 33, 6749 (2008).

[121] J. Philip, T. Jaykumar, P. Kalyanasundaram, B. Raj. Meas. Sci. Technol. 14, 1289 (2003).

[122] A. Mishra, P. Tripathy, S. Ram, H.J. Fecht. J. Nanosci. Nanotechnol. 9, 4342 (2009).

[123] W. Yu, H.Q. Xie, D. Bao, Nanotechnology, 21, 055705 (2010).

[124] W. Yu, H.Q. Xie, W. Chen, J. Appl. Phys. 107, 094317 (2010).

[125] Z.H. Han, F.Y. Cao, B. Yang, Appl. Phys. Lett. 92, 243104 (2008). 


\title{
Thermal Conductivity of Nanoparticles Filled Polymers
}

\author{
Hassan Ebadi-Dehaghani and Monireh Nazempour \\ Shahreza Branch, Islamic Azad University \\ Iran
}

\section{Introduction}

Thermal conductivity of polymers is an important thermal property for both polymer applications and processing. Polymers typically have intrinsic thermal conductivity much lower than those for metals or ceramic materials, and therefore are good thermal insulators. Further enhancement of this thermal insulating quality can be achieved by foaming polymers. In other applications which require higher thermal conductivity, such as in electronic packaging and encapsulations, satellite devices, and in areas where good heat dissipation, low thermal expansion and light weight are needed, polymers reinforced with fillers, organic or inorganic, are becoming more and more common in producing advanced polymer composites for these applications (Hodgin \& Estes, 1999; Tavman, 2004; Lee \& Eun, 2004; Liu \& Mather, 2004; Ishida \& Heights, 1999; Frank \& Phillip, 2002; Hermansen, 2001; Ishida, 2000). Most polymeric materials are processed and fabricated at elevated temperatures, often above their melting temperatures. This process may be long and expensive because of the low thermal conductivity of polymers. Subsequently, the cooling process or annealing may also be controlled by heat transport properties of polymers, which eventually affect the physical properties of the materials. One example is crystalline polymers, for which the structural and morphological features may be significantly changed with the speed of cooling. Careful consideration in designing polymer processing is vital to achieve desired properties.

For one-dimensional and rectilinear heat flow, the steady-state heat transfer in polymeric materials can be described by the Fourier's law of heat conduction:

$$
\mathrm{q}=-\mathrm{k} \frac{\mathrm{dt}}{\mathrm{dx}}
$$

where $\mathrm{q}$ is the heat flux (i.e., the heat transfer rate per unit area normal to the direction of flow), $x$ is the thickness of the material, $d T / d x$ is the temperature gradient per unit length, and the proportionality constant $\mathrm{k}$ is known as the thermal conductivity. The units for thermal conductivity $\mathrm{k}$ are expressed as $\mathrm{W} /(\mathrm{m} \mathrm{K})$ in SI units, Btu in./ $\left(\mathrm{ft}^{2} \mathrm{~h}{ }^{\circ} \mathrm{F}\right)$ in English units, and $\mathrm{cal} /\left(\mathrm{cm} \mathrm{s}^{\circ} \mathrm{C}\right)$ in cgs units. The corresponding units for heat flux are expressed as $\mathrm{W} /\left(\mathrm{m}^{2}\right), \mathrm{Btu} /\left(\mathrm{ft}^{2} \mathrm{~h}\right)$, and cal $/\left(\mathrm{cm}^{2} \mathrm{~s}\right)$, respectively.

Heat transfer involves the transport of energy from one place to another by energy carriers. In a gas phase, gas molecules carry energy either by random molecular motion (diffusion) or by 
an overall drift of the molecules in a certain direction (advection). In liquids, energy can be transported by diffusion and advection of molecules. In solids, phonons, electrons, or photons transport energy. Phonons, quantized modes of vibration occurring in a rigid crystal lattice, are the primary mechanism of heat conduction in most polymers since free movement of electrons is not possible (Majumdar, 1998). In view of theoretical prediction, the Debye equation is usually used to calculate the thermal conductivity of polymers (Han \& Fina, 2010).

$$
\lambda=\frac{C_{p} v l}{3}
$$

where $C_{p}$ is the specific heat capacity per unit volume; $v$ is the average phonon velocity; and $l$ is the phonon mean free path.

For amorphous polymers, $l$ is an extremely small constant (i.e. a few angstroms) due to phonon scattering from numerous defects, leading to a very low thermal conductivity of polymers (Agari et al., 1997). Table 1 displays the thermal conductivities of some polymers (T'Joen et al., 2009), (Hu et al., 2007) and (Speight, 2005).

\begin{tabular}{|c|c|}
\hline Material & $\begin{array}{l}\text { Thermal Conductivity at } 25^{\circ} \mathrm{C} \\
(\mathrm{W} / \mathrm{m} \mathrm{K})\end{array}$ \\
\hline Low density polyethylene (LDPE) & 0.30 \\
\hline High density polyethylene (HDPE) & 0.44 \\
\hline Polypropylene (PP) & 0.11 \\
\hline Polystyrene (PS) & 0.14 \\
\hline Polymethylmethacrylate (PMMA) & 0.21 \\
\hline Nylon-6 (PA6) & 0.25 \\
\hline Nylon-6.6 (PA66) & 0.26 \\
\hline Poly(ethylene terephthalate) (PET) & 0.15 \\
\hline Poly(butylene terephthalate) (PBT) & 0.29 \\
\hline Polycarbonate (PC) & 0.20 \\
\hline $\begin{array}{l}\text { Poly(acrylonitrile-butadiene-styrene) } \\
\text { copolymer (ABS) }\end{array}$ & 0.33 \\
\hline Polyetheretherketone (PEEK) & 0.25 \\
\hline Polyphenylene sulfide (PPS) & 0.30 \\
\hline Polysulfone (PSU) & 0.22 \\
\hline Polyphenylsulfone (PPSU) & 0.35 \\
\hline Polyvinyl chloride (PVC) & 0.19 \\
\hline Polyvinylidene difluoride (PVDF) & 0.19 \\
\hline Polytetrafluoroethylene (PTFE) & 0.27 \\
\hline Poly(ethylene vinyl acetate) (EVA) & 0.34 \\
\hline Polyimide, Thermoplastic (PI) & 0.11 \\
\hline Poly(dimethylsiloxane) (PDMS) & 0.25 \\
\hline Epoxy resin & 0.19 \\
\hline
\end{tabular}

Table 1. Thermal conductivities of some polymers (T’Joen et al., 2009), (Hu et al., 2007) and (Speight, 2005). 


\section{Thermal conductivity - measurement and modeling}

\subsection{Methods for thermal conductivity measurements}

Several methods, as reviewed elsewhere (Tritt \& Weston, 2004) and (Rides et al., 2009), have been proposed and used for measurement of the thermal conductivity of polymers and composites. Classical steady-state methods measure the temperature difference across the specimens in response to an applied heating power, either as an absolute value or by comparison with a reference material put in series or in parallel to the sample to be measured. However, these methods are often time consuming and require relatively bulky specimens.

Several non steady-state methods have also been developed, including hot wire and hot plate methods, temperature wave method and laser flash techniques (Nunes dos Santos, 2007). Among these, laser-flash thermal diffusivity measurement is widely used, being a relatively fast method, using small specimens (Nunes dos Santos, 2007), (Nunes dos Santos, 2005) and (Gaal et al., 2004). In this method, the sample surface is irradiated with a very short laser pulse and the temperature rise is measured on the opposite side of the specimen, permitting calculation of the thermal diffusivity of the material, after proper mathematical elaboration. The thermal conductivity $\mathrm{k}$ is then calculated according to Eq. (3):

$$
k=a C_{p} \rho
$$

where $a, C_{p}$ and $\rho$ are the thermal diffusivity, heat capacity and density, respectively.

Differential scanning calorimetry (DSC) methods may also be used, applying an oscillary (Marcus \& Blaine, 1994) or step temperature profile (Merzlyakov \& Schick, 2001) and analyzing the dynamic response.

Significant experimental error may be involved in thermal conductivity measurements, due to difficulties in controlling the test conditions, such as the thermal contact resistance with the sample, leading to accuracy of thermal conductivity measurements typically in the range of $5-10 \%$. In indirect methods, such as those calculating the thermal conductivity from the thermal diffusivity, experimental errors on density and heat capacity values will also contribute to the experimental error in the thermal conductivity.

\subsection{Modeling of thermal conductivity in composites}

Several different models developed to predict the thermal conductivity of traditional polymer composites are reviewed elsewhere (Bigg, 1995), (Zhou et al., 2007), (Zeng et al., 2009) and (Wang et al., 2008). The fundamentals are recalled in this section.

The two basic models representing the upper bound and the lower bound for thermal conductivity of composites are the rule of mixture and the so-called series model, respectively. In the rule of mixture model, also referred to as the parallel model, each phase is assumed to contribute independently to the overall conductivity, proportionally to its volume fraction (Eq. (4)):

$$
\mathrm{k}_{\mathrm{c}}=\mathrm{k}_{\mathrm{p}} \Phi_{\mathrm{p}}+\mathrm{k}_{\mathrm{m}} \Phi_{\mathrm{m}}
$$


where $k_{c}, k_{p}, k_{m}$ are the thermal conductivity of the composite, particle, matrix, respectively, and $\Phi_{p}, \Phi_{m}$ volume fractions of particles and matrix, respectively. The parallel model maximizes the contribution of the conductive phase and implicitly assumes perfect contact between particles in a fully percolating network. This model has some relevance to the case of continuous fiber composites in the direction parallel to fibers, but generally results in very large overestimation for other types of composites.

On the other hand, the basic series model assumes no contact between particles and thus the contribution of particles is confined to the region of matrix embedding the particle. The conductivity of composites accordingly with the series model is predicted by Eq. (5):

$$
k_{c}=\frac{1}{\left(\phi_{m}+k_{m}\right)+\left(\phi_{p}+k_{p}\right)}
$$

Most of the experimental results were found to fall in between the two models. However, the lower bound model is usually closer to the experimental data compared to the rule of mixture (Ebadi-Dehaghani et al., 2011; Bigg, 1995), which brought to a number of different models derived from the basic series model, generally introducing some more complex weighted averages on thermal conductivities and volume fractions of particles and matrix. These so-called second-order models including equations by Hashin and Shtrikman, Hamilton and Crosser, Hatta and Taya, Agari, Cheng and Vachon as well as by Nielsen (Bigg, 1995), (Zhou et al., 2007) and (Okamoto \& Ishida 1999), appear to reasonably fit most of the experimental data for composites based on isotropic particles as well as short fibers and flakes with limited aspect ratio, up to loadings of about $30 \%$ in volume.

In the case of the geometric mean model, the effective thermal conductivity of the composite is given by:

$$
K_{c}=K_{m}^{\phi_{m}}+K_{f}^{\phi_{f}}
$$

Lewis and Nielsen modified the Halpin-Tsai equation (Nielsen et al., 1994) to include the effect of the shape of the particles and the orientation or type of packing for a two-phase system.

$$
K_{c}=K_{m}\left[\frac{1+A B \phi_{f}}{1-B \psi \phi_{f}}\right]
$$

Where

$$
B=\frac{\frac{K_{f}}{K_{m}}-1}{\frac{K_{f}}{K_{m}}-A} \psi=1+\left(\frac{1-\phi_{\max }}{\phi_{\max }{ }^{2}}\right) \phi_{f}
$$

The values of $\mathrm{A}$ and $\Phi_{\max }$ were given for many geometric shapes and orientations (Weidenfeller et al., 2004).

This model appears to reasonably fit most of the experimental data for composites based on isotropic particles as well as short fibers and flakes with limited aspect ratio, up to loading of about $30 \%$ in volume. For higher loadings, the Nielsen's model appear to best fit the rapid increase of thermal conductivity above $30 \mathrm{vol} . \%$, thanks for the introduction of the maximum packing factor into the fitting equation, despite the evaluation of maximum packing factor in 
real composites may present difficulties due to particle size distribution and particle dispersion in the matrix. However, the basic assumption of separated particles in the effective medium approach is not valid in principle for highly filled composites, where contacts are likely to occur, possibly leading to thermally conductive paths (Tavman, 1996).

Maxwell, using potential theory, obtained an exact solution for the conductivity of randomly distributed and non-interacting homogeneous spheres in a homogeneous medium:

$$
K_{c}=K_{m}\left[\frac{K_{f}+2 K_{m}+2 \phi_{f}\left(K_{f}-K_{m}\right)}{K_{f}+2 K_{m}-\phi_{f}\left(K_{f}-K_{m}\right)}\right]
$$

Other theoretical models have attempted to explain the thermal conductivity of two-phased composites. Some of these models, such as those by Bruggeman, Botcher, De Loor, and Ce Wen Nan et al., equations 6 to 9 respectively, have been used for prediction of thermal conductivity of carbon nanotube composites (Bruggeman, 1935; Böttcher, 1952; deLoor, 1956; Nan et al., 2004).

$$
\begin{gathered}
K_{c}=\frac{K_{m}}{\left(1-\phi_{f}\right)^{3}} \\
K_{c}=\frac{K_{m}}{\left(1-\phi_{f}\right)} \\
K_{c}=\frac{K_{m}\left(1+\phi_{f}\right)}{1-2 \phi_{f}} \\
K_{c}=\frac{K_{m}\left[3+\phi_{f}\left(\frac{K_{f}}{K_{m}}\right)\right]}{3-2 \phi_{f}}
\end{gathered}
$$

In order to take into account fluctuations in thermal conductivity in the composites, Zhi et al. (Zhi et al., 2009) proposed the concept of heat-transfer passages, to model the conduction in regions where interparticle distance is low, applying the series model to "packed-belt" of conductive particles.

Even though these macroscopic approaches may be of interest from the engineering point of view, they deliver little or no information about the physical background of the observed behavior. As an example, very limited interpretation is given to the rapidly increasing conductivity with filler content above a certain filler loading (typically above 30 vol.\%), or why the experimental results are so far away from the upper bound conductivity, even for highly percolated systems.

Attempts to model thermal conductivity taking into account the interfacial thermal resistance between conductive particles and matrix have been reported by several research groups (Nan et al., 1997), (Every et al., 1992), (Dunn \& Taya, 1993), (Lipton \& Vernescu, 1996) and (Torquato \& Rintoul, 1995) and applied particles with different geometries and topologies, including aligned continuous fibers, laminated flat plates, spheres, as well as disoriented ellipsoidal particles. In general, these models provided an improved fit with experimental data for ceramic based composites than models not accounting for interface thermal resistance. These approaches generally assume conductive particles to be isolated in the matrix and take into account the thermal resistance in heat transfer between conductive 
particle and matrix, also known as Kapitza resistance, from the name of the discoverer of the temperature discontinuity at the metal-liquid interface. A very simple proof of thermal interfacial resistance is the fact that a thermal conductivity lower than the reference matrix was experimentally found with some composites containing particles with thermal conductivity higher than the matrix (Nan et al., 1997) and (Every et al., 1992). This phenomenon is explained by the very low efficiency of heat transfer between particles and matrix, so that the higher thermal conductivity of the filler cannot be taken into advantage and the composite behaves like a hollow material, thus reducing its conductivity compared to the dense reference matrix. Evaluation of the effective thermal conductivity of composite polymers by considering the filler size distribution law was investigated by Holotescu et al (Holotescu et al., 2009).

They presented an empirical model for the effective thermal conductivity (ETC) of a polymer composite that includes dependency on the filler size distribution-chosen as the Rosin-Rammler distribution. The ETC is determined based on certain hypotheses that connect the behavior of a real composite material A, to that of a model composite material B, filled with mono-dimensional filler. The application of these hypotheses to the Maxwell model for ETC is presented. The validation of the new model and its characteristic equation was carried out using experimental data from the reference. The comparison showed that by using the size distribution law a very good fit between the equation of the new model (the size distribution model for the ETC) and the reference experimental results is obtained, even for high volume fractions, up to about $50 \%$.

\section{Crystallinity and temperature dependence}

Polymer crystallinity strongly affects their thermal conductivity, which roughly varies from $0.2 \mathrm{~W} / \mathrm{m} \mathrm{K}$ for amorphous polymers such as polymethylmethacrylate (PMMA) or polystyrene (PS), to $0.5 \mathrm{~W} / \mathrm{m} \mathrm{K}$ for highly crystalline polymers as high-density polyethylene (HDPE) (Hu et al., 2007). The thermal conductivity of semi-crystalline polymers is reported to increase with crystallinity. As an example, the thermal conductivity of polytetrafluoroethylene (PTFE) was found to increase linearly with crystallinity at $232{ }^{\circ} \mathrm{C}$ (Price \& Jarratt, 2002).

However, there is a large scatter in the reported experimental data of thermal conductivity of crystalline polymers, even including some contradictory results. It should be noticed that the thermal conductivities of polymers depend on many factors, such as chemical constituents, bond strength, structure type, side group molecular weight, molecular density distribution, type and strength of defects or structural faults, size of intermediate range order, processing conditions and temperature. Furthermore, due to the phonon scattering at the interface between the amorphous and crystalline phase and complex factors on crystallinity of polymer, the prediction of the thermal conductivity vs. crystallinity presents a significant degree of complexity (Han \& Fina, 2010).

Semicrystalline and amorphous polymers also vary considerably in the temperature dependence of the thermal conductivity. At low temperature, semicrystalline polymers display a temperature dependence similar to that obtained from highly imperfect crystals, having a maximum in the temperature range near $100 \mathrm{~K}$, shifting to lower temperatures and higher thermal conductivities as the crystallinity increases (Greig \& Hardy, 1981) and (Yano 
\& Yamaoka, 1995), while amorphous polymers display temperature dependence similar to that obtained for inorganic glasses with no maximum, but a significant plateau region at low temperature range (Reese, 1969). The thermal conductivity of an amorphous polymer increases with increasing temperature to the glass transition temperature $\left(T_{g}\right)$, while it decreases above $T_{g}$ (Zhong et al., 2001) and (Dashora \& Gupta, 1996). The study of the thermal conductivity of some amorphous and partially crystalline polymers (PE, PS, PTFE and epoxy resin) as a function of temperature in a common-use range (273-373 K) indicates that the conductivity of amorphous polymers increases with temperature and that the conductivity is significantly higher in crystalline than amorphous regions (Kline, 1961).

From the general overview given in the preceding, it appears that very limited thermal conductivity is usually characteristic of polymers. On the other hand, there are many reasons to increase thermal conductivity of polymer-based materials in various industrial applications including circuit boards in power electronics, heat exchangers, electronics appliances and machinery. This justifies the recent significant research efforts on thermally conductive composite materials to overcome the limitations of traditional polymers.

\section{Fillers for thermally conductive composites}

Many applications would benefit from the use of polymers with enhanced thermal conductivity. For example, when used as heat sinks in electric or electronic systems, composites with a thermal conductivity approximately from 1 to $30 \mathrm{~W} / \mathrm{m} \mathrm{K}$ are required (King et al., 1999). The thermal conductivity of polymers has been traditionally enhanced by the addition of thermally conductive fillers, including graphite, carbon black, carbon fibers, ceramic or metal particles (see Table 2) (Pierson, 1993), (Wypych, 2000), (Fischer, 2006),

\begin{tabular}{|l|c|}
\hline Material & $\begin{array}{c}\text { Thermal Conductivity at } 25^{\circ} \mathbf{C} \\
(\mathbf{W} / \mathbf{m} \text { K) }\end{array}$ \\
\hline Graphite & $100 \sim 400$ (on plane) \\
\hline Carbon black & $6 \sim 174$ \\
\hline Carbon Nanotubes & $2000 \sim 6000$ \\
\hline Diamond & 2000 \\
\hline PAN-based Carbon Fibre & $8 \sim 70$ (along the axis) \\
\hline Pitch-based Carbon Fibre & $530 \sim 1100$ (along the axis) \\
\hline Copper & 483 \\
\hline Silver & 450 \\
\hline Gold & 345 \\
\hline Aluminum & 204 \\
\hline Nickel & 158 \\
\hline Boron Nitride & $250 \sim 300$ \\
\hline Aluminum nitride & 200 \\
\hline Beryllium oxide & 260 \\
\hline Aluminum oxide & $20 \sim 29$ \\
\hline
\end{tabular}

Table 2. Thermal conductivities of some thermally conductive fillers (Pierson, 1993), (Wypych, 2000), (Fischer, 2006), (Wolff \& Wang, 1993) and (Kelly, 1981). 
(Wolff \& Wang, 1993) and (Kelly, 1981). It is worth noticing that significant scatter of data are typically reported for thermal conductivity of fillers. This is caused by several factors, including filler purity, crystallinity, particle size and measurement method. It is also important to point out that some materials, typically fibers and layers, are highly anisotropic and can show much higher conductivity along a main axis or on a plane, compared to perpendicular direction.

High filler loadings ( $>30 \mathrm{vol} . \%$ ) are typically necessary to achieve the appropriate level of thermal conductivity in thermally conductive polymer composites, which represents a significant processing challenge. Indeed, the processing requirements, such as possibility to be extruded and injection molded, often limit the amount of fillers in the formulation and, consequently, the thermal conductivity performance (King et al., 2008). Moreover, high inorganic filler loading dramatically alters the polymer mechanical behavior and density. For these reasons, obtaining composites having thermal conductivities higher than $4 \mathrm{~W} / \mathrm{m} \mathrm{K}$ and usual polymer processability is very challenging at present (Han \& Fina, 2010).

\subsection{Carbon-based fillers}

Carbon-based fillers appear to be the best promising fillers, coupling high thermal conductivity and lightweight. Graphite, carbon fiber and carbon black are well-known traditional carbon-based fillers. Graphite is usually recognized as the best conductive filler because of its good thermal conductivity, low cost and fair dispersability in polymer matrix (Causin et al., 2006) and (Tu \& Ye, 2009). Single graphene sheets constituting graphite show intrinsically high thermal conductivity of about $800 \mathrm{~W} / \mathrm{m} \mathrm{K}$ (Liu et al., 2008) or higher (theoretically estimated to be as high as $5300 \mathrm{~W} / \mathrm{m} \mathrm{K}$ ( Veca et al., 2009) and (Stankovich et al., 2006)), this determining the high thermal conductivity of graphite, usually reported in the range from 100 to $400 \mathrm{~W} / \mathrm{m} \mathrm{K}$. Expanded graphite (EG), an exfoliated form of graphite with layers of 20-100 nm thickness, has also been used in polymer composites (Ganguli et al., 2008), for which the thermal conductivity depends on the exfoliation degree (Park et al., 2008), its dispersion in matrix (Mu \& Feng, 2007) and the aspect ratio of the EG (Kalaitzidou et al., 2007).

Thermal conductivity of exfoliated graphite nanocomposites was investigated by Fukushima et al. (Fukushima et al., 2006). Since the late 1990's, research has been reported where intercalated, expanded, and/or exfoliated graphite nanoflakes could also be used as reinforcements in polymer systems. The key point to utilizing graphite as a platelet nanoreinforcement is in the ability to exfoliate graphite using Graphite Intercalated Compounds (GICs). Natural graphite is still abundant and its cost is quite low compared to the other nano-size carbon materials, the cost of producing graphite nanoplatelets is expected to be $\sim \$ 5 / \mathrm{lb}$. This is significantly less expensive than single wall nanotubes $(\mathrm{SWNT})(>\$ 45000 / \mathrm{lb})$ or vapor grown carbon fiber (VGCF) $(\$ 40-50 / 1 b)$, yet the mechanical, electrical, and thermal properties of crystalline graphite flakes are comparable to those of SWNT and VGCF. The use of exfoliated graphite flakes $(x \mathrm{GnP})$ opens up many new applications where electromagnetic shielding, high thermal conductivity, gas barrier resistance or low flammability are required. A special thermal treatment was developed to exfoliate graphite flakes for the production of nylon and high density polypropylene nanocomposites. X-ray diffraction (XRD), scanning electron microscopy (SEM) and transmission electron microscopy (TEM) were used to assess the degree of exfoliation of the 
graphite platelets and the morphology of the nanocomposites. The thermal conductivity of these composites was investigated by three different methods, namely, by DSC, modified hot wire, and halogen flash lamp methods. The addition of small amounts of exfoliated graphite flakes showed a marked improvement in thermal and electrical conductivity of the composites.

Carbon fiber, typically vapor grown carbon fiber (VGCF), is another important carbon-based filler. Polymer/VGCF composites have been reviewed by Tibbetts et al. (Tibbetts et al., 2007). Since VGCF is composed of an annular geometry parallel to the fiber axis, thermal conductive properties along the fiber axis are very different from the transverse direction (estimated up to $2000 \mathrm{~W} / \mathrm{m} \mathrm{K}$ in the axial direction vs. $10-110 \mathrm{~W} / \mathrm{m} \mathrm{K}$ in the transverse direction (Chen \& Ting, 2002) and (Zhang et al, 2000)), directly affecting the thermal conductivity of aligned composites (Mohammed \& Uttandaraman, 2009) and (Kuriger et al., 2002).

Carbon black particles are aggregates of graphite microcrystals and characteristic of their particle size $(10-500 \mathrm{~nm})$ and surface area $\left(25-150 \mathrm{~m}^{2} / \mathrm{g}\right.$ ) (Pierson, 1993). Carbon black is reported to contribute to electrical conductivity rather than thermal conductivity (Wong et al., 2001), (Abdel-Aal et al., 2008) and (King et al., 2006).

\subsection{Metallic fillers}

The filling of a polymer with metallic particles may result in both increase of thermal conductivity and electrical conductivity in the composites. However, a density increase is also obtained when adding significant metal loadings to the polymer matrix, thus limiting applications when lightweight is required. Metallic particles used for thermal conductivity improvement include powders of aluminum, silver, copper and nickel. Boudenne et al studied the thermal behavior of polypropylene filled with copper particles (Kumlutaş et al., 2003). In this work thermal conductivity, diffusivity, effusivity and specific heat of polypropylene matrix filled with copper particles of two different sizes were investigated. A parallel study of the evolution of the electrical conductivity was also carried out. The highest heat transport ability was observed for the composites filled with the smaller particles. The Agari's model provides a good estimation of the thermal conductivity of composites for all filler concentrations. Polymers modified with the inclusion of metallic particles include polyethylene (Kumlutaş et al., 2003), polypropylene (Boudenne et al., 2005), polyamide (Tekce et al., 2007), polyvinylchloride and epoxy resins (Mamunya et al., 2002), showing thermal conductivity performance depending on the thermal conductivity of the metallic fillers, the particle shape and size, the volume fraction and spatial arrangement in the polymer matrix. Thermal conductivity of metal powder-polymer feedstock for powder injection moulding was studied by Kowalski et al. (Kowalski et al., 1999).

Thermal conductivity of a powder injection molding feedstock (mixture of metal powders and polymers) in solid and molten states has been measured by using the laser flash method. The filler material was 316L stainless steel powder and its content in the mixture amounted $60 \%$ by volume. An attempt has been made to employ two most promising existing mathematical models (theoretical Maxwell- and semi-theoretical Lewis \& Nielsen model) to calculate the thermal conductivity of the mixture (see section 1.3.2). Comparison of the experimental and calculated results has revealed that the Lewis \& Nielsen model 
predicts better than Maxwell model the thermal conductivity of the feedstock. As the difference between the calculated (Maxwell model) and the measured results amounts to $15-85 \%$, it is suggested that it can only be used for preliminary assessment of the thermal conductivity of so highly filled composite material. If accurate thermal conductivity data are required (as in case of numerical simulation of the powder injection moulding process), measurement of this property has to be performed if meaningful simulation results are to be expected.

\subsection{Ceramic fillers}

Ceramic powder reinforced polymer materials have been used extensively as electronic materials. Being aware of the high electrical conductivity of metallic particles, several ceramic materials such as aluminum nitride $(\mathrm{AlN})$, boron nitride $(\mathrm{BN})$, silicon carbide $(\mathrm{SiC})$ and beryllium oxide $(\mathrm{BeO})$ gained more attention as thermally conductive fillers due to their high thermal conductivity and electrical resistivity (Nu et al., 2008) and (Ishida \& Rimdusit, 1998). Thermal conductivities of composites with ceramic filler are influenced by filler packing density(Ohashi et al., 2005), particle size and size distribution (Yu et al., 2002) and (Mu et al., 2007), surface treatment (Gu et al., 2009) and mixing methods (Zhou et al., 2007). Models and theories for predicting the thermal conductivity of polymer composites were discussed. Effective Medium Theory (EMT), Agari model and Nielsen model respectively are introduced and are applied as predictions for the thermal conductivity of ceramic particle filled polymer composites. Thermal conductivity of experimentally prepared $\mathrm{Si}_{3} \mathrm{~N}_{4}$ /epoxy composite and some data cited from the literature are discussed using the above theories. Feasibility of the three methods as a prediction in the whole volume fraction region of the filler from 0 to 1 was evaluated for a comparison. As a conclusion: both EMT and Nielsen model can give a well prediction for the thermal conductivity at a low volume fraction of the filler; Agari model give a better prediction in the whole range, but with larger error percentage (He et al., 2007).

\section{Nanocomposites for thermal conductivity}

Polymer nanocomposites are commonly defined as the combination of a polymer matrix and additives that have at least one dimension in the nanometer range. The additives can be one-dimensional (examples include nanotubes and fibres), two-dimensional (which include layered minerals like clay), or three-dimensional (including spherical particles). Over the past decade, polymer nanocomposites have attracted considerable interests in both academia and industry, owing to their outstanding mechanical properties like elastic stiffness and strength with only a small amount of the nanoadditives. This is caused by the large surface area to volume ratio of nanoadditives when compared to the micro- and macro-additives. Other superior properties of polymer nano-composites include barrier resistance, flame retardancy, scratch/wear resistance, as well as optical, magnetic, thermal conductivity and electrical properties. Polymer based nanocomposites can be obtained by the addition of nanoscale particles which are classified into three categories depending on their dimensions: nanoparticles, nanotubes and nanolayers. The interest in using nanoscaled fillers in polymer matrices is the potential for unique properties deriving from the nanoscopic dimensions and inherent extreme aspect ratios of the nanofillers (Mai et al., 2006). Kumar et al. (Kumar et al., 2009) summarized six interrelated characteristics of 
nanocomposites over conventional micro-composites: (1) low-percolation threshold (about $0.1-2$ vol.\%), (2) particle-particle correlation (orientation and position) arising at lowvolume fractions (less than 0.001), (3) large number density of particles per particle volume (106 to $10^{8}$ particles $\left./ \mu^{3}\right)$, (4) extensive interfacial area per volume of particles $\left(10^{3}\right.$ to $\left.10^{4} \mathrm{~m}^{2} / \mathrm{ml}\right)$, (5) short distances between particles (10-50 $\mathrm{nm}$ at 1-8 vol.\%) and (6) comparable size scales among the rigid nanoparticles inclusion, distance between particles, and the relaxation volume of polymer chains (Han \& Fina, 2010).

Different nanoparticles have been used to improve thermal conductivity of polymers. As a few examples, HDPE filled with 7 vol.\% nanometer size expanded graphite has a thermal conductivity of $1.59 \mathrm{~W} / \mathrm{m} \mathrm{K}$, twice that of microcomposites $(0.78 \mathrm{~W} / \mathrm{m} \mathrm{K})$ at the same volume content (Ye et al., 2006). Poly(vinyl butyral) (PVB), PS, PMMA and poly(ethylene vinyl alcohol) (PEVA) based nanocomposites with $24 \mathrm{wt}$ \% boron nitride nanotubes (BNNT) have thermal conductivities of 1.80, 3.61, 3.16 and $2.50 \mathrm{~W} / \mathrm{m} \mathrm{K}$, respectively (Zhi et al., 2009). Carbon nanofiber was also reported to improve the thermal conductivity of polymer composites (Sui et al., 2008) and (Elgafy \& Lafdi, 2005). However, the most widely used and studied nanoparticles for thermal conductivity are certainly carbon nanotubes (either single wall-SWCNT or multiwall-MWCNT), which have attracted growing research interest. Indeed, CNT couples very high thermal conductivity with outstanding aspect ratio, thus forming percolating network at very low loadings.

Droval and co-workers (Droval et al., 2006) investigated the effect of boron nitride (BN), talc $\left(\mathrm{Mg}_{3} \mathrm{Si}_{4} \mathrm{O}_{10}(\mathrm{OH})_{2}\right)$, aluminum nitride $(\mathrm{AlN})$ and aluminum oxide $\left(\mathrm{Al}_{2} \mathrm{O}_{3}\right)$ particles, and their impact on thermal properties. Lewis and Nielson, Cheng and Vachon, Agari and Uno models were used to predict the evolution of thermal conductivity with filler content and were found to describe correctly thermal conductivity. Only BN shows a real exponential increase of conductivity over $20 \% \mathrm{v} / \mathrm{v}$ filler. Consequently, in best conditions introducing $30 \% \mathrm{v} / \mathrm{v}$ of BN allows the thermal conductivity to be multiplied by six.

A technology has been developed for making carbon-ceramic composite refractories by combining carbon fibers as reinforcing component with a mixture matrix, which allows one to make refractory components of various sizes and geometry, including thin-walled large constructions (Chernenko et al., 2009). The heat resistance of these composite refractories increases with the bulk silicization during ceramic production on a carbon-carbon substrate. The degree of silicization is determined by the volume of the open microporosity of transport type, which is formed by pyrolysis of a polymer coke-forming matrix in the initial carbon plastic. The transport micropores are produced by a modification of the phenolformaldehyde resin additive treatment, which does not give rise to coke on pyrolysis. As a result, the content of open pores in the carbon framework attains $55 \%$, which enables one to make a silicized composite refractory of density up to $2.7 \mathrm{~g} / \mathrm{cm}^{3}$ with a compressive strength of $250-300 \mathrm{MPa}$, bending strength 120-140, and tensile strength $60-80 \mathrm{MPa}$, elastic modulus 120-140 GPa, linear expansion coefficient $3.5 \times 10^{-6}-4.5 \times 10^{-6} \mathrm{~K}^{-1}$, and thermal conductivity $6-8 \mathrm{~W} /(\mathrm{m} \mathrm{K})$. These refractories are widely used in various branches of industry. Thermal conductivity of particle filled polyethylene composite materials was investigated by Kumlutas et al. (Kumlutas et al., 2003). In this study, the effective thermal conductivity of aluminum filled high-density polyethylene composites is investigated numerically as a function of filler concentration. The obtained values are compared with experimental results and the existing theoretical and empirical models. The thermal 
conductivity is measured by a modified hot-wire technique. For numerical study, the effective thermal conductivity of particle-filled composite was calculated numerically using the micro structural images of them. By identifying each pixel with a finite difference equation and accompanying appropriate image processing, the effective thermal conductivity of composite material is determined numerically. As a result of this study, numerical results, experimental values and all the models are close to each other at low particle content. For particle content greater than $10 \%$, the effective thermal conductivity is exponentially formed. All the models fail to predict thermal conductivity in this region. But, numerical results give satisfactory values in the whole range of aluminum particle content.

\subsection{Nanocomposites using inorganic fillers}

Thermally conductive polymer nanocomposites based on polypropylene has been studied (Vakili et al., 2011; Ebadi-Dehaghani et al., 2011). In this study three nanocomposite containing 5 to $15 \mathrm{wt} \%$ of $\mathrm{ZnO}$ and $\mathrm{CaCO} 3$ nanoparticles prepared by extrusion were used. The thermal conductivity (TC) of compression moulded polypropylene (PP) and PP filled nanoparticles was studied using thermal conductivity analyser (TCA). The effect of nanoparticle content and crystallinity on thermal conductivity was investigated using conventional methods like SEM, XRD and DSC. The incorporation of nanoparticles improved crystallinity and thermal conductivity simultaneously. The experimental TC values of PP nanocomposites with different level of nanoparticles concentration showed a linear increase with an increase in crystallinity.

\subsubsection{Differential Scanning Calorimetry (DSC)}

DSC measurements were investigated by conventional differential scanning calorimeter Labsys TG (Setaram Instumentation, Caluire, France). A pellet of extruded sample, with a weight of 8-10 mg, was placed into an alumina pan in the presence of air as the furnace atmosphere. Measurements were performed from ambient temperature up to $200^{\circ} \mathrm{C}$ with heating rate of $10^{\circ} \mathrm{C} / \mathrm{min}$. The DSC results for pure PP and nanocomposites, The Tm (peak temperature of melting) and $\Delta \mathrm{Hm}$ (enthalpy of melting), are listed in Table 3.

The degree of crystallinity of a specimen can be calculated from the melting heat of crystallization according to the following equation:

$$
X_{\varepsilon}=\frac{\Delta H_{m}}{\Delta H_{v}\left(1-W_{f}\right)} \times 100
$$

Where $\mathrm{w}_{\mathrm{f}}$ is the weight fraction of nanofiller and $\Delta \mathrm{H}_{0}=207.1 \mathrm{Jg}^{-1}$ is the melting heat of $100 \%$ crystalline PP (Bai et al., 1999).

The DSC results indicated that the addition of both nanoparticles to the PP caused only a marginal effect on melting temperature $\left(\mathrm{T}_{\mathrm{m}}\right)$ and no correlation of the results with the filler concentration could be established. The calculated degree of crystallinity of the PP phase increased with increasing content of both nanoparticles, indicating that the nanofillers nucleated the crystallization process. (Frormann et al., 2008) This implies that the existence of nanoparticles facilitates the crystallization of PP and this effect becomes more evident with higher nanoparticle content (Zhao \& Li, 2006). Similar results for $\mathrm{PP} / \mathrm{CaCO} 3$ 
nanocomposites, $\mathrm{PP} /$ carbon nanotube composites and $\mathrm{PP} /$ nanoclay composites (Han \& Fina , 2011; Frormann et al., 2008; Vakili et al., 2011) have been reported. However there are some contradicting results in the literature (Zhao \& Li, 2006).

\begin{tabular}{llccc}
\hline Neat PP & $\begin{array}{c}\text { PP+5wt\% } \\
\text { nanofiller }\end{array}$ & $\begin{array}{c}\text { PP+10wt\% } \\
\text { nanofiller }\end{array}$ & $\begin{array}{c}\text { PP+15wt\% } \\
\text { nanofiller }\end{array}$ \\
\hline $\mathrm{T}_{\mathrm{m}}\left({ }^{\circ} \mathrm{C}\right)$ & 167.8 & 168.5 & 168.8 & 167.0 \\
$\Delta \mathrm{H}_{\mathrm{m}}(\mathrm{Jg}-1)$ & 78.0 & 91.2 & 108.9 & 107.7 \\
$\mathrm{X}_{\mathrm{c}}(\%)$ & 37.7 & 44.0 & 52.6 & 52.0
\end{tabular}

a)

\begin{tabular}{lcccc}
\hline & Neat PP & $\begin{array}{c}\text { PP+5wt\% } \\
\text { nanofiller }\end{array}$ & $\begin{array}{c}\text { PP+10wt\% } \\
\text { nanofiller }\end{array}$ & $\begin{array}{c}\text { PP+15wt\% } \\
\text { nanofiller }\end{array}$ \\
\hline $\mathrm{T}_{\mathrm{m}}\left({ }^{\circ} \mathrm{C}\right)$ & 167.8 & 168.6 & 168.1 & 168.4 \\
$\Delta \mathrm{H}_{\mathrm{m}}\left(\mathrm{Jg}^{-1}\right)$ & 78.0 & 81.4 & 85.8 & 104.6 \\
$\mathrm{X}_{\mathrm{c}}(\%)$ & 37.7 & 39.3 & 41.4 & 50.5
\end{tabular}

b)

Table 3. Crystallization parameters of neat PP and nanocomposites. a) PP/ZnO nanocomposite. b) $\mathrm{PP} / \mathrm{CaCO}_{3}$ nanocomposite.

\subsubsection{Thermal conductivity measurement}

Thermal conductivity was measured using a TCA Thermal Conductivity Analyser (TCA200LT-A, Netzsch, Selb, Germany) with the guarded heat flow meter method. Each compression molded sample $(30 \mathrm{~cm} \times 30 \mathrm{~cm}$ sheets with $10 \mathrm{~mm}$ thickness $)$ was placed between two heated surface controlled at different temperatures with a flow of heat from the hot to the cold surface. When thermal equilibrium was attained thermal conductivity data were taken within an accuracy of $3 \%$. Fig. 1 compares the effect of the nanoparticles' content on the thermal conductivity of the nanocomposites. As seen, the value of thermal conductivity increased with an increase in the nanoparticle concentration up to $64 \%$ and $82 \%$ for $\mathrm{CaCO}_{3}$ and $\mathrm{ZnO}$ respectively. The increase in $\mathrm{TC}$ for $\mathrm{ZnO}$ nanoparticles is more than $\mathrm{CaCO}_{3}$ due to the nature of nanofiller and also crystallinity degree regarding to DSC results (Table 3). These increases in TC for both nanoparticles are higher than that of reported values for $\mathrm{CNF}$ in a PP matrix (Frormann et al., 2008).

The values obtained from the experimental study of PP nanocomposites were compared with several TC models (Figs 2.a and 2.b). As seen the experimental results were found to fall in between the Series and Parallel models. However, the lower bound model (series) is usually closer to the experimental data.

Maxwell, Lewis \& Nielson, Bruggeman, Bottcher and De Loor models predict fairly well thermal conductivity values up to $10 \mathrm{wt} \%$ for $\mathrm{PP} / \mathrm{ZnO}$ nanocomposites (Fig. 2.a). In the concentration of $15 \mathrm{wt} \%$ no model could predict well the TC values and all of the mentioned models underestimated the TC values of nanocomposite whereas in the case $5 \mathrm{wt} \%$ all models overestimated the TC values. 


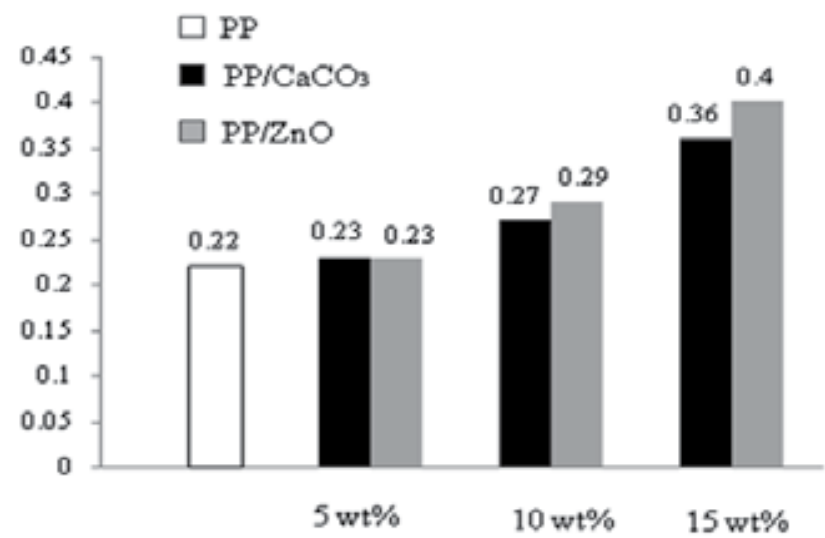

Fig. 1. Comparing the effect of nanoparticles on the TC of PP.

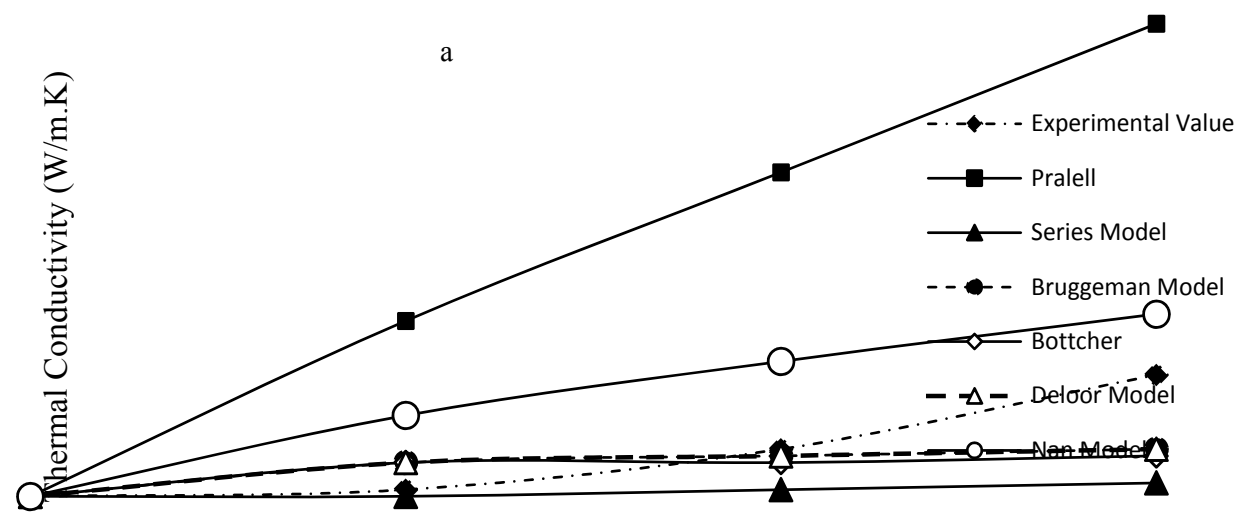

$\mathrm{wt} \%$ of Nanofiller

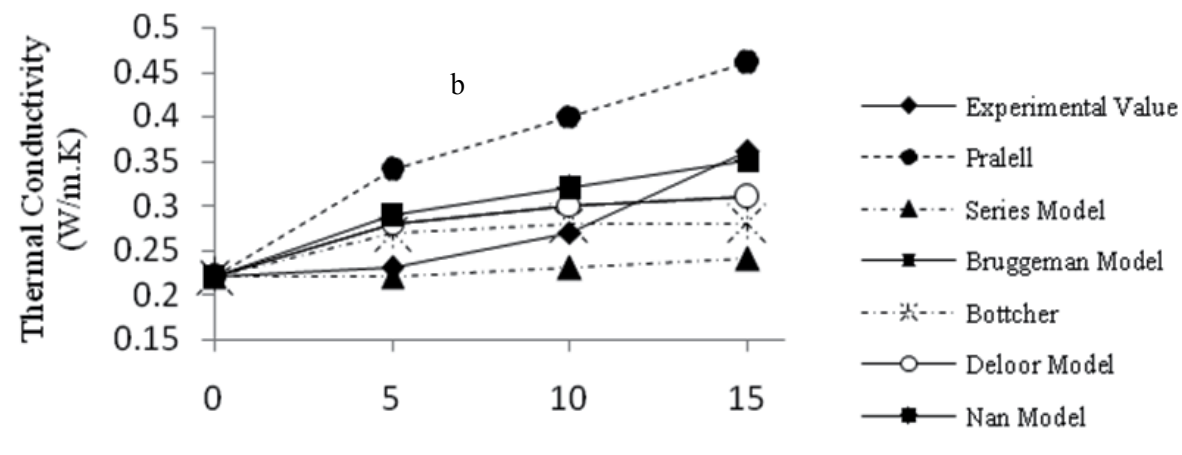

wt $\%$ of Nanofiller

Fig. 2. Comparing the experimental TC values vs nanoparticle content with theoretical models. a) $\mathrm{PP} / \mathrm{ZnO}$ nanocomposite b) $\mathrm{PP} / \mathrm{CaCO}_{3}$ nanocomposite. 
This fact can be attributed to the intrinsic thermal conductivity of both nanoparticles and their large surface area which even at lower loadings of nanofillers they are still effective to transfer heat through the samples (Frormann et al., 2008). At a higher volume fraction, this effect becomes stronger. Fig. 2.b the values obtained from the experimental study for $\mathrm{PP} / \mathrm{CaCO}_{3}$ nanocomposites are compared with a number of TC models. As seen the Ce Wen Nan model predicts fairly well the thermal conductivity values up to $15 \mathrm{wt} \%$. For the concentration of $10 \mathrm{wt} \%$ all the models predict the TC values well. In the case of $15 \mathrm{wt} \%$ other models underestimated the TC values of nanocomposites except for the Ce Wen Nan model, whereas for $5 \mathrm{wt} \%$ all models overestimated the TC value. The predicted TC values by the models depend on the nature of nanofiller and their relative concentrations (Weidenfeller et al., 2004; Frormann et al., 2008).

The TC improvement in $\mathrm{PP} / \mathrm{ZnO}$ nanocomposite is greater than that of $\mathrm{PP} /$ calcium carbonate nanocomposites. This fact can be attributed to intrinsic thermal conductivity of the $\mathrm{ZnO}$ nanoparticles. Several models have been used for prediction of TC in the nanocomposites (see section 3.2). In the PP/ZnO nanocomposites TC values correlated well with the values predicted by Series, Maxwell, Lewis \& Nielson, Bruggeman and De Loor models up to $10 \mathrm{wt} \%$.

\section{Conclusions}

As electronic devices tend to become slimmer and more integrated, heat management become a central task for device design and application. Similar issues are faced in several other applications, including electric motors and generators, heat exchangers in power generation, automotive, etc. Metallic materials are widely used as heat dissipation materials, but there have been many attempts to replace the metallic materials with highly thermally conductive polymer based composites due to their lightweight, corrosion resistance, easy processing and lower manufacturing cost.

Thermally conductive polymer based composites are tentatively prepared by the incorporation of thermally conductive fillers. The outstanding thermal conductivity of mentioned fillers makes them a promising candidates to obtain highly thermally conductive polymer based composites.

PP nanocomposites were prepared by melt extrusion in a twin screw extruder. The introduction of nanoparticles resulted in an increase in crystallinity. Scanning electron microscopy (SEM) indicated a good dispersion of the nanofillers within the PP matrix that might enhance the thermal conductivity of the nanocomposites even at lower nanofiller loadings owing to enhanced filler-matrix interaction. The thermal conductivity of $\mathrm{PP} / \mathrm{ZnO}$ nanocomposites had an increase of $82 \%$ at $15 \mathrm{wt} \%$ concentration comparing to that of pure $\mathrm{PP}$, while for $\mathrm{PP} / \mathrm{CaCO}_{3}$ nanocomposite with same level of nanoparticle content it was $64 \%$, so it is concluded that $\mathrm{ZnO}$ nanoparticles had more intrinsic potential to improve thermal conductivity of PP comparing to $\mathrm{CaCO}_{3}$ nanoparticles regarding to its nature and crystallinity.

The thermal conductivity was increased from $\mathrm{K}=0.22 \mathrm{~W} / \mathrm{mK}$ for pure PP by $64 \%$ for the sample with $15 \mathrm{wt} \%$ of $\mathrm{CaCO}_{3}$ nanoparticles. These results for both nanocomposites $\left(\mathrm{PP} / \mathrm{ZnO}\right.$ and $\left.\mathrm{PP} / \mathrm{CaCO}_{3}\right)$ are higher than the values which reported for $\mathrm{CNF}$ in a PP matrix 
Frormann L, Iqbal A, Abdullah S.A. 2008. The measured values were also compared with various models in the investigated range of nanofiller concentration. The Series, Maxwell, Lewis \& Nielson, Bruggeman, Bottcher, De Loor and Ce Wen Nan models predicted fairly well the thermal conductivity values for the samples containing more than $5 \mathrm{wt} \%$ of nanoparticles. The experimental TC values of PP nanocomposites showed a linear increase with an increase in concentration and crystallinity.

\section{Abbreviations}

ABS poly(acrylonitrile-butadiene-styrene) copolymer

AlN aluminum nitride

$\mathrm{BeO}$ beryllium oxide

BN boron nitride

BNNT boron nitride nanotubes

CNT carbon nanotube

$C_{p} \quad$ heat capacity

DSC differential scanning calorimetry

DWCNT double-walled carbon nanotube

EG expanded graphite

EPDM ethylene propylene diene rubber

EVA poly(ethylene vinyl acetate)

GNP graphite nanoplatelet

HDPE high density polyethylene

$K$ thermal conductivity (in some figures taken from literature referred as $K_{e}=$ effective thermal conductivity)

$k_{c} \quad$ thermal conductivity of composite

$k_{m} \quad$ thermal conductivity of matrix

$k_{p} \quad$ thermal conductivity of particle

$l \quad$ phonon mean free path

$L \quad$ length parameter

LDPE low density polyethylene

MWCNT multi-walled carbon nanotube

PA6 polyamide 6

PA66 polyamide 6-6

PBT poly(butylene terephthalate)

PC polycarbonate

PDMS poly(dimethylsiloxane)

PE polyethylene

PEEK polyetheretherketone

PET poly(ethylene terephthalate)

PEVA poly(ethylene vinyl alcohol)

PI polyimide

PMDA pyromellitic dianhydride

PMMA polymethylmethacrylate

PP polypropylene

PPS polyphenylene sulfide

PPSU polyphenylsulfone 


$\begin{array}{ll}\text { PS } & \text { polystyrene } \\ \text { PSU } & \text { polysulfone } \\ \text { PTFE } & \begin{array}{l}\text { polytetrafluoroethylene } \\ \text { PU }\end{array} \\ \text { polyurethane } \\ \text { PVB } & \text { poly(vinyl butyral) } \\ \text { PVC } & \text { polyvinyl chloride } \\ \text { PVDF } & \text { polyvinylidene difluoride } \\ R_{k} & \text { interfacial resistance } \\ \text { SiC } & \text { silicon carbide } \\ \text { SWCNT } & \text { single-walled carbon nanotube } \\ T_{g} & \text { glass transition temperature } \\ v & \text { average phonon velocity } \\ \text { VGCF } & \text { vapor grown carbon fiber } \\ A & \text { thermal diffusivity } \\ \rho & \text { density of the material } \\ \Phi_{m} & \text { volume fractions of matrix } \\ \Phi_{p} & \text { volume fractions of particles } \\ \mathrm{W}_{\mathrm{f}} & \text { weight fraction of particles }\end{array}$

\section{References}

Abdel-Aal N.; El-Tantawy F.; Al-Hajry A. \& Bououdina M. (2008), Epoxy resin/plasticized carbon black composites. Part I. Electrical and thermal properties and their applications, Polymer Composites, vol.29 pp. 511-517.

Agari Y., Ueda A., Omura Y. \& Nagai S. (1997), Thermal diffusivity and conductivity of PMMA-PC blends, Polymer, vol.38, pp. 801-807.

Bai F.; Li F.; Calhoun B.; Quirk R.P. \& Cheng S.Z.D. (1999), Polymer Handbook, 4th edition. In: John Wiley ESons, Inc., New York.

Bigg D.M. (1995), Thermal conductivity of heterophase polymer compositions, Advanced Polymer Science, vol.119, pp. 1-30.

Böttcher C. (1952), Theory of Electric Polarization, In: Elsevier. Amsterdam, Netherlands.

Boudenne A.; Ibos L.; Fois M.; Majeste J.C. \& Géhin E. (2005), Electrical and thermal behavior of polypropylene filled with copper particles, Composites: part A, vol.36, pp. 1545-1554.

Bruggeman D. (1935), Dielectric constant and conductivity of mixtures of isotropic materials. Annual Physics, vol.24, pp. 636-645.

Causin V.; C. Marega;A. Marigo; G. Ferrara \& A. Ferraro (2006), Morphological and structural characterization of polypropylene/conductive graphite nanocomposites, European Polymer Journal, vol.42, pp. 3153-3161.

Chen Y. \& Ting J. (2002), Ultra high thermal conductivity polymer composites, Carbon, vol.40, pp. 359-362.

Chernenko N. M.; Yu. N.; Beilina A. \& Sokolov I. (2009), TECHNOLOGICAL ASPECTS OF HEAT RESISTANCE IN CARBON-CERAMIC COMPOSITE REFRACTORIES. Refractories and Industrial Ceramics, Vol. 50, No. 2.

Dashora P. \& Gupta G. (1996), On the temperature dependence of the thermal conductivity of linear amorphous polymers, Polymer, vol.37, pp. 231-234. 
DeLoor G. (1956), Dielectric Proberties of heterogeneous mixtures. PhD Thesis, University of Leiden, NL. Leiden.

Droval G.; Feller J.F., Salagnac P. \& Glouannec P. (2006), Thermal conductivity enhancement of electrically insulating syndiotactic poly(styrene) matrix for diphasic conductive polymer composites. Polymers for Advanced Technology, vol.17, pp.732-745.

Dunn M.L. \& Taya M. (1993), The effective thermal conductivity of composites with coated reinforcement and the application to imperfect interfaces, Journal of Applied Physics. Vol.73, pp. 1711-1722.

Elgafy A. \& Lafdi K. (2005), Effect of carbon nanofiber additives on thermal behavior of phase change materials, Carbon, vol. 43, pp. 3067-3074.

Every A.G.; Tzou Y.; Hasselman D.P.H. \& Ray R. (1992), The effect of particle size on the thermal conductivity of ZnS/diamond composites, Acta Metallurgica et Materialia, vol.40, pp. 123-129.

Ebadi-Dehaghani H.; Reiszadeh M.; Chavoshi A. \& Nazempour M. (2011), A study on the effect of zinc oxide $(\mathrm{ZnO})$ and calcium carbonate $\left(\mathrm{CaCO}_{3}\right)$ nanoparticles on the thermal conductivity (TC) of polypropylene. Composites: part B (revised)

Fischer J.E. (2006), Carbon nanotubes: structure and properties. Gogotsi Y., Carbon nanomaterials, Taylor and Francis Group, New York, pp. 51-58.

Frank H. R. \& Phillip D. S. (2002), Enhanced Boron Nitride Composition and Polymer Based High Thermal Conductivity Molding Compound, EP 0794227 B1.

Frormann L.; Iqbal A. \& Abdullah S.A. (2008), Thermo-viscoelastic behavior of PCNF-filled polypropylene nanocomposites. Journal of Applied Polymer Science; 107: 2695-2703.

Fukushima H.; Drzal L. T.; Rook B. P. \& Rich M. J. (2006), Thermal conductivity of exfoliated Graphite nanocomposites. Journal of Thermal Analysis and Calorimetry, Vol. 85, pp.235-238.

Gaal P.S.; Thermitus M.A. \& Stroe D.E. (2004), Thermal conductivity measurements using the flash method, Journal of Thermal Analysis and Calorimetry, vol.78, pp. 185-189.

Ganguli S.; Roy A.K. \& Anderson D.P. (2008), Improved thermal conductivity for chemically functionalized exfoliated graphite/epoxy composites, Carbon, vol.46, pp. 806-817.

Greig D. \& Hardy N.D. (1981), The thermal conductivity of semicrystalline polymers at very low temperatures, Journal of Physics Colloq, vol.42, pp. 69-71.

Gu J.; Zhang Q.; Dang J.; Zhang J. \& Yang Z. (2009), Thermal conductivity and mechanical properties of aluminum nitride filled linear low-density polyethylene composites, Polymer Engineering Science, vol.49, pp. 1030-1034.

Han Z. \& Fina A. (2011). Thermal conductivity of carbon nanotubes and their polymer nanocomposites: A review, Progress in Polymer Science, vol.36, Issue 7, pp.914-944.

He H.; Fu R.; Han Y.; Shen Y. \& Song X (2007),Thermal conductivity of ceramic particle filled polymer composites and theoretical predictions. Journal of Material Science, vol.42, pp.6749-6754. DOI 10.1007/s10853-006-1480-y.

Hermansen R. D. (2001), Room-Temperature Stable, One-Component, ThermallyConductive, Flexible Epoxy Adhesives, EP 0754741 B1.

Hodgin M. J. \& Estes R. H. (1999). Proceedings of the Technical Programs, NEPCO WEST Conference, pp. 359-366.Anaheim, CA.

Holotescu S. \& Stoian F. D. (2009), Evaluation of the effective thermal conductivity of composite polymers by considering the filler size distribution law. Journal of Zhejiang University SCIENCE A. vol. 10(5) pp.704-709. 
Hu M.; Yu D. \& Wei J. (2007), Thermal conductivity determination of small polymer samples by differential scanning calorimetry. Polymer Testing, vol.26, pp.333-337.

Ishida H. (2000), Surface Treated Boron Nitride for Forming A Low Viscosity High Thermal Conductivity Polymer Based on Boron Nitride Composition and Method, US Patent, 6,160,042.

Ishida H. \& Heights S. (1999), Composition for Forming High Thermal Conductivity Polybenzoxazine-Based Material and Method, US Patent 5,900,447.

Ishida H. \& Rimdusit S. (1998), Very high thermal conductivity obtained by boron nitridefilled polybenzoxazine, Thermochimica Acta, vol.320, pp. 177-186.

Kalaitzidou K.; Fukushima H. \& Drzal L.T. (2007), Multifunctional polypropylene composites produced by incorporation of exfoliated graphite nanoplatelets, Carbon, vol.45, pp. 1446-1452.

Kelly B.T. (1981), Physics of graphite, Applied Science Publishers, Barking (UK).

King J.A.; Barton R.L., Hauser R.A. \& Keith J.M. (2008), Synergistic effects of carbon fillers in electrically and thermally conductive liquid crystal polymer based resins, Polymer Composites, vol.29, pp. 421-428.

King J.A.; Morrison F.A.; Keith J.M.; Miller M.G.; Smith R.C.; Cruz M.; Neuhalfen A.M. \& Barton R.L. (2006), Electrical conductivity and rheology of carbon-filled liquid crystal polymer composites, Journal of Applied Polymer Science, vol.101, pp. 26802688.

King J.A.; Tucker K.W.; Vogt B.D.; Weber E.H. \& Quan C. (1999), Electrically and thermally conductive nylon 6,6, Polymer Composites, vol.20, pp. 643-654.

Kline D.E. (1961), Thermal conductivity studies of polymers, Journal of Polymer Science, vol.50, pp. 441-450.

Kowalski L.; Duszczyk J. \& Katgerman L(1999), Thermal conductivity of metal powderpolymer feedstock for powder injection moulding. Journal of Material Science, vol.34, pp.1- 5 .

Kumar A.P.; Depan D.; Tomer N.S. \& Singh R.P. (2009), Nanoscale particles for polymer degradation and stabilization-trends and future perspectives, Progress Polymer Science, vol.34, pp. 479-515.

Kumlutas D.; Tavman I. H. \& Coban M.T. (2003). Thermal conductivity of particle filled polyethylene composite materials. Composites Science and Technology, vol.63, pp.113117.

Kuriger R.J.; Alam M.K.; Anderson D.P. \& Jacobsen R.L. (2002), Processing and characterization of aligned vapor grown carbon fiber reinforced polypropylene, Composites: PartA, vol.33, pp. 53-62.

Lee H. \& Eun S. (2004). In Composites 2004 Convention and Trade Show, American Composites Manufacturers Association, Tampa, Florida.

Lipton R. \& Vernescu B. (1996), Composites with imperfect interface, Proc $R$ Soc Lond A, vol.452, pp. 329-358.

Liu C. \& Mather T. (2004), ANTEC 2004, Society of Plastic Engineers, pp. 3080-3084.

Liu Z., Guo Q., Shi J., Zhai G. \& Liu L. (2008), Graphite blocks with high thermal conductivity derived from natural graphite flake, Carbon, vol.46, pp. 414-421.

Mai Y. \& Yu Z. (2006), Polymer nanocomposites. Woodhead publishing limited, Cambridge England. 
Majumdar A. (1998), Microscale transport phenomena. Rohsenow W.M.; Hartnett J.R. \& Cho Y.I., Handbook of heat transfer, (3rd ed.), McGraw-Hill, New York, pp. 8.1-8.8.

Mamunya Y.P.; Davydenko V.V.; Pissis P. \& Lebedev E.V. (2002), Electrical and thermal conductivity of polymers filled with metal powders, European Polymer Journal, vol.38, pp. 1887-1897.

Marcus S.M. \& Blaine R.L. (1994), Thermal conductivity of polymers, glasses and ceramics by modulated DSC, Thermochimica Acta, vol.243, pp. 231-239.

Merzlyakov M. \& Schick C. (2001), Thermal conductivity from dynamical response of DSC, Thermochimica Acta, vol.377, pp. 183-191.

Mohammed H.A. \& Uttandaraman S. (2009), A review of vapor grown carbon nanofiber/polymer conductive composites, Carbon, vol.47, pp. 2-22.

Momentive Performance Materials, Boron nitride finds new applications in thermoplastic compounds. (2008), Plastics, Additives and Compounding, (May/June), pp. 26-31.

$\mathrm{Mu}$ Q.; Feng S. \& Diao G. (2007), Thermal conductivity of silicone rubber filled with ZnO, Polymer Composites, vol.28, pp. 125-130.

Mu Q. and Feng S. (2007), Thermal conductivity of graphite/silicone rubber prepared by solution intercalation, Thermochimica Acta, vol.462, pp. 70-75.

Nan C.W.; Birringer R.; Clarke D.R. \& Gleiter H. (1997), Effective thermal conductivity of particulate composites with interfacial thermal resistance, Journal of Applied Physics, vol. 10, pp. 6692-6699.

Nan C.W.; Liu G.; Lin Y. \& Li M. (2004), Interface effect on thermal conductivity of carbon nanotube composites. Applied Physics Letter, vol. 85, pp.3549-3555.

Nielsen E.; Landel R.F. (1994), Mechanical Properties of Polymers and Composites. 2nd edition, Marcel Dekker Inc., New York.

Nunes dos Santos W., Mummery P. \& Wallwork A. (2005), Thermal diffusivity of polymers by the laser flash technique, Polymer Testing, vol.24, pp. 628-634.

Nunes dos Santos W. (2007), Thermal properties of polymers by non-steady-state techniques, Polymer Testing, vol.26, pp. 556-566.

Ohashi M.; Kawakami S. \& Yokogawa Y. (2005), Spherical aluminum nitride fillers for heatconducting plastic packages, Journal of American Ceramic Society, vol.88, pp. 26152618.

Okamoto S. \& Ishida H. (1999), A new theoretical equation for thermal conductivity of twophase systems, Journal of Applied Polymer Science, vol.72, pp. 1689-1697.

Park S.H.; Hong C.M.; Kim S. \& Lee Y.J. (2008), Effect of fillers shape factor on the performance of thermally conductive polymer composites, ANTEC Plastics - Annual Technical Conference Proceedings 2008, pp. 39-43.

Price D.M. \& Jarratt M. (2002), Thermal conductivity of PTFE and PTFE composites, Thermochimiuca Acta, vol.392-393, pp. 231-236.

Pierson H.O. (1993), Handbook of carbon, graphite, diamond and fullerenes: properties. Processing and applications, Noyes Publications, New Jersey.

Reese W. (1969), Thermal properties of polymers at low temperatures, Journal of Macromolecular Science A, vol.3, pp. 1257-1295.

Rides M.; Morikawa J.; Halldahl L.; Hay B.; Lobo H.; Dawson A. \& Allen C. (2009), Intercomparison of thermal conductivity and thermal diffusivity methods for plastics, Polymer Testing, vol.28, pp. 480-489. 
Speight J.G. (2005), Lange's handbook of chemistry (16th ed.), McGraw-Hill, New York pp. 2794-2797.

Stankovich S.; Dikin D.A.; Dommett G.H.B.; Kohlhaas K.M.; Zimney E.J.; Stach E.A.; Piner R.D.; Nguyen S.T. \& Ruoff R.S., Graphene-based composite materials, Nature, vol.442 (2006), pp. 282-286.

Sui G.; Jana S.; Zhong W.H.; Fuqua M.A. \& Ulven C.A. (2008), Dielectric properties and conductivity of carbon nanofiber/semi-crystalline polymer composites, Acta Material, vol.56, pp. 2381-2388.

Tavman I.H. (1996), Thermal and mechanical properties of aluminum powder-filled highdensity polyethylene composites, Journal of Applied Polymer Science, vol. 62, pp. 2161-2167.

Tavman I. H. (2004). in Nanoengineered Nanofibrous Materials, NATO Science Series II, Mathematics, Physics and Chemistry, Guceri S. I.; Gogotsi Y. \& Kuzentsov V., Kluwer Academic Book Publication, Dordrecht, Netherlands, Vol. 169, p. 449.

Tekce H.S.; Kumlutas D. \& Tavman I.H. (2007), Effect of particle shape on thermal conductivity of copper reinforced polymer composites, Journal of Reinforced Plastics and Composites, vol.26. pp. 113-121.

Thostenson E.K.; Li C. \& Chou T.W. (2005), Nanocomposites in context, Composite Science Technology, vol.65, pp. 491-516.

Tibbetts G.G.; Lake M.L.; Strong K.L. \& Rice B.P. (2007), A review of the fabrication and properties of vapor-grown carbon nanofiber/polymer composites, Composite Science Technology, vol.67, pp. 1709-1718.

T'Joen C.; Park Y.; Wang Q.; Sommers A.; Han X. \& Jacobi A. (2009), A review on polymer heat exchangers for HVAC\&R applications, International Journal of Refrigeration, vol.32, pp. 763-779.

Torquato S. \& Rintoul M.D. (1995), Effect of interface on the properties of composite media, Physical Review Letters, vol.75, pp. 4067-4070.

Tritt T.M. \& Weston D. (2004), Measurement techniques and considerations for determining thermal conductivity of bulk materials. Tritt T.M., Thermal conductivity theory, properties, and applications, Kluwer Academic/Plenum Publishers, New York, pp. 187-203.

Tu H. \& Ye L. (2009), Thermal conductive PS/graphite composites, Polymer Advanced Technology, vol.20, pp. 21-27.

Vakili M.H.; Ebadi-Dehaghani H.; Haghshenas-Fard M. (2011), Crystallization and Thermal Conductivity of $\mathrm{CaCO}_{3}$ Nanoparticle Filled Polypropylene. Journal of Macromolocular Science: Part B. Physics, vol.50, pp.1637-1645.

Veca M.L.; Meziani M.J.; Wang W.; Wang X.; Lu F.; Zhang P.; Lin Y., Fee R.; Connell J.W. \& Sun Y. (2009), Carbon nanosheets for polymeric nanocomposites with high thermal conductivity, Advanced Material, vol. 21, pp. 2088-2092.

Wang J.; Carson J.K.; North M.F. \& Cleland D.J. (2008), A new structural model of effective thermal conductivity for heterogeneous materials with cocontinuous phases, International Journal of Heat and Mass Transfer, vol.51, pp. 2389-2397.

Weidenfeller B.; Hofer M \& Schilling F. R. (2004), Thermal conductivity, thermal diffusivity, and specific heat capacity of particle filled polypropylene. Composites: Part A, vol.35, pp. 423-429. 
Wolff S. \& Wang M.J. (1993), Carbon black science \& technology (2nd ed.), Marcel Dekker, New York.

Wong Y.W.; Lo K.L. \& Shin F.G. (2001), Electrical and thermal properties of composite of liquid crystalline polymer filled with carbon black, Journal of Applied Polymer Science, vol.82, pp. 1549-1555.

Wypych G. (2000), Handbook of fillers: physical properties of fillers and filled materials, ChemTec Publishing, Toronto.

Yano O. \& Yamaoka H. (1995), Cryogenic properties of polymers, Progress Polymer Science, vol.20, pp. 585-613.

Ye C.M.; Shentu B.Q. \& Weng Z.X. (2006), Thermal conductivity of high density polyethylene filled with graphite, Journal of Applied Polymer Science, vol.101, pp. 3806-3810.

Yu S.; Hing P. \& Hu X. (2002), Thermal conductivity of polystyrene-aluminum nitride composite, Composites: Part A, vol.33, pp. 289-292.

Zhang X.; Fujiwara S. \& Fujii M. (2000), Measurements of thermal conductivity and electrical conductivity of a single carbon fiber, International Journal of Thermophysics, vol.21, pp. 965-980.

Zhao H. \& Li R.K.Y. (2006), A study on the photo-degradation of zinc oxide ( $\mathrm{ZnO}$ ) filled polypropylene nanocomposites. Polymer; vol.47, pp.3207-3217.

Zeng J.; Fu R.; Agathopoulos S.; Zhang S.; Song X. \& He H. (2009), Numerical simulation of thermal conductivity of particle filled epoxy composites, Journal of Electronic Packaging, vol.131, pp.1-7.

Zhi C.; Bando Y.; Terao T.; Tang C.; Kuwahara H. \& Golberg D. (2009), Towards thermoconductive, electrically insulating polymeric composites with boron nitride nanotubes as fillers, Advanced Functional Materials, vol.19, pp. 1857-1862.

Zhong C.; Yang Q. \& Wang W. (2001), Correlation and prediction of the thermal conductivity of amorphous polymers, Fluid Phase Equilibria, vol.181, pp. 195-202.

Zhou H.; Zhang S. \& Yang M. (2007), The effect of heat-transfer passages on the effective thermal conductivity of high filler loading composite materials, Composites Science and Technology, vol.67, pp. 1035-1040.

Zhou W.; Qi S.; An Q.; Zhao H. \& Liu N. (2007), Thermal conductivity of boron nitride reinforced polyethylene composites, Materials Research Bulletin, vol.42, pp. 18631873. 


\title{
Magnetic Properties and Size Effects of Spin-1/2 and Spin-1 Models of Core-Surface Nanoparticles in Different Type Lattices
}

\author{
Orhan Yalçın'1, Rıza Erdem² and Zafer Demir ${ }^{3}$ \\ ${ }^{1}$ Department of Physics, Niğde University, Niğde \\ ${ }^{2}$ Department of Physics, Akdeniz University, Antalya \\ 3Institute of Graduate School of Natural and Applied Sciences, Niğde University, Niğde
}

Turkey

\section{Introduction}

Dimension in the range of 1 to $100 \mathrm{~nm}$, is called the nano regime. In recent years, nanoparticles/quantum dots are in a class of magnetic nanostructures (Aktaş et al., 2003, 2006; Kartopu \& Yalçın, 2010). Nanoparticles (NPs) have been steadily interesting in Physics, Chemistry, Biology, Biomedicine, Spintronics, etc. As the dimensions of magnetic NPs decrease down to the nanometer scale, these core-surface NPs start to exhibit new and interesting physical properties mainly due to quantum size effects. Even the intrinsic physical characteristics of NPs are observed to change drastically compared to their macroscopic counterparts. The potential applications of NPs are very attractive for magnetosensor, bio-sensor, magneto-electronics, data storage media, computer hard disks, microwave electronic devices, nano-transistors, etc. Especially, the studies of core-surface NPs are extremely important for technology because of transmission of data at high density to optical computer, nanorobot to assemble, compose rigid disk. The nanoparticles have relevance to thin film devices in the new breed of magnetoelectronics, spin-valve, spintransistors, spin-dependence tunneling devices and etc. (Babin, et al., 2003). The hysteresis in fine magnetic particles applied to new technologies such as Magnetic Random Access Memory (MRAM).

In generally, a nanoparticle is divided into the inner, outer and intermediate regions. These zones are called core (C), surface (S) and core-surface (CS), respectively. The size effects of core-surface NPs are very important for technological and biomedicine applications (Fraerman et al., 2001; Pankhurst et al., 2003). Especially, superparamagnetic (singledomain) NPs are important for non surgecial interfere of human body. The ferromagnetic (FM) orders in magnetic systems were dominated as mono-domain (or single-domain) nanoparticles consisting of FM surface and antiferromagnetic (AFM) core regions which couple with each other (Rego \& Figueiredo, 2001; Leite \& Figueiredo, 2004). At the lower temperatures, the FM surface and AFM core are only ordered in the noninteracting (monodomain) NPs. Stoner-Wohlfarth (Stoner \& Wohlfarth, 1948) and Heisenberg model (Heisenberg, 1928) to describe the fine structure were fistly used in detail. Magnetic 
evolutions with temperatures (Babin, et al., 2003; Szlaferek, 2004; Usov \& Gudoshnikov, 2005), thermodynamic properties (Vargas et al., 2002) and experimental techniques (Wernsdorfer et al., 1995; Wernsdorfer et al., 2000) were performed by different type works for the core-surface NPs. A simple (Bakuzis \& Morais, 2004) and the first atomic-scale models of the ferrimagnetic and heterogeneous systems in which the exchange energy plays a central role in determining the magnetization of the NPs, were studied (Kodama et al., 1996, 1999; Kodama \& Berkowitz, 1999).

Ising models and real magnets have provided a rich and productive field for the interaction between theory and experiment over the past 86 years (Ising, 1925; Peierls, 1936). Ising models (Erdem, 1995; Keskin, \& Erdem 1997; Erdem \& Keskin, 2001; Erdem, 2009; Erdem, 2008; Chen \& Levy, 1973) and thier variants such as Blume-Capel (Blume, 1966; Capel, 1966; Bakchich, et al., 1994), Blume-Emery-Griffiths (Blume, et al., 1971; Achiam, 1985; Hoston, \& Berker, 1991; Bakkali, 1996; Goveas \& Mukhopadhyay, 1997; Keskin, et al., 1999; Temizer, 2008) and mixed spin (Benayad \& Dakhama 1997; Kaneyoshi, 1998; Albayrak, \& Yigit, 2005; Albayrak, \& Yigit, 2006; Albayrak, 2007; Albayrak, 2007; Deviren, et al., 2009) models were regarded as theoretical simplifications, designed to model the essential aspects of cooperative system (Kikuchi,1951) without detailed correspondence to specific materials.

In the scope of this chapter, we give a detailed analysis for both spin $S=1 / 2$ and $S=1$ Ising models of homegeneous and core-surface composite NPs to describe the magnetic properties of these particles. These models are based on the pair approximation in the Kikuchi version (Kikuchi, 1974; Keskin, 1986; Erdinç \& Keskin, 2002; Yalçın, et al. 2008, Özüm, 2010; Çiftçi, 2011). Incorporating the pair correlations between the spins inside the NPs, we calculated the free energy and minimized with respect to pair variables to obtain the field-cooled magnetization. The field cooling magnetization $(M)$ curves of homogeneous and composite NPs are given as a function of the reduced temperature with different radius and different type lattices. Hysteresis loops and coercive fields with their linear fit to the data were plotted as a function of radius and temperature of different NPs. We compared our result with other works (Kaneyoshi, 2005; Kodama, 1999; Usov \& Gudoshnikov, 2005).

\section{Theoretical model}

\subsection{Ising model}

Ising model, which was introduced in the field of magnetism, is one of the most studied models in modern statistical physics. Although its greatest success during the last century has been in the theory of phase transitions, the model today is viewed as a mathematical structure which can represent a variaty of different physical phenomena. In this section, we give a brief summary for the basics of the model before its application to the nanoparticle (NP) magnetism.

Ising model is considered on a regular lattice where each interior site has the same number of nearest-neighbour sites. This is called the coordination number of the lattice and will be denoted by $\gamma$. The system under consideration is composed of the magnetic atoms (also called the spins) located at the lattice sites. It is assumed that, in the thermodynamic limit, boundary sites can be disregarded and that, with $N$ sites, the number of nearest-neighbour site pairs is $N \gamma / 2$. The standard Hamiltonian for the the simplest Ising model is given by 


$$
\mathrm{H}\left\{S_{i}\right\}=-J \sum_{\langle i j\rangle} S_{i} S_{j}-h \sum_{\langle i j\rangle}\left(S_{i}+S_{j}\right), \text { with } S_{i}= \pm 1,
$$

where $h$ is the external magnetic field at the site $i$ and the summation is performed for nearest-neighbour sites. $J$ is the exchange interaction between neighbouring sites $\langle i j\rangle$. Two distinctive cases corresponding to different signs of intersite interaction is considered, i.e., $J<0$ (ferromagnetic (FM) coupling) and $J>0$ (antiferromagnetic (AFM) coupling). The fractions of $S_{i}= \pm 1$ spins given by $X_{i}$ are called the point (or state) variables. The $X_{i}$ are normalized by $\sum_{i=1}^{2} X_{i}=1$. The long-range order parameter in the model is called the magnetization $(M)$ and it is defined by $M=X_{1}-X_{2}$. From this definition and the normalization condition the point variables can be written as

$$
X_{1}=\frac{1}{2}(1+M), X_{2}=\frac{1}{2}(1-M) .
$$

On the other hand, Eq. (1) may be extended by allowing values $s=0, \pm 1, \pm 2, \ldots, \pm S$ for the variables. It is then possible to consider higher order interactions such as $K \sum_{\langle i j\rangle} S_{i}^{2} S_{j}^{2}$ or a chemical potential such as $\Delta \sum_{i} S_{i}^{2}$. These generalizations are regarded as extensions of the Blume-Emery-Griffiths (BEG) model (Blume et al., 1971). Recently, there have been many theoretical studies of mixed spin Ising systems. These are of interest because they have less translational symmetry than their single-spin counterparts since they consist of two interpenetrating inequivalent sublattices. The latter property is very important to study a certain type of ferrimagnetism, namely molecular-based magnetic materials which are of current interest (Kaneyoshi et al., 1998).

\subsection{Pair approximation}

In the pair approximation, we consider the pair correlations between the spins. Besides the point variables $\left(X_{i}\right)$, we introduce new variables $\left(Y_{i j}\right)$, indicating the average number of the states in which the first member of the nearest-neighbour pair is in state $i$ and the second member in state $j$. These will be called the pair or bond variables. The bond variables are normalized by $\sum_{i, j=1}^{n} Y_{i j}=1$ and related to the state varibales by the relations $X_{i}=\sum_{j=1}^{n} Y_{i j}$. Here $n$ is the number of spin states in the given spin $S$ model. The interaction energy $E$ and entropy $S_{E}$ can be written in terms of $Y_{i j}$ as

$$
\begin{gathered}
\beta E=N \frac{\gamma}{2} \sum_{i, j}^{n} \eta_{i j} Y_{i j} \\
S_{E}=N k\left((\gamma-1) \sum_{i, j=1}^{n} X_{i} \ln \left(X_{i}\right)-\frac{\gamma}{2} \sum_{i, j=1}^{n} Y_{i j} \ln \left(Y_{j i}\right)\right),
\end{gathered}
$$

where $\beta=1 / k T$ ( $k$ Boltzmann's constant and $T$ temperature). In Eq. (3), the parameters $\eta_{i j}$ are called the bond energies for the spin pairs $(i, j)$ and determined from Eq. (1). The free energy per site $\Phi$ can be found from 


$$
\Phi=\frac{\beta F}{N}=\frac{\beta}{N}\left(E-T S_{E}\right) .
$$

For the system at equilibrium, the minimization of Eq. (5) with respect to $Y_{i j}\left(\partial \Phi / \partial Y_{i j}=0\right)$ leads to the following set of self-consistent equations:

$$
Y_{i j}=\frac{1}{Z}\left(X_{i} X_{j}\right)^{\bar{\gamma}} e^{-\beta \eta_{i j}} \equiv \frac{e_{i j}}{Z},
$$

where $\bar{\gamma}=(\gamma-1) / \gamma$ and $Z$ is the partition function:

$$
Z=\exp (2 \beta \lambda / \gamma)=\sum_{i, j=1}^{n} e_{i j} .
$$

In Eq. (7), $\lambda$ is introduced to maintain the normalization condition. Applications of the above formulation to $S=1 / 2$ and $S=1$ Ising systems can be found in many works in the

\begin{tabular}{|c|c|c|}
\hline & $S=1 / 2$ & $S=1$ \\
\hline Spin state variables $\left(X_{i}\right)$ & $X_{1}, X_{2}$ & $X_{1}, X_{2}, X_{3}$ \\
\hline Spin values $\left(S_{i}\right)$ & $+1,-1$ & $+1,0,-1$ \\
\hline $\begin{array}{l}\text { Bond variables } \\
Y_{i j}\left(S_{i}, S_{j}\right)\end{array}$ & $\begin{array}{l}Y_{11}(+1,+1), Y_{12}(+1,-1) \\
Y_{21}(-1,+1), Y_{22}(-1,-1)\end{array}$ & $\begin{array}{l}Y_{11}(+1,+1), Y_{12}(+1,0), Y_{13}(+1,-1) \\
Y_{21}(0,+1), Y_{22}(0,0), Y_{23}(0,-1) \\
Y_{31}(-1,+1), Y_{32}(-1,0), Y_{33}(-1,-1)\end{array}$ \\
\hline Normalization & $\sum_{i=1}^{2} X_{i}=1, \sum_{i, j=1}^{2} Y_{i j}=1$ & $\sum_{i=1}^{3} X_{i}=1, \sum_{i, j=1}^{3} Y_{i j}=1$ \\
\hline \multirow{2}{*}{$\begin{array}{l}\text { Relations between point } \\
\text { variables and } \\
\text { pair variables }\end{array}$} & $X_{i}=\sum_{j=1}^{2} Y_{i j}$ & $X_{i}=\sum_{j=1}^{3} Y_{i j}$ \\
\hline & $\begin{array}{l}X_{1}=Y_{11}+Y_{12} \\
X_{2}=Y_{21}+Y_{22}\end{array}$ & $\begin{array}{l}X_{1}=Y_{11}+Y_{12}+Y_{13} \\
X_{2}=Y_{21}+Y_{22}+Y_{23} \\
X_{3}=Y_{31}+Y_{32}+Y_{33}\end{array}$ \\
\hline \multirow{2}{*}{$\begin{array}{l}\text { Avarage } \\
\text { magnetization }\left(M \equiv\left\langle S_{i}\right\rangle\right)\end{array}$} & $M=X_{1}-X_{2}$ & $M=X_{1}-X_{3}$ \\
\hline & $M=Y_{11}+Y_{12}-\left(Y_{21}+Y_{22}\right)$ & $\begin{array}{l}M=Y_{11}+Y_{12}+Y_{13} \\
-\left(Y_{31}+Y_{32}+Y_{33}\right)\end{array}$ \\
\hline \multirow{2}{*}{$\begin{array}{l}\text { Quadrupole moment } \\
Q \equiv\langle Q\rangle \equiv\left\langle S_{i}{ }^{2}\right\rangle\end{array}$} & \multirow{2}{*}{----- } & $Q \equiv X_{1}+X_{3}$ \\
\hline & & $Q=Y_{11}+Y_{12}+Y_{13}+Y_{31}+Y_{32}+Y_{33}$ \\
\hline
\end{tabular}
literature (Meijer et al., 1986; Keskin \& Meijer, 1986; Keskin \& Erdinç, 1995; Erdinç \& Keskin, 2002). These applications are summerized for comparison in Table 1.

Table 1. Comparison of the $S=1 / 2$ and $S=1$ Ising models under the pair approximation. 


\section{Magnetic properties of $S=1 / 2$ and $S=1$ Ising nanoparticles}

The magnetic particles become single domain below a critical size in contrast with the usual multidomain structure of the bulk materials. Therefore, in the scope of this section, we study size effects and magnetic properties of monodomain NPs manifestations. We consider a noninteracting monodomain NP with Ising spins on both hexagonal and square lattices for any two-dimensional (2D) regular arrays which can also be extended to hexagonal closed packed (hcp) and simple cubic (sc) lattices for the three-dimensional (3D) case as in Fig.1. The shells and their numbers originate from the nearest-neighbor pair interactions for the hexagonal and square lattices in 2D. In this structure, number of shells for hexagonal and square lattices can be associated with radius $(R)$ of the NPs. This behaviour can be seen explicitly in Fig. 2 for hexagonal lattice and in Fig. 3 for square lattice. The value of $R$ includes number of shells and the size of a NP increases as the number of shells increses. Therefore, we have considered Ising spins in three parts that are core $(C)$, core-surface $(C S)$ and surface $(S)$ within the NP. Each of these parts contain core spin number $\left(N_{C}\right)$, coresurface spin number $\left(N_{C S}\right)$ and surface spin number $\left(N_{S}\right)$, respectively. The total number of spins $(N)$ in a single NP involves core and surface spin numbers, i.e. $N=N_{C}+N_{S}$. The $C$ and $S$ spins interact ferromagnetically $(J<0)$ or antiferromagnetically $(J>0)$. The $S=1 / 2$ and $S=1$ Ising model Hamiltonians with dipol-dipol interaction $(J)$ for a NP is given by

$$
\mathrm{H}=\mathrm{H}_{\mathrm{C}}+\mathrm{H}_{\mathrm{CS}}+\mathrm{H}_{\mathrm{S}}
$$

with

$$
\begin{gathered}
\mathrm{H}_{C}=-J_{C} \sum_{\langle i, j\rangle} S_{i} S_{j}-h \sum_{\langle i, j\rangle}\left(S_{i}+S_{j}\right), \\
\mathrm{H}_{C S}=-J_{C S} \sum_{\langle i, j\rangle} S_{i} \sigma_{j}, \\
\mathrm{H}_{S}=-J_{S} \sum_{\langle i, j\rangle} \sigma_{i} \sigma_{j}-h \sum_{\langle i, j\rangle}\left(\sigma_{i}+\sigma_{j}\right),
\end{gathered}
$$

where $J_{C}, J_{C S}$ and $J_{S}$ represent exchange interactions for $C, C S$ and $S$ atoms, respectively. If $J_{C}=J_{C S}=J_{S}$, the NP is known as a homegeneous NP. It is called a composite NP when $J_{C} \neq J_{C S} \neq J_{S}, J_{C}=J_{C S} \neq J_{S}, J_{C} \neq J_{C S}=J_{S}$ or $J_{C S} \neq J_{C}=J_{S}$. In Eqs. (9), $S_{i}$ is called the core spin values and $\sigma_{i}$ is the surface spin values. These variables take on the values \pm 1 for $S=1 / 2$ and $0, \pm 1$ for $S=1$ Ising systems.

The interaction energies for $S=1 / 2$ and $S=1$ models of an Ising NP in 2D can be written shortly in term of $Y_{i j}$ as

$$
\beta E=\sum_{\langle i, j\rangle}\left(N_{P}^{C} \eta_{i j}^{C}+N_{P}^{C S} \eta_{i j}^{C S}+N_{P}^{S} \eta_{i j}^{S}\right) Y_{i j}
$$


where the numbers of spin pairs for $C, C S$ and $S$ regions are defined by $N_{P}^{C}=\left(N_{C} \gamma_{C} / 2\right)-N_{C S}, N_{P}^{C S}=2 N_{C S} \gamma_{C S} / 2$ and $N_{P}^{S}=N_{S} \gamma_{S} / 2$, respectively. Similarly $\gamma_{C}$, $\gamma_{C S}, \gamma_{S}$ denote the coordination numbers for these regions. Since we consider the arrays of Ising spins for a structure made up of bigger particles in 2D, we choose $\gamma_{C}=6, \gamma_{C S}=\gamma_{S}=2$ for hexagonal lattice and $\gamma_{C}=4, \gamma_{S}=0, \gamma_{C S}=2$ for square lattice, as depicted in Figs. 2 and 3, respectively. The values of these numbers for both suructures in $2 \mathrm{D}$ are given in Table 2. The expressions for the bond energies $\eta_{i j}^{C}, \eta_{i j}^{C S}$ and $\eta_{i j}^{S}$ of three regions are found using Eq. (9) for both models, as listed in Table 3.

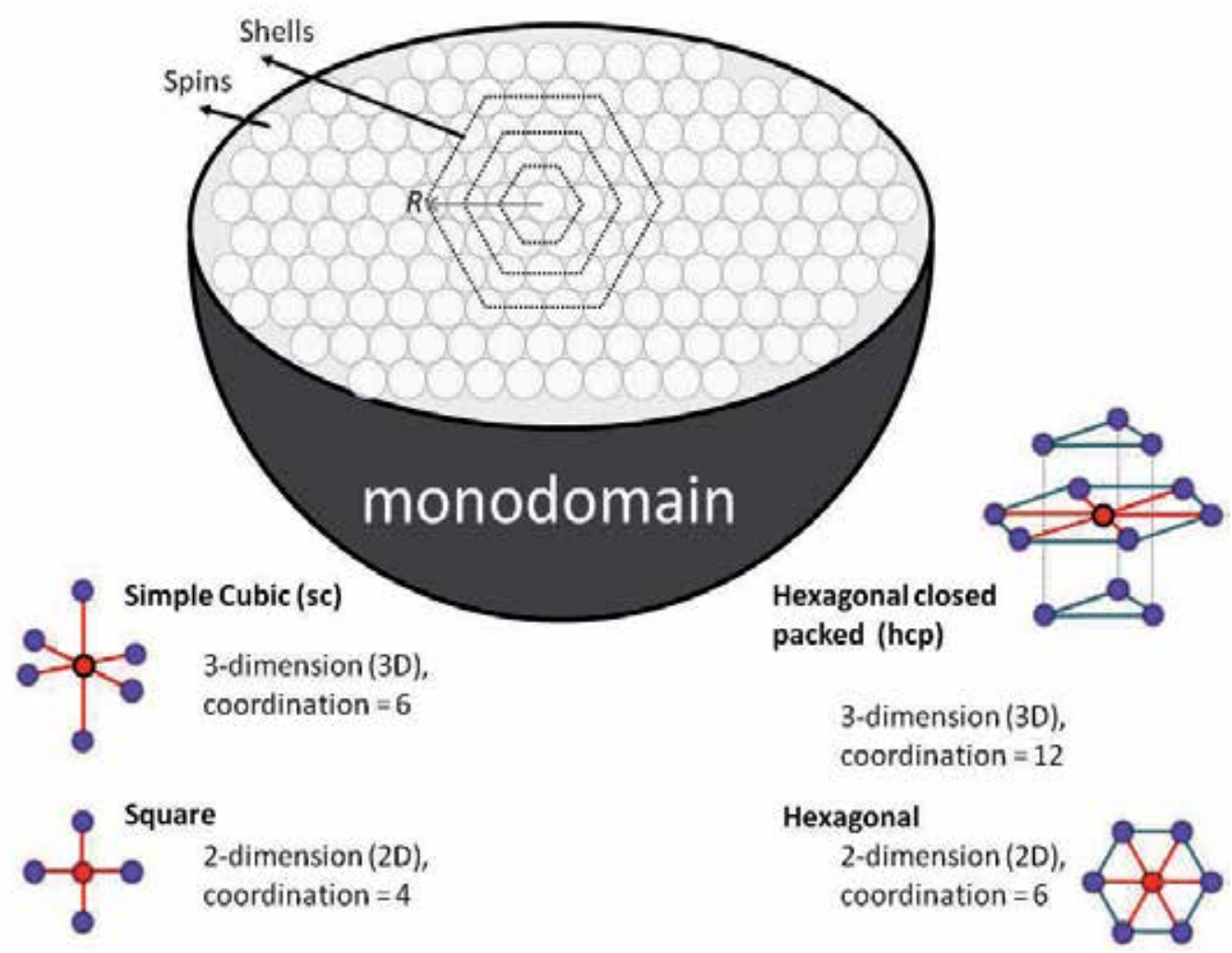

Fig. 1. A spherical monodomain magnetic NP spaced coherently in a form of 3D arrays. The shape of a single NP consists of the hexagonal lattice. The dashed lines displayed shells of spins in a 2D finite arrays. The radius of NP $(R)$ includes shell numbers. The insets exhibit coordination numbers $(\gamma)$ of hexagonal closed packed (hcp) and simple cubic (sc) lattices in $3 \mathrm{D}$ as well as hexagonal and square lattices in 2D structure. 


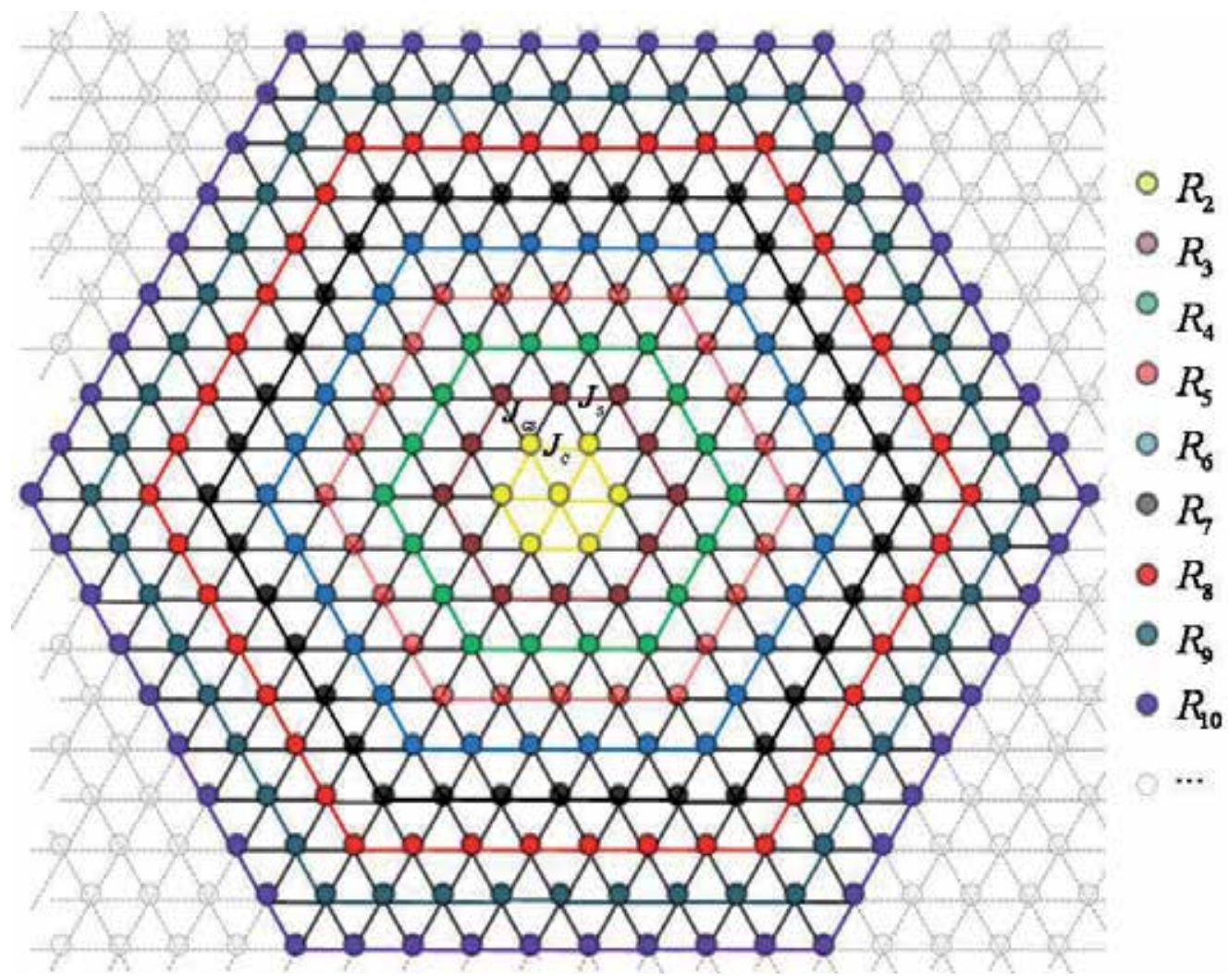

Fig. 2. Schematic representation of a NP on a hexagonal lattice in 2D exhibiting nine shells of spins. Small full coloured circles correspond to ten radius of the NP. Solid grey lines are number of the core-shell pairs. Solid coloured lines are number of shell pair (this line corresponds to core and shell number for $R=2$ ). 


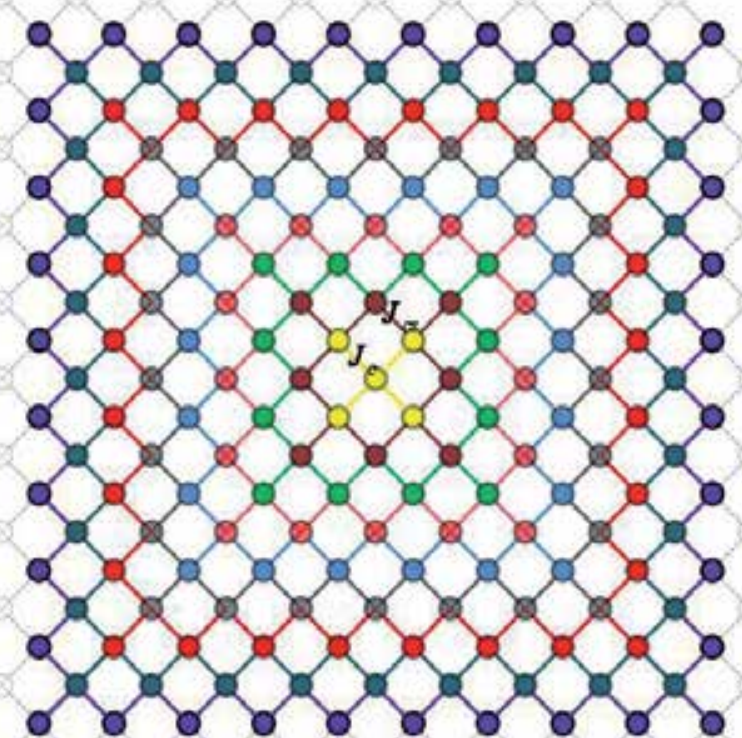

$\circ R_{2}$

- $R_{3}$

○ $R_{4}$

- $R_{5}$

$\circ R_{6}$

- $R_{7}$

- $R_{8}$

- $R_{9}$

- $R_{10}$

Fig. 3. Same as Fig. 2 but for the NP on square lattice in 2D.

\begin{tabular}{|l|c|c|c|c|c|c|c|c|c|c|}
\hline Lattice Type & $\mathrm{R}$ & $\mathbf{2}$ & $\mathbf{3}$ & $\mathbf{4}$ & $\mathbf{5}$ & $\mathbf{6}$ & $\mathbf{7}$ & $\mathbf{8}$ & $\mathbf{9}$ & $\mathbf{1 0}$ \\
\hline \multirow{5}{*}{$\begin{array}{l}\text { Hexagonal } \\
\text { Lattice in 2D }\end{array}$} & $N_{C}$ & 7 & 19 & 37 & 61 & 91 & 127 & 169 & 217 & 271 \\
\cline { 2 - 12 } & $N_{S}$ & 12 & 18 & 24 & 30 & 36 & 42 & 48 & 54 & 60 \\
\cline { 2 - 12 } & $N_{C S}$ & 9 & 15 & 21 & 27 & 33 & 39 & 45 & 51 & 57 \\
\cline { 2 - 11 } & $N_{P}^{C}$ & 12 & 42 & 90 & 156 & 240 & 342 & 462 & 600 & 756 \\
\cline { 2 - 11 } & $N_{P}^{S}$ & 12 & 18 & 24 & 30 & 36 & 42 & 48 & 54 & 60 \\
\cline { 2 - 11 } & $N_{P}^{C S}$ & 18 & 30 & 42 & 54 & 66 & 78 & 90 & 102 & 114 \\
\hline \multirow{4}{*}{$\begin{array}{l}\text { Square Lattice in } \\
\text { 2D }\end{array}$} & $N_{C}$ & 5 & 13 & 25 & 41 & 61 & 85 & 113 & 145 & 181 \\
\cline { 2 - 11 } & $N_{S}$ & 8 & 12 & 16 & 20 & 24 & 28 & 32 & 36 & 40 \\
\cline { 2 - 11 } & $N_{C S}$ & 6 & 10 & 14 & 18 & 22 & 26 & 30 & 34 & 38 \\
\cline { 2 - 10 } & $N_{P}^{C}$ & 4 & 16 & 36 & 64 & 100 & 144 & 196 & 256 & 324 \\
\cline { 2 - 10 } & $N_{P}^{C S}$ & 12 & 20 & 28 & 36 & 44 & 52 & 60 & 68 & 76 \\
\hline
\end{tabular}

Table 2. Numbers of the spins and spin pairs within the $C$, CS and $S$ regions (Yalçın, et al., 2008). 


\begin{tabular}{|c|c|c|c|c|}
\hline Spin Model & Pair & $\begin{array}{l}\text { Bond energy } \\
\text { for Core } \\
\left(\eta_{i j}^{C}\right)\end{array}$ & $\begin{array}{l}\text { Bond energy for } \\
\text { Core -Surface } \\
\qquad\left(\eta_{i j}^{C S}\right)\end{array}$ & $\begin{array}{c}\text { Bond energy } \\
\text { for Surface } \\
\left(\eta_{i j}^{S}\right)\end{array}$ \\
\hline \multirow{4}{*}{$\begin{array}{l}S=1 / 2 \\
(n=2)\end{array}$} & $\eta_{11}$ & $-J_{C}-2 h$ & $-J_{C S}$ & $-J_{S}-2 h$ \\
\hline & $\eta_{12}$ & $+J_{C}$ & $+J_{C S}$ & $+J_{S}$ \\
\hline & $\eta_{21}$ & $+J_{C}$ & $+J_{C S}$ & $+J_{S}$ \\
\hline & $\eta_{22}$ & $-J_{C}+2 h$ & $-J_{C S}$ & $-J_{S}+2 h$ \\
\hline \multirow{9}{*}{$\begin{array}{l}S=1 \\
(n=3)\end{array}$} & $\eta_{11}$ & $-J_{C}-2 h$ & $-J_{C S}$ & $-J_{S}-2 h$ \\
\hline & $\eta_{12}$ & $-h$ & 0 & $-h$ \\
\hline & $\eta_{13}$ & $+J_{C}$ & $+J_{C S}$ & $+J_{S}$ \\
\hline & $\eta_{21}$ & $-h$ & 0 & $-h$ \\
\hline & $\eta_{22}$ & 0 & 0 & 0 \\
\hline & $\eta_{23}$ & $+h$ & 0 & $+h$ \\
\hline & $\eta_{31}$ & $+J_{C}$ & $+J_{C S}$ & $+J_{S}$ \\
\hline & $\eta_{32}$ & $+h$ & 0 & $+h$ \\
\hline & $\eta_{33}$ & $-J_{C}+2 h$ & $-J_{C S}$ & $-J_{S}+2 h$ \\
\hline
\end{tabular}

Table 3. Bond energies for $S=1 / 2$ and $S=1$ models.

Using Eq. (6) we obtain four self-consistent equations of $Y_{i j}$ for $S=1 / 2$ model of coresurface NPs:

$$
\begin{aligned}
& Y_{11}=\frac{1}{Z}\left(X_{1} X_{1}\right)^{\bar{\gamma}} \exp \left[-\beta\left(N_{P}^{C} \eta_{11}^{C}+N_{P}^{C S} \eta_{11}^{C S}+N_{P}^{S} \eta_{11}^{S}\right)\right] \equiv \frac{e_{11}}{Z}, \\
& Y_{12}=\frac{1}{Z}\left(X_{1} X_{2}\right)^{\bar{\gamma}} \exp \left[-\beta\left(N_{P}^{C} \eta_{12}^{C}+N_{P}^{C S} \eta_{12}^{C S}+N_{P}^{S} \eta_{12}^{S}\right)\right] \equiv \frac{e_{12}}{Z}, \\
& Y_{21}=\frac{1}{Z}\left(X_{2} X_{1}\right)^{\bar{\gamma}} \exp \left[-\beta\left(N_{P}^{C} \eta_{21}^{C}+N_{P}^{C S} \eta_{21}^{C S}+N_{P}^{S} \eta_{21}^{S}\right)\right] \equiv \frac{e_{21}}{Z}, \\
& Y_{22}=\frac{1}{Z}\left(X_{2} X_{2}\right)^{\bar{\gamma}} \exp \left[-\beta\left(N_{P}^{C} \eta_{22}^{C}+N_{P}^{C S} \eta_{22}^{C S}+N_{P}^{S} \eta_{22}^{S}\right)\right] \equiv \frac{e_{22}}{Z} .
\end{aligned}
$$

Similarly, nine self-consistent equations of $Y_{i j}$ for $S=1$ model of these particles are 


$$
\begin{aligned}
& Y_{11}=\frac{1}{Z}\left(X_{1} X_{1}\right)^{\bar{\gamma}} \exp \left[-\beta\left(N_{P}^{C} \eta_{11}^{C}+N_{P}^{C S} \eta_{11}^{C S}+N_{P}^{S} \eta_{11}^{S}\right)\right] \equiv \frac{e_{11}}{Z} \\
& Y_{12}=\frac{1}{Z}\left(X_{1} X_{2}\right)^{\bar{\gamma}} \exp \left[-\beta\left(N_{P}^{C} \eta_{12}^{C}+N_{P}^{C S} \eta_{12}^{C S}+N_{P}^{S} \eta_{12}^{S}\right)\right] \equiv \frac{e_{12}}{Z} \\
& Y_{13}=\frac{1}{Z}\left(X_{1} X_{3}\right)^{\bar{\gamma}} \exp \left[-\beta\left(N_{P}^{C} \eta_{13}^{C}+N_{P}^{C S} \eta_{13}^{C S}+N_{P}^{S} \eta_{13}^{S}\right)\right] \equiv \frac{e_{13}}{Z} \\
& Y_{21}=\frac{1}{Z}\left(X_{2} X_{1}\right)^{\bar{\gamma}} \exp \left[-\beta\left(N_{P}^{C} \eta_{21}^{C}+N_{P}^{C S} \eta_{21}^{C S}+N_{P}^{S} \eta_{21}^{S}\right)\right] \equiv \frac{e_{21}}{Z} \\
& Y_{22}=\frac{1}{Z}\left(X_{2} X_{2}\right)^{\bar{\gamma}} \exp \left[-\beta\left(N_{P}^{C} \eta_{22}^{C}+N_{P}^{C S} \eta_{22}^{C S}+N_{P}^{S} \eta_{22}^{S}\right)\right] \equiv \frac{e_{22}}{Z}, \\
& Y_{23}=\frac{1}{Z}\left(X_{2} X_{3}\right)^{\bar{\gamma}} \exp \left[-\beta\left(N_{P}^{C} \eta_{23}^{C}+N_{P}^{C S} \eta_{23}^{C S}+N_{P}^{S} \eta_{23}^{S}\right)\right] \equiv \frac{e_{23}}{Z}, \\
& Y_{31}=\frac{1}{Z}\left(X_{3} X_{1}\right)^{\bar{\gamma}} \exp \left[-\beta\left(N_{P}^{C} \eta_{31}^{C}+N_{P}^{C S} \eta_{31}^{C S}+N_{P}^{S} \eta_{31}^{S}\right)\right] \equiv \frac{e_{31}}{Z}, \\
& Y_{32}=\frac{1}{Z}\left(X_{3} X_{2}\right)^{\bar{\gamma}} \exp \left[-\beta\left(N_{P}^{C} \eta_{32}^{C}+N_{P}^{C S} \eta_{32}^{C S}+N_{P}^{S} \eta_{32}^{S}\right)\right] \equiv \frac{e_{32}}{Z}, \\
& Y_{33}=\frac{1}{Z}\left(X_{3} X_{3} \cdot\right)^{\bar{\gamma}} \exp \left[-\beta\left(N_{P}^{C} \eta_{33}^{C}+N_{P}^{C S} \eta_{33}^{C S}+N_{P}^{S} \eta_{33}^{S}\right)\right] \equiv \frac{e_{33}}{Z} .
\end{aligned}
$$

Eqs. (11) and (12) are solved numerically using Newton-Raphson method and normalized magnetization $(M)$ is easily calculated for both $S=1 / 2$ and $S=1$ models of homegeneous and core-surface composite NPs. Results are shown as the magnetization curves and hysteresis loops in Figs. 4-9.

\section{Result and discussions}

\subsection{Magnetization}

The evolution of normalized magnetization $(M)$ as a function of the reduced temperature $\left(k_{B} T / J_{0}\right)$ and particle size dependence of the transition temperature $T_{C}$ from $\mathrm{FM}$ to paramagnetic (PM) phases for homogeneous and composite Ising NPs are shown in Figs. 4 and 5, respectively. The magnetization curves in Fig. 4 are plotted for $S=1 / 2$ and $S=1$ models of homogeneous NPs using the FM core $\left(J_{0}=1, J_{C}=1\right)$, FM surface $\left(J_{S}=J_{0}\right)$ and FM core-surface $\left(J_{C S}=J_{0}\right)$ interactions and the curves in Fig. 5 are obtained for both models of the composite NPs based on FM core $\left(J_{C}=J_{0}\right)$, FM surface $\left(J_{S}=J_{0}\right)$ and AFM coresurface $\left(J_{C S}=-J_{0}\right)$ interactions. In the plots, different values for the applied magnetic field are considered $(h=0.0-0.1)$. The solid curves in the figures correspond to hexagonal lattice while dotted ones denote the square lattice. As seen from the figures, the changes in the magnetization with the reduced temperature point out an interesting aspect for NPs on the hexagonal and square lattices in 2D. The magnetization curves are decreasing from one (1) to zero (0) value while the reduced temperature is increasing (Figs. 4(a), 4(b), 5(a), 5(b)). These decreases terminate at the phase transition temperature (or Curie temperature, $T_{C}$ ) from FM phase to PM phase for $h=0.0$, seen in Figs. 4(c) and 5(c). To show the size dependence of the critical temperature we plot $\sqrt{T_{C}}$ vs $R$ in Figs. $4(\mathrm{~d})$ and $5(\mathrm{~d})$. All critical temperature values follow a linear increase with the particle radius. With increase in the 
particle radius it approaches to the Crue temperatures of the bulk materials. This is consistent with the mean-field approximation for the magnetic structure of Heisenberg NP (Usov \& Gudoshnikov, 2005). On the other hand, it is interesting that composite $S=1 / 2$ and $S=1$ Ising NPs show smaller transition temperatures than their corresponding homegeneous NPs. This can easily be seen by comparing the same coloured fits in Figs. 4(d) and $5(\mathrm{~d})$.
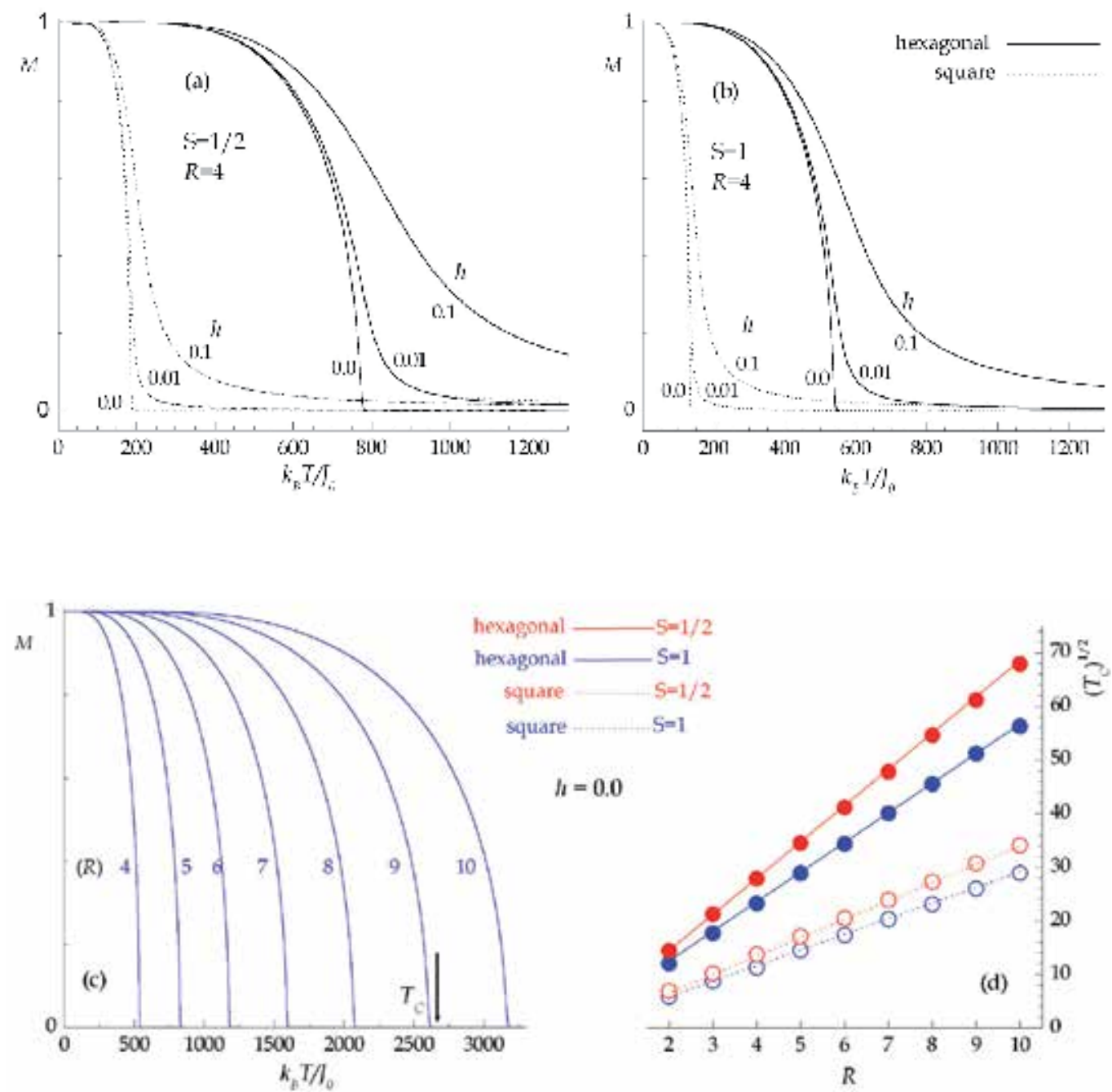

Fig. 4. Normalized magnetization $(M)$ vs. reduced temperature $\left(k_{B} T / J_{0}\right)$ and particle size dependence of the transition temperature $T_{C}$ from FM to PM phases for homogeneous $S=1 / 2$ and $S=1$ Ising NPs on the hexagonal and square lattices. $J_{0}=J_{C}=J_{C S}=J_{S}=1$ and $h=0.0-0.1$. 

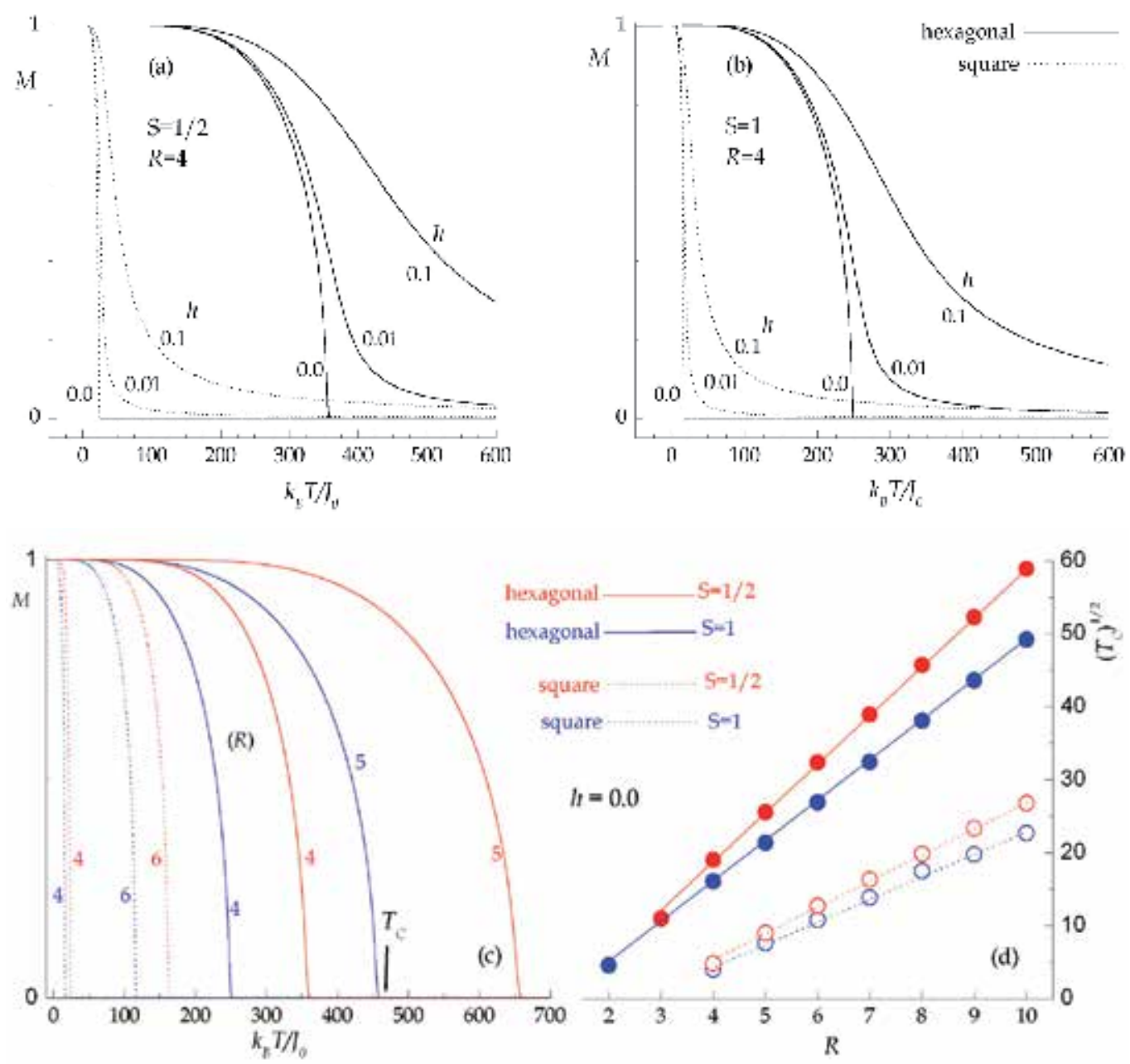

Fig. 5. Same as Fig. 4 but for the core-surface composite NPs with $J_{0}=J_{C}=J_{S}=1, J_{C S}=-J_{0}=-1$.

\subsection{Hysteresis loops}

The magnetic field evolution of normalized magnetization (or hysteresis loops) for the homegeneous $S=1 / 2$ and $S=1$ Ising NPs which has different particle sizes and their corresponding coercive field vs. $R^{-2}$ variation are given Figs. 6 and 7 , respectively. We consider a FM coupling in core $\left(J_{C}=J_{0}\right)$, surface $\left(J_{S}=J_{0}\right)$ and core-surface $\left(J_{C S}=J_{0}\right)$ regions with $J_{0}=1$ on the hexagonal and square lattice structures. The hysteresis curves of small diameters, namely with radius $R=2,4,5$ in Figs. 6(a)-6(d), are approximately the same. These behaviours are called superparamegnetic (SP) regime. However, the loops strongly depend on the size of NP. The hysteresis curves of high diamater values change sharply, as also shown in Figs. 6(a)-6(d). Moreover, the hysteresis curves for this type of NPs are broadening while the diamater of NPs is increasing so that it approaches to bulk 
materials. The size dependence of the coercive fields $h_{C}$ is determined from the hysteresis loops in Fig. 7. In Fig. 7, the full red and blue circles correspond to the curves obtained for $R=2-9$ in the Figs. 6(a) and 6(c), respectively. Similarly, the open red and blue circles correspond to the curves obtained for $R=3-11$ and $R=4-10$ in Figs. 6(b) and 6(d), respectively. The straight solid and dotted lines are the results from a linear fit to the calculated data. From this fit, it is obvious that the coercive field $\left(h_{C}\right)$ depends linearly on $1 / R^{2}$
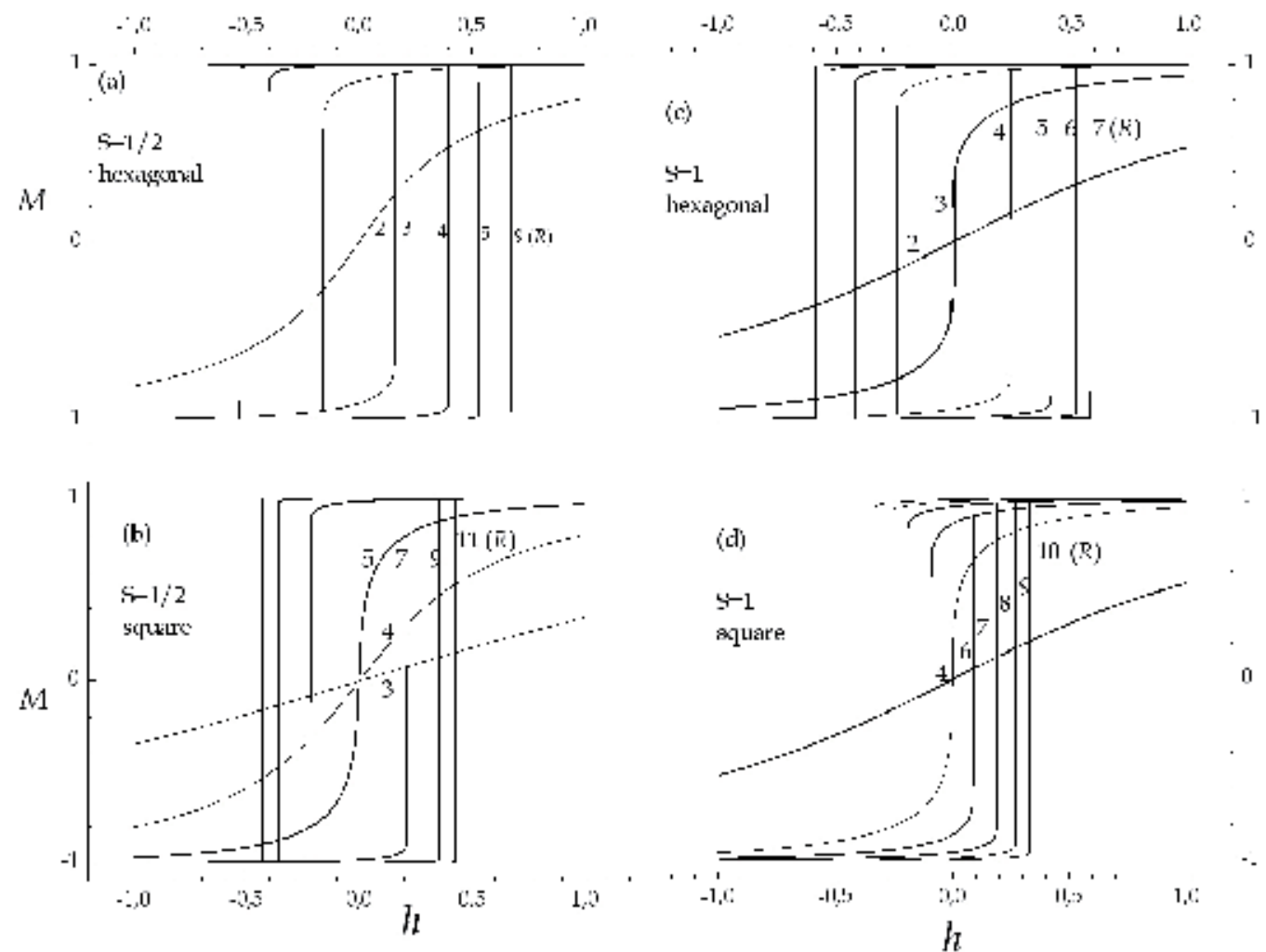

Fig. 6. (a) Hysteresis loops of a homegeneous $S=1 / 2$ Ising NP on the hexagonal lattice for various sizes. (b) Same as Fig. 6(a) but for NP on the square lattice. (c) Hysteresis loops of a homegeneous $S=1$ Ising NP on the hexagonal lattice for different sizes. (d) Same as Fig. 6(c) but for NP on the square lattice. $J_{0}=J_{C}=J_{C S}=J_{S}=1$ and $T=300 J_{0} / k_{B}$.

Magnetic hysteresis loops of composite $S=1 / 2$ and $S=1$ Ising NPs on the hexagonal and square lattice (in 2D) structures for various values of particle sizes are shown in Fig. 8. The exchange interactions in the $C$ and $S$ regions are FM, i.e. $J_{0}=J_{C}=J_{S}$, while the coupling 
between $C$ and $S$ is an AFM exchange constant $J_{C S}=-J_{0}$ for each type of NP. From the figure, it is clear that the hysteresis loops strongly depend on the particle size. The loops for the $S=1 / 2$ and $S=1$ Ising NPs on the hexagonal lattice change suddenly in low radius values while those for the $S=1 / 2$ and $S=1$ Ising NPs on the square lattice in high radius values.

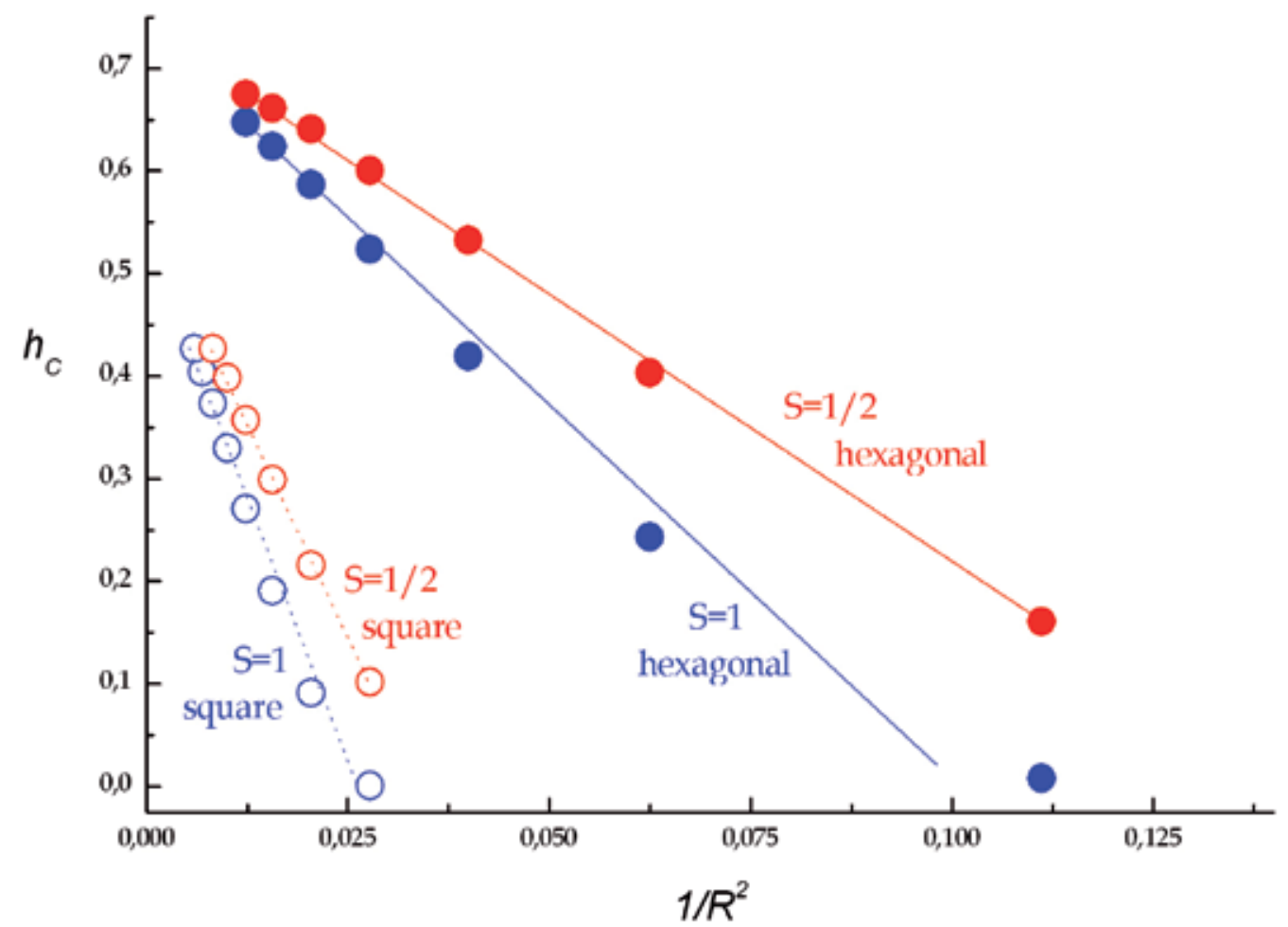

Fig. 7. The coercive field $\left(h_{C}\right)$ plotted as a function of $\mathrm{R}^{-2}$ for the hysteresis loops of the homegeneous NP in Fig. 6. 

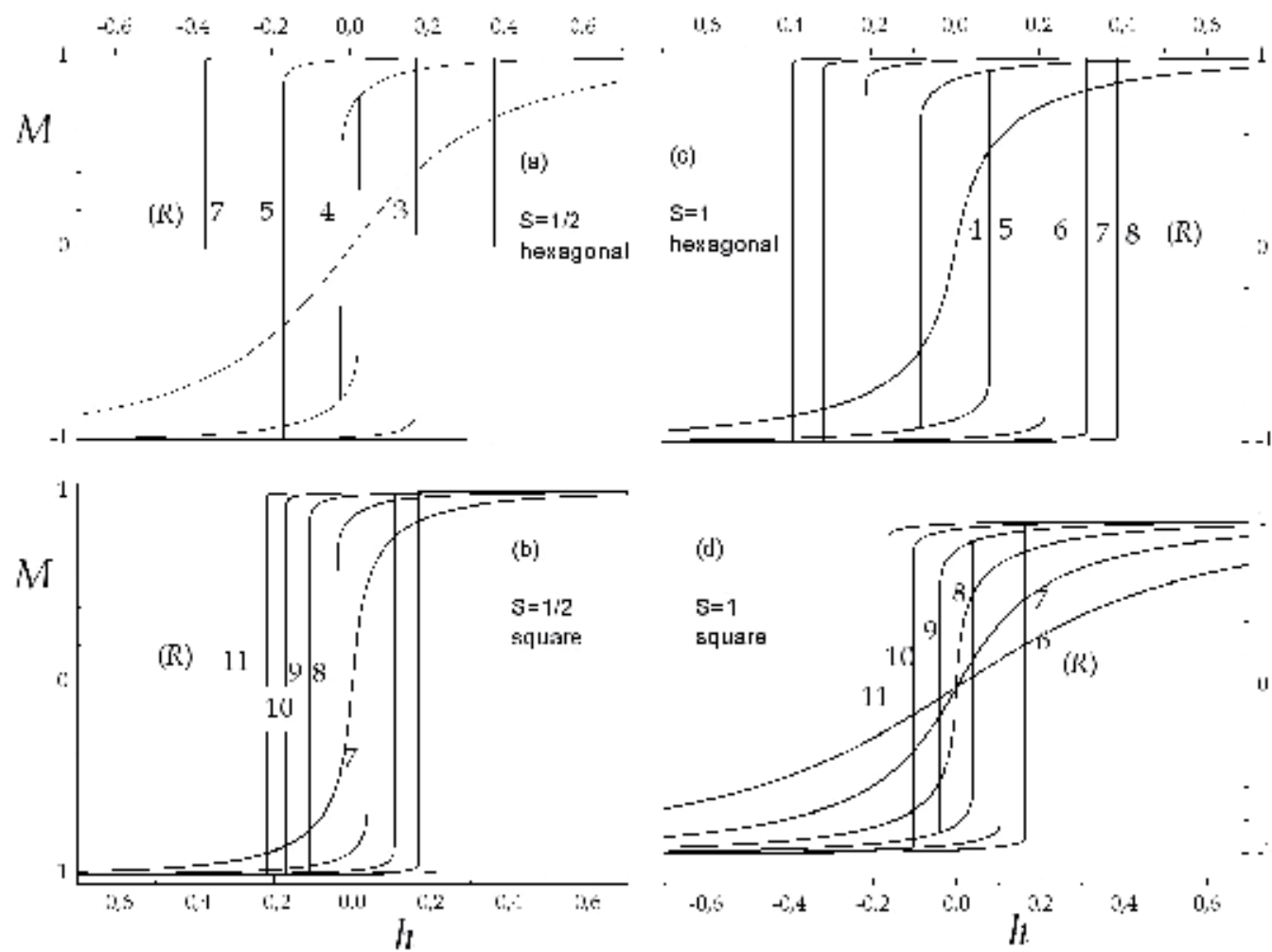

Fig. 8. Same as Fig. 6 but for the composite NP. $J_{0}=J_{C}=J_{S}=1, J_{C S}=-J_{0}$.

Finally, the evolutions of hysteresis loops and their coercive field according to the temperature of composite Ising NPs are seen to change monotically as the temperature increases, illustrated in Fig. 9(a) and 9(b), respectively. Since the loops for both models of NPs on the hexagonal and square lattices display the same behaviour we have drawn only the loops of $S=1 / 2$ Ising NP on the hexagonal lattice. In this case, hysteresis for the NP is in superparamagnetic (SP) regime at $700 J_{0} / k_{B}$. But, the loops for the temperature regime between $150 J_{0} / k_{B}-600 J_{0} / k_{B}$ belong to the FM phase (Fig. 9(a)). The tempereture dependence of the coercivity $\left(h_{C}\right)$ are determined from the hysteresis loops of Fig. 9(a), as given in Fig. 9(b). 

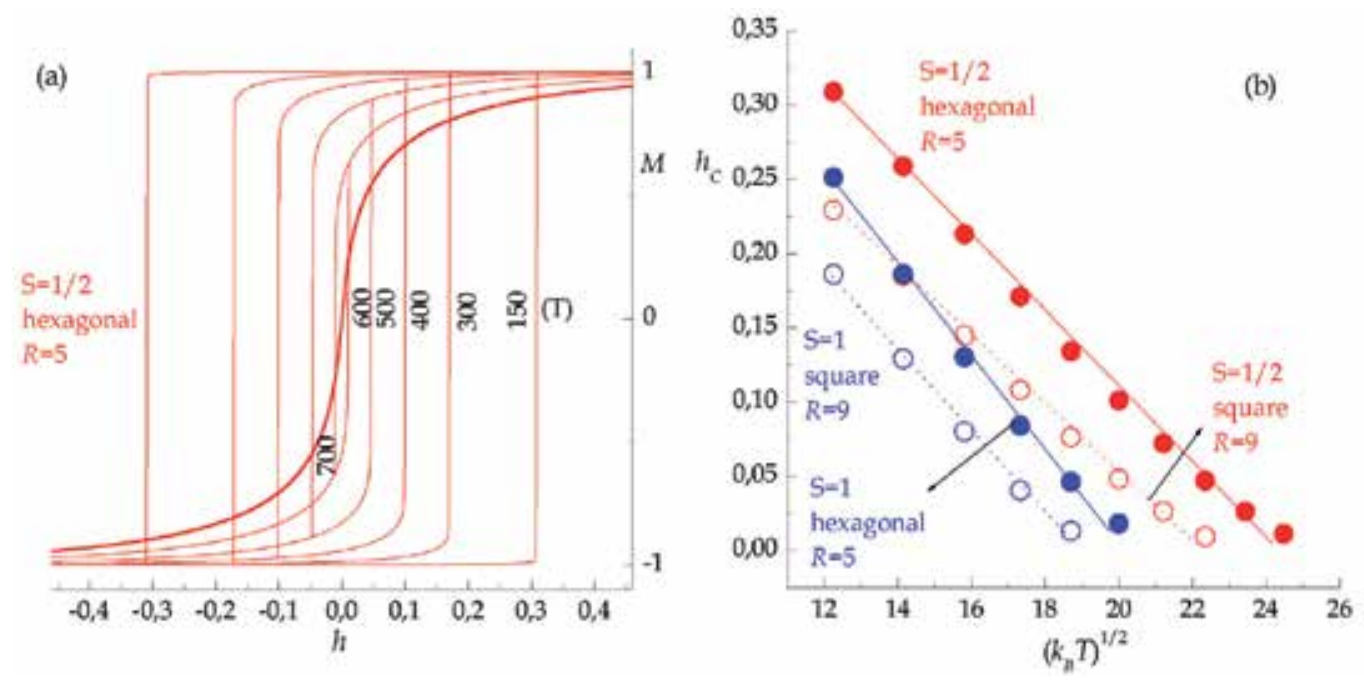

Fig. 9. (a) Temperature dependence of the hysteresis loops for the $S=1 / 2$ Ising NP on the hexagonal lattice exhibiting five shells of spins $(R=5)$. (b) The coercive field $\left(h_{C}\right)$ plotted as function of $\left(k_{B} T\right)^{1 / 2}$ for two models of NP on both structures studied above. $J_{0}=J_{C}=J_{S}=1, J_{C S}=-J_{0}$.

\section{Conclusion}

In the scope of this chapter, we have focused on the magnetic properties with size effects for homogeneous and core-surface composite NPs which have Ising spins (1, 1/2 ) on 2D lattice structures (hexagonal, square). The transition for all NPs corresponds to a second-order phase transition in the absence of magnetic field $(h \approx 0)$. The spin disorder can be caused by lower coordination of the surface atoms in core-surface NPs broken exchange interactions that produce spin-glass (SG) like state of spatially disordered spin in the surface captions with inhomogeneous surface effects (Kodama, 1999; Kaneyoshi, 2005). Our theoretical observations are scrutinized below briefly.

i. All critical temperature $\left(\sqrt{T_{C}}\right)$ values of both types of Ising NPs on 2D lattice structures follow a linear increase with the particle size. With increase in the NP size it approaches to the Crue temperature of the bulk materials. These results agree with the mean-field magnetic structure of Heisenberg NPs (Usov \& Gudoshnikov, 2005).

ii. From the hysteresis loops for the homegeneous $S=1 / 2$ and $S=1$ Ising NPs which have different sizes and corresponding coercive field $\left(h_{C}\right)$ vs. $R^{-2}$ variations, it is clearly seen that the coercivity strongly depends on the particle size. Due to the superparamegnetic regime the hysteresis curves of small diameters are almost independent of each other while the curves of big diameters sharply change. This shows that the NP approaches to bulk materials.

iii. The hysteresis loops at different temperatures show a monotonic change in the coercive field of composite Ising NPs on 2D lattice structures. This property probably is an important aspect in the future high-density magnetic data storage. 


\section{Acknowledgements}

One of us (Orhan Yalçın) would like to express his gratitude to "The Scientific and Technological Research Council of Turkey" (TÜBITAK) for financial support (Grant No. 107T635) during the this work.

\section{References}

Achiam, Y. (1985). Critical Relaxation of the One-Dimensional Blume-Emery-Griffiths Model. Physical Review B, Vol.31, pp.260-265, ISSN:1095-3795.

Aktaş, B.; Tagirov, L. \& Mikailov, F. (October, 2006). Magnetic Nanostructures, Springer Series in materials science, Vol. 94, ISBN 978-3-540-49334-1.

Aktaş, B.; Tagirov, L. \& Mikailov, F. (2004). Nanostructures Magnetic Materials and Their Applications., Kluwer Academic Publisher. Nato Science Series. Mathematics, Physics and Chemistry. Vol. 143. ISBN 1-4020-2004-X.

Albayrak, E. (2007). Mixed Spin-2 and Spin-5/2 Blume-Emery-Griffiths Model. Physica A, Vol. 375, pp. 174-184, ISSN:0378-4371.

Albayrak, E. (2007). The Critical and Compensation Temperatures for the Mixed Spin-3/2 and Spin-2 Ising Model. Physica B, Vol. 391, pp. 47-53, ISSN: 0921-4526.

Albayrak, E. \& Yigit, A. (2005). The Critical Behavior of the Mixed Spin-1 and Spin-2 Ising Ferromagnetic System on the Bethe lattice. Physica A, Vol. 349, pp. 471-486, ISSN:0378-4371.

Albayrak, E. \& Yigit, A. (2006). Mixed Spin-3/2 and Spin-5/2 Ising System on the Bethe Lattice. Physics Letters A, Vol. 353, pp. 121-129, ISSN: 0375-9601.

Bakkali, A.; Kerouad, M. \& Saber, M. (1996). The Spin-3/2 Blume-Emery-Griffiths Model. Physica A,Vol. 229, No.3-4, pp.563-573, ISSN:0378-4371.

Babin, V.; Garstecki, P. \& Holyst, R. (2003). Multiple Photonic Band Gaps in the Structures Composed of Core-Shell Particles. Journal of Applied Physics, Vol. 94, pp. 4244, ISSN: 1089-7550.

Benayad, N. \& Dakhama, A. (1997). Magnetic Properties of the Mixed-Spin Ising Ferromagnet with a Ferrimagnet Surface. Physical Review B, Vol. 55, No.18, pp. 12276-12289, ISSN:1095-3795.

Bakchich, A.; Bekhechi, S. \& Benyoussef, A. (1994). Multicritical Behavior of the Antiferromagnetic Spin-3/2 Blume-Capel Model. Physica A, Vol. 210, pp. 415-423, ISSN:0378-4371.

Bakuzis, A.F. \& Morais, P.C. (2004). Magnetic nanoparticle systems: an Ising model approximation Journal of Magnetism and Magnetic Materials, Vol.272-276, pp. e1161e1163 ISSN: 0304-8853.

Blume, M. (January 1966). Theory of the First-Order Magnetic Phase Change in $\mathrm{UO}_{2}$, Physical Review, Vol. 141, No.2, pp. 517-524, ISSN 1094-1622.

Blume, M.; Emery, V. J. \& Griffiths, R. B. (September 1971). Ising Model for the $\lambda$ Transition and Phase Separation in $\mathrm{He}^{3}-\mathrm{He}^{4}$ Mixtures, Physical Review A, Vol. 4, No.3, pp. 1071-1077, ISSN 1094-1622.

Capel, H. W., (1966). On the Possibility of First-Order Phase transitions in Ising Systems of Triplet Ions with Zero-Field Splitting. Physica Vol. 32, pp. 966-988.

Chen, H.H. \& Levy, P.M. (1973). High Temperature Series Expansions for a Spin-1 Model of Ferromagnetism, Physical Review B, Vol. 7, pp. 4284-4289, ISSN: 1538-4446. 
Çiftçi, N. (July, 2011). Magnetic properties of a monodomain nanoparticle with dipole-quadropole interaction, Master of Science Thesis. Thesis Supervisor, R. Erdem. Gaziosmanpaşa University, Turkey.

Deviren, B.; Keskin, M.\& Canko, O. (2009). Kinetics of a Mixed Spin-1/2 and Spin-3/2 Ising Ferrimagnetic Model. Journal of Magnetism and Magnetic Materials, Vol. 321, pp. 458466,. ISSN: 0304-8853.

Deviren, B.; Keskin, M. \& Canko, O. (2009). Magnetic Properties of an Anti-ferromagnetic and ferrimagnetic Mixed Spin-1/2 and Spin-5/2 Ising Model in the Longitudinal Magnetic Field within the Effective-Field Approximation. Physica A, Vol. 388, pp. 1835-1848, ISSN:0378-4371.

Erdem, R. (2009). Frequency Dependence of the Complex Susceptibility for a Spin-1 Ising Model. Journal of Magnetism and Magnetic Materials, Vol. 321, pp. 2592-25595,. ISSN: 0304-8853.

Erdem, R. \& Keskin, M. (2001). Dynamics of a Spin-1 Ising System in the neighborhood of Equilibrium States. Physical Review E, Vol. 64, pp. 0261102-1-9, ISSN 1550-2376.

Erdem, R. (2008). Magnetic Relaxation in a Spin-1 Ising Model near the second-order Phase Transition Point. Journal of Magnetism and Magnetic Materials, Vol. 320, pp. 22732278,. ISSN: 0304-8853.

Erdem, R. (September, 1995) A study of the dynamics of a spin-1 Ising model with bilinear and biquadratic interactions. Science Thesis. Thesis Supervisor, M. Keskin. Gaziosmanpaşa University, Turkey.

Erdinç, A. \& Keskin, M. (May 2002). Equilibrium and Nonequilibrium Behavior of the Spin1 Ising Model in the Quadrupole Phase. Physica A, Vol. 307, No. 3-4, pp. 453-468, ISSN:0378-4371.

Fraerman, A. A.; Gusev, S.A.; Nefedov, I.M.; Nozdrin, Y.N.; Karetnikova, I.R.; Mazo L.A.; Sapozhnikov, M.V.; Shereshevsky, I.A. \& Suhodoev, L.V. (2001). Journal of Physics Condensed Matter, Vol. 13, pp. 683-689. ISSN 1361-648X.

Goveas, N. \& Mukhopadhyay, G. (1997). Study of Blume-Emery-Griffiths Model by a Modified Bethe-Peierls Method. Physica Scripta, Vol. 56, pp.661-666, ISSN: 00318949.

Heisenberg, W. (1928). Theory of ferromagnetism Zeitschrift für Physik. Vol. 49, pp. 619-636.

Hoston, W. \& Berker, A. N. (1991). Multicritical Phase Diagrams of the Blume-EmeryGriffiths Model with Republisive Biquadratic Coupling. Physical Review Letters, Vol.67, pp.1027-1030. ISSN:0031-9007.

Ising, E. (1925). Beitrag zur Theorie des Ferromagnetismus. Zeitschrift für Physik, Vol. 31, pp. 253-258.

Kaneyoshi, T.; Nakamura, Y. \& Shin, S. (1998). A Diluted Mixed Spin-2 and Spin-5/2 Ferrimagnetic Ising System; A Study of a Molecular-Based Magnet. Journal of Physics: Condensed Matter, Vol.10, pp. 7025-7035. ISSN: 1361-648X.

Kartopu G. \& Yalçın O., (February, 2010). Electrodeposited Nanowires and their Applications, In: Electrodeposited Nanowires and Their Applications, Nicolate Lupu, pp. 113-140. ISBN 978-953-7619-88-6.

Keskin, M.; Ekiz, C. \&Yalcin, O. (1999). Stable, Metastable and Unstable Solutions of the Blume-Emery-Griffiths Model. Physica A, Vol. 267, pp. 392-405, ISSN:0378-4371. 
Keskin, M. (1986). A Model for Quenching Via Hidden Variables Non-Equilibrium Behaviour of a system with Two Range Order Parameters II. Influence of a Magnetic Field. Physica A, Vol. 135, pp. 226-236, ISSN:0378-4371.

Keskin, M. \& Erdem, R. (1997). Dynamic Behavior of a Spin-1 Ising Model. I. Relaxation of Order Parameters and the 'Flatness' Property of Metastable States. Journal of Statistical Physics, Vol. 89, pp. 1035-1046, ISSN: 1572-9613.

Keskin, M. \& Erdinç, A. (1995). The Spin-1 Ising Model on the Body-Centered Cubic Lattice Using the Pair Approximation. Turkish Journal of Physics, Vol.19, pp. 88-100, ISSN 1010-7630.

Keskin, M. \& Meijer, P. H. E. (December 1986). Dynamics of a Spin-1 Model with the Pair Correlation. Journal of Chemical Physics, Vol. 85, pp.7324-7333. ISSN: 1520-5207.

Kikuchi, R. (1951). A Theory of Cooperative Phenomena. Physical Review, Vol. 81, pp. 9881002, ISSN: 1536-6065.

Kaneyoshi, T. (2005). Phase Diagrams of a Nanoparticle Described by the Transverse Ising Model. Physica Status Solidi (b) Vol.242, pp. 2938-2948, ISSN: 1521-3951.

Kikuchi, R. (1974). Superposition Approximation and Natural İteration Calculation in Cluster-Variation Method. Journal of Chemical Physics, Vol. 60, pp. 1071, ISSN:10897690.

Kodama, R.H. (1999)., Magnetic Nanoparticles. Journal of Magnetism and Magnetic Materials, Vol.200, pp. 359-372, ISSN:0304-8853.

Kodama, R.H. \& Berkowitz, A.E. (March 1999). Atomic-Scale Magnetic Modeling of Oxide Nanoparticles. Physical Review B, Vol.59, No. 9, pp. 6321-6336, ISSN:0163-1829.

Kodama, R.H.; Berkowitz, A.E.; McNiff Jr. E.J. \& Foner S. (July 1996). Surface Spin Disorder in $\mathrm{NiFe}_{2} \mathrm{O}_{4}$ Nanoparticles. Physical Review Letters, Vol.77, No.2, pp.394-397, ISSN:1079-7114.

Leite, V. S. \& Figueiredo, W. (2004). Monte Carlo Simulations of Antiferromagnetic Small Particles. Brazilian Journal of Physics, Vol. 34, No. 2a, pp.452-454, ISSN 0103-9733.

Meijer, P. H. E.; Keskin, M. \& Bodegom, E. (October 1986). A Simple Model for the Dynamics Towards Metastable States. Journal of Statistical Physics, Vol. 45, No1-2. pp. 215-232. ISSN: 1572-9613.

Özüm, S. (July, 2010). A study with pair approximation of spin- Ising model of noninteracting nanoparticles with quadratic and crystal field interactions, Master of Science Thesis. Thesis Supervisor, Yalçın, O. Bozok University, Turkey.

Peierls, R. (1936). On Isings Model of Ferromagnetism. Proceedings of the Cambridge Philosophical Society,.Vol. 32, pp. 477-481, ISSN: 1469-8064.

Pankhurst, Q. A.; Connolly, J.; Jones, S.K. \& Dobson, J. (2003). Applications of magnetic nanoparticles in biomedicine. Journal of Physics D Applied Physics, Vol. 36,pp. R167, ISSN:1361-6463.

Rego, L. G. C. \& Figueiredo, W. (September 2001). Magnetic Properties of Nanoparticles in the Bethe-Peierls Aproximation. Physical Review B, Vol. 64, pp. 144424-1-7, ISSN: 1538-4446.

Stoner, E. C. \& Wohlfarth, E. P. (1948). A Mechanism of Magnetic Hysteresis in Heterogeneous Alloys. Philosophical Transactions of the Royal Society of London Series A, Vol. 240,pp. 599-642, ISSN:0261-0523.

Szlaferek, A. (May 2004). Model Exchange-Spring Nanocomposite Magnetic Grains. Physica Status Solidi B, Vol. 241, pp. 1312-1315, ISSN: 1521-3951. 
Temizer, U.; Kantar, E.; Keskin, M. \& Canko, O. (2008). Multicritical Dynamical Phase Diagrams of the Kinetic Blume-Emery-Griffiths Model with Repulsive Biquadratic Coupling in an Oscillating Field. Journal of Magnetism and Magnetic Materials, Vol. 320, pp. 1787-1801, ISSN: 0304-8853.

Usov, N. A. \& Gudoshnikov, S. A. (2005). Magnetic Structure of a Nanoparticle in MeanField Approximation. Journal of Magnetism and Magnetic Materials, Vol.290, pp. 727730, ISSN: 0304-8853.

Vargas, P.; Altbir, D.; Knobel, M. \& Laroze, D. (May 2002). Thermodynamics of TwoDimensional Magnetic Nanoparticles. Europhysics Letters, Vol.58, No. 4, pp. 603-609, ISSN:1286-4854.

Wernsdorfer, W.; Hasselbach, K.; Mailly, D.; Barbara, B.; Benoit, A.; Thomas, L. \& Suran, G. (1995). DC-SQUID Magnetization Measurements of Single Magnetic Particles. Journal of Magnetism and Magnetic Materials, Vol.145, pp. 33, ISSN:0304-8853

Wernsdorfer, W.; Mailly, D. \& Benoit, A. (May 2000). Single Nanoparticle Measurement Techniques. Journal of Applied Physics, Vol. 87, No. 9, pp. 5094-5096, ISSN: 10897550.

Yalçın, O.; Erdem, R. \& Övünç, S. (2008). Spin-1 Model of Noninteracting Nanoparticles. Acta Physica Polonica A, Vol. 114, No.4, pp. 835-844, ISSN: 0587-4246. 


\title{
Organic Semiconductor Nanoparticle Film: Preparation and Application
}

\author{
Xinjun $\mathrm{Xu}$ and Lidong $\mathrm{Li}$ \\ School of Materials Science and Engineering, \\ University of Science and Technology Beijing, \\ Beijing, \\ P. R. China
}

\section{Introduction}

Organic semiconductors usually comprising $\pi$-conjugated structure in their molecules can exhibit excellent optical and electronic properties. They have advantages of simple fabrication and ease of tuning the chemical structure to give desired features. So they can serve as attractive candidates for applications in bio/chemical sensors and optoelectronic devices. 1,2 To meet the requirement of domains including information, energy and healthcare, nanoscale materials have emerged as new building blocks for optoelectronic devices, bioimaging agents, and drug delivery carriers in recent years. $3^{3-5}$ These nanomaterials especially nanoparticles have already shown great potential to offer exciting opportunities in these areas.

Currently, most of the relevant works have been focused on inorganic semiconductor nanoparticles. Besides inorganic ones, organic semiconductor nanoparticles (OSNs) are desirable for a number of reasons. Their properties can be easily tuned for desired applications through the choice of functional molecules and surface modification. Additionally, their facile synthesis, good processability, high photoluminescence (PL) efficiency, high reaction activity, tunable properties, low toxicity and good biocompatibility further make them complementary to the inorganic nanomaterials and highly attractive in the material choice. As a result, OSNs have captured more and more interests. These OSNs can exhibit unique optical and electrical properties different from both the bulk solid samples and their molecular precursors. In comparison with molecule dispersed systems, OSNs are expected to show improved photostability and enhanced emission in various media. ${ }^{6,7}$ These properties are essentially important in fluorescent labeling applications, such as fluorescence bioimaging and single molecular spectroscopy. For example, single molecules of most commercial dyes undergo photo-bleaching in a few milliseconds under typical excitation conditions under the radiation of a laser beam. On the contrary, because large numbers of chromophores are incorporated in single nanoparticles, they can show bright fluorescence even at a low excitation power. Thus, the fluorescent nanoparticles do not undergo rapid photo-bleaching and give less emission blinking which are generally observed in single molecule experiment. ${ }^{8}$ 
Up to now, most of the OSNs are used in aqueous solutions to serve as biological labels,, 910 chemical sensors, 11 and photocatalysis materials. ${ }^{12}$ To expand the application area of OSNs, there is an increasing effort to prepare OSNs as an active solid film in chemo/biosensors and optical and electronic devices. ${ }^{13-16}$ Compared with bulk solid samples, nanoparticle films provide larger contact interface area, which is highly desired for chemical and biological sensing in sensors. So OSN based functional films tend to become a promising research area for applications in biosensing, energy conversion, photonic and optoelectronic devices.

In this review, after a brief introduction of organic semiconductor materials, we will summarize the methods for preparation OSN films. Then, its application in optical/electronic devices and chemo-/biosensors will be described. We hope this review can cast light on the advances and main problems in the research field of nanoparticle-based devices and sensors.

\section{Organic semiconductor materials}

Organic semiconductor materials are mainly classified into two categories. One is small molecules and the other is polymers made from repeated small conjugated monomer units.

\subsection{Small molecule semiconductors}

Polyphenyl derivatives (1-3),17,18 fused aromatic rings (4-8), 19,20 porphyrin derivatives $(\mathbf{9}, \mathbf{1 0}), 21,22$ metal phthalocyanines $(\mathbf{1 1}),{ }^{23}$ fullerenes $(\mathbf{1 2}),{ }^{24}$ and some fluorescent dyes $(\mathbf{1 3}, \mathbf{1 4})$ have been made into nanoparticles. ${ }^{25,26}$ Their molecular structures are illustrated in Figure 1. Small molecules are more easily packed to form crystals than polymers, so in some cases the nanoparticles of small-molecule semiconductors can transform to nanorods, nanotubes and nanoflakes.
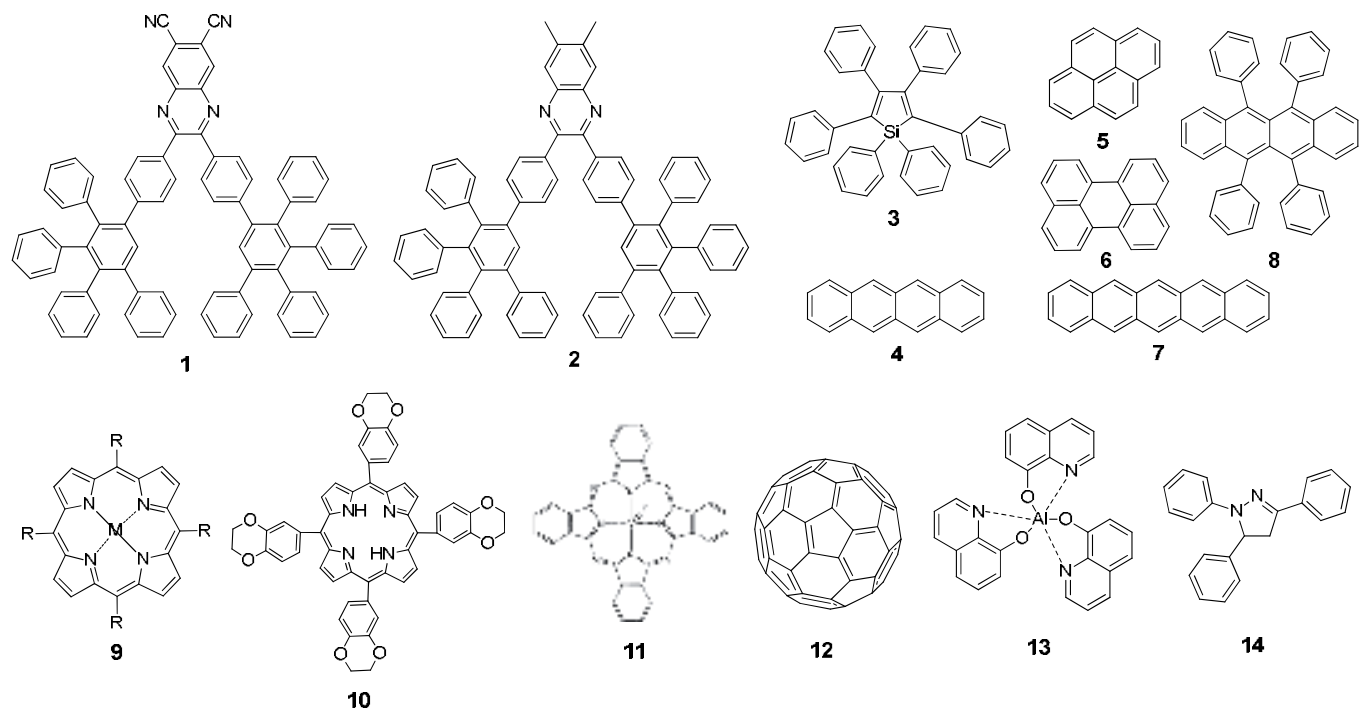

Fig. 1. Molecular structures of some small molecule semiconductors for synthesizing OSNs. 


\subsection{Polymer semiconductors}

Poly(phenylene vinylene)s (15), ${ }^{27}$ polyfluorenes $(\mathbf{1 6 - 1 8}),{ }^{28^{-30}}$ polythiophenes (19), ${ }^{31}$ laddertype poly(para-phenylene)s (LPPP) (20), ${ }^{32}$ poly(phenylene ethynylene)s (PPEs) $(\mathbf{2 1 , 2 2 ) , 3 3}$ polyanilines (PANIs) (23), ${ }^{34}$ and some copolymers $(\mathbf{2 4}, \mathbf{2 5})$ have been utilized to prepare nanoparticles. ${ }^{35,36}$ Their molecular structures are shown in Figure 2. Polymers have longer chains than small molecules, so it is possible for polymers to form nanoparticles even with a single molecule, which is advantageous for researches on single molecule behavior.

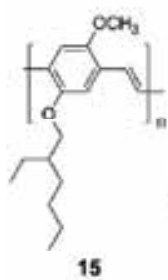

15

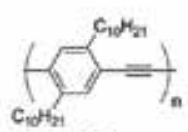

22

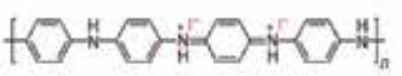

23

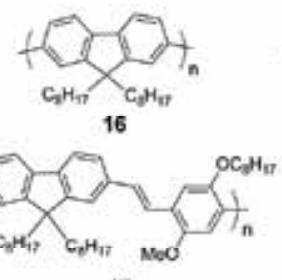

17

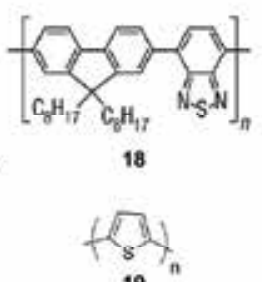

19

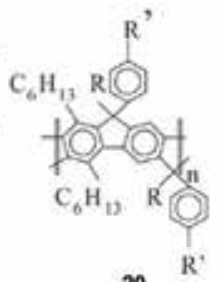

20

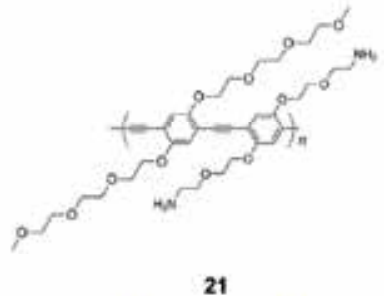

21

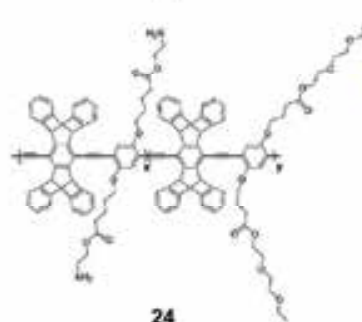

24

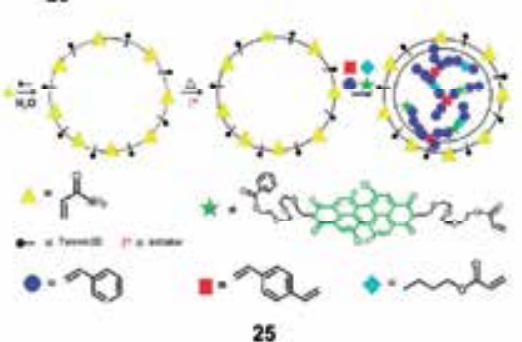

Fig. 2. Molecular structures of some polymer semiconductors for synthesizing OSNs.

\section{Methods for synthesizing organic semiconductor nanoparticles}

\subsection{Reprecipitation}

In 1992, Nakanishi and co-workers proposed the reprecipitation method and demonstrated the nanoparticles with the particle size less than $100 \mathrm{~nm}$ dispersed in water. ${ }^{37}$ Since then, this method has been widely used in nanoparticle preparation for various kinds of molecules. In this method, a hydrophobic organic semiconductor material is dissolved in a good solvent (e.g., THF) for it and poured into a poor solvent (e.g., water), which is miscible with the good solvent. The resulting mixture is stirred vigorously using a magnetic stir bar or a sonicator to assist the formation of nanoparticles. After the nanoparticle formation the organic solvent is removed either by partial vacuum evaporation or by repeated dialysis process to leave behind water-dispersible nanoparticles. The main driving force for the formation of nanoparticles is the hydrophobic effect. When the solution of an organic semiconductor material in organic solvent is added to water, the compound molecules tend to avoid contacting with water. Consequently, in order to achieve minimum exposure they fold or packed into spherical shapes. The preparation does not involve the use of any additives such as surfactants and can be applied to a wide variety of organic semiconductors including both polymers and small molecules given that they are soluble in organic solvents. Moreover, using this method, it is possible to tune the size of nanoparticles by adjusting the concentration and the temperature of the solutions. 


\subsection{Miniemulsion}

This is another commonly used method in the synthesis of OSNs. Using this method, Landfester and co-workers prepared nanoparticles from various polymers. ${ }^{38}$ To prepare OSNs, the compound is dissolved in a water immiscible organic solvent and then the resulting solution is injected into an aqueous solution of an appropriate surfactant. The mixture is stirred vigorously by ultrasonicating to form stable miniemulsions containing small droplets of the polymer solution. The organic solvent is then evaporated to obtain a stable dispersion of polymer nanoparticles in water. The size of nanoparticles could vary from $30 \mathrm{~nm}$ to $500 \mathrm{~nm}$ depending on the concentration of the polymer solution. However, the droplets could also be destabilized by Ostwald ripening as well as the flocculation caused by the coalescence of droplets. To prevent flocculation appropriate surfactants are needed, while Ostwald ripening can be suppressed by addition of a hydrophobic agent (hydrophobe) to the dispersed phase. The hydrophobic agent promotes the formation of an osmotic pressure inside the droplets that counteracts the Laplace pressure (the pressure difference between the inside and the outside of a droplet) preventing diffusion from one droplet to the surrounding aqueous medium.

\subsection{Pulsed-laser ablation}

In this method, OSNs are formed by pulsed-laser ablation of large, several-micrometersized, organic crystals suspended in a liquid. 39,40 The powder of organic semiconductors was added to an aqueous solution containing surfactants such as sodium dodecyl sulfate (SDS). Then, the suspension was sonicated for a while. The mixture was put into a quartz cuvette, stirred vigorously with a magnetic stirrer, and then simultaneously exposed to the second harmonic of a nanosecond YAG laser. The spot area was approximately tens of $\mathrm{mm}^{2}$, and the laser intensity was adjusted using a polarizer. The laser ablation mechanism for nanosecond laser ablation is based on photothermalization. The organic crystals in solutions absorb the laser light leading to a local increase in temperature and evaporation of a small amount of material from the crystal surface. The vaporized material is rapidly cooled by the surrounding liquid to form nanoparticles.

For nanosecond photothermal ablation in a solvent, rapid temperature elevation upon pulse excitation is compensated by a cooling process due to thermal diffusion to the solvent, and its balance gives the transient temperature determining the nanoparticle size. Higher fluence gives higher effective transient temperature, leading to efficient fragmentation to smaller particles. One advantage of the laser ablation method is its high controllability of size and phase of nanoparticles by tuning laser pulse width, wavelength, fluence, and shot number. However, this method is limited to fabricate OSNs based on small molecules only.

\subsection{Direct condensation of organic vapor}

Due to the fact that in the reprecipitation or miniemulsion process a solution of organic material (of typically about millimolar concentration) is added to a large excess of nonsolvent, only very dilute particle dispersions can be obtained. That is one main disadvantage of these methods. The second one is that the reprecipitation or miniemulsion method is not applicable for organic materials that are poorly soluble in organic solvents (such as pentacene). As for laser ablation, more concentrated nanoparticle dispersions can be 
prepared compared to the repreciptation and miniemulsion method. However, as particle formation only occurs within the narrow laser beam, only small amounts of these nanoparticle dispersions can be prepared. Furthermore, the intense laser light may cause severe photochemical damage especially in the case of rather sensitive organic materials.

To overcome the above mentioned drawbacks, an approach for preparation of concentrated dispersions of organic nanoparticles by direct condensation the vapor of an organic semiconductor material into a liquid dispersion medium has been developed. ${ }^{19}$ This approach combines elements from the physical vapor deposition (PVD) technique with cooling and condensation of the vapor directly inside a liquid. An illustration of the apparatus used in the direct condensation method is shown in Figure 3. The apparatus consists of four main parts: a tube furnace, a double-walled heated-vapor injection tube, a condensation and receiving vessel, and a vacuum pumping system. Temperatures in the different zones are adjusted according to the organic material to be evaporated, and were maintained such that no condensation of the organic materials occurred in the tubes. The evaporated organic material will be carried by the inert gas flow to the vapor-injection tube, which guides the organic material into a liquid condensation medium. The condensation liquid typically consists of an aqueous solution containing surfactants or polymeric stabilizers. It rapidly cools down the gas leading to condensation of the organic vapor and formation of nanoparticles. These nanoparticles are subsequently stabilized in situ by the surfactant or polymeric additive at the bubble/liquid interface to form a stable dispersion. The size of OSNs prepared by this method is in the range of 100-200 nm for fused aromatic hydrocarbons such as pentacene, rubrene, and tetracene.

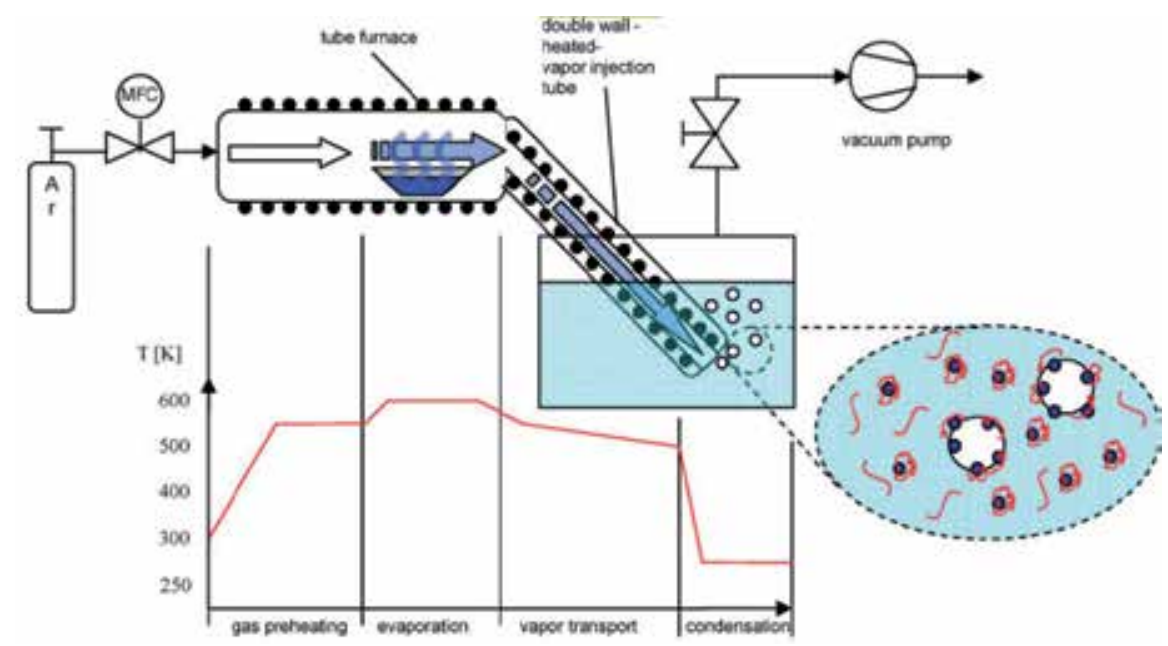

Fig. 3. Apparatus for direct condensation of organic vapor (Reproduced from Ref. 19, Copyright 2009 Wiley-VCH Verlag GmbH \& Co.)

\subsection{Template-based approaches}

\subsubsection{Soft templates}

Micelles can be used as soft templates to conduct the polymerization in the aqueous heterophase system. By dispersing the appropriate monomers, surfactant, solvent, and 
catalysts in an aqueous medium, the Glaser coupling reaction can be carried out exclusively within the hydrophobic interior of surfactant micelles to produce the poly(arylene diethynylenes) nanoparticles. ${ }^{41}$ Similarly, poly(p-phenyleneethynylene) nanoparticles can also be prepared by this method. ${ }^{42}$

The molecular structure of surfactant used in the aqueous heterophase system has a big influence on the shape of the formed nanoparticles. Using dodecylbenzene sulfonic acid as a surfactant and doping agent for poly(3,4-ethylenedioxythiophene) (PEDOT) yielded amorphous and polydisperse particles with diameters in the range of 35-100 nm.43 Short chain alcohol ethoxylate surfactants yielded more spherical particles, but significant amounts of surfactant residue were trapped on the PEDOT latex, and secondary nucleation could not be completely suppressed. ${ }^{44}$

These examples show that the soft template approach has been a versatile method for preparing conjugated polymer nanoparticles. However, control over important parameters such as particle diameter and polydispersity by this method is often not easy. Many of these issues can be addressed by the use of a hard template.

\subsubsection{Hard templates}

Due to the shape persistence of hard templates, they typically offer a more reliable way of directing the shape of conjugated polymer nanostructures. Monodisperse nanoparticles such as silica and polystyrene particles can be used as a hard template for preparing core-shell structures. Conjugated polymers such as polypyrrole, PANI and PEDOT, highly fluorescent polymers such as PPE have also been attached to the surface of colloidal particles. ${ }^{45}$ The conjugated polymers can either be polymerized in situ from monomers absorbed on the surface of the particle templates or be deposited from a layer-by-layer technique through electrostatic interactions. ${ }^{46}$

\section{Methods for preparing nanoparticles film}

Since OSNs are usually synthesized in solution with a low concentration, conventional thin film forming processes such as spin-coating or dip-casting are not appropriate for preparing OSN films. So other methods have been developed to prepare good OSN films.

\subsection{Electrophoresis deposition}

Electrophoretic deposition, which is based on the electrical collection of small, charged particles dispersed in dielectric liquids, is one of the most widely used coating methods capable of patterning. It has been reported that the phosphors for a cathode ray tube, ${ }^{47}$ the oxide superconductors, 48 and the carbon nanotubes for a cold cathode have been successfully coated by electrophoretic deposition. ${ }^{49}$ As for OSNs in solutions, they often carried charges on their surface. Such surface charges are generated according to Coehn's empirical rule. That is, the electrostatic charge separation may occur when two dielectrics are in intimate contact. The substance with the higher dielectric constant will receive the positive charge, while the other one will receive the negative charge. As illustrated in Figure 4, a DC voltage (usually hundreds of volts) was applied between two ITO-coated glass plates soaked in the nanoparticle suspension. Then nanoparticles will move towards the 
corresponding electrode under the driving of electric field force. ${ }^{50}$ The films thus obtained were washed with clean solvent and dried in air.

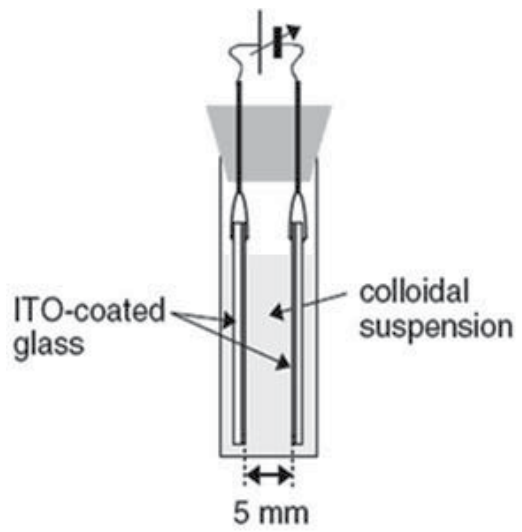

Fig. 4. Apparatus for electrophoretic deposition of OSNs

\subsection{Rapid expansion of supercritical solution (RESS) technique}

This technique for prepare OSN film is based on a rapid expansion process of supercritical solution (e.g. $\mathrm{CO}_{2}$ ) which contains dissolved organic semiconductors. ${ }^{51}$ By using an apparatus illustrated in Figure 5, organic semiconductors dispersed in supercritical $\mathrm{CO}^{2}$ solution can be sprayed on the substrate through a long stainless steel capillary tube attached to the chamber. After the rapid evaporation of $\mathrm{CO}_{2}, \mathrm{OSNs}$ are precipitated on the surface of substrates. Using process conditions of compressed-fluid precipitation and formulation, it appears possible to produce organic nanoparticles with tunable sizes and optical properties. This capability opens up avenues to create devices and functional films using organic nanoparticles as building blocks, which may be tailored for the application.

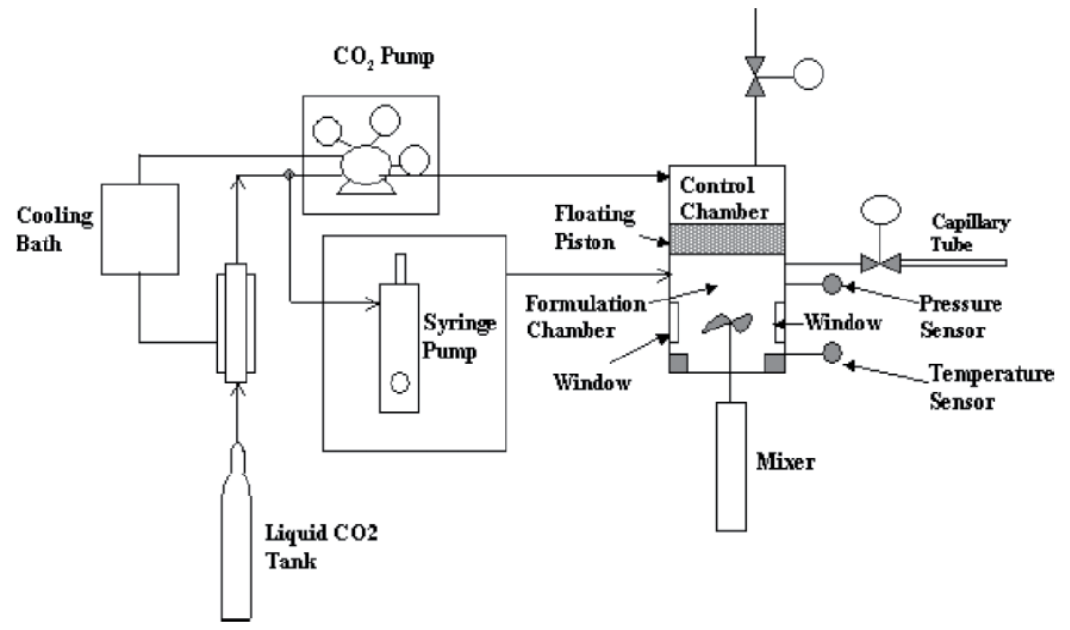

Fig. 5. Apparatus for preparation of OSN films by RESS technique (Reproduced from Ref. 51, Copyright 2006 Wiley-VCH Verlag GmbH \& Co.) 
Additionally, it is possible to mix building blocks of organic nanoparticles or combine different molecules within a building block. However, the limitation of this technique is that only small molecule based OSN film can be prepared while preparation of polymer based OSN films by this technique is not available. Also, surfactants such as the ammoniumexchanged Fluorolink $7004\left(\mathrm{Cl}\left(\mathrm{CF}_{2} \mathrm{CF}\left(\mathrm{CF}_{3}\right) \mathrm{O}\right)_{n} \mathrm{CF}_{2} \mathrm{COO}-\mathrm{NH}_{4}{ }^{+}\right)$need to be introduced into the supercritical solution to adjust the size of ultimate nanoparticles. ${ }^{51}$

\subsection{Solvent-evaporation induced self-assembly}

The evaporation behavior during the drying process of a solution plays a vital role in controlling the film morphology and the distribution of solute in the final films. It is well known that when a liquid drop containing dispersed solids evaporates on a surface, it commonly leaves a dense, ring-like deposit along the perimeter. The reason is that the contact line is pinned during the drying process, leading to a fixed contact area on the substrate. Therefore, a capillary flow of the solvent occurs from the center of the drop to the contact line to replenish the evaporation loss, and this flow transports the solutes to its periphery.52 As far as the OSNs solution is concerned, such phenomenon will result in an undesirably uneven distribution of nanoparticles across the deposited films. However, if another flow which has an opposite direction to the capillary flow is introduced into the OSNs solutions during the drying process, the transportation of nanoparticles towards the contact line by the capillary flow is expected to be counteracted. Marangoni effect is usually observed in a solution containing two kinds of solvents with different surface tensions and boiling points, and a flow is induced by the surface tension gradient existed in the solution caused by solvent evaporation. Such a flow is named as the Marangoni flow, and its direction can be controlled to be the same as the spreading of a drop on a solid surface (outward) or opposite to the spreading (inward), depending on the boiling points and surface tensions of the two solvents to be mixed. Consequently, by proper introduction of a second solvent into the solution, a Marangoni flow with an opposite direction to the capillary flow can be achieved.

The solvent-evaporation induced self-assembly method for preparing the thin nanoparticles films from their OSNs solutions is illustrated in Figure 6. ${ }^{53}$ By using ethylene glycol (EG) as the second solvent with a high boiling point but a low surface tension, the capillary flow in the solution can be counterbalanced by the Marangoni flow. The self-assembly of nanoparticles on the substrate can thus be achieved through the nanoparticle-substrate and nanoparticle-nanoparticle van der Waals interactions.

\subsection{Vapor-driven self-assembly}

The vapor-driven self-assembly process is based on the selective phase demixing and selfassembled aggregate formation. Such behaviors occur from a molecularly dispersed solid solution of specific fluorescent molecules in a polymer matrix when it is exposed to volatile organic solvent vapors. ${ }^{4}$ After solvent exposure, the supramolecular self-assembly of organic semiconductor materials leads to the formation of spherical nanoparticles (see Figure 7). The advantage of this method is to form nanoparticles films in situ on the substrate. Nevertheless, this kind of method is only appropriate for small-molecule 


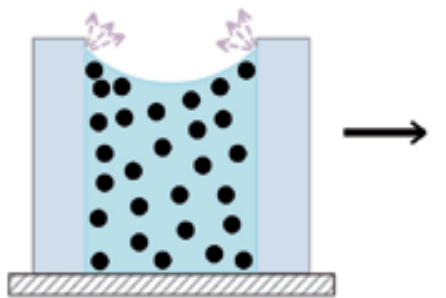

(a)

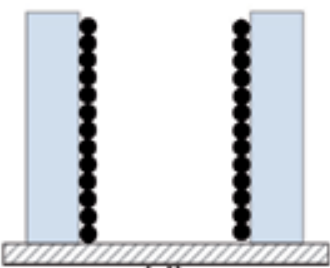

(d)

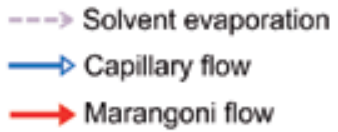

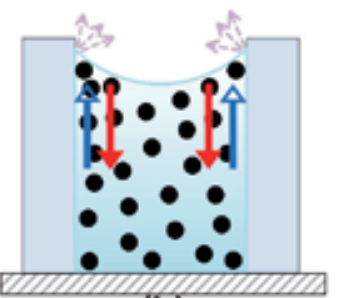

(b)
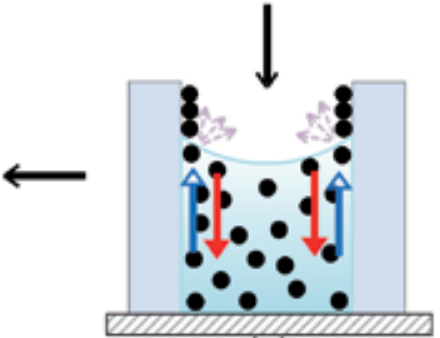

(c)

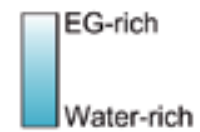

Fig. 6. Scheme for the solvent-evaporation-induced self-assembly of OSNs on the substrates to form films. (Reproduced from Ref. 53, Copyright 2010 The American Chemical Society)

compounds with certain structures and is not a universal method for most polymer semiconducting materials. In addition, OSNs formed by this method are discrete and continuous OSNs films can not be obtained.
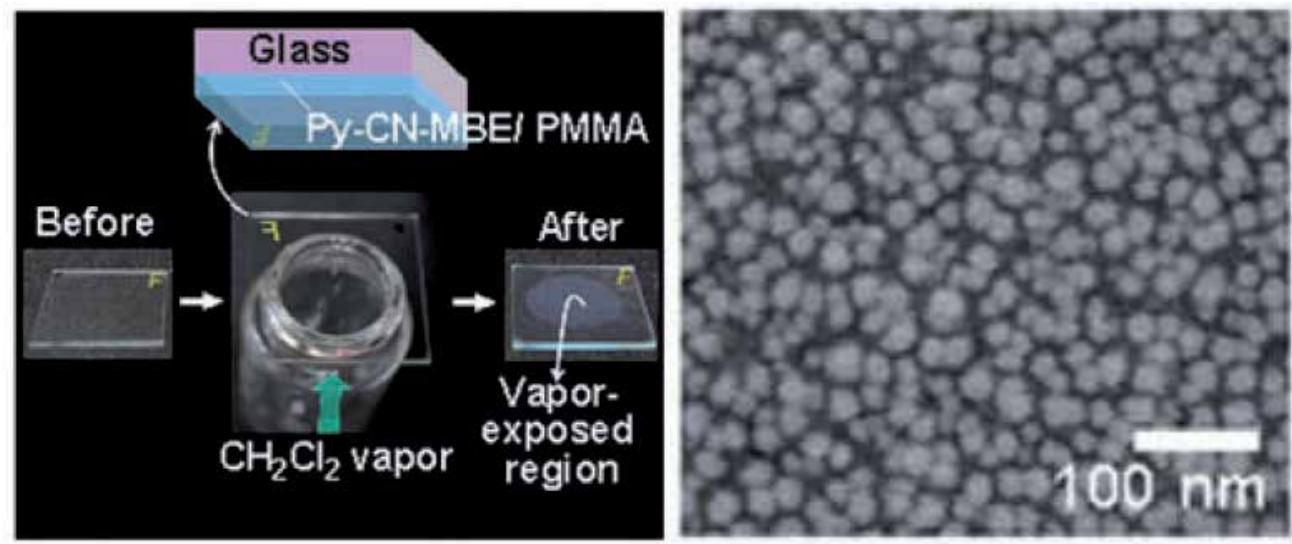

Fig. 7. Photograph of the 1-cyano-trans-1-(4'-methylbiphenyl)-2-[4'-(2'-pyridyl) phenyl]ethylene (Py-CN-MBE)/ poly(methyl methacrylate) (PMMA) film before and after exposure to dichloromethane vapor (Left panel) and the SEM image of the Py-CN-MBE nanoparticles formed by the vapor-driven self-assembly process (Right panel). (Reproduced from Ref. 54, Copyright 2007 Wiley-VCH Verlag GmbH \& Co.) 


\subsection{Inkjet printing}

As mentioned above, when a droplet of OSN solution is dripped on the surface of a substrate, the OSNs tend to form coffee-stains after the evaporation of the solvent. So direct inkjet printing of OSN solutions can not provide good film morphology. To avoid this drawback, an aqueous dispersion of semiconducting polymer nanospheres is deposited by inkjet printing onto a polymer surface patterned by soft embossing. ${ }^{55}$ By interaction between the spheres and the undulated surface a self assembly process is triggered, resulting in the formation of OSN nanostructures determined by the template.
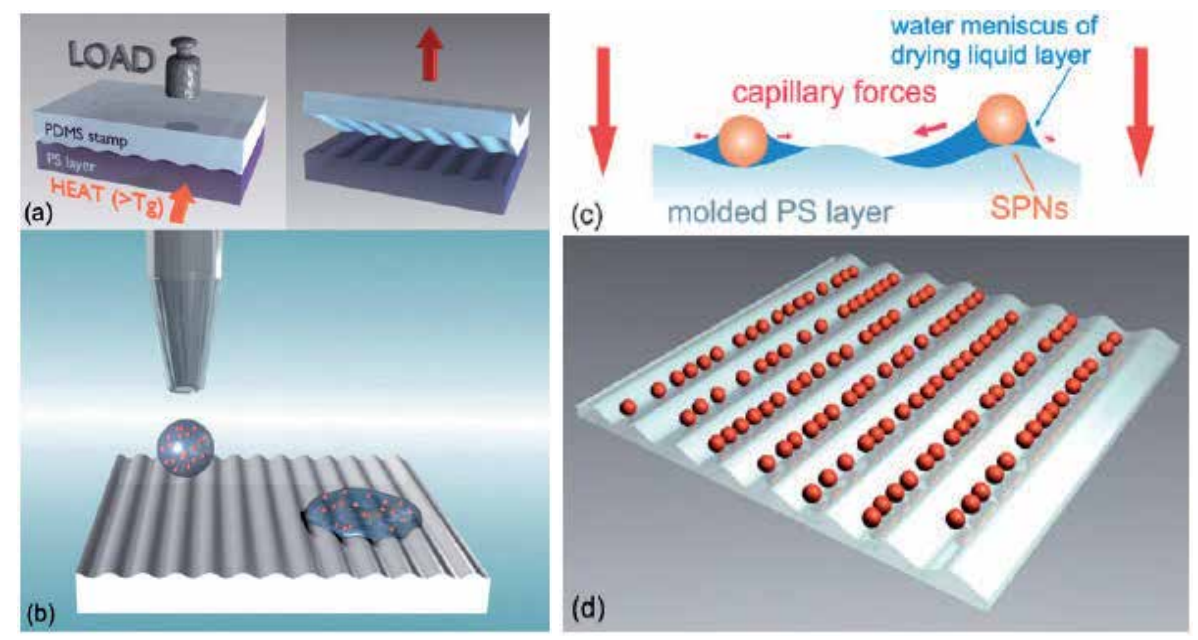

Fig. 8. The fabrication process for functional nanostructures from inkjet printing.

Reprodcued from Ref. 55 (Copyright 2008 The Royal Society of Chemistry).

As shown in Figure 8, after a droplet of the OSN solution was printed on the surface of the structured polymeric template layer, OSNs assemble in the grooves of the embossed surface. This method relies on the application of a polymer template layer, so that the patterned structure that is formed with the OSNs can be incorporated into a device.

\subsection{Spin-coating}

Due to the very low concentration of OSN solutions, good film can hardly be formed by spin-coating or dip-coating method without any additives. As a result, auxiliary underlayers or additives such as surfactants or polymer matrix have to be introduced to assist the deposition of nanoparticle films. As mentioned above, nanoparticles usually carry charges on their surfaces when they are dispersed in solutions. Therefore, negatively charged nanoparticles can be formed on polycationic films with the help of electrostatic interactions via spin-coating and vice versa. Layers of LPPP nanoparticles were spin-coated on poly(allylamine hydrochloride) (PAH) can exhibit a homogeneous fluorescence over large areas. ${ }^{38}$ Similarly, conjugated polymer nanoparticles such as polyfluorene derivatives and LPPP spin-coated on poly(3,4-ethylenedioxythiophene):poly(4-styrenesulfonate) (PEDOT:PSS) film can also exhibit a good film morphology. ${ }^{32,56}$ Besides auxiliary underlayers, polymer matrix can also been utilized to act as a binder to improve the film quality deposited from OSN solutions. This kind of binder for the nanoparticles can also assist in the reduction of electric field 
singularities around the particles that may result in regions of pinhole formation in electronic devices. Poly(vinyl alcohol), ${ }^{57}$ hexadecyl-modified poly(ethylene oxide) (PEO),, 5 and PEDOT:PSS $58^{-60}$ are polymer matrixes used for this purpose. These binders have virtually no effect on the color characteristics of the electroluminescence spectrum since PVA, PEO, and PEDOT:PSS have a negligible absorption in the luminance regime of OSNs. Although by this means the film quality is improved, the additives remained in the nanoparticles films will be disadvantages to the optical and electrical properties of OSNs. For example, when using PEDOT:PSS as an additive to the OSNs aqueous solution for preparing thin nanoparticles films by spin-coating, the acidity of PEDOT:PSS will deteriorate the luminescent properties of the conjugated compounds largely.

\section{Applications of OSN film in optical and electronic devices}

\subsection{Organic light-emitting diodes}

LPPP, poly(9-vinylcarbazole) (PVK), 2-(4-tert-butylphenyl)-5-(4-biphenylyl)-1,3,4-oxadiazole (tBu-PBD), coumarins, nile red, nanoparticles prepared by miniemulsion method, core-shell nanoparticles with perylene as the core and poly[methyl methacrylate-co-vinylcarbazole-co2-(3'-nitrophenyl)-5-(4'-acryloylphenyl)-1,3,4-oxadiazole] as the shell formed by emulsion copolymerization, poly(3-octadecylthiophene) nanoparticles prepared by reprecipitation method, multi-component nanoparticles prepared by RESS process or miniemulsion method have been reported to serve as an active layer in organic light-emitting diodes.61 For nanoparticles synthesized by both miniemulsion and RESS methods, surfactants, stabilizing agents or hydrophobes are necessary and can hardly be removed. Such additives will be disadvantageous to the native optoelectronic properties of OSNs in devices. It would be still interesting to fabricate optoelectronic devices from OSNs prepared via the reprecipitation method in which there would be no additives including surfactants, stabilizing agents and hydrophobes. Electrophoretic deposition of OSN films from reprecipitation-processed nanoparticle solutions has been employed in fabricating OLEDs. ${ }^{62}$ Although an electroluminescent emission from the device could be observed, the emission is not uniform because the nanoporosity of the OSN film prepared by electrophoretic deposition probably causes fatal pin-holes. As a result, an approach for preparing high-quality OSN films from reprecipitation-processed nanoparticle solutions is highly desired. Fortunately, the solventevaporation induced self-assembly method introduced above can meet this requirement. Currently, the main drawback of this method for fabricating OLEDs is that the solvent evaporation period is time-consuming. If there are some ways are found to overcome this drawback, this method is very promising for fabricating OSN based OLEDs.

\subsection{Organic field-effect transistors}

Organic field-effect transistors (OFETs) fabricated using solution-deposition techqiques are particularly well-suited for large-area electronic devices. For meaningful practical applications, the organic semiconductors need to provide FET mobilities close to that of amorphous silicon. This will necessitate establishment of proper molecular order in the semiconductors to achieve high mobilities, since charge-carrier transport in organic semiconductors is dominated by hopping and disordered materials are not efficient chargetransporting media. In nanoparticles, molecules are closely packed and they are usually 
highly ordered. For example, both poy(3-hexylthiophene) (P3HT) and poly(9,9'dioctylfluorene) (PFO) nanoparticles can exhibit highly ordered structures and can be distinguished from the UV absorption spectrum with occurrence of a new peak.31,63 Poly(3,3"'-dialkylquarterthiophene)s (PQTs) nanoparticles have been explored for using in OFETs. ${ }^{64}$ As expected, the presence of lamellar $\pi$-stacking order in the nanoparticles can be verified by XRD and UV spectrum data. OFETs based on PQT nanoparticles show a $50 \%$ improvement in mobility on bare $\mathrm{SiO}_{2}$ dielectric layer and an order of magnitude improvement in mobility on surface modified $\mathrm{SiO}_{2}$ dielectric layer relative to those based on normal films.

\subsection{Organic solar cells}

It is well known that excitons formed in the active layer of organic solar cells usually have a migration distance less than $20 \mathrm{~nm} .65$ So in organic solar cells, the distance for excitons diffused to the interface of electron donors and acceptors should be smaller than $20 \mathrm{~nm}$ to ensure good light conversion efficiency. However, because the entropy of mixing is generally low for polymers, solid polymer blends tend to phase-separate at the macroscopic scale. Moreover, when a thin layer of immiscible polymers is deposited from solution, the resulting morphology strongly depends on various parameters, such as the individual solubility of the polymers in the solvent used, the interaction with the substrate surface, the layer thickness and the method of deposition, drying and annealing. Therefore, controlling of the lengths of phase separation in thin layers is important for organic solar cells to avoid large-scale phase separation. Kietzke et al. have reported that by using the blend of poly $(9,9-$ dioctylfluorene-co-benzothiadiazole) (F8BT) and poly(9,9-dioctylfluorene-co-N,N-bis(4butylphenyl)-N,Ndiphenyl-1,4-phenylenediamine) (PFB) nanoparticles as the active layer the phase separation in organic solar cells can be controlled on the nanoscale..$^{29,56}$

\subsection{Photonic crystals}

If a photonic crystal is constructed from a material with sufficiently high refractive index, it can exhibit a photonic bandgap, a frequency range in which the mode density is zero and photons cannot propagate in any direction. Although this property is desirable since it would allow the inhibition of spontaneous emission and the ability to manipulate the flow of light, it is difficult to be achieved with organic semiconductors due to their relatively low refractive index. Instead, an organic photonic crystal would more likely have a partial bandgap, a frequency range where light can propagate in a limited number of directions. However, even in this case, the mode density for forbidden directions can be strongly modified by the photonic crystal. For frequencies just outside the partial bandgap, the mode density along these directions can be higher than in free space. This increase indicates that more optical modes are available to interact with electronic excitations. Thus, by combining organic semiconductors with photonic crystals, this enhanced interaction with light can be used to further improve optoelectronic properties.

One of the simplest ways of preparing photonic crystals is by the self assembly of monodisperse spheres, for instance, by the self-assembly of colloidal silica or polystyrene microspheres widely reported in the literatures. These spheres can then act as secondary 
templates for the fabrication of conjugated polymer inverse photonic crystals, where the interstitial voids of the sphere template have been filled with conjugated polymer. This approach has been successfully used in the preparation of poly(p-phenylenevinylene) (PPV) inverse photonic crystal films. 66

\section{Applications of OSN film in chemo-/biosensors}

Although lots of applications of organic nanoparticles in chemo-/biosensors have been explored in recent year, most of them are carried out in solutions. Compared with solutions, solid-state samples can be more convenient for storage and transport which are highly desired for off-site laboratory analysis. Here we will introduce some applications of OSN film in chemical and biosensors.

\subsection{Chemical sensors}

Hydroxyl radical is one of the most important reactive oxygen species, which is recognized to play an important role in physiological and pathological processes of the organisms. In addition, hydroxyl radical is also involved in many chemical, environmental, and pharmaceutical processes such as semiconductor photocatalysis in aqueous solution, wastewater treatment, and tumor cell killing. By using a binary nanoparticle system combining PFO nanoparticles and MEH-PPV nanoparticles, a linear relationship between the concentration of hydroxyl radical and the intensity ratio (Band I to Band III) of PFO nanoparticles can be found in the deposited nanoparticle film. ${ }^{67}$ The synergy between MEHPPV NPs and PFO NPs are crucial to the response of free radicals in this kind of binary NP system. When exposed to free radicals, MEH-PPV NPs undergo molecular structure changes in the outer shell. As a result, a broad-sense polarity vector across the whole NP pointing from the weak-polarity core to the strong-polarity shell is established. Such a polarity vector will influence on the vibronic coupling among different electronic states of PFO molecules when the core-shell MEH-PPV NPs are adjacent to PFO NPs, which will change the relative PL emission intensity between bands I and III of PFO.

\subsection{Biosensors}

In general, conducting polymer nanoparticles are dispersed on the surface of the electrode to increase the area/volume ratio and to favor the adsorption of bio-molecules. By this means, uniform electrostatic adsorption of protein was enabled, thereby exhibiting higher signal-to-background ratios and shorter response times than electrochemically prepared films. ${ }^{68}$ Taking advantage of conducting polymer nanoparticles, sufficient amounts of enzyme were firmly immobilized during the fabrication of a phosphate biosensor. The response time of the biosensors was about $6 \mathrm{~s}$. A linear response was observed between $1.0 \mu \mathrm{M}$ and $100 \mu \mathrm{M}$ and the detection limit was determined to be about $0.3 \mu \mathrm{M} .69$ Besides, an ascorbic acid sensor has been fabricated via the drop-casting of PANI nanoparticles onto a screen-printed carbon-paste electrode. ${ }^{70}$ The PANI nanoparticles not only enhanced the catalytic reaction, but also allowed the detection of ascorbate at the reduced applied potential of $0 \mathrm{~V}$ and operation at neutral $\mathrm{pH}$, avoiding the problem of sample interference. 


\section{Conclusions and prospects}

Most of the organic semiconductors including both small molecules and conjugated polymers can be utilized for preparing OSNs, thus ensuring a very wide material selectivity for their applications. Various approaches have been reported for synthesizing OSNs. Besides reprecipitation method, additives such as surfactants are usually employed in other methods, which may be disadvantageous to the optical and electronic properties of OSNs. When depositing the OSN solution onto the substrate to form nanoparticle films, auxiliary underlayer and binders such as polymer matrix or surfactants are often used to improve the film quality. An exception is the vapor-driven self-assembly and solvent-evaporation induced self-assembly methods, which can prepare clean nanoparticle films and is highly desired for the optical and electronic applications.

Although OSNs have been proved to be effective building blocks in both optoelectronic devices and chemo/biosensors, a number of challenges and avenues of exploration remain. The interface between nanoparticles and the surroundings is crucial to its optical, electrical, and catalytic properties. So surface modification of OSNs can not only improve their contact properties but also endow them with a new function. However, the surface modification of OSNs is seldom reported yet.

The field of photonic crystals has recently provided a number of novel insights into the manipulation of light. These photonic properties have yet to be fully combined with the optoelectronic properties of OSNs, and the development of this area remains a very active area of research. Additionally, the ability to precisely control the morphology and alignment of OSNs is of importance to all fields of organic electronics.

In the field of electronic devices, OSN based OLEDs have been widely explored. However, OSN based OFETs still need to be paid more attention. By appropriate design, OFETs using OSNs as an active layer may be served as multifunctional optoelectronic devices.

With the great advantages of OSNs, they are believed to play an important role in more and more application fields and will provide new scientific insights in the coming years.

\section{References}

[1] J. Janata, M. Josowicz, Nat. Mater. 2003, 2, 19-24.

[2] J. E. Anthony, A. Facchetti, M. Heeney, S. R. Marder, X. Zhan, Adv. Mater. 2010, 22, 38763892.

[3] T. Sagawa, S. Yoshikawa, H. Imahori, J. Phys. Chem. Lett. 2010, 1, 1020-1025.

[4] L. Xia, Z. Wei, M. Wan, J. Colloid Interface Sci. 2010, 341, 1-11.

[5] X. Feng, F. Lv, L. Liu, H. Tang, C. Xing, Q. Yang, S. Wang, ACS Appl. Mater. Interfaces 2010, 2, 2429-2435.

[6] B. -K. An, S.-K. Kwon, S.-D. Jung, S. Y. Park, J. Am. Chem. Soc. 2002, 124, 14410-14415.

[7] J. Chen, C. C. W. Law, J. W. Y. Lam, Y. Dong, S. M. F. Lo, I. D. Williams, D. Zhu, B. Tang, Chem. Mater. 2003, 15, 1535-1546.

[8] J. N. Clifford, T. D. M. Bell, P. Tinnefeld, M. Heilemann, S. M. Melnikov, J. Hotta, M. Sliwa, P. Dedecker, M. Sauer, J. Hofkens, E. K. L. Yeow, J. Phys. Chem. B 2007, 111, 6987-6991. 
[9] Y. Zhou, G. Bian, L. Wang, L. Dong, L. Wang, J. Kan, Spectrochim. Acta, Part A 2005, 61, 1841-1845.

[10] X. Xu, S. Chen, L. Li, G. Yu, C. Di, Y. Liu, J. Mater. Chem. 2008, 18, 2555-2561.

[11] L. Wang, L. Dong, G. Bian, L. Wang, T. Xia, H. Chen, Anal. Bioanal. Chem. 2005, 382, 1300-1303.

[12] H. Y. Kim, T. G. Bjorklund, S.-H. Lim, C. J. Bardeen, Langmuir 2003, 19, 3941-3946.

[13] J. Jang, J. Ha, J. Cho, Adv. Mater. 2007, 19, 1772-1775.

[14] T. Matsuya, K. Otake, S. Tashiro, N. Hoshino, M. Katada, T. Okuyama, Anal. Bioanal. Chem. 2006, 385, 797-806.

[15] S.-J. Lim, B.-K. An, S. D. Jung, M.-A. Chung, S. Y. Park, Angew. Chem. Int. Ed. 2004, 43, 6346-6350.

[16] F. Wang, M.-Y. Han, K. Y. Mya, Y. Wang, Y.-H. Lai, J. Am. Chem. Soc. 2005, 127, 1035010355.

[17] X. Xu, S. Chen, L. Li, G. Yu, C. Di, Y. Liu, J. Mater. Chem. 2008, 18, 2555-2561.

[18] Y. Liu, Y. Tang, N. N. Barashkov, I. S. Irgibaeva, J. W. Y. Lam, R. Hu, D. Birimzhanova, Y. Yu, B. Z. Tang, J. Am. Chem. Soc. 2010, 132, 13951-13953.

[19] S. Köstler, A. Rudorfer, A. Haase, V. Satzinger, G. Jakopic, V. Ribitsch, Adv. Mater. 2009, 21, 2505-2510.

[20] E. H. Cho, M. S. Kim, D. H. Park, H. Jung, J. Bang, J. Kim, J. Joo, Adv. Funct. Mater. 2011, 21, 3056-3063.

[21] X. Gong, T. Milic, C. Xu, J. D. Batteas, C. M. Drain, J. Am. Chem. Soc. 2002, 124, 14290 14291.

[22] C. Huang, Y. Li, Y. Song, Y. Li, H. Liu, D. Zhu, Adv. Mater. 2010, 22, 3532-3536.

[23] X. Zhang, Y. Wang, Y. Ma, Y. Ye, Y. Wang, K. Wu, Langmuir 2006, 22, 344-348.

[24] K. L. Chen, M. Elimelech, Langmuir 2006, 22, 10994-11001.

[25] A. M. Collins, S. N. Olof, J. M. Mitchels, S. Mann, J. Mater. Chem. 2009, 19, 3950-3954.

[26] Y. S. Zhao, H. Fu, A. Peng, Y. Ma, Q. Liao, J. Yao, Acc. Chem. Res. 2010, 43, 409-418.

[27] C. Szymanski, C. Wu, J. Hooper, M. A. Salazar, A. Perdomo, A. Dukes, J. McNeill, J. Phys. Chem. B 2005, 109, 8543-8546.

[28] C. Wu, C. Szymanski, Z. Cain and J. McNeill, J. Am. Chem. Soc. 2007, 129, 12904-12905.

[29] T. Kietzke, D. Neher, K. Landfester, R. Montenegro, R. Güntner, U. Scherf, Nat. Mater. 2003, 2, 408-412.

[30] Y.-L. Chang, R. E. Palacios, F.-R. F. Fan, A. J. Bard, P. F. Barbara, J. Am. Chem. Soc. 2008, 130, 8906-8907.

[31] J. E. Millstone, D. F. J. Kavulak, C. H. Woo, T. W. Holcombe, E. J. Westling, A. L. Briseno, M. F. Toney, J. M. J. Fréchet, Langmuir 2010, 26, 13056-13061.

[32] T. Piok, S. Gamerith, C. Gadermaier, H. Plank, F. P. Wenzl, S. Patil, R. Montenegro, T. Kietzke, D. Neher, U. Scherf, et al. Adv. Mater. 2003, 15, 800-804.

[33] N. A. A. Rahim, W. McDaniel, K. Bardon, S. Srinivasan, V. Vickerman, P. T. C. So, J. H. Moon, Adv. Mater. 2009, 21, 3492-3496.

[34] J. Yang, J. Choi, D. Bang, E. Kim, E.-K. Lim, H. Park, J.-S. Suh, K. Lee, K.-H. Yoo, E.-K. Kim, Y.-M. Huh, S. Haam, Angew. Chem. Int. Ed. 2011, 50, 441-444.

[35] J. H. Moon, R. Deans, E. Krueger and L. F. Hancock, Chem. Commun. 2003, 104-105.

[36] Z. Tian, A. D. Shallerz, A. D. Q. Li, Chem. Commun. 2009, 180-182.

[37] H. Kasai, H. S. Nalwa, H. Oikawa, S. Okada, H. Matsuda, N. Minami, A. Kakuta, K. Ono, A. Mukoh, H. Nakanishi, Jpn. J. Appl. Phys. 1992, 31, L1132-L1134. 
[38] K. Landfester, R. Montenegro, U. Scherf, R. Güntner, U. Asawapirom, S. Patil, D. Neher, T. Kietzke, Adv. Mater. 2002, 14, 651-655.

[39] T. Asahi, T. Sugiyama, H. Masuhara, Acc. Chem. Res. 2008, 41, 1790-1798.

[40] R. Yasukuni, T. Asahi, T. Sugiyama, H. Masuhara, M. Sliwa, J. Hofkens, F.C. De Schryver, M. Van der Auweraer, A. Herrmann, K. Müllen, Appl. Phys. A 2008, 93, 5-9.

[41] M. C. Baier, J. Huber, S. Mecking, J. Am. Chem. Soc. 2009, 131, 14267-14273.

[42] E. Hittinger, A. Kokil, C. Weder, Angew. Chem. Int. Ed. 2004, 43, 1808-1811.

[43] J. W. Choi, M. G. Han, S. Y. Kim, S. G. Oh, S. S. Im, Synth. Met. 2004, 141, 293-299.

[44] A. M. J. Henderson, J. M. Saunders, J. Mrkic, P. Kent, J. Gore, B. R. Saunders, J. Mater. Chem. 2001, 11, 3037-3042.

[45] T. L. Kelly, M. O. Wolf, Chem. Soc. Rev. 2010, 39, 1526-1535.

[46] J. H. Wosnick, J. H. Liao, T. M. Swager, Macromolecules 2005, 38, 9287-9290.

[47] P. F. Grosso, R. E. Rutherford, D. E. Sargent, Jr. J. Electrochem. Soc. 1970, 117, 1456-1459.

[48] C. T. Chu, B. Dunn, Appl. Phys. Lett. 1989, 55, 492-494.

[49] B. Gao, G. Z. Yue, Q. Qiu, Y. Cheng, H. Shimoda, L. Fleming, O. Zhou, Adv. Mater. 2001, 13, 1770-1773.

[50] K. Tada, M. Onoda, Adv. Funct. Mater. 2002, 12, 420-424.

[51] R. Jagannathan, G. Irvin, T. Blanton, S. Jagannathan, Adv. Funct. Mater. 2006, 16, 747753.

[52] R. D. Deegan, O. Bakajin, T. F. Dupont, G. Huber, S. R. Nagel, T. A. Witten, Nature 1997, 389, 827-829.

[53] C. Zheng, X. Xu, F. He, L. Li, B. Wu, G. Yu, Y. Liu, Langmuir 2010, 26, 16730-16736.

[54] B.-K. An, S.-K. Kwon, S. Y. Park, Angew. Chem. Int. Ed. 2007, 46, 1978 -1982.

[55] E. Fisslthaler, A. Blümel, K. Landfester, U. Scherfd, E. J. W. List, Soft Matter 2008, 4, 2448-2453.

[56] T. Kietzke, D. Neher, M. Kumke, R. Montenegro, K. Landfester, U. Scherf, Macromolecules 2004, 37, 4882-4890.

[57] J.-S. Heo, N.-H. Park, J.-H. Ryu, K.-D. Suh, Adv. Mater. 2005, 17, 822-826.

[58] C. F. Huebner, J. B. Carroll, D. D. Evanoff, Jr., Y. Ying, B. J. Stevenson,J. R. Lawrence, J. M. Houchins, A. L. Foguth, J. Sperry, S. H. Foulger, J. Mater. Chem. 2008, 18, 4942-4948.

[59] C. F. Huebner, S. H. Foulger, Langmuir 2010, 26, 2945-2950.

[60] C. F. Huebner, R. D. Roeder, S. H. Foulger, Adv. Funct. Mater. 2009, 19, 3604-3609.

[61] D. Tuncel, H. V. Demirb, Nanoscale, 2010, 2, 484-494.

[62] K. Tada, M. Onoda, Thin Solid Films 2003, 438, 365-368.

[63] S. Moynihan, D. Iacopino, D. O'Carroll, P. Lovera, G. Redmond, Chem. Mater. 2008, 20, 996-1003.

[64] B. S. Ong, Y. Wu, P. Liu, S. Gardner, Adv. Mater. 2005, 17, 1141-1144.

[65] H. Hoppe, N. S. Sariciftci, Adv. Polym. Sci. 2008, 214, 1-86.

[66] M. Deutsch, Yu. A. Vlasov, D. J. Norris, Adv. Mater. 2000, 12, 1176-1180.

[67] J. Wang, X. Xu, Y. Zhao, C. Zheng, L. Li, J. Mater. Chem. 2011, 21, 18696-18703

[68] A. Morrin, O. Ngamna, A. J. Killard, S. E. Moulton, M. R. Smyth, G. G. Wallace, Electroanalysis 2005, 17, 423-430.

[69] M. A. Rahman, D. S. Park, S. C. Chang, C. J. McNeil, Y. B. Shim, Biosens. Bioelectron. 2006, 21, 1116-1124.

[70] A. Ambrosi, A. Morrin, M.R. Smyth, A.J. Killard, Anal. Chim. Acta 2008, 609, 37-43. 

